2003 PACIFIC NORTHWEST

LOADS AND RESOURCES STUDY

Technical Appendix

Volume 1

Energy Analysis

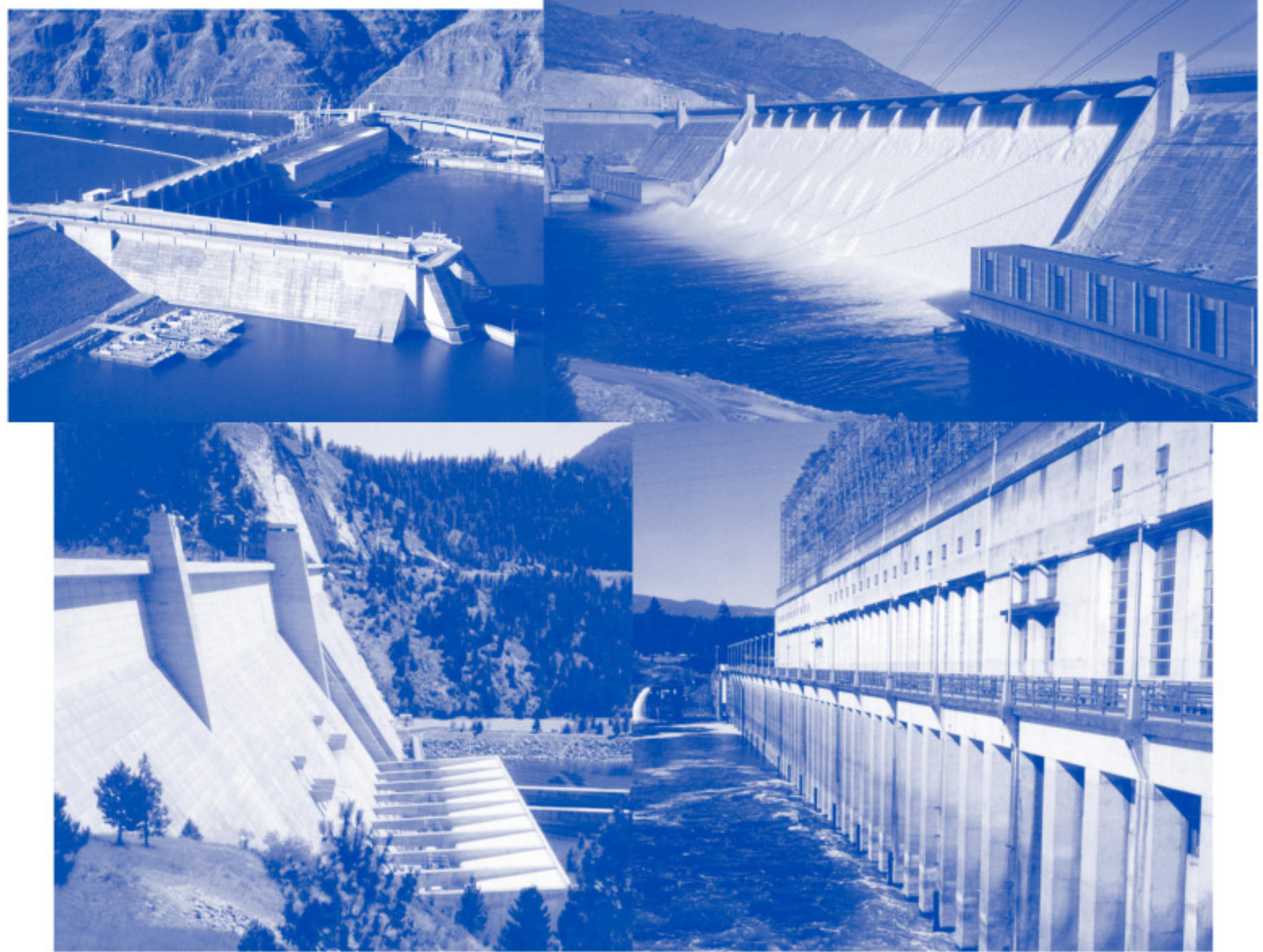




\title{
2003 PACIFIC NORTHWEST LOADS AND RESOURCES STUDY
}

\section{THE WHITE BOOK}

\section{TECHNICAL APPENDIX}

VOLUME 1

\author{
ENERGY ANALYSIS
}




\section{Cover Photo Montage:}

(Top left clockwise)

Photographs provided by John Hyde P.E. Hydraulic Engineer, BPA, Regional Coordination \& Operations Planning Group

Lower Granite Dam is located at the head of Lake Bryan in southeastern Washington. Lower Granite dam is owned and operated by the U.S. Corps of Engineers. Construction for this project began in July 1964, and was completed in 1984. The installed capacity of Lower Granite dam is $810 \mathrm{MW}$.

Grand Coulee Dam spans the Columbia River and is 90 miles west of Spokane, Washington. The U.S. Bureau of Reclamation began operating this storage project in 1933. Grand Coulee dam has an installed capacity of $6,465 \mathrm{MW}$.

Bonneville Second Power House is part of Bonneville Dam and is located on the Washington side of the Columbia River. The U.S. Army Corps of Engineers began operating this unit in 1982. The installed capacity of the Second Power House is 532 MW. Total capacity of Bonneville Dam is 1,093 MW.

Libby Dam is located on the Kootenai River in northwestern Montana. Its reservoir, Koocanusa, extends 42 miles into British Columbia, Canada. The Columbia River Treaty allowed the construction of Libby dam, which was completion in 1972 . The installed capacity is $525 \mathrm{MW}$. 


\section{ACKNOWLEDGMENTS}

Preparation of the annual Pacific Northwest loads and resources study is a complex, multidisciplinary effort. BPA wishes to acknowledge the team-BPA staff and otherswhose diligence and dedication result in a reliable, high quality document.

\section{Bonneville Power Administration}

Generation Supply: Regional Coordination Group

Office of General Counsel

\section{Pacific Northwest Utilities Conference Committee Northwest Power \& Conservation Council}

Reports Powered by:

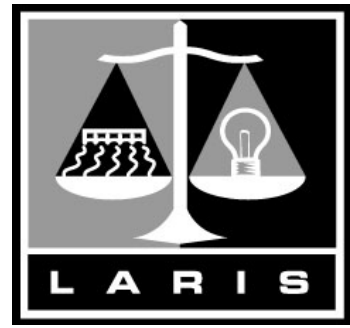

Loads and Resources Information System 


\section{Pacific Northwest Loads and Resources Study \\ Technical Appendix Volume 1}

\section{TABLE OF CONTENTS}

\section{Annual Energy Analysis}

\section{SECTION 1: Annual Federal Loads and Resources Table, Average Energy}

SECTION 2: Annual Regional Loads and Resources Table, Average Energy

SECTION 3: Annual A-Tables, Average Energy

Total Retail Loads

Exports

Regulated Hydro

Independent Hydro

Imports

Small Thermal \& Miscellaneous

Combustion Turbines

Renewable Resources

Cogeneration

Large Thermal

Restoration

Canadian Entitlement Return For CSPE

Columbia Storage Power Exchange Purchase (CSPE)

Supplemental \& Entitlement Capacity

Canadian Entitlement Return For Canada

Intra Regional Transfers

Capacity Reserves

Wells \& Chelan Allocation

Rocky Reach \& Rock Island Allocation

Wanapum \& Priest Rapids Allocation

Swift \#1 \& Swift \#2 Allocation

BPA Power Sales Contracts

Non-Utility Generating Resources

Regional Non-Utility Generating Resources By Project

Dedicated Resources

Other Dedicated Resources

Table A-1

Table A-2

Table A-3

Table A-4

Table A-5

Table A-6

Table A-7

Table A-8

Table A-9

Table A-10

Table A-11

Table A-12

Table A-13

Table A-14

Table A-15

Table A-16

Table A-17

Table A-18

Table A-19

Table A-20

Table A-21

Table A-22

Table A-23

Table A-24

Table A-25

Table A-26 
Monthly Energy for OY 2005-2014

SECTION 4: Monthly Federal Loads and Resources Table, Average Energy OY 2005-2014

SECTION 5: Monthly Regional Loads and Resources Table, Average Energy OY 2005-2014

SECTION 6: Monthly Federal 50 Water Year Table, Average Energy OY 2005-2014

SECTION 7: Monthly Regional 50 Water Year Table, Average Energy OY 2005-2014

SECTION 8: Monthly A-Tables, Average Energy

Total Retail Loads

Exports

Regulated Hydro

Independent Hydro

Imports

Small Thermal \& Miscellaneous

Combustion Turbines

Renewable Resources

Cogeneration

Large Thermal

Restoration

Canadian Entitlement Return For CSPE

Columbia Storage Power Exchange Purchase (CSPE)

Supplemental \& Entitlement Capacity

Canadian Entitlement Return For Canada

Intra Regional Transfers

Capacity Reserves

Wells \& Chelan Allocation

Rocky Reach \& Rock Island Allocation

Wanapum \& Priest Rapids Allocation

Swift \#1 \& Swift \#2 Allocation

BPA Power Sales Contracts

Non-Utility Generating Resources

Regional Non-Utility Generating Resources By Project

Dedicated Resources

Other Dedicated Resources

Table A-1

Table A-2

Table A-3

Table A-4

Table A-5

Table A-6

Table A-7

Table A-8

Table A-9

Table A-10

Table A-11

Table A-12

Table A-13

Table A-14

Table A-15

Table A-16

Table A-17

Table A-18

Table A-19

Table A-20

Table A-21

Table A-22

Table A-23

Table A-24

Table A-25

Table A-26 
Section 1

Annual Federal Table

Loads \& Resources

Average Energy in Megawatts 
Loads and Resources - Federal System

PNW Loads and Resources Study

2005 - 2014 Operating Years

1937 Water Year

2003 White Book

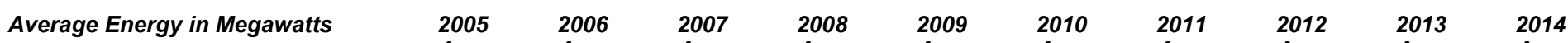

Avg. Avg. Avg. Avg. Avg. Avg. Avg. Avg. Avg. Avg.

Non-Utility Obligations

Federal Agencies 1981 PSC Sale

USBR 1981 PSC Sale

DSI 1981 PSC Sale

Federal Agencies 2002 PSC Sale

USBR 2002 PSC Sale

DSI 2002 PSC Sale

$\begin{array}{rrrrrrrrrr}0 & 0 & 0 & 0 & 0 & 0 & 0 & 0 & 0 & 0 \\ 0 & 0 & 0 & 0 & 0 & 0 & 0 & 0 & 0 & 0 \\ 0 & 0 & 0 & 0 & 0 & 0 & 0 & 0 & 0 & 0 \\ 119 & 119 & 120 & 120 & 121 & 121 & 122 & 119 & 118 & 118 \\ 149 & 149 & 149 & 149 & 149 & 149 & 149 & 149 & 149 & 149 \\ 267 & 271 & 45 & 0 & 0 & 0 & 0 & 0 & 0 & 0 \\ & & & & & & & & & \\ & 535 & 539 & 314 & 270 & 270 & 271 & 271 & 268 & 268\end{array}$

Total Firm Non-Utility Obligations

535

539

$\begin{array}{rrrrr}0 & 0 & 0 & 0 & 0 \\ 0 & 0 & 0 & 0 & 0 \\ 0 & 0 & 0 & 0 & 0 \\ 2716 & 2755 & 2917 & 2974 & 3009 \\ 2142 & 2126 & 2367 & 2431 & 2443 \\ 607 & 621 & 614 & 627 & 618 \\ 1011 & 1034 & 1022 & 1044 & 1030 \\ 382 & 382 & 64 & 0 & 0 \\ 1007 & 968 & 783 & 697 & 633 \\ 512 & 413 & 380 & 370 & 370 \\ 0 & 0 & 0 & 0 & 0 \\ 0 & 0 & 0 & 0 & 0 \\ 0 & 0 & 0 & 0 & 0\end{array}$

Transfers Out

NGP 1981 PSC Sale

GPU 1981 PSC Sale

IOU 1981 PSC Sale

NGP 2002 PSC Sale

GPU 2002 PSC Sale

NGP Slice Sale

GPU Slice Sale

IOU 2002 PSC Sale

Exports

Intra-Regional Transfers (Out)

Supp \& Entitlement Capacity (Out)

CSPE To West Group Utilities

Federal Diversity

\begin{tabular}{|c|c|c|c|c|c|c|c|c|c|c|}
\hline Total Transfers Out & 8376 & 8300 & 8146 & 8143 & 8103 & 8252 & 8261 & 8168 & 8172 & 8227 \\
\hline Total Firm Obligations & 8911 & 8839 & 8460 & 8413 & 8373 & 8523 & 8532 & 8437 & 8439 & 8494 \\
\hline \multicolumn{11}{|l|}{ Hydro Resources } \\
\hline Regulated Hydro & 6446 & 6464 & 6492 & 6527 & 6545 & 6562 & 6577 & 6589 & 6603 & 6614 \\
\hline Independent Hydro & 397 & 397 & 398 & 398 & 398 & 398 & 398 & 398 & 398 & 398 \\
\hline Sustained Peaking Adjustment & 0 & 0 & 0 & 0 & 0 & 0 & 0 & 0 & 0 & 0 \\
\hline Non-Fed CER (CSPE) & 0 & 0 & 0 & 0 & 0 & 0 & 0 & 0 & 0 & 0 \\
\hline Non-Fed CER (Canada) & 142 & 139 & 134 & 131 & 130 & 133 & 139 & 137 & 135 & 133 \\
\hline Restoration & 0 & 0 & 0 & 0 & 0 & 0 & 0 & 0 & 0 & 0 \\
\hline
\end{tabular}

Total Hydro Resources

6985

$7000 \quad 702$

7056

$7072 \quad 7092$

$\begin{array}{rr}0 & \\ 0 & \\ 0 & \\ 3044 & 3078 \\ 2454 & 2463 \\ 630 & 621 \\ 1049 & 1034 \\ 0 & \\ 705 & 6 \\ 370 & 370 \\ 0 & \\ 0 & \\ 0 & \end{array}$

$\begin{array}{rr}0 & \\ 0 & \\ 0 & \\ 3078 & 3103 \\ 2463 & 2494 \\ 621 & 632 \\ 1034 & 1053 \\ 0 & \\ 694 & 68 \\ 370 & 199\end{array}$

$\begin{array}{rrr}0 & 0 & 0 \\ 0 & 0 & 0 \\ 0 & 0 & 0 \\ 3103 & 3137 & 3169\end{array}$

$2494 \quad 2511 \quad 2518$

$623 \quad 634$

$\begin{array}{rrr}053 & 1038 & 1056 \\ 0 & 0 & 0\end{array}$

$686 \quad 679 \quad 672$

$199 \quad 184 \quad 178$

Other Resources

Small Thermal \& Misc

Combustion Turbines

Renewables

Cogeneration

Imports

Intra-Regional Transfers (In)

Supp \& Entitlement Capacity (In)

Large Thermal

Non-Utility Generation

Resource Acquisition

$\begin{array}{rrrrrr}0 & 0 & 0 & 0 & 0 & \\ 0 & 0 & 0 & 0 & 0 & \\ 29 & 29 & 29 & 29 & 29 & 29 \\ 0 & 0 & 0 & 0 & 0 & \\ 256 & 246 & 226 & 199 & 184 & 183 \\ 1367 & 1210 & 510 & 276 & 276 & 270 \\ 0 & 0 & 0 & 0 & 0 & \\ 861 & 1000 & 877 & 1000 & 877 & 1000 \\ 92 & 92 & 110 & 119 & 119 & 119 \\ 0 & 0 & 0 & 0 & 0 & \end{array}$

$\begin{array}{rr}0 & \\ 0 & \\ 29 & 29 \\ 0 & \\ 183 & 175 \\ 276 & 276 \\ 0 & \\ 1000 & 877 \\ 119 & 119 \\ 0 & \end{array}$

$\begin{array}{rr}0 & 0 \\ 0 & 0 \\ 29 & 29 \\ 0 & 0 \\ 175 & 175 \\ 276 & 105 \\ 0 & 0 \\ 877 & 1000 \\ 119 & 119 \\ 0 & 0\end{array}$

$\begin{array}{rr}0 & \\ 0 & \\ 29 & 29 \\ 0 & \\ 175 & 175 \\ 105 & 90 \\ 0 & \\ 1000 & 877 \\ 119 & 119 \\ 0 & \end{array}$

$\begin{array}{rr}0 & 0 \\ 0 & 0 \\ 29 & 29 \\ 0 & 0 \\ 175 & 175 \\ 90 & 90 \\ 0 & 0 \\ 877 & 1000 \\ 119 & 119 \\ 0 & 0\end{array}$

Total Other Resources

2605

$2577 \quad 1751$

1622

$1485 \quad 1607$

1476

$1428 \quad 1289 \quad 1413$

Total Resources

9590

9578

8775

8678

8557

8699

8589

8551

8425

8558

Reserves \& Maintenance

Hydro Reserves

Small Thermal \& Misc. Reserves

Contract Reserves

Large Thermal Reserves

Federal Hydro Maintenance

Spinning Reserves

Federal Transmission Losses

$\begin{array}{lll}0 & 0 & 0 \\ 0 & 0 & 0\end{array}$

Total Reserves, Maintenance \& Losses

$\begin{array}{llll}-270 & -270 & -247 & -245\end{array}$

0
0
0

$\begin{array}{rr}0 & 0 \\ 0 & 0 \\ 0 & 0 \\ 0 & 0 \\ 0 & 0 \\ 0 & 0 \\ -241 & -245\end{array}$

$\begin{array}{rr}0 & 0 \\ 0 & 0 \\ 0 & 0 \\ 0 & 0 \\ 0 & 0 \\ 0 & 0 \\ -245 & -242\end{array}$

$\begin{array}{rrrr}0 & 0 & 0 & 0 \\ 0 & 0 & 0 & 0 \\ 0 & 0 & 0 & 0 \\ 0 & 0 & 0 & 0 \\ 0 & 0 & 0 & 0 \\ 0 & 0 & 0 & 0 \\ -242 & -241 & -238 & -241\end{array}$

Total Net Resources

$9320 \quad 9307 \quad 8527$

8433

$-241 \quad-245$

$-242$

$-241-238$

$-241$

67

$20-58$

8454

8347

$\begin{array}{lll}8310 & 8187 & 8317\end{array}$ 


\section{Section 2 \\ Annual Regional Table \\ Loads \& Resources \\ Average Energy in Megawatts}


Loads and Resources - Pacific Northwest Region

PNW Loads and Resources Study

2005 - 2014 Operating Years

1937 Water Year

2003 White Book

2005

2006

2007

2008

2009

2010

2011

2012

20132014 Avg. Avg. Avg. Avg. Avg.

Avg. Avg.

Avg.

Avg.

Firm Regional Loads

Regional Firm Loads

Exports

Federal Diversity

$\begin{array}{rrr}19666 & 19957 & 20575 \\ 1468 & 1307 & 1077 \\ 0 & 0 & 0\end{array}$

20914

21246

21556
909
0

21864

22160

22537

22849

21135

$21264 \quad 21652$

22465

22762

$23049 \quad 23400 \quad 23698$

Non-Firm Regional Loads

Regional Non-Firm Loads

$\begin{array}{llllllllll}0 & 0 & 0 & 0 & 0 & 0 & 0 & 0 & 0 & 0\end{array}$

Total Non-Firm Regional Loads

0

Total Loads

21135

21264

22086

22465

22762

23049

23400

23698

Hydro Resources

Regulated Hydro

Independent Hydro

Sustained Peaking Adjustment

\begin{tabular}{|c|c|c|c|c|c|c|c|c|c|}
\hline 10605 & 10622 & 10650 & 10686 & 10703 & 10721 & 10736 & 10748 & 10762 & 10773 \\
\hline 1084 & 1084 & 1085 & 1085 & 1085 & 1085 & 1085 & 1085 & 1085 & 10 \\
\hline 0 & 0 & 0 & 0 & 0 & 0 & 0 & 0 & 0 & \\
\hline
\end{tabular}

Total Hydro Resources

11688

11706

11735

11770

11788

11805

11821

11832

11846

11858

Other Resources

Small Thermal \& Misc.

Combustion Turbines

Renewables

Cogeneration

Imports

Large Thermal

Non-Utility Generation

$\begin{array}{rr}17 & 17 \\ 1946 & 1976 \\ 80 & 80 \\ 1999 & 1999 \\ 603 & 597 \\ 5930 & 6034 \\ 1296 & 1292 \\ 0 & 0\end{array}$

$\begin{array}{rrr}17 & 17 & 17 \\ 1967 & 1937 & 1941 \\ 80 & 80 & 80 \\ 1999 & 1999 & 1999 \\ 514 & 484 & 288 \\ 5766 & 6026 & 5873 \\ 1329 & 1337 & 1337 \\ 0 & 0 & 0\end{array}$

$\begin{array}{rr}17 & 17 \\ 1941 & 1921 \\ 80 & 80 \\ 1999 & 1999 \\ 288 & 280 \\ 5873 & 5973 \\ 1337 & 1338 \\ 0 & 0\end{array}$

$\begin{array}{rr}17 & 17 \\ 1921 & 1914 \\ 80 & 80 \\ 1999 & 1999 \\ 280 & 226 \\ 5973 & 5878 \\ 1338 & 1340 \\ 0 & \end{array}$

$\begin{array}{rr}17 & 17 \\ 1914 & 1923 \\ 80 & 80 \\ 1999 & 1999 \\ 226 & 226 \\ 5878 & 6009 \\ 1340 & 1340 \\ 0 & 0\end{array}$

$\begin{array}{rrr}17 & 17 & 17 \\ 1923 & 1918 & 1922 \\ 80 & 80 & 80 \\ 1999 & 1999 & 1999 \\ 226 & 223 & 223 \\ 6009 & 5910 & 6047 \\ 1340 & 1341 & 1341 \\ 0 & 0 & 0\end{array}$

Total Other Resources

$11870 \quad 1199$

$11672 \quad 11880$

$1880 \quad 11535 \quad 1160$

\begin{tabular}{lllll}
11607 & 11454 & 11596 & 11488 & 11629 \\
\hline 23412 & 23275 & 23428 & 23335 & 23487
\end{tabular}

Reserves \& Maintenance

Hydro Reserves

Small Thermal \& Misc. Reserves

Contract Reserves

Large Thermal Reserves

Regional Hydro Maintenance

Spinning Reserves

Regional Transmission Losses

23559

$23702 \quad 23407$

$23650 \quad 23323$

$\begin{array}{rrr}0 & 0 & \\ 0 & 0 & \\ 0 & 0 & \\ 0 & 0 & \\ -12 & -12 & -12 \\ 0 & 0 & \\ -664 & -668 & -660\end{array}$

$\begin{array}{rr}0 & \\ 0 & \\ 0 & \\ 0 & \\ -12 & 0 \\ 0 & \\ -660 & -12\end{array}$

0

Total Reserves, Maintenance \& Losses

$-676 \quad-680 \quad-672$

$-672-679$

0
0
0
0
-12
0
-657

0
0
0
0
-12
0
-660

0

$$
\begin{aligned}
& 0 \\
& 0 \\
& 0
\end{aligned}
$$

$-12$

Total Net Resources

22883

$23022 \quad 22735$

$-669 \quad-672$

$-672-668$

$-656$

$\begin{array}{lll}0 & 0 & 0 \\ 0 & 0\end{array}$

Surplus/Deficits

Firm Surplus/Defici

\begin{tabular}{llllllllll}
1748 & 1758 & 1083 & 1112 & 567 & 275 & -155 & -294 & -735 & -885 \\
\hline 1748 & 1758 & 1083 & 1112 & 567 & 275 & -155 & -294 & -735 & -885
\end{tabular}




\section{Section 3 \\ Annual OY 2005 - 2014 \\ Average Energy in Megawatts}

$\underline{\text { Table Number }}$

Total Retail Loads

A-1

Exports

A-2

Regulated Hydro Projects

A-3

Independent Hydro Projects

A-4

Imports

A-5

Small Thermal \& Miscellaneous

A-6

Combustion Turbines

A-7

Renewable Resources

A-8

Cogeneration

A-9

Large Thermal

A-10

Restoration

A-11

Canadian Entitlement Return For CSPE

A-12

Columbia Storage Power Exchange Purchase (CSPE)

A-13

Supplemental \& Entitlement Capacity

A-14

Canadian Entitlement Return For Canada

A-15

Intra-Regional Transfers

A-16

Capacity Reserves

A-17

Wells \& Chelan Allocation

A-18

Rocky Reach \& Rock Island Allocation

A-19

Wanapum \& Priest Rapids Allocation

A-20

Swift \#1 \& Swift \#2 Allocation

A-21

BPA Power Sales Contracts

A-22

Non Utility Generating Resources

Regional Non Utility Generating Resources By Project

A-23

Dedicated Resources

A-24

Other Dedicated Resources

A-25

A-26 
Table A-1: Pacific Northwest Total Retail Loads

PNW Loads and Resources Study
2005 - 2014 Operating Years

\section{White Book}

Avg. Avg. Avg.

2010

2011

2012

2013

2014

Avg. Avg. Avg. Avg. Avg.

Firm Loads

-Federal Entities-

1 Federal Entities

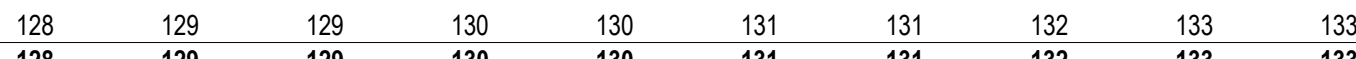

2 Total Federal Entities

128

129

$130 \quad 130$

131

131

$132 \quad 133 \quad 133$

U.S. Bureau of Reclamatiom-

3 U.S. Bureau of Reclamation

\begin{tabular}{llllllllll}
149 & 149 & 149 & 149 & 149 & 149 & 149 & 149 & 149 & 149 \\
\hline 149 & 149 & 149 & 149 & 149 & 149 & 149 & 149 & 149 & 149
\end{tabular}

4 Total U.S. Bureau of Reclamation

149

-Direct Service Industry-

5 Direct Sevice Industry

\begin{tabular}{llllllllll}
292 & 296 & 611 & 674 & 674 & 674 & 674 & 674 & 674 & 674 \\
\hline 292 & 296 & 611 & 674 & 674 & 674 & 674 & 674 & 674 & 674
\end{tabular}

6 Total Direct Service Industry

296

-Generating Public Entities-

7 Generating Public Entities

\begin{tabular}{llllllllll}
4866 & 4936 & 5029 & 5104 & 5195 & 5268 & 5336 & 5431 & 5525 & 5592 \\
\hline 4866 & 4936 & 5029 & 5104 & 5195 & 5268 & 5336 & 5431 & 5525 & 5592
\end{tabular}

8 Total Generating Public Entities

$4866 \quad 4936$

$5029 \quad 5104 \quad 5195$

$5268 \quad 5336$

$5431 \quad 5525 \quad 5592$

-Non-Generating Public Entities-

9 Non-Generating Public Entities

\begin{tabular}{llllllllll}
3414 & 3462 & 3506 & 3541 & 3596 & 3646 & 3693 & 3721 & 3773 & 3819 \\
\hline 3414 & 3462 & 3506 & 3541 & 3596 & 3646 & 3693 & 3721 & 3773 & 3819
\end{tabular}

10 Total Non-Generating Public Entities

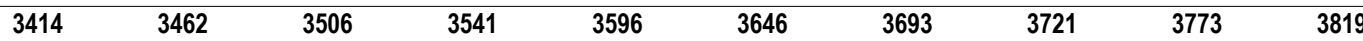

-Investor-Owned Entities-

11 Investor-Owned Entities

12 Total Investor-Owned Entities

10636

$\begin{array}{llll}10636 & 10805 & 10970 & 11136 \\ 10636 & 10805 & 10970 & 11136\end{array}$

$\begin{array}{lllllll}1136 & 11322 & 11508 & 11700 & 11872 & 12103 & 12318 \\ 11136 & 11322 & 11508 & 11700 & 11872 & 12103 & 12318\end{array}$

-Other Firm Loads-

13 Other PNW Entities

$180 \quad 180$

14 Total Other Firm Loads

$180 \quad 180$

$180 \quad 180$

- Total Regional Firm Loads-

15 Federal Entities

16 Direct Service Industry

17 Public Entities

18 Investor-Owned Entities

19 Other Entities

20 Total Regional Firm Loads

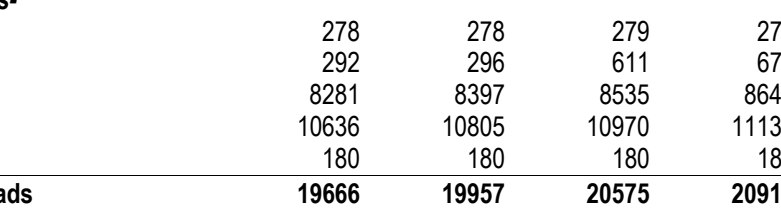

\begin{tabular}{rr}
180 & 180 \\
\hline 180 & 180 \\
& \\
279 & 280 \\
674 & 674 \\
645 & 879 \\
136 & 1132 \\
180 & 180 \\
\hline 914 & 2124
\end{tabular}

$\begin{array}{llllllllll}0 & 0 & 0 & 0 & 0 & 0 & 0 & 0 & 0 & 0 \\ 0 & 0 & 0 & 0 & 0 & 0 & 0 & 0 & 0 & 0\end{array}$

Non-Firm Loads

-Investor-Owned Entities-

21 Investor-Owned Entities

22 Total Investor-Owned Entities

- Total Regional Non-Firm Loads-

23 Federal Entities

24 Direct Service Industry

25 Public Entities

26 Investor-Owned Entities

27 Total Regional Non-Firm Loads

- Total Regional Medium Loads-

28 Federal Entities

29 Direct Service Industry

30 Public Entities

31 Investor-Owned Entities

32 Other Entities

33 Total Regional Medium Loads

\begin{tabular}{llll} 
& 0 & 0 & 0 \\
& 0 & 0 & 0 \\
& 0 & 0 & 0 \\
0 & 0 & 0 \\
\hline 0 & 0 & 0 & 0
\end{tabular}

0
0
0
0
0

$\begin{array}{ll}0 & 0 \\ 0 & 0 \\ 0 & 0 \\ 0 & 0 \\ 0 & 0\end{array}$

$\begin{array}{llll}0 & 0 & 0 & 0 \\ 0 & 0 & 0 & 0 \\ 0 & 0 & 0 & 0 \\ 0 & 0 & 0 & 0 \\ 0 & 0 & 0 & 0\end{array}$

\begin{tabular}{rrrrrrrrrr}
278 & 278 & 279 & 279 & 280 & 280 & 281 & 281 & 282 & 283 \\
292 & 296 & 611 & 674 & 674 & 674 & 674 & 674 & 674 & 674 \\
8281 & 8397 & 8535 & 8645 & 8791 & 8914 & 9028 & 9152 & 9298 & 9410 \\
10636 & 10805 & 10970 & 11136 & 11322 & 11508 & 11700 & 11872 & 12103 & 12318 \\
180 & 180 & 180 & 180 & 180 & 180 & 180 & 180 & 180 & 165 \\
\hline 19666 & 19957 & $\mathbf{2 0 5 7 5}$ & $\mathbf{2 0 9 1 4}$ & $\mathbf{2 1 2 4 6}$ & $\mathbf{2 1 5 5 6}$ & $\mathbf{2 1 8 6 4}$ & $\mathbf{2 2 1 6 0}$ & $\mathbf{2 2 5 3 7}$ & $\mathbf{2 2 8 4 9}$
\end{tabular}


Table A-2: Regional Exports

PNW Loads and Resources Study

2005 - 2014 Operating Years 2003 White Book

East of Continental Divide-

$\begin{array}{llllllllll}2005 & 2006 & 2007 & 2008 & 2009 & 2010 & 2011 & 2012 & 2013 & 2014 \\ \text { Avg. } & \text { Avg. } & \text { Avg. } & \text { Avg. } & \text { Avg. } & \text { Avg. } & \text { Avg. } & \text { Avg. } & \text { Avg. } & \text { Avg. }\end{array}$

-Exports East of Continental Divide-

2 BPA To Other Entities

3 Total Exports To ECD

\begin{tabular}{|c|c|c|c|c|c|c|c|}
\hline 9 & 9 & 9 & 2.3 & 0 & 0 & 0 & 0 \\
\hline 81 & 84 & 88 & 91 & 95 & 99 & 102 & 107 \\
\hline 90 & 93 & 97 & 93 & 95 & 99 & 102 & 107 \\
\hline
\end{tabular}

-Exports to Pacific Southwest-

4 BPA To ANHM PwrX

5 BPA To BART PwrS

6 BPA To BURB C/N/X

7 BPA To GLEN C/N/X

8 BPA To MSR PwrS

9 BPA To Other Entities

10 BPA TO PASA C/N/X

11 BPA To PASA C/N/X

12 BPA TO PASA S/N/X

13 BPA To RVSD CapS

14 BPA To RVSD C/N/X

15 BPA To RVSD C/N/X

16 BPA To RVSD S/Pwr/X

17 IPC To CLTN PwrS

18 PGE To GLEN PwrS

19 PGE To GLEN S/Pwr/X

20 PPL To CDWR PwrS

21 PPL To SCE PwrS

22 PPL To SMUD PwrS

23 PSE To PGS S/Pwr/X

24 SCL To NCPA S/Pwr/X

25 SDGE To SDGE PwrS

26 SHPD To SMUD PwrS

27 TID To TID PwrS

28 BPA NW-SW Intertie Losses

29 Total Exports To PSW

\begin{tabular}{|c|c|c|c|c|c|c|c|c|c|}
\hline 3.3 & 0 & 0 & 0 & 0 & 0 & 0 & 0 & 0 & 0 \\
\hline 40 & 37 & 0 & 0 & 0 & 0 & 0 & 0 & 0 & 0 \\
\hline 3.8 & 3.8 & 3.8 & 2.5 & 0 & 0 & 0 & 0 & 0 & 0 \\
\hline 4.2 & 4.2 & 4.2 & 2.7 & 0 & 0 & 0 & 0 & 0 & 0 \\
\hline 75 & 15 & 0 & 0 & 0 & 0 & 0 & 0 & 0 & 0 \\
\hline 177 & 204 & 118 & 42 & 0 & 0 & 0 & 0 & 0 & 0 \\
\hline 2.5 & 2.5 & 2.5 & 1.6 & 0 & 0 & 0 & 0 & 0 & 0 \\
\hline 1.1 & 1.1 & 1.1 & 1.1 & 1.1 & 1.1 & 1.1 & 1.1 & 1.1 & 1.1 \\
\hline 0.4 & 0.4 & 0.4 & 0.4 & 0.4 & 0.4 & 0.4 & 0.4 & 0.4 & 0.4 \\
\hline 3.0 & 3.0 & 3.0 & 3.0 & 3.0 & 3.0 & 0 & 0 & 0 & 0 \\
\hline 2.8 & 2.8 & 2.8 & 2.8 & 2.8 & 1.7 & 0 & 0 & 0 & 0 \\
\hline 4.9 & 4.9 & 4.9 & 4.9 & 4.9 & 4.9 & 4.9 & 4.9 & 4.9 & 4.9 \\
\hline 4.3 & 4.3 & 4.3 & 4.3 & 4.3 & 4.3 & 4.3 & 4.3 & 4.3 & 4.3 \\
\hline 2.6 & 0 & 0 & 0 & 0 & 0 & 0 & 0 & 0 & 0 \\
\hline 20 & 20 & 20 & 20 & 20 & 20 & 20 & 20 & 3.3 & 0 \\
\hline 3.0 & 3.0 & 3.0 & 3.0 & 3.0 & 3.0 & 3.0 & 1.5 & 0 & 0 \\
\hline 30 & 0 & 0 & 0 & 0 & 0 & 0 & 0 & 0 & 0 \\
\hline 54 & 54 & 9 & 0 & 0 & 0 & 0 & 0 & 0 & 0 \\
\hline 40 & 40 & 40 & 40 & 40 & 40 & 40 & 40 & 40 & 40 \\
\hline 43 & 43 & 43 & 28 & 0 & 0 & 0 & 0 & 0 & 0 \\
\hline 10 & 10 & 10 & 10 & 10 & 10 & 10 & 10 & 10 & 5.3 \\
\hline 57 & 57 & 57 & 57 & 57 & 57 & 57 & 57 & 57 & 57 \\
\hline 29 & 29 & 29 & 6 & 0 & 0 & 0 & 0 & 0 & 0 \\
\hline 39 & 39 & 39 & 39 & 39 & 39 & 39 & 39 & 39 & 39 \\
\hline 9.7 & 8.5 & 4.4 & 1.9 & 0.5 & 0.5 & 0.3 & 0.3 & 0.3 & 0.3 \\
\hline 660 & 586 & 399 & 270 & 186 & 185 & 180 & 178 & 160 & 152 \\
\hline
\end{tabular}

-Exports to Inland Southwest-

30 BPA To SPP PwrS

31 LVFBR To WAPA PwrS

32 PPL To WAPA PwrS

\begin{tabular}{rrrrrrrrrr}
60 & 60 & 60 & 60 & 60 & 60 & 60 & 60 & 60 & 60 \\
36 & 36 & 36 & 36 & 36 & 36 & 36 & 36 & 36 & 36 \\
89 & 0 & 0 & 0 & 0 & 0 & 0 & 0 & 0 & 0 \\
\hline 185 & 96 & 96 & 96 & 96 & 96 & 96 & 96 & 96 & 96
\end{tabular}

-Exports to Canada-

34 BPA To BCHP CanEnt

35 Total Exports To Canada

-Exports to Other Entities-

36 Total Exports To Other Entities

537

$535-488 \quad 483$

465

$534 \quad 524$

\begin{tabular}{llll}
524 & 513 & 501 & 490 \\
\hline 524 & $\mathbf{5 1 3}$ & $\mathbf{5 0 1}$ & $\mathbf{4 9 0}$
\end{tabular}

-Total Exports-

37 Federal Entities

38 Public Entities

39 Investor-Owned Entities

40 Other Entities

41 Total Exports.

$\begin{array}{llllllllll}0 & 0 & 0 & 0 & 0 & 0 & 0 & 0 & 0 & 0\end{array}$

\begin{tabular}{lrrrrrrrrrr} 
& 1007 & 968 & 783 & 697 & 633 & 705 & 694 & 686 & 679 & 672 \\
& 78 & 78 & 78 & 55 & 49 & 49 & 49 & 49 & 49 & 44 \\
& 347 & 226 & 181 & 157 & 122 & 120 & 120 & 118 & 100 & 97 \\
& 36 & 36 & 36 & 36 & 36 & 36 & 36 & 36 & 36 & 36 \\
\hline & $\mathbf{1 4 6 8}$ & $\mathbf{1 3 0 7}$ & $\mathbf{1 0 7 7}$ & $\mathbf{9 4 5}$ & $\mathbf{8 4 0}$ & $\mathbf{9 0 9}$ & $\mathbf{8 9 8}$ & $\mathbf{8 9 0}$ & $\mathbf{8 6 4}$ & $\mathbf{8 4 8}$
\end{tabular}


Table A-3: Regional Regulated Hydro Projects

PNW Loads and Resources Study

2005 - 2014 Operating Years

1937 Water Year

2003 White Book

Average Energy in Megawatts $\quad 2005 \quad 2006 \quad 2007 \quad 2008 \quad 2009 \quad 2010 \quad 2011 \quad 2012 \quad 2013 \quad 2014$

Avg. Avg. Avg. Avg. Avg. Avg. Avg. Avg. Avg. Avg.

-Regulated Hydro Projects-

1 Albeni Falls

2 Alder

3 Bonneville

4 Boundary

5 Box Canyon

6 Brownlee

7 Cabinet Gorge

8 Chelan

9 Chief Joseph

10 Cushman 1

11 Cushman 2

12 Diablo

13 Dworshak

14 Faraday

15 Gorge

16 Grand Coulee

17 Hells Canyon

18 Hungry Horse

19 Ice Harbor

20 John Day

21 La Grande

22 Libby

23 Little Falls

24 Little Goose

25 Long Lake

26 Lower Baker

27 Lower Granite

28 Lower Monumental

29 Mayfield

30 McNary

31 Merwin

32 Monroe Street

33 Mossyrock

34 Nine Mile

35 North Fork

36 Noxon

37 Oak Grove

38 Oxbow

39 Pelton

40 Post Falls

41 Priest Rapids

42 River Mill

43 Rock Island PH\#1

44 Rock Island PH\#2

45 Rocky Reach

46 Ross

47 Round Butte

48 Swift 1

49 Swift 2

50 The Dalles

51 Upper Baker

$\begin{array}{rr}25 & \\ 21 & \\ 341 & 3 \\ 332 & 332 \\ 48 & \\ 235 & 238 \\ 85 & \\ 44 & \\ 1062 & 10\end{array}$

25
21
343
332
48
235
85
44
1066
14

25

$25 \quad 25$

43

332

48
235

235
85

44
073

14

14

1073
14

$\begin{array}{ll}29 & 29 \\ 79 & 79\end{array}$

$126 \quad 126$

20

91
1938

20

29
79

79
126

126
20

91
1952

$189 \quad 189$

1961

$$
\begin{array}{r}
77 \\
137
\end{array}
$$

$138 \quad 137$

$797 \quad 800$

34
168

77

137

801

34
169

19

215

42

35
218

218
221

68

$\begin{array}{rr}68 & 68 \\ 518 & 52 \\ 52 & 52\end{array}$

527

21
345

25
21
345

25
21
345
332

25
21
345

$\begin{array}{rrr}25 & 25 & 25 \\ 21 & 21 & 21 \\ 345 & 345 & 345\end{array}$

$332 \quad 332$

$\begin{array}{rr}332 & 332 \\ 48 & 48 \\ 235 & 235\end{array}$

332

$\begin{array}{lll}235 & 235 & 235\end{array}$

$\begin{array}{lrr}85 & 85 & 85 \\ 44 & 44 & 44\end{array}$

48
235

$1089 \quad 1095 \quad 1101$

$\begin{array}{rrr}1089 & 1095 & 1101 \\ 14 & 14 & 14\end{array}$

$\begin{array}{lll}29 & 29 & 29 \\ 79 & 79 & 79\end{array}$

$\begin{array}{lll}127 & 127 & 127\end{array}$

$\begin{array}{lll}20 & 20 & 20 \\ 91 & 91 & 91\end{array}$

$1975 \quad 1975$

$\begin{array}{rr}189 & 189 \\ 78 & 78\end{array}$

$136 \quad 136$

805

34
169
19

$19 \quad 19$

213

42
35
216

$$
216
$$

219
68

68
530
52

52
11

$\begin{array}{lll}11 & 11 & 11 \\ 95 & 95 & 95\end{array}$

$\begin{array}{lll}13 & 13 & 13 \\ 23 & 23 & 23\end{array}$

$\begin{array}{rrr}23 & 23 & 23 \\ 131 & 131 & 131\end{array}$

25

97
36

25
97

$$
\begin{array}{rr}
36 & 36 \\
7.8 & 7.8
\end{array}
$$$$
337
$$

11
172

$$
\begin{array}{r}
337 \\
11
\end{array}
$$

11
172

$116 \quad 116$

$548 \quad 548$

548
83

548
83

$83 \quad 83$

$\begin{array}{ll}89 & 89 \\ 61 & 61\end{array}$

$\begin{array}{rr}22 & 22 \\ 601 & 601 \\ 34 & 34\end{array}$

52 Upper Falls

53 Wanapum

54 Wells

55 White

56 Yale

$601 \quad 601$

$\begin{array}{rrr}34 & 34 & 34 \\ 8.4 & 8.4 & 8.4\end{array}$

$\begin{array}{lll}362 & 362 & 362\end{array}$

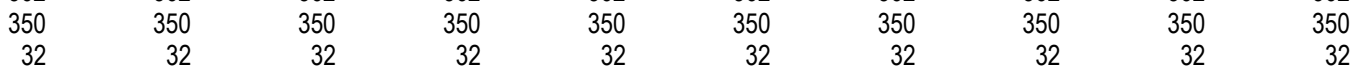

\begin{tabular}{rrr}
32 & 32 & 32 \\
57 & 57 & 57 \\
\hline 10686 & 10703 & 10721
\end{tabular}

85
44

1105

14

29
79

79
127

127
20

91
1975

189
78

136

805

34
169

19

213

42
35

35
216

219

219
68

564
52

52
11

95

13
23

131

25
97

97
36
7.8

7.8
337

337
11

11
172

172
116

548

83
89

345
332

332
48
235

332

345
332

$235 \quad 235$
-85

$\begin{array}{lll}85 & 85 & 85 \\ 44 & 44 & 44\end{array}$

$\begin{array}{rrr}4105 & 1105 & 44\end{array}$

$\begin{array}{lll}14 & 14 & 14 \\ 29 & 29 & 29\end{array}$

$\begin{array}{lll}29 & 29 & 29 \\ 79 & 79 & 79\end{array}$

$\begin{array}{rrr}127 & 127 & 127 \\ 20 & 20 & 20\end{array}$

$\begin{array}{rrr}91 & 91 & 91 \\ 1975 & 1976 & 1976\end{array}$

$\begin{array}{rrr}189 & 189 & 189 \\ 78 & 78 & 78\end{array}$

$\begin{array}{lll}136 & 136 & 136\end{array}$

$805 \quad 805 \quad 805$

$\begin{array}{rrr}34 & 34 & 34 \\ 169 & 169 & 169\end{array}$

$\begin{array}{lll}19 & 19 & 19\end{array}$

$\begin{array}{rrr}213 & 213 & 213 \\ 42 & 42\end{array}$

$\begin{array}{lll}42 & 42 & 42 \\ 35 & 35 & 35\end{array}$

$216 \quad 216 \quad 216$

$\begin{array}{rrr}219 & 219 & 219 \\ 68 & 68 & 68\end{array}$

$\begin{array}{rrr}68 & 68 & 68 \\ 576 & 587 & 599\end{array}$

$\begin{array}{lll}52 & 52 & 52 \\ 11 & 11 & 11\end{array}$

$\begin{array}{lll}95 & 95 & 95\end{array}$

$\begin{array}{lll}13 & 13 & 13 \\ 23 & 23 & 23\end{array}$

$\begin{array}{lll}131 & 131 & 131\end{array}$

$\begin{array}{lll}25 & 25 & 25 \\ 97 & 97 & 97\end{array}$

$\begin{array}{rrr}36 & 36 & 36 \\ 7.8 & 7.8 & 7.8\end{array}$

$\begin{array}{lll}7.8 & 7.8 & 7.8 \\ 337 & 337\end{array}$

$\begin{array}{rrr}337 & 337 & 337 \\ 11 & 11 & 11\end{array}$

$\begin{array}{rrr}11 & 11 & 11 \\ 172 & 172 & 172\end{array}$

$\begin{array}{lll}172 & 172 & 172 \\ 116 & 116 & 116\end{array}$

$\begin{array}{lll}116 & 116 & 116 \\ 548 & 548 & 548\end{array}$

$83 \quad 83 \quad 83$

$\begin{array}{llll}61 & 61 & 61 & 61\end{array}$

$\begin{array}{rrrr}22 & 22 & 22 & 22 \\ 601 & 601 & 601 & 601\end{array}$

$\begin{array}{lllll}34 & 34 & 34 & 34 & 34\end{array}$

$\begin{array}{rrrrr}8.4 & 8.4 & 8.4 & 8.4 & 8.4 \\ 362 & 362 & 362 & 362 & 362\end{array}$

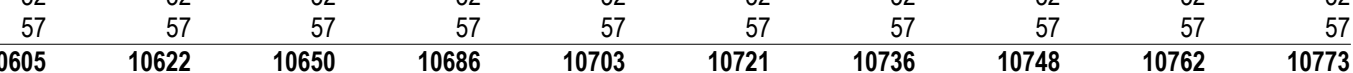

-Total Regulated Hydro W/Enc.-

57 Federal System

58 Public Entities

59 Investor-Owned Entities

60 Other Entities

61 Total Regulated Hydro w/Enc.

\begin{tabular}{rrrrrrrrrr}
6446 & 6464 & 6492 & 6527 & 6545 & 6562 & 6577 & 6589 & 6603 & 6614 \\
1681 & 1734 & 1755 & 1755 & 1755 & 1799 & 1815 & 1818 & 1821 & 1825 \\
2311 & 2257 & 2236 & 2236 & 2236 & 2192 & 2176 & 2174 & 2170 & 2167 \\
167 & 167 & 167 & 167 & 167 & 167 & 167 & 167 & 167 & 167 \\
\hline $\mathbf{1 0 6 0 5}$ & $\mathbf{1 0 6 2 2}$ & $\mathbf{1 0 6 5 0}$ & $\mathbf{1 0 6 8 6}$ & $\mathbf{1 0 7 0 3}$ & $\mathbf{1 0 7 2 1}$ & $\mathbf{1 0 7 3 6}$ & $\mathbf{1 0 7 4 8}$ & $\mathbf{1 0 7 6 2}$ & $\mathbf{1 0 7 7 3}$
\end{tabular}


Table A-4: Regional Independent Hydro Projects

PNW Loads and Resources Study

2005 - 2014 Operating Years

1937 Water Year

2003 White Book

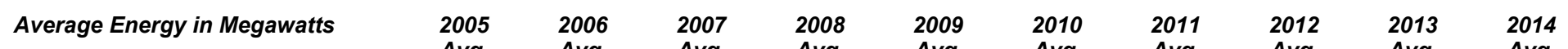

Avg. Avg. Avg. Avg. Avg. Avg. Avg. Avg. Avg.

-Independent Hydro Projects-

1 American Falls

2 Anderson Ranch

3 Big Cliff

4 Big Creek

5 Black Canyon

6 Bliss

7 Blue River

8 Bonneville Fishway

9 Bull Run

10 Calispel Creek

11 Carmen-Smith

12 Cascade

13 Cedar Falls \& Newhalem

14 Chandler

15 CJ Strike

16 Condit Sm Plts

17 Cougar

18 Cowlitz Falls

19 Detroit

20 Dexter

21 Felt

22 Foster

$23 \mathrm{Gem}$ State Hydro

24 Green Peter

25 Green Springs - USBR

26 Hills Creek

27 Hood Street

28 Idaho Falls - City Plant

29 Idaho Falls - Lower Plant

30 Idaho Falls - Upper Plant

31 Island Park

32 Jackson

33 Klamath

34 Lake Creek

35 Leaburg

36 Lookout Point

37 Lost Creek

38 Lower Salmon

39 McNary Fishway

40 Mill Creek (WA)

41 Milltown/Flint Creek

42 Milner

43 Minidoka

44 Morse Creek

45 Moyie Springs

46 North Umpqua

47 Packwood

48 Palisades

49 Reader Gulch

50 Rogue

51 Roza

52 Shoshone Falls

53 Snoq Elect \& Nooksack

54 Soda Creek

55 South Fork Tolt

56 Spring Plants

57 Stone Creek

58 Strawberry Creek

59 Swan Falls

60 The Dalles Fishway

61 Trail Bridge

62 TW Sullivan

63 Twin Falls

64 Upper Salmon 1 \& 2

65 Upper Salmon 3 \& 4

66 Walterville

$\begin{array}{lll}28 & 28 & 28\end{array}$

8.1

42

2.7

10

0.3

16

4.5

12

$8.6 \quad 8.6$

26

26

9.3

0.7

12

14

7.3

18

0.6

5.9

6.3

6.3

4.8

43

35

9.5

35

30

26
7.2

0.4

0.3

$2-0$

16

0.4
2

107

9.9

66

0.4

$36 \quad 36$

7.9

9.2

46

0.5

28

6.5

1.5

16

27

$3.5 \quad 3.5$

$\begin{array}{rr}14 & 14 \\ 5.6 & 5.6\end{array}$

$18 \quad 18$

$15 \quad 15$

$6.2 \quad 6.2$

$4.3 \quad 4.3$

9.4

1084

1084

397

397

397
166

398
166

398

398
166

398
166

69 Federal System
70 Public Entities

166

166

$28 \quad 28$

$\begin{array}{ll}28 & 28 \\ 16 & 16 \\ 11 & 11\end{array}$

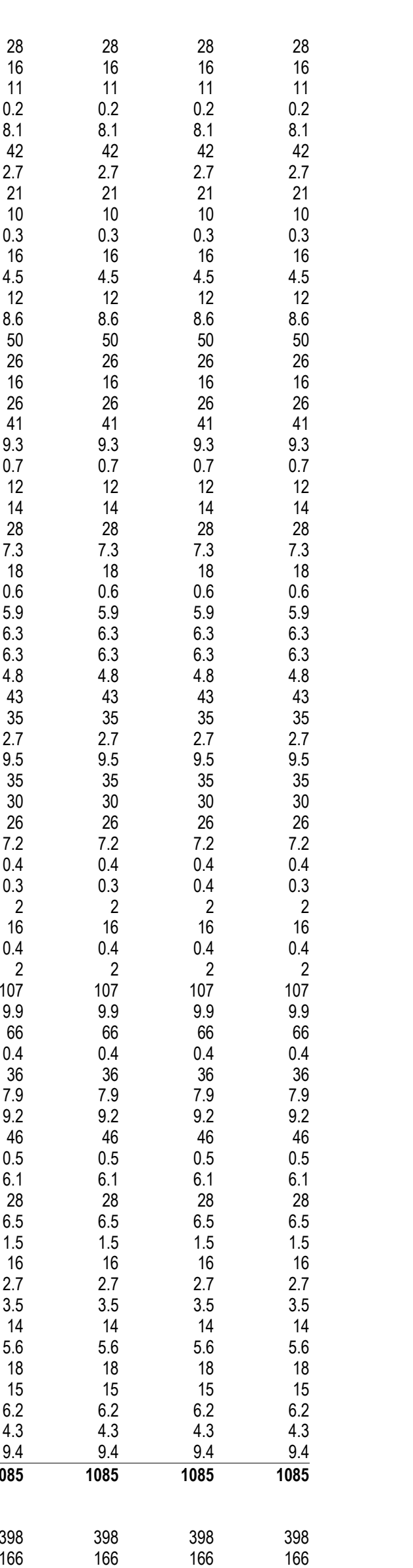

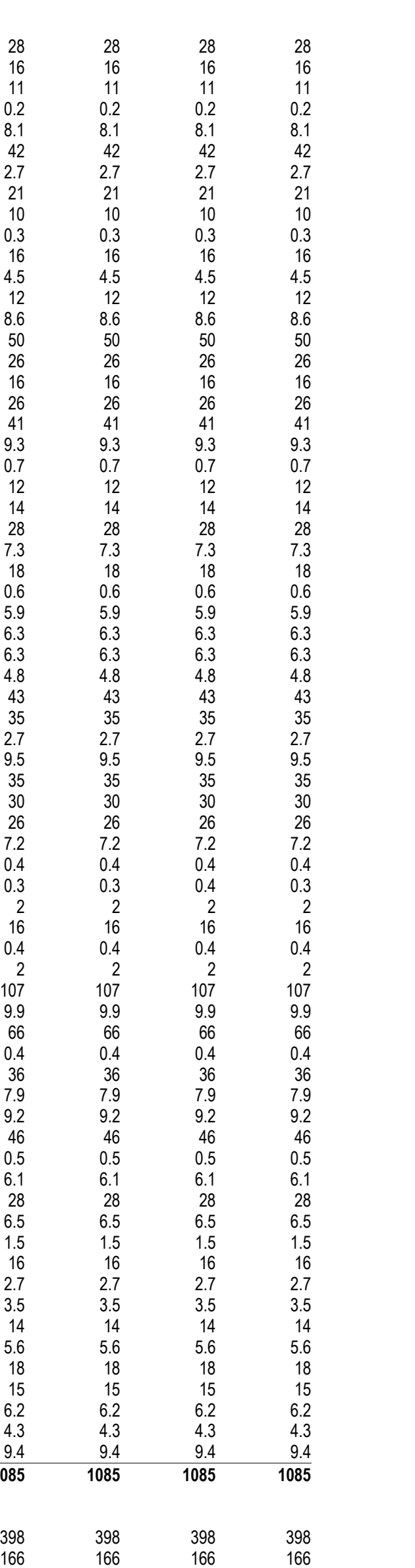

20.2

$8.1 \quad 8$

$\begin{array}{ll}42 & 42 \\ 2.7 & 27\end{array}$

0.2

$42 \quad 42$

$\begin{array}{rr}2.7 & 2.7 \\ 21 & 21\end{array}$

$\begin{array}{rr}10 & 10 \\ 0.3 & 0.3\end{array}$

16

$\begin{array}{rr}16 & 16 \\ 4.5 & 4.5\end{array}$

$\begin{array}{ll}12 & 12 \\ 8.6 & 8.6\end{array}$

$\begin{array}{rrr}8.6 & 8.6 & 8.6 \\ 50 & 50\end{array}$

$\begin{array}{ll}26 & 26 \\ 16 & 16\end{array}$

$26 \quad 26 \quad 26 \quad 26$

$\begin{array}{lll}41 & 41 & 41 \\ 9.3 & 9.3 & 9.3\end{array}$

$\begin{array}{ll}0.7 & 0.7\end{array}$

$\begin{array}{ll}12 & 12 \\ 14 & 14\end{array}$

12
14

28

18

0.6

5.9

6.3

6.3

4.8

43
35

35
2.7

9.5

35
30

30
26

26
7.2
0.4

7.2
0.4
0.3

0.3

2
16

16
0.4

2
107

9.9
66

66
0.4
36

36

7.9

9.2

46
0.5

0.5
6.1

6.1
28

6.5

1.5

1.5
16
27

2.7

3.5

14
5.6

5.6
18
15

15

6.2

4.3

1085

1085

1085

398
166

398
166

\begin{tabular}{|c|c|c|c|}
\hline 28 & 28 & 28 & 28 \\
\hline 16 & 16 & 16 & 16 \\
\hline 11 & 11 & 11 & 11 \\
\hline 0.2 & 0.2 & 0.2 & 0.2 \\
\hline 8.1 & 8.1 & 8.1 & 8.1 \\
\hline 42 & 42 & 42 & 42 \\
\hline 2.7 & 2.7 & 2.7 & 2.7 \\
\hline 21 & 21 & 21 & 21 \\
\hline 10 & 10 & 10 & 10 \\
\hline 0.3 & 0.3 & 0.3 & 0.3 \\
\hline 16 & 16 & 16 & 16 \\
\hline 4.5 & 4.5 & 4.5 & 4.5 \\
\hline 12 & 12 & 12 & 12 \\
\hline 8.6 & 8.6 & 8.6 & 8.6 \\
\hline 50 & 50 & 50 & 50 \\
\hline 26 & 26 & 26 & 26 \\
\hline 16 & 16 & 16 & 16 \\
\hline 26 & 26 & 26 & 26 \\
\hline 41 & 41 & 41 & 41 \\
\hline 9.3 & 9.3 & 9.3 & 9.3 \\
\hline 0.7 & 0.7 & 0.7 & 0.7 \\
\hline 12 & 12 & 12 & 12 \\
\hline 14 & 14 & 14 & 14 \\
\hline 28 & 28 & 28 & 28 \\
\hline 7.3 & 7.3 & 7.3 & 7.3 \\
\hline 18 & 18 & 18 & 18 \\
\hline 0.6 & 0.6 & 0.6 & 0.6 \\
\hline 5.9 & 5.9 & 5.9 & 5.9 \\
\hline 6.3 & 6.3 & 6.3 & 6.3 \\
\hline 6.3 & 6.3 & 6.3 & 6.3 \\
\hline 4.8 & 4.8 & 4.8 & 4.8 \\
\hline 43 & 43 & 43 & 43 \\
\hline 35 & 35 & 35 & 35 \\
\hline 2.7 & 2.7 & 2.7 & 2.7 \\
\hline 9.5 & 9.5 & 9.5 & 9.5 \\
\hline 35 & 35 & 35 & 35 \\
\hline 30 & 30 & 30 & 30 \\
\hline 26 & 26 & 26 & 26 \\
\hline 7.2 & 7.2 & 7.2 & 7.2 \\
\hline 0.4 & 0.4 & 0.4 & 0.4 \\
\hline 0.3 & 0.3 & 0.4 & 0.3 \\
\hline 2 & 2 & 2 & 2 \\
\hline 16 & 16 & 16 & 16 \\
\hline 0.4 & 0.4 & 0.4 & 0.4 \\
\hline 2 & 2 & 2 & 2 \\
\hline 107 & 107 & 107 & 107 \\
\hline 9.9 & 9.9 & 9.9 & 9.9 \\
\hline 66 & 66 & 66 & 66 \\
\hline 0.4 & 0.4 & 0.4 & 0.4 \\
\hline 36 & 36 & 36 & 36 \\
\hline 7.9 & 7.9 & 7.9 & 7.9 \\
\hline 9.2 & 9.2 & 9.2 & 9.2 \\
\hline 46 & 46 & 46 & 46 \\
\hline 0.5 & 0.5 & 0.5 & 0.5 \\
\hline 6.1 & 6.1 & 6.1 & 6.1 \\
\hline 28 & 28 & 28 & 28 \\
\hline 6.5 & 6.5 & 6.5 & 6.5 \\
\hline 1.5 & 1.5 & 1.5 & 1.5 \\
\hline 16 & 16 & 16 & 16 \\
\hline 2.7 & 2.7 & 2.7 & 2.7 \\
\hline 3.5 & 3.5 & 3.5 & 3.5 \\
\hline 14 & 14 & 14 & 14 \\
\hline 5.6 & 5.6 & 5.6 & 5.6 \\
\hline 18 & 18 & 18 & 18 \\
\hline 15 & 15 & 15 & 15 \\
\hline 6.2 & 6.2 & 6.2 & 6.2 \\
\hline 4.3 & 4.3 & 4.3 & 4.3 \\
\hline 9.4 & 9.4 & 9.4 & 9.4 \\
\hline 085 & 1085 & 1085 & 1085 \\
\hline 398 & 398 & 398 & 398 \\
\hline 166 & 166 & 166 & 166 \\
\hline
\end{tabular}

166 
Table A-4: Regional Independent Hydro Projects

Continued

PNW Loads and Resources Study

2005 - 2014 Operating Years

1937 Water Year

2003 White Book

\begin{tabular}{|c|c|c|c|c|c|c|c|c|c|c|}
\hline Average Energy in Megawatts & $\begin{array}{l}2005 \\
\text { Avg. }\end{array}$ & $\begin{array}{l}2006 \\
\text { Avg. }\end{array}$ & $\begin{array}{l}2007 \\
\text { Avg. }\end{array}$ & $\begin{array}{l}2008 \\
\text { Avg. }\end{array}$ & $\begin{array}{l}2009 \\
\text { Avg. }\end{array}$ & $\begin{array}{l}2010 \\
\text { Avg. }\end{array}$ & $\begin{array}{l}2011 \\
\text { Avg. }\end{array}$ & $\begin{array}{l}2012 \\
\text { Avg. }\end{array}$ & $\begin{array}{l}2013 \\
\text { Avg. }\end{array}$ & $\begin{array}{l}2014 \\
\text { Avg. }\end{array}$ \\
\hline 71 Investor-Owned Entities & 521 & 521 & 521 & 521 & 521 & 521 & 521 & 521 & 521 & 521 \\
\hline 72 Other Entities & 0 & 0 & 0 & 0 & 0 & 0 & 0 & 0 & 0 & 0 \\
\hline 73 Total Independent Hydro w/Enc. & 1084 & 1084 & 1085 & 1085 & 1085 & 1085 & 1085 & 1085 & 1085 & 1085 \\
\hline
\end{tabular}


Table A-5: Regional Imports

PNW Loads and Resources Study

2005 - 2014 Operating Years 2003 White Book

-

1 BEPC To NWE PwrS

2 NWE To NWE PwrS

3 PPL To BPA PwrS

4 PPL To PPL PwrS

5 PPLM To IPC PwrS

$\begin{array}{llllllllll}2005 & 2006 & 2007 & 2008 & 2009 & 2010 & 2011 & 2012 & 2013 & 2014 \\ \text { Avg. } & \text { Avg. } & \text { Avg. } & \text { Avg. } & \text { Avg. } & \text { Avg. } & \text { Avg. } & \text { Avg. } & \text { Avg. } & \text { Avg. }\end{array}$

6 Total Imports From ECD

-Imports From Pacific Southwest-

7 ANHM To BPA PwrX

8 BURB To BPA XchgNrg

9 BURB To BPA PkRepl

10 GLEN To BPA XchgNrg

11 GLEN To BPA PkRepl

12 GLEN To PGE S/Pwr/X

13 NCPA To SCL S/Pwr/X

14 PASA To BPA XchgNrg

15 PASA To BPA PkRepl

16 PASA To BPA XchgNrg

17 PASA To BPA S/N/X

18 PASA To BPA PkRepl

19 PG\&E To PSE S/Pwr/X

20 RVSD To BPA XchgNrg

21 RVSD To BPA PkRepl

22 RVSD To BPA PkRepl

23 RVSD To BPA XchgNrg

24 RVSD To BPA S/Pwr/X

25 RVSD To BPA PkRepl

26 Total Imports From PSW

\begin{tabular}{rrrrrrrrrr}
42 & 42 & 42 & 42 & 42 & 42 & 0 & 0 & 0 & 0 \\
0 & 0 & 0 & 0 & 0 & 0 & 0 & 0 & 0 & 0 \\
94 & 94 & 94 & 94 & 94 & 94 & 94 & 94 & 94 & 94 \\
196 & 197 & 138 & 153 & 0 & 0 & 0 & 0 & 0 & 0 \\
8.6 & 11 & 11 & 11 & 11 & 3.7 & 0 & 0 & 0 & 0 \\
\hline $\mathbf{3 4 1}$ & $\mathbf{3 4 5}$ & $\mathbf{2 8 6}$ & $\mathbf{3 0 1}$ & $\mathbf{1 4 8}$ & $\mathbf{1 4 0}$ & $\mathbf{9 4}$ & $\mathbf{9 4}$ & $\mathbf{9 4}$ & $\mathbf{9 4}$
\end{tabular}

-Imports From Inland Southwest-

27 Other Entities To BPA

28 SPP To BPA PwrS

29 WAPA To GLEC ARP

30 Total Imports From ISW

$\begin{array}{rrrrrrrrrr}10 & 0 & 0 & 0 & 0 & 0 & 0 & 0 & 0 & 0 \\ 3.6 & 3.6 & 3.6 & 2.9 & 0 & 0 & 0 & 0 & 0 & 0 \\ 3.8 & 3.8 & 3.8 & 2.5 & 0 & 0 & 0 & 0 & 0 & 0 \\ 4 & 4 & 4 & 3.2 & 0 & 0 & 0 & 0 & 0 & 0 \\ 4.2 & 4.2 & 4.2 & 2.7 & 0 & 0 & 0 & 0 & 0 & 0 \\ 3.0 & 3.0 & 3.0 & 3.0 & 3.0 & 3.0 & 3.0 & 3.0 & 0 & 0 \\ 12 & 12 & 12 & 12 & 12 & 12 & 12 & 12 & 12 & 12 \\ 2.4 & 2.4 & 2.4 & 1.9 & 0 & 0 & 0 & 0 & 0 & 0 \\ 2.5 & 2.5 & 2.5 & 1.6 & 0 & 0 & 0 & 0 & 0 & 0 \\ 1.9 & 1.9 & 1.9 & 1.9 & 1.9 & 1.9 & 1.9 & 1.9 & 1.9 & 1.9 \\ 0.4 & 0.4 & 0.4 & 0.4 & 0.4 & 0.4 & 0.4 & 0.4 & 0.4 & 0.4 \\ 1.1 & 1.1 & 1.1 & 1.1 & 1.1 & 1.1 & 1.1 & 1.1 & 1.1 & 1.1 \\ 46 & 46 & 46 & 27 & 0 & 0 & 0 & 0 & 0 & 0 \\ 3.1 & 3.1 & 3.1 & 3.1 & 3.1 & 3.1 & 0 & 0 & 0 & 0 \\ 3.0 & 3.0 & 3.0 & 3.0 & 3.0 & 3.0 & 0 & 0 & 0 & 0 \\ 2.8 & 2.8 & 2.8 & 2.8 & 2.8 & 1.7 & 0 & 0 & 0 & 0 \\ 7.3 & 7.3 & 7.3 & 7.3 & 7.3 & 7.3 & 7.3 & 7.3 & 7.3 & 7.3 \\ 4.2 & 4.2 & 4.2 & 4.2 & 4.2 & 4.2 & 4.2 & 4.2 & 4.2 & 4.2 \\ 4.9 & 4.9 & 4.9 & 4.9 & 4.9 & 4.9 & 4.9 & 4.9 & 4.9 & 4.9 \\ 121 & 111 & 111 & 86 & 44 & 43 & 35 & 35 & 32 & 32\end{array}$

-Imports From Canada-

31 BCHP To BPA PwrS

32 BCHP To SCL PwrS

33 Total Imports From Canada

\begin{tabular}{|c|c|c|c|c|c|c|c|c|c|}
\hline 42 & 42 & 21 & 0 & 0 & 0 & 0 & 0 & 0 & 0 \\
\hline 60 & 60 & 60 & 60 & 60 & 60 & 60 & 60 & 60 & 60 \\
\hline 3.3 & 3.3 & 0.5 & 0 & 0 & 0 & 0 & 0 & 0 & 0 \\
\hline
\end{tabular}

-Imports From Other Entities-

34 Total Imports From Other Entities

-Total Imports

35 Federal System

36 Public Entities

37 Investor-Owned Entities

38 Other Entities

39 Total Imports.

\begin{tabular}{|c|c|c|c|c|c|c|c|}
\hline 1 & 1 & 1 & 1 & 1 & 1 & 1 & 1 \\
\hline 36 & 36 & 36 & 36 & 36 & 36 & 36 & 36 \\
\hline 37 & 37 & 37 & 37 & 37 & 37 & 37 & 37 \\
\hline
\end{tabular}

$\begin{array}{llllllllll}0 & 0 & 0 & 0 & 0 & 0 & 0 & 0 & 0 & 0\end{array}$

$\begin{array}{rrrrrrrrrr}256 & 246 & 226 & 199 & 184 & 183 & 175 & 175 & 175 & 175 \\ 51 & 51 & 49 & 48 & 48 & 48 & 48 & 48 & 48 & 48 \\ 295 & 300 & 240 & 237 & 56 & 49 & 3.0 & 3.0 & 0 & 0 \\ 0 & 0 & 0 & 0 & 0 & 0 & 0 & 0 & 0 & 0 \\ \mathbf{6 0 3} & \mathbf{5 9 7} & \mathbf{5 1 4} & \mathbf{4 8 4} & \mathbf{2 8 8} & \mathbf{2 8 0} & \mathbf{2 2 6} & \mathbf{2 2 6} & \mathbf{2 2 3} & \mathbf{2 2 3}\end{array}$


Table A-6: Regional Small Thermal and Miscellaneous

PNW Loads and Resources Study

2005 - 2014 Operating Years

2003 White Book

-Federal System-

1 Bangor Base \#1 Diesel Genorators

2 Bangor Base \#2 Diesel Generators

3 Puget Sound Shipyards Diesel Generators

4 Total Federal System

$\begin{array}{llllllllll}2005 & 2006 & 2007 & 2008 & 2009 & 2010 & 2011 & 2012 & 2013 & 2014 \\ \text { Avg. } & \text { Avg. } & \text { Avg. } & \text { Avg. } & \text { Avg. } & \text { Avg. } & \text { Avg. } & \text { Avg. } & \text { Avg. } & \text { Avg. }\end{array}$

-Public Entities-

5 Total Public Entities

0

0

0

$\begin{array}{llll}0 & 0 & 0 & 0\end{array}$

-Investor-Owned Entities-

6 Crystal Mountain

7 Energy Mgmt Systems

8 Total Investor-Owned Entities

\begin{tabular}{rrrrrrrrrr}
0.1 & 0.1 & 0.1 & 0.1 & 0.1 & 0.1 & 0.1 & 0.1 & 0.1 & 0.1 \\
17 & 17 & 17 & 17 & 17 & 17 & 17 & 17 & 17 & 17 \\
\hline 17 & 17 & 17 & 17 & 17 & 17 & 17 & 17 & 17 & 17
\end{tabular}

-Other Entities-

9 Total Other Entities

0

0

0

$0 \quad 0 \quad 0$

-Small Thermal and Miscellaneous-

10 Federal System

11 Public Entities

12 Investor-Owned Entities

13 Other Entities

14 Total Small Termal \& Misc.

$\begin{array}{rrrrrrrrrr}0 & 0 & 0 & 0 & 0 & 0 & 0 & 0 & 0 & 0 \\ 0 & 0 & 0 & 0 & 0 & 0 & 0 & 0 & 0 & 0 \\ 17 & 17 & 17 & 17 & 17 & 17 & 17 & 17 & 17 & 17 \\ 0 & 0 & 0 & 0 & 0 & 0 & 0 & 0 & 0 \\ 17 & 17 & 17 & 17 & 17 & 17 & 17 & 17 & 17 & 17\end{array}$




$\begin{array}{llllllllll}2005 & 2006 & 2007 & 2008 & 2009 & 2010 & 2011 & 2012 & 2013 & 2014 \\ \text { Avg. } & \text { Avg. } & \text { Avg. } & \text { Avg. } & \text { Avg. } & \text { Avg. } & \text { Avg. } & \text { Avg. } & \text { Avg. } & \text { Avg. }\end{array}$

-Federal System-

1 Total Federal System

Avg.

Avg.

Avg.

Avg.

Avg.

Avg.

Avg.

Avg.

Avg.

Avg.

-Public Entities-

2 Benton CT

3 Frederickson CT

4 Hoquiam Diesels

5 Olympic View Generating Station

6 Pasco Harbor Generating Station

7 Randolph Road Diesel Farm

8 River Road

9 Roosevelt Landfill

10 Springfield Gen Farm

11 Wauna Peaking Project

12 Total Public Entities

-Investor-Owned Entities-

13 Basin Creek Project

14 Beaver

15 Beaver 8

16 Big Hanaford CCCT

17 Boulder Park

18 Danskin

19 Encogen

20 Frederickson CT

21 Fredonia $1 \& 2$

22 Fredonia $3 \& 4$

23 Fredrickson $1 \& 2$

24 Kettle Falls CT

25 Northeastern 1 \& 2

26 Whitehorn 2 \& 3

27 Total Investor-Owned Entities

-Other Entities-

28 Chehalis Generation Facility CCCT

29 Frederickson CT

30 Klamath Peaking Unit

31 Lancaster Power Project (Rathdrum)

32 Total Other Entities

-Combustion Turbines-

33 Federal System

34 Public Entities

35 Investor-Owned Entities

36 Other Entities

37 Total Combustion Turbines

$\begin{array}{rrr}0 & 0 & 0 \\ & & \\ 0.8 & 0.8 & 0.8 \\ 73 & 73 & 73 \\ 0 & 0 & 0 \\ 0 & 0 & 0 \\ 4 & 4 & 4 \\ 0 & 0 & 0 \\ 221 & 235 & 188 \\ 8 & 8 & 8 \\ 0 & 0 & 0 \\ 2.3 & 2.3 & 2.3 \\ 309 & 323 & 276\end{array}$

0
0.8
73
0
0
4
0
172
8
0
2.3
260

0

0

\begin{tabular}{rrrrrr}
0.8 & 0.8 & 0.8 & 0.8 & 0.8 & 0.8 \\
73 & 73 & 73 & 73 & 73 & 73 \\
0 & 0 & 0 & 0 & 0 & 0 \\
0 & 0 & 0 & 0 & 0 & 0 \\
4 & 4 & 4 & 4 & 4 & 4 \\
0 & 0 & 0 & 0 & 0 & 0 \\
179 & 179 & 172 & 180 & 191 & 201 \\
8 & 8 & 8 & 8 & 8 & 8 \\
0 & 0 & 0 & 0 & 0 & 0 \\
2.3 & 2.3 & 2.3 & 2.3 & 2.3 & 2.3 \\
\hline 267 & 267 & $\mathbf{2 6 0}$ & $\mathbf{2 6 8}$ & $\mathbf{2 7 9}$ & $\mathbf{2 8 9}$
\end{tabular}

\begin{tabular}{|c|c|c|c|c|c|c|c|c|c|}
\hline 3.3 & 4 & 4 & 4 & 4 & 4 & 4 & 4 & 4 & 4 \\
\hline 453 & 452 & 449 & 450 & 452 & 449 & 450 & 454 & 448 & 451 \\
\hline 0 & 0 & 0 & 0 & 0 & 0 & 0 & 0 & 0 & 0 \\
\hline 224 & 224 & 224 & 224 & 224 & 224 & 224 & 224 & 224 & 224 \\
\hline 23 & 23 & 23 & 23 & 23 & 23 & 23 & 23 & 23 & 23 \\
\hline 4 & 4 & 4 & 4 & 4 & 4 & 4 & 4 & 4 & 4 \\
\hline 85 & 96 & 104 & 106 & 105 & 103 & 97 & 94 & 89 & 89 \\
\hline 73 & 73 & 73 & 73 & 73 & 73 & 73 & 73 & 73 & 73 \\
\hline 31 & 24 & 20 & 16 & 16 & 0 & 16 & 16 & 16 & 15 \\
\hline 14 & 23 & 31 & 32 & 30 & 31 & 26 & 29 & 23 & 18 \\
\hline 0.8 & 4 & 28 & 13 & 14 & 17 & 11 & 8.4 & 9.3 & 6.3 \\
\hline 6.9 & 6.9 & 6.9 & 6.9 & 6.9 & 6.9 & 6.9 & 6.9 & 6.9 & 6.9 \\
\hline 44 & 44 & 44 & 44 & 44 & 44 & 44 & 44 & 44 & 44 \\
\hline 0 & 0.5 & 6 & 6.3 & 4 & 0 & 0 & 0 & 0 & 0 \\
\hline 961 & 977 & 1015 & 1002 & 999 & 978 & 978 & 980 & 963 & 957 \\
\hline
\end{tabular}

\begin{tabular}{rrrrrrrrrr}
17 & 417 & 417 & 417 & 417 & 417 & 417 & 417 & 417 & 417 \\
0.2 & 0.2 & 0.2 & 0.2 & 0.2 & 0.2 & 0.2 & 0.2 & 0.2 & 0.2 \\
14 & 14 & 14 & 14 & 14 & 14 & 14 & 14 & 14 & 14 \\
44 & 244 & 244 & 244 & 244 & 244 & 244 & 244 & 244 & 244 \\
\hline 76 & 676 & 676 & 676 & 676 & 676 & 676 & 676 & 676 & 676
\end{tabular}

$\begin{array}{rrrrrrrrrr}0 & 0 & 0 & 0 & 0 & 0 & 0 & 0 & 0 \\ 309 & 323 & 276 & 260 & 267 & 267 & 260 & 268 & 279 & 289 \\ 961 & 977 & 1015 & 1002 & 999 & 978 & 978 & 980 & 963 & 957 \\ 676 & 676 & 676 & 676 & 676 & 676 & 676 & 676 & 676 & 676 \\ 1946 & 1976 & 1967 & 1937 & 1941 & 1921 & 1914 & 1923 & 1918 & 1922\end{array}$




$\begin{array}{llllllllll}2005 & 2006 & 2007 & 2008 & 2009 & 2010 & 2011 & 2012 & 2013 & 2014 \\ \text { Avg. } & \text { Avg. } & \text { Avg. } & \text { Avg. } & \text { Avg. } & \text { Avg. } & \text { Avg. } & \text { Avg. } & \text { Avg. } & \text { Avg. }\end{array}$

-Federal System-

\begin{tabular}{lllllllllll}
\hline T Total Federal System & 0 & 0 & 0 & 0 & 0 & 0 & 0 & 0 & 0 & 0
\end{tabular}

-Public Entities-

2 WEYCO Energy Center

3 Total Public Entities

$14 \quad 14$

$14 \quad 14$

-Investor-Owned Entities-

4 Coyote Springs \#1

5 Coyote Springs \#2

6 Georgia-Pacific Paper (Camas)

7 Hermiston Cogeneration

8 Thompson River Co-Gen

9 Total Investor-Owned Entities

14

-Other Entities-

10 Coyote Springs \#2

11 Hermiston Power Project

12 Klamath Cogneration Project

13 Longview Fibre

14 Total Other Entities

\begin{tabular}{|c|c|c|c|c|c|c|c|c|c|}
\hline 233 & 233 & 233 & 233 & 233 & 233 & 233 & 233 & 233 & 233 \\
\hline 126 & 126 & 126 & 126 & 126 & 126 & 126 & 126 & 126 & 126 \\
\hline 44 & 44 & 44 & 44 & 44 & 44 & 44 & 44 & 44 & 44 \\
\hline 406 & 406 & 406 & 406 & 406 & 406 & 406 & 406 & 406 & 406 \\
\hline 9.6 & 9.6 & 9.6 & 9.6 & 9.6 & 9.6 & 9.6 & 9.6 & 9.6 & 9.6 \\
\hline 819 & 819 & 819 & 819 & 819 & 819 & 819 & 819 & 819 & 819 \\
\hline
\end{tabular}

-Cogeneration-

15 Federal System

16 Public Entities

17 Investor-Owned Entities

18 Other Entities

\begin{tabular}{|c|c|c|c|c|c|c|c|c|}
\hline 126 & 126 & 126 & 126 & 126 & 126 & 126 & 126 & 126 \\
\hline 568 & 568 & 568 & 568 & 568 & 568 & 568 & 568 & 568 \\
\hline 436 & 436 & 436 & 436 & 436 & 436 & 436 & 436 & 436 \\
\hline 36 & 36 & 36 & 36 & 36 & 36 & 36 & 36 & 36 \\
\hline 1166 & 1166 & 1166 & 1166 & 1166 & 1166 & 1166 & 1166 & 1166 \\
\hline
\end{tabular}

19 Total Cogeneration

$\begin{array}{rrrrrrrrrr}0 & 0 & 0 & 0 & 0 & 0 & 0 & 0 & 0 & 0 \\ 14 & 14 & 14 & 14 & 14 & 14 & 14 & 14 & 14 \\ 819 & 819 & 819 & 819 & 819 & 819 & 819 & 819 & 819 & 819 \\ 1166 & 1166 & 1166 & 1166 & 1166 & 1166 & 1166 & 1166 & 1166 & 1166 \\ 1999 & 1999 & 1999 & 1999 & 1999 & 1999 & 1999 & 1999 & 1999 & 1999\end{array}$


-Boardman: Coal-

1 Idaho Power Company

2 Portland General Electric

3 San Diego Gas \& Electric

4 Turlock Irrigation District

5 Boardman: Regional Total

$2006 \quad 2007 \quad 2008 \quad 2009$

Avg. Avg. Avg.

2010

2011

2012

2013

2014

Avg. Avg. Avg.

Avg.

Avg.

$\begin{array}{rrrrrrrrrr}43 & 43 & 43 & 43 & 43 & 43 & 43 & 43 & 43 & 43 \\ 283 & 283 & 283 & 283 & 283 & 283 & 283 & 283 & 283 & 283 \\ 64 & 64 & 64 & 64 & 64 & 64 & 64 & 64 & 64 & 64 \\ 43 & 43 & 43 & 43 & 43 & 43 & 43 & 43 & 43 & 43 \\ 434 & 434 & 434 & 434 & 434 & 434 & 434 & 434 & 434\end{array}$

-Centralia \#1: Coal-

6 Trans Alta Utilities Corp

\begin{tabular}{llllllllll}
626 & 577 & 626 & 577 & 626 & 554 & 626 & 577 & 626 & 577 \\
\hline 626 & 577 & 626 & 577 & 626 & 554 & 626 & 577 & 626 & 577
\end{tabular}

7 Centralia \#1: Regional Tota

626

$577 \quad 626$

577

$626 \quad 554$

626

554

626

577

626

8 Trans Alta Utilities Corp

554

$626 \quad 577$

$626 \quad 577$

$626 \quad 55$

626

$577 \quad 626$

-Colstrip \#1: Coal-

10 Puget Sound Energy

\begin{tabular}{llllllllll}
136 & 136 & 136 & 136 & 136 & 136 & 136 & 136 & 136 & 136 \\
\hline 136 & 136 & 136 & 136 & 136 & 136 & 136 & 136 & 136 & 136
\end{tabular}

11 Colstrip \#1: Regional Total

136

136

$136 \quad 136$

$126 \quad 132$

$132 \quad 131$

$131 \quad 117$

$117 \quad 125$

$125 \quad 125$

125

125

125

133 125

13 Colstrip \#2: Regional Total

$\begin{array}{rrrr}99 & 96 & 87 & 99 \\ 66 & 64 & 58 & 66 \\ 132 & 129 & 116 & 132 \\ 165 & 161 & 145 & 165 \\ \mathbf{4 6 2} & \mathbf{4 5 0} & \mathbf{4 0 6} & \mathbf{4 6 2}\end{array}$

$\begin{array}{rrr}99 & 93 & \\ 66 & 62 & \\ 132 & 124 & 124 \\ 165 & 155 & 155 \\ 462 & 433 & 433\end{array}$

$\begin{array}{rr}93 & 99 \\ 62 & 66 \\ 124 & 132 \\ 155 & 165 \\ 433 & 462\end{array}$

\begin{tabular}{rrrr}
99 & 93 & 99 & 93 \\
66 & 62 & 66 & 62 \\
132 & 124 & 132 & 124 \\
165 & 155 & 165 & 155 \\
\hline 462 & 433 & 462 & $\mathbf{4 3 3}$
\end{tabular}

-Colstrip \#4: Coal-

19 Avista Corp (WWP Division)

20 Northwestern Energy LLC (MPC)

21 Pacific Power

22 Portland General Electric

23 Puget Sound Energy

$\begin{array}{rrrrrrrrrr}96 & 88 & 92 & 94 & 92 & 92 & 92 & 92 & 92 & 92 \\ 192 & 176 & 184 & 187 & 184 & 184 & 184 & 184 & 184 & 184 \\ 64 & 59 & 61 & 62 & 61 & 61 & 61 & 61 & 61 & 61 \\ 128 & 117 & 123 & 125 & 123 & 123 & 123 & 123 & 123 & 123 \\ 160 & 147 & 153 & 156 & 153 & 153 & 153 & 153 & 153 & 153 \\ \mathbf{6 4 0} & \mathbf{5 8 7} & \mathbf{6 1 3} & \mathbf{6 2 4} & \mathbf{6 1 3} & \mathbf{6 1 3} & \mathbf{6 1 3} & \mathbf{6 1 3} & \mathbf{6 1 3} & \mathbf{6 1 3}\end{array}$

-Columbia Generating Station: Uranium-

25 BPA - Power Business

861

26 Columbia Generating Station: Regional Total

$861 \quad 1000$

$\begin{array}{lll}1000 & 877 & 1000\end{array}$

877

$877 \quad 1000$

$1000 \quad 877$

\begin{tabular}{llll}
877 & 1000 & 877 & 1000 \\
\hline 877 & 1000 & 877 & 1000
\end{tabular}

-Jim Bridger \#1: Coal-

27 Idaho Power Company

28 Pacific Power

29 Jim Bridger \#1: Regional Total

\begin{tabular}{llll}
161 & 161 & 137 & 161 \\
321 & 321 & 274 & 321 \\
\hline 482 & 482 & 411 & 482
\end{tabular}

$\begin{array}{lll}161 & 157 & 161 \\ 321 & 313 & 321 \\ 482 & 470 & 482\end{array}$

\begin{tabular}{lllll}
161 & 148 & 161 & 158 & 161 \\
321 & 295 & 321 & 315 & 321 \\
\hline 482 & 443 & 482 & $\mathbf{4 7 3}$ & $\mathbf{4 8 2}$
\end{tabular}

-Jim Bridger \#2: Coal-

30 Idaho Power Company

31 Pacific Power

$\begin{array}{lllll}482 & 482 & 411 & 482 & 470\end{array}$

$482 \quad 443$

482

32 Jim Bridger \#2: Regional Total

\begin{tabular}{llllllllll}
163 & 163 & 159 & 163 & 150 & 163 & 159 & 163 & 149 & 163 \\
325 & 325 & 317 & 325 & 299 & 325 & 317 & 325 & 298 & 325 \\
\hline $\mathbf{4 8 8}$ & $\mathbf{4 8 8}$ & $\mathbf{4 7 6}$ & $\mathbf{4 8 8}$ & $\mathbf{4 4 9}$ & $\mathbf{4 8 8}$ & $\mathbf{4 7 6}$ & $\mathbf{4 8 8}$ & $\mathbf{4 4 6}$ & $\mathbf{4 8 8}$
\end{tabular}

-Jim Bridger \#3: Coal-

33 Idaho Power Company

34 Pacific Power

35 Jim Bridger \#3: Regional Total

$\begin{array}{lll}155 & 159 & 141 \\ 310 & 318 & 282 \\ \mathbf{4 6 5} & \mathbf{4 7 7} & \mathbf{4 2 2}\end{array}$

\begin{tabular}{ll}
159 & 159 \\
318 & 318 \\
\hline 477 & $\mathbf{4 7 7}$
\end{tabular}

$\begin{array}{ll}146 & 159 \\ 292 & 318 \\ 437 & 477\end{array}$

\begin{tabular}{llll}
159 & 159 & 159 & 159 \\
318 & 318 & 318 & 318 \\
\hline $\mathbf{4 7 7}$ & $\mathbf{4 7 7}$ & $\mathbf{4 7 7}$ & $\mathbf{4 7 7}$
\end{tabular}

-Jim Bridger\#4: Coal-

36 Idaho Power Company

37 Pacific Power

38 Jim Bridger \#4: Regional Total

\begin{tabular}{llllllllll}
154 & 150 & 154 & 136 & 154 & 150 & 154 & 141 & 154 & 154 \\
307 & 300 & 307 & 272 & 307 & 300 & 307 & 282 & 307 & 307 \\
\hline $\mathbf{4 6 1}$ & $\mathbf{4 4 9}$ & $\mathbf{4 6 1}$ & $\mathbf{4 0 8}$ & $\mathbf{4 6 1}$ & $\mathbf{4 5 0}$ & $\mathbf{4 6 1}$ & $\mathbf{4 2 4}$ & $\mathbf{4 6 1}$ & $\mathbf{4 6 1}$
\end{tabular}

-Valmy \#1: Coal-

39 Idaho Power Company

40 Valmy \#1: Regional Total

$\begin{array}{llllllllll}98 & 98 & 97 & 98 & 98 & 98 & 98 & 97 & 98 & 97 \\ \mathbf{9 8} & \mathbf{9 8} & \mathbf{9 7} & \mathbf{9 8} & \mathbf{9 8} & \mathbf{9 8} & \mathbf{9 8} & \mathbf{9 7} & \mathbf{9 8} & \mathbf{9 7}\end{array}$

-Valmy \#2: Coal-

41 Idaho Power Company

42 Valmy \#2: Regional Total

\begin{tabular}{rrrrrrrrrr}
97 & 97 & 98 & 97 & 97 & 97 & 97 & 97 & 97 & 97 \\
\hline 97 & 97 & 98 & 97 & 97 & 97 & 97 & 97 & 97 & 97 \\
& & & & & & & & & \\
861 & 1000 & 877 & 1000 & 877 & 1000 & 877 & 1000 & 877 & 1000 \\
43 & 43 & 43 & 43 & 43 & 43 & 43 & 43 & 43 & 43 \\
5026 & 4991 & 4846 & 4983 & 4953 & 4929 & 4958 & 4966 & 4990 & 5004
\end{tabular}

-Total Large Thermal-

43 Federal System

44 Public Entities

45 Investor-Owned Entities

$\begin{array}{lllll}5026 & 4991 & 4846 & 4983 & 4953\end{array}$

4929

4958

4966

5004 


\section{Continued}

Table A-10: Regional Large Thermal

PNW Loads and Resources Study

Average Energy in Megawatts

46 Other Entities

47 Non-NW Entities

48 Total Large Thermal
2005

Avg.

2006

Avg.

2007

Avg.

2008

Avg.

2009

Avg.

5930

6034

$\begin{array}{rrr}0 & 0 & 0 \\ 5766 & 6026 & 5873\end{array}$

2005 - 2014 Operating Years

2003 White Book

$3 / 31 / 2004$

$\begin{array}{lllll}2010 & 2011 & 2012 & 2013 & 2014\end{array}$

Avg.

Avg

Avg.

Avg.

Avg.

0
0
5873

5973

0
0
5878

0
0
6009

$0 \quad 0$

$\begin{array}{rrrr}5878 & 6009 & 5910 & 6047\end{array}$



2003 White Book

$\begin{array}{llllllllll}2005 & 2006 & 2007 & 2008 & 2009 & 2010 & 2011 & 2012 & 2013 & 2014\end{array}$

Avg. Avg. Avg. Avg. Avg. Avg. Avg. Avg. Avg.

-Federal System-

1 Total Federal System

$\begin{array}{llllllllll}0 & 0 & 0 & 0 & 0 & 0 & 0 & 0 & 0 & 0\end{array}$

-Public Entities-

2 Total Public Entities

0

0

0

0

$\begin{array}{lll}0 & 0 & 0\end{array}$

-Investor-Owned Entities-

3 Total Investor-Owned Entities

0

0

0

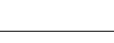

$\begin{array}{llll} & & & \\ 0 & 0 & 0\end{array}$

-Other Entities-

4 Total Other Entities

0

$0 \quad 0$

0

$0 \quad 0$

0

0

0

0

-Restoration-

5 Federal System

6 Public Entities

7 Investor-Owned Entities

8 Other Entities

9 Total Restoration

$\begin{array}{llllllllll}0 & 0 & 0 & 0 & 0 & 0 & 0 & 0 & 0 & 0 \\ 0 & 0 & 0 & 0 & 0 & 0 & 0 & 0 & 0 \\ 0 & 0 & 0 & 0 & 0 & 0 & 0 & 0 & 0 & 0 \\ 0 & 0 & 0 & 0 & 0 & 0 & 0 & 0 & 0 \\ 0 & 0 & 0 & 0 & 0 & 0 & 0 & 0 & 0 & 0\end{array}$


Table A-12: Canadian Entitlement Return For CSPE

PNW Loads and Resources Study

2005 - 2014 Operating Years

Avg. Avg.

$\begin{array}{lll}2007 & 2008 & 2009 \\ \text { Avg. } & \text { Avg. } & \text { Avg }\end{array}$

Avg.

2010

2011

2012

2013

2014

-BPA CER for CSPE-

$1 \mathrm{BPA}$ : Regional Totals

0

0

0

$0 \quad 0$

$\begin{array}{lll}0 & 0 & 0\end{array}$

-Priest Rapids CER for CSPE.

2 Priest Rapids: Regional Totals

0

0

$\begin{array}{llll}0 & 0 & 0 & 0\end{array}$

-Wanapum CER for CSPE-

3 Wanapum: Regional Totals

0

0

0

$+2$

ock Island P.H. \#1 CER for CSPE-

4 Rock Island P.H. \#1: Regional Totals

$\begin{array}{lllllllll}0 & 0 & 0 & 0 & 0 & 0 & 0 & 0 & 0\end{array}$

0

0

-Rock Island P.H. \#2 CER for CSPE-

5 Rock Island P.H. \#2: Regional Totals

$\begin{array}{rrrrrrrrr}0 & 0 & 0 & 0 & 0 & 0 & 0 & 0 & 0\end{array}$

-Rocky Reach CER for CSPE-

6 Rocky Reach: Regional Totals

0

$0 \quad 0$

0

0

0

$0 \quad 0$

0

-Wells CER for CSPE-

7 Wells: Regional Totals

$0 \quad 0$

0

0

0

0

$\begin{array}{lll}0 & 0 & 0\end{array}$

-Total CER For CSPE-

8 Federal System

9 Public Agencies

10 Investor-Owned Utilities

11 Other Entities

12 Non-NW Utilities

13 Total CER For CSPE

\begin{tabular}{llllllllll}
0 & 0 & 0 & 0 & 0 & 0 & 0 & 0 & 0 & 0 \\
0 & 0 & 0 & 0 & 0 & 0 & 0 & 0 & 0 \\
0 & 0 & 0 & 0 & 0 & 0 & 0 & 0 & 0 \\
0 & 0 & 0 & 0 & 0 & 0 & 0 & 0 & 0 \\
0 & 0 & 0 & 0 & 0 & 0 & 0 & 0 & 0 \\
0 & 0 & 0 & 0 & 0 & 0 & 0 & 0 & 0 \\
\hline 0
\end{tabular}


Table A-13: Columbia Storage Power Exchange Purchase (CSPE)

PNW Loads and Resources Study

2005 - 2014 Operating Years 2003 White Book

-Generating Public Entities-

1 BPA to
2 Total Generating Public Entities

Avg.

200

2007

2008
Avg.

2009

2010

2011

2012

2013

2014

Small \& Non-Gen Public Entities-

3 BPA to

4 Total Small \& Non-Gen Public Entities

0

$\begin{array}{ll}0 & 0 \\ 0 & 0\end{array}$

0

0

$\begin{array}{lllll}0 & 0 & 0 & 0 & 0 \\ 0 & 0 & 0 & 0 & 0\end{array}$

-Investor-Owned Entities-

\begin{tabular}{llllllllll}
5 BPA to & 0 & 0 & 0 & 0 & 0 & 0 & 0 & 0 & 0 \\
\hline 6 Total Investor-Owned Entities & 0 & 0 & 0 & 0 & 0 & 0 & 0 & 0 & 0 \\
\hline
\end{tabular}

-Other Entities-

7 BPA tc

0

$\begin{array}{lll}0 & 0 & 0 \\ 0 & 0\end{array}$

0

0

$\begin{array}{lllll}0 & 0 & 0 & 0 & 0 \\ 0 & 0 & 0 & 0 & 0\end{array}$

9 Generating Public Agencies

10 Small \& Non-Gen Public Agencies

11 Investor-Owned Utilities

12 Other Entities

13 Total CSPE

0
0
0
0

$\begin{array}{ll}0 & 0 \\ 0 & 0 \\ 0 & 0 \\ 0 & 0 \\ 0 & 0\end{array}$

\begin{tabular}{lllllll}
0 & 0 & 0 & 0 & 0 & 0 & 0 \\
0 & 0 & 0 & 0 & 0 & 0 & 0 \\
0 & 0 & 0 & 0 & 0 & 0 & 0 \\
0 & 0 & 0 & 0 & 0 & 0 & 0 \\
\hline 0 & 0 & 0 & 0 & 0 & 0 & 0
\end{tabular}


Table A-14: Supplemental \& Entitlement Capacity

PNW Loads and Resources Study

2005 - 2014 Operating Years

$3 / 31 / 2004$

Average Energy in Megawatts

2005

Avg.
2006

Avg.

Supplemental \& Entitlement Capacity

-Deliveries-

2003 White Book

1 Total Deliveries

2007

Avg.
2008

Avg.
2009
Avg.
2010

Avg.
2011

Avg.
2012

Avg.
2013

Avg.
2014

Avg.
-Replacement-

2 Total Replacement

-Total Deliveries-

3 Federal Entities

4 Public Entities

5 Investor-Owned Entities

6 Other Entities

7 Total Deliveries

-Total Replacement-

8 Federal Entities

9 Public Entities

10 Investor-Owned Entities

11 Other Entities

12 Total Replacement
0

0

0

0

0

0

0

0

0

$\begin{array}{lllllll}0 & 0 & 0 & 0 & 0 & 0 & 0 \\ 0 & 0 & 0 & 0 & 0 & 0 & 0 \\ 0 & 0 & 0 & 0 & 0 & 0 & 0 \\ 0 & 0 & 0 & 0 & 0 & 0 & 0 \\ 0 & 0 & 0 & 0 & 0 & 0 & 0\end{array}$

$\begin{array}{llll}0 & 0 & 0 & 0 \\ 0 & 0 & 0 & 0 \\ 0 & 0 & 0 & 0 \\ 0 & 0 & 0 & 0 \\ 0 & 0 & 0 & 0\end{array}$

\begin{tabular}{llllllllll}
0 & 0 & 0 & 0 & 0 & 0 & 0 & 0 & 0 & 0 \\
0 & 0 & 0 & 0 & 0 & 0 & 0 & 0 & 0 & 0 \\
0 & 0 & 0 & 0 & 0 & 0 & 0 & 0 & 0 & 0 \\
0 & 0 & 0 & 0 & 0 & 0 & 0 & 0 & 0 & 0 \\
\hline 0 & $\mathbf{0}$ & $\mathbf{0}$ & $\mathbf{0}$ & $\mathbf{0}$ & $\mathbf{0}$ & $\mathbf{0}$ & $\mathbf{0}$ & $\mathbf{0}$ & $\mathbf{0}$
\end{tabular}


Table A-15: Canadian Entitlement Return For Canada

PNW Loads and Resources Study

2005 - 2014 Operating Years

Avg. Avg.

Avg. Avg. Avg.

$2009 \quad 2010$

Avg.

2011

2012

2013

2014

-BPA Canada-

1 BPA: Regional Totals

0

0

0

-Priest Rapids CER for Canada-

2 AVWP to BPA

3 COPD to BPA

4 CWPC to BPA

5 EWEB to BPA

6 FGRV to BPA

7 FREC to BPA

8 GCPD to BPA

9 ICLP to BPA

$10 \mathrm{KITT}$ to BPA

$11 \mathrm{KOOT}$ to BPA

12 LREC to BPA

13 LVE to BPA

$14 \mathrm{MCMN}$ to BPA

15 MTFR to BPA

16 NLEC to BPA

17 PGE to BPA

$18 \mathrm{PPL}$ to BPA

19 PSE to BPA

20 RREC to BPA

21 SCL to BPA

22 SLEC to BPA

23 TPU to BPA

24 UNEC to BPA

25 Priest Rapids: Regional Totals

$\begin{array}{rrrrrrrrrr}1.9 & 1.1 & 0.8 & 0.8 & 0.8 & 1.2 & 1.3 & 1.3 & 1.2 & 1.2 \\ 0.6 & 0.4 & 0.3 & 0.3 & 0.3 & 0.4 & 0.4 & 0.4 & 0.4 & 0.4 \\ 0 & 0 & 0 & 0 & 0 & 0 & 0 & 0 & 0 & 0 \\ 0.5 & 0.3 & 0.2 & 0.2 & 0.2 & 0.3 & 0.4 & 0.4 & 0.3 & 0.3 \\ 0.2 & 0.1 & 0.1 & 0.1 & 0.1 & 0.1 & 0.1 & 0.1 & 0.1 & 0.1 \\ 0 & 0 & 0 & 0 & 0 & 0 & 0 & 0 & 0 & 0 \\ 11 & 19 & 20 & 20 & 20 & 18 & 18 & 18 & 18 & 18 \\ 0 & 0 & 0 & 0 & 0 & 0 & 0 & 0 & 0 & 0 \\ 0.1 & 0.1 & 0 & 0 & 0 & 0 & 0 & 0 & 0 & 0 \\ 0 & 0 & 0 & 0 & 0 & 0.1 & 0.1 & 0.1 & 0.1 & 0.1 \\ 0 & 0 & 0 & 0 & 0 & 0 & 0 & 0 & 0 & 0 \\ 0 & 0 & 0 & 0 & 0 & 0.1 & 0.1 & 0.1 & 0.1 & 0.1 \\ 0.2 & 0.1 & 0.1 & 0.1 & 0.1 & 0.1 & 0.1 & 0.1 & 0.1 & 0.1 \\ 0.2 & 0.1 & 0.1 & 0.1 & 0.1 & 0.1 & 0.1 & 0.1 & 0.1 & 0.1 \\ 0 & 0 & 0 & 0 & 0 & 0 & 0.1 & 0 & 0 & 0 \\ 4.3 & 2.5 & 1.9 & 1.9 & 1.8 & 2.6 & 3.0 & 2.9 & 2.8 & 2.7 \\ 4.3 & 2.5 & 1.9 & 1.9 & 1.8 & 2.6 & 3.0 & 2.9 & 2.8 & 2.7 \\ 2.5 & 1.5 & 1.1 & 1.1 & 1.1 & 1.5 & 1.7 & 1.7 & 1.6 & 1.6 \\ 0 & 0 & 0 & 0 & 0 & 0 & 0 & 0 & 0 & 0 \\ 2.5 & 1.4 & 1 & 1.0 & 1.0 & 0.8 & 0.7 & 0.7 & 0.7 & 0.7 \\ 0 & 0 & 0 & 0 & 0 & 0 & 0 & 0 & 0 & 0 \\ 2.5 & 1.4 & 1 & 1.0 & 1.0 & 0.8 & 0.7 & 0.7 & 0.7 & 0.7 \\ 0 & 0 & 0 & 0 & 0 & 0 & 0 & 0 & 0 & 0 \\ 31 & 30 & 29 & 28 & \mathbf{2 8} & \mathbf{2 9} & \mathbf{3 0} & \mathbf{3 0} & \mathbf{2 9} & \mathbf{2 9}\end{array}$

-Wanapum CER for Canada-

26 AVWP to BPA

27 COPD to BPA

28 CWPC to BPA

29 EWEB to BPA

30 FGRV to BPA

31 FREC to BPA

32 GCPD to BPA

33 ICLP to BPA

$34 \mathrm{KITT}$ to BPA

$35 \mathrm{KOOT}$ to BPA

36 LREC to BPA

37 LVE to BPA

$38 \mathrm{MCMN}$ to BPA

39 MTFR to BPA

40 NLEC to BPA

41 PGE to BPA

$42 \mathrm{PPL}$ to BPA

43 PSE to BPA

44 RREC to BPA

$45 \mathrm{SCL}$ to BPA

46 SLEC to BPA

47 TPU to BPA

48 UNEC to BPA

49 Wanapum: Regional Totals

(1)

-Rock Island P.H. \#1 CER for Canada-

$50 \mathrm{CHPD}$ to BPA

51 PSE to BPA

52 Rock Island P.H. \#1: Regional Totals

$\begin{array}{rrrrrrrrrr}2.4 & 2.4 & 2.3 & 2.2 & 2.2 & 1.5 & 1.3 & 1.2 & 1.2 & 1.1 \\ 0.8 & 0.8 & 0.7 & 0.7 & 0.7 & 0.5 & 0.4 & 0.4 & 0.4 & 0.4 \\ 0 & 0 & 0 & 0 & 0 & 0 & 0 & 0 & 0 & 0 \\ 0.7 & 0.7 & 0.6 & 0.6 & 0.6 & 0.4 & 0.4 & 0.3 & 0.3 & 0.3 \\ 0.2 & 0.2 & 0.2 & 0.2 & 0.2 & 0.1 & 0.1 & 0.1 & 0.1 & 0.1 \\ 0 & 0 & 0 & 0 & 0 & 0 & 0 & 0 & 0 & 0 \\ 11 & 11 & 10 & 9.9 & 9.8 & 15 & 17 & 17 & 17 & 17 \\ 0 & 0 & 0 & 0 & 0 & 0 & 0 & 0 & 0 & 0 \\ 0 & 0 & 0 & 0 & 0 & 0 & 0 & 0 & 0 & 0 \\ 0 & 0 & 0 & 0 & 0 & 0 & 0.1 & 0.1 & 0.1 & 0 \\ 0 & 0 & 0 & 0 & 0 & 0 & 0 & 0 & 0 & 0 \\ 0 & 0 & 0 & 0 & 0 & 0 & 0.1 & 0.1 & 0.1 & 0.1 \\ 0.2 & 0.2 & 0.2 & 0.2 & 0.2 & 0.1 & 0.1 & 0.1 & 0.1 & 0.1 \\ 0.2 & 0.2 & 0.2 & 0.2 & 0.2 & 0.1 & 0.1 & 0.1 & 0.1 & 0.1 \\ 0 & 0 & 0 & 0 & 0 & 0 & 0 & 0 & 0 & 0 \\ 5.5 & 5.4 & 5.2 & 5.1 & 5 & 3.3 & 2.9 & 2.8 & 2.7 & 2.6 \\ 5.5 & 5.4 & 5.2 & 5.1 & 5 & 3.3 & 2.9 & 2.8 & 2.7 & 2.6 \\ 3.2 & 3.1 & 3.0 & 2.9 & 2.9 & 1.9 & 1.6 & 1.6 & 1.6 & 1.5 \\ 0 & 0 & 0 & 0 & 0 & 0 & 0 & 0 & 0 & 0 \\ 0 & 0 & 0 & 0 & 0 & 0.5 & 0.7 & 0.7 & 0.7 & 0.6 \\ 0 & 0 & 0 & 0 & 0 & 0 & 0 & 0 & 0 & 0 \\ 0 & 0 & 0 & 0 & 0 & 0.5 & 0.7 & 0.7 & 0.7 & 0.6 \\ 0 & 0 & 0 & 0 & 0 & 0 & 0 & 0 & 0 & 0 \\ 29 & 29 & \mathbf{2 8} & \mathbf{2 7} & \mathbf{2 7} & \mathbf{2 7} & \mathbf{2 9} & \mathbf{2 7} & \mathbf{2 8} & \mathbf{2 7}\end{array}$

-Rock Island P.H. \#2 CER for Canada-

$53 \mathrm{CHPD}$ to BPA

54 PSE to BPA

55 Rock Island P.H. \#2: Regional Totals

\begin{tabular}{|c|c|c|c|c|c|c|c|c|c|}
\hline $\begin{array}{l}5.7 \\
5.7\end{array}$ & $\begin{array}{l}5.6 \\
5.6\end{array}$ & $\begin{array}{l}5.4 \\
5.4\end{array}$ & $\begin{array}{l}5.2 \\
5.2\end{array}$ & $\begin{array}{l}5.2 \\
5.2\end{array}$ & $\begin{array}{l}5.3 \\
5.3\end{array}$ & $\begin{array}{l}5.5 \\
5.5\end{array}$ & $\begin{array}{l}5.5 \\
5.5\end{array}$ & $\begin{array}{l}5.4 \\
5.4\end{array}$ & $\begin{array}{l}5.3 \\
5.3\end{array}$ \\
\hline 11 & 11 & 11 & 10 & 10 & 11 & 11 & 11 & 11 & 11 \\
\hline
\end{tabular}

-Rocky Reach CER for Canada-

56 AVWP to BPA

$57 \mathrm{CHPD}$ to BPA

58 CLKM to BPA

59 DOPD to BPA

60 PGE to BPA

$61 \mathrm{PPL}$ to BPA

62 PSE to BPA

\begin{tabular}{llllllllll}
3.6 & 3.6 & 3.4 & 3.3 & 3.3 & 3.4 & 3.5 & 3.5 & 3.4 & 3.4 \\
3.6 & 3.6 & 3.4 & 3.3 & 3.3 & 3.4 & 3.5 & 3.5 & 3.4 & 3.4 \\
\hline $\mathbf{7 . 3}$ & $\mathbf{7 . 1}$ & $\mathbf{6 . 8}$ & $\mathbf{6 . 7}$ & $\mathbf{6 . 6}$ & $\mathbf{6 . 8}$ & $\mathbf{7 . 1}$ & $\mathbf{7 . 0}$ & $\mathbf{6 . 9}$ & $\mathbf{6 . 8}$
\end{tabular}

\begin{tabular}{rrrrrrrrrr}
1.1 & 1.1 & 1.1 & 1 & 1 & 1 & 1.1 & 1.1 & 1.1 & 1 \\
5.8 & 5.7 & 5.5 & 5.4 & 5.3 & 5.4 & 5.7 & 5.6 & 5.5 & 5.5 \\
8.9 & 8.7 & 8.4 & 8.2 & 8.1 & 8.3 & 8.7 & 8.5 & 8.4 & 8.3 \\
1 & 1 & 1.0 & 1.0 & 1.0 & 1.0 & 1 & 1 & 1.0 & 1.0 \\
4.7 & 4.6 & 4.4 & 4.3 & 4.3 & 4.4 & 4.6 & 4.5 & 4.4 & 4.4 \\
2 & 2 & 1.9 & 1.9 & 1.9 & 1.9 & 2.0 & 2.0 & 1.9 & 1.9 \\
15 & 15 & 14 & 14 & 14 & 14 & 15 & 14 & 14 & 14 \\
\hline 39 & $\mathbf{3 8}$ & $\mathbf{3 6}$ & $\mathbf{3 6}$ & $\mathbf{3 5}$ & $\mathbf{3 6}$ & $\mathbf{3 8}$ & $\mathbf{3 7}$ & $\mathbf{3 7}$ & $\mathbf{3 6}$
\end{tabular}


Table A-15: Canadian Entitlement Return For Canada

PNW Loads and Resources Study

2005 - 2014 Operating Years

2003 White Book

-Wells CER for Canada-

64 AVWP to BPA

65 DOPD to BPA

66 OKPD to BPA

67 PGE to BPA

$68 \mathrm{PPL}$ to BPA

69 PSE to BPA

70 Wells: Regional Totals

\begin{tabular}{rrrrrrrrrr}
$\mathbf{2 0 0 5}$ & $\mathbf{2 0 0 6}$ & $\mathbf{2 0 0 7}$ & $\mathbf{2 0 0 8}$ & $\mathbf{2 0 0 9}$ & $\mathbf{2 0 1 0}$ & $\mathbf{2 0 1 1}$ & $\mathbf{2 0 1 2}$ & $\mathbf{2 0 1 3}$ & $\mathbf{2 0 1 4}$ \\
Avg. & Avg. & Avg. & Avg. & Avg. & Avg. & Avg. & Avg. & Avg. & Avg. \\
& & & & & & & & & \\
\\
0.9 & 0.9 & 0.8 & 0.8 & 0.8 & 0.8 & 0.9 & 0.8 & 0.8 & 0.8 \\
7.5 & 7.3 & 7.1 & 6.9 & 6.8 & 7.0 & 7.3 & 7.2 & 7.1 & 7 \\
2 & 2.0 & 1.9 & 1.8 & 1.8 & 1.9 & 1.9 & 1.9 & 1.9 & 1.9 \\
5.1 & 5.0 & 4.8 & 4.7 & 4.6 & 4.7 & 4.9 & 4.9 & 4.8 & 4.7 \\
1.7 & 1.7 & 1.6 & 1.6 & 1.6 & 1.6 & 1.7 & 1.7 & 1.6 & 1.6 \\
7.8 & 7.7 & 7.4 & 7.2 & 7.1 & 7.3 & 7.6 & 7.5 & 7.4 & 7.3 \\
\hline $\mathbf{2 5}$ & $\mathbf{2 4}$ & $\mathbf{2 4}$ & $\mathbf{2 3}$ & $\mathbf{2 3}$ & $\mathbf{2 3}$ & $\mathbf{2 4}$ & $\mathbf{2 4}$ & $\mathbf{2 4}$ & $\mathbf{2 3}$
\end{tabular}

-Total CER For Canada-

71 Federal System

72 Public Entities

73 Investor-Owned Entities

74 Other Entities

75 Non-NW Entities

76 Total CER For Canada

$\begin{array}{rrrr}0 & 0 & 0 & \\ 56 & 60 & 60 & \\ 77 & 71 & 66 & \\ 8.9 & 8.7 & 8.4 & \\ 0 & 0 & 0 & \\ 0 & 139 & 134 & 131\end{array}$

$\begin{array}{rrrr}0 & 0 & 0 & \\ 58 & 58 & 62 & 66 \\ 65 & 64 & 62 & 64 \\ 8.2 & 8.1 & 8.3 & 8.7 \\ 0 & 0 & 0 & \\ 131 & 130 & 133 & 139\end{array}$

\begin{tabular}{rrrr}
0 & 0 & 0 & 0 \\
66 & 65 & 65 & 64 \\
64 & 63 & 62 & 61 \\
8.7 & 8.5 & 8.4 & 8.3 \\
0 & 0 & 0 & 0 \\
\hline $\mathbf{3 9}$ & $\mathbf{1 3 7}$ & $\mathbf{1 3 5}$ & $\mathbf{1 3 3}$
\end{tabular}


Table A-16: Regional Intra-Regional Transfers

PNW Loads and Resources Study

2005 - 2014 Operating Years

2003 White Book

2006

2007

2008

2009

2010

2011

2012

2013

2014

-Intra-Regional Transfers.

1 AVWP To BPA WP3Set

2 AVWP To PGE CapS

3 BPA To AVWP WP3Set

4 BPA TO EWEB PwrS

5 BPA To Other Entities

6 BPA To PPL S/N/X

7 BPA To PPL CapS

8 BPA To PPL S/Pwr/X

9 BPA TO PPL PwrS

10 BPA To PSE PwrS

11 BPA To PSE WP3Set

12 CHPD To PGE C/N/X

13 CIDF To UPL PwrS

14 FPLEV To PGE PwrS

15 GCPD To PPL PwrS

16 NWCP To PSE PwrS

17 NWE To MVP PwrS

18 NWE To PSE PwrS

19 Other Entities To AVWP

20 Other Entities To BPA

21 Other Entities To GHPD

22 PGE To AVWP PkRepl

23 PGE To CHPD PkRepl

24 PGE To CHPD XchgNrg

25 PGE To PPL Cove Repl

26 PKE To SCL PwrS

27 POPD To SCL PwrS

28 PPL To AVWP S/Pwr/X

29 PPL To BPA S/Pwr/X

$30 \mathrm{PPL}$ To BPA S/N/X

31 PPL To BPA PkRepl

32 PPL To FLHD PwrS

33 PPL To SUB PwrS

34 PSE To BPA WP3Set

35 SCL To POPD PwrS

36 SHPD To PSE PwrS

37 TAUC To AVWP PwrS

38 TPU To BPA PwrX

39 WSPE To PGE PwrS

40 GCPD To AVWP PwrS

41 GCPD To COPD PwrS

42 GCPD To EWEB PwrS

43 GCPD To FGRV PwrS

44 GCPD To FREC PwrS

45 GCPD To KITT PwrS

46 GCPD To KOOT PwrS

47 GCPD To LVE PwrS

48 GCPD To LVE PwrS

49 GCPD To MCMN PwrS

50 GCPD To MTFR PwrS

51 GCPD To PGE PwrS

52 GCPD To PPL PwrS

53 GCPD To PSE PwrS

54 GCPD To TPU PwrS

\begin{tabular}{|c|c|c|c|c|c|c|c|c|c|}
\hline 42 & 42 & 42 & 42 & 42 & 42 & 42 & 42 & 42 & 42 \\
\hline 45 & 45 & 45 & 45 & 45 & 45 & 45 & 45 & 45 & 45 \\
\hline 42 & 42 & 42 & 42 & 42 & 42 & 42 & 42 & 42 & 42 \\
\hline 2 & 2 & 0.3 & 0 & 0 & 0 & 0 & 0 & 0 & 0 \\
\hline 139 & 41 & 8.9 & 0 & 0 & 0 & 0 & 0 & 0 & 0 \\
\hline 0 & 0 & 0 & 0 & 0 & 0 & 0 & 0 & 0 & 0 \\
\hline 186 & 186 & 186 & 186 & 186 & 186 & 186 & 15 & 0 & 0 \\
\hline 5.7 & 5.7 & 5.7 & 5.7 & 5.7 & 5.7 & 5.7 & 5.7 & 5.7 & 0 \\
\hline 94 & 94 & 94 & 94 & 94 & 94 & 94 & 94 & 94 & 94 \\
\hline 0 & 0 & 0 & 0 & 0 & 0 & 0 & 0 & 0 & 0 \\
\hline 42 & 42 & 42 & 42 & 42 & 42 & 42 & 42 & 42 & 42 \\
\hline 10 & 10 & 10 & 10 & 10 & 10 & 6 & 0 & 0 & 0 \\
\hline 4.3 & 4.3 & 4.3 & 4.3 & 4.3 & 4.3 & 4.3 & 4.3 & 4.3 & 4.3 \\
\hline 7.4 & 7.4 & 7.4 & 7.4 & 7.4 & 7.4 & 7.4 & 7.4 & 7.4 & 7.4 \\
\hline 10.0 & 10.0 & 10.0 & 10.0 & 10.0 & 10.0 & 10.0 & 10.0 & 10.0 & 10.0 \\
\hline 4.4 & 4.4 & 4.4 & 4.4 & 4.4 & 4.4 & 4.4 & 4.4 & 1.9 & 0 \\
\hline 9.6 & 9.6 & 9.6 & 9.6 & 9.6 & 9.6 & 9.6 & 9.6 & 9.6 & 9.6 \\
\hline 89 & 82 & 89 & 89 & 82 & 89 & 37 & 0 & 0 & 0 \\
\hline 100 & 100 & 100 & 100 & 100 & 100 & 42 & 0 & 0 & 0 \\
\hline 1064 & 907 & 228 & 0 & 0 & 0 & 0 & 0 & 0 & 0 \\
\hline 15 & 15 & 2.5 & 0 & 0 & 0 & 0 & 0 & 0 & 0 \\
\hline 45 & 45 & 45 & 45 & 45 & 45 & 45 & 45 & 45 & 45 \\
\hline 7.4 & 7.4 & 7.4 & 7.4 & 7.4 & 7.4 & 4.5 & 0 & 0 & 0 \\
\hline 12 & 12 & 12 & 12 & 12 & 12 & 8.3 & 0 & 0 & 0 \\
\hline 1 & 1 & 1 & 1 & 1 & 0.9 & 0 & 0 & 0 & 0 \\
\hline 95 & 95 & 40 & 0 & 0 & 0 & 0 & 0 & 0 & 0 \\
\hline 9 & 0 & 0 & 0 & 0 & 0 & 0 & 0 & 0 & 0 \\
\hline 0 & 0 & 0 & 0 & 0 & 0 & 0 & 0 & 0 & 0 \\
\hline 5.8 & 5.8 & 5.8 & 5.8 & 5.8 & 5.8 & 5.8 & 5.8 & 5.8 & 5.8 \\
\hline 0 & 0 & 0 & 0 & 0 & 0 & 0 & 0 & 0 & 0 \\
\hline 186 & 186 & 186 & 186 & 186 & 186 & 186 & 15 & 0 & 0 \\
\hline 16 & 16 & 2.7 & 0 & 0 & 0 & 0 & 0 & 0 & 0 \\
\hline 28 & 28 & 28 & 28 & 28 & 28 & 28 & 28 & 28 & 28 \\
\hline 42 & 42 & 42 & 42 & 42 & 42 & 42 & 42 & 42 & 42 \\
\hline 32 & 43 & 43 & 43 & 43 & 43 & 43 & 0 & 0 & 0 \\
\hline 11 & 11 & 11 & 11 & 11 & 6.8 & 0 & 0 & 0 & 0 \\
\hline 174 & 181 & 181 & 181 & 181 & 177 & 174 & 181 & 181 & 181 \\
\hline 26 & 26 & 5.8 & 0 & 0 & 0 & 0 & 0 & 0 & 0 \\
\hline 41 & 41 & 41 & 41 & 41 & 41 & 41 & 41 & 41 & 41 \\
\hline 0 & 15 & 22 & 22 & 22 & 21 & 20 & 3 & 0 & 0 \\
\hline 0 & 5.2 & 7.2 & 7.3 & 7.3 & 6.8 & 6.8 & 1 & 0 & 0 \\
\hline 0 & 4.2 & 6.1 & 6.2 & 6.2 & 5.9 & 5.8 & 0.8 & 0 & 0 \\
\hline 0 & 1.2 & 1.9 & 1.9 & 2 & 1.6 & 1.4 & 0.3 & 0 & 0 \\
\hline 0 & 0.3 & 0.5 & 0.7 & 0.7 & 0.7 & 0.7 & 0.2 & 0 & 0 \\
\hline 0 & 0.9 & 1.2 & 1.2 & 1.2 & 0.8 & 0.6 & 0.1 & 0 & 0 \\
\hline 0 & 0.8 & 1 & 1 & 1 & 1 & 1 & 0.2 & 0 & 0 \\
\hline 0 & 0.8 & 1 & 1 & 1 & 1 & 1 & 0.2 & 0 & 0 \\
\hline 0 & 0.8 & 1 & 1 & 0.2 & 0 & 0 & 0 & 0 & 0 \\
\hline 0 & 1.2 & 1.9 & 1.9 & 2 & 1.6 & 1.4 & 0.3 & 0 & 0 \\
\hline 0 & 1.2 & 1.9 & 1.9 & 2 & 1.6 & 1.4 & 0.3 & 0 & 0 \\
\hline 0 & 34 & 50 & 51 & 51 & 48 & 47 & 6.8 & 0 & 0 \\
\hline 0 & 34 & 50 & 51 & 51 & 48 & 47 & 6.8 & 0 & 0 \\
\hline 0 & 20 & 28 & 29 & 29 & 28 & 27 & 3.9 & 0 & 0 \\
\hline 0 & 16 & 23 & 23 & 23 & 14 & 12 & 1.7 & 0 & 0 \\
\hline 2645 & 2527 & 1780 & 1495 & 1488 & 1468 & 1330 & 705 & 647 & 639 \\
\hline
\end{tabular}

-Total Contracts Out-

56 Federal System

57 Public Entities

58 Investor-Owned Entities

59 Other Entities

\begin{tabular}{rrrr}
512 & 413 & 380 & \\
107 & 244 & 284 & \\
898 & 896 & 729 & \\
129 & 973 & 386 & \\
\hline 645 & $\mathbf{2 5 2 7}$ & $\mathbf{1 7 8 0}$ & $\mathbf{1 4 9 5}$
\end{tabular}

60 Total Contracts Out

2645

2527

$\begin{array}{rrr}1376 & 1220 & 519 \\ 221 & 252 & 183 \\ 928 & 1018 & 1069 \\ 120 & 38 & 8.3\end{array}$

\begin{tabular}{rrrrrrr}
370 & 370 & 370 & 370 & 199 & 184 & 178 \\
282 & 281 & 258 & 242 & 44 & 16 & 14 \\
694 & 687 & 691 & 627 & 413 & 398 & 398 \\
149 & 149 & 149 & 91 & 49 & 49 & 49 \\
\hline 1495 & 1488 & 1468 & 1330 & $\mathbf{7 0 5}$ & $\mathbf{6 4 7}$ & $\mathbf{6 3 9}$
\end{tabular}

-Total Contracts In-

61 Federal System

62 Public Entities

63 Investor-Owned Entities

64 Other Entities

2645

$2527 \quad 1780$

$1780 \quad 1495$

$\begin{array}{r}285 \\ 139 \\ 1071 \\ 0 \\ \hline\end{array}$

$\begin{array}{rrr}286 & 286 & 286 \\ 138 & 127 & 116 \\ 1064 & 1056 & 928 \\ 0 & 0 & \end{array}$

$\begin{array}{rrrr}286 & 115 & 100 & 100 \\ 116 & 33 & 28 & 28 \\ 928 & 557 & 519 & 511 \\ 0 & 0 & 0 & 0 \\ 330 & 705 & 647 & \mathbf{6 3 9}\end{array}$


Table A-17: Regional Capacity Reserves

PNW Loads and Resources Study

2005 - 2014 Operating Years

1937 Water Year

2003 White Book

Average Energy in Megawatts $\quad 2005 \quad 2006 \quad 2007 \quad 2008 \quad 2009 \quad 2010 \quad 2011 \quad 2012 \quad 2013 \quad 2014$ Avg. Avg. Avg. Avg. Avg. Avg. Avg. Avg. Avg. Avg.

Hydro Reserves

-Federal Entities-

10 BPA - Power Business

$\begin{array}{llllllllll}0 & 0 & 0 & 0 & 0 & 0 & 0 & 0 & 0 & 0 \\ 0 & 0 & 0 & 0 & 0 & 0 & 0 & 0 & 0 & 0\end{array}$

-Generating Public Entities-

120 Chelan County PUD \#1

121 Clark County PUD \#1

122 Cowlitz County PUD \#1

123 Douglas County PUD \#1

124 Eugene Water \& Electric Board

125 Grant County PUD \#2

126 Grays Harbor PUD \#1

127 Okanogan County PUD \#1

128 Pend Oreille County PUD \#1

129 Seattle City Light

130 Snohomish County PUD \#1

131 Tacoma Public Utilities

132 Total Generating Public Entities

\begin{tabular}{|c|c|c|c|c|c|c|c|c|c|}
\hline 0 & 0 & 0 & 0 & 0 & 0 & 0 & 0 & & 0 \\
\hline 0 & 0 & 0 & 0 & 0 & 0 & 0 & 0 & & 0 \\
\hline 0 & 0 & 0 & 0 & 0 & 0 & 0 & 0 & & 0 \\
\hline 0 & 0 & 0 & 0 & 0 & 0 & 0 & 0 & & 0 \\
\hline 0 & 0 & 0 & 0 & 0 & 0 & 0 & 0 & & 0 \\
\hline 0 & 0 & 0 & 0 & 0 & 0 & 0 & 0 & & 0 \\
\hline 0 & 0 & 0 & 0 & 0 & 0 & 0 & 0 & & 0 \\
\hline 0 & 0 & 0 & 0 & 0 & 0 & 0 & 0 & & 0 \\
\hline 0 & 0 & 0 & 0 & 0 & 0 & 0 & 0 & & 0 \\
\hline 0 & 0 & 0 & 0 & 0 & 0 & 0 & 0 & & 0 \\
\hline 0 & 0 & 0 & 0 & 0 & 0 & 0 & 0 & & 0 \\
\hline 0 & 0 & 0 & 0 & 0 & 0 & 0 & 0 & & 0 \\
\hline 0 & 0 & 0 & 0 & 0 & 0 & 0 & 0 & & 0 \\
\hline
\end{tabular}

-Non-Generating Public Entities-

430 Ashland, City of

431 Benton County PUD \#1

432 Bonners Ferry, City of

433 Centralia, City of

434 Clallam County PUD \#1

435 Clearwater Power

436 Consumers Power

437 Emerald County PUD

438 Energy Northwest

439 Fall River Elec Coop

440 Forest Grove, City of

441 Franklin County PUD \#1

442 Idaho County L \& P

443 Idaho Falls, City of

444 Kittitas County PUD \#1

445 Klickitat County PUD \#1

446 Kootenai Electric Coop

447 Lewis County PUD \#1

448 Lost River Elec Coop

449 Lower Valley Energy

450 Mason County PUD \#1

451 McMinnville, City of

452 Milton Freewater, City of

453 Northern Lights

454 Northern Wasco County PUD

455 Oregon Trail Coop

456 Port Angeles, City of

457 Raft River Elec Coop

458 Salmon River Elec Coop

459 Soda Springs, City of

460 Tanner Elec Coop

461 Tillamook PUD \#1

462 United Electric Coop

463 Total Non-Generating Public Entities

$\begin{array}{llllllllll}0 & 0 & 0 & 0 & 0 & 0 & 0 & 0 & 0 & 0 \\ 0 & 0 & 0 & 0 & 0 & 0 & 0 & 0 & 0 & 0 \\ 0 & 0 & 0 & 0 & 0 & 0 & 0 & 0 & 0 & 0 \\ 0 & 0 & 0 & 0 & 0 & 0 & 0 & 0 & 0 & 0 \\ 0 & 0 & 0 & 0 & 0 & 0 & 0 & 0 & 0 & 0 \\ 0 & 0 & 0 & 0 & 0 & 0 & 0 & 0 & 0 & 0 \\ 0 & 0 & 0 & 0 & 0 & 0 & 0 & 0 & 0 & 0 \\ 0 & 0 & 0 & 0 & 0 & 0 & 0 & 0 & 0 & 0 \\ 0 & 0 & 0 & 0 & 0 & 0 & 0 & 0 & 0 & 0 \\ 0 & 0 & 0 & 0 & 0 & 0 & 0 & 0 & 0 & 0 \\ 0 & 0 & 0 & 0 & 0 & 0 & 0 & 0 & 0 & 0 \\ 0 & 0 & 0 & 0 & 0 & 0 & 0 & 0 & 0 & 0 \\ 0 & 0 & 0 & 0 & 0 & 0 & 0 & 0 & 0 & 0 \\ 0 & 0 & 0 & 0 & 0 & 0 & 0 & 0 & 0 & 0 \\ 0 & 0 & 0 & 0 & 0 & 0 & 0 & 0 & 0 & 0 \\ 0 & 0 & 0 & 0 & 0 & 0 & 0 & 0 & 0 & 0 \\ 0 & 0 & 0 & 0 & 0 & 0 & 0 & 0 & 0 & 0 \\ 0 & 0 & 0 & 0 & 0 & 0 & 0 & 0 & 0 & 0 \\ 0 & 0 & 0 & 0 & 0 & 0 & 0 & 0 & 0 & 0 \\ 0 & 0 & 0 & 0 & 0 & 0 & 0 & 0 & 0 & 0 \\ 0 & 0 & 0 & 0 & 0 & 0 & 0 & 0 & 0 & 0 \\ 0 & 0 & 0 & 0 & 0 & 0 & 0 & 0 & 0 & 0 \\ 0 & 0 & 0 & 0 & 0 & 0 & 0 & 0 & 0 & 0 \\ 0 & 0 & 0 & 0 & 0 & 0 & 0 & 0 & 0 & 0 \\ 0 & 0 & 0 & 0 & 0 & 0 & 0 & 0 & 0 & 0 \\ 0 & 0 & 0 & 0 & 0 & 0 & 0 & 0 & 0 & 0 \\ 0 & 0 & 0 & 0 & 0 & 0 & 0 & 0 & 0 & 0 \\ 0 & 0 & 0 & 0 & 0 & 0 & 0 & 0 & 0 & 0 \\ 0 & 0 & 0 & 0 & 0 & 0 & 0 & 0 & 0 & 0 \\ 0 & 0 & 0 & 0 & 0 & 0 & 0 & 0 & 0 & 0 \\ 0 & 0 & 0 & 0 & 0 & 0 & 0 & 0 & 0 & 0 \\ 0 & 0 & 0 & 0 & 0 & 0 & 0 & 0 & 0 & 0 \\ 0 & 0 & 0 & 0 & 0 & 0 & 0 & 0 & 0 & 0 \\ 0 & 0 & 0 & 0 & 0 & 0 & 0 & 0 & 0 \\ 0 & 0 & 0 & 0 & 0 & 0 & 0 & 0 \\ 0\end{array}$

-Investor-Owned Entities-

518 Avista Corp (WWP Division)

519 Idaho Power Company

520 Northwestern Energy LLC (MPC)

521 Pacific Power

522 Portland General Electric

523 Puget Sound Energy

524 Total Investor-Owned Entities

-Other Entities-

552 Colockum Transmission Company

553 Longview Fibre

554 Warm Springs Power Enterprise

555 Total Other Entities

\begin{tabular}{lllllllllll} 
& 0 & 0 & 0 & 0 & 0 & 0 & 0 & 0 & 0 & 0 \\
& 0 & 0 & 0 & 0 & 0 & 0 & 0 & 0 & 0 & 0 \\
& 0 & 0 & 0 & 0 & 0 & 0 & 0 & 0 & 0 & 0 \\
& 0 & 0 & 0 & 0 & 0 & 0 & 0 & 0 & 0 & 0 \\
& 0 & 0 & 0 & 0 & 0 & 0 & 0 & 0 & 0 & 0 \\
& 0 & 0 & 0 & 0 & 0 & 0 & 0 & 0 & 0 & 0 \\
\hline & 0 & 0 & 0 & 0 & 0 & 0 & 0 & 0 & 0 & $\mathbf{0}$
\end{tabular}

Small Thermal \& Misc. Reserves

-Federal Entities-

583 BPA - Power Business

0

0

0

0

0

0

0

0

0 
2006 2003 White Book

584 U.S. Naval Station, Everett (Jim Creek) 585 U.S. Naval Submarine Base, Bangor 586 Total Federal Entities

2007

2008

2009

2010

2011

2012

2013

2014 Avg. Avg. Avg. Avg. Avg.

-Generating Public Entities695 Chelan County PUD \#1 696 Clark County PUD \#1 697 Cowlitz County PUD \#1 698 Douglas County PUD \#1 699 Eugene Water \& Electric Board 700 Grant County PUD \#2

701 Grays Harbor PUD \#1

702 Okanogan County PUD \#1

703 Seattle City Light

704 Snohomish County PUD \#1

705 Springfield Utility Board

706 Tacoma Public Utilities

707 Total Generating Public Entities

\begin{tabular}{llllllllll}
0 & 0 & 0 & 0 & 0 & 0 & 0 & 0 & 0 & 0 \\
0 & 0 & 0 & 0 & 0 & 0 & 0 & 0 & 0 & 0 \\
\hline & 0 & 0 & 0 & 0 & 0 & 0 & 0 & 0 & 0
\end{tabular}

-Non-Generating Public Entities-

852 Ashland, City of

853 Benton County PUD \#1

854 Clallam County PUD \#1

855 Clatskanie PUD

856 Consumers Power

857 Emerald County PUD

858 Energy Northwest

859 Fall River Elec Coop

860 Franklin County PUD \#1

861 Klickitat County PUD \#1

862 Lewis County PUD \#1

863 Mason County PUD \#1

864 Mason County PUD \#3

865 Oregon Trail Coop

866 Tanner Elec Coop

867 Tillamook PUD \#1

868 Total Non-Generating Public Entities

$\begin{array}{llllllllll}0 & 0 & 0 & 0 & 0 & 0 & 0 & 0 & 0 & 0 \\ 0 & 0 & 0 & 0 & 0 & 0 & 0 & 0 & 0 & 0 \\ 0 & 0 & 0 & 0 & 0 & 0 & 0 & 0 & 0 & 0 \\ 0 & 0 & 0 & 0 & 0 & 0 & 0 & 0 & 0 & 0 \\ 0 & 0 & 0 & 0 & 0 & 0 & 0 & 0 & 0 & 0 \\ 0 & 0 & 0 & 0 & 0 & 0 & 0 & 0 & 0 & 0 \\ 0 & 0 & 0 & 0 & 0 & 0 & 0 & 0 & 0 & 0 \\ 0 & 0 & 0 & 0 & 0 & 0 & 0 & 0 & 0 & 0 \\ 0 & 0 & 0 & 0 & 0 & 0 & 0 & 0 & 0 & 0 \\ 0 & 0 & 0 & 0 & 0 & 0 & 0 & 0 & 0 & 0 \\ 0 & 0 & 0 & 0 & 0 & 0 & 0 & 0 & 0 & 0 \\ 0 & 0 & 0 & 0 & 0 & 0 & 0 & 0 & 0 & 0 \\ 0 & 0 & 0 & 0 & 0 & 0 & 0 & 0 & 0 & 0\end{array}$

-Investor-Owned Entities-

941 Avista Corp (WWP Division)

942 Idaho Power Company

943 Northwestern Energy LLC (MPC)

944 Pacific Power

945 Portland General Electric

946 Puget Sound Energy

947 Trans Alta Utilities Corp

948 Utah Power

949 Total Investor-Owned Entities

$\begin{array}{llllllllll}0 & 0 & 0 & 0 & 0 & 0 & 0 & 0 & 0 & 0 \\ 0 & 0 & 0 & 0 & 0 & 0 & 0 & 0 & 0 & 0 \\ 0 & 0 & 0 & 0 & 0 & 0 & 0 & 0 & 0 & 0 \\ 0 & 0 & 0 & 0 & 0 & 0 & 0 & 0 & 0 & 0 \\ 0 & 0 & 0 & 0 & 0 & 0 & 0 & 0 & 0 & 0 \\ 0 & 0 & 0 & 0 & 0 & 0 & 0 & 0 & 0 & 0 \\ 0 & 0 & 0 & 0 & 0 & 0 & 0 & 0 & 0 & 0 \\ 0 & 0 & 0 & 0 & 0 & 0 & 0 & 0 & 0 & 0 \\ 0 & 0 & 0 & 0 & 0 & 0 & 0 & 0 & 0 & 0 \\ 0 & 0 & 0 & 0 & 0 & 0 & 0 & 0 & 0 & 0 \\ 0 & 0 & 0 & 0 & 0 & 0 & 0 & 0 & 0 & 0 \\ 0 & 0 & 0 & 0 & 0 & 0 & 0 & 0 & 0 & 0 \\ 0 & 0 & 0 & 0 & 0 & 0 & 0 & 0 & 0 & 0 \\ 0 & 0 & 0 & 0 & 0 & 0 & 0 & 0 & 0 & 0 \\ 0 & 0 & 0 & 0 & 0 & 0 & 0 & 0 & 0 & 0 \\ 0 & 0 & 0 & 0 & 0 & 0 & 0 & 0 & 0 & 0 \\ 0 & 0 & \mathbf{0} & \mathbf{0} & \mathbf{0} & \mathbf{0} & \mathbf{0} & \mathbf{0} & \mathbf{0} & \mathbf{0}\end{array}$

-Other Entities-

1040 Avista Energy Inc.

1041 Chehalis Power Inc.

1042 FPL Energy Vansycle LLC

1043 Frederickson Power LP,

1044 Hermiston Power Partnership

1045 Klamath Pacific Energy

1046 Longview Fibre

1047 Mirant Americas Energy Marketing, L.P.

1048 PPM Energy

1049 SP Newsprin

1050 Total Other Entities

$\begin{array}{llllllllll}0 & 0 & 0 & 0 & 0 & 0 & 0 & 0 & 0 & 0 \\ 0 & 0 & 0 & 0 & 0 & 0 & 0 & 0 & 0 & 0 \\ 0 & 0 & 0 & 0 & 0 & 0 & 0 & 0 & 0 & 0 \\ 0 & 0 & 0 & 0 & 0 & 0 & 0 & 0 & 0 & 0 \\ 0 & 0 & 0 & 0 & 0 & 0 & 0 & 0 & 0 & 0 \\ 0 & 0 & 0 & 0 & 0 & 0 & 0 & 0 & 0 & 0 \\ 0 & 0 & 0 & 0 & 0 & 0 & 0 & 0 & 0 & 0 \\ 0 & 0 & 0 & 0 & 0 & 0 & 0 & 0 & 0 & 0 \\ 0 & 0 & 0 & 0 & 0 & 0 & 0 & 0 & 0 & 0\end{array}$

Large Thermal Reserves

-Federal Entities-

1060 BPA - Power Business

1061 Total Federal Entities

$\begin{array}{llllllllll}0 & 0 & 0 & 0 & 0 & 0 & 0 & 0 & 0 & 0 \\ 0 & 0 & 0 & 0 & 0 & 0 & 0 & 0 & 0 & 0 \\ 0 & 0 & 0 & 0 & 0 & 0 & 0 & 0 & 0 & 0 \\ 0 & 0 & 0 & 0 & 0 & 0 & 0 & 0 & 0 & 0 \\ 0 & 0 & 0 & 0 & 0 & 0 & 0 & 0 & 0 & 0 \\ 0 & 0 & 0 & 0 & 0 & 0 & 0 & 0 & 0 & 0 \\ 0 & 0 & 0 & 0 & 0 & 0 & 0 & 0 & 0 & 0 \\ 0 & 0 & 0 & 0 & 0 & 0 & 0 & 0 & 0 & 0 \\ 0 & 0 & 0 & 0 & 0 & 0 & 0 & 0 & 0 & 0 \\ 0 & 0 & 0 & 0 & 0 & 0 & 0 & 0 & 0 & 0 \\ 0 & 0 & 0 & 0 & 0 & 0 & 0 & 0 & \mathbf{0} & \mathbf{0}\end{array}$

-Generating Public Entities-

1071 Turlock Irrigation District

1072 Total Generating Public Entities

-Non-Generating Public Entities-

1073 Total Non-Generating Public Entities

0

0

0

0

0

0 


\section{White Book}

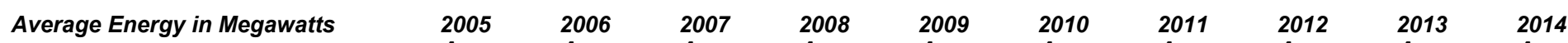

\section{nvestor-Owned Entities}

1147 Idaho Power Company

1148 Northwestern Energy LLC (MPC)

1149 Pacific Power

1150 Portland General Electric

1151 Puget Sound Energy

1152 San Diego Gas \& Electric

1153 Trans Alta Utilities Corp

1154 Total Investor-Owned Entities
1146 Avista Corp (WWP Division)

$\begin{array}{llllllllll}0 & 0 & 0 & 0 & 0 & 0 & 0 & 0 & 0 & 0 \\ 0 & 0 & 0 & 0 & 0 & 0 & 0 & 0 & 0 & 0 \\ 0 & 0 & 0 & 0 & 0 & 0 & 0 & 0 & 0 & 0 \\ 0 & 0 & 0 & 0 & 0 & 0 & 0 & 0 & 0 & 0 \\ 0 & 0 & 0 & 0 & 0 & 0 & 0 & 0 & 0 & 0 \\ 0 & 0 & 0 & 0 & 0 & 0 & 0 & 0 & 0 & 0 \\ 0 & 0 & 0 & 0 & 0 & 0 & 0 & 0 & 0 & 0 \\ 0 & 0 & 0 & 0 & 0 & 0 & 0 & 0 & 0 & 0 \\ 0 & 0 & 0 & 0 & 0 & 0 & 0 & 0 & 0 & 0\end{array}$

-Other Entities-

1155 Total Other Entities

0

0

0

Contract Reserves

-Federal Entities-

1156 Total Federal Entities

0

0

0

0

-Generating Public Entities-

1157 Total Generating Public Entities

0

0

$0 \quad 0$

0

0

0

0

0

-Non-Generating Public Entities-

1158 Total Non-Generating Public Entities

$0 \quad 0$

$0 \quad 0$

0

-Investor-Owned Entities-

1177 Northwestern Energy LLC (MPC)

1178 Pacific Power

1179 Total Investor-Owned Entities

\begin{tabular}{llllllllll}
0 & 0 & 0 & 0 & 0 & 0 & 0 & 0 & 0 & 0 \\
0 & 0 & 0 & 0 & 0 & 0 & 0 & 0 & 0 & 0 \\
\hline $\mathbf{0}$ & 0 & 0 & 0 & 0 & 0 & 0 & 0 & 0 & 0
\end{tabular}

-Other Entities-

1180 Total Other Entities

0

0

0

$\begin{array}{llllllllll}\mathbf{0} & \mathbf{0} & \mathbf{0} & \mathbf{0} & \mathbf{0} & \mathbf{0} & \mathbf{0} & \mathbf{0} & \mathbf{0} & \mathbf{0} \\ & & & & & & & & & \\ 0 & 0 & 0 & 0 & 0 & 0 & 0 & 0 & 0 & 0 \\ 0 & 0 & 0 & 0 & 0 & 0 & 0 & 0 & 0 & 0 \\ 0 & 0 & 0 & 0 & 0 & 0 & 0 & 0 & 0 & 0 \\ 0 & 0 & 0 & 0 & 0 & 0 & 0 & 0 & 0 & 0 \\ \mathbf{0} & \mathbf{0} & \mathbf{0} & \mathbf{0} & \mathbf{0} & \mathbf{0} & \mathbf{0} & \mathbf{0} & \mathbf{0} & \mathbf{0}\end{array}$

-Total Capacity Reserves-

1181 Total Hydro Reserves

1182 Total Small Thermal \& Misc Reserves

1183 Total Large Thermal Reserves

1184 Total Contract Reserves

1185 Total Capacity Reserves 
Table A-18: Wells and Chelan Allocation

PNW Loads and Resources Study

2005 - 2014 Operating Years

1937 Water Year

2003 White Book

\begin{tabular}{|c|c|c|c|c|c|c|c|c|c|c|}
\hline Average Energy in Megawatts & $\begin{array}{c}2005 \\
\text { Avg. }\end{array}$ & $\begin{array}{l}2006 \\
\text { Avg. }\end{array}$ & $\begin{array}{l}2007 \\
\text { Avg. }\end{array}$ & $\begin{array}{l}2008 \\
\text { Avg. }\end{array}$ & $\begin{array}{l}2009 \\
\text { Avg. }\end{array}$ & $\begin{array}{l}2010 \\
\text { Avg. }\end{array}$ & $\begin{array}{l}2011 \\
\text { Avg. }\end{array}$ & $\begin{array}{l}2012 \\
\text { Avg. }\end{array}$ & $\begin{array}{l}2013 \\
\text { Avg. }\end{array}$ & $\begin{array}{l}2014 \\
\text { Avg. }\end{array}$ \\
\hline \multicolumn{8}{|l|}{-Wells- } & & & \\
\hline 2 Wells Before Enc & 395 & 395 & 395 & 395 & 395 & 395 & 395 & 395 & 395 & 395 \\
\hline \multicolumn{11}{|l|}{-Wells- } \\
\hline 3 AVWP Share & 13 & 13 & 13 & 13 & 13 & 13 & 13 & 13 & 13 & 13 \\
\hline 4 DOPD Share & 97 & 97 & 97 & 97 & 97 & 97 & 97 & 97 & 97 & 97 \\
\hline 5 OKPD Share & 28 & 28 & 28 & 28 & 28 & 28 & 28 & 28 & 28 & 28 \\
\hline 6 PGE Share & 74 & 74 & 74 & 74 & 74 & 74 & 74 & 74 & 74 & 74 \\
\hline 7 PPL Share & 25 & 25 & 25 & 25 & 25 & 25 & 25 & 25 & 25 & 25 \\
\hline 8 PSE Share & 113 & 113 & 113 & 113 & 113 & 113 & 113 & 113 & 113 & 113 \\
\hline 9 Wells After Enc & 350 & 350 & 350 & 350 & 350 & 350 & 350 & 350 & 350 & 350 \\
\hline \multicolumn{11}{|l|}{-Chelan- } \\
\hline 10 Chelan & 41 & 41 & 41 & 41 & 41 & 41 & 41 & 41 & 41 & 41 \\
\hline 11 Chelan Before Enc & 41 & 41 & 41 & 41 & 41 & 41 & 41 & 41 & 41 & $\overline{41}$ \\
\hline \multicolumn{11}{|l|}{-Chelan- } \\
\hline 12 CHPD Share & 44 & 44 & 44 & 44 & 44 & 44 & 44 & 44 & 44 & 44 \\
\hline 13 Chelan After Enc & 44 & 44 & 44 & 44 & 44 & 44 & 44 & 44 & 44 & $\overline{44}$ \\
\hline
\end{tabular}


Table A-19: Rocky Reach and Rock Island Allocation

PNW Loads and Resources Study

2005 - 2014 Operating Years

1937 Water Year

2003 White Book

Average Energy in Megawatts $\quad 2005 \quad 2006 \quad 2007 \quad 2008 \quad 2009 \quad 2010 \quad 2011 \quad 2012 \quad 2013 \quad 2014$

Avg. Avg. Avg. Avg. Avg. Avg. Avg. Avg. Avg. Avg.

-Rocky Reach-

1 Rocky Reach

$531-531$

2 Rocky Reach Before Enc

$531 \quad 531$

$531 \quad 531 \quad 531$

$531 \quad 531$

$\begin{array}{llll}531 & 531 & 531 & 531 \\ 531 & \mathbf{5 3 1} & \mathbf{5 3 1} & \mathbf{5 3 1}\end{array}$

-Rocky Reach-

3 AVWP Share

4 CHPD Share

5 CLKM Share

6 DOPD Share

7 PGE Share

8 PPL Share

9 PSE Share

$531 \quad 531$

10 Rocky Reach After Enc

\begin{tabular}{rrrrrrrrrr}
16 & 16 & 16 & 16 & 16 & 16 & 16 & 16 & 16 & 16 \\
83 & 83 & 83 & 83 & 83 & 83 & 83 & 83 & 83 & 83 \\
126 & 126 & 126 & 126 & 126 & 126 & 126 & 126 & 126 & 126 \\
15 & 15 & 15 & 15 & 15 & 15 & 15 & 15 & 15 & 15 \\
66 & 66 & 66 & 66 & 66 & 66 & 66 & 66 & 66 & 66 \\
29 & 29 & 29 & 29 & 29 & 29 & 29 & 29 & 29 & 29 \\
213 & 213 & 213 & 213 & 213 & 213 & 213 & 213 & 213 & 213 \\
\hline $\mathbf{5 4 8}$ & $\mathbf{5 4 8}$ & $\mathbf{5 4 8}$ & $\mathbf{5 4 8}$ & $\mathbf{5 4 8}$ & $\mathbf{5 4 8}$ & $\mathbf{5 4 8}$ & $\mathbf{5 4 8}$ & $\mathbf{5 4 8}$ & $\mathbf{5 4 8}$
\end{tabular}

-Rock Island PH\#1-

11 Rock Island PH\#1

12 Rock Island PH\#1 Before Enc

$131 \quad 131$

131

$131 \quad 131 \quad 131$

$131 \quad 131 \quad 131$

\begin{tabular}{llll}
131 & 131 & 131 & 131 \\
\hline 131 & 131 & 131 & 131
\end{tabular}

-Rock Island PH\#1-

13 CHPD Share

14 PSE Share

\begin{tabular}{|c|c|c|c|c|c|c|c|c|}
\hline $\begin{array}{l}86 \\
86\end{array}$ & $\begin{array}{l}86 \\
86\end{array}$ & $\begin{array}{l}86 \\
86\end{array}$ & $\begin{array}{l}86 \\
86\end{array}$ & $\begin{array}{l}86 \\
86\end{array}$ & $\begin{array}{l}86 \\
86\end{array}$ & $\begin{array}{l}86 \\
86\end{array}$ & $\begin{array}{l}86 \\
86\end{array}$ & $\begin{array}{l}86 \\
86\end{array}$ \\
\hline 172 & 172 & 172 & 172 & 172 & 172 & 172 & 172 & 172 \\
\hline
\end{tabular}

-Rock Island PH\#2-

16 Rock Island PH\#2

$\begin{array}{llllllllll}90 & 90 & 90 & 90 & 90 & 90 & 90 & 90 & 90 & 90 \\ 90 & 90 & 90 & 90 & 90 & 90 & 90 & 90 & 90 & 90\end{array}$

-Rock Island PH\#2-

18 CHPD Share

19 PSE Share

20 Rock Island PH\#2 After Enc

\begin{tabular}{rrrrrrrrrr}
58 & 58 & 58 & 58 & 58 & 58 & 58 & 58 & 58 & 58 \\
58 & 58 & 58 & 58 & 58 & 58 & 58 & 58 & 58 & 58 \\
\hline 116 & 116 & 116 & 116 & 116 & 116 & 116 & 116 & 116 & 116
\end{tabular}


Table A-20: Wanapum and Priest Rapids Allocation

PNW Loads and Resources Study

2005 - 2014 Operating Years

1937 Water Year

2003 White Book

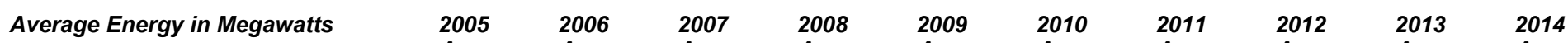
Avg. Avg. Avg. Avg. Avg. Avg. Avg. Avg. Avg.

-Wanapum-

1 Wanapum

$433 \quad 433$

2 Wanapum Before Enc

433

433

433

433

433

433

433

433

433
433

-Wanapum-

3 AVWP Share

4 COPD Share
5 CWPC Share

6 EWEB Share

7 FGRV Share

8 FREC Share

9 GCPD Share

10 ICLP Share

11 KITT Share

12 KOOT Share

13 LREC Share

14 LVE Share

15 MCMN Share

16 MTFR Share

17 NLEC Share

18 PGE Share

19 PPL Share

20 PSE Share

21 RREC Share

22 SCL Share

23 SLEC Share

24 TPU Share

25 UNEC Share

26 Wanapum After Enc

\begin{tabular}{|c|c|c|c|c|c|c|c|c|c|}
\hline 30 & 30 & 30 & 30 & 30 & 20 & 16 & 16 & 15 & 15 \\
\hline 9.8 & 9.8 & 9.8 & 9.8 & 9.8 & 6.4 & 5.2 & 5.2 & 5.1 & 5.0 \\
\hline 0 & 0 & 0 & 0 & 0 & 0.3 & 0.4 & 0.4 & 0.4 & 0.4 \\
\hline 8.3 & 8.3 & 8.3 & 8.3 & 8.3 & 5.5 & 4.4 & 4.4 & 4.3 & 4.2 \\
\hline 2.5 & 2.5 & 2.5 & 2.5 & 2.5 & 1.6 & 1.3 & 1.3 & 1.3 & 1.3 \\
\hline 0 & 0 & 0 & 0 & 0 & 0.3 & 0.5 & 0.4 & 0.4 & 0.4 \\
\hline 132 & 132 & 132 & 132 & 132 & 195 & 217 & 219 & 222 & 224 \\
\hline 0 & 0 & 0 & 0 & 0 & 0.1 & 0.1 & 0.1 & 0.1 & 0.1 \\
\hline 0 & 0 & 0 & 0 & 0 & 0.3 & 0.4 & 0.4 & 0.4 & 0.4 \\
\hline 0 & 0 & 0 & 0 & 0 & 0.5 & 0.7 & 0.7 & 0.7 & 0.6 \\
\hline 0 & 0 & 0 & 0 & 0 & 0.1 & 0.1 & 0.1 & 0.1 & 0.1 \\
\hline 0 & 0 & 0 & 0 & 0 & 0.6 & 0.8 & 0.8 & 0.8 & 0.8 \\
\hline 2.5 & 2.5 & 2.5 & 2.5 & 2.5 & 1.6 & 1.3 & 1.3 & 1.3 & 1.3 \\
\hline 2.5 & 2.5 & 2.5 & 2.5 & 2.5 & 1.6 & 1.3 & 1.3 & 1.3 & 1.3 \\
\hline 0 & 0 & 0 & 0 & 0 & 0.5 & 0.6 & 0.6 & 0.6 & 0.6 \\
\hline 68 & 68 & 68 & 68 & 68 & 44 & 36 & 36 & 35 & 34 \\
\hline 68 & 68 & 68 & 68 & 68 & 44 & 36 & 36 & 35 & 35 \\
\hline 39 & 39 & 39 & 39 & 39 & 26 & 21 & 21 & 20 & 20 \\
\hline 0 & 0 & 0 & 0 & 0 & 0.1 & 0.1 & 0.1 & 0.1 & 0.1 \\
\hline 0 & 0 & 0 & 0 & 0 & 6.7 & 8.9 & 8.8 & 8.6 & 8.5 \\
\hline 0 & 0 & 0 & 0 & 0 & 0.1 & 0.1 & 0.1 & 0.1 & 0.1 \\
\hline 0 & 0 & 0 & 0 & 0 & 6.7 & 8.9 & 8.8 & 8.6 & 8.5 \\
\hline 0 & 0 & 0 & 0 & 0 & 0.2 & 0.2 & 0.2 & 0.2 & 0.2 \\
\hline 362 & 362 & 362 & 362 & 362 & 362 & 362 & 362 & 362 & \\
\hline
\end{tabular}

-Priest Rapids-

27 Priest Rapids

28 Priest Rapids Before Enc

353

$353 \quad 353$

353

353

$353 \quad 353$

353 353

353

353

-Priest Rapids-

29 AVWP Share

30 COPD Share

31 CWPC Share

32 EWEB Share

33 FGRV Share

34 FREC Share

35 GCPD Share

36 ICLP Share

37 KITT Share

38 KOOT Share

39 LREC Share

40 LVE Share

41 MCMN Share

42 MTFR Share

43 NLEC Share

44 PGE Share

45 PPL Share

46 PSE Share

47 RREC Share

48 SCL Share

49 SLEC Share

50 TPU Share

51 UNEC Share

52 Priest Rapids After Enc

\begin{tabular}{|c|c|c|c|c|c|c|c|c|c|}
\hline 21 & 13 & 9.7 & 9.7 & 9.7 & 13 & 15 & 15 & 14 & 14 \\
\hline 6.7 & 4.2 & 3.2 & 3.2 & 3.2 & 4.4 & 4.9 & 4.8 & 4.7 & 4.6 \\
\hline 0 & 0.2 & 0.3 & 0.3 & 0.3 & 0.3 & 0.4 & 0.4 & 0.4 & 0.3 \\
\hline 5.7 & 3.6 & 2.7 & 2.7 & 2.7 & 3.8 & 4.1 & 4.1 & 4 & 3.9 \\
\hline 1.7 & 1 & 0.8 & 0.8 & 0.8 & 1.1 & 1.2 & 1.2 & 1.2 & 1.2 \\
\hline 0 & 0.2 & 0.3 & 0.3 & 0.3 & 0.4 & 0.4 & 0.4 & 0.4 & 0.4 \\
\hline 123 & 204 & 236 & 236 & 236 & 211 & 203 & 204 & 207 & 209 \\
\hline 0 & 0.1 & 0.1 & 0.1 & 0.1 & 0.1 & 0.1 & 0.1 & 0.1 & 0.1 \\
\hline 1.3 & 0.8 & 0.6 & 0.6 & 0.6 & 0.5 & 0.4 & 0.4 & 0.4 & 0.4 \\
\hline 0 & 0.3 & 0.4 & 0.4 & 0.4 & 0.6 & 0.6 & 0.6 & 0.6 & 0.6 \\
\hline 0 & 0 & 0.1 & 0.1 & 0.1 & 0.1 & 0.1 & 0.1 & 0.1 & 0.1 \\
\hline 0 & 0.4 & 0.5 & 0.5 & 0.5 & 0.7 & 0.7 & 0.7 & 0.7 & 0.7 \\
\hline 1.7 & 1 & 0.8 & 0.8 & 0.8 & 1.1 & 1.2 & 1.2 & 1.2 & 1.2 \\
\hline 1.7 & 1 & 0.8 & 0.8 & 0.8 & 1.1 & 1.2 & 1.2 & 1.2 & 1.2 \\
\hline 0 & 0.3 & 0.4 & 0.4 & 0.4 & 0.5 & 0.6 & 0.6 & 0.5 & 0.5 \\
\hline 47 & 29 & 22 & 22 & 22 & 31 & 34 & 33 & 33 & 32 \\
\hline 47 & 29 & 22 & 22 & 22 & 31 & 34 & 33 & 33 & 32 \\
\hline 27 & 17 & 13 & 13 & 13 & 18 & 19 & 19 & 19 & 19 \\
\hline 0 & 0.1 & 0.1 & 0.1 & 0.1 & 0.1 & 0.1 & 0.1 & 0.1 & 0.1 \\
\hline 27 & 16 & 12 & 12 & 12 & 9.3 & 8.3 & 8.2 & 8 & 7.9 \\
\hline 0 & 0.1 & 0.1 & 0.1 & 0.1 & 0.1 & 0.1 & 0.1 & 0.1 & 0.1 \\
\hline 27 & 16 & 12 & 12 & 12 & 9.3 & 8.3 & 8.2 & 8 & 7.9 \\
\hline 0 & 0.1 & 0.1 & 0.1 & 0.1 & 0.2 & 0.2 & 0.2 & 0.2 & 0.2 \\
\hline 337 & 337 & 337 & 337 & 337 & 337 & 337 & 337 & 337 & 337 \\
\hline
\end{tabular}


Table A-21: Swift \#1 and Swift \#2 Allocation

PNW Loads and Resources Study

2005 - 2014 Operating Years

1937 Water Year

2003 White Book

\begin{tabular}{|c|c|c|}
\hline Average Energy in Megawatts & $\begin{array}{l}2005 \\
A v q\end{array}$ & 2007 \\
\hline
\end{tabular}

-Swift 1-

1 Swift 1

2 Swift 1 Before Enc

$61 \quad 61$

$61 \quad 61$

$61 \quad 61$

$61 \quad 61 \quad 61 \quad 61$

\begin{tabular}{llll}
61 & 61 & 61 & 61 \\
\hline 61 & 61 & 61 & 61
\end{tabular}

-Swift $1-$

3 COPD Share

4 PPL Share

5 Swift 1 After Enc

\begin{tabular}{llllllllll}
16 & 16 & 16 & 16 & 16 & 16 & 16 & 16 & 16 & 16 \\
$\mathbf{4 6}$ & 46 & 46 & 46 & 46 & 46 & 46 & 46 & 46 & 46 \\
\hline $\mathbf{6 1}$ & $\mathbf{6 1}$ & $\mathbf{6 1}$ & $\mathbf{6 1}$ & $\mathbf{6 1}$ & $\mathbf{6 1}$ & $\mathbf{6 1}$ & $\mathbf{6 1}$ & $\mathbf{6 1}$ & $\mathbf{6 1}$
\end{tabular}

-Swift 2-

6 Swift

7 Swift 2 Before Enc

\begin{tabular}{llllllllll}
22 & 22 & 22 & 22 & 22 & 22 & 22 & 22 & 22 & 22 \\
\hline 22 & 22 & 22 & 22 & 22 & 22 & 22 & 22 & 22 & $\mathbf{2 2}$
\end{tabular}

-Swift 2-

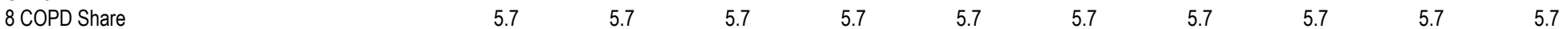
9 PPL Share

10 Swift 2 After Enc

\begin{tabular}{rrrrrrrrrr}
5.7 & 5.7 & 5.7 & 5.7 & 5.7 & 5.7 & 5.7 & 5.7 & 5.7 & 5.7 \\
16 & 16 & 16 & 16 & 16 & 16 & 16 & 16 & 16 & 16 \\
\hline $\mathbf{2 2}$ & $\mathbf{2 2}$ & $\mathbf{2 2}$ & $\mathbf{2 2}$ & $\mathbf{2 2}$ & $\mathbf{2 2}$ & $\mathbf{2 2}$ & $\mathbf{2 2}$ & $\mathbf{2 2}$ & $\mathbf{2 2}$
\end{tabular}




\begin{tabular}{|c|c|c|c|c|c|c|c|c|c|}
\hline Average Energy in Megawatts & $\begin{array}{l}2005 \\
\text { Avg. }\end{array}$ & $\begin{array}{l}2006 \\
\text { Avg. }\end{array}$ & $\begin{array}{l}2007 \\
\text { Avg. }\end{array}$ & $\begin{array}{l}2008 \\
\text { Avg. }\end{array}$ & $\begin{array}{l}2009 \\
\text { Avg. }\end{array}$ & $\begin{array}{l}2010 \\
\text { Avg. }\end{array}$ & $\begin{array}{l}2011 \\
\text { Avg. }\end{array}$ & $\begin{array}{l}2012 \\
\text { Avg. }\end{array}$ & $\begin{array}{l}2013 \\
\text { Avg. }\end{array}$ \\
\hline
\end{tabular}

-Federal Entities-

1 BPA To Federal Entities

$\begin{array}{llllllllll}119 & 119 & 120 & 120 & 121 & 121 & 122 & 119 & 118 & 118 \\ 119 & 119 & \mathbf{1 2 0} & \mathbf{1 2 0} & \mathbf{1 2 1} & \mathbf{1 2 1} & \mathbf{1 2 2} & \mathbf{1 1 9} & \mathbf{1 1 8} & 118\end{array}$

2 Total Federal Entities

-U.S. Bureau of Reclamation-

3 BPA To U.S. Bureau of Reclamation

$\begin{array}{llllllllll}149 & 149 & 149 & 149 & 149 & 149 & 149 & 149 & 149 & 149 \\ 149 & 149 & 149 & 149 & 149 & 149 & 149 & 149 & 149 & 149\end{array}$

4 Total U.S. Bureau of Reclamation

$\begin{array}{llllllllll}267 & 271 & 45 & 0 & 0 & 0 & 0 & 0 & 0 & 0 \\ 267 & 271 & 45 & 0 & 0 & 0 & 0 & 0 & 0 & 0\end{array}$

5 BPA To Direct Service Industry

6 Total Direct Service Industry

267

-Generating Public Entities-

7 BPA To Generating Public Entities

\begin{tabular}{llllllllll}
2142 & 2126 & 2367 & 2431 & 2443 & 2454 & 2463 & 2494 & 2511 & 2518 \\
\hline 2142 & 2126 & 2367 & 2431 & 2443 & 2454 & 2463 & 2494 & 2511 & 2518
\end{tabular}

8 Total Generating Public Entities

$2142 \quad 2126$

$2367 \quad 2431$

-Generating Public Entities (Slice)-

9 BPA To Generating Public Entities (Slice)

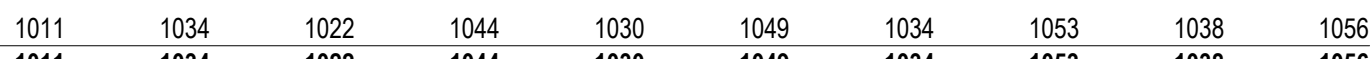

10 Total Generating Public Entities (Slice)

$\begin{array}{llllllllll}1011 & 1034 & 1022 & 1044 & 1030 & 1049 & 1034 & 1053 & 1038 & 1056\end{array}$

-Non-Generating Public Entities-

11 BPA To Non-Generating Public Entities

12 Total Non-Generating Public Entities

$\begin{array}{llllllllll}2716 & 2755 & 2917 & 2974 & 3009 & 3044 & 3078 & 3103 & 3137 & 3169 \\ \mathbf{2 7 1 6} & \mathbf{2 7 5 5} & \mathbf{2 9 1 7} & \mathbf{2 9 7 4} & \mathbf{3 0 0 9} & \mathbf{3 0 4 4} & \mathbf{3 0 7 8} & \mathbf{3 1 0 3} & \mathbf{3 1 3 7} & \mathbf{3 1 6 9}\end{array}$

-Non-Generating Public Entities (Slice)-

13 BPA To Non-Generating Public Entities (Slice

\begin{tabular}{llllllllll}
607 & 621 & 614 & 627 & 618 & 630 & 621 & 632 & 623 & 634 \\
\hline 607 & 621 & 614 & 627 & 618 & 630 & 621 & 632 & 623 & 634
\end{tabular}

14 Total Non-Generating Public Entities (Slice

$\begin{array}{llllllllll}607 & 621 & 614 & 627 & 618 & 630 & 621 & 632 & 623 & 634\end{array}$

-Investor-Owned Entities-

15 BPA To Investor-Owned Entities

$\begin{array}{llllllllll}382 & 382 & 64 & 0 & 0 & 0 & 0 & 0 & 0 & 0 \\ 382 & 382 & 64 & 0 & 0 & 0 & 0 & 0 & 0 & 0\end{array}$

-Other Entities-

17 BPA To Other PNW Entities

18 Total Other Entities

0

-Power Sales Contracts

19 Federal Entities

20 U.S. Bureau of Reclamation

21 Direct Service Industry

22 Generating Public Entities

23 Non-Generating Public Entities

24 Investor-Owned Entities

25 Other Entities

26 Total Power Sales Contracts

$\begin{array}{rrrrrrrrrr}119 & 119 & 120 & 120 & 121 & 121 & 122 & 119 & 118 & 118 \\ 149 & 149 & 149 & 149 & 149 & 149 & 149 & 149 & 149 & 149 \\ 267 & 271 & 45 & 0 & 0 & 0 & 0 & 0 & 0 & 0 \\ 3152 & 3160 & 3389 & 3475 & 3472 & 3503 & 3498 & 3547 & 3549 & 3574 \\ 3322 & 3376 & 3530 & 3601 & 3627 & 3674 & 3699 & 3735 & 3760 & 3803 \\ 382 & 382 & 64 & 0 & 0 & 0 & 0 & 0 & 0 & 0 \\ 0 & 0 & 0 & 0 & 0 & 0 & 0 & 0 & 0 & 0 \\ \mathbf{7 3 9 1} & \mathbf{7 4 5 8} & \mathbf{7 2 9 8} & \mathbf{7 3 4 6} & \mathbf{7 3 7 0} & \mathbf{7 4 4 8} & \mathbf{7 4 6 8} & \mathbf{7 5 5 1} & \mathbf{7 5 7 7} & \mathbf{7 6 4 4}\end{array}$


Table A-23: Regional Non Utility Generating Resources By Owner

PNW Loads and Resources Study

2005 - 2014 Operating Years

2003 White Book

-Federal Entities-

1 Federal Entities

2005

2006

$2007 \quad 2008 \quad 2009$

2009

2010

2011

2012

2013

2014

2 Total Federal Entities

92

$\begin{array}{lll}92 & 110 & 119 \\ 92 & 110\end{array}$

$232 \quad 232$

Generating Public Entities

3 Generating Public Entities

$232 \quad 232$

$232 \quad 232$

232

$19 \quad 119$

$119 \quad 119 \quad 119$

\begin{tabular}{llll}
119 & 119 & 119 & 119 \\
\hline 119 & 119 & 119 & 119
\end{tabular}

-Non-Generating Public Entities-

5 Non-Generating Public Entities

6 Total Non-Generating Public Entities

$10 \quad 6.8$

-Investor-Owned Entities-

7 Investor-Owned Entities

8 Total Investor-owned Entities

$\begin{array}{ll}876 & 87 \\ 876 & 876\end{array}$

$6.8 \quad 4.4$

$\begin{array}{llll}4.4 & 4.4 & 4.4 & 4.3 \\ 4.4 & 4.4 & 4.4\end{array}$

$4.3 \quad 4.3$

\begin{tabular}{llll}
4.1 & 4.1 & 4.1 & 4.1 \\
\hline 4.1 & 4.1 & 4.1 & 4.1
\end{tabular}

-Other Entities-

9 Other Entities

$876 \quad 896$

$896 \quad 896$

$896 \quad 896$

$896 \quad 897$

$897 \quad 997 \quad 900$

$\begin{array}{llll}900 & 900 & 901 & 901 \\ 900 & 900 & 901 & 901\end{array}$

10 Total Other Entities

$\begin{array}{lll}85 & 85 & 85 \\ 85 & 85 & 85\end{array}$

$\begin{array}{ll}85 & 85 \\ 85 & 85\end{array}$

$85 \quad 85 \quad 85$

-Total Non-Utility Generating Resources-

11 Federal Entities

12 Public Entities

13 Investor-Owned Entities

14 Other Entities

15 Total Non-Utility Generation

$\begin{array}{ll}85 & 85 \\ 85 & 85\end{array}$

$\begin{array}{rrrrrrrrrr}85 & 85 & 85 & 85 & 85 & 85 & 85 & 85 & 85 \\ & & & & & & & & & \\ 92 & 92 & 110 & 119 & 119 & 119 & 119 & 119 & 119 \\ 242 & 239 & 237 & 237 & 237 & 237 & 237 & 237 & 237 \\ 876 & 876 & 896 & 896 & 896 & 897 & 900 & 900 & 901 & 237 \\ 85 & 85 & 85 & 85 & 85 & 85 & 85 & 85 & 85 & 85 \\ 1296 & 1292 & 1329 & 1337 & 1337 & 1338 & 1340 & 1340 & 1341 & 1341\end{array}$


Table A-24: Regional Non Utility Generating Resources By Project

PNW Loads and Resources Study

2005 - 2014 Operating Years

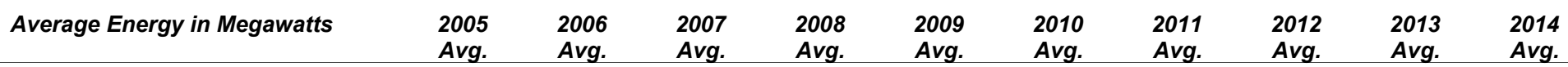

Federal Entities

-NUG: Hydro-

1 Clearwater - State of ID DWR (BPA)

2 Dworshak Small Hydropower (BPA)

3 Elwah Hydro (BPA)

4 Glines Hydro (BPA)

5 Federal -Total NUG: Hydro

\begin{tabular}{|c|c|c|c|c|c|c|c|c|c|}
\hline 1 & 1 & 1 & 1 & 1 & 1 & 1 & 1 & 1 & 1 \\
\hline 2.6 & 2.6 & 2.6 & 2.6 & 2.6 & 2.6 & 2.6 & 2.6 & 2.6 & 2.6 \\
\hline 8.6 & 8.6 & 0 & 0 & 0 & 0 & 0 & 0 & 0 & 0 \\
\hline 15 & 15 & 0 & 0 & 0 & 0 & 0 & 0 & 0 & 0 \\
\hline 27 & 27 & 3.6 & 3.6 & 3.6 & 3.6 & 3.6 & 3.6 & 3.6 & 3.6 \\
\hline
\end{tabular}

-NUG: Renewables-

6 Ashland Solar Project (BPA)

7 Condon Wind Project (BPA)

8 Foote Creek 1 (BPA)

9 Foote Creek 2 (BPA)

10 Foote Creek 4 (BPA)

11 Klondike Phase 1 (BPA)

12 Stateline Wind Project (BPA)

13 Fourmile Hill Geothermal (BPA)

14 Federal -Total NUG: Renewables

Generating Public Entities

-NUG: Hydro-

15 Don Steffen (SHPD)

16 Eltopia Br Canal 4.6 (SCL)

17 Eltopia Br Canal 4.6 (TPU)

18 John Llewellyn (SHPD)

19 Kevin Duncan (SHPD)

20 Lucky Peak (SCL)

21 Main Canal Headworks (SCL)

22 Main Canal Headworks (TPU)

23 PEC Headworks (GCPD)

24 Pickering Micro Hydro (CKPD)

25 Potholes E Canal 66.0 (SCL)

26 Potholes E Canal 66.0 (TPU)

27 Quincy Chute (GCPD)

28 Russel D. Smith (SCL)

29 Russel D. Smith (TPU)

30 Smith Creek (EWEB)

31 Summer Falls (SCL)

32 Summer Falls (TPU)

33 Woods Creek (SHPD)

34 GPU - Total NUG: Hydro

$\begin{array}{rrrrrrrrrr}0 & 0 & 0 & 0 & 0 & 0 & 0 & 0 & 0 & 0 \\ 12 & 12 & 12 & 12 & 12 & 12 & 12 & 12 & 12 & 12 \\ 6.4 & 6.4 & 6.4 & 6.4 & 6.4 & 6.4 & 6.4 & 6.4 & 6.4 & 6.4 \\ 0.8 & 0.8 & 0.8 & 0.8 & 0.8 & 0.8 & 0.8 & 0.8 & 0.8 & 0.8 \\ 7.4 & 7.4 & 7.4 & 7.4 & 7.4 & 7.4 & 7.4 & 7.4 & 7.4 & 7.4 \\ 8.1 & 8.1 & 8.1 & 8.1 & 8.1 & 8.1 & 8.1 & 8.1 & 8.1 & 8.1 \\ 30 & 30 & 30 & 30 & 30 & 30 & 30 & 30 & 30 & 30 \\ 0 & 0 & 42 & 50 & 50 & 50 & 50 & 50 & 50 & 50 \\ \mathbf{6 5} & \mathbf{6 5} & 107 & 115 & 115 & 115 & 115 & 115 & 115 & 115\end{array}$

-NUG: Small Thermal \& Misc.

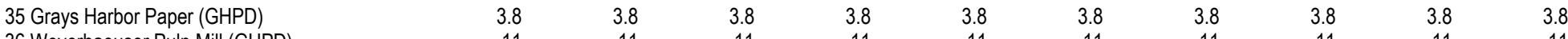

36 Weyerhaeuser Pulp Mill (GHPD)

37 GPU - Total NUG: Small Thermal \& Misc.

$\begin{array}{rrrrrrrrrr}0 & 0 & 0 & 0 & 0 & 0 & 0 & 0 & 0 & 0 \\ 0.5 & 0.5 & 0.5 & 0.5 & 0.5 & 0.5 & 0.5 & 0.5 & 0.5 & 0.5 \\ 0.5 & 0.5 & 0.5 & 0.5 & 0.5 & 0.5 & 0.5 & 0.5 & 0.5 & 0.5 \\ 0 & 0 & 0 & 0 & 0 & 0 & 0 & 0 & 0 & 0 \\ 0 & 0 & 0 & 0 & 0 & 0 & 0 & 0 & 0 & 0 \\ 38 & 38 & 38 & 38 & 38 & 38 & 38 & 38 & 38 & 38 \\ 4.9 & 4.9 & 4.9 & 4.9 & 4.9 & 4.9 & 4.9 & 4.9 & 4.9 & 4.9 \\ 4.9 & 4.9 & 4.9 & 4.9 & 4.9 & 4.9 & 4.9 & 4.9 & 4.9 & 4.9 \\ 2.9 & 2.9 & 2.9 & 2.9 & 2.9 & 2.9 & 2.9 & 2.9 & 2.9 & 2.9 \\ 0 & 0 & 0 & 0 & 0 & 0 & 0 & 0 & 0 & 0 \\ 0.5 & 0.5 & 0.5 & 0.5 & 0.5 & 0.5 & 0.5 & 0.5 & 0.5 & 0.5 \\ 0.5 & 0.5 & 0.5 & 0.5 & 0.5 & 0.5 & 0.5 & 0.5 & 0.5 & 0.5 \\ 3.7 & 3.7 & 3.7 & 3.7 & 3.7 & 3.7 & 3.7 & 3.7 & 3.7 & 3.7 \\ 0.9 & 0.9 & 0.9 & 0.9 & 0.9 & 0.9 & 0.9 & 0.9 & 0.9 & 0.9 \\ 0.9 & 0.9 & 0.9 & 0.9 & 0.9 & 0.9 & 0.9 & 0.9 & 0.9 & 0.9 \\ 9.2 & 9.2 & 9.2 & 9.2 & 9.2 & 9.2 & 9.2 & 9.2 & 9.2 & 9.2 \\ 18 & 18 & 18 & 18 & 18 & 18 & 18 & 18 & 18 & 18 \\ 18 & 18 & 18 & 18 & 18 & 18 & 18 & 18 & 18 & 18 \\ 0.1 & 0.1 & 0.1 & 0.1 & 0.1 & 0.1 & 0.1 & 0.1 & 0.1 & 0.1 \\ 105 & 105 & 105 & 105 & 105 & 105 & 105 & 105 & 105 & 105\end{array}$

-NUG: Co-Generation-

38 Metro Westpoint (SCL)

39 Scott Paper (SHPD)

$40 \mathrm{GPU}$ - Total NUG: Co-Generation

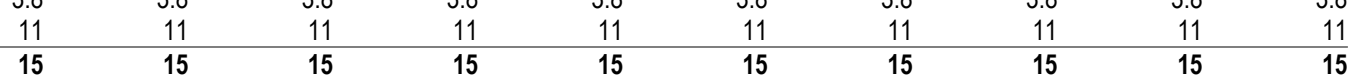

-NUG: Renewables-

41 Foote Creek 1 (EWEB)

42 Nine Canyon Wind Project (CHPD)

43 Nine Canyon Wind Project (COPD)

44 Nine Canyon Wind Project (DOPD)

45 Nine Canyon Wind Project (GCPD)

46 Nine Canyon Wind Project (GHPD)

47 Nine Canyon Wind Project (OKPD)

48 Stateline Wind Project (EWEB)

49 Stateline Wind Project (SCL)

50 GPU - Total NUG: Renewables

\begin{tabular}{|c|c|c|c|c|c|c|c|c|c|}
\hline 1.2 & 1.2 & 1.2 & 1.2 & 1.2 & 1.2 & 1.2 & 1.2 & 1.2 & 1.2 \\
\hline 33 & 33 & 33 & 32 & 32 & 32 & 32 & 32 & 32 & 32 \\
\hline 34 & 34 & 34 & 34 & 34 & 34 & 34 & 34 & 34 & 34 \\
\hline
\end{tabular}

Non-Generating Public Entities

-NUG: Hydro-

51 Buffalo Hydro (FREC)

52 Burr \& Bouchard (TNEC)

53 City of Cove (OTEC)

54 Falls Creek (CCPD)

55 Lilliwaup Falls Generating Co. (MCPD1)

56 Thomas Burnside (TNEC)

57 NGP - Total NUG: Hydro

\begin{tabular}{rrrrrrrrrr}
33 & 33 & 33 & 32 & 32 & 32 & 32 & 32 & 32 & 32 \\
\hline 34 & $\mathbf{3 4}$ & $\mathbf{3 4}$ & $\mathbf{3 4}$ & $\mathbf{3 4}$ & $\mathbf{3 4}$ & $\mathbf{3 4}$ & $\mathbf{3 4}$ & $\mathbf{3 4}$ & $\mathbf{3 4}$ \\
& & & & & & & & & \\
2.7 & 2.7 & 2.7 & 2.7 & 2.7 & 2.7 & 2.7 & 2.7 & 2.7 & 2.7 \\
2.5 & 2.5 & 2.5 & 2.5 & 2.5 & 2.5 & 2.5 & 2.5 & 2.5 & 2.5 \\
0.6 & 0.6 & 0.6 & 0.6 & 0.6 & 0.6 & 0.6 & 0.6 & 0.6 & 0.6 \\
3 & 3 & 3 & 3 & 3 & 3 & 3 & 3 & 3 & 3 \\
3.7 & 3.7 & 3.7 & 3.7 & 3.7 & 3.7 & 3.7 & 3.7 & 3.7 & 3.7 \\
2.5 & 2.5 & 2.5 & 2.5 & 2.5 & 2.5 & 2.5 & 2.5 & 2.5 & 2.5 \\
4.9 & 4.9 & 4.9 & 4.9 & 4.9 & 4.9 & 4.9 & 4.9 & 4.9 & 4.9 \\
8.6 & 8.6 & 8.6 & 8.6 & 8.6 & 8.6 & 8.6 & 8.6 & 8.6 & 8.6 \\
50 & 50 & 50 & 50 & 50 & 50 & 50 & 50 & 50 & 50 \\
79 & $\mathbf{7 9}$ & $\mathbf{7 9}$ & $\mathbf{7 9}$ & $\mathbf{7 9}$ & $\mathbf{7 9}$ & $\mathbf{7 9}$ & $\mathbf{7 9}$ & $\mathbf{7 9}$ & $\mathbf{7 9}$
\end{tabular}
34 


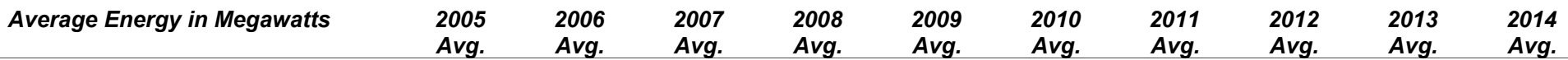

-NUG: Small Thermal \& Misc.

58 Methane Energy Agricultural D. (TKPD)

0.8

$\begin{array}{lllllllll}0.8 & 0.8 & 0.8 & 0.8 & 0.8 & 0.8 & 0.8 & 0.8 & 0.8 \\ \mathbf{0 . 8} & \mathbf{0 . 8} & \mathbf{0 . 8} & \mathbf{0 . 8} & \mathbf{0 . 8} & \mathbf{0 . 8} & \mathbf{0 . 8} & \mathbf{0 . 8} & \mathbf{0 . 8}\end{array}$

-NUG: Co-Generation-

60 Cogen Co (OTEC)

61 NGP - Total NUG: Co-Generation

$5.6 \quad 2.3$

$\begin{array}{rrrrrrrrr}2.3 & 0 & 0 & 0 & 0 & 0 & 0 & 0 & 0 \\ 2.3 & 0 & 0 & 0 & 0 & 0 & 0 & 0 & 0\end{array}$

-NUG: Renewables-

62 Ashland Solar Project (ASHL)

63 Nine Canyon Wind Project (BCPD)

64 Nine Canyon Wind Project (ENW)

65 Nine Canyon Wind Project (LCPD)

66 Nine Canyon Wind Project (MCPD3)

67 White Bluffs Solar (ENW)

\begin{tabular}{|c|c|c|c|c|c|c|c|c|c|}
\hline 0 & 0 & 0 & 0 & 0 & 0 & 0 & 0 & 0 & 0 \\
\hline 0.9 & 0.9 & 0.9 & 0.9 & 0.9 & 0.9 & 0.9 & 0.9 & 0.9 & 0.9 \\
\hline 0.6 & 0.6 & 0.6 & 0.6 & 0.6 & 0.6 & 0.6 & 0.6 & 0.6 & 0.6 \\
\hline 0.3 & 0.3 & 0.3 & 0.3 & 0.3 & 0.3 & 0.3 & 0.3 & 0.3 & 0.3 \\
\hline 0.6 & 0.6 & 0.6 & 0.6 & 0.6 & 0.6 & 0.6 & 0.6 & 0.6 & 0.6 \\
\hline 0 & 0 & 0 & 0 & 0 & 0 & 0 & 0 & 0 & 0 \\
\hline 2.5 & 2.5 & 2.5 & 2.5 & 2.5 & 2.5 & 2.5 & 2.5 & 2.5 & 2.5 \\
\hline
\end{tabular}

Investor-Owned Entities

-NUG: Hydro-

69 Barber Dam (IPC)

70 Barney Creek (NWE)

71 Beaverton (PGE)

72 Bell Mountain (UPL)

73 Bingham Engineering (NWE)

74 Birch Creek (IPC)

75 Birch Creek (UPL)

76 Black Canyon \#3 (IPC)

77 Black Creek (AVWP)

78 Blind Canyon Hydro (IPC)

79 Box Canyon (IPC)

80 Boyd James (PPL)

81 Briggs Creek (IPC)

82 Broadwater Dam (NWE)

83 Bypass (IPC)

84 Canyon Springs (IPC)

85 Carlton (PGE)

86 Cascade Creek (NWE)

87 CDM Hydro - Bonneville Pacific (PPL)

88 Cedar Draw (IPC)

89 Central OR Irrigation District (PPL)

90 Clear Springs Trout (IPC)

91 Corbett (PGE)

92 Cornelius (PGE)

93 Crystal Springs (IPC)

94 Curry Cattle Co. (IPC)

95 Dairy Creek (PGE)

96 Deep Creek (AVWP)

97 Derr Creek (AVWP)

98 Dietrich Drop (IPC)

99 Donald Jenni (NWE)

100 Eagle Creek (PGE)

101 Eagle Point Irrigation Dist. (PPL)

102 Elk Creek (IPC)

103 Estacada (PGE)

104 Falls Creek (PPL)

105 Falls River (IPC)

106 Farmers Irrig. (PPL)

107 Faulkner Ranch (IPC)

108 Fisheries Development Co (IPC)

109 Galesville Dam (PPL)

110 Geobon 2 (IPC)

111 Georgetown Power (UPL)

112 Hailey Cspp (IPC)

113 Hazelton A (IPC)

114 Hazelton B (IPC)

115 Horseshoe Bend (IPC)

116 Hutchinson Creek (PSE)

117 Ingram Warm Springs (UPL)

$118 \mathrm{Jim}$ Ford Creek (AVWP)

119 Jim Knight (IPC)

120 John Day Creek (AVWP)

121 Joseph Hydro (PPL)

122 Kasel \& Witherspoon (IPC)

123 Koma Kulshan (PSE)

124 Koyle (IPC)

\begin{tabular}{|c|c|c|c|c|c|c|c|c|c|}
\hline 2.3 & 2.3 & 2.3 & 2.3 & 2.3 & 2.3 & 2.3 & 2.3 & 2.3 & 2.3 \\
\hline 0 & 0 & 0 & 0 & 0 & 0 & 0 & 0 & 0 & 0 \\
\hline 0 & 0 & 0 & 0 & 0 & 0 & 0 & 0 & 0 & 0 \\
\hline 0.1 & 0.1 & 0.1 & 0.1 & 0.1 & 0.1 & 0.1 & 0.1 & 0.1 & 0.1 \\
\hline 0.1 & 0.1 & 0.1 & 0.1 & 0.1 & 0.1 & 0.1 & 0.1 & 0.1 & 0.1 \\
\hline 0 & 0 & 0 & 0 & 0 & 0 & 0 & 0 & 0 & 0 \\
\hline 1.2 & 1.2 & 1.2 & 1.2 & 1.2 & 1.2 & 1.2 & 1.2 & 1.2 & 1.2 \\
\hline 0.1 & 0.1 & 0.1 & 0.1 & 0.1 & 0.1 & 0.1 & 0.1 & 0.1 & 0.1 \\
\hline 2.9 & 2.8 & 2.9 & 2.9 & 3.1 & 3 & 2.9 & 2.8 & 2.8 & 3.0 \\
\hline 0.6 & 0.6 & 0.6 & 0.6 & 0.6 & 0.6 & 0.6 & 0.6 & 0.6 & 0.6 \\
\hline 0.4 & 0.4 & 0.4 & 0.4 & 0.4 & 0.4 & 0.4 & 0.4 & 0.4 & 0.4 \\
\hline 0.3 & 0.3 & 0.3 & 0.3 & 0.3 & 0.3 & 0.3 & 0.3 & 0.3 & 0.3 \\
\hline 0.6 & 0.6 & 0.6 & 0.6 & 0.6 & 0.6 & 0.6 & 0.6 & 0.6 & 0.6 \\
\hline 1.6 & 1.6 & 1.7 & 1.7 & 1.7 & 1.8 & 1.9 & 2.0 & 2 & 2 \\
\hline 3.8 & 3.8 & 3.8 & 3.8 & 3.8 & 3.8 & 3.8 & 3.8 & 3.8 & 3.8 \\
\hline 0.1 & 0.1 & 0.1 & 0.1 & 0.1 & 0.1 & 0.1 & 0.1 & 0.1 & 0.1 \\
\hline 0 & 0 & 0 & 0 & 0 & 0 & 0 & 0 & 0 & 0 \\
\hline 0 & 0 & 0 & 0 & 0 & 0 & 0 & 0 & 0 & 0 \\
\hline 3.1 & 3.1 & 3.1 & 3.1 & 3.1 & 3.1 & 3.1 & 3.1 & 3.1 & 3.1 \\
\hline 0.7 & 0.7 & 0.7 & 0.7 & 0.7 & 0.7 & 0.7 & 0.7 & 0.7 & 0.7 \\
\hline 2.1 & 2.1 & 2.1 & 2.1 & 2.1 & 2.1 & 2.1 & 2.1 & 2.1 & 2.1 \\
\hline 0.4 & 0.4 & 0.4 & 0.4 & 0.4 & 0.4 & 0.4 & 0.4 & 0.4 & 0.4 \\
\hline 0 & 0 & 0 & 0 & 0 & 0 & 0 & 0 & 0 & 0 \\
\hline 0 & 0 & 0 & 0 & 0 & 0 & 0 & 0 & 0 & 0 \\
\hline 1.1 & 1.1 & 1.1 & 1.1 & 1.1 & 1.1 & 1.1 & 1.1 & 1.1 & 1.1 \\
\hline 0.1 & 0.1 & 0.1 & 0.1 & 0.1 & 0.1 & 0.1 & 0.1 & 0.1 & 0.1 \\
\hline 0 & 0 & 0 & 0 & 0 & 0 & 0 & 0 & 0 & 0 \\
\hline 0 & 0 & 0 & 0 & 0 & 0 & 0 & 0 & 0 & 0 \\
\hline 0.1 & 0.1 & 0.1 & 0.1 & 0.1 & 0.1 & 0.1 & 0.1 & 0.1 & 0.1 \\
\hline 2.5 & 2.5 & 2.5 & 2.5 & 2.5 & 2.5 & 2.5 & 2.5 & 2.5 & 2.5 \\
\hline 0.1 & 0.1 & 0.1 & 0.1 & 0.1 & 0.1 & 0.1 & 0.1 & 0.1 & 0.1 \\
\hline 0 & 0 & 0 & 0 & 0 & 0 & 0 & 0 & 0 & 0 \\
\hline 0.4 & 0.4 & 0.4 & 0.4 & 0.4 & 0.4 & 0.4 & 0.4 & 0.4 & 0.4 \\
\hline 0.6 & 0.6 & 0.6 & 0.6 & 0.6 & 0.6 & 0.6 & 0.6 & 0.6 & 0.6 \\
\hline 0.1 & 0.1 & 0.1 & 0.1 & 0.1 & 0.1 & 0.1 & 0.1 & 0.1 & 0.1 \\
\hline 2.0 & 2.0 & 2.0 & 2.0 & 2.0 & 2.0 & 2.0 & 2.0 & 2.0 & 2.0 \\
\hline 5.9 & 5.9 & 5.9 & 5.9 & 5.9 & 5.9 & 5.9 & 5.9 & 5.9 & 5.9 \\
\hline 2.4 & 2.4 & 2.4 & 2.4 & 2.4 & 2.4 & 2.4 & 2.4 & 2.4 & 2.4 \\
\hline 0.6 & 0.6 & 0.6 & 0.6 & 0.6 & 0.6 & 0.6 & 0.6 & 0.6 & 0.6 \\
\hline 0.3 & 0.3 & 0.3 & 0.3 & 0.3 & 0.3 & 0.3 & 0.3 & 0.3 & 0.3 \\
\hline 0.3 & 0.3 & 0.3 & 0.3 & 0.3 & 0.3 & 0.3 & 0.3 & 0.3 & 0.3 \\
\hline 0.5 & 0.5 & 0.5 & 0.5 & 0.5 & 0.5 & 0.5 & 0.5 & 0.5 & 0.5 \\
\hline 0.2 & 0.2 & 0.2 & 0.2 & 0.2 & 0.2 & 0.2 & 0.2 & 0.2 & 0.2 \\
\hline 0.1 & 0.1 & 0.1 & 0.1 & 0.1 & 0.1 & 0.1 & 0.1 & 0.1 & 0.1 \\
\hline 3.2 & 3.2 & 3.2 & 3.2 & 3.2 & 3.2 & 3.2 & 3.2 & 3.2 & 3.2 \\
\hline 3 & 3 & 3 & 3 & 3 & 3 & 3 & 3 & 3 & 3 \\
\hline 6.6 & 6.6 & 6.6 & 6.6 & 6.6 & 6.6 & 6.6 & 6.6 & 6.6 & 6.6 \\
\hline 0.7 & 0.7 & 0.7 & 0.7 & 0.7 & 0.7 & 0.7 & 0.7 & 0.7 & 0.7 \\
\hline 0.4 & 0.4 & 0.4 & 0.4 & 0.4 & 0.4 & 0.4 & 0.4 & 0.4 & 0.4 \\
\hline 0.5 & 0.5 & 0.5 & 0.5 & 0.5 & 0.5 & 0.5 & 0.5 & 0.5 & 0.5 \\
\hline 0.2 & 0.2 & 0.2 & 0.2 & 0.2 & 0.2 & 0.2 & 0.2 & 0.2 & 0.2 \\
\hline 0.2 & 0.2 & 0.2 & 0.2 & 0.2 & 0.2 & 0.2 & 0.2 & 0.2 & 0.2 \\
\hline 0.6 & 0.6 & 0.6 & 0.6 & 0.6 & 0.6 & 0.6 & 0.6 & 0.6 & 0.6 \\
\hline 0.7 & 0.7 & 0.7 & 0.7 & 0.7 & 0.7 & 0.7 & 0.7 & 0.7 & 0.7 \\
\hline 6.4 & 6.4 & 6.4 & 6.4 & 6.4 & 6.4 & 6.4 & 6.4 & 6.4 & 6.4 \\
\hline 0.7 & 0.7 & 0.7 & 0.7 & 0.7 & 0.7 & 0.7 & 0.7 & 0.7 & 0.7 \\
\hline
\end{tabular}


$125 \mathrm{~L}$. Fery (PPL)

126 Lacomb Irrig. (PPL)

127 Lake Oswego (PGE)

128 Lateral \#10 (IPC)

129 Lee Tavenner (NWE)

130 Lemoyne (IPC)

131 Little Wood River Res. (IPC)

132 Littlewood/Arkoosh (IPC)

133 Lowline \#2 (IPC)

134 Lowline Canal (IPC)

135 Magic Reservoir (IPC)

136 Malad River (IPC)

137 Marcos Ranches (IPC)

138 Marsh Valley (UPL)

139 Meyers Falls (AVWP)

140 Middlefork Irrig. (PPL)

141 Mile 28 (IPC)

142 Minikahda (PGE)

143 Mink Creek (UPL)

144 Mitchell Butte (IPC)

145 Mountain Energy (PPL)

$146 \mathrm{Mt}$. Tabor (PGE)

147 Mud Creek/S\&S (IPC)

148 Mud Creek/White (IPC)

149 Nichols Gap (PPL)

150 Nicholson Sunnybar (UPL)

151 North Fork Sprague (PPL)

152 O.J. Power (UPL)

153 Odell Creek (PPL)

154 Opal Springs (PPL)

155 Owyhee Dam (IPC)

156 Pancheri (UPL)

157 Pelton Rereg. Dam (PPL)

158 Phillips Ranch (AVWP)

159 Phillipsburg (NWE)

160 Pigeon Cove (IPC)

161 Pine Creek (NWE)

162 Port Townsend Paper Hydro (PSE)

163 Portland (PGE)

164 Portland Hydro Project (PGE)

165 Preston City (UPL)

166 Pristine Springs (IPC)

167 Pristine Springs \#3 (IPC)

168 Reynolds Irrigation (IPC)

169 Rim View (IPC)

170 Rock Creek \#1 (IPC)

171 Rock Creek \#2 (IPC)

172 Sagebrush (IPC)

173 Salem (PGE)

174 Schaffner (IPC)

175 Sheep Creek (AVWP)

176 Shingle Creek (IPC)

177 Shoshone (IPC)

178 Shoshone II (IPC)

179 Snake River Pottery (IPC)

180 Snedigar Ranch (IPC)

181 Solar Research (PPL)

182 South Dry Creek (NWE)

183 Spokane Upriver (AVWP)

184 Stauffer Dry Creek (UPL)

185 Strawberry Creek (NWE)

186 Sunshine Power \#2 (IPC)

187 Sygitowicz Creek Small Hydro (PSE)

188 TGS/Briggs (UPL)

189 Trout Co. (IPC)

190 Tunnel \#1 (IPC)

191 Twin Falls (PSE)

192 Walla Walla (PPL)

193 Water Street / Santiam (PPL)

194 Weeks Falls (PSE)

195 White James (PPL)

196 White Water Ranch (IPC

197 Wilson Lake Hydro (IPC)

198 Wisconsin Creek (NWE)

199 Yakima-Tieton (PPL) $\begin{array}{llllllll}2007 & 2008 & 2009 & 2010 & 2011 & 2012 & 2013 & 2014 \\ \text { Avg. } & \text { Avg. } & \text { Avg. } & \text { Avg. } & \text { Avg. } & \text { Avg. } & \text { Avg. } & \text { Avg. }\end{array}$

Avg. Avg.

vg.

0

0.5

0.4

0.1

0.9

0.5

1.8

5.3

3.5
0.4

0.4
0.9

0.4

0.9

2.2
0.7

0

0.9

0.6

0.1

0.4

0.1

0.2

0.2

0.2
0.1

$\begin{array}{rr}0 & 0.1 \\ 0 & 0\end{array}$

$2.9 \quad 2.9$

$\begin{array}{rr}1.8 & 1.8 \\ 0 & 0\end{array}$

7.8

0

1.2

0.1

0.3

12

0.4

0.1

0.2

0.2

0.2

1.3

0.3

0.3

0.7

0.1

0.3

0.3

0.2

0

0.3

9.7

0.8

0.1

0.1

0.2

0.2

$0.1 \quad 0.1$

$2.7 \quad 2.7$

$9.6 \quad 9.6$

$1.5 \quad 1.5$

0.1

1.7

0.2

0.1

3.1

0.9

$\begin{array}{rrrr}0 & 0 & 0 & 0 \\ 0.5 & 0.5 & 0.5 & 0.5 \\ 0.4 & 0.4 & 0.4 & 0.4 \\ 1.7 & 1.7 & 1.7 & 1.7 \\ 0.1 & 0.1 & 0.1 & 0.1 \\ 0 & 0 & 0 & 0 \\ 0.9 & 0.9 & 0.9 & 0.9\end{array}$

0
0.5
0.4
1.7

$\begin{array}{rrr}0 & 0 & \\ 0.5 & 0.5 & 0.5 \\ 0.4 & 0.4 & 0.4\end{array}$

$\begin{array}{lll}1.7 & 1.7 & 1.7 \\ 0.1 & 0.1 & 0.1\end{array}$

$\begin{array}{rrrr}0 & 0 & 0 & 0\end{array}$

$\begin{array}{llll}0.9 & 0.9 & 0.9 & 0.9 \\ 0.5 & 0.5 & 0.5 & 0.5\end{array}$

$\begin{array}{llllllll}1.8 & 1.8 & 1.8 & 1.8 & 1.8 & 1.8 & 1.8 & 1.8 \\ 5.3 & 5.3 & 5.3 & 5.3 & 5.3 & 5.3 & 5.3 & 5.3\end{array}$

$\begin{array}{llllllll}3.5 & 3.5 & 3.5 & 3.5 & 3.5 & 3.5 & 3.5 & 3.5\end{array}$

$\begin{array}{llllllll}0.4 & 0.4 & 0.4 & 0.4 & 0.4 & 0.4 & 0.4 & 0.4\end{array}$

$\begin{array}{llllllll}0.4 & 0.4 & 0.4 & 0.4 & 0.4 & 0.4 & 0.4 & 0.4\end{array}$

$\begin{array}{llllllll}0.9 & 0.9 & 0.9 & 0.9 & 0.9 & 0.9 & 0.9 & 0.9\end{array}$

$\begin{array}{llllllll}2.2 & 2.2 & 2.2 & 2.2 & 2.2 & 2.2 & 2.2 & 2.2\end{array}$

$\begin{array}{rrrrrrrr}0.7 & 0.7 & 0.7 & 0.7 & 0.7 & 0.7 & 0.7 & 0.7 \\ 0 & 0 & 0 & 0 & 0 & 0 & 0 & 0\end{array}$

$\begin{array}{llllllll}0.9 & 0.9 & 0.9 & 0.9 & 0.9 & 0.9 & 0.9 & 0.9\end{array}$

$\begin{array}{rrrrrrrr}0.6 & 0.6 & 0.6 & 0.6 & 0.6 & 0.6 & 0.6 & 0.6 \\ 0 & 0 & 0 & 0 & 0 & 0 & 0 & 0\end{array}$

$\begin{array}{llllllll}0.1 & 0.1 & 0.1 & 0.1 & 0.1 & 0.1 & 0.1 & 0.1 \\ 0.4 & 0.4 & 0.4 & 0.4 & 0.4 & 0.4 & 0.4 & 0.4\end{array}$

$\begin{array}{llllllll}0.4 & 0.4 & 0.4 & 0.4 & 0.4 & 0.4 & 0.4 & 0.4 \\ 0.1 & 0.1 & 0.1 & 0.1 & 0.1 & 0.1 & 0.1 & 0.1\end{array}$

$\begin{array}{llllllll}0.2 & 0.2 & 0.2 & 0.2 & 0.2 & 0.2 & 0.2 & 0.2 \\ 0.2 & 0.2 & 0.2 & 0.2 & 0.2 & 0.2 & 0.2\end{array}$

$\begin{array}{llllllll}0.2 & 0.2 & 0.2 & 0.2 & 0.2 & 0.2 & 0.2 & 0.2\end{array}$

$\begin{array}{llllllll}0.2 & 0.2 & 0.2 & 0.2 & 0.2 & 0.2 & 0.2 & 0.2 \\ 0.1 & 0.1 & 0.1 & 0.1 & 0.1 & 0.1 & 0.1 & 0.1\end{array}$

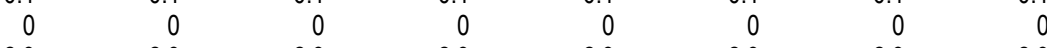

$\begin{array}{llll}2.9 & 2.9 & 2.9 & 2.9\end{array}$

1.8

7.8

$1.8 \quad 1.8$

$\begin{array}{ll}7.8 & 7.8 \\ 0 & \end{array}$

$\begin{array}{rrr}0 & 0 & 0 \\ 12 & 12 & 12\end{array}$

$1.2 \quad 1.2$

$\begin{array}{lll}0.1 & 0.1 & 0.1 \\ 0.3 & 0.3 & 0.3\end{array}$

$\begin{array}{rrr}0.3 & 0.3 & 0.3 \\ 0 & 0 & 0\end{array}$

12

$\begin{array}{lll}0.4 & 0.4 & 0.4\end{array}$

$0.1-0.1 \quad 0.1$

$\begin{array}{lll}0.2 & 0.2 & 0.2\end{array}$

$\begin{array}{lll}0.2 & 0.2 & 0.2\end{array}$

$\begin{array}{lll}1.3 & 1.3 & 1.3\end{array}$

$\begin{array}{lll}1.6 & 1.6 & 1.6\end{array}$

$0.3 \quad 0.3$

$\begin{array}{ll}0.3 & 0.3\end{array}$

0.7

$\begin{array}{lll}0.1 & 0.1 & 0.1\end{array}$


Average Energy in Megawatts $\quad 2005 \quad 2006 \quad 2007 \quad 2008 \quad 2009 \quad 2010 \quad 2011 \quad 2012 \quad 2013 \quad 2014$ Avg. Avg. Avg. Avg. Avg. Avg. Avg. Avg. Avg. Avg.

\begin{tabular}{|c|c|c|c|c|c|c|c|c|c|c|}
\hline 200 IOU - Total NUG: Hydro & 148 & 148 & 148 & 148 & 148 & 148 & 148 & 148 & 148 & 148 \\
\hline \multicolumn{11}{|l|}{-NUG: Small Thermal \& Misc.- } \\
\hline 201 Marion Solid Waste (PGE) & 9.3 & 9.3 & 9.3 & 9.3 & 9.3 & 9.3 & 9.3 & 9.3 & 9.3 & 9.3 \\
\hline 202 Minnesota Methane (AVWP) & 0.8 & 0.8 & 0.8 & 0.8 & 0.8 & 0.8 & 0.8 & 0.8 & 0.8 & 0.8 \\
\hline 203 Montana One (NWE) & 7.7 & 7.7 & 8.1 & 8.0 & 8 & 8.3 & 9.3 & 9.4 & 9.7 & 9.6 \\
\hline 204 Pocatello Waste (IPC) & 0.1 & 0.1 & 0.1 & 0.1 & 0.1 & 0.1 & 0.1 & 0.1 & 0.1 & 0.1 \\
\hline 205 Spokane MSW (PSE) & 15 & 15 & 15 & 15 & 15 & 15 & 15 & 15 & 15 & 15 \\
\hline 206 West Boise Waste (IPC) & 0.1 & 0.1 & 0.1 & 0.1 & 0.1 & 0.1 & 0.1 & 0.1 & 0.1 & 0.1 \\
\hline 207 IOU - Total NUG: Small Thermal \& Misc. & 33 & 33 & 34 & 34 & 34 & 34 & 35 & 35 & 35 & 35 \\
\hline
\end{tabular}

-NUG: Combustion Turbines -

208 March Point \#1 Cogeneration (PSE)

209 March Point \#2 Cogeneration (PSE)

210 Rathdrum- Boekel Rd. \#1 (AVWP)

211 Rathdrum -Boekel Rd. \#2 (AVWP)

212 Tenaska Gas Cogen (PSE)

$\begin{array}{rrr}77 & 77 & 77 \\ 58 & 58 & 58 \\ 62 & 62 & 62 \\ 62 & 62 & 62 \\ 215 & 215 & 235 \\ 475 & 475 & 494\end{array}$

213 IOU - Total NUG: Combustion Turbines

$475 \quad 475$

\begin{tabular}{rr}
77 & \\
58 & \\
62 & \\
62 & \\
235 & 235 \\
\hline 494 & 494 \\
\hline
\end{tabular}

\begin{tabular}{rrr}
77 & 77 & 77 \\
58 & 58 & 58 \\
62 & 62 & 62 \\
62 & 62 & 62 \\
235 & 235 & 235 \\
\hline 494 & 494 & 494
\end{tabular}

\begin{tabular}{rrrr}
77 & 77 & 77 & 77 \\
58 & 58 & 58 & 58 \\
62 & 62 & 62 & 62 \\
62 & 62 & 62 & 62 \\
235 & 235 & 235 & 235 \\
\hline 494 & 494 & 494 & 494
\end{tabular}

-NUG: Co-Generation-

214 Billings Generation (NWE)

215 Biomass One (PPL)

216 Boise Cascade Medford (PPL)

217 Champion (PPL)

218 DR Johnson - Co-Gen II (PPL)

219 Magic Valley (IPC)

220 Magic West (IPC)

221 PERC Pierce, Wa (PSE)

222 Simplot Pocatello (IPC)

223 Sumas Energy (PSE)

224 Tamarack (IPC)

225 TASCO Nampa ID (IPC)

226 TASCO Twin Falls ID (IPC)

227 Vaagen Bros. (IPC)

228 Warm Springs (PPL)

$\begin{array}{rrrrrrrrrr}13 & 13 & 14 & 14 & 14 & 14 & 16 & 16 & 17 & 17 \\ 19 & 19 & 19 & 19 & 19 & 19 & 19 & 19 & 19 & 19 \\ 8.5 & 8.5 & 8.5 & 8.5 & 8.5 & 8.5 & 8.5 & 8.5 & 8.5 & 8.5 \\ 3.1 & 3.1 & 3.1 & 3.1 & 3.1 & 3.1 & 3.1 & 3.1 & 3.1 & 3.1 \\ 7.2 & 7.2 & 7.2 & 7.2 & 7.2 & 7.2 & 7.2 & 7.2 & 7.2 & 7.2 \\ 9.5 & 9.5 & 9.5 & 9.5 & 9.5 & 9.5 & 9.5 & 9.5 & 9.5 & 9.5 \\ 9.5 & 9.5 & 9.5 & 9.5 & 9.5 & 9.5 & 9.5 & 9.5 & 9.5 & 9.5 \\ 1.8 & 1.8 & 1.8 & 1.8 & 1.8 & 1.8 & 1.8 & 1.8 & 1.8 & 1.8 \\ 9.0 & 9.0 & 9.0 & 9.0 & 9.0 & 9.0 & 9.0 & 9.0 & 9.0 & 9.0 \\ 120 & 120 & 120 & 120 & 120 & 120 & 120 & 120 & 120 & 120 \\ 4.1 & 4.1 & 4.1 & 4.1 & 4.1 & 4.1 & 4.1 & 4.1 & 4.1 & 4.1 \\ 0.2 & 0.2 & 0.2 & 0.2 & 0.2 & 0.2 & 0.2 & 0.2 & 0.2 & 0.2 \\ 0 & 0 & 0 & 0 & 0 & 0 & 0 & 0 & 0 & 0 \\ 3.3 & 3.3 & 3.3 & 3.3 & 3.3 & 3.3 & 3.3 & 3.3 & 3.3 & 3.3 \\ 0.1 & 0.1 & 0.1 & 0.1 & 0.1 & 0.1 & 0.1 & 0.1 & 0.1 & 0.1 \\ \mathbf{2 0 9} & \mathbf{2 0 9} & \mathbf{2 1 0} & \mathbf{2 1 0} & \mathbf{2 1 0} & \mathbf{2 1 0} & \mathbf{2 1 2} & \mathbf{2 1 2} & \mathbf{2 1 3} & \mathbf{2 1 3}\end{array}$

-NUG: Renewables-

230 Foote Creek 1 (PPL)

231 Healow \#2 (NWE)

232 Josef Staufer (NWE)

233 Lewandowski Farms (IPC)

234 Livingston (NWE)

235 Puyallup Energy Recovery (PSE)

236 Stateline Wind Project (AVWP)

237 Windmill (PGE)

238 IOU - Total NUG: Renewables

$\begin{array}{rrrrrrrrrr}8.1 & 8.1 & 8.1 & 8.1 & 8.1 & 8.1 & 8.1 & 8.1 & 8.1 & 8.1 \\ 0 & 0 & 0 & 0 & 0 & 0 & 0 & 0 & 0 & 0 \\ 0 & 0 & 0 & 0 & 0 & 0 & 0 & 0 & 0 & 0 \\ 0 & 0 & 0 & 0 & 0 & 0 & 0 & 0 & 0 & 0 \\ 0 & 0 & 0 & 0 & 0 & 0 & 0 & 0 & 0 & 0 \\ 2 & 2 & 2 & 2 & 2 & 2 & 2 & 2 & 2 & 2 \\ 0.3 & 0.3 & 0.3 & 0.3 & 0.3 & 0.3 & 0.3 & 0.3 & 0.3 & 0.3 \\ 0 & 0 & 0 & 0 & 0 & 0 & 0 & 0 & 0 & 0 \\ 10 & 10 & 10 & 10 & 10 & 10 & 10 & 10 & 10 & 10\end{array}$

Other Entities

-NUG: Co-Generation-

239 SP Newsprint cogen (SPN)

240 Other - Total NUG: Co-Generation

$25 \quad 25$

-NUG: Renewables-

241 Stateline Wind Project (PPME)

242 Vansycle Wind (FPLEV)

243 Other - Total NUG: Renewables

25

$\begin{array}{lll}25 & 25 & 25 \\ 25 & 25 & 25\end{array}$

$25 \quad 25$

$\begin{array}{llllll}25 & 25 & 25 & 25 & 25 & 25 \\ \mathbf{2 5} & \mathbf{2 5} & \mathbf{2 5} & \mathbf{2 5} & \mathbf{2 5} & \mathbf{2 5}\end{array}$

-Total Non-Utility Generating Resources-

244 Federal Entities

245 Generating Public Entities

246 Non Generating Public Entities

247 Investor-Owned Entities

248 Other Entities

249 Total Non-Utility Generation

\begin{tabular}{|c|c|c|c|c|c|c|c|c|c|}
\hline $\begin{array}{r}53 \\
7.4\end{array}$ & $\begin{array}{r}53 \\
7.4\end{array}$ & $\begin{array}{r}53 \\
7.4\end{array}$ & $\begin{array}{r}53 \\
7.4\end{array}$ & $\begin{array}{r}53 \\
7.4\end{array}$ & $\begin{array}{r}53 \\
7.4\end{array}$ & $\begin{array}{r}53 \\
7.4\end{array}$ & $\begin{array}{r}53 \\
7.4\end{array}$ & $\begin{array}{r}53 \\
7.4\end{array}$ & $\begin{array}{r}53 \\
7.4\end{array}$ \\
\hline 60 & 60 & 60 & 60 & 60 & 60 & 60 & 60 & 60 & 60 \\
\hline
\end{tabular}

\begin{tabular}{|c|c|c|c|c|c|c|c|c|c|}
\hline $\begin{array}{r}53 \\
7.4\end{array}$ & $\begin{array}{r}53 \\
7.4\end{array}$ & $\begin{array}{r}53 \\
7.4\end{array}$ & $\begin{array}{r}53 \\
7.4\end{array}$ & $\begin{array}{r}53 \\
7.4\end{array}$ & $\begin{array}{r}53 \\
7.4\end{array}$ & $\begin{array}{r}53 \\
7.4\end{array}$ & $\begin{array}{r}53 \\
7.4\end{array}$ & $\begin{array}{r}53 \\
7.4\end{array}$ & $\begin{array}{r}53 \\
7.4\end{array}$ \\
\hline 60 & 60 & 60 & 60 & 60 & 60 & 60 & 60 & 60 & 60 \\
\hline
\end{tabular}

\begin{tabular}{|c|c|c|c|c|c|c|c|c|c|}
\hline $\begin{array}{r}53 \\
7.4\end{array}$ & $\begin{array}{r}53 \\
7.4\end{array}$ & $\begin{array}{r}53 \\
7.4\end{array}$ & $\begin{array}{r}53 \\
7.4\end{array}$ & $\begin{array}{r}53 \\
7.4\end{array}$ & $\begin{array}{r}53 \\
7.4\end{array}$ & $\begin{array}{r}53 \\
7.4\end{array}$ & $\begin{array}{r}53 \\
7.4\end{array}$ & $\begin{array}{r}53 \\
7.4\end{array}$ & $\begin{array}{r}53 \\
7.4\end{array}$ \\
\hline 60 & 60 & 60 & 60 & 60 & 60 & 60 & 60 & 60 & 60 \\
\hline
\end{tabular}

\begin{tabular}{|c|c|c|c|c|c|c|c|c|c|}
\hline $\begin{array}{r}53 \\
7.4\end{array}$ & $\begin{array}{r}53 \\
7.4\end{array}$ & $\begin{array}{r}53 \\
7.4\end{array}$ & $\begin{array}{r}53 \\
7.4\end{array}$ & $\begin{array}{r}53 \\
7.4\end{array}$ & $\begin{array}{r}53 \\
7.4\end{array}$ & $\begin{array}{r}53 \\
7.4\end{array}$ & $\begin{array}{r}53 \\
7.4\end{array}$ & $\begin{array}{r}53 \\
7.4\end{array}$ & $\begin{array}{r}53 \\
7.4\end{array}$ \\
\hline 60 & 60 & 60 & 60 & 60 & 60 & 60 & 60 & 60 & 60 \\
\hline
\end{tabular}

229 IOU - Total NUG: Co-Generation

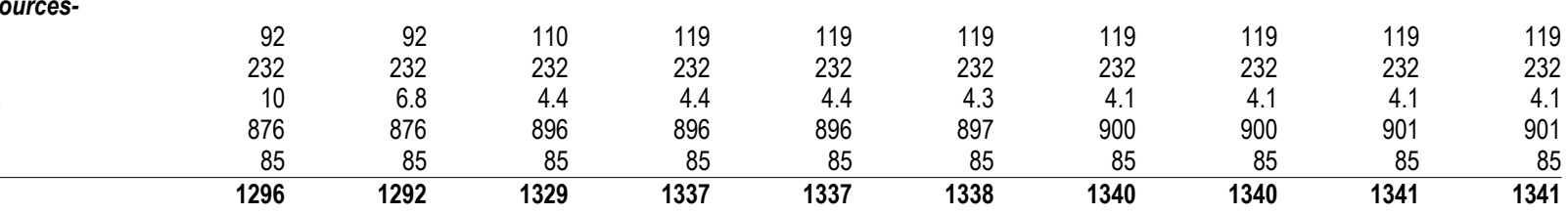


Table A-25: Dedicated Resources

PNW Loads and Resources Study

2005 - 2014 Operating Years

1937 Water Year

2003 White Book

\begin{tabular}{|c|c|c|c|c|c|c|c|c|c|c|}
\hline Average Energy in Megawatts & $\begin{array}{l}2005 \\
\text { Avg. }\end{array}$ & $\begin{array}{l}2006 \\
\text { Avg. }\end{array}$ & $\begin{array}{l}2007 \\
\text { Avg. }\end{array}$ & $\begin{array}{l}2008 \\
\text { Avg. }\end{array}$ & $\begin{array}{l}2009 \\
\text { Avg. }\end{array}$ & $\begin{array}{l}2010 \\
\text { Avg. }\end{array}$ & $\begin{array}{l}2011 \\
\text { Avg. }\end{array}$ & $\begin{array}{l}2012 \\
\text { Avg. }\end{array}$ & $\begin{array}{l}2013 \\
\text { Avg. }\end{array}$ & $\begin{array}{l}2014 \\
\text { Avg. }\end{array}$ \\
\hline $\begin{array}{l}\text {-Generating Public Entities- } \\
1 \text { Generating Public Enities }\end{array}$ & 2274 & 2109 & 2019 & 2027 & 2041 & 2002 & 1978 & 2141 & 2175 & 2186 \\
\hline 2 Total Generating Public Entities & 2274 & 2109 & 2019 & 2027 & 2041 & 2002 & 1978 & 2141 & 2175 & 2186 \\
\hline $\begin{array}{l}\text {-Non-Generating Public Entities- } \\
3 \text { Small \& Non Generating Public Enities }\end{array}$ & 83 & 85 & 70 & 67 & 66 & 66 & 66 & 59 & 58 & 58 \\
\hline 4 Total Non-Generating Public Entities & 83 & 85 & 70 & 67 & 66 & 66 & 66 & 59 & 58 & 58 \\
\hline $\begin{array}{l}\text {-Investor-Owned Entities- } \\
5 \text { Investor-Owned Enities }\end{array}$ & 6804 & 6879 & 6781 & 6869 & 6871 & 6800 & 6743 & 6650 & 6626 & 6662 \\
\hline 6 Total Investor-owned Entities & 6804 & 6879 & 6781 & 6869 & 6871 & 6800 & 6743 & 6650 & 6626 & 6662 \\
\hline -Total Dedicated Resources- & & & & & & & & & & \\
\hline $\begin{array}{l}7 \text { Generating Public Entities } \\
8 \text { Small \& Non Generating Entities }\end{array}$ & $\begin{array}{r}2274 \\
83\end{array}$ & $\begin{array}{r}2109 \\
85\end{array}$ & $\begin{array}{r}2019 \\
70\end{array}$ & $\begin{array}{r}2027 \\
67\end{array}$ & $\begin{array}{r}2041 \\
66\end{array}$ & $\begin{array}{r}2002 \\
66\end{array}$ & $\begin{array}{r}1978 \\
66\end{array}$ & $\begin{array}{r}2141 \\
59\end{array}$ & $\begin{array}{r}2175 \\
58\end{array}$ & $\begin{array}{r}2186 \\
58\end{array}$ \\
\hline 9 Investor-Owned Entities & 6804 & 6879 & 6781 & 6869 & 6871 & 6800 & 6743 & 6650 & 6626 & 6662 \\
\hline 10 Regional Total Dedicated Resources & 9161 & 9072 & 8870 & 8963 & 8978 & 8868 & 8787 & 8850 & 8859 & 8906 \\
\hline
\end{tabular}


Table A-26: Other Dedicated Resources

PNW Loads and Resources Study

2005 - 2014 Operating Years

1937 Water Year

2003 White Book

\begin{tabular}{|c|c|c|c|c|c|c|c|c|c|c|}
\hline Average Energy in Megawatts & $\begin{array}{l}2005 \\
\text { Avg. }\end{array}$ & $\begin{array}{l}2006 \\
\text { Avg. }\end{array}$ & $\begin{array}{l}2007 \\
\text { Avg. }\end{array}$ & $\begin{array}{l}2008 \\
\text { Avg. }\end{array}$ & $\begin{array}{l}2009 \\
\text { Avg. }\end{array}$ & $\begin{array}{l}2010 \\
\text { Avg. }\end{array}$ & $\begin{array}{c}2011 \\
\text { Avg. }\end{array}$ & $\begin{array}{c}2012 \\
\text { Avg. }\end{array}$ & $\begin{array}{c}2013 \\
\text { Avg. }\end{array}$ & $\begin{array}{l}2014 \\
\text { Avg. }\end{array}$ \\
\hline \multicolumn{11}{|l|}{-Generating Public Entities- } \\
\hline 2 Total Generating Public Entities & $\begin{array}{l}4450 \\
4458\end{array}$ & 4501 & 4002 & 4730 & 4713 & 4767 & 4751 & 4769 & 4755 & 4804 \\
\hline $\begin{array}{l}\text {-Non-Generating Public Entities- } \\
3 \text { Small \& Non Generating Public Enities }\end{array}$ & 3979 & 4047 & 4194 & 4278 & 4296 & 4354 & 4370 & 4417 & 4436 & 4491 \\
\hline 4 Total Non-Generating Public Entities & 3979 & 4047 & 4194 & 4278 & 4296 & 4354 & 4370 & 4417 & 4436 & 4491 \\
\hline \multicolumn{11}{|l|}{-Investor-Owned Entities- } \\
\hline 6 Total Investor-owned Entities & 3413 & 3499 & 3179 & 3173 & 3146 & 3122 & 3079 & 3022 & 3044 & 3009 \\
\hline \multicolumn{11}{|l|}{-Total Dedicated Resources- } \\
\hline 7 Generating Public Entities & 4458 & 4501 & 4662 & 4730 & 4713 & 4767 & 4751 & 4769 & 4755 & 4804 \\
\hline 8 Non Generating Entities & 3979 & 4047 & 4194 & 4278 & 4296 & 4354 & 4370 & 4417 & 4436 & 4491 \\
\hline 9 Investor-Owned Entities & 3413 & 3499 & 3179 & 3173 & 3146 & 3122 & 3079 & 3022 & 3044 & 3009 \\
\hline 10 Regional Total Dedicated Resources & 11850 & 12046 & 12035 & 12181 & 12155 & 12243 & 12199 & 12208 & 12235 & 12304 \\
\hline
\end{tabular}




\section{Section 4 \\ Monthly Federal Tables \\ Loads \& Resources \\ Average Energy in Megawatts}


Loads and Resources - Federal System

PNW Loads and Resources Study

2004 - 2005 Operating Year

1937 Water Year

2003 White Book

Non-Utility Obligations

Federal Agencies 1981 PSC Sale

USBR 1981 PSC Sale

DSI 1981 PSC Sale

Federal Agencies 2002 PSC Sale

USBR 2002 PSC Sale

DSI 2002 PSC Sale

\begin{tabular}{|c|c|c|c|c|c|c|c|c|c|c|c|c|c|c|}
\hline 0 & 0 & 0 & 0 & 0 & 0 & 0 & 0 & 0 & 0 & 0 & 0 & 0 & 0 & 0 \\
\hline 0 & 0 & 0 & 0 & 0 & 0 & 0 & 0 & 0 & 0 & 0 & 0 & 0 & 0 & 0 \\
\hline 0 & 0 & 0 & 0 & 0 & 0 & 0 & 0 & 0 & 0 & 0 & 0 & 0 & 0 & 0 \\
\hline 120 & 120 & 108 & 106 & 122 & 136 & 137 & 131 & 123 & 110 & 110 & 108 & 108 & 115 & 119 \\
\hline 309 & 275 & 166 & 129 & 0 & 0 & 0 & 1.0 & 23 & 252 & 315 & 268 & 298 & 332 & 149 \\
\hline 277 & 276 & 274 & 271 & 269 & 266 & 263 & 262 & 262 & 265 & 265 & 265 & 265 & 265 & 267 \\
\hline 706 & 671 & 548 & 506 & 391 & 402 & 400 & 394 & 408 & 627 & 690 & 641 & 671 & 712 & 535 \\
\hline
\end{tabular}

\begin{tabular}{|c|c|c|c|c|c|c|c|c|c|c|c|c|c|c|}
\hline 0 & 0 & 0 & 0 & 0 & 0 & 0 & 0 & 0 & 0 & 0 & 0 & 0 & 0 & 0 \\
\hline 0 & 0 & 0 & 0 & 0 & 0 & 0 & 0 & 0 & 0 & 0 & 0 & 0 & 0 & 0 \\
\hline 0 & 0 & 0 & 0 & 0 & 0 & 0 & 0 & 0 & 0 & 0 & 0 & 0 & 0 & 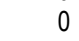 \\
\hline 2618 & 2618 & 2399 & 2386 & 2678 & 3076 & 3175 & 3035 & 2765 & 2602 & 2597 & 2540 & 2600 & 2717 & 2716 \\
\hline 1731 & 1731 & 1895 & 1925 & 2290 & 2581 & 2622 & 2601 & 2382 & 2147 & 2141 & 2030 & 1763 & 1733 & 2142 \\
\hline 840 & 645 & 499 & 609 & 665 & 670 & 549 & 524 & 571 & 542 & 523 & 556 & 638 & 724 & 607 \\
\hline 1399 & 1074 & 831 & 1014 & 1108 & 1117 & 915 & 873 & 951 & 903 & 870 & 927 & 1063 & 1206 & 1011 \\
\hline 382 & 382 & 382 & 382 & 382 & 382 & 382 & 382 & 382 & 382 & 382 & 382 & 382 & 382 & 382 \\
\hline 1070 & 1071 & 1062 & 974 & 961 & 995 & 991 & 987 & 950 & 974 & 974 & 992 & 1070 & 1064 & 1007 \\
\hline 1248 & 1277 & 461 & 346 & 647 & 660 & 658 & 602 & 450 & 367 & 368 & 172 & 232 & 282 & 512 \\
\hline 0 & 0 & 0 & 0 & 0 & 0 & 0 & 0 & 0 & 0 & 0 & 0 & 0 & 0 & \\
\hline 0 & 0 & 0 & 0 & 0 & 0 & 0 & 0 & 0 & 0 & 0 & 0 & 0 & 0 & \\
\hline 0 & 0 & 0 & 0 & 0 & 0 & 0 & 0 & 0 & 0 & 0 & 0 & 0 & 0 & \\
\hline
\end{tabular}

Total Firm Non-Utility Obligations

$\begin{array}{lllllllllllllll}9288 & 8798 & 7528 & 7637 & 8731 & 9481 & 9293 & 9004 & 8451 & 7918 & 7855 & 7599 & 7750 & 8107 & 8376\end{array}$

Transfers Out

GPU 1981 PSC Sale

IOU 1981 PSC Sale

NGP 2002 PSC Sale

GPU 2002 PSC Sale

NGP Slice Sale

GPU Slice Sale

IOU 2002 PSC Sale

Exports

Intra-Regional Transfers (Out)

Supp \& Entitlement Capacity (Out)

CSPE To West Group Utilities

Federal Diversity

Total Transfers Out

$9993 \quad 9468$

$8076 \quad 8143$

91229883

$9692 \quad 9398 \quad 8858$

$8546 \quad 8545$

8240

8421

$8819 \quad 8911$

Hydro Resources

Regulated Hydro

Independent Hydro

Sustained Peaking Adjustment

Non-Fed CER (CSPE)

Non-Fed CER (Canada)

Restoration

\begin{tabular}{|c|c|c|c|c|c|c|c|c|c|c|c|c|c|c|}
\hline 9248 & 6885 & 5135 & 6320 & 7020 & 7142 & 5731 & 5407 & 5916 & 5561 & 5238 & 6095 & 7314 & 7806 & 6446 \\
\hline 437 & 435 & 370 & 386 & 305 & 237 & 176 & 196 & 275 & 434 & 515 & 711 & 746 & 451 & 397 \\
\hline 0 & 0 & 0 & 0 & 0 & 0 & 0 & 0 & 0 & 0 & 0 & 0 & 0 & 0 & \\
\hline 0 & 0 & 0 & 0 & 0 & 0 & 0 & 0 & 0 & 0 & 0 & 0 & 0 & 0 & \\
\hline 143 & 143 & 143 & 143 & 143 & 143 & 143 & 143 & 143 & 141 & 141 & 141 & 141 & 141 & 142 \\
\hline 0 & 0 & 0 & 0 & 0 & 0 & 0 & 0 & 0 & 0 & 0 & 0 & 0 & 0 & \\
\hline
\end{tabular}

Total Hydro Resources

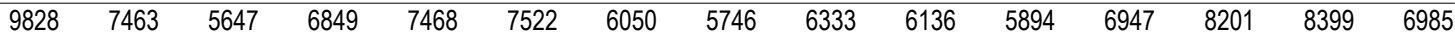

Other Resources

Small Thermal \& Misc.

Combustion Turbines

Renewables

Cogeneration

Imports

Intra-Regional Transfers (In)

Supp \& Entitlement Capacity (In)

Large Thermal

Non-Utility Generation

Resource Acquisition

$\begin{array}{rrrr}0 & 0 & 0 & 0 \\ 0 & 0 & 0 & 0 \\ 27 & 27 & 27 & 28 \\ 0 & 0 & 0 & 0 \\ 183 & 183 & 216 & 270 \\ 1448 & 1452 & 1623 & 1521 \\ 0 & 0 & 0 & 0 \\ 1000 & 1000 & 1000 & 1000 \\ 83 & 81 & 58 & 76 \\ 0 & 0 & 0 & 0\end{array}$

$\begin{array}{rr}0 & 0 \\ 0 & 0 \\ 29 & 31 \\ 0 & 0 \\ 338 & 395 \\ 1567 & 1503 \\ 0 & 0 \\ 1000 & 1000 \\ 101 & 104 \\ 0 & 0\end{array}$

$\begin{array}{rr}0 & \\ 0 & \\ 32 & \\ 0 & \\ 369 & 325 \\ 1562 & 1550 \\ 0 & \\ 1000 & 1000 \\ 107 & \\ 0 & \end{array}$

0
0
31
0
295
1529
0
1000
91
0

$\begin{array}{rr}0 & 0 \\ 0 & 0 \\ 30 & 30 \\ 0 & 0 \\ 276 & 238 \\ 1273 & 873 \\ 0 & 0 \\ 1000 & 1000 \\ 113 & 113 \\ 0 & 0\end{array}$

0
0
27
0
101
754
0
194
107
0

$\begin{array}{rrr}0 & 0 & 0 \\ 0 & 0 & 0 \\ 27 & 27 & 29 \\ 0 & 0 & 0 \\ 123 & 204 & 256 \\ 875 & 1396 & 1367 \\ 0 & 0 & 0 \\ 133 & 1000 & 861 \\ 94 & 75 & 92 \\ 0 & 0 & 0\end{array}$

Total Other Resources

$\begin{array}{rrrrrrrrrrrrrrr}2741 & 2743 & 2924 & 2895 & 3035 & 3034 & 3069 & 3004 & 2946 & 2692 & 2254 & 1183 & 1253 & 2702 & 2605 \\ 12569 & 10206 & 8572 & 9743 & 10502 & 10555 & 9119 & 8750 & 9279 & 8828 & 8149 & 8130 & 9454 & 11101 & 9590\end{array}$

Total Resources

Reserves \& Maintenance

Hydro Reserves

Small Thermal \& Misc. Reserves

Contract Reserves

Large Thermal Reserves

Federal Hydro Maintenance

Spinning Reserves

Federal Transmission Losses

Total Reserves, Maintenance \& Losses

Total Net Resources

$12215 \quad 9918$

\begin{tabular}{rrrrrrrrrrrrrrr}
0 & 0 & 0 & 0 & 0 & 0 & 0 & 0 & 0 & 0 & 0 & 0 & 0 & 0 & 0 \\
0 & 0 & 0 & 0 & 0 & 0 & 0 & 0 & 0 & 0 & 0 & 0 & 0 & 0 & 0 \\
0 & 0 & 0 & 0 & 0 & 0 & 0 & 0 & 0 & 0 & 0 & 0 & 0 & 0 & 0 \\
0 & 0 & 0 & 0 & 0 & 0 & 0 & 0 & 0 & 0 & 0 & 0 & 0 & 0 & 0 \\
0 & 0 & 0 & 0 & 0 & 0 & 0 & 0 & 0 & 0 & 0 & 0 & 0 & 0 & 0 \\
0 & 0 & 0 & 0 & 0 & 0 & 0 & 0 & 0 & 0 & 0 & 0 & 0 & 0 & 0 \\
-354 & -288 & -242 & -275 & -296 & -298 & -257 & -247 & -262 & -249 & -230 & -229 & -267 & -313 & -270 \\
\hline-354 & -288 & -242 & -275 & -296 & -298 & -257 & -247 & -262 & -249 & -230 & -229 & -267 & -313 & -270 \\
& & & & & & & & & & & & & & \\
\hline
\end{tabular}

222

$\begin{array}{llll}450 & 254 & 1326 & 1084\end{array}$

$\begin{array}{lll}375 & -830 & -895\end{array}$

159

$\begin{array}{lll}34 & -626 & -339\end{array}$

$\begin{array}{lll}767 & 1969 \quad 409\end{array}$ 
Loads and Resources - Federal System

PNW Loads and Resources Study

2005 - 2006 Operating Year

1937 Water Year

2003 White Book

Non-Utility Obligations

Federal Agencies 1981 PSC Sale

USBR 1981 PSC Sale

DSI 1981 PSC Sale

Federal Agencies 2002 PSC Sale

USBR 2002 PSC Sale

DSI 2002 PSC Sale

\begin{tabular}{|c|c|c|c|c|c|c|c|c|c|c|c|c|c|c|}
\hline $\lg 1$ & Aug16 & Sep & Oct & Nov & Dec & Jan & $F e b$ & Mar & Apr1 & Apr16 & May & Jun & Jul & Avg \\
\hline 0 & 0 & 0 & 0 & 0 & 0 & 0 & 0 & 0 & 0 & 0 & 0 & 0 & 0 & 0 \\
\hline 0 & 0 & 0 & 0 & 0 & 0 & 0 & 0 & 0 & 0 & 0 & 0 & 0 & 0 & 0 \\
\hline 0 & 0 & 0 & 0 & 0 & 0 & 0 & 0 & 0 & 0 & 0 & 0 & 0 & 0 & 0 \\
\hline 120 & 120 & 109 & 106 & 122 & 137 & 137 & 132 & 123 & 111 & 111 & 108 & 109 & 116 & 119 \\
\hline 309 & 275 & 166 & 129 & 0 & 0 & 0 & 1.0 & 23 & 252 & 315 & 268 & 298 & 332 & 149 \\
\hline 265 & 265 & 265 & 272 & 272 & 272 & 272 & 272 & 272 & 272 & 272 & 272 & 272 & 272 & 271 \\
\hline 694 & 660 & 540 & 508 & 394 & 409 & 409 & 405 & 418 & 635 & 698 & 648 & 679 & 720 & 539 \\
\hline 0 & 0 & 0 & 0 & 0 & 0 & 0 & 0 & 0 & 0 & 0 & 0 & 0 & 0 & 0 \\
\hline 0 & 0 & 0 & 0 & 0 & 0 & 0 & 0 & 0 & 0 & 0 & 0 & 0 & 0 & 0 \\
\hline 0 & 0 & 0 & 0 & 0 & 0 & 0 & 0 & 0 & 0 & 0 & 0 & 0 & 0 & 0 \\
\hline 2654 & 2654 & 2432 & 2416 & 2712 & 3116 & 3223 & 3082 & 2808 & 2644 & 2637 & 2580 & 2640 & 2759 & 2755 \\
\hline 1723 & 1723 & 1897 & 1935 & 2299 & 2585 & 2626 & 2580 & 2388 & 2101 & 2095 & 1980 & 1715 & 1685 & 2126 \\
\hline 846 & 651 & 504 & 613 & 670 & 675 & 553 & 528 & 574 & 546 & 526 & 609 & 713 & 726 & 621 \\
\hline 1410 & 1084 & 840 & 1021 & 1116 & 1124 & 921 & 879 & 957 & 909 & 875 & 1015 & 1188 & 1210 & 1034 \\
\hline 382 & 382 & 382 & 382 & 382 & 382 & 382 & 382 & 382 & 382 & 382 & 382 & 382 & 382 & 382 \\
\hline 1036 & 1036 & 1027 & 935 & 939 & 954 & 952 & 948 & 940 & 964 & 963 & 983 & 991 & 945 & 968 \\
\hline 252 & 253 & 292 & 346 & 647 & 660 & 658 & 602 & 450 & 367 & 368 & 172 & 232 & 282 & 413 \\
\hline 0 & 0 & 0 & 0 & 0 & 0 & 0 & 0 & 0 & 0 & 0 & 0 & 0 & 0 & 0 \\
\hline 0 & 0 & 0 & 0 & 0 & 0 & 0 & 0 & 0 & 0 & 0 & 0 & 0 & 0 & 0 \\
\hline 0 & 0 & 0 & 0 & 0 & 0 & 0 & 0 & 0 & 0 & 0 & 0 & 0 & 0 & 0 \\
\hline 8303 & 7782 & 7374 & 7649 & 8765 & 9496 & 9316 & 9001 & 8499 & 7913 & 7847 & 7722 & 7862 & 7989 & 8300 \\
\hline 8998 & 8443 & 7914 & 8157 & 9159 & 9905 & 9725 & 9405 & 8917 & 8548 & 8545 & 8370 & 8541 & 8709 & 8839 \\
\hline 9278 & 6907 & 5151 & 6342 & 7050 & 7171 & 5752 & 5427 & 5931 & 5577 & 5248 & 6096 & 7328 & 7811 & 6464 \\
\hline 437 & 435 & 370 & 386 & 305 & 237 & 176 & 196 & 275 & 434 & 515 & 711 & 747 & 451 & 397 \\
\hline 0 & 0 & 0 & 0 & 0 & 0 & 0 & 0 & 0 & 0 & 0 & 0 & 0 & 0 & 0 \\
\hline 0 & 0 & 0 & 0 & 0 & 0 & 0 & 0 & 0 & 0 & 0 & 0 & 0 & 0 & 0 \\
\hline 141 & 141 & 141 & 141 & 141 & 141 & 141 & 141 & 141 & 136 & 136 & 136 & 136 & 136 & 139 \\
\hline 0 & 0 & 0 & 0 & 0 & 0 & 0 & 0 & 0 & 0 & 0 & 0 & 0 & 0 & 0 \\
\hline 9856 & 7484 & 5663 & 6869 & 7497 & 7550 & 6069 & 5765 & 6347 & 6146 & 5899 & 6942 & 8210 & 8398 & 7000 \\
\hline 0 & 0 & 0 & 0 & 0 & 0 & 0 & 0 & 0 & 0 & 0 & 0 & 0 & 0 & 0 \\
\hline 0 & 0 & 0 & 0 & 0 & 0 & 0 & 0 & 0 & 0 & 0 & 0 & 0 & 0 & 0 \\
\hline 27 & 27 & 27 & 28 & 29 & 31 & 32 & 31 & 31 & 30 & 30 & 27 & 27 & 27 & 29 \\
\hline 0 & 0 & 0 & 0 & 0 & 0 & 0 & 0 & 0 & 0 & 0 & 0 & 0 & 0 & 0 \\
\hline 183 & 183 & 216 & 250 & 318 & 375 & 349 & 305 & 275 & 276 & 238 & 101 & 123 & 204 & 246 \\
\hline 1422 & 1425 & 1567 & 1520 & 1568 & 1503 & 1161 & 1151 & 1128 & 873 & 871 & 756 & 874 & 996 & 1210 \\
\hline 0 & 0 & 0 & 0 & 0 & 0 & 0 & 0 & 0 & 0 & 0 & 0 & 0 & 0 & 0 \\
\hline 1000 & 1000 & 1000 & 1000 & 1000 & 1000 & 1000 & 1000 & 1000 & 1000 & 1000 & 1000 & 1000 & 1000 & 1000 \\
\hline 83 & 81 & 58 & 76 & 101 & 104 & 107 & 98 & 91 & 113 & 113 & 107 & 94 & 75 & 92 \\
\hline 0 & 0 & 0 & 0 & 0 & 0 & 0 & 0 & 0 & 0 & 0 & 0 & 0 & 0 & 0 \\
\hline 715 & 2716 & 2869 & 2874 & 3016 & 3014 & 2648 & 2585 & 2525 & 2292 & 2252 & 1991 & 2119 & 2302 & 2577 \\
\hline 571 & 10200 & 8532 & 9743 & 10512 & 10563 & 8717 & 8350 & 8871 & 8438 & 8151 & 8933 & 10329 & 10700 & 9578 \\
\hline 0 & 0 & 0 & 0 & 0 & 0 & 0 & 0 & 0 & 0 & 0 & 0 & 0 & 0 & 0 \\
\hline
\end{tabular}

\begin{tabular}{|c|c|c|c|c|c|c|c|c|c|c|c|c|c|c|}
\hline Aug1 & Aug16 & Sep & Oct & Nov & Dec & Jan & $F e b$ & Mar & Apr1 & Apr16 & May & Jun & Jul & Avg \\
\hline 0 & 0 & 0 & 0 & 0 & 0 & 0 & 0 & 0 & 0 & 0 & 0 & 0 & 0 & 0 \\
\hline 0 & 0 & 0 & 0 & 0 & 0 & 0 & 0 & 0 & 0 & 0 & 0 & 0 & 0 & 0 \\
\hline 0 & 0 & 0 & 0 & 0 & 0 & 0 & 0 & 0 & 0 & 0 & 0 & 0 & 0 & 0 \\
\hline 120 & 120 & 109 & 106 & 122 & 137 & 137 & 132 & 123 & 111 & 111 & 108 & 109 & 116 & 119 \\
\hline 309 & 275 & 166 & 129 & 0 & 0 & 0 & 1.0 & 23 & 252 & 315 & 268 & 298 & 332 & 149 \\
\hline 265 & 265 & 265 & 272 & 272 & 272 & 272 & 272 & 272 & 272 & 272 & 272 & 272 & 272 & 271 \\
\hline 694 & 660 & 540 & 508 & 394 & 409 & 409 & 405 & 418 & 635 & 698 & 648 & 679 & 720 & 539 \\
\hline 0 & 0 & 0 & 0 & 0 & 0 & 0 & 0 & 0 & 0 & 0 & 0 & 0 & 0 & 0 \\
\hline 0 & 0 & 0 & 0 & 0 & 0 & 0 & 0 & 0 & 0 & 0 & 0 & 0 & 0 & 0 \\
\hline 0 & 0 & 0 & 0 & 0 & 0 & 0 & 0 & 0 & 0 & 0 & 0 & 0 & 0 & 0 \\
\hline 2654 & 2654 & 2432 & 2416 & 2712 & 3116 & 3223 & 3082 & 2808 & 2644 & 2637 & 2580 & 2640 & 2759 & 2755 \\
\hline 1723 & 1723 & 1897 & 1935 & 2299 & 2585 & 2626 & 2580 & 2388 & 2101 & 2095 & 1980 & 1715 & 1685 & 2126 \\
\hline 846 & 651 & 504 & 613 & 670 & 675 & 553 & 528 & 574 & 546 & 526 & 609 & 713 & 726 & 621 \\
\hline 1410 & 1084 & 840 & 1021 & 1116 & 1124 & 921 & 879 & 957 & 909 & 875 & 1015 & 1188 & 1210 & 1034 \\
\hline 382 & 382 & 382 & 382 & 382 & 382 & 382 & 382 & 382 & 382 & 382 & 382 & 382 & 382 & 382 \\
\hline 1036 & 1036 & 1027 & 935 & 939 & 954 & 952 & 948 & 940 & 964 & 963 & 983 & 991 & 945 & 968 \\
\hline 252 & 253 & 292 & 346 & 647 & 660 & 658 & 602 & 450 & 367 & 368 & 172 & 232 & 282 & 413 \\
\hline 0 & 0 & 0 & 0 & 0 & 0 & 0 & 0 & 0 & 0 & 0 & 0 & 0 & 0 & 0 \\
\hline 0 & 0 & 0 & 0 & 0 & 0 & 0 & 0 & 0 & 0 & 0 & 0 & 0 & 0 & 0 \\
\hline 0 & 0 & 0 & 0 & 0 & 0 & 0 & 0 & 0 & 0 & 0 & 0 & 0 & 0 & 0 \\
\hline 8303 & 7782 & 7374 & 7649 & 8765 & 9496 & 9316 & 9001 & 8499 & 7913 & 7847 & 7722 & 7862 & 7989 & 8300 \\
\hline 8998 & 8443 & 7914 & 8157 & 9159 & 9905 & 9725 & 9405 & 8917 & 8548 & 8545 & 8370 & 8541 & 8709 & 8839 \\
\hline 9278 & 6907 & 5151 & 6342 & 7050 & 7171 & 5752 & 5427 & 5931 & 5577 & 5248 & 6096 & 7328 & 7811 & 6464 \\
\hline 437 & 435 & 370 & 386 & 305 & 237 & 176 & 196 & 275 & 434 & 515 & 711 & 747 & 451 & 397 \\
\hline 0 & 0 & 0 & 0 & 0 & 0 & 0 & 0 & 0 & 0 & 0 & 0 & 0 & 0 & 0 \\
\hline 0 & 0 & 0 & 0 & 0 & 0 & 0 & 0 & 0 & 0 & 0 & 0 & 0 & 0 & 0 \\
\hline 141 & 141 & 141 & 141 & 141 & 141 & 141 & 141 & 141 & 136 & 136 & 136 & 136 & 136 & 139 \\
\hline 0 & 0 & 0 & 0 & 0 & 0 & 0 & 0 & 0 & 0 & 0 & 0 & 0 & 0 & 0 \\
\hline 9856 & 7484 & 5663 & 6869 & 7497 & 7550 & 6069 & 5765 & 6347 & 6146 & 5899 & 6942 & 8210 & 8398 & 7000 \\
\hline 0 & 0 & 0 & 0 & 0 & 0 & 0 & 0 & 0 & 0 & 0 & 0 & 0 & 0 & 0 \\
\hline 0 & 0 & 0 & 0 & 0 & 0 & 0 & 0 & 0 & 0 & 0 & 0 & 0 & 0 & 0 \\
\hline 27 & 27 & 27 & 28 & 29 & 31 & 32 & 31 & 31 & 30 & 30 & 27 & 27 & 27 & 29 \\
\hline 0 & 0 & 0 & 0 & 0 & 0 & 0 & 0 & 0 & 0 & 0 & 0 & 0 & 0 & 0 \\
\hline 183 & 183 & 216 & 250 & 318 & 375 & 349 & 305 & 275 & 276 & 238 & 101 & 123 & 204 & 246 \\
\hline 1422 & 1425 & 1567 & 1520 & 1568 & 1503 & 1161 & 1151 & 1128 & 873 & 871 & 756 & 874 & 996 & 1210 \\
\hline 0 & 0 & 0 & 0 & 0 & 0 & 0 & 0 & 0 & 0 & 0 & 0 & 0 & 0 & 0 \\
\hline 1000 & 1000 & 1000 & 1000 & 1000 & 1000 & 1000 & 1000 & 1000 & 1000 & 1000 & 1000 & 1000 & 1000 & 1000 \\
\hline 83 & 81 & 58 & 76 & 101 & 104 & 107 & 98 & 91 & 113 & 113 & 107 & 94 & 75 & 92 \\
\hline 0 & 0 & 0 & 0 & 0 & 0 & 0 & 0 & 0 & 0 & 0 & 0 & 0 & 0 & 0 \\
\hline 2715 & 2716 & 2869 & 2874 & 3016 & 3014 & 2648 & 2585 & 2525 & 2292 & 2252 & 1991 & 2119 & 2302 & 2577 \\
\hline 12571 & 10200 & 8532 & 9743 & 10512 & 10563 & 8717 & 8350 & 8871 & 8438 & 8151 & 8933 & 10329 & 10700 & 9578 \\
\hline 0 & 0 & 0 & 0 & 0 & 0 & 0 & 0 & 0 & 0 & 0 & 0 & 0 & 0 & 0 \\
\hline
\end{tabular}

Total Transfers Ou

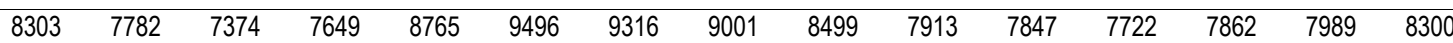

Total Firm Obligations

8998

$8443 \quad 7914 \quad 8157$

$9159 \quad 9905$

$9725 \quad 9405 \quad 8917$

$8548 \quad 8545$

$\begin{array}{llll}8370 & 8541 & 8709 & 8839\end{array}$

Hydro Resources

Regulated Hydro

Independent Hydro

Sustained Peaking Adjustment

Non-Fed CER (CSPE)

Non-Fed CER (Canada)

Restoration

\begin{tabular}{|c|c|c|c|c|c|c|c|c|c|c|c|c|c|c|}
\hline Aug1 & Aug16 & Sep & Oct & Nov & $D e c$ & Jan & $F e b$ & Mar & Apr1 & Apr16 & May & Jun & Jul & Avg \\
\hline 0 & 0 & 0 & 0 & 0 & 0 & 0 & 0 & 0 & 0 & 0 & 0 & 0 & 0 & 0 \\
\hline 0 & 0 & 0 & 0 & 0 & 0 & 0 & 0 & 0 & 0 & 0 & 0 & 0 & 0 & 0 \\
\hline 0 & 0 & 0 & 0 & 0 & 0 & 0 & 0 & 0 & 0 & 0 & 0 & 0 & 0 & 0 \\
\hline 120 & 120 & 109 & 106 & 122 & 137 & 137 & 132 & 123 & 111 & 111 & 108 & 109 & 116 & 119 \\
\hline 309 & 275 & 166 & 129 & 0 & 0 & 0 & 1.0 & 23 & 252 & 315 & 268 & 298 & 332 & 149 \\
\hline 265 & 265 & 265 & 272 & 272 & 272 & 272 & 272 & 272 & 272 & 272 & 272 & 272 & 272 & 271 \\
\hline 694 & 660 & 540 & 508 & 394 & 409 & 409 & 405 & 418 & 635 & 698 & 648 & 679 & 720 & 539 \\
\hline 0 & 0 & 0 & 0 & 0 & 0 & 0 & 0 & 0 & 0 & 0 & 0 & 0 & 0 & 0 \\
\hline 0 & 0 & 0 & 0 & 0 & 0 & 0 & 0 & 0 & 0 & 0 & 0 & 0 & 0 & 0 \\
\hline 0 & 0 & 0 & 0 & 0 & 0 & 0 & 0 & 0 & 0 & 0 & 0 & 0 & 0 & 0 \\
\hline 2654 & 2654 & 2432 & 2416 & 2712 & 3116 & 3223 & 3082 & 2808 & 2644 & 2637 & 2580 & 2640 & 2759 & 2755 \\
\hline 1723 & 1723 & 1897 & 1935 & 2299 & 2585 & 2626 & 2580 & 2388 & 2101 & 2095 & 1980 & 1715 & 1685 & 2126 \\
\hline 846 & 651 & 504 & 613 & 670 & 675 & 553 & 528 & 574 & 546 & 526 & 609 & 713 & 726 & 621 \\
\hline 1410 & 1084 & 840 & 1021 & 1116 & 1124 & 921 & 879 & 957 & 909 & 875 & 1015 & 1188 & 1210 & 1034 \\
\hline 382 & 382 & 382 & 382 & 382 & 382 & 382 & 382 & 382 & 382 & 382 & 382 & 382 & 382 & 382 \\
\hline 1036 & 1036 & 1027 & 935 & 939 & 954 & 952 & 948 & 940 & 964 & 963 & 983 & 991 & 945 & 968 \\
\hline 252 & 253 & 292 & 346 & 647 & 660 & 658 & 602 & 450 & 367 & 368 & 172 & 232 & 282 & 413 \\
\hline 0 & 0 & 0 & 0 & 0 & 0 & 0 & 0 & 0 & 0 & 0 & 0 & 0 & 0 & 0 \\
\hline 0 & 0 & 0 & 0 & 0 & 0 & 0 & 0 & 0 & 0 & 0 & 0 & 0 & 0 & 0 \\
\hline 0 & 0 & 0 & 0 & 0 & 0 & 0 & 0 & 0 & 0 & 0 & 0 & 0 & 0 & 0 \\
\hline 8303 & 7782 & 7374 & 7649 & 8765 & 9496 & 9316 & 9001 & 8499 & 7913 & 7847 & 7722 & 7862 & 7989 & 8300 \\
\hline 8998 & 8443 & 7914 & 8157 & 9159 & 9905 & 9725 & 9405 & 8917 & 8548 & 8545 & 8370 & 8541 & 8709 & 8839 \\
\hline 9278 & 6907 & 5151 & 6342 & 7050 & 7171 & 5752 & 5427 & 5931 & 5577 & 5248 & 6096 & 7328 & 7811 & 6464 \\
\hline 437 & 435 & 370 & 386 & 305 & 237 & 176 & 196 & 275 & 434 & 515 & 711 & 747 & 451 & 397 \\
\hline 0 & 0 & 0 & 0 & 0 & 0 & 0 & 0 & 0 & 0 & 0 & 0 & 0 & 0 & 0 \\
\hline 0 & 0 & 0 & 0 & 0 & 0 & 0 & 0 & 0 & 0 & 0 & 0 & 0 & 0 & 0 \\
\hline 141 & 141 & 141 & 141 & 141 & 141 & 141 & 141 & 141 & 136 & 136 & 136 & 136 & 136 & 139 \\
\hline 0 & 0 & 0 & 0 & 0 & 0 & 0 & 0 & 0 & 0 & 0 & 0 & 0 & 0 & 0 \\
\hline 9856 & 7484 & 5663 & 6869 & 7497 & 7550 & 6069 & 5765 & 6347 & 6146 & 5899 & 6942 & 8210 & 8398 & 7000 \\
\hline 0 & 0 & 0 & 0 & 0 & 0 & 0 & 0 & 0 & 0 & 0 & 0 & 0 & 0 & 0 \\
\hline 0 & 0 & 0 & 0 & 0 & 0 & 0 & 0 & 0 & 0 & 0 & 0 & 0 & 0 & 0 \\
\hline 27 & 27 & 27 & 28 & 29 & 31 & 32 & 31 & 31 & 30 & 30 & 27 & 27 & 27 & 29 \\
\hline 0 & 0 & 0 & 0 & 0 & 0 & 0 & 0 & 0 & 0 & 0 & 0 & 0 & 0 & 0 \\
\hline 183 & 183 & 216 & 250 & 318 & 375 & 349 & 305 & 275 & 276 & 238 & 101 & 123 & 204 & 246 \\
\hline 1422 & 1425 & 1567 & 1520 & 1568 & 1503 & 1161 & 1151 & 1128 & 873 & 871 & 756 & 874 & 996 & 1210 \\
\hline 0 & 0 & 0 & 0 & 0 & 0 & 0 & 0 & 0 & 0 & 0 & 0 & 0 & 0 & 0 \\
\hline 1000 & 1000 & 1000 & 1000 & 1000 & 1000 & 1000 & 1000 & 1000 & 1000 & 1000 & 1000 & 1000 & 1000 & 1000 \\
\hline 83 & 81 & 58 & 76 & 101 & 104 & 107 & 98 & 91 & 113 & 113 & 107 & 94 & 75 & 92 \\
\hline 0 & 0 & 0 & 0 & 0 & 0 & 0 & 0 & 0 & 0 & 0 & 0 & 0 & 0 & 0 \\
\hline 2715 & 2716 & 2869 & 2874 & 3016 & 3014 & 2648 & 2585 & 2525 & 2292 & 2252 & 1991 & 2119 & 2302 & 2577 \\
\hline 12571 & 10200 & 8532 & 9743 & 10512 & 10563 & 8717 & 8350 & 8871 & 8438 & 8151 & 8933 & 10329 & 10700 & 9578 \\
\hline 0 & 0 & 0 & 0 & 0 & 0 & 0 & 0 & 0 & 0 & 0 & 0 & 0 & 0 & o \\
\hline
\end{tabular}

\begin{tabular}{|c|c|c|c|c|c|c|c|c|c|c|c|c|c|}
\hline Aug16 & Sep & Oct & Nov & $D e c$ & Jan & $F e b$ & Mar & Apr1 & Apr16 & May & Jun & Jul & Avg \\
\hline 0 & 0 & 0 & 0 & 0 & 0 & 0 & 0 & 0 & 0 & 0 & 0 & 0 & 0 \\
\hline 0 & 0 & 0 & 0 & 0 & 0 & 0 & 0 & 0 & 0 & 0 & 0 & 0 & 0 \\
\hline 0 & 0 & 0 & 0 & 0 & 0 & 0 & 0 & 0 & 0 & 0 & 0 & 0 & 0 \\
\hline 120 & 109 & 106 & 122 & 137 & 137 & 132 & 123 & 111 & 111 & 108 & 109 & 116 & 119 \\
\hline 275 & 166 & 129 & 0 & 0 & 0 & 1.0 & 23 & 252 & 315 & 268 & 298 & 332 & 149 \\
\hline 265 & 265 & 272 & 272 & 272 & 272 & 272 & 272 & 272 & 272 & 272 & 272 & 272 & 271 \\
\hline 660 & 540 & 508 & 394 & 409 & 409 & 405 & 418 & 635 & 698 & 648 & 679 & 720 & 539 \\
\hline 0 & 0 & 0 & 0 & 0 & 0 & 0 & 0 & 0 & 0 & 0 & 0 & 0 & 0 \\
\hline 0 & 0 & 0 & 0 & 0 & 0 & 0 & 0 & 0 & 0 & 0 & 0 & 0 & 0 \\
\hline 0 & 0 & 0 & 0 & 0 & 0 & 0 & 0 & 0 & 0 & 0 & 0 & 0 & 0 \\
\hline 2654 & 2432 & 2416 & 2712 & 3116 & 3223 & 3082 & 2808 & 2644 & 2637 & 2580 & 2640 & 2759 & 2755 \\
\hline 1723 & 1897 & 1935 & 2299 & 2585 & 2626 & 2580 & 2388 & 2101 & 2095 & 1980 & 1715 & 1685 & 2126 \\
\hline 651 & 504 & 613 & 670 & 675 & 553 & 528 & 574 & 546 & 526 & 609 & 713 & 726 & 621 \\
\hline 1084 & 840 & 1021 & 1116 & 1124 & 921 & 879 & 957 & 909 & 875 & 1015 & 1188 & 1210 & 1034 \\
\hline 382 & 382 & 382 & 382 & 382 & 382 & 382 & 382 & 382 & 382 & 382 & 382 & 382 & 382 \\
\hline 1036 & 1027 & 935 & 939 & 954 & 952 & 948 & 940 & 964 & 963 & 983 & 991 & 945 & 968 \\
\hline 253 & 292 & 346 & 647 & 660 & 658 & 602 & 450 & 367 & 368 & 172 & 232 & 282 & 413 \\
\hline 0 & 0 & 0 & 0 & 0 & 0 & 0 & 0 & 0 & 0 & 0 & 0 & 0 & 0 \\
\hline 0 & 0 & 0 & 0 & 0 & 0 & 0 & 0 & 0 & 0 & 0 & 0 & 0 & 0 \\
\hline 0 & 0 & 0 & 0 & 0 & 0 & 0 & 0 & 0 & 0 & 0 & 0 & 0 & 0 \\
\hline 7782 & 7374 & 7649 & 8765 & 9496 & 9316 & 9001 & 8499 & 7913 & 7847 & 7722 & 7862 & 7989 & 8300 \\
\hline 8443 & 7914 & 8157 & 9159 & 9905 & 9725 & 9405 & 8917 & 8548 & 8545 & 8370 & 8541 & 8709 & 8839 \\
\hline 6907 & 5151 & 6342 & 7050 & 7171 & 5752 & 5427 & 5931 & 5577 & 5248 & 6096 & 7328 & 7811 & 6464 \\
\hline 435 & 370 & 386 & 305 & 237 & 176 & 196 & 275 & 434 & 515 & 711 & 747 & 451 & 397 \\
\hline 0 & 0 & 0 & 0 & 0 & 0 & 0 & 0 & 0 & 0 & 0 & 0 & 0 & 0 \\
\hline 0 & 0 & 0 & 0 & 0 & 0 & 0 & 0 & 0 & 0 & 0 & 0 & 0 & 0 \\
\hline 141 & 141 & 141 & 141 & 141 & 141 & 141 & 141 & 136 & 136 & 136 & 136 & 136 & 139 \\
\hline 0 & 0 & 0 & 0 & 0 & 0 & 0 & 0 & 0 & 0 & 0 & 0 & 0 & 0 \\
\hline 7484 & 5663 & 6869 & 7497 & 7550 & 6069 & 5765 & 6347 & 6146 & 5899 & 6942 & 8210 & 8398 & 7000 \\
\hline 0 & 0 & 0 & 0 & 0 & 0 & 0 & 0 & 0 & 0 & 0 & 0 & 0 & 0 \\
\hline 0 & 0 & 0 & 0 & 0 & 0 & 0 & 0 & 0 & 0 & 0 & 0 & 0 & 0 \\
\hline 27 & 27 & 28 & 29 & 31 & 32 & 31 & 31 & 30 & 30 & 27 & 27 & 27 & 29 \\
\hline 0 & 0 & 0 & 0 & 0 & 0 & 0 & 0 & 0 & 0 & 0 & 0 & 0 & 0 \\
\hline 183 & 216 & 250 & 318 & 375 & 349 & 305 & 275 & 276 & 238 & 101 & 123 & 204 & 246 \\
\hline 1425 & 1567 & 1520 & 1568 & 1503 & 1161 & 1151 & 1128 & 873 & 871 & 756 & 874 & 996 & 1210 \\
\hline 0 & 0 & 0 & 0 & 0 & 0 & 0 & 0 & 0 & 0 & 0 & 0 & 0 & 0 \\
\hline 1000 & 1000 & 1000 & 1000 & 1000 & 1000 & 1000 & 1000 & 1000 & 1000 & 1000 & 1000 & 1000 & 1000 \\
\hline 81 & 58 & 76 & 101 & 104 & 107 & 98 & 91 & 113 & 113 & 107 & 94 & 75 & 92 \\
\hline 0 & 0 & 0 & 0 & 0 & 0 & 0 & 0 & 0 & 0 & 0 & 0 & 0 & 0 \\
\hline 2716 & 2869 & 2874 & 3016 & 3014 & 2648 & 2585 & 2525 & 2292 & 2252 & 1991 & 2119 & 2302 & 2577 \\
\hline 10200 & 8532 & 9743 & 10512 & 10563 & 8717 & 8350 & 8871 & 8438 & 8151 & 8933 & 10329 & 10700 & 9578 \\
\hline 0 & 0 & 0 & 0 & 0 & 0 & 0 & 0 & 0 & 0 & 0 & 0 & 0 & 0 \\
\hline
\end{tabular}

\begin{tabular}{|c|c|c|c|c|c|c|c|c|c|c|c|c|c|}
\hline Aug16 & Sep & Oct & Nov & $D e c$ & Jan & $F e b$ & Mar & Apr1 & Apr16 & May & Jun & Jul & Avg \\
\hline 0 & 0 & 0 & 0 & 0 & 0 & 0 & 0 & 0 & 0 & 0 & 0 & 0 & 0 \\
\hline 0 & 0 & 0 & 0 & 0 & 0 & 0 & 0 & 0 & 0 & 0 & 0 & 0 & 0 \\
\hline 0 & 0 & 0 & 0 & 0 & 0 & 0 & 0 & 0 & 0 & 0 & 0 & 0 & 0 \\
\hline 120 & 109 & 106 & 122 & 137 & 137 & 132 & 123 & 111 & 111 & 108 & 109 & 116 & 119 \\
\hline 275 & 166 & 129 & 0 & 0 & 0 & 1.0 & 23 & 252 & 315 & 268 & 298 & 332 & 149 \\
\hline 265 & 265 & 272 & 272 & 272 & 272 & 272 & 272 & 272 & 272 & 272 & 272 & 272 & 271 \\
\hline 660 & 540 & 508 & 394 & 409 & 409 & 405 & 418 & 635 & 698 & 648 & 679 & 720 & 539 \\
\hline 0 & 0 & 0 & 0 & 0 & 0 & 0 & 0 & 0 & 0 & 0 & 0 & 0 & 0 \\
\hline 0 & 0 & 0 & 0 & 0 & 0 & 0 & 0 & 0 & 0 & 0 & 0 & 0 & 0 \\
\hline 0 & 0 & 0 & 0 & 0 & 0 & 0 & 0 & 0 & 0 & 0 & 0 & 0 & 0 \\
\hline 2654 & 2432 & 2416 & 2712 & 3116 & 3223 & 3082 & 2808 & 2644 & 2637 & 2580 & 2640 & 2759 & 2755 \\
\hline 1723 & 1897 & 1935 & 2299 & 2585 & 2626 & 2580 & 2388 & 2101 & 2095 & 1980 & 1715 & 1685 & 2126 \\
\hline 651 & 504 & 613 & 670 & 675 & 553 & 528 & 574 & 546 & 526 & 609 & 713 & 726 & 621 \\
\hline 1084 & 840 & 1021 & 1116 & 1124 & 921 & 879 & 957 & 909 & 875 & 1015 & 1188 & 1210 & 1034 \\
\hline 382 & 382 & 382 & 382 & 382 & 382 & 382 & 382 & 382 & 382 & 382 & 382 & 382 & 382 \\
\hline 1036 & 1027 & 935 & 939 & 954 & 952 & 948 & 940 & 964 & 963 & 983 & 991 & 945 & 968 \\
\hline 253 & 292 & 346 & 647 & 660 & 658 & 602 & 450 & 367 & 368 & 172 & 232 & 282 & 413 \\
\hline 0 & 0 & 0 & 0 & 0 & 0 & 0 & 0 & 0 & 0 & 0 & 0 & 0 & 0 \\
\hline 0 & 0 & 0 & 0 & 0 & 0 & 0 & 0 & 0 & 0 & 0 & 0 & 0 & 0 \\
\hline 0 & 0 & 0 & 0 & 0 & 0 & 0 & 0 & 0 & 0 & 0 & 0 & 0 & 0 \\
\hline 7782 & 7374 & 7649 & 8765 & 9496 & 9316 & 9001 & 8499 & 7913 & 7847 & 7722 & 7862 & 7989 & 8300 \\
\hline 8443 & 7914 & 8157 & 9159 & 9905 & 9725 & 9405 & 8917 & 8548 & 8545 & 8370 & 8541 & 8709 & 8839 \\
\hline 6907 & 5151 & 6342 & 7050 & 7171 & 5752 & 5427 & 5931 & 5577 & 5248 & 6096 & 7328 & 7811 & 6464 \\
\hline 435 & 370 & 386 & 305 & 237 & 176 & 196 & 275 & 434 & 515 & 711 & 747 & 451 & 397 \\
\hline 0 & 0 & 0 & 0 & 0 & 0 & 0 & 0 & 0 & 0 & 0 & 0 & 0 & 0 \\
\hline 0 & 0 & 0 & 0 & 0 & 0 & 0 & 0 & 0 & 0 & 0 & 0 & 0 & 0 \\
\hline 141 & 141 & 141 & 141 & 141 & 141 & 141 & 141 & 136 & 136 & 136 & 136 & 136 & 139 \\
\hline 0 & 0 & 0 & 0 & 0 & 0 & 0 & 0 & 0 & 0 & 0 & 0 & 0 & 0 \\
\hline 7484 & 5663 & 6869 & 7497 & 7550 & 6069 & 5765 & 6347 & 6146 & 5899 & 6942 & 8210 & 8398 & 7000 \\
\hline 0 & 0 & 0 & 0 & 0 & 0 & 0 & 0 & 0 & 0 & 0 & 0 & 0 & 0 \\
\hline 0 & 0 & 0 & 0 & 0 & 0 & 0 & 0 & 0 & 0 & 0 & 0 & 0 & 0 \\
\hline 27 & 27 & 28 & 29 & 31 & 32 & 31 & 31 & 30 & 30 & 27 & 27 & 27 & 29 \\
\hline 0 & 0 & 0 & 0 & 0 & 0 & 0 & 0 & 0 & 0 & 0 & 0 & 0 & 0 \\
\hline 183 & 216 & 250 & 318 & 375 & 349 & 305 & 275 & 276 & 238 & 101 & 123 & 204 & 246 \\
\hline 1425 & 1567 & 1520 & 1568 & 1503 & 1161 & 1151 & 1128 & 873 & 871 & 756 & 874 & 996 & 1210 \\
\hline 0 & 0 & 0 & 0 & 0 & 0 & 0 & 0 & 0 & 0 & 0 & 0 & 0 & 0 \\
\hline 1000 & 1000 & 1000 & 1000 & 1000 & 1000 & 1000 & 1000 & 1000 & 1000 & 1000 & 1000 & 1000 & 1000 \\
\hline 81 & 58 & 76 & 101 & 104 & 107 & 98 & 91 & 113 & 113 & 107 & 94 & 75 & 92 \\
\hline 0 & 0 & 0 & 0 & 0 & 0 & 0 & 0 & 0 & 0 & 0 & 0 & 0 & 0 \\
\hline 2716 & 2869 & 2874 & 3016 & 3014 & 2648 & 2585 & 2525 & 2292 & 2252 & 1991 & 2119 & 2302 & 2577 \\
\hline 10200 & 8532 & 9743 & 10512 & 10563 & 8717 & 8350 & 8871 & 8438 & 8151 & 8933 & 10329 & 10700 & 9578 \\
\hline 0 & 0 & 0 & 0 & 0 & 0 & 0 & 0 & 0 & 0 & 0 & 0 & 0 & 0 \\
\hline
\end{tabular}

\begin{tabular}{|c|c|c|c|c|c|c|c|c|c|c|c|c|c|}
\hline Aug16 & Sep & Oct & Nov & $D e c$ & Jan & $F e b$ & Mar & Apr1 & Apr16 & May & Jun & Jul & Avg \\
\hline 0 & 0 & 0 & 0 & 0 & 0 & 0 & 0 & 0 & 0 & 0 & 0 & 0 & 0 \\
\hline 0 & 0 & 0 & 0 & 0 & 0 & 0 & 0 & 0 & 0 & 0 & 0 & 0 & 0 \\
\hline 0 & 0 & 0 & 0 & 0 & 0 & 0 & 0 & 0 & 0 & 0 & 0 & 0 & 0 \\
\hline 120 & 109 & 106 & 122 & 137 & 137 & 132 & 123 & 111 & 111 & 108 & 109 & 116 & 119 \\
\hline 275 & 166 & 129 & 0 & 0 & 0 & 1.0 & 23 & 252 & 315 & 268 & 298 & 332 & 149 \\
\hline 265 & 265 & 272 & 272 & 272 & 272 & 272 & 272 & 272 & 272 & 272 & 272 & 272 & 271 \\
\hline 660 & 540 & 508 & 394 & 409 & 409 & 405 & 418 & 635 & 698 & 648 & 679 & 720 & 539 \\
\hline 0 & 0 & 0 & 0 & 0 & 0 & 0 & 0 & 0 & 0 & 0 & 0 & 0 & 0 \\
\hline 0 & 0 & 0 & 0 & 0 & 0 & 0 & 0 & 0 & 0 & 0 & 0 & 0 & 0 \\
\hline 0 & 0 & 0 & 0 & 0 & 0 & 0 & 0 & 0 & 0 & 0 & 0 & 0 & 0 \\
\hline 2654 & 2432 & 2416 & 2712 & 3116 & 3223 & 3082 & 2808 & 2644 & 2637 & 2580 & 2640 & 2759 & 2755 \\
\hline 1723 & 1897 & 1935 & 2299 & 2585 & 2626 & 2580 & 2388 & 2101 & 2095 & 1980 & 1715 & 1685 & 2126 \\
\hline 651 & 504 & 613 & 670 & 675 & 553 & 528 & 574 & 546 & 526 & 609 & 713 & 726 & 621 \\
\hline 1084 & 840 & 1021 & 1116 & 1124 & 921 & 879 & 957 & 909 & 875 & 1015 & 1188 & 1210 & 1034 \\
\hline 382 & 382 & 382 & 382 & 382 & 382 & 382 & 382 & 382 & 382 & 382 & 382 & 382 & 382 \\
\hline 1036 & 1027 & 935 & 939 & 954 & 952 & 948 & 940 & 964 & 963 & 983 & 991 & 945 & 968 \\
\hline 253 & 292 & 346 & 647 & 660 & 658 & 602 & 450 & 367 & 368 & 172 & 232 & 282 & 413 \\
\hline 0 & 0 & 0 & 0 & 0 & 0 & 0 & 0 & 0 & 0 & 0 & 0 & 0 & 0 \\
\hline 0 & 0 & 0 & 0 & 0 & 0 & 0 & 0 & 0 & 0 & 0 & 0 & 0 & 0 \\
\hline 0 & 0 & 0 & 0 & 0 & 0 & 0 & 0 & 0 & 0 & 0 & 0 & 0 & 0 \\
\hline 7782 & 7374 & 7649 & 8765 & 9496 & 9316 & 9001 & 8499 & 7913 & 7847 & 7722 & 7862 & 7989 & 8300 \\
\hline 8443 & 7914 & 8157 & 9159 & 9905 & 9725 & 9405 & 8917 & 8548 & 8545 & 8370 & 8541 & 8709 & 8839 \\
\hline 6907 & 5151 & 6342 & 7050 & 7171 & 5752 & 5427 & 5931 & 5577 & 5248 & 6096 & 7328 & 7811 & 6464 \\
\hline 435 & 370 & 386 & 305 & 237 & 176 & 196 & 275 & 434 & 515 & 711 & 747 & 451 & 397 \\
\hline 0 & 0 & 0 & 0 & 0 & 0 & 0 & 0 & 0 & 0 & 0 & 0 & 0 & 0 \\
\hline 0 & 0 & 0 & 0 & 0 & 0 & 0 & 0 & 0 & 0 & 0 & 0 & 0 & 0 \\
\hline 141 & 141 & 141 & 141 & 141 & 141 & 141 & 141 & 136 & 136 & 136 & 136 & 136 & 139 \\
\hline 0 & 0 & 0 & 0 & 0 & 0 & 0 & 0 & 0 & 0 & 0 & 0 & 0 & 0 \\
\hline 7484 & 5663 & 6869 & 7497 & 7550 & 6069 & 5765 & 6347 & 6146 & 5899 & 6942 & 8210 & 8398 & 7000 \\
\hline 0 & 0 & 0 & 0 & 0 & 0 & 0 & 0 & 0 & 0 & 0 & 0 & 0 & 0 \\
\hline 0 & 0 & 0 & 0 & 0 & 0 & 0 & 0 & 0 & 0 & 0 & 0 & 0 & 0 \\
\hline 27 & 27 & 28 & 29 & 31 & 32 & 31 & 31 & 30 & 30 & 27 & 27 & 27 & 29 \\
\hline 0 & 0 & 0 & 0 & 0 & 0 & 0 & 0 & 0 & 0 & 0 & 0 & 0 & 0 \\
\hline 183 & 216 & 250 & 318 & 375 & 349 & 305 & 275 & 276 & 238 & 101 & 123 & 204 & 246 \\
\hline 1425 & 1567 & 1520 & 1568 & 1503 & 1161 & 1151 & 1128 & 873 & 871 & 756 & 874 & 996 & 1210 \\
\hline 0 & 0 & 0 & 0 & 0 & 0 & 0 & 0 & 0 & 0 & 0 & 0 & 0 & 0 \\
\hline 1000 & 1000 & 1000 & 1000 & 1000 & 1000 & 1000 & 1000 & 1000 & 1000 & 1000 & 1000 & 1000 & 1000 \\
\hline 81 & 58 & 76 & 101 & 104 & 107 & 98 & 91 & 113 & 113 & 107 & 94 & 75 & 92 \\
\hline 0 & 0 & 0 & 0 & 0 & 0 & 0 & 0 & 0 & 0 & 0 & 0 & 0 & 0 \\
\hline 2716 & 2869 & 2874 & 3016 & 3014 & 2648 & 2585 & 2525 & 2292 & 2252 & 1991 & 2119 & 2302 & 2577 \\
\hline 10200 & 8532 & 9743 & 10512 & 10563 & 8717 & 8350 & 8871 & 8438 & 8151 & 8933 & 10329 & 10700 & 9578 \\
\hline 0 & 0 & 0 & 0 & 0 & 0 & 0 & 0 & 0 & 0 & 0 & 0 & 0 & 0 \\
\hline
\end{tabular}

\begin{tabular}{|c|c|c|c|c|c|c|c|c|c|c|c|c|c|c|}
\hline Aug1 & Aug16 & Sep & Oct & Nov & $D e c$ & Jan & $F e b$ & Mar & Apr1 & Apr16 & May & Jun & Jul & Avg \\
\hline 0 & 0 & 0 & 0 & 0 & 0 & 0 & 0 & 0 & 0 & 0 & 0 & 0 & 0 & 0 \\
\hline 0 & 0 & 0 & 0 & 0 & 0 & 0 & 0 & 0 & 0 & 0 & 0 & 0 & 0 & 0 \\
\hline 0 & 0 & 0 & 0 & 0 & 0 & 0 & 0 & 0 & 0 & 0 & 0 & 0 & 0 & 0 \\
\hline 120 & 120 & 109 & 106 & 122 & 137 & 137 & 132 & 123 & 111 & 111 & 108 & 109 & 116 & 119 \\
\hline 309 & 275 & 166 & 129 & 0 & 0 & 0 & 1.0 & 23 & 252 & 315 & 268 & 298 & 332 & 149 \\
\hline 265 & 265 & 265 & 272 & 272 & 272 & 272 & 272 & 272 & 272 & 272 & 272 & 272 & 272 & 271 \\
\hline 694 & 660 & 540 & 508 & 394 & 409 & 409 & 405 & 418 & 635 & 698 & 648 & 679 & 720 & 539 \\
\hline 0 & 0 & 0 & 0 & 0 & 0 & 0 & 0 & 0 & 0 & 0 & 0 & 0 & 0 & 0 \\
\hline 0 & 0 & 0 & 0 & 0 & 0 & 0 & 0 & 0 & 0 & 0 & 0 & 0 & 0 & 0 \\
\hline 0 & 0 & 0 & 0 & 0 & 0 & 0 & 0 & 0 & 0 & 0 & 0 & 0 & 0 & 0 \\
\hline 2654 & 2654 & 2432 & 2416 & 2712 & 3116 & 3223 & 3082 & 2808 & 2644 & 2637 & 2580 & 2640 & 2759 & 2755 \\
\hline 1723 & 1723 & 1897 & 1935 & 2299 & 2585 & 2626 & 2580 & 2388 & 2101 & 2095 & 1980 & 1715 & 1685 & 2126 \\
\hline 846 & 651 & 504 & 613 & 670 & 675 & 553 & 528 & 574 & 546 & 526 & 609 & 713 & 726 & 621 \\
\hline 1410 & 1084 & 840 & 1021 & 1116 & 1124 & 921 & 879 & 957 & 909 & 875 & 1015 & 1188 & 1210 & 1034 \\
\hline 382 & 382 & 382 & 382 & 382 & 382 & 382 & 382 & 382 & 382 & 382 & 382 & 382 & 382 & 382 \\
\hline 1036 & 1036 & 1027 & 935 & 939 & 954 & 952 & 948 & 940 & 964 & 963 & 983 & 991 & 945 & 968 \\
\hline 252 & 253 & 292 & 346 & 647 & 660 & 658 & 602 & 450 & 367 & 368 & 172 & 232 & 282 & 413 \\
\hline 0 & 0 & 0 & 0 & 0 & 0 & 0 & 0 & 0 & 0 & 0 & 0 & 0 & 0 & 0 \\
\hline 0 & 0 & 0 & 0 & 0 & 0 & 0 & 0 & 0 & 0 & 0 & 0 & 0 & 0 & 0 \\
\hline 0 & 0 & 0 & 0 & 0 & 0 & 0 & 0 & 0 & 0 & 0 & 0 & 0 & 0 & 0 \\
\hline 8303 & 7782 & 7374 & 7649 & 8765 & 9496 & 9316 & 9001 & 8499 & 7913 & 7847 & 7722 & 7862 & 7989 & 8300 \\
\hline 8998 & 8443 & 7914 & 8157 & 9159 & 9905 & 9725 & 9405 & 8917 & 8548 & 8545 & 8370 & 8541 & 8709 & 8839 \\
\hline 9278 & 6907 & 5151 & 6342 & 7050 & 7171 & 5752 & 5427 & 5931 & 5577 & 5248 & 6096 & 7328 & 7811 & 6464 \\
\hline 437 & 435 & 370 & 386 & 305 & 237 & 176 & 196 & 275 & 434 & 515 & 711 & 747 & 451 & 397 \\
\hline 0 & 0 & 0 & 0 & 0 & 0 & 0 & 0 & 0 & 0 & 0 & 0 & 0 & 0 & 0 \\
\hline 0 & 0 & 0 & 0 & 0 & 0 & 0 & 0 & 0 & 0 & 0 & 0 & 0 & 0 & 0 \\
\hline 141 & 141 & 141 & 141 & 141 & 141 & 141 & 141 & 141 & 136 & 136 & 136 & 136 & 136 & 139 \\
\hline 0 & 0 & 0 & 0 & 0 & 0 & 0 & 0 & 0 & 0 & 0 & 0 & 0 & 0 & 0 \\
\hline 9856 & 7484 & 5663 & 6869 & 7497 & 7550 & 6069 & 5765 & 6347 & 6146 & 5899 & 6942 & 8210 & 8398 & 7000 \\
\hline 0 & 0 & 0 & 0 & 0 & 0 & 0 & 0 & 0 & 0 & 0 & 0 & 0 & 0 & 0 \\
\hline 0 & 0 & 0 & 0 & 0 & 0 & 0 & 0 & 0 & 0 & 0 & 0 & 0 & 0 & 0 \\
\hline 27 & 27 & 27 & 28 & 29 & 31 & 32 & 31 & 31 & 30 & 30 & 27 & 27 & 27 & 29 \\
\hline 0 & 0 & 0 & 0 & 0 & 0 & 0 & 0 & 0 & 0 & 0 & 0 & 0 & 0 & 0 \\
\hline 183 & 183 & 216 & 250 & 318 & 375 & 349 & 305 & 275 & 276 & 238 & 101 & 123 & 204 & 246 \\
\hline 1422 & 1425 & 1567 & 1520 & 1568 & 1503 & 1161 & 1151 & 1128 & 873 & 871 & 756 & 874 & 996 & 1210 \\
\hline 0 & 0 & 0 & 0 & 0 & 0 & 0 & 0 & 0 & 0 & 0 & 0 & 0 & 0 & 0 \\
\hline 1000 & 1000 & 1000 & 1000 & 1000 & 1000 & 1000 & 1000 & 1000 & 1000 & 1000 & 1000 & 1000 & 1000 & 1000 \\
\hline 83 & 81 & 58 & 76 & 101 & 104 & 107 & 98 & 91 & 113 & 113 & 107 & 94 & 75 & 92 \\
\hline 0 & 0 & 0 & 0 & 0 & 0 & 0 & 0 & 0 & 0 & 0 & 0 & 0 & 0 & 0 \\
\hline 2715 & 2716 & 2869 & 2874 & 3016 & 3014 & 2648 & 2585 & 2525 & 2292 & 2252 & 1991 & 2119 & 2302 & 2577 \\
\hline 12571 & 10200 & 8532 & 9743 & 10512 & 10563 & 8717 & 8350 & 8871 & 8438 & 8151 & 8933 & 10329 & 10700 & 9578 \\
\hline 0 & 0 & 0 & 0 & 0 & 0 & 0 & 0 & 0 & 0 & 0 & 0 & 0 & 0 & 0 \\
\hline
\end{tabular}

\begin{tabular}{|c|c|c|c|c|c|c|c|c|c|c|c|c|c|}
\hline Aug16 & Sep & Oct & Nov & $D e c$ & Jan & $F e b$ & Mar & Apr1 & Apr16 & May & Jun & Jul & Avg \\
\hline 0 & 0 & 0 & 0 & 0 & 0 & 0 & 0 & 0 & 0 & 0 & 0 & 0 & 0 \\
\hline 0 & 0 & 0 & 0 & 0 & 0 & 0 & 0 & 0 & 0 & 0 & 0 & 0 & 0 \\
\hline 0 & 0 & 0 & 0 & 0 & 0 & 0 & 0 & 0 & 0 & 0 & 0 & 0 & 0 \\
\hline 120 & 109 & 106 & 122 & 137 & 137 & 132 & 123 & 111 & 111 & 108 & 109 & 116 & 119 \\
\hline 275 & 166 & 129 & 0 & 0 & 0 & 1.0 & 23 & 252 & 315 & 268 & 298 & 332 & 149 \\
\hline 265 & 265 & 272 & 272 & 272 & 272 & 272 & 272 & 272 & 272 & 272 & 272 & 272 & 271 \\
\hline 660 & 540 & 508 & 394 & 409 & 409 & 405 & 418 & 635 & 698 & 648 & 679 & 720 & 539 \\
\hline 0 & 0 & 0 & 0 & 0 & 0 & 0 & 0 & 0 & 0 & 0 & 0 & 0 & 0 \\
\hline 0 & 0 & 0 & 0 & 0 & 0 & 0 & 0 & 0 & 0 & 0 & 0 & 0 & 0 \\
\hline 0 & 0 & 0 & 0 & 0 & 0 & 0 & 0 & 0 & 0 & 0 & 0 & 0 & 0 \\
\hline 2654 & 2432 & 2416 & 2712 & 3116 & 3223 & 3082 & 2808 & 2644 & 2637 & 2580 & 2640 & 2759 & 2755 \\
\hline 1723 & 1897 & 1935 & 2299 & 2585 & 2626 & 2580 & 2388 & 2101 & 2095 & 1980 & 1715 & 1685 & 2126 \\
\hline 651 & 504 & 613 & 670 & 675 & 553 & 528 & 574 & 546 & 526 & 609 & 713 & 726 & 621 \\
\hline 1084 & 840 & 1021 & 1116 & 1124 & 921 & 879 & 957 & 909 & 875 & 1015 & 1188 & 1210 & 1034 \\
\hline 382 & 382 & 382 & 382 & 382 & 382 & 382 & 382 & 382 & 382 & 382 & 382 & 382 & 382 \\
\hline 1036 & 1027 & 935 & 939 & 954 & 952 & 948 & 940 & 964 & 963 & 983 & 991 & 945 & 968 \\
\hline 253 & 292 & 346 & 647 & 660 & 658 & 602 & 450 & 367 & 368 & 172 & 232 & 282 & 413 \\
\hline 0 & 0 & 0 & 0 & 0 & 0 & 0 & 0 & 0 & 0 & 0 & 0 & 0 & 0 \\
\hline 0 & 0 & 0 & 0 & 0 & 0 & 0 & 0 & 0 & 0 & 0 & 0 & 0 & 0 \\
\hline 0 & 0 & 0 & 0 & 0 & 0 & 0 & 0 & 0 & 0 & 0 & 0 & 0 & 0 \\
\hline 7782 & 7374 & 7649 & 8765 & 9496 & 9316 & 9001 & 8499 & 7913 & 7847 & 7722 & 7862 & 7989 & 8300 \\
\hline 8443 & 7914 & 8157 & 9159 & 9905 & 9725 & 9405 & 8917 & 8548 & 8545 & 8370 & 8541 & 8709 & 8839 \\
\hline 6907 & 5151 & 6342 & 7050 & 7171 & 5752 & 5427 & 5931 & 5577 & 5248 & 6096 & 7328 & 7811 & 6464 \\
\hline 435 & 370 & 386 & 305 & 237 & 176 & 196 & 275 & 434 & 515 & 711 & 747 & 451 & 397 \\
\hline 0 & 0 & 0 & 0 & 0 & 0 & 0 & 0 & 0 & 0 & 0 & 0 & 0 & 0 \\
\hline 0 & 0 & 0 & 0 & 0 & 0 & 0 & 0 & 0 & 0 & 0 & 0 & 0 & 0 \\
\hline 141 & 141 & 141 & 141 & 141 & 141 & 141 & 141 & 136 & 136 & 136 & 136 & 136 & 139 \\
\hline 0 & 0 & 0 & 0 & 0 & 0 & 0 & 0 & 0 & 0 & 0 & 0 & 0 & 0 \\
\hline 7484 & 5663 & 6869 & 7497 & 7550 & 6069 & 5765 & 6347 & 6146 & 5899 & 6942 & 8210 & 8398 & 7000 \\
\hline 0 & 0 & 0 & 0 & 0 & 0 & 0 & 0 & 0 & 0 & 0 & 0 & 0 & 0 \\
\hline 0 & 0 & 0 & 0 & 0 & 0 & 0 & 0 & 0 & 0 & 0 & 0 & 0 & 0 \\
\hline 27 & 27 & 28 & 29 & 31 & 32 & 31 & 31 & 30 & 30 & 27 & 27 & 27 & 29 \\
\hline 0 & 0 & 0 & 0 & 0 & 0 & 0 & 0 & 0 & 0 & 0 & 0 & 0 & 0 \\
\hline 183 & 216 & 250 & 318 & 375 & 349 & 305 & 275 & 276 & 238 & 101 & 123 & 204 & 246 \\
\hline 1425 & 1567 & 1520 & 1568 & 1503 & 1161 & 1151 & 1128 & 873 & 871 & 756 & 874 & 996 & 1210 \\
\hline 0 & 0 & 0 & 0 & 0 & 0 & 0 & 0 & 0 & 0 & 0 & 0 & 0 & 0 \\
\hline 1000 & 1000 & 1000 & 1000 & 1000 & 1000 & 1000 & 1000 & 1000 & 1000 & 1000 & 1000 & 1000 & 1000 \\
\hline 81 & 58 & 76 & 101 & 104 & 107 & 98 & 91 & 113 & 113 & 107 & 94 & 75 & 92 \\
\hline 0 & 0 & 0 & 0 & 0 & 0 & 0 & 0 & 0 & 0 & 0 & 0 & 0 & 0 \\
\hline 2716 & 2869 & 2874 & 3016 & 3014 & 2648 & 2585 & 2525 & 2292 & 2252 & 1991 & 2119 & 2302 & 2577 \\
\hline 10200 & 8532 & 9743 & 10512 & 10563 & 8717 & 8350 & 8871 & 8438 & 8151 & 8933 & 10329 & 10700 & 9578 \\
\hline 0 & 0 & 0 & 0 & 0 & 0 & 0 & 0 & 0 & 0 & 0 & 0 & 0 & 0 \\
\hline
\end{tabular}

\begin{tabular}{|c|c|c|c|c|c|c|c|c|c|c|c|c|c|c|}
\hline Aug1 & Aug16 & Sep & Oct & Nov & $D e c$ & Jan & $F e b$ & Mar & Apr1 & Apr16 & May & Jun & Jul & Avg \\
\hline 0 & 0 & 0 & 0 & 0 & 0 & 0 & 0 & 0 & 0 & 0 & 0 & 0 & 0 & 0 \\
\hline 0 & 0 & 0 & 0 & 0 & 0 & 0 & 0 & 0 & 0 & 0 & 0 & 0 & 0 & 0 \\
\hline 0 & 0 & 0 & 0 & 0 & 0 & 0 & 0 & 0 & 0 & 0 & 0 & 0 & 0 & 0 \\
\hline 120 & 120 & 109 & 106 & 122 & 137 & 137 & 132 & 123 & 111 & 111 & 108 & 109 & 116 & 119 \\
\hline 309 & 275 & 166 & 129 & 0 & 0 & 0 & 1.0 & 23 & 252 & 315 & 268 & 298 & 332 & 149 \\
\hline 265 & 265 & 265 & 272 & 272 & 272 & 272 & 272 & 272 & 272 & 272 & 272 & 272 & 272 & 271 \\
\hline 694 & 660 & 540 & 508 & 394 & 409 & 409 & 405 & 418 & 635 & 698 & 648 & 679 & 720 & 539 \\
\hline 0 & 0 & 0 & 0 & 0 & 0 & 0 & 0 & 0 & 0 & 0 & 0 & 0 & 0 & 0 \\
\hline 0 & 0 & 0 & 0 & 0 & 0 & 0 & 0 & 0 & 0 & 0 & 0 & 0 & 0 & 0 \\
\hline 0 & 0 & 0 & 0 & 0 & 0 & 0 & 0 & 0 & 0 & 0 & 0 & 0 & 0 & 0 \\
\hline 2654 & 2654 & 2432 & 2416 & 2712 & 3116 & 3223 & 3082 & 2808 & 2644 & 2637 & 2580 & 2640 & 2759 & 2755 \\
\hline 1723 & 1723 & 1897 & 1935 & 2299 & 2585 & 2626 & 2580 & 2388 & 2101 & 2095 & 1980 & 1715 & 1685 & 2126 \\
\hline 846 & 651 & 504 & 613 & 670 & 675 & 553 & 528 & 574 & 546 & 526 & 609 & 713 & 726 & 621 \\
\hline 1410 & 1084 & 840 & 1021 & 1116 & 1124 & 921 & 879 & 957 & 909 & 875 & 1015 & 1188 & 1210 & 1034 \\
\hline 382 & 382 & 382 & 382 & 382 & 382 & 382 & 382 & 382 & 382 & 382 & 382 & 382 & 382 & 382 \\
\hline 1036 & 1036 & 1027 & 935 & 939 & 954 & 952 & 948 & 940 & 964 & 963 & 983 & 991 & 945 & 968 \\
\hline 252 & 253 & 292 & 346 & 647 & 660 & 658 & 602 & 450 & 367 & 368 & 172 & 232 & 282 & 413 \\
\hline 0 & 0 & 0 & 0 & 0 & 0 & 0 & 0 & 0 & 0 & 0 & 0 & 0 & 0 & 0 \\
\hline 0 & 0 & 0 & 0 & 0 & 0 & 0 & 0 & 0 & 0 & 0 & 0 & 0 & 0 & 0 \\
\hline 0 & 0 & 0 & 0 & 0 & 0 & 0 & 0 & 0 & 0 & 0 & 0 & 0 & 0 & 0 \\
\hline 8303 & 7782 & 7374 & 7649 & 8765 & 9496 & 9316 & 9001 & 8499 & 7913 & 7847 & 7722 & 7862 & 7989 & 8300 \\
\hline 8998 & 8443 & 7914 & 8157 & 9159 & 9905 & 9725 & 9405 & 8917 & 8548 & 8545 & 8370 & 8541 & 8709 & 8839 \\
\hline 9278 & 6907 & 5151 & 6342 & 7050 & 7171 & 5752 & 5427 & 5931 & 5577 & 5248 & 6096 & 7328 & 7811 & 6464 \\
\hline 437 & 435 & 370 & 386 & 305 & 237 & 176 & 196 & 275 & 434 & 515 & 711 & 747 & 451 & 397 \\
\hline 0 & 0 & 0 & 0 & 0 & 0 & 0 & 0 & 0 & 0 & 0 & 0 & 0 & 0 & 0 \\
\hline 0 & 0 & 0 & 0 & 0 & 0 & 0 & 0 & 0 & 0 & 0 & 0 & 0 & 0 & 0 \\
\hline 141 & 141 & 141 & 141 & 141 & 141 & 141 & 141 & 141 & 136 & 136 & 136 & 136 & 136 & 139 \\
\hline 0 & 0 & 0 & 0 & 0 & 0 & 0 & 0 & 0 & 0 & 0 & 0 & 0 & 0 & 0 \\
\hline 9856 & 7484 & 5663 & 6869 & 7497 & 7550 & 6069 & 5765 & 6347 & 6146 & 5899 & 6942 & 8210 & 8398 & 7000 \\
\hline 0 & 0 & 0 & 0 & 0 & 0 & 0 & 0 & 0 & 0 & 0 & 0 & 0 & 0 & 0 \\
\hline 0 & 0 & 0 & 0 & 0 & 0 & 0 & 0 & 0 & 0 & 0 & 0 & 0 & 0 & 0 \\
\hline 27 & 27 & 27 & 28 & 29 & 31 & 32 & 31 & 31 & 30 & 30 & 27 & 27 & 27 & 29 \\
\hline 0 & 0 & 0 & 0 & 0 & 0 & 0 & 0 & 0 & 0 & 0 & 0 & 0 & 0 & 0 \\
\hline 183 & 183 & 216 & 250 & 318 & 375 & 349 & 305 & 275 & 276 & 238 & 101 & 123 & 204 & 246 \\
\hline 1422 & 1425 & 1567 & 1520 & 1568 & 1503 & 1161 & 1151 & 1128 & 873 & 871 & 756 & 874 & 996 & 1210 \\
\hline 0 & 0 & 0 & 0 & 0 & 0 & 0 & 0 & 0 & 0 & 0 & 0 & 0 & 0 & 0 \\
\hline 1000 & 1000 & 1000 & 1000 & 1000 & 1000 & 1000 & 1000 & 1000 & 1000 & 1000 & 1000 & 1000 & 1000 & 1000 \\
\hline 83 & 81 & 58 & 76 & 101 & 104 & 107 & 98 & 91 & 113 & 113 & 107 & 94 & 75 & 92 \\
\hline 0 & 0 & 0 & 0 & 0 & 0 & 0 & 0 & 0 & 0 & 0 & 0 & 0 & 0 & 0 \\
\hline 2715 & 2716 & 2869 & 2874 & 3016 & 3014 & 2648 & 2585 & 2525 & 2292 & 2252 & 1991 & 2119 & 2302 & 2577 \\
\hline 12571 & 10200 & 8532 & 9743 & 10512 & 10563 & 8717 & 8350 & 8871 & 8438 & 8151 & 8933 & 10329 & 10700 & 9578 \\
\hline 0 & 0 & 0 & 0 & 0 & 0 & 0 & 0 & 0 & 0 & 0 & 0 & 0 & 0 & 0 \\
\hline
\end{tabular}

\begin{tabular}{|c|c|c|c|c|c|c|c|c|c|c|c|c|c|}
\hline Aug16 & Sep & Oct & Nov & $D e c$ & Jan & $F e b$ & Mar & Apr1 & Apr16 & May & Jun & Jul & Avg \\
\hline 0 & 0 & 0 & 0 & 0 & 0 & 0 & 0 & 0 & 0 & 0 & 0 & 0 & 0 \\
\hline 0 & 0 & 0 & 0 & 0 & 0 & 0 & 0 & 0 & 0 & 0 & 0 & 0 & 0 \\
\hline 0 & 0 & 0 & 0 & 0 & 0 & 0 & 0 & 0 & 0 & 0 & 0 & 0 & 0 \\
\hline 120 & 109 & 106 & 122 & 137 & 137 & 132 & 123 & 111 & 111 & 108 & 109 & 116 & 119 \\
\hline 275 & 166 & 129 & 0 & 0 & 0 & 1.0 & 23 & 252 & 315 & 268 & 298 & 332 & 149 \\
\hline 265 & 265 & 272 & 272 & 272 & 272 & 272 & 272 & 272 & 272 & 272 & 272 & 272 & 271 \\
\hline 660 & 540 & 508 & 394 & 409 & 409 & 405 & 418 & 635 & 698 & 648 & 679 & 720 & 539 \\
\hline 0 & 0 & 0 & 0 & 0 & 0 & 0 & 0 & 0 & 0 & 0 & 0 & 0 & 0 \\
\hline 0 & 0 & 0 & 0 & 0 & 0 & 0 & 0 & 0 & 0 & 0 & 0 & 0 & 0 \\
\hline 0 & 0 & 0 & 0 & 0 & 0 & 0 & 0 & 0 & 0 & 0 & 0 & 0 & 0 \\
\hline 2654 & 2432 & 2416 & 2712 & 3116 & 3223 & 3082 & 2808 & 2644 & 2637 & 2580 & 2640 & 2759 & 2755 \\
\hline 1723 & 1897 & 1935 & 2299 & 2585 & 2626 & 2580 & 2388 & 2101 & 2095 & 1980 & 1715 & 1685 & 2126 \\
\hline 651 & 504 & 613 & 670 & 675 & 553 & 528 & 574 & 546 & 526 & 609 & 713 & 726 & 621 \\
\hline 1084 & 840 & 1021 & 1116 & 1124 & 921 & 879 & 957 & 909 & 875 & 1015 & 1188 & 1210 & 1034 \\
\hline 382 & 382 & 382 & 382 & 382 & 382 & 382 & 382 & 382 & 382 & 382 & 382 & 382 & 382 \\
\hline 1036 & 1027 & 935 & 939 & 954 & 952 & 948 & 940 & 964 & 963 & 983 & 991 & 945 & 968 \\
\hline 253 & 292 & 346 & 647 & 660 & 658 & 602 & 450 & 367 & 368 & 172 & 232 & 282 & 413 \\
\hline 0 & 0 & 0 & 0 & 0 & 0 & 0 & 0 & 0 & 0 & 0 & 0 & 0 & 0 \\
\hline 0 & 0 & 0 & 0 & 0 & 0 & 0 & 0 & 0 & 0 & 0 & 0 & 0 & 0 \\
\hline 0 & 0 & 0 & 0 & 0 & 0 & 0 & 0 & 0 & 0 & 0 & 0 & 0 & 0 \\
\hline 7782 & 7374 & 7649 & 8765 & 9496 & 9316 & 9001 & 8499 & 7913 & 7847 & 7722 & 7862 & 7989 & 8300 \\
\hline 8443 & 7914 & 8157 & 9159 & 9905 & 9725 & 9405 & 8917 & 8548 & 8545 & 8370 & 8541 & 8709 & 8839 \\
\hline 6907 & 5151 & 6342 & 7050 & 7171 & 5752 & 5427 & 5931 & 5577 & 5248 & 6096 & 7328 & 7811 & 6464 \\
\hline 435 & 370 & 386 & 305 & 237 & 176 & 196 & 275 & 434 & 515 & 711 & 747 & 451 & 397 \\
\hline 0 & 0 & 0 & 0 & 0 & 0 & 0 & 0 & 0 & 0 & 0 & 0 & 0 & 0 \\
\hline 0 & 0 & 0 & 0 & 0 & 0 & 0 & 0 & 0 & 0 & 0 & 0 & 0 & 0 \\
\hline 141 & 141 & 141 & 141 & 141 & 141 & 141 & 141 & 136 & 136 & 136 & 136 & 136 & 139 \\
\hline 0 & 0 & 0 & 0 & 0 & 0 & 0 & 0 & 0 & 0 & 0 & 0 & 0 & 0 \\
\hline 7484 & 5663 & 6869 & 7497 & 7550 & 6069 & 5765 & 6347 & 6146 & 5899 & 6942 & 8210 & 8398 & 7000 \\
\hline 0 & 0 & 0 & 0 & 0 & 0 & 0 & 0 & 0 & 0 & 0 & 0 & 0 & 0 \\
\hline 0 & 0 & 0 & 0 & 0 & 0 & 0 & 0 & 0 & 0 & 0 & 0 & 0 & 0 \\
\hline 27 & 27 & 28 & 29 & 31 & 32 & 31 & 31 & 30 & 30 & 27 & 27 & 27 & 29 \\
\hline 0 & 0 & 0 & 0 & 0 & 0 & 0 & 0 & 0 & 0 & 0 & 0 & 0 & 0 \\
\hline 183 & 216 & 250 & 318 & 375 & 349 & 305 & 275 & 276 & 238 & 101 & 123 & 204 & 246 \\
\hline 1425 & 1567 & 1520 & 1568 & 1503 & 1161 & 1151 & 1128 & 873 & 871 & 756 & 874 & 996 & 1210 \\
\hline 0 & 0 & 0 & 0 & 0 & 0 & 0 & 0 & 0 & 0 & 0 & 0 & 0 & 0 \\
\hline 1000 & 1000 & 1000 & 1000 & 1000 & 1000 & 1000 & 1000 & 1000 & 1000 & 1000 & 1000 & 1000 & 1000 \\
\hline 81 & 58 & 76 & 101 & 104 & 107 & 98 & 91 & 113 & 113 & 107 & 94 & 75 & 92 \\
\hline 0 & 0 & 0 & 0 & 0 & 0 & 0 & 0 & 0 & 0 & 0 & 0 & 0 & 0 \\
\hline 2716 & 2869 & 2874 & 3016 & 3014 & 2648 & 2585 & 2525 & 2292 & 2252 & 1991 & 2119 & 2302 & 2577 \\
\hline 10200 & 8532 & 9743 & 10512 & 10563 & 8717 & 8350 & 8871 & 8438 & 8151 & 8933 & 10329 & 10700 & 9578 \\
\hline 0 & 0 & 0 & 0 & 0 & 0 & 0 & 0 & 0 & 0 & 0 & 0 & 0 & 0 \\
\hline
\end{tabular}

\begin{tabular}{|c|c|c|c|c|c|c|c|c|c|c|c|c|c|}
\hline Aug16 & Sep & Oct & Nov & $D e c$ & Jan & $F e b$ & Mar & Apr1 & Apr16 & May & Jun & Jul & Avg \\
\hline 0 & 0 & 0 & 0 & 0 & 0 & 0 & 0 & 0 & 0 & 0 & 0 & 0 & 0 \\
\hline 0 & 0 & 0 & 0 & 0 & 0 & 0 & 0 & 0 & 0 & 0 & 0 & 0 & 0 \\
\hline 0 & 0 & 0 & 0 & 0 & 0 & 0 & 0 & 0 & 0 & 0 & 0 & 0 & 0 \\
\hline 120 & 109 & 106 & 122 & 137 & 137 & 132 & 123 & 111 & 111 & 108 & 109 & 116 & 119 \\
\hline 275 & 166 & 129 & 0 & 0 & 0 & 1.0 & 23 & 252 & 315 & 268 & 298 & 332 & 149 \\
\hline 265 & 265 & 272 & 272 & 272 & 272 & 272 & 272 & 272 & 272 & 272 & 272 & 272 & 271 \\
\hline 660 & 540 & 508 & 394 & 409 & 409 & 405 & 418 & 635 & 698 & 648 & 679 & 720 & 539 \\
\hline 0 & 0 & 0 & 0 & 0 & 0 & 0 & 0 & 0 & 0 & 0 & 0 & 0 & 0 \\
\hline 0 & 0 & 0 & 0 & 0 & 0 & 0 & 0 & 0 & 0 & 0 & 0 & 0 & 0 \\
\hline 0 & 0 & 0 & 0 & 0 & 0 & 0 & 0 & 0 & 0 & 0 & 0 & 0 & 0 \\
\hline 2654 & 2432 & 2416 & 2712 & 3116 & 3223 & 3082 & 2808 & 2644 & 2637 & 2580 & 2640 & 2759 & 2755 \\
\hline 1723 & 1897 & 1935 & 2299 & 2585 & 2626 & 2580 & 2388 & 2101 & 2095 & 1980 & 1715 & 1685 & 2126 \\
\hline 651 & 504 & 613 & 670 & 675 & 553 & 528 & 574 & 546 & 526 & 609 & 713 & 726 & 621 \\
\hline 1084 & 840 & 1021 & 1116 & 1124 & 921 & 879 & 957 & 909 & 875 & 1015 & 1188 & 1210 & 1034 \\
\hline 382 & 382 & 382 & 382 & 382 & 382 & 382 & 382 & 382 & 382 & 382 & 382 & 382 & 382 \\
\hline 1036 & 1027 & 935 & 939 & 954 & 952 & 948 & 940 & 964 & 963 & 983 & 991 & 945 & 968 \\
\hline 253 & 292 & 346 & 647 & 660 & 658 & 602 & 450 & 367 & 368 & 172 & 232 & 282 & 413 \\
\hline 0 & 0 & 0 & 0 & 0 & 0 & 0 & 0 & 0 & 0 & 0 & 0 & 0 & 0 \\
\hline 0 & 0 & 0 & 0 & 0 & 0 & 0 & 0 & 0 & 0 & 0 & 0 & 0 & 0 \\
\hline 0 & 0 & 0 & 0 & 0 & 0 & 0 & 0 & 0 & 0 & 0 & 0 & 0 & 0 \\
\hline 7782 & 7374 & 7649 & 8765 & 9496 & 9316 & 9001 & 8499 & 7913 & 7847 & 7722 & 7862 & 7989 & 8300 \\
\hline 8443 & 7914 & 8157 & 9159 & 9905 & 9725 & 9405 & 8917 & 8548 & 8545 & 8370 & 8541 & 8709 & 8839 \\
\hline 6907 & 5151 & 6342 & 7050 & 7171 & 5752 & 5427 & 5931 & 5577 & 5248 & 6096 & 7328 & 7811 & 6464 \\
\hline 435 & 370 & 386 & 305 & 237 & 176 & 196 & 275 & 434 & 515 & 711 & 747 & 451 & 397 \\
\hline 0 & 0 & 0 & 0 & 0 & 0 & 0 & 0 & 0 & 0 & 0 & 0 & 0 & 0 \\
\hline 0 & 0 & 0 & 0 & 0 & 0 & 0 & 0 & 0 & 0 & 0 & 0 & 0 & 0 \\
\hline 141 & 141 & 141 & 141 & 141 & 141 & 141 & 141 & 136 & 136 & 136 & 136 & 136 & 139 \\
\hline 0 & 0 & 0 & 0 & 0 & 0 & 0 & 0 & 0 & 0 & 0 & 0 & 0 & 0 \\
\hline 7484 & 5663 & 6869 & 7497 & 7550 & 6069 & 5765 & 6347 & 6146 & 5899 & 6942 & 8210 & 8398 & 7000 \\
\hline 0 & 0 & 0 & 0 & 0 & 0 & 0 & 0 & 0 & 0 & 0 & 0 & 0 & 0 \\
\hline 0 & 0 & 0 & 0 & 0 & 0 & 0 & 0 & 0 & 0 & 0 & 0 & 0 & 0 \\
\hline 27 & 27 & 28 & 29 & 31 & 32 & 31 & 31 & 30 & 30 & 27 & 27 & 27 & 29 \\
\hline 0 & 0 & 0 & 0 & 0 & 0 & 0 & 0 & 0 & 0 & 0 & 0 & 0 & 0 \\
\hline 183 & 216 & 250 & 318 & 375 & 349 & 305 & 275 & 276 & 238 & 101 & 123 & 204 & 246 \\
\hline 1425 & 1567 & 1520 & 1568 & 1503 & 1161 & 1151 & 1128 & 873 & 871 & 756 & 874 & 996 & 1210 \\
\hline 0 & 0 & 0 & 0 & 0 & 0 & 0 & 0 & 0 & 0 & 0 & 0 & 0 & 0 \\
\hline 1000 & 1000 & 1000 & 1000 & 1000 & 1000 & 1000 & 1000 & 1000 & 1000 & 1000 & 1000 & 1000 & 1000 \\
\hline 81 & 58 & 76 & 101 & 104 & 107 & 98 & 91 & 113 & 113 & 107 & 94 & 75 & 92 \\
\hline 0 & 0 & 0 & 0 & 0 & 0 & 0 & 0 & 0 & 0 & 0 & 0 & 0 & 0 \\
\hline 2716 & 2869 & 2874 & 3016 & 3014 & 2648 & 2585 & 2525 & 2292 & 2252 & 1991 & 2119 & 2302 & 2577 \\
\hline 10200 & 8532 & 9743 & 10512 & 10563 & 8717 & 8350 & 8871 & 8438 & 8151 & 8933 & 10329 & 10700 & 9578 \\
\hline 0 & 0 & 0 & 0 & 0 & 0 & 0 & 0 & 0 & 0 & 0 & 0 & 0 & 0 \\
\hline
\end{tabular}

\begin{tabular}{|c|c|c|c|c|c|c|c|c|c|c|c|c|c|}
\hline Aug16 & Sep & Oct & Nov & $D e c$ & Jan & $F e b$ & Mar & Apr1 & Apr16 & May & Jun & Jul & Avg \\
\hline 0 & 0 & 0 & 0 & 0 & 0 & 0 & 0 & 0 & 0 & 0 & 0 & 0 & 0 \\
\hline 0 & 0 & 0 & 0 & 0 & 0 & 0 & 0 & 0 & 0 & 0 & 0 & 0 & 0 \\
\hline 0 & 0 & 0 & 0 & 0 & 0 & 0 & 0 & 0 & 0 & 0 & 0 & 0 & 0 \\
\hline 120 & 109 & 106 & 122 & 137 & 137 & 132 & 123 & 111 & 111 & 108 & 109 & 116 & 119 \\
\hline 275 & 166 & 129 & 0 & 0 & 0 & 1.0 & 23 & 252 & 315 & 268 & 298 & 332 & 149 \\
\hline 265 & 265 & 272 & 272 & 272 & 272 & 272 & 272 & 272 & 272 & 272 & 272 & 272 & 271 \\
\hline 660 & 540 & 508 & 394 & 409 & 409 & 405 & 418 & 635 & 698 & 648 & 679 & 720 & 539 \\
\hline 0 & 0 & 0 & 0 & 0 & 0 & 0 & 0 & 0 & 0 & 0 & 0 & 0 & 0 \\
\hline 0 & 0 & 0 & 0 & 0 & 0 & 0 & 0 & 0 & 0 & 0 & 0 & 0 & 0 \\
\hline 0 & 0 & 0 & 0 & 0 & 0 & 0 & 0 & 0 & 0 & 0 & 0 & 0 & 0 \\
\hline 2654 & 2432 & 2416 & 2712 & 3116 & 3223 & 3082 & 2808 & 2644 & 2637 & 2580 & 2640 & 2759 & 2755 \\
\hline 1723 & 1897 & 1935 & 2299 & 2585 & 2626 & 2580 & 2388 & 2101 & 2095 & 1980 & 1715 & 1685 & 2126 \\
\hline 651 & 504 & 613 & 670 & 675 & 553 & 528 & 574 & 546 & 526 & 609 & 713 & 726 & 621 \\
\hline 1084 & 840 & 1021 & 1116 & 1124 & 921 & 879 & 957 & 909 & 875 & 1015 & 1188 & 1210 & 1034 \\
\hline 382 & 382 & 382 & 382 & 382 & 382 & 382 & 382 & 382 & 382 & 382 & 382 & 382 & 382 \\
\hline 1036 & 1027 & 935 & 939 & 954 & 952 & 948 & 940 & 964 & 963 & 983 & 991 & 945 & 968 \\
\hline 253 & 292 & 346 & 647 & 660 & 658 & 602 & 450 & 367 & 368 & 172 & 232 & 282 & 413 \\
\hline 0 & 0 & 0 & 0 & 0 & 0 & 0 & 0 & 0 & 0 & 0 & 0 & 0 & 0 \\
\hline 0 & 0 & 0 & 0 & 0 & 0 & 0 & 0 & 0 & 0 & 0 & 0 & 0 & 0 \\
\hline 0 & 0 & 0 & 0 & 0 & 0 & 0 & 0 & 0 & 0 & 0 & 0 & 0 & 0 \\
\hline 7782 & 7374 & 7649 & 8765 & 9496 & 9316 & 9001 & 8499 & 7913 & 7847 & 7722 & 7862 & 7989 & 8300 \\
\hline 8443 & 7914 & 8157 & 9159 & 9905 & 9725 & 9405 & 8917 & 8548 & 8545 & 8370 & 8541 & 8709 & 8839 \\
\hline 6907 & 5151 & 6342 & 7050 & 7171 & 5752 & 5427 & 5931 & 5577 & 5248 & 6096 & 7328 & 7811 & 6464 \\
\hline 435 & 370 & 386 & 305 & 237 & 176 & 196 & 275 & 434 & 515 & 711 & 747 & 451 & 397 \\
\hline 0 & 0 & 0 & 0 & 0 & 0 & 0 & 0 & 0 & 0 & 0 & 0 & 0 & 0 \\
\hline 0 & 0 & 0 & 0 & 0 & 0 & 0 & 0 & 0 & 0 & 0 & 0 & 0 & 0 \\
\hline 141 & 141 & 141 & 141 & 141 & 141 & 141 & 141 & 136 & 136 & 136 & 136 & 136 & 139 \\
\hline 0 & 0 & 0 & 0 & 0 & 0 & 0 & 0 & 0 & 0 & 0 & 0 & 0 & 0 \\
\hline 7484 & 5663 & 6869 & 7497 & 7550 & 6069 & 5765 & 6347 & 6146 & 5899 & 6942 & 8210 & 8398 & 7000 \\
\hline 0 & 0 & 0 & 0 & 0 & 0 & 0 & 0 & 0 & 0 & 0 & 0 & 0 & 0 \\
\hline 0 & 0 & 0 & 0 & 0 & 0 & 0 & 0 & 0 & 0 & 0 & 0 & 0 & 0 \\
\hline 27 & 27 & 28 & 29 & 31 & 32 & 31 & 31 & 30 & 30 & 27 & 27 & 27 & 29 \\
\hline 0 & 0 & 0 & 0 & 0 & 0 & 0 & 0 & 0 & 0 & 0 & 0 & 0 & 0 \\
\hline 183 & 216 & 250 & 318 & 375 & 349 & 305 & 275 & 276 & 238 & 101 & 123 & 204 & 246 \\
\hline 1425 & 1567 & 1520 & 1568 & 1503 & 1161 & 1151 & 1128 & 873 & 871 & 756 & 874 & 996 & 1210 \\
\hline 0 & 0 & 0 & 0 & 0 & 0 & 0 & 0 & 0 & 0 & 0 & 0 & 0 & 0 \\
\hline 1000 & 1000 & 1000 & 1000 & 1000 & 1000 & 1000 & 1000 & 1000 & 1000 & 1000 & 1000 & 1000 & 1000 \\
\hline 81 & 58 & 76 & 101 & 104 & 107 & 98 & 91 & 113 & 113 & 107 & 94 & 75 & 92 \\
\hline 0 & 0 & 0 & 0 & 0 & 0 & 0 & 0 & 0 & 0 & 0 & 0 & 0 & 0 \\
\hline 2716 & 2869 & 2874 & 3016 & 3014 & 2648 & 2585 & 2525 & 2292 & 2252 & 1991 & 2119 & 2302 & 2577 \\
\hline 10200 & 8532 & 9743 & 10512 & 10563 & 8717 & 8350 & 8871 & 8438 & 8151 & 8933 & 10329 & 10700 & 9578 \\
\hline 0 & 0 & 0 & 0 & 0 & 0 & 0 & 0 & 0 & 0 & 0 & 0 & 0 & 0 \\
\hline
\end{tabular}

\begin{tabular}{|c|c|c|c|c|c|c|c|c|c|c|c|c|c|}
\hline Aug16 & Sep & Oct & Nov & $D e c$ & Jan & $F e b$ & Mar & Apr1 & Apr16 & May & Jun & Jul & Avg \\
\hline 0 & 0 & 0 & 0 & 0 & 0 & 0 & 0 & 0 & 0 & 0 & 0 & 0 & 0 \\
\hline 0 & 0 & 0 & 0 & 0 & 0 & 0 & 0 & 0 & 0 & 0 & 0 & 0 & 0 \\
\hline 0 & 0 & 0 & 0 & 0 & 0 & 0 & 0 & 0 & 0 & 0 & 0 & 0 & 0 \\
\hline 120 & 109 & 106 & 122 & 137 & 137 & 132 & 123 & 111 & 111 & 108 & 109 & 116 & 119 \\
\hline 275 & 166 & 129 & 0 & 0 & 0 & 1.0 & 23 & 252 & 315 & 268 & 298 & 332 & 149 \\
\hline 265 & 265 & 272 & 272 & 272 & 272 & 272 & 272 & 272 & 272 & 272 & 272 & 272 & 271 \\
\hline 660 & 540 & 508 & 394 & 409 & 409 & 405 & 418 & 635 & 698 & 648 & 679 & 720 & 539 \\
\hline 0 & 0 & 0 & 0 & 0 & 0 & 0 & 0 & 0 & 0 & 0 & 0 & 0 & 0 \\
\hline 0 & 0 & 0 & 0 & 0 & 0 & 0 & 0 & 0 & 0 & 0 & 0 & 0 & 0 \\
\hline 0 & 0 & 0 & 0 & 0 & 0 & 0 & 0 & 0 & 0 & 0 & 0 & 0 & 0 \\
\hline 2654 & 2432 & 2416 & 2712 & 3116 & 3223 & 3082 & 2808 & 2644 & 2637 & 2580 & 2640 & 2759 & 2755 \\
\hline 1723 & 1897 & 1935 & 2299 & 2585 & 2626 & 2580 & 2388 & 2101 & 2095 & 1980 & 1715 & 1685 & 2126 \\
\hline 651 & 504 & 613 & 670 & 675 & 553 & 528 & 574 & 546 & 526 & 609 & 713 & 726 & 621 \\
\hline 1084 & 840 & 1021 & 1116 & 1124 & 921 & 879 & 957 & 909 & 875 & 1015 & 1188 & 1210 & 1034 \\
\hline 382 & 382 & 382 & 382 & 382 & 382 & 382 & 382 & 382 & 382 & 382 & 382 & 382 & 382 \\
\hline 1036 & 1027 & 935 & 939 & 954 & 952 & 948 & 940 & 964 & 963 & 983 & 991 & 945 & 968 \\
\hline 253 & 292 & 346 & 647 & 660 & 658 & 602 & 450 & 367 & 368 & 172 & 232 & 282 & 413 \\
\hline 0 & 0 & 0 & 0 & 0 & 0 & 0 & 0 & 0 & 0 & 0 & 0 & 0 & 0 \\
\hline 0 & 0 & 0 & 0 & 0 & 0 & 0 & 0 & 0 & 0 & 0 & 0 & 0 & 0 \\
\hline 0 & 0 & 0 & 0 & 0 & 0 & 0 & 0 & 0 & 0 & 0 & 0 & 0 & 0 \\
\hline 7782 & 7374 & 7649 & 8765 & 9496 & 9316 & 9001 & 8499 & 7913 & 7847 & 7722 & 7862 & 7989 & 8300 \\
\hline 8443 & 7914 & 8157 & 9159 & 9905 & 9725 & 9405 & 8917 & 8548 & 8545 & 8370 & 8541 & 8709 & 8839 \\
\hline 6907 & 5151 & 6342 & 7050 & 7171 & 5752 & 5427 & 5931 & 5577 & 5248 & 6096 & 7328 & 7811 & 6464 \\
\hline 435 & 370 & 386 & 305 & 237 & 176 & 196 & 275 & 434 & 515 & 711 & 747 & 451 & 397 \\
\hline 0 & 0 & 0 & 0 & 0 & 0 & 0 & 0 & 0 & 0 & 0 & 0 & 0 & 0 \\
\hline 0 & 0 & 0 & 0 & 0 & 0 & 0 & 0 & 0 & 0 & 0 & 0 & 0 & 0 \\
\hline 141 & 141 & 141 & 141 & 141 & 141 & 141 & 141 & 136 & 136 & 136 & 136 & 136 & 139 \\
\hline 0 & 0 & 0 & 0 & 0 & 0 & 0 & 0 & 0 & 0 & 0 & 0 & 0 & 0 \\
\hline 7484 & 5663 & 6869 & 7497 & 7550 & 6069 & 5765 & 6347 & 6146 & 5899 & 6942 & 8210 & 8398 & 7000 \\
\hline 0 & 0 & 0 & 0 & 0 & 0 & 0 & 0 & 0 & 0 & 0 & 0 & 0 & 0 \\
\hline 0 & 0 & 0 & 0 & 0 & 0 & 0 & 0 & 0 & 0 & 0 & 0 & 0 & 0 \\
\hline 27 & 27 & 28 & 29 & 31 & 32 & 31 & 31 & 30 & 30 & 27 & 27 & 27 & 29 \\
\hline 0 & 0 & 0 & 0 & 0 & 0 & 0 & 0 & 0 & 0 & 0 & 0 & 0 & 0 \\
\hline 183 & 216 & 250 & 318 & 375 & 349 & 305 & 275 & 276 & 238 & 101 & 123 & 204 & 246 \\
\hline 1425 & 1567 & 1520 & 1568 & 1503 & 1161 & 1151 & 1128 & 873 & 871 & 756 & 874 & 996 & 1210 \\
\hline 0 & 0 & 0 & 0 & 0 & 0 & 0 & 0 & 0 & 0 & 0 & 0 & 0 & 0 \\
\hline 1000 & 1000 & 1000 & 1000 & 1000 & 1000 & 1000 & 1000 & 1000 & 1000 & 1000 & 1000 & 1000 & 1000 \\
\hline 81 & 58 & 76 & 101 & 104 & 107 & 98 & 91 & 113 & 113 & 107 & 94 & 75 & 92 \\
\hline 0 & 0 & 0 & 0 & 0 & 0 & 0 & 0 & 0 & 0 & 0 & 0 & 0 & 0 \\
\hline 2716 & 2869 & 2874 & 3016 & 3014 & 2648 & 2585 & 2525 & 2292 & 2252 & 1991 & 2119 & 2302 & 2577 \\
\hline 10200 & 8532 & 9743 & 10512 & 10563 & 8717 & 8350 & 8871 & 8438 & 8151 & 8933 & 10329 & 10700 & 9578 \\
\hline 0 & 0 & 0 & 0 & 0 & 0 & 0 & 0 & 0 & 0 & 0 & 0 & 0 & 0 \\
\hline
\end{tabular}

\begin{tabular}{|c|c|c|c|c|c|c|c|c|c|c|c|c|c|}
\hline Aug16 & Sep & Oct & Nov & $D e c$ & Jan & $F e b$ & Mar & Apr1 & Apr16 & May & Jun & Jul & Avg \\
\hline 0 & 0 & 0 & 0 & 0 & 0 & 0 & 0 & 0 & 0 & 0 & 0 & 0 & 0 \\
\hline 0 & 0 & 0 & 0 & 0 & 0 & 0 & 0 & 0 & 0 & 0 & 0 & 0 & 0 \\
\hline 0 & 0 & 0 & 0 & 0 & 0 & 0 & 0 & 0 & 0 & 0 & 0 & 0 & 0 \\
\hline 120 & 109 & 106 & 122 & 137 & 137 & 132 & 123 & 111 & 111 & 108 & 109 & 116 & 119 \\
\hline 275 & 166 & 129 & 0 & 0 & 0 & 1.0 & 23 & 252 & 315 & 268 & 298 & 332 & 149 \\
\hline 265 & 265 & 272 & 272 & 272 & 272 & 272 & 272 & 272 & 272 & 272 & 272 & 272 & 271 \\
\hline 660 & 540 & 508 & 394 & 409 & 409 & 405 & 418 & 635 & 698 & 648 & 679 & 720 & 539 \\
\hline 0 & 0 & 0 & 0 & 0 & 0 & 0 & 0 & 0 & 0 & 0 & 0 & 0 & 0 \\
\hline 0 & 0 & 0 & 0 & 0 & 0 & 0 & 0 & 0 & 0 & 0 & 0 & 0 & 0 \\
\hline 0 & 0 & 0 & 0 & 0 & 0 & 0 & 0 & 0 & 0 & 0 & 0 & 0 & 0 \\
\hline 2654 & 2432 & 2416 & 2712 & 3116 & 3223 & 3082 & 2808 & 2644 & 2637 & 2580 & 2640 & 2759 & 2755 \\
\hline 1723 & 1897 & 1935 & 2299 & 2585 & 2626 & 2580 & 2388 & 2101 & 2095 & 1980 & 1715 & 1685 & 2126 \\
\hline 651 & 504 & 613 & 670 & 675 & 553 & 528 & 574 & 546 & 526 & 609 & 713 & 726 & 621 \\
\hline 1084 & 840 & 1021 & 1116 & 1124 & 921 & 879 & 957 & 909 & 875 & 1015 & 1188 & 1210 & 1034 \\
\hline 382 & 382 & 382 & 382 & 382 & 382 & 382 & 382 & 382 & 382 & 382 & 382 & 382 & 382 \\
\hline 1036 & 1027 & 935 & 939 & 954 & 952 & 948 & 940 & 964 & 963 & 983 & 991 & 945 & 968 \\
\hline 253 & 292 & 346 & 647 & 660 & 658 & 602 & 450 & 367 & 368 & 172 & 232 & 282 & 413 \\
\hline 0 & 0 & 0 & 0 & 0 & 0 & 0 & 0 & 0 & 0 & 0 & 0 & 0 & 0 \\
\hline 0 & 0 & 0 & 0 & 0 & 0 & 0 & 0 & 0 & 0 & 0 & 0 & 0 & 0 \\
\hline 0 & 0 & 0 & 0 & 0 & 0 & 0 & 0 & 0 & 0 & 0 & 0 & 0 & 0 \\
\hline 7782 & 7374 & 7649 & 8765 & 9496 & 9316 & 9001 & 8499 & 7913 & 7847 & 7722 & 7862 & 7989 & 8300 \\
\hline 8443 & 7914 & 8157 & 9159 & 9905 & 9725 & 9405 & 8917 & 8548 & 8545 & 8370 & 8541 & 8709 & 8839 \\
\hline 6907 & 5151 & 6342 & 7050 & 7171 & 5752 & 5427 & 5931 & 5577 & 5248 & 6096 & 7328 & 7811 & 6464 \\
\hline 435 & 370 & 386 & 305 & 237 & 176 & 196 & 275 & 434 & 515 & 711 & 747 & 451 & 397 \\
\hline 0 & 0 & 0 & 0 & 0 & 0 & 0 & 0 & 0 & 0 & 0 & 0 & 0 & 0 \\
\hline 0 & 0 & 0 & 0 & 0 & 0 & 0 & 0 & 0 & 0 & 0 & 0 & 0 & 0 \\
\hline 141 & 141 & 141 & 141 & 141 & 141 & 141 & 141 & 136 & 136 & 136 & 136 & 136 & 139 \\
\hline 0 & 0 & 0 & 0 & 0 & 0 & 0 & 0 & 0 & 0 & 0 & 0 & 0 & 0 \\
\hline 7484 & 5663 & 6869 & 7497 & 7550 & 6069 & 5765 & 6347 & 6146 & 5899 & 6942 & 8210 & 8398 & 7000 \\
\hline 0 & 0 & 0 & 0 & 0 & 0 & 0 & 0 & 0 & 0 & 0 & 0 & 0 & 0 \\
\hline 0 & 0 & 0 & 0 & 0 & 0 & 0 & 0 & 0 & 0 & 0 & 0 & 0 & 0 \\
\hline 27 & 27 & 28 & 29 & 31 & 32 & 31 & 31 & 30 & 30 & 27 & 27 & 27 & 29 \\
\hline 0 & 0 & 0 & 0 & 0 & 0 & 0 & 0 & 0 & 0 & 0 & 0 & 0 & 0 \\
\hline 183 & 216 & 250 & 318 & 375 & 349 & 305 & 275 & 276 & 238 & 101 & 123 & 204 & 246 \\
\hline 1425 & 1567 & 1520 & 1568 & 1503 & 1161 & 1151 & 1128 & 873 & 871 & 756 & 874 & 996 & 1210 \\
\hline 0 & 0 & 0 & 0 & 0 & 0 & 0 & 0 & 0 & 0 & 0 & 0 & 0 & 0 \\
\hline 1000 & 1000 & 1000 & 1000 & 1000 & 1000 & 1000 & 1000 & 1000 & 1000 & 1000 & 1000 & 1000 & 1000 \\
\hline 81 & 58 & 76 & 101 & 104 & 107 & 98 & 91 & 113 & 113 & 107 & 94 & 75 & 92 \\
\hline 0 & 0 & 0 & 0 & 0 & 0 & 0 & 0 & 0 & 0 & 0 & 0 & 0 & 0 \\
\hline 2716 & 2869 & 2874 & 3016 & 3014 & 2648 & 2585 & 2525 & 2292 & 2252 & 1991 & 2119 & 2302 & 2577 \\
\hline 10200 & 8532 & 9743 & 10512 & 10563 & 8717 & 8350 & 8871 & 8438 & 8151 & 8933 & 10329 & 10700 & 9578 \\
\hline 0 & 0 & 0 & 0 & 0 & 0 & 0 & 0 & 0 & 0 & 0 & 0 & 0 & 0 \\
\hline
\end{tabular}

\begin{tabular}{|c|c|c|c|c|c|c|c|c|c|c|c|c|c|}
\hline Aug16 & Sep & Oct & Nov & $D e c$ & Jan & $F e b$ & Mar & Apr1 & Apr16 & May & Jun & Jul & Avg \\
\hline 0 & 0 & 0 & 0 & 0 & 0 & 0 & 0 & 0 & 0 & 0 & 0 & 0 & 0 \\
\hline 0 & 0 & 0 & 0 & 0 & 0 & 0 & 0 & 0 & 0 & 0 & 0 & 0 & 0 \\
\hline 0 & 0 & 0 & 0 & 0 & 0 & 0 & 0 & 0 & 0 & 0 & 0 & 0 & 0 \\
\hline 120 & 109 & 106 & 122 & 137 & 137 & 132 & 123 & 111 & 111 & 108 & 109 & 116 & 119 \\
\hline 275 & 166 & 129 & 0 & 0 & 0 & 1.0 & 23 & 252 & 315 & 268 & 298 & 332 & 149 \\
\hline 265 & 265 & 272 & 272 & 272 & 272 & 272 & 272 & 272 & 272 & 272 & 272 & 272 & 271 \\
\hline 660 & 540 & 508 & 394 & 409 & 409 & 405 & 418 & 635 & 698 & 648 & 679 & 720 & 539 \\
\hline 0 & 0 & 0 & 0 & 0 & 0 & 0 & 0 & 0 & 0 & 0 & 0 & 0 & 0 \\
\hline 0 & 0 & 0 & 0 & 0 & 0 & 0 & 0 & 0 & 0 & 0 & 0 & 0 & 0 \\
\hline 0 & 0 & 0 & 0 & 0 & 0 & 0 & 0 & 0 & 0 & 0 & 0 & 0 & 0 \\
\hline 2654 & 2432 & 2416 & 2712 & 3116 & 3223 & 3082 & 2808 & 2644 & 2637 & 2580 & 2640 & 2759 & 2755 \\
\hline 1723 & 1897 & 1935 & 2299 & 2585 & 2626 & 2580 & 2388 & 2101 & 2095 & 1980 & 1715 & 1685 & 2126 \\
\hline 651 & 504 & 613 & 670 & 675 & 553 & 528 & 574 & 546 & 526 & 609 & 713 & 726 & 621 \\
\hline 1084 & 840 & 1021 & 1116 & 1124 & 921 & 879 & 957 & 909 & 875 & 1015 & 1188 & 1210 & 1034 \\
\hline 382 & 382 & 382 & 382 & 382 & 382 & 382 & 382 & 382 & 382 & 382 & 382 & 382 & 382 \\
\hline 1036 & 1027 & 935 & 939 & 954 & 952 & 948 & 940 & 964 & 963 & 983 & 991 & 945 & 968 \\
\hline 253 & 292 & 346 & 647 & 660 & 658 & 602 & 450 & 367 & 368 & 172 & 232 & 282 & 413 \\
\hline 0 & 0 & 0 & 0 & 0 & 0 & 0 & 0 & 0 & 0 & 0 & 0 & 0 & 0 \\
\hline 0 & 0 & 0 & 0 & 0 & 0 & 0 & 0 & 0 & 0 & 0 & 0 & 0 & 0 \\
\hline 0 & 0 & 0 & 0 & 0 & 0 & 0 & 0 & 0 & 0 & 0 & 0 & 0 & 0 \\
\hline 7782 & 7374 & 7649 & 8765 & 9496 & 9316 & 9001 & 8499 & 7913 & 7847 & 7722 & 7862 & 7989 & 8300 \\
\hline 8443 & 7914 & 8157 & 9159 & 9905 & 9725 & 9405 & 8917 & 8548 & 8545 & 8370 & 8541 & 8709 & 8839 \\
\hline 6907 & 5151 & 6342 & 7050 & 7171 & 5752 & 5427 & 5931 & 5577 & 5248 & 6096 & 7328 & 7811 & 6464 \\
\hline 435 & 370 & 386 & 305 & 237 & 176 & 196 & 275 & 434 & 515 & 711 & 747 & 451 & 397 \\
\hline 0 & 0 & 0 & 0 & 0 & 0 & 0 & 0 & 0 & 0 & 0 & 0 & 0 & 0 \\
\hline 0 & 0 & 0 & 0 & 0 & 0 & 0 & 0 & 0 & 0 & 0 & 0 & 0 & 0 \\
\hline 141 & 141 & 141 & 141 & 141 & 141 & 141 & 141 & 136 & 136 & 136 & 136 & 136 & 139 \\
\hline 0 & 0 & 0 & 0 & 0 & 0 & 0 & 0 & 0 & 0 & 0 & 0 & 0 & 0 \\
\hline 7484 & 5663 & 6869 & 7497 & 7550 & 6069 & 5765 & 6347 & 6146 & 5899 & 6942 & 8210 & 8398 & 7000 \\
\hline 0 & 0 & 0 & 0 & 0 & 0 & 0 & 0 & 0 & 0 & 0 & 0 & 0 & 0 \\
\hline 0 & 0 & 0 & 0 & 0 & 0 & 0 & 0 & 0 & 0 & 0 & 0 & 0 & 0 \\
\hline 27 & 27 & 28 & 29 & 31 & 32 & 31 & 31 & 30 & 30 & 27 & 27 & 27 & 29 \\
\hline 0 & 0 & 0 & 0 & 0 & 0 & 0 & 0 & 0 & 0 & 0 & 0 & 0 & 0 \\
\hline 183 & 216 & 250 & 318 & 375 & 349 & 305 & 275 & 276 & 238 & 101 & 123 & 204 & 246 \\
\hline 1425 & 1567 & 1520 & 1568 & 1503 & 1161 & 1151 & 1128 & 873 & 871 & 756 & 874 & 996 & 1210 \\
\hline 0 & 0 & 0 & 0 & 0 & 0 & 0 & 0 & 0 & 0 & 0 & 0 & 0 & 0 \\
\hline 1000 & 1000 & 1000 & 1000 & 1000 & 1000 & 1000 & 1000 & 1000 & 1000 & 1000 & 1000 & 1000 & 1000 \\
\hline 81 & 58 & 76 & 101 & 104 & 107 & 98 & 91 & 113 & 113 & 107 & 94 & 75 & 92 \\
\hline 0 & 0 & 0 & 0 & 0 & 0 & 0 & 0 & 0 & 0 & 0 & 0 & 0 & 0 \\
\hline 2716 & 2869 & 2874 & 3016 & 3014 & 2648 & 2585 & 2525 & 2292 & 2252 & 1991 & 2119 & 2302 & 2577 \\
\hline 10200 & 8532 & 9743 & 10512 & 10563 & 8717 & 8350 & 8871 & 8438 & 8151 & 8933 & 10329 & 10700 & 9578 \\
\hline 0 & 0 & 0 & 0 & 0 & 0 & 0 & 0 & 0 & 0 & 0 & 0 & 0 & 0 \\
\hline
\end{tabular}

Total Other Resources

$2715 \quad 27$

\begin{tabular}{|c|c|c|c|c|c|c|c|c|c|c|c|c|c|}
\hline Aug16 & Sep & Oct & Nov & $D e c$ & Jan & $F e b$ & Mar & Apr1 & Apr16 & May & Jun & Jul & Avg \\
\hline 0 & 0 & 0 & 0 & 0 & 0 & 0 & 0 & 0 & 0 & 0 & 0 & 0 & 0 \\
\hline 0 & 0 & 0 & 0 & 0 & 0 & 0 & 0 & 0 & 0 & 0 & 0 & 0 & 0 \\
\hline 0 & 0 & 0 & 0 & 0 & 0 & 0 & 0 & 0 & 0 & 0 & 0 & 0 & 0 \\
\hline 120 & 109 & 106 & 122 & 137 & 137 & 132 & 123 & 111 & 111 & 108 & 109 & 116 & 119 \\
\hline 275 & 166 & 129 & 0 & 0 & 0 & 1.0 & 23 & 252 & 315 & 268 & 298 & 332 & 149 \\
\hline 265 & 265 & 272 & 272 & 272 & 272 & 272 & 272 & 272 & 272 & 272 & 272 & 272 & 271 \\
\hline 660 & 540 & 508 & 394 & 409 & 409 & 405 & 418 & 635 & 698 & 648 & 679 & 720 & 539 \\
\hline 0 & 0 & 0 & 0 & 0 & 0 & 0 & 0 & 0 & 0 & 0 & 0 & 0 & 0 \\
\hline 0 & 0 & 0 & 0 & 0 & 0 & 0 & 0 & 0 & 0 & 0 & 0 & 0 & 0 \\
\hline 0 & 0 & 0 & 0 & 0 & 0 & 0 & 0 & 0 & 0 & 0 & 0 & 0 & 0 \\
\hline 2654 & 2432 & 2416 & 2712 & 3116 & 3223 & 3082 & 2808 & 2644 & 2637 & 2580 & 2640 & 2759 & 2755 \\
\hline 1723 & 1897 & 1935 & 2299 & 2585 & 2626 & 2580 & 2388 & 2101 & 2095 & 1980 & 1715 & 1685 & 2126 \\
\hline 651 & 504 & 613 & 670 & 675 & 553 & 528 & 574 & 546 & 526 & 609 & 713 & 726 & 621 \\
\hline 1084 & 840 & 1021 & 1116 & 1124 & 921 & 879 & 957 & 909 & 875 & 1015 & 1188 & 1210 & 1034 \\
\hline 382 & 382 & 382 & 382 & 382 & 382 & 382 & 382 & 382 & 382 & 382 & 382 & 382 & 382 \\
\hline 1036 & 1027 & 935 & 939 & 954 & 952 & 948 & 940 & 964 & 963 & 983 & 991 & 945 & 968 \\
\hline 253 & 292 & 346 & 647 & 660 & 658 & 602 & 450 & 367 & 368 & 172 & 232 & 282 & 413 \\
\hline 0 & 0 & 0 & 0 & 0 & 0 & 0 & 0 & 0 & 0 & 0 & 0 & 0 & 0 \\
\hline 0 & 0 & 0 & 0 & 0 & 0 & 0 & 0 & 0 & 0 & 0 & 0 & 0 & 0 \\
\hline 0 & 0 & 0 & 0 & 0 & 0 & 0 & 0 & 0 & 0 & 0 & 0 & 0 & 0 \\
\hline 7782 & 7374 & 7649 & 8765 & 9496 & 9316 & 9001 & 8499 & 7913 & 7847 & 7722 & 7862 & 7989 & 8300 \\
\hline 8443 & 7914 & 8157 & 9159 & 9905 & 9725 & 9405 & 8917 & 8548 & 8545 & 8370 & 8541 & 8709 & 8839 \\
\hline 6907 & 5151 & 6342 & 7050 & 7171 & 5752 & 5427 & 5931 & 5577 & 5248 & 6096 & 7328 & 7811 & 6464 \\
\hline 435 & 370 & 386 & 305 & 237 & 176 & 196 & 275 & 434 & 515 & 711 & 747 & 451 & 397 \\
\hline 0 & 0 & 0 & 0 & 0 & 0 & 0 & 0 & 0 & 0 & 0 & 0 & 0 & 0 \\
\hline 0 & 0 & 0 & 0 & 0 & 0 & 0 & 0 & 0 & 0 & 0 & 0 & 0 & 0 \\
\hline 141 & 141 & 141 & 141 & 141 & 141 & 141 & 141 & 136 & 136 & 136 & 136 & 136 & 139 \\
\hline 0 & 0 & 0 & 0 & 0 & 0 & 0 & 0 & 0 & 0 & 0 & 0 & 0 & 0 \\
\hline 7484 & 5663 & 6869 & 7497 & 7550 & 6069 & 5765 & 6347 & 6146 & 5899 & 6942 & 8210 & 8398 & 7000 \\
\hline 0 & 0 & 0 & 0 & 0 & 0 & 0 & 0 & 0 & 0 & 0 & 0 & 0 & 0 \\
\hline 0 & 0 & 0 & 0 & 0 & 0 & 0 & 0 & 0 & 0 & 0 & 0 & 0 & 0 \\
\hline 27 & 27 & 28 & 29 & 31 & 32 & 31 & 31 & 30 & 30 & 27 & 27 & 27 & 29 \\
\hline 0 & 0 & 0 & 0 & 0 & 0 & 0 & 0 & 0 & 0 & 0 & 0 & 0 & 0 \\
\hline 183 & 216 & 250 & 318 & 375 & 349 & 305 & 275 & 276 & 238 & 101 & 123 & 204 & 246 \\
\hline 1425 & 1567 & 1520 & 1568 & 1503 & 1161 & 1151 & 1128 & 873 & 871 & 756 & 874 & 996 & 1210 \\
\hline 0 & 0 & 0 & 0 & 0 & 0 & 0 & 0 & 0 & 0 & 0 & 0 & 0 & 0 \\
\hline 1000 & 1000 & 1000 & 1000 & 1000 & 1000 & 1000 & 1000 & 1000 & 1000 & 1000 & 1000 & 1000 & 1000 \\
\hline 81 & 58 & 76 & 101 & 104 & 107 & 98 & 91 & 113 & 113 & 107 & 94 & 75 & 92 \\
\hline 0 & 0 & 0 & 0 & 0 & 0 & 0 & 0 & 0 & 0 & 0 & 0 & 0 & 0 \\
\hline 2716 & 2869 & 2874 & 3016 & 3014 & 2648 & 2585 & 2525 & 2292 & 2252 & 1991 & 2119 & 2302 & 2577 \\
\hline 10200 & 8532 & 9743 & 10512 & 10563 & 8717 & 8350 & 8871 & 8438 & 8151 & 8933 & 10329 & 10700 & 9578 \\
\hline 0 & 0 & 0 & 0 & 0 & 0 & 0 & 0 & 0 & 0 & 0 & 0 & 0 & 0 \\
\hline
\end{tabular}

\section{Reserves \& Maintenance}

Hydro Reserves

Small Thermal \& Misc. Reserves

Contract Reserves

Large Thermal Reserves

Federal Hydro Maintenance

Spinning Reserves

Federal Transmission Losses

$12571 \quad 10200$

\section{0}

Total Reserves, Maintenance \& Losses

\begin{tabular}{rrrrrrrrrrrrrrr}
0 & 0 & 0 & 0 & 0 & 0 & 0 & 0 & 0 & 0 & 0 & 0 & 0 & 0 & 0 \\
0 & 0 & 0 & 0 & 0 & 0 & 0 & 0 & 0 & 0 & 0 & 0 & 0 & 0 & 0 \\
0 & 0 & 0 & 0 & 0 & 0 & 0 & 0 & 0 & 0 & 0 & 0 & 0 & 0 & 0 \\
0 & 0 & 0 & 0 & 0 & 0 & 0 & 0 & 0 & 0 & 0 & 0 & 0 & 0 & 0 \\
0 & 0 & 0 & 0 & 0 & 0 & 0 & 0 & 0 & 0 & 0 & 0 & 0 & 0 & 0 \\
0 & 0 & 0 & 0 & 0 & 0 & 0 & 0 & 0 & 0 & 0 & 0 & 0 & 0 & 0 \\
-354 & -288 & -241 & -275 & -296 & -298 & -246 & -235 & -250 & -238 & -230 & -252 & -291 & -302 & -270 \\
& & & & & & & & & & & & & & \\
-354 & -288 & -241 & -275 & -296 & -298 & -246 & -235 & -250 & -238 & -230 & -252 & -291 & -302 & -270 \\
& & & & & & & & & & & & & & \\
\hline
\end{tabular}


Loads and Resources - Federal System

PNW Loads and Resources Study

2006 - 2007 Operating Year

1937 Water Year

2003 White Book

Non-Utility Obligations

Federal Agencies 1981 PSC Sale

USBR 1981 PSC Sale

DSI 1981 PSC Sale

Federal Agencies 2002 PSC Sale

USBR 2002 PSC Sale

DSI 2002 PSC Sale
Oct Nov Dec Jan Feb

\begin{tabular}{|c|c|c|c|c|c|c|c|c|c|c|c|c|c|c|}
\hline 0 & 0 & 0 & 0 & 0 & 0 & 0 & 0 & 0 & 0 & 0 & 0 & 0 & 0 & 0 \\
\hline 0 & 0 & 0 & 0 & 0 & 0 & 0 & 0 & 0 & 0 & 0 & 0 & 0 & 0 & 0 \\
\hline 0 & 0 & 0 & 0 & 0 & 0 & 0 & 0 & 0 & 0 & 0 & 0 & 0 & 0 & 0 \\
\hline 121 & 121 & 109 & 107 & 123 & 137 & 138 & 132 & 124 & 111 & 111 & 109 & 109 & 116 & 120 \\
\hline 309 & 275 & 166 & 129 & 0 & 0 & 0 & 1.0 & 23 & 252 & 315 & 268 & 298 & 332 & 149 \\
\hline 272 & 272 & 272 & 0 & 0 & 0 & 0 & 0 & 0 & 0 & 0 & 0 & 0 & 0 & 45 \\
\hline
\end{tabular}

\begin{tabular}{|c|c|c|c|c|c|c|c|c|c|c|c|c|c|c|}
\hline 0 & 0 & 0 & 0 & 0 & 0 & 0 & 0 & 0 & 0 & 0 & 0 & 0 & 0 & 0 \\
\hline 0 & 0 & 0 & 0 & 0 & 0 & 0 & 0 & 0 & 0 & 0 & 0 & 0 & 0 & 0 \\
\hline 0 & 0 & 0 & 0 & 0 & 0 & 0 & 0 & 0 & 0 & 0 & 0 & 0 & 0 & \\
\hline 2695 & 2695 & 2480 & 2580 & 2906 & 3270 & 3419 & 3274 & 2967 & 2817 & 2809 & 2772 & 2856 & 2972 & 2917 \\
\hline 1686 & 1686 & 1860 & 2273 & 2651 & 2970 & 2984 & 2929 & 2710 & 2349 & 2342 & 2151 & 1877 & 1971 & 2367 \\
\hline 851 & 655 & 508 & 615 & 673 & 678 & 556 & 530 & 577 & 549 & 528 & 553 & 653 & 730 & 614 \\
\hline 1417 & 1091 & 846 & 1025 & 1120 & 1129 & 925 & 882 & 960 & 914 & 879 & 921 & 1088 & 1215 & 1022 \\
\hline 382 & 382 & 382 & 0 & 0 & 0 & 0 & 0 & 0 & 0 & 0 & 0 & 0 & 0 & 64 \\
\hline 891 & 891 & 884 & 737 & 741 & 757 & 755 & 751 & 743 & 766 & 766 & 784 & 793 & 787 & 783 \\
\hline 252 & 253 & 292 & 291 & 592 & 605 & 602 & 547 & 394 & 361 & 362 & 167 & 227 & 226 & 380 \\
\hline 0 & 0 & 0 & 0 & 0 & 0 & 0 & 0 & 0 & 0 & 0 & 0 & 0 & 0 & \\
\hline 0 & 0 & 0 & 0 & 0 & 0 & 0 & 0 & 0 & 0 & 0 & 0 & 0 & 0 & \\
\hline 0 & 0 & 0 & 0 & 0 & 0 & 0 & 0 & 0 & 0 & 0 & 0 & 0 & 0 & \\
\hline
\end{tabular}

Transfers Out

NGP 1981 PSC Sale

GPU 1981 PSC Sale

IOU 1981 PSC Sale

NGP 2002 PSC Sale

GPU 2002 PSC Sale

NGP Slice Sale

GPU Slice Sale

IOU 2002 PSC Sale

Exports

Intra-Regional Transfers (Out)

Supp \& Entitlement Capacity (Out)

CSPE To West Group Utilities

Federal Diversity

\begin{tabular}{|c|}
\hline $3173 \quad 7652$ \\
\hline
\end{tabular}

8875

8320

7799

$7758 \quad 8806 \quad 9546$

$9380 \quad 90$

$\begin{array}{rrrrrr}9303 & 6930 & 5175 & 6370 & 7083 & 7 \\ 438 & 436 & 371 & 386 & 306 & \\ 0 & 0 & 0 & 0 & 0 \\ 0 & 0 & 0 & 0 & 0 \\ 136 & 136 & 136 & 136 & 136 \\ 0 & 0 & 0 & 0 & 0\end{array}$

$\begin{array}{rr}7205 & 5778 \\ 237 & 176 \\ 0 & \\ 0 & 0 \\ 136 & 136 \\ 0 & \end{array}$

$8498 \quad 8118$

8112

7726

7901

$8349 \quad 8460$
Regulated Hydro

Independent Hydro

Sustained Peaking Adjustment

-Fed CER (CSPE)

Restoration

$9877 \quad 7501$

$\begin{array}{rrrr}0 & 0 & 0 & 0 \\ 0 & 0 & 0 & 0 \\ 27 & 27 & 27 & 28 \\ 0 & 0 & 0 & 0 \\ 183 & 183 & 216 & 250 \\ 1024 & 1022 & 1167 & 693 \\ 0 & 0 & 0 & 0 \\ 1000 & 1000 & 1000 & 1000 \\ 59 & 59 & 52 & 116 \\ 0 & 0 & 0 & 0\end{array}$

$\begin{array}{rr}0 & 0 \\ 0 & 0 \\ 29 & 31 \\ 0 & 0 \\ 318 & 375 \\ 739 & 674 \\ 0 & 0 \\ 1000 & 1000 \\ 127 & 127 \\ 0 & 0\end{array}$

0
0
32
0
299
324
0
1000
130
0

$\begin{array}{rr}0 & 0 \\ 0 & 0 \\ 31 & 31 \\ 0 & 0 \\ 255 & 225 \\ 312 & 290 \\ 0 & 0 \\ 1000 & 1000 \\ 125 & 119 \\ 0 & 0\end{array}$

\begin{tabular}{|c|c|c|c|c|c|c|}
\hline Mar & Apr1 & Apr16 & May & Jun & Jul & Avg \\
\hline 0 & 0 & 0 & 0 & 0 & 0 & 0 \\
\hline 0 & 0 & 0 & 0 & 0 & 0 & 0 \\
\hline 0 & 0 & 0 & 0 & 0 & 0 & 0 \\
\hline 124 & 111 & 111 & 109 & 109 & 116 & 120 \\
\hline 23 & 252 & 315 & 268 & 298 & 332 & 149 \\
\hline 0 & 0 & 0 & 0 & 0 & 0 & 45 \\
\hline 147 & 363 & 426 & 377 & 407 & 448 & 314 \\
\hline 0 & 0 & 0 & 0 & 0 & 0 & 0 \\
\hline 0 & 0 & 0 & 0 & 0 & 0 & 0 \\
\hline 0 & 0 & 0 & 0 & 0 & 0 & 0 \\
\hline 2967 & 2817 & 2809 & 2772 & 2856 & 2972 & 2917 \\
\hline 2710 & 2349 & 2342 & 2151 & 1877 & 1971 & 2367 \\
\hline 577 & 549 & 528 & 553 & 653 & 730 & 614 \\
\hline 960 & 914 & 879 & 921 & 1088 & 1215 & 1022 \\
\hline 0 & 0 & 0 & 0 & 0 & 0 & 64 \\
\hline 743 & 766 & 766 & 784 & 793 & 787 & 783 \\
\hline 394 & 361 & 362 & 167 & 227 & 226 & 380 \\
\hline 0 & 0 & 0 & 0 & 0 & 0 & 0 \\
\hline 0 & 0 & 0 & 0 & 0 & 0 & 0 \\
\hline 0 & 0 & 0 & 0 & 0 & 0 & 0 \\
\hline 351 & 7755 & 7686 & 7349 & 7494 & 7901 & 8146 \\
\hline 3498 & 8118 & 8112 & 7726 & 7901 & 8349 & 8460 \\
\hline 5956 & 5604 & 5272 & 6121 & 7360 & 7846 & 6492 \\
\hline 275 & 434 & 516 & 712 & 748 & 452 & 398 \\
\hline 0 & 0 & 0 & 0 & 0 & 0 & 0 \\
\hline 0 & 0 & 0 & 0 & 0 & 0 & 0 \\
\hline 136 & 132 & 132 & 132 & 132 & 132 & 134 \\
\hline 0 & 0 & 0 & 0 & 0 & 0 & 0 \\
\hline 3367 & 6170 & 5920 & 6965 & 8239 & 8430 & 7024 \\
\hline 0 & 0 & 0 & 0 & 0 & 0 & 0 \\
\hline 0 & 0 & 0 & 0 & 0 & 0 & 0 \\
\hline 31 & 30 & 30 & 27 & 27 & 27 & 29 \\
\hline 0 & 0 & 0 & 0 & 0 & 0 & 0 \\
\hline 225 & 226 & 188 & 101 & 123 & 154 & 226 \\
\hline 290 & 285 & 285 & 167 & 286 & 159 & 510 \\
\hline 0 & 0 & 0 & 0 & 0 & 0 & 0 \\
\hline 1000 & 1000 & 1000 & 355 & 167 & 1000 & 877 \\
\hline 119 & 121 & 121 & 118 & 120 & 109 & 110 \\
\hline 0 & 0 & 0 & 0 & 0 & 0 & 0 \\
\hline 1664 & 1662 & 1624 & 768 & 724 & 1449 & 1751 \\
\hline 3031 & 7832 & 7544 & 7733 & 8963 & 9879 & 8775 \\
\hline 0 & 0 & 0 & 0 & 0 & 0 & \\
\hline
\end{tabular}

\begin{tabular}{|c|c|c|c|c|c|c|}
\hline Mar & Apr1 & Apr16 & May & Jun & Jul & Avg \\
\hline 0 & 0 & 0 & 0 & 0 & 0 & 0 \\
\hline 0 & 0 & 0 & 0 & 0 & 0 & 0 \\
\hline 0 & 0 & 0 & 0 & 0 & 0 & 0 \\
\hline 124 & 111 & 111 & 109 & 109 & 116 & 120 \\
\hline 23 & 252 & 315 & 268 & 298 & 332 & 149 \\
\hline 0 & 0 & 0 & 0 & 0 & 0 & 45 \\
\hline 147 & 363 & 426 & 377 & 407 & 448 & 314 \\
\hline 0 & 0 & 0 & 0 & 0 & 0 & 0 \\
\hline 0 & 0 & 0 & 0 & 0 & 0 & 0 \\
\hline 0 & 0 & 0 & 0 & 0 & 0 & 0 \\
\hline 2967 & 2817 & 2809 & 2772 & 2856 & 2972 & 2917 \\
\hline 2710 & 2349 & 2342 & 2151 & 1877 & 1971 & 2367 \\
\hline 577 & 549 & 528 & 553 & 653 & 730 & 614 \\
\hline 960 & 914 & 879 & 921 & 1088 & 1215 & 1022 \\
\hline 0 & 0 & 0 & 0 & 0 & 0 & 64 \\
\hline 743 & 766 & 766 & 784 & 793 & 787 & 783 \\
\hline 394 & 361 & 362 & 167 & 227 & 226 & 380 \\
\hline 0 & 0 & 0 & 0 & 0 & 0 & 0 \\
\hline 0 & 0 & 0 & 0 & 0 & 0 & 0 \\
\hline 0 & 0 & 0 & 0 & 0 & 0 & 0 \\
\hline 351 & 7755 & 7686 & 7349 & 7494 & 7901 & 8146 \\
\hline 3498 & 8118 & 8112 & 7726 & 7901 & 8349 & 8460 \\
\hline 5956 & 5604 & 5272 & 6121 & 7360 & 7846 & 6492 \\
\hline 275 & 434 & 516 & 712 & 748 & 452 & 398 \\
\hline 0 & 0 & 0 & 0 & 0 & 0 & 0 \\
\hline 0 & 0 & 0 & 0 & 0 & 0 & 0 \\
\hline 136 & 132 & 132 & 132 & 132 & 132 & 134 \\
\hline 0 & 0 & 0 & 0 & 0 & 0 & 0 \\
\hline 3367 & 6170 & 5920 & 6965 & 8239 & 8430 & 7024 \\
\hline 0 & 0 & 0 & 0 & 0 & 0 & 0 \\
\hline 0 & 0 & 0 & 0 & 0 & 0 & 0 \\
\hline 31 & 30 & 30 & 27 & 27 & 27 & 29 \\
\hline 0 & 0 & 0 & 0 & 0 & 0 & 0 \\
\hline 225 & 226 & 188 & 101 & 123 & 154 & 226 \\
\hline 290 & 285 & 285 & 167 & 286 & 159 & 510 \\
\hline 0 & 0 & 0 & 0 & 0 & 0 & 0 \\
\hline 1000 & 1000 & 1000 & 355 & 167 & 1000 & 877 \\
\hline 119 & 121 & 121 & 118 & 120 & 109 & 110 \\
\hline 0 & 0 & 0 & 0 & 0 & 0 & 0 \\
\hline 1664 & 1662 & 1624 & 768 & 724 & 1449 & 1751 \\
\hline 3031 & 7832 & 7544 & 7733 & 8963 & 9879 & 8775 \\
\hline 0 & 0 & 0 & 0 & 0 & 0 & \\
\hline
\end{tabular}

\begin{tabular}{|c|c|c|c|c|c|c|}
\hline Mar & Apr1 & Apr16 & May & Jun & Jul & Avg \\
\hline 0 & 0 & 0 & 0 & 0 & 0 & 0 \\
\hline 0 & 0 & 0 & 0 & 0 & 0 & 0 \\
\hline 0 & 0 & 0 & 0 & 0 & 0 & 0 \\
\hline 124 & 111 & 111 & 109 & 109 & 116 & 120 \\
\hline 23 & 252 & 315 & 268 & 298 & 332 & 149 \\
\hline 0 & 0 & 0 & 0 & 0 & 0 & 45 \\
\hline 147 & 363 & 426 & 377 & 407 & 448 & 314 \\
\hline 0 & 0 & 0 & 0 & 0 & 0 & 0 \\
\hline 0 & 0 & 0 & 0 & 0 & 0 & 0 \\
\hline 0 & 0 & 0 & 0 & 0 & 0 & 0 \\
\hline 2967 & 2817 & 2809 & 2772 & 2856 & 2972 & 2917 \\
\hline 2710 & 2349 & 2342 & 2151 & 1877 & 1971 & 2367 \\
\hline 577 & 549 & 528 & 553 & 653 & 730 & 614 \\
\hline 960 & 914 & 879 & 921 & 1088 & 1215 & 1022 \\
\hline 0 & 0 & 0 & 0 & 0 & 0 & 64 \\
\hline 743 & 766 & 766 & 784 & 793 & 787 & 783 \\
\hline 394 & 361 & 362 & 167 & 227 & 226 & 380 \\
\hline 0 & 0 & 0 & 0 & 0 & 0 & 0 \\
\hline 0 & 0 & 0 & 0 & 0 & 0 & 0 \\
\hline 0 & 0 & 0 & 0 & 0 & 0 & 0 \\
\hline 351 & 7755 & 7686 & 7349 & 7494 & 7901 & 8146 \\
\hline 3498 & 8118 & 8112 & 7726 & 7901 & 8349 & 8460 \\
\hline 5956 & 5604 & 5272 & 6121 & 7360 & 7846 & 6492 \\
\hline 275 & 434 & 516 & 712 & 748 & 452 & 398 \\
\hline 0 & 0 & 0 & 0 & 0 & 0 & 0 \\
\hline 0 & 0 & 0 & 0 & 0 & 0 & 0 \\
\hline 136 & 132 & 132 & 132 & 132 & 132 & 134 \\
\hline 0 & 0 & 0 & 0 & 0 & 0 & 0 \\
\hline 3367 & 6170 & 5920 & 6965 & 8239 & 8430 & 7024 \\
\hline 0 & 0 & 0 & 0 & 0 & 0 & 0 \\
\hline 0 & 0 & 0 & 0 & 0 & 0 & 0 \\
\hline 31 & 30 & 30 & 27 & 27 & 27 & 29 \\
\hline 0 & 0 & 0 & 0 & 0 & 0 & 0 \\
\hline 225 & 226 & 188 & 101 & 123 & 154 & 226 \\
\hline 290 & 285 & 285 & 167 & 286 & 159 & 510 \\
\hline 0 & 0 & 0 & 0 & 0 & 0 & 0 \\
\hline 1000 & 1000 & 1000 & 355 & 167 & 1000 & 877 \\
\hline 119 & 121 & 121 & 118 & 120 & 109 & 110 \\
\hline 0 & 0 & 0 & 0 & 0 & 0 & 0 \\
\hline 1664 & 1662 & 1624 & 768 & 724 & 1449 & 1751 \\
\hline 3031 & 7832 & 7544 & 7733 & 8963 & 9879 & 8775 \\
\hline 0 & 0 & 0 & 0 & 0 & 0 & \\
\hline
\end{tabular}

Total Other Resources

$2293 \quad 2291$

$2291 \quad 2463 \quad 2086$

\begin{tabular}{rrrrrrrrrrrr}
2086 & 2213 & 2207 & 1784 & 1722 & 1664 & 1662 & 1624 & 768 & 724 & 1449 & 1751 \\
\hline 8979 & 9738 & 9785 & 7874 & 7506 & 8031 & 7832 & 7544 & 7733 & 8963 & 9879 & 8775
\end{tabular}

Reserves \& Maintenance

Hydro Reserves

Small Thermal \& Misc. Reserves

Contract Reserves

Large Thermal Reserves

Federal Hydro Maintenance

Spinning Reserves

Federal Transmission Losses

$12169 \quad 9793 \quad 8143 \quad 8979$

Total Reserves, Maintenance \& Losses

$\begin{array}{cccccccc}0 & 0 & 0 & 0 & 0 & 0 & 0 & 0 \\ 0 & 0 & 0 & 0 & 0 & 0 & 0 & 0 \\ 0 & 0 & 0 & 0 & 0 & 0 & 0 & 0 \\ 0 & 0 & 0 & 0 & 0 & 0 & 0 & 0 \\ 0 & 0 & 0 & 0 & 0 & 0 & 0 & 0 \\ 0 & 0 & 0 & 0 & 0 & 0 & 0 & 0\end{array}$

$-343$

$\begin{array}{rr}0 & 0 \\ -276 & -230\end{array}$

$-343 \quad-276$

$-230 \quad-253$

$\begin{array}{llll}11826 & 9516 & 7914 & 87\end{array}$

$2951 \quad 119$

$114 \quad 967 \quad 657$

$\begin{array}{lll}-37 & -1728 & -175\end{array}$

$7294 \quad 7804$

Total Firm Surplus/Deficit

\begin{abstract}
$967 \quad 657$
\end{abstract}

$\begin{array}{lllll}-275 & -276 & -222 & -212 & -226\end{array}$




Loads and Resources - Federal System

PNW Loads and Resources Study

2007 - 2008 Operating Year

1937 Water Year

2003 White Book

Non-Utility Obligations

Federal Agencies 1981 PSC Sale

USBR 1981 PSC Sale

DSI 1981 PSC Sale

Federal Agencies 2002 PSC Sale

USBR 2002 PSC Sale

DSI 2002 PSC Sale
Oct Nov Dec Jan Fe
Mar Apr1 Apr16
Jun

Jul Avg

Total Firm Non-Utility Obligations

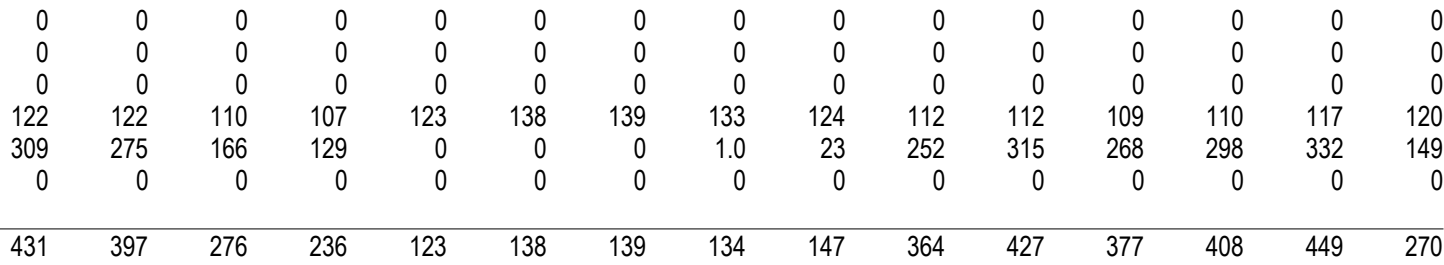

Transfers Out

NGP 1981 PSC Sale

GPU 1981 PSC Sale

IOU 1981 PSC Sale

NGP 2002 PSC Sale

GPU 2002 PSC Sale

NGP Slice Sale

GPU Slice Sale

IOU 2002 PSC Sale

Exports

Intra-Regional Transfers (Out)

Supp \& Entitlement Capacity (Out)

CSPE To West Group Utilities

Federal Diversity

\begin{tabular}{|c|c|c|c|c|c|c|c|c|c|c|c|c|c|c|}
\hline 0 & 0 & 0 & 0 & 0 & 0 & 0 & 0 & 0 & 0 & 0 & 0 & 0 & 0 & \\
\hline 0 & 0 & 0 & 0 & 0 & 0 & 0 & 0 & 0 & 0 & 0 & 0 & 0 & 0 & \\
\hline 0 & 0 & 0 & 0 & 0 & 0 & 0 & 0 & 0 & 0 & 0 & 0 & 0 & 0 & \\
\hline 2924 & 2924 & 2649 & 2607 & 2938 & 3306 & 3457 & 3275 & 3000 & 2847 & 2839 & 2802 & 2886 & 3003 & 29 \\
\hline 2039 & 2039 & 2180 & 2282 & 2663 & 2983 & 2997 & 2923 & 2723 & 2357 & 2350 & 2161 & 1887 & 1979 & 2431 \\
\hline 855 & 658 & 511 & 619 & 677 & 682 & 559 & 533 & 579 & 551 & 530 & 613 & 719 & 734 & \\
\hline 1425 & 1097 & 850 & 1030 & 1127 & 1136 & 930 & 887 & 964 & 918 & 883 & 1021 & 1198 & 1222 & \\
\hline 0 & 0 & 0 & 0 & 0 & 0 & 0 & 0 & 0 & 0 & 0 & 0 & 0 & 0 & \\
\hline 775 & 775 & 768 & 734 & 739 & 756 & 650 & 646 & 638 & 661 & 653 & 667 & 672 & 667 & 69 \\
\hline 197 & 197 & 236 & 291 & 592 & 605 & 602 & 540 & 394 & 361 & 362 & 167 & 227 & 226 & \\
\hline 0 & 0 & 0 & 0 & 0 & 0 & 0 & 0 & 0 & 0 & 0 & 0 & 0 & 0 & \\
\hline 0 & 0 & 0 & 0 & 0 & 0 & 0 & 0 & 0 & 0 & 0 & 0 & 0 & 0 & \\
\hline 0 & 0 & 0 & 0 & 0 & 0 & 0 & 0 & 0 & 0 & 0 & 0 & 0 & 0 & \\
\hline
\end{tabular}

Total Transfers Ou

$7690 \quad 7194 \quad 7564$

$9468 \quad 9196$

$196 \quad 8804 \quad 8299$

$\begin{array}{llll}8645 & 8087 & 7470 & 7800\end{array}$

$8858 \quad 9606$

9334

8938

8446

8058

8044

7809

7997

$8279 \quad 8413$

Hydro Resources

Regulated Hydro

Independent Hydro

Sustained Peaking Adjustment

Non-Fed CER (CSPE)

Non-Fed CER (Canada)

Restoration

$\begin{array}{rrrrrr}9358 & 6971 & 5203 & 6407 & 7131 & 7252 \\ 438 & 436 & 371 & 386 & 306 & 237 \\ 0 & 0 & 0 & 0 & 0 & 0 \\ 0 & 0 & 0 & 0 & 0 & 0 \\ 132 & 132 & 132 & 132 & 132 & 132 \\ 0 & 0 & 0 & 0 & 0\end{array}$

$\begin{array}{rr}7252 & 5812 \\ 237 & 176 \\ 0 & 0 \\ 0 & 0 \\ 132 & 132 \\ 0 & 0\end{array}$

$\begin{array}{llll}9928 & 7539 & 5706 & 6925\end{array}$

$6925 \quad 7568 \quad 7621$

$7621 \quad 6120 \quad 5812 \quad 6391$

$\begin{array}{rrrr}0 & 0 & 0 & 0 \\ 0 & 0 & 0 & 0 \\ 27 & 27 & 27 & 28 \\ 0 & 0 & 0 & 0 \\ 133 & 133 & 166 & 200 \\ 184 & 184 & 329 & 318 \\ 0 & 0 & 0 & 0 \\ 1000 & 1000 & 1000 & 1000 \\ 109 & 109 & 102 & 116 \\ 0 & 0 & 0 & 0\end{array}$

$\begin{array}{rr}0 & 0 \\ 0 & 0 \\ 29 & 31 \\ 0 & 0 \\ 268 & 325 \\ 364 & 300 \\ 0 & 0 \\ 1000 & 1000 \\ 127 & 127 \\ 0 & 0\end{array}$

$\begin{array}{rrr}0 & 0 & 0 \\ 0 & 0 & 0 \\ 32 & 31 & 31 \\ 0 & 0 & 0 \\ 299 & 255 & 225 \\ 324 & 305 & 290 \\ 0 & 0 & 0 \\ 1000 & 1000 & 1000 \\ 130 & 125 & 119 \\ 0 & 0 & 0\end{array}$

0
0
30
0
226
285
0
1000
121
0

0
0
30
0
173
285
0
1000
121
0

0
0
27
0
83
167
0
1000
118
0

$\begin{array}{rrr}0 & 0 & 0 \\ 0 & 0 & 0 \\ 27 & 27 & 29 \\ 0 & 0 & 0 \\ 101 & 132 & 199 \\ 286 & 159 & 276 \\ 0 & 0 & 0 \\ 1000 & 1000 & 1000 \\ 120 & 109 & 119 \\ 0 & 0 & 0\end{array}$

Total Other Resources

Total Resources

$11380 \quad 8992$

\begin{tabular}{llllllllllllll}
1453 & 1624 & 1661 & 1788 & 1783 & 1784 & 1716 & 1664 & 1662 & 1609 & 1395 & 1535 & 1427 & 1622 \\
\hline 8992 & 7330 & 8586 & 9356 & 9404 & 7905 & 7527 & 8055 & 7859 & 7551 & 8373 & 9804 & 9903 & 8678
\end{tabular}

Reserves \& Maintenance

Hydro Reserves

Small Thermal \& Misc. Reserves

Contract Reserves

Large Thermal Reserves

Federal Hydro Maintenance

Spinning Reserves

Federal Transmission Losses

$\begin{array}{lllllll}0 & 0 & 0 & 0 & 0 & 0 & 0 \\ 0 & 0 & 0 & 0 & 0 & 0 & 0 \\ 0 & 0 & 0 & 0 & 0 & 0 & 0 \\ 0 & 0 & 0 & 0 & 0 & 0 & 0 \\ 0 & 0 & 0 & 0 & 0 & 0 & 0 \\ 0 & 0 & 0 & 0 & 0 & 0 & 0\end{array}$

$\begin{array}{llll}0 & 0 & 0 & 0 \\ 0 & 0 & 0 & 0 \\ 0 & 0 & 0 & 0 \\ 0 & 0 & 0 & 0 \\ 0 & 0 & 0 & 0 \\ 0 & 0 & 0 & 0\end{array}$

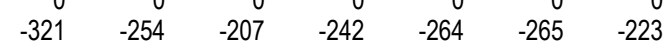

$-212 \quad-227 \quad-222$

$\begin{array}{llllllllll}-321 & -254 & -207 & -242 & -264 & -265 & -223 & -212 & -227 & -222\end{array}$

$-213-236$

$\begin{array}{rrrr}0 & 0 & 0 & 0 \\ 0 & 0 & 0 & 0 \\ 0 & 0 & 0 & 0 \\ 0 & 0 & 0 & 0 \\ 0 & 0 & 0 & 0 \\ 0 & 0 & 0 & 0\end{array}$

Total Reserves, Maintenance \& Losses

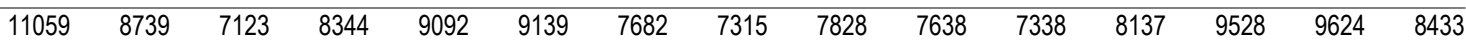

Total Net Resources

2414

652

544

$234 \quad-467 \quad-1653 \quad-1623$

$-617$

$-420 \quad-706$

$\begin{array}{llll}328 & 1531 & 1345 \quad 20\end{array}$ 
Loads and Resources - Federal System

PNW Loads and Resources Study

2008 - 2009 Operating Year

1937 Water Year

2003 White Book

Non-Utility Obligations

Federal Agencies 1981 PSC Sale

USBR 1981 PSC Sale

DSI 1981 PSC Sale

Federal Agencies 2002 PSC Sale

USBR 2002 PSC Sale

DSI 2002 PSC Sale
Oct Nov Dec Jan Fe
Mar Apr1 Apr16
Jun

Jul Avg

Total Firm Non-Utility Obligations

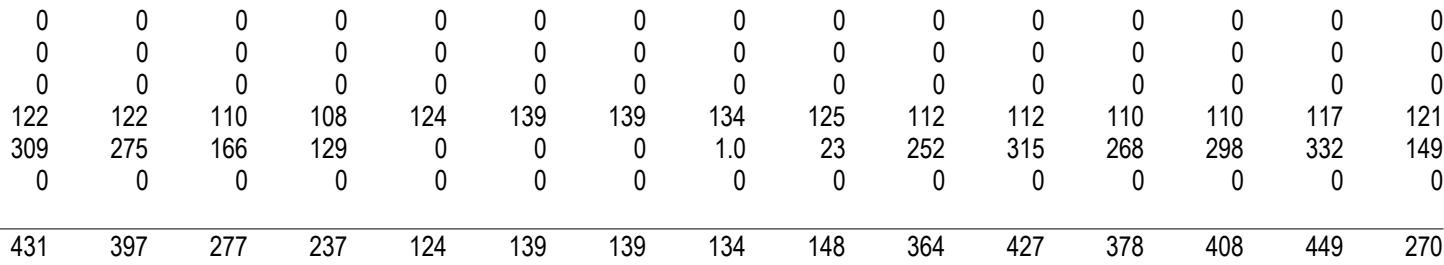

Transfers Out

NGP 1981 PSC Sale

GPU 1981 PSC Sale

IOU 1981 PSC Sale

NGP 2002 PSC Sale

GPU 2002 PSC Sale

NGP Slice Sale

GPU Slice Sale

IOU 2002 PSC Sale

Exports

Intra-Regional Transfers (Out)

Supp \& Entitlement Capacity (Out)

CSPE To West Group Utilities

Federal Diversity

\begin{tabular}{|c|c|c|c|c|c|c|c|c|c|c|c|c|c|c|}
\hline 0 & 0 & 0 & 0 & 0 & 0 & 0 & 0 & 0 & 0 & 0 & 0 & 0 & 0 & \\
\hline 0 & 0 & 0 & 0 & 0 & 0 & 0 & 0 & 0 & 0 & 0 & 0 & 0 & 0 & \\
\hline 0 & 0 & 0 & 0 & 0 & 0 & 0 & 0 & 0 & 0 & 0 & 0 & 0 & 0 & \\
\hline 2955 & 2955 & 2677 & 2634 & 2969 & 3343 & 3494 & 3348 & 3034 & 2878 & 2871 & 2832 & 2916 & 3034 & 30 \\
\hline 2049 & 2049 & 2188 & 2292 & 2675 & 2994 & 3011 & 2954 & 2734 & 2367 & 2360 & 2172 & 1894 & 1987 & 24 \\
\hline 858 & 660 & 512 & 620 & 679 & 684 & 560 & 534 & 581 & 553 & 531 & 556 & 657 & 736 & \\
\hline 1428 & 1099 & 853 & 1033 & 1130 & 1139 & 933 & 889 & 967 & 920 & 885 & 926 & 1095 & 1225 & \\
\hline 0 & 0 & 0 & 0 & 0 & 0 & 0 & 0 & 0 & 0 & 0 & 0 & 0 & 0 & \\
\hline 641 & 641 & 634 & 605 & 614 & 631 & 629 & 625 & 616 & 639 & 638 & 652 & 657 & 652 & \\
\hline 197 & 197 & 236 & 291 & 592 & 605 & 602 & 547 & 394 & 361 & 362 & 167 & 227 & 226 & \\
\hline 0 & 0 & 0 & 0 & 0 & 0 & 0 & 0 & 0 & 0 & 0 & 0 & 0 & 0 & \\
\hline 0 & 0 & 0 & 0 & 0 & 0 & 0 & 0 & 0 & 0 & 0 & 0 & 0 & 0 & \\
\hline 0 & 0 & 0 & 0 & 0 & 0 & 0 & 0 & 0 & 0 & 0 & 0 & 0 & 0 & \\
\hline
\end{tabular}

Total Transfers Ou

$\begin{array}{lllllllllllllll}8127 & 7602 & 7100 & 7475 & 8659 & 9395 & 9229 & 8897 & 8325 & 7718 & 7648 & 7305 & 7447 & 7861 & 8103\end{array}$

Total Firm Obligations

8558

$7999 \quad 7377 \quad 7712$

$9534 \quad 9368$

$9031 \quad 8473$

$8473 \quad 8083$

$8075 \quad 7682$

7855

$8310 \quad 8373$

Hydro Resources

Regulated Hydro

Independent Hydro

Sustained Peaking Adjustment

Non-Fed CER (CSPE)

Non-Fed CER (Canada)

Restoration

\begin{tabular}{|c|c|c|c|c|c|c|c|c|c|c|c|c|c|c|}
\hline 9383 & 6991 & 5217 & 6424 & 7151 & 7272 & 5829 & 5498 & 6001 & 5650 & 5309 & 6149 & 7409 & 7918 & 6545 \\
\hline 438 & 436 & 371 & 386 & 306 & 237 & 176 & 197 & 275 & 434 & 516 & 712 & 748 & 452 & 398 \\
\hline 0 & 0 & 0 & 0 & 0 & 0 & 0 & 0 & 0 & 0 & 0 & 0 & 0 & 0 & 0 \\
\hline 0 & 0 & 0 & 0 & 0 & 0 & 0 & 0 & 0 & 0 & 0 & 0 & 0 & 0 & $v$ \\
\hline 130 & 130 & 130 & 130 & 130 & 130 & 130 & 130 & 130 & 129 & 129 & 129 & 129 & 129 & 130 \\
\hline & 0 & 0 & 0 & 0 & 0 & 0 & 0 & 0 & 0 & 0 & 0 & 0 & 0 & 0 \\
\hline
\end{tabular}

Total Hydro Resources

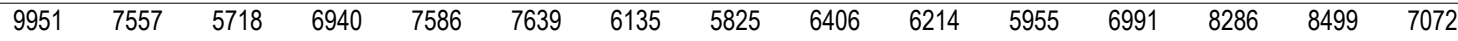

Other Resources

Small Thermal \& Misc.

Combustion Turbines

Renewables

Cogeneration

Imports

Intra-Regional Transfers (In)

Supp \& Entitlement Capacity (In)

Large Thermal

Non-Utility Generation

Resource Acquisition

$\begin{array}{rrrr}0 & 0 & 0 & 0 \\ 0 & 0 & 0 & 0 \\ 27 & 27 & 27 & 28 \\ 0 & 0 & 0 & 0 \\ 111 & 111 & 144 & 182 \\ 184 & 184 & 329 & 318 \\ 0 & 0 & 0 & 0 \\ 1000 & 1000 & 1000 & 1000 \\ 109 & 109 & 102 & 116 \\ 0 & 0 & 0 & 0\end{array}$

0
0
29
0
249
364
0
1000
127
0

$\begin{array}{rrrr}0 & 0 & 0 & \\ 0 & 0 & 0 & 0 \\ 31 & 32 & 31 & 3 \\ 0 & 0 & 0 & 0 \\ 304 & 277 & 234 & 203 \\ 300 & 324 & 312 & 290 \\ 0 & 0 & 0 & 0 \\ 1000 & 1000 & 1000 & 1000 \\ 127 & 130 & 125 & 119 \\ 0 & 0 & 0 & 0\end{array}$

$\begin{array}{rr}0 & \\ 0 & \\ 31 & 30 \\ 0 & 0 \\ 203 & 204 \\ 290 & 285 \\ 0 & 0 \\ 1000 & 1000 \\ 119 & 121 \\ 0 & \end{array}$

$\begin{array}{rrr}0 & 0 & 0 \\ 0 & 0 & 0 \\ 30 & 30 & 27 \\ 0 & 0 & 0 \\ 204 & 173 & 83 \\ 285 & 285 & 167 \\ 0 & 0 & \\ 1000 & 1000 & 35 \\ 121 & 121 & 118 \\ 0 & 0 & \end{array}$

$\begin{array}{rrrr}0 & 0 & 0 & 0 \\ 0 & 0 & 0 & 0 \\ 27 & 27 & 27 & 29 \\ 0 & 0 & 0 & 0 \\ 83 & 101 & 132 & 184 \\ 167 & 286 & 159 & 276 \\ 0 & 0 & 0 & 0 \\ 355 & 167 & 1000 & 877 \\ 118 & 120 & 109 & 119 \\ 0 & 0 & 0 & 0\end{array}$

Total Other Resources

Total Resources

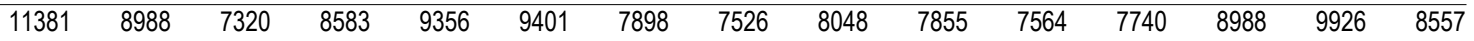

Reserves \& Maintenance

Hydro Reserves

Small Thermal \& Misc. Reserves

Contract Reserves

Large Thermal Reserves

Federal Hydro Maintenance

Spinning Reserves

Federal Transmission Losses

$\begin{array}{lllllll}0 & 0 & 0 & 0 & 0 & 0 & 0 \\ 0 & 0 & 0 & 0 & 0 & 0 & 0 \\ 0 & 0 & 0 & 0 & 0 & 0 & 0 \\ 0 & 0 & 0 & 0 & 0 & 0 & 0 \\ 0 & 0 & 0 & 0 & 0 & 0 & 0 \\ 0 & 0 & 0 & 0 & 0 & 0 & 0\end{array}$

Total Reserves, Maintenance \& Losses

Total Net Resources

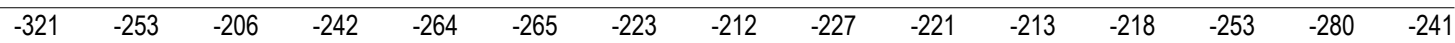

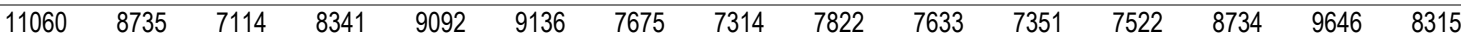

Total Firm Surplus/Deficit

$\begin{array}{llll}310 & -398 & -1693 & -1717\end{array}$

$-652$

$\begin{array}{lll}-449 & -724 & -161\end{array}$

$879 \quad 1336 \quad-58$ 
Loads and Resources - Federal System

PNW Loads and Resources Study

2009 - 2010 Operating Year

1937 Water Year

2003 White Book

Non-Utility Obligations

Federal Agencies 1981 PSC Sale

USBR 1981 PSC Sale

DSI 1981 PSC Sale

Federal Agencies 2002 PSC Sale

USBR 2002 PSC Sale

DSI 2002 PSC Sale
Oct Nov Dec Jan Fe
Mar Apr1 Apr16
May Jun

Jul Avg

Total Firm Non-Utility Obligations

$\begin{array}{rrrrrrrrrrrrrrr}0 & 0 & 0 & 0 & 0 & 0 & 0 & 0 & 0 & 0 & 0 & 0 & 0 & 0 & 0 \\ 0 & 0 & 0 & 0 & 0 & 0 & 0 & 0 & 0 & 0 & 0 & 0 & 0 & 0 & 0 \\ 0 & 0 & 0 & 0 & 0 & 0 & 0 & 0 & 0 & 0 & 0 & 0 & 0 & 0 & 0 \\ 123 & 123 & 111 & 108 & 124 & 139 & 140 & 134 & 125 & 113 & 112 & 110 & 111 & 118 & 121 \\ 309 & 275 & 166 & 129 & 0 & 0 & 0 & 1.0 & 23 & 252 & 315 & 268 & 298 & 332 & 149 \\ 0 & 0 & 0 & 0 & 0 & 0 & 0 & 0 & 0 & 0 & 0 & 0 & 0 & 0 & 0 \\ & & & & & & & & & & & & & & \\ 432 & 398 & 277 & 237 & 124 & 139 & 140 & 135 & 148 & 365 & 427 & 378 & 409 & 450 & 271\end{array}$

Transfers Out

NGP 1981 PSC Sale

GPU 1981 PSC Sale

IOU 1981 PSC Sale

NGP 2002 PSC Sale

GPU 2002 PSC Sale

NGP Slice Sale

GPU Slice Sale

IOU 2002 PSC Sale

Exports

Intra-Regional Transfers (Out)

Supp \& Entitlement Capacity (Out)

CSPE To West Group Utilities

Federal Diversity

\begin{tabular}{|c|c|c|c|c|c|c|c|c|c|c|c|c|c|c|}
\hline 0 & 0 & 0 & 0 & 0 & 0 & 0 & 0 & 0 & 0 & 0 & 0 & 0 & 0 & 0 \\
\hline 0 & 0 & 0 & 0 & 0 & 0 & 0 & 0 & 0 & 0 & 0 & 0 & 0 & 0 & 0 \\
\hline 0 & 0 & 0 & 0 & 0 & 0 & 0 & 0 & 0 & 0 & 0 & 0 & 0 & 0 & 0 \\
\hline 2985 & 2985 & 2706 & 2664 & 3002 & 3381 & 3540 & 3388 & 3071 & 2914 & 2905 & 2866 & 2950 & 3069 & 3044 \\
\hline 2057 & 2057 & 2197 & 2303 & 2687 & 3007 & 3026 & 2966 & 2745 & 2378 & 2371 & 2182 & 1903 & 1996 & 2454 \\
\hline 860 & 662 & 513 & 622 & 680 & 685 & 562 & 535 & 582 & 554 & 533 & 616 & 722 & 738 & 630 \\
\hline 1432 & 1103 & 855 & 1035 & 1133 & 1142 & 935 & 892 & 969 & 923 & 887 & 1025 & 1203 & 1229 & 1049 \\
\hline 0 & 0 & 0 & 0 & 0 & 0 & 0 & 0 & 0 & 0 & 0 & 0 & 0 & 0 & \\
\hline 714 & 714 & 706 & 677 & 687 & 705 & 703 & 698 & 689 & 711 & 711 & 724 & 722 & 718 & 705 \\
\hline 197 & 197 & 236 & 291 & 592 & 605 & 602 & 547 & 394 & 361 & 362 & 167 & 227 & 226 & 370 \\
\hline 0 & 0 & 0 & 0 & 0 & 0 & 0 & 0 & 0 & 0 & 0 & 0 & 0 & 0 & \\
\hline 0 & 0 & 0 & 0 & 0 & 0 & 0 & 0 & 0 & 0 & 0 & 0 & 0 & 0 & \\
\hline 0 & 0 & 0 & 0 & 0 & 0 & 0 & 0 & 0 & 0 & 0 & 0 & 0 & 0 & \\
\hline
\end{tabular}

Total Transfers Ou

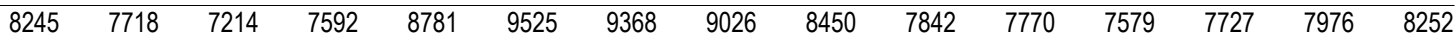

Total Firm Obligations

$8116 \quad 7491 \quad 7829$

$8906 \quad 9664$

$9508 \quad 9161$

8598

$8206 \quad 8197$

$8197 \quad 7958$

8136

$8426 \quad 8523$

Hydro Resources

Regulated Hydro

Independent Hydro

Sustained Peaking Adjustment

Non-Fed CER (CSPE)

Non-Fed CER (Canada)

Restoration

$\begin{array}{rrr}9408 & 7011 & 5231 \\ 438 & 436 & 371 \\ 0 & 0 & 0 \\ 0 & 0 & 0 \\ 129 & 129 & 129 \\ 0 & 0 & 0\end{array}$

$\begin{array}{rrr}231 & 6441 & 7171 \\ 371 & 386 & 306 \\ 0 & 0 & 0 \\ 0 & 0 & 0 \\ 129 & 129 & 129 \\ 0 & 0 & 0\end{array}$

$\begin{array}{rrr}7171 & 7293 & 5845 \\ 306 & 237 & 176 \\ 0 & 0 & 0 \\ 0 & 0 & 0 \\ 129 & 129 & 129 \\ 0 & 0 & 0\end{array}$

$9975 \quad 7577 \quad 5731 \quad 6956$

$6956 \quad 7606 \quad 7659$

$\begin{array}{rrrr}0 & 0 & 0 & 0 \\ 0 & 0 & 0 & 0 \\ 27 & 27 & 27 & 28 \\ 0 & 0 & 0 & 0 \\ 111 & 111 & 144 & 182 \\ 184 & 184 & 329 & 318 \\ 0 & 0 & 0 & 0 \\ 1000 & 1000 & 1000 & 1000 \\ 109 & 109 & 102 & 116 \\ 0 & 0 & 0 & 0\end{array}$

$\begin{array}{rr}0 & 0 \\ 0 & 0 \\ 29 & 31 \\ 0 & 0 \\ 249 & 304 \\ 364 & 300 \\ 0 & 0 \\ 1000 & 1000 \\ 127 & 127 \\ 0 & 0\end{array}$

$\begin{array}{rrr}0 & 0 & 0 \\ 0 & 0 & 0 \\ 32 & 31 & 31 \\ 0 & 0 & 0 \\ 277 & 234 & 203 \\ 324 & 312 & 290 \\ 0 & 0 & 0 \\ 1000 & 1000 & 1000 \\ 130 & 125 & 119 \\ 0 & 0 & 0\end{array}$

$\begin{array}{rr}0 & \\ 0 & \\ 30 & 30 \\ 0 & \\ 204 & 173 \\ 285 & 285 \\ 0 & \\ 1000 & 100 \\ 121 & 121 \\ 0 & \end{array}$

$\begin{array}{rr}0 & 0 \\ 0 & 0 \\ 30 & 27 \\ 0 & 0 \\ 173 & 83 \\ 285 & 167 \\ 0 & 0 \\ 1000 & 1000 \\ 121 & 118 \\ 0 & \end{array}$

0
0
27
0
83
167
0
1000
118
0

$\begin{array}{rrr}0 & 0 & 0 \\ 0 & 0 & 0 \\ 27 & 27 & 29 \\ 0 & 0 & 0 \\ 94 & 125 & 183 \\ 286 & 159 & 276 \\ 0 & 0 & 0 \\ 1000 & 1000 & 1000 \\ 120 & 109 & 119 \\ 0 & 0 & 0\end{array}$

Total Other Resources

Total Resources

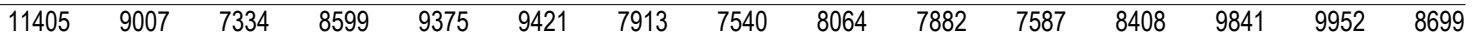

Reserves \& Maintenance

Hydro Reserves

Small Thermal \& Misc. Reserves

Contract Reserves

Large Thermal Reserves

Federal Hydro Maintenance

Spinning Reserves

Federal Transmission Losses

Total Reserves, Maintenance \& Losses

Total Net Resources

$11083 \quad 8753$

$\begin{array}{rrrrrrrrrrrrrrr}0 & 0 & 0 & 0 & 0 & 0 & 0 & 0 & 0 & 0 & 0 & 0 & 0 & 0 & 0 \\ 0 & 0 & 0 & 0 & 0 & 0 & 0 & 0 & 0 & 0 & 0 & 0 & 0 & 0 & 0 \\ 0 & 0 & 0 & 0 & 0 & 0 & 0 & 0 & 0 & 0 & 0 & 0 & 0 & 0 & 0 \\ 0 & 0 & 0 & 0 & 0 & 0 & 0 & 0 & 0 & 0 & 0 & 0 & 0 & 0 & 0 \\ 0 & 0 & 0 & 0 & 0 & 0 & 0 & 0 & 0 & 0 & 0 & 0 & 0 & 0 & 0 \\ 0 & 0 & 0 & 0 & 0 & 0 & 0 & 0 & 0 & 0 & 0 & 0 & 0 & 0 & 0 \\ -322 & -254 & -207 & -242 & -264 & -266 & -223 & -213 & -227 & -222 & -214 & -237 & -278 & -281 & -245 \\ & & & & & & & & & & & & & & -245 \\ -322 & -254 & -207 & -242 & -264 & -266 & -223 & -213 & -227 & -222 & -214 & -237 & -278 & -281 & -245 \\ & & & & & & & & & & & & & & \\ \end{array}$

$637 \quad-364$

528

205

$\begin{array}{lll}-509 & -1817 & -1833\end{array}$

$-762 \quad-547$

$-824$

$213 \quad 1427$

$1246-69$ 
Loads and Resources - Federal System

PNW Loads and Resources Study

2010 - 2011 Operating Year

1937 Water Year

2003 White Book

Non-Utility Obligations

Federal Agencies 1981 PSC Sale

USBR 1981 PSC Sale

DSI 1981 PSC Sale

Federal Agencies 2002 PSC Sale

USBR 2002 PSC Sale

DSI 2002 PSC Sale
Oct Nov Dec Jan Feb
Mar Apr1 Apr16
May Jun

Jul Avg

Total Firm Non-Utility Obligations

\begin{tabular}{|c|c|c|c|c|c|c|c|c|c|c|c|c|c|c|}
\hline 0 & 0 & 0 & 0 & 0 & 0 & 0 & 0 & 0 & 0 & 0 & 0 & 0 & 0 & 0 \\
\hline 0 & 0 & 0 & 0 & 0 & 0 & 0 & 0 & 0 & 0 & 0 & 0 & 0 & 0 & 0 \\
\hline 0 & 0 & 0 & 0 & 0 & 0 & 0 & 0 & 0 & 0 & 0 & 0 & 0 & 0 & 0 \\
\hline 124 & 124 & 112 & 109 & 125 & 140 & 140 & 135 & 126 & 113 & 113 & 111 & 111 & 119 & 122 \\
\hline 309 & 275 & 166 & 129 & 0 & 0 & 0 & 1.0 & 23 & 252 & 315 & 268 & 298 & 332 & 149 \\
\hline 0 & 0 & 0 & 0 & 0 & 0 & 0 & 0 & 0 & 0 & 0 & 0 & 0 & 0 & 0 \\
\hline
\end{tabular}

Transfers Out

NGP 1981 PSC Sale

GPU 1981 PSC Sale

IOU 1981 PSC Sale

NGP 2002 PSC Sale

GPU 2002 PSC Sale

NGP Slice Sale

GPU Slice Sale

IOU 2002 PSC Sale

Exports

Intra-Regional Transfers (Out)

Supp \& Entitlement Capacity (Out)

CSPE To West Group Utilities

Federal Diversity

\begin{tabular}{|c|c|c|c|c|c|c|c|c|c|c|c|c|c|c|}
\hline 0 & 0 & 0 & 0 & 0 & 0 & 0 & 0 & 0 & 0 & 0 & 0 & 0 & 0 & 0 \\
\hline 0 & 0 & 0 & 0 & 0 & 0 & 0 & 0 & 0 & 0 & 0 & 0 & 0 & 0 & 0 \\
\hline 0 & 0 & 0 & 0 & 0 & 0 & 0 & 0 & 0 & 0 & 0 & 0 & 0 & 0 & 0 \\
\hline 3020 & 3020 & 2739 & 2696 & 3038 & 3421 & 3578 & 3424 & 3103 & 2944 & 2936 & 2896 & 2980 & 3101 & 3078 \\
\hline 2065 & 2065 & 2205 & 2313 & 2696 & 3019 & 3039 & 2978 & 2756 & 2387 & 2381 & 2191 & 1911 & 2004 & 2463 \\
\hline 862 & 663 & 514 & 623 & 682 & 687 & 563 & 536 & 583 & 555 & 533 & 558 & 660 & 739 & 621 \\
\hline 1435 & 1105 & 856 & 1037 & 1135 & 1144 & 937 & 893 & 971 & 925 & 888 & 929 & 1100 & 1232 & 1034 \\
\hline 0 & 0 & 0 & 0 & 0 & 0 & 0 & 0 & 0 & 0 & 0 & 0 & 0 & 0 & \\
\hline 700 & 700 & 692 & 663 & 676 & 695 & 692 & 687 & 678 & 700 & 700 & 710 & 716 & 712 & 694 \\
\hline 197 & 197 & 236 & 291 & 592 & 605 & 602 & 547 & 394 & 361 & 362 & 167 & 227 & 226 & 370 \\
\hline 0 & 0 & 0 & 0 & 0 & 0 & 0 & 0 & 0 & 0 & 0 & 0 & 0 & 0 & \\
\hline 0 & 0 & 0 & 0 & 0 & 0 & 0 & 0 & 0 & 0 & 0 & 0 & 0 & 0 & \\
\hline 0 & 0 & 0 & 0 & 0 & 0 & 0 & 0 & 0 & 0 & 0 & 0 & 0 & 0 & \\
\hline
\end{tabular}

Total Transfers Ou

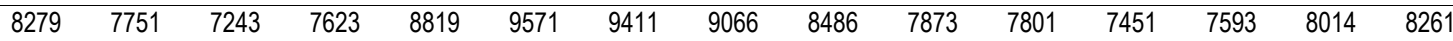

Total Firm Obligations

$8150 \quad 7520 \quad 7860$

$8944 \quad 9711 \quad 955$

$9201 \quad 8635$

8238

$8229 \quad 7829$

$8002 \quad 8464 \quad 8532$

Hydro Resources

Regulated Hydro

Independent Hydro

Sustained Peaking Adjustment

Non-Fed CER (CSPE)

Non-Fed CER (Canada)

Restoration

$\begin{array}{rrrrrr}9429 & 7029 & 5244 & 6456 & 7188 & 731 \\ 438 & 436 & 371 & 386 & 306 & \\ 0 & 0 & 0 & 0 & 0 \\ 0 & 0 & 0 & 0 & 0 \\ 139 & 139 & 139 & 139 & 139 \\ 0 & 0 & 0 & 0 & 0\end{array}$

$\begin{array}{rrr}7311 & 5859 & 552 \\ 237 & 176 & \\ 0 & 0 \\ 0 & 0 \\ 139 & 139 \\ 0 & 0\end{array}$

$\begin{array}{rr}5527 & 6032 \\ 197 & 27 \\ 0 & 0 \\ 0 & 0 \\ 139 & 139 \\ 0 & \end{array}$

$10006 \quad 7604 \quad 5754 \quad 6981$

$\begin{array}{llllll}6981 & 7633 & 7687 & 6174 & 5862 & 6446\end{array}$

$\begin{array}{rrrr}0 & 0 & 0 & 0 \\ 0 & 0 & 0 & 0 \\ 27 & 27 & 27 & 28 \\ 0 & 0 & 0 & 0 \\ 104 & 104 & 137 & 169 \\ 184 & 184 & 329 & 318 \\ 0 & 0 & 0 & 0 \\ 1000 & 1000 & 1000 & 1000 \\ 109 & 109 & 102 & 116 \\ 0 & 0 & 0 & 0\end{array}$

$\begin{array}{rr}0 & 0 \\ 0 & 0 \\ 29 & 31 \\ 0 & 0 \\ 239 & 293 \\ 364 & 300 \\ 0 & 0 \\ 1000 & 1000 \\ 127 & 127 \\ 0 & 0\end{array}$

$\begin{array}{rrr}0 & 0 & 0 \\ 0 & 0 & 0 \\ 32 & 31 & 31 \\ 0 & 0 & 0 \\ 267 & 223 & 193 \\ 324 & 312 & 290 \\ 0 & 0 & 0 \\ 1000 & 1000 & 1000 \\ 130 & 125 & 119 \\ 0 & 0 & 0\end{array}$

$\begin{array}{rr}0 & 0 \\ 0 & 0 \\ 30 & 30 \\ 0 & 0 \\ 194 & 168 \\ 285 & 285 \\ 0 & 0 \\ 1000 & 1000 \\ 121 & 121 \\ 0 & 0\end{array}$

0
0
27
0
76
167
0
355
118
0

$\begin{array}{rrr}0 & 0 & 0 \\ 0 & 0 & 0 \\ 27 & 27 & 29 \\ 0 & 0 & 0 \\ 94 & 125 & 175 \\ 286 & 159 & 276 \\ 0 & 0 & 0 \\ 167 & 1000 & 877 \\ 120 & 109 & 119 \\ 0 & 0 & 0\end{array}$

Total Other Resources

$1423 \quad 1424$

\begin{tabular}{lllllllllll}
1759 & 1751 & 1752 & 1690 & 1632 & 1630 & 1605 & 743 & 695 & 1420 & 1476 \\
\hline 9392 & 9438 & 7927 & 7553 & 8078 & 7885 & 7593 & 7765 & 9021 & 9971 & 8589
\end{tabular}

\section{Reserves \& Maintenance}

Hydro Reserves

Small Thermal \& Misc. Reserves

Contract Reserves

Large Thermal Reserves

Federal Hydro Maintenance

Spinning Reserves

Federal Transmission Losses

$\begin{array}{llll}11430 & 9028 & 7349 & 8612\end{array}$

Total Reserves, Maintenance \& Losses

$\begin{array}{llllll}0 & 0 & 0 & 0 & 0 & 0 \\ 0 & 0 & 0 & 0 & 0 & 0 \\ 0 & 0 & 0 & 0 & 0 & 0 \\ 0 & 0 & 0 & 0 & 0 & 0 \\ 0 & 0 & 0 & 0 & 0 & 0 \\ 0 & 0 & 0 & 0 & 0 & 0\end{array}$

$\begin{array}{ll}0 & 0 \\ 0 & 0 \\ 0 & 0 \\ 0 & 0 \\ 0 & 0 \\ 0 & 0\end{array}$

$\begin{array}{lll}-322 & -255 & -207\end{array}$

$-243-265$

$\begin{array}{lllll}-322 & -255 & -207 & -243 & -265\end{array}$

$-266 \quad-224$

$-213-228$

$\begin{array}{rr}0 & 0 \\ 0 & 0 \\ 0 & 0 \\ 0 & 0 \\ 0 & 0 \\ 0 & 0 \\ -228 & -222\end{array}$

$\begin{array}{rr}0 & 0 \\ 0 & 0 \\ 0 & 0 \\ 0 & 0 \\ 0 & 0 \\ 0 & 0 \\ -222 & -214\end{array}$

0
0
0
0
0
0

$\begin{array}{lll}0 & 0 & 0 \\ 0 & 0 & 0 \\ 0 & 0 & 0 \\ 0 & 0 & 0 \\ 0 & 0 & 0 \\ 0 & 0 & 0\end{array}$

Total Net Resources

$\begin{array}{lllllll}11107 & 8774 & 7142 & 8369 & 9127 & 9172 & 7703\end{array}$

$\begin{array}{lll}-224 & -213 \quad-228\end{array}$

$-222$

$\begin{array}{lllll}-214 & -219 & -254 & -281 & -242\end{array}$

$\begin{array}{lllllllllll}183 & -539 & -1848 & -1862 & -785 & -576 & -850 & -283 & 764 & 1226 & -185\end{array}$


Loads and Resources - Federal System

PNW Loads and Resources Study

2011 - 2012 Operating Year

1937 Water Year

2003 White Book

Non-Utility Obligations

Federal Agencies 1981 PSC Sale

USBR 1981 PSC Sale

DSI 1981 PSC Sale

Federal Agencies 2002 PSC Sale

USBR 2002 PSC Sale

DSI 2002 PSC Sale
Oct Nov Dec Jan Fe
Mar Apr1 Apr16
Jun

Jul Avg

Total Firm Non-Utility Obligations

$\begin{array}{rrrrrrrrrrrrrrr}0 & 0 & 0 & 0 & 0 & 0 & 0 & 0 & 0 & 0 & 0 & 0 & 0 & 0 & 0 \\ 0 & 0 & 0 & 0 & 0 & 0 & 0 & 0 & 0 & 0 & 0 & 0 & 0 & 0 & 0 \\ 0 & 0 & 0 & 0 & 0 & 0 & 0 & 0 & 0 & 0 & 0 & 0 & 0 & 0 & 0 \\ 124 & 124 & 112 & 105 & 124 & 136 & 139 & 130 & 122 & 110 & 110 & 107 & 108 & 111 & 119 \\ 309 & 275 & 166 & 129 & 0 & 0 & 0 & 1.0 & 23 & 252 & 315 & 268 & 298 & 332 & 149 \\ 0 & 0 & 0 & 0 & 0 & 0 & 0 & 0 & 0 & 0 & 0 & 0 & 0 & 0 & 0 \\ & & & & & & & & & & & & & & \\ 433 & 399 & 278 & 234 & 124 & 136 & 139 & 131 & 145 & 362 & 425 & 375 & 406 & 443 & 268\end{array}$

Transfers Out

NGP 1981 PSC Sale

GPU 1981 PSC Sale

IOU 1981 PSC Sale

NGP 2002 PSC Sale

GPU 2002 PSC Sale

NGP Slice Sale

GPU Slice Sale

IOU 2002 PSC Sale

Exports

Intra-Regional Transfers (Out)

Supp \& Entitlement Capacity (Out)

CSPE To West Group Utilities

Federal Diversity

\begin{tabular}{|c|c|c|c|c|c|c|c|c|c|c|c|c|c|c|}
\hline 0 & 0 & 0 & 0 & 0 & 0 & 0 & 0 & 0 & 0 & 0 & 0 & 0 & 0 & 0 \\
\hline 0 & 0 & 0 & 0 & 0 & 0 & 0 & 0 & 0 & 0 & 0 & 0 & 0 & 0 & 0 \\
\hline 0 & 0 & 0 & 0 & 0 & 0 & 0 & 0 & 0 & 0 & 0 & 0 & 0 & 0 & 0 \\
\hline 3047 & 3047 & 2763 & 2745 & 3099 & 3498 & 3567 & 3402 & 3106 & 2942 & 2934 & 2913 & 3023 & 3141 & 3103 \\
\hline 2072 & 2072 & 2213 & 2352 & 2754 & 3086 & 3087 & 2940 & 2800 & 2426 & 2419 & 2229 & 1948 & 2030 & 2494 \\
\hline 863 & 664 & 515 & 624 & 683 & 688 & 563 & 537 & 584 & 556 & 534 & 617 & 724 & 741 & 632 \\
\hline 1437 & 1107 & 858 & 1039 & 1137 & 1146 & 938 & 895 & 973 & 927 & 889 & 1028 & 1206 & 1234 & 1053 \\
\hline 0 & 0 & 0 & 0 & 0 & 0 & 0 & 0 & 0 & 0 & 0 & 0 & 0 & 0 & \\
\hline 692 & 692 & 684 & 656 & 669 & 689 & 686 & 681 & 672 & 693 & 693 & 703 & 708 & 704 & 686 \\
\hline 197 & 197 & 42 & 108 & 363 & 418 & 391 & 347 & 217 & 188 & 190 & 0.1 & 53 & 67 & 19 \\
\hline 0 & 0 & 0 & 0 & 0 & 0 & 0 & 0 & 0 & 0 & 0 & 0 & 0 & 0 & \\
\hline 0 & 0 & 0 & 0 & 0 & 0 & 0 & 0 & 0 & 0 & 0 & 0 & 0 & 0 & \\
\hline 0 & 0 & 0 & 0 & 0 & 0 & 0 & 0 & 0 & 0 & 0 & 0 & 0 & 0 & \\
\hline
\end{tabular}

Total Transfers Ou

$7074-7523-8705$

$9524 \quad 9233$

$8741 \quad 81$

$7353 \quad 7757$

$8829 \quad 9660$

$9372 \quad 893$

$8803 \quad 8351$

$8351 \quad 7733 \quad 7659$

$7659 \quad 7490$

$\begin{array}{llll}7490 & 7663 & 7916 & 8168\end{array}$

\begin{tabular}{|c|c|c|c|c|c|c|c|c|c|c|c|c|c|c|}
\hline 9445 & 7042 & 5253 & 6467 & 7201 & 7324 & 5870 & 5536 & 6043 & 5695 & 5343 & 6181 & 7451 & 7978 & 6589 \\
\hline 438 & 436 & 371 & 386 & 306 & 237 & 176 & 197 & 275 & 434 & 516 & 712 & 748 & 452 & 398 \\
\hline 0 & 0 & 0 & 0 & 0 & 0 & 0 & 0 & 0 & 0 & 0 & 0 & 0 & 0 & \\
\hline 0 & 0 & 0 & 0 & 0 & 0 & 0 & 0 & 0 & 0 & 0 & 0 & 0 & 0 & \\
\hline 138 & 138 & 138 & 138 & 138 & 138 & 138 & 138 & 138 & 135 & 135 & 135 & 135 & 135 & 137 \\
\hline 0 & 0 & 0 & 0 & 0 & 0 & 0 & 0 & 0 & 0 & 0 & 0 & 0 & 0 & \\
\hline
\end{tabular}

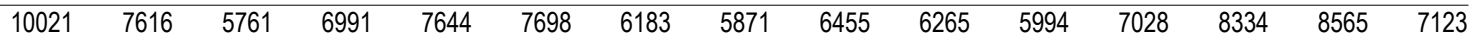

\section{Other Resources}

Small Thermal \& Misc.

Combustion Turbines

Renewables

Cogeneration

Imports

Intra-Regional Transfers (In)

Supp \& Entitlement Capacity (In)

Large Thermal

Non-Utility Generation

Resource Acquisition

$\begin{array}{rrrr}0 & 0 & 0 & 0 \\ 0 & 0 & 0 & 0 \\ 27 & 27 & 27 & 28 \\ 0 & 0 & 0 & 0 \\ 104 & 104 & 137 & 169 \\ 184 & 184 & 135 & 135 \\ 0 & 0 & 0 & 0 \\ 1000 & 1000 & 1000 & 1000 \\ 109 & 109 & 102 & 116 \\ 0 & 0 & 0 & 0\end{array}$

$\begin{array}{rr}0 & 0 \\ 0 & 0 \\ 29 & 31 \\ 0 & 0 \\ 239 & 293 \\ 135 & 112 \\ 0 & 0 \\ 1000 & 1000 \\ 127 & 127 \\ 0 & 0\end{array}$

$\begin{array}{rrr}0 & 0 & 0 \\ 0 & 0 & 0 \\ 32 & 31 & 31 \\ 0 & 0 & 0 \\ 267 & 223 & 193 \\ 112 & 112 & 112 \\ 0 & 0 & 0 \\ 1000 & 1000 & 1000 \\ 130 & 125 & 119 \\ 0 & 0 & 0\end{array}$

$\begin{array}{rr}0 & 0 \\ 0 & 0 \\ 30 & 30 \\ 0 & 0 \\ 194 & 168 \\ 112 & 112 \\ 0 & 0 \\ 1000 & 1000 \\ 121 & 121 \\ 0 & 0\end{array}$

0
0
27
0
76
0
0
1000
118
0

$\begin{array}{rrr}0 & 0 & 0 \\ 0 & 0 & 0 \\ 27 & 27 & 29 \\ 0 & 0 & 0 \\ 94 & 125 & 175 \\ 112 & 0 & 105 \\ 0 & 0 & 0 \\ 1000 & 1000 & 1000 \\ 120 & 109 & 119 \\ 0 & 0 & 0\end{array}$

Total Other Resources

$1423 \quad 1424$

\begin{tabular}{llllllllllllll}
1424 & 1402 & 1448 & 1530 & 1564 & 1541 & 1491 & 1455 & 1457 & 1432 & 1221 & 1354 & 1261 & 1428 \\
\hline 040 & 7163 & 8438 & 9174 & 9262 & 7724 & 7362 & 7910 & 7722 & 7426 & 8249 & 9688 & 9827 & 8551
\end{tabular}

Reserves \& Maintenance

Hydro Reserves

Small Thermal \& Misc. Reserves

Contract Reserves

Large Thermal Reserves

Federal Hydro Maintenance

Spinning Reserves

Federal Transmission Losses

114449040

\section{0}

$\begin{array}{lllllllllllllll}0 & 0 & 0 & 0 & 0 & 0 & 0 & 0 & 0 & 0 & 0 & 0 & 0 & 0 & 0 \\ 0 & 0 & 0 & 0 & 0 & 0 & 0 & 0 & 0 & 0 & 0 & 0 & 0 & 0 & 0 \\ 0 & 0 & 0 & 0 & 0 & 0 & 0 & 0 & 0 & 0 & 0 & 0 & 0 & 0 & 0 \\ 0 & 0 & 0 & 0 & 0 & 0 & 0 & 0 & 0 & 0 & 0 & 0 & 0 & 0 & 0 \\ 0 & 0 & 0 & 0 & 0 & 0 & 0 & 0 & 0 & 0 & 0 & 0 & 0 & 0 & 0 \\ 0 & 0 & 0 & 0 & 0 & 0 & 0 & 0 & 0 & 0 & 0 & 0 & 0 & 0 & 0\end{array}$

Total Reserves, Maintenance \& Losses

$\begin{array}{lllllllllllllll}-323 & -255 & -202 & -238 & -259 & -261 & -218 & -208 & -223 & -218 & -209 & -233 & -273 & -277 & -241\end{array}$

Total Net Resources

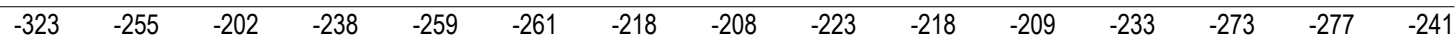

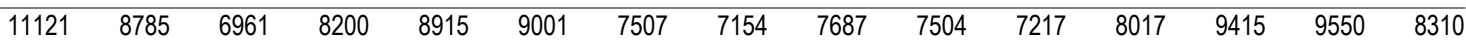


Loads and Resources - Federal System

PNW Loads and Resources Study

2012 - 2013 Operating Year

1937 Water Year

2003 White Book

Oc

Nov Dec Jan Feb

\begin{tabular}{|c|c|c|c|c|c|c|c|c|c|c|c|c|c|}
\hline \multirow{3}{*}{$\begin{array}{l}0 \\
0\end{array}$} & 0 & 0 & 0 & 0 & 0 & 0 & 0 & 0 & 0 & 0 & 0 & 0 & 0 \\
\hline & 0 & 0 & 0 & 0 & 0 & 0 & 0 & 0 & 0 & 0 & 0 & 0 & 0 \\
\hline & 0 & 0 & 0 & 0 & 0 & 0 & 0 & 0 & 0 & 0 & 0 & 0 & 0 \\
\hline 114 & 114 & 105 & 106 & 125 & 137 & 139 & 133 & 122 & 110 & 110 & 107 & 108 & 111 \\
\hline 309 & 275 & 166 & 129 & 0 & 0 & 0 & 1.0 & 23 & 252 & 315 & 268 & 298 & 332 \\
\hline 0 & 0 & 0 & 0 & 0 & 0 & 0 & 0 & 0 & 0 & 0 & 0 & 0 & 0 \\
\hline
\end{tabular}

Non-Utility Obligation

Federal Agencies 1981 PSC Sale

USBR 1981 PSC Sale

DSI 1981 PSC Sale

Federal Agencies 2002 PSC Sale

USBR 2002 PSC Sale

DSI 2002 PSC Sale

Total Firm Non-Utility Obligations

$\begin{array}{rrr}0 & 0 \\ 0 & 0 \\ 0 & 0 & \\ 3092 & 3092 & 27 \\ 2092 & 2092 & 2232 \\ 864 & 666 & \\ 1440 & 1109 \\ 0 & 0 \\ 684 & 684 \\ 13 & 13 \\ 0 & 0 \\ 0 & 0 \\ 0 & 0\end{array}$

$\begin{array}{rr}0 & \\ 0 & \\ 0 & \\ 2790 & 2761 \\ 2232 & 2356 \\ 516 & 625 \\ 859 & 1041 \\ 0 & \\ 676 & 647 \\ 42 & 108 \\ 0 & \\ 0 & 0 \\ 0 & \end{array}$

$\begin{array}{rrr}0 & 0 & \\ 0 & 0 & 0 \\ 0 & 0 & \\ 2761 & 3118 & 3518 \\ 2356 & 2760 & 3092 \\ 625 & 684 & 689 \\ 1041 & 1139 & 1 \\ 0 & 0 & \\ 647 & 662 & 0 \\ 108 & 363 & 418 \\ 0 & 0 & \\ 0 & 0 & \\ 0 & 0 & \end{array}$

$\begin{array}{rrr}0 & 0 & \\ 0 & 0 & \\ 0 & 0 & \\ 3518 & 3603 & 3492 \\ 3092 & 3094 & 3048 \\ 689 & 565 & 538 \\ 1148 & 940 & 896 \\ 0 & 0 & \\ 682 & 679 & 674 \\ 418 & 391 & 347 \\ 0 & 0 & \\ 0 & 0 & \\ 0 & 0 & \end{array}$

\begin{tabular}{|c|c|}
\hline 0 & 0 \\
\hline 0 & 0 \\
\hline 0 & 0 \\
\hline 3492 & 3138 \\
\hline 3048 & 2806 \\
\hline 538 & 585 \\
\hline 896 & 975 \\
\hline 0 & 0 \\
\hline 674 & 664 \\
\hline 347 & 217 \\
\hline 0 & 0 \\
\hline 0 & 0 \\
\hline 0 & 0 \\
\hline
\end{tabular}

$\begin{array}{rr}0 & \\ 0 & \\ 0 & \\ 2972 & 2964 \\ 2432 & 2425 \\ 558 & 535 \\ 929 & 891 \\ 0 & \\ 685 & 685 \\ 188 & 190 \\ 0 & \\ 0 & \\ 0 & \end{array}$

$\begin{array}{rr}0 & \\ 0 & \\ 0 & \\ 2964 & 29 \\ 2425 & 2235 \\ 535 & 559 \\ 891 & 931 \\ 0 & \\ 685 & 694 \\ 190 & 0.1 \\ 0 & \\ 0 & \\ 0 & \end{array}$

$\begin{array}{rrrr}0 & 0 & 0 & 0 \\ 0 & 0 & 0 & 0 \\ 0 & 0 & 0 & 0 \\ 2942 & 3052 & 3172 & 3137 \\ 2235 & 1954 & 2035 & 2511 \\ 559 & 662 & 742 & 623 \\ 931 & 1103 & 1236 & 1038 \\ 0 & 0 & 0 & 0 \\ 694 & 700 & 696 & 679 \\ 0.1 & 53 & 67 & 184 \\ 0 & 0 & 0 & 0 \\ 0 & 0 & 0 & 0 \\ 0 & 0 & 0 & 0\end{array}$

Total Transfers Ou

$8184 \quad 7655$

$\begin{array}{lll}7115 & 7538 \quad 8725\end{array}$

$9547 \quad 927$

$8996 \quad 8384$

$8384 \quad 7764 \quad 7690$

$\begin{array}{lllll}7690 & 7362 & 7524 & 7949 & 8172\end{array}$

$8607 \quad 8044 \quad 7386$

$7774 \quad 8850 \quad 9$

9411913

$9130 \quad 8529$

8126

$8115 \quad 7737$

7930

$8393 \quad 8439$

Hydro Resources

Regulated Hydro

Independent Hydro

Sustained Peaking Adjustment

Non-Fed CER (CSPE)

Non-Fed CER (Canada)

Restoration

$\begin{array}{rrrrrr}9465 & 7058 & 5265 & 6481 & 7216 & 7340 \\ 438 & 436 & 371 & 386 & 306 & \\ 0 & 0 & 0 & 0 & 0 \\ 0 & 0 & 0 & 0 & 0 \\ 135 & 135 & 135 & 135 & 135 \\ 0 & 0 & 0 & 0 & 0\end{array}$

$\begin{array}{rrr}7340 & 5883 & 5540 \\ 237 & 176 \\ 0 & 0 \\ 0 & 0 \\ 135 & 135 \\ 0 & 0\end{array}$

$\begin{array}{rrrr}5549 & 6056 & 5710 & 5354 \\ 197 & 275 & 435 & 516 \\ 0 & 0 & 0 \\ 0 & 0 & 0 \\ 135 & 135 & 134 \\ 0 & 0 & 0\end{array}$

$\begin{array}{rr}5354 & 619 \\ 516 & 7 \\ 0 & \\ 0 & \\ 134 & \\ 0 & \end{array}$

$\begin{array}{rrrr}6191 & 7465 & 7997 & 6603 \\ 713 & 748 & 452 & 398 \\ 0 & 0 & 0 & 0 \\ 0 & 0 & 0 & 0 \\ 134 & 134 & 134 & 135 \\ 0 & 0 & 0 & 0\end{array}$

Total Hydro Resources

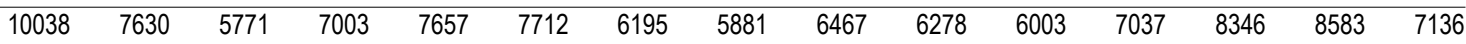

Other Resources

Small Thermal \& Misc.

Combustion Turbines

Renewables

Cogeneration

Imports

Intra-Regional Transfers (In)

Supp \& Entitlement Capacity (In)

Large Thermal

Non-Utility Generation

Resource Acquisition

$\begin{array}{rrrr}0 & 0 & 0 & 0 \\ 0 & 0 & 0 & 0 \\ 27 & 27 & 27 & 28 \\ 0 & 0 & 0 & 0 \\ 104 & 104 & 137 & 169 \\ 0 & 0 & 135 & 135 \\ 0 & 0 & 0 & 0 \\ 1000 & 1000 & 1000 & 1000 \\ 109 & 109 & 102 & 116 \\ 0 & 0 & 0 & 0\end{array}$

$\begin{array}{rr}0 & 0 \\ 0 & 0 \\ 29 & 31 \\ 0 & 0 \\ 239 & 293 \\ 135 & 112 \\ 0 & 0 \\ 1000 & 1000 \\ 127 & 127 \\ 0 & 0\end{array}$

$\begin{array}{rrr}0 & 0 & 0 \\ 0 & 0 & 0 \\ 32 & 31 & 31 \\ 0 & 0 & 0 \\ 267 & 223 & 193 \\ 112 & 112 & 112 \\ 0 & 0 & 0 \\ 1000 & 1000 & 1000 \\ 130 & 125 & 119 \\ 0 & 0 & 0\end{array}$

$\begin{array}{rr}0 & 0 \\ 0 & 0 \\ 30 & 30 \\ 0 & 0 \\ 194 & 168 \\ 112 & 112 \\ 0 & 0 \\ 1000 & 1000 \\ 121 & 121 \\ 0 & 0\end{array}$

0
0
27
0
76
0
0
355
118
0

$\begin{array}{rrr}0 & 0 & 0 \\ 0 & 0 & 0 \\ 27 & 27 & 29 \\ 0 & 0 & 0 \\ 94 & 125 & 175 \\ 112 & 0 & 90 \\ 0 & 0 & 0 \\ 167 & 1000 & 877 \\ 120 & 109 & 119 \\ 0 & 0 & 0\end{array}$

Total Other Resources

$1240 \quad 1240$

\begin{tabular}{llllllllllllll}
\hline 240 & 1402 & 1448 & 1530 & 1564 & 1541 & 1491 & 1455 & 1457 & 1432 & 576 & 521 & 1261 & 1289 \\
& & & & & & & & & & & & & \\
\hline 869 & 7173 & 8450 & 9188 & 9276 & 7736 & 7372 & 7922 & 7735 & 7435 & 7613 & 8867 & 9844 & 8425
\end{tabular}

Total Resources

$11278 \quad 88$

Reserves \& Maintenance

Hydro Reserves

Small Thermal \& Misc. Reserves

Contract Reserves

Large Thermal Reserves

Federal Hydro Maintenance

Spinning Reserves

Federal Transmission Losses

$\begin{array}{rrrrr}0 & 0 & 0 & 0 & 0 \\ 0 & 0 & 0 & 0 & 0 \\ 0 & 0 & 0 & 0 & 0 \\ 0 & 0 & 0 & 0 & 0 \\ 0 & 0 & 0 & 0 & 0 \\ 0 & 0 & 0 & 0 & 0 \\ -318 & -250 & -202 & -238 & -259\end{array}$

$\begin{array}{rr}0 & 0 \\ 0 & 0 \\ 0 & 0 \\ 0 & 0 \\ 0 & 0 \\ 0 & 0 \\ -262 & -218\end{array}$

$\begin{array}{rr}0 & 0 \\ 0 & 0 \\ 0 & 0 \\ 0 & 0 \\ 0 & 0 \\ 0 & 0 \\ -208 & -223\end{array}$

$\begin{array}{rr}0 & 0 \\ 0 & 0 \\ 0 & 0 \\ 0 & 0 \\ 0 & 0 \\ 0 & 0\end{array}$

$\begin{array}{lllll}-318 & -250 & -202 & -238 & -259\end{array}$

$-262 \quad-218$

$-208 \quad-223$

$\begin{array}{lllllllll}10960 & 8619 & 6971 & 8212 & 8929 & 9014 & 7517 & 7164 & 7698\end{array}$

Total Net Resources

$2352 \quad 575$

$-416 \quad 438$

$\begin{array}{llll}78 & -670 & -1894 & -1966\end{array}$

$\begin{array}{lll}-831 & -609 & -889\end{array}$

$-339$

$\begin{array}{lll}687 & 1174 & -252\end{array}$ 
Loads and Resources - Federal System

PNW Loads and Resources Study

2013 - 2014 Operating Year

1937 Water Year

2003 White Book

Non-Utility Obligations

Federal Agencies 1981 PSC Sale

USBR 1981 PSC Sale

DSI 1981 PSC Sale

Federal Agencies 2002 PSC Sale

USBR 2002 PSC Sale

DSI 2002 PSC Sale
Oct Nov Dec Jan Feb
Mar Apr1 Apr16
Jun

Jul Avg

Total Firm Non-Utility Obligations

$\begin{array}{rrrrrrrrrrrrrrr}0 & 0 & 0 & 0 & 0 & 0 & 0 & 0 & 0 & 0 & 0 & 0 & 0 & 0 & 0 \\ 0 & 0 & 0 & 0 & 0 & 0 & 0 & 0 & 0 & 0 & 0 & 0 & 0 & 0 & 0 \\ 0 & 0 & 0 & 0 & 0 & 0 & 0 & 0 & 0 & 0 & 0 & 0 & 0 & 0 & 0 \\ 114 & 114 & 105 & 107 & 126 & 137 & 139 & 134 & 122 & 110 & 110 & 107 & 108 & 112 & 118 \\ 309 & 275 & 166 & 129 & 0 & 0 & 0 & 1.0 & 23 & 252 & 315 & 268 & 298 & 332 & 149 \\ 0 & 0 & 0 & 0 & 0 & 0 & 0 & 0 & 0 & 0 & 0 & 0 & 0 & 0 & 0 \\ & & & & & & & & & & & & & & \\ 423 & 389 & 271 & 236 & 126 & 137 & 139 & 135 & 145 & 362 & 425 & 375 & 406 & 444 & 268\end{array}$

Transfers Out

NGP 1981 PSC Sale

GPU 1981 PSC Sale

IOU 1981 PSC Sale

NGP 2002 PSC Sale

GPU 2002 PSC Sale

NGP Slice Sale

GPU Slice Sale

IOU 2002 PSC Sale

Exports

Intra-Regional Transfers (Out)

Supp \& Entitlement Capacity (Out)

CSPE To West Group Utilities

Federal Diversity

\begin{tabular}{|c|c|c|c|c|c|c|c|c|c|c|c|c|c|c|}
\hline 0 & 0 & 0 & 0 & 0 & 0 & 0 & 0 & 0 & 0 & 0 & 0 & 0 & 0 & 0 \\
\hline 0 & 0 & 0 & 0 & 0 & 0 & 0 & 0 & 0 & 0 & 0 & 0 & 0 & 0 & 0 \\
\hline 0 & 0 & 0 & 0 & 0 & 0 & 0 & 0 & 0 & 0 & 0 & 0 & 0 & 0 & 0 \\
\hline 3122 & 3122 & 2819 & 2788 & 3150 & 3555 & 3640 & 3527 & 3171 & 3002 & 2994 & 2971 & 3082 & 3202 & 3169 \\
\hline 2098 & 2098 & 2238 & 2363 & 2767 & 3099 & 3102 & 3056 & 2813 & 2439 & 2432 & 2241 & 1960 & 2041 & 2518 \\
\hline 866 & 667 & 517 & 626 & 685 & 690 & 565 & 539 & 586 & 559 & 536 & 618 & 726 & 744 & 634 \\
\hline 1442 & 1110 & 860 & 1042 & 1141 & 1150 & 942 & 898 & 976 & 930 & 892 & 1030 & 1209 & 1238 & 1056 \\
\hline 0 & 0 & 0 & 0 & 0 & 0 & 0 & 0 & 0 & 0 & 0 & 0 & 0 & 0 & \\
\hline 677 & 677 & 668 & 640 & 655 & 676 & 673 & 668 & 658 & 678 & 678 & 687 & 693 & 690 & 672 \\
\hline 13 & 13 & 42 & 108 & 363 & 418 & 391 & 347 & 217 & 188 & 190 & 0.1 & 18 & 34 & 178 \\
\hline 0 & 0 & 0 & 0 & 0 & 0 & 0 & 0 & 0 & 0 & 0 & 0 & 0 & 0 & \\
\hline 0 & 0 & 0 & 0 & 0 & 0 & 0 & 0 & 0 & 0 & 0 & 0 & 0 & 0 & \\
\hline 0 & 0 & 0 & 0 & 0 & 0 & 0 & 0 & 0 & 0 & 0 & 0 & 0 & 0 & \\
\hline
\end{tabular}

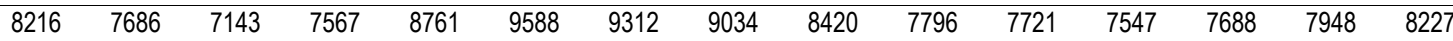

Total Firm Obligations

$8640 \quad 8075$

$\begin{array}{lll}7414 & 7803\end{array}$

8887

$9725 \quad 945$

$9169 \quad 85$

$8565 \quad 815$

8158

8146

$7922 \quad 8094$

$8392 \quad 8494$

Hydro Resources

Regulated Hydro

Independent Hydro

Sustained Peaking Adjustment

Non-Fed CER (CSPE)

Non-Fed CER (Canada)

Restoration

$\begin{array}{rrrrrr}9481 & 7071 & 5275 & 6492 & 7229 & 733 \\ 438 & 436 & 371 & 386 & 306 & \\ 0 & 0 & 0 & 0 & 0 \\ 0 & 0 & 0 & 0 & 0 & \\ 134 & 134 & 134 & 134 & 134 & \\ 0 & 0 & 0 & 0 & 0 & \end{array}$

$\begin{array}{rr}7353 & 5894 \\ 237 & 176 \\ 0 & 0 \\ 0 & 0 \\ 134 & 134 \\ 0 & 0\end{array}$

$\begin{array}{rr}5559 & 6067 \\ 197 & 275 \\ 0 & \\ 0 & \\ 134 & 134 \\ 0 & \end{array}$

$\begin{array}{rrr}6067 & 5722 & 5362 \\ 275 & 435 & 516 \\ 0 & 0 & 0 \\ 0 & 0 & 0 \\ 134 & 132 & \\ 0 & 0 & 0\end{array}$

$\begin{array}{rrrrr}5362 & 6199 & 7476 & 8013 & 6614 \\ 516 & 713 & 748 & 452 & 398 \\ 0 & 0 & 0 & 0 & 0 \\ 0 & 0 & 0 & 0 & 0 \\ 132 & 132 & 132 & 132 & 133 \\ 0 & 0 & 0 & 0 & 0\end{array}$

Total Hydro Resources

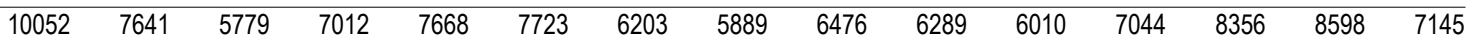

Other Resources

Small Thermal \& Misc.

Combustion Turbines

Renewables

Cogeneration

Imports

Intra-Regional Transfers (In)

Supp \& Entitlement Capacity (In)

Large Thermal

Non-Utility Generation

Resource Acquisition

$\begin{array}{rrrr}0 & 0 & 0 & 0 \\ 0 & 0 & 0 & 0 \\ 27 & 27 & 27 & 28 \\ 0 & 0 & 0 & 0 \\ 104 & 104 & 137 & 169 \\ 0 & 0 & 135 & 135 \\ 0 & 0 & 0 & 0 \\ 1000 & 1000 & 1000 & 1000 \\ 109 & 109 & 102 & 116 \\ 0 & 0 & 0 & 0\end{array}$

0
0
29
0
239
135
0
1000
127
0

$\begin{array}{rr}0 & \\ 0 & \\ 31 & \\ 0 & \\ 293 & 267 \\ 112 & 112 \\ 0 & \\ 1000 & 1000 \\ 127 & 130 \\ 0 & \end{array}$

$\begin{array}{rrr}0 & 0 & 0 \\ 0 & 0 & 0 \\ 32 & 31 & 31 \\ 0 & 0 & 0 \\ 267 & 223 & 193 \\ 112 & 112 & 112 \\ 0 & 0 & 0 \\ 1000 & 1000 & 1000 \\ 130 & 125 & 119 \\ 0 & 0 & 0\end{array}$

$\begin{array}{rr}0 & \\ 0 & \\ 30 & \\ 0 & \\ 194 & 168 \\ 112 & 112 \\ 0 & \\ 1000 & 1000 \\ 121 & 121 \\ 0 & \end{array}$

$\begin{array}{rr}0 & 0 \\ 0 & 0 \\ 30 & 27 \\ 0 & 0 \\ 168 & 76 \\ 112 & 0 \\ 0 & 0 \\ 1000 & 1000 \\ 121 & 118 \\ 0 & 0\end{array}$

$\begin{array}{rrrr}0 & 0 & 0 & 0 \\ 0 & 0 & 0 & 0 \\ 27 & 27 & 27 & 29 \\ 0 & 0 & 0 & 0 \\ 76 & 94 & 125 & 175 \\ 0 & 112 & 0 & 90 \\ 0 & 0 & 0 & 0 \\ 1000 & 1000 & 1000 & 1000 \\ 118 & 120 & 109 & 119 \\ 0 & 0 & 0 & 0\end{array}$

Total Other Resources

Total Resources

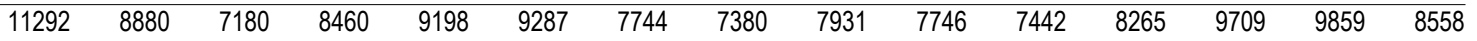

Reserves \& Maintenance

Hydro Reserves

Small Thermal \& Misc. Reserves

Contract Reserves

Large Thermal Reserves

Federal Hydro Maintenance

Spinning Reserves

Federal Transmission Losses

$\begin{array}{rrrrr}0 & 0 & 0 & 0 & 0 \\ 0 & 0 & 0 & 0 & 0 \\ 0 & 0 & 0 & 0 & 0 \\ 0 & 0 & 0 & 0 & 0 \\ 0 & 0 & 0 & 0 & 0 \\ 0 & 0 & 0 & 0 & 0 \\ -318 & -250 & -202 & -239 & -259\end{array}$

$\begin{array}{rr}0 & 0 \\ 0 & 0 \\ 0 & 0 \\ 0 & 0 \\ 0 & 0 \\ 0 & 0 \\ -262 & -218\end{array}$

0
0
0
0
0
0

$\begin{array}{rr}0 & 0 \\ 0 & 0 \\ 0 & 0 \\ 0 & 0 \\ 0 & 0 \\ 0 & 0 \\ -224 & -218\end{array}$

0
0
0
0
0
0

0
0
0
0
0
0
-233

$\begin{array}{rrr}0 & 0 & 0 \\ 0 & 0 & 0 \\ 0 & 0 & 0 \\ 0 & 0 & 0 \\ 0 & 0 & 0 \\ 0 & 0 & 0 \\ -274 & -278 & -241\end{array}$

Total Reserves, Maintenance \& Losses

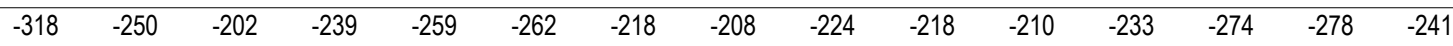

Total Net Resources

$\begin{array}{lllllll}10973 & 8630 & 6978 & 8221 & 8939 & 9025 & 7526\end{array}$

$7172 \quad 7707$

$\begin{array}{llllllll}2334 & 555 & -436 & 418 & 52 & -700 & -1925 & -1997\end{array}$

$-858$

$\begin{array}{llllll}7527 & 7232 & 8032 & 9436 & 9581 & 8317\end{array}$

Total Firm Surplus/Deficit

$-631-914$

$\begin{array}{llll}109 & 1341 & 1189 & -178\end{array}$ 


\section{Section 5 \\ Monthly Regional Tables \\ Loads \& Resources \\ Average Energy in Megawatts}


Average Energy in Megawatts

Firm Regional Loads

Regional Firm Loads

Exports

Federal Diversity

\section{White Book}

Total Firm Regional Loads

Aug1 Aug16 S

$\begin{array}{lll}18969 & 18934 \quad 17728\end{array}$

1753
Oct

Nov Dec Jan

207
Non-Firm Regional Loads

Regional Non-Firm Loads
Total Non-Firm Regional Loads

Total Loads

Hydro Resources

Regulated Hydro

Independent Hydro

Sustained Peaking Adjustment

Total Hydro Resources

Other Resources

Small Thermal \& Misc.

Combustion Turbines

Renewables

Cogeneration

Imports

Large Thermal

Non-Utility Generation

Resource Acquisition

$0 \quad 0$

$0 \quad 0$

0

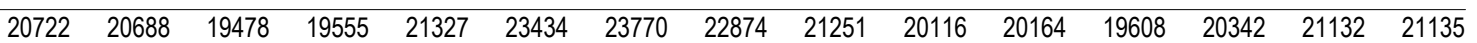

Total Other Resources

13476

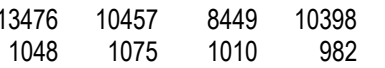

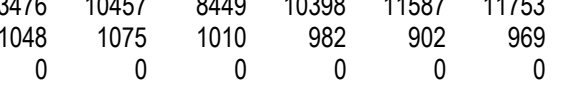

$14524 \quad 11532 \quad 9459$

$59 \quad 11380$

$12489 \quad 12722$

\begin{tabular}{|c|c|c|c|c|c|c|c|c|c|c|c|c|c|c|}
\hline 17 & 17 & 17 & 17 & 17 & 17 & 17 & 17 & 17 & 17 & 17 & 17 & 17 & 17 & \\
\hline 2206 & 2206 & 2269 & 2191 & 2137 & 2119 & 2252 & 2002 & 1841 & 1594 & 1541 & 1266 & 1372 & 2131 & \\
\hline 79 & 79 & 79 & 81 & 84 & 86 & 87 & 85 & 84 & 82 & 82 & 57 & 79 & 79 & \\
\hline 2126 & 2126 & 2130 & 2132 & 2139 & 2136 & 2144 & 2141 & 1518 & 2133 & 2133 & 1137 & 2128 & 2119 & \\
\hline 424 & 424 & 35 & 441 & 789 & 1004 & 951 & 806 & 656 & 585 & 548 & 329 & 417 & 499 & \\
\hline 6268 & 6268 & 6268 & 6268 & 6268 & 6268 & 6268 & 6268 & 6137 & 5055 & 5124 & 4615 & 5174 & 6268 & \\
\hline 1392 & 1390 & 1265 & 1213 & 1232 & 1212 & 1204 & 1206 & 1223 & 1402 & 1402 & 1192 & 1550 & 1459 & \\
\hline & & & & & & & & & & & & & & \\
\hline
\end{tabular}

Total Resources

$27036 \quad 24042$

\section{Reserves \& Maintenance}

Hydro Reserves

Small Thermal \& Misc. Reserves

Contract Reserves

Large Thermal Reserves

Regional Hydro Maintenance

Spinning Reserves

Regional Transmission Losses

$\begin{array}{rrrrrrrrrrrrrrr}0 & 0 & 0 & 0 & 0 & 0 & 0 & 0 & 0 & 0 & 0 & 0 & 0 & 0 & 0 \\ 0 & 0 & 0 & 0 & 0 & 0 & 0 & 0 & 0 & 0 & 0 & 0 & 0 & 0 & 0 \\ 0 & 0 & 0 & 0 & 0 & 0 & 0 & 0 & 0 & 0 & 0 & 0 & 0 & 0 & 0 \\ 0 & 0 & 0 & 0 & 0 & 0 & 0 & 0 & 0 & 0 & 0 & 0 & 0 & 0 & 0 \\ -30 & -25 & -8.6 & -9 & -3.8 & 0 & 0 & 0 & -5.2 & -7.4 & -7.6 & -20 & -14 & -49 & -12 \\ 0 & 0 & 0 & 0 & 0 & 0 & 0 & 0 & 0 & 0 & 0 & 0 & 0 & 0 & 0 \\ -762 & -677 & -616 & -669 & -709 & -721 & -667 & -619 & -626 & -611 & -605 & -563 & -714 & -738 & -664\end{array}$

Total Reserves, Maintenance \& Losses

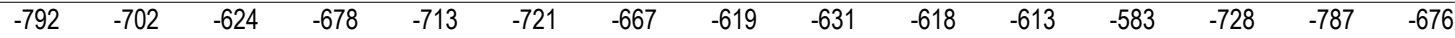

Total Net Resources

$\begin{array}{lllllllllllllll}26244 & 23340 & 21219 & 23045 & 24441 & 24842 & 22992 & 21317 & 21581 & 21041 & 20859 & 19395 & 24601 & 25417 & 22883\end{array}$

Surplus/Deficits

Firm Surplus/Deficit

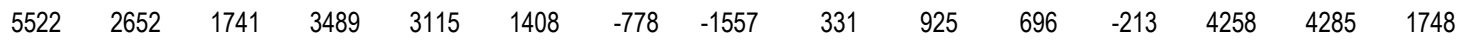

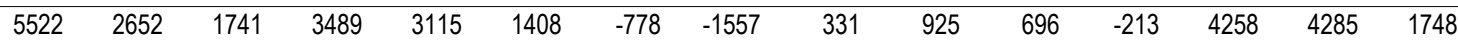

Total Surplus/Deficit 
Loads and Resources - Pacific Northwest Region

PNW Loads and Resources Study

2005 - 2006 Operating Year

1937 Water Year

Average Energy in Megawatts

2003 White Book

Firm Regional Loads

Regional Firm Loads

Exports

Federal Diversity

Aug1 Aug16 S

Oct Nov Dec Jan F

May Jun Jul Avg

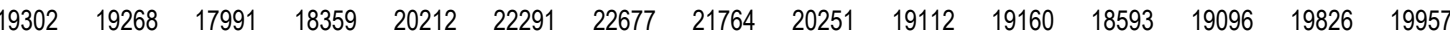

$\begin{array}{llrrrrrrrrrrrrr}1530 & 1530 & 1529 & 1234 & 1223 & 1238 & 1236 & 1232 & 1225 & 1248 & 1248 & 1219 & 1369 & 1404 & 1307\end{array}$

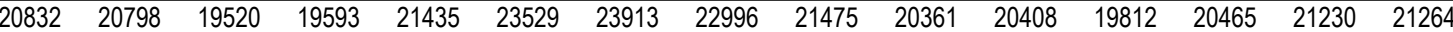

Total Firm Regional Loads

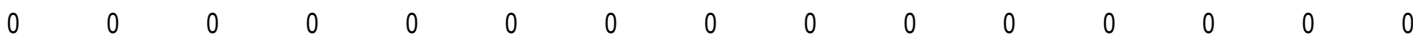
Regional Non-Firm Loads

Total Non-Firm Regional Loads

$\begin{array}{lllllllllllllll}0 & 0 & 0 & 0 & 0 & 0 & 0 & 0 & 0 & 0 & 0 & 0 & 0 & 0 & 0\end{array}$

Total Loads

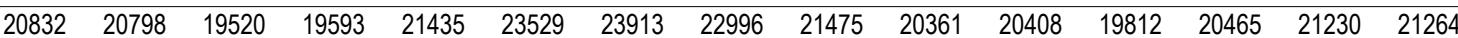

Hydro Resources

Regulated Hydro

Independent Hydro

Sustained Peaking Adjustment

Total Hydro Resources

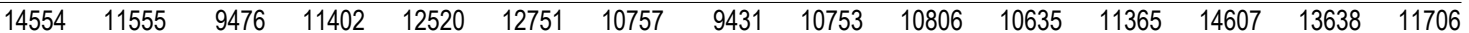

Other Resources

Small Thermal \& Misc.

Combustion Turbines

Renewables

Cogeneration

Imports

Large Thermal

Non-Utility Generation

Resource Acquisition

\begin{tabular}{|c|c|c|c|c|c|c|c|c|c|c|c|c|c|c|}
\hline 17 & 17 & 17 & 17 & 17 & 17 & 17 & 17 & 17 & 17 & 17 & 17 & 17 & 17 & 17 \\
\hline 2211 & 2205 & 2326 & 2264 & 2101 & 2172 & 2276 & 1878 & 1924 & 1572 & 1591 & 1419 & 1369 & 2194 & \\
\hline 79 & 79 & 79 & 81 & 84 & 86 & 87 & 85 & 84 & 82 & 82 & 57 & 79 & 79 & \\
\hline 2126 & 2126 & 2130 & 2132 & 2139 & 2136 & 2144 & 2141 & 1518 & 2133 & 2133 & 1137 & 2128 & 2119 & \\
\hline 466 & 46 & 42 & 462 & 830 & 976 & 926 & 778 & 613 & 568 & 530 & 251 & 415 & 476 & \\
\hline 267 & 6267 & 6267 & 6267 & 6267 & 6267 & 6267 & 6267 & 6134 & 5753 & 5538 & 4866 & 6267 & 5627 & \\
\hline 392 & 1391 & 1266 & 1213 & 1232 & 1213 & 1198 & 1199 & 1216 & 1397 & 1396 & 1187 & 1544 & 1454 & \\
\hline & U & & 0 & U & 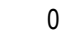 & & 0 & & 0 & & & & & \\
\hline
\end{tabular}

Total Other Resources

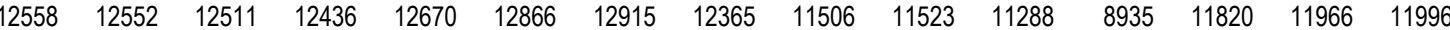

Total Resources

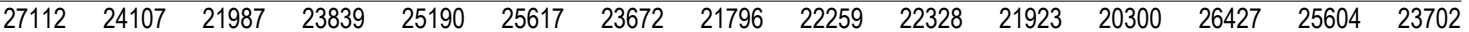

Reserves \& Maintenance

Hydro Reserves

Small Thermal \& Misc. Reserves

Contract Reserves

Large Thermal Reserves

Regional Hydro Maintenance

Spinning Reserves

Regional Transmission Losses

Total Reserves, Maintenance \& Losses

$2631823403 \quad 21358 \quad 23158 \quad 24476 \quad 24895 \quad 23005 \quad 21182 \quad 21626 \quad 21692 \quad 21297 \quad 19708 \quad 25668 \quad 24834 \quad 23022$

Total Net Resources

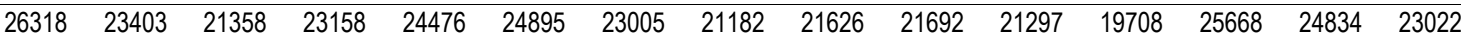

Surplus/Deficits

Firm Surplus/Deficit

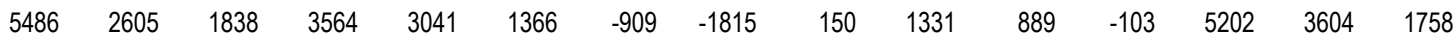

Total Surplus/Deficit

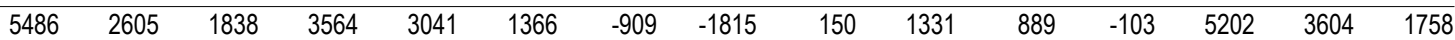


Loads and Resources - Pacific Northwest Region

PNW Loads and Resources Study

2006 - 2007 Operating Year

1937 Water Year

Average Energy in Megawatts

2003 White Book

Firm Regional Loads

Regional Firm Loads

Exports

Federal Diversity

Aug1 Aug16 S

Oct Nov Dec Jan Feb

eb

Mar Apr1 Apr16

May Jun Jul Avg

Total Firm Regional Loads

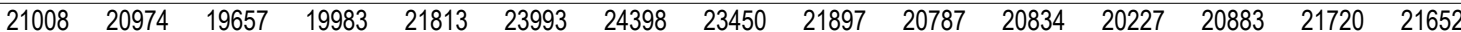

Non-Firm Regional Loads

Regional Non-Firm Loads

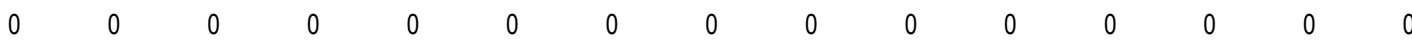

Total Non-Firm Regional Loads

$\begin{array}{lllllllllllllll}0 & 0 & 0 & 0 & 0 & 0 & 0 & 0 & 0 & 0 & 0 & 0 & 0 & 0 & 0\end{array}$

Total Loads

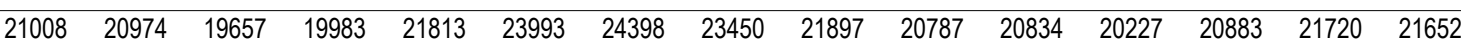

Hydro Resources

Regulated Hydro

Independent Hydro

Sustained Peaking Adjustment

Total Hydro Resources

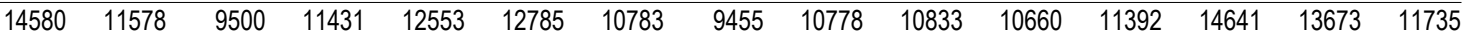

Other Resources

Small Thermal \& Misc.

Combustion Turbines

Renewables

Cogeneration

Imports

Large Thermal

Non-Utility Generation

Resource Acquisition

Total Other Resources

\begin{tabular}{|c|c|c|c|c|c|c|c|c|c|c|c|c|c|c|}
\hline 17 & 17 & 17 & 17 & 17 & 17 & 17 & 17 & 17 & 17 & 17 & 17 & 17 & 17 & 17 \\
\hline 2393 & 2415 & 2533 & 2250 & 2147 & 2144 & 2076 & 1887 & 1793 & 1516 & 1510 & 1413 & 1299 & 2145 & \\
\hline 79 & 79 & 79 & 81 & 84 & 86 & 87 & 85 & 84 & 82 & 82 & 57 & 79 & 79 & \\
\hline 2126 & 2126 & 2130 & 2132 & 2139 & 2136 & 2144 & 2141 & 1518 & 2133 & 2133 & 1137 & 2128 & 2119 & \\
\hline 377 & 377 & 369 & 401 & 761 & 914 & 806 & 661 & 513 & 465 & 427 & 215 & 348 & 361 & \\
\hline 5627 & 6267 & 6267 & 6267 & 6267 & 6267 & 6267 & 6267 & 6211 & 5377 & 5016 & 3571 & 4663 & 5997 & \\
\hline 1368 & 1368 & 1259 & 1252 & 1258 & 1235 & 1226 & 1231 & 1249 & 1409 & 1408 & 1382 & 1583 & 1493 & \\
\hline & 0 & 0 & 0 & U & 0 & & 0 & & 0 & & & & & \\
\hline
\end{tabular}

Total Resources

$\begin{array}{lllllllllllllll}26567 & 24227 & 22154 & 23831 & 25227 & 25585 & 23407 & 21745 & 22163 & 21833 & 21252 & 19185 & 24759 & 25884 & 23407\end{array}$

Reserves \& Maintenance

Hydro Reserves

Small Thermal \& Misc. Reserves

Contract Reserves

Large Thermal Reserves

Regional Hydro Maintenance

Spinning Reserves

Regional Transmission Losses

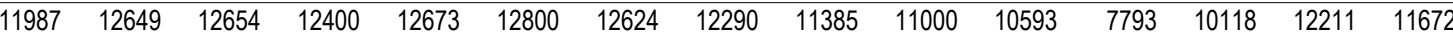

Total Reserves, Maintenance \& Losses

Total Net Resources

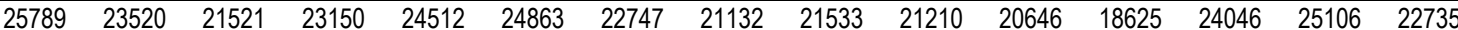

Surplus/Deficits

Firm Surplus/Deficit

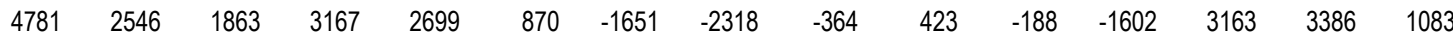

Total Surplus/Deficit

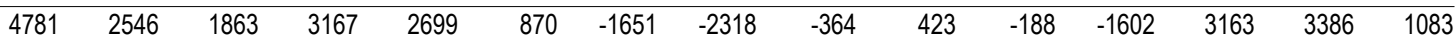


1937 Water Year

2003 White Book

Average Energy in Megawatts

Firm Regional Loads

Regional Firm Loads

Exports

Federal Diversity

Aug1 Aug16 Sep Oct Nov Dec Jan

Feb

Mar Apr1 Apr16

May Jun

Jul Avg

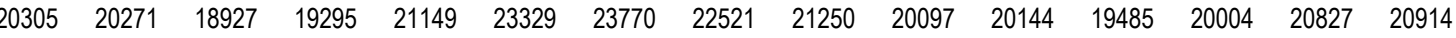

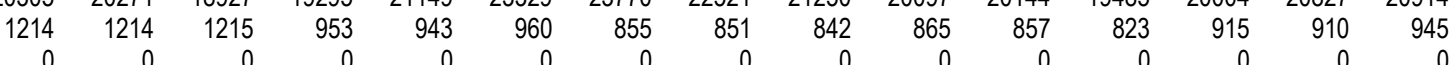

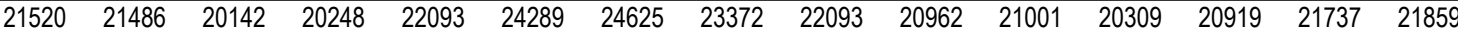

Total Firm Regional Loads

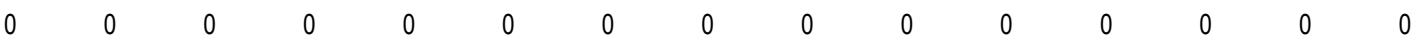
Regional Non-Firm Loads

Total Non-Firm Regional Load

$\begin{array}{rrrrrrrrrrrrrrrr}0 & 0 & 0 & 0 & 0 & 0 & 0 & 0 & 0 & 0 & 0 & 0 & 0 & 0 & 0\end{array}$

Total Loads

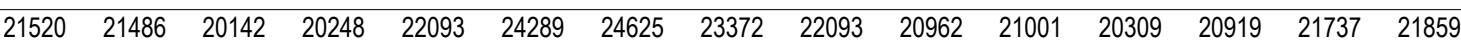

Hydro Resources

Regulated Hydro

Independent Hydro

Sustained Peaking Adjustment

Total Hydro Resources

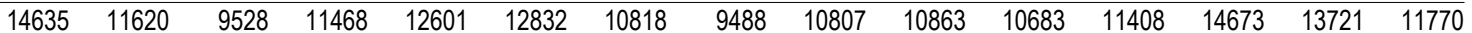

Other Resources

Small Thermal \& Misc.

Combustion Turbines

Renewables

Cogeneration

Imports

Large Thermal

Non-Utility Generation

Resource Acquisition

Total Other Resources

\begin{tabular}{|c|c|c|c|c|c|c|c|c|c|c|c|c|c|c|}
\hline 17 & 17 & 17 & 17 & 17 & 17 & 17 & 17 & 17 & 17 & 17 & 17 & 17 & 17 & 17 \\
\hline 328 & 2324 & 2382 & 2241 & 2162 & 2044 & 2090 & 1824 & 1881 & 1509 & 1502 & 1328 & 1304 & 2160 & 1937 \\
\hline 79 & 79 & 79 & 81 & 84 & 86 & 87 & 85 & 84 & 82 & 82 & 57 & 79 & 79 & 80 \\
\hline 2126 & 2126 & 2130 & 2132 & 2139 & 2136 & 2144 & 2141 & 1518 & 2133 & 2133 & 1137 & 2128 & 2119 & 1999 \\
\hline 357 & 357 & 277 & 355 & 704 & 889 & 620 & 627 & 543 & 489 & 437 & 243 & 351 & 379 & 484 \\
\hline 2274 & 6274 & 6274 & 6274 & 6274 & 6274 & 6274 & 6274 & 6214 & 5781 & 5681 & 4414 & 5765 & 6274 & 6026 \\
\hline 418 & 1418 & 1309 & 1253 & 1259 & 1235 & 1226 & 1232 & 1250 & 1409 & 1408 & 1383 & 1575 & 1491 & 1337 \\
\hline & & 0 & 0 & 0 & & & & 0 & & & & & & \\
\hline
\end{tabular}

Total Resources

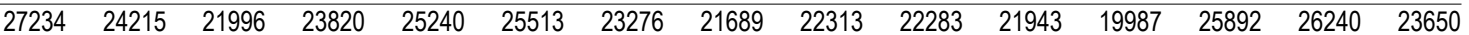

Reserves \& Maintenance

Hydro Reserves

Small Thermal \& Misc. Reserves

Contract Reserves

Large Thermal Reserves

Regional Hydro Maintenance

Spinning Reserves

Regional Transmission Losses

13587

$2599 \quad 12595 \quad 12468 \quad 12352 \quad 1263$

\begin{tabular}{lllllllllll}
2639 & 12681 & 12458 & 12201 & 11506 & 11420 & 11260 & 8579 & 11219 & 12519 & 11880 \\
& & & & & & & & & & \\
\hline 5240 & 25513 & 23276 & 21689 & 22313 & 22283 & 21943 & 19987 & 25892 & 26240 & 23650
\end{tabular}

Total Reserves, Maintenance \& Losses

$\begin{array}{rrrrrrrrrrrrrrr}0 & 0 & 0 & 0 & 0 & 0 & 0 & 0 & 0 & 0 & 0 & 0 & 0 & 0 & 0 \\ 0 & 0 & 0 & 0 & 0 & 0 & 0 & 0 & 0 & 0 & 0 & 0 & 0 & 0 & 0 \\ 0 & 0 & 0 & 0 & 0 & 0 & 0 & 0 & 0 & 0 & 0 & 0 & 0 & 0 & 0 \\ 0 & 0 & 0 & 0 & 0 & 0 & 0 & 0 & 0 & 0 & 0 & 0 & 0 & 0 & 0 \\ -30 & -25 & -8.6 & -9 & -3.8 & 0 & 0 & 0 & -5.2 & -7.4 & -7.6 & -20 & -14 & -49 & -12 \\ 0 & 0 & 0 & 0 & 0 & 0 & 0 & 0 & 0 & 0 & 0 & 0 & 0 & 0 & 0 \\ -767 & -682 & -620 & -671 & -712 & -719 & -656 & -612 & -629 & -628 & -619 & -563 & -730 & -739 & -667\end{array}$

Total Net Resources $\begin{array}{lllllllllllllll}-797 & -707 & -629 & -680 & -715 & -719 & -656 & -612 & -634 & -636 & -626 & -583 & -744 & -788 & -679\end{array}$

Surplus/Deficits

Firm Surplus/Deficit

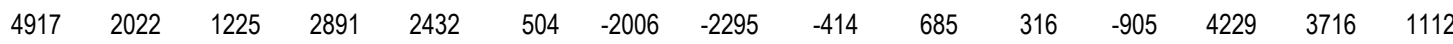

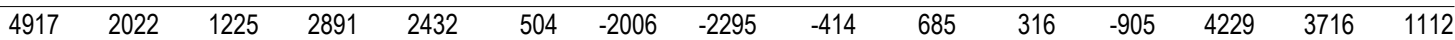


Loads and Resources - Pacific Northwest Region

PNW Loads and Resources Study

2008 - 2009 Operating Year

1937 Water Year

Average Energy in Megawatts

2003 White Book

Firm Regional Loads

Regional Firm Loads

Exports

Federal Diversity

Aug1 Aug16 Sep Oct Nov Dec Jan Feb

Feb

Mar Apr1 Apr16

May Jun Jul Avg

Total Firm Regional Loads

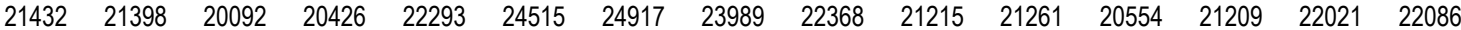

Non-Firm Regional Loads

Regional Non-Firm Loads

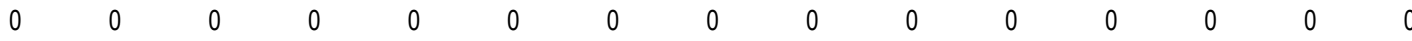

Total Non-Firm Regional Loads

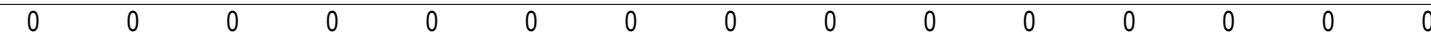

Total Loads

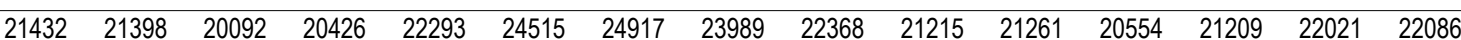

Hydro Resources

Regulated Hydro

Independent Hydro

Sustained Peaking Adjustment

Total Hydro Resources

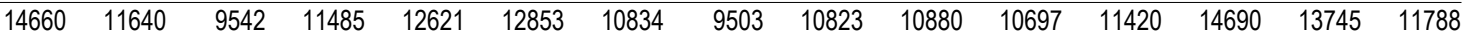

Other Resources

Small Thermal \& Misc.

Combustion Turbines

Renewables

Cogeneration

Imports

Large Thermal

Non-Utility Generation

Resource Acquisition

\begin{tabular}{|c|c|c|c|c|c|c|c|c|c|c|c|c|c|c|}
\hline 17 & 17 & 17 & 17 & 17 & 17 & 17 & 17 & 17 & 17 & 17 & 17 & 17 & 17 & 17 \\
\hline 2309 & 2313 & 2375 & 2252 & 2044 & 2105 & 2091 & 1875 & 1875 & 1490 & 1490 & 1417 & 1316 & 2147 & 1941 \\
\hline 79 & 79 & 79 & 81 & 84 & 86 & 87 & 85 & 84 & 82 & 82 & 57 & 79 & 79 & 80 \\
\hline 2126 & 2126 & 2130 & 2132 & 2139 & 2136 & 2144 & 2141 & 1518 & 2133 & 2133 & 1137 & 2128 & 2119 & 1999 \\
\hline 173 & 173 & 157 & 200 & 401 & 472 & 458 & 424 & 37 & 322 & 291 & 109 & 184 & 200 & 288 \\
\hline 274 & 6274 & 6274 & 6274 & 6274 & 6274 & 6274 & 6274 & 6211 & 5785 & 5392 & 3458 & 5021 & 6274 & 5873 \\
\hline 417 & 1419 & 1309 & 1253 & 1259 & 1236 & 1227 & 1232 & 1250 & 1409 & 1409 & 1383 & 1575 & 1492 & 1337 \\
\hline & & 0 & 0 & 0 & & & & 0 & & & & & & \\
\hline
\end{tabular}

Total Other Resources

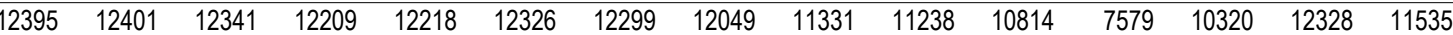

Total Resources

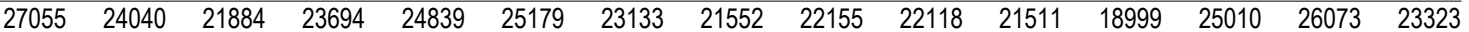

Reserves \& Maintenance

Hydro Reserves

Small Thermal \& Misc. Reserves

Contract Reserves

Large Thermal Reserves

Regional Hydro Maintenance

Spinning Reserves

Regional Transmission Losses

$\begin{array}{rrrrrrrrrrrrrrr}0 & 0 & 0 & 0 & 0 & 0 & 0 & 0 & 0 & 0 & 0 & 0 & 0 & 0 & 0 \\ 0 & 0 & 0 & 0 & 0 & 0 & 0 & 0 & 0 & 0 & 0 & 0 & 0 & 0 & 0 \\ 0 & 0 & 0 & 0 & 0 & 0 & 0 & 0 & 0 & 0 & 0 & 0 & 0 & 0 & 0 \\ 0 & 0 & 0 & 0 & 0 & 0 & 0 & 0 & 0 & 0 & 0 & 0 & 0 & 0 & 0 \\ -30 & -25 & -8.6 & -9 & -3.8 & 0 & 0 & 0 & -5.2 & -7.4 & -7.6 & -20 & -14 & -49 & -12 \\ 0 & 0 & 0 & 0 & 0 & 0 & 0 & 0 & 0 & 0 & 0 & 0 & 0 & 0 & 0 \\ -762 & -677 & -617 & -668 & -700 & -710 & -652 & -608 & -625 & -624 & -606 & -535 & -705 & -734 & -657\end{array}$

Total Reserves, Maintenance \& Losses

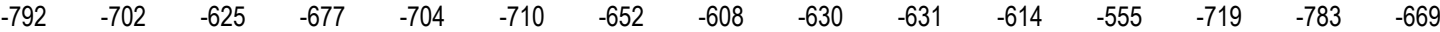

Total Net Resources

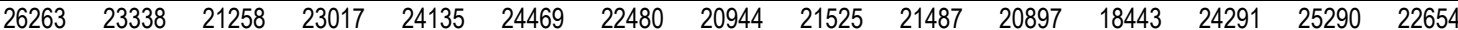

Surplus/Deficits

Firm Surplus/Deficit

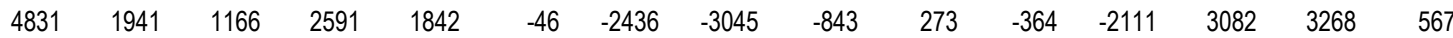

Total Surplus/Deficit

$\begin{array}{lllllllllllllll}4831 & 1941 & 1166 & 2591 & 1842 & -46 & -2436 & -3045 & -843 & 273 & -364 & -2111 & 3082 & 3268 & 567\end{array}$


1937 Water Year

2003 White Book

Average Energy in Megawatts

Firm Regional Loads

Regional Firm Loads

Exports

Federal Diversity
Aug1 Aug16 S

$20860 \quad 20826 \quad 19$

937

Total Firm Regional Loads

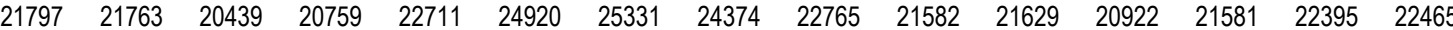

Non-Firm Regional Loads

Regional Non-Firm Loads

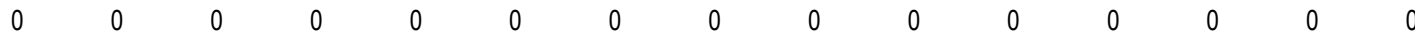

Total Non-Firm Regional Loads

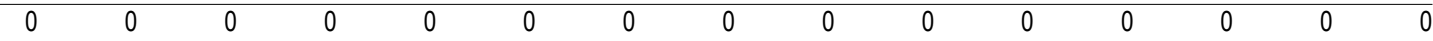

Total Loads

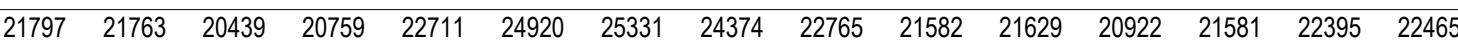

Hydro Resources

Regulated Hydro

Independent Hydro

Sustained Peaking Adjustment

Total Hydro Resources

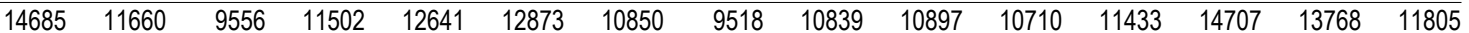

Other Resources

Small Thermal \& Misc.

Combustion Turbines

Renewables

Cogeneration

Imports

Large Thermal

Non-Utility Generation

Resource Acquisition

Total Other Resources

\begin{tabular}{|c|c|c|c|c|c|c|c|c|c|c|c|c|c|c|}
\hline 17 & 17 & 17 & 17 & 17 & 17 & 17 & 17 & 17 & 17 & 17 & 17 & 17 & 17 & \\
\hline 2264 & 2307 & 2392 & 2125 & 2105 & 2093 & 2083 & 1822 & 1785 & 1490 & 1484 & 1429 & 1320 & 2126 & \\
\hline 79 & 79 & 79 & 81 & 84 & 86 & 87 & 85 & 84 & 82 & 82 & 57 & 79 & 79 & \\
\hline 2126 & 2126 & 2130 & 2132 & 2139 & 2136 & 2144 & 2141 & 1518 & 2133 & 2133 & 1137 & 2128 & 2119 & \\
\hline 173 & 173 & 15 & 200 & 401 & 472 & 458 & 424 & 377 & 322 & 291 & 109 & 130 & 149 & \\
\hline 6268 & 6268 & 6268 & 6268 & 6268 & 6268 & 6268 & 6268 & 6137 & 4974 & 5066 & 4956 & 5419 & 6268 & \\
\hline 1417 & 1418 & 1308 & 1252 & 1259 & 1236 & 1227 & 1232 & 1250 & 1410 & 1409 & 1383 & 1583 & 1493 & \\
\hline 0 & & & & & & & & & & 0 & & & & \\
\hline
\end{tabular}

Total Resources

$\begin{array}{lllllllllllllll}12344 & 12388 & 12350 & 12075 & 12273 & 12308 & 12284 & 11989 & 11168 & 10428 & 10482 & 9089 & 10676 & 12250 & 11607\end{array}$

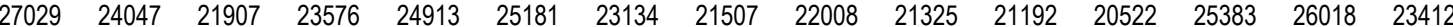

Reserves \& Maintenance

Hydro Reserves

Small Thermal \& Misc. Reserves

Contract Reserves

Large Thermal Reserves

Regional Hydro Maintenance

Spinning Reserves

Regional Transmission Losses

$\begin{array}{rrrrrrrrr}0 & 0 & 0 & 0 & 0 & 0 & 0 & 0 & \\ 0 & 0 & 0 & 0 & 0 & 0 & 0 & 0 & \\ 0 & 0 & 0 & 0 & 0 & 0 & 0 & 0 & \\ 0 & 0 & 0 & 0 & 0 & 0 & 0 & 0 & \\ -30 & -25 & -8.6 & -9 & -3.8 & 0 & 0 & 0 & -5.2 \\ 0 & 0 & 0 & 0 & 0 & 0 & 0 & 0 & 0 \\ -701 & -677 & -618 & -065 & -702 & -710 & -052 & -607 & -620\end{array}$

$\begin{array}{rrrrrrr}0 & 0 & 0 & 0 & 0 & 0 & 0 \\ 0 & 0 & 0 & 0 & 0 & 0 & 0 \\ 0 & 0 & 0 & 0 & 0 & 0 & 0 \\ 0 & 0 & 0 & 0 & 0 & 0 & 0 \\ -5.2 & -7.4 & -7.6 & -20 & -14 & -49 & -12 \\ 0 & 0 & 0 & 0 & 0 & 0 & 0 \\ -620 & -601 & -597 & -578 & -715 & -732 & -660\end{array}$

Total Reserves, Maintenance \&

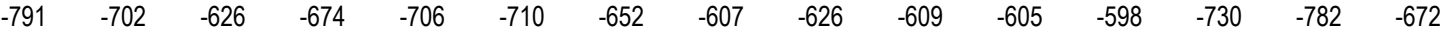

Total Net Resources

$\begin{array}{lllllllllllllll}26237 & 23345 & 21281 & 22903 & 24207 & 24470 & 22482 & 20901 & 21382 & 20717 & 20587 & 19923 & 24653 & 25236 & 22740\end{array}$

Surplus/Deficits

Firm Surplus/Deficit

$\begin{array}{lllllllllllllll}4440 & 1582 & 842 & 2144 & 1496 & -450 & -2850 & -3474 & -1383 & -866 & -1041 & -999 & 3072 & 2841 & 275\end{array}$

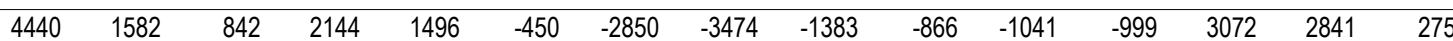


1937 Water Year

2003 White Book

Average Energy in Megawatts

Firm Regional Loads

Regional Firm Loads

Exports

Federal Diversity
Aug1 Aug16 Sep Oct Nov Dec Jan

Feb

Mar Apr1 Apr16

May Jun

Jul Avg

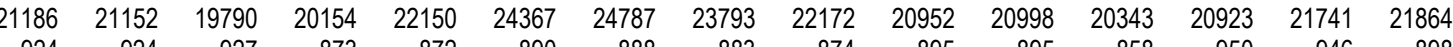

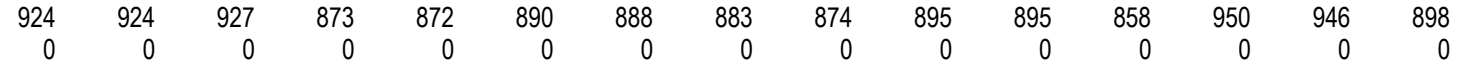

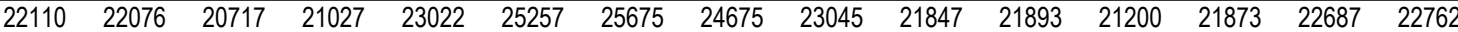

Total Firm Regional Loads

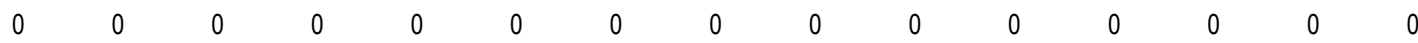
Regional Non-Firm Loads

Total Non-Firm Regional Load

$\begin{array}{lllllllllllllll}0 & 0 & 0 & 0 & 0 & 0 & 0 & 0 & 0 & 0 & 0 & 0 & 0 & 0 & 0\end{array}$

Total Loads

$20717 \quad 21027$

$23022 \quad 25257 \quad 25675$

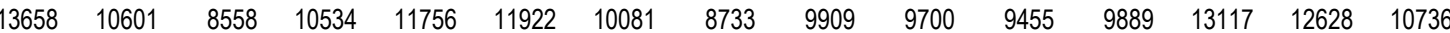

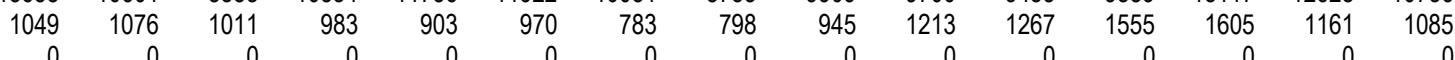

Regulated Hydro

Independent Hydro

Sustained Peaking Adjustment

Total Hydro Resources

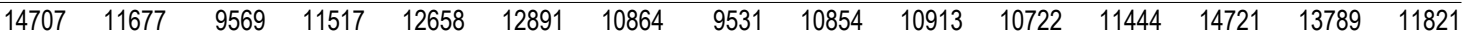

Other Resources

Small Thermal \& Misc.

Combustion Turbines

Renewables

Cogeneration

Imports

Large Thermal

Non-Utility Generation

Resource Acquisition

Total Other Resources

\begin{tabular}{|c|c|c|c|c|c|c|c|c|c|c|c|c|c|c|}
\hline 17 & 17 & 17 & 17 & 17 & 17 & 17 & 17 & 17 & 17 & 17 & 17 & 17 & 17 & 17 \\
\hline 2302 & 2312 & 2223 & 2189 & 2082 & 2148 & 2113 & 1871 & 1865 & 1493 & 1515 & 1261 & 1289 & 2115 & 1914 \\
\hline 79 & 79 & 79 & 81 & 84 & 86 & 87 & 85 & 84 & 82 & 82 & 57 & 79 & 79 & 80 \\
\hline 2126 & 2126 & 2130 & 2132 & 2139 & 2136 & 2144 & 2141 & 1518 & 2133 & 2133 & 1137 & 2128 & 2119 & 1999 \\
\hline 122 & 122 & 15 & 188 & 306 & 377 & 364 & 329 & 28 & 227 & 202 & 102 & 130 & 149 & 226 \\
\hline 268 & 6268 & 6268 & 6268 & 6268 & 6268 & 6268 & 6268 & 6191 & 4827 & 4854 & 4348 & 5015 & 6268 & 5878 \\
\hline 418 & 1418 & 1308 & 1253 & 1266 & 1241 & 1233 & 1240 & 1258 & 1416 & 1416 & 1384 & 1576 & 1493 & 1340 \\
\hline & & 0 & 0 & 0 & 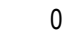 & 0 & & & & & & & & \\
\hline
\end{tabular}

Total Resources

$\begin{array}{lllllllllllllll}27038 & 24019 & 21744 & 23643 & 24820 & 25164 & 23091 & 21482 & 22068 & 21109 & 20942 & 19749 & 24956 & 26028 & 23275\end{array}$

Reserves \& Maintenance

Hydro Reserves

Small Thermal \& Misc. Reserves

Contract Reserves

Large Thermal Reserves

Regional Hydro Maintenance

Spinning Reserves

Regional Transmission Losses

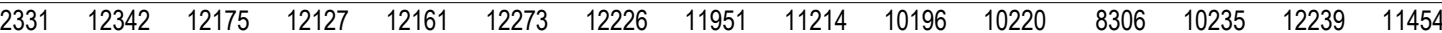

Total Reserves, Maintenance \& Losses

Total Net Resources

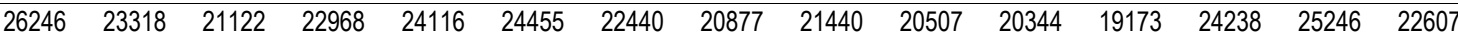

Surplus/Deficits

Firm Surplus/Defici

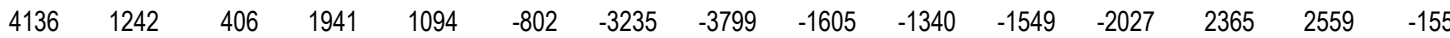


Loads and Resources - Pacific Northwest Region

PNW Loads and Resources Study

2011 - 2012 Operating Year

1937 Water Year

Average Energy in Megawatts

2003 White Book

Firm Regional Loads

Regional Firm Loads

Exports

Federal Diversity

Aug1 Aug16 Sep Oct Nov Dec Jan

Feb

Mar Apr1 Apr16

May Jun Jul Avg

Total Firm Regional Loads

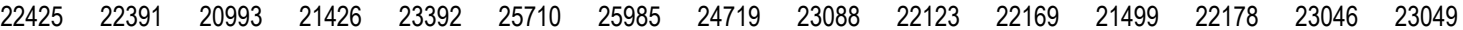

Non-Firm Regional Loads

Regional Non-Firm Loads

$\begin{array}{lllllllllllllllllll}0 & 0 & 0 & 0 & 0 & 0 & 0 & 0 & 0 & 0 & 0 & 0 & 0 & 0 & 0\end{array}$

Total Non-Firm Regional Loads

$\begin{array}{llllllllllllllll}0 & 0 & 0 & 0 & 0 & 0 & 0 & 0 & 0 & 0 & 0 & 0 & 0 & 0 & 0\end{array}$

Total Loads

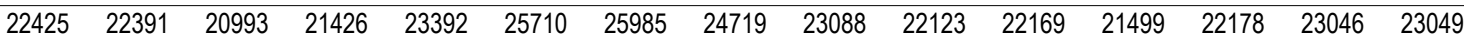

Hydro Resources

Regulated Hydro

Independent Hydro

Sustained Peaking Adjustment

Total Hydro Resources

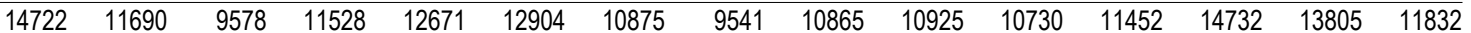

Other Resources

Small Thermal \& Misc.

Combustion Turbines

Renewables

Cogeneration

Imports

Large Thermal

Non-Utility Generation

Resource Acquisition

\begin{tabular}{|c|c|c|c|c|c|c|c|c|c|c|c|c|c|c|}
\hline 17 & 17 & 17 & 17 & 17 & 17 & 17 & 17 & 17 & 17 & 17 & 17 & 17 & 17 & 17 \\
\hline 2260 & 2184 & 2254 & 2199 & 2164 & 2146 & 2121 & 1875 & 1863 & 1520 & 1516 & 1219 & 1339 & 2161 & 1923 \\
\hline 2126 & 2126 & 2130 & 2132 & 2139 & 2136 & 2144 & 2141 & 1518 & 2133 & 2133 & 1137 & 2128 & 2119 & 1999 \\
\hline 6267 & 6267 & 6267 & 6267 & 6267 & 6267 & 6267 & 6267 & 6207 & 5729 & 5681 & 4262 & 5801 & 6267 & 6009 \\
\hline 1418 & 1418 & 1309 & 1253 & 1266 & 1241 & 1234 & 1240 & 1256 & 1416 & 1416 & 1384 & 1576 & 1493 & 1340 \\
\hline
\end{tabular}

Total Other Resources

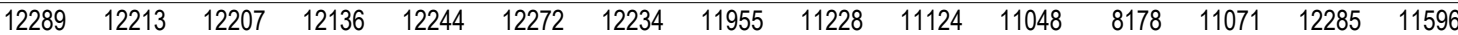

Total Resources

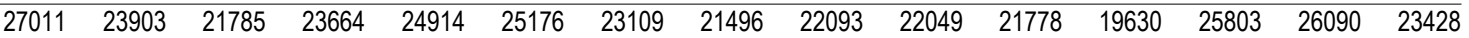

Reserves \& Maintenance

Hydro Reserves

Small Thermal \& Misc. Reserves

Contract Reserves

Large Thermal Reserves

Regional Hydro Maintenance

Spinning Reserves

Regional Transmission Losses

$\begin{array}{rrrrrrrrrrrrrrr}0 & 0 & 0 & 0 & 0 & 0 & 0 & 0 & 0 & 0 & 0 & 0 & 0 & 0 & 0 \\ 0 & 0 & 0 & 0 & 0 & 0 & 0 & 0 & 0 & 0 & 0 & 0 & 0 & 0 & 0 \\ 0 & 0 & 0 & 0 & 0 & 0 & 0 & 0 & 0 & 0 & 0 & 0 & 0 & 0 & 0 \\ 0 & 0 & 0 & 0 & 0 & 0 & 0 & 0 & 0 & 0 & 0 & 0 & 0 & 0 & 0 \\ -30 & -25 & -8.6 & -9 & -3.8 & 0 & 0 & 0 & -5.2 & -7.4 & -7.6 & -20 & -14 & -49 & -12 \\ 0 & 0 & 0 & 0 & 0 & 0 & 0 & 0 & 0 & 0 & 0 & 0 & 0 & 0 & 0 \\ -761 & -673 & -614 & -667 & -702 & -710 & -652 & -606 & -623 & -622 & -614 & -553 & -727 & -734 & -660\end{array}$

Total Reserves, Maintenance \& Losses

$\begin{array}{llllllllllllllll}-791 & -698 & -623 & -676 & -706 & -710 & -652 & -606 & -628 & -629 & -622 & -573 & -742 & -784 & -672\end{array}$

Total Net Resources

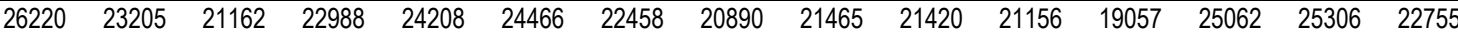

Surplus/Deficits

Firm Surplus/Defici

$\begin{array}{lllllllllllllll}3795 & 814 & 169 & 1562 & 816 & -1245 & -3527 & -3829 & -1623 & -703 & -1013 & -2443 & 2883 & 2260 & -294\end{array}$

$\begin{array}{lllllllllllllll}3795 & 814 & 169 & 1562 & 816 & -1245 & -3527 & -3829 & -1623 & -703 & -1013 & -2443 & 2883 & 2260 & -294\end{array}$


Loads and Resources - Pacific Northwest Region

PNW Loads and Resources Stud
2012 - 2013 Operating Year
1937 Water Year
2003 White Book

Average Energy in Megawatts

Firm Regional Loads

Regional Firm Loads

Exports

Federal Diversity

\section{Aug1 Aug16}

Sep

Oct Nov

$21904 \quad 21870$

$898 \quad 898$

$22802 \quad 22768$

$0 \quad 0$

Total Non-Firm Regional Loads

\begin{tabular}{llllllllllllll}
0 & 0 & 0 & 0 & 0 & 0 & 0 & 0 & 0 & 0 & 0 & 0 & 0 & 0 \\
\hline
\end{tabular}

Total Loads

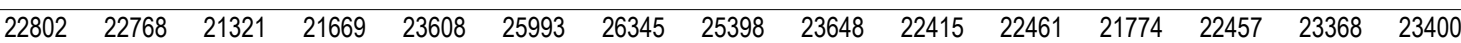

Hydro Resources

Regulated Hydro

Independent Hydro

Sustained Peaking Adjustment

Total Hydro Resources

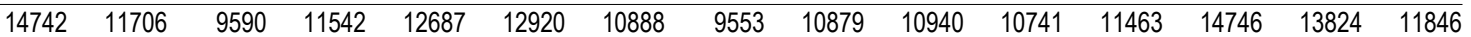

Other Resources

Small Thermal \& Misc.

Combustion Turbines

Renewables

Cogeneration

Imports

Large Thermal

Non-Utility Generation

Resource Acquisition

\begin{tabular}{|c|c|c|c|c|c|c|c|c|c|c|c|c|c|c|}
\hline 17 & 17 & 17 & 17 & 17 & 17 & 17 & 17 & 17 & 17 & 17 & 17 & 17 & 17 & 17 \\
\hline 2175 & 2236 & 2281 & 2195 & 2108 & 2123 & 2125 & 1867 & 1893 & 1522 & 1486 & 1338 & 1344 & 2033 & 1918 \\
\hline 79 & 79 & 79 & 81 & 84 & 86 & 87 & 85 & 84 & 82 & 82 & 57 & 79 & 79 & 80 \\
\hline 126 & 2126 & 2130 & 2132 & 2139 & 2136 & 2144 & 2141 & 1518 & 2133 & 2133 & 1137 & 2128 & 2119 & 1999 \\
\hline 122 & 122 & 150 & 188 & 297 & 369 & 355 & 320 & 282 & 227 & 202 & 102 & 130 & 149 & 223 \\
\hline 6274 & 6274 & 6274 & 6274 & 6274 & 6274 & 6274 & 6274 & 6085 & 5252 & 5325 & 4241 & 5113 & 6274 & 5910 \\
\hline 419 & 1419 & 1309 & 1253 & 1266 & 1243 & 1234 & 1240 & 1257 & 1416 & 1416 & 1383 & 1583 & 1494 & 1341 \\
\hline & 0 & 0 & 0 & 0 & 0 & 0 & U & 0 & 0 & 0 & 0 & 0 & & \\
\hline
\end{tabular}

Total Other Resources

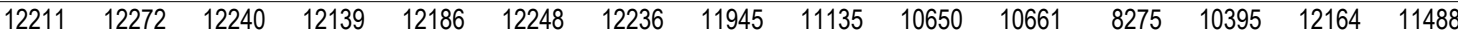

Total Resources

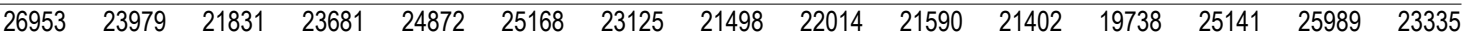

Reserves \& Maintenance

Hydro Reserves

Small Thermal \& Misc. Reserves

Contract Reserves

Large Thermal Reserves

Regional Hydro Maintenance

Spinning Reserves

Regional Transmission Losses

$\begin{array}{rrrrr}0 & 0 & 0 & 0 & 0 \\ 0 & 0 & 0 & 0 & 0 \\ 0 & 0 & 0 & 0 & 0 \\ 0 & 0 & 0 & 0 & 0 \\ -30 & -25 & -8.6 & -9 & -3.8 \\ 0 & 0 & 0 & 0 & 0\end{array}$

$\begin{array}{rr}0 & 0 \\ 0 & 0 \\ 0 & 0 \\ 0 & 0 \\ 0 & 0 \\ 0 & 0 \\ -710 & -652\end{array}$

$\begin{array}{rr}0 & 0 \\ 0 & 0 \\ 0 & 0 \\ 0 & 0 \\ 0 & 0 \\ 0 & 0 \\ 52 & -606\end{array}$

$-789 \quad-700 \quad-62$

$-677 \quad-705$

$-710 \quad-652 \quad-606$

0
0
0
0
-5.2
0
-621

$\begin{array}{rr}0 & 0 \\ 0 & 0 \\ 0 & 0 \\ 0 & 0 \\ -7.4 & -7.6 \\ 0 & 0 \\ -609 & -603\end{array}$

$\begin{array}{rr}0 & 0 \\ 0 & 0 \\ 0 & 0 \\ 0 & 0 \\ -7.6 & -20 \\ 0 & 0 \\ -603 & -556\end{array}$

$\begin{array}{rrrr}0 & 0 & 0 & 0 \\ 0 & 0 & 0 & 0 \\ 0 & 0 & 0 & 0 \\ 0 & 0 & 0 & 0 \\ -20 & -14 & -49 & -12 \\ 0 & 0 & 0 & 0 \\ -556 & -709 & -731 & -658\end{array}$

Total Reserves, Maintenance \& Losses

$\begin{array}{lllllllllllllll}26164 & 23279 & 21207 & 23005 & 24167 & 24458 & 22473 & 20892 & 21389 & 20974 & 20791 & 19162 & 24418 & 25208 & 22665\end{array}$

Surplus/Deficits

Firm Surplus/Deficit

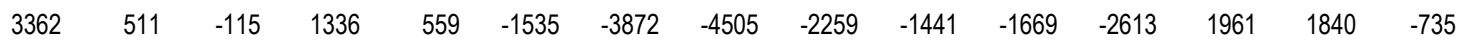

\begin{tabular}{|c|}
\hline $3362 \quad 511$ \\
\hline
\end{tabular}

Total Surplus/Deficit

$511-115 \quad 1336$ 
Loads and Resources - Pacific Northwest Region

PNW Loads and Resources Study

2013 - 2014 Operating Year

1937 Water Year

Average Energy in Megawatts

2003 White Book

Firm Regional Loads

Regional Firm Loads

Exports

Federal Diversity

Aug1 Aug16 Sep

Oct Nov Dec Jan

Feb

May Jun Jul Avg

Total Firm Regional Loads

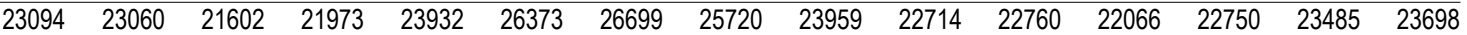

Non-Firm Regional Loads

Regional Non-Firm Loads

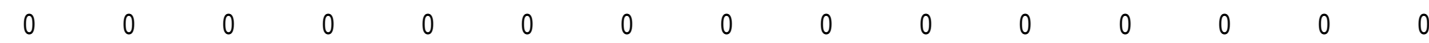

Total Non-Firm Regional Loads

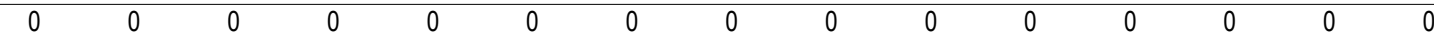

Total Loads

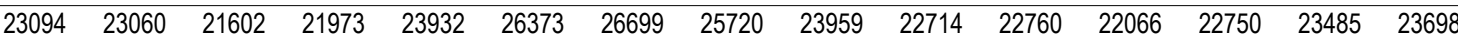

Hydro Resources

Regulated Hydro

Independent Hydro

Sustained Peaking Adjustment

Total Hydro Resources

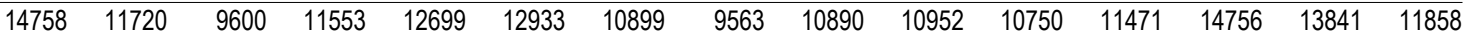

Other Resources

Small Thermal \& Misc.

Combustion Turbines

Renewables

Cogeneration

Imports

Large Thermal

Non-Utility Generation

Resource Acquisition

\begin{tabular}{|c|c|c|c|c|c|c|c|c|c|c|c|c|c|c|}
\hline 17 & 17 & 17 & 17 & 17 & 17 & 17 & 17 & 17 & 17 & 17 & 17 & 17 & 17 & 17 \\
\hline 211 & 2211 & 2317 & 2176 & 2118 & 2122 & 2129 & 1900 & 1900 & 1496 & 1539 & 1348 & 1228 & 2091 & 1922 \\
\hline 79 & 79 & 79 & 81 & 84 & 86 & 87 & 85 & 84 & 82 & 82 & 57 & 79 & 79 & 80 \\
\hline 2126 & 2126 & 2130 & 2132 & 2139 & 2136 & 2144 & 2141 & 1518 & 2133 & 2133 & 1137 & 2128 & 2119 & 1999 \\
\hline 122 & 122 & 150 & 188 & 297 & 369 & 355 & 320 & 2 & 227 & 202 & 102 & 130 & 149 & 223 \\
\hline 2274 & 6274 & 6274 & 6274 & 6274 & 6274 & 6274 & 6274 & 6211 & 5733 & 5681 & 4600 & 5854 & 6274 & 6047 \\
\hline 419 & 1419 & 1309 & 1254 & 1267 & 1243 & 1235 & 1241 & 1258 & 1417 & 1416 & 1383 & 1576 & 1493 & 1341 \\
\hline & & 0 & 0 & 0 & & 0 & & & & & & & & \\
\hline
\end{tabular}

Total Other Resources

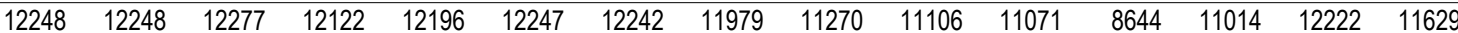

Total Resources

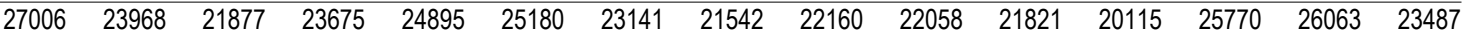

Reserves \& Maintenance

Hydro Reserves

Small Thermal \& Misc. Reserves

Contract Reserves

Large Thermal Reserves

Regional Hydro Maintenance

Spinning Reserves

Regional Transmission Losses

$\begin{array}{rrrrrrrrrrrrrrr}0 & 0 & 0 & 0 & 0 & 0 & 0 & 0 & 0 & 0 & 0 & 0 & 0 & 0 & 0 \\ 0 & 0 & 0 & 0 & 0 & 0 & 0 & 0 & 0 & 0 & 0 & 0 & 0 & 0 & 0 \\ 0 & 0 & 0 & 0 & 0 & 0 & 0 & 0 & 0 & 0 & 0 & 0 & 0 & 0 & 0 \\ 0 & 0 & 0 & 0 & 0 & 0 & 0 & 0 & 0 & 0 & 0 & 0 & 0 & 0 & 0 \\ -30 & -25 & -8.6 & -9 & -3.8 & 0 & 0 & 0 & -5.2 & -7.4 & -7.6 & -20 & -14 & -49 & -12 \\ 0 & 0 & 0 & 0 & 0 & 0 & 0 & 0 & 0 & 0 & 0 & 0 & 0 & 0 & 0 \\ -761 & -675 & -617 & -667 & -702 & -710 & -653 & -607 & -625 & -622 & -615 & -567 & -726 & -734 & -662\end{array}$

Total Reserves, Maintenance \& Losses

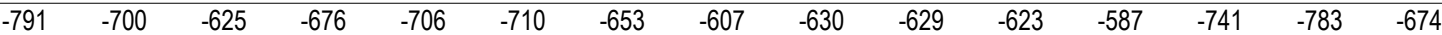

Total Net Resources

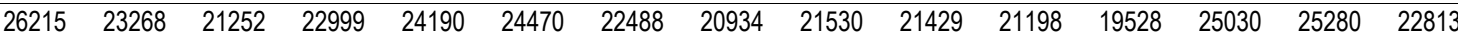

Surplus/Deficits

Firm Surplus/Deficit

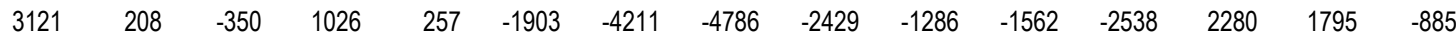

Total Surplus/Deficit

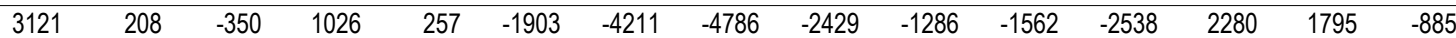


Section 6

Monthly Federal 50 Water Year Surplus/Deficit

Average Energy in Megawatts 
Federal Surplus/Deficit by Water Year

PNW Loads and Resources Study

2004 - 2005 Operating Year

$3 / 31 / 2004$ 2003 White Book

\begin{tabular}{|c|c|c|c|c|c|c|c|c|c|c|c|c|c|c|c|}
\hline Average Energy in Megawatts & Aug1 & Aug16 & Sep & Oct & Nov & Dec & Jan & $\mathrm{Feb}$ & Mar & Apr1 & Apr16 & May & Jun & Jul & Avg \\
\hline 1929 Federal Surplus/Deficit & 2657 & 642 & 330 & 1151 & 1012 & 415 & 977 & -1098 & 167 & -183 & -901 & -768 & 1378 & 2398 & 589 \\
\hline 1930 Federal Surplus/Deficit & 1321 & 418 & 216 & 1336 & 1160 & 388 & -1086 & -137 & 213 & 187 & -475 & -821 & 1265 & 2609 & 489 \\
\hline 1931 Federal Surplus/Deficit & 1777 & 674 & 342 & 1285 & 1128 & 429 & -1039 & -1078 & -7.4 & 711 & -1115 & -319 & 762 & 1702 & 352 \\
\hline 1932 Federal Surplus/Deficit & 1644 & 209 & 363 & 1190 & 1017 & 355 & -1006 & -1230 & 1396 & 3587 & 4900 & 4596 & 4073 & 3527 & 1621 \\
\hline 1933 Federal Surplus/Deficit & 2939 & 2021 & 557 & 1336 & 771 & 1278 & 3431 & 2586 & 164 & 1034 & 1505 & 2948 & 6351 & 5862 & 2419 \\
\hline 1934 Federal Surplus/Deficit & 2470 & 2755 & 1137 & 2435 & 2504 & 5567 & 6560 & 4883 & 3411 & 6258 & 5164 & 4015 & 611 & 3150 & 3550 \\
\hline 1935 Federal Surplus/Deficit & 1054 & -289 & 163 & 1090 & 736 & 829 & 2892 & 3237 & -350 & 855 & 2201 & 2246 & 2957 & 3501 & 1601 \\
\hline 1936 Federal Surplus/Deficit & 2465 & 687 & 342 & 1277 & 1036 & 393 & -353 & -1064 & 394 & 70 & 1924 & 5738 & 2579 & 2479 & 1283 \\
\hline 1937 Federal Surplus/Deficit & 2221 & 450 & 254 & 1326 & 1084 & 375 & -830 & -895 & 159 & 34 & -626 & -339 & 767 & 1969 & 409 \\
\hline 1938 Federal Surplus/Deficit & 1825 & 61 & 241 & 1488 & 953 & 471 & 3700 & 581 & 2416 & 2951 & 2689 & 4781 & 3340 & 3094 & 2069 \\
\hline 1939 Federal Surplus/Deficit & 1582 & 290 & 645 & 1398 & 1040 & 377 & 1344 & -788 & 735 & 902 & 1288 & 3475 & 1275 & 2592 & 1177 \\
\hline 1940 Federal Surplus/Deficit & 2395 & 569 & 259 & 1509 & 976 & 585 & 723 & 14 & 2637 & 1830 & 2000 & 2159 & 2018 & 1973 & 1354 \\
\hline 1941 Federal Surplus/Deficit & 1488 & 140 & 209 & 1299 & 860 & 978 & 1287 & -1036 & 260 & -343 & -762 & 250 & 1596 & 1345 & 609 \\
\hline 1942 Federal Surplus/Deficit & 1267 & 286 & 588 & 1250 & 748 & 2568 & 3820 & -417 & -325 & 387 & 738 & 1890 & 4526 & 4043 & 1669 \\
\hline 1943 Federal Surplus/Deficit & 2907 & 1972 & 548 & 1299 & 874 & 811 & 3080 & 2312 & 3077 & 5928 & 4836 & 4716 & 4335 & 4173 & 2754 \\
\hline 1944 Federal Surplus/Deficit & 2709 & 1885 & 552 & 1405 & 1075 & 359 & 1349 & -1021 & -252 & -126 & -1076 & -801 & 527 & 1187 & 506 \\
\hline 1945 Federal Surplus/Deficit & 1357 & -218 & 325 & 1121 & 1039 & 357 & -960 & -604 & 35 & -58 & -786 & 2152 & 3591 & 2522 & 810 \\
\hline 1946 Federal Surplus/Deficit & 1535 & 1049 & 138 & 1426 & 1048 & 429 & 1793 & 1706 & 3157 & 3407 & 3927 & 5062 & 3706 & 3988 & 2284 \\
\hline 1947 Federal Surplus/Deficit & 3000 & 1669 & 770 & 1288 & 1024 & 3406 & 4052 & 3215 & 3423 & 1795 & 2803 & 4228 & 3388 & 3621 & 2754 \\
\hline 1948 Federal Surplus/Deficit & 3020 & 1099 & 603 & 3443 & 2008 & 2277 & 3947 & 794 & 2017 & 2035 & 3743 & 6208 & 7053 & 4399 & 3141 \\
\hline 1949 Federal Surplus/Deficit & 2604 & 3055 & 1482 & 1574 & 941 & 1046 & 1900 & 639 & 4241 & 2100 & 4639 & 5117 & 3232 & 1872 & 2354 \\
\hline 1950 Federal Surplus/Deficit & 1323 & -177 & -55 & 1317 & 861 & 145 & 2153 & 3629 & 4981 & 4482 & 3836 & 3979 & 6149 & 4536 & 2702 \\
\hline 1951 Federal Surplus/Deficit & 2230 & 2512 & 930 & 2297 & 2512 & 4350 & 5429 & 5376 & 3662 & 4336 & 4364 & 5108 & 2608 & 4445 & 3620 \\
\hline 1952 Federal Surplus/Deficit & 2702 & 2617 & 1221 & 2909 & 1192 & 2700 & 3720 & 2133 & 2436 & 4569 & 4561 & 6113 & 3538 & 2863 & 3004 \\
\hline 1953 Federal Surplus/Deficit & 2721 & 956 & 483 & 1278 & 1127 & 384 & 1165 & 3239 & 637 & -70 & 1168 & 3666 & 5931 & 4615 & 2076 \\
\hline 1954 Federal Surplus/Deficit & 2844 & 1727 & 802 & 1551 & 1110 & 1743 & 2759 & 3476 & 2012 & 3758 & 2641 & 4444 & 5391 & 5316 & 2841 \\
\hline 1955 Federal Surplus/Deficit & 3820 & 2816 & 3374 & 2135 & 1622 & 1864 & 786 & -704 & -193 & 876 & -3.5 & 1632 & 6706 & 6368 & 2279 \\
\hline 1956 Federal Surplus/Deficit & 2616 & 2986 & 694 & 2010 & 1910 & 4028 & 5969 & 2643 & 4382 & 4516 & 6023 & 6159 & 6704 & 4776 & 3946 \\
\hline 1957 Federal Surplus/Deficit & 2974 & 2228 & 746 & 1676 & 911 & 1912 & 2463 & -63 & 1649 & 5112 & 2924 & 5703 & 6416 & 3062 & 2591 \\
\hline 1958 Federal Surplus/Deficit & 1882 & 517 & 394 & 1416 & 1038 & 524 & 1983 & 2193 & 2222 & 1110 & 3541 & 5079 & 4877 & 2634 & 2157 \\
\hline 1959 Federal Surplus/Deficit & 1912 & 788 & 490 & 1304 & 1529 & 2665 & 5452 & 3802 & 2257 & 3254 & 2061 & 3340 & 5357 & 5142 & 2946 \\
\hline 1960 Federal Surplus/Deficit & 2713 & 2049 & 3161 & 4077 & 2991 & 3525 & 3693 & 828 & 2075 & 6348 & 3863 & 2895 & 3725 & 3648 & 3175 \\
\hline 1961 Federal Surplus/Deficit & 3007 & 663 & 553 & 1453 & 1246 & 1011 & 2511 & 2862 & 2910 & 3294 & 2899 & 3593 & 6267 & 3197 & 2545 \\
\hline 1962 Federal Surplus/Deficit & 2082 & 1149 & 203 & 1372 & 1023 & 267 & 2396 & -119 & 207 & 4709 & 4557 & 3050 & 2868 & 3871 & 1782 \\
\hline 1963 Federal Surplus/Deficit & 2528 & 1570 & 437 & 1874 & 1669 & 2637 & 2707 & 1431 & 1342 & 959 & 825 & 3890 & 3496 & 3590 & 2168 \\
\hline 1964 Federal Surplus/Deficit & 3236 & 1785 & 874 & 1198 & 1080 & 637 & 2262 & 637 & 160 & 2057 & 1708 & 2659 & 6391 & 5890 & 2182 \\
\hline 1965 Federal Surplus/Deficit & 2749 & 2459 & 1472 & 2259 & 1200 & 4269 & 6491 & 4995 & 3925 & 3047 & 4473 & 4892 & 4300 & 3168 & 3611 \\
\hline 1966 Federal Surplus/Deficit & 2815 & 2241 & 832 & 1805 & 1250 & 1841 & 3368 & 362 & 461 & 3727 & 1544 & 2973 & 2325 & 3954 & 2028 \\
\hline 1967 Federal Surplus/Deficit & 3042 & 1407 & 299 & 1269 & 1019 & 978 & 3775 & 4394 & 1391 & 2659 & 1050 & 1735 & 5937 & 5223 & 2508 \\
\hline 1968 Federal Surplus/Deficit & 2808 & 2129 & 932 & 1621 & 1142 & 1675 & 3273 & 2488 & 2200 & -353 & 926 & 1884 & 4093 & 4886 & 2246 \\
\hline 1969 Federal Surplus/Deficit & 2969 & 2271 & 1865 & 2383 & 2047 & 2516 & 5311 & 3935 & 2692 & 5510 & 4599 & 5952 & 3951 & 3831 & 3513 \\
\hline 1970 Federal Surplus/Deficit & 2918 & 1003 & 478 & 1391 & 1142 & 803 & 2909 & 2426 & 664 & 99 & 1714 & 2917 & 4480 & 1990 & 1839 \\
\hline 1971 Federal Surplus/Deficit & 1735 & 670 & 216 & 1398 & 1246 & 679 & 4544 & 5965 & 3437 & 3781 & 4082 & 6315 & 6170 & 5107 & 3351 \\
\hline 1972 Federal Surplus/Deficit & 2927 & 3140 & 1099 & 1476 & 1159 & 1821 & 4345 & 5575 & 5994 & 5996 & 2550 & 6016 & 6606 & 5511 & 3909 \\
\hline 1973 Federal Surplus/Deficit & 3471 & 2834 & 1550 & 1641 & 1026 & 2053 & 3730 & -746 & 98 & -499 & -853 & 114 & 1538 & 2322 & 1317 \\
\hline 1974 Federal Surplus/Deficit & 1623 & -36 & 112 & 1030 & 732 & 2866 & 6978 & 5915 & 4742 & 5313 & 5517 & 5440 & 6733 & 6753 & 3959 \\
\hline 1975 Federal Surplus/Deficit & 2993 & 3110 & 1494 & 1162 & 1048 & 911 & 3148 & 1985 & 3454 & 2115 & 1979 & 3792 & 4926 & 5796 & 2735 \\
\hline 1976 Federal Surplus/Deficit & 1829 & 1111 & 873 & 2048 & 2323 & 5119 & 5336 & 3817 & 2852 & 5255 & 3637 & 5526 & 3009 & 5333 & 3513 \\
\hline 1977 Federal Surplus/Deficit & 4375 & 4035 & 3996 & 1792 & 1053 & 538 & 1373 & -1115 & -581 & -627 & -1369 & -51 & 169 & 1155 & 961 \\
\hline 1978 Federal Surplus/Deficit & 1661 & -224 & 83 & 855 & 839 & 733 & 2493 & 908 & 3307 & 2112 & 2459 & 3307 & 2675 & 3983 & 1849 \\
\hline \multicolumn{16}{|l|}{-Ranked Averages- } \\
\hline Top Ten Percent & 2429 & 2212 & 862 & 1814 & 1503 & 3467 & 5842 & 4901 & 4541 & 4642 & 4585 & 5523 & 5390 & 4931 & 3809 \\
\hline Middle Eighty Percent & 2448 & 1366 & 842 & 1634 & 1228 & 1490 & 2738 & 1565 & 1734 & 2424 & 2379 & 3602 & 4026 & 3761 & 2244 \\
\hline Bottom Ten Percent & 2137 & 814 & 339 & 1300 & 1092 & 393 & -126 & -846 & 56 & 125 & -839 & -610 & 940 & 1973 & 469 \\
\hline
\end{tabular}


Federal Surplus/Deficit by Water Year

PNW Loads and Resources Study

2005 - 2006 Operating Year

$3 / 31 / 2004$ 2003 White Book

\begin{tabular}{|c|c|c|c|c|c|c|c|c|c|c|c|c|c|c|c|}
\hline Average Energy in Megawatts & Aug1 & Aug16 & Sep & Oct & Nov & Dec & Jan & $\mathrm{Feb}$ & Mar & Apr1 & Apr16 & May & Jun & Jul & Avg \\
\hline 1929 Federal Surplus/Deficit & 3660 & 1661 & 454 & 1137 & 981 & 397 & 556 & -1497 & -291 & -566 & -898 & -114 & 2107 & 2113 & 648 \\
\hline 1930 Federal Surplus/Deficit & 2315 & 1438 & 341 & 1320 & 1132 & 372 & -1512 & -535 & -243 & -197 & -478 & -166 & 2002 & 2332 & 548 \\
\hline 1931 Federal Surplus/Deficit & 2775 & 1696 & 466 & 1267 & 1100 & 416 & -1465 & -1476 & -464 & 329 & -1113 & 340 & 1506 & 1430 & 414 \\
\hline 1932 Federal Surplus/Deficit & 2642 & 1230 & 489 & 1174 & 987 & 337 & -1433 & -1628 & 936 & 3212 & 4925 & 5266 & 4817 & 3248 & 1683 \\
\hline 1933 Federal Surplus/Deficit & 3941 & 3046 & 682 & 1323 & 740 & 1262 & 3023 & 2207 & -289 & 665 & 1516 & 3621 & 7092 & 5597 & 2487 \\
\hline 1934 Federal Surplus/Deficit & 3473 & 3787 & 1267 & 2429 & 2473 & 5560 & 6159 & 4509 & 2966 & 5888 & 5182 & 4698 & 1349 & 2876 & 3621 \\
\hline 1935 Federal Surplus/Deficit & 2051 & 732 & 291 & 1074 & 704 & 812 & 2483 & 2860 & -798 & 474 & 2210 & 2907 & 3693 & 3227 & 1666 \\
\hline 1936 Federal Surplus/Deficit & 3469 & 1710 & 469 & 1264 & 1008 & 380 & -774 & -1461 & -64 & -309 & 1921 & 6408 & 3326 & 2202 & 1346 \\
\hline 1937 Federal Surplus/Deficit & 3219 & 1470 & 377 & 1311 & 1057 & 361 & -1254 & -1291 & -296 & -347 & -624 & 311 & 1497 & 1690 & 468 \\
\hline 1938 Federal Surplus/Deficit & 2823 & 1084 & 366 & 1474 & 926 & 454 & 3289 & 192 & 1962 & 2575 & 2697 & 5455 & 4074 & 2814 & 2133 \\
\hline 1939 Federal Surplus/Deficit & 2579 & 1306 & 771 & 1384 & 1009 & 358 & 930 & -1184 & 276 & 519 & 1288 & 4138 & 2012 & 2313 & 1238 \\
\hline 1940 Federal Surplus/Deficit & 3396 & 1589 & 383 & 1497 & 949 & 569 & 301 & -386 & 2184 & 1444 & 2014 & 2815 & 2754 & 1695 & 1415 \\
\hline 1941 Federal Surplus/Deficit & 2484 & 1161 & 333 & 1284 & 831 & 963 & 862 & -1435 & -197 & -719 & -748 & 910 & 2328 & 1059 & 669 \\
\hline 1942 Federal Surplus/Deficit & 2260 & 1305 & 710 & 1233 & 716 & 2547 & 3417 & -815 & -783 & 1.2 & 739 & 2549 & 5264 & 3769 & 1730 \\
\hline 1943 Federal Surplus/Deficit & 3912 & 2995 & 672 & 1285 & 842 & 790 & 2663 & 1911 & 2619 & 5549 & 4841 & 5391 & 5067 & 3889 & 2815 \\
\hline 1944 Federal Surplus/Deficit & 3708 & 2911 & 673 & 1390 & 1043 & 337 & 928 & -1421 & -709 & -511 & -1071 & -142 & 1257 & 899 & 564 \\
\hline 1945 Federal Surplus/Deficit & 2350 & 800 & 449 & 1104 & 1008 & 340 & -1388 & -1006 & -424 & -443 & -787 & 2807 & 4323 & 2243 & 868 \\
\hline 1946 Federal Surplus/Deficit & 2527 & 2069 & 261 & 1409 & 1017 & 408 & 1368 & 1311 & 2703 & 3033 & 3936 & 5740 & 4454 & 3710 & 2347 \\
\hline 1947 Federal Surplus/Deficit & 4004 & 2693 & 894 & 1272 & 991 & 3377 & 3647 & 2820 & 2973 & 1424 & 2817 & 4895 & 4135 & 3343 & 2818 \\
\hline 1948 Federal Surplus/Deficit & 4020 & 2118 & 727 & 3437 & 1974 & 2262 & 3533 & 394 & 1568 & 1661 & 3755 & 6879 & 7798 & 4130 & 3207 \\
\hline 1949 Federal Surplus/Deficit & 3605 & 4087 & 1608 & 1561 & 910 & 1026 & 1485 & 239 & 3787 & 1720 & 4653 & 5782 & 3974 & 1589 & 2416 \\
\hline 1950 Federal Surplus/Deficit & 2317 & 840 & 68 & 1301 & 830 & 127 & 1740 & 3237 & 4532 & 4101 & 3850 & 4652 & 6892 & 4256 & 2766 \\
\hline 1951 Federal Surplus/Deficit & 3228 & 3540 & 1054 & 2285 & 2476 & 4334 & 5025 & 4992 & 3209 & 3958 & 4380 & 5785 & 3344 & 4171 & 3686 \\
\hline 1952 Federal Surplus/Deficit & 3704 & 3645 & 1348 & 2901 & 1162 & 2679 & 3308 & 1740 & 1980 & 4185 & 4565 & 6783 & 4275 & 2581 & 3067 \\
\hline 1953 Federal Surplus/Deficit & 3722 & 1973 & 606 & 1262 & 1097 & 367 & 734 & 2849 & 185 & -442 & 1176 & 4337 & 6667 & 4335 & 2138 \\
\hline 1954 Federal Surplus/Deficit & 3846 & 2751 & 927 & 1539 & 1081 & 1724 & 2345 & 3090 & 1562 & 3384 & 2645 & 5121 & 6145 & 5046 & 2908 \\
\hline 1955 Federal Surplus/Deficit & 4828 & 3846 & 3512 & 2126 & 1592 & 1851 & 365 & -1102 & -648 & 495 & 7.9 & 2296 & 7458 & 6105 & 2345 \\
\hline 1956 Federal Surplus/Deficit & 3618 & 4017 & 819 & 2000 & 1876 & 4009 & 5564 & 2253 & 3925 & 4145 & 6036 & 6831 & 7451 & 4502 & 4011 \\
\hline 1957 Federal Surplus/Deficit & 3976 & 3255 & 870 & 1663 & 879 & 1891 & 2048 & -464 & 1193 & 4731 & 2937 & 6374 & 7163 & 2778 & 2654 \\
\hline 1958 Federal Surplus/Deficit & 2877 & 1534 & 517 & 1401 & 1008 & 503 & 1568 & 1795 & 1775 & 731 & 3548 & 5742 & 5626 & 2348 & 2219 \\
\hline 1959 Federal Surplus/Deficit & 2907 & 1808 & 613 & 1290 & 1495 & 2645 & 5044 & 3423 & 1803 & 2885 & 2077 & 4017 & 6104 & 4868 & 3012 \\
\hline 1960 Federal Surplus/Deficit & 3716 & 3076 & 3296 & 4069 & 2958 & 3514 & 3291 & 437 & 1619 & 5985 & 3888 & 3569 & 4466 & 3371 & 3244 \\
\hline 1961 Federal Surplus/Deficit & 4010 & 1681 & 679 & 1441 & 1216 & 993 & 2100 & 2475 & 2463 & 2927 & 2915 & 4275 & 7024 & 2922 & 2613 \\
\hline 1962 Federal Surplus/Deficit & 3081 & 2172 & 326 & 1359 & 991 & 248 & 1982 & -513 & -251 & 4329 & 4579 & 3723 & 3607 & 3593 & 1846 \\
\hline 1963 Federal Surplus/Deficit & 3531 & 2592 & 561 & 1859 & 1637 & 2619 & 2298 & 1035 & 884 & 581 & 837 & 4549 & 4230 & 3313 & 2230 \\
\hline 1964 Federal Surplus/Deficit & 4240 & 2807 & 1000 & 1184 & 1048 & 618 & 1849 & 246 & -296 & 1670 & 1718 & 3324 & 7130 & 5624 & 2245 \\
\hline 1965 Federal Surplus/Deficit & 3751 & 3487 & 1598 & 2249 & 1172 & 4239 & 6082 & 4606 & 3471 & 2669 & 4481 & 5560 & 5027 & 2879 & 3673 \\
\hline 1966 Federal Surplus/Deficit & 3816 & 3264 & 955 & 1792 & 1220 & 1818 & 2956 & -25 & 2.8 & 3356 & 1556 & 3640 & 3065 & 3682 & 2092 \\
\hline 1967 Federal Surplus/Deficit & 4048 & 2427 & 423 & 1256 & 988 & 960 & 3365 & 4013 & 943 & 2294 & 1064 & 2400 & 6681 & 4947 & 2574 \\
\hline 1968 Federal Surplus/Deficit & 3812 & 3157 & 1058 & 1607 & 1111 & 1654 & 2859 & 2098 & 1749 & -736 & 939 & 2542 & 4836 & 4609 & 2309 \\
\hline 1969 Federal Surplus/Deficit & 3972 & 3298 & 1994 & 2371 & 2014 & 2499 & 4900 & 3551 & 2233 & 5137 & 4616 & 6627 & 4702 & 3552 & 3580 \\
\hline 1970 Federal Surplus/Deficit & 3917 & 2024 & 602 & 1379 & 1112 & 783 & 2484 & 2032 & 211 & -274 & 1736 & 3579 & 5208 & 1705 & 1900 \\
\hline 1971 Federal Surplus/Deficit & 2731 & 1687 & 335 & 1381 & 1213 & 655 & 4124 & 5578 & 2980 & 3401 & 4096 & 6984 & 6907 & 4831 & 3412 \\
\hline 1972 Federal Surplus/Deficit & 3930 & 4171 & 1223 & 1462 & 1126 & 1798 & 3928 & 5189 & 5544 & 5631 & 2569 & 6686 & 7347 & 5246 & 3975 \\
\hline 1973 Federal Surplus/Deficit & 4477 & 3865 & 1676 & 1628 & 993 & 2031 & 3314 & -1145 & -360 & -877 & -841 & 770 & 2276 & 2042 & 1378 \\
\hline 1974 Federal Surplus/Deficit & 2621 & 983 & 236 & 1011 & 696 & 2840 & 6570 & 5533 & 4290 & 4937 & 5529 & 6107 & 7474 & 6486 & 4023 \\
\hline 1975 Federal Surplus/Deficit & 3996 & 4141 & 1621 & 1146 & 1016 & 889 & 2734 & 1596 & 2999 & 1742 & 1990 & 4459 & 5656 & 5520 & 2798 \\
\hline 1976 Federal Surplus/Deficit & 2827 & 2131 & 997 & 2034 & 2287 & 5100 & 4927 & 3432 & 2397 & 4878 & 3650 & 6194 & 3741 & 5063 & 3576 \\
\hline 1977 Federal Surplus/Deficit & 5386 & 5068 & 4136 & 1780 & 1022 & 518 & 953 & -1514 & -1037 & -1008 & -1360 & 614 & 906 & 876 & 1025 \\
\hline 1978 Federal Surplus/Deficit & 2658 & 797 & 208 & 838 & 807 & 705 & 2070 & 506 & 2856 & 1744 & 2476 & 3978 & 3407 & 3702 & 1909 \\
\hline \multicolumn{16}{|l|}{-Ranked Averages- } \\
\hline Top Ten Percent & 3430 & 3240 & 986 & 1802 & 1469 & 3444 & 5434 & 4514 & 4088 & 4268 & 4599 & 6194 & 6129 & 4657 & 3874 \\
\hline ghty Percent & 3448 & 2389 & 967 & 1620 & 1197 & 1471 & 2323 & 1172 & 1280 & 2048 & 2391 & 4270 & 4766 & 3484 & 2307 \\
\hline Bottom Ten Percent & 3135 & 1835 & 462 & 1285 & 1063 & 377 & -549 & -1244 & -401 & -259 & -837 & 46 & 1674 & 1693 & 528 \\
\hline
\end{tabular}


Federal Surplus/Deficit by Water Year

PNW Loads and Resources Study

2006 - 2007 Operating Year 2003 White Book

\begin{tabular}{|c|c|c|c|c|c|c|c|c|c|c|c|c|c|c|c|}
\hline Average Energy in Megawatts & Aug1 & Aug16 & Sep & Oct & Nov & Dec & Jan & $\mathrm{Feb}$ & Mar & Apr1 & Apr16 & May & Jun & Jul & Avg \\
\hline 1929 Federal Surplus/Deficit & 3401 & 1388 & 192 & 795 & 578 & -2.1 & 88 & -1961 & -690 & -727 & -1054 & -631 & 1424 & 1673 & 247 \\
\hline 1930 Federal Surplus/Deficit & 2045 & 1165 & 78 & 974 & 733 & -25 & -1987 & -993 & -640 & -356 & -635 & -685 & 1320 & 1895 & 148 \\
\hline 1931 Federal Surplus/Deficit & 2508 & 1424 & 203 & 921 & 700 & 18 & -1939 & -1938 & -862 & 171 & -1272 & -177 & 825 & 994 & 13 \\
\hline 1932 Federal Surplus/Deficit & 2376 & 960 & 226 & 829 & 586 & -65 & -1909 & -2091 & 541 & 3064 & 4790 & 4764 & 4145 & 2815 & 1286 \\
\hline 1933 Federal Surplus/Deficit & 3676 & 2774 & 420 & 981 & 340 & 863 & 2566 & 1763 & -683 & 514 & 1366 & 3112 & 6427 & 5174 & 2094 \\
\hline 1934 Federal Surplus/Deficit & 3209 & 3522 & 1011 & 2092 & 2072 & 5178 & 5715 & 4073 & 2584 & 5750 & 5050 & 4201 & 661 & 2440 & 3233 \\
\hline 1935 Federal Surplus/Deficit & 1785 & 462 & 31 & 729 & 303 & 411 & 2024 & 2418 & -1190 & 319 & 2064 & 2396 & 3013 & 2796 & 1270 \\
\hline 1936 Federal Surplus/Deficit & 3207 & 1440 & 207 & 921 & 608 & -18 & -1243 & -1924 & -461 & -470 & 1775 & 5908 & 2649 & 1766 & 949 \\
\hline 1937 Federal Surplus/Deficit & 2951 & 1197 & 114 & 967 & 657 & -37 & -1728 & -1752 & -693 & -507 & -781 & -211 & 809 & 1252 & 67 \\
\hline 1938 Federal Surplus/Deficit & 2556 & 814 & 104 & 1130 & 527 & 56 & 2830 & -259 & 1573 & 2424 & 2552 & 4955 & 3395 & 2381 & 1739 \\
\hline 1939 Federal Surplus/Deficit & 2309 & 1027 & 510 & 1042 & 607 & -42 & 469 & -1642 & -119 & 364 & 1139 & 3632 & 1328 & 1876 & 840 \\
\hline 1940 Federal Surplus/Deficit & 3132 & 1318 & 121 & 1155 & 550 & 170 & -168 & -845 & 1798 & 1289 & 1876 & 2304 & 2071 & 1257 & 1018 \\
\hline 1941 Federal Surplus/Deficit & 2215 & 891 & 70 & 942 & 432 & 566 & 390 & -1896 & -595 & -874 & -896 & 397 & 1644 & 618 & 270 \\
\hline 1942 Federal Surplus/Deficit & 1989 & 1031 & 447 & 889 & 315 & 2148 & 2967 & -1273 & -1182 & -156 & 590 & 2038 & 4591 & 3340 & 1334 \\
\hline 1943 Federal Surplus/Deficit & 3649 & 2723 & 410 & 943 & 440 & 389 & 2206 & 1459 & 2233 & 5407 & 4704 & 4892 & 4392 & 3458 & 2422 \\
\hline 1944 Federal Surplus/Deficit & 3439 & 2638 & 410 & 1048 & 640 & -62 & 460 & -1885 & -1110 & -673 & -1224 & -657 & 571 & 456 & 163 \\
\hline 1945 Federal Surplus/Deficit & 2079 & 526 & 186 & 759 & 606 & -60 & -1864 & -1468 & -823 & -604 & -947 & 2299 & 3647 & 1810 & 468 \\
\hline 1946 Federal Surplus/Deficit & 2254 & 1793 & -2.1 & 1064 & 616 & 11 & 901 & 859 & 2319 & 2882 & 3795 & 5242 & 3780 & 3278 & 1953 \\
\hline 1947 Federal Surplus/Deficit & 3740 & 2422 & 634 & 930 & 590 & 2978 & 3198 & 2375 & 2588 & 1272 & 2672 & 4392 & 3463 & 2911 & 2426 \\
\hline 1948 Federal Surplus/Deficit & 3752 & 1841 & 465 & 3105 & 1573 & 1869 & 3079 & -61 & 1181 & 1507 & 3612 & 6385 & 7137 & 3707 & 2816 \\
\hline 1949 Federal Surplus/Deficit & 3338 & 3819 & 1349 & 1221 & 509 & 626 & 1023 & -220 & 3405 & 1569 & 4514 & 5280 & 3295 & 1150 & 2021 \\
\hline 1950 Federal Surplus/Deficit & 2045 & 563 & -196 & 957 & 429 & -271 & 1280 & 2788 & 4154 & 3955 & 3710 & 4149 & 6227 & 3828 & 2374 \\
\hline 1951 Federal Surplus/Deficit & 2957 & 3270 & 792 & 1948 & 2074 & 3946 & 4578 & 4558 & 2826 & 3811 & 4242 & 5287 & 2664 & 3743 & 3296 \\
\hline 1952 Federal Surplus/Deficit & 3437 & 3375 & 1089 & 2566 & 762 & 2284 & 2852 & 1294 & 1589 & 4038 & 4426 & 6289 & 3599 & 2149 & 2676 \\
\hline 1953 Federal Surplus/Deficit & 3455 & 1694 & 343 & 919 & 696 & -31 & 261 & 2407 & -205 & -598 & 1025 & 3830 & 5998 & 3907 & 1743 \\
\hline 1954 Federal Surplus/Deficit & 3580 & 2477 & 666 & 1199 & 680 & 1325 & 1887 & 2648 & 1173 & 3234 & 2499 & 4621 & 5477 & 4621 & 2516 \\
\hline 1955 Federal Surplus/Deficit & 4567 & 3578 & 3263 & 1788 & 1192 & 1455 & -103 & -1563 & -1047 & 341 & -135 & 1780 & 6794 & 5685 & 1952 \\
\hline 1956 Federal Surplus/Deficit & 3353 & 3750 & 558 & 1661 & 1473 & 3619 & 5118 & 1810 & 3541 & 4001 & 5904 & 6336 & 6788 & 4075 & 3624 \\
\hline 1957 Federal Surplus/Deficit & 3710 & 2983 & 609 & 1323 & 478 & 1493 & 1588 & -923 & 804 & 4584 & 2795 & 5881 & 6498 & 2342 & 2261 \\
\hline 1958 Federal Surplus/Deficit & 2606 & 1255 & 254 & 1059 & 607 & 102 & 1107 & 1344 & 1390 & 576 & 3404 & 5241 & 4959 & 1910 & 1824 \\
\hline 1959 Federal Surplus/Deficit & 2636 & 1533 & 351 & 949 & 1094 & 2249 & 4595 & 2985 & 1412 & 2737 & 1932 & 3514 & 5438 & 4438 & 2620 \\
\hline 1960 Federal Surplus/Deficit & 3452 & 2805 & 3046 & 3738 & 2559 & 3124 & 2842 & -14 & 1230 & 5849 & 3749 & 3061 & 3790 & 2939 & 2853 \\
\hline 1961 Federal Surplus/Deficit & 3746 & 1405 & 419 & 1101 & 816 & 593 & 1640 & 2031 & 2078 & 2777 & 2766 & 3774 & 6359 & 2487 & 2220 \\
\hline 1962 Federal Surplus/Deficit & 2815 & 1901 & 63 & 1017 & 589 & -152 & 1521 & -967 & -649 & 4180 & 4445 & 3216 & 2928 & 3162 & 1450 \\
\hline 1963 Federal Surplus/Deficit & 3266 & 2319 & 298 & 1520 & 1236 & 2224 & 1842 & 588 & 487 & 428 & 692 & 4041 & 3557 & 2882 & 1836 \\
\hline 1964 Federal Surplus/Deficit & 3975 & 2532 & 740 & 842 & 646 & 217 & 1388 & -206 & -694 & 1514 & 1568 & 2815 & 6465 & 5203 & 1851 \\
\hline 1965 Federal Surplus/Deficit & 3484 & 3215 & 1340 & 1913 & 773 & 3843 & 5638 & 4170 & 3086 & 2517 & 4343 & 5061 & 4353 & 2445 & 3283 \\
\hline 1966 Federal Surplus/Deficit & 3548 & 2988 & 696 & 1452 & 821 & 1418 & 2498 & -474 & -394 & 3213 & 1411 & 3134 & 2386 & 3252 & 1697 \\
\hline 1967 Federal Surplus/Deficit & 3787 & 2154 & 161 & 913 & 586 & 560 & 2909 & 3575 & 553 & 2145 & 913 & 1890 & 6016 & 4519 & 2182 \\
\hline 1968 Federal Surplus/Deficit & 3548 & 2887 & 798 & 1267 & 711 & 1255 & 2402 & 1654 & 1362 & -895 & 792 & 2027 & 4166 & 4178 & 1915 \\
\hline 1969 Federal Surplus/Deficit & 3709 & 3027 & 1739 & 2035 & 1613 & 2105 & 4451 & 3114 & 1844 & 4994 & 4480 & 6132 & 4032 & 3119 & 3191 \\
\hline 1970 Federal Surplus/Deficit & 3650 & 1750 & 340 & 1038 & 711 & 382 & 2020 & 1587 & -182 & -431 & 1592 & 3070 & 4532 & 1268 & 1504 \\
\hline 1971 Federal Surplus/Deficit & 2460 & 1407 & 71 & 1038 & 810 & 256 & 3670 & 5144 & 2594 & 3251 & 3957 & 6490 & 6241 & 4405 & 3021 \\
\hline 1972 Federal Surplus/Deficit & 3665 & 3902 & 964 & 1121 & 726 & 1399 & 3472 & 4753 & 5169 & 5492 & 2427 & 6191 & 6682 & 4825 & 3587 \\
\hline 1973 Federal Surplus/Deficit & 4215 & 3597 & 1417 & 1287 & 592 & 1633 & 2857 & -1606 & -758 & -1034 & -990 & 252 & 1594 & 1604 & 981 \\
\hline 1974 Federal Surplus/Deficit & 2353 & 710 & -28 & 665 & 294 & 2441 & 6128 & 5099 & 3911 & 4794 & 5396 & 5610 & 6810 & 6066 & 3635 \\
\hline 1975 Federal Surplus/Deficit & 3729 & 3872 & 1363 & 804 & 613 & 489 & 2276 & 1150 & 2611 & 1591 & 1842 & 3954 & 4983 & 5097 & 2405 \\
\hline 1976 Federal Surplus/Deficit & 2557 & 1853 & 738 & 1695 & 1886 & 4715 & 4480 & 2992 & 2013 & 4736 & 3510 & 5697 & 3062 & 4638 & 3187 \\
\hline 1977 Federal Surplus/Deficit & 5126 & 4801 & 3889 & 1441 & 619 & 119 & 486 & -1977 & -1437 & -1170 & -1513 & 98 & 219 & 434 & 626 \\
\hline 1978 Federal Surplus/Deficit & 2391 & 526 & -55 & 492 & 404 & 302 & 1606 & 50 & 2471 & 1595 & 2331 & 3473 & 2725 & 3271 & 1513 \\
\hline \multicolumn{16}{|l|}{-Ranked Averages- } \\
\hline Top Ten Percent & 3163 & 2970 & 726 & 1461 & 1068 & 3050 & 4987 & 4078 & 3707 & 4123 & 4462 & 5697 & 5459 & 4231 & 3485 \\
\hline Middle Eighty Percent & 3182 & 2116 & 707 & 1279 & 796 & 1073 & 1863 & 722 & 889 & 1897 & 2246 & 3766 & 4092 & 3053 & 1913 \\
\hline Bottom Ten Percent & 2869 & 1562 & 199 & 941 & 662 & -22 & -1021 & -1706 & -799 & -419 & -993 & -472 & 990 & 1254 & 128 \\
\hline
\end{tabular}


Federal Surplus/Deficit by Water Year

PNW Loads and Resources Study

2007 - 2008 Operating Year 2003 White Book

\begin{tabular}{|c|c|c|c|c|c|c|c|c|c|c|c|c|c|c|c|}
\hline Average Energy in Megawatts & Aug1 & Aug16 & Sep & Oct & Nov & Dec & Jan & $\mathrm{Feb}$ & Mar & Apr1 & Apr16 & May & Jun & Jul & Avg \\
\hline 1929 Federal Surplus/Deficit & 2871 & 842 & -269 & 373 & 149 & -436 & 168 & -1836 & -618 & -644 & -978 & -86 & 2147 & 1764 & 200 \\
\hline 1930 Federal Surplus/Deficit & 1502 & 620 & -381 & 549 & 310 & -456 & -1915 & -865 & -566 & -273 & -564 & -140 & 2052 & 1991 & 102 \\
\hline 1931 Federal Surplus/Deficit & 1971 & 882 & -257 & 493 & 275 & -410 & -1866 & -1811 & -788 & 256 & -1199 & 374 & 1565 & 1091 & -31 \\
\hline 1932 Federal Surplus/Deficit & 1838 & 414 & -232 & 404 & 161 & -501 & -1837 & -1964 & 613 & 3164 & 4900 & 5335 & 4890 & 2918 & 1245 \\
\hline 1933 Federal Surplus/Deficit & 3145 & 2238 & -38 & 560 & -87 & 430 & 2666 & 1918 & -603 & 617 & 1453 & 3679 & 7174 & 5294 & 2060 \\
\hline 1934 Federal Surplus/Deficit & 2680 & 2997 & 561 & 1680 & 1645 & 4763 & 5827 & 4238 & 2679 & 5863 & 5155 & 4786 & 1391 & 2537 & 3205 \\
\hline 1935 Federal Surplus/Deficit & 1245 & -84 & -422 & 303 & -126 & -24 & 2122 & 2576 & -1105 & 409 & 2152 & 2952 & 3744 & 2901 & 1232 \\
\hline 1936 Federal Surplus/Deficit & 2679 & 898 & -249 & 499 & 184 & -446 & -1163 & -1797 & -388 & -382 & 1851 & 6480 & 3392 & 1864 & 908 \\
\hline 1937 Federal Surplus/Deficit & 2414 & 652 & -347 & 544 & 234 & -467 & -1653 & -1623 & -617 & -420 & -706 & 328 & 1531 & 1345 & 20 \\
\hline 1938 Federal Surplus/Deficit & 2018 & 272 & -355 & 706 & 104 & -377 & 2926 & -117 & 1654 & 2521 & 2640 & 5531 & 4126 & 2483 & 1701 \\
\hline 1939 Federal Surplus/Deficit & 1772 & 480 & 53 & 620 & 180 & -477 & 561 & -1511 & -45 & 452 & 1216 & 4193 & 2058 & 1971 & 797 \\
\hline 1940 Federal Surplus/Deficit & 2600 & 773 & -339 & 734 & 127 & -262 & -89 & -718 & 1881 & 1372 & 1972 & 2856 & 2801 & 1350 & 975 \\
\hline 1941 Federal Surplus/Deficit & 1675 & 346 & -389 & 520 & 7.0 & 135 & 465 & -1770 & -522 & -780 & -804 & 952 & 2371 & 707 & 224 \\
\hline 1942 Federal Surplus/Deficit & 1446 & 487 & -15 & 462 & -114 & 1711 & 3075 & -1144 & -1110 & -73 & 667 & 2590 & 5329 & 3449 & 1292 \\
\hline 1943 Federal Surplus/Deficit & 3123 & 2186 & -50 & 521 & 12 & -50 & 2297 & 1588 & 2312 & 5508 & 4794 & 5469 & 5124 & 3557 & 2382 \\
\hline 1944 Federal Surplus/Deficit & 2908 & 2106 & -54 & 626 & 211 & -500 & 541 & -1762 & -1039 & -592 & -1144 & -106 & 1294 & 542 & 116 \\
\hline 1945 Federal Surplus/Deficit & 1537 & -22 & -274 & 333 & 179 & -494 & -1795 & -1345 & -752 & -521 & -877 & 2849 & 4377 & 1912 & 421 \\
\hline 1946 Federal Surplus/Deficit & 1710 & 1250 & -463 & 638 & 189 & -425 & 978 & 993 & 2404 & 2982 & 3888 & 5823 & 4527 & 3383 & 1913 \\
\hline 1947 Federal Surplus/Deficit & 3214 & 1884 & 175 & 506 & 160 & 2532 & 3306 & 2514 & 2677 & 1373 & 2765 & 4960 & 4211 & 3016 & 2390 \\
\hline 1948 Federal Surplus/Deficit & 3220 & 1296 & 5.8 & 2696 & 1144 & 1441 & 3175 & 68 & 1268 & 1604 & 3706 & 6962 & 7889 & 3827 & 2782 \\
\hline 1949 Federal Surplus/Deficit & 2810 & 3295 & 892 & 801 & 80 & 188 & 1113 & -94 & 3492 & 1661 & 4613 & 5845 & 4033 & 1242 & 1982 \\
\hline 1950 Federal Surplus/Deficit & 1503 & 13 & -658 & 531 & 1 & -704 & 1374 & 2928 & 4248 & 4050 & 3807 & 4723 & 6976 & 3937 & 2337 \\
\hline 1951 Federal Surplus/Deficit & 2424 & 2741 & 333 & 1530 & 1640 & 3519 & 4687 & 4715 & 2912 & 3910 & 4343 & 5867 & 3396 & 3854 & 3263 \\
\hline 1952 Federal Surplus/Deficit & 2908 & 2846 & 633 & 2153 & 336 & 1849 & 2949 & 1434 & 1667 & 4131 & 4515 & 6865 & 4333 & 2251 & 2639 \\
\hline 1953 Federal Surplus/Deficit & 2925 & 1148 & -119 & 495 & 270 & -463 & 330 & 2553 & -123 & -501 & 1109 & 4398 & 6737 & 4015 & 1703 \\
\hline 1954 Federal Surplus/Deficit & 3052 & 1942 & 208 & 779 & 255 & 889 & 1980 & 2798 & 1258 & 3334 & 2582 & 5200 & 6234 & 4737 & 2483 \\
\hline 1955 Federal Surplus/Deficit & 4046 & 3052 & 2824 & 1373 & 766 & 1028 & -22 & -1436 & -972 & 430 & -44 & 2336 & 7550 & 5808 & 1916 \\
\hline 1956 Federal Surplus/Deficit & 2824 & 3223 & 100 & 1245 & 1041 & 3188 & 5227 & 1955 & 3624 & 4109 & 6006 & 6913 & 7540 & 4189 & 3592 \\
\hline 1957 Federal Surplus/Deficit & 3182 & 2451 & 150 & 903 & 49 & 1056 & 1680 & -798 & 883 & 4679 & 2889 & 6458 & 7251 & 2438 & 2223 \\
\hline 1958 Federal Surplus/Deficit & 2067 & 708 & -206 & 635 & 180 & -337 & 1197 & 1477 & 1481 & 667 & 3491 & 5806 & 5712 & 2003 & 1785 \\
\hline 1959 Federal Surplus/Deficit & 2096 & 989 & -109 & 529 & 664 & 1815 & 4700 & 3144 & 1492 & 2842 & 2028 & 4090 & 6189 & 4547 & 2587 \\
\hline 1960 Federal Surplus/Deficit & 2924 & 2273 & 2603 & 3328 & 2130 & 2700 & 2952 & 125 & 1309 & 5967 & 3855 & 3630 & 4529 & 3043 & 2822 \\
\hline 1961 Federal Surplus/Deficit & 3219 & 859 & -37 & 681 & 390 & 157 & 1736 & 2177 & 2168 & 2883 & 2858 & 4357 & 7119 & 2586 & 2187 \\
\hline 1962 Federal Surplus/Deficit & 2280 & 1360 & -397 & 597 & 161 & -588 & 1613 & -832 & -577 & 4274 & 4553 & 3785 & 3663 & 3269 & 1411 \\
\hline 1963 Federal Surplus/Deficit & 2738 & 1779 & -162 & 1100 & 808 & 1792 & 1940 & 725 & 560 & 521 & 783 & 4596 & 4291 & 2987 & 1796 \\
\hline 1964 Federal Surplus/Deficit & 3448 & 1993 & 283 & 420 & 218 & -219 & 1481 & -66 & -620 & 1596 & 1654 & 3376 & 7211 & 5324 & 1813 \\
\hline 1965 Federal Surplus/Deficit & 2957 & 2685 & 886 & 1498 & 349 & 3398 & 5744 & 4320 & 3171 & 2612 & 4436 & 5632 & 5082 & 2542 & 3247 \\
\hline 1966 Federal Surplus/Deficit & 3020 & 2454 & 237 & 1032 & 396 & 980 & 2594 & -330 & -321 & 3320 & 1502 & 3699 & 3123 & 3359 & 1660 \\
\hline 1967 Federal Surplus/Deficit & 3262 & 1609 & -299 & 492 & 159 & 126 & 3008 & 3733 & 640 & 2254 & 1003 & 2450 & 6766 & 4632 & 2148 \\
\hline 1968 Federal Surplus/Deficit & 3021 & 2357 & 342 & 847 & 284 & 817 & 2495 & 1799 & 1448 & -810 & 883 & 2577 & 4912 & 4284 & 1878 \\
\hline 1969 Federal Surplus/Deficit & 3184 & 2496 & 1288 & 1619 & 1184 & 1674 & 4553 & 3270 & 1922 & 5101 & 4582 & 6711 & 4784 & 3223 & 3159 \\
\hline 1970 Federal Surplus/Deficit & 3118 & 1208 & -119 & 618 & 284 & -55 & 2098 & 1727 & -101 & -336 & 1694 & 3628 & 5260 & 1362 & 1462 \\
\hline 1971 Federal Surplus/Deficit & 1922 & 860 & -394 & 613 & 379 & -184 & 3761 & 5298 & 2674 & 3344 & 4055 & 7065 & 6985 & 4514 & 2983 \\
\hline 1972 Federal Surplus/Deficit & 3139 & 3377 & 506 & 698 & 296 & 960 & 3563 & 4907 & 5265 & 5608 & 2527 & 6767 & 7429 & 4946 & 3555 \\
\hline 1973 Federal Surplus/Deficit & 3693 & 3072 & 961 & 867 & 163 & 1196 & 2949 & -1479 & -685 & -943 & -902 & 799 & 2328 & 1698 & 938 \\
\hline 1974 Federal Surplus/Deficit & 1815 & 163 & -488 & 236 & -138 & 1998 & 6235 & 5258 & 4002 & 4898 & 5498 & 6182 & 7558 & 6187 & 3601 \\
\hline 1975 Federal Surplus/Deficit & 3203 & 3348 & 908 & 380 & 184 & 50 & 2368 & 1294 & 2692 & 1690 & 1930 & 4521 & 5715 & 5209 & 2367 \\
\hline 1976 Federal Surplus/Deficit & 2024 & 1315 & 279 & 1275 & 1453 & 4286 & 4586 & 3145 & 2096 & 4839 & 3606 & 6270 & 3790 & 4753 & 3152 \\
\hline 1977 Federal Surplus/Deficit & 4611 & 4280 & 3453 & 1023 & 190 & -317 & 569 & -1852 & -1364 & -1086 & -1429 & 656 & 949 & 522 & 585 \\
\hline 1978 Federal Surplus/Deficit & 1851 & -19 & -515 & 64 & -26 & -145 & 1686 & 176 & 2559 & 1702 & 2428 & 4043 & 3451 & 3374 & 1471 \\
\hline \multicolumn{16}{|l|}{-Ranked Averages- } \\
\hline Top Ten Percent & 2632 & 2438 & 267 & 1042 & 638 & 2613 & 5091 & 4231 & 3795 & 4227 & 4562 & 6272 & 6201 & 4344 & 3452 \\
\hline Middle Eighty Percent & 2650 & 1578 & 250 & 858 & 368 & 639 & 1955 & 861 & 970 & 1993 & 2338 & 4333 & 4832 & 3157 & 1875 \\
\hline Bottom Ten Percent & 2333 & 1020 & -261 & 517 & 236 & -454 & -945 & -1579 & -726 & -335 & -918 & 74 & 1718 & 1347 & 81 \\
\hline
\end{tabular}


Federal Surplus/Deficit by Water Year

PNW Loads and Resources Study

2008 - 2009 Operating Year 2003 White Book

\begin{tabular}{|c|c|c|c|c|c|c|c|c|c|c|c|c|c|c|c|}
\hline Average Energy in Megawatts & Aug1 & Aug16 & Sep & Oct & Nov & Dec & Jan & $\mathrm{Feb}$ & Mar & Apr1 & Apr16 & May & Jun & Jul & Avg \\
\hline 1929 Federal Surplus/Deficit & 2960 & 925 & -185 & 460 & 223 & -368 & 131 & -1931 & -653 & -675 & -997 & -572 & 1497 & 1755 & 122 \\
\hline 1930 Federal Surplus/Deficit & 1588 & 703 & -298 & 633 & 386 & -387 & -1957 & -958 & -600 & -302 & -583 & -627 & 1402 & 1983 & 23 \\
\hline 1931 Federal Surplus/Deficit & 2059 & 967 & -174 & 577 & 351 & -340 & -1907 & -1906 & -823 & 229 & -1219 & -111 & 917 & 1084 & -110 \\
\hline 1932 Federal Surplus/Deficit & 1926 & 498 & -149 & 489 & 236 & -434 & -1879 & -2059 & 581 & 3139 & 4893 & 4858 & 4246 & 2911 & 1169 \\
\hline 1933 Federal Surplus/Deficit & 3234 & 2325 & 46 & 646 & -11 & 499 & 2635 & 1834 & -635 & 592 & 1440 & 3201 & 6531 & 5290 & 1986 \\
\hline 1934 Federal Surplus/Deficit & 2769 & 3087 & 648 & 1770 & 1721 & 4840 & 5798 & 4156 & 2653 & 5841 & 5148 & 4310 & 739 & 2529 & 3132 \\
\hline 1935 Federal Surplus/Deficit & 1333 & 0.6 & -337 & 388 & -51 & 44 & 2091 & 2494 & -1136 & 382 & 2140 & 2470 & 3097 & 2895 & 1157 \\
\hline 1936 Federal Surplus/Deficit & 2768 & 984 & -165 & 585 & 260 & -377 & -1201 & -1892 & -422 & -411 & 1840 & 6003 & 2747 & 1856 & 832 \\
\hline 1937 Federal Surplus/Deficit & 2502 & 736 & -263 & 629 & 310 & -398 & -1693 & -1717 & -652 & -449 & -724 & -161 & 879 & 1336 & -58 \\
\hline 1938 Federal Surplus/Deficit & 2106 & 358 & -271 & 791 & 180 & -308 & 2894 & -205 & 1625 & 2496 & 2629 & 5055 & 3479 & 2477 & 1626 \\
\hline 1939 Federal Surplus/Deficit & 1859 & 563 & 138 & 706 & 254 & -409 & 528 & -1604 & -78 & 425 & 1203 & 3713 & 1407 & 1963 & 720 \\
\hline 1940 Federal Surplus/Deficit & 2688 & 857 & -256 & 821 & 204 & -194 & -127 & -811 & 1853 & 1345 & 1964 & 2374 & 2151 & 1342 & 899 \\
\hline 1941 Federal Surplus/Deficit & 1762 & 431 & -306 & 606 & 83 & 205 & 425 & -1864 & -556 & -806 & -817 & 468 & 1721 & 697 & 147 \\
\hline 1942 Federal Surplus/Deficit & 1533 & 572 & 69 & 548 & -38 & 1779 & 3047 & -1236 & -1145 & -100 & 654 & 2109 & 4685 & 3444 & 1216 \\
\hline 1943 Federal Surplus/Deficit & 3212 & 2273 & 35 & 607 & 86 & 18 & 2265 & 1500 & 2284 & 5485 & 4785 & 4993 & 4479 & 3550 & 2308 \\
\hline 1944 Federal Surplus/Deficit & 2996 & 2193 & 30 & 712 & 285 & -432 & 504 & -1857 & -1075 & -623 & -1161 & -591 & 642 & 531 & 38 \\
\hline 1945 Federal Surplus/Deficit & 1623 & 61 & -191 & 417 & 254 & -426 & -1837 & -1440 & -787 & -552 & -897 & 2369 & 3731 & 1905 & 343 \\
\hline 1946 Federal Surplus/Deficit & 1796 & 1336 & -380 & 722 & 264 & -356 & 941 & 905 & 2376 & 2957 & 3879 & 5347 & 3884 & 3376 & 1839 \\
\hline 1947 Federal Surplus/Deficit & 3303 & 1971 & 260 & 592 & 235 & 2600 & 3278 & 2429 & 2650 & 1348 & 2756 & 4483 & 3567 & 3009 & 2316 \\
\hline 1948 Federal Surplus/Deficit & 3308 & 1381 & 90 & 2787 & 1219 & 1513 & 3145 & -23 & 1240 & 1578 & 3696 & 6486 & 7245 & 3823 & 2709 \\
\hline 1949 Federal Surplus/Deficit & 2899 & 3384 & 978 & 889 & 155 & 256 & 1080 & -187 & 3465 & 1636 & 4604 & 5367 & 3387 & 1234 & 1907 \\
\hline 1950 Federal Surplus/Deficit & 1589 & 96 & -575 & 616 & 76 & -635 & 1341 & 2841 & 4222 & 4026 & 3798 & 4247 & 6333 & 3932 & 2263 \\
\hline 1951 Federal Surplus/Deficit & 2512 & 2829 & 417 & 1619 & 1715 & 3594 & 4659 & 4634 & 2885 & 3885 & 4335 & 5391 & 2750 & 3849 & 3191 \\
\hline 1952 Federal Surplus/Deficit & 2997 & 2934 & 719 & 2243 & 412 & 1919 & 2919 & 1349 & 1636 & 4106 & 4505 & 6389 & 3688 & 2245 & 2566 \\
\hline 1953 Federal Surplus/Deficit & 3014 & 1233 & -35 & 580 & 345 & -394 & 290 & 2470 & -152 & -527 & 1096 & 3920 & 6094 & 4009 & 1628 \\
\hline 1954 Federal Surplus/Deficit & 3141 & 2029 & 293 & 867 & 331 & 958 & 1949 & 2714 & 1230 & 3309 & 2571 & 4724 & 5591 & 4733 & 2410 \\
\hline 1955 Federal Surplus/Deficit & 4137 & 3141 & 2914 & 1462 & 842 & 1098 & -59 & -1531 & -1007 & 404 & -53 & 1853 & 6907 & 5804 & 1842 \\
\hline 1956 Federal Surplus/Deficit & 2913 & 3312 & 185 & 1333 & 1116 & 3262 & 5198 & 1871 & 3596 & 4086 & 5999 & 6437 & 6897 & 4184 & 3519 \\
\hline 1957 Federal Surplus/Deficit & 3272 & 2539 & 235 & 991 & 124 & 1125 & 1647 & -891 & 854 & 4654 & 2880 & 5982 & 6608 & 2430 & 2148 \\
\hline 1958 Federal Surplus/Deficit & 2155 & 792 & -123 & 721 & 255 & -270 & 1164 & 1388 & 1454 & 641 & 3481 & 5328 & 5070 & 1994 & 1710 \\
\hline 1959 Federal Surplus/Deficit & 2183 & 1074 & -25 & 616 & 739 & 1885 & 4671 & 3062 & 1462 & 2818 & 2018 & 3614 & 5547 & 4542 & 2513 \\
\hline 1960 Federal Surplus/Deficit & 3013 & 2361 & 2693 & 3419 & 2206 & 2773 & 2923 & 36 & 1279 & 5946 & 3847 & 3152 & 3885 & 3036 & 2749 \\
\hline 1961 Federal Surplus/Deficit & 3309 & 942 & 48 & 768 & 466 & 225 & 1703 & 2093 & 2141 & 2859 & 2847 & 3881 & 6477 & 2579 & 2113 \\
\hline 1962 Federal Surplus/Deficit & 2368 & 1445 & -314 & 683 & 235 & -521 & 1581 & -921 & -612 & 4248 & 4546 & 3307 & 3017 & 3263 & 1335 \\
\hline 1963 Federal Surplus/Deficit & 2828 & 1866 & -78 & 1188 & 884 & 1863 & 1910 & 639 & 526 & 495 & 771 & 4117 & 3646 & 2981 & 1721 \\
\hline 1964 Federal Surplus/Deficit & 3537 & 2080 & 368 & 506 & 292 & -151 & 1448 & -155 & -655 & 1568 & 1642 & 2897 & 6568 & 5320 & 1738 \\
\hline 1965 Federal Surplus/Deficit & 3046 & 2773 & 972 & 1588 & 425 & 3468 & 5715 & 4238 & 3143 & 2586 & 4428 & 5156 & 4437 & 2534 & 3174 \\
\hline 1966 Federal Surplus/Deficit & 3110 & 2541 & 322 & 1120 & 472 & 1048 & 2563 & -417 & -355 & 3296 & 1492 & 3221 & 2476 & 3353 & 1585 \\
\hline 1967 Federal Surplus/Deficit & 3352 & 1694 & -216 & 578 & 234 & 194 & 2978 & 3651 & 611 & 2230 & 991 & 1972 & 6123 & 4627 & 2074 \\
\hline 1968 Federal Surplus/Deficit & 3111 & 2445 & 427 & 934 & 360 & 885 & 2465 & 1715 & 1421 & -839 & 871 & 2094 & 4269 & 4278 & 1803 \\
\hline 1969 Federal Surplus/Deficit & 3274 & 2584 & 1376 & 1708 & 1259 & 1745 & 4524 & 3188 & 1893 & 5078 & 4574 & 6234 & 4141 & 3217 & 3087 \\
\hline 1970 Federal Surplus/Deficit & 3206 & 1293 & -35 & 706 & 359 & 12 & 2063 & 1642 & -133 & -363 & 1684 & 3148 & 4614 & 1354 & 1387 \\
\hline 1971 Federal Surplus/Deficit & 2010 & 943 & -311 & 698 & 453 & -116 & 3730 & 5216 & 2646 & 3319 & 4047 & 6589 & 6341 & 4508 & 2910 \\
\hline 1972 Federal Surplus/Deficit & 3229 & 3466 & 591 & 785 & 371 & 1028 & 3533 & 4825 & 5239 & 5587 & 2518 & 6291 & 6787 & 4942 & 3483 \\
\hline 1973 Federal Surplus/Deficit & 3783 & 3161 & 1047 & 954 & 237 & 1265 & 2918 & -1573 & -719 & -971 & -916 & 313 & 1679 & 1689 & 862 \\
\hline 1974 Federal Surplus/Deficit & 1903 & 246 & -406 & 320 & -63 & 2067 & 6206 & 5177 & 3975 & 4875 & 5491 & 5706 & 6915 & 6183 & 3528 \\
\hline 1975 Federal Surplus/Deficit & 3293 & 3437 & 994 & 466 & 258 & 117 & 2336 & 1209 & 2663 & 1665 & 1919 & 4044 & 5071 & 5205 & 2293 \\
\hline 1976 Federal Surplus/Deficit & 2112 & 1401 & 365 & 1363 & 1528 & 4362 & 4557 & 3062 & 2069 & 4816 & 3597 & 5794 & 3144 & 4748 & 3080 \\
\hline 1977 Federal Surplus/Deficit & 4702 & 4370 & 3544 & 1110 & 264 & -249 & 532 & -1947 & -1400 & -1117 & -1445 & 171 & 297 & 511 & 507 \\
\hline 1978 Federal Surplus/Deficit & 1938 & 65 & -431 & 148 & 49 & -79 & 1651 & 85 & 2532 & 1678 & 2418 & 3566 & 2805 & 3368 & 1395 \\
\hline \multicolumn{16}{|l|}{-Ranked Averages- } \\
\hline Top Ten Percent & 2721 & 2525 & 352 & 1129 & 713 & 2684 & 5062 & 4149 & 3768 & 4204 & 4554 & 5796 & 5557 & 4338 & 3379 \\
\hline Middle Eighty Percent & 2739 & 1664 & 335 & 945 & 443 & 708 & 1922 & 773 & 939 & 1967 & 2327 & 3854 & 4187 & 3151 & 1801 \\
\hline Bottom Ten Percent & 2421 & 1105 & -178 & 602 & 311 & -385 & -984 & -1674 & -760 & -364 & -937 & -412 & 1067 & 1338 & 3.1 \\
\hline
\end{tabular}


Federal Surplus/Deficit by Water Year

PNW Loads and Resources Study

2009 - 2010 Operating Year 2003 White Book

\begin{tabular}{|c|c|c|c|c|c|c|c|c|c|c|c|c|c|c|c|}
\hline Average Energy in Megawatts & Aug1 & Aug16 & Sep & Oct & Nov & Dec & Jan & $\mathrm{Feb}$ & Mar & Apr1 & Apr16 & May & Jun & Jul & Avg \\
\hline 1929 Federal Surplus/Deficit & 2866 & 826 & -286 & 360 & 117 & -480 & 11 & -2048 & -764 & -774 & -1097 & -196 & 2047 & 1664 & 111 \\
\hline 1930 Federal Surplus/Deficit & 1491 & 604 & -398 & 531 & 281 & -498 & -2082 & -1072 & -710 & -399 & -684 & -252 & 1952 & 1893 & 13 \\
\hline 1931 Federal Surplus/Deficit & 1963 & 869 & -275 & 475 & 246 & -451 & -2032 & -2023 & -934 & 133 & -1321 & 265 & 1468 & 994 & -120 \\
\hline 1932 Federal Surplus/Deficit & 1831 & 399 & -249 & 387 & 131 & -548 & -2005 & -2176 & 473 & 3045 & 4804 & 5243 & 4802 & 2822 & 1160 \\
\hline 1933 Federal Surplus/Deficit & 3140 & 2228 & -53 & 547 & -116 & 387 & 2522 & 1729 & -743 & 499 & 1345 & 3584 & 7088 & 5204 & 1980 \\
\hline 1934 Federal Surplus/Deficit & 2675 & 2993 & 551 & 1673 & 1616 & 4738 & 5686 & 4053 & 2550 & 5751 & 5060 & 4696 & 1287 & 2439 & 3127 \\
\hline 1935 Federal Surplus/Deficit & 1237 & -98 & -436 & 286 & -156 & -69 & 1976 & 2389 & -1243 & 287 & 2047 & 2851 & 3649 & 2807 & 1149 \\
\hline 1936 Federal Surplus/Deficit & 2674 & 887 & -265 & 484 & 156 & -487 & -1323 & -2008 & -532 & -508 & 1746 & 6388 & 3302 & 1766 & 823 \\
\hline 1937 Federal Surplus/Deficit & 2406 & 637 & -364 & 528 & 205 & -509 & -1817 & -1833 & -762 & -547 & -824 & 213 & 1427 & 1246 & -69 \\
\hline 1938 Federal Surplus/Deficit & 2011 & 260 & -372 & 690 & 76 & -419 & 2779 & -315 & 1520 & 2402 & 2537 & 5441 & 4033 & 2388 & 1619 \\
\hline 1939 Federal Surplus/Deficit & 1764 & 464 & 38 & 607 & 148 & -521 & 412 & -1719 & -187 & 330 & 1107 & 4096 & 1956 & 1873 & 711 \\
\hline 1940 Federal Surplus/Deficit & 2594 & 759 & -356 & 721 & 100 & -305 & -249 & -926 & 1749 & 1249 & 1874 & 2755 & 2701 & 1251 & 890 \\
\hline 1941 Federal Surplus/Deficit & 1666 & 332 & -406 & 507 & -21 & 95 & 302 & -1980 & -667 & -901 & -911 & 847 & 2272 & 606 & 137 \\
\hline 1942 Federal Surplus/Deficit & 1437 & 473 & -32 & 447 & -142 & 1667 & 2935 & -1350 & -1256 & -196 & 559 & 2489 & 5240 & 3356 & 1207 \\
\hline 1943 Federal Surplus/Deficit & 3119 & 2176 & -66 & 507 & -20 & -94 & 2151 & 1389 & 2179 & 5393 & 4695 & 5379 & 5034 & 3462 & 2301 \\
\hline 1944 Federal Surplus/Deficit & 2902 & 2097 & -71 & 612 & 179 & -544 & 383 & -1975 & -1187 & -722 & -1260 & -213 & 1191 & 438 & 27 \\
\hline 1945 Federal Surplus/Deficit & 1526 & -38 & -291 & 315 & 148 & -538 & -1963 & -1557 & -899 & -650 & -999 & 2751 & 4285 & 1816 & 332 \\
\hline 1946 Federal Surplus/Deficit & 1699 & 1238 & -481 & 621 & 159 & -467 & 821 & 794 & 2273 & 2863 & 3788 & 5733 & 4439 & 3288 & 1831 \\
\hline 1947 Federal Surplus/Deficit & 3209 & 1874 & 161 & 492 & 130 & 2488 & 3165 & 2321 & 2546 & 1254 & 2664 & 4868 & 4123 & 2921 & 2310 \\
\hline 1948 Federal Surplus/Deficit & 3213 & 1283 & -9.8 & 2691 & 1114 & 1404 & 3031 & -136 & 1137 & 1483 & 3605 & 6872 & 7801 & 3737 & 2703 \\
\hline 1949 Federal Surplus/Deficit & 2805 & 3289 & 879 & 790 & 50 & 143 & 963 & -302 & 3361 & 1541 & 4514 & 5751 & 3941 & 1143 & 1900 \\
\hline 1950 Federal Surplus/Deficit & 1492 & -4.1 & -676 & 515 & -29 & -746 & 1226 & 2733 & 4120 & 3933 & 3708 & 4632 & 6890 & 3845 & 2256 \\
\hline 1951 Federal Surplus/Deficit & 2417 & 2734 & 318 & 1522 & 1609 & 3488 & 4547 & 4530 & 2781 & 3792 & 4245 & 5777 & 3304 & 3762 & 3186 \\
\hline 1952 Federal Surplus/Deficit & 2903 & 2839 & 621 & 2147 & 308 & 1809 & 2805 & 1242 & 1530 & 4013 & 4414 & 6775 & 4243 & 2157 & 2560 \\
\hline 1953 Federal Surplus/Deficit & 2920 & 1134 & -136 & 479 & 240 & -505 & 167 & 2364 & -258 & -622 & 1001 & 4304 & 6650 & 3922 & 1620 \\
\hline 1954 Federal Surplus/Deficit & 3047 & 1933 & 194 & 768 & 226 & 846 & 1834 & 2609 & 1125 & 3216 & 2478 & 5110 & 6148 & 4647 & 2404 \\
\hline 1955 Federal Surplus/Deficit & 4045 & 3047 & 2820 & 1365 & 738 & 989 & -180 & -1647 & -1119 & 309 & -145 & 2233 & 7464 & 5718 & 1834 \\
\hline 1956 Federal Surplus/Deficit & 2819 & 3217 & 85 & 1236 & 1010 & 3155 & 5086 & 1765 & 3492 & 3995 & 5911 & 6823 & 7454 & 4097 & 3514 \\
\hline 1957 Federal Surplus/Deficit & 3178 & 2443 & 136 & 892 & 18 & 1015 & 1531 & -1006 & 749 & 4560 & 2788 & 6368 & 7165 & 2341 & 2141 \\
\hline 1958 Federal Surplus/Deficit & 2059 & 692 & -223 & 621 & 149 & -383 & 1047 & 1278 & 1351 & 546 & 3389 & 5712 & 5627 & 1904 & 1702 \\
\hline 1959 Federal Surplus/Deficit & 2087 & 976 & -126 & 516 & 634 & 1775 & 4559 & 2959 & 1355 & 2725 & 1927 & 4000 & 6104 & 4454 & 2507 \\
\hline 1960 Federal Surplus/Deficit & 2920 & 2265 & 2599 & 3323 & 2102 & 2666 & 2811 & -74 & 1173 & 5856 & 3758 & 3536 & 4439 & 2948 & 2744 \\
\hline 1961 Federal Surplus/Deficit & 3215 & 843 & -51 & 669 & 361 & 112 & 1588 & 1987 & 2037 & 2766 & 2753 & 4267 & 7034 & 2490 & 2107 \\
\hline 1962 Federal Surplus/Deficit & 2273 & 1348 & -415 & 584 & 129 & -634 & 1464 & -1033 & -723 & 4154 & 4457 & 3691 & 3571 & 3175 & 1327 \\
\hline 1963 Federal Surplus/Deficit & 2734 & 1769 & -178 & 1090 & 779 & 1753 & 1797 & 531 & 415 & 400 & 678 & 4499 & 4201 & 2892 & 1714 \\
\hline 1964 Federal Surplus/Deficit & 3443 & 1983 & 269 & 406 & 186 & -264 & 1332 & -266 & -765 & 1472 & 1548 & 3281 & 7125 & 5234 & 1730 \\
\hline 1965 Federal Surplus/Deficit & 2953 & 2678 & 874 & 1491 & 320 & 3357 & 5603 & 4134 & 3039 & 2492 & 4337 & 5542 & 4991 & 2445 & 3169 \\
\hline 1966 Federal Surplus/Deficit & 3016 & 2446 & 223 & 1021 & 368 & 936 & 2449 & -527 & -465 & 3204 & 1399 & 3605 & 3029 & 3265 & 1578 \\
\hline 1967 Federal Surplus/Deficit & 3259 & 1595 & -316 & 478 & 128 & 82 & 2864 & 3547 & 506 & 2138 & 896 & 2355 & 6680 & 4540 & 2067 \\
\hline 1968 Federal Surplus/Deficit & 3017 & 2350 & 328 & 835 & 256 & 774 & 2350 & 1609 & 1317 & -936 & 777 & 2473 & 4826 & 4190 & 1797 \\
\hline 1969 Federal Surplus/Deficit & 3181 & 2489 & 1280 & 1611 & 1154 & 1636 & 4412 & 3084 & 1788 & 4987 & 4485 & 6620 & 4697 & 3128 & 3082 \\
\hline 1970 Federal Surplus/Deficit & 3111 & 1194 & -136 & 607 & 254 & -101 & 1944 & 1535 & -240 & -459 & 1593 & 3531 & 5168 & 1264 & 1379 \\
\hline 1971 Federal Surplus/Deficit & 1914 & 843 & -412 & 597 & 347 & -228 & 3616 & 5113 & 2541 & 3225 & 3957 & 6975 & 6898 & 4421 & 2903 \\
\hline 1972 Federal Surplus/Deficit & 3136 & 3372 & 492 & 685 & 266 & 916 & 3418 & 4721 & 5138 & 5497 & 2428 & 6677 & 7344 & 4856 & 3477 \\
\hline 1973 Federal Surplus/Deficit & 3691 & 3067 & 948 & 855 & 132 & 1154 & 2804 & -1689 & -830 & -1068 & -1012 & 690 & 2229 & 1599 & 853 \\
\hline 1974 Federal Surplus/Deficit & 1808 & 146 & -507 & 217 & -170 & 1955 & 6094 & 5073 & 3873 & 4783 & 5402 & 6092 & 7472 & 6097 & 3522 \\
\hline 1975 Federal Surplus/Deficit & 3199 & 3343 & 896 & 365 & 151 & 4.7 & 2221 & 1101 & 2558 & 1571 & 1826 & 4428 & 5625 & 5119 & 2287 \\
\hline 1976 Federal Surplus/Deficit & 2017 & 1305 & 266 & 1265 & 1423 & 4258 & 4445 & 2958 & 1965 & 4724 & 3507 & 6180 & 3697 & 4662 & 3074 \\
\hline 1977 Federal Surplus/Deficit & 4610 & 4276 & 3451 & 1012 & 158 & -361 & 412 & -2064 & -1513 & -1216 & -1543 & 548 & 845 & 418 & 497 \\
\hline 1978 Federal Surplus/Deficit & 1842 & -34 & -532 & 46 & -57 & -193 & 1532 & -28 & 2429 & 1586 & 2327 & 3951 & 3357 & 3280 & 1387 \\
\hline \multicolumn{16}{|l|}{-Ranked Averages- } \\
\hline Top Ten Percent & 2626 & 2429 & 252 & 1030 & 607 & 2574 & 4950 & 4044 & 3665 & 4112 & 4465 & 6182 & 6113 & 4252 & 3374 \\
\hline Middle Eighty Percent & 2644 & 1567 & 236 & 846 & 338 & 597 & 1806 & 663 & 833 & 1873 & 2235 & 4238 & 4742 & 3062 & 1793 \\
\hline Bottom Ten Percent & 2326 & 1007 & -279 & 501 & 206 & -496 & -1108 & -1790 & -871 & -462 & -1037 & -37 & 1617 & 1247 & -7.8 \\
\hline
\end{tabular}


Federal Surplus/Deficit by Water Year

PNW Loads and Resources Study

2010 - 2011 Operating Year 2003 White Book

\begin{tabular}{|c|c|c|c|c|c|c|c|c|c|c|c|c|c|c|c|}
\hline Average Energy in Megawatts & Aug1 & Aug16 & Sep & Oct & Nov & Dec & Jan & $\mathrm{Feb}$ & Mar & Apr1 & Apr16 & May & Jun & Jul & Avg \\
\hline 1929 Federal Surplus/Deficit & 2857 & 812 & -300 & 342 & 93 & -510 & -17 & -2078 & -788 & -804 & -1124 & -690 & 1386 & 1643 & -4.1 \\
\hline 1930 Federal Surplus/Deficit & 1479 & 590 & -413 & 511 & 259 & -528 & -2113 & -1099 & -734 & -428 & -712 & -747 & 1291 & 1874 & -103 \\
\hline 1931 Federal Surplus/Deficit & 1953 & 856 & -290 & 454 & 223 & -481 & -2063 & -2052 & -958 & 106 & -1349 & -228 & 807 & 975 & -236 \\
\hline 1932 Federal Surplus/Deficit & 1820 & 385 & -264 & 367 & 108 & -580 & -2036 & -2205 & 451 & 3020 & 4788 & 4757 & 4146 & 2803 & 1046 \\
\hline 1933 Federal Surplus/Deficit & 3130 & 2217 & -67 & 528 & -138 & 357 & 2500 & 1710 & -765 & 474 & 1324 & 3097 & 6433 & 5187 & 1868 \\
\hline 1934 Federal Surplus/Deficit & 2666 & 2983 & 540 & 1658 & 1594 & 4715 & 5666 & 4035 & 2533 & 5728 & 5043 & 4211 & 624 & 2420 & 3017 \\
\hline 1935 Federal Surplus/Deficit & 1226 & -112 & -449 & 266 & -179 & -101 & 1953 & 2371 & -1264 & 261 & 2027 & 2361 & 2991 & 2789 & 1037 \\
\hline 1936 Federal Surplus/Deficit & 2665 & 875 & -278 & 466 & 133 & -517 & -1351 & -2037 & -555 & -536 & 1726 & 5902 & 2646 & 1747 & 710 \\
\hline 1937 Federal Surplus/Deficit & 2396 & 624 & -378 & 509 & 183 & -539 & -1848 & -1862 & -785 & -576 & -850 & -283 & 764 & 1226 & -185 \\
\hline 1938 Federal Surplus/Deficit & 2000 & 248 & -386 & 671 & 54 & -449 & 2756 & -338 & 1501 & 2377 & 2518 & 4956 & 3376 & 2370 & 1507 \\
\hline 1939 Federal Surplus/Deficit & 1753 & 451 & 25 & 588 & 125 & -552 & 388 & -1745 & -209 & 303 & 1085 & 3609 & 1294 & 1853 & 598 \\
\hline 1940 Federal Surplus/Deficit & 2584 & 745 & -370 & 702 & 78 & -336 & -277 & -953 & 1731 & 1223 & 1856 & 2266 & 2040 & 1232 & 776 \\
\hline 1941 Federal Surplus/Deficit & 1656 & 319 & -420 & 488 & -43 & 65 & 272 & -2008 & -690 & -927 & -932 & 356 & 1611 & 585 & 23 \\
\hline 1942 Federal Surplus/Deficit & 1425 & 460 & -46 & 428 & -165 & 1637 & 2914 & -1376 & -1280 & -223 & 537 & 2000 & 4583 & 3339 & 1094 \\
\hline 1943 Federal Surplus/Deficit & 3109 & 2165 & -79 & 489 & -43 & -125 & 2129 & 1366 & 2161 & 5369 & 4677 & 4894 & 4377 & 3443 & 2189 \\
\hline 1944 Federal Surplus/Deficit & 2892 & 2086 & -86 & 594 & 155 & -575 & 355 & -2004 & -1212 & -752 & -1285 & -706 & 529 & 417 & -88 \\
\hline 1945 Federal Surplus/Deficit & 1515 & -52 & -306 & 295 & 125 & -569 & -1995 & -1586 & -924 & -680 & -1026 & 2262 & 3628 & 1797 & 217 \\
\hline 1946 Federal Surplus/Deficit & 1688 & 1227 & -495 & 601 & 136 & -497 & 794 & 770 & 2255 & 2838 & 3770 & 5247 & 3783 & 3269 & 1719 \\
\hline 1947 Federal Surplus/Deficit & 3200 & 1863 & 148 & 474 & 107 & 2457 & 3145 & 2301 & 2529 & 1229 & 2646 & 4381 & 3468 & 2903 & 2199 \\
\hline 1948 Federal Surplus/Deficit & 3204 & 1270 & -23 & 2676 & 1091 & 1376 & 3010 & -161 & 1119 & 1457 & 3587 & 6387 & 7146 & 3720 & 2592 \\
\hline 1949 Federal Surplus/Deficit & 2795 & 3280 & 867 & 773 & 27 & 112 & 939 & -328 & 3344 & 1516 & 4497 & 5265 & 3284 & 1123 & 1787 \\
\hline 1950 Federal Surplus/Deficit & 1481 & -19 & -691 & 496 & -52 & -776 & 1203 & 2712 & 4104 & 3907 & 3690 & 4146 & 6235 & 3827 & 2144 \\
\hline 1951 Federal Surplus/Deficit & 2407 & 2724 & 305 & 1506 & 1586 & 3462 & 4526 & 4513 & 2764 & 3767 & 4228 & 5291 & 2646 & 3744 & 3076 \\
\hline 1952 Federal Surplus/Deficit & 2894 & 2828 & 609 & 2131 & 285 & 1780 & 2784 & 1222 & 1510 & 3988 & 4396 & 6290 & 3586 & 2138 & 2449 \\
\hline 1953 Federal Surplus/Deficit & 2910 & 1121 & -150 & 460 & 217 & -535 & 137 & 2345 & -278 & -648 & 980 & 3817 & 5994 & 3904 & 1508 \\
\hline 1954 Federal Surplus/Deficit & 3038 & 1922 & 181 & 751 & 203 & 816 & 1812 & 2590 & 1107 & 3190 & 2459 & 4624 & 5493 & 4630 & 2293 \\
\hline 1955 Federal Surplus/Deficit & 4036 & 3037 & 2812 & 1349 & 716 & 960 & -208 & -1676 & -1143 & 283 & -163 & 1743 & 6809 & 5702 & 1722 \\
\hline 1956 Federal Surplus/Deficit & 2809 & 3207 & 72 & 1220 & 987 & 3128 & 5066 & 1746 & 3474 & 3971 & 5894 & 6337 & 6799 & 4079 & 3404 \\
\hline 1957 Federal Surplus/Deficit & 3168 & 2433 & 123 & 875 & -5.3 & 985 & 1507 & -1032 & 730 & 4535 & 2770 & 5883 & 6510 & 2321 & 2029 \\
\hline 1958 Federal Surplus/Deficit & 2048 & 678 & -238 & 603 & 126 & -415 & 1023 & 1255 & 1334 & 519 & 3370 & 5226 & 4972 & 1884 & 1590 \\
\hline 1959 Federal Surplus/Deficit & 2076 & 963 & -140 & 499 & 611 & 1746 & 4539 & 2941 & 1336 & 2701 & 1908 & 3514 & 5449 & 4436 & 2396 \\
\hline 1960 Federal Surplus/Deficit & 2910 & 2255 & 2590 & 3308 & 2080 & 2640 & 2791 & -97 & 1153 & 5833 & 3740 & 3049 & 3783 & 2930 & 2633 \\
\hline 1961 Federal Surplus/Deficit & 3206 & 829 & -64 & 652 & 338 & 80 & 1564 & 1968 & 2020 & 2741 & 2732 & 3781 & 6379 & 2471 & 1995 \\
\hline 1962 Federal Surplus/Deficit & 2263 & 1335 & -429 & 566 & 105 & -666 & 1440 & -1057 & -747 & 4128 & 4441 & 3205 & 2914 & 3156 & 1214 \\
\hline 1963 Federal Surplus/Deficit & 2725 & 1758 & -193 & 1073 & 756 & 1725 & 1775 & 511 & 392 & 374 & 657 & 4011 & 3544 & 2874 & 1602 \\
\hline 1964 Federal Surplus/Deficit & 3434 & 1972 & 256 & 387 & 162 & -296 & 1308 & -290 & -789 & 1445 & 1527 & 2793 & 6470 & 5218 & 1617 \\
\hline 1965 Federal Surplus/Deficit & 2944 & 2667 & 862 & 1476 & 298 & 3328 & 5582 & 4116 & 3021 & 2466 & 4320 & 5057 & 4334 & 2426 & 3058 \\
\hline 1966 Federal Surplus/Deficit & 3007 & 2435 & 211 & 1004 & 346 & 905 & 2427 & -549 & -489 & 3180 & 1379 & 3118 & 2371 & 3247 & 1466 \\
\hline 1967 Federal Surplus/Deficit & 3250 & 1582 & -330 & 459 & 105 & 51 & 2843 & 3529 & 486 & 2113 & 875 & 1868 & 6025 & 4523 & 1956 \\
\hline 1968 Federal Surplus/Deficit & 3008 & 2340 & 315 & 818 & 233 & 743 & 2328 & 1590 & 1299 & -965 & 756 & 1982 & 4171 & 4172 & 1685 \\
\hline 1969 Federal Surplus/Deficit & 3172 & 2479 & 1270 & 1596 & 1132 & 1607 & 4392 & 3066 & 1769 & 4963 & 4468 & 6135 & 4042 & 3110 & 2972 \\
\hline 1970 Federal Surplus/Deficit & 3101 & 1181 & -150 & 589 & 230 & -132 & 1918 & 1516 & -261 & -485 & 1574 & 3043 & 4511 & 1245 & 1266 \\
\hline 1971 Federal Surplus/Deficit & 1904 & 829 & -427 & 578 & 323 & -259 & 3594 & 5095 & 2523 & 3199 & 3939 & 6489 & 6243 & 4404 & 2792 \\
\hline 1972 Federal Surplus/Deficit & 3127 & 3362 & 479 & 667 & 243 & 885 & 3396 & 4703 & 5122 & 5474 & 2410 & 6191 & 6688 & 4840 & 3367 \\
\hline 1973 Federal Surplus/Deficit & 3682 & 3058 & 935 & 838 & 108 & 1124 & 2782 & -1717 & -854 & -1096 & -1035 & 197 & 1569 & 1579 & 739 \\
\hline 1974 Federal Surplus/Deficit & 1797 & 132 & -522 & 197 & -193 & 1924 & 6074 & 5056 & 3856 & 4759 & 5386 & 5607 & 6816 & 6080 & 3411 \\
\hline 1975 Federal Surplus/Deficit & 3190 & 3334 & 883 & 347 & 127 & -27 & 2199 & 1081 & 2539 & 1546 & 1806 & 3942 & 4969 & 5102 & 2175 \\
\hline 1976 Federal Surplus/Deficit & 2007 & 1293 & 253 & 1249 & 1399 & 4234 & 4424 & 2940 & 1947 & 4700 & 3489 & 5695 & 3039 & 4645 & 2964 \\
\hline 1977 Federal Surplus/Deficit & 4602 & 4267 & 3443 & 995 & 134 & -392 & 385 & -2093 & -1538 & -1246 & -1568 & 55 & 182 & 395 & 383 \\
\hline 1978 Federal Surplus/Deficit & 1831 & -48 & -547 & 26 & -81 & -225 & 1506 & -53 & 2411 & 1561 & 2309 & 3465 & 2700 & 3262 & 1274 \\
\hline \multicolumn{16}{|l|}{-Ranked Averages- } \\
\hline Top Ten Percent & 2617 & 2418 & 239 & 1013 & 584 & 2545 & 4929 & 4027 & 3647 & 4088 & 4447 & 5696 & 5457 & 4234 & 3263 \\
\hline ghty Percent & 2635 & 1555 & 223 & 828 & 315 & 567 & 1782 & 640 & 812 & 1847 & 2216 & 3751 & 4085 & 3044 & 1681 \\
\hline Bottom Ten Percent & 2315 & 994 & -293 & 482 & 183 & -527 & -1137 & -1819 & -895 & -491 & -1064 & -531 & 955 & 1227 & -123 \\
\hline
\end{tabular}


Federal Surplus/Deficit by Water Year

PNW Loads and Resources Study

2011 - 2012 Operating Year 2003 White Book

\begin{tabular}{|c|c|c|c|c|c|c|c|c|c|c|c|c|c|c|c|}
\hline Average Energy in Megawatts & Aug1 & Aug16 & Sep & Oct & Nov & Dec & Jan & $\mathrm{Feb}$ & Mar & Apr1 & Apr16 & May & Jun & Jul & Avg \\
\hline 1929 Federal Surplus/Deficit & 2841 & 793 & -313 & 277 & -4.8 & -631 & -32 & -1996 & -812 & -819 & -1142 & -253 & 1970 & 1607 & 54 \\
\hline 1930 Federal Surplus/Deficit & 1462 & 572 & -427 & 445 & 162 & -648 & -2131 & -1015 & -757 & -443 & -730 & -311 & 1874 & 1838 & -45 \\
\hline 1931 Federal Surplus/Deficit & 1937 & 838 & -304 & 389 & 126 & -601 & -2080 & -1971 & -982 & 93 & -1368 & 209 & 1390 & 939 & -178 \\
\hline 1932 Federal Surplus/Deficit & 1804 & 366 & -278 & 302 & 11 & -702 & -2054 & -2124 & 430 & 3007 & 4776 & 5199 & 4732 & 2768 & 1105 \\
\hline 1933 Federal Surplus/Deficit & 3114 & 2201 & -79 & 464 & -235 & 236 & 2490 & 1799 & -787 & 461 & 1311 & 3539 & 7019 & 5152 & 1928 \\
\hline 1934 Federal Surplus/Deficit & 2650 & 2966 & 529 & 1596 & 1498 & 4600 & 5655 & 4124 & 2514 & 5715 & 5032 & 4653 & 1205 & 2384 & 3078 \\
\hline 1935 Federal Surplus/Deficit & 1210 & -130 & -461 & 201 & -276 & -222 & 1942 & 2461 & -1285 & 248 & 2014 & 2801 & 3576 & 2753 & 1097 \\
\hline 1936 Federal Surplus/Deficit & 2649 & 858 & -291 & 401 & 37 & -638 & -1366 & -1955 & -578 & -550 & 1715 & 6343 & 3232 & 1712 & 769 \\
\hline 1937 Federal Surplus/Deficit & 2380 & 606 & -392 & 443 & 86 & -659 & -1865 & -1779 & -808 & -591 & -867 & 152 & 1346 & 1190 & -127 \\
\hline 1938 Federal Surplus/Deficit & 1984 & 231 & -399 & 606 & -42 & -569 & 2745 & -252 & 1481 & 2364 & 2507 & 5398 & 3962 & 2334 & 1567 \\
\hline 1939 Federal Surplus/Deficit & 1737 & 433 & 12 & 524 & 28 & -673 & 376 & -1662 & -231 & 290 & 1071 & 4050 & 1876 & 1818 & 657 \\
\hline 1940 Federal Surplus/Deficit & 2568 & 727 & -384 & 638 & -19 & -457 & -292 & -870 & 1712 & 1210 & 1845 & 2706 & 2622 & 1196 & 836 \\
\hline 1941 Federal Surplus/Deficit & 1640 & 301 & -434 & 424 & -139 & -54 & 256 & -1926 & -714 & -940 & -946 & 794 & 2195 & 549 & 82 \\
\hline 1942 Federal Surplus/Deficit & 1409 & 442 & -59 & 363 & -261 & 1517 & 2904 & -1292 & -1304 & -236 & 525 & 2440 & 5170 & 3303 & 1154 \\
\hline 1943 Federal Surplus/Deficit & 3093 & 2148 & -92 & 424 & -140 & -246 & 2118 & 1454 & 2142 & 5356 & 4665 & 5336 & 4964 & 3407 & 2250 \\
\hline 1944 Federal Surplus/Deficit & 2876 & 2070 & -99 & 529 & 57 & -696 & 341 & -1922 & -1236 & -767 & -1301 & -268 & 1111 & 380 & -30 \\
\hline 1945 Federal Surplus/Deficit & 1499 & -71 & -320 & 230 & 27 & -690 & -2012 & -1504 & -948 & -695 & -1044 & 2704 & 4214 & 1761 & 276 \\
\hline 1946 Federal Surplus/Deficit & 1671 & 1210 & -509 & 536 & 39 & -617 & 780 & 857 & 2236 & 2825 & 3758 & 5689 & 4370 & 3234 & 1779 \\
\hline 1947 Federal Surplus/Deficit & 3184 & 1846 & 136 & 410 & 9.6 & 2338 & 3135 & 2390 & 2510 & 1216 & 2635 & 4823 & 4054 & 2867 & 2259 \\
\hline 1948 Federal Surplus/Deficit & 3187 & 1253 & -36 & 2614 & 995 & 1258 & 2999 & -76 & 1100 & 1444 & 3576 & 6828 & 7731 & 3685 & 2652 \\
\hline 1949 Federal Surplus/Deficit & 2779 & 3263 & 856 & 709 & -70 & -9.5 & 927 & -244 & 3325 & 1503 & 4486 & 5707 & 3870 & 1088 & 1848 \\
\hline 1950 Federal Surplus/Deficit & 1464 & -37 & -704 & 431 & -149 & -895 & 1191 & 2799 & 4084 & 3895 & 3679 & 4588 & 6821 & 3792 & 2205 \\
\hline 1951 Federal Surplus/Deficit & 2391 & 2708 & 292 & 1443 & 1490 & 3347 & 4516 & 4602 & 2745 & 3754 & 4217 & 5733 & 3233 & 3709 & 3137 \\
\hline 1952 Federal Surplus/Deficit & 2878 & 2812 & 597 & 2069 & 189 & 1662 & 2773 & 1310 & 1489 & 3975 & 4385 & 6732 & 4172 & 2102 & 2510 \\
\hline 1953 Federal Surplus/Deficit & 2894 & 1104 & -164 & 395 & 120 & -656 & 122 & 2434 & -298 & -661 & 968 & 4259 & 6580 & 3868 & 1568 \\
\hline 1954 Federal Surplus/Deficit & 3022 & 1906 & 168 & 687 & 107 & 695 & 1801 & 2680 & 1087 & 3177 & 2448 & 5066 & 6079 & 4595 & 2354 \\
\hline 1955 Federal Surplus/Deficit & 4020 & 3021 & 2802 & 1286 & 620 & 840 & -223 & -1594 & -1167 & 270 & -174 & 2183 & 7396 & 5666 & 1781 \\
\hline 1956 Federal Surplus/Deficit & 2793 & 3191 & 59 & 1157 & 890 & 3012 & 5055 & 1835 & 3455 & 3958 & 5883 & 6779 & 7385 & 4044 & 3465 \\
\hline 1957 Federal Surplus/Deficit & 3152 & 2416 & 110 & 812 & -102 & 865 & 1495 & -949 & 711 & 4522 & 2759 & 6324 & 7096 & 2286 & 2089 \\
\hline 1958 Federal Surplus/Deficit & 2032 & 659 & -251 & 539 & 29 & -536 & 1011 & 1342 & 1314 & 506 & 3358 & 5667 & 5558 & 1848 & 1650 \\
\hline 1959 Federal Surplus/Deficit & 2060 & 946 & -153 & 435 & 515 & 1628 & 4528 & 3030 & 1315 & 2688 & 1897 & 3956 & 6035 & 4400 & 2457 \\
\hline 1960 Federal Surplus/Deficit & 2894 & 2238 & 2580 & 3246 & 1983 & 2522 & 2781 & -11 & 1133 & 5820 & 3729 & 3491 & 4369 & 2894 & 2694 \\
\hline 1961 Federal Surplus/Deficit & 3190 & 811 & -77 & 588 & 241 & -41 & 1552 & 2057 & 2001 & 2728 & 2720 & 4223 & 6965 & 2435 & 2056 \\
\hline 1962 Federal Surplus/Deficit & 2247 & 1318 & -443 & 501 & 7.5 & -788 & 1428 & -972 & -771 & 4115 & 4430 & 3647 & 3500 & 3121 & 1274 \\
\hline 1963 Federal Surplus/Deficit & 2709 & 1741 & -206 & 1010 & 660 & 1606 & 1765 & 600 & 368 & 361 & 644 & 4453 & 4131 & 2838 & 1663 \\
\hline 1964 Federal Surplus/Deficit & 3418 & 1955 & 244 & 323 & 65 & -417 & 1296 & -205 & -812 & 1432 & 1515 & 3235 & 7057 & 5182 & 1677 \\
\hline 1965 Federal Surplus/Deficit & 2927 & 2651 & 851 & 1414 & 202 & 3209 & 5572 & 4205 & 3002 & 2454 & 4308 & 5498 & 4921 & 2391 & 3119 \\
\hline 1966 Federal Surplus/Deficit & 2991 & 2418 & 199 & 941 & 250 & 784 & 2416 & -463 & -512 & 3167 & 1367 & 3560 & 2956 & 3211 & 1526 \\
\hline 1967 Federal Surplus/Deficit & 3234 & 1564 & -344 & 395 & 7.5 & -69 & 2833 & 3618 & 466 & 2100 & 861 & 2310 & 6611 & 4488 & 2016 \\
\hline 1968 Federal Surplus/Deficit & 2992 & 2323 & 302 & 754 & 137 & 623 & 2318 & 1679 & 1280 & -979 & 742 & 2421 & 4757 & 4136 & 1746 \\
\hline 1969 Federal Surplus/Deficit & 3156 & 2462 & 1260 & 1533 & 1035 & 1488 & 4381 & 3155 & 1750 & 4950 & 4456 & 6577 & 4628 & 3074 & 3033 \\
\hline 1970 Federal Surplus/Deficit & 3085 & 1163 & -163 & 525 & 133 & -254 & 1906 & 1605 & -282 & -498 & 1562 & 3485 & 5097 & 1209 & 1326 \\
\hline 1971 Federal Surplus/Deficit & 1888 & 811 & -441 & 513 & 225 & -379 & 3584 & 5184 & 2504 & 3186 & 3928 & 6931 & 6829 & 4368 & 2852 \\
\hline 1972 Federal Surplus/Deficit & 3111 & 3345 & 467 & 603 & 146 & 764 & 3386 & 4792 & 5103 & 5461 & 2399 & 6633 & 7275 & 4804 & 3428 \\
\hline 1973 Federal Surplus/Deficit & 3666 & 3041 & 923 & 774 & 11 & 1004 & 2771 & -1634 & -877 & -1111 & -1050 & 634 & 2152 & 1543 & 798 \\
\hline 1974 Federal Surplus/Deficit & 1781 & 113 & -536 & 131 & -290 & 1804 & 6063 & 5145 & 3837 & 4747 & 5375 & 6048 & 7402 & 6045 & 3471 \\
\hline 1975 Federal Surplus/Deficit & 3174 & 3317 & 871 & 282 & 30 & -148 & 2188 & 1169 & 2520 & 1533 & 1795 & 4384 & 5555 & 5067 & 2236 \\
\hline 1976 Federal Surplus/Deficit & 1991 & 1277 & 241 & 1186 & 1303 & 4119 & 4414 & 3029 & 1928 & 4687 & 3477 & 6137 & 3626 & 4609 & 3026 \\
\hline 1977 Federal Surplus/Deficit & 4585 & 4251 & 3433 & 931 & 36 & -512 & 370 & -2011 & -1563 & -1262 & -1584 & 492 & 763 & 358 & 441 \\
\hline 1978 Federal Surplus/Deficit & 1815 & -67 & -560 & -40 & -178 & -346 & 1493 & 32 & 2392 & 1548 & 2298 & 3906 & 3286 & 3226 & 1334 \\
\hline \multicolumn{16}{|l|}{-Ranked Averages- } \\
\hline Top Ten Percent & 2601 & 2401 & 227 & 949 & 487 & 2427 & 4918 & 4116 & 3628 & 4075 & 4436 & 6138 & 6043 & 4198 & 3324 \\
\hline Middle Eighty Percent & 2618 & 1537 & 210 & 764 & 218 & 447 & 1770 & 727 & 792 & 1834 & 2203 & 4192 & 4670 & 3008 & 1741 \\
\hline Bottom Ten Percent & 2299 & 976 & -307 & 417 & 85 & -647 & -1153 & -1737 & -919 & -505 & -1081 & -94 & 1538 & 1191 & -65 \\
\hline
\end{tabular}


Federal Surplus/Deficit by Water Year

PNW Loads and Resources Study

2012 - 2013 Operating Year 2003 White Book

\begin{tabular}{|c|c|c|c|c|c|c|c|c|c|c|c|c|c|c|c|}
\hline Average Energy in Megawatts & Aug1 & Aug16 & Sep & Oct & Nov & Dec & Jan & $\mathrm{Feb}$ & Mar & Apr1 & Apr16 & May & Jun & Jul & Avg \\
\hline 1929 Federal Surplus/Deficit & 2813 & 762 & -337 & 273 & -14 & -642 & -57 & -2183 & -835 & -839 & -1165 & -743 & 1313 & 1591 & -71 \\
\hline 1930 Federal Surplus/Deficit & 1433 & 541 & -451 & 440 & 154 & -659 & -2161 & -1200 & -780 & -461 & -753 & -801 & 1215 & 1822 & -170 \\
\hline 1931 Federal Surplus/Deficit & 1909 & 808 & -328 & 383 & 118 & -611 & -2109 & -2158 & -1005 & 76 & -1392 & -281 & 731 & 923 & -303 \\
\hline 1932 Federal Surplus/Deficit & 1776 & 335 & -302 & 296 & 2.5 & -715 & -2083 & -2311 & 410 & 2992 & 4761 & 4716 & 4079 & 2752 & 981 \\
\hline 1933 Federal Surplus/Deficit & 3086 & 2172 & -102 & 460 & -242 & 226 & 2469 & 1620 & -807 & 445 & 1293 & 3055 & 6367 & 5137 & 1807 \\
\hline 1934 Federal Surplus/Deficit & 2623 & 2939 & 508 & 1594 & 1490 & 4598 & 5636 & 3947 & 2498 & 5701 & 5017 & 4170 & 544 & 2368 & 2958 \\
\hline 1935 Federal Surplus/Deficit & 1182 & -160 & -484 & 195 & -284 & -233 & 1921 & 2283 & -1305 & 232 & 1997 & 2315 & 2921 & 2738 & 974 \\
\hline 1936 Federal Surplus/Deficit & 2622 & 829 & -314 & 396 & 29 & -648 & -1393 & -2142 & -600 & -568 & 1699 & 5861 & 2578 & 1696 & 646 \\
\hline 1937 Federal Surplus/Deficit & 2352 & 575 & -416 & 438 & 78 & -670 & -1894 & -1966 & -831 & -609 & -889 & -339 & 687 & 1174 & -252 \\
\hline 1938 Federal Surplus/Deficit & 1956 & 201 & -423 & 601 & -50 & -578 & 2723 & -434 & 1463 & 2348 & 2491 & 4915 & 3308 & 2318 & 1445 \\
\hline 1939 Federal Surplus/Deficit & 1709 & 403 & -10 & 521 & 19 & -683 & 353 & -1848 & -252 & 274 & 1053 & 3565 & 1216 & 1801 & 534 \\
\hline 1940 Federal Surplus/Deficit & 2541 & 697 & -407 & 634 & -26 & -467 & -319 & -1055 & 1695 & 1194 & 1829 & 2221 & 1964 & 1180 & 712 \\
\hline 1941 Federal Surplus/Deficit & 1612 & 271 & -457 & 420 & -146 & -64 & 229 & -2112 & -736 & -956 & -965 & 307 & 1538 & 532 & -42 \\
\hline 1942 Federal Surplus/Deficit & 1381 & 412 & -83 & 359 & -268 & 1507 & 2884 & -1476 & -1327 & -253 & 507 & 1955 & 4516 & 3287 & 1031 \\
\hline 1943 Federal Surplus/Deficit & 3066 & 2120 & -115 & 420 & -149 & -256 & 2098 & 1273 & 2125 & 5341 & 4651 & 4853 & 4310 & 3392 & 2128 \\
\hline 1944 Federal Surplus/Deficit & 2849 & 2042 & -123 & 526 & 48 & -706 & 315 & -2110 & -1260 & -787 & -1323 & -757 & 452 & 362 & -155 \\
\hline 1945 Federal Surplus/Deficit & 1470 & -102 & -344 & 224 & 19 & -701 & -2042 & -1690 & -971 & -714 & -1068 & 2220 & 3560 & 1745 & 151 \\
\hline 1946 Federal Surplus/Deficit & 1643 & 1181 & -532 & 530 & 31 & -627 & 756 & 675 & 2219 & 2809 & 3743 & 5207 & 3716 & 3218 & 1657 \\
\hline 1947 Federal Surplus/Deficit & 3157 & 1818 & 113 & 407 & 1.5 & 2329 & 3115 & 2212 & 2493 & 1200 & 2619 & 4340 & 3401 & 2851 & 2138 \\
\hline 1948 Federal Surplus/Deficit & 3160 & 1223 & -59 & 2613 & 987 & 1251 & 2979 & -259 & 1083 & 1428 & 3560 & 6347 & 7079 & 3670 & 2531 \\
\hline 1949 Federal Surplus/Deficit & 2752 & 3235 & 834 & 707 & -79 & -20 & 904 & -429 & 3309 & 1487 & 4470 & 5224 & 3216 & 1071 & 1726 \\
\hline 1950 Federal Surplus/Deficit & 1436 & -68 & -728 & 426 & -157 & -905 & 1169 & 2620 & 4068 & 3880 & 3664 & 4105 & 6169 & 3776 & 2083 \\
\hline 1951 Federal Surplus/Deficit & 2363 & 2680 & 270 & 1441 & 1483 & 3342 & 4496 & 4425 & 2728 & 3739 & 4202 & 5251 & 2578 & 3693 & 3017 \\
\hline 1952 Federal Surplus/Deficit & 2851 & 2784 & 575 & 2068 & 181 & 1654 & 2753 & 1132 & 1470 & 3960 & 4370 & 6250 & 3518 & 2087 & 2389 \\
\hline 1953 Federal Surplus/Deficit & 2867 & 1074 & -187 & 390 & 111 & -666 & 95 & 2257 & -317 & -677 & 950 & 3775 & 5927 & 3853 & 1446 \\
\hline 1954 Federal Surplus/Deficit & 2995 & 1878 & 145 & 684 & 99 & 685 & 1781 & 2502 & 1069 & 3162 & 2432 & 4584 & 5427 & 4580 & 2232 \\
\hline 1955 Federal Surplus/Deficit & 3993 & 2993 & 2783 & 1285 & 612 & 831 & -249 & -1780 & -1191 & 254 & -190 & 1697 & 6743 & 5651 & 1659 \\
\hline 1956 Federal Surplus/Deficit & 2766 & 3163 & 36 & 1155 & 882 & 3007 & 5036 & 1657 & 3439 & 3943 & 5868 & 6297 & 6733 & 4029 & 3345 \\
\hline 1957 Federal Surplus/Deficit & 3125 & 2388 & 88 & 809 & -110 & 856 & 1472 & -1134 & 693 & 4507 & 2743 & 5843 & 6444 & 2269 & 1968 \\
\hline 1958 Federal Surplus/Deficit & 2004 & 629 & -274 & 535 & 20 & -547 & 989 & 1161 & 1297 & 490 & 3343 & 5185 & 4905 & 1832 & 1528 \\
\hline 1959 Federal Surplus/Deficit & 2032 & 916 & -176 & 432 & 507 & 1620 & 4509 & 2853 & 1297 & 2672 & 1881 & 3473 & 5382 & 4385 & 2336 \\
\hline 1960 Federal Surplus/Deficit & 2867 & 2210 & 2561 & 3245 & 1976 & 2517 & 2761 & -193 & 1114 & 5806 & 3714 & 3008 & 3716 & 2878 & 2573 \\
\hline 1961 Federal Surplus/Deficit & 3163 & 781 & -99 & 585 & 233 & -52 & 1530 & 1879 & 1984 & 2712 & 2702 & 3740 & 6312 & 2419 & 1934 \\
\hline 1962 Federal Surplus/Deficit & 2219 & 1288 & -467 & 498 & -1.4 & -799 & 1405 & -1154 & -794 & 4100 & 4415 & 3163 & 2846 & 3105 & 1151 \\
\hline 1963 Federal Surplus/Deficit & 2681 & 1713 & -230 & 1008 & 652 & 1599 & 1745 & 421 & 346 & 345 & 627 & 3970 & 3477 & 2823 & 1541 \\
\hline 1964 Federal Surplus/Deficit & 3391 & 1927 & 221 & 319 & 56 & -428 & 1273 & -387 & -835 & 1416 & 1498 & 2751 & 6404 & 5167 & 1555 \\
\hline 1965 Federal Surplus/Deficit & 2900 & 2623 & 829 & 1412 & 194 & 3202 & 5553 & 4028 & 2986 & 2438 & 4294 & 5016 & 4267 & 2375 & 2999 \\
\hline 1966 Federal Surplus/Deficit & 2964 & 2390 & 177 & 938 & 242 & 774 & 2395 & -644 & -534 & 3152 & 1350 & 3077 & 2301 & 3196 & 1404 \\
\hline 1967 Federal Surplus/Deficit & 3207 & 1533 & -368 & 390 & -1 & -80 & 2812 & 3441 & 447 & 2085 & 843 & 1826 & 5959 & 4472 & 1894 \\
\hline 1968 Federal Surplus/Deficit & 2965 & 2295 & 280 & 752 & 130 & 613 & 2298 & 1501 & 1263 & -998 & 724 & 1935 & 4104 & 4121 & 1624 \\
\hline 1969 Federal Surplus/Deficit & 3129 & 2434 & 1240 & 1532 & 1028 & 1480 & 4362 & 2978 & 1733 & 4935 & 4441 & 6095 & 3975 & 3058 & 2912 \\
\hline 1970 Federal Surplus/Deficit & 3058 & 1133 & -187 & 522 & 124 & -265 & 1882 & 1427 & -302 & -515 & 1546 & 3001 & 4443 & 1193 & 1204 \\
\hline 1971 Federal Surplus/Deficit & 1860 & 780 & -465 & 509 & 216 & -389 & 3564 & 5008 & 2487 & 3171 & 3913 & 6449 & 6176 & 4353 & 2731 \\
\hline 1972 Federal Surplus/Deficit & 3084 & 3317 & 445 & 600 & 138 & 754 & 3366 & 4615 & 5087 & 5446 & 2383 & 6151 & 6622 & 4789 & 3307 \\
\hline 1973 Federal Surplus/Deficit & 3639 & 3013 & 901 & 771 & 2.6 & 995 & 2751 & -1820 & -900 & -1129 & -1070 & 145 & 1495 & 1526 & 674 \\
\hline 1974 Federal Surplus/Deficit & 1753 & 81 & -561 & 125 & -299 & 1795 & 6044 & 4968 & 3821 & 4732 & 5360 & 5566 & 6750 & 6031 & 3350 \\
\hline 1975 Federal Surplus/Deficit & 3147 & 3289 & 849 & 278 & 21 & -158 & 2167 & 990 & 2502 & 1517 & 1778 & 3900 & 4902 & 5052 & 2114 \\
\hline 1976 Federal Surplus/Deficit & 1964 & 1248 & 219 & 1184 & 1295 & 4117 & 4395 & 2851 & 1911 & 4673 & 3462 & 5654 & 2972 & 4594 & 2905 \\
\hline 1977 Federal Surplus/Deficit & 4559 & 4223 & 3414 & 928 & 27 & -522 & 345 & -2198 & -1587 & -1283 & -1606 & 2.5 & 103 & 339 & 316 \\
\hline 1978 Federal Surplus/Deficit & 1786 & -98 & -584 & -45 & -186 & -357 & 1469 & -151 & 2375 & 1533 & 2282 & 3423 & 2632 & 3210 & 1211 \\
\hline \multicolumn{16}{|l|}{-Ranked Averages- } \\
\hline Top Ten Percent & 2573 & 2373 & 204 & 947 & 480 & 2420 & 4899 & 3939 & 3612 & 4060 & 4421 & 5656 & 5390 & 4183 & 3204 \\
\hline Middle Eighty Percent & 2591 & 1508 & 188 & 761 & 210 & 437 & 1748 & 545 & 772 & 1818 & 2187 & 3708 & 4016 & 2992 & 1619 \\
\hline Bottom Ten Percent & 2271 & 946 & -331 & 412 & 77 & -657 & -1181 & -1923 & -942 & -524 & -1105 & -584 & 880 & 1174 & -190 \\
\hline
\end{tabular}


Federal Surplus/Deficit by Water Year

PNW Loads and Resources Study

2013 - 2014 Operating Year 2003 White Book

\begin{tabular}{|c|c|c|c|c|c|c|c|c|c|c|c|c|c|c|c|}
\hline Average Energy in Megawatts & Aug1 & Aug16 & Sep & Oct & Nov & Dec & Jan & $\mathrm{Feb}$ & Mar & Apr1 & Apr16 & May & Jun & Jul & Avg \\
\hline 1929 Federal Surplus/Deficit & 2795 & 741 & -358 & 254 & -41 & -672 & -87 & -2215 & -861 & -862 & -1190 & -293 & 1968 & 1605 & 3.5 \\
\hline 1930 Federal Surplus/Deficit & 1413 & 520 & -472 & 419 & 128 & -689 & -2193 & -1230 & -806 & -483 & -779 & -353 & 1870 & 1837 & -96 \\
\hline 1931 Federal Surplus/Deficit & 1891 & 787 & -350 & 362 & 92 & -641 & -2141 & -2190 & -1033 & 57 & -1419 & 169 & 1386 & 938 & -229 \\
\hline 1932 Federal Surplus/Deficit & 1758 & 313 & -323 & 276 & -24 & -747 & -2115 & -2343 & 386 & 2972 & 4742 & 5170 & 4737 & 2767 & 1056 \\
\hline 1933 Federal Surplus/Deficit & 3068 & 2153 & -123 & 441 & -268 & 195 & 2444 & 1595 & -833 & 426 & 1272 & 3510 & 7025 & 5152 & 1883 \\
\hline 1934 Federal Surplus/Deficit & 2604 & 2920 & 490 & 1577 & 1465 & 4573 & 5611 & 3923 & 2475 & 5681 & 4998 & 4625 & 1197 & 2383 & 3035 \\
\hline 1935 Federal Surplus/Deficit & 1164 & -181 & -504 & 175 & -311 & -264 & 1895 & 2258 & -1330 & 212 & 1976 & 2767 & 3578 & 2753 & 1050 \\
\hline 1936 Federal Surplus/Deficit & 2603 & 810 & -334 & 377 & 2.5 & -678 & -1423 & -2174 & -626 & -589 & 1680 & 6315 & 3236 & 1710 & 722 \\
\hline 1937 Federal Surplus/Deficit & 2334 & 555 & -436 & 418 & 52 & -700 & -1925 & -1997 & -858 & -631 & -914 & 109 & 1341 & 1189 & -178 \\
\hline 1938 Federal Surplus/Deficit & 1938 & 181 & -444 & 581 & -76 & -608 & 2697 & -461 & 1440 & 2329 & 2472 & 5370 & 3966 & 2333 & 1522 \\
\hline 1939 Federal Surplus/Deficit & 1691 & 382 & -30 & 502 & -7.7 & -714 & 326 & -1878 & -278 & 255 & 1032 & 4019 & 1870 & 1816 & 609 \\
\hline 1940 Federal Surplus/Deficit & 2522 & 677 & -428 & 615 & -52 & -498 & -349 & -1085 & 1672 & 1174 & 1810 & 2673 & 2618 & 1194 & 788 \\
\hline 1941 Federal Surplus/Deficit & 1593 & 250 & -478 & 401 & -172 & -93 & 198 & -2143 & -763 & -976 & -986 & 758 & 2194 & 546 & 32 \\
\hline 1942 Federal Surplus/Deficit & 1362 & 391 & -104 & 339 & -294 & 1477 & 2859 & -1506 & -1354 & -272 & 487 & 2408 & 5174 & 3302 & 1107 \\
\hline 1943 Federal Surplus/Deficit & 3048 & 2101 & -136 & 401 & -176 & -286 & 2073 & 1247 & 2102 & 5322 & 4631 & 5307 & 4968 & 3407 & 2205 \\
\hline 1944 Federal Surplus/Deficit & 2830 & 2023 & -144 & 506 & 21 & -737 & 286 & -2142 & -1288 & -809 & -1348 & -307 & 1106 & 376 & -81 \\
\hline 1945 Federal Surplus/Deficit & 1451 & -123 & -365 & 204 & -8 & -732 & -2074 & -1722 & -999 & -736 & -1094 & 2674 & 4219 & 1760 & 225 \\
\hline 1946 Federal Surplus/Deficit & 1623 & 1161 & -553 & 510 & 4.7 & -657 & 727 & 647 & 2197 & 2790 & 3724 & 5661 & 4374 & 3233 & 1733 \\
\hline 1947 Federal Surplus/Deficit & 3138 & 1799 & 94 & 388 & -25 & 2299 & 3090 & 2188 & 2471 & 1180 & 2600 & 4795 & 4059 & 2866 & 2215 \\
\hline 1948 Federal Surplus/Deficit & 3141 & 1203 & -80 & 2596 & 962 & 1223 & 2954 & -287 & 1060 & 1409 & 3541 & 6801 & 7736 & 3685 & 2608 \\
\hline 1949 Federal Surplus/Deficit & 2733 & 3216 & 815 & 688 & -105 & -52 & 877 & -458 & 3286 & 1468 & 4451 & 5678 & 3874 & 1086 & 1802 \\
\hline 1950 Federal Surplus/Deficit & 1417 & -89 & -749 & 406 & -184 & -935 & 1143 & 2594 & 4046 & 3860 & 3645 & 4560 & 6827 & 3791 & 2160 \\
\hline 1951 Federal Surplus/Deficit & 2345 & 2661 & 250 & 1424 & 1457 & 3316 & 4471 & 4401 & 2706 & 3719 & 4183 & 5705 & 3237 & 3708 & 3094 \\
\hline 1952 Federal Surplus/Deficit & 2832 & 2765 & 556 & 2051 & 156 & 1625 & 2728 & 1107 & 1446 & 3940 & 4351 & 6704 & 4177 & 2102 & 2466 \\
\hline 1953 Federal Surplus/Deficit & 2848 & 1054 & -208 & 371 & 85 & -696 & 65 & 2232 & -341 & -697 & 930 & 4230 & 6586 & 3868 & 1522 \\
\hline 1954 Federal Surplus/Deficit & 2976 & 1858 & 125 & 666 & 73 & 655 & 1755 & 2478 & 1046 & 3142 & 2413 & 5038 & 6085 & 4595 & 2309 \\
\hline 1955 Federal Surplus/Deficit & 3975 & 2974 & 2766 & 1267 & 587 & 802 & -278 & -1812 & -1218 & 234 & -209 & 2149 & 7401 & 5666 & 1735 \\
\hline 1956 Federal Surplus/Deficit & 2747 & 3144 & 16 & 1137 & 856 & 2980 & 5011 & 1633 & 3416 & 3924 & 5849 & 6751 & 7391 & 4044 & 3422 \\
\hline 1957 Federal Surplus/Deficit & 3106 & 2369 & 68 & 791 & -137 & 827 & 1446 & -1163 & 670 & 4487 & 2724 & 6297 & 7102 & 2284 & 2044 \\
\hline 1958 Federal Surplus/Deficit & 1986 & 608 & -295 & 515 & -6.5 & -578 & 962 & 1134 & 1275 & 470 & 3324 & 5639 & 5564 & 1847 & 1604 \\
\hline 1959 Federal Surplus/Deficit & 2014 & 896 & -197 & 413 & 481 & 1591 & 4484 & 2829 & 1273 & 2653 & 1862 & 3927 & 6041 & 4400 & 2413 \\
\hline 1960 Federal Surplus/Deficit & 2848 & 2191 & 2544 & 3228 & 1951 & 2489 & 2736 & -220 & 1091 & 5786 & 3695 & 3462 & 4374 & 2893 & 2651 \\
\hline 1961 Federal Surplus/Deficit & 3144 & 760 & -120 & 566 & 207 & -84 & 1503 & 1855 & 1961 & 2693 & 2682 & 4195 & 6971 & 2434 & 2011 \\
\hline 1962 Federal Surplus/Deficit & 2201 & 1268 & -488 & 479 & -28 & -831 & 1378 & -1182 & -821 & 4080 & 4396 & 3617 & 3504 & 3120 & 1227 \\
\hline 1963 Federal Surplus/Deficit & 2663 & 1693 & -251 & 990 & 626 & 1570 & 1719 & 396 & 319 & 326 & 606 & 4424 & 4135 & 2838 & 1618 \\
\hline 1964 Federal Surplus/Deficit & 3372 & 1908 & 201 & 300 & 29 & -460 & 1246 & -415 & -862 & 1397 & 1478 & 3206 & 7063 & 5182 & 1631 \\
\hline 1965 Federal Surplus/Deficit & 2882 & 2604 & 810 & 1395 & 168 & 3173 & 5528 & 4004 & 2963 & 2418 & 4274 & 5470 & 4925 & 2390 & 3076 \\
\hline 1966 Federal Surplus/Deficit & 2945 & 2371 & 157 & 920 & 217 & 744 & 2369 & -671 & -561 & 3133 & 1330 & 3531 & 2958 & 3211 & 1480 \\
\hline 1967 Federal Surplus/Deficit & 3188 & 1513 & -389 & 371 & -28 & -111 & 2787 & 3417 & 424 & 2065 & 821 & 2280 & 6617 & 4487 & 1971 \\
\hline 1968 Federal Surplus/Deficit & 2947 & 2276 & 260 & 733 & 104 & 582 & 2272 & 1477 & 1241 & -1019 & 702 & 2386 & 4762 & 4135 & 1700 \\
\hline 1969 Federal Surplus/Deficit & 3110 & 2415 & 1222 & 1515 & 1002 & 1451 & 4337 & 2954 & 1710 & 4915 & 4422 & 6549 & 4633 & 3073 & 2990 \\
\hline 1970 Federal Surplus/Deficit & 3039 & 1113 & -208 & 504 & 98 & -296 & 1855 & 1403 & -327 & -535 & 1526 & 3455 & 5102 & 1208 & 1280 \\
\hline 1971 Federal Surplus/Deficit & 1842 & 759 & -487 & 489 & 189 & -420 & 3539 & 4983 & 2464 & 3151 & 3894 & 6903 & 6835 & 4368 & 2807 \\
\hline 1972 Federal Surplus/Deficit & 3065 & 3298 & 425 & 581 & 111 & 724 & 3341 & 4591 & 5065 & 5427 & 2364 & 6605 & 7280 & 4804 & 3384 \\
\hline 1973 Federal Surplus/Deficit & 3621 & 2994 & 882 & 752 & -24 & 965 & 2726 & -1851 & -927 & -1150 & -1093 & 595 & 2150 & 1541 & 750 \\
\hline 1974 Federal Surplus/Deficit & 1735 & 59 & -582 & 105 & -325 & 1765 & 6019 & 4944 & 3799 & 4712 & 5341 & 6020 & 7408 & 6045 & 3427 \\
\hline 1975 Federal Surplus/Deficit & 3128 & 3270 & 830 & 258 & -6.6 & -189 & 2141 & 965 & 2480 & 1498 & 1759 & 4355 & 5560 & 5067 & 2191 \\
\hline 1976 Federal Surplus/Deficit & 1945 & 1229 & 199 & 1166 & 1269 & 4092 & 4370 & 2827 & 1889 & 4653 & 3443 & 6109 & 3630 & 4609 & 2983 \\
\hline 1977 Federal Surplus/Deficit & 4540 & 4204 & 3397 & 910 & 0.1 & -553 & 316 & -2230 & -1616 & -1306 & -1630 & 452 & 756 & 351 & 391 \\
\hline 1978 Federal Surplus/Deficit & 1767 & -119 & -606 & -66 & -213 & -387 & 1441 & -179 & 2353 & 1513 & 2262 & 3878 & 3290 & 3225 & 1287 \\
\hline \multicolumn{16}{|l|}{-Ranked Averages- } \\
\hline Top Ten Percent & 2555 & 2353 & 184 & 928 & 453 & 2392 & 4874 & 3914 & 3590 & 4040 & 4402 & 6110 & 6048 & 4198 & 3281 \\
\hline Middle Eighty Percent & 2572 & 1488 & 168 & 742 & 184 & 407 & 1721 & 518 & 748 & 1798 & 2167 & 4162 & 4674 & 3007 & 1695 \\
\hline Bottom Ten Percent & 2253 & 925 & -352 & 392 & 50 & -688 & -1212 & -1955 & -969 & -546 & -1130 & -135 & 1534 & 1189 & -116 \\
\hline
\end{tabular}


Section 7

Monthly Regional 50 Water Year Surplus/Deficit

Average Energy in Megawatts 
Regional Surplus/Deficit by Water Year

PNW Loads and Resources Study

2004 - 2005 Operating Year

$3 / 31 / 2004$ 2003 White Book

\begin{tabular}{|c|c|c|c|c|c|c|c|c|c|c|c|c|c|c|c|}
\hline Average Energy in Megawatts & Aug1 & Aug16 & Sep & Oct & Nov & Dec & Jan & $\mathrm{Feb}$ & Mar & Apr1 & Apr16 & May & Jun & Jul & Avg \\
\hline 1929 Regional Surplus/Deficit & 7206 & 3415 & 2140 & 3749 & 3577 & 1596 & 2029 & -1613 & 449 & 206 & 718 & -174 & 5277 & 4685 & 2291 \\
\hline 1930 Regional Surplus/Deficit & 3888 & 2447 & 1536 & 3506 & 3171 & 1305 & -849 & 614 & 458 & 871 & 756 & -745 & 3848 & 4692 & 1793 \\
\hline 1931 Regional Surplus/Deficit & 4592 & 2830 & 1800 & 3482 & 3234 & 1099 & -560 & -1684 & 241 & 1126 & 231 & -554 & 2995 & 3440 & 1490 \\
\hline 1932 Regional Surplus/Deficit & 4211 & 1919 & 1589 & 3170 & 3008 & 1180 & -338 & -1495 & 3198 & 6984 & 10654 & 8808 & 10084 & 7240 & 4027 \\
\hline 1933 Regional Surplus/Deficit & 7270 & 5498 & 2211 & 3727 & 3895 & 3568 & 7282 & 4997 & 600 & 3017 & 4180 & 6021 & 14323 & 12549 & 5763 \\
\hline 1934 Regional Surplus/Deficit & 6905 & 6914 & 3545 & 6861 & 6982 & 11705 & 13602 & 10293 & 7372 & 11928 & 10760 & 7974 & 3475 & 5839 & 7992 \\
\hline 1935 Regional Surplus/Deficit & 3552 & 1364 & 1417 & 3512 & 3960 & 2858 & 6724 & 6948 & -392 & 1893 & 5060 & 4968 & 8071 & 7217 & 4268 \\
\hline 1936 Regional Surplus/Deficit & 6112 & 3488 & 1847 & 3538 & 3177 & 1164 & 290 & -1321 & 981 & 862 & 5225 & 11086 & 7411 & 4900 & 3410 \\
\hline 1937 Regional Surplus/Deficit & 5522 & 2652 & 1741 & 3489 & 3115 & 1408 & -778 & -1557 & 331 & 925 & 696 & -213 & 4258 & 4285 & 1748 \\
\hline 1938 Regional Surplus/Deficit & 4867 & 1943 & 1608 & 4059 & 3968 & 2356 & 8054 & 1779 & 4813 & 5720 & 7366 & 9861 & 8725 & 6662 & 5153 \\
\hline 1939 Regional Surplus/Deficit & 4540 & 2288 & 2482 & 3829 & 3481 & 1795 & 3758 & -666 & 1603 & 2252 & 3955 & 6997 & 4322 & 5368 & 3291 \\
\hline 1940 Regional Surplus/Deficit & 5883 & 2792 & 1694 & 3957 & 3088 & 2238 & 2282 & 1368 & 5353 & 3838 & 5208 & 4136 & 4762 & 3392 & 3427 \\
\hline 1941 Regional Surplus/Deficit & 3984 & 2101 & 1509 & 3610 & 2958 & 2488 & 2860 & -1094 & 535 & -99 & 730 & 472 & 4201 & 2643 & 1962 \\
\hline 1942 Regional Surplus/Deficit & 3795 & 2213 & 1926 & 3359 & 2423 & 5723 & 7005 & 46 & -600 & 1283 & 2905 & 3913 & 10043 & 8061 & 3916 \\
\hline 1943 Regional Surplus/Deficit & 6921 & 5391 & 2132 & 3543 & 3812 & 2959 & 6633 & 5406 & 6088 & 12168 & 10860 & 9868 & 10690 & 9652 & 6538 \\
\hline 1944 Regional Surplus/Deficit & 7189 & 5628 & 2402 & 4001 & 3539 & 1901 & 2946 & -1088 & -212 & 233 & 409 & -942 & 2583 & 2472 & 2028 \\
\hline 1945 Regional Surplus/Deficit & 4090 & 1498 & 1557 & 2848 & 2825 & 1322 & -249 & -304 & 183 & 488 & 781 & 4702 & 8632 & 5385 & 2527 \\
\hline 1946 Regional Surplus/Deficit & 4392 & 3487 & 1607 & 4120 & 3912 & 2418 & 4994 & 4426 & 6531 & 7047 & 9335 & 10596 & 9575 & 8383 & 5724 \\
\hline 1947 Regional Surplus/Deficit & 7416 & 5079 & 2778 & 3927 & 4163 & 7904 & 8617 & 7269 & 7019 & 4641 & 7610 & 8054 & 8567 & 7237 & 6492 \\
\hline 1948 Regional Surplus/Deficit & 7364 & 3819 & 2497 & 8804 & 6125 & 5324 & 8513 & 2366 & 3692 & 4581 & 8452 & 11887 & 15299 & 9288 & 7159 \\
\hline 1949 Regional Surplus/Deficit & 7069 & 7903 & 4197 & 4610 & 3785 & 2920 & 4042 & 2351 & 8519 & 4754 & 10305 & 10305 & 8583 & 4436 & 5730 \\
\hline 1950 Regional Surplus/Deficit & 4565 & 1731 & 1376 & 4072 & 4152 & 1707 & 5575 & 7790 & 9990 & 9241 & 9384 & 8176 & 14102 & 10517 & 6660 \\
\hline 1951 Regional Surplus/Deficit & 6809 & 7356 & 3307 & 6521 & 7481 & 10284 & 11535 & 12123 & 7832 & 9160 & 10176 & 10573 & 7770 & 9487 & 8639 \\
\hline 1952 Regional Surplus/Deficit & 6988 & 7002 & 3813 & 7677 & 4494 & 6436 & 7795 & 5344 & 5167 & 8792 & 10236 & 12060 & 9132 & 6232 & 7055 \\
\hline 1953 Regional Surplus/Deficit & 6980 & 3553 & 2225 & 3554 & 3434 & 1474 & 4440 & 7197 & 1557 & 567 & 4032 & 8031 & 13402 & 9910 & 5233 \\
\hline 1954 Regional Surplus/Deficit & 7502 & 5294 & 2828 & 4480 & 4192 & 4995 & 6719 & 7764 & 4685 & 8017 & 6729 & 8770 & 12368 & 11900 & 6873 \\
\hline 1955 Regional Surplus/Deficit & 9150 & 7641 & 7772 & 5629 & 5402 & 4500 & 2009 & -307 & -236 & 2613 & 1435 & 3583 & 14557 & 13421 & 5563 \\
\hline 1956 Regional Surplus/Deficit & 7096 & 7581 & 2707 & 6167 & 6369 & 9009 & 12521 & 6367 & 8777 & 9642 & 13094 & 12245 & 15369 & 10172 & 9034 \\
\hline 1957 Regional Surplus/Deficit & 7765 & 6413 & 2912 & 5222 & 3806 & 5331 & 5716 & 1512 & 3900 & 9761 & 7038 & 11440 & 14063 & 6132 & 6293 \\
\hline 1958 Regional Surplus/Deficit & 5239 & 2814 & 2029 & 4010 & 3559 & 2336 & 5405 & 5588 & 4611 & 2916 & 7816 & 10408 & 11332 & 5430 & 5342 \\
\hline 1959 Regional Surplus/Deficit & 5248 & 3401 & 2303 & 3959 & 5356 & 6510 & 11266 & 8126 & 4552 & 7219 & 6284 & 7118 & 12210 & 10667 & 6929 \\
\hline 1960 Regional Surplus/Deficit & 6885 & 5911 & 7769 & 10061 & 8032 & 7760 & 7526 & 2791 & 4433 & 12439 & 9135 & 6443 & 9626 & 7622 & 7437 \\
\hline 1961 Regional Surplus/Deficit & 7381 & 3606 & 2405 & 4125 & 4615 & 2971 & 6323 & 6810 & 5836 & 6639 & 6428 & 7250 & 13349 & 6586 & 6025 \\
\hline 1962 Regional Surplus/Deficit & 5510 & 3948 & 1634 & 3982 & 3661 & 1751 & 5598 & 618 & 512 & 9305 & 10437 & 6610 & 7623 & 7758 & 4529 \\
\hline 1963 Regional Surplus/Deficit & 6715 & 4682 & 2173 & 5094 & 5461 & 6489 & 5881 & 4506 & 2773 & 2832 & 3457 & 7651 & 8653 & 7227 & 5396 \\
\hline 1964 Regional Surplus/Deficit & 7665 & 5112 & 2933 & 3749 & 4225 & 2364 & 5665 & 1938 & 575 & 4730 & 4892 & 5931 & 14768 & 12455 & 5483 \\
\hline 1965 Regional Surplus/Deficit & 7363 & 6936 & 4297 & 5807 & 4257 & 9407 & 13262 & 10685 & 7689 & 6388 & 10452 & 9951 & 10524 & 7019 & 8206 \\
\hline 1966 Regional Surplus/Deficit & 7288 & 6608 & 3099 & 4850 & 4169 & 4392 & 7504 & 1240 & 1387 & 7524 & 4588 & 6304 & 6786 & 8106 & 5070 \\
\hline 1967 Regional Surplus/Deficit & 7200 & 4769 & 1950 & 3771 & 3828 & 3477 & 8448 & 9310 & 2751 & 6210 & 4000 & 4572 & 13735 & 10666 & 6133 \\
\hline 1968 Regional Surplus/Deficit & 6930 & 5927 & 3009 & 5079 & 4273 & 4292 & 7592 & 6360 & 5075 & 262 & 3305 & 4089 & 9909 & 9519 & 5617 \\
\hline 1969 Regional Surplus/Deficit & 7122 & 6595 & 5168 & 6485 & 6249 & 6202 & 11019 & 8197 & 5535 & 11233 & 10609 & 12097 & 9780 & 7690 & 8017 \\
\hline 1970 Regional Surplus/Deficit & 7214 & 3660 & 2439 & 4214 & 3983 & 2612 & 6736 & 5802 & 1535 & 1358 & 4542 & 6419 & 11021 & 4565 & 4809 \\
\hline 1971 Regional Surplus/Deficit & 4976 & 2910 & 1861 & 4079 & 4254 & 2679 & 10155 & 12785 & 7148 & 7845 & 9296 & 12287 & 14243 & 11341 & 7779 \\
\hline 1972 Regional Surplus/Deficit & 7810 & 8029 & 3499 & 4503 & 4481 & 4839 & 9692 & 12062 & 12836 & 13175 & 7624 & 11869 & 14976 & 11989 & 9089 \\
\hline 1973 Regional Surplus/Deficit & 8774 & 7471 & 4379 & 4590 & 3906 & 5529 & 7954 & -481 & 561 & -229 & 1152 & 768 & 4681 & 4455 & 3744 \\
\hline 1974 Regional Surplus/Deficit & 4087 & 1646 & 1292 & 3198 & 3530 & 7156 & 14709 & 12989 & 9683 & 10860 & 11938 & 11024 & 15484 & 14498 & 8986 \\
\hline 1975 Regional Surplus/Deficit & 8051 & 7977 & 4190 & 3515 & 3847 & 3470 & 7201 & 4428 & 6635 & 4376 & 5350 & 8284 & 12008 & 12297 & 6563 \\
\hline 1976 Regional Surplus/Deficit & 5790 & 4597 & 3203 & 5734 & 7012 & 11542 & 11748 & 8377 & 5787 & 10551 & 8715 & 11149 & 8590 & 11314 & 8274 \\
\hline 1977 Regional Surplus/Deficit & 10428 & 9704 & 8835 & 4658 & 3436 & 1806 & 2871 & -1202 & -740 & -642 & -410 & -111 & 1825 & 2236 & 2763 \\
\hline 1978 Regional Surplus/Deficit & 4066 & 1512 & 1092 & 2463 & 3443 & 2773 & 5604 & 2418 & 6468 & 4672 & 6395 & 7584 & 7401 & 8449 & 4668 \\
\hline \multicolumn{16}{|l|}{-Ranked Averages- } \\
\hline Top Ten Percent & 6318 & 5842 & 2801 & 5225 & 5775 & 8566 & 12041 & 10384 & 8983 & 10678 & 10309 & 11372 & 12438 & 11492 & 8804 \\
\hline Middle Eighty Percent & 6465 & 4605 & 2943 & 4568 & 4205 & 3957 & 6288 & 4027 & 3695 & 5143 & 6249 & 7368 & 9873 & 7812 & 5497 \\
\hline Bottom Ten Percent & 5035 & 3132 & 1798 & 3618 & 3203 & 1640 & 724 & -962 & 270 & 611 & 564 & -396 & 3577 & 3507 & 1804 \\
\hline
\end{tabular}


Regional Surplus/Deficit by Water Year

PNW Loads and Resources Study

2005 - 2006 Operating Year 2003 White Book

\begin{tabular}{|c|c|c|c|c|c|c|c|c|c|c|c|c|c|c|c|}
\hline Average Energy in Megawatts & Aug1 & Aug16 & Sep & Oct & Nov & Dec & Jan & $\mathrm{Feb}$ & Mar & Apr1 & Apr16 & May & Jun & Jul & Avg \\
\hline 1929 Regional Surplus/Deficit & 7178 & 3367 & 2238 & 3826 & 3498 & 1548 & 1901 & -1876 & 264 & 609 & 913 & -59 & 6219 & 3996 & 2299 \\
\hline 1930 Regional Surplus/Deficit & 3848 & 2400 & 1635 & 3579 & 3097 & 1261 & -982 & 353 & 276 & 1272 & 944 & -628 & 4801 & 4013 & 1803 \\
\hline 1931 Regional Surplus/Deficit & 4557 & 2785 & 1898 & 3553 & 3158 & 1058 & -693 & -1945 & 58 & 1529 & 425 & -433 & 3956 & 2768 & 1502 \\
\hline 1932 Regional Surplus/Deficit & 4176 & 1874 & 1689 & 3244 & 2931 & 1132 & -472 & -1756 & 3010 & 7398 & 10877 & 8943 & 11046 & 6559 & 4041 \\
\hline 1933 Regional Surplus/Deficit & 7239 & 5456 & 2311 & 3804 & 3817 & 3523 & 7171 & 4760 & 422 & 3439 & 4386 & 6160 & 15281 & 11885 & 5783 \\
\hline 1934 Regional Surplus/Deficit & 6876 & 6883 & 3652 & 6946 & 6903 & 11671 & 13500 & 10063 & 7205 & 12347 & 10974 & 8127 & 4428 & 5165 & 8017 \\
\hline 1935 Regional Surplus/Deficit & 3515 & 1318 & 1522 & 3586 & 3881 & 2811 & 6611 & 6714 & -564 & 2300 & 5262 & 5093 & 9021 & 6543 & 4285 \\
\hline 1936 Regional Surplus/Deficit & 6084 & 3445 & 1949 & 3615 & 3103 & 1123 & 162 & -1582 & 797 & 1270 & 5412 & 11222 & 8375 & 4221 & 3424 \\
\hline 1937 Regional Surplus/Deficit & 5486 & 2605 & 1838 & 3564 & 3041 & 1366 & -909 & -1815 & 150 & 1331 & 889 & -103 & 5202 & 3604 & 1758 \\
\hline 1938 Regional Surplus/Deficit & 4832 & 1899 & 1707 & 4134 & 3895 & 2310 & 7940 & 1530 & 4634 & 6133 & 7567 & 10002 & 9673 & 5980 & 5168 \\
\hline 1939 Regional Surplus/Deficit & 4504 & 2236 & 2582 & 3905 & 3402 & 1745 & 3640 & -924 & 1418 & 2656 & 4146 & 7125 & 5275 & 4687 & 3302 \\
\hline 1940 Regional Surplus/Deficit & 5851 & 2745 & 1792 & 4034 & 3015 & 2193 & 2153 & 1105 & 5176 & 4237 & 5417 & 4254 & 5713 & 2712 & 3439 \\
\hline 1941 Regional Surplus/Deficit & 3946 & 2055 & 1608 & 3686 & 2882 & 2444 & 2727 & -1356 & 351 & 312 & 938 & 595 & 5147 & 1953 & 1972 \\
\hline 1942 Regional Surplus/Deficit & 3754 & 2165 & 2022 & 3431 & 2342 & 5671 & 6901 & -214 & -784 & 1683 & 3097 & 4034 & 10998 & 7386 & 3928 \\
\hline 1943 Regional Surplus/Deficit & 6894 & 5349 & 2230 & 3620 & 3732 & 2906 & 6511 & 5141 & 5904 & 12576 & 11058 & 10010 & 11635 & 8964 & 6549 \\
\hline 1944 Regional Surplus/Deficit & 7156 & 5589 & 2497 & 4076 & 3460 & 1848 & 2818 & -1353 & -396 & 633 & 608 & -820 & 3527 & 1778 & 2036 \\
\hline 1945 Regional Surplus/Deficit & 4050 & 1448 & 1656 & 2921 & 2747 & 1275 & -386 & -570 & -2.2 & 889 & 971 & 4817 & 9578 & 4704 & 2535 \\
\hline 1946 Regional Surplus/Deficit & 4350 & 3440 & 1704 & 4192 & 3834 & 2367 & 4861 & 4168 & 6353 & 7462 & 9538 & 10741 & 10542 & 7703 & 5738 \\
\hline 1947 Regional Surplus/Deficit & 7389 & 5037 & 2876 & 4000 & 4082 & 7841 & 8510 & 7013 & 6846 & 5059 & 7818 & 8185 & 9532 & 6556 & 6508 \\
\hline 1948 Regional Surplus/Deficit & 7331 & 3770 & 2594 & 8891 & 6043 & 5281 & 8394 & 2103 & 3519 & 4997 & 8659 & 12024 & 16262 & 8620 & 7176 \\
\hline 1949 Regional Surplus/Deficit & 7038 & 7870 & 4297 & 4688 & 3706 & 2869 & 3921 & 2087 & 8339 & 5161 & 10514 & 10433 & 9542 & 3749 & 5744 \\
\hline 1950 Regional Surplus/Deficit & 4524 & 1680 & 1473 & 4145 & 4073 & 1660 & 5457 & 7537 & 9818 & 9647 & 9592 & 8316 & 15062 & 9835 & 6675 \\
\hline 1951 Regional Surplus/Deficit & 6774 & 7320 & 3404 & 6600 & 7396 & 10240 & 11430 & 11880 & 7654 & 9570 & 10388 & 10717 & 8722 & 8811 & 8657 \\
\hline 1952 Regional Surplus/Deficit & 6958 & 6966 & 3915 & 7760 & 4417 & 6384 & 7679 & 5090 & 4984 & 9193 & 10431 & 12196 & 10084 & 5548 & 7069 \\
\hline 1953 Regional Surplus/Deficit & 6948 & 3503 & 2322 & 3628 & 3357 & 1428 & 4299 & 6947 & 1381 & 984 & 4233 & 8168 & 14353 & 9228 & 5245 \\
\hline 1954 Regional Surplus/Deficit & 7473 & 5253 & 2927 & 4558 & 4116 & 4946 & 6601 & 7519 & 4511 & 8432 & 6925 & 8914 & 13343 & 11230 & 6892 \\
\hline 1955 Regional Surplus/Deficit & 9127 & 7607 & 7888 & 5712 & 5326 & 4459 & 1882 & -568 & -417 & 3019 & 1640 & 3710 & 15528 & 12761 & 5582 \\
\hline 1956 Regional Surplus/Deficit & 7066 & 7548 & 2806 & 6247 & 6287 & 8960 & 12414 & 6117 & 8594 & 10061 & 13301 & 12383 & 16334 & 9497 & 9052 \\
\hline 1957 Regional Surplus/Deficit & 7735 & 6375 & 3010 & 5299 & 3726 & 5279 & 5597 & 1246 & 3718 & 10167 & 7245 & 11577 & 15028 & 5445 & 6307 \\
\hline 1958 Regional Surplus/Deficit & 5200 & 2764 & 2126 & 4084 & 3481 & 2283 & 5285 & 5328 & 4441 & 3325 & 8017 & 10535 & 12300 & 4740 & 5355 \\
\hline 1959 Regional Surplus/Deficit & 5209 & 3353 & 2401 & 4035 & 5274 & 6459 & 11155 & 7889 & 4372 & 7641 & 6496 & 7262 & 13176 & 9991 & 6947 \\
\hline 1960 Regional Surplus/Deficit & 6856 & 5873 & 7881 & 10146 & 7951 & 7721 & 7424 & 2539 & 4252 & 12867 & 9358 & 6583 & 10583 & 6944 & 7458 \\
\hline 1961 Regional Surplus/Deficit & 7353 & 3557 & 2506 & 4204 & 4538 & 2923 & 6209 & 6562 & 5666 & 7063 & 6639 & 7401 & 14327 & 5910 & 6046 \\
\hline 1962 Regional Surplus/Deficit & 5477 & 3905 & 1732 & 4060 & 3582 & 1703 & 5480 & 361 & 328 & 9712 & 10656 & 6749 & 8578 & 7079 & 4544 \\
\hline 1963 Regional Surplus/Deficit & 6686 & 4638 & 2271 & 5169 & 5380 & 6441 & 5768 & 4247 & 2589 & 3241 & 3664 & 7772 & 9601 & 6549 & 5409 \\
\hline 1964 Regional Surplus/Deficit & 7637 & 5068 & 3033 & 3825 & 4145 & 2314 & 5548 & 1687 & 392 & 5127 & 5096 & 6060 & 15723 & 11789 & 5498 \\
\hline 1965 Regional Surplus/Deficit & 7333 & 6898 & 4398 & 5890 & 4181 & 9344 & 13151 & 10435 & 7509 & 6798 & 10653 & 10084 & 11464 & 6326 & 8218 \\
\hline 1966 Regional Surplus/Deficit & 7256 & 6565 & 3196 & 4927 & 4092 & 4338 & 7388 & 993 & 1203 & 7943 & 4794 & 6436 & 7743 & 7435 & 5086 \\
\hline 1967 Regional Surplus/Deficit & 7175 & 4722 & 2048 & 3847 & 3750 & 3429 & 8335 & 9071 & 2579 & 6637 & 4209 & 4700 & 14697 & 9988 & 6151 \\
\hline 1968 Regional Surplus/Deficit & 6901 & 5890 & 3110 & 5155 & 4195 & 4240 & 7474 & 6109 & 4901 & 666 & 3513 & 4208 & 10868 & 8842 & 5632 \\
\hline 1969 Regional Surplus/Deficit & 7094 & 6557 & 5272 & 6565 & 6168 & 6156 & 10904 & 7954 & 5350 & 11650 & 10821 & 12238 & 10751 & 7009 & 8036 \\
\hline 1970 Regional Surplus/Deficit & 7181 & 3615 & 2537 & 4292 & 3906 & 2562 & 6603 & 5546 & 1357 & 1774 & 4762 & 6545 & 11962 & 3875 & 4821 \\
\hline 1971 Regional Surplus/Deficit & 4939 & 2858 & 1954 & 4151 & 4172 & 2623 & 10029 & 12537 & 6964 & 8251 & 9504 & 12422 & 15195 & 10663 & 7791 \\
\hline 1972 Regional Surplus/Deficit & 7781 & 7995 & 3598 & 4578 & 4401 & 4786 & 9569 & 11815 & 12663 & 13601 & 7839 & 12005 & 15932 & 11326 & 9107 \\
\hline 1973 Regional Surplus/Deficit & 8749 & 7438 & 4480 & 4667 & 3826 & 5476 & 7832 & -744 & 377 & 180 & 1358 & 885 & 5635 & 3773 & 3756 \\
\hline 1974 Regional Surplus/Deficit & 4051 & 1598 & 1390 & 3268 & 3446 & 7097 & 14598 & 12748 & 9506 & 11272 & 12146 & 11157 & 16442 & 13832 & 9001 \\
\hline 1975 Regional Surplus/Deficit & 8021 & 7945 & 4292 & 3589 & 3766 & 3417 & 7082 & 4179 & 6453 & 4792 & 5555 & 8417 & 12951 & 11619 & 6577 \\
\hline 1976 Regional Surplus/Deficit & 5755 & 4550 & 3301 & 5811 & 6927 & 11493 & 11636 & 8133 & 5607 & 10962 & 8923 & 11282 & 9536 & 10644 & 8289 \\
\hline 1977 Regional Surplus/Deficit & 10409 & 9673 & 8954 & 4737 & 3357 & 1756 & 2745 & -1465 & -922 & -237 & -207 & 18 & 2777 & 1554 & 2778 \\
\hline 1978 Regional Surplus/Deficit & 4029 & 1466 & 1191 & 2535 & 3362 & 2712 & 5474 & 2152 & 6292 & 5094 & 6608 & 7720 & 8346 & 7765 & 4679 \\
\hline \multicolumn{16}{|l|}{-Ranked Averages- } \\
\hline Top Ten Percent & 6285 & 5802 & 2900 & 5301 & 5691 & 8515 & 11930 & 10139 & 8805 & 11093 & 10519 & 11509 & 13393 & 10822 & 8821 \\
\hline Middle Eighty Percent & 6433 & 4562 & 3043 & 4645 & 4127 & 3908 & 6168 & 3773 & 3516 & 5555 & 6454 & 7501 & 10830 & 7133 & 5512 \\
\hline Bottom Ten Percent & 4999 & 3087 & 1895 & 3692 & 3127 & 1595 & 592 & -1223 & 88 & 1016 & 761 & -278 & 4526 & 2823 & 1814 \\
\hline
\end{tabular}


Regional Surplus/Deficit by Water Year

PNW Loads and Resources Study

2006 - 2007 Operating Year

$3 / 31 / 2004$ 2003 White Book

\begin{tabular}{|c|c|c|c|c|c|c|c|c|c|c|c|c|c|c|c|}
\hline Average Energy in Megawatts & Aug1 & Aug16 & Sep & Oct & Nov & Dec & Jan & Feb & Mar & Apr1 & Apr16 & May & Jun & Jul & Avg \\
\hline 1929 Regional Surplus/Deficit & 6483 & 3308 & 2263 & 3431 & 3152 & 1051 & 1166 & -2382 & -251 & -301 & -164 & -1552 & 4185 & 3776 & 1625 \\
\hline 1930 Regional Surplus/Deficit & 3139 & 2341 & 1661 & 3181 & 2755 & 766 & -1726 & -147 & -238 & 365 & -134 & -2124 & 2769 & 3798 & 1129 \\
\hline 1931 Regional Surplus/Deficit & 3852 & 2729 & 1923 & 3153 & 2815 & 564 & -1436 & -2449 & -457 & 625 & -656 & -1925 & 1926 & 2554 & 829 \\
\hline 1932 Regional Surplus/Deficit & 3473 & 1819 & 1715 & 2845 & 2588 & 631 & -1217 & -2261 & 2501 & 6506 & 9827 & 7471 & 9027 & 6348 & 3372 \\
\hline 1933 Regional Surplus/Deficit & 6538 & 5400 & 2338 & 3409 & 3474 & 3026 & 6451 & 4279 & -87 & 2542 & 3317 & 4677 & 13271 & 11687 & 5119 \\
\hline 1934 Regional Surplus/Deficit & 6176 & 6834 & 3685 & 6559 & 6560 & 11196 & 12796 & 9592 & 6711 & 11468 & 9928 & 6660 & 2389 & 4950 & 7359 \\
\hline 1935 Regional Surplus/Deficit & 2812 & 1263 & 1551 & 3187 & 3536 & 2312 & 5889 & 6236 & -1071 & 1399 & 4198 & 3607 & 6992 & 6334 & 3617 \\
\hline 1936 Regional Surplus/Deficit & 5386 & 3390 & 1976 & 3219 & 2760 & 628 & -575 & -2087 & 283 & 361 & 4348 & 9750 & 6350 & 4007 & 2755 \\
\hline 1937 Regional Surplus/Deficit & 4781 & 2546 & 1863 & 3167 & 2699 & 870 & -1651 & -2318 & -364 & 423 & -188 & -1602 & 3163 & 3386 & 1083 \\
\hline 1938 Regional Surplus/Deficit & 4128 & 1845 & 1733 & 3737 & 3554 & 1814 & 7217 & 1040 & 4131 & 5236 & 6505 & 8531 & 7645 & 5769 & 4502 \\
\hline 1939 Regional Surplus/Deficit & 3796 & 2170 & 2610 & 3511 & 3058 & 1248 & 2914 & -1424 & 908 & 1755 & 3079 & 5646 & 3239 & 4471 & 2632 \\
\hline 1940 Regional Surplus/Deficit & 5150 & 2688 & 1818 & 3640 & 2673 & 1696 & 1417 & 605 & 4676 & 3335 & 4363 & 2770 & 3679 & 2495 & 2770 \\
\hline 1941 Regional Surplus/Deficit & 3240 & 2000 & 1633 & 3291 & 2540 & 1950 & 1987 & -1859 & -163 & -589 & -127 & -893 & 3113 & 1732 & 1300 \\
\hline 1942 Regional Surplus/Deficit & 3044 & 2106 & 2047 & 3033 & 1999 & 5175 & 6189 & -714 & -1300 & 779 & 2029 & 2549 & 8977 & 7180 & 3260 \\
\hline 1943 Regional Surplus/Deficit & 6195 & 5291 & 2257 & 3225 & 3387 & 2408 & 5790 & 4649 & 5404 & 11691 & 10005 & 8540 & 9613 & 8757 & 5885 \\
\hline 1944 Regional Surplus/Deficit & 6449 & 5531 & 2521 & 3681 & 3114 & 1351 & 2083 & -1859 & -914 & -277 & -466 & -2311 & 1490 & 1555 & 1361 \\
\hline 1945 Regional Surplus/Deficit & 3340 & 1388 & 1681 & 2522 & 2402 & 777 & -1131 & -1074 & -519 & -20 & -110 & 3336 & 7553 & 4493 & 1862 \\
\hline 1946 Regional Surplus/Deficit & 3638 & 3378 & 1729 & 3793 & 3490 & 1872 & 4128 & 3676 & 5855 & 6566 & 8482 & 9274 & 8520 & 7493 & 5072 \\
\hline 1947 Regional Surplus/Deficit & 6689 & 4981 & 2904 & 3605 & 3739 & 7345 & 7800 & 6530 & 6349 & 4162 & 6756 & 6711 & 7513 & 6347 & 5845 \\
\hline 1948 Regional Surplus/Deficit & 6627 & 3706 & 2622 & 8509 & 5700 & 4792 & 7678 & 1607 & 3019 & 4096 & 7600 & 10561 & 14256 & 8422 & 6515 \\
\hline 1949 Regional Surplus/Deficit & 6334 & 7819 & 4327 & 4295 & 3362 & 2370 & 3195 & 1586 & 7846 & 4264 & 9460 & 8960 & 7514 & 3531 & 5077 \\
\hline 1950 Regional Surplus/Deficit & 3814 & 1616 & 1497 & 3748 & 3729 & 1164 & 4733 & 7050 & 9329 & 8756 & 8537 & 6842 & 13051 & 9631 & 6011 \\
\hline 1951 Regional Surplus/Deficit & 6065 & 7265 & 3431 & 6212 & 7051 & 9757 & 10723 & 11411 & 7159 & 8679 & 9335 & 9250 & 6692 & 8607 & 7997 \\
\hline 1952 Regional Surplus/Deficit & 6254 & 6911 & 3945 & 7375 & 4074 & 5892 & 6960 & 4606 & 4479 & 8302 & 9377 & 10733 & 8060 & 5337 & 6407 \\
\hline 1953 Regional Surplus/Deficit & 6244 & 3436 & 2347 & 3231 & 3014 & 932 & 3559 & 6467 & 876 & 81 & 3164 & 6688 & 12337 & 9023 & 4578 \\
\hline 1954 Regional Surplus/Deficit & 6769 & 5193 & 2955 & 4165 & 3773 & 4449 & 5879 & 7040 & 4008 & 7537 & 5862 & 7444 & 11329 & 11030 & 6229 \\
\hline 1955 Regional Surplus/Deficit & 8431 & 7555 & 7931 & 5323 & 4983 & 3967 & 1147 & -1072 & -932 & 2119 & 581 & 2220 & 13520 & 12567 & 4916 \\
\hline 1956 Regional Surplus/Deficit & 6365 & 7497 & 2834 & 5858 & 5941 & 8475 & 11708 & 5636 & 8098 & 9174 & 12256 & 10919 & 14326 & 9293 & 8394 \\
\hline 1957 Regional Surplus/Deficit & 7032 & 6317 & 3038 & 4907 & 3382 & 4784 & 4872 & 746 & 3215 & 9275 & 6187 & 10115 & 13018 & 5230 & 5643 \\
\hline 1958 Regional Surplus/Deficit & 4491 & 2697 & 2151 & 3689 & 3137 & 1785 & 4559 & 4837 & 3943 & 2423 & 6956 & 9062 & 10288 & 4523 & 4688 \\
\hline 1959 Regional Surplus/Deficit & 4499 & 3293 & 2427 & 3642 & 4930 & 5967 & 10445 & 7416 & 3867 & 6748 & 5434 & 5787 & 11164 & 9785 & 6285 \\
\hline 1960 Regional Surplus/Deficit & 6155 & 5817 & 7923 & 9765 & 7610 & 7235 & 6713 & 2048 & 3749 & 11990 & 8303 & 5102 & 8560 & 6734 & 6798 \\
\hline 1961 Regional Surplus/Deficit & 6653 & 3494 & 2535 & 3811 & 4195 & 2424 & 5485 & 6081 & 5168 & 6168 & 5572 & 5929 & 12317 & 5696 & 5382 \\
\hline 1962 Regional Surplus/Deficit & 4774 & 3849 & 1757 & 3666 & 3237 & 1204 & 4754 & -133 & -187 & 8817 & 9608 & 5269 & 6550 & 6870 & 3876 \\
\hline 1963 Regional Surplus/Deficit & 5985 & 4579 & 2297 & 4778 & 5037 & 5950 & 5049 & 3762 & 2076 & 2342 & 2602 & 6291 & 7581 & 6340 & 4743 \\
\hline 1964 Regional Surplus/Deficit & 6936 & 5007 & 3062 & 3430 & 3800 & 1815 & 4822 & 1195 & -123 & 4224 & 4027 & 4578 & 13713 & 11594 & 4832 \\
\hline 1965 Regional Surplus/Deficit & 6629 & 6842 & 4430 & 5502 & 3840 & 8850 & 12446 & 9964 & 7012 & 5901 & 9600 & 8614 & 9442 & 6113 & 7558 \\
\hline 1966 Regional Surplus/Deficit & 6550 & 6502 & 3226 & 4536 & 3751 & 3841 & 6667 & 505 & 690 & 7057 & 3732 & 4957 & 5714 & 7227 & 4420 \\
\hline 1967 Regional Surplus/Deficit & 6478 & 4663 & 2073 & 3452 & 3405 & 2932 & 7616 & 8597 & 2075 & 5742 & 3139 & 3216 & 12687 & 9784 & 5488 \\
\hline 1968 Regional Surplus/Deficit & 6201 & 5836 & 3139 & 4763 & 3852 & 3743 & 6752 & 5628 & 4400 & -241 & 2448 & 2719 & 8852 & 8633 & 4967 \\
\hline 1969 Regional Surplus/Deficit & 6395 & 6501 & 5308 & 6177 & 5825 & 5665 & 10194 & 7482 & 4847 & 10764 & 9770 & 10775 & 8734 & 6799 & 7377 \\
\hline 1970 Regional Surplus/Deficit & 6476 & 3555 & 2563 & 3900 & 3562 & 2063 & 5872 & 5063 & 850 & 870 & 3701 & 5062 & 9938 & 3660 & 4153 \\
\hline 1971 Regional Surplus/Deficit & 4229 & 2791 & 1978 & 3755 & 3827 & 2127 & 9313 & 12069 & 6466 & 7356 & 8450 & 10958 & 13184 & 10461 & 7129 \\
\hline 1972 Regional Surplus/Deficit & 7079 & 7943 & 3627 & 4185 & 4057 & 4289 & 8851 & 11345 & 12178 & 12720 & 6781 & 10541 & 13923 & 11130 & 8449 \\
\hline 1973 Regional Surplus/Deficit & 8051 & 7386 & 4510 & 4274 & 3481 & 4981 & 7112 & -1246 & -137 & -724 & 290 & -608 & 3603 & 3556 & 3086 \\
\hline 1974 Regional Surplus/Deficit & 3346 & 1539 & 1414 & 2868 & 3101 & 6601 & 13896 & 12280 & 9016 & 10386 & 11099 & 9689 & 14434 & 13639 & 8344 \\
\hline 1975 Regional Surplus/Deficit & 7318 & 7891 & 4324 & 3194 & 3421 & 2919 & 6360 & 3694 & 5952 & 3895 & 4489 & 6940 & 10932 & 11421 & 5913 \\
\hline 1976 Regional Surplus/Deficit & 5046 & 4485 & 3331 & 5420 & 6583 & 11014 & 10929 & 7657 & 5110 & 10077 & 7867 & 9815 & 7508 & 10443 & 7629 \\
\hline 1977 Regional Surplus/Deficit & 9714 & 9622 & 9000 & 4347 & 3011 & 1259 & 2012 & -1970 & -1439 & -1148 & -1280 & -1473 & 739 & 1332 & 2106 \\
\hline 1978 Regional Surplus/Deficit & 3324 & 1410 & 1216 & 2135 & 3016 & 2210 & 4743 & 1655 & 5795 & 4201 & 5546 & 6243 & 6315 & 7556 & 4011 \\
\hline \multicolumn{16}{|l|}{-Ranked Averages- } \\
\hline Top Ten Percent & 5580 & 5746 & 2928 & 4908 & 5347 & 8027 & 11221 & 9666 & 8312 & 10207 & 9467 & 10043 & 11377 & 10623 & 8163 \\
\hline Middle Eighty Percent & 5730 & 4504 & 3072 & 4252 & 3783 & 3413 & 5444 & 3284 & 3011 & 4657 & 5393 & 6024 & 8809 & 6924 & 4846 \\
\hline Bottom Ten Percent & 4292 & 3029 & 1920 & 3295 & 2785 & 1100 & -148 & -1727 & -427 & 110 & -314 & -1771 & 2492 & 2605 & 1140 \\
\hline
\end{tabular}


Regional Surplus/Deficit by Water Year

PNW Loads and Resources Study

2007 - 2008 Operating Year 2003 White Book

\begin{tabular}{|c|c|c|c|c|c|c|c|c|c|c|c|c|c|c|c|}
\hline Average Energy in Megawatts & Aug1 & Aug16 & Sep & Oct & Nov & Dec & Jan & $\mathrm{Feb}$ & Mar & Apr1 & Apr16 & May & Jun & Jul & Avg \\
\hline 1929 Regional Surplus/Deficit & 6628 & 2783 & 1626 & 3157 & 2877 & 679 & 819 & -2364 & -307 & -43 & 342 & -846 & 5252 & 4103 & 1654 \\
\hline 1930 Regional Surplus/Deficit & 3268 & 1817 & 1025 & 2901 & 2488 & 398 & -2084 & -124 & -290 & 624 & 365 & -1418 & 3847 & 4131 & 1159 \\
\hline 1931 Regional Surplus/Deficit & 3989 & 2209 & 1286 & 2872 & 2547 & 200 & -1793 & -2429 & -509 & 885 & -153 & -1212 & 3015 & 2888 & 861 \\
\hline 1932 Regional Surplus/Deficit & 3608 & 1294 & 1081 & 2568 & 2317 & 257 & -1577 & -2241 & 2445 & 6785 & 10377 & 8209 & 10122 & 6690 & 3409 \\
\hline 1933 Regional Surplus/Deficit & 6682 & 4887 & 1703 & 3136 & 3202 & 2656 & 6128 & 4336 & -133 & 2826 & 3837 & 5412 & 14368 & 12052 & 5165 \\
\hline 1934 Regional Surplus/Deficit & 6322 & 6337 & 3061 & 6298 & 6287 & 10849 & 12490 & 9662 & 6685 & 11764 & 10472 & 7417 & 3465 & 5284 & 7412 \\
\hline 1935 Regional Surplus/Deficit & 2945 & 739 & 921 & 2909 & 3262 & 1939 & 5563 & 6297 & -1110 & 1665 & 4719 & 4327 & 8069 & 6679 & 3658 \\
\hline 1936 Regional Surplus/Deficit & 5534 & 2870 & 1343 & 2945 & 2493 & 264 & -923 & -2066 & 229 & 625 & 4855 & 10490 & 7443 & 4343 & 2792 \\
\hline 1937 Regional Surplus/Deficit & 4917 & 2022 & 1225 & 2891 & 2432 & 504 & -2006 & -2295 & -414 & 685 & 316 & -905 & 4229 & 3716 & 1112 \\
\hline 1938 Regional Surplus/Deficit & 4263 & 1325 & 1098 & 3460 & 3287 & 1444 & 6889 & 1079 & 4087 & 5512 & 7025 & 9277 & 8722 & 6111 & 4543 \\
\hline 1939 Regional Surplus/Deficit & 3931 & 1643 & 1976 & 3236 & 2785 & 874 & 2581 & -1397 & 854 & 2018 & 3586 & 6371 & 4316 & 4803 & 2666 \\
\hline 1940 Regional Surplus/Deficit & 5293 & 2165 & 1181 & 3368 & 2407 & 1327 & 1068 & 626 & 4636 & 3594 & 4895 & 3483 & 4756 & 2825 & 2804 \\
\hline 1941 Regional Surplus/Deficit & 3372 & 1476 & 997 & 3016 & 2271 & 1583 & 1632 & -1839 & -217 & -318 & 398 & -175 & 4184 & 2056 & 1331 \\
\hline 1942 Regional Surplus/Deficit & 3173 & 1582 & 1407 & 2753 & 1725 & 4799 & 5878 & -691 & -1356 & 1036 & 2536 & 3265 & 10064 & 7531 & 3295 \\
\hline 1943 Regional Surplus/Deficit & 6346 & 4777 & 1620 & 2950 & 3113 & 2029 & 5456 & 4673 & 5358 & 11972 & 10530 & 9287 & 10692 & 9094 & 5924 \\
\hline 1944 Regional Surplus/Deficit & 6592 & 5023 & 1880 & 3406 & 2839 & 974 & 1736 & -1843 & -971 & -22 & 46 & -1597 & 2557 & 1875 & 1390 \\
\hline 1945 Regional Surplus/Deficit & 3469 & 861 & 1044 & 2242 & 2130 & 406 & -1493 & -1059 & -575 & 236 & 388 & 4048 & 8629 & 4833 & 1890 \\
\hline 1946 Regional Surplus/Deficit & 3766 & 2857 & 1090 & 3513 & 3218 & 1498 & 3775 & 3707 & 5817 & 6845 & 9009 & 10025 & 9618 & 7838 & 5111 \\
\hline 1947 Regional Surplus/Deficit & 6839 & 4466 & 2269 & 3328 & 3464 & 6958 & 7488 & 6566 & 6315 & 4442 & 7284 & 7445 & 8611 & 6691 & 5888 \\
\hline 1948 Regional Surplus/Deficit & 6768 & 3183 & 1985 & 8252 & 5424 & 4428 & 7350 & 1630 & 2983 & 4372 & 8128 & 11307 & 15361 & 8787 & 6561 \\
\hline 1949 Regional Surplus/Deficit & 6481 & 7321 & 3694 & 4024 & 3088 & 1994 & 2859 & 1605 & 7809 & 4534 & 9994 & 9692 & 8599 & 3859 & 5116 \\
\hline 1950 Regional Surplus/Deficit & 3943 & 1086 & 857 & 3469 & 3456 & 793 & 4402 & 7088 & 9302 & 9030 & 9069 & 7584 & 14151 & 9981 & 6054 \\
\hline 1951 Regional Surplus/Deficit & 6205 & 6762 & 2795 & 5943 & 6770 & 9395 & 10412 & 11471 & 7122 & 8956 & 9873 & 9999 & 7771 & 8960 & 8045 \\
\hline 1952 Regional Surplus/Deficit & 6401 & 6407 & 3314 & 7113 & 3804 & 5519 & 6633 & 4644 & 4431 & 8573 & 9899 & 11478 & 9142 & 5679 & 6450 \\
\hline 1953 Regional Surplus/Deficit & 6389 & 2911 & 1708 & 2954 & 2743 & 563 & 3196 & 6513 & 835 & 356 & 3679 & 7423 & 13426 & 9372 & 4617 \\
\hline 1954 Regional Surplus/Deficit & 6917 & 4682 & 2321 & 3895 & 3503 & 4076 & 5548 & 7091 & 3970 & 7816 & 6377 & 8192 & 12439 & 11389 & 6277 \\
\hline 1955 Regional Surplus/Deficit & 8588 & 7056 & 7321 & 5058 & 4712 & 3604 & 800 & -1051 & -984 & 2385 & 1106 & 2938 & 14629 & 12936 & 4961 \\
\hline 1956 Regional Surplus/Deficit & 6511 & 6997 & 2199 & 5590 & 5663 & 8108 & 11397 & 5681 & 8056 & 9463 & 12795 & 11666 & 15430 & 9649 & 8444 \\
\hline 1957 Regional Surplus/Deficit & 7180 & 5810 & 2402 & 4635 & 3107 & 4408 & 4539 & 764 & 3169 & 9549 & 6716 & 10861 & 14123 & 5564 & 5683 \\
\hline 1958 Regional Surplus/Deficit & 4625 & 2171 & 1513 & 3413 & 2866 & 1407 & 4224 & 4865 & 3912 & 2691 & 7476 & 9793 & 11393 & 4852 & 4727 \\
\hline 1959 Regional Surplus/Deficit & 4632 & 2770 & 1790 & 3369 & 4654 & 5594 & 10129 & 7478 & 3821 & 7034 & 5965 & 6533 & 12268 & 10135 & 6331 \\
\hline 1960 Regional Surplus/Deficit & 6303 & 5310 & 7309 & 9507 & 7336 & 6876 & 6404 & 2085 & 3703 & 12293 & 8848 & 5839 & 9648 & 7077 & 6847 \\
\hline 1961 Regional Surplus/Deficit & 6802 & 2968 & 1903 & 3540 & 3925 & 2051 & 5156 & 6127 & 5136 & 6455 & 6099 & 6683 & 13432 & 6034 & 5429 \\
\hline 1962 Regional Surplus/Deficit & 4912 & 3330 & 1119 & 3394 & 2963 & 829 & 4422 & -101 & -242 & 9089 & 10155 & 6006 & 7632 & 7217 & 3915 \\
\hline 1963 Regional Surplus/Deficit & 6133 & 4062 & 1660 & 4506 & 4764 & 5580 & 4725 & 3795 & 2022 & 2613 & 3127 & 7009 & 8662 & 6685 & 4781 \\
\hline 1964 Regional Surplus/Deficit & 7084 & 4491 & 2429 & 3156 & 3526 & 1441 & 4491 & 1232 & -176 & 4481 & 4546 & 5304 & 14810 & 11960 & 4873 \\
\hline 1965 Regional Surplus/Deficit & 6778 & 6337 & 3800 & 5237 & 3572 & 8465 & 12132 & 10016 & 6972 & 6173 & 10129 & 9354 & 10516 & 6447 & 7602 \\
\hline 1966 Regional Surplus/Deficit & 6699 & 5993 & 2590 & 4264 & 3481 & 3463 & 6339 & 549 & 636 & 7344 & 4257 & 5688 & 6799 & 7574 & 4461 \\
\hline 1967 Regional Surplus/Deficit & 6630 & 4140 & 1437 & 3178 & 3133 & 2560 & 7292 & 8658 & 2039 & 6033 & 3663 & 3942 & 13789 & 10138 & 5533 \\
\hline 1968 Regional Surplus/Deficit & 6351 & 5331 & 2507 & 4491 & 3581 & 3366 & 6422 & 5672 & 4363 & 19 & 2973 & 3431 & 9949 & 8979 & 5008 \\
\hline 1969 Regional Surplus/Deficit & 6546 & 5995 & 4683 & 5910 & 5549 & 5298 & 9874 & 7539 & 4799 & 11052 & 10309 & 11524 & 9838 & 7142 & 7426 \\
\hline 1970 Regional Surplus/Deficit & 6618 & 3035 & 1927 & 3628 & 3290 & 1687 & 5522 & 5101 & 805 & 1142 & 4239 & 5784 & 11010 & 3990 & 4189 \\
\hline 1971 Regional Surplus/Deficit & 4365 & 2264 & 1334 & 3476 & 3550 & 1747 & 8978 & 12125 & 6421 & 7627 & 8984 & 11703 & 14277 & 10811 & 7170 \\
\hline 1972 Regional Surplus/Deficit & 7230 & 7445 & 2993 & 3911 & 3782 & 3911 & 8517 & 11400 & 12153 & 13021 & 7317 & 11286 & 15021 & 11496 & 8498 \\
\hline 1973 Regional Surplus/Deficit & 8207 & 6888 & 3878 & 4002 & 3206 & 4604 & 6779 & -1226 & -191 & -456 & 812 & 99 & 4684 & 3886 & 3121 \\
\hline 1974 Regional Surplus/Deficit & 3481 & 1013 & 776 & 2584 & 2822 & 6218 & 13583 & 12342 & 8984 & 10670 & 11638 & 10430 & 15533 & 14004 & 8390 \\
\hline 1975 Regional Surplus/Deficit & 7468 & 7395 & 3693 & 2918 & 3145 & 2540 & 6028 & 3737 & 5908 & 4174 & 5009 & 7673 & 12010 & 11776 & 5954 \\
\hline 1976 Regional Surplus/Deficit & 5187 & 3970 & 2696 & 5148 & 6304 & 10650 & 10613 & 7711 & 5070 & 10361 & 8398 & 10557 & 8581 & 10801 & 7674 \\
\hline 1977 Regional Surplus/Deficit & 9878 & 9130 & 8394 & 4077 & 2736 & 885 & 1667 & -1952 & -1494 & -888 & -764 & -752 & 1814 & 1656 & 2143 \\
\hline 1978 Regional Surplus/Deficit & 3457 & 886 & 579 & 1854 & 2741 & 1822 & 4396 & 1675 & 5761 & 4488 & 6078 & 6981 & 7386 & 7899 & 4046 \\
\hline \multicolumn{16}{|l|}{-Ranked Averages- } \\
\hline Top Ten Percent & 5723 & 5237 & 2292 & 4635 & 5068 & 7656 & 10904 & 9721 & 8277 & 10494 & 10004 & 10788 & 12467 & 10982 & 8210 \\
\hline Middle Eighty Percent & 5874 & 3988 & 2439 & 3980 & 3511 & 3039 & 5111 & 3320 & 2966 & 4931 & 5918 & 6757 & 9898 & 7268 & 4887 \\
\hline Bottom Ten Percent & 4428 & 2509 & 1283 & 3017 & 2515 & 732 & -503 & -1706 & -480 & 371 & 194 & -1061 & 3566 & 2933 & 1171 \\
\hline
\end{tabular}


Regional Surplus/Deficit by Water Year

PNW Loads and Resources Study

2008 - 2009 Operating Year

$3 / 31 / 2004$ 2003 White Book

\begin{tabular}{|c|c|c|c|c|c|c|c|c|c|c|c|c|c|c|c|}
\hline Average Energy in Megawatts & Aug1 & Aug16 & Sep & Oct & Nov & Dec & Jan & Feb & Mar & Apr1 & Apr16 & May & Jun & Jul & Avg \\
\hline 1929 Regional Surplus/Deficit & 6545 & 2700 & 1567 & 2859 & 2285 & 128 & 392 & -3115 & -737 & -458 & -339 & -2049 & 4108 & 3654 & 1110 \\
\hline 1930 Regional Surplus/Deficit & 3180 & 1735 & 965 & 2600 & 1898 & -152 & -2517 & -872 & -719 & 211 & -317 & -2622 & 2703 & 3685 & 614 \\
\hline 1931 Regional Surplus/Deficit & 3903 & 2128 & 1226 & 2570 & 1956 & -350 & -2225 & -3180 & -939 & 474 & -836 & -2414 & 1872 & 2442 & 316 \\
\hline 1932 Regional Surplus/Deficit & 3522 & 1212 & 1021 & 2267 & 1726 & -297 & -2009 & -2992 & 2018 & 6377 & 9712 & 7018 & 8985 & 6245 & 2866 \\
\hline 1933 Regional Surplus/Deficit & 6598 & 4809 & 1645 & 2838 & 2612 & 2105 & 5711 & 3599 & -559 & 2418 & 3163 & 4219 & 13233 & 11610 & 4626 \\
\hline 1934 Regional Surplus/Deficit & 6238 & 6262 & 3007 & 6004 & 5697 & 10310 & 12074 & 8928 & 6266 & 11361 & 9807 & 6227 & 2318 & 4837 & 6875 \\
\hline 1935 Regional Surplus/Deficit & 2859 & 657 & 865 & 2608 & 2671 & 1387 & 5144 & 5563 & -1535 & 1255 & 4048 & 3130 & 6929 & 6235 & 3117 \\
\hline 1936 Regional Surplus/Deficit & 5450 & 2791 & 1285 & 2646 & 1902 & -286 & -1350 & -2817 & -200 & 213 & 4183 & 9299 & 6305 & 3896 & 2250 \\
\hline 1937 Regional Surplus/Deficit & 4831 & 1941 & 1166 & 2591 & 1842 & -46 & -2436 & -3045 & -843 & 273 & -364 & -2111 & 3082 & 3268 & 567 \\
\hline 1938 Regional Surplus/Deficit & 4177 & 1246 & 1039 & 3160 & 2697 & 893 & 6470 & 337 & 3665 & 5104 & 6355 & 8087 & 7583 & 5666 & 4003 \\
\hline 1939 Regional Surplus/Deficit & 3845 & 1561 & 1919 & 2938 & 2193 & 323 & 2161 & -2145 & 427 & 1608 & 2912 & 5177 & 3170 & 4356 & 2123 \\
\hline 1940 Regional Surplus/Deficit & 5208 & 2084 & 1122 & 3069 & 1818 & 776 & 640 & -123 & 4214 & 3183 & 4228 & 2286 & 3611 & 2378 & 2262 \\
\hline 1941 Regional Surplus/Deficit & 3286 & 1395 & 938 & 2718 & 1682 & 1033 & 1203 & -2589 & -646 & -727 & -275 & -1374 & 3040 & 1607 & 788 \\
\hline 1942 Regional Surplus/Deficit & 3086 & 1501 & 1348 & 2454 & 1135 & 4248 & 5463 & -1438 & -1786 & 625 & 1862 & 2067 & 8926 & 7087 & 2753 \\
\hline 1943 Regional Surplus/Deficit & 6262 & 4699 & 1562 & 2652 & 2521 & 1477 & 5037 & 3930 & 4936 & 11567 & 9862 & 8097 & 9554 & 8649 & 5384 \\
\hline 1944 Regional Surplus/Deficit & 6508 & 4945 & 1820 & 3108 & 2246 & 422 & 1310 & -2595 & -1402 & -437 & -633 & -2798 & 1410 & 1425 & 845 \\
\hline 1945 Regional Surplus/Deficit & 3382 & 778 & 984 & 1941 & 1538 & -146 & -1926 & -1810 & -1006 & -178 & -294 & 2853 & 7490 & 4388 & 1346 \\
\hline 1946 Regional Surplus/Deficit & 3678 & 2778 & 1031 & 3213 & 2627 & 947 & 3350 & 2964 & 5396 & 6437 & 8340 & 8835 & 8482 & 7393 & 4571 \\
\hline 1947 Regional Surplus/Deficit & 6755 & 4388 & 2212 & 3029 & 2872 & 6406 & 7073 & 5828 & 5894 & 4034 & 6615 & 6254 & 7475 & 6247 & 5349 \\
\hline 1948 Regional Surplus/Deficit & 6683 & 3102 & 1927 & 7959 & 4833 & 3881 & 6933 & 884 & 2562 & 3963 & 7459 & 10117 & 14225 & 8346 & 6023 \\
\hline 1949 Regional Surplus/Deficit & 6397 & 7246 & 3638 & 3727 & 2497 & 1442 & 2438 & 857 & 7389 & 4126 & 9327 & 8499 & 7460 & 3412 & 4576 \\
\hline 1950 Regional Surplus/Deficit & 3856 & 1003 & 797 & 3169 & 2865 & 243 & 3982 & 6347 & 8884 & 8623 & 8401 & 6394 & 13016 & 9538 & 5515 \\
\hline 1951 Regional Surplus/Deficit & 6120 & 6685 & 2737 & 5648 & 6178 & 8851 & 9997 & 10737 & 6702 & 8549 & 9206 & 8809 & 6632 & 8517 & 7507 \\
\hline 1952 Regional Surplus/Deficit & 6317 & 6331 & 3258 & 6819 & 3214 & 4970 & 6216 & 3906 & 4007 & 8166 & 9230 & 10288 & 8003 & 5235 & 5911 \\
\hline 1953 Regional Surplus/Deficit & 6305 & 2830 & 1649 & 2655 & 2152 & 12 & 2766 & 5777 & 412 & -53 & 3006 & 6230 & 12289 & 8928 & 4076 \\
\hline 1954 Regional Surplus/Deficit & 6833 & 4604 & 2263 & 3598 & 2912 & 3524 & 5130 & 6355 & 3548 & 7409 & 5706 & 7002 & 11304 & 10948 & 5738 \\
\hline 1955 Regional Surplus/Deficit & 8506 & 6981 & 7270 & 4763 & 4122 & 3055 & 373 & -1801 & -1414 & 1975 & 437 & 1740 & 13494 & 12494 & 4420 \\
\hline 1956 Regional Surplus/Deficit & 6427 & 6921 & 2141 & 5295 & 5071 & 7563 & 10981 & 4944 & 7635 & 9059 & 12130 & 10475 & 14295 & 9207 & 7906 \\
\hline 1957 Regional Surplus/Deficit & 7096 & 5733 & 2345 & 4339 & 2516 & 3858 & 4119 & 16 & 2746 & 9141 & 6047 & 9671 & 12988 & 5118 & 5144 \\
\hline 1958 Regional Surplus/Deficit & 4538 & 2088 & 1454 & 3115 & 2274 & 854 & 3803 & 4123 & 3492 & 2281 & 6806 & 8601 & 10258 & 4405 & 4186 \\
\hline 1959 Regional Surplus/Deficit & 4545 & 2690 & 1731 & 3072 & 4063 & 5046 & 9714 & 6744 & 3397 & 6628 & 5296 & 5343 & 11133 & 9692 & 5793 \\
\hline 1960 Regional Surplus/Deficit & 6219 & 5233 & 7258 & 9214 & 6746 & 6331 & 5989 & 1342 & 3279 & 11890 & 8182 & 4647 & 8510 & 6633 & 6309 \\
\hline 1961 Regional Surplus/Deficit & 6718 & 2886 & 1845 & 3243 & 3334 & 1498 & 4737 & 5390 & 4716 & 6049 & 5427 & 5493 & 12297 & 5588 & 4890 \\
\hline 1962 Regional Surplus/Deficit & 4827 & 3250 & 1060 & 3096 & 2371 & 276 & 4001 & -846 & -672 & 8681 & 9489 & 4813 & 6493 & 6773 & 3374 \\
\hline 1963 Regional Surplus/Deficit & 6049 & 3983 & 1601 & 4210 & 4173 & 5032 & 4308 & 3056 & 1592 & 2204 & 2455 & 5815 & 7524 & 6240 & 4241 \\
\hline 1964 Regional Surplus/Deficit & 7001 & 4412 & 2372 & 2857 & 2934 & 888 & 4070 & 489 & -605 & 4070 & 3874 & 4110 & 13675 & 11519 & 4332 \\
\hline 1965 Regional Surplus/Deficit & 6694 & 6260 & 3744 & 4943 & 2982 & 7915 & 11716 & 9281 & 6552 & 5765 & 9461 & 8163 & 9378 & 6002 & 7064 \\
\hline 1966 Regional Surplus/Deficit & 6615 & 5915 & 2533 & 3968 & 2891 & 2911 & 5921 & -193 & 207 & 6939 & 3586 & 4496 & 5659 & 7131 & 3921 \\
\hline 1967 Regional Surplus/Deficit & 6547 & 4058 & 1377 & 2880 & 2541 & 2009 & 6875 & 7924 & 1616 & 5627 & 2990 & 2748 & 12653 & 9696 & 4994 \\
\hline 1968 Regional Surplus/Deficit & 6268 & 5255 & 2449 & 4194 & 2991 & 2815 & 6004 & 4935 & 3942 & -394 & 2300 & 2232 & 8813 & 8535 & 4469 \\
\hline 1969 Regional Surplus/Deficit & 6463 & 5919 & 4629 & 5616 & 4959 & 4750 & 9459 & 6805 & 4377 & 10647 & 9643 & 10334 & 8703 & 6698 & 6889 \\
\hline 1970 Regional Surplus/Deficit & 6533 & 2954 & 1868 & 3331 & 2699 & 1134 & 5099 & 4363 & 380 & 732 & 3570 & 4589 & 9871 & 3544 & 3648 \\
\hline 1971 Regional Surplus/Deficit & 4279 & 2181 & 1274 & 3177 & 2957 & 1195 & 8560 & 11391 & 5999 & 7219 & 8317 & 10513 & 13141 & 10368 & 6631 \\
\hline 1972 Regional Surplus/Deficit & 7147 & 7369 & 2936 & 3613 & 3191 & 3359 & 8099 & 10666 & 11735 & 12618 & 6649 & 10096 & 13886 & 11055 & 7961 \\
\hline 1973 Regional Surplus/Deficit & 8124 & 6813 & 3821 & 3705 & 2615 & 4054 & 6361 & -1975 & -621 & -868 & 137 & -1103 & 3540 & 3439 & 2578 \\
\hline 1974 Regional Surplus/Deficit & 3395 & 930 & 716 & 2283 & 2230 & 5666 & 13168 & 11609 & 8566 & 10266 & 10972 & 9240 & 14397 & 13563 & 7851 \\
\hline 1975 Regional Surplus/Deficit & 7384 & 7320 & 3637 & 2618 & 2553 & 1988 & 5609 & 2999 & 5485 & 3766 & 4339 & 6481 & 10872 & 11334 & 5415 \\
\hline 1976 Regional Surplus/Deficit & 5102 & 3891 & 2639 & 4852 & 5712 & 10108 & 10198 & 6976 & 4648 & 9956 & 7730 & 9367 & 7441 & 10359 & 7137 \\
\hline 1977 Regional Surplus/Deficit & 9797 & 9056 & 8345 & 3780 & 2144 & 333 & 1241 & -2703 & -1925 & -1303 & -1441 & -1953 & 667 & 1205 & 1599 \\
\hline 1978 Regional Surplus/Deficit & 3370 & 804 & 520 & 1553 & 2149 & 1268 & 3972 & 929 & 5341 & 4082 & 5409 & 5789 & 6245 & 7455 & 3504 \\
\hline \multicolumn{16}{|l|}{-Ranked Averages- } \\
\hline Top Ten Percent & 5638 & 5159 & 2234 & 4338 & 4477 & 7109 & 10489 & 8986 & 7857 & 10089 & 9337 & 9598 & 11330 & 10540 & 7672 \\
\hline Middle Eighty Percent & 5789 & 3909 & 2382 & 3682 & 2919 & 2489 & 4691 & 2578 & 2542 & 4523 & 5248 & 5564 & 8760 & 6823 & 4347 \\
\hline Bottom Ten Percent & 4342 & 2429 & 1223 & 2718 & 1925 & 182 & -933 & -2456 & -910 & -41 & -485 & -2264 & 2421 & 2485 & 626 \\
\hline
\end{tabular}


Regional Surplus/Deficit by Water Year

PNW Loads and Resources Study

2009 - 2010 Operating Year 2003 White Book

\begin{tabular}{|c|c|c|c|c|c|c|c|c|c|c|c|c|c|c|c|}
\hline Average Energy in Megawatts & Aug1 & Aug16 & Sep & Oct & Nov & Dec & Jan & $\mathrm{Feb}$ & Mar & Apr1 & Apr16 & May & Jun & Jul & Avg \\
\hline 1929 Regional Surplus/Deficit & 6155 & 2341 & 1243 & 2413 & 1937 & -277 & -16 & -3545 & -1278 & -1598 & -1017 & -934 & 4101 & 3227 & 818 \\
\hline 1930 Regional Surplus/Deficit & 2786 & 1376 & 641 & 2152 & 1552 & -556 & -2931 & -1299 & -1259 & -927 & -996 & -1508 & 2697 & 3258 & 322 \\
\hline 1931 Regional Surplus/Deficit & 3512 & 1770 & 901 & 2121 & 1610 & -754 & -2639 & -3610 & -1481 & -662 & -1516 & -1298 & 1866 & 2017 & 24 \\
\hline 1932 Regional Surplus/Deficit & 3131 & 852 & 697 & 1819 & 1380 & -704 & -2424 & -3422 & 1481 & 5244 & 9049 & 8145 & 8987 & 5820 & 2576 \\
\hline 1933 Regional Surplus/Deficit & 6207 & 4453 & 1322 & 2392 & 2266 & 1701 & 5311 & 3184 & -1096 & 1286 & 2493 & 5344 & 13236 & 11189 & 4339 \\
\hline 1934 Regional Surplus/Deficit & 5849 & 5909 & 2687 & 5562 & 5351 & 9916 & 11677 & 8516 & 5736 & 10232 & 9144 & 7356 & 2308 & 4412 & 6591 \\
\hline 1935 Regional Surplus/Deficit & 2467 & 298 & 543 & 2160 & 2324 & 980 & 4743 & 5149 & -2070 & 120 & 3379 & 4251 & 6926 & 5811 & 2829 \\
\hline 1936 Regional Surplus/Deficit & 5061 & 2434 & 963 & 2199 & 1557 & -690 & -1760 & -3246 & -739 & -925 & 3514 & 10426 & 6305 & 3471 & 1961 \\
\hline 1937 Regional Surplus/Deficit & 4440 & 1582 & 842 & 2144 & 1496 & -450 & -2850 & -3474 & -1383 & -866 & -1041 & -999 & 3072 & 2841 & 275 \\
\hline 1938 Regional Surplus/Deficit & 3786 & 889 & 715 & 2713 & 2352 & 490 & 6068 & -84 & 3131 & 3971 & 5688 & 9216 & 7581 & 5242 & 3716 \\
\hline 1939 Regional Surplus/Deficit & 3453 & 1201 & 1596 & 2492 & 1846 & -83 & 1758 & -2572 & -112 & 473 & 2240 & 6302 & 3162 & 3929 & 1834 \\
\hline 1940 Regional Surplus/Deficit & 4818 & 1725 & 798 & 2623 & 1473 & 371 & 230 & -550 & 3682 & 2047 & 3563 & 3408 & 3604 & 1951 & 1972 \\
\hline 1941 Regional Surplus/Deficit & 2894 & 1036 & 614 & 2272 & 1337 & 630 & 791 & -3018 & -1187 & -1861 & -945 & -255 & 3034 & 1179 & 497 \\
\hline 1942 Regional Surplus/Deficit & 2693 & 1143 & 1024 & 2006 & 789 & 3843 & 5065 & -1864 & -2327 & -511 & 1192 & 3189 & 8926 & 6664 & 2464 \\
\hline 1943 Regional Surplus/Deficit & 5873 & 4344 & 1238 & 2206 & 2174 & 1072 & 4636 & 3509 & 4404 & 10436 & 9197 & 9226 & 9553 & 8224 & 5097 \\
\hline 1944 Regional Surplus/Deficit & 6117 & 4590 & 1496 & 2662 & 1898 & 17 & 901 & -3026 & -1944 & -1578 & -1308 & -1681 & 1402 & 995 & 553 \\
\hline 1945 Regional Surplus/Deficit & 2988 & 418 & 660 & 1493 & 1191 & -551 & -2341 & -2240 & -1548 & -1318 & -974 & 3976 & 7489 & 3963 & 1054 \\
\hline 1946 Regional Surplus/Deficit & 3284 & 2421 & 707 & 2765 & 2280 & 543 & 2942 & 2542 & 4864 & 5304 & 7675 & 9964 & 8483 & 6969 & 4283 \\
\hline 1947 Regional Surplus/Deficit & 6366 & 4032 & 1890 & 2584 & 2525 & 6001 & 6675 & 5411 & 5363 & 2901 & 5949 & 7381 & 7477 & 5823 & 5063 \\
\hline 1948 Regional Surplus/Deficit & 6293 & 2744 & 1604 & 7519 & 4487 & 3480 & 6533 & 459 & 2030 & 2828 & 6794 & 11246 & 14226 & 7925 & 5737 \\
\hline 1949 Regional Surplus/Deficit & 6007 & 6893 & 3317 & 3283 & 2150 & 1036 & 2034 & 430 & 6858 & 2992 & 8663 & 9626 & 7459 & 2985 & 4288 \\
\hline 1950 Regional Surplus/Deficit & 3462 & 642 & 473 & 2721 & 2519 & -161 & 3580 & 5928 & 8354 & 7490 & 7737 & 7522 & 13019 & 9115 & 5228 \\
\hline 1951 Regional Surplus/Deficit & 5729 & 6332 & 2414 & 5205 & 5832 & 8454 & 9599 & 10325 & 6170 & 7416 & 8542 & 9938 & 6630 & 8095 & 7223 \\
\hline 1952 Regional Surplus/Deficit & 5927 & 5977 & 2937 & 6378 & 2868 & 4568 & 5817 & 3488 & 3471 & 7033 & 8565 & 11417 & 8003 & 4810 & 5626 \\
\hline 1953 Regional Surplus/Deficit & 5915 & 2472 & 1325 & 2208 & 1806 & -392 & 2354 & 5362 & -123 & -1187 & 2336 & 7356 & 12291 & 8505 & 3788 \\
\hline 1954 Regional Surplus/Deficit & 6444 & 4249 & 1941 & 3153 & 2567 & 3120 & 4729 & 5940 & 3015 & 6276 & 5038 & 8131 & 11307 & 10527 & 5453 \\
\hline 1955 Regional Surplus/Deficit & 8119 & 6628 & 6955 & 4320 & 3777 & 2652 & -36 & -2231 & -1956 & 840 & -229 & 2861 & 13497 & 12073 & 4133 \\
\hline 1956 Regional Surplus/Deficit & 6037 & 6567 & 1819 & 4852 & 4724 & 7164 & 10584 & 4529 & 7103 & 7929 & 11467 & 11604 & 14298 & 8784 & 7622 \\
\hline 1957 Regional Surplus/Deficit & 6706 & 5379 & 2022 & 3895 & 2169 & 3454 & 3716 & -411 & 2212 & 8007 & 5381 & 10800 & 12991 & 4691 & 4856 \\
\hline 1958 Regional Surplus/Deficit & 4146 & 1728 & 1130 & 2669 & 1927 & 447 & 3399 & 3701 & 2962 & 1146 & 6139 & 9727 & 10261 & 3978 & 3898 \\
\hline 1959 Regional Surplus/Deficit & 4153 & 2332 & 1407 & 2627 & 3717 & 4643 & 9316 & 6331 & 2862 & 5495 & 4630 & 6471 & 11135 & 9268 & 5507 \\
\hline 1960 Regional Surplus/Deficit & 5829 & 4878 & 6942 & 8774 & 6400 & 5932 & 5591 & 920 & 2744 & 10762 & 7518 & 5773 & 8510 & 6209 & 6024 \\
\hline 1961 Regional Surplus/Deficit & 6329 & 2526 & 1523 & 2798 & 2988 & 1092 & 4334 & 4974 & 4184 & 4916 & 4758 & 6622 & 12300 & 5163 & 4604 \\
\hline 1962 Regional Surplus/Deficit & 4436 & 2893 & 735 & 2650 & 2023 & -130 & 3598 & -1269 & -1214 & 7547 & 8827 & 5940 & 6492 & 6349 & 3085 \\
\hline 1963 Regional Surplus/Deficit & 5660 & 3627 & 1277 & 3766 & 3827 & 4631 & 3908 & 2638 & 1052 & 1069 & 1786 & 6939 & 7524 & 5816 & 3954 \\
\hline 1964 Regional Surplus/Deficit & 6611 & 4056 & 2050 & 2411 & 2587 & 482 & 3667 & 67 & -1146 & 2934 & 3204 & 5236 & 13677 & 11098 & 4044 \\
\hline 1965 Regional Surplus/Deficit & 6305 & 5906 & 3424 & 4502 & 2637 & 7511 & 11319 & 8868 & 6020 & 4631 & 8796 & 9292 & 9377 & 5576 & 6779 \\
\hline 1966 Regional Surplus/Deficit & 6226 & 5560 & 2212 & 3524 & 2546 & 2506 & 5520 & -613 & -333 & 5808 & 2919 & 5622 & 5656 & 6707 & 3634 \\
\hline 1967 Regional Surplus/Deficit & 6158 & 3700 & 1054 & 2434 & 2194 & 1603 & 6475 & 7511 & 1083 & 4496 & 2320 & 3873 & 12656 & 9274 & 4708 \\
\hline 1968 Regional Surplus/Deficit & 5879 & 4900 & 2127 & 3750 & 2645 & 2410 & 5603 & 4520 & 3411 & -1532 & 1631 & 3352 & 8816 & 8111 & 4182 \\
\hline 1969 Regional Surplus/Deficit & 6075 & 5565 & 4312 & 5174 & 4613 & 4348 & 9061 & 6392 & 3843 & 9517 & 8979 & 11462 & 8705 & 6273 & 6604 \\
\hline 1970 Regional Surplus/Deficit & 6143 & 2596 & 1545 & 2886 & 2352 & 728 & 4692 & 3947 & -156 & -403 & 2904 & 5713 & 9870 & 3118 & 3359 \\
\hline 1971 Regional Surplus/Deficit & 3887 & 1821 & 949 & 2730 & 2609 & 790 & 8160 & 10978 & 5466 & 6085 & 7652 & 11641 & 13143 & 9945 & 6345 \\
\hline 1972 Regional Surplus/Deficit & 6759 & 7016 & 2614 & 3167 & 2844 & 2953 & 7699 & 10253 & 11206 & 11489 & 5984 & 11224 & 13889 & 10634 & 7676 \\
\hline 1973 Regional Surplus/Deficit & 7736 & 6461 & 3500 & 3260 & 2268 & 3649 & 5960 & -2404 & -1162 & -2005 & -535 & 13 & 3535 & 3012 & 2288 \\
\hline 1974 Regional Surplus/Deficit & 3004 & 569 & 391 & 1834 & 1883 & 5261 & 12770 & 11196 & 8035 & 9135 & 10310 & 10368 & 14400 & 13142 & 7566 \\
\hline 1975 Regional Surplus/Deficit & 6995 & 6967 & 3316 & 2172 & 2205 & 1582 & 5207 & 2581 & 4952 & 2633 & 3671 & 7608 & 10872 & 10913 & 5128 \\
\hline 1976 Regional Surplus/Deficit & 4711 & 3535 & 2317 & 4409 & 5366 & 9712 & 9800 & 6562 & 4116 & 8825 & 7065 & 10496 & 7439 & 9937 & 6852 \\
\hline 1977 Regional Surplus/Deficit & 9409 & 8704 & 8030 & 3336 & 1796 & -72 & 834 & -3133 & -2468 & -2444 & -2116 & -836 & 658 & 774 & 1308 \\
\hline 1978 Regional Surplus/Deficit & 2978 & 445 & 196 & 1104 & 1801 & 860 & 3565 & 504 & 4809 & 2951 & 4744 & 6917 & 6243 & 7031 & 3216 \\
\hline \multicolumn{16}{|l|}{-Ranked Averages- } \\
\hline Top Ten Percent & 5248 & 4804 & 1911 & 3894 & 4130 & 6709 & 10090 & 8573 & 7326 & 8959 & 8674 & 10726 & 11331 & 10118 & 7388 \\
\hline Middle Eighty Percent & 5399 & 3553 & 2060 & 3237 & 2573 & 2084 & 4288 & 2157 & 2007 & 3389 & 4580 & 6689 & 8759 & 6399 & 4059 \\
\hline Bottom Ten Percent & 3950 & 2071 & 899 & 2270 & 1579 & -223 & -1345 & -2885 & -1451 & -1179 & -1161 & -1148 & 2414 & 2058 & 334 \\
\hline
\end{tabular}


Regional Surplus/Deficit by Water Year

PNW Loads and Resources Study

2010 - 2011 Operating Year 2003 White Book

\begin{tabular}{|c|c|c|c|c|c|c|c|c|c|c|c|c|c|c|c|}
\hline Average Energy in Megawatts & Aug1 & Aug16 & Sep & Oct & Nov & Dec & Jan & Feb & Mar & Apr1 & Apr16 & May & Jun & Jul & Avg \\
\hline 1929 Regional Surplus/Deficit & 5853 & 2000 & 807 & 2211 & 1533 & -630 & -398 & -3872 & -1501 & -2075 & -1526 & -1960 & 3397 & 2944 & 388 \\
\hline 1930 Regional Surplus/Deficit & 2480 & 1035 & 205 & 1948 & 1150 & -908 & -3318 & -1622 & -1481 & -1402 & -1505 & -2535 & 1992 & 2977 & -107 \\
\hline 1931 Regional Surplus/Deficit & 3208 & 1430 & 464 & 1917 & 1208 & -1106 & -3025 & -3936 & -1703 & -1134 & -2026 & -2323 & 1163 & 1735 & -406 \\
\hline 1932 Regional Surplus/Deficit & 2827 & 511 & 260 & 1615 & 977 & -1059 & -2810 & -3749 & 1261 & 4774 & 8553 & 7130 & 8288 & 5540 & 2149 \\
\hline 1933 Regional Surplus/Deficit & 5904 & 4116 & 887 & 2191 & 1863 & 1348 & 4938 & 2871 & -1316 & 816 & 1991 & 4327 & 12539 & 10911 & 3914 \\
\hline 1934 Regional Surplus/Deficit & 5546 & 5574 & 2255 & 5365 & 4949 & 9574 & 11304 & 8205 & 5523 & 9765 & 8648 & 6341 & 1600 & 4130 & 6168 \\
\hline 1935 Regional Surplus/Deficit & 2163 & -42 & 109 & 1956 & 1921 & 626 & 4368 & 4837 & -2288 & -352 & 2879 & 3231 & 6224 & 5531 & 2403 \\
\hline 1936 Regional Surplus/Deficit & 4758 & 2096 & 527 & 1997 & 1154 & -1042 & -2143 & -3572 & -961 & -1399 & 3014 & 9411 & 5606 & 3189 & 1534 \\
\hline 1937 Regional Surplus/Deficit & 4136 & 1242 & 406 & 1941 & 1094 & -802 & -3235 & -3799 & -1605 & -1340 & -1549 & -2027 & 2365 & 2559 & -155 \\
\hline 1938 Regional Surplus/Deficit & 3482 & 550 & 279 & 2510 & 1951 & 138 & 5693 & -402 & 2915 & 3501 & 5189 & 8201 & 6881 & 4961 & 3291 \\
\hline 1939 Regional Surplus/Deficit & 3149 & 861 & 1162 & 2291 & 1442 & -436 & 1381 & -2896 & -332 & 1.2 & 1737 & 5284 & 2455 & 3648 & 1406 \\
\hline 1940 Regional Surplus/Deficit & 4515 & 1385 & 362 & 2422 & 1071 & 18 & -153 & -874 & 3467 & 1575 & 3066 & 2388 & 2899 & 1669 & 1545 \\
\hline 1941 Regional Surplus/Deficit & 2589 & 696 & 178 & 2071 & 935 & 279 & 406 & -3343 & -1409 & -2332 & -1446 & -1278 & 2330 & 896 & 68 \\
\hline 1942 Regional Surplus/Deficit & 2388 & 803 & 587 & 1804 & 387 & 3490 & 4693 & -2186 & -2549 & -983 & 690 & 2169 & 8227 & 6385 & 2038 \\
\hline 1943 Regional Surplus/Deficit & 5571 & 4006 & 803 & 2004 & 1770 & 718 & 4262 & 3190 & 4189 & 9967 & 8699 & 8211 & 8854 & 7944 & 4672 \\
\hline 1944 Regional Surplus/Deficit & 5814 & 4253 & 1060 & 2460 & 1494 & -336 & 520 & -3352 & -2168 & -2054 & -1815 & -2705 & 695 & 710 & 123 \\
\hline 1945 Regional Surplus/Deficit & 2683 & 77 & 223 & 1289 & 787 & -905 & -2728 & -2566 & -1772 & -1794 & -1484 & 2957 & 6789 & 3682 & 625 \\
\hline 1946 Regional Surplus/Deficit & 2978 & 2082 & 270 & 2561 & 1877 & 191 & 2561 & 2223 & 4650 & 4834 & 7177 & 8949 & 7784 & 6688 & 3858 \\
\hline 1947 Regional Surplus/Deficit & 6064 & 3694 & 1455 & 2382 & 2122 & 5648 & 6303 & 5096 & 5149 & 2430 & 5451 & 6365 & 6779 & 5542 & 4638 \\
\hline 1948 Regional Surplus/Deficit & 5989 & 2404 & 1168 & 7322 & 4084 & 3131 & 6160 & 138 & 1816 & 2357 & 6296 & 10231 & 13528 & 7646 & 5312 \\
\hline 1949 Regional Surplus/Deficit & 5704 & 6558 & 2884 & 3082 & 1747 & 681 & 1657 & 107 & 6644 & 2521 & 8166 & 8609 & 6759 & 2703 & 3862 \\
\hline 1950 Regional Surplus/Deficit & 3157 & 301 & 36 & 2518 & 2115 & -512 & 3204 & 5612 & 8141 & 7020 & 7239 & 6507 & 12322 & 8836 & 4803 \\
\hline 1951 Regional Surplus/Deficit & 5426 & 5995 & 1980 & 5007 & 5429 & 8108 & 9227 & 10014 & 5956 & 6946 & 8046 & 8923 & 5930 & 7815 & 6799 \\
\hline 1952 Regional Surplus/Deficit & 5624 & 5640 & 2503 & 6180 & 2466 & 4217 & 5443 & 3174 & 3254 & 6563 & 8067 & 10402 & 7303 & 4530 & 5202 \\
\hline 1953 Regional Surplus/Deficit & 5612 & 2132 & 888 & 2005 & 1402 & -744 & 1970 & 5048 & -340 & -1658 & 1835 & 6340 & 11592 & 8226 & 3362 \\
\hline 1954 Regional Surplus/Deficit & 6141 & 3912 & 1506 & 2953 & 2164 & 2767 & 4354 & 5627 & 2800 & 5806 & 4539 & 7116 & 10610 & 10248 & 5029 \\
\hline 1955 Regional Surplus/Deficit & 7817 & 6292 & 6526 & 4122 & 3375 & 2302 & -418 & -2556 & -2179 & 369 & -727 & 1839 & 12800 & 11795 & 3707 \\
\hline 1956 Regional Surplus/Deficit & 5734 & 6231 & 1384 & 4653 & 4320 & 6816 & 10212 & 4215 & 6888 & 7460 & 10971 & 10589 & 13601 & 8505 & 7198 \\
\hline 1957 Regional Surplus/Deficit & 6404 & 5042 & 1588 & 3695 & 1765 & 3102 & 3339 & -734 & 1996 & 7537 & 4883 & 9785 & 12294 & 4410 & 4431 \\
\hline 1958 Regional Surplus/Deficit & 3842 & 1387 & 694 & 2467 & 1524 & 93 & 3022 & 3383 & 2748 & 675 & 5640 & 8711 & 9564 & 3696 & 3473 \\
\hline 1959 Regional Surplus/Deficit & 3848 & 1992 & 971 & 2426 & 3314 & 4293 & 8944 & 6020 & 2645 & 5026 & 4132 & 5456 & 10438 & 8989 & 5083 \\
\hline 1960 Regional Surplus/Deficit & 5527 & 4542 & 6513 & 8577 & 5998 & 5585 & 5219 & 601 & 2527 & 10294 & 7021 & 4756 & 7811 & 5929 & 5601 \\
\hline 1961 Regional Surplus/Deficit & 6027 & 2186 & 1089 & 2597 & 2585 & 738 & 3958 & 4661 & 3970 & 4446 & 4257 & 5607 & 11603 & 4882 & 4179 \\
\hline 1962 Regional Surplus/Deficit & 4132 & 2554 & 299 & 2449 & 1619 & -485 & 3221 & -1589 & -1437 & 7076 & 8331 & 4923 & 5792 & 6069 & 2659 \\
\hline 1963 Regional Surplus/Deficit & 5357 & 3289 & 841 & 3567 & 3425 & 4280 & 3535 & 2323 & 830 & 598 & 1285 & 5921 & 6825 & 5536 & 3529 \\
\hline 1964 Regional Surplus/Deficit & 6309 & 3718 & 1615 & 2210 & 2183 & 128 & 3290 & -253 & -1368 & 2461 & 2703 & 4218 & 12980 & 10820 & 3618 \\
\hline 1965 Regional Surplus/Deficit & 6003 & 5569 & 2991 & 4304 & 2235 & 7160 & 10946 & 8556 & 5805 & 4160 & 8299 & 8277 & 8678 & 5295 & 6355 \\
\hline 1966 Regional Surplus/Deficit & 5924 & 5224 & 1778 & 3324 & 2144 & 2153 & 5146 & -931 & -555 & 5339 & 2419 & 4605 & 4955 & 6428 & 3208 \\
\hline 1967 Regional Surplus/Deficit & 5856 & 3359 & 617 & 2232 & 1791 & 1250 & 6102 & 7199 & 866 & 4027 & 1819 & 2856 & 11959 & 8995 & 4283 \\
\hline 1968 Regional Surplus/Deficit & 5577 & 4564 & 1693 & 3549 & 2243 & 2057 & 5229 & 4206 & 3196 & -2006 & 1130 & 2330 & 8118 & 7831 & 3757 \\
\hline 1969 Regional Surplus/Deficit & 5773 & 5228 & 3881 & 4976 & 4210 & 3997 & 8688 & 6080 & 3628 & 9049 & 8483 & 10447 & 8008 & 5993 & 6181 \\
\hline 1970 Regional Surplus/Deficit & 5839 & 2256 & 1109 & 2686 & 1949 & 373 & 4313 & 3633 & -375 & -875 & 2405 & 4695 & 9170 & 2836 & 2933 \\
\hline 1971 Regional Surplus/Deficit & 3583 & 1480 & 512 & 2527 & 2205 & 437 & 7786 & 10667 & 5251 & 5615 & 7155 & 10626 & 12446 & 9666 & 5920 \\
\hline 1972 Regional Surplus/Deficit & 6457 & 6680 & 2180 & 2966 & 2441 & 2600 & 7324 & 9941 & 10994 & 11022 & 5487 & 10209 & 13191 & 10356 & 7252 \\
\hline 1973 Regional Surplus/Deficit & 7435 & 6125 & 3066 & 3059 & 1864 & 3297 & 5586 & -2729 & -1384 & -2478 & -1039 & -1012 & 2831 & 2729 & 1861 \\
\hline 1974 Regional Surplus/Deficit & 2700 & 227 & -46 & 1629 & 1479 & 4908 & 12398 & 10885 & 7822 & 8667 & 9814 & 9353 & 13702 & 12863 & 7141 \\
\hline 1975 Regional Surplus/Deficit & 6693 & 6631 & 2882 & 1970 & 1801 & 1228 & 4832 & 2266 & 4736 & 2162 & 3171 & 6592 & 10173 & 10635 & 4704 \\
\hline 1976 Regional Surplus/Deficit & 4408 & 3198 & 1883 & 4210 & 4963 & 9368 & 9428 & 6250 & 3902 & 8356 & 6567 & 9481 & 6739 & 9659 & 6429 \\
\hline 1977 Regional Surplus/Deficit & 9108 & 8369 & 7602 & 3136 & 1391 & -425 & 453 & -3459 & -2692 & -2921 & -2622 & -1861 & -49 & 489 & 879 \\
\hline 1978 Regional Surplus/Deficit & 2673 & 104 & -241 & 900 & 1398 & 505 & 3186 & 182 & 4595 & 2482 & 4246 & 5901 & 5542 & 6751 & 2789 \\
\hline \multicolumn{16}{|l|}{-Ranked Averages- } \\
\hline Top Ten Percent & 4945 & 4466 & 1476 & 3693 & 3726 & 6360 & 9718 & 8261 & 7112 & 8490 & 8177 & 9711 & 10633 & 9840 & 6964 \\
\hline Middle Eighty Percent & 5096 & 3214 & 1625 & 3036 & 2170 & 1732 & 3911 & 1838 & 1789 & 2918 & 4080 & 5672 & 8059 & 6118 & 3634 \\
\hline Bottom Ten Percent & 3646 & 1731 & 462 & 2068 & 1176 & -575 & -1730 & -3211 & -1673 & -1653 & -1669 & -2174 & 1709 & 1775 & -95 \\
\hline
\end{tabular}


Regional Surplus/Deficit by Water Year

PNW Loads and Resources Study

2011 - 2012 Operating Year 2003 White Book

\begin{tabular}{|c|c|c|c|c|c|c|c|c|c|c|c|c|c|c|c|}
\hline Average Energy in Megawatts & Aug1 & Aug16 & Sep & Oct & Nov & Dec & Jan & Feb & Mar & Apr1 & Apr16 & May & Jun & Jul & Avg \\
\hline 1929 Regional Surplus/Deficit & 5511 & 1571 & 571 & 1834 & 1254 & -1073 & -687 & -3903 & -1519 & -1438 & -990 & -2374 & 3917 & 2644 & 249 \\
\hline 1930 Regional Surplus/Deficit & 2137 & 607 & -32 & 1569 & 872 & -1351 & -3611 & -1650 & -1499 & -764 & -970 & -2950 & 2511 & 2677 & -247 \\
\hline 1931 Regional Surplus/Deficit & 2866 & 1003 & 228 & 1538 & 930 & -1548 & -3317 & -3967 & -1722 & -494 & -1492 & -2737 & 1681 & 1436 & -545 \\
\hline 1932 Regional Surplus/Deficit & 2485 & 83 & 24 & 1236 & 699 & -1504 & -3103 & -3780 & 1246 & 5414 & 9097 & 6722 & 8812 & 5240 & 2011 \\
\hline 1933 Regional Surplus/Deficit & 5563 & 3690 & 652 & 1813 & 1586 & 905 & 4654 & 2849 & -1332 & 1456 & 2532 & 3920 & 13063 & 10612 & 3779 \\
\hline 1934 Regional Surplus/Deficit & 5205 & 5148 & 2022 & 4990 & 4672 & 9139 & 11020 & 8183 & 5511 & 10405 & 9192 & 5933 & 2117 & 3831 & 6033 \\
\hline 1935 Regional Surplus/Deficit & 1821 & -470 & -126 & 1577 & 1643 & 183 & 4084 & 4815 & -2304 & 289 & 3421 & 2821 & 6747 & 5232 & 2267 \\
\hline 1936 Regional Surplus/Deficit & 4417 & 1670 & 292 & 1619 & 877 & -1484 & -2433 & -3603 & -978 & -760 & 3558 & 9003 & 6130 & 2890 & 1396 \\
\hline 1937 Regional Surplus/Deficit & 3795 & 814 & 169 & 1562 & 816 & -1245 & -3527 & -3829 & -1623 & -703 & -1013 & -2443 & 2883 & 2260 & -294 \\
\hline 1938 Regional Surplus/Deficit & 3140 & 123 & 43 & 2132 & 1673 & -304 & 5408 & -427 & 2902 & 4141 & 5733 & 7794 & 7405 & 4662 & 3155 \\
\hline 1939 Regional Surplus/Deficit & 2808 & 434 & 927 & 1914 & 1164 & -879 & 1095 & -2925 & -348 & 641 & 2278 & 4876 & 2973 & 3348 & 1269 \\
\hline 1940 Regional Surplus/Deficit & 4174 & 957 & 126 & 2044 & 794 & -425 & -443 & -903 & 3455 & 2215 & 3610 & 1979 & 3418 & 1369 & 1408 \\
\hline 1941 Regional Surplus/Deficit & 2248 & 269 & -58 & 1694 & 658 & -163 & 115 & -3373 & -1427 & -1692 & -906 & -1689 & 2851 & 596 & -70 \\
\hline 1942 Regional Surplus/Deficit & 2047 & 375 & 352 & 1426 & 110 & 3048 & 4409 & -2214 & -2568 & -343 & 1232 & 1760 & 8751 & 6085 & 1901 \\
\hline 1943 Regional Surplus/Deficit & 5229 & 3580 & 568 & 1626 & 1492 & 276 & 3978 & 3167 & 4176 & 10607 & 9242 & 7804 & 9378 & 7644 & 4536 \\
\hline 1944 Regional Surplus/Deficit & 5473 & 3828 & 824 & 2083 & 1215 & -779 & 231 & -3383 & -2188 & -1417 & -1278 & -3118 & 1214 & 409 & -16 \\
\hline 1945 Regional Surplus/Deficit & 2341 & -352 & -13 & 910 & 509 & -1348 & -3021 & -2596 & -1791 & -1156 & -949 & 2549 & 7313 & 3383 & 486 \\
\hline 1946 Regional Surplus/Deficit & 2636 & 1656 & 34 & 2182 & 1599 & -251 & 2273 & 2198 & 4638 & 5474 & 7721 & 8542 & 8308 & 6389 & 3721 \\
\hline 1947 Regional Surplus/Deficit & 5722 & 3268 & 1221 & 2005 & 1844 & 5206 & 6019 & 5074 & 5137 & 3071 & 5994 & 5958 & 7303 & 5243 & 4503 \\
\hline 1948 Regional Surplus/Deficit & 5648 & 1978 & 933 & 6947 & 3807 & 2692 & 5876 & 112 & 1803 & 2997 & 6839 & 9824 & 14051 & 7347 & 5177 \\
\hline 1949 Regional Surplus/Deficit & 5363 & 6132 & 2650 & 2706 & 1469 & 238 & 1371 & 79 & 6631 & 3161 & 8710 & 8202 & 7283 & 2403 & 3726 \\
\hline 1950 Regional Surplus/Deficit & 2815 & -128 & -200 & 2140 & 1837 & -954 & 2919 & 5589 & 8129 & 7660 & 7783 & 6099 & 12845 & 8537 & 4667 \\
\hline 1951 Regional Surplus/Deficit & 5084 & 5570 & 1745 & 4632 & 5151 & 7671 & 8943 & 9992 & 5943 & 7587 & 8589 & 8516 & 6453 & 7516 & 6665 \\
\hline 1952 Regional Surplus/Deficit & 5283 & 5215 & 2269 & 5805 & 2189 & 3777 & 5159 & 3152 & 3239 & 7203 & 8610 & 9994 & 7827 & 4231 & 5066 \\
\hline 1953 Regional Surplus/Deficit & 5271 & 1705 & 652 & 1627 & 1124 & -1187 & 1680 & 5027 & -354 & -1018 & 2377 & 5932 & 12116 & 7926 & 3226 \\
\hline 1954 Regional Surplus/Deficit & 5799 & 3486 & 1271 & 2576 & 1886 & 2324 & 4070 & 5606 & 2786 & 6446 & 5082 & 6709 & 11134 & 9949 & 4893 \\
\hline 1955 Regional Surplus/Deficit & 7476 & 5867 & 6295 & 3746 & 3098 & 1861 & -707 & -2587 & -2198 & 1009 & -183 & 1430 & 13324 & 11495 & 3570 \\
\hline 1956 Regional Surplus/Deficit & 5393 & 5806 & 1148 & 4277 & 4043 & 6379 & 9928 & 4194 & 6876 & 8100 & 11515 & 10181 & 14125 & 8206 & 7064 \\
\hline 1957 Regional Surplus/Deficit & 6062 & 4616 & 1353 & 3319 & 1487 & 2661 & 3053 & -763 & 1984 & 8177 & 5427 & 9378 & 12817 & 4110 & 4295 \\
\hline 1958 Regional Surplus/Deficit & 3500 & 959 & 458 & 2089 & 1246 & -351 & 2736 & 3359 & 2735 & 1315 & 6183 & 8303 & 10088 & 3396 & 3337 \\
\hline 1959 Regional Surplus/Deficit & 3507 & 1565 & 735 & 2049 & 3037 & 3853 & 8660 & 5998 & 2631 & 5666 & 4675 & 5049 & 10962 & 8689 & 4948 \\
\hline 1960 Regional Surplus/Deficit & 5185 & 4116 & 6282 & 8202 & 5721 & 5147 & 4935 & 576 & 2513 & 10935 & 7565 & 4349 & 8335 & 5629 & 5466 \\
\hline 1961 Regional Surplus/Deficit & 5685 & 1758 & 854 & 2220 & 2308 & 294 & 3672 & 4639 & 3958 & 5087 & 4799 & 5200 & 12126 & 4582 & 4043 \\
\hline 1962 Regional Surplus/Deficit & 3790 & 2127 & 62 & 2071 & 1340 & -929 & 2935 & -1615 & -1455 & 7716 & 8875 & 4516 & 6316 & 5770 & 2522 \\
\hline 1963 Regional Surplus/Deficit & 5016 & 2864 & 605 & 3191 & 3147 & 3840 & 3251 & 2301 & 812 & 1238 & 1827 & 5514 & 7349 & 5237 & 3393 \\
\hline 1964 Regional Surplus/Deficit & 5967 & 3293 & 1381 & 1832 & 1904 & -316 & 3004 & -279 & -1386 & 3101 & 3245 & 3811 & 13504 & 10520 & 3482 \\
\hline 1965 Regional Surplus/Deficit & 5661 & 5143 & 2757 & 3929 & 1957 & 6720 & 10662 & 8534 & 5793 & 4801 & 8843 & 7870 & 9201 & 4996 & 6220 \\
\hline 1966 Regional Surplus/Deficit & 5582 & 4798 & 1544 & 2948 & 1867 & 1710 & 4861 & -956 & -573 & 5979 & 2961 & 4198 & 5477 & 6128 & 3072 \\
\hline 1967 Regional Surplus/Deficit & 5515 & 2932 & 381 & 1854 & 1512 & 807 & 5818 & 7178 & 852 & 4667 & 2360 & 2449 & 12483 & 8696 & 4147 \\
\hline 1968 Regional Surplus/Deficit & 5235 & 4138 & 1458 & 3173 & 1966 & 1615 & 4945 & 4185 & 3184 & -1368 & 1670 & 1919 & 8642 & 7532 & 3621 \\
\hline 1969 Regional Surplus/Deficit & 5431 & 4802 & 3649 & 4602 & 3933 & 3557 & 8405 & 6059 & 3615 & 9689 & 9027 & 10040 & 8531 & 5694 & 6047 \\
\hline 1970 Regional Surplus/Deficit & 5498 & 1828 & 873 & 2309 & 1670 & -70 & 4026 & 3611 & -390 & -235 & 2948 & 4288 & 9694 & 2537 & 2797 \\
\hline 1971 Regional Surplus/Deficit & 3242 & 1052 & 275 & 2149 & 1926 & -5.3 & 7502 & 10646 & 5238 & 6255 & 7699 & 10219 & 12970 & 9367 & 5784 \\
\hline 1972 Regional Surplus/Deficit & 6115 & 6255 & 1945 & 2589 & 2163 & 2157 & 7040 & 9920 & 10981 & 11662 & 6031 & 9802 & 13715 & 10056 & 7117 \\
\hline 1973 Regional Surplus/Deficit & 7093 & 5699 & 2831 & 2683 & 1586 & 2856 & 5302 & -2759 & -1403 & -1840 & -500 & -1425 & 3351 & 2429 & 1723 \\
\hline 1974 Regional Surplus/Deficit & 2358 & -202 & -283 & 1250 & 1201 & 4466 & 12114 & 10864 & 7810 & 9307 & 10357 & 8946 & 14225 & 12564 & 7006 \\
\hline 1975 Regional Surplus/Deficit & 6352 & 6206 & 2648 & 1592 & 1521 & 785 & 4547 & 2243 & 4723 & 2803 & 3715 & 6184 & 10697 & 10335 & 4568 \\
\hline 1976 Regional Surplus/Deficit & 4066 & 2772 & 1649 & 3834 & 4685 & 8934 & 9144 & 6229 & 3889 & 8997 & 7111 & 9073 & 7263 & 9359 & 6294 \\
\hline 1977 Regional Surplus/Deficit & 8767 & 7943 & 7370 & 2760 & 1113 & -867 & 164 & -3490 & -2712 & -2285 & -2085 & -2274 & 468 & 187 & 741 \\
\hline 1978 Regional Surplus/Deficit & 2331 & -325 & -477 & 520 & 1119 & 62 & 2899 & 156 & 4583 & 3122 & 4789 & 5493 & 6066 & 6452 & 2653 \\
\hline \multicolumn{16}{|l|}{-Ranked Averages- } \\
\hline Top Ten Percent & 4603 & 4040 & 1241 & 3316 & 3449 & 5921 & 9434 & 8240 & 7100 & 9131 & 8721 & 9304 & 11156 & 9540 & 6829 \\
\hline Middle Eighty Percent & 4754 & 2788 & 1390 & 2659 & 1892 & 1290 & 3625 & 1813 & 1774 & 3557 & 4623 & 5264 & 8582 & 5819 & 3497 \\
\hline Bottom Ten Percent & 3304 & 1304 & 226 & 1689 & 898 & -1017 & -2022 & -3241 & -1692 & -1014 & -1132 & -2587 & 2228 & 1476 & -234 \\
\hline
\end{tabular}


Regional Surplus/Deficit by Water Year

PNW Loads and Resources Study

2012 - 2013 Operating Year 2003 White Book

\begin{tabular}{|c|c|c|c|c|c|c|c|c|c|c|c|c|c|c|c|}
\hline Average Energy in Megawatts & Aug1 & Aug16 & Sep & Oct & Nov & Dec & Jan & $\mathrm{Feb}$ & Mar & Apr1 & Apr16 & May & Jun & Jul & Avg \\
\hline 1929 Regional Surplus/Deficit & 5078 & 1267 & 287 & 1608 & 995 & -1364 & -1028 & -4580 & -2156 & -2178 & -1648 & -2541 & 2998 & 2223 & -191 \\
\hline 1930 Regional Surplus/Deficit & 1702 & 303 & -316 & 1341 & 615 & -1641 & -3957 & -2324 & -2135 & -1503 & -1628 & -3119 & 1590 & 2257 & -688 \\
\hline 1931 Regional Surplus/Deficit & 2433 & 700 & -57 & 1310 & 672 & -1838 & -3662 & -4644 & -2359 & -1230 & -2151 & -2904 & 761 & 1016 & -986 \\
\hline 1932 Regional Surplus/Deficit & 2052 & -222 & -261 & 1009 & 441 & -1797 & -3449 & -4457 & 614 & 4680 & 8450 & 6563 & 7898 & 4821 & 1572 \\
\hline 1933 Regional Surplus/Deficit & 5130 & 3390 & 369 & 1588 & 1329 & 615 & 4320 & 2183 & -1965 & 721 & 1881 & 3760 & 12149 & 10194 & 3342 \\
\hline 1934 Regional Surplus/Deficit & 4772 & 4848 & 1742 & 4767 & 4416 & 8858 & 10688 & 7519 & 4882 & 9672 & 8546 & 5775 & 1194 & 3411 & 5598 \\
\hline 1935 Regional Surplus/Deficit & 1388 & -773 & -409 & 1350 & 1385 & -109 & 3748 & 4151 & -2937 & -447 & 2771 & 2658 & 5830 & 4813 & 1829 \\
\hline 1936 Regional Surplus/Deficit & 3984 & 1369 & 8.9 & 1393 & 619 & -1775 & -2774 & -4280 & -1613 & -1498 & 2910 & 8844 & 5215 & 2470 & 958 \\
\hline 1937 Regional Surplus/Deficit & 3362 & 511 & -115 & 1336 & 559 & -1535 & -3872 & -4505 & -2259 & -1441 & -1669 & -2613 & 1961 & 1840 & -735 \\
\hline 1938 Regional Surplus/Deficit & 2707 & -179 & -241 & 1905 & 1416 & -594 & 5073 & -1097 & 2272 & 3406 & 5085 & 7635 & 6489 & 4242 & 2718 \\
\hline 1939 Regional Surplus/Deficit & 2375 & 131 & 644 & 1688 & 905 & -1170 & 758 & -3600 & -982 & -94 & 1627 & 4715 & 2051 & 2928 & 830 \\
\hline 1940 Regional Surplus/Deficit & 3741 & 654 & -158 & 1819 & 537 & -716 & -785 & -1577 & 2826 & 1480 & 2962 & 1816 & 2497 & 949 & 969 \\
\hline 1941 Regional Surplus/Deficit & 1815 & -34 & -342 & 1468 & 401 & -452 & -228 & -4049 & -2063 & -2427 & -1557 & -1854 & 1931 & 175 & -510 \\
\hline 1942 Regional Surplus/Deficit & 1613 & 72 & 68 & 1200 & -147 & 2758 & 4076 & -2888 & -3204 & -1079 & 582 & 1597 & 7836 & 5666 & 1463 \\
\hline 1943 Regional Surplus/Deficit & 4797 & 3280 & 284 & 1400 & 1234 & -15 & 3644 & 2498 & 3547 & 9874 & 8596 & 7645 & 8463 & 7225 & 4100 \\
\hline 1944 Regional Surplus/Deficit & 5040 & 3527 & 540 & 1857 & 956 & -1070 & -110 & -4061 & -2826 & -2157 & -1934 & -3284 & 292 & -13 & -457 \\
\hline 1945 Regional Surplus/Deficit & 1907 & -656 & -297 & 682 & 251 & -1640 & -3367 & -3272 & -2428 & -1896 & -1608 & 2389 & 6398 & 2963 & 46 \\
\hline 1946 Regional Surplus/Deficit & 2202 & 1355 & -250 & 1955 & 1342 & -541 & 1934 & 1528 & 4009 & 4739 & 7074 & 8383 & 7393 & 5970 & 3284 \\
\hline 1947 Regional Surplus/Deficit & 5290 & 2968 & 939 & 1780 & 1586 & 4917 & 5686 & 4409 & 4508 & 2336 & 5346 & 5799 & 6388 & 4823 & 4067 \\
\hline 1948 Regional Surplus/Deficit & 5215 & 1676 & 650 & 6725 & 3550 & 2404 & 5543 & -560 & 1174 & 2262 & 6192 & 9666 & 13138 & 6929 & 4741 \\
\hline 1949 Regional Surplus/Deficit & 4930 & 5832 & 2369 & 2482 & 1211 & -53 & 1034 & -594 & 6003 & 2426 & 8063 & 8043 & 6367 & 1983 & 3289 \\
\hline 1950 Regional Surplus/Deficit & 2381 & -432 & -485 & 1913 & 1580 & -1244 & 2583 & 4922 & 7501 & 6927 & 7136 & 5940 & 11932 & 8118 & 4231 \\
\hline 1951 Regional Surplus/Deficit & 4651 & 5270 & 1462 & 4409 & 4895 & 7388 & 8611 & 9329 & 5315 & 6853 & 7942 & 8357 & 5538 & 7097 & 6230 \\
\hline 1952 Regional Surplus/Deficit & 4850 & 4914 & 1988 & 5583 & 1932 & 3489 & 4825 & 2486 & 2608 & 6469 & 7964 & 9836 & 6912 & 3811 & 4631 \\
\hline 1953 Regional Surplus/Deficit & 4838 & 1402 & 368 & 1400 & 866 & -1478 & 1338 & 4363 & -985 & -1754 & 1726 & 5772 & 11203 & 7507 & 2788 \\
\hline 1954 Regional Surplus/Deficit & 5367 & 3186 & 989 & 2352 & 1629 & 2034 & 3736 & 4941 & 2156 & 5712 & 4435 & 6550 & 10220 & 9531 & 4457 \\
\hline 1955 Regional Surplus/Deficit & 7044 & 5567 & 6017 & 3524 & 2841 & 1572 & -1048 & -3263 & -2835 & 274 & -831 & 1267 & 12410 & 11078 & 3132 \\
\hline 1956 Regional Surplus/Deficit & 4960 & 5506 & 865 & 4054 & 3785 & 6095 & 9596 & 3529 & 6247 & 7367 & 10869 & 10023 & 13212 & 7787 & 6629 \\
\hline 1957 Regional Surplus/Deficit & 5630 & 4316 & 1070 & 3095 & 1229 & 2372 & 2717 & -1437 & 1354 & 7444 & 4780 & 9220 & 11904 & 3691 & 3858 \\
\hline 1958 Regional Surplus/Deficit & 3067 & 655 & 175 & 1864 & 987 & -643 & 2400 & 2690 & 2106 & 580 & 5536 & 8145 & 9174 & 2976 & 2899 \\
\hline 1959 Regional Surplus/Deficit & 3074 & 1263 & 452 & 1825 & 2780 & 3565 & 8327 & 5334 & 2000 & 4932 & 4027 & 4889 & 10048 & 8270 & 4512 \\
\hline 1960 Regional Surplus/Deficit & 4753 & 3816 & 6004 & 7980 & 5465 & 4862 & 4602 & -94 & 1882 & 10202 & 6918 & 4188 & 7420 & 5210 & 5030 \\
\hline 1961 Regional Surplus/Deficit & 5253 & 1454 & 571 & 1996 & 2050 & 1.9 & 3336 & 3975 & 3329 & 4352 & 4149 & 5040 & 11213 & 4163 & 3607 \\
\hline 1962 Regional Surplus/Deficit & 3357 & 1825 & -222 & 1846 & 1081 & -1221 & 2598 & -2286 & -2092 & 6982 & 8228 & 4356 & 5400 & 5351 & 2084 \\
\hline 1963 Regional Surplus/Deficit & 4583 & 2563 & 321 & 2968 & 2890 & 3553 & 2917 & 1636 & 176 & 503 & 1176 & 5354 & 6434 & 4817 & 2957 \\
\hline 1964 Regional Surplus/Deficit & 5535 & 2992 & 1098 & 1606 & 1645 & -608 & 2667 & -950 & -2022 & 2366 & 2595 & 3651 & 12591 & 10102 & 3044 \\
\hline 1965 Regional Surplus/Deficit & 5229 & 4843 & 2476 & 3707 & 1700 & 6433 & 10331 & 7871 & 5164 & 4066 & 8196 & 7711 & 8287 & 4576 & 5785 \\
\hline 1966 Regional Surplus/Deficit & 5150 & 4497 & 1262 & 2724 & 1610 & 1420 & 4527 & -1625 & -1209 & 5246 & 2312 & 4038 & 4560 & 5709 & 2635 \\
\hline 1967 Regional Surplus/Deficit & 5082 & 2629 & 97 & 1628 & 1254 & 516 & 5484 & 6514 & 221 & 3932 & 1708 & 2288 & 11569 & 8277 & 3710 \\
\hline 1968 Regional Surplus/Deficit & 4803 & 3838 & 1175 & 2949 & 1709 & 1325 & 4611 & 3520 & 2555 & -2106 & 1019 & 1755 & 7728 & 7113 & 3185 \\
\hline 1969 Regional Surplus/Deficit & 4999 & 4502 & 3369 & 4379 & 3676 & 3269 & 8072 & 5395 & 2986 & 8955 & 8380 & 9882 & 7617 & 5274 & 5611 \\
\hline 1970 Regional Surplus/Deficit & 5065 & 1525 & 589 & 2085 & 1412 & -362 & 3689 & 2946 & -1023 & -971 & 2300 & 4128 & 8779 & 2117 & 2360 \\
\hline 1971 Regional Surplus/Deficit & 2809 & 748 & -9.2 & 1923 & 1667 & -296 & 7169 & 9982 & 4610 & 5521 & 7052 & 10061 & 12056 & 8948 & 5348 \\
\hline 1972 Regional Surplus/Deficit & 5683 & 5955 & 1663 & 2364 & 1905 & 1867 & 6707 & 9256 & 10354 & 10929 & 5383 & 9644 & 12802 & 9638 & 6681 \\
\hline 1973 Regional Surplus/Deficit & 6661 & 5400 & 2550 & 2458 & 1327 & 2567 & 4968 & -3434 & -2039 & -2579 & -1154 & -1592 & 2431 & 2009 & 1284 \\
\hline 1974 Regional Surplus/Deficit & 1925 & -506 & -568 & 1022 & 943 & 4177 & 11782 & 10200 & 7182 & 8574 & 9711 & 8788 & 13312 & 12146 & 6570 \\
\hline 1975 Regional Surplus/Deficit & 5919 & 5906 & 2366 & 1366 & 1263 & 494 & 4213 & 1577 & 4094 & 2068 & 3066 & 6025 & 9783 & 9917 & 4131 \\
\hline 1976 Regional Surplus/Deficit & 3634 & 2471 & 1367 & 3611 & 4428 & 8653 & 8812 & 5565 & 3261 & 8263 & 6464 & 8915 & 6348 & 8941 & 5860 \\
\hline 1977 Regional Surplus/Deficit & 8335 & 7643 & 7093 & 2536 & 854 & -1158 & -177 & -4167 & -3351 & -3025 & -2741 & -2442 & -455 & -237 & 300 \\
\hline 1978 Regional Surplus/Deficit & 1898 & -629 & -762 & 292 & 861 & -229 & 2561 & -515 & 3954 & 2388 & 4141 & 5334 & 5150 & 6033 & 2215 \\
\hline \multicolumn{16}{|l|}{-Ranked Averages- } \\
\hline Top Ten Percent & 4171 & 3739 & 958 & 3092 & 3191 & 5636 & 9101 & 7576 & 6472 & 8397 & 8074 & 9145 & 10242 & 9122 & 6394 \\
\hline Middle Eighty Percent & 4322 & 2486 & 1107 & 2434 & 1634 & 1000 & 3289 & 1144 & 1142 & 2822 & 3974 & 5104 & 7667 & 5399 & 3060 \\
\hline Bottom Ten Percent & 2870 & 1001 & -58 & 1462 & 641 & -1307 & -2366 & -3917 & -2328 & -1752 & -1788 & -2755 & 1307 & 1055 & -675 \\
\hline
\end{tabular}


Regional Surplus/Deficit by Water Year

PNW Loads and Resources Study

2013 - 2014 Operating Year 2003 White Book

\begin{tabular}{|c|c|c|c|c|c|c|c|c|c|c|c|c|c|c|c|}
\hline Average Energy in Megawatts & Aug1 & Aug16 & Sep & Oct & Nov & Dec & Jan & $\mathrm{Feb}$ & Mar & Apr1 & Apr16 & May & Jun & Jul & Avg \\
\hline 1929 Regional Surplus/Deficit & 4838 & 964 & 51 & 1299 & 692 & -1732 & -1364 & -4862 & -2326 & -2024 & -1541 & -2465 & 3318 & 2178 & -341 \\
\hline 1930 Regional Surplus/Deficit & 1460 & 0.3 & -552 & 1030 & 313 & -2009 & -4297 & -2603 & -2305 & -1347 & -1522 & -3044 & 1909 & 2212 & -837 \\
\hline 1931 Regional Surplus/Deficit & 2193 & 398 & -293 & 999 & 370 & -2206 & -4001 & -4925 & -2530 & -1072 & -2046 & -2828 & 1079 & 971 & -1136 \\
\hline 1932 Regional Surplus/Deficit & 1812 & -525 & -497 & 698 & 139 & -2167 & -3788 & -4739 & 447 & 4839 & 8564 & 6646 & 8221 & 4776 & 1423 \\
\hline 1933 Regional Surplus/Deficit & 4890 & 3089 & 135 & 1279 & 1028 & 247 & 3990 & 1911 & -2133 & 879 & 1993 & 3842 & 12473 & 10149 & 3195 \\
\hline 1934 Regional Surplus/Deficit & 4531 & 4548 & 1510 & 4461 & 4115 & 8498 & 10358 & 7248 & 4718 & 9831 & 8660 & 5857 & 1511 & 3366 & 5452 \\
\hline 1935 Regional Surplus/Deficit & 1147 & -1076 & -643 & 1039 & 1083 & -478 & 3417 & 3879 & -3104 & -288 & 2884 & 2737 & 6152 & 4768 & 1682 \\
\hline 1936 Regional Surplus/Deficit & 3744 & 1068 & -226 & 1083 & 318 & -2142 & -3111 & -4561 & -1783 & -1341 & 3024 & 8927 & 5539 & 2425 & 810 \\
\hline 1937 Regional Surplus/Deficit & 3121 & 208 & -350 & 1026 & 257 & -1903 & -4211 & -4786 & -2429 & -1286 & -1562 & -2538 & 2280 & 1795 & -885 \\
\hline 1938 Regional Surplus/Deficit & 2467 & -480 & -476 & 1595 & 1115 & -961 & 4741 & -1372 & 2107 & 3565 & 5199 & 7717 & 6813 & 4197 & 2571 \\
\hline 1939 Regional Surplus/Deficit & 2134 & -171 & 410 & 1380 & 603 & -1538 & 425 & -3879 & -1151 & 64 & 1739 & 4796 & 2369 & 2883 & 682 \\
\hline 1940 Regional Surplus/Deficit & 3500 & 352 & -393 & 1510 & 236 & -1084 & -1121 & -1857 & 2661 & 1638 & 3077 & 1897 & 2816 & 904 & 821 \\
\hline 1941 Regional Surplus/Deficit & 1574 & -336 & -577 & 1159 & 100 & -819 & -565 & -4329 & -2233 & -2269 & -1446 & -1776 & 2252 & 129 & -658 \\
\hline 1942 Regional Surplus/Deficit & 1372 & -230 & -167 & 891 & -448 & 2391 & 3746 & -3166 & -3375 & -920 & 694 & 1678 & 8160 & 5622 & 1316 \\
\hline 1943 Regional Surplus/Deficit & 4556 & 2980 & 50 & 1091 & 931 & -383 & 3314 & 2224 & 3383 & 10032 & 8710 & 7727 & 8787 & 7181 & 3954 \\
\hline 1944 Regional Surplus/Deficit & 4800 & 3227 & 305 & 1548 & 653 & -1438 & -446 & -4342 & -2997 & -2002 & -1827 & -3207 & 611 & -59 & -606 \\
\hline 1945 Regional Surplus/Deficit & 1666 & -959 & -533 & 372 & -52 & -2009 & -3706 & -3553 & -2599 & -1740 & -1502 & 2471 & 6722 & 2919 & -103 \\
\hline 1946 Regional Surplus/Deficit & 1961 & 1054 & -485 & 1645 & 1040 & -908 & 1599 & 1253 & 3845 & 4898 & 7188 & 8466 & 7717 & 5925 & 3137 \\
\hline 1947 Regional Surplus/Deficit & 5049 & 2667 & 705 & 1472 & 1284 & 4551 & 5356 & 4137 & 4344 & 2494 & 5461 & 5881 & 6712 & 4779 & 3921 \\
\hline 1948 Regional Surplus/Deficit & 4975 & 1374 & 415 & 6419 & 3249 & 2040 & 5212 & -837 & 1010 & 2420 & 6306 & 9748 & 13461 & 6884 & 4595 \\
\hline 1949 Regional Surplus/Deficit & 4690 & 5532 & 2136 & 2175 & 909 & -422 & 701 & -873 & 5839 & 2585 & 8177 & 8126 & 6691 & 1938 & 3143 \\
\hline 1950 Regional Surplus/Deficit & 2140 & -735 & -720 & 1603 & 1278 & -1611 & 2251 & 4648 & 7337 & 7085 & 7250 & 6022 & 12256 & 8073 & 4084 \\
\hline 1951 Regional Surplus/Deficit & 4411 & 4969 & 1228 & 4102 & 4593 & 7026 & 8281 & 9057 & 5151 & 7011 & 8057 & 8440 & 5862 & 7052 & 6085 \\
\hline 1952 Regional Surplus/Deficit & 4610 & 4614 & 1755 & 5277 & 1631 & 3123 & 4495 & 2214 & 2442 & 6627 & 8078 & 9919 & 7236 & 3766 & 4485 \\
\hline 1953 Regional Surplus/Deficit & 4598 & 1101 & 133 & 1091 & 563 & -1845 & 1001 & 4091 & -1151 & -1595 & 1839 & 5855 & 11526 & 7462 & 2641 \\
\hline 1954 Regional Surplus/Deficit & 5126 & 2885 & 754 & 2044 & 1327 & 1666 & 3405 & 4670 & 1991 & 5870 & 4549 & 6632 & 10544 & 9486 & 4311 \\
\hline 1955 Regional Surplus/Deficit & 6803 & 5266 & 5786 & 3217 & 2540 & 1205 & -1384 & -3544 & -3006 & 432 & -717 & 1347 & 12734 & 11033 & 2985 \\
\hline 1956 Regional Surplus/Deficit & 4720 & 5206 & 631 & 3747 & 3483 & 5732 & 9265 & 3258 & 6083 & 7525 & 10983 & 10105 & 13535 & 7742 & 6483 \\
\hline 1957 Regional Surplus/Deficit & 5389 & 4016 & 836 & 2788 & 927 & 2005 & 2384 & -1715 & 1189 & 7602 & 4894 & 9302 & 12228 & 3646 & 3712 \\
\hline 1958 Regional Surplus/Deficit & 2826 & 352 & -61 & 1555 & 685 & -1012 & 2067 & 2415 & 1942 & 738 & 5650 & 8227 & 9498 & 2932 & 2753 \\
\hline 1959 Regional Surplus/Deficit & 2833 & 961 & 217 & 1517 & 2478 & 3200 & 7997 & 5063 & 1835 & 5090 & 4142 & 4972 & 10372 & 8226 & 4366 \\
\hline 1960 Regional Surplus/Deficit & 4512 & 3515 & 5773 & 7674 & 5164 & 4498 & 4272 & -369 & 1716 & 10360 & 7032 & 4271 & 7744 & 5165 & 4885 \\
\hline 1961 Regional Surplus/Deficit & 5012 & 1152 & 337 & 1687 & 1749 & -367 & 3004 & 3703 & 3165 & 4510 & 4262 & 5123 & 11537 & 4118 & 3460 \\
\hline 1962 Regional Surplus/Deficit & 3117 & 1524 & -458 & 1537 & 778 & -1590 & 2265 & -2562 & -2262 & 7140 & 8343 & 4438 & 5724 & 5306 & 1937 \\
\hline 1963 Regional Surplus/Deficit & 4343 & 2262 & 86 & 2661 & 2589 & 3188 & 2587 & 1363 & 6.1 & 662 & 1289 & 5436 & 6758 & 4772 & 2810 \\
\hline 1964 Regional Surplus/Deficit & 5294 & 2692 & 864 & 1297 & 1342 & -976 & 2334 & -1226 & -2192 & 2525 & 2708 & 3733 & 12914 & 10058 & 2897 \\
\hline 1965 Regional Surplus/Deficit & 4988 & 4543 & 2243 & 3400 & 1399 & 6068 & 10000 & 7599 & 5000 & 4224 & 8310 & 7793 & 8611 & 4531 & 5640 \\
\hline 1966 Regional Surplus/Deficit & 4909 & 4197 & 1028 & 2416 & 1309 & 1052 & 4196 & -1900 & -1378 & 5404 & 2425 & 4120 & 4882 & 5664 & 2488 \\
\hline 1967 Regional Surplus/Deficit & 4842 & 2327 & -138 & 1319 & 951 & 148 & 5154 & 6242 & 55 & 4091 & 1819 & 2371 & 11893 & 8232 & 3564 \\
\hline 1968 Regional Surplus/Deficit & 4562 & 3538 & 941 & 2641 & 1407 & 957 & 4281 & 3249 & 2390 & -1950 & 1130 & 1834 & 8052 & 7068 & 3038 \\
\hline 1969 Regional Surplus/Deficit & 4758 & 4202 & 3138 & 4073 & 3375 & 2903 & 7742 & 5124 & 2821 & 9113 & 8494 & 9964 & 7941 & 5229 & 5466 \\
\hline 1970 Regional Surplus/Deficit & 4824 & 1223 & 354 & 1777 & 1109 & -731 & 3355 & 2675 & -1190 & -813 & 2413 & 4210 & 9103 & 2072 & 2213 \\
\hline 1971 Regional Surplus/Deficit & 2568 & 445 & -245 & 1613 & 1365 & -663 & 6839 & 9711 & 4446 & 5679 & 7167 & 10143 & 12380 & 8903 & 5202 \\
\hline 1972 Regional Surplus/Deficit & 5442 & 5654 & 1429 & 2055 & 1604 & 1499 & 6377 & 8984 & 10190 & 11087 & 5497 & 9726 & 13125 & 9593 & 6535 \\
\hline 1973 Regional Surplus/Deficit & 6421 & 5099 & 2316 & 2150 & 1025 & 2200 & 4638 & -3714 & -2209 & -2422 & -1044 & -1515 & 2751 & 1964 & 1136 \\
\hline 1974 Regional Surplus/Deficit & 1685 & -810 & -805 & 711 & 640 & 3810 & 11452 & 9929 & 7018 & 8732 & 9826 & 8870 & 13635 & 12102 & 6423 \\
\hline 1975 Regional Surplus/Deficit & 5679 & 5606 & 2133 & 1057 & 960 & 125 & 3882 & 1304 & 3929 & 2226 & 3180 & 6107 & 10107 & 9872 & 3985 \\
\hline 1976 Regional Surplus/Deficit & 3393 & 2171 & 1133 & 3304 & 4126 & 8293 & 8482 & 5293 & 3097 & 8422 & 6578 & 8998 & 6671 & 8896 & 5715 \\
\hline 1977 Regional Surplus/Deficit & 8095 & 7343 & 6862 & 2228 & 551 & -1526 & -512 & -4448 & -3523 & -2871 & -2633 & -2366 & -138 & -285 & 151 \\
\hline 1978 Regional Surplus/Deficit & 1656 & -932 & -997 & -18 & 559 & -597 & 2227 & -792 & 3790 & 2546 & 4256 & 5416 & 5474 & 5988 & 2068 \\
\hline \multicolumn{16}{|l|}{-Ranked Averages- } \\
\hline Top Ten Percent & 3930 & 3438 & 723 & 2784 & 2889 & 5272 & 8771 & 7304 & 6308 & 8555 & 8188 & 9228 & 10566 & 9077 & 6248 \\
\hline Middle Eighty Percent & 4081 & 2185 & 873 & 2125 & 1333 & 633 & 2956 & 869 & 976 & 2980 & 4087 & 5185 & 7990 & 5354 & 2913 \\
\hline Bottom Ten Percent & 2630 & 699 & -294 & 1153 & 339 & -1675 & -2704 & -4197 & -2499 & -1595 & -1681 & -2679 & 1626 & 1010 & -824 \\
\hline
\end{tabular}




\section{Section 8 Monthly OY 2005 - 2014 Average Energy in Megawatts}

$\underline{\text { Table Number }}$

Total Retail Loads

A-1

Exports

A-2

Regulated Hydro

A-3

Independent Hydro

A-4

Imports

A-5

Small Thermal \& Miscellaneous

A-6

Combustion Turbines

A-7

Renewable Resources

A-8

Cogeneration

A-9

Large Thermal

A-10

Restoration

A-11

Canadian Entitlement Return For CSPE

A-12

Columbia Storage Power Exchange Purchase (CSPE)

Supplemental \& Entitlement Capacity

A-14

Canadian Entitlement Return For Canada

A-15

Intra Regional Transfers

A-16

Capacity Reserves

A-17

Wells \& Chelan Allocation

Rocky Reach \& Rock Island Allocation

A-18

Wanapum \& Priest Rapids Allocation

A-19

Swift \#1 \& Swift \#2 Allocation

A-20

BPA Power Sales Contracts

A-21

Non-Utility Generating Resources

A-22

Regional Non-Utility Generating Resources By Project

A-23

Dedicated Resources

A-24

Other Dedicated Resources

A-25

A-26 
Aug1 Aug16 Sep Oct Nov

Dec Jan Fe

Feb Mar Apr1 Apr16 May Jun Jul Avg

Firm Loads

-Federal Entities-

1 Federal Entities

2 Total Federal Entities

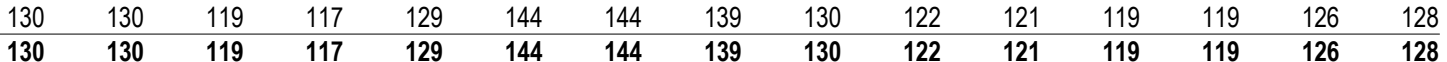

-U.S. Bureau of Reclamation-

3 U.S. Bureau of Reclamation

$\begin{array}{lllllllllllllll}309 & 275 & 166 & 129 & 0 & 0 & 0 & 1.0 & 23 & 252 & 315 & 268 & 298 & 332 & 149 \\ \mathbf{3 0 9} & \mathbf{2 7 5} & \mathbf{1 6 6} & \mathbf{1 2 9} & \mathbf{0} & \mathbf{0} & \mathbf{0} & \mathbf{1 . 0} & \mathbf{2 3} & \mathbf{2 5 2} & \mathbf{3 1 5} & \mathbf{2 6 8} & \mathbf{2 9 8} & \mathbf{3 3 2} & \mathbf{1 4 9}\end{array}$

-Direct Service Industry-

5 Direct Sevice Industry

\begin{tabular}{lllllllllllllll}
302 & 301 & 299 & 296 & 294 & 291 & 288 & 287 & 287 & 290 & 290 & 290 & 290 & 290 & 292 \\
\hline $\mathbf{3 0 2}$ & $\mathbf{3 0 1}$ & $\mathbf{2 9 9}$ & $\mathbf{2 9 6}$ & $\mathbf{2 9 4}$ & $\mathbf{2 9 1}$ & $\mathbf{2 8 8}$ & $\mathbf{2 8 7}$ & $\mathbf{2 8 7}$ & $\mathbf{2 9 0}$ & $\mathbf{2 9 0}$ & $\mathbf{2 9 0}$ & $\mathbf{2 9 0}$ & $\mathbf{2 9 0}$ & $\mathbf{2 9 2}$
\end{tabular}

6 Total Direct Service Industry

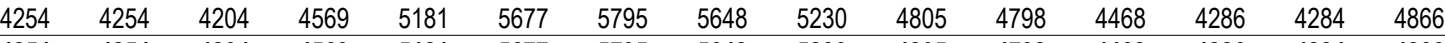

-Generating Public Entities

7 Generating Public Entities

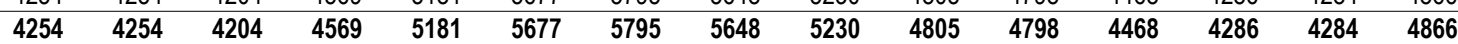

-Non-Generating Public Entities-

9 Non-Generating Public Entities

$\begin{array}{lllllllllllllll}3364 & 3364 & 3042 & 3014 & 3388 & 3881 & 3923 & 3738 & 3386 & 3232 & 3223 & 3208 & 3340 & 3461 & 3414 \\ \mathbf{3 3 6 4} & \mathbf{3 3 6 4} & \mathbf{3 0 4 2} & \mathbf{3 0 1 4} & \mathbf{3 3 8 8} & \mathbf{3 8 8 1} & \mathbf{3 9 2 3} & \mathbf{3 7 3 8} & \mathbf{3 3 8 6} & \mathbf{3 2 3 2} & \mathbf{3 2 2 3} & \mathbf{3 2 0 8} & \mathbf{3 3 4 0} & \mathbf{3 4 6 1} & \mathbf{3 4 1 4}\end{array}$

-Investor-Owned Entities-

11 Investor-Owned Entities

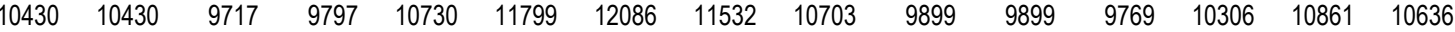

12 Total Investor-Owned Entities

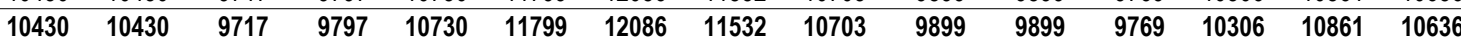

-Other Firm Loads-

13 Other PNW Entities

14 Total Other Firm Loads

$\begin{array}{lllllllllllllll}180 & 180 & 180 & 180 & 180 & 180 & 180 & 180 & 180 & 180 & 180 & 180 & 180 & 180 & 180 \\ 1 \mathbf{1 8 0} & \mathbf{1 8 0} & \mathbf{1 8 0} & \mathbf{1 8 0} & \mathbf{1 8 0} & \mathbf{1 8 0} & \mathbf{1 8 0} & \mathbf{1 8 0} & \mathbf{1 8 0} & \mathbf{1 8 0} & \mathbf{1 8 0} & \mathbf{1 8 0} & \mathbf{1 8 0} & \mathbf{1 8 0} & \mathbf{1 8 0}\end{array}$

- Total Regional Firm Loads-

15 Federal Entities

16 Direct Service Industry

17 Public Entities

18 Investor-Owned Entities

19 Other Entities

20 Total Regional Firm Loads

Non-Firm Loads

-Investor-Owned Entities-

21 Investor-Owned Entities

22 Total Investor-Owned Entities

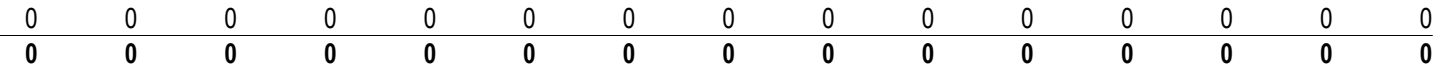

- Total Regional Non-Firm Loads-

23 Federal Entities

24 Direct Service Industry

25 Public Entities

26 Investor-Owned Entities

27 Other Entities

28 Total Regional Non-Firm Loads

- Total Regional Medium Loads

29 Federal Entities

30 Direct Service Industry

31 Public Entities

32 Investor-Owned Entities

33 Other Entities

34 Total Regional Medium Loads

\begin{tabular}{|c|c|c|c|c|c|c|c|c|c|c|c|c|c|c|}
\hline 439 & 405 & 285 & 246 & 129 & 144 & 144 & 139 & 153 & 374 & 436 & 387 & 417 & 458 & 278 \\
\hline 302 & 301 & 299 & 296 & 294 & 291 & 288 & 287 & 287 & 290 & 290 & 290 & 290 & 290 & 92 \\
\hline 7618 & 7618 & 7246 & 7583 & 8569 & 9558 & 9718 & 9386 & 8616 & 8037 & 8022 & 7676 & 7626 & 7745 & 281 \\
\hline 043 & 10430 & 9717 & 9797 & 10730 & 11799 & 12086 & 11532 & 10703 & 9899 & 9899 & 9769 & 10306 & 10861 & 10 \\
\hline 180 & 180 & 180 & 180 & 180 & 180 & 180 & 180 & 180 & 180 & 180 & 180 & 180 & 180 & \\
\hline 96 & 1893 & 7728 & 3102 & 9903 & 21973 & 22416 & 21525 & 19939 & 18780 & 18827 & 18302 & 18819 & 19534 & \\
\hline
\end{tabular}

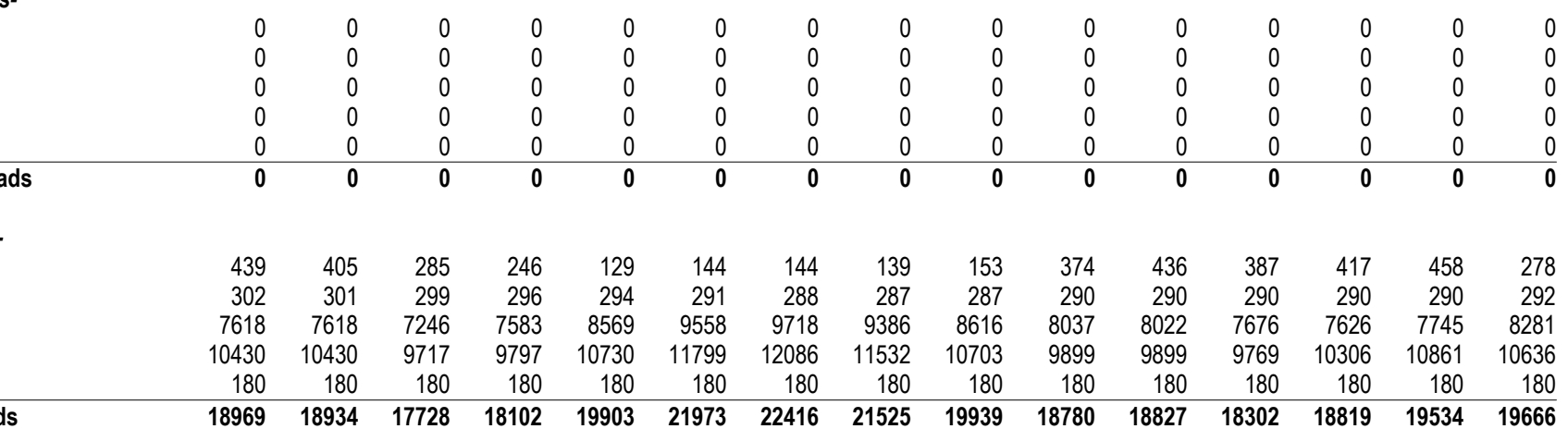


Aug1 Aug16 Sep Oct Nov 2003 White Book

Firm Loads

-Federal Entities-

1 Federal Entities

2 Total Federal Entities

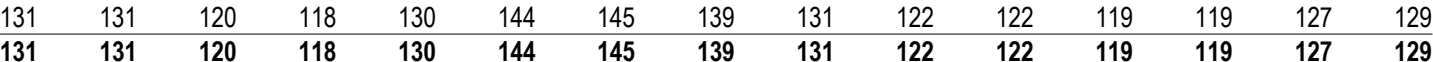

-U.S. Bureau of Reclamation-

3 U.S. Bureau of Reclamation

$\begin{array}{lllllllllllllll}309 & 275 & 166 & 129 & 0 & 0 & 0 & 1.0 & 23 & 252 & 315 & 268 & 298 & 332 & 149 \\ \mathbf{3 0 9} & \mathbf{2 7 5} & \mathbf{1 6 6} & \mathbf{1 2 9} & \mathbf{0} & \mathbf{0} & \mathbf{0} & \mathbf{1 . 0} & \mathbf{2 3} & \mathbf{2 5 2} & \mathbf{3 1 5} & \mathbf{2 6 8} & \mathbf{2 9 8} & \mathbf{3 3 2} & \mathbf{1 4 9}\end{array}$

-Direct Service Industry-

5 Direct Sevice Industry

\begin{tabular}{lllllllllllllll}
290 & 290 & 290 & 297 & 297 & 297 & 297 & 297 & 297 & 297 & 297 & 297 & 297 & 297 & 296 \\
\hline 290 & $\mathbf{2 9 0}$ & $\mathbf{2 9 0}$ & $\mathbf{2 9 7}$ & $\mathbf{2 9 7}$ & $\mathbf{2 9 7}$ & $\mathbf{2 9 7}$ & $\mathbf{2 9 7}$ & $\mathbf{2 9 7}$ & $\mathbf{2 9 7}$ & $\mathbf{2 9 7}$ & $\mathbf{2 9 7}$ & $\mathbf{2 9 7}$ & $\mathbf{2 9 7}$ & $\mathbf{2 9 6}$
\end{tabular}

6 Total Direct Service Industry

\begin{tabular}{lllllllllllllll}
4312 & 4312 & 4257 & 4623 & 5230 & 5730 & 5880 & 5710 & 5326 & 4897 & 4891 & 4540 & 4365 & 4363 & 4936 \\
\hline $\mathbf{4 3 1 2}$ & $\mathbf{4 3 1 2}$ & $\mathbf{4 2 5 7}$ & $\mathbf{4 6 2 3}$ & $\mathbf{5 2 3 0}$ & $\mathbf{5 7 3 0}$ & $\mathbf{5 8 8 0}$ & $\mathbf{5 7 1 0}$ & $\mathbf{5 3 2 6}$ & $\mathbf{4 8 9 7}$ & $\mathbf{4 8 9 1}$ & $\mathbf{4 5 4 0}$ & $\mathbf{4 3 6 5}$ & $\mathbf{4 3 6 3}$ & $\mathbf{4 9 3 6}$
\end{tabular}

-Generating Public Entities.

7 Generating Public Entities

-Non-Generating Public Entities-

9 Non-Generating Public Entities

10 Total Non-Generating Public Entities

\begin{tabular}{lllllllllllllll}
3404 & 3404 & 3076 & 3057 & 3438 & 3938 & 3992 & 3786 & 3439 & 3277 & 3268 & 3246 & 3387 & 3504 & 3462 \\
\hline 3404 & $\mathbf{3 4 0 4}$ & $\mathbf{3 0 7 6}$ & $\mathbf{3 0 5 7}$ & $\mathbf{3 4 3 8}$ & $\mathbf{3 9 3 8}$ & $\mathbf{3 9 9 2}$ & $\mathbf{3 7 8 6}$ & $\mathbf{3 4 3 9}$ & $\mathbf{3 2 7 7}$ & $\mathbf{3 2 6 8}$ & $\mathbf{3 2 4 6}$ & $\mathbf{3 3 8 7}$ & $\mathbf{3 5 0 4}$ & $\mathbf{3 4 6 2}$
\end{tabular}

-Investor-Owned Entities-

11 Investor-Owned Entities

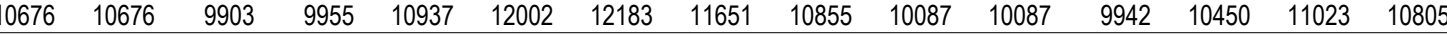

12 Total Investor-Owned Entities

$\begin{array}{lllllllllllllll}10676 & 10676 & 9903 & 9955 & 10937 & 12002 & 12183 & 11651 & 10855 & 10087 & 10087 & 9942 & 10450 & 11023 & 10805\end{array}$

-Other Firm Loads-

13 Other PNW Entities

14 Total Other Firm Loads

$180 \quad 180 \quad 180$

- Total Regional Firm Loads-

15 Federal Entities

16 Direct Service Industry

17 Public Entities

18 Investor-Owned Entities

19 Other Entities

20 Total Regional Firm Loads

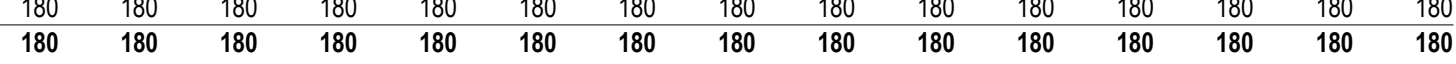

Non-Firm Loads

-Investor-Owned Entities-

21 Investor-Owned Entities

22 Total Investor-Owned Entities

- Total Regional Non-Firm Loads-

23 Federal Entities

24 Direct Service Industry

25 Public Entities

26 Investor-Owned Entities

27 Other Entities

28 Total Regional Non-Firm Loads

- Total Regional Medium Loads

29 Federal Entities

30 Direct Service Industry

31 Public Entities

32 Investor-Owned Entities

33 Other Entities

34 Total Regional Medium Loads

\begin{tabular}{|c|c|c|c|c|c|c|c|c|c|c|c|c|c|c|}
\hline 440 & 406 & 286 & 247 & 130 & 144 & 145 & 140 & 154 & 374 & 437 & 387 & 417 & 459 & \\
\hline 290 & 290 & 290 & 297 & 297 & 297 & 297 & 297 & 297 & 297 & 297 & 297 & 297 & 297 & \\
\hline 7716 & 7716 & 7333 & 7680 & 8668 & 9668 & 9873 & 9496 & 8765 & 8174 & & 7786 & 7752 & 7867 & \\
\hline 676 & 10676 & 990 & 9955 & 10937 & 12002 & 1218 & 11651 & 108 & 10087 & 10 & & 10450 & 023 & \\
\hline 18 & 18 & $1 \varepsilon$ & 180 & $1 \varepsilon$ & 180 & 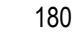 & 180 & & 180 & & 100 & & 30 & \\
\hline 30 & 9268 & 001 & 325 & 210 & 2291 & 2677 & 1764 & 251 & 19112 & 0 & 8593 & 9096 & 19826 & \\
\hline
\end{tabular}

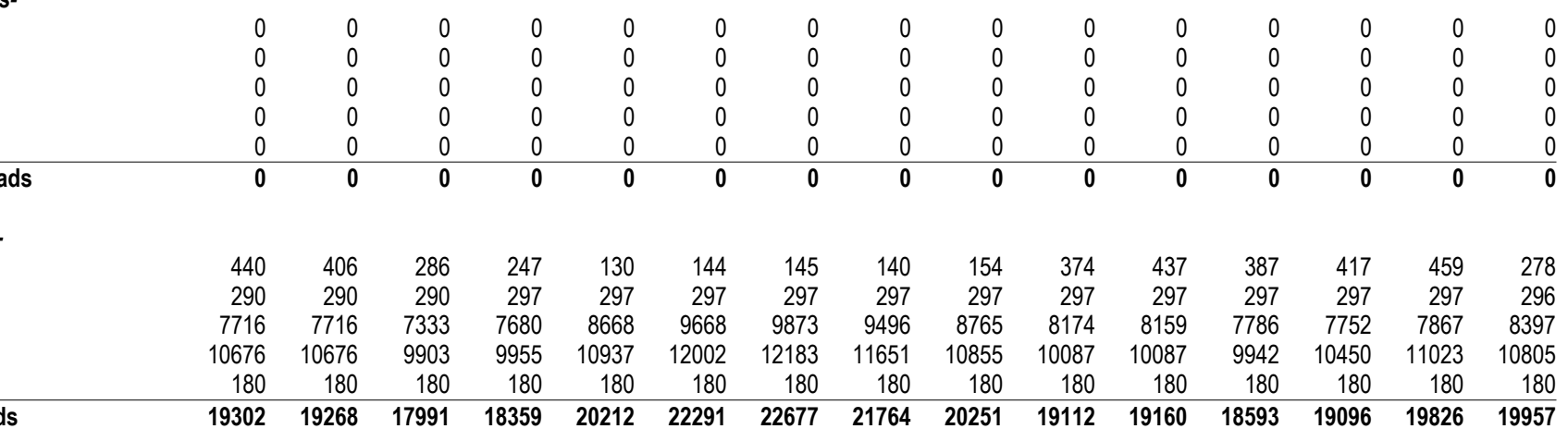



2003 White Book

Firm Loads

-Federal Entities-

1 Federal Entities

2 Total Federal Entities

\begin{tabular}{|c|c|c|c|c|c|c|c|c|c|c|c|c|}
\hline 132 & 121 & 118 & 130 & 145 & 145 & 140 & 131 & 123 & 122 & 120 & 120 & 127 \\
\hline 132 & 121 & 118 & 130 & 145 & 145 & 140 & 131 & 123 & 122 & 120 & 120 & 127 \\
\hline
\end{tabular}

-U.S. Bureau of Reclamation-

3 U.S. Bureau of Reclamation

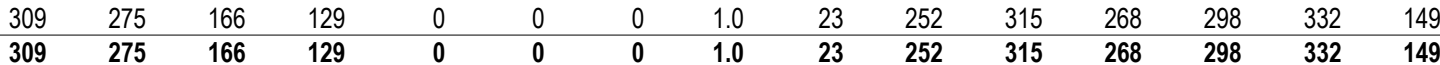

-Direct Service Industry-

5 Direct Sevice Industry

\begin{tabular}{lllllllllllllll}
297 & 297 & 297 & 674 & 674 & 674 & 674 & 674 & 674 & 674 & 674 & 674 & 674 & 674 & 611 \\
\hline 297 & $\mathbf{2 9 7}$ & $\mathbf{2 9 7}$ & $\mathbf{6 7 4}$ & $\mathbf{6 7 4}$ & $\mathbf{6 7 4}$ & $\mathbf{6 7 4}$ & $\mathbf{6 7 4}$ & $\mathbf{6 7 4}$ & $\mathbf{6 7 4}$ & $\mathbf{6 7 4}$ & $\mathbf{6 7 4}$ & $\mathbf{6 7 4}$ & $\mathbf{6 7 4}$ & $\mathbf{6 1 1}$
\end{tabular}

6 Total Direct Service Industry

-Generating Public Entities-

7 Generating Public Entities

\begin{tabular}{|c|c|c|c|c|c|c|c|c|c|c|c|c|c|c|}
\hline 375 & 4375 & 4324 & 4714 & 5332 & 5841 & 5990 & 5820 & 5429 & 4994 & 4987 & 4630 & 4450 & 4448 & 5029 \\
\hline 437 & 4375 & 4324 & 4714 & 5332 & 5841 & 5990 & 5820 & 5429 & 4994 & 4987 & 4630 & 4450 & & \\
\hline
\end{tabular}

8 Total Generating Public Entities

-Non-Generating Public Entities-

9 Non-Generating Public Entities

10 Total Non-Generating Public Entities

\begin{tabular}{lllllllllllllll}
3461 & 3461 & 3125 & 3095 & 3473 & 3980 & 4038 & 3837 & 3482 & 3318 & 3310 & 3286 & 3432 & 3547 & 3506 \\
\hline 3461 & 3461 & $\mathbf{3 1 2 5}$ & $\mathbf{3 0 9 5}$ & $\mathbf{3 4 7 3}$ & $\mathbf{3 9 8 0}$ & $\mathbf{4 0 3 8}$ & $\mathbf{3 8 3 7}$ & $\mathbf{3 4 8 2}$ & $\mathbf{3 3 1 8}$ & $\mathbf{3 3 1 0}$ & $\mathbf{3 2 8 6}$ & $\mathbf{3 4 3 2}$ & $\mathbf{3 5 4 7}$ & $\mathbf{3 5 0 6}$
\end{tabular}

-Investor-Owned Entities-

11 Investor-Owned Entities

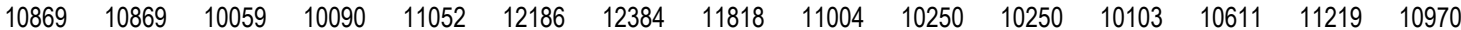

12 Total Investor-Owned Entities

$\begin{array}{lllllllllllllll}10869 & 10869 & 10059 & 10090 & 11052 & 12186 & 12384 & 11818 & 11004 & 10250 & 10250 & 10103 & 10611 & 11219 & 10970\end{array}$

-Other Firm Loads-

13 Other PNW Entities

14 Total Other Firm Loads

$180 \quad 180 \quad 180$

- Total Regional Firm Loads-

15 Federal Entities

16 Direct Service Industry

17 Public Entities

18 Investor-Owned Entities

19 Other Entities

20 Total Regional Firm Loads

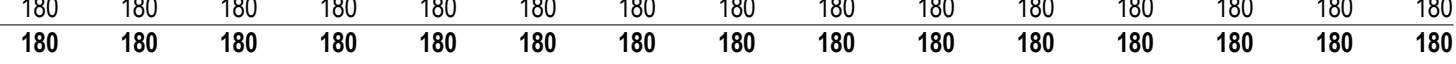

Non-Firm Loads

-Investor-Owned Entities-

21 Investor-Owned Entities

22 Total Investor-Owned Entities

- Total Regional Non-Firm Loads-

23 Federal Entities

24 Direct Service Industry

25 Public Entities

26 Investor-Owned Entities

27 Other Entities

28 Total Regional Non-Firm Loads

- Total Regional Medium Loads.

29 Federal Entities

30 Direct Service Industry

31 Public Entities

32 Investor-Owned Entities

33 Other Entities

34 Total Regional Medium Loads

\begin{tabular}{|c|c|c|c|c|c|c|c|c|c|c|c|c|c|c|}
\hline 441 & 407 & 287 & 247 & 130 & 145 & 145 & 141 & 154 & 375 & 437 & 388 & 418 & 459 & $27 \varsigma$ \\
\hline 297 & 297 & 297 & 674 & 674 & 674 & 674 & 674 & 674 & 674 & 674 & 674 & 674 & 674 & \\
\hline 7837 & 7837 & 7449 & 7809 & 8806 & 9821 & 10029 & 9656 & 8911 & 8312 & 8297 & 7916 & 7883 & 7996 & \\
\hline 86 & 10869 & 10059 & 10090 & 110 & 12186 & 1238 & 118 & 11004 & 50 & 0 & 3 & 1 & 19 & \\
\hline 18 & $1 \varepsilon$ & 18 & 180 & $1 \varepsilon$ & 18 & 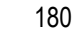 & 18 & 18 & 18 & & 180 & & 180 & \\
\hline $5 ?$ & 958 & 18271 & 9 & 084 & 3005 & 3412 & 22469 & 20923 & 9790 & 9838 & 19260 & 9766 & 20528 & \\
\hline
\end{tabular}

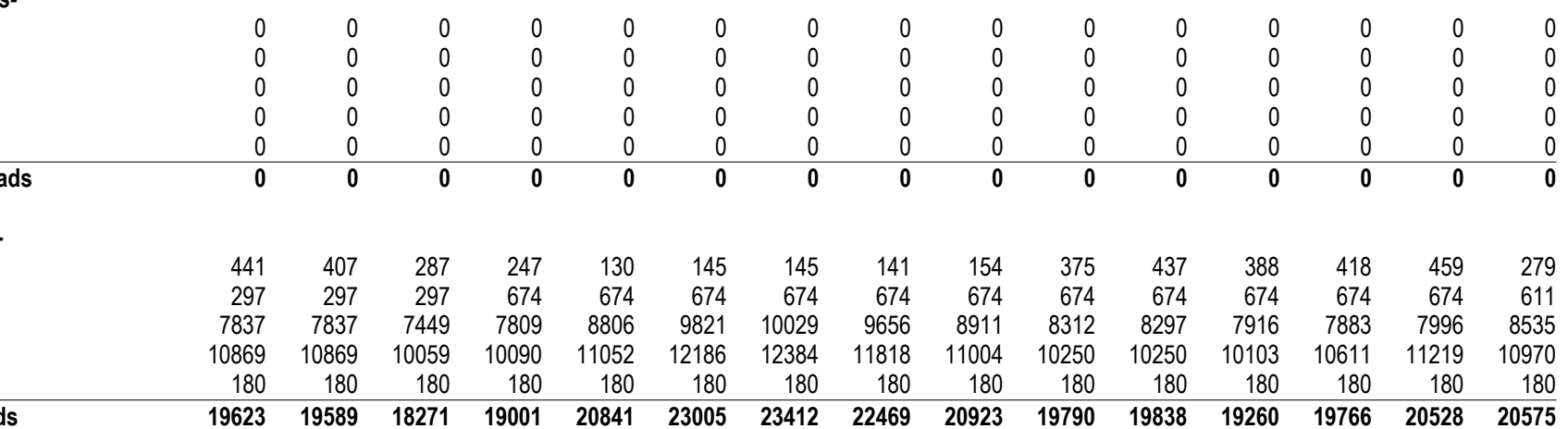



2003 White Book

Aug1 Aug16 Sep Oct Nov

Dec

Jan Feb

Mar Apr1 Apr16 May Jun Jul Avg

Firm Loads

-Federal Entities-

1 Federal Entities

2 Total Federal Entities

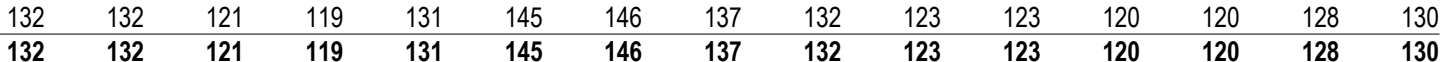

-U.S. Bureau of Reclamation-

3 U.S. Bureau of Reclamation

$\begin{array}{lllllllllllllll}309 & 275 & 166 & 129 & 0 & 0 & 0 & 1.0 & 23 & 252 & 315 & 268 & 298 & 332 & 149 \\ \mathbf{3 0 9} & \mathbf{2 7 5} & \mathbf{1 6 6} & \mathbf{1 2 9} & \mathbf{0} & \mathbf{0} & \mathbf{0} & \mathbf{1 . 0} & \mathbf{2 3} & \mathbf{2 5 2} & \mathbf{3 1 5} & \mathbf{2 6 8} & \mathbf{2 9 8} & \mathbf{3 3 2} & \mathbf{1 4 9}\end{array}$

-Direct Service Industry-

5 Direct Sevice Industry

$\begin{array}{lllllllllllllll}674 & 674 & 674 & 674 & 674 & 674 & 674 & 674 & 674 & 674 & 674 & 674 & 674 & 674 & 674 \\ \mathbf{6 7 4} & \mathbf{6 7 4} & \mathbf{6 7 4} & \mathbf{6 7 4} & \mathbf{6 7 4} & \mathbf{6 7 4} & \mathbf{6 7 4} & \mathbf{6 7 4} & \mathbf{6 7 4} & \mathbf{6 7 4} & \mathbf{6 7 4} & \mathbf{6 7 4} & \mathbf{6 7 4} & \mathbf{6 7 4} & \mathbf{6 7 4}\end{array}$

6 Total Direct Service Industry

-Generating Public Entities-

7 Generating Public Entities

\begin{tabular}{|c|c|c|c|c|c|c|c|c|c|c|c|c|c|}
\hline 4459 & 4459 & 4409 & 4798 & 5427 & 5944 & 6083 & 5803 & 5515 & 5073 & 5066 & 4702 & 4518 & 4516 \\
\hline 145 & 445 & 4409 & 4798 & 5427 & 5944 & 6083 & 5803 & 5515 & 5073 & 5066 & 4702 & 4518 & 4516 \\
\hline
\end{tabular}

8 Total Generating Public Entities

-Non-Generating Public Entities-

9 Non-Generating Public Entities

10 Total Non-Generating Public Entities

\begin{tabular}{lllllllllllllll}
3503 & 3503 & 3163 & 3135 & 3517 & 4034 & 4086 & 3770 & 3526 & 3360 & 3351 & 3333 & 3478 & 3596 & 3541 \\
\hline 3503 & 3503 & 3163 & 3135 & 3517 & 4034 & 4086 & 3770 & 3526 & 3360 & 3351 & 3333 & 3478 & 3596 & 3541
\end{tabular}

-Investor-Owned Entities-

11 Investor-Owned Entities

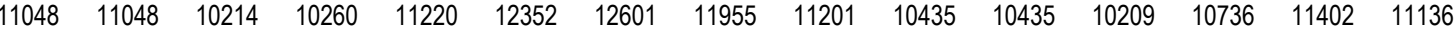

12 Total Investor-Owned Entities

$\begin{array}{lllllllllllllll}11048 & 11048 & 10214 & 10260 & 11220 & 12352 & 12601 & 11955 & 11201 & 10435 & 10435 & 10209 & 10736 & 11402 & 11136\end{array}$

-Other Firm Loads-

13 Other PNW Entities

14 Total Other Firm Loads

$180 \quad 180 \quad 180$

- Total Regional Firm Loads-

15 Federal Entities

16 Direct Service Industry

17 Public Entities

18 Investor-Owned Entities

19 Other Entities

20 Total Regional Firm Loads

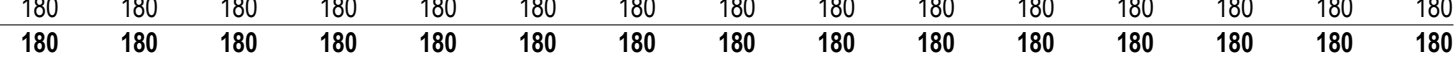

Non-Firm Loads

-Investor-Owned Entities-

21 Investor-Owned Entities

22 Total Investor-Owned Entities

- Total Regional Non-Firm Loads-

23 Federal Entities

24 Direct Service Industry

25 Public Entities

26 Investor-Owned Entities

27 Other Entities

28 Total Regional Non-Firm Loads

\begin{tabular}{|c|c|c|c|c|c|c|c|c|c|c|c|c|c|c|}
\hline 441 & 407 & 287 & 248 & 131 & 145 & 146 & 138 & 155 & 375 & 438 & 388 & 418 & 460 & \\
\hline 674 & 674 & 674 & 674 & 674 & 674 & 674 & 674 & 674 & 674 & & & & & \\
\hline 963 & 7963 & 7572 & $7 \mathrm{~s}$ & 8945 & 9978 & 0169 & 9574 & 9041 & 8433 & 8 & 8034 & 6 & 8112 & \\
\hline 048 & 11048 & 10214 & 10260 & 11220 & 12352 & 12601 & 11955 & 11201 & 10435 & 10435 & 10209 & 10736 & 11402 & \\
\hline 180 & 180 & 180 & 180 & 180 & 180 & 180 & 180 & 180 & 180 & 180 & 180 & 180 & 180 & \\
\hline & 27 & 3927 & 295 & 1149 & 3329 & 3770 & 2521 & 1250 & 0097 & 014 & 9485 & 0004 & 0827 & \\
\hline
\end{tabular}

- Total Regional Medium Loads.

29 Federal Entities

30 Direct Service Industry

31 Public Entities

32 Investor-Owned Entities

33 Other Entities

34 Total Regional Medium Loads

$\begin{array}{lllllllllllllll}0 & 0 & 0 & 0 & 0 & 0 & 0 & 0 & 0 & 0 & 0 & 0 & 0 & 0 & 0 \\ 0 & 0 & 0 & 0 & 0 & 0 & 0 & 0 & 0 & 0 & 0 & 0 & 0 & 0 & 0\end{array}$

$\begin{array}{rrrrrrrrrrrrrrrr} & 0 & 0 & 0 & 0 & 0 & 0 & 0 & 0 & 0 & 0 & 0 & 0 & 0 & 0 & 0 \\ & 0 & 0 & 0 & 0 & 0 & 0 & 0 & 0 & 0 & 0 & 0 & 0 & 0 & 0 & 0 \\ & 0 & 0 & 0 & 0 & 0 & 0 & 0 & 0 & 0 & 0 & 0 & 0 & 0 & 0 & 0 \\ & 0 & 0 & 0 & 0 & 0 & 0 & 0 & 0 & 0 & 0 & 0 & 0 & 0 & 0 & 0 \\ & 0 & 0 & 0 & 0 & 0 & 0 & 0 & 0 & 0 & 0 & 0 & 0 & 0 & 0 & 0 \\ & 0 & 0 & 0 & 0 & 0 & \mathbf{0} & \mathbf{0} & \mathbf{0} & \mathbf{0} & \mathbf{0} & \mathbf{0} & \mathbf{0} & \mathbf{0} & \mathbf{0} & \mathbf{0} \\ & & & & & & & & & & & & & & & \\ & 441 & 407 & 287 & 248 & 131 & 145 & 146 & 138 & 155 & 375 & 438 & 388 & 418 & 460 & 279 \\ & 674 & 674 & 674 & 674 & 674 & 674 & 674 & 674 & 674 & 674 & 674 & 674 & 674 & 674 & 674 \\ & 7963 & 7963 & 7572 & 7934 & 8945 & 9978 & 10169 & 9574 & 9041 & 8433 & 8418 & 8034 & 7996 & 8112 & 8645 \\ & 11048 & 11048 & 10214 & 10260 & 11220 & 12352 & 12601 & 11955 & 11201 & 10435 & 10435 & 10209 & 10736 & 11402 & 11136 \\ & 180 & 180 & 180 & 180 & 180 & 180 & 180 & 180 & 180 & 180 & 180 & 180 & 180 & 180 & 180 \\ & 20305 & 20271 & 18927 & 19295 & \mathbf{2 1 1 4 9} & \mathbf{2 3 3 2 9} & \mathbf{2 3 7 7 0} & \mathbf{2 2 5 2 1} & \mathbf{2 1 2 5 0} & \mathbf{2 0 0 9 7} & \mathbf{2 0 1 4 4} & \mathbf{1 9 4 8 5} & \mathbf{2 0 0 0 0 4} & \mathbf{2 0 8 2 7} & \mathbf{2 0 9 9 1 4}\end{array}$


Aug1 Aug16 Sep Oct Nov

Dec Jan Feb

Feb Mar Apr1 Apr16 May Jun Jul Avg

Firm Loads

-Federal Entities-

1 Federal Entities

2 Total Federal Entities

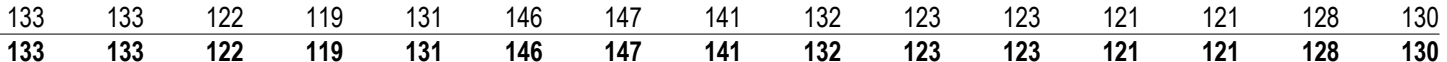

-U.S. Bureau of Reclamation-

3 U.S. Bureau of Reclamation

$\begin{array}{lllllllllllllll}309 & 275 & 166 & 129 & 0 & 0 & 0 & 1.0 & 23 & 252 & 315 & 268 & 298 & 332 & 149 \\ 309 & \mathbf{2 7 5} & \mathbf{1 6 6} & \mathbf{1 2 9} & \mathbf{0} & \mathbf{0} & \mathbf{0} & \mathbf{1 . 0} & \mathbf{2 3} & \mathbf{2 5 2} & \mathbf{3 1 5} & \mathbf{2 6 8} & \mathbf{2 9 8} & \mathbf{3 3 2} & \mathbf{1 4 9}\end{array}$

-Direct Service Industry-

5 Direct Sevice Industry

$\begin{array}{lllllllllllllll}674 & 674 & 674 & 674 & 674 & 674 & 674 & 674 & 674 & 674 & 674 & 674 & 674 & 674 & 674 \\ \mathbf{6 7 4} & \mathbf{6 7 4} & \mathbf{6 7 4} & \mathbf{6 7 4} & \mathbf{6 7 4} & \mathbf{6 7 4} & \mathbf{6 7 4} & \mathbf{6 7 4} & \mathbf{6 7 4} & \mathbf{6 7 4} & \mathbf{6 7 4} & \mathbf{6 7 4} & \mathbf{6 7 4} & \mathbf{6 7 4} & \mathbf{6 7 4}\end{array}$

6 Total Direct Service Industry

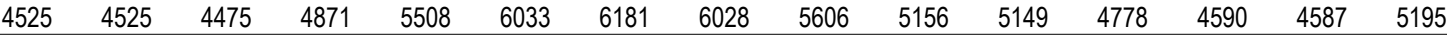

Generating Public Entities

7 Generating Public Entities

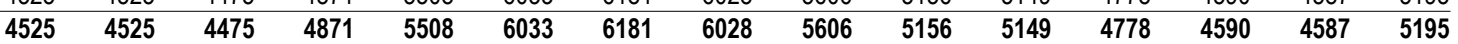

-Non-Generating Public Entities-

9 Non-Generating Public Entities

\begin{tabular}{lllllllllllllll}
3543 & 3543 & 3203 & 3174 & 3563 & 4088 & 4134 & 3938 & 3570 & 3406 & 3397 & 3376 & 3523 & 3644 & 3596 \\
\hline 3543 & $\mathbf{3 5 4 3}$ & $\mathbf{3 2 0 3}$ & $\mathbf{3 1 7 4}$ & $\mathbf{3 5 6 3}$ & $\mathbf{4 0 8 8}$ & $\mathbf{4 1 3 4}$ & $\mathbf{3 9 3 8}$ & $\mathbf{3 5 7 0}$ & $\mathbf{3 4 0 6}$ & $\mathbf{3 3 9 7}$ & $\mathbf{3 3 7 6}$ & $\mathbf{3 5 2 3}$ & $\mathbf{3 6 4 4}$ & $\mathbf{3 5 9 6}$
\end{tabular}

-Investor-Owned Entities-

11 Investor-Owned Entities

$\begin{array}{lllllllllllllll}11194 & 11194 & 10395 & 10455 & 11427 & 12568 & 12776 & 12206 & 11370 & 10589 & 10589 & 10359 & 10932 & 11590 & 11322\end{array}$

12 Total Investor-Owned Entities

$\begin{array}{lllllllllllllll}11194 & 11194 & 10395 & 10455 & 11427 & 12568 & 12776 & 12206 & 11370 & 10589 & 10589 & 10359 & 10932 & 11590 & 11322\end{array}$

-Other Firm Loads-

13 Other PNW Entities

14 Total Other Firm Loads

$180 \quad 180 \quad 180$

- Total Regional Firm Loads-

15 Federal Entities

16 Direct Service Industry

17 Public Entities

18 Investor-Owned Entities

19 Other Entities

20 Total Regional Firm Loads

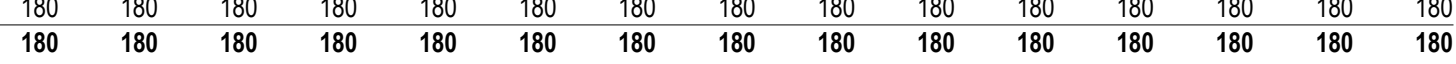

Non-Firm Loads

-Investor-Owned Entities-

21 Investor-Owned Entities

22 Total Investor-Owned Entities

- Total Regional Non-Firm Loads-

23 Federal Entities

24 Direct Service Industry

25 Public Entities

26 Investor-Owned Entities

27 Other Entities

28 Total Regional Non-Firm Loads

\begin{tabular}{|c|c|c|c|c|c|c|c|c|c|c|c|c|c|c|}
\hline 442 & 408 & 288 & 248 & 131 & 146 & 147 & 142 & 155 & 375 & 438 & 389 & 419 & 460 & 280 \\
\hline 674 & 674 & 674 & 674 & 674 & 674 & 674 & 674 & 674 & 674 & 674 & 674 & 674 & 674 & 674 \\
\hline 8068 & 8068 & 7678 & 8045 & 9071 & 10120 & 10315 & 9967 & 9177 & 8562 & 8546 & 8154 & 8113 & 8231 & \\
\hline 180 & 180 & 180 & 180 & 180 & 180 & 180 & 180 & 180 & 180 & 180 & 180 & 180 & 180 & \\
\hline 50 & 20524 & 19215 & 9602 & 21484 & 888 & 24092 & 23169 & 21556 & 20381 & 20427 & 19755 & 0318 & 21135 & 212 \\
\hline
\end{tabular}

- Total Regional Medium Loads.

29 Federal Entities

30 Direct Service Industry

31 Public Entities

32 Investor-Owned Entities

33 Other Entities

34 Total Regional Medium Loads

$\begin{array}{lllllllllllllll}0 & 0 & 0 & 0 & 0 & 0 & 0 & 0 & 0 & 0 & 0 & 0 & 0 & 0 & 0 \\ 0 & 0 & 0 & 0 & 0 & 0 & 0 & 0 & 0 & 0 & 0 & 0 & 0 & 0 & 0\end{array}$

\begin{tabular}{|c|c|c|c|c|c|c|c|c|c|c|c|c|c|c|}
\hline 0 & 0 & 0 & 0 & 0 & 0 & 0 & 0 & 0 & 0 & 0 & 0 & 0 & 0 & 0 \\
\hline 0 & 0 & 0 & 0 & 0 & 0 & 0 & 0 & 0 & 0 & 0 & 0 & 0 & 0 & 0 \\
\hline 0 & 0 & 0 & 0 & 0 & 0 & 0 & 0 & 0 & 0 & 0 & 0 & 0 & 0 & 0 \\
\hline 0 & 0 & 0 & 0 & 0 & 0 & 0 & 0 & 0 & 0 & 0 & 0 & 0 & 0 & . \\
\hline 0 & 0 & 0 & 0 & 0 & 0 & 0 & 0 & 0 & 0 & 0 & 0 & 0 & 0 & \\
\hline 0 & 0 & 0 & 0 & 0 & 0 & 0 & 0 & 0 & 0 & 0 & 0 & 0 & 0 & \\
\hline 442 & 408 & 288 & 248 & 131 & 146 & 147 & 142 & 155 & 375 & 438 & 389 & 419 & 460 & 280 \\
\hline 674 & 674 & 674 & 674 & 674 & 674 & 674 & 674 & 674 & 674 & 674 & 674 & 674 & 674 & 674 \\
\hline 8068 & 8068 & 7678 & 8045 & 9071 & 10120 & 10315 & 9967 & 9177 & 8562 & 8546 & 8154 & 8113 & 8231 & 8791 \\
\hline 11194 & 11194 & 10395 & 10455 & 11427 & 12568 & 12776 & 12206 & 11370 & 10589 & 10589 & 10359 & 10932 & 11590 & 11322 \\
\hline 180 & 180 & 180 & 180 & 180 & 180 & 180 & 180 & 180 & 180 & 180 & 180 & 180 & 180 & 180 \\
\hline 20558 & 20524 & 19215 & 19602 & 21484 & 23688 & 24092 & 23169 & 21556 & 20381 & 20427 & 19755 & 20318 & 21135 & 21246 \\
\hline
\end{tabular}


Aug1 Aug16 Sep 2003 White Book

Firm Loads

-Federal Entities-

1 Federal Entities

2 Total Federal Entities

\begin{tabular}{|c|c|c|c|c|c|c|c|c|c|c|c|c|}
\hline 134 & 122 & 119 & 132 & 147 & 147 & 141 & 133 & 124 & 124 & 121 & 121 & 129 \\
\hline 134 & 122 & 119 & 132 & 147 & 147 & 141 & 133 & 124 & 124 & 121 & 121 & 129 \\
\hline
\end{tabular}

-U.S. Bureau of Reclamation-

3 U.S. Bureau of Reclamation

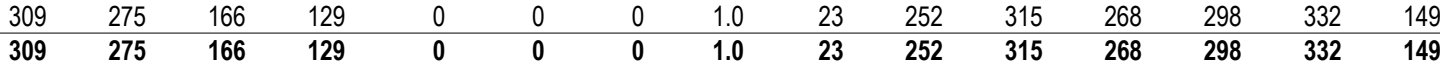

-Direct Service Industry-

5 Direct Sevice Industry

$\begin{array}{lllllllllllllll}674 & 674 & 674 & 674 & 674 & 674 & 674 & 674 & 674 & 674 & 674 & 674 & 674 & 674 & 674 \\ \mathbf{6 7 4} & \mathbf{6 7 4} & \mathbf{6 7 4} & \mathbf{6 7 4} & \mathbf{6 7 4} & \mathbf{6 7 4} & \mathbf{6 7 4} & \mathbf{6 7 4} & \mathbf{6 7 4} & \mathbf{6 7 4} & \mathbf{6 7 4} & \mathbf{6 7 4} & \mathbf{6 7 4} & \mathbf{6 7 4} & \mathbf{6 7 4}\end{array}$

6 Total Direct Service Industry

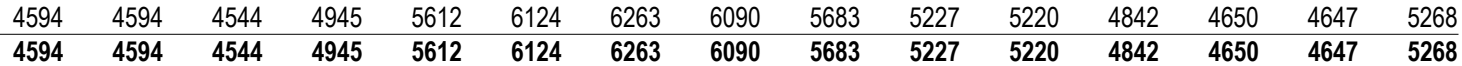

-Generating Public Entities.

7 Generating Public Entities

-Non-Generating Public Entities-

9 Non-Generating Public Entities

\begin{tabular}{lllllllllllllll}
3587 & 3587 & 3244 & 3216 & 3612 & 4145 & 4193 & 3993 & 3620 & 3454 & 3444 & 3424 & 3570 & 3696 & 3646 \\
\hline 3587 & 3587 & 3244 & 3216 & $\mathbf{3 6 1 2}$ & $\mathbf{4 1 4 5}$ & $\mathbf{4 1 9 3}$ & $\mathbf{3 9 9 3}$ & $\mathbf{3 6 2 0}$ & $\mathbf{3 4 5 4}$ & $\mathbf{3 4 4 4}$ & $\mathbf{3 4 2 4}$ & $\mathbf{3 5 7 0}$ & $\mathbf{3 6 9 6}$ & $\mathbf{3 6 4 6}$
\end{tabular}

-Investor-Owned Entities-

11 Investor-Owned Entities

\begin{tabular}{lllllllllllllll}
11383 & 11383 & 10569 & 10609 & 11619 & 12750 & 12976 & 12401 & 11568 & 10765 & 10765 & 10541 & 11131 & 11785 & 11508 \\
\hline
\end{tabular}

12 Total Investor-Owned Entities

$\begin{array}{lllllllllllllll}11383 & 11383 & 10569 & 10609 & 11619 & 12750 & 12976 & 12401 & 11568 & 10765 & 10765 & 10541 & 11131 & 11785 & 11508\end{array}$

-Other Firm Loads-

13 Other PNW Entities

14 Total Other Firm Loads

$180 \quad 180 \quad 180$

- Total Regional Firm Loads-

15 Federal Entities

16 Direct Service Industry

17 Public Entities

18 Investor-Owned Entities

19 Other Entities

20 Total Regional Firm Loads

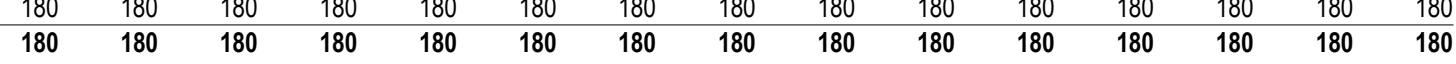

Non-Firm Loads

-Investor-Owned Entities-

21 Investor-Owned Entities

22 Total Investor-Owned Entities

- Total Regional Non-Firm Loads-

23 Federal Entities

24 Direct Service Industry

25 Public Entities

26 Investor-Owned Entities

27 Other Entities

28 Total Regional Non-Firm Loads

$\begin{array}{rrrrrrrrrrrrrrr}443 & 409 & 288 & 248 & 132 & 147 & 147 & 142 & 156 & 376 & 439 & 389 & 419 & 461 & 280 \\ 674 & 674 & 674 & 674 & 674 & 674 & 674 & 674 & 674 & 674 & 674 & 674 & 674 & 674 & 674 \\ 8181 & 8181 & 7787 & 8160 & 9224 & 10269 & 10456 & 10084 & 9303 & 8681 & 8664 & 8266 & 8220 & 8342 & 8914 \\ 1383 & 11383 & 10569 & 10609 & 11619 & 12750 & 12976 & 12401 & 11568 & 10765 & 10765 & 10541 & 11131 & 11785 & 11508 \\ 180 & 180 & 180 & 180 & 180 & 180 & 180 & 180 & 180 & 180 & 180 & 180 & 180 & 180 & 180 \\ \mathbf{2 0 8 6 0} & \mathbf{2 0 8 2 6} & \mathbf{1 9 4 9 8} & \mathbf{1 9 8 7 2} & \mathbf{2 1 8 2 8} & \mathbf{2 4 0 2 0} & \mathbf{2 4 4 3 3} & \mathbf{2 3 4 8 1} & \mathbf{2 1 8 8 0} & \mathbf{2 0 6 7 6} & \mathbf{2 0 7 2 2} & \mathbf{2 0 0 5 1} & \mathbf{2 0 6 2 5} & \mathbf{2 1 4 4 3} & \mathbf{2 1 5 5 6}\end{array}$

- Total Regional Medium Loads-

29 Federal Entities

30 Direct Service Industry

31 Public Entities

32 Investor-Owned Entities

33 Other Entities

34 Total Regional Medium Loads

208

\begin{tabular}{|c|c|c|c|c|c|c|c|c|c|c|c|c|c|c|}
\hline 0 & 0 & 0 & 0 & 0 & 0 & 0 & 0 & 0 & 0 & 0 & 0 & 0 & 0 & 0 \\
\hline 0 & 0 & 0 & 0 & 0 & 0 & 0 & 0 & 0 & 0 & 0 & 0 & 0 & 0 & 0 \\
\hline 0 & 0 & 0 & 0 & 0 & 0 & 0 & 0 & 0 & 0 & 0 & 0 & 0 & 0 & 0 \\
\hline 0 & 0 & 0 & 0 & 0 & 0 & 0 & 0 & 0 & 0 & 0 & 0 & 0 & 0 & 0 \\
\hline 0 & 0 & 0 & 0 & 0 & 0 & 0 & 0 & 0 & 0 & 0 & 0 & 0 & 0 & 0 \\
\hline 0 & 0 & 0 & 0 & 0 & 0 & 0 & 0 & 0 & 0 & 0 & 0 & 0 & 0 & 0 \\
\hline 443 & 409 & 288 & 248 & 132 & 147 & 147 & 142 & 156 & 376 & 439 & 389 & 419 & 461 & 280 \\
\hline 674 & 674 & 674 & 674 & 674 & 674 & 674 & 674 & 674 & 674 & 674 & 674 & 674 & 674 & 674 \\
\hline 8181 & 8181 & 7787 & 8160 & 9224 & 10269 & 10456 & 10084 & 9303 & 8681 & 8664 & 8266 & 8220 & 8342 & 8914 \\
\hline 11383 & 11383 & 10569 & 10609 & 11619 & 12750 & 12976 & 12401 & 11568 & 10765 & 10765 & 10541 & 11131 & 11785 & 11508 \\
\hline 180 & 180 & 180 & 180 & 180 & 180 & 180 & 180 & 180 & 180 & 180 & 180 & 180 & 180 & 180 \\
\hline 20860 & 20826 & 19498 & 19872 & 21828 & 24020 & 24433 & 23481 & 21880 & 20676 & 20722 & 20051 & 20625 & 21443 & 21556 \\
\hline
\end{tabular}


Aug1 Aug16 Sep Oct Nov 2003 White Book

Firm Loads

-Federal Entities-

1 Federal Entities

2 Total Federal Entities

\begin{tabular}{|c|c|c|c|c|c|c|c|c|c|c|c|c|}
\hline 134 & 123 & 120 & 132 & 147 & 148 & 142 & 133 & 124 & 124 & 122 & 122 & 130 \\
\hline 134 & 123 & 120 & 132 & 147 & 148 & 142 & 133 & 124 & 124 & 122 & 122 & 130 \\
\hline
\end{tabular}

-U.S. Bureau of Reclamation-

3 U.S. Bureau of Reclamation

$\begin{array}{lllllllllllllll}309 & 275 & 166 & 129 & 0 & 0 & 0 & 1.0 & 23 & 252 & 315 & 268 & 298 & 332 & 149 \\ \mathbf{3 0 9} & \mathbf{2 7 5} & \mathbf{1 6 6} & \mathbf{1 2 9} & \mathbf{0} & \mathbf{0} & \mathbf{0} & \mathbf{1 . 0} & \mathbf{2 3} & \mathbf{2 5 2} & \mathbf{3 1 5} & \mathbf{2 6 8} & \mathbf{2 9 8} & \mathbf{3 3 2} & \mathbf{1 4 9}\end{array}$

-Direct Service Industry-

5 Direct Sevice Industry

$\begin{array}{lllllllllllllll}674 & 674 & 674 & 674 & 674 & 674 & 674 & 674 & 674 & 674 & 674 & 674 & 674 & 674 & 674 \\ \mathbf{6 7 4} & \mathbf{6 7 4} & \mathbf{6 7 4} & \mathbf{6 7 4} & \mathbf{6 7 4} & \mathbf{6 7 4} & \mathbf{6 7 4} & \mathbf{6 7 4} & \mathbf{6 7 4} & \mathbf{6 7 4} & \mathbf{6 7 4} & \mathbf{6 7 4} & \mathbf{6 7 4} & \mathbf{6 7 4} & \mathbf{6 7 4}\end{array}$

6 Total Direct Service Industry

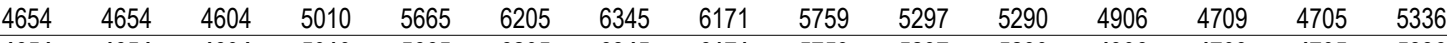

Generating Public Entities.

7 Generating Public Entities

$\begin{array}{lllllllllllllll}4654 & 4654 & 4604 & 5010 & 5665 & 6205 & 6345 & 6171 & 5759 & 5297 & 5290 & 4906 & 4709 & 4705 & 5336\end{array}$

-Non-Generating Public Entities-

9 Non-Generating Public Entities

10 Total Non-Generating Public Entities

\begin{tabular}{lllllllllllllll}
3635 & 3635 & 3287 & 3258 & 3663 & 4200 & 4247 & 4044 & 3662 & 3497 & 3487 & 3469 & 3613 & 3743 & 3693 \\
\hline 3635 & $\mathbf{3 6 3 5}$ & $\mathbf{3 2 8 7}$ & $\mathbf{3 2 5 8}$ & $\mathbf{3 6 6 3}$ & $\mathbf{4 2 0 0}$ & $\mathbf{4 2 4 7}$ & $\mathbf{4 0 4 4}$ & $\mathbf{3 6 6 2}$ & $\mathbf{3 4 9 7}$ & $\mathbf{3 4 8 7}$ & $\mathbf{3 4 6 9}$ & $\mathbf{3 6 1 3}$ & $\mathbf{3 7 4 3}$ & $\mathbf{3 6 9 3}$
\end{tabular}

-Investor-Owned Entities-

11 Investor-Owned Entities

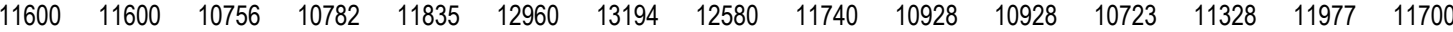

12 Total Investor-Owned Entities

$\begin{array}{lllllllllllllll}11600 & 11600 & 10756 & 10782 & 11835 & 12960 & 13194 & 12580 & 11740 & 10928 & 10928 & 10723 & 11328 & 11977 & 11700\end{array}$

-Other Firm Loads-

13 Other PNW Entities

14 Total Other Firm Loads

$180 \quad 180 \quad 180$

- Total Regional Firm Loads-

15 Federal Entities

16 Direct Service Industry

17 Public Entities

18 Investor-Owned Entities

19 Other Entities

20 Total Regional Firm Loads

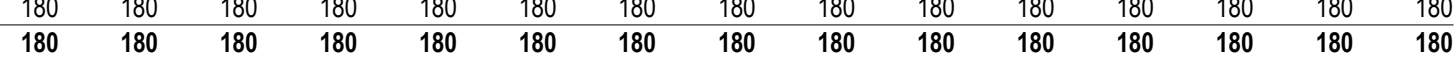

Non-Firm Loads

-Investor-Owned Entities-

21 Investor-Owned Entities

22 Total Investor-Owned Entities

- Total Regional Non-Firm Loads-

23 Federal Entities

24 Direct Service Industry

25 Public Entities

26 Investor-Owned Entities

27 Other Entities

28 Total Regional Non-Firm Loads

\begin{tabular}{|c|c|c|c|c|c|c|c|c|c|c|c|c|c|}
\hline 409 & 289 & 249 & 132 & 147 & 148 & 143 & 156 & 376 & 439 & 390 & 420 & 462 & \\
\hline 674 & 674 & 674 & 674 & 674 & 674 & 674 & 674 & 674 & 64 & $6 /$ & 674 & 674 & \\
\hline 8289 & 7891 & 8268 & 9329 & 10405 & 10591 & 10215 & 9421 & 8794 & 8777 & 6 & 8322 & 8449 & \\
\hline 11600 & 10756 & 10782 & 11835 & 12960 & 13194 & 12580 & 11740 & 10928 & 10928 & 10723 & 11328 & 11977 & \\
\hline 180 & 180 & 180 & 180 & 180 & 180 & 180 & 180 & 180 & 180 & 180 & 180 & 180 & \\
\hline 152 & 19790 & 20154 & 22150 & 24367 & 24787 & 23793 & 22172 & 0952 & 0998 & 0343 & 0923 & 174 & \\
\hline
\end{tabular}

- Total Regional Medium Loads.

29 Federal Entities

30 Direct Service Industry

31 Public Entities

32 Investor-Owned Entities

33 Other Entities

34 Total Regional Medium Loads

21186

\begin{tabular}{|c|c|c|c|c|c|c|c|c|c|c|c|c|c|c|}
\hline 0 & 0 & 0 & 0 & 0 & 0 & 0 & 0 & 0 & 0 & 0 & 0 & 0 & 0 & 0 \\
\hline 0 & 0 & 0 & 0 & 0 & 0 & 0 & 0 & 0 & 0 & 0 & 0 & 0 & 0 & 0 \\
\hline 0 & 0 & 0 & 0 & 0 & 0 & 0 & 0 & 0 & 0 & 0 & 0 & 0 & 0 & 0 \\
\hline 0 & 0 & 0 & 0 & 0 & 0 & 0 & 0 & 0 & 0 & 0 & 0 & 0 & 0 & 0 \\
\hline 0 & 0 & 0 & 0 & 0 & 0 & 0 & 0 & 0 & 0 & 0 & 0 & 0 & 0 & 0 \\
\hline 0 & 0 & 0 & 0 & 0 & 0 & 0 & 0 & 0 & 0 & 0 & 0 & 0 & 0 & 0 \\
\hline 443 & 409 & 289 & 249 & 132 & 147 & 148 & 143 & 156 & 376 & 439 & 390 & 420 & 462 & 281 \\
\hline 674 & 674 & 674 & 674 & 674 & 674 & 674 & 674 & 674 & 674 & 674 & 674 & 674 & 674 & 674 \\
\hline 8289 & 8289 & 7891 & 8268 & 9329 & 10405 & 10591 & 10215 & 9421 & 8794 & 8777 & 8376 & 8322 & 8449 & 9028 \\
\hline 11600 & 11600 & 10756 & 10782 & 11835 & 12960 & 13194 & 12580 & 11740 & 10928 & 10928 & 10723 & 11328 & 11977 & 11700 \\
\hline 180 & 180 & 180 & 180 & 180 & 180 & 180 & 180 & 180 & 180 & 180 & 180 & 180 & 180 & 180 \\
\hline 21186 & 21152 & 19790 & 20154 & 22150 & 24367 & 24787 & 23793 & 22172 & 20952 & 20998 & 20343 & 20923 & 21741 & 21864 \\
\hline
\end{tabular}


Aug1 Aug16 Sep Oct Nov 2003 White Book

Firm Loads

-Federal Entities-

1 Federal Entities

2 Total Federal Entities

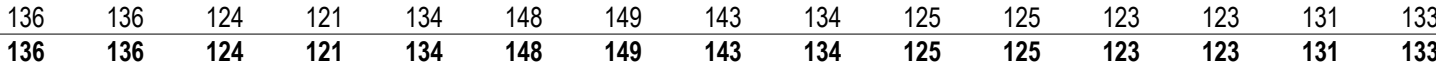

-U.S. Bureau of Reclamation-

3 U.S. Bureau of Reclamation

$\begin{array}{lllllllllllllll}309 & 275 & 166 & 129 & 0 & 0 & 0 & 1.0 & 23 & 252 & 315 & 268 & 298 & 332 & 149 \\ \mathbf{3 0 9} & \mathbf{2 7 5} & \mathbf{1 6 6} & \mathbf{1 2 9} & \mathbf{0} & \mathbf{0} & \mathbf{0} & \mathbf{1 . 0} & \mathbf{2 3} & \mathbf{2 5 2} & \mathbf{3 1 5} & \mathbf{2 6 8} & \mathbf{2 9 8} & \mathbf{3 3 2} & \mathbf{1 4 9}\end{array}$

-Direct Service Industry-

5 Direct Sevice Industry

$\begin{array}{lllllllllllllll}674 & 674 & 674 & 674 & 674 & 674 & 674 & 674 & 674 & 674 & 674 & 674 & 674 & 674 & 674 \\ \mathbf{6 7 4} & \mathbf{6 7 4} & \mathbf{6 7 4} & \mathbf{6 7 4} & \mathbf{6 7 4} & \mathbf{6 7 4} & \mathbf{6 7 4} & \mathbf{6 7 4} & \mathbf{6 7 4} & \mathbf{6 7 4} & \mathbf{6 7 4} & \mathbf{6 7 4} & \mathbf{6 7 4} & \mathbf{6 7 4} & \mathbf{6 7 4}\end{array}$

6 Total Direct Service Industry

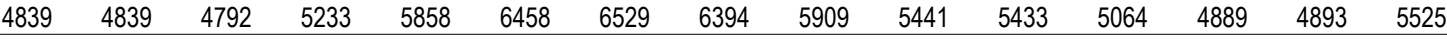

Generating Public Entities.

7 Generating Public Entities

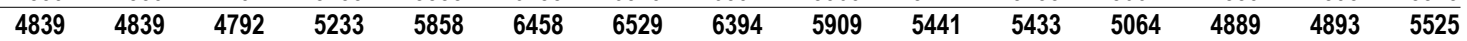

-Non-Generating Public Entities-

9 Non-Generating Public Entities

$\begin{array}{lllllllllllllll}3733 & 3733 & 3371 & 3349 & 3743 & 4313 & 4303 & 4133 & 3728 & 3539 & 3530 & 3528 & 3701 & 3836 & 3773 \\ \mathbf{3 7 3 3} & \mathbf{3 7 3 3} & \mathbf{3 3 7 1} & \mathbf{3 3 4 9} & \mathbf{3 7 4 3} & \mathbf{4 3 1 3} & \mathbf{4 3 0 3} & \mathbf{4 1 3 3} & \mathbf{3 7 2 8} & \mathbf{3 5 3 9} & \mathbf{3 5 3 0} & \mathbf{3 5 2 8} & \mathbf{3 7 0 1} & \mathbf{3 8 3 6} & \mathbf{3 7 7 3}\end{array}$

-Investor-Owned Entities-

11 Investor-Owned Entities

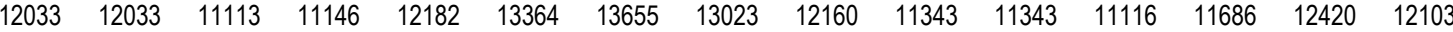

12 Total Investor-Owned Entities

$\begin{array}{lllllllllllllll}12033 & 12033 & 11113 & 11146 & 12182 & 13364 & 13655 & 13023 & 12160 & 11343 & 11343 & 11116 & 11686 & 12420 & 12103\end{array}$

-Other Firm Loads-

13 Other PNW Entities

14 Total Other Firm Loads

$180 \quad 180 \quad 180$

- Total Regional Firm Loads-

15 Federal Entities

16 Direct Service Industry

17 Public Entities

18 Investor-Owned Entities

19 Other Entities

20 Total Regional Firm Loads

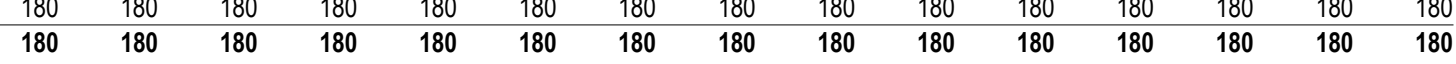

Non-Firm Loads

-Investor-Owned Entities-

21 Investor-Owned Entities

22 Total Investor-Owned Entities

- Total Regional Non-Firm Loads-

23 Federal Entities

24 Direct Service Industry

25 Public Entities

26 Investor-Owned Entities

27 Other Entities

28 Total Regional Non-Firm Loads

$\begin{array}{rrrrrrrrrrrrrrr}445 & 411 & 290 & 250 & 134 & 148 & 149 & 144 & 157 & 377 & 440 & 391 & 421 & 463 & 282 \\ 674 & 674 & 674 & 674 & 674 & 674 & 674 & 674 & 674 & 674 & 674 & 674 & 674 & 674 & 674 \\ 8572 & 8572 & 8164 & 8582 & 9601 & 10771 & 10833 & 10527 & 9637 & 8980 & 8963 & 8592 & 8590 & 8729 & 9298 \\ 2033 & 12033 & 11113 & 11146 & 12182 & 13364 & 13655 & 13023 & 12160 & 11343 & 11343 & 11116 & 11686 & 12420 & 12103 \\ 180 & 180 & 180 & 180 & 180 & 180 & 180 & 180 & 180 & 180 & 180 & 180 & 180 & 180 & 180 \\ 21904 & \mathbf{2 1 8 7 0} & \mathbf{2 0 4 2 0} & \mathbf{2 0 8 3 2} & \mathbf{2 2 7 7 1} & \mathbf{2 5 1 3 6} & \mathbf{2 5 4 9 0} & \mathbf{2 4 5 4 8} & \mathbf{2 2 8 0 8} & \mathbf{2 1 5 5 4} & \mathbf{2 1 6 0 0} & \mathbf{2 0 9 5 3} & \mathbf{2 1 5 5 1} & \mathbf{2 2 4 6 6} & \mathbf{2 2 5 3 7}\end{array}$

- Total Regional Medium Loads.

29 Federal Entities

30 Direct Service Industry

31 Public Entities

32 Investor-Owned Entities

33 Other Entities

34 Total Regional Medium Loads

21904

\begin{tabular}{|c|c|c|c|c|c|c|c|c|c|c|c|c|c|c|}
\hline 0 & 0 & 0 & 0 & 0 & 0 & 0 & 0 & 0 & 0 & 0 & 0 & 0 & 0 & 0 \\
\hline 0 & 0 & 0 & 0 & 0 & 0 & 0 & 0 & 0 & 0 & 0 & 0 & 0 & 0 & 0 \\
\hline 0 & 0 & 0 & 0 & 0 & 0 & 0 & 0 & 0 & 0 & 0 & 0 & 0 & 0 & 0 \\
\hline 0 & 0 & 0 & 0 & 0 & 0 & 0 & 0 & 0 & 0 & 0 & 0 & 0 & 0 & 0 \\
\hline 0 & 0 & 0 & 0 & 0 & 0 & 0 & 0 & 0 & 0 & 0 & 0 & 0 & 0 & 0 \\
\hline 0 & 0 & 0 & 0 & 0 & 0 & 0 & 0 & 0 & 0 & 0 & 0 & 0 & 0 & 0 \\
\hline 445 & 411 & 290 & 250 & 134 & 148 & 149 & 144 & 157 & 377 & 440 & 391 & 421 & 463 & 282 \\
\hline 674 & 674 & 674 & 674 & 674 & 674 & 674 & 674 & 674 & 674 & 674 & 674 & 674 & 674 & 674 \\
\hline 8572 & 8572 & 8164 & 8582 & 9601 & 10771 & 10833 & 10527 & 9637 & 8980 & 8963 & 8592 & 8590 & 8729 & 9298 \\
\hline 12033 & 12033 & 11113 & 11146 & 12182 & 13364 & 13655 & 13023 & 12160 & 11343 & 11343 & 11116 & 11686 & 12420 & 12103 \\
\hline 180 & 180 & 180 & 180 & 180 & 180 & 180 & 180 & 180 & 180 & 180 & 180 & 180 & 180 & 180 \\
\hline 21904 & 21870 & 20420 & 20832 & 22771 & 25136 & 25490 & 24548 & 22808 & 21554 & 21600 & 20953 & 21551 & 22466 & 22537 \\
\hline
\end{tabular}


Aug1 Aug16 Sep Oct Nov

Dec Jan Feb

Feb Mar Apr1 Apr16 May Jun Jul Avg

Firm Loads

-Federal Entities-

1 Federal Entities

2 Total Federal Entities

\begin{tabular}{|c|c|c|c|c|c|c|c|c|c|c|c|c|c|}
\hline 136 & 125 & 122 & 134 & 149 & 150 & 144 & 135 & 126 & 125 & 123 & 123 & 132 & 133 \\
\hline 136 & 125 & 122 & 134 & 149 & 150 & 144 & 135 & 126 & 125 & 123 & 123 & 132 & 33 \\
\hline
\end{tabular}

-U.S. Bureau of Reclamation-

3 U.S. Bureau of Reclamation

$\begin{array}{lllllllllllllll}309 & 275 & 166 & 129 & 0 & 0 & 0 & 1.0 & 23 & 252 & 315 & 268 & 298 & 332 & 149 \\ \mathbf{3 0 9} & \mathbf{2 7 5} & \mathbf{1 6 6} & \mathbf{1 2 9} & \mathbf{0} & \mathbf{0} & \mathbf{0} & \mathbf{1 . 0} & \mathbf{2 3} & \mathbf{2 5 2} & \mathbf{3 1 5} & \mathbf{2 6 8} & \mathbf{2 9 8} & \mathbf{3 3 2} & \mathbf{1 4 9}\end{array}$

-Direct Service Industry-

5 Direct Sevice Industry

$\begin{array}{lllllllllllllll}674 & 674 & 674 & 674 & 674 & 674 & 674 & 674 & 674 & 674 & 674 & 674 & 674 & 674 & 674 \\ \mathbf{6 7 4} & \mathbf{6 7 4} & \mathbf{6 7 4} & \mathbf{6 7 4} & \mathbf{6 7 4} & \mathbf{6 7 4} & \mathbf{6 7 4} & \mathbf{6 7 4} & \mathbf{6 7 4} & \mathbf{6 7 4} & \mathbf{6 7 4} & \mathbf{6 7 4} & \mathbf{6 7 4} & \mathbf{6 7 4} & \mathbf{6 7 4}\end{array}$

6 Total Direct Service Industry

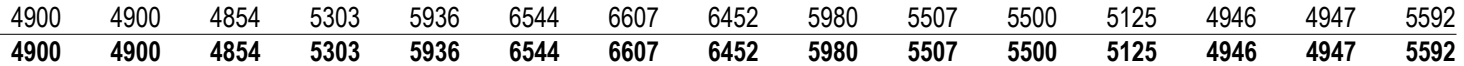

-Generating Public Entities.

7 Generating Public Entities

-Non-Generating Public Entities-

9 Non-Generating Public Entities

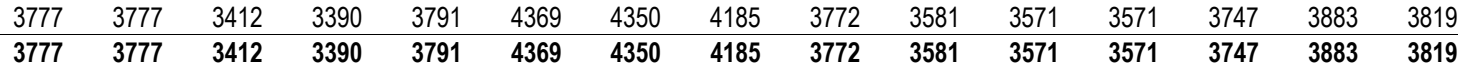

-Investor-Owned Entities-

11 Investor-Owned Entities

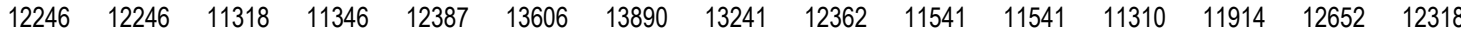

12 Total Investor-Owned Entities

$\begin{array}{lllllllllllllll}12246 & 12246 & 11318 & 11346 & 12387 & 13606 & 13890 & 13241 & 12362 & 11541 & 11541 & 11310 & 11914 & 12652 & 12318\end{array}$

-Other Firm Loads-

13 Other PNW Entities

14 Total Other Firm Loads

$180 \quad 180 \quad 180$

- Total Regional Firm Loads-

15 Federal Entities

16 Direct Service Industry

17 Public Entities

18 Investor-Owned Entities

19 Other Entities

20 Total Regional Firm Loads

180

Non-Firm Loads

-Investor-Owned Entities-

21 Investor-Owned Entities

22 Total Investor-Owned Entities

- Total Regional Non-Firm Loads-

23 Federal Entities

24 Direct Service Industry

25 Public Entities

26 Investor-Owned Entities

27 Other Entities

28 Total Regional Non-Firm Loads

\begin{tabular}{|c|c|c|c|c|c|c|c|c|c|c|c|c|c|}
\hline 411 & 291 & 251 & 134 & 149 & 150 & 145 & 158 & 378 & 440 & 391 & 421 & 464 & \\
\hline 674 & 674 & 674 & 674 & 674 & 674 & 674 & 674 & 67 & 674 & $b_{1}$ & 674 & 674 & \\
\hline 8678 & 8266 & 8693 & 9727 & 10913 & 10957 & 10637 & 9752 & 9088 & 9071 & 8697 & 8693 & 8830 & \\
\hline 12246 & 11318 & 11346 & 12387 & 13606 & 13890 & 13241 & 12362 & 11541 & 11541 & 11310 & 11914 & 12652 & \\
\hline 180 & 180 & 180 & 180 & 180 & 180 & 180 & 180 & 180 & 180 & 180 & 180 & 0 & \\
\hline $18 \mathrm{~s}$ & 072 & 21143 & 23102 & 25521 & 25850 & 24877 & 23126 & 1861 & 1906 & 21252 & 1882 & & \\
\hline
\end{tabular}

- Total Regional Medium Loads.

29 Federal Entities

30 Direct Service Industry

31 Public Entities

32 Investor-Owned Entities

33 Other Entities

34 Total Regional Medium Loads

$\begin{array}{lllllllllllllll}0 & 0 & 0 & 0 & 0 & 0 & 0 & 0 & 0 & 0 & 0 & 0 & 0 & 0 & 0 \\ 0 & 0 & 0 & 0 & 0 & 0 & 0 & 0 & 0 & 0 & 0 & 0 & 0 & 0 & 0\end{array}$

\begin{tabular}{|c|c|c|c|c|c|c|c|c|c|c|c|c|c|c|}
\hline 0 & 0 & 0 & 0 & 0 & 0 & 0 & 0 & 0 & 0 & 0 & 0 & 0 & 0 & 0 \\
\hline 0 & 0 & 0 & 0 & 0 & 0 & 0 & 0 & 0 & 0 & 0 & 0 & 0 & 0 & 0 \\
\hline 0 & 0 & 0 & 0 & 0 & 0 & 0 & 0 & 0 & 0 & 0 & 0 & 0 & 0 & 0 \\
\hline 0 & 0 & 0 & 0 & 0 & 0 & 0 & 0 & 0 & 0 & 0 & 0 & 0 & 0 & 0 \\
\hline 0 & 0 & 0 & 0 & 0 & 0 & 0 & 0 & 0 & 0 & 0 & 0 & 0 & 0 & 0 \\
\hline 0 & 0 & 0 & 0 & 0 & 0 & 0 & 0 & 0 & 0 & 0 & 0 & 0 & 0 & \\
\hline 445 & 411 & 291 & 251 & 134 & 149 & 150 & 145 & 158 & 378 & 440 & 391 & 421 & 464 & 283 \\
\hline 674 & 674 & 674 & 674 & 674 & 674 & 674 & 674 & 674 & 674 & 674 & 674 & 674 & 674 & 674 \\
\hline 8678 & 8678 & 8266 & 8693 & 9727 & 10913 & 10957 & 10637 & 9752 & 9088 & 9071 & 8697 & 8693 & 8830 & 9410 \\
\hline 12246 & 12246 & 11318 & 11346 & 12387 & 13606 & 13890 & 13241 & 12362 & 11541 & 11541 & 11310 & 11914 & 12652 & 12318 \\
\hline 180 & 180 & 180 & 180 & 180 & 180 & 180 & 180 & 180 & 180 & 180 & 180 & 180 & 0 & 165 \\
\hline 22223 & 22189 & 20729 & 21143 & 23102 & 25521 & 25850 & 24877 & 23126 & 21861 & 21906 & 21252 & 21882 & 22620 & 22849 \\
\hline
\end{tabular}


Table A-2: Regional Exports

PNW Loads and Resources Study

2004 - 2005 Operating Year 2003 White Book

Aug1 Aug16 Sep Oct Nov Dec Jan Feb Mar Apr1 Apr16 May Jun Jul Avg

-Exports East of Continental Divide -

1 AVWP to PPLM PwrS

2 BPA to Other Entities

3 Total Exports to ECD

$\begin{array}{rrrrrrrrrrrrrrr}9 & 9 & 9 & 9 & 9 & 9 & 9 & 9 & 9 & 9 & 9 & 9 & 9 & 9 & 9 \\ 67 & 67 & 61 & 66 & 82 & 97 & 95 & 91 & 84 & 78 & 78 & 65 & 70 & 76 & 78 \\ 76 & 76 & 70 & 75 & \mathbf{9 2} & \mathbf{1 0 6} & \mathbf{1 0 4} & \mathbf{1 0 0} & \mathbf{9 3} & \mathbf{8 7} & \mathbf{8 6} & \mathbf{7 4} & \mathbf{7 9} & \mathbf{8 6} & \mathbf{8 7}\end{array}$

-Exports to Pacific Southwest-

4 BPA to ANHM PwrX

5 BPA to BART PwrS

$6 \mathrm{BPA}$ to BURB $\mathrm{C} / \mathrm{N} / \mathrm{X}$

7 BPA to GLEN C/N/X

8 BPA to MSR PwrS

9 BPA to Other Entities

10 BPA to PASA C/N/X

11 BPA to PASA C/N/X

12 BPA to PASA S/N/X

13 BPA to RVSD CapS

$14 \mathrm{BPA}$ to RVSD C/N/X

$15 \mathrm{BPA}$ to RVSD $\mathrm{C} / \mathrm{N} / \mathrm{X}$

16 BPA to RVSD S/Pwr/X

17 IPC to CLTN PwrS

18 PGE to GLEN PwrS

19 PGE to GLEN S/Pwr/X

20 PPL to CDWR PwrS

$21 \mathrm{PPL}$ to SCE PwrS

22 PPL to SMUD PwrS

23 PSE to PGS S/Pwr/X

24 SCL to NCPA S/Pwr/X

25 SDGE to SDGE PwrS

26 SHPD to SMUD PwrS

27 TID to TID PwrS

28 BPA NW-SW Intertie Losses

29 Total Exports To PSW

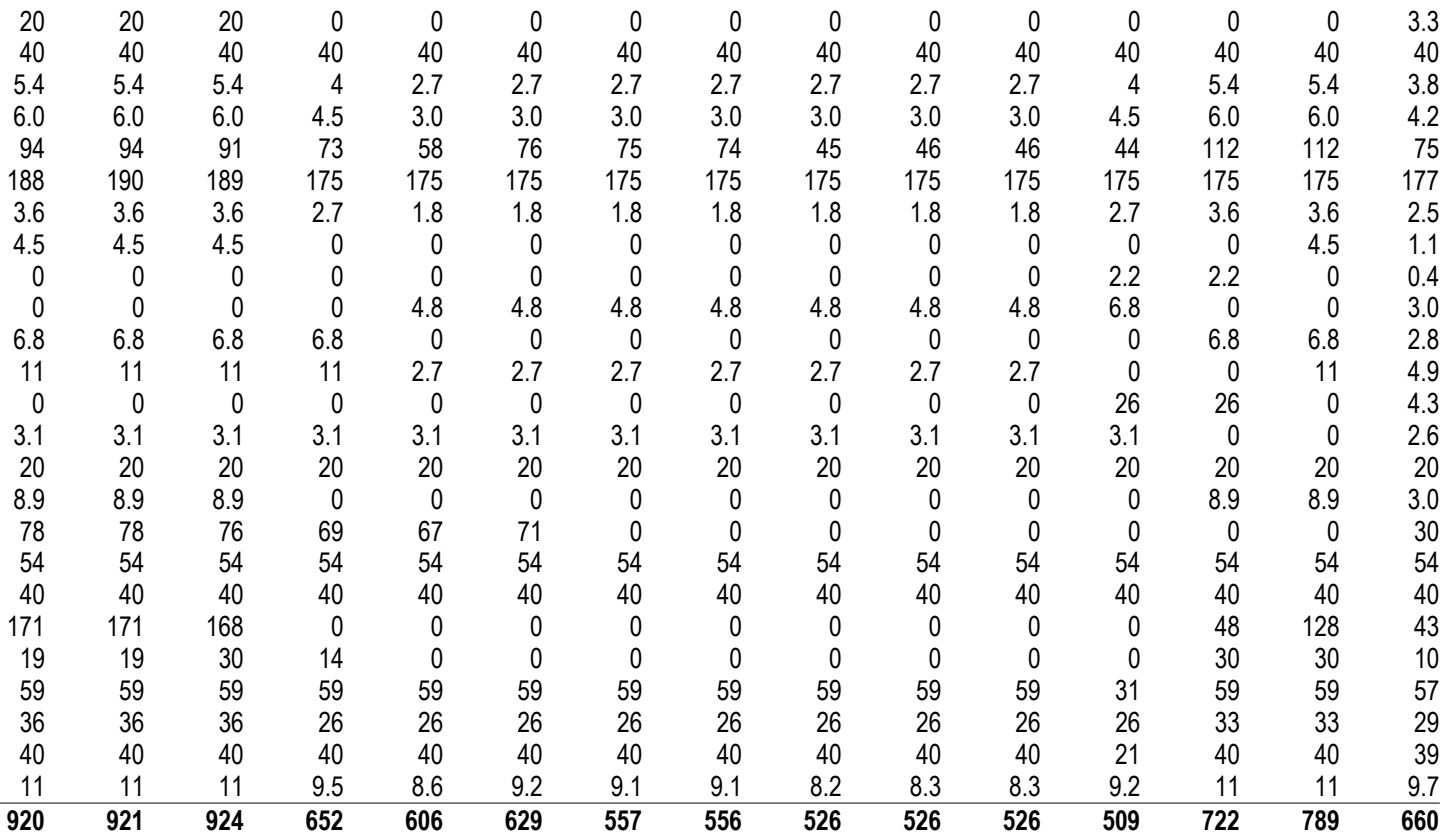

-Exports to Inland Southwest-

30 BPA to SPP PwrS

31 LVFBR to WAPA PwrS

32 PPL to WAPA PwrS

33 Total Exports To ISW

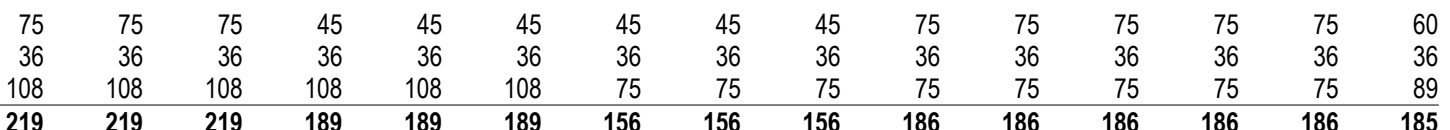

-Exports to Canada-

34 BPA to BCHP CanEnt

$\begin{array}{lllllllllllllll}537 & 537 & 537 & 537 & 537 & 537 & 537 & 537 & 537 & 537 & 537 & 537 & 537 & 537 & 537 \\ \mathbf{5 3 7} & \mathbf{5 3 7} & \mathbf{5 3 7} & \mathbf{5 3 7} & \mathbf{5 3 7} & \mathbf{5 3 7} & \mathbf{5 3 7} & \mathbf{5 3 7} & \mathbf{5 3 7} & \mathbf{5 3 7} & \mathbf{5 3 7} & \mathbf{5 3 7} & \mathbf{5 3 7} & \mathbf{5 3 7} & \mathbf{5 3 7}\end{array}$

35 Total Exports To Canada

$537 \quad 537$

-Exports to Other Entities-

36 Total Exports To Other Entities

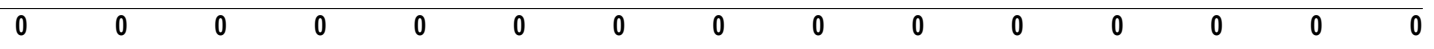

-Total Exports-

37 Federal Entities

38 Public Entities

39 Investor-Owned Entities

40 Other Entities

\begin{tabular}{|c|c|c|c|c|c|c|c|c|c|c|c|c|c|}
\hline 1071 & 1062 & 974 & 961 & 995 & 991 & 987 & 950 & 974 & 974 & 992 & 1070 & 1064 & 1007 \\
\hline 95 & 106 & 81 & 66 & 66 & 66 & 66 & 66 & 66 & 66 & 47 & 103 & 103 & 78 \\
\hline 551 & 546 & 362 & 360 & 364 & 260 & 260 & 260 & 260 & 260 & 232 & 314 & 395 & 347 \\
\hline 36 & 36 & 36 & 36 & 36 & 36 & 36 & 36 & 36 & 36 & 36 & 36 & 36 & 36 \\
\hline 1754 & 1750 & 1453 & 1424 & 1461 & 1354 & 1349 & 1312 & 1336 & 1336 & 1306 & 1524 & 598 & 46 \\
\hline
\end{tabular}


Table A-2: Regional Exports

PNW Loads and Resources Study

2005 - 2006 Operating Year

Aug1 Aug16 Sep Oct Nov Dec Jan $F$ Feb Mar Apr1 Apr16 May Jun Jul Avg -Exports East of Continental Divide -

1 AVWP to PPLM PwrS

$2 \mathrm{BPA}$ to Other Entities

3 Total Exports to ECD

\begin{tabular}{|c|c|c|c|c|c|c|c|c|c|c|c|c|c|c|}
\hline 9 & 9 & 9 & 9 & 9 & 9 & 9 & 9 & 9 & 9 & 9 & 9 & 9 & 9 & 9 \\
\hline 70 & 70 & 64 & 68 & 86 & 101 & 99 & 95 & 88 & 81 & 80 & 68 & 72 & 80 & 81 \\
\hline 79 & 79 & 73 & 77 & 95 & 110 & 108 & 104 & 96 & 90 & 90 & 77 & 81 & 89 & 90 \\
\hline
\end{tabular}

-Exports to Pacific Southwest-

4 BPA to BART PwrS

5 BPA to BURB $C / N / X$

6 BPA to GLEN C/N/X

7 BPA to MSR PwrS

$8 \mathrm{BPA}$ to Other Entities

$9 \mathrm{BPA}$ to PASA C/N/X

$10 \mathrm{BPA}$ to PASA $\mathrm{C} / \mathrm{N} / \mathrm{X}$

11 BPA to PASA S/N/X

12 BPA to RVSD CapS

$13 \mathrm{BPA}$ to RVSD C/N/X

$14 \mathrm{BPA}$ to RVSD C/N/X

15 BPA to RVSD S/Pwr/X

16 PGE to GLEN PwrS

17 PGE to GLEN S/Pwr/X

18 PPL to SCE PwrS

19 PPL to SMUD PwrS

20 PSE to PGS S/Pwr/X

21 SCL to NCPA S/Pwr/X

22 SDGE to SDGE PwrS

23 SHPD to SMUD PwrS

24 TID to TID PwrS

25 BPA NW-SW Intertie Losses

26 Total Exports To PSW

\begin{tabular}{|c|c|c|c|c|c|c|c|c|c|c|c|c|c|c|}
\hline 40 & 40 & 40 & 40 & 40 & 40 & 40 & 40 & 40 & 40 & 40 & 40 & 40 & 1.3 & 37 \\
\hline 5.4 & 5.4 & 5.4 & 4 & 2.7 & 2.7 & 2.7 & 2.7 & 2.7 & 2.7 & 2.7 & 4 & 5.4 & 5.4 & 3.8 \\
\hline 6.0 & 6.0 & 6.0 & 4.5 & 3.0 & 3.0 & 3.0 & 3.0 & 3.0 & 3.0 & 3.0 & 4.5 & 6.0 & 6.0 & 4.2 \\
\hline 94 & 94 & 91 & 0 & 0 & 0 & 0 & 0 & 0 & 0 & 0 & 0 & 0 & 0 & 15 \\
\hline 175 & 175 & 175 & 210 & 210 & 210 & 210 & 210 & 210 & 210 & 210 & 210 & 210 & 210 & 204 \\
\hline 3.6 & 3.6 & 3.6 & 2.7 & 1.8 & 1.8 & 1.8 & 1.8 & 1.8 & 1.8 & 1.8 & 2.7 & 3.6 & 3.6 & 2.5 \\
\hline 4.5 & 4.5 & 4.5 & 0 & 0 & 0 & 0 & 0 & 0 & 0 & 0 & 0 & 0 & 4.5 & 1.1 \\
\hline 0 & 0 & 0 & 0 & 0 & 0 & 0 & 0 & 0 & 0 & 0 & 2.2 & 2.2 & 0 & 0.4 \\
\hline 0 & 0 & 0 & 0 & 4.8 & 4.8 & 4.8 & 4.8 & 4.8 & 4.8 & 4.8 & 6.8 & 0 & 0 & 3.0 \\
\hline 6.8 & 6.8 & 6.8 & 6.8 & 0 & 0 & 0 & 0 & 0 & 0 & 0 & 0 & 6.8 & 6.8 & 2.8 \\
\hline 11 & 11 & 11 & 11 & 2.7 & 2.7 & 2.7 & 2.7 & 2.7 & 2.7 & 2.7 & 0 & 0 & 11 & 4.9 \\
\hline 0 & 0 & 0 & 0 & 0 & 0 & 0 & 0 & 0 & 0 & 0 & 26 & 26 & 0 & 4.3 \\
\hline 20 & 20 & 20 & 20 & 20 & 20 & 20 & 20 & 20 & 20 & 20 & 20 & 20 & 20 & 20 \\
\hline 8.9 & 8.9 & 8.9 & 0 & 0 & 0 & 0 & 0 & 0 & 0 & 0 & 0 & 8.9 & 8.9 & 3.0 \\
\hline 54 & 54 & 54 & 54 & 54 & 54 & 54 & 54 & 54 & 54 & 54 & 54 & 54 & 54 & 54 \\
\hline 40 & 40 & 40 & 40 & 40 & 40 & 40 & 40 & 40 & 40 & 40 & 40 & 40 & 40 & 40 \\
\hline 171 & 171 & 168 & 0 & 0 & 0 & 0 & 0 & 0 & 0 & 0 & 0 & 48 & 128 & 43 \\
\hline 19 & 19 & 30 & 14 & 0 & 0 & 0 & 0 & 0 & 0 & 0 & 0 & 30 & 30 & 10 \\
\hline 59 & 59 & 59 & 59 & 59 & 59 & 59 & 59 & 59 & 59 & 59 & 31 & 59 & 59 & 57 \\
\hline 36 & 36 & 36 & 26 & 26 & 26 & 26 & 26 & 26 & 26 & 26 & 26 & 33 & 33 & 29 \\
\hline 40 & 40 & 40 & 40 & 40 & 40 & 40 & 40 & 40 & 40 & 40 & 21 & 40 & 40 & 39 \\
\hline 10 & 10 & 10 & 8.4 & 7.9 & 7.9 & 7.9 & 7.9 & 7.9 & 7.9 & 7.9 & 8.9 & 9.0 & 7.4 & 8.5 \\
\hline 805 & 805 & 810 & 541 & 512 & 512 & 512 & 512 & 512 & 512 & 512 & 496 & 642 & 669 & 586 \\
\hline
\end{tabular}

-Exports to Inland Southwest-

27 BPA to SPP PwrS
28 LVFBR to WAPA PwrS

29 Total Exports To ISW

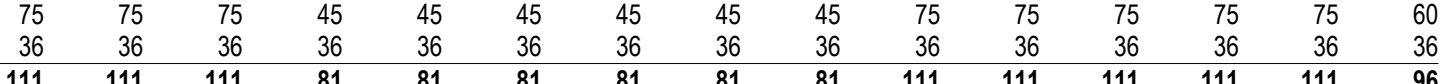

-Exports to Canada-

30 BPA to BCHP CanEnt

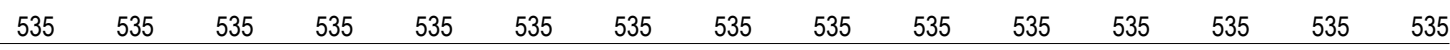

31 Total Exports To Canada

$535 \quad 535 \quad 535$

-Exports to Other Entities-

32 Total Exports To Other Entities

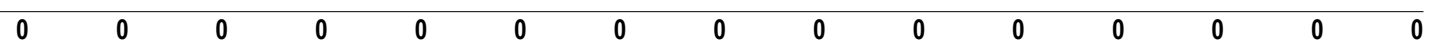

-Total Exports-

33 Federal Entities

34 Public Entities

35 Investor-Owned Entities

36 Other Entities

37 Total Exports.

\begin{tabular}{|c|c|c|c|c|c|c|c|c|c|c|c|c|c|c|}
\hline 1036 & 1036 & 1027 & 935 & 939 & 954 & 952 & 948 & 940 & 964 & 963 & 983 & 991 & 945 & 968 \\
\hline 95 & 95 & 106 & 81 & 66 & 66 & 66 & 66 & 66 & 66 & 66 & 47 & 103 & 103 & 78 \\
\hline 362 & 362 & 359 & 182 & 182 & 182 & 182 & 182 & 182 & 182 & 182 & 154 & 239 & 320 & 226 \\
\hline 36 & 36 & 36 & 36 & 36 & 36 & 36 & 36 & 36 & 36 & 36 & 36 & 36 & 36 & 36 \\
\hline 1530 & 1530 & 1529 & 1234 & 1223 & 1238 & 1236 & 1232 & 1225 & 1248 & 1248 & 1219 & 1369 & 1404 & 1307 \\
\hline
\end{tabular}


Table A-2: Regional Exports

PNW Loads and Resources Study

2006 - 2007 Operating Year Dec Jan Fe

Feb Mar Apr1 Apr16 May Jun Jul Avg

-Exports East of Continental Divide -

1 AVWP to PPLM PwrS

2 BPA to Other Entities

3 Total Exports to ECD

\begin{tabular}{|c|c|c|c|c|c|c|c|c|c|c|c|c|c|c|}
\hline 9 & 9 & 9 & 9 & 9 & 9 & 9 & 9 & 9 & 9 & 9 & 9 & 9 & 9 & 9 \\
\hline 73 & 73 & 66 & 71 & 89 & 105 & 103 & 99 & 91 & 84 & 84 & 71 & 75 & 83 & 84 \\
\hline 82 & 82 & 76 & 80 & 98 & 114 & 112 & 108 & 100 & 93 & 93 & 80 & 84 & 92 & 93 \\
\hline
\end{tabular}

-Exports to Pacific Southwest-

$4 \mathrm{BPA}$ to BURB $\mathrm{C} / \mathrm{N} / \mathrm{X}$

5 BPA to GLEN C/N/X

6 BPA to Other Entities

7 BPA to PASA C/N/X

$8 \mathrm{BPA}$ to PASA $\mathrm{C} / \mathrm{N} / \mathrm{X}$

9 BPA to PASA S/N/X

10 BPA to RVSD CapS

$11 \mathrm{BPA}$ to RVSD C/N/X

$12 \mathrm{BPA}$ to RVSD $\mathrm{C} / \mathrm{N} / \mathrm{X}$

13 BPA to RVSD S/Pwr/X

14 PGE to GLEN PwrS

15 PGE to GLEN S/Pwr/X

16 PPL to SCE PwrS

17 PPL to SMUD PwrS

18 PSE to PGS S/Pwr/X

19 SCL to NCPA S/Pwr/X

20 SDGE to SDGE PwrS

21 SHPD to SMUD PwrS

22 TID to TID PwrS

23 BPA NW-SW Intertie Losses

24 Total Exports To PSW

$\begin{array}{rrrrrrrrrrrrrrr}5.4 & 5.4 & 5.4 & 4 & 2.7 & 2.7 & 2.7 & 2.7 & 2.7 & 2.7 & 2.7 & 4 & 5.4 & 5.4 & 3.8 \\ 6.0 & 6.0 & 6.0 & 4.5 & 3.0 & 3.0 & 3.0 & 3.0 & 3.0 & 3.0 & 3.0 & 4.5 & 6.0 & 6.0 & 4.2 \\ 210 & 210 & 210 & 100 & 100 & 100 & 100 & 100 & 100 & 100 & 100 & 100 & 100 & 100 & 118 \\ 3.6 & 3.6 & 3.6 & 2.7 & 1.8 & 1.8 & 1.8 & 1.8 & 1.8 & 1.8 & 1.8 & 2.7 & 3.6 & 3.6 & 2.5 \\ 4.5 & 4.5 & 4.5 & 0 & 0 & 0 & 0 & 0 & 0 & 0 & 0 & 0 & 0 & 4.5 & 1.1 \\ 0 & 0 & 0 & 0 & 0 & 0 & 0 & 0 & 0 & 0 & 0 & 2.2 & 2.2 & 0 & 0.4 \\ 0 & 0 & 0 & 0 & 4.8 & 4.8 & 4.8 & 4.8 & 4.8 & 4.8 & 4.8 & 6.8 & 0 & 0 & 3.0 \\ 6.8 & 6.8 & 6.8 & 6.8 & 0 & 0 & 0 & 0 & 0 & 0 & 0 & 0 & 6.8 & 6.8 & 2.8 \\ 11 & 11 & 11 & 11 & 2.7 & 2.7 & 2.7 & 2.7 & 2.7 & 2.7 & 2.7 & 0 & 0 & 11 & 4.9 \\ 0 & 0 & 0 & 0 & 0 & 0 & 0 & 0 & 0 & 0 & 0 & 26 & 26 & 0 & 4.3 \\ 20 & 20 & 20 & 20 & 20 & 20 & 20 & 20 & 20 & 20 & 20 & 20 & 20 & 20 & 20 \\ 8.9 & 8.9 & 8.9 & 0 & 0 & 0 & 0 & 0 & 0 & 0 & 0 & 0 & 8.9 & 8.9 & 3.0 \\ 54 & 54 & 54 & 0 & 0 & 0 & 0 & 0 & 0 & 0 & 0 & 0 & 0 & 0 & 9 \\ 40 & 40 & 40 & 40 & 40 & 40 & 40 & 40 & 40 & 40 & 40 & 40 & 40 & 40 & 40 \\ 171 & 171 & 168 & 0 & 0 & 0 & 0 & 0 & 0 & 0 & 0 & 0 & 48 & 128 & 43 \\ 19 & 19 & 30 & 14 & 0 & 0 & 0 & 0 & 0 & 0 & 0 & 0 & 30 & 30 & 10 \\ 59 & 59 & 59 & 59 & 59 & 59 & 59 & 59 & 59 & 59 & 59 & 31 & 59 & 59 & 57 \\ 36 & 36 & 36 & 26 & 26 & 26 & 26 & 26 & 26 & 26 & 26 & 26 & 33 & 33 & 29 \\ 40 & 40 & 40 & 40 & 40 & 40 & 40 & 40 & 40 & 40 & 40 & 21 & 40 & 40 & 39 \\ 7.4 & 7.4 & 7.4 & 3.9 & 3.4 & 3.4 & 3.4 & 3.4 & 3.4 & 3.4 & 3.4 & 4.4 & 4.5 & 4.1 & 4.4 \\ 703 & 703 & \mathbf{7 1 1} & \mathbf{3 3 3} & \mathbf{3 0 4} & \mathbf{3 0 4} & \mathbf{3 0 4} & \mathbf{3 0 4} & \mathbf{3 0 4} & \mathbf{3 0 4} & \mathbf{3 0 4} & \mathbf{2 8 7} & \mathbf{4 3 3} & \mathbf{5 0 1} & \mathbf{3 0 4}\end{array}$

-Exports to Inland Southwest-

25 BPA to SPP PwrS
26 LVFBR to WAPA PwrS

27 Total Exports To ISW

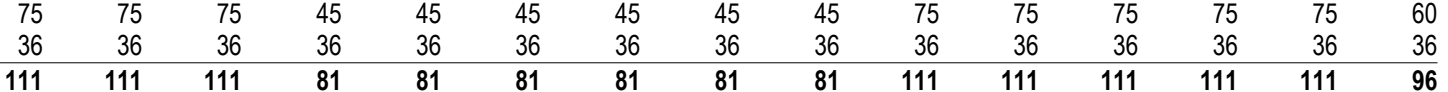

-Exports to Canada-

28 BPA to BCHP CanEnt

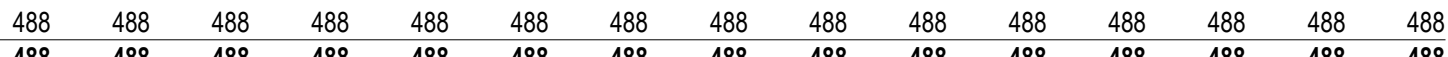

29 Total Exports To Canada

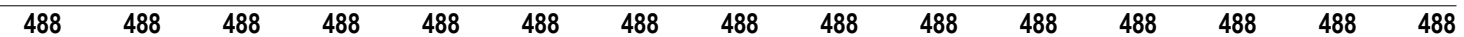

-Exports to Other Entities-

30 Total Exports To Other Entities

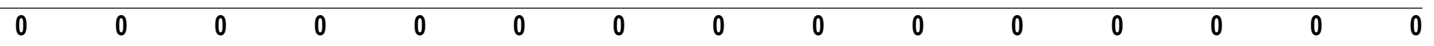

-Total Exports-

31 Federal Entities

32 Public Entities

33 Investor-Owned Entities

34 Other Entities

35 Total Exports.

\begin{tabular}{|c|c|c|c|c|c|c|c|c|c|c|c|c|c|c|}
\hline 891 & 891 & 884 & 737 & 741 & 757 & 755 & 751 & 743 & 766 & 766 & 784 & 793 & 787 & 783 \\
\hline 95 & 95 & 106 & 81 & 66 & 66 & 66 & 66 & 66 & 66 & 66 & 47 & 103 & 103 & 78 \\
\hline 362 & 362 & 359 & 128 & 128 & 128 & 128 & 128 & 128 & 128 & 128 & 100 & 185 & 266 & 181 \\
\hline 36 & 36 & 36 & 36 & 36 & 36 & 36 & 36 & 36 & 36 & 36 & 36 & 36 & 36 & 36 \\
\hline & 384 & 1386 & 982 & 972 & 988 & 985 & 981 & 974 & 996 & 996 & 967 & 1117 & 1192 & 10 \\
\hline
\end{tabular}


Table A-2: Regional Exports

PNW Loads and Resources Study

2007 - 2008 Operating Year 2003 White Book

-Exports East of Continental Divide -

1 AVWP to PPLM PwrS

$2 \mathrm{BPA}$ to Other Entities

3 Total Exports to ECD

\begin{tabular}{|c|c|c|c|c|c|c|c|c|c|c|c|c|c|c|}
\hline 9 & 9 & 9 & 9 & 9 & 9 & 9 & 9 & 9 & 9 & 9 & 9 & 9 & 9 & 9 \\
\hline 76 & 76 & 69 & 74 & 93 & 110 & 107 & 103 & 95 & 88 & 87 & 74 & 78 & 86 & 88 \\
\hline 85 & 85 & 78 & 83 & 102 & 118 & 116 & 112 & 104 & 96 & 96 & 83 & 87 & 95 & 97 \\
\hline
\end{tabular}

-Exports to Pacific Southwest-

$4 \mathrm{BPA}$ to BURB $\mathrm{C} / \mathrm{N} / \mathrm{X}$

5 BPA to GLEN $C / N / X$

$6 \mathrm{BPA}$ to Other Entities

7 BPA to PASA C/N/X

$8 \mathrm{BPA}$ to PASA $\mathrm{C} / \mathrm{N} / \mathrm{X}$

9 BPA to PASA S/N/X

10 BPA to RVSD CapS

$11 \mathrm{BPA}$ to RVSD C/N/X

$12 \mathrm{BPA}$ to RVSD $\mathrm{C} / \mathrm{N} / \mathrm{X}$

13 BPA to RVSD S/Pwr/X

14 PGE to GLEN PwrS

15 PGE to GLEN S/Pwr/X

16 PPL to SMUD PwrS

17 PSE to PGS S/Pwr/X

18 SCL to NCPA S/Pwr/X

19 SDGE to SDGE PwrS

20 SHPD to SMUD PwrS

21 TID to TID PwrS

22 BPA NW-SW Intertie Losses

23 Total Exports To PSW

\begin{tabular}{rrrrrrrrrrrrrrr}
5.4 & 5.4 & 5.4 & 4 & 2.7 & 2.7 & 2.7 & 2.7 & 2.7 & 2.7 & 0 & 0 & 0 & 0 & 2.5 \\
6.0 & 6.0 & 6.0 & 4.5 & 3.0 & 3.0 & 3.0 & 3.0 & 3.0 & 3.0 & 0 & 0 & 0 & 0 & 2.7 \\
100 & 100 & 100 & 100 & 100 & 100 & 0 & 0 & 0 & 0 & 0 & 0 & 0 & 0 & 42 \\
3.6 & 3.6 & 3.6 & 2.7 & 1.8 & 1.8 & 1.8 & 1.8 & 1.8 & 1.8 & 0 & 0 & 0 & 0 & 1.6 \\
4.5 & 4.5 & 4.5 & 0 & 0 & 0 & 0 & 0 & 0 & 0 & 0 & 0 & 0 & 4.5 & 1.1 \\
0 & 0 & 0 & 0 & 0 & 0 & 0 & 0 & 0 & 0 & 0 & 2.2 & 2.2 & 0 & 0.4 \\
0 & 0 & 0 & 0 & 4.8 & 4.8 & 4.8 & 4.8 & 4.8 & 4.8 & 4.8 & 6.8 & 0 & 0 & 3.0 \\
6.8 & 6.8 & 6.8 & 6.8 & 0 & 0 & 0 & 0 & 0 & 0 & 0 & 0 & 6.8 & 6.8 & 2.8 \\
11 & 11 & 11 & 11 & 2.7 & 2.7 & 2.7 & 2.7 & 2.7 & 2.7 & 2.7 & 0 & 0 & 11 & 4.9 \\
0 & 0 & 0 & 0 & 0 & 0 & 0 & 0 & 0 & 0 & 0 & 26 & 26 & 0 & 4.3 \\
20 & 20 & 20 & 20 & 20 & 20 & 20 & 20 & 20 & 20 & 20 & 20 & 20 & 20 & 20 \\
8.9 & 8.9 & 8.9 & 0 & 0 & 0 & 0 & 0 & 0 & 0 & 0 & 0 & 8.9 & 8.9 & 3.0 \\
40 & 40 & 40 & 40 & 40 & 40 & 40 & 40 & 40 & 40 & 40 & 40 & 40 & 40 & 40 \\
171 & 171 & 168 & 0 & 0 & 0 & 0 & 0 & 0 & 0 & 0 & 0 & 0 & 0 & 28 \\
19 & 19 & 30 & 14 & 0 & 0 & 0 & 0 & 0 & 0 & 0 & 0 & 30 & 30 & 10 \\
59 & 59 & 59 & 59 & 59 & 59 & 59 & 59 & 59 & 59 & 59 & 31 & 59 & 59 & 57 \\
36 & 36 & 36 & 0 & 0 & 0 & 0 & 0 & 0 & 0 & 0 & 0 & 0 & 0 & 6 \\
40 & 40 & 40 & 40 & 40 & 40 & 40 & 40 & 40 & 40 & 40 & 21 & 40 & 40 & 39 \\
4.1 & 4.1 & 4.1 & 3.9 & 3.4 & 3.4 & 0.4 & 0.4 & 0.4 & 0.4 & 0.2 & 1 & 1 & 0.7 & 1.9 \\
\hline 536 & $\mathbf{5 3 6}$ & $\mathbf{5 4 3}$ & $\mathbf{3 0 7}$ & $\mathbf{2 7 8}$ & $\mathbf{2 7 8}$ & $\mathbf{1 7 5}$ & $\mathbf{1 7 5}$ & $\mathbf{1 7 5}$ & $\mathbf{1 7 5}$ & $\mathbf{1 6 7}$ & $\mathbf{1 4 7}$ & $\mathbf{2 3 4}$ & $\mathbf{2 2 1}$ & $\mathbf{2 7 0}$
\end{tabular}

-Exports to Inland Southwest-

24 BPA to SPP PwrS

25 LVFBR to WAPA PwrS

26 Total Exports To ISW

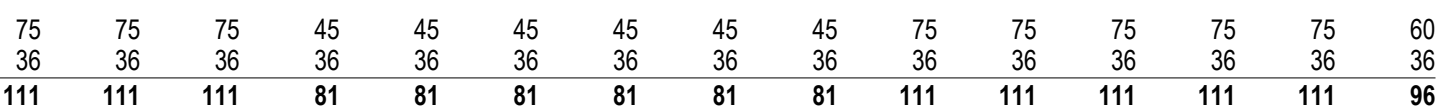

-Exports to Canada-

27 BPA to BCHP CanEnt

$483 \quad 483 \quad 483$

28 Total Exports To Canada

-Exports to Other Entities-

29 Total Exports To Other Entities

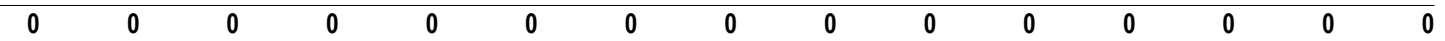

-Total Exports-

30 Federal Entities

31 Public Entities

32 Investor-Owned Entities

33 Other Entities

34 Total Exports.

\begin{tabular}{|c|c|c|c|c|c|c|c|c|c|c|c|c|c|c|}
\hline 775 & 775 & 768 & 734 & 739 & 756 & 650 & 646 & 638 & 661 & 653 & 667 & 672 & 667 & 697 \\
\hline 95 & 95 & 106 & 55 & 40 & 40 & 40 & 40 & 40 & 40 & 40 & 21 & 70 & 70 & 55 \\
\hline 308 & 308 & 305 & 128 & 128 & 128 & 128 & 128 & 128 & 128 & 128 & 100 & 137 & 137 & 157 \\
\hline 36 & 36 & 36 & 36 & 36 & 36 & 36 & 36 & 36 & 36 & 36 & 36 & 36 & 36 & 36 \\
\hline
\end{tabular}


Table A-2: Regional Exports

PNW Loads and Resources Study

2008 - 2009 Operating Year 2003 White Book

Aug1 Aug16 Sep Oct Nov Dec Jan Feb Mar Apr1 Apr16 May Jun Jul Avg

-Exports East of Continental Divide -

1 AVWP to PPLM PwrS

2 BPA to Other Entities

3 Total Exports to ECD

\begin{tabular}{|c|c|c|c|c|c|c|c|c|c|c|c|c|c|c|}
\hline 9 & 9 & 9 & 9 & 0 & 0 & 0 & 0 & 0 & 0 & 0 & 0 & 0 & 0 & 2.3 \\
\hline 79 & 79 & 72 & 77 & 96 & 114 & 112 & 107 & 99 & 91 & 91 & 77 & 82 & 90 & 91 \\
\hline 88 & 88 & 81 & 86 & 96 & 114 & 112 & 107 & 99 & 91 & 91 & 77 & 82 & 90 & 93 \\
\hline
\end{tabular}

-Exports to Pacific Southwest-

4 BPA to PASA C/N/X

5 BPA to PASA S/N/X

6 BPA to RVSD CapS

7 BPA to RVSD C/N/X

$8 \mathrm{BPA}$ to RVSD $\mathrm{C} / \mathrm{N} / \mathrm{X}$

9 BPA to RVSD S/Pwr/X

10 PGE to GLEN PwrS

11 PGE to GLEN S/Pwr/X

12 PPL to SMUD PwrS

13 SCL to NCPA S/Pwr/X

14 SDGE to SDGE PwrS

15 TID to TID PwrS

16 BPA NW-SW Intertie Losses

17 Total Exports To PSW

\begin{tabular}{rrrrrrrrrrrrrrr}
4.5 & 4.5 & 4.5 & 0 & 0 & 0 & 0 & 0 & 0 & 0 & 0 & 0 & 0 & 4.5 & 1.1 \\
0 & 0 & 0 & 0 & 0 & 0 & 0 & 0 & 0 & 0 & 0 & 2.2 & 2.2 & 0 & 0.4 \\
0 & 0 & 0 & 0 & 4.8 & 4.8 & 4.8 & 4.8 & 4.8 & 4.8 & 4.8 & 6.8 & 0 & 0 & 3.0 \\
6.8 & 6.8 & 6.8 & 6.8 & 0 & 0 & 0 & 0 & 0 & 0 & 0 & 0 & 6.8 & 6.8 & 2.8 \\
11 & 11 & 11 & 11 & 2.7 & 2.7 & 2.7 & 2.7 & 2.7 & 2.7 & 2.7 & 0 & 0 & 11 & 4.9 \\
0 & 0 & 0 & 0 & 0 & 0 & 0 & 0 & 0 & 0 & 0 & 26 & 26 & 0 & 4.3 \\
20 & 20 & 20 & 20 & 20 & 20 & 20 & 20 & 20 & 20 & 20 & 20 & 20 & 20 & 20 \\
8.9 & 8.9 & 8.9 & 0 & 0 & 0 & 0 & 0 & 0 & 0 & 0 & 0 & 8.9 & 8.9 & 3.0 \\
40 & 40 & 40 & 40 & 40 & 40 & 40 & 40 & 40 & 40 & 40 & 40 & 40 & 40 & 40 \\
19 & 19 & 30 & 14 & 0 & 0 & 0 & 0 & 0 & 0 & 0 & 0 & 30 & 30 & 10 \\
59 & 59 & 59 & 59 & 59 & 59 & 59 & 59 & 59 & 59 & 59 & 31 & 59 & 59 & 57 \\
40 & 40 & 40 & 40 & 40 & 40 & 40 & 40 & 40 & 40 & 40 & 21 & 40 & 40 & 39 \\
0.7 & 0.7 & 0.7 & 0.5 & 0.2 & 0.2 & 0.2 & 0.2 & 0.2 & 0.2 & 0.2 & 1 & 1 & 0.7 & 0.5 \\
\hline $\mathbf{2 1 0}$ & $\mathbf{2 1 0}$ & $\mathbf{2 2 1}$ & $\mathbf{1 9 2}$ & $\mathbf{1 6 7}$ & $\mathbf{1 6 7}$ & $\mathbf{1 6 7}$ & $\mathbf{1 6 7}$ & $\mathbf{1 6 7}$ & $\mathbf{1 6 7}$ & $\mathbf{1 6 7}$ & $\mathbf{1 4 7}$ & $\mathbf{2 3 4}$ & $\mathbf{2 2 1}$ & $\mathbf{1 8 6}$
\end{tabular}

-Exports to Inland Southwest-

19 LVFBR to WAPA PwrS

20 Total Exports To ISW

\begin{tabular}{|c|c|c|c|c|c|c|c|c|c|c|c|c|c|}
\hline 75 & 75 & 75 & 45 & 45 & 45 & 45 & 45 & 45 & 75 & 75 & 75 & 75 & 75 \\
\hline 36 & 36 & 36 & 36 & 36 & 36 & 36 & 36 & 36 & 36 & 36 & 36 & 36 & 36 \\
\hline 11 & 111 & 111 & 81 & 81 & 81 & 81 & 81 & 81 & 111 & 111 & 111 & 111 & 111 \\
\hline
\end{tabular}

-Exports to Canada-

21 BPA to BCHP CanEnt

$465 \quad 465 \quad 465$

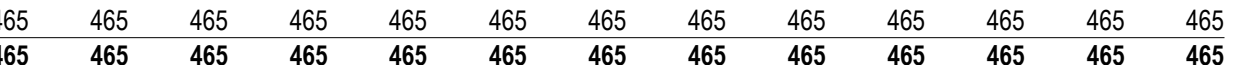

-Exports to Other Entities-

23 Total Exports To Other Entities

$465 \quad 465$

-Total Exports-

24 Federal Entities

25 Public Entities

26 Investor-Owned Entities

27 Other Entities

28 Total Exports.

\begin{tabular}{|c|c|c|c|c|c|c|c|c|c|c|c|c|c|c|}
\hline 641 & 641 & 634 & 605 & 614 & 631 & 629 & 625 & 616 & 639 & 638 & 652 & 657 & 652 & 633 \\
\hline 59 & 59 & 70 & 55 & 40 & 40 & 40 & 40 & 40 & 40 & 40 & 21 & 70 & 70 & 49 \\
\hline 137 & 137 & 137 & 128 & 119 & 119 & 119 & 119 & 119 & 119 & 119 & 91 & 128 & 128 & 122 \\
\hline 36 & 36 & 36 & 36 & 36 & 36 & 36 & 36 & 36 & 36 & 36 & 36 & 36 & 36 & 36 \\
\hline
\end{tabular}


Table A-2: Regional Exports

PNW Loads and Resources Study

2009 - 2010 Operating Year 2003 White Book

Aug1 Aug16 Sep Oct Nov Dec Jan Feb Mar Apr1 Apr16 May Jun Jul Avg

-Exports East of Continental Divide -

$1 \mathrm{BPA}$ to Other Entities

\begin{tabular}{|c|c|c|c|c|c|c|c|c|c|c|c|c|c|}
\hline 82 & 82 & 75 & 80 & 100 & 118 & 116 & 111 & 102 & 94 & 94 & 80 & 85 & 93 \\
\hline 82 & 82 & 75 & 80 & 100 & 118 & 116 & 111 & 102 & 94 & 94 & 80 & 85 & 93 \\
\hline
\end{tabular}

2 Total Exports to ECD

$\begin{array}{rrrrrrrrrrrrrrr}82 & \mathbf{8 2} & \mathbf{7 5} & \mathbf{8 0} & \mathbf{1 0 0} & \mathbf{1 1 8} & \mathbf{1 1 6} & \mathbf{1 1 1} & \mathbf{1 0 2} & \mathbf{9 4} & \mathbf{9 4} & \mathbf{8 0} & \mathbf{8 5} & \mathbf{9 3} & \mathbf{9 5} \\ & & & & & & & & & & & & & & \\ 4.5 & 4.5 & 4.5 & 0 & 0 & 0 & 0 & 0 & 0 & 0 & 0 & 0 & 0 & 4.5 & 1.1 \\ 0 & 0 & 0 & 0 & 0 & 0 & 0 & 0 & 0 & 0 & 0 & 2.2 & 2.2 & 0 & 0.4 \\ 0 & 0 & 0 & 0 & 4.8 & 4.8 & 4.8 & 4.8 & 4.8 & 4.8 & 4.8 & 6.8 & 0 & 0 & 3.0 \\ 6.8 & 6.8 & 6.8 & 6.8 & 0 & 0 & 0 & 0 & 0 & 0 & 0 & 0 & 0 & 0 & 1.7 \\ 11 & 11 & 11 & 11 & 2.7 & 2.7 & 2.7 & 2.7 & 2.7 & 2.7 & 2.7 & 0 & 0 & 11 & 4.9 \\ 0 & 0 & 0 & 0 & 0 & 0 & 0 & 0 & 0 & 0 & 0 & 26 & 26 & 0 & 4.3 \\ 20 & 20 & 20 & 20 & 20 & 20 & 20 & 20 & 20 & 20 & 20 & 20 & 20 & 20 & 20 \\ 8.9 & 8.9 & 8.9 & 0 & 0 & 0 & 0 & 0 & 0 & 0 & 0 & 0 & 8.9 & 8.9 & 3.0 \\ 40 & 40 & 40 & 40 & 40 & 40 & 40 & 40 & 40 & 40 & 40 & 40 & 40 & 40 & 40 \\ 19 & 19 & 30 & 14 & 0 & 0 & 0 & 0 & 0 & 0 & 0 & 0 & 30 & 30 & 10 \\ 59 & 59 & 59 & 59 & 59 & 59 & 59 & 59 & 59 & 59 & 59 & 31 & 59 & 59 & 57 \\ 40 & 40 & 40 & 40 & 40 & 40 & 40 & 40 & 40 & 40 & 40 & 21 & 40 & 40 & 39 \\ 0.7 & 0.7 & 0.7 & 0.5 & 0.2 & 0.2 & 0.2 & 0.2 & 0.2 & 0.2 & 0.2 & 1 & 0.8 & 0.5 & 0.5 \\ 210 & \mathbf{2 1 0} & \mathbf{2 2 1} & 192 & 167 & 167 & 167 & 167 & 167 & 167 & 167 & \mathbf{1 4 7} & \mathbf{2 2 7} & \mathbf{2 1 4} & \mathbf{1 8 7}\end{array}$

-Exports to Pacific Southwest-

3 BPA to PASA C/N/X

4 BPA to PASA S/N/X

5 BPA to RVSD CapS

$6 \mathrm{BPA}$ to RVSD $\mathrm{C} / \mathrm{N} / \mathrm{X}$

7 BPA to RVSD C/N/X

8 BPA to RVSD S/Pwr/X

9 PGE to GLEN PwrS

10 PGE to GLEN S/Pwr/X

$11 \mathrm{PPL}$ to SMUD PwrS

12 SCL to NCPA S/Pwr/X

13 SDGE to SDGE PwrS

14 TID to TID PwrS

15 BPA NW-SW Intertie Losses

16 Total Exports To PSW

$210 \quad 210$

-Exports to Inland Southwest.

17 BPA to SPP PwrS

18 LVFBR to WAPA PwrS

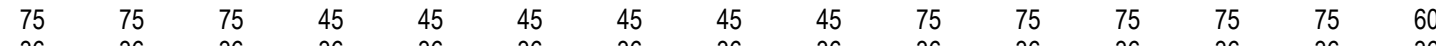

19 Total Exports To ISW

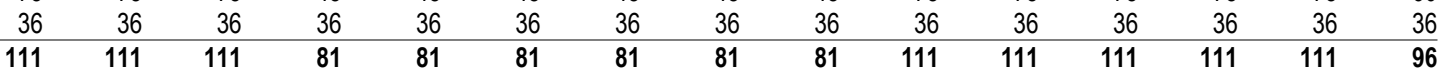

-Exports to Canada-

20 BPA to BCHP CanEnt

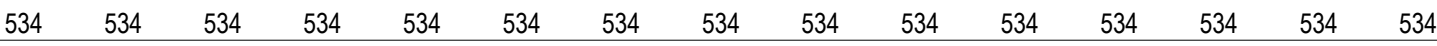

21 Total Exports To Canada

$534 \quad 534 \quad 534$

-Exports to Other Entities-

22 Total Exports To Other Entities

-Total Exports-

23 Federal Entities

24 Public Entities

25 Investor-Owned Entities

26 Other Entities

27 Total Exports.

$\begin{array}{rrrrrrrrrrrrrrr}\mathbf{0} & \mathbf{0} & \mathbf{0} & \mathbf{0} & \mathbf{0} & \mathbf{0} & \mathbf{0} & \mathbf{0} & \mathbf{0} & \mathbf{0} & \mathbf{0} & \mathbf{0} & \mathbf{0} & \mathbf{0} & \mathbf{0} \\ & & & & & & & & & & & & & & \\ 714 & 714 & 706 & 677 & 687 & 705 & 703 & 698 & 689 & 711 & 711 & 724 & 722 & 718 & 705 \\ 59 & 59 & 70 & 55 & 40 & 40 & 40 & 40 & 40 & 40 & 40 & 21 & 70 & 70 & 49 \\ 128 & 128 & 128 & 119 & 119 & 119 & 119 & 119 & 119 & 119 & 119 & 91 & 128 & 128 & 120 \\ 36 & 36 & 36 & 36 & 36 & 36 & 36 & 36 & 36 & 36 & 36 & 36 & 36 & 36 & 36 \\ \mathbf{9 3 7} & \mathbf{9 3 7} & \mathbf{9 4 1} & \mathbf{8 8 7} & \mathbf{8 8 3} & \mathbf{9 0 0} & \mathbf{8 9 8} & \mathbf{8 9 3} & \mathbf{8 8 5} & \mathbf{9 0 7} & \mathbf{9 0 6} & \mathbf{8 7 2} & \mathbf{9 5 7} & \mathbf{9 5 2} & \mathbf{9 0 9}\end{array}$


Table A-2: Regional Exports

PNW Loads and Resources Study

2010 - 2011 Operating Year 2003 White Book

Aug1 Aug16 Sep Oct Nov Dec Jan Feb Mar Apr1 Apr16 May Jun Jul Avg

-Exports East of Continental Divide -

$1 \mathrm{BPA}$ to Other Entities

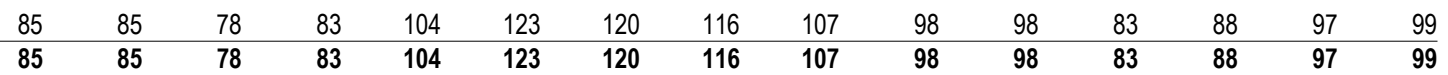

2 Total Exports to ECD

\begin{tabular}{rrrrrrrrrrrrrrr}
$\mathbf{8 5}$ & $\mathbf{8 5}$ & $\mathbf{7 8}$ & $\mathbf{8 3}$ & $\mathbf{1 0 4}$ & $\mathbf{1 2 3}$ & $\mathbf{1 2 0}$ & $\mathbf{1 1 6}$ & $\mathbf{1 0 7}$ & $\mathbf{9 8}$ & $\mathbf{9 8}$ & $\mathbf{8 3}$ & $\mathbf{8 8}$ & $\mathbf{9 7}$ & $\mathbf{9 9}$ \\
& & & & & & & & & & & & & & \\
4.5 & 4.5 & 4.5 & 0 & 0 & 0 & 0 & 0 & 0 & 0 & 0 & 0 & 0 & 4.5 & 1.1 \\
0 & 0 & 0 & 0 & 0 & 0 & 0 & 0 & 0 & 0 & 0 & 2.2 & 2.2 & 0 & 0.4 \\
11 & 11 & 11 & 11 & 2.7 & 2.7 & 2.7 & 2.7 & 2.7 & 2.7 & 2.7 & 0 & 0 & 11 & 4.9 \\
0 & 0 & 0 & 0 & 0 & 0 & 0 & 0 & 0 & 0 & 0 & 26 & 26 & 0 & 4.3 \\
20 & 20 & 20 & 20 & 20 & 20 & 20 & 20 & 20 & 20 & 20 & 20 & 20 & 20 & 20 \\
8.9 & 8.9 & 8.9 & 0 & 0 & 0 & 0 & 0 & 0 & 0 & 0 & 0 & 8.9 & 8.9 & 3.0 \\
40 & 40 & 40 & 40 & 40 & 40 & 40 & 40 & 40 & 40 & 40 & 40 & 40 & 40 & 40 \\
19 & 19 & 30 & 14 & 0 & 0 & 0 & 0 & 0 & 0 & 0 & 0 & 30 & 30 & 10 \\
59 & 59 & 59 & 59 & 59 & 59 & 59 & 59 & 59 & 59 & 59 & 31 & 59 & 59 & 57 \\
40 & 40 & 40 & 40 & 40 & 40 & 40 & 40 & 40 & 40 & 40 & 21 & 40 & 40 & 39 \\
0.5 & 0.5 & 0.5 & 0.3 & 0.1 & 0.1 & 0.1 & 0.1 & 0.1 & 0.1 & 0.1 & 0.8 & 0.8 & 0.5 & 0.3 \\
\hline $\mathbf{2 0 3}$ & $\mathbf{2 0 3}$ & $\mathbf{2 1 4}$ & $\mathbf{1 8 5}$ & $\mathbf{1 6 2}$ & $\mathbf{1 6 2}$ & $\mathbf{1 6 2}$ & $\mathbf{1 6 2}$ & $\mathbf{1 6 2}$ & $\mathbf{1 6 2}$ & $\mathbf{1 6 2}$ & $\mathbf{1 4 0}$ & $\mathbf{2 2 7}$ & $\mathbf{2 1 4}$ & $\mathbf{1 8 0}$
\end{tabular}

-Exports to Pacific Southwest-

3 BPA to PASA C/N/X

4 BPA to PASA S/N/X

5 BPA to RVSD C/N/X

6 BPA to RVSD S/Pwr/X

7 PGE to GLEN PwrS

8 PGE to GLEN S/Pwr/X

9 PPL to SMUD PwrS

10 SCL to NCPA S/Pwr/X

11 SDGE to SDGE PwrS

12 TID to TID PwrS

13 BPA NW-SW Intertie Losses

14 Total Exports To PSW

203

-Exports to Inland Southwest.

15 BPA to SPP PwrS

16 LVFBR to WAPA PwrS

17 Total Exports To ISW

\begin{tabular}{|c|c|c|c|c|c|c|c|c|c|c|c|c|c|}
\hline 75 & 75 & 45 & 45 & 45 & 45 & 45 & 45 & 75 & 75 & 75 & 75 & 75 & 60 \\
\hline 36 & 36 & 36 & 36 & 36 & 36 & 36 & 36 & 36 & 36 & 36 & 36 & 36 & 36 \\
\hline 111 & 111 & 81 & 81 & 81 & 81 & 81 & 81 & 111 & 111 & 111 & 111 & 111 & 96 \\
\hline
\end{tabular}

-Exports to Canada-

18 BPA to BCHP CanEnt

\begin{tabular}{lllllllllllllll}
524 & 524 & 524 & 524 & 524 & 524 & 524 & 524 & 524 & 524 & 524 & 524 & 524 & 524 & 524 \\
\hline
\end{tabular}

19 Total Exports To Canada

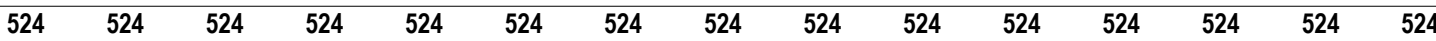

-Exports to Other Entities-

20 Total Exports To Other Entities

0

0

-Total Exports-

21 Federal Entities

22 Public Entities

23 Investor-Owned Entities

24 Other Entities

25 Total Exports.

\begin{tabular}{rrrrrrrrrrrrrrr}
700 & 700 & 692 & 663 & 676 & 695 & 692 & 687 & 678 & 700 & 700 & 710 & 716 & 712 & 694 \\
59 & 59 & 70 & 55 & 40 & 40 & 40 & 40 & 40 & 40 & 40 & 21 & 70 & 70 & 49 \\
128 & 128 & 128 & 119 & 119 & 119 & 119 & 119 & 119 & 119 & 119 & 91 & 128 & 128 & 120 \\
36 & 36 & 36 & 36 & 36 & 36 & 36 & 36 & 36 & 36 & 36 & 36 & 36 & 36 & 36 \\
\hline $\mathbf{9 2 4}$ & $\mathbf{9 2 4}$ & $\mathbf{9 2 7}$ & $\mathbf{8 7 3}$ & $\mathbf{8 7 2}$ & $\mathbf{8 9 0}$ & $\mathbf{8 8 8}$ & $\mathbf{8 8 3}$ & $\mathbf{8 7 4}$ & $\mathbf{8 9 5}$ & $\mathbf{8 9 5}$ & $\mathbf{8 5 8}$ & $\mathbf{9 5 0}$ & $\mathbf{9 4 6}$ & $\mathbf{8 9 8}$
\end{tabular}


Table A-2: Regional Exports

PNW Loads and Resources Study

2011 - 2012 Operating Year 2003 White Book

May Jun Jul Avg

-Exports East of Continental Divide -

$1 \mathrm{BPA}$ to Other Entities

Dec Jan Feb Mar Apr1 Apr16

2 Total Exports to ECD

\begin{tabular}{|c|c|c|c|c|c|c|c|c|c|c|c|c|c|c|}
\hline 89 & 89 & 81 & 86 & 109 & 128 & 125 & 120 & 111 & 102 & 102 & 86 & 92 & 101 & 102 \\
\hline 89 & 89 & 81 & 86 & 109 & 128 & 125 & 120 & 111 & 102 & 102 & 86 & 92 & 101 & 102 \\
\hline
\end{tabular}

-Exports to Pacific Southwest-

3 BPA to PASA C/N/X

4 BPA to PASA S/N/X

5 BPA to RVSD C/N/X

6 BPA to RVSD S/Pwr/X

7 PGE to GLEN PwrS

8 PGE to GLEN S/Pwr/X

9 PPL to SMUD PwrS

10 SCL to NCPA S/Pwr/X

11 SDGE to SDGE PwrS

12 TID to TID PwrS

13 BPA NW-SW Intertie Losses

14 Total Exports To PSW

\begin{tabular}{rrrrrrrrrrrrrrr}
4.5 & 4.5 & 4.5 & 0 & 0 & 0 & 0 & 0 & 0 & 0 & 0 & 0 & 0 & 4.5 & 1.1 \\
0 & 0 & 0 & 0 & 0 & 0 & 0 & 0 & 0 & 0 & 0 & 2.2 & 2.2 & 0 & 0.4 \\
11 & 11 & 11 & 11 & 2.7 & 2.7 & 2.7 & 2.7 & 2.7 & 2.7 & 2.7 & 0 & 0 & 11 & 4.9 \\
0 & 0 & 0 & 0 & 0 & 0 & 0 & 0 & 0 & 0 & 0 & 26 & 26 & 0 & 4.3 \\
20 & 20 & 20 & 20 & 20 & 20 & 20 & 20 & 20 & 20 & 20 & 20 & 20 & 20 & 20 \\
8.9 & 8.9 & 8.9 & 0 & 0 & 0 & 0 & 0 & 0 & 0 & 0 & 0 & 0 & 0 & 1.5 \\
40 & 40 & 40 & 40 & 40 & 40 & 40 & 40 & 40 & 40 & 40 & 40 & 40 & 40 & 40 \\
19 & 19 & 30 & 14 & 0 & 0 & 0 & 0 & 0 & 0 & 0 & 0 & 30 & 30 & 10 \\
59 & 59 & 59 & 59 & 59 & 59 & 59 & 59 & 59 & 59 & 59 & 31 & 59 & 59 & 57 \\
40 & 40 & 40 & 40 & 40 & 40 & 40 & 40 & 40 & 40 & 40 & 21 & 40 & 40 & 39 \\
0.5 & 0.5 & 0.5 & 0.3 & 0.1 & 0.1 & 0.1 & 0.1 & 0.1 & 0.1 & 0.1 & 0.8 & 0.8 & 0.5 & 0.3 \\
\hline 203 & $\mathbf{2 0 3}$ & $\mathbf{2 1 4}$ & $\mathbf{1 8 5}$ & 162 & $\mathbf{1 6 2}$ & $\mathbf{1 6 2}$ & 162 & 162 & 162 & 162 & $\mathbf{1 4 0}$ & $\mathbf{2 1 8}$ & $\mathbf{2 0 5}$ & $\mathbf{1 6 0}$
\end{tabular}

-Exports to Inland Southwest-

15 BPA to SPP PWS

16 LVFBR to WAPA PwrS

17 Total Exports To ISW

\begin{tabular}{|c|c|c|c|c|c|c|c|c|c|c|c|c|c|}
\hline 75 & 75 & 45 & 45 & 45 & 45 & 45 & 45 & 75 & 75 & 75 & 75 & 75 & 60 \\
\hline 36 & 36 & 36 & 36 & 36 & 36 & 36 & 36 & 36 & 36 & 36 & 36 & 36 & 36 \\
\hline 111 & 111 & 81 & 81 & 81 & 81 & 81 & 81 & 111 & 111 & 111 & 111 & 111 & 96 \\
\hline
\end{tabular}

-Exports to Canada-

18 BPA to BCHP CanEnt

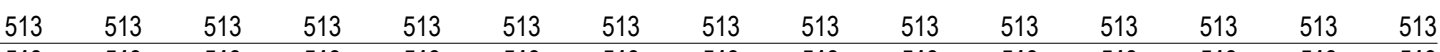

19 Total Exports To Canada

\begin{tabular}{|c|}
\hline 13 \\
\hline
\end{tabular}

-Exports to Other Entities-

20 Total Exports To Other Entities

0

$0 \quad 0$

0

-Total Exports-

21 Federal Entities

22 Public Entities

23 Investor-Owned Entities

24 Other Entities

25 Total Exports.

\begin{tabular}{|c|c|c|c|c|c|c|c|c|c|c|c|c|c|}
\hline 692 & 684 & 656 & 669 & 689 & 686 & 681 & 672 & 693 & 693 & 703 & 708 & 704 & 686 \\
\hline 59 & 70 & 55 & 40 & 40 & 40 & 40 & 40 & 40 & 40 & 21 & 70 & 70 & 49 \\
\hline 128 & 128 & 119 & 119 & 119 & 119 & 119 & 119 & 119 & 119 & 91 & 119 & 119 & 118 \\
\hline 36 & 36 & 36 & 36 & 36 & 36 & 36 & 36 & 36 & 36 & 36 & 36 & 36 & 36 \\
\hline 916 & 919 & 865 & 865 & 884 & 881 & 877 & 867 & 888 & 888 & 850 & 934 & 930 & 890 \\
\hline
\end{tabular}


Table A-2: Regional Exports

PNW Loads and Resources Study

2012 - 2013 Operating Year

2003 White Book

Aug1 Aug16 Sep Oct Nov Dec Jan Feb Mar Apr1 Apr16

May Jun Jul Avg

-Exports East of Continental Divide -

$1 \mathrm{BPA}$ to Other Entities

\begin{tabular}{|c|c|c|c|c|c|c|c|c|c|c|c|c|c|c|}
\hline 92 & 92 & 84 & 90 & 113 & 133 & 130 & 125 & 115 & 106 & 106 & 90 & 95 & 105 & 107 \\
\hline 92 & 92 & 84 & 90 & 113 & 133 & 130 & 125 & 115 & 106 & 106 & 90 & 95 & 105 & 107 \\
\hline
\end{tabular}

2 Total Exports to ECD

$\begin{array}{rrrrrrrrrrrrrrr}92 & 92 & \mathbf{8 4} & \mathbf{9 0} & \mathbf{1 1 3} & \mathbf{1 3 3} & \mathbf{1 3 0} & \mathbf{1 2 5} & \mathbf{1 1 5} & \mathbf{1 0 6} & \mathbf{1 0 6} & \mathbf{9 0} & \mathbf{9 5} & \mathbf{1 0 5} & \mathbf{1 0 7} \\ & & & & & & & & & & & & & & \\ 4.5 & 4.5 & 4.5 & 0 & 0 & 0 & 0 & 0 & 0 & 0 & 0 & 0 & 0 & 4.5 & 1.1 \\ 0 & 0 & 0 & 0 & 0 & 0 & 0 & 0 & 0 & 0 & 0 & 2.2 & 2.2 & 0 & 0.4 \\ 11 & 11 & 11 & 11 & 2.7 & 2.7 & 2.7 & 2.7 & 2.7 & 2.7 & 2.7 & 0 & 0 & 11 & 4.9 \\ 0 & 0 & 0 & 0 & 0 & 0 & 0 & 0 & 0 & 0 & 0 & 26 & 26 & 0 & 4.3 \\ 20 & 20 & 20 & 0 & 0 & 0 & 0 & 0 & 0 & 0 & 0 & 0 & 0 & 0 & 3.3 \\ 0 & 0 & 0 & 0 & 0 & 0 & 0 & 0 & 0 & 0 & 0 & 0 & 0 & 0 & 0 \\ 40 & 40 & 40 & 40 & 40 & 40 & 40 & 40 & 40 & 40 & 40 & 40 & 40 & 40 & 40 \\ 19 & 19 & 30 & 14 & 0 & 0 & 0 & 0 & 0 & 0 & 0 & 0 & 30 & 30 & 10 \\ 59 & 59 & 59 & 59 & 59 & 59 & 59 & 59 & 59 & 59 & 59 & 31 & 59 & 59 & 57 \\ 40 & 40 & 40 & 40 & 40 & 40 & 40 & 40 & 40 & 40 & 40 & 21 & 40 & 40 & 39 \\ 0.5 & 0.5 & 0.5 & 0.3 & 0.1 & 0.1 & 0.1 & 0.1 & 0.1 & 0.1 & 0.1 & 0.8 & 0.8 & 0.5 & 0.3 \\ 194 & 194 & \mathbf{2 0 5} & 165 & 142 & 142 & 142 & 142 & 142 & 142 & 142 & \mathbf{1 2 0} & \mathbf{1 9 8} & \mathbf{1 8 5} & \mathbf{1 6 0}\end{array}$

-Exports to Pacific Southwest-

3 BPA to PASA C/N/X

4 BPA to PASA S/N/X

5 BPA to RVSD C/N/X

6 BPA to RVSD S/Pwr/X

7 PGE to GLEN PwrS

8 PGE to GLEN S/Pwr/X

9 PPL to SMUD PwrS

10 SCL to NCPA S/Pwr/X

11 SDGE to SDGE PwrS

12 TID to TID PwrS

13 BPA NW-SW Intertie Losses

14 Total Exports To PSW

-Exports to Inland Southwest-

15 BPA to SPP PWS

16 LVFBR to WAPA PwrS

17 Total Exports To ISW

\begin{tabular}{|c|c|c|c|c|c|c|c|c|c|c|c|c|c|}
\hline 75 & 75 & 45 & 45 & 45 & 45 & 45 & 45 & 75 & 75 & 75 & 75 & 75 & 60 \\
\hline 36 & 36 & 36 & 36 & 36 & 36 & 36 & 36 & 36 & 36 & 36 & 36 & 36 & 36 \\
\hline 111 & 111 & 81 & 81 & 81 & 81 & 81 & 81 & 111 & 111 & 111 & 111 & 111 & 96 \\
\hline
\end{tabular}

-Exports to Canada-

18 BPA to BCHP CanEnt

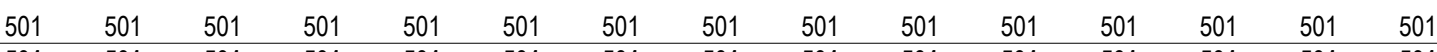

19 Total Exports To Canada

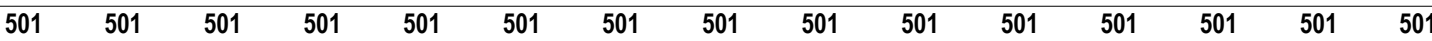

-Exports to Other Entities-

20 Total Exports To Other Entities

-Total Exports.

21 Federal Entities

22 Public Entities

23 Investor-Owned Entities

24 Other Entities

25 Total Exports.

$\begin{array}{lllllllllllllll}0 & 0 & 0 & 0 & 0 & 0 & 0 & 0 & 0 & 0 & 0 & 0 & 0 & 0 & 0\end{array}$

\begin{tabular}{|c|c|c|c|c|c|c|c|c|c|c|c|c|c|}
\hline 684 & 676 & 647 & 662 & 682 & 679 & 674 & 664 & 685 & 685 & 694 & 700 & 696 & 679 \\
\hline 59 & 70 & 55 & 40 & 40 & 40 & 40 & 40 & 40 & 40 & 21 & 70 & 70 & 49 \\
\hline 119 & 119 & 99 & 99 & 99 & 99 & 99 & 99 & 99 & 99 & 71 & 99 & 99 & 100 \\
\hline 36 & 36 & 36 & 36 & 36 & 36 & 36 & 36 & 36 & 36 & 36 & 36 & 36 & 36 \\
\hline 898 & 901 & 837 & 837 & 857 & 854 & 849 & 839 & 860 & 860 & 821 & 905 & 902 & 864 \\
\hline
\end{tabular}


Table A-2: Regional Exports

PNW Loads and Resources Study

2013 - 2014 Operating Year 2003 White Book

May Jun Jul Avg

-Exports East of Continental Divide -

1 BPA to Other Entities Dec Jan Feb Mar Apr1 Apr16

2 Total Exports to ECD

\begin{tabular}{|c|c|c|c|c|c|c|c|c|c|c|c|c|c|c|}
\hline 96 & 96 & 87 & 94 & 117 & 138 & 135 & 130 & 120 & 110 & 110 & 93 & 99 & 109 & 111 \\
\hline 96 & 96 & 87 & 94 & 117 & 138 & 135 & 130 & 120 & 110 & 110 & 93 & 99 & 109 & 111 \\
\hline
\end{tabular}

-Exports to Pacific Southwest-

3 BPA to PASA C/N/X

4 BPA to PASA S/N/X

5 BPA to RVSD C/N/X

6 BPA to RVSD S/Pwr/X

7 PPL to SMUD PwrS

8 SCL to NCPA S/Pwr/X

9 SDGE to SDGE PwrS

10 TID to TID PwrS

11 BPA NW-SW Intertie Losses

12 Total Exports To PSW

$\begin{array}{rrrrrrrrrrrrrrr}4.5 & 4.5 & 4.5 & 0 & 0 & 0 & 0 & 0 & 0 & 0 & 0 & 0 & 0 & 4.5 & 1.1 \\ 0 & 0 & 0 & 0 & 0 & 0 & 0 & 0 & 0 & 0 & 0 & 2.2 & 2.2 & 0 & 0.4 \\ 11 & 11 & 11 & 11 & 2.7 & 2.7 & 2.7 & 2.7 & 2.7 & 2.7 & 2.7 & 0 & 0 & 11 & 4.9 \\ 0 & 0 & 0 & 0 & 0 & 0 & 0 & 0 & 0 & 0 & 0 & 26 & 26 & 0 & 4.3 \\ 40 & 40 & 40 & 40 & 40 & 40 & 40 & 40 & 40 & 40 & 40 & 40 & 40 & 40 & 40 \\ 19 & 19 & 30 & 14 & 0 & 0 & 0 & 0 & 0 & 0 & 0 & 0 & 0 & 0 & 5.3 \\ 59 & 59 & 59 & 59 & 59 & 59 & 59 & 59 & 59 & 59 & 59 & 31 & 59 & 59 & 57 \\ 40 & 40 & 40 & 40 & 40 & 40 & 40 & 40 & 40 & 40 & 40 & 21 & 40 & 40 & 39 \\ 0.5 & 0.5 & 0.5 & 0.3 & 0.1 & 0.1 & 0.1 & 0.1 & 0.1 & 0.1 & 0.1 & 0.8 & 0.8 & 0.5 & 0.3 \\ 174 & 174 & 185 & 165 & 142 & 142 & 142 & 142 & 142 & 142 & 142 & 120 & 168 & 155 & 152\end{array}$

-Exports to Inland Southwest-

13 BPA to SPP PwrS

14 LVFBR to WAPA PwrS

15 Total Exports To ISW

\begin{tabular}{|c|c|c|c|c|c|c|c|c|c|c|c|c|c|}
\hline 75 & 75 & 45 & 45 & 45 & 45 & 45 & 45 & 75 & 75 & 75 & 75 & 75 & 60 \\
\hline 36 & 36 & 36 & 36 & 36 & 36 & 36 & 36 & 36 & 36 & 36 & 36 & 36 & 36 \\
\hline 111 & 111 & 81 & 81 & 81 & 81 & 81 & 81 & 111 & 111 & 111 & 111 & 111 & 96 \\
\hline
\end{tabular}

-Exports to Canada-

16 BPA to BCHP CanEnt

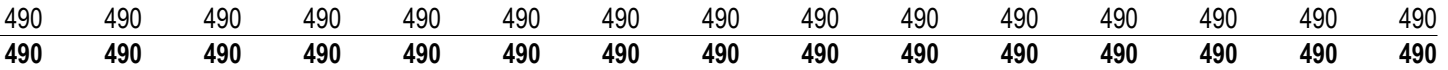

-Exports to Other Entities-

18 Total Exports To Other Entities

\section{0}

0

\begin{tabular}{|c|c|c|c|c|c|c|c|c|c|c|c|c|c|c|}
\hline 677 & 677 & 668 & 640 & 655 & 676 & 673 & 668 & 658 & 678 & 678 & 687 & 693 & 690 & 672 \\
\hline 59 & 59 & 70 & 55 & 40 & 40 & 40 & 40 & 40 & 40 & 40 & 21 & 40 & 40 & 44 \\
\hline 99 & 99 & 99 & 99 & 99 & 99 & 99 & 99 & 99 & 99 & 99 & 71 & 99 & 99 & 97 \\
\hline 36 & 36 & 36 & 36 & 36 & 36 & 36 & 36 & 36 & 36 & 36 & 36 & 36 & 36 & 36 \\
\hline 871 & 871 & 873 & 829 & 831 & 851 & 849 & 843 & 833 & 854 & 853 & 814 & 868 & 865 & 848 \\
\hline
\end{tabular}

-Total Exports-

19 Federal Entities

20 Public Entities

21 Investor-Owned Entities

22 Other Entities

23 Total Exports. 
2003 White Book

-Regulated Hydro Projects-

1 Albeni Falls

2 Alder

3 Bonneville

4 Boundary

5 Box Canyon

6 Brownlee

7 Cabinet Gorge

8 Chelan

9 Chief Joseph

10 Cushman 1

11 Cushman 2

12 Diablo

13 Dworshak

14 Faraday

15 Gorge

16 Grand Coulee

17 Hells Canyon

18 Hungry Horse

19 lce Harbor

20 John Day

21 La Grande

22 Libby

23 Little Falls

24 Little Goose

25 Long Lake

26 Lower Baker

27 Lower Granite

28 Lower Monumental

29 Mayfield

30 McNary

31 Merwin

32 Monroe Street

33 Mossyrock

34 Nine Mile

35 North Fork

36 Noxon

37 Oak Grove

38 Oxbow

39 Pelton

40 Post Falls

41 Priest Rapids

42 River Mill

43 Rock Island PH\#1

44 Rock Island PH\#2

45 Rocky Reach

46 Ross

47 Round Butte

48 Swift 1

49 Swift 2

50 The Dalles

51 Upper Baker

52 Upper Falls

53 Wanapum

54 Wells

55 White

56 Yale

Total Regulated Hydro w/Enc.
Sep

Oct
Nov Dec Jan Feb

\begin{tabular}{|c|c|c|c|c|c|c|c|c|c|c|c|c|c|c|}
\hline 23 & 18 & 27 & 37 & 18 & 18 & 16 & 14 & 17 & 18 & 18 & 28 & 45 & 38 & 25 \\
\hline 14 & 10 & 11 & 12 & 6 & 25 & 45 & 21 & 25 & 30 & 7.1 & 23 & 50 & 8 & 21 \\
\hline 170 & 136 & 396 & 463 & 519 & 543 & 455 & 446 & 462 & 289 & 133 & 132 & 181 & 130 & 341 \\
\hline 190 & 150 & 238 & 503 & 266 & 263 & 223 & 186 & 248 & 281 & 417 & 530 & 606 & 399 & 332 \\
\hline 31 & 25 & 37 & 69 & 41 & 41 & 35 & 30 & 38 & 44 & 60 & 72 & 74 & 55 & 48 \\
\hline 232 & 259 & 238 & 240 & 155 & 158 & 252 & 146 & 258 & 324 & 324 & 305 & 192 & 312 & 235 \\
\hline 51 & 39 & 41 & 65 & 74 & 72 & 66 & 50 & 64 & 52 & 85 & 176 & 188 & 112 & 85 \\
\hline 31 & 23 & 20 & 55 & 55 & 55 & 54 & 54 & 54 & 52 & 32 & 9.9 & 51 & 57 & 44 \\
\hline 1729 & 1297 & 803 & 1038 & 1264 & 1244 & 945 & 891 & 885 & 890 & 897 & 846 & 1118 & 1300 & 1062 \\
\hline 3.3 & 2.3 & 19 & 1.8 & 27 & 25 & 29 & 20 & 1.1 & 1.3 & 24 & 1.3 & 28 & 1.3 & 14 \\
\hline 2.4 & 0.7 & 30 & 0.1 & 49 & 50 & 66 & 55 & 0.4 & 0.2 & 59 & 0.1 & 63 & 0 & 29 \\
\hline 74 & 56 & 76 & 35 & 106 & 102 & 1.5 & 15 & 72 & 91 & 71 & 74 & 172 & 154 & 79 \\
\hline 438 & 203 & 51 & 51 & 51 & 51 & 51 & 50 & 50 & 51 & 53 & 56 & 284 & 440 & 126 \\
\hline 8.1 & 7.5 & 8.2 & 8 & 6.2 & 16 & 8.9 & 17 & 31 & 43 & 42 & 43 & 39 & 14 & 20 \\
\hline 86 & 68 & 87 & 43 & 118 & 118 & 2.7 & 19 & 86 & 109 & 86 & 92 & 182 & 168 & 91 \\
\hline 3226 & 2372 & 1472 & 1952 & 2354 & 2248 & 1733 & 1654 & 1576 & 1499 & 1473 & 1456 & 2074 & 2457 & 1938 \\
\hline 193 & 218 & 202 & 208 & 133 & 127 & 198 & 114 & 194 & 244 & 245 & 239 & 152 & 246 & 189 \\
\hline 91 & 80 & 76 & 85 & 90 & 90 & 92 & 92 & 88 & 67 & 28 & 29 & 30 & 118 & 77 \\
\hline 94 & 65 & 127 & 145 & 101 & 108 & 130 & 111 & 190 & 131 & 137 & 233 & 175 & 126 & 138 \\
\hline 912 & 688 & 661 & 794 & 915 & 901 & 734 & 710 & 814 & 775 & 714 & 750 & 891 & 843 & 797 \\
\hline 21 & 15 & 15 & 18 & 8.3 & 39 & 63 & 44 & 56 & 62 & 12 & 37 & 63 & 12 & 34 \\
\hline 519 & 354 & 113 & 153 & 88 & 298 & 81 & 79 & 78 & 77 & 77 & 172 & 292 & 143 & 168 \\
\hline 9.3 & 9.2 & 15 & 14 & 13 & 21 & 8 & 11 & 24 & 31 & 30 & 30 & 34 & 14 & 19 \\
\hline 235 & 174 & 138 & 145 & 102 & 106 & 132 & 97 & 183 & 259 & 313 & 528 & 396 & 295 & 218 \\
\hline 22 & 22 & 34 & 34 & 30 & 46 & 18 & 24 & 51 & 69 & 72 & 72 & 72 & 33 & 42 \\
\hline 29 & 23 & 22 & 24 & 30 & 60 & 56 & 11 & 28 & 39 & 37 & 1.3 & 67 & 59 & 35 \\
\hline 241 & 178 & 145 & 143 & 103 & 108 & 134 & 98 & 185 & 252 & 321 & 533 & 406 & 303 & 221 \\
\hline 237 & 176 & 132 & 147 & 101 & 108 & 131 & 112 & 194 & 275 & 329 & 535 & 409 & 305 & 224 \\
\hline 28 & 25 & 41 & 55 & 55 & 55 & 172 & 85 & 70 & 70 & 171 & 8.4 & 82 & 47 & 68 \\
\hline 723 & 687 & 455 & 546 & 600 & 606 & 506 & 475 & 539 & 406 & 266 & 292 & 415 & 745 & 518 \\
\hline 18 & 15 & 16 & 28 & 67 & 83 & 103 & 35 & 66 & 109 & 92 & 13 & 71 & 24 & 52 \\
\hline 5.5 & 5.8 & 12 & 12 & 9.9 & 15 & 5.1 & 6.7 & 15 & 15 & 15 & 15 & 15 & 11 & 11 \\
\hline 56 & 48 & 74 & 91 & 92 & 68 & 286 & 75 & 52 & 55 & 207 & 0 & 129 & 85 & 95 \\
\hline 5.6 & 5.7 & 10 & 10 & 9.3 & 11 & 4.6 & 7 & 15 & 23 & 23 & 22 & 24 & 10 & 13 \\
\hline 9 & 8.4 & 9.1 & 8.9 & 7 & 18 & 9.9 & 18 & 35 & 54 & 47 & 54 & 43 & 15 & 23 \\
\hline 69 & 57 & 58 & 99 & 114 & 110 & 101 & 72 & 96 & 86 & 116 & 286 & 308 & 158 & 131 \\
\hline 21 & 20 & 24 & 28 & 17 & 20 & 17 & 18 & 24 & 34 & 34 & 34 & 34 & 25 & 25 \\
\hline 101 & 114 & 106 & 109 & 69 & 65 & 103 & 58 & 100 & 125 & 125 & 120 & 75 & 128 & 97 \\
\hline 36 & 35 & 35 & 31 & 31 & 34 & 38 & 35 & 35 & 33 & 54 & 36 & 39 & 36 & 36 \\
\hline 2.2 & 2.5 & 6 & 6.5 & 5.4 & 7.6 & 2.9 & 3.6 & 10 & 15 & 14 & 14 & 15 & 5.2 & 7.8 \\
\hline 385 & 301 & 347 & 452 & 541 & 533 & 416 & 389 & 389 & 55 & 55 & 55 & 212 & 317 & 337 \\
\hline 4.6 & 4.3 & 4.7 & 4.6 & 3.6 & 9.2 & 5.1 & 9.5 & 18 & 23 & 23 & 23 & 22 & 7.7 & 11 \\
\hline 174 & 187 & 144 & 171 & 185 & 184 & 163 & 156 & 156 & 159 & 171 & 175 & 189 & 193 & 172 \\
\hline 197 & 146 & 101 & 134 & 162 & 159 & 122 & 113 & 113 & 96 & 42 & 27 & 120 & 104 & 116 \\
\hline 827 & 660 & 420 & 547 & 656 & 646 & 504 & 474 & 475 & 476 & 416 & 394 & 642 & 627 & 548 \\
\hline 52 & 40 & 70 & 33 & 121 & 107 & 0 & 17 & 71 & 90 & 64 & 61 & 236 & 151 & 83 \\
\hline 91 & 87 & 88 & 76 & 76 & 85 & 94 & 85 & 86 & 80 & 138 & 89 & 100 & 89 & 89 \\
\hline 31 & 27 & 24 & 34 & 131 & 74 & 72 & 26 & 45 & 154 & 14 & 19 & 152 & 47 & 61 \\
\hline 8.6 & 7.3 & 6.8 & 9.8 & 42 & 27 & 32 & 12 & 20 & 69 & 5.9 & 7 & 46 & 13 & 22 \\
\hline 610 & 457 & 539 & 622 & 715 & 714 & 592 & 578 & 654 & 572 & 481 & 503 & 599 & 562 & 595 \\
\hline 29 & 23 & 20 & 21 & 29 & 47 & 31 & 8.9 & 22 & 34 & 32 & 0.3 & 103 & 60 & 34 \\
\hline 4.9 & 5.2 & 9.8 & 9.8 & 8.8 & 10 & 4.6 & 5.8 & 10 & 9.5 & 8.6 & 8.3 & 9.3 & 9.6 & 8.4 \\
\hline 484 & 351 & 290 & 398 & 497 & 488 & 359 & 333 & 333 & 229 & 229 & 229 & 395 & 375 & 362 \\
\hline 543 & 430 & 269 & 346 & 420 & 412 & 316 & 296 & 294 & 297 & 275 & 267 & 407 & 405 & 350 \\
\hline 27 & 20 & 14 & 10 & 5.9 & 34 & 28 & 18 & 36 & 47 & 53 & 53 & 64 & 48 & 32 \\
\hline 23 & 20 & 22 & 24 & 94 & 87 & 107 & 32 & 60 & 106 & 91 & 0 & 112 & 30 & 57 \\
\hline 3476 & 10457 & 8449 & 10398 & 11587 & 11753 & 9953 & 8614 & 9793 & 9578 & 9358 & 9811 & 12989 & 12472 & 10605 \\
\hline
\end{tabular}

-Total Regulated Hydro w/Enc.-

57 Federal System

58 Public Entities

10457

$8449 \quad 1039$

$11587 \quad 11753 \quad 9953$

59 Investor-Owned Entities

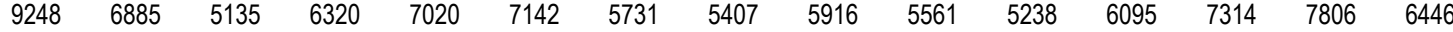

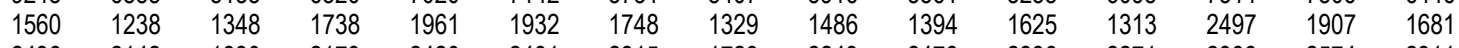

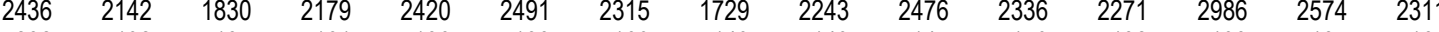

60 Other Entities

$\begin{array}{lllllll}13476 & 10457 & 8449 & 10398 & 11587 & 11753 & 9953\end{array}$

$8614-9793$

$\begin{array}{rrrrrr}9578 & 9358 & 9811 & 12989 & 12472 & 10605\end{array}$ 
2003 White Book

Average Energy in Megawatts

Aug1 Aug16

Sep

Oct

\begin{abstract}
Dec
\end{abstract}

\begin{tabular}{|c|c|c|c|c|c|c|c|c|c|c|c|c|c|c|}
\hline 23 & 18 & 27 & 37 & 18 & 18 & 16 & 14 & 17 & 18 & 18 & 28 & 45 & 38 & 25 \\
\hline 14 & 10 & 11 & 12 & 6 & 25 & 45 & 21 & 25 & 30 & 7.1 & 23 & 50 & 8 & 21 \\
\hline 171 & 137 & 398 & 465 & 521 & 546 & 457 & 448 & 463 & 291 & 133 & 133 & 182 & 131 & 343 \\
\hline 190 & 150 & 238 & 503 & 266 & 263 & 223 & 186 & 248 & 281 & 417 & 530 & 606 & 399 & 332 \\
\hline 31 & 25 & 37 & 69 & 41 & 41 & 35 & 30 & 38 & 44 & 60 & 72 & 74 & 55 & 48 \\
\hline 232 & 259 & 238 & 240 & 155 & 158 & 252 & 146 & 258 & 324 & 324 & 305 & 192 & 312 & 235 \\
\hline 51 & 39 & 41 & 65 & 74 & 72 & 66 & 50 & 64 & 52 & 85 & 176 & 188 & 112 & 85 \\
\hline 31 & 23 & 20 & 55 & 55 & 55 & 54 & 54 & 54 & 52 & 32 & 9.9 & 51 & 57 & 44 \\
\hline 1736 & 1302 & 806 & 1042 & 1270 & 1249 & 949 & 894 & 889 & 894 & 901 & 850 & 1122 & 1306 & 1066 \\
\hline 3.3 & 2.3 & 19 & 1.8 & 27 & 25 & 29 & 20 & 1.1 & 1.3 & 24 & 1.3 & 28 & 1.3 & 14 \\
\hline 2.4 & 0.7 & 30 & 0.1 & 49 & 50 & 66 & 55 & 0.4 & 0.2 & 59 & 0.1 & 63 & 0 & 29 \\
\hline 74 & 56 & 76 & 35 & 106 & 102 & 1.5 & 15 & 72 & 91 & 71 & 74 & 172 & 154 & 79 \\
\hline 440 & 204 & 52 & 52 & 51 & 51 & 51 & 50 & 51 & 51 & 53 & 56 & 285 & 442 & 126 \\
\hline 8.1 & 7.5 & 8.2 & 8 & 6.2 & 16 & 8.9 & 17 & 31 & 43 & 42 & 43 & 39 & 14 & 20 \\
\hline 86 & 68 & 87 & 43 & 118 & 118 & 2.7 & 19 & 86 & 109 & 86 & 92 & 182 & 168 & 91 \\
\hline 3246 & 2387 & 1482 & 1965 & 2370 & 2264 & 1745 & 1665 & 1586 & 1509 & 1483 & 1466 & 2089 & 2474 & 1952 \\
\hline 193 & 218 & 202 & 208 & 133 & 127 & 198 & 114 & 194 & 244 & 245 & 239 & 152 & 246 & 189 \\
\hline 92 & 80 & 76 & 85 & 90 & 90 & 92 & 92 & 88 & 67 & 28 & 29 & 30 & 119 & 77 \\
\hline 93 & 64 & 125 & 143 & 100 & 106 & 128 & 110 & 188 & 130 & 136 & 232 & 174 & 125 & 137 \\
\hline 915 & 691 & 664 & 798 & 919 & 905 & 738 & 713 & 818 & 779 & 717 & 753 & 894 & 846 & 800 \\
\hline 21 & 15 & 15 & 18 & 8.3 & 39 & 63 & 44 & 56 & 62 & 12 & 37 & 63 & 12 & 34 \\
\hline 520 & 355 & 113 & 154 & 90 & 298 & 81 & 79 & 78 & 77 & 77 & 172 & 293 & 145 & 168 \\
\hline 9.3 & 9.2 & 15 & 14 & 13 & 21 & 8 & 11 & 24 & 31 & 30 & 30 & 34 & 14 & 19 \\
\hline 232 & 172 & 136 & 143 & 101 & 105 & 130 & 96 & 180 & 255 & 309 & 522 & 391 & 286 & 215 \\
\hline 22 & 22 & 34 & 34 & 30 & 46 & 18 & 24 & 51 & 69 & 72 & 72 & 72 & 33 & 42 \\
\hline 29 & 23 & 22 & 24 & 30 & 60 & 56 & 11 & 28 & 39 & 37 & 1.3 & 67 & 59 & 35 \\
\hline 238 & 176 & 143 & 141 & 101 & 106 & 132 & 97 & 183 & 249 & 317 & 526 & 401 & 293 & 218 \\
\hline 234 & 173 & 130 & 145 & 100 & 106 & 130 & 110 & 192 & 272 & 325 & 529 & 404 & 295 & 220 \\
\hline 28 & 25 & 41 & 55 & 55 & 55 & 172 & 85 & 70 & 70 & 171 & 8.4 & 82 & 47 & 68 \\
\hline 726 & 689 & 457 & 548 & 603 & 609 & 508 & 477 & 541 & 408 & 268 & 294 & 417 & 748 & 521 \\
\hline 18 & 15 & 16 & 28 & 67 & 83 & 103 & 35 & 66 & 109 & 92 & 13 & 71 & 24 & 52 \\
\hline 5.5 & 5.8 & 12 & 12 & 9.9 & 15 & 5.1 & 6.7 & 15 & 15 & 15 & 15 & 15 & 11 & 11 \\
\hline 56 & 48 & 74 & 91 & 92 & 68 & 286 & 75 & 52 & 55 & 207 & 0 & 129 & 85 & 95 \\
\hline 5.6 & 5.7 & 10 & 10 & 9.3 & 11 & 4.6 & 7 & 15 & 23 & 23 & 22 & 24 & 10 & 13 \\
\hline 9 & 8.4 & 9.1 & 8.9 & 7 & 18 & 9.9 & 18 & 35 & 54 & 47 & 54 & 43 & 15 & 23 \\
\hline 69 & 57 & 58 & 99 & 114 & 110 & 101 & 72 & 96 & 86 & 116 & 286 & 308 & 158 & 131 \\
\hline 21 & 20 & 24 & 28 & 17 & 20 & 17 & 18 & 24 & 34 & 34 & 34 & 34 & 25 & 25 \\
\hline 101 & 114 & 106 & 109 & 69 & 65 & 103 & 58 & 100 & 125 & 125 & 120 & 75 & 128 & 97 \\
\hline 36 & 35 & 35 & 31 & 31 & 34 & 38 & 35 & 35 & 33 & 54 & 36 & 39 & 36 & 36 \\
\hline 2.2 & 2.5 & 6 & 6.5 & 5.4 & 7.6 & 2.9 & 3.6 & 10 & 15 & 14 & 14 & 15 & 5.2 & 7.8 \\
\hline 385 & 301 & 347 & 452 & 541 & 533 & 416 & 389 & 389 & 55 & 55 & 55 & 212 & 317 & 337 \\
\hline 4.6 & 4.3 & 4.7 & 4.6 & 3.6 & 9.2 & 5.1 & 9.5 & 18 & 23 & 23 & 23 & 22 & 7.7 & 11 \\
\hline 174 & 187 & 144 & 171 & 185 & 184 & 163 & 156 & 156 & 159 & 171 & 175 & 189 & 193 & 172 \\
\hline 197 & 146 & 101 & 134 & 162 & 159 & 122 & 113 & 113 & 96 & 42 & 27 & 120 & 104 & 116 \\
\hline 827 & 660 & 420 & 547 & 656 & 646 & 504 & 474 & 475 & 476 & 416 & 394 & 642 & 627 & 548 \\
\hline 52 & 40 & 70 & 33 & 121 & 107 & 0 & 17 & 71 & 90 & 64 & 61 & 236 & 151 & 83 \\
\hline 91 & 87 & 88 & 76 & 76 & 85 & 94 & 85 & 86 & 80 & 138 & 89 & 100 & 89 & 89 \\
\hline 31 & 27 & 24 & 34 & 131 & 74 & 72 & 26 & 45 & 154 & 14 & 19 & 152 & 47 & 61 \\
\hline 8.6 & 7.3 & 6.8 & 9.8 & 42 & 27 & 32 & 12 & 20 & 69 & 5.9 & 7 & 46 & 13 & 22 \\
\hline 612 & 459 & 542 & 625 & 718 & 717 & 595 & 580 & 656 & 575 & 483 & 505 & 601 & 564 & 597 \\
\hline 29 & 23 & 20 & 21 & 29 & 47 & 31 & 8.9 & 22 & 34 & 32 & 0.3 & 103 & 60 & 34 \\
\hline 4.9 & 5.2 & 9.8 & 9.8 & 8.8 & 10 & 4.6 & 5.8 & 10 & 9.5 & 8.6 & 8.3 & 9.3 & 9.6 & 8.4 \\
\hline 484 & 351 & 290 & 398 & 497 & 488 & 359 & 333 & 333 & 229 & 229 & 229 & 395 & 375 & 362 \\
\hline 543 & 430 & 269 & 346 & 420 & 412 & 316 & 296 & 294 & 297 & 275 & 267 & 407 & 405 & 350 \\
\hline 27 & 20 & 14 & 10 & 5.9 & 34 & 28 & 18 & 36 & 47 & 53 & 53 & 64 & 48 & 32 \\
\hline 23 & 20 & 22 & 24 & 94 & 87 & 107 & 32 & 60 & 106 & 91 & 0 & 112 & 30 & 57 \\
\hline 13506 & 10480 & 8466 & 10420 & 11617 & 11782 & 9974 & 8634 & 9808 & 9594 & 9368 & 9812 & 13004 & 12477 & 10622 \\
\hline 9278 & 6907 & 5151 & 6342 & 7050 & 7171 & 5752 & 5427 & 5931 & 5577 & 5248 & 6096 & 7328 & 7811 & 6464 \\
\hline
\end{tabular}

-Regulated Hydro Projects-

1 Albeni Falls

3 Bonneville

4 Boundary

5 Box Canyon

6 Brownlee

7 Cabinet Gorge

8 Chelan

9 Chief Joseph

10 Cushman 1

11 Cushman 2

12 Diablo

13 Dworshak

14 Faraday

15 Gorge

16 Grand Coulee

17 Hells Canyon

18 Hungry Horse

19 Ice Harbor

20 John Day

21 La Grande

22 Libby

23 Little Falls

24 Little Goose

25 Long Lake

26 Lower Baker

27 Lower Granite

28 Lower Monumental

29 Mayfield

30 McNary

31 Merwin

32 Monroe Street

33 Mossyrock

34 Nine Mile

35 North Fork

36 Noxon

37 Oak Grove

38 Oxbow

39 Pelton

40 Post Falls

41 Priest Rapids

42 River Mill

43 Rock Island PH\#1

44 Rock Island PH\#2

45 Rocky Reach

46 Ross

47 Round Butte

48 Swift 1

49 Swift 2

50 The Dalles

51 Upper Baker

52 Upper Falls

53 Wanapum

54 Wells

55 White

56 Yale

$13506 \quad 10480 \quad 8466 \quad 10420$

1617

$8634 \quad 9808$

95949368

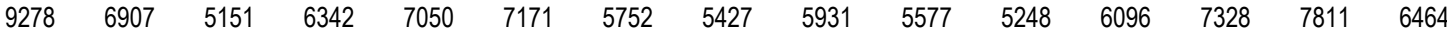
$\begin{array}{lllllllllllllll}1560 & 1238 & 1348 & 1738 & 2081 & 2050 & 1840 & 1415 & 1572 & 1406 & 1637 & 1326 & 2544 & 1977 & 1734\end{array}$

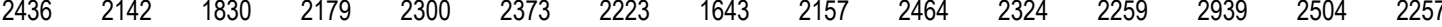

-Total Regulated Hydro w/Enc.-

57 Federal System

58 Public Entities

59 Investor-Owned Entities

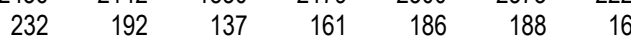

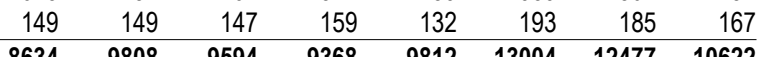

13506 
2003 White Book

-Regulated Hydro Projects-

1 Albeni Falls

2 Alder

3 Bonneville

4 Boundary

5 Box Canyon

6 Brownlee

7 Cabinet Gorge

8 Chelan

9 Chief Joseph

10 Cushman 1

11 Cushman 2

12 Diablo

13 Dworshak

14 Faraday

15 Gorge

16 Grand Coulee

17 Hells Canyon

18 Hungry Horse

19 lce Harbor

20 John Day

$21 \mathrm{La}$ Grande

22 Libby

23 Little Falls

24 Little Goose

25 Long Lake

26 Lower Baker

27 Lower Granite

28 Lower Monumental

29 Mayfield

30 McNary

31 Merwin

32 Monroe Street

33 Mossyrock

34 Nine Mile

35 North Fork

36 Noxon

37 Oak Grove

38 Oxbow

39 Pelton

40 Post Falls

41 Priest Rapids

42 River Mill

44 Rock Island PH\#2

45 Rocky Reach

46 Ross

48 Swift 1

49 Swift 2

50 The Dalles

51 Upper Baker

52 Upper Falls

53 Wanapum

54 Wells

55 White

56 Yale

Total Regulated Hydro w/Enc.
43 Rock Island PH\#1

47 Round Butte
Sep

Oct
$2.4 \quad 0.7$

$\begin{array}{rr}74 & 56 \\ 441 & 204\end{array}$

$\begin{array}{rr}8.1 & 7.5 \\ 86 & 68\end{array}$

$\begin{array}{rr}3261 & 2398 \\ 193 & 218\end{array}$

92
93 6

$916 \quad 692$

$\begin{array}{rr}21 & 15 \\ 521 & 355\end{array}$

$\begin{array}{rr}521 & 355 \\ 9.3 & 9.2\end{array}$

228

22

29

$233 \quad 174$

$230 \quad 171$

$\begin{array}{rr}28 & 25 \\ 735 & 697\end{array}$

$\begin{array}{rr}18 & 15 \\ 5.5 & 5.8\end{array}$

$56 \quad 48$

$5.6 \quad 5.7$

$\begin{array}{rr}9 & 8.4 \\ 69 & 57\end{array}$

$\begin{array}{rr}21 & 20 \\ 101 & 114\end{array}$

$36 \quad 35$

$\begin{array}{rr}2.2 & 2.5 \\ 385 & 301\end{array}$

$\begin{array}{rr}385 & 301 \\ 4.6 & 4.3\end{array}$

$174 \quad 187$

$\begin{array}{lllllll}197 & 146 & 144 & 171 & 185 & 184 & 163\end{array}$

$\begin{array}{lllllll}827 & 660 & 420 & 547 & 656 & 646 & 504\end{array}$

$\begin{array}{llllll}52 & 40 & 70 & 33 & 121 & 107\end{array}$

$\begin{array}{rrrrrrr}91 & 87 & 88 & 76 & 76 & 85 & 94\end{array}$

$31 \quad 27$

$\begin{array}{rrrrrrr}8.6 & 7.3 & 6.8 & 9.8 & 42 & 27 & 32\end{array}$

$\begin{array}{rrrrrrr}613 & 460 & 543 & 626 & 719 & 718 & 596 \\ 29 & 23 & 20 & 21 & 29 & 47 & 31\end{array}$

$\begin{array}{llllll}4.9 & 5.2 & 9.8 & 9.8 & 8.8 & 10\end{array}$

$\begin{array}{lllllll}484 & 351 & 290 & 398 & 497 & 488 & 359\end{array}$

$\begin{array}{lllllll}543 & 430 & 269 & 346 & 420 & 412 & 316\end{array}$

$\begin{array}{rrrr}23 & 18 & 27 & 37 \\ 14 & 10 & 11 & 12 \\ 171 & 137 & 399 & 466 \\ 190 & 150 & 238 & 503 \\ 31 & 25 & 37 & 69 \\ 232 & 259 & 238 & 240 \\ 51 & 39 & 41 & 65 \\ 31 & 23 & 20 & 55 \\ 1746 & 1310 & 811 & 1048\end{array}$

Feb
Mar Apr1 Apr16

May

Jun

Jul Avg

-Total Regulated Hydro w/Enc.-

57 Federal System

58 Public Entities

59 Investor-Owned Entities

60 Other Entities
$23 \quad 20$

\section{25}

18
30
292

18
7.1
133

$\begin{array}{rrrr}28 & 45 & 38 & 25 \\ 23 & 50 & 8 & 21 \\ 133 & 182 & 131 & 343 \\ 530 & 606 & 399 & 332 \\ 72 & 74 & 55 & 48\end{array}$

$\begin{array}{rr}44 & 60 \\ 324 & 324\end{array}$

$\begin{array}{rr}52 & 32 \\ 900 & 906\end{array}$

900

0.2

9

51

43

31
86

1594

1594
194

109

1516
244

244
67

130
781

819

56

78
24

77

31

256

310

51

28

184

193

39
250

250
273

70

415

109

15

$55 \quad 207$

$\begin{array}{ll}55 & 207 \\ 23 & 23\end{array}$

$54 \quad 47$

$86 \quad 116$

$34 \quad 34$

125

33

15

55

23
159

159

96
476

476

$\begin{array}{rr}476 & 416 \\ 90 & 64\end{array}$

$\begin{array}{rr}80 & 138\end{array}$

$154 \quad 14$

$\begin{array}{rr}154 & 14 \\ 69 & 5.9\end{array}$

$576 \quad 484$

$\begin{array}{rr}576 & 484 \\ 34 & 32\end{array}$

$9.5 \quad 8.6$

$\begin{array}{ll}229 & 229 \\ 297 & 275\end{array}$

$\begin{array}{rrr}5.9 & 34 & 28 \\ 94 & 87 & 107\end{array}$

$8489 \quad 10448$

11651

$11816 \quad 1000$

$\begin{array}{rrrr}305 & 192 & 312 & 235\end{array}$

$\begin{array}{llll}176 & 188 & 112 & 85\end{array}$

$\begin{array}{llrr}9.9 & 51 & 57 & 44\end{array}$

$\begin{array}{llll}855 & 1130 & 1314 & 1073\end{array}$

1.3
0.1

74
56

28

63

172
285

$\begin{array}{rr}1.3 & 14 \\ 0 & 29\end{array}$

$154 \quad 79$

$\begin{array}{rr}154 & 79 \\ 443 & 126\end{array}$

$\begin{array}{rrrr}43 & 39 & 14 & 20 \\ 92 & 182 & 168 & 91\end{array}$

$\begin{array}{llll}1473 & 2100 & 2486 & 1961\end{array}$

$\begin{array}{llll}239 & 152 & 246 & 189\end{array}$

$\begin{array}{rrrr}29 & 30 & 119 & 77 \\ 232 & 174 & 125 & 137\end{array}$

$\begin{array}{llll}754 & 896 & 847 & 801\end{array}$

$\begin{array}{rrrr}37 & 63 & 12 & 34\end{array}$

$\begin{array}{llll}173 & 293 & 146 & 169\end{array}$

$\begin{array}{rrrr}523 & 34 & 14 & 19 \\ 792 & 72 & 33 & 42\end{array}$

$\begin{array}{llll}72 & 72 & 33 & 42\end{array}$

$\begin{array}{llll}1.3 & 67 & 59 & 35\end{array}$

$\begin{array}{llll}528 & 402 & 293 & 218\end{array}$

$\begin{array}{llll}531 & 406 & 295 & 221\end{array}$

$\begin{array}{rrrr}8.4 & 82 & 47 & 68 \\ 298 & 423 & 758 & 527\end{array}$

$\begin{array}{lll}13 & 71 & 24\end{array}$

$\begin{array}{llll}15 & 15 & 11 & 11\end{array}$

$\begin{array}{rrrr}0 & 129 & 85 & 95 \\ 22 & 24 & 10 & 13\end{array}$

$\begin{array}{llll}54 & 43 & 15 & 23\end{array}$

$\begin{array}{llll}286 & 308 & 158 & 131\end{array}$

$\begin{array}{llll}34 & 34 & 25 & 25\end{array}$

$\begin{array}{rrrr}120 & 75 & 128 & 97\end{array}$

$\begin{array}{rrrr}14 & 15 & 5.2 & 7.8\end{array}$

$\begin{array}{llll}55 & 212 & 317 & 337\end{array}$

$\begin{array}{llll}23 & 22 & 7.7 & 11\end{array}$

$\begin{array}{llll}175 & 189 & 193 & 172\end{array}$

$\begin{array}{rrrr}27 & 120 & 104 & 116 \\ 394 & 642 & 627 & 548\end{array}$

$\begin{array}{llll}61 & 236 & 151 & 83\end{array}$

$\begin{array}{llll}89 & 100 & 89 & 89\end{array}$

$\begin{array}{llll}19 & 152 & 47 & 61\end{array}$

$\begin{array}{rrrr}7 & 46 & 13 & 22 \\ 506 & 602 & 565 & 598\end{array}$

$\begin{array}{llll}0.3 & 103 & 60 & 34\end{array}$

$\begin{array}{llll}8.3 & 9.3 & 9.6 & 8.4\end{array}$

$\begin{array}{llll}229 & 395 & 375 & 362\end{array}$

$\begin{array}{llll}267 & 407 & 405 & 350\end{array}$
$52 \quad 85$

\begin{tabular}{|c|c|c|c|c|c|c|c|c|c|c|c|c|c|}
\hline 6930 & 5175 & 6370 & 7083 & 7205 & 5778 & 5451 & 5956 & 5604 & 5272 & 6121 & 7360 & 7846 & 6492 \\
\hline 1305 & 1424 & 1838 & 208 & 2050 & 1840 & 1415 & 1572 & 140 & 1637 & 1326 & 25 & 1977 & \\
\hline 207 & 1753 & 207 & 230 & 237 & 222 & 1643 & 21 & 246 & 23 & 2259 & 9 & 2504 & \\
\hline 19 & 137 & 161 & 186 & 188 & 160 & 149 & 149 & 147 & 159 & 132 & 193 & 185 & \\
\hline 507 & 188 & 448 & 451 & 1816 & 0000 & 6658 & 834 & 621 & 392 & 9837 & 3036 & 2512 & \\
\hline
\end{tabular}

61 Total Regulated Hydro w/Enc. 
2003 White Book

Average Energy in Megawatts

Aug1 Aug16

Sep

Oct

\begin{abstract}
Dec Jan
Feb
\end{abstract}

\begin{tabular}{|c|c|c|c|c|c|c|c|c|c|c|c|c|c|c|}
\hline 23 & 19 & 27 & 37 & 18 & 18 & 16 & 14 & 17 & 18 & 18 & 29 & 45 & 38 & 25 \\
\hline 14 & 10 & 11 & 12 & 6 & 25 & 45 & 21 & 25 & 30 & 7.1 & 23 & 50 & 8 & 21 \\
\hline 172 & 138 & 401 & 468 & 524 & 549 & 460 & 451 & 465 & 295 & 134 & 134 & 183 & 132 & 345 \\
\hline 190 & 150 & 238 & 503 & 266 & 263 & 223 & 186 & 248 & 281 & 417 & 530 & 606 & 399 & 332 \\
\hline 31 & 25 & 37 & 69 & 41 & 41 & 35 & 30 & 38 & 44 & 60 & 72 & 74 & 55 & 48 \\
\hline 232 & 259 & 238 & 240 & 155 & 158 & 252 & 146 & 258 & 324 & 324 & 305 & 192 & 312 & 235 \\
\hline 51 & 39 & 41 & 65 & 74 & 72 & 66 & 50 & 64 & 52 & 85 & 176 & 188 & 112 & 85 \\
\hline 31 & 23 & 20 & 55 & 55 & 55 & 54 & 54 & 54 & 52 & 32 & 9.9 & 51 & 57 & 44 \\
\hline 1769 & 1327 & 823 & 1064 & 1297 & 1277 & 970 & 913 & 908 & 913 & 920 & 867 & 1147 & 1332 & 1089 \\
\hline 3.3 & 2.3 & 19 & 1.8 & 27 & 25 & 29 & 20 & 1.1 & 1.3 & 24 & 1.3 & 28 & 1.3 & 14 \\
\hline 2.4 & 0.7 & 30 & 0.1 & 49 & 50 & 66 & 55 & 0.4 & 0.2 & 59 & 0.1 & 63 & 0 & 29 \\
\hline 74 & 56 & 76 & 35 & 106 & 102 & 1.5 & 15 & 72 & 91 & 71 & 74 & 172 & 154 & 79 \\
\hline 443 & 205 & 52 & 52 & 52 & 51 & 51 & 51 & 51 & 52 & 53 & 56 & 287 & 445 & 127 \\
\hline 8.1 & 7.5 & 8.2 & 8 & 6.2 & 16 & 8.9 & 17 & 31 & 43 & 42 & 43 & 39 & 14 & $\angle 0$ \\
\hline 86 & 68 & 87 & 43 & 118 & 118 & 2.7 & 19 & 86 & 109 & 86 & 92 & 182 & 168 & $y$ \\
\hline 3281 & 2414 & 1500 & 1989 & 2399 & 2293 & 1767 & 1685 & 1605 & 1527 & 1502 & 1484 & 2116 & 2503 & 1975 \\
\hline 193 & 218 & 202 & 208 & 133 & 127 & 198 & 114 & 194 & 244 & 245 & 239 & 152 & 246 & 189 \\
\hline 92 & 81 & 77 & 86 & 90 & 91 & 93 & 93 & 89 & 67 & 28 & 29 & 31 & 120 & 78 \\
\hline 93 & 64 & 124 & 142 & 99 & 105 & 127 & 109 & 186 & 130 & 136 & 232 & 173 & 124 & 136 \\
\hline 920 & 695 & 668 & 803 & 925 & 911 & 742 & 718 & 823 & 785 & 722 & 757 & 899 & 851 & 805 \\
\hline 21 & 15 & 15 & 18 & 8.3 & 39 & 63 & 44 & 56 & 62 & 12 & 37 & 63 & 12 & 32 \\
\hline 522 & 357 & 114 & 155 & 91 & 299 & 81 & 80 & 78 & 78 & 78 & 173 & 293 & 149 & 169 \\
\hline 9.3 & 9.2 & 15 & 14 & 13 & 21 & 8 & 11 & 24 & 31 & 30 & 30 & 34 & 14 & 19 \\
\hline 227 & 169 & 136 & 142 & 101 & 104 & 129 & 95 & 179 & 254 & 307 & 518 & 388 & 285 & 213 \\
\hline 22 & 22 & 34 & 34 & 30 & 46 & 18 & 24 & 51 & 69 & 72 & 72 & 72 & 33 & 4 \\
\hline 29 & 23 & 22 & 24 & 30 & 60 & 56 & 11 & 28 & 39 & 37 & 1.3 & 67 & 59 & 00 \\
\hline 233 & 173 & 142 & 140 & 101 & 106 & 131 & 96 & 182 & 248 & 315 & 523 & 398 & 292 & 216 \\
\hline 229 & 170 & 130 & 144 & 99 & 106 & 129 & 109 & 191 & 270 & 323 & 526 & 402 & 294 & 219 \\
\hline 28 & 25 & 41 & 55 & 55 & 55 & 172 & 85 & 70 & 70 & 171 & 8.4 & 82 & 47 & 68 \\
\hline 739 & 700 & 464 & 557 & 613 & 619 & 517 & 485 & 550 & 418 & 274 & 300 & 426 & 761 & 530 \\
\hline 18 & 15 & 16 & 28 & 67 & 83 & 103 & 35 & 66 & 109 & 92 & 13 & 71 & 24 & 5 \\
\hline 5.5 & 5.8 & 12 & 12 & 9.9 & 15 & 5.1 & 6.7 & 15 & 15 & 15 & 15 & 15 & 11 & \\
\hline 56 & 48 & 74 & 91 & 92 & 68 & 286 & 75 & 52 & 55 & 207 & 0 & 129 & 85 & \\
\hline 5.6 & 5.7 & 10 & 10 & 9.3 & 11 & 4.6 & 7 & 15 & 23 & 23 & 22 & 24 & 10 & \\
\hline 9 & 8.4 & 9.1 & 8.9 & 7 & 18 & 9.9 & 18 & 35 & 54 & 47 & 54 & 43 & 15 & 23 \\
\hline 69 & 57 & 58 & 99 & 114 & 110 & 101 & 72 & 96 & 86 & 116 & 286 & 308 & 158 & 131 \\
\hline 21 & 20 & 24 & 28 & 17 & 20 & 17 & 18 & 24 & 34 & 34 & 34 & 34 & 25 & \\
\hline 101 & 114 & 106 & 109 & 69 & 65 & 103 & 58 & 100 & 125 & 125 & 120 & 75 & 128 & 97 \\
\hline 36 & 35 & 35 & 31 & 31 & 34 & 38 & 35 & 35 & 33 & 54 & 36 & 39 & 36 & \\
\hline 2.2 & 2.5 & 6 & 6.5 & 5.4 & 7.6 & 2.9 & 3.6 & 10 & 15 & 14 & 14 & 15 & 5.2 & 7.8 \\
\hline 385 & 301 & 347 & 452 & 541 & 533 & 416 & 389 & 389 & 55 & 55 & 55 & 212 & 317 & 337 \\
\hline 4.6 & 4.3 & 4.7 & 4.6 & 3.6 & 9.2 & 5.1 & 9.5 & 18 & 23 & 23 & 23 & 22 & 7.7 & 1 \\
\hline 174 & 187 & 144 & 171 & 185 & 184 & 163 & 156 & 156 & 159 & 171 & 175 & 189 & 193 & 172 \\
\hline 197 & 146 & 101 & 134 & 162 & 159 & 122 & 113 & 113 & 96 & 42 & 27 & 120 & 104 & 116 \\
\hline 827 & 660 & 420 & 547 & 656 & 646 & 504 & 474 & 475 & 476 & 416 & 394 & 642 & 627 & 54 \\
\hline 52 & 40 & 70 & 33 & 121 & 107 & 0 & 17 & 71 & 90 & 64 & 61 & 236 & 151 & \\
\hline 91 & 87 & 88 & 76 & 76 & 85 & 94 & 85 & 86 & 80 & 138 & 89 & 100 & 89 & 8 \\
\hline 31 & 27 & 24 & 34 & 131 & 74 & 72 & 26 & 45 & 154 & 14 & 19 & 152 & 47 & 6 \\
\hline 8.6 & 7.3 & 6.8 & 9.8 & 42 & 27 & 32 & 12 & 20 & 69 & 5.9 & 7 & 46 & 13 & 2 \\
\hline 615 & 461 & 545 & 629 & 722 & 721 & 599 & 584 & 661 & 579 & 487 & 509 & 605 & 567 & 601 \\
\hline 29 & 23 & 20 & 21 & 29 & 47 & 31 & 8.9 & 22 & 34 & 32 & 0.3 & 103 & 60 & \\
\hline 4.9 & 5.2 & 9.8 & 9.8 & 8.8 & 10 & 4.6 & 5.8 & 10 & 9.5 & 8.6 & 8.3 & 9.3 & 9.6 & 8. \\
\hline 484 & 351 & 290 & 398 & 497 & 488 & 359 & 333 & 333 & 229 & 229 & 229 & 395 & 375 & 362 \\
\hline 543 & 430 & 269 & 346 & 420 & 412 & 316 & 296 & 294 & 297 & 275 & 267 & 407 & 405 & 350 \\
\hline 27 & 20 & 14 & 10 & 5.9 & 34 & 28 & 18 & 36 & 47 & 53 & 53 & 64 & 48 & 3 \\
\hline 23 & 20 & 22 & 24 & 94 & 87 & 107 & 32 & 60 & 106 & 91 & 0 & 112 & 30 & 5 \\
\hline
\end{tabular}

-Regulated Hydro Projects-

1 Albeni Falls

2 Alder

3 Bonneville

4 Boundary

5 Box Canyon

6 Brownlee

7 Cabinet Gorge

8 Chelan

9 Chief Joseph

10 Cushman 1

11 Cushman 2

12 Diablo

13 Dworshak

14 Faraday

15 Gorge

16 Grand Coulee

17 Hells Canyon

18 Hungry Horse

19 Ice Harbor

20 John Day

21 La Grande

22 Libby

23 Little Falls

24 Little Goose

25 Long Lake

26 Lower Baker

27 Lower Granite

28 Lower Monumental

29 Mayfield

30 McNary

31 Merwin

32 Monroe Street

33 Mossyrock

34 Nine Mile

35 North Fork

36 Noxon

37 Oak Grove

38 Oxbow

39 Pelton

40 Post Falls

42 River Mill

44 Rock Island PH\#2

45 Rocky Reach

46 Ross

48 Swift 1

49 Swift 2

50 The Dalles

51 Upper Baker

52 Upper Falls

53 Wanapum

54 Wells

55 White

56 Yale

Total Regulated Hydro w/Enc.
41 Priest Rapids

43 Rock Island PH\#1

47 Round Butte
$13587 \quad 10544$

$8518 \quad 10484$
$11698 \quad 11863 \quad 1003$
Ma

Apr1 Apr16

Jun

Jul Avg

-Total Regulated Hydro w/Enc.-

57 Federal System

58 Public Entities

$\begin{array}{lllllllllllllll}9358 & 6971 & 5203 & 6407 & 7131 & 7252 & 5812 & 5483 & 5984 & 5633 & 5296 & 6136 & 7392 & 7894 & 6527\end{array}$

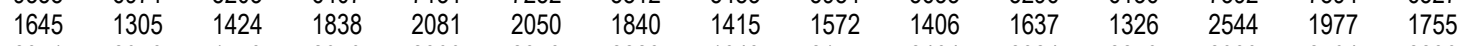

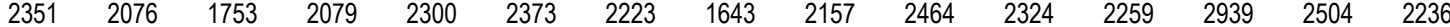

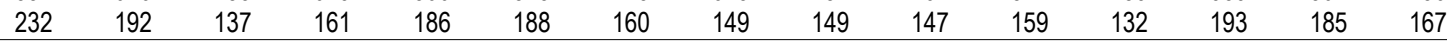

60 Other Entities

$8518 \quad 10484 \quad 11698 \quad 11863 \quad 10034$

86909862 
2003 White Book

-Regulated Hydro Projects-

1 Albeni Falls

2 Alder

3 Bonneville

4 Boundary

5 Box Canyon

6 Brownlee

7 Cabinet Gorge

8 Chelan

9 Chief Joseph

10 Cushman 1

11 Cushman 2

12 Diablo

13 Dworshak

14 Faraday

15 Gorge

16 Grand Coulee

17 Hells Canyon

18 Hungry Horse

19 lce Harbor

20 John Day

$21 \mathrm{La}$ Grande

22 Libby

23 Little Falls

25 Long Lake

26 Lower Baker

27 Lower Granite

29 Mayfield

30 McNary

31 Merwin

32 Monroe Street

33 Mossyrock

34 Nine Mile

35 North Fork

36 Noxon

37 Oak Grove

38 Oxbow

39 Pelton

40 Post Falls

41 Priest Rapids

42 River Mill

44 Rock Island PH\#2

45 Rocky Reach

46 Ross

48 Swift 1

49 Swift 2

50 The Dalles

51 Upper Baker

52 Upper Falls

53 Wanapum

54 Wells

55 White

56 Yale

Total Regulated Hydro w/Enc.
24 Little Goose

28 Lower Monumental

43 Rock Island PH\#1

47 Round Butte
Sep

Oct
Nov Dec Jan Feb

$\begin{array}{rrrr}23 & 19 & 27 & 37 \\ 14 & 10 & 11 & 12 \\ 172 & 138 & 401 & 468 \\ 190 & 150 & 238 & 503 \\ 31 & 25 & 37 & 69 \\ 232 & 259 & 238 & 240 \\ 51 & 39 & 41 & 65 \\ 31 & 23 & 20 & 55 \\ 1778 & 1334 & 828 & 1070\end{array}$

$$
3.3
$$

$2.4 \quad 0.7$

$\begin{array}{rr}74 & 56 \\ 443 & 205\end{array}$

$\begin{array}{rr}8.1 & 7.5 \\ 86 & 68 \\ 3281 & 2414\end{array}$

$\begin{array}{rr}3281 & 2414 \\ 193 & 218\end{array}$

$\begin{array}{rr}92 & 81 \\ 93 & 64 \\ 920 & 695\end{array}$

$\begin{array}{rr}920 & 695 \\ 522 & 357\end{array}$

$\begin{array}{rr}522 & 357 \\ 227 & 9.2\end{array}$

$22 \quad 22$

$29 \quad 23$

$233 \quad 173$

$\begin{array}{rr}28 & 25 \\ 754 & 713\end{array}$

$\begin{array}{rr}18 & 15 \\ 5.5 & 5.8\end{array}$

$56 \quad 48$

$5.6 \quad 5.7$

$69 \quad 57$

$\begin{array}{rr}21 & 20 \\ 101 & 114\end{array}$

$36 \quad 35$

$\begin{array}{rr}2.2 & 2.5 \\ 385 & 301\end{array}$

$\begin{array}{llllll}301 & 347 & 452 & 541 & 533 & 416\end{array}$

$\begin{array}{rrrrrrr}4.6 & 4.3 & 4.7 & 4.6 & 3.6 & 9.2 & 5.1 \\ 174 & 187 & 144 & 171 & 185 & 184 & 163\end{array}$

$\begin{array}{lllllll}174 & 187 & 144 & 171 & 185 & 184 & 163 \\ 197 & 146 & 101 & 134 & 162 & 159 & 122 \\ 827 & 660 & 420 & 547 & 656 & 646 & 504\end{array}$

$\begin{array}{rrrrrrr}91 & 87 & 88 & 33 & 121 & 107 & \\ 31 & 27 & 24 & 34 & 76 & 85 & 94\end{array}$

$\begin{array}{rrrrrrr}31 & 27 & 24 & 34 & 131 & 74 & 72 \\ 8.6 & 7.3 & 6.8 & 9.8 & 42 & 27 & 32\end{array}$

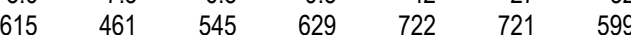

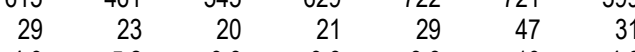

$\begin{array}{rrrrrrr}4.9 & 5.2 & 9.8 & 9.8 & 8.8 & 10 & 4.6 \\ 484 & 351 & 290 & 398 & 497 & 488 & 359\end{array}$

$\begin{array}{lllllll}543 & 430 & 269 & 346 & 420 & 412 & 316\end{array}$
Mar Apr1 Apr16

May

Jun

Jul Avg

-Total Regulated Hydro w/Enc.-

57 Federal System

58 Public Entities

59 Investor-Owned Entities

60 Other Entities
$13611 \quad 10564$

$\begin{array}{rrrrr}18 & 18 & 16 & 14 & 17 \\ 6 & 25 & 45 & 21 & 25 \\ 524 & 549 & 460 & 451 & 465 \\ 266 & 263 & 223 & 186 & 248 \\ 41 & 41 & 35 & 30 & 38 \\ 155 & 158 & 252 & 146 & 258\end{array}$

$66 \quad 50 \quad 64$

975

29
66

1.5
51

8.9
2.7

1767

198

93
127
742

742
63

81
8

129
18

56

131
129

172
527

103
5.1

286

4.6

101

17
103

38

2.9

5.1

$163 \quad 156$

113

474

$\begin{array}{ll}94 & 85\end{array}$

26
12

584

584
8.9

$\begin{array}{ll}5.8 & 10\end{array}$

$\begin{array}{ll}333 & 333 \\ 296 & 294\end{array}$

86
1605

1605
194

823
56

78
24

51

28
182
18
30
295

$\begin{array}{rrr}5.9 & 34 & 28 \\ 94 & 87 & 107\end{array}$

$8532 \quad 10501$

$\begin{array}{rrrrr}94 & 87 & 107 & 32 & 60\end{array}$
18
7.1
134

$281 \quad 417$

44
320

$324 \quad 324$

$52 \quad 32$

$919 \quad 925$

1.3

0.2

91
52

43

109

$1527 \quad 1502$

$244 \quad 245$

$67 \quad 28$

$130 \quad 136$

$785 \quad 722$

78

31

254

307

39
248

248
270

323

70

430

109

15

$55 \quad 207$

23

54

$\begin{array}{ll}86 & 416\end{array}$

$34 \quad 34$

125

33

$\begin{array}{ll}15 & 14\end{array}$

$55 \quad 55$

23

159

96

476

$90-416$

$\begin{array}{rr}80 & 138\end{array}$

$154 \quad 14$

$\begin{array}{rr}154 & 14 \\ 69 & 5.9\end{array}$

$579 \quad 487$

$\begin{array}{rr}579 & 487 \\ 34 & 32\end{array}$

$9.5 \quad 8.6$

$\begin{array}{ll}229 & 229 \\ 297 & 275\end{array}$

$\begin{array}{rrrr}29 & 45 & 38 & 25 \\ 23 & 50 & 8 & 21 \\ 134 & 183 & 132 & 345 \\ 530 & 606 & 399 & 332 \\ 72 & 74 & 55 & 48 \\ 305 & 192 & 312 & 235 \\ 176 & 188 & 112 & 85 \\ 9.9 & 51 & 57 & 44\end{array}$

$\begin{array}{llll}872 & 1153 & 1339 & 1095\end{array}$

$1.3 \quad 28$

0.1

$74 \quad 172$

56

43
92

92
1484

1484
239

232

232
757

37

173

30
518

518

72
1.3
523

523
526

526

8.4
308

13
15
0

$0 \quad 129$

$22 \quad 24$

54

286

34

120

36

14
55

23

175

27
394

$61 \quad 236$

$89 \quad 100$

$19 \quad 152$

$7 \quad 46$

$509 \quad 605$

$0.3 \quad 103$

$8.3 \quad 9.3$

229
267

$\begin{array}{rr}47 & 53 \\ 106 & 91\end{array}$

9667

28
63

172
287

$\begin{array}{lll}39 & 445 \quad 127\end{array}$

$\begin{array}{rrr}182 & 168 & 91\end{array}$

$1116 \quad 2503 \quad 1975$

$\begin{array}{rrr}31 & 246 & 189\end{array}$

$\begin{array}{lll}173 & 124 & 136\end{array}$

$851 \quad 805$

$\begin{array}{lll}293 & 149 & 169\end{array}$

$\begin{array}{lll}34 & 14 & 19\end{array}$

$285 \quad 213$

$\begin{array}{lll}67 & 59 & 35\end{array}$

$398 \quad 292 \quad 216$

$\begin{array}{lll}294 & 219\end{array}$

$\begin{array}{rrr}82 & 47 & 68 \\ 36 & 777 & 541\end{array}$

$71-24 \quad 52$

$\begin{array}{lll}5 & 11 & 11\end{array}$

$10 \quad 13$

$34 \quad 25 \quad 25$

$\begin{array}{lll}75 & 128 & 97\end{array}$

$36 \quad 36$

$\begin{array}{lll}212 & 317 & 7.8\end{array}$

$\begin{array}{lll}22 & 7.7 & 11\end{array}$

$\begin{array}{lll}89 & 193 & 172\end{array}$

$\begin{array}{lll}20 & 104 & 116\end{array}$

$627 \quad 548$ 
2003 White Book

-Regulated Hydro Projects-

1 Albeni Falls

2 Alder

3 Bonneville

4 Boundary

5 Box Canyon

6 Brownlee

7 Cabinet Gorge

8 Chelan

9 Chief Joseph

10 Cushman 1

11 Cushman 2

12 Diablo

13 Dworshak

14 Faraday

15 Gorge

16 Grand Coulee

17 Hells Canyon

18 Hungry Horse

19 lce Harbor

20 John Day

21 La Grande

22 Libby

23 Little Falls

24 Little Goose

25 Long Lake

26 Lower Baker

27 Lower Granite

28 Lower Monumental

29 Mayfield

30 McNary

31 Merwin

32 Monroe Street

33 Mossyrock

34 Nine Mile

35 North Fork

36 Noxon

37 Oak Grove

38 Oxbow

39 Pelton

40 Post Falls

41 Priest Rapids

42 River Mill

43 Rock Island PH\#1

44 Rock Island PH\#2

45 Rocky Reach

46 Ross

47 Round Butte

48 Swift 1

49 Swift 2

50 The Dalles

51 Upper Baker

52 Upper Falls

53 Wanapum

54 Wells

55 White

56 Yale

Total Regulated Hydro w/Enc.
Sep

Oct
Nov Dec Jan Feb

\begin{tabular}{|c|c|c|c|c|c|c|c|c|c|c|c|c|c|c|}
\hline 23 & 19 & 27 & 37 & 18 & 18 & 16 & 14 & 17 & 18 & 18 & 29 & 45 & 38 & 25 \\
\hline 14 & 10 & 11 & 12 & 6 & 25 & 45 & 21 & 25 & 30 & 7.1 & 23 & 50 & 8 & 21 \\
\hline 172 & 138 & 401 & 468 & 524 & 549 & 460 & 451 & 465 & 295 & 134 & 134 & 183 & 132 & 345 \\
\hline 190 & 150 & 238 & 503 & 266 & 263 & 223 & 186 & 248 & 281 & 417 & 530 & 606 & 399 & 332 \\
\hline 31 & 25 & 37 & 69 & 41 & 41 & 35 & 30 & 38 & 44 & 60 & 72 & 74 & 55 & 48 \\
\hline 232 & 259 & 238 & 240 & 155 & 158 & 252 & 146 & 258 & 324 & 324 & 305 & 192 & 312 & 235 \\
\hline 51 & 39 & 41 & 65 & 74 & 72 & 66 & 50 & 64 & 52 & 85 & 176 & 188 & 112 & 85 \\
\hline 31 & 23 & 20 & 55 & 55 & 55 & 54 & 54 & 54 & 52 & 32 & 9.9 & 51 & 57 & 44 \\
\hline 1786 & 1341 & 833 & 1075 & 1312 & 1292 & 981 & 924 & 918 & 924 & 930 & 877 & 1160 & 1346 & 1101 \\
\hline 3.3 & 2.3 & 19 & 1.8 & 27 & 25 & 29 & 20 & 1.1 & 1.3 & 24 & 1.3 & 28 & 1.3 & 14 \\
\hline 2.4 & 0.7 & 30 & 0.1 & 49 & 50 & 66 & 55 & 0.4 & 0.2 & 59 & 0.1 & 63 & 0 & 29 \\
\hline 74 & 56 & 76 & 35 & 106 & 102 & 1.5 & 15 & 72 & 91 & 71 & 74 & 172 & 154 & 79 \\
\hline 443 & 205 & 52 & 52 & 52 & 51 & 51 & 51 & 51 & 52 & 53 & 56 & 287 & 445 & 127 \\
\hline 8.1 & 7.5 & 8.2 & 8 & 6.2 & 16 & 8.9 & 17 & 31 & 43 & 42 & 43 & 39 & 14 & 20 \\
\hline 86 & 68 & 87 & 43 & 118 & 118 & 2.7 & 19 & 86 & 109 & 86 & 92 & 182 & 168 & 91 \\
\hline 3281 & 2414 & 1500 & 1989 & 2399 & 2293 & 1767 & 1685 & 1605 & 1527 & 1502 & 1484 & 2116 & 2503 & 1975 \\
\hline 193 & 218 & 202 & 208 & 133 & 127 & 198 & 114 & 194 & 244 & 245 & 239 & 152 & 246 & 189 \\
\hline 92 & 81 & 77 & 86 & 90 & 91 & 93 & 93 & 89 & 67 & 28 & 29 & 31 & 120 & 78 \\
\hline 93 & 64 & 124 & 142 & 99 & 105 & 127 & 109 & 186 & 130 & 136 & 232 & 173 & 124 & 136 \\
\hline 920 & 695 & 668 & 803 & 925 & 911 & 742 & 718 & 823 & 785 & 722 & 757 & 899 & 851 & 805 \\
\hline 21 & 15 & 15 & 18 & 8.3 & 39 & 63 & 44 & 56 & 62 & 12 & 37 & 63 & 12 & 34 \\
\hline 522 & 357 & 114 & 155 & 91 & 299 & 81 & 80 & 78 & 78 & 78 & 173 & 293 & 149 & 169 \\
\hline 9.3 & 9.2 & 15 & 14 & 13 & 21 & 8 & 11 & 24 & 31 & 30 & 30 & 34 & 14 & 19 \\
\hline 227 & 169 & 136 & 142 & 101 & 104 & 129 & 95 & 179 & 254 & 307 & 518 & 388 & 285 & 213 \\
\hline 22 & 22 & 34 & 34 & 30 & 46 & 18 & 24 & 51 & 69 & 72 & 72 & 72 & 33 & 42 \\
\hline 29 & 23 & 22 & 24 & 30 & 60 & 56 & 11 & 28 & 39 & 37 & 1.3 & 67 & 59 & 35 \\
\hline 233 & 173 & 142 & 140 & 101 & 106 & 131 & 96 & 182 & 248 & 315 & 523 & 398 & 292 & 216 \\
\hline 229 & 170 & 130 & 144 & 99 & 106 & 129 & 109 & 191 & 270 & 323 & 526 & 402 & 294 & 219 \\
\hline 28 & 25 & 41 & 55 & 55 & 55 & 172 & 85 & 70 & 70 & 171 & 8.4 & 82 & 47 & 68 \\
\hline 770 & 726 & 483 & 579 & 638 & 645 & 538 & 505 & 573 & 442 & 291 & 316 & 447 & 794 & 553 \\
\hline 18 & 15 & 16 & 28 & 67 & 83 & 103 & 35 & 66 & 109 & 92 & 13 & 71 & 24 & 52 \\
\hline 5.5 & 5.8 & 12 & 12 & 9.9 & 15 & 5.1 & 6.7 & 15 & 15 & 15 & 15 & 15 & 11 & 11 \\
\hline 56 & 48 & 74 & 91 & 92 & 68 & 286 & 75 & 52 & 55 & 207 & 0 & 129 & 85 & 95 \\
\hline 5.6 & 5.7 & 10 & 10 & 9.3 & 11 & 4.6 & 7 & 15 & 23 & 23 & 22 & 24 & 10 & 13 \\
\hline 9 & 8.4 & 9.1 & 8.9 & 7 & 18 & 9.9 & 18 & 35 & 54 & 47 & 54 & 43 & 15 & 23 \\
\hline 69 & 57 & 58 & 99 & 114 & 110 & 101 & 72 & 96 & 86 & 116 & 286 & 308 & 158 & 131 \\
\hline 21 & 20 & 24 & 28 & 17 & 20 & 17 & 18 & 24 & 34 & 34 & 34 & 34 & 25 & 25 \\
\hline 101 & 114 & 106 & 109 & 69 & 65 & 103 & 58 & 100 & 125 & 125 & 120 & 75 & 128 & 97 \\
\hline 36 & 35 & 35 & 31 & 31 & 34 & 38 & 35 & 35 & 33 & 54 & 36 & 39 & 36 & 36 \\
\hline 2.2 & 2.5 & 6 & 6.5 & 5.4 & 7.6 & 2.9 & 3.6 & 10 & 15 & 14 & 14 & 15 & 5.2 & 7.8 \\
\hline 385 & 301 & 347 & 452 & 541 & 533 & 416 & 389 & 389 & 55 & 55 & 55 & 212 & 317 & 337 \\
\hline 4.6 & 4.3 & 4.7 & 4.6 & 3.6 & 9.2 & 5.1 & 9.5 & 18 & 23 & 23 & 23 & 22 & 7.7 & 11 \\
\hline 174 & 187 & 144 & 171 & 185 & 184 & 163 & 156 & 156 & 159 & 171 & 175 & 189 & 193 & 172 \\
\hline 197 & 146 & 101 & 134 & 162 & 159 & 122 & 113 & 113 & 96 & 42 & 27 & 120 & 104 & 116 \\
\hline 827 & 660 & 420 & 547 & 656 & 646 & 504 & 474 & 475 & 476 & 416 & 394 & 642 & 627 & 548 \\
\hline 52 & 40 & 70 & 33 & 121 & 107 & 0 & 17 & 71 & 90 & 64 & 61 & 236 & 151 & 83 \\
\hline 91 & 87 & 88 & 76 & 76 & 85 & 94 & 85 & 86 & 80 & 138 & 89 & 100 & 89 & 89 \\
\hline 31 & 27 & 24 & 34 & 131 & 74 & 72 & 26 & 45 & 154 & 14 & 19 & 152 & 47 & 61 \\
\hline 8.6 & 7.3 & 6.8 & 9.8 & 42 & 27 & 32 & 12 & 20 & 69 & 5.9 & 7 & 46 & 13 & 22 \\
\hline 615 & 461 & 545 & 629 & 722 & 721 & 599 & 584 & 661 & 579 & 487 & 509 & 605 & 567 & 601 \\
\hline 29 & 23 & 20 & 21 & 29 & 47 & 31 & 8.9 & 22 & 34 & 32 & 0.3 & 103 & 60 & 34 \\
\hline 4.9 & 5.2 & 9.8 & 9.8 & 8.8 & 10 & 4.6 & 5.8 & 10 & 9.5 & 8.6 & 8.3 & 9.3 & 9.6 & 8.4 \\
\hline 484 & 351 & 290 & 398 & 497 & 488 & 359 & 333 & 333 & 229 & 229 & 229 & 395 & 375 & 362 \\
\hline 543 & 430 & 269 & 346 & 420 & 412 & 316 & 296 & 294 & 297 & 275 & 267 & 407 & 405 & 350 \\
\hline 27 & 20 & 14 & 10 & 5.9 & 34 & 28 & 18 & 36 & 47 & 53 & 53 & 64 & 48 & 32 \\
\hline 23 & 20 & 22 & 24 & 94 & 87 & 107 & 32 & 60 & 106 & 91 & 0 & 112 & 30 & 57 \\
\hline
\end{tabular}

-Total Regulated Hydro w/Enc.-

57 Federal System

58 Public Entities

$\begin{array}{lll}10584 & 8546 & 1051\end{array}$

$1738-11904$

$8720 \quad 989$

9685

$\begin{array}{rrrrr}9443 & 9878 & 13102 & 12607 & 10721\end{array}$

59 Investor-Owned Entities

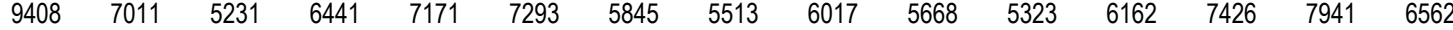

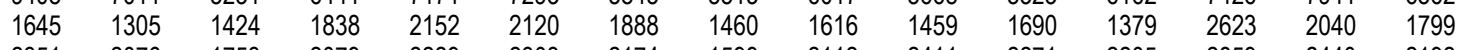

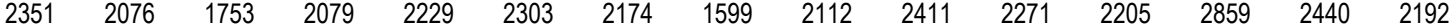

60 Other Entities

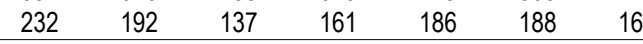

$149 \quad 149$

\begin{tabular}{rrrrrr}
147 & 159 & 132 & 193 & 185 & 167 \\
\hline 9685 & 9443 & 9878 & 13102 & 12607 & 10721
\end{tabular} 
2003 White Book

-Regulated Hydro Projects-

1 Albeni Falls

2 Alder

3 Bonneville

4 Boundary

5 Box Canyon

6 Brownlee

7 Cabinet Gorge

8 Chelan

9 Chief Joseph

10 Cushman 1

11 Cushman 2

12 Diablo

13 Dworshak

14 Faraday

15 Gorge

16 Grand Coulee

17 Hells Canyon

18 Hungry Horse

19 lce Harbor

20 John Day

$21 \mathrm{La}$ Grande

22 Libby

23 Little Falls

24 Little Goose

25 Long Lake

26 Lower Baker

27 Lower Granite

28 Lower Monumental

29 Mayfield

30 McNary

31 Merwin

32 Monroe Street

33 Mossyrock

34 Nine Mile

35 North Fork

36 Noxon

37 Oak Grove

38 Oxbow

39 Pelton

40 Post Falls

41 Priest Rapids

42 River Mill

43 Rock Island PH\#1

44 Rock Island PH\#2

45 Rocky Reach

46 Ross

47 Round Butte

48 Swift 1

49 Swift 2

50 The Dalles

51 Upper Baker

52 Upper Falls

53 Wanapum

54 Wells

55 White

56 Yale

Total Regulated Hydro w/Enc.
Sep

Oct
Nov Dec Jan Feb

\begin{tabular}{|c|c|c|c|c|c|c|c|c|c|c|c|c|c|c|}
\hline 23 & 19 & 27 & 37 & 18 & 18 & 16 & 14 & 17 & 18 & 18 & 29 & 45 & 38 & 25 \\
\hline 14 & 10 & 11 & 12 & 6 & 25 & 45 & 21 & 25 & 30 & 7.1 & 23 & 50 & 8 & 21 \\
\hline 172 & 138 & 401 & 468 & 524 & 549 & 460 & 451 & 465 & 295 & 134 & 134 & 183 & 132 & 345 \\
\hline 190 & 150 & 238 & 503 & 266 & 263 & 223 & 186 & 248 & 281 & 417 & 530 & 606 & 399 & 332 \\
\hline 31 & 25 & 37 & 69 & 41 & 41 & 35 & 30 & 38 & 44 & 60 & 72 & 74 & 55 & 48 \\
\hline 232 & 259 & 238 & 240 & 155 & 158 & 252 & 146 & 258 & 324 & 324 & 305 & 192 & 312 & 235 \\
\hline 51 & 39 & 41 & 65 & 74 & 72 & 66 & 50 & 64 & 52 & 85 & 176 & 188 & 112 & 85 \\
\hline 31 & 23 & 20 & 55 & 55 & 55 & 54 & 54 & 54 & 52 & 32 & 9.9 & 51 & 57 & 4 \\
\hline 1792 & 1345 & 836 & 1079 & 1317 & 1297 & 985 & 927 & 921 & 927 & 934 & 880 & 1164 & 1351 & 1105 \\
\hline 3.3 & 2.3 & 19 & 1.8 & 27 & 25 & 29 & 20 & 1.1 & 1.3 & 24 & 1.3 & 28 & 1.3 & 14 \\
\hline 2.4 & 0.7 & 30 & 0.1 & 49 & 50 & 66 & 55 & 0.4 & 0.2 & 59 & 0.1 & 63 & 0 & 29 \\
\hline 74 & 56 & 76 & 35 & 106 & 102 & 1.5 & 15 & 72 & 91 & 71 & 74 & 172 & 154 & 79 \\
\hline 443 & 205 & 52 & 52 & 52 & 51 & 51 & 51 & 51 & 52 & 53 & 56 & 287 & 445 & 127 \\
\hline 8.1 & 7.5 & 8.2 & 8 & 6.2 & 16 & 8.9 & 17 & 31 & 43 & 42 & 43 & 39 & 14 & 20 \\
\hline 86 & 68 & 87 & 43 & 118 & 118 & 2.7 & 19 & 86 & 109 & 86 & 92 & 182 & 168 & 91 \\
\hline 3281 & 2414 & 1500 & 1989 & 2399 & 2293 & 1767 & 1685 & 1605 & 1527 & 1502 & 1484 & 2116 & 2503 & 1975 \\
\hline 193 & 218 & 202 & 208 & 133 & 127 & 198 & 114 & 194 & 244 & 245 & 239 & 152 & 246 & 189 \\
\hline 92 & 81 & 77 & 86 & 90 & 91 & 93 & 93 & 89 & 67 & 28 & 29 & 31 & 120 & 78 \\
\hline 93 & 64 & 124 & 142 & 99 & 105 & 127 & 109 & 186 & 130 & 136 & 232 & 173 & 124 & 136 \\
\hline 920 & 695 & 668 & 803 & 925 & 911 & 742 & 718 & 823 & 785 & 722 & 757 & 899 & 851 & 805 \\
\hline 21 & 15 & 15 & 18 & 8.3 & 39 & 63 & 44 & 56 & 62 & 12 & 37 & 63 & 12 & 34 \\
\hline 522 & 357 & 114 & 155 & 91 & 299 & 81 & 80 & 78 & 78 & 78 & 173 & 293 & 149 & 169 \\
\hline 9.3 & 9.2 & 15 & 14 & 13 & 21 & 8 & 11 & 24 & 31 & 30 & 30 & 34 & 14 & 19 \\
\hline 227 & 169 & 136 & 142 & 101 & 104 & 129 & 95 & 179 & 254 & 307 & 518 & 388 & 285 & 213 \\
\hline 22 & 22 & 34 & 34 & 30 & 46 & 18 & 24 & 51 & 69 & 72 & 72 & 72 & 33 & 42 \\
\hline 29 & 23 & 22 & 24 & 30 & 60 & 56 & 11 & 28 & 39 & 37 & 1.3 & 67 & 59 & 35 \\
\hline 233 & 173 & 142 & 140 & 101 & 106 & 131 & 96 & 182 & 248 & 315 & 523 & 398 & 292 & 216 \\
\hline 229 & 170 & 130 & 144 & 99 & 106 & 129 & 109 & 191 & 270 & 323 & 526 & 402 & 294 & 219 \\
\hline 28 & 25 & 41 & 55 & 55 & 55 & 172 & 85 & 70 & 70 & 171 & 8.4 & 82 & 47 & 68 \\
\hline 786 & 739 & 493 & 591 & 650 & 658 & 549 & 515 & 584 & 454 & 299 & 324 & 457 & 810 & 564 \\
\hline 18 & 15 & 16 & 28 & 67 & 83 & 103 & 35 & 66 & 109 & 92 & 13 & 71 & 24 & 52 \\
\hline 5.5 & 5.8 & 12 & 12 & 9.9 & 15 & 5.1 & 6.7 & 15 & 15 & 15 & 15 & 15 & 11 & 11 \\
\hline 56 & 48 & 74 & 91 & 92 & 68 & 286 & 75 & 52 & 55 & 207 & 0 & 129 & 85 & 95 \\
\hline 5.6 & 5.7 & 10 & 10 & 9.3 & 11 & 4.6 & 7 & 15 & 23 & 23 & 22 & 24 & 10 & 13 \\
\hline 9 & 8.4 & 9.1 & 8.9 & 7 & 18 & 9.9 & 18 & 35 & 54 & 47 & 54 & 43 & 15 & 23 \\
\hline 69 & 57 & 58 & 99 & 114 & 110 & 101 & 72 & 96 & 86 & 116 & 286 & 308 & 158 & 131 \\
\hline 21 & 20 & 24 & 28 & 17 & 20 & 17 & 18 & 24 & 34 & 34 & 34 & 34 & 25 & 25 \\
\hline 101 & 114 & 106 & 109 & 69 & 65 & 103 & 58 & 100 & 125 & 125 & 120 & 75 & 128 & 97 \\
\hline 36 & 35 & 35 & 31 & 31 & 34 & 38 & 35 & 35 & 33 & 54 & 36 & 39 & 36 & 36 \\
\hline 2.2 & 2.5 & 6 & 6.5 & 5.4 & 7.6 & 2.9 & 3.6 & 10 & 15 & 14 & 14 & 15 & 5.2 & 7.8 \\
\hline 385 & 301 & 347 & 452 & 541 & 533 & 416 & 389 & 389 & 55 & 55 & 55 & 212 & 317 & 337 \\
\hline 4.6 & 4.3 & 4.7 & 4.6 & 3.6 & 9.2 & 5.1 & 9.5 & 18 & 23 & 23 & 23 & 22 & 7.7 & 11 \\
\hline 174 & 187 & 144 & 171 & 185 & 184 & 163 & 156 & 156 & 159 & 171 & 175 & 189 & 193 & 172 \\
\hline 197 & 146 & 101 & 134 & 162 & 159 & 122 & 113 & 113 & 96 & 42 & 27 & 120 & 104 & 116 \\
\hline 827 & 660 & 420 & 547 & 656 & 646 & 504 & 474 & 475 & 476 & 416 & 394 & 642 & 627 & 548 \\
\hline 52 & 40 & 70 & 33 & 121 & 107 & 0 & 17 & 71 & 90 & 64 & 61 & 236 & 151 & 83 \\
\hline 91 & 87 & 88 & 76 & 76 & 85 & 94 & 85 & 86 & 80 & 138 & 89 & 100 & 89 & 89 \\
\hline 31 & 27 & 24 & 34 & 131 & 74 & 72 & 26 & 45 & 154 & 14 & 19 & 152 & 47 & 61 \\
\hline 8.6 & 7.3 & 6.8 & 9.8 & 42 & 27 & 32 & 12 & 20 & 69 & 5.9 & 7 & 46 & 13 & 22 \\
\hline 615 & 461 & 545 & 629 & 722 & 721 & 599 & 584 & 661 & 579 & 487 & 509 & 605 & 567 & 601 \\
\hline 29 & 23 & 20 & 21 & 29 & 47 & 31 & 8.9 & 22 & 34 & 32 & 0.3 & 103 & 60 & 3 \\
\hline 4.9 & 5.2 & 9.8 & 9.8 & 8.8 & 10 & 4.6 & 5.8 & 10 & 9.5 & 8.6 & 8.3 & 9.3 & 9.6 & 8.4 \\
\hline 484 & 351 & 290 & 398 & 497 & 488 & 359 & 333 & 333 & 229 & 229 & 229 & 395 & 375 & 362 \\
\hline 543 & 430 & 269 & 346 & 420 & 412 & 316 & 296 & 294 & 297 & 275 & 267 & 407 & 405 & 350 \\
\hline 27 & 20 & 14 & 10 & 5.9 & 34 & 28 & 18 & 36 & 47 & 53 & 53 & 64 & 48 & 32 \\
\hline 23 & 20 & 22 & 24 & 94 & 87 & 107 & 32 & 60 & 106 & 91 & 0 & 112 & 30 & 57 \\
\hline 3658 & 10601 & 8558 & 10534 & 11756 & 11922 & 10081 & 8733 & 9909 & 9700 & 9455 & 9889 & 13117 & 12628 & 10736 \\
\hline
\end{tabular}

-Total Regulated Hydro w/Enc.-

57 Federal System

58 Public Entities

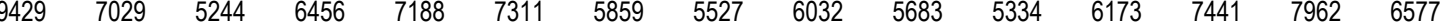

59 Investor-Owned Entities

$\begin{array}{lllllllllllllll}1730 & 1363 & 1462 & 1893 & 2157 & 2124 & 1892 & 1463 & 1620 & 1461 & 1692 & 1380 & 2626 & 2043 & 1815 \\ 2267 & 2017 & 1715 & 2024 & 2225 & 2298 & 2171 & 1595 & 2109 & 2410 & 2269 & 2204 & 2856 & 2437 & 2176\end{array}$

60 Other Entities

$\begin{array}{llll}232 & 192 & 137 & 161\end{array}$

$\begin{array}{lrr}186 & 188 & 160\end{array}$

149149

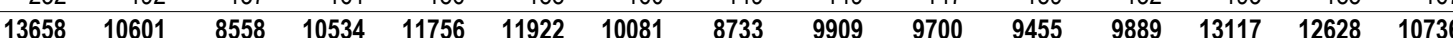


2003 White Book

Average Energy in Megawatts

Aug1 Aug16

Sep

Oct

\begin{abstract}
Dec Jan
Feb
\end{abstract}

\begin{tabular}{|c|c|c|c|c|c|c|c|c|c|c|c|c|c|c|}
\hline 23 & 19 & 27 & 37 & 18 & 18 & 16 & 14 & 17 & 18 & 18 & 29 & 45 & 38 & 25 \\
\hline 14 & 10 & 11 & 12 & 6 & 25 & 45 & 21 & 25 & 30 & 7.1 & 23 & 50 & 8 & 21 \\
\hline 172 & 138 & 401 & 468 & 524 & 549 & 460 & 451 & 465 & 295 & 134 & 134 & 183 & 132 & 345 \\
\hline 190 & 150 & 238 & 503 & 266 & 263 & 223 & 186 & 248 & 281 & 417 & 530 & 606 & 399 & 332 \\
\hline 31 & 25 & 37 & 69 & 41 & 41 & 35 & 30 & 38 & 44 & 60 & 72 & 74 & 55 & 48 \\
\hline 232 & 259 & 238 & 240 & 155 & 158 & 252 & 146 & 258 & 324 & 324 & 305 & 192 & 312 & 235 \\
\hline 51 & 39 & 41 & 65 & 74 & 72 & 66 & 50 & 64 & 52 & 85 & 176 & 188 & 112 & 85 \\
\hline 31 & 23 & 20 & 55 & 55 & 55 & 54 & 54 & 54 & 52 & 32 & 9.9 & 51 & 57 & 44 \\
\hline 1792 & 1345 & 836 & 1079 & 1317 & 1297 & 985 & 927 & 921 & 927 & 934 & 880 & 1164 & 1351 & 1105 \\
\hline 3.3 & 2.3 & 19 & 1.8 & 27 & 25 & 29 & 20 & 1.1 & 1.3 & 24 & 1.3 & 28 & 1.3 & 14 \\
\hline 2.4 & 0.7 & 30 & 0.1 & 49 & 50 & 66 & 55 & 0.4 & 0.2 & 59 & 0.1 & 63 & 0 & 29 \\
\hline 74 & 56 & 76 & 35 & 106 & 102 & 1.5 & 15 & 72 & 91 & 71 & 74 & 172 & 154 & 79 \\
\hline 443 & 205 & 52 & 52 & 52 & 51 & 51 & 51 & 51 & 52 & 53 & 56 & 287 & 445 & 127 \\
\hline 8.1 & 7.5 & 8.2 & 8 & 6.2 & 16 & 8.9 & 17 & 31 & 43 & 42 & 43 & 39 & 14 & 20 \\
\hline 86 & 68 & 87 & 43 & 118 & 118 & 2.7 & 19 & 86 & 109 & 86 & 92 & 182 & 168 & 91 \\
\hline 3281 & 2414 & 1500 & 1989 & 2399 & 2293 & 1767 & 1685 & 1605 & 1527 & 1502 & 1484 & 2116 & 2503 & 1975 \\
\hline 193 & 218 & 202 & 208 & 133 & 127 & 198 & 114 & 194 & 244 & 245 & 239 & 152 & 246 & 189 \\
\hline 92 & 81 & 77 & 86 & 90 & 91 & 93 & 93 & 89 & 67 & 28 & 29 & 31 & 120 & 78 \\
\hline 93 & 64 & 124 & 142 & 99 & 105 & 127 & 109 & 186 & 130 & 136 & 232 & 173 & 124 & 136 \\
\hline 920 & 695 & 668 & 803 & 925 & 911 & 742 & 718 & 823 & 785 & 722 & 757 & 899 & 851 & 805 \\
\hline 21 & 15 & 15 & 18 & 8.3 & 39 & 63 & 44 & 56 & 62 & 12 & 37 & 63 & 12 & 34 \\
\hline 522 & 357 & 114 & 155 & 91 & 299 & 81 & 80 & 78 & 78 & 78 & 173 & 293 & 149 & 169 \\
\hline 9.3 & 9.2 & 15 & 14 & 13 & 21 & 8 & 11 & 24 & 31 & 30 & 30 & 34 & 14 & 19 \\
\hline 227 & 169 & 136 & 142 & 101 & 104 & 129 & 95 & 179 & 254 & 307 & 518 & 388 & 285 & 213 \\
\hline 22 & 22 & 34 & 34 & 30 & 46 & 18 & 24 & 51 & 69 & 72 & 72 & 72 & 33 & 42 \\
\hline 29 & 23 & 22 & 24 & 30 & 60 & 56 & 11 & 28 & 39 & 37 & 1.3 & 67 & 59 & 35 \\
\hline 233 & 173 & 142 & 140 & 101 & 106 & 131 & 96 & 182 & 248 & 315 & 523 & 398 & 292 & 216 \\
\hline 229 & 170 & 130 & 144 & 99 & 106 & 129 & 109 & 191 & 270 & 323 & 526 & 402 & 294 & 219 \\
\hline 28 & 25 & 41 & 55 & 55 & 55 & 172 & 85 & 70 & 70 & 171 & 8.4 & 82 & 47 & 68 \\
\hline 802 & 753 & 502 & 602 & 663 & 671 & 559 & 525 & 595 & 466 & 308 & 332 & 467 & 826 & 576 \\
\hline 18 & 15 & 16 & 28 & 67 & 83 & 103 & 35 & 66 & 109 & 92 & 13 & 71 & 24 & 52 \\
\hline 5.5 & 5.8 & 12 & 12 & 9.9 & 15 & 5.1 & 6.7 & 15 & 15 & 15 & 15 & 15 & 11 & 11 \\
\hline 56 & 48 & 74 & 91 & 92 & 68 & 286 & 75 & 52 & 55 & 207 & 0 & 129 & 85 & 95 \\
\hline 5.6 & 5.7 & 10 & 10 & 9.3 & 11 & 4.6 & 7 & 15 & 23 & 23 & 22 & 24 & 10 & 13 \\
\hline 9 & 8.4 & 9.1 & 8.9 & 7 & 18 & 9.9 & 18 & 35 & 54 & 47 & 54 & 43 & 15 & 23 \\
\hline 69 & 57 & 58 & 99 & 114 & 110 & 101 & 72 & 96 & 86 & 116 & 286 & 308 & 158 & 131 \\
\hline 21 & 20 & 24 & 28 & 17 & 20 & 17 & 18 & 24 & 34 & 34 & 34 & 34 & 25 & 25 \\
\hline 101 & 114 & 106 & 109 & 69 & 65 & 103 & 58 & 100 & 125 & 125 & 120 & 75 & 128 & 97 \\
\hline 36 & 35 & 35 & 31 & 31 & 34 & 38 & 35 & 35 & 33 & 54 & 36 & 39 & 36 & 36 \\
\hline 2.2 & 2.5 & 6 & 6.5 & 5.4 & 7.6 & 2.9 & 3.6 & 10 & 15 & 14 & 14 & 15 & 5.2 & 7.8 \\
\hline 385 & 301 & 347 & 452 & 541 & 533 & 416 & 389 & 389 & 55 & 55 & 55 & 212 & 317 & 337 \\
\hline 4.6 & 4.3 & 4.7 & 4.6 & 3.6 & 9.2 & 5.1 & 9.5 & 18 & 23 & 23 & 23 & 22 & 7.7 & 11 \\
\hline 174 & 187 & 144 & 171 & 185 & 184 & 163 & 156 & 156 & 159 & 171 & 175 & 189 & 193 & 172 \\
\hline 197 & 146 & 101 & 134 & 162 & 159 & 122 & 113 & 113 & 96 & 42 & 27 & 120 & 104 & 116 \\
\hline 827 & 660 & 420 & 547 & 656 & 646 & 504 & 474 & 475 & 476 & 416 & 394 & 642 & 627 & 548 \\
\hline 52 & 40 & 70 & 33 & 121 & 107 & 0 & 17 & 71 & 90 & 64 & 61 & 236 & 151 & \\
\hline 91 & 87 & 88 & 76 & 76 & 85 & 94 & 85 & 86 & 80 & 138 & 89 & 100 & 89 & \\
\hline 31 & 27 & 24 & 34 & 131 & 74 & 72 & 26 & 45 & 154 & 14 & 19 & 152 & 47 & 61 \\
\hline 8.6 & 7.3 & 6.8 & 9.8 & 42 & 27 & 32 & 12 & 20 & 69 & 5.9 & 7 & 46 & 13 & 22 \\
\hline 615 & 461 & 545 & 629 & 722 & 721 & 599 & 584 & 661 & 579 & 487 & 509 & 605 & 567 & 601 \\
\hline 29 & 23 & 20 & 21 & 29 & 47 & 31 & 8.9 & 22 & 34 & 32 & 0.3 & 103 & 60 & \\
\hline 4.9 & 5.2 & 9.8 & 9.8 & 8.8 & 10 & 4.6 & 5.8 & 10 & 9.5 & 8.6 & 8.3 & 9.3 & 9.6 & 8.6 \\
\hline 484 & 351 & 290 & 398 & 497 & 488 & 359 & 333 & 333 & 229 & 229 & 229 & 395 & 375 & 362 \\
\hline 543 & 430 & 269 & 346 & 420 & 412 & 316 & 296 & 294 & 297 & 275 & 267 & 407 & 405 & 350 \\
\hline 27 & 20 & 14 & 10 & 5.9 & 34 & 28 & 18 & 36 & 47 & 53 & 53 & 64 & 48 & 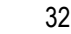 \\
\hline 23 & 20 & 22 & 24 & 94 & 87 & 107 & 32 & 60 & 106 & 91 & 0 & 112 & 30 & 57 \\
\hline 13674 & 10614 & 8568 & 10545 & 11768 & 11934 & 10092 & 8743 & 9920 & 9712 & 9463 & 9897 & 13127 & 12644 & 10748 \\
\hline
\end{tabular}

1 Albeni Falls

2 Alder

3 Bonneville

4 Boundary

5 Box Canyon

6 Brownlee

7 Cabinet Gorge

8 Chelan

9 Chief Joseph

10 Cushman 1

11 Cushman 2

12 Diablo

13 Dworshak

14 Faraday

15 Gorge

16 Grand Coulee

17 Hells Canyon

18 Hungry Horse

19 Ice Harbor

20 John Day

$21 \mathrm{La}$ Grande

22 Libby

23 Little Falls

24 Little Goose

25 Long Lake

26 Lower Baker

27 Lower Granite

28 Lower Monumental

29 Mayfield

30 McNary

31 Merwin

32 Monroe Street

33 Mossyrock

34 Nine Mile

35 North Fork

36 Noxon

37 Oak Grove

38 Oxbow

39 Pelton

40 Post Falls

41 Priest Rapids

42 River Mill

43 Rock Island PH\#1

45 Rocky Reach

46 Ross

47 Round Butte

48 Swift 1

49 Swift 2

50 The Dalles

51 Upper Baker

52 Upper Falls

53 Wanapum

54 Wells

55 White

56 Yale

Total Regulated Hydro w/Enc.
-Regulated Hydro Projects-

44 Rock Island PH\#2
$13674 \quad 10614$

85681054
Ma

Apr1 Apr16

May

Jun

Jul Avg

-Total Regulated Hydro w/Enc.-

57 Federal System

58 Public Entities

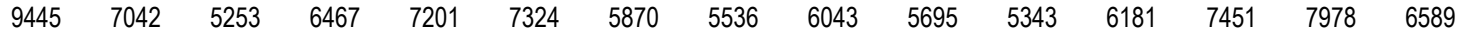

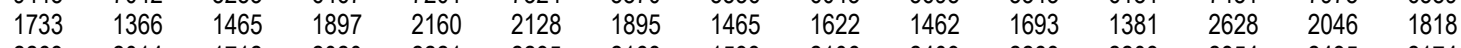

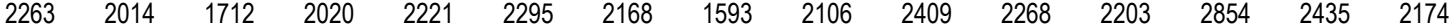

60 Other Entities

$13674 \quad 10614$

$8568 \quad 10545$

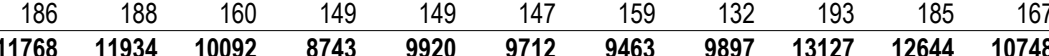


2003 White Book

-Regulated Hydro Projects-

1 Albeni Falls

2 Alder

3 Bonneville

4 Boundary

5 Box Canyon

6 Brownlee

7 Cabinet Gorge

8 Chelan

9 Chief Joseph

10 Cushman 1

11 Cushman 2

12 Diablo

13 Dworshak

14 Faraday

15 Gorge

16 Grand Coulee

17 Hells Canyon

18 Hungry Horse

19 lce Harbor

20 John Day

$21 \mathrm{La}$ Grande

22 Libby

23 Little Falls

25 Long Lake

26 Lower Baker

27 Lower Granite

29 Mayfield

30 McNary

31 Merwin

32 Monroe Street

33 Mossyrock

34 Nine Mile

35 North Fork

36 Noxon

37 Oak Grove

38 Oxbow

39 Pelton

40 Post Falls

41 Priest Rapids

42 River Mill

44 Rock Island PH\#2

45 Rocky Reach

46 Ross

47 Round Butte

48 Swift 1

49 Swift 2

50 The Dalles

51 Upper Baker

52 Upper Falls

53 Wanapum

54 Wells

55 White

56 Yale

Total Regulated Hydro w/Enc.
24 Little Goose

28 Lower Monumental

43 Rock Island PH\#1
Sep

Oct
Nov Dec Jan Dec

$\begin{array}{rrrrrrr}23 & 19 & 27 & 37 & 18 & 18 & 16 \\ 14 & 10 & 11 & 12 & 6 & 25 & 45 \\ 172 & 138 & 401 & 468 & 525 & 550 & 460 \\ 190 & 150 & 238 & 503 & 266 & 263 & 223 \\ 31 & 25 & 37 & 69 & 41 & 41 & 35 \\ 232 & 259 & 238 & 240 & 155 & 158 & 252 \\ 51 & 39 & 41 & 65 & 74 & 72 & 66 \\ 31 & 23 & 20 & 55 & 55 & 55 & 54 \\ 1793 & 1346 & 836 & 1080 & 1317 & 1297 & 985 \\ 3.3 & 2.3 & 19 & 1.8 & 27 & 25 & 29 \\ 2.4 & 0.7 & 30 & 0.1 & 49 & 50 & 66 \\ 74 & 56 & 76 & 35 & 106 & 102 & 1.5 \\ 443 & 205 & 52 & 52 & 52 & 51 & 51 \\ 8.1 & 7.5 & 8.2 & 8 & 6.2 & 16 & 8.9 \\ 86 & 68 & 87 & 43 & 118 & 118 & 2.7\end{array}$

$\begin{array}{rr}3283 & 2415 \\ 193 & 218\end{array}$

$\begin{array}{llll}92 & 81 & 77 & 86\end{array}$

$\begin{array}{llll}93 & 64 & 124 & 142\end{array}$

$920 \quad 695$

$\begin{array}{rr}21 & 15 \\ 522 & 357\end{array}$

$9.3 \quad 9.2$

$227 \quad 169$

22

$29 \quad 23$

$\begin{array}{ll}233 & 173 \\ 229 & 170\end{array}$

$\begin{array}{rr}28 & 25 \\ 818 & 766\end{array}$

$\begin{array}{rr}18 & 15 \\ 5.5 & 5.8\end{array}$

$56 \quad 48$

$5.6 \quad 5.7$

$69 \quad 57$

$\begin{array}{rr}21 & 20 \\ 101 & 114\end{array}$

$36 \quad 35$

$\begin{array}{rr}2.2 & 2.5 \\ 385 & 301\end{array}$

$\begin{array}{llllll}-301 & 347 & 452 & 541 & 533 & 416\end{array}$

$\begin{array}{rrrrrrr}4.6 & 4.3 & 4.7 & 4.6 & 3.6 & 9.2 & 5.1 \\ 174 & 187 & 144 & 171 & 185 & 184 & 163\end{array}$

$\begin{array}{lllllll}197 & 187 & 144 & 171 & 185 & 184 & 163 \\ 146 & 101 & 134 & 162 & 159 & 122\end{array}$

$\begin{array}{lllllll}827 & 660 & 420 & 547 & 656 & 646 & 504\end{array}$

$\begin{array}{rrrrrrr}52 & 40 & 70 & 33 & 121 & 107 & 0 \\ 91 & 87 & 88 & 76 & 76 & 85 & 94\end{array}$

$\begin{array}{lllllll}31 & 27 & 24 & 34 & 131 & 74 & 72\end{array}$

$\begin{array}{rrrrrrr}8.6 & 7.3 & 6.8 & 9.8 & 42 & 27 & 32\end{array}$

$\begin{array}{rrrrrrr}616 & 462 & 545 & 629 & 723 & 722 & 599 \\ 29 & 23 & 20 & 21 & 29 & 47 & 31\end{array}$

$\begin{array}{lllllll}4.9 & 52 & 98 & 98 & 88 & 10 & 4.6\end{array}$

$\begin{array}{rrrrrrr}484 & 351 & 290 & 398 & 497 & 488 & 359 \\ 543 & 430 & 269 & 346 & 420 & 412 & 316\end{array}$
Feb
Mar Apr1 Apr16

May

Jun

Jul Avg

-Total Regulated Hydro w/Enc.-

57 Federal System

58 Public Entities

59 Investor-Owned Entities

60 Other Entities

$\begin{array}{rrrrrrr}23 & 20 & 22 & 24 & 94 & 87 & 107\end{array}$

$13693 \quad 10630$

$8580 \quad 10559$ $\begin{array}{rr}14 & \\ 21 & \\ 451 & 465 \\ 186 & 248 \\ 30 & 38 \\ 146 & 258\end{array}$

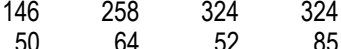

54
927

$927 \quad 922$

15

51

19

1686
114

114
93

109
718
44

44

80

129

95

24

11

96
110

110

85
535

535
35

35
6.7

75

7
18

18

72

18

$\begin{array}{rr}58 & 100 \\ 35\end{array}$

35

3.6

389

9.5
156

156

113
474

474
17

17
85

26
12

12

$584 \quad 661$

$\begin{array}{rr}584 & 661 \\ 8.9 & 22\end{array}$

5.8

$\begin{array}{ll}333 & 333 \\ 296 & 294\end{array}$

$928 \quad 934$

$\begin{array}{rr}43 & 42 \\ 109 & 86\end{array}$

$\begin{array}{lll}605 & 1527 & 1503\end{array}$

$\begin{array}{ll}194 & 244 \\ 8 & 245\end{array}$

$\begin{array}{rrr}89 & 67 & 28 \\ 186 & 130 & 136\end{array}$

823

24
179

51

182
191

191

70
606

66
15

15
52

15

35
96

35
10
389

389

18
156

113

475

476

$\begin{array}{rrr}86 & 80 & 138\end{array}$
$55 \quad 0.4$

$\begin{array}{rrr}18 & 18 & 29 \\ 30 & 7.1 & 23 \\ 295 & 134 & 134 \\ 281 & 417 & 530 \\ 44 & 60 & 72 \\ 324 & 324 & 305 \\ 52 & 85 & 176 \\ 52 & 32 & 9.9\end{array}$

$\begin{array}{rrr}45 & 38 & 25 \\ 50 & 8 & 21 \\ 183 & 132 & 345 \\ 606 & 399 & 332 \\ 74 & 55 & 48 \\ 192 & 312 & 235\end{array}$

$\begin{array}{lll}188 & 112 & 85\end{array}$

$\begin{array}{rr}54 & 487 \\ 9 & 32\end{array}$

32

9934
1.3
0.2

91
52

43

785

62

$78 \quad 78$

254

307

$154 \quad 14$

69
6.9

$579 \quad 487$

$9.5 \quad 8.6$

$229 \quad 229$

$297 \quad 275$

229
297
47

$\begin{array}{rr}47 & 53 \\ 106 & 91\end{array}$

$880 \quad 1165$

$1.3 \quad 28$

0.1

74

57

43
92

1484

239

232

232

758
37

173

30
519

519

72
1.3

523
526

8.4
340

13
15
0

$0 \quad 129$

$22 \quad 24$

54

286

34

120

36

14
55

55

23
175

27
394

$\begin{array}{ll}61 & 236 \\ 89 & 100\end{array}$

$\begin{array}{ll}19 & 152\end{array}$

746

$509 \quad 605$

$0.3 \quad 103$

$8.3 \quad 9.3$

229

267

9727

9474 $\begin{array}{rr}57 & 44 \\ 1352 & 1105\end{array}$

$\begin{array}{rr}1.3 & 14 \\ 0 & 29\end{array}$

$\begin{array}{ll}445 & 127\end{array}$

$39 \quad 14 \quad 20$

$168 \quad 91$

$\begin{array}{rrr}152 & 246 & 189\end{array}$

$31 \quad 120 \quad 78$

$124 \quad 136$

$\begin{array}{lll}63 & 12 & 34\end{array}$

$149 \quad 169$

$\begin{array}{lll}67 & 59 & 35\end{array}$

$\begin{array}{lll}402 & 294 & 219\end{array}$

$82 \quad 47 \quad 68$

$\begin{array}{lll}478 & 842 & 587\end{array}$

$71 \quad 24 \quad 52$

$11 \quad 11$

85
-10

$\begin{array}{lll}308 & 158 & 131\end{array}$

$\begin{array}{lll}34 & 25 & 25\end{array}$

$\begin{array}{rrr}39 & 128 & 36\end{array}$

$\begin{array}{lll}15 & 5.2 & 7.8\end{array}$

$\begin{array}{lll}112 & 317 & 337\end{array}$

$\begin{array}{lll}22 & 7.7 & 11\end{array}$

$193 \quad 172$

$\begin{array}{lll}120 & 104 & 116\end{array}$

627548

83
$\quad 89 \quad 89$
$154 \quad 79$

$285 \quad 213$ 
2003 White Book

-Regulated Hydro Projects-

1 Albeni Falls

2 Alder

3 Bonneville

4 Boundary

5 Box Canyon

6 Brownlee

7 Cabinet Gorge

8 Chelan

9 Chief Joseph

10 Cushman 1

11 Cushman 2

12 Diablo

13 Dworshak

14 Faraday

15 Gorge

16 Grand Coulee

17 Hells Canyon

18 Hungry Horse

19 lce Harbor

20 John Day

21 La Grande

22 Libby

23 Little Falls

24 Little Goose

25 Long Lake

26 Lower Baker

27 Lower Granite

28 Lower Monumental

29 Mayfield

30 McNary

31 Merwin

32 Monroe Street

33 Mossyrock

34 Nine Mile

35 North Fork

36 Noxon

37 Oak Grove

38 Oxbow

39 Pelton

40 Post Falls

41 Priest Rapids

42 River Mill

43 Rock Island PH\#1

44 Rock Island PH\#2

45 Rocky Reach

46 Ross

47 Round Butte

48 Swift 1

49 Swift 2

50 The Dalles

51 Upper Baker

52 Upper Falls

53 Wanapum

54 Wells

55 White

56 Yale

Total Regulated Hydro w/Enc.
Sep

Oct
Nov Dec Jan Feb

$\begin{array}{rrrr}23 & 19 & 27 & 37 \\ 14 & 10 & 11 & 12 \\ 172 & 138 & 401 & 468 \\ 190 & 150 & 238 & 503 \\ 31 & 25 & 37 & 69 \\ 232 & 259 & 238 & 240 \\ 51 & 39 & 41 & 65 \\ 31 & 23 & 20 & 55 \\ 1793 & 1346 & 836 & 1080\end{array}$

$3.3 \quad 2.3$

$2.4 \quad 0.7$

$\begin{array}{rr}74 & 56 \\ 443 & 205\end{array}$

$\begin{array}{lll}8.1 & 7.5 & 82\end{array}$

86
3283
193

$\begin{array}{rr}92 & 81 \\ 93 & 64 \\ 920 & 695\end{array}$

21
522
9

9.3

227

$22 \quad 22$

$29 \quad 23$

$\begin{array}{ll}233 & 173 \\ 229 & 170\end{array}$

28

834

$\begin{array}{rr}834 & 779 \\ 5.5 & 15.8\end{array}$

$5.5 \quad 5.8$

$56 \quad 48$

$69 \quad 57$

$\begin{array}{rr}21 & 20 \\ 101 & 114\end{array}$

$36 \quad 35$

$2.2 \quad 2.5$

385
4.6

$174 \quad 187$

$\begin{array}{lllllll}197 & 146 & 144 & 171 & 185 & 184 & 163\end{array}$

$\begin{array}{llllll}52 & 40 & 70 & 33 & 121 & 107\end{array}$

$\begin{array}{rrrrrrr}91 & 87 & 88 & 76 & 76 & 85 & 94\end{array}$

$\begin{array}{rrrrrrr}31 & 27 & 24 & 34 & 131 & 74 & 72 \\ 8.6 & 7.3 & 6.8 & 9.8 & 42 & 27 & 32\end{array}$

$\begin{array}{lllllll}616 & 462 & 545 & 629 & 723 & 722 & 599\end{array}$

$\begin{array}{rrrrrrr}29 & 23 & 20 & 21 & 29 & 47 & 31\end{array}$

$\begin{array}{rrrrrrr}4.9 & 5.2 & 9.8 & 9.8 & 8.8 & 10 & 4.6 \\ 484 & 351 & 290 & 398 & 497 & 488 & 359\end{array}$

$\begin{array}{lllllll}543 & 430 & 269 & 346 & 420 & 412 & 316\end{array}$
$920-695 \quad 668-803$

$5.6 \quad 5.7$

$\begin{array}{lllllll}827 & 660 & 420 & 547 & 656 & 646 & 504\end{array}$

Mar Apr1 Apr16

May

Jun

Jul Avg

\begin{tabular}{|c|c|c|c|c|c|c|c|c|c|c|}
\hline 18 & 18 & 16 & 14 & 17 & 18 & 18 & 29 & 45 & 38 & 25 \\
\hline 6 & 25 & 45 & 21 & 25 & 30 & 7.1 & 23 & 50 & 8 & 21 \\
\hline 525 & 550 & 460 & 451 & 465 & 295 & 134 & 134 & 183 & 132 & 345 \\
\hline 266 & 263 & 223 & 186 & 248 & 281 & 417 & 530 & 606 & 399 & 332 \\
\hline 41 & 41 & 35 & 30 & 38 & 44 & 60 & 72 & 74 & 55 & 48 \\
\hline 155 & 158 & 252 & 146 & 258 & 324 & 324 & 305 & 192 & 312 & 235 \\
\hline 74 & 72 & 66 & 50 & 64 & 52 & 85 & 176 & 188 & 112 & 85 \\
\hline 55 & 55 & 54 & 54 & 54 & 52 & 32 & 9.9 & 51 & 57 & 44 \\
\hline 1317 & 1297 & 985 & 927 & 922 & 928 & 934 & 880 & 1165 & 1352 & 1105 \\
\hline 27 & 25 & 29 & 20 & 1.1 & 1.3 & 24 & 1.3 & 28 & 1.3 & 14 \\
\hline 49 & 50 & 66 & 55 & 0.4 & 0.2 & 59 & 0.1 & 63 & 0 & 29 \\
\hline 106 & 102 & 1.5 & 15 & 72 & 91 & 71 & 74 & 172 & 154 & 70 \\
\hline 52 & 51 & 51 & 51 & 51 & 52 & 53 & 57 & 287 & 445 & 127 \\
\hline 6.2 & 16 & 8.9 & 17 & 31 & 43 & 42 & 43 & 39 & 14 & 20 \\
\hline 118 & 118 & 2.7 & 19 & 86 & 109 & 86 & 92 & 182 & 168 & 91 \\
\hline 2400 & 2294 & 1768 & 1686 & 1605 & 1527 & 1503 & 1484 & 2117 & 2504 & 1976 \\
\hline 133 & 127 & 198 & 114 & 194 & 244 & 245 & 239 & 152 & 246 & 189 \\
\hline 91 & 91 & 93 & 93 & 89 & 67 & 28 & 29 & 31 & 120 & 78 \\
\hline 99 & 105 & 127 & 109 & 186 & 130 & 136 & 232 & 173 & 124 & 136 \\
\hline 925 & 911 & 743 & 718 & 823 & 785 & 722 & 758 & 900 & 851 & 805 \\
\hline 8.3 & 39 & 63 & 44 & 56 & 62 & 12 & 37 & 63 & 12 & 34 \\
\hline 92 & 299 & 82 & 80 & 78 & 78 & 78 & 173 & 293 & 149 & 169 \\
\hline 13 & 21 & 8 & 11 & 24 & 31 & 30 & 30 & 34 & 14 & 19 \\
\hline 101 & 104 & 129 & 95 & 179 & 254 & 307 & 519 & 388 & 285 & 213 \\
\hline 30 & 46 & 18 & 24 & 51 & 69 & 72 & 72 & 72 & 33 & 42 \\
\hline 30 & 60 & 56 & 11 & 28 & 39 & 37 & 1.3 & 67 & 59 & 35 \\
\hline 101 & 106 & 131 & 96 & 182 & 248 & 315 & 523 & 398 & 293 & 216 \\
\hline 99 & 106 & 129 & 110 & 191 & 270 & 323 & 526 & 402 & 294 & 219 \\
\hline 55 & 55 & 172 & 85 & 70 & 70 & 171 & 8.4 & 82 & 47 & 68 \\
\hline 688 & 697 & 581 & 544 & 618 & 491 & 325 & 348 & 489 & 858 & 599 \\
\hline 67 & 83 & 103 & 35 & 66 & 109 & 92 & 13 & 71 & 24 & 52 \\
\hline 9.9 & 15 & 5.1 & 6.7 & 15 & 15 & 15 & 15 & 15 & 11 & 1 \\
\hline 92 & 68 & 286 & 75 & 52 & 55 & 207 & 0 & 129 & 85 & 9 \\
\hline 9.3 & 11 & 4.6 & 7 & 15 & 23 & 23 & 22 & 24 & 10 & 13 \\
\hline 7 & 18 & 9.9 & 18 & 35 & 54 & 47 & 54 & 43 & 15 & 23 \\
\hline 114 & 110 & 101 & 72 & 96 & 86 & 116 & 286 & 308 & 158 & 131 \\
\hline 17 & 20 & 17 & 18 & 24 & 34 & 34 & 34 & 34 & 25 & 2 \\
\hline 69 & 65 & 103 & 58 & 100 & 125 & 125 & 120 & 75 & 128 & 9 \\
\hline 31 & 34 & 38 & 35 & 35 & 33 & 54 & 36 & 39 & 36 & 36 \\
\hline 5.4 & 7.6 & 2.9 & 3.6 & 10 & 15 & 14 & 14 & 15 & 5.2 & 7.8 \\
\hline 541 & 533 & 416 & 389 & 389 & 55 & 55 & 55 & 212 & 317 & 337 \\
\hline 3.6 & 9.2 & 5.1 & 9.5 & 18 & 23 & 23 & 23 & 22 & 7.7 & 11 \\
\hline 185 & 184 & 163 & 156 & 156 & 159 & 171 & 175 & 189 & 193 & 172 \\
\hline 162 & 159 & 122 & 113 & 113 & 96 & 42 & 27 & 120 & 104 & 116 \\
\hline 656 & 646 & 504 & 474 & 475 & 476 & 416 & 394 & 642 & 627 & $54 \varepsilon$ \\
\hline 121 & 107 & 0 & 17 & 71 & 90 & 64 & 61 & 236 & 151 & 8 \\
\hline 76 & 85 & 94 & 85 & 86 & 80 & 138 & 89 & 100 & 89 & 8 \\
\hline 131 & 74 & 72 & 26 & 45 & 154 & 14 & 19 & 152 & 47 & 61 \\
\hline 42 & 27 & 32 & 12 & 20 & 69 & 5.9 & 7 & 46 & 13 & 2 \\
\hline 723 & 722 & 599 & 584 & 661 & 579 & 487 & 509 & 605 & 568 & 60 \\
\hline 29 & 47 & 31 & 8.9 & 22 & 34 & 32 & 0.3 & 103 & 60 & 0 \\
\hline 8.8 & 10 & 4.6 & 5.8 & 10 & 9.5 & 8.6 & 8.3 & 9.3 & 9.6 & 8. \\
\hline 497 & 488 & 359 & 333 & 333 & 229 & 229 & 229 & 395 & 375 & 36 \\
\hline 420 & 412 & 316 & 296 & 294 & 297 & 275 & 267 & 407 & 405 & 350 \\
\hline 5.9 & 34 & 28 & 18 & 36 & 47 & 53 & 53 & 64 & 48 & 3 \\
\hline 94 & 87 & 107 & 32 & 60 & 106 & 91 & 0 & 112 & 30 & 57 \\
\hline 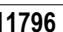 & 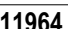 & 0116 & 8765 & 9945 & 9739 & 9482 & 9915 & 13151 & 12679 & 10773 \\
\hline
\end{tabular}

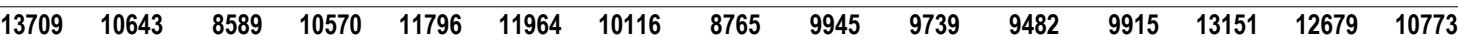

Tydro w/Enc.

57 Federal System

58 Public Entities

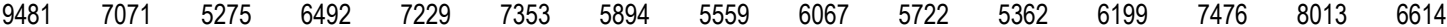

59 Investor-Owned Entities

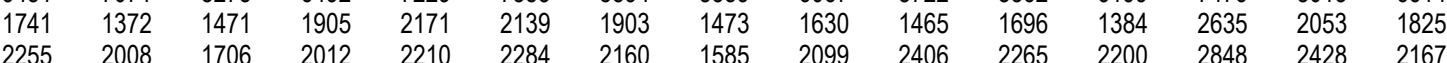

60 Other Entities

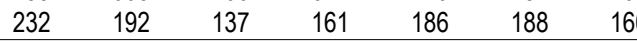

149149

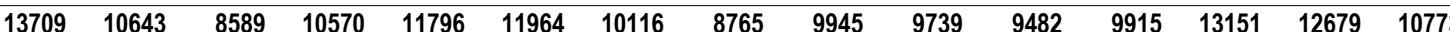


5 Black Canyon

6 Bliss

7 Blue River

8 Bonneville Fishway

9 Bull Run

10 Calispel Creek

11 Carmen-Smith

12 Cascade

13 Cedar Falls \& Newhalem

14 Chandler

15 CJ Strike

16 Condit Sm Plts

17 Cougar

18 Cowlitz Falls

19 Detroit

20 Dexter

21 Felt

22 Foster

23 Gem State Hydro

24 Green Peter

25 Green Springs - USBR

26 Hills Creek

27 Hood Street

28 Idaho Falls - City Plant

29 Idaho Falls - Lower Plant

30 Idaho Falls - Upper Plant

31 Island Park

32 Jackson

33 Klamath

34 Lake Creek

35 Leaburg

36 Lookout Point

37 Lost Creek

38 Lower Salmon

39 McNary Fishway

40 Mill Creek (WA)

41 Milltown/Flint Creek

42 Milner

43 Minidoka

44 Morse Creek

45 Moyie Springs

46 North Umpqua

47 Packwood

48 Palisades

49 Reader Gulch

50 Rogue

51 Roza

52 Shoshone Falls

53 Snoq Elect \& Nooksack

54 Soda Creek

55 South Fork Tolt

56 Spring Plants

57 Stone Creek

58 Strawberry Creek

59 Swan Falls

60 The Dalles Fishway

61 Trail Bridge

62 TW Sullivar

63 Twin Falls

64 Upper Salmon $1 \& 2$

65 Upper Salmon 3 \& 4

66 Walterville

67 Wynochee

68 Yelm

Total Independent Hydro w/Enc.
55

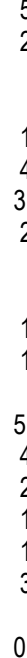

0.7

15

$22 \quad 32$

7.3

0.1

6

4.8
18

$18 \quad 18$

$12 \quad 32$

$\begin{array}{ll}2.7 & 2.7 \\ 7.5 & 7.5\end{array}$

$25 \quad 22$

44

$26 \quad 26$

$0.4 \quad 0.4$

0.3

0
31

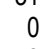

2

93

6.1

$131 \quad 131$

$0.4 \quad 0.4$

33

$13 \quad 13$

$\begin{array}{lll}10 & 10 & 12\end{array}$

$37 \quad 34$

$0.5 \quad 0.5$

$\begin{array}{rr}0 & 1 \\ 16 & 16\end{array}$

$\begin{array}{rrr}13 & 10 & 2.4 \\ 2 & 2.8 & 7.8\end{array}$

$4.1 \quad 6.7$

44
52 54

$10 \quad 7.9$

32

$\begin{array}{ll}6 & 12\end{array}$

4

$4 \quad 26$

$\begin{array}{rr}7.3 & 7.3 \\ 25 & 23\end{array}$

$$
0.7
$$$$
7
$$

7
4.8

2.7
7.5

28

72

0.3

12

$$
2
$$

2
85

85
3.7
$55 \quad 35 \quad 13$

$\begin{array}{rrrrrr}18 & 11 & 4.3 & 5.4 & 11 & 16 \\ 7 & 9 & 13 & 15 & 7 & 4 \\ 0 & 0 & 0 & 0 & 1 & 0 \\ 10 & 7.9 & 9 & 5.9 & 7.1 & 8.8 \\ 41 & 46 & 44 & 46 & 46 & 43\end{array}$

$\begin{array}{rrr}3.4 & 2.2 & 2.2 \\ 5 & 24 & 24\end{array}$

$\begin{array}{lll}23 & 23 & 20 \\ 24 & 12 & 25\end{array}$

$23 \quad 27$

$\begin{array}{lll}0.7 & 0.7 & 0.7 \\ 6 & 6\end{array}$

21

53

75
40

$40 \quad 26$

0.4

$55-2.7$

$0.4 \quad 0.4$

$27 \quad 26$

1

$2.2 \quad 3.2$

$0.4 \quad 0.4$
3.2

$13 \quad 16$

12

59
19

24

$3.1 \quad 23$

$\begin{array}{ll}16 & 20 \\ 0.7 & 0.7\end{array}$

0.7

0.7

14
12

34

6
0.7

0.7
5

5
5
4.8

$\begin{array}{rr}4.8 & 4.8 \\ 80 & 33\end{array}$

$52 \quad 55$

$2.7 \quad 2.7$

7.1

9

$\begin{array}{ll}18 & 17\end{array}$

$\begin{array}{rr}30 & 27 \\ 72 & 72\end{array}$

7.2

0.2

10

$\begin{array}{lll}3.1 & 9 & 28\end{array}$

$0.5 \quad 0.5$

2
82

$2.7 \quad 5.9$

$15 \quad 15$

$28 \quad 25$

5

$12 \quad 12$

$\begin{array}{rr}56 & 28 \\ 0.5 & 0.5\end{array}$

$0.5 \quad 0.5$

$\begin{array}{rr}5 & 5 \\ 31 & 30\end{array}$

$31 \quad 30$

$\begin{array}{ll}1.8 & 7.1 \\ 1.5 & 1.5\end{array}$

$19 \quad 17$

$\begin{array}{rr}19 & 17 \\ 2.7 & 2.7\end{array}$

$\begin{array}{ll}2.7 & 2.7 \\ 3.3 & 3\end{array}$

14

$14 \quad 14$

$\begin{array}{ll}14 & 10 \\ 19 & 19\end{array}$

$\begin{array}{lll}18 & 16 & 15\end{array}$

$\begin{array}{rrr}18 & 16 & 15 \\ 5 & 3.1 & 7.2\end{array}$

Feb Mar Apr1 Apr16

May Jun

Jul Avg

-Total Independent Hydro W/Enc.-

69 Federal System

70 Public Entities

71 Investor-Owned Entities

$\begin{array}{llll}437 & 435 & 370 \quad 386\end{array}$

$\begin{array}{llll}127 & 138 & 121 & 127 \\ 484 & 502 & 519 & 470\end{array}$ $\begin{array}{rr}0 & \\ 16 & 5 \\ 7 & \\ 0 & 7 \\ 4.8 & 7.9 \\ 40 & 40\end{array}$

$\begin{array}{lllllll}5.8 & 20 & 20 & 67 & 70 & 75 & 28\end{array}$ $\begin{array}{rrrrrrr}7 & 25 & 27 & 11 & 38 & 27 & 16 \\ 0 & 0 & 17 & 21 & 21 & 8 & 11 \\ 7.9 & 10 & 10 & 0 & 1 & 0 & 0.2\end{array}$

$\begin{array}{llll}21 & 19 & 25 & 25\end{array}$

$\begin{array}{llll}24 & 24 & 24 & 24\end{array}$

14

$14 \quad 17$

$\begin{array}{ll}1.8 & 1.1 \\ 6.6 & 8.6\end{array}$

$9.3-13-10$

$\begin{array}{lll}51 & 51 & 49\end{array}$

$\begin{array}{llll}21 & 31 & 36 & 36\end{array}$

$\begin{array}{rrr}6 & 7 & 8 \\ 14 & 33 & 48\end{array}$

$18 \quad 20$

$\begin{array}{lll}5 & 5 & 10\end{array}$

$0.7 \quad 0.7$

21
15

$\begin{array}{lll}33 & 89 & 19 \\ 7.3 & 7.3 & 7.3\end{array}$

$\begin{array}{lll}7.3 & 7.3 & 7.3\end{array}$

$\begin{array}{rrr}5 & 19 & 31 \\ 0.7 & 0.7 & 0.7\end{array}$

$\begin{array}{rr}0.7 & 0.7 \\ 5 & 5\end{array}$

$$
5
$$

5
5
4.8

4.8
42

50

2.7

13
15

$13 \quad 14$

24

7.2

0.4
0.4

0.4

2.2

0.5

2
95

95
4.8

4.8
27
0.4

0.4

40

$4.3 \quad 13$

127.4

$\begin{array}{rr}39 & 59 \\ 0.5 & 0.5\end{array}$

$\begin{array}{lll}5 & 62\end{array}$

$30 \quad 27$

0.6

1.5

17

$2.7 \quad 2.7$

3.23 .8

$14 \quad 14$

$\begin{array}{rrrrr}8 & 0 & 0 & 0 & 0 \\ 18 & 16 & 16 & 16 & 16\end{array}$

$\begin{array}{rrrr}7.2 & 8 & 6.3 & 6.3\end{array}$

$\begin{array}{lll}6.3 & 6.3 & 9.5\end{array}$

14
9.5

$\begin{array}{rr}10 & 10 \\ 797 & 944\end{array}$

$\begin{array}{rrrr}10 & 8.1 & 7.5 & 8.1 \\ 38 & 38 & 37 & 42\end{array}$

$\begin{array}{llll}2.9 & 2.4 & 2.6 & 2.7\end{array}$

$\begin{array}{llll}24 & 24 & 24 & 21\end{array}$

$\begin{array}{llll}15 & 15 & 12 & 10\end{array}$

$\begin{array}{rr}0 & 0.3 \\ 17 & 16\end{array}$

$11 \quad 4.5$

$2.2 \quad 12$

$\begin{array}{rr}4.6 & 8.6 \\ 38 & 50\end{array}$

$28 \quad 26$

$11 \quad 16$

$\begin{array}{ll}29 & 26 \\ 30 & 41\end{array}$

$\begin{array}{rr}6 & 9.3 \\ 0.7 & 0.7\end{array}$

$\begin{array}{rr}0.7 & 0.7 \\ 6 & 12\end{array}$

$\begin{array}{ll}19 & 14 \\ 10 & 28\end{array}$

$\begin{array}{ll}7.3 & 7.3\end{array}$

$14 \quad 18$

$\begin{array}{ll}0.3 & 0.6 \\ 7 & 5.9\end{array}$

$\begin{array}{ll}7 & 6.3\end{array}$

$\begin{array}{ll}7 & 6.3\end{array}$

$4.8 \quad 4.8$

$46 \quad 43$ 
Table A-4: Regional Independent Hydro Projects

PNW Loads and Resources Study

2004 - 2005 Operating Year

1937 Water Year

2003 White Book

Average Energy in Megawatts

Aug1 Aug16

Oct Nov Dec Jan

Mar Apr1 Apr1

May Jun

Jul Avg

72 Other Entities

$\begin{array}{rr}0 & 0 \\ 1048 & 1075\end{array}$

$\begin{array}{rrr}0 & 0 & 0 \\ 1010 & 982 & 902\end{array}$

$\begin{array}{rrrrrrrrrr}0 & 0 & 0 & 0 & 0 & 0 & 0 & 0 & 0 & 0 \\ 969 & 783 & 797 & 944 & 1212 & 1266 & 1553 & 1603 & 1160 & 1084\end{array}$


$127 \quad 138$ $484 \quad 502$

$\begin{array}{rr}35 & 13 \\ 11 & 4.3 \\ 9 & 13 \\ 0 & 0 \\ 7.9 & 9 \\ 46 & 44 \\ 2.2 & 2.2 \\ 24 & 24 \\ 4 & 2 \\ 1 & 0 \\ 16 & 14 \\ 10 & 2.4 \\ 2.8 & 7.8 \\ 4.1 & 6.7 \\ 52 & 54 \\ 23 & 20 \\ 12 & 25 \\ 7.9 & 6.2 \\ 32 & 44 \\ 6 & 12 \\ 0.7 & 0.7 \\ 4 & 8 \\ 11 & 10 \\ 4 & 26 \\ 7.3 & 7.3 \\ 25 & 23 \\ 0.3 & 0.7 \\ 6 & 6\end{array}$

0
5.4
15
0
5.9
46

$\begin{array}{lllll}0 & 0 & 0 & 5.8 & 20\end{array}$ $\begin{array}{rrrr}11 & 16 & 16 & 5.1 \\ 7 & 4 & 7\end{array}$

$\begin{array}{rrrr}7.1 & 0 & 0 & 0 \\ 46 & 8.8 & 4.8 & 79\end{array}$ $\begin{array}{lllll}3.2 & 3.6 & 3.4 & 2.1 & 19\end{array}$ $\begin{array}{llll}12 & 12 & 24 & 24\end{array}$ 115

7

$13 \quad 16$

3.2

18

0
14

$\begin{array}{ll}5.6 & 1.8\end{array}$

$9.6 \quad 6.6$

$9.5 \quad 9.3$

$\begin{array}{lllllll}59 & 55 & 51 & 51 & 49 & 49 & 45 \\ 23 & 20 & 21 & 31 & 36 & 36 & 37\end{array}$

$\begin{array}{ll}36 & 37 \\ 29 & 29\end{array}$

48

66

16
0.7

23

19

45

$16 \quad 34$

27

0.7

$\begin{array}{rr}6 & 0.7 \\ 5 & 0.7\end{array}$

7.3
6
0.7
6

$\begin{array}{ll}7.3 & 7.3\end{array}$

4
0.7

0.7

$\begin{array}{rrrr}6 & 6 & 5 & \\ 7 & 6 & 5 & \\ 7 & 6 & 5 & \\ 4.8 & 4.8 & 4.8 & 4.8\end{array}$

5

5

19

31
0.7

0.7
5

5
6

$\begin{array}{rrrr}5 & 6 & 6 & 7 \\ 4.8 & 4.8 & 4.8 & 4.8\end{array}$

4.8
65

12

2.7

13
64

$15 \quad 56$

24

7.2

0.4

0.2

0
14
0.5

0.5

2
141

141
8.6

$\begin{array}{rr}8.6 & 19 \\ 38 & 95\end{array}$

$0.4 \quad 0.4$

$50 \quad 51$

$13 \quad 13$

$\begin{array}{ll}68 & 68\end{array}$

$\begin{array}{ll}0.5 & 0.5\end{array}$

28

4.7

1.5

16

2.7

3.8

14

$\begin{array}{rr}14 & 14 \\ 0 & 0\end{array}$

$\begin{array}{rr}16 & 0 \\ 14 & 16\end{array}$

$14 \quad 14-14$

$\begin{array}{ll}6.3 & 9.5\end{array}$

$4.3 \quad 4.3$
Jun

Ju

Avg 
Table A-4: Regional Independent Hydro Projects

PNW Loads and Resources Study

2005 - 2006 Operating Year

1937 Water Year

2003 White Book

Average Energy in Megawatts

Aug1 Aug16

Oct Nov Dec Jan

Mar Apr1 Apr16

May Jun

Jul Avg

72 Other Entities

$\begin{array}{rr}0 & 0 \\ 1048 & 1075\end{array}$

0

902

$\begin{array}{rrrrrrrrrr}0 & 0 & 0 & 0 & 0 & 0 & 0 & 0 & 0 & 0 \\ 969 & 783 & 797 & 945 & 1212 & 1266 & 1554 & 1604 & 1161 & 1084\end{array}$


1 American Falls

2 Anderson Ranch

3 Big Cliff

4 Big Creek

5 Black Canyon

6 Bliss

7 Blue River

8 Bonneville Fishway

9 Bull Run

10 Calispel Creek

11 Carmen-Smith

12 Cascade

13 Cedar Falls \& Newhalem

14 Chandler

15 CJ Strike

16 Condit Sm Plts

17 Cougar

18 Cowlitz Falls

19 Detroit

20 Dexter

21 Felt

22 Foster

23 Gem State Hydro

24 Green Peter

25 Green Springs - USBR

26 Hills Creek

27 Hood Street

28 Idaho Falls - City Plant

29 Idaho Falls - Lower Plant

30 Idaho Falls - Upper Plant

31 Island Park

32 Jackson

33 Klamath

34 Lake Creek

35 Leaburg

36 Lookout Point

37 Lost Creek

38 Lower Salmon

39 McNary Fishway

40 Mill Creek (WA)

41 Milltown/Flint Creek

42 Milner

43 Minidoka

44 Morse Creek

45 Moyie Springs

46 North Umpqua

47 Packwood

48 Palisades

49 Reader Gulch

50 Rogue

51 Roza

52 Shoshone Falls

53 Snoq Elect \& Nooksack

54 Soda Creek

55 South Fork Tolt

56 Spring Plants

57 Stone Creek

58 Strawberry Creek

59 Swan Falls

60 The Dalles Fishway

61 Trail Bridge

62 TW Sullivar

63 Twin Falls

64 Upper Salmon 1 \& 2

65 Upper Salmon 3 \& 4

66 Walterville

67 Wynochee

68 Yelm

Total Independent Hydro w/Enc.
Mar Apr1 Apr16

May Jun

Jul Avg
-Total Independent Hydro W/Enc.-

69 Federal System

70 Public Entities

71 Investor-Owned Entities

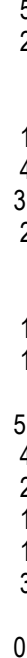

0.7

15

$22 \quad 32$

7.3

0.1

6

4.8
18

$18 \quad 18$

$12 \quad 32$

$\begin{array}{ll}2.7 & 2.7 \\ 7.5 & 7.5\end{array}$

$25 \quad 22$

44

$26 \quad 26$

0.3

0
31
0

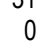

2

93

6.1

$132 \quad 132$

$0.4 \quad 0.4$

33

$13 \quad 13$

10

$37 \quad 34$

$0.5 \quad 0.5$ $\begin{array}{ll}7.2 & 7.2 \\ 0.4 & 0.4\end{array}$ $\begin{array}{lll}55 & 35 & 13\end{array}$

$\begin{array}{rrrrrr}18 & 11 & 4.3 & 5.4 & 11 & 16 \\ 7 & 9 & 13 & 15 & 7 & 4 \\ 0 & 0 & 0 & 0 & 1 & 0 \\ 10 & 7.9 & 9 & 5.9 & 7.1 & 8.8 \\ 41 & 46 & 44 & 46 & 46 & 43\end{array}$

$\begin{array}{rrr}3.4 & 2.2 & 2.2 \\ 24 & 24 & 24\end{array}$

$\begin{array}{rr}0 & 1 \\ 16 & 16\end{array}$

$\begin{array}{rrr}13 & 10 & 2.4 \\ 2 & 2.8 & 7.8\end{array}$

$\begin{array}{ll}2.8 & 6.7 \\ 52 & 54\end{array}$

$\begin{array}{lll}44 & 52 & 54\end{array}$

$23 \quad 23 \quad 20$

$\begin{array}{lll}24 & 12 & 25\end{array}$

7.9

32

44

$12 \quad 16$

0.7

10

26

7.3

$$
0.7
$$

0.7
6

6

$\begin{array}{rrrr}7 & 6 & 5 & 6 \\ 4.8 & 4.8 & 4.8 & 4.8\end{array}$

21

$53 \quad 55$

2.7

7.5

45

$40 \quad 26$

$\begin{array}{lll}28 & 30 & 30\end{array}$

7.2

0.4

0.3

0.4

0.2

6
3.1

0.5

$$
2
$$

2
85

85
3.7

$\begin{array}{ll}3.7 & 2.7 \\ 55 & 15\end{array}$

55
0.4

27

3.6

8.4

$\begin{array}{rrrr}30 & 19 & 56 & 28 \\ 0.5 & 0.5 & 0.5 & 0.5\end{array}$

Feb

\begin{tabular}{|c|c|}
\hline & \\
\hline 16 & \\
\hline 7 & \\
\hline 0 & \\
\hline 4.8 & \\
\hline 40 & \\
\hline 2.1 & \\
\hline 24 & \\
\hline 14 & \\
\hline 0 & \\
\hline 14 & \\
\hline 1.8 & \\
\hline 6.6 & \\
\hline 9.3 & \\
\hline 51 & \\
\hline 21 & \\
\hline 6 & \\
\hline 14 & \\
\hline 18 & \\
\hline 5 & \\
\hline 0.7 & \\
\hline 9 & \\
\hline 11 & \\
\hline $\begin{array}{r}1 \\
73\end{array}$ & \\
\hline $\begin{array}{r}7.3 \\
4\end{array}$ & \\
\hline 0.7 & \\
\hline 5 & \\
\hline 6 & \\
\hline 6 & \\
\hline 4.8 & \\
\hline 16 & \\
\hline 53 & \\
\hline 2.7 & \\
\hline 13 & \\
\hline 12 & \\
\hline 12 & \\
\hline 25 & \\
\hline 7.2 & \\
\hline 0.4 & \\
\hline 0.2 & \\
\hline $\begin{array}{r}3 \\
24\end{array}$ & \\
\hline $\begin{array}{l}2.4 \\
0.5\end{array}$ & \\
\hline 2 & \\
\hline 92 & \\
\hline 3.7 & \\
\hline 23 & \\
\hline 0.4 & \\
\hline 27 & \\
\hline 4.3 & \\
\hline 12 & \\
\hline 39 & \\
\hline 0.5 & \\
\hline $\begin{array}{r}5 \\
30\end{array}$ & \\
\hline $\begin{array}{c}30 \\
0.6\end{array}$ & \\
\hline $\begin{array}{l}0.6 \\
1.5\end{array}$ & \\
\hline 17 & \\
\hline 2.7 & \\
\hline 3.2 & \\
\hline 14 & \\
\hline 8 & \\
\hline 18 & \\
\hline 15 & \\
\hline $\begin{array}{l}7.2 \\
43\end{array}$ & \\
\hline $\begin{array}{r}4.3 \\
10\end{array}$ & \\
\hline
\end{tabular}

28
$-12 \quad 12$

$\begin{array}{llllll}20 & 20 & 67 & 70 & 75 & 28\end{array}$ $\begin{array}{rrrrrr}25 & 27 & 11 & 39 & 28 & 16 \\ 10 & 17 & 21 & 21 & 8 & 11 \\ 0 & 0 & 0 & 1 & 0 & 0.2\end{array}$

$\begin{array}{lll}1 & 0.2 \\ 8.1 & 8.5 & 8.1\end{array}$

$\begin{array}{lll}38 & 37 & 42\end{array}$

$2.6-2.7$

$\begin{array}{lll}15 & 12 & 10\end{array}$

$\begin{array}{rrr}0 & 0 & 0.3 \\ 17 & 17 & 16\end{array}$

$\begin{array}{lll}1.2 & 11 & 4.5\end{array}$

$\begin{array}{lll}30 & 2.2 & 12\end{array}$

$\begin{array}{lll}43 & 38 & 50\end{array}$

$\begin{array}{lll}37 & 28 & 26\end{array}$

$\begin{array}{lll}29 & 11 & 16\end{array}$

$\begin{array}{lll}91 & 30 & 41\end{array}$

$\begin{array}{lll}17 & 6 & 9.3 \\ 0.7 & 0.7 & 0.7\end{array}$

$\begin{array}{lll}23 & 6 & 0.7\end{array}$

$\begin{array}{lll}17 & 19 & 14\end{array}$

$10 \quad 28$

$\begin{array}{lll}35 & 14 & 18\end{array}$

$\begin{array}{lll}0.7 & 0.3 & 0.6\end{array}$

$\begin{array}{lll}7 & 7 & 5.9 \\ 7 & 7 & 6.3\end{array}$

$\begin{array}{lll}7 & 7 & 6.3\end{array}$

$\begin{array}{lll}4.8 & 4.8 & 4.8\end{array}$

$8246 \quad 43$

$\begin{array}{lll}2.7 & 2.7 & 2.7\end{array}$

$\begin{array}{lll}13 & 10 & 9.5\end{array}$

$24 \quad 35$

$46 \quad 30$

$\begin{array}{ll}7.2 & 7.2\end{array}$

$\begin{array}{ll}0.4 & 0.4\end{array}$

$31 \quad 16$

$\begin{array}{ll}0.5 & 0.4\end{array}$

$104-107$

$20 \quad 9.9$

$132 \quad 66$

$0.4-0.4$

$13 \quad 7.9$

$6.3 \quad 9.2$

$0.5-0.5$

$\begin{array}{ll}9 & 6.1\end{array}$

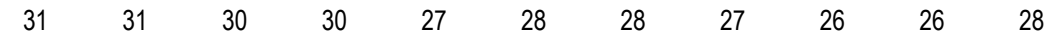

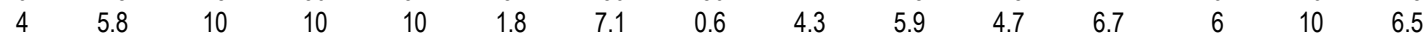

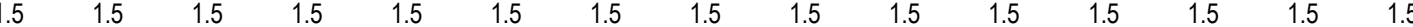

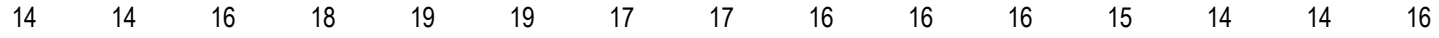

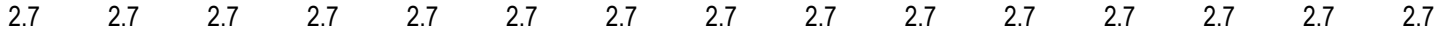

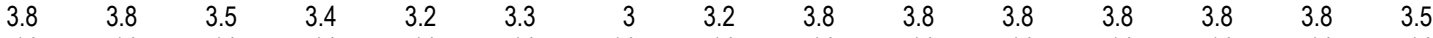

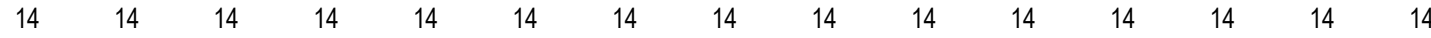

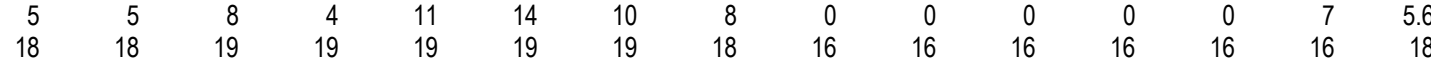

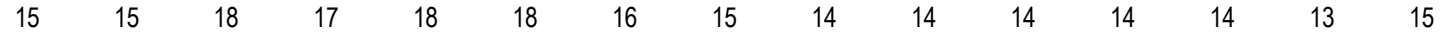

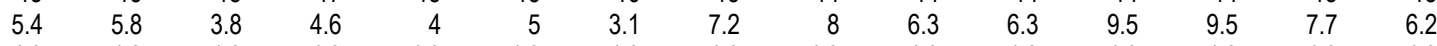

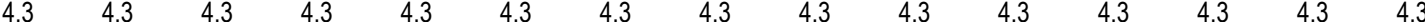

$\begin{array}{lllllllllllllll}1049 & 1076 & 1011 & 983 & 903 & 969 & 783 & 798 & 945 & 1213 & 1267 & 1555 & 1605 & 1161 & 1085\end{array}$ 
Table A-4: Regional Independent Hydro Projects

PNW Loads and Resources Study

2006 - 2007 Operating Year

1937 Water Year

2003 White Book

Average Energy in Megawatts

Aug1 Aug16

Oct Nov Dec Jan

Mar Apr1 Apr1

May Jun

Jul Avg

72 Other Entities

$\begin{array}{rr}0 & 0 \\ 1049 & 1076\end{array}$

0

$\begin{array}{rrrrrrrrrrr}0 & 0 & 0 & 0 & 0 & 0 & 0 & 0 & 0 & 0 & 0 \\ 903 & 969 & 783 & 798 & 945 & 1213 & 1267 & 1555 & 1605 & 1161 & 1085\end{array}$


$438 \quad 436 \quad 371-386$

$127 \quad 138$ $484 \quad 502$

$\begin{array}{rrrrr}13 & 0 & 0 & 0 & \\ 4.3 & 5.4 & 11 & 16 & \\ 13 & 15 & 7 & 4 & \\ 0 & 0 & 1 & 0 & \\ 9 & 5.9 & 7.1 & 8.8 & 4.8 \\ 44 & 46 & 46 & 43 & 40 \\ 2.2 & 3.2 & 3.6 & 3.4 & 2.1 \\ 24 & 12 & 12 & 12 & 24 \\ 2 & 1 & 15 & 7 & 14 \\ 0 & 0 & 1 & 0 & \\ 14 & 13 & 16 & 14 & 14.8 \\ 2.4 & 2.2 & 3.2 & 5.6 & 1.8 \\ 7.8 & 11 & 18 & 9.6 & 6.6 \\ 6.7 & 12 & 13 & 9.5 & 9.3 \\ 54 & 59 & 59 & 55 & 51 \\ 20 & 19 & 23 & 20 & 21 \\ 25 & 24 & 6 & 4 & \\ 6.2 & 3.1 & 23 & 10 & 14 \\ 44 & 46 & 20 & 11 & \\ 12 & 16 & 4 & 4 & \end{array}$

0.7

0.7

4
12

0.7

9
11

$\begin{array}{rr}1 & 33 \\ 7.3 & 7.3\end{array}$

7.3

6
0.7

4
0.7

0.7
5

5
6
6

5

5
5
4.8

4.8
42

50

2.7

13

15

$13 \quad 14$

24

7.2

0.4
0.4

0.4
0

2.2

3.1

0.5

$\begin{array}{rr}9 & 2.8 \\ 0.5 & 0.5\end{array}$

2.4

0.5

95

95
4.8

4.8
27

0.4

40

13

7.4

59

0.5
Apr1 Apr16

May
Jun

Ju

Jul Avg 
Table A-4: Regional Independent Hydro Projects

Continued PNW Loads and Resources Study

2007 - 2008 Operating Year

1937 Water Year

2003 White Book

Average Energy in Megawatts

Aug1 Aug16

Oct Nov Dec Jan

Mar Apr1 Apr16

May Jun

Jul Avg

72 Other Entities

$\begin{array}{rr}0 & 0 \\ 1049 & 1076\end{array}$

0

0
903

$\begin{array}{rrrrrrrrrr}0 & 0 & 0 & 0 & 0 & 0 & 0 & 0 & 0 & 0 \\ 969 & 783 & 798 & 945 & 1213 & 1267 & 1555 & 1605 & 1161 & 1085\end{array}$


-Independent Hydro Projects-

1 American Falls

2 Anderson Ranch

3 Big Cliff

4 Big Creek

5 Black Canyon

6 Bliss

7 Blue River

8 Bonneville Fishway

9 Bull Run

10 Calispel Creek

11 Carmen-Smith

12 Cascade

13 Cedar Falls \& Newhalem

14 Chandler

15 CJ Strike

16 Condit Sm Plts

17 Cougar

18 Cowlitz Falls

19 Detroit

20 Dexter

21 Felt

22 Foster

23 Gem State Hydro

24 Green Peter

25 Green Springs - USBR

26 Hills Creek

27 Hood Street

28 Idaho Falls - City Plant

29 Idaho Falls - Lower Plant

30 Idaho Falls - Upper Plant

31 Island Park

32 Jackson

33 Klamath

34 Lake Creek

35 Leaburg

36 Lookout Point

37 Lost Creek

38 Lower Salmon

39 McNary Fishway

40 Mill Creek (WA)

41 Milltown/Flint Creek

42 Milner

43 Minidoka

44 Morse Creek

45 Moyie Springs

46 North Umpqua

47 Packwood

48 Palisades

49 Reader Gulch

50 Rogue

51 Roza

52 Shoshone Falls

53 Snoq Elect \& Nooksack

54 Soda Creek

55 South Fork Tolt

56 Spring Plants

57 Stone Creek

58 Strawberry Creek

59 Swan Falls

60 The Dalles Fishway

61 Trail Bridge

62 TW Sullivar

63 Twin Falls

64 Upper Salmon 1 \& 2

65 Upper Salmon 3 \& 4

66 Walterville

67 Wynochee

68 Yelm

\begin{tabular}{|c|c|c|c|c|c|c|c|c|c|c|c|c|c|}
\hline 55 & 35 & 13 & 0 & 0 & 0 & 0 & 5.8 & 20 & 20 & 67 & 70 & 75 & 28 \\
\hline 23 & 11 & 4.3 & 5.4 & 11 & 16 & 16 & 5.1 & 25 & 27 & 11 & 39 & 28 & 16 \\
\hline 8 & 9 & 13 & 15 & 7 & 4 & 7 & 7 & 10 & 17 & 21 & 21 & 8 & 11 \\
\hline 0 & 0 & 0 & 0 & 1 & 0 & 0 & 0 & 0 & 0 & 0 & 1 & 0 & 0.2 \\
\hline 10 & 7.9 & 9 & 5.9 & 7.1 & 8.8 & 4.8 & 7.9 & 10 & 10 & 10 & 8.1 & 7.5 & 8.1 \\
\hline 41 & 46 & 44 & 46 & 46 & 43 & 40 & 40 & 39 & 39 & 38 & 38 & 37 & 42 \\
\hline 3.4 & 2.2 & 2.2 & 3.2 & 3.6 & 3.4 & 2.1 & 1.9 & 2.5 & 2.5 & 2.9 & 2.4 & 2.6 & 2.7 \\
\hline 24 & 24 & 24 & 12 & 12 & 12 & 24 & 24 & 24 & 24 & 24 & 24 & 24 & 21 \\
\hline 5 & 4 & 2 & 1 & 15 & 7 & 14 & 15 & 15 & 15 & 15 & 15 & 12 & 10 \\
\hline 0 & 1 & 0 & 0 & 1 & 0 & 0 & 1 & 0 & 0 & 1 & 0 & 0 & 0.3 \\
\hline 17 & 16 & 14 & 13 & 16 & 14 & 14 & 17 & 17 & 17 & 17 & 17 & 17 & 16 \\
\hline 13 & 10 & 2.4 & 2.2 & 3.2 & 5.6 & 1.8 & 1.1 & 1.1 & 1.1 & 1.1 & 1.2 & 11 & 4.5 \\
\hline 2 & 2.8 & 7.8 & 11 & 18 & 9.6 & 6.6 & 8.6 & 18 & 18 & 25 & 30 & 2.2 & 12 \\
\hline 5.2 & 4.1 & 6.7 & 12 & 13 & 9.5 & 9.3 & 13 & 10 & 10 & 8.4 & 8.1 & 4.6 & 8.6 \\
\hline 44 & 52 & 54 & 59 & 59 & 55 & 51 & 51 & 49 & 49 & 45 & 43 & 38 & 50 \\
\hline 23 & 23 & 20 & 19 & 23 & 20 & 21 & 31 & 36 & 36 & 37 & 37 & 28 & 26 \\
\hline 17 & 12 & 25 & 24 & 6 & 4 & 6 & 7 & 8 & 29 & 29 & 29 & 11 & 16 \\
\hline 10 & 7.9 & 6.2 & 3.1 & 23 & 10 & 14 & 33 & 48 & 48 & 64 & 66 & 29 & 26 \\
\hline 30 & 32 & 44 & 46 & 20 & 11 & 18 & 20 & 33 & 66 & 106 & 91 & 30 & 41 \\
\hline 6 & 6 & 12 & 16 & 4 & 4 & 5 & 5 & 10 & 16 & 17 & 17 & 6 & 9.3 \\
\hline 0.7 & 0.7 & 0.7 & 0.7 & 0.7 & 0.7 & 0.7 & 0.7 & 0.7 & 0.7 & 0.7 & 0.7 & 0.7 & 0.7 \\
\hline 10 & 4 & 8 & 6 & 14 & 4 & 9 & 21 & 22 & 23 & 23 & 23 & 6 & 12 \\
\hline 15 & 11 & 10 & 11 & 12 & 12 & 11 & 15 & 19 & 19 & 18 & 17 & 19 & 14 \\
\hline 22 & 4 & 26 & 16 & 34 & 8 & 1 & 33 & 89 & 45 & 59 & 49 & 10 & 28 \\
\hline 7.3 & 7.3 & 7.3 & 7.3 & 7.3 & 7.3 & 7.3 & 7.3 & 7.3 & 7.3 & 7.3 & 7.3 & 7.3 & 7.3 \\
\hline 7 & 25 & 23 & 27 & 6 & 6 & 4 & 5 & 19 & 31 & 35 & 35 & 14 & 18 \\
\hline 0.1 & 0.3 & 0.7 & 0.7 & 0.7 & 0.7 & 0.7 & 0.7 & 0.7 & 0.7 & 0.7 & 0.7 & 0.3 & 0.6 \\
\hline 6 & 6 & 6 & 6 & 5 & 6 & 5 & 5 & 5 & 5 & 7 & 7 & 7 & 5.9 \\
\hline 6 & 7 & 7 & 6 & 5 & 6 & 6 & 5 & 6 & 6 & 7 & 7 & 7 & 6.3 \\
\hline 7 & 7 & 7 & 6 & 5 & 6 & 6 & 5 & 6 & 6 & 7 & 7 & 7 & 6.3 \\
\hline 4.8 & 4.8 & 4.8 & 4.8 & 4.8 & 4.8 & 4.8 & 4.8 & 4.8 & 4.8 & 4.8 & 4.8 & 4.8 & 4.8 \\
\hline 18 & 20 & 21 & 19 & 80 & 33 & 16 & 42 & 65 & 65 & 74 & 82 & 46 & 43 \\
\hline 12 & 51 & 53 & 55 & 52 & 55 & 53 & 50 & 44 & 12 & 1 & 0 & 2 & 35 \\
\hline 2.7 & 2.7 & 2.7 & 2.7 & 2.7 & 2.7 & 2.7 & 2.7 & 2.7 & 2.7 & 2.7 & 2.7 & 2.7 & 2.7 \\
\hline 7.5 & 5.5 & 7.5 & 7.5 & 7.1 & 4 & 13 & 13 & 13 & 13 & 13 & 13 & 10 & 9.5 \\
\hline 25 & 24 & 45 & 47 & 9 & 9 & 12 & 15 & 38 & 64 & 80 & 75 & 24 & 35 \\
\hline 44 & 23 & 40 & 26 & 18 & 17 & 12 & 13 & 14 & 15 & 56 & 56 & 46 & 30 \\
\hline 26 & 30 & 28 & 30 & 30 & 27 & 25 & 24 & 24 & 24 & 24 & 24 & 23 & 26 \\
\hline 7.2 & 7.2 & 7.2 & 7.2 & 7.2 & 7.2 & 7.2 & 7.2 & 7.2 & 7.2 & 7.2 & 7.2 & 7.2 & 7.2 \\
\hline 0.4 & 0.4 & 0.4 & 0.4 & 0.4 & 0.4 & 0.4 & 0.4 & 0.4 & 0.4 & 0.4 & 0.4 & 0.4 & 0.4 \\
\hline 0.3 & 0.3 & 0.3 & 0.2 & 0.3 & 0.3 & 0.2 & 0.4 & 0.2 & 0.2 & 0.3 & 0.6 & 0 & 0.3 \\
\hline 0 & 0 & 0 & 6 & 10 & 5 & 3 & 0 & 0 & 0 & 0 & 0 & 0 & 2 \\
\hline 31 & 29 & 12 & 3.1 & 9 & 2.8 & 2.4 & 2.2 & 14 & 14 & 31 & 31 & 31 & 16 \\
\hline 0 & 0 & 0 & 0.5 & 0.5 & 0.5 & 0.5 & 0.5 & 0.5 & 0.5 & 0.5 & 0.5 & 0.5 & 0.4 \\
\hline 2 & 2 & 2 & 2 & 2 & 2 & 2 & 2 & 2 & 2 & 2 & 2 & 2 & 2 \\
\hline 93 & 89 & 85 & 82 & 86 & 82 & 92 & 95 & 141 & 141 & 171 & 162 & 104 & 107 \\
\hline 6.1 & 5.3 & 3.7 & 2.7 & 5.9 & 4 & 3.7 & 4.8 & 8.6 & 8.6 & 19 & 30 & 20 & 9.9 \\
\hline 132 & 119 & 55 & 15 & 15 & 20 & 23 & 27 & 21 & 38 & 95 & 132 & 132 & 66 \\
\hline 0.4 & 0.4 & 0.4 & 0.4 & 0.4 & 0.4 & 0.4 & 0.4 & 0.4 & 0.4 & 0.4 & 0.4 & 0.4 & 0.4 \\
\hline 33 & 30 & 27 & 26 & 28 & 25 & 27 & 40 & 50 & 50 & 51 & 51 & 45 & 36 \\
\hline 13 & 0 & 3.6 & 0 & 5 & 4 & 4.3 & 13 & 13 & 13 & 13 & 13 & 13 & 7.9 \\
\hline 10 & 12 & 8.4 & 12 & 12 & 12 & 12 & 7.4 & 6.5 & 6.5 & 5.7 & 5.4 & 6.3 & 9.2 \\
\hline 37 & 34 & 30 & 19 & 56 & 28 & 39 & 59 & 62 & 68 & 68 & 68 & 55 & 46 \\
\hline 0.5 & 0.5 & 0.5 & 0.5 & 0.5 & 0.5 & 0.5 & 0.5 & 0.5 & 0.5 & 0.5 & 0.5 & 0.5 & 0.5 \\
\hline 9 & 6 & 5 & 5 & 5 & 5 & 5 & 5 & 5 & 5 & 6 & 8 & 9 & 6.1 \\
\hline 26 & 28 & 30 & 31 & 31 & 30 & 30 & 27 & 28 & 28 & 27 & 26 & 26 & 28 \\
\hline 5.8 & 10 & 10 & 10 & 1.8 & 7.1 & 0.6 & 4.3 & 5.9 & 4.7 & 6.7 & 6 & 10 & 6.5 \\
\hline 1.5 & 1.5 & 1.5 & 1.5 & 1.5 & 1.5 & 1.5 & 1.5 & 1.5 & 1.5 & 1.5 & 1.5 & 1.5 & 1.5 \\
\hline 14 & 16 & 18 & 19 & 19 & 17 & 17 & 16 & 16 & 16 & 15 & 14 & 14 & 16 \\
\hline 2.7 & 2.7 & 2.7 & 2.7 & 2.7 & 2.7 & 2.7 & 2.7 & 2.7 & 2.7 & 2.7 & 2.7 & 2.7 & 2.7 \\
\hline 3.8 & 3.5 & 3.4 & 3.2 & 3.3 & 3 & 3.2 & 3.8 & 3.8 & 3.8 & 3.8 & 3.8 & 3.8 & 3.5 \\
\hline 14 & 14 & 14 & 14 & 14 & 14 & 14 & 14 & 14 & 14 & 14 & 14 & 14 & 14 \\
\hline 5 & 8 & 4 & 11 & 14 & 10 & 8 & 0 & 0 & 0 & 0 & 0 & 7 & 5.6 \\
\hline 18 & 19 & 19 & 19 & 19 & 19 & 18 & 16 & 16 & 16 & 16 & 16 & 16 & 18 \\
\hline 15 & 18 & 17 & 18 & 18 & 16 & 15 & 14 & 14 & 14 & 14 & 14 & 13 & 15 \\
\hline 5.8 & 3.8 & 4.6 & 4 & 5 & 3.1 & 7.2 & 8 & 6.3 & 6.3 & 9.5 & 9.5 & 7.7 & 6.2 \\
\hline 4.3 & 4.3 & 4.3 & 4.3 & 4.3 & 4.3 & 4.3 & 4.3 & 4.3 & 4.3 & 4.3 & 4.3 & 4.3 & 4.3 \\
\hline 9 & 6 & 10 & 10 & 10 & 10 & 10 & 10 & 10 & 10 & 10 & 10 & 8 & 9.4 \\
\hline 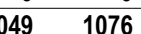 & & 093 & 003 & 060 & 723 & 709 & 0,15 & & 267 & 1555 & 1605 & 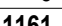 & \\
\hline
\end{tabular}

-Total Independent Hydro W/Enc.-

69 Federal System

70 Public Entitie

71 Investor-Owned Entities $\begin{array}{llll}484 & 502 & 519 & 470\end{array}$ $\begin{array}{ll}306 & 237 \\ 126 & 196 \\ 471 & 5\end{array}$

237
196
536

176
135
472

197
120

$120 \quad 161$

161
509 $\begin{array}{ll}434 & 516 \\ 202 & 201 \\ 570 & 551\end{array}$

576
$551 \quad 610$
May Jun

Jul

Avg 
Table A-4: Regional Independent Hydro Projects

PNW Loads and Resources Study

2008 - 2009 Operating Year

1937 Water Year

2003 White Book

Average Energy in Megawatts

Aug1 Aug16

Oct Nov Dec Jan

Mar Apr1 Apr1

May Jun

Jul Avg

72 Other Entities

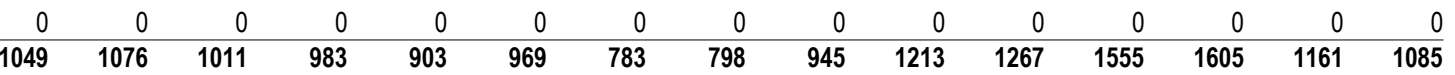


1 American Falls

2 Anderson Ranch

3 Big Cliff

4 Big Creek

5 Black Canyon

6 Bliss

7 Blue River

8 Bonneville Fishway

9 Bull Run

10 Calispel Creek

11 Carmen-Smith

12 Cascade

13 Cedar Falls \& Newhalem

14 Chandler

15 CJ Strike

16 Condit Sm Plts

17 Cougar

18 Cowlitz Falls

19 Detroit

20 Dexter

21 Felt

22 Foster

23 Gem State Hydro

24 Green Peter

25 Green Springs - USBR

26 Hills Creek

27 Hood Street

28 Idaho Falls - City Plant

29 Idaho Falls - Lower Plant

30 Idaho Falls - Upper Plant

31 Island Park

32 Jackson

33 Klamath

34 Lake Creek

35 Leaburg

36 Lookout Point

37 Lost Creek

38 Lower Salmon

39 McNary Fishway

40 Mill Creek (WA)

41 Milltown/Flint Creek

42 Milner

43 Minidoka

44 Morse Creek

45 Moyie Springs

46 North Umpqua

47 Packwood

48 Palisades

49 Reader Gulch

50 Rogue

51 Roza

52 Shoshone Falls

53 Snoq Elect \& Nooksack

54 Soda Creek

55 South Fork Tolt

56 Spring Plants

57 Stone Creek

58 Strawberry Creek

59 Swan Falls

60 The Dalles Fishway

61 Trail Bridge

62 TW Sullivar

63 Twin Falls

64 Upper Salmon 1 \& 2

65 Upper Salmon 3 \& 4

66 Walterville

67 Wynochee

68 Yelm

Total Independent Hydro w/Enc.
Mar Apr1 Apr16

May Jun

Jul Avg
-Total Independent Hydro W/Enc.-

69 Federal System

70 Public Entities

71 Investor-Owned Entities

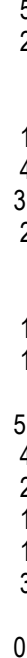

0.7

15

$22 \quad 32$

7.3

0.1

6

4.8
18

$18 \quad 18$

$12 \quad 32$

$\begin{array}{ll}2.7 & 2.7 \\ 7.5 & 7.5\end{array}$

$25 \quad 22$

44

$26 \quad 26$

0.3

0
31
0

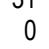

2

93

6.1

$132 \quad 132$

$0.4 \quad 0.4$

33

$13 \quad 13$

10

$37 \quad 34$

$0.5 \quad 0.5$ $\begin{array}{ll}7.2 & 7.2 \\ 0.4 & 0.4\end{array}$ $\begin{array}{lll}55 & 35 & 13\end{array}$

$\begin{array}{rrrrrr}18 & 11 & 4.3 & 5.4 & 11 & 16 \\ 7 & 9 & 13 & 15 & 7 & 4 \\ 0 & 0 & 0 & 0 & 1 & 0 \\ 10 & 7.9 & 9 & 5.9 & 7.1 & 8.8 \\ 41 & 46 & 44 & 46 & 46 & 43\end{array}$

$\begin{array}{rrr}3.4 & 2.2 & 2.2 \\ 24 & 24 & 24\end{array}$

$\begin{array}{rr}0 & 1 \\ 16 & 16\end{array}$

$\begin{array}{rrr}13 & 10 & 2.4 \\ 2 & 2.8 & 7.8\end{array}$

$\begin{array}{ll}2.8 & 6.7 \\ 52 & 54\end{array}$

$\begin{array}{ll}44 & 52 \quad 54\end{array}$

$23 \quad 23 \quad 20$

$\begin{array}{lll}24 & 12 & 25\end{array}$

7.9

32

44

$12 \quad 16$

0.7

10

26

7.3

$$
0.7
$$

0.7
6

6

4

$48 \quad 4.8$

21

$53 \quad 55$

2.7

7.5

45

$40 \quad 26$

$\begin{array}{lll}28 & 30 & 30\end{array}$

7.2

0.4

0.3

0.4

0.2

6
3.1

0.5

$$
2
$$

2
85

85
3.7

$\begin{array}{ll}3.7 & 2.7 \\ 5 & 15\end{array}$

55
0.4

27

3.6

8.4

$\begin{array}{rrrr}30 & 19 & 56 & 28 \\ 0.5 & 0.5 & 0.5 & 0.5\end{array}$

Feb

\begin{tabular}{|c|c|}
\hline & \\
\hline 16 & \\
\hline 7 & \\
\hline 0 & \\
\hline 4.8 & \\
\hline 40 & \\
\hline 2.1 & \\
\hline 24 & \\
\hline 14 & \\
\hline 0 & \\
\hline 14 & \\
\hline 1.8 & \\
\hline 6.6 & \\
\hline 9.3 & \\
\hline 51 & \\
\hline 21 & \\
\hline 6 & \\
\hline 14 & \\
\hline 18 & \\
\hline 5 & \\
\hline 0.7 & \\
\hline 9 & \\
\hline 11 & \\
\hline $\begin{array}{r}1 \\
7.3\end{array}$ & \\
\hline 4 & \\
\hline 0.7 & \\
\hline 5 & \\
\hline 6 & \\
\hline 6 & \\
\hline 4.8 & \\
\hline 16 & \\
\hline 53 & \\
\hline 2.7 & \\
\hline 13 & \\
\hline 12 & \\
\hline 12 & \\
\hline 25 & \\
\hline 7.2 & \\
\hline 0.4 & \\
\hline 0.3 & \\
\hline 3 & \\
\hline $\begin{array}{l}2.4 \\
0.5\end{array}$ & \\
\hline 2 & \\
\hline 92 & \\
\hline 3.7 & \\
\hline 23 & \\
\hline 0.4 & \\
\hline 27 & \\
\hline 4.3 & \\
\hline 12 & \\
\hline 39 & \\
\hline $\begin{array}{r}0.5 \\
5\end{array}$ & \\
\hline $\begin{array}{r}5 \\
30\end{array}$ & \\
\hline $\begin{array}{l}30 \\
0.6\end{array}$ & \\
\hline $\begin{array}{l}1.5 \\
\end{array}$ & \\
\hline 17 & \\
\hline 2.7 & \\
\hline 3.2 & \\
\hline 14 & \\
\hline 8 & \\
\hline 18 & \\
\hline 15 & \\
\hline $\begin{array}{l}7.2 \\
43\end{array}$ & \\
\hline $\begin{array}{r}4.3 \\
10\end{array}$ & \\
\hline
\end{tabular}

28
$-12-12$

$\begin{array}{llllll}20 & 20 & 67 & 70 & 75 & 28\end{array}$ $\begin{array}{rrrrrr}25 & 27 & 11 & 39 & 28 & 16 \\ 10 & 17 & 21 & 21 & 8 & 11 \\ 0 & 0 & 0 & 1 & 0 & 0.2\end{array}$

$\begin{array}{lll}1 & 0.2 \\ 8.1 & 8.5\end{array}$

$\begin{array}{lll}38 & 37 & 42\end{array}$

$2.6-2.7$

$\begin{array}{lll}15 & 12 & 10\end{array}$

$\begin{array}{rrr}0 & 0 & 0.3 \\ 17 & 17 & 16\end{array}$

$\begin{array}{lll}1.2 & 11 & 4.5\end{array}$

$\begin{array}{lll}30 & 2.2 & 12\end{array}$

$\begin{array}{lll}43 & 38 & 50\end{array}$

$\begin{array}{lll}37 & 28 & 26\end{array}$

$\begin{array}{lll}29 & 11 & 16\end{array}$

$\begin{array}{lll}91 & 30 & 41\end{array}$

$\begin{array}{lll}17 & 6 & 9.3 \\ 0.7 & 0.7 & 0.7\end{array}$

$\begin{array}{lll}23 & 6 & 0.7\end{array}$

$\begin{array}{lll}17 & 19 & 14\end{array}$

$10 \quad 28$

$\begin{array}{lll}35 & 14 & 18\end{array}$

$\begin{array}{lll}0.7 & 0.3 & 0.6\end{array}$

$\begin{array}{lll}7 & 7 & 5.9 \\ 7 & 7 & 6.3\end{array}$

$\begin{array}{lll}7 & 7 & 6.3\end{array}$

$\begin{array}{lll}4.8 & 4.8 & 4.8\end{array}$

$82 \quad 46 \quad 43$

$\begin{array}{lll}2.7 & 2.7 & 2.7\end{array}$

$\begin{array}{lll}13 & 10 & 9.5\end{array}$

$24 \quad 35$

$46 \quad 30$

$\begin{array}{ll}7.2 & 7.2\end{array}$

$\begin{array}{ll}0.4 & 0.4\end{array}$

$31 \quad 16$

$\begin{array}{ll}0.5 & 0.4\end{array}$

$104 \quad 107$

$20 \quad 9.9$

$132 \quad 66$

$\begin{array}{rr}0.4 & 0.4 \\ 45 & 36\end{array}$

$13 \quad 7.9$

$6.3 \quad 9.2$

$0.5-0.5$

$\begin{array}{ll}9 & 6.1\end{array}$

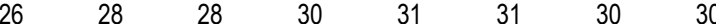

$\begin{array}{rrrrrrr}4 & 5.8 & 10 & 10 & 10 & 1.8 & -7.1 \\ 1.5 & 1.5 & 1.5 & 1.5 & 1.5 & 1.5 & 1.5\end{array}$

$\begin{array}{rrrrrrr}14 & 14 & 16 & 18 & 19 & 19 & 17 \\ 27 & 27 & 27 & 27 & 27 & 27 & 2.7\end{array}$

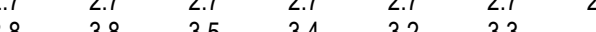

$\begin{array}{lllrrrr}14 & 14 & 14 & 14 & 14 & 14 & 14\end{array}$

$5 \quad 5$

$18 \quad 18$

$\begin{array}{lllllll}15 & 15 & 18 & 17 & 18 & 18 & 16\end{array}$

$\begin{array}{rrrrrrr}5.4 & 5.8 & 3.8 & 4.6 & 4 & 5 & 3.1\end{array}$

$\begin{array}{lllllll}4.3 & 4.3 & 4.3 & 4.3 & 4.3 & 4.3 & 4.3\end{array}$

$\begin{array}{rrr}10 & 10 \quad 10\end{array}$

945 $\begin{array}{rrr}10 & 10\end{array}$

\begin{tabular}{|c|c|c|c|c|c|c|c|c|c|c|c|c|}
\hline 436 & 371 & 386 & 306 & 237 & 176 & 197 & 275 & 434 & 516 & 712 & 748 & 452 \\
\hline 138 & 121 & 127 & 126 & 196 & 135 & 120 & 161 & 202 & 201 & 232 & 256 & 182 \\
\hline 502 & 519 & 470 & 471 & 536 & 472 & 481 & 509 & 576 & 551 & 610 & 601 & 527 \\
\hline
\end{tabular}


Table A-4: Regional Independent Hydro Projects

PNW Loads and Resources Study

2009 - 2010 Operating Year

1937 Water Year

2003 White Book

Average Energy in Megawatts

Aug1 Aug16

Oct Nov Dec Jan

Mar Apr1 Apr1

May Jun

Jul Avg

72 Other Entities

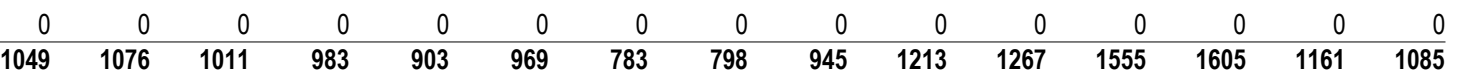


$438 \quad 436 \quad 371-386$

$127 \quad 138$ $484 \quad 502$

$\begin{array}{rrrrr}13 & 0 & 0 & 0 & \\ 4.3 & 5.4 & 11 & 16 & 16 \\ 13 & 15 & 7 & 4 & \\ 0 & 0 & 1 & 0 & \\ 9 & 5.9 & 7.1 & 8.8 & 4.8 \\ 44 & 46 & 46 & 43 & 40 \\ 2.2 & 3.2 & 3.6 & 3.4 & 2.1 \\ 24 & 12 & 12 & 12 & 24 \\ 2 & 1 & 15 & 7 & 14 \\ 0 & 0 & 1 & 0 & \\ 14 & 13 & 16 & 14 & 14 \\ 2.4 & 2.2 & 3.2 & 5.6 & 1.8 \\ 7.8 & 11 & 18 & 9.6 & 6 \\ 6.7 & 12 & 13 & 9.5 & 9.3 \\ 54 & 59 & 59 & 55 & 51 \\ 20 & 19 & 23 & 20 & 21 \\ 25 & 24 & 6 & 4 & \\ 6.2 & 3.1 & 23 & 10 & 14 \\ 44 & 46 & 20 & 11 & 18 \\ 12 & 16 & 4 & 4 & 5 \\ 0.7 & 0.7 & 0.7 & 0.7 & 0.7\end{array}$

0.7

0.7

$\begin{array}{rrr}0.7 & 0.7 & 0.7 \\ 8 & 6 & 14\end{array}$

10
26

7.3

$16 \quad 34$

4.8

$18 \quad 18$

$\begin{array}{rr}12 & 32 \\ 2.7 & 27\end{array}$

7.5

$25 \quad 22$

44
26

$7.2 \quad 7.2$

$0.4 \quad 0.4$

$\begin{array}{rr}0 & 0 \\ 31 & 31 \\ 0 & 0\end{array}$
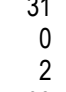

93

$6.1 \quad 16$

$\begin{array}{rr}132 & 132 \\ 0.4 & 0.4\end{array}$

$\begin{array}{rr}0 & 5.8 \\ 16 & 5.1 \\ 7 & 7 \\ 0 & 0 \\ 4.8 & 7.9 \\ 40 & 40\end{array}$

$2.1 \quad 1.9$

$24 \quad 24$

$5.8 \quad 20$ $\begin{array}{rrr}5.1 & 25 \\ 7 & 10\end{array}$

$\begin{array}{lllll}20 & 67 & 70 & 75 & 28\end{array}$ $\begin{array}{rrrrr}27 & 11 & 39 & 28 & 16 \\ 17 & 21 & 21 & 8 & 11\end{array}$

$\begin{array}{rrrrr}10 & 0 & 1 & 0 & 0.2 \\ 30 & 30 & 8.1 & 7.5 & 8.1\end{array}$

$\begin{array}{lllll}10 & 10 & 8.1 & 7.5 & 8.1 \\ 39 & 38 & 38 & 37 & 42\end{array}$

7.2 $\begin{array}{rrr}0 & 0 & 0 \\ 16 & 16 & 16\end{array}$ 
Table A-4: Regional Independent Hydro Projects

Continued PNW Loads and Resources Study

2010 - 2011 Operating Year

1937 Water Year

2003 White Book

Average Energy in Megawatts

Aug1 Aug16

Oct Nov Dec Jan

Mar Apr1 Apr1

May Jun

Jul Avg

72 Other Entities

\begin{tabular}{|c|c|c|c|c|c|c|c|c|c|c|c|c|c|}
\hline 0 & 0 & 0 & 0 & 0 & 0 & 0 & 0 & 0 & 0 & 0 & 0 & 0 & 0 \\
\hline 1076 & 1011 & 983 & 903 & 970 & 783 & 798 & 945 & 1213 & 1267 & 1555 & 1605 & 1161 & 85 \\
\hline
\end{tabular}


$438 \quad 436 \quad 371-386$

$127 \quad 138$ $484 \quad 502$

$\begin{array}{rrrrr}13 & 0 & 0 & 0 & \\ 4.3 & 5.4 & 11 & 16 & 16 \\ 13 & 15 & 7 & 4 & \\ 0 & 0 & 1 & 0 & \\ 9 & 5.9 & 7.1 & 8.8 & 4.8 \\ 44 & 46 & 46 & 43 & 40 \\ 2.2 & 3.2 & 3.6 & 3.4 & 2.1 \\ 24 & 12 & 12 & 12 & 24 \\ 2 & 1 & 15 & 7 & 14 \\ 0 & 0 & 1 & 0 & \\ 14 & 13 & 16 & 14 & 14 \\ 2.4 & 2.2 & 3.2 & 5.6 & 1.8 \\ 7.8 & 11 & 18 & 9.6 & 6 \\ 6.7 & 12 & 13 & 9.5 & 9.3 \\ 54 & 59 & 59 & 55 & 51 \\ 20 & 19 & 23 & 20 & 21 \\ 25 & 24 & 6 & 4 & \\ 6.2 & 3.1 & 23 & 10 & 14 \\ 44 & 46 & 20 & 11 & 18 \\ 12 & 16 & 4 & 4 & 5 \\ 0.7 & 0.7 & 0.7 & 0.7 & 0.7\end{array}$

0.7

0.7

4
12

0.7

9
11

1
7.3

7.3

6
0.7

4
0.7

0.7
5

5
6
6

5

5
5
4.8

$4.8 \quad 4.8$

42

50

2.7

13
15

$13 \quad 14$

24

7.2

0.4
0.6

7.2

0.4

0.3

0.4

0.3

0.3

0

2.2

$\begin{array}{lll}3.1 & 9 & 2.8\end{array}$

2.4

0.5
2

2
95
4.8

27
0.4
40

40
13

7.4

7.4
0.5

0.5
Apr1 Apr16

May
Jun

Ju

Jul Avg 
Table A-4: Regional Independent Hydro Projects

PNW Loads and Resources Study

2011 - 2012 Operating Year

1937 Water Year

2003 White Book

Average Energy in Megawatts

Aug1 Aug16

Oct Nov Dec Jan

Mar Apr1 Apr1

May Jun

Jul Avg

72 Other Entities

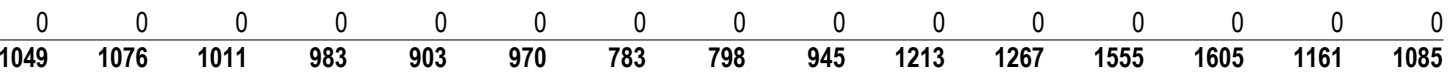


$\begin{array}{llll}438 & 436 & 371 & 386\end{array}$

$127 \quad 138$ $484 \quad 502$

$\begin{array}{rrrrr}13 & 0 & 0 & 0 & \\ 4.3 & 5.4 & 11 & 16 & \\ 13 & 15 & 7 & 4 & \\ 0 & 0 & 1 & 0 & \\ 9 & 5.9 & 7.1 & 8.8 & 4.8 \\ 44 & 46 & 46 & 43 & 40 \\ 2.2 & 3.2 & 3.6 & 3.4 & 2.1 \\ 24 & 12 & 12 & 12 & 24 \\ 2 & 1 & 15 & 7 & 14 \\ 0 & 0 & 1 & 0 & \\ 14 & 13 & 16 & 14 & 14.8 \\ 2.4 & 2.2 & 3.2 & 5.6 & 1.8 \\ 7.8 & 11 & 18 & 9.6 & 6.6 \\ 6.7 & 12 & 13 & 9.5 & 9.3 \\ 54 & 59 & 59 & 55 & 51 \\ 20 & 19 & 23 & 20 & 21 \\ 25 & 24 & 6 & 4 & \\ 6.2 & 3.1 & 23 & 10 & 14 \\ 44 & 46 & 20 & 11 & \\ 12 & 16 & 4 & 4 & \end{array}$

0.7

4

$\begin{array}{lll}8 & 6 & 14\end{array}$

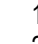

$\begin{array}{lllllll}5.8 & 20 & 20 & 67 & 70 & 75 & 28\end{array}$ $\begin{array}{rrrrr}27 & 11 & 39 & 28 & 16 \\ 17 & 21 & 21 & 8 & 11\end{array}$

$\begin{array}{rrrrr}10 & 10 & 8.1 & 7.5 & 8.1\end{array}$

$\begin{array}{lllll}39 & 38 & 38 & 37 & 42\end{array}$

$\begin{array}{lllll}2.5 & 2.9 & 2.4 & 2.6 & 2.7\end{array}$

$\begin{array}{lllll}15 & 15 & 15 & 12 & 10\end{array}$

$\begin{array}{rrrrr}0 & 1 & 0 & 0 & 0.3 \\ 17 & 17 & 17 & 17 & 16\end{array}$

$\begin{array}{lllll}1.1 & 1.1 & 1.2 & 11 & 4.5\end{array}$

$\begin{array}{lllll}18 & 25 & 30 & 2.2 & 12\end{array}$

$\begin{array}{rrrrr}10 & 8.4 & 8.1 & 4.6 & 8.6 \\ 49 & 45 & 43 & 38 & 50\end{array}$

$\begin{array}{lllll}36 & 37 & 37 & 28 & 26\end{array}$

$\begin{array}{lllll}29 & 29 & 29 & 11 & 16 \\ 48 & 64 & 66 & 29 & 26\end{array}$

$\begin{array}{rrrrr}66 & 106 & 91 & 30 & 41\end{array}$

$\begin{array}{rrrrr}16 & 17 & 17 & 6 & 9.3 \\ 0.7 & 0.7 & 0.7 & 0.7 & 0.7\end{array}$

$\begin{array}{lllll}23 & 23 & 23 & 6 & 12\end{array}$

$\begin{array}{lllll}45 & 59 & 49 & 10 & 28\end{array}$

$\begin{array}{lllll}7.3 & 7.3 & 7.3 & 7.3 & 7.3\end{array}$

$\begin{array}{rrrrr}31 & 35 & 35 & 14 & 18 \\ 0.7 & 0.7 & 0.7 & 0.3 & 0.6\end{array}$

$\begin{array}{rrr}0.7 & 0.3 & 0.6 \\ 7 & 7 & 5.9\end{array}$

$\begin{array}{lll}7 & 7 & 6.3\end{array}$

$\begin{array}{ll}7 & 6.3 \\ 7 & 6.3\end{array}$

$4.8 \quad 4.8$

$46 \quad 43$

$\begin{array}{rr}2 & 35 \\ 2.7 & 2.7\end{array}$

$10 \quad 9.5$

$24 \quad 35$

$46 \quad 30$

$\begin{array}{rr}46 & 26 \\ 72 & 72\end{array}$

$\begin{array}{ll}0.4 & 0.4 \\ 0 & 0.4\end{array}$

$0 \quad 2$

$31 \quad 16$

$\begin{array}{rrr}0.5 & 0.5 & 0.4 \\ 2 & 2 & 2\end{array}$

$\begin{array}{lll}162 & 104 & 107\end{array}$

$\begin{array}{lll}30 & 20 & 9.9\end{array}$

$\begin{array}{lll}132 & 132 & 66\end{array}$

$\begin{array}{lll}0.4 & 0.4 & 0.4\end{array}$

$\begin{array}{lll}51 & 45 & 36 \\ 13 & 13 & 7.9\end{array}$

$\begin{array}{lll}5.4 & 6.3 & 9.2\end{array}$

$\begin{array}{lll}68 & 55 & 46\end{array}$

$\begin{array}{lll}0.5 & 0.5 & 0.5\end{array}$

$\begin{array}{rrr}8 & 9 & 6.1 \\ 26 & 26 & 28\end{array}$

$\begin{array}{lll}6 & 10 & 6.5\end{array}$

$\begin{array}{lll}1.5 & 1.5 & 1.5\end{array}$

$\begin{array}{lll}14 & 14 & 16\end{array}$

$\begin{array}{lll}2.7 & 2.7 & 2.7\end{array}$

$\begin{array}{lll}3.8 & 3.8 & 3.5\end{array}$

$\begin{array}{lll}14 & 14 & 14\end{array}$

$\begin{array}{rrr}0 & 7 & 5.6\end{array}$

$\begin{array}{lll}14 & 16 & 18\end{array}$

$\begin{array}{rrr}14 & 13 & 15 \\ 9.5 & 7.7 & 6.2 \\ 4.3 & 4.3 & 4.3\end{array}$

\begin{tabular}{rrr}
105 & 8 & 9.4 \\
\hline & 1161 & 1085
\end{tabular}


Table A-4: Regional Independent Hydro Projects

Continued

PNW Loads and Resources Study

2012 - 2013 Operating Year

1937 Water Year

2003 White Book

Average Energy in Megawatts

Aug1 Aug16

Oct Nov Dec Jan

Mar Apr1 Apr1

May Jun

Jul Avg

72 Other Entities

$\begin{array}{rrrrr}0 & 0 & 0 & 0 & 0 \\ 1049 & 1076 & 1011 & 983 & 903\end{array}$

$\begin{array}{rrrrrrrrrr}0 & 0 & 0 & 0 & 0 & 0 & 0 & 0 & 0 & 0 \\ 970 & 783 & 798 & 945 & 1213 & 1267 & 1555 & 1605 & 1161 & 1085\end{array}$


$\begin{array}{llll}438 & 436 & 371 & 386\end{array}$

$127 \quad 138$ $484 \quad 502$

$\begin{array}{rrrrr}13 & 0 & 0 & 0 & \\ 4.3 & 5.4 & 11 & 16 & \\ 13 & 15 & 7 & 4 & \\ 0 & 0 & 1 & 0 & \\ 9 & 5.9 & 7.1 & 8.8 & 4.8 \\ 44 & 46 & 46 & 43 & 40 \\ 2.2 & 3.2 & 3.6 & 3.4 & 2.1 \\ 24 & 12 & 12 & 12 & 24 \\ 2 & 1 & 15 & 7 & 14 \\ 0 & 0 & 1 & 0 & \\ 14 & 13 & 16 & 14 & 14.8 \\ 2.4 & 2.2 & 3.2 & 5.6 & 1.8 \\ 7.8 & 11 & 18 & 9.6 & 6.6 \\ 6.7 & 12 & 13 & 9.5 & 9.3 \\ 54 & 59 & 59 & 55 & 51 \\ 20 & 19 & 23 & 20 & 21 \\ 25 & 24 & 6 & 4 & \\ 6.2 & 3.1 & 23 & 10 & 14 \\ 44 & 46 & 20 & 11 & \\ 12 & 16 & 4 & 4 & \end{array}$

0.7

0.7

0.7

4
12

0.7

9
11

$\begin{array}{rr}1 & 33 \\ 7.3 & 7.3\end{array}$

7.3

0.7

4
0.7

0.7
5

0.7

0.7
5

$$
\begin{array}{r}
5 \\
5 \\
4.8
\end{array}
$$

5
4.8
42

42

50
2.7

13

15

$13 \quad 14$

24

7.2
0.4

0.6

0.3

6
3.1

3.1
0.5
2

82
27

2.7

$15-15$

0.4

$28 \quad 25$

27

4.3

127.4

0
2.2

2.2
0.5

2

95
4.8
27

27
0.4

$0.4 \quad 0.4$

$40 \quad 50 \quad 50$

7.4
7.4

$\begin{array}{ll}59 & 62\end{array}$

0.5 10

24
24
15

18

10
49

23

0.7

$$
\begin{array}{r}
6 \\
6 \\
4.8
\end{array}
$$

0.3 $\begin{array}{llllll}20 & 20 & 67 & 70 & 75 & 28\end{array}$ $\begin{array}{rrrrrr}10 & 27 & 11 & 39 & 28 & 16 \\ 0 & 17 & 21 & 21 & 8 & 11\end{array}$

$\begin{array}{lllll}10 & 10 & 8.1 & 7.5 & 8.2\end{array}$

$\begin{array}{lllll}10 & 10 & 8.1 & 7.5 & 8.1\end{array}$

2.5

0

17

1.1

$36 \quad 37$

$29 \quad 29$

48

66

16
0.7

19

7.3

31

5

4.8

65

12
2.7

13

64

15
24

7.2

0.4

0
14
0.5

0.5

2
141

141
8.6

$38-19$

$0.4 \quad 0.4$

$\begin{array}{ll}50 & 51 \\ 13 & 13\end{array}$

$6.5 \quad 5.7$

$68 \quad 68$

$0.5 \quad 0.5$

5

$28 \quad 27$

$\begin{array}{rrrr}27 & 28 & 28 & 27 \\ 4.3 & 5.9 & 4.7 & 6.7\end{array}$

$1.5 \quad 1.5$

$\begin{array}{rr}16 & 15 \\ 27 & 27 \\ 3.8 & 3.8\end{array}$

$2.7-2.7$

$\begin{array}{rr}14 & 14 \\ 0 & 0\end{array}$

000

$16 \quad 16$

$\begin{array}{lll}14 & 14 & 16\end{array}$

6.3

9.5

4.3 $\begin{array}{rrr}38 & 37 & 42 \\ 2.4 & 26 & 27\end{array}$

$\begin{array}{lll}24 & 24 & 21\end{array}$

$\begin{array}{lll}15 & 12 & 10\end{array}$

$\begin{array}{rrr}17 & 17 & 0.3 \\ 1 & 11 & 4.5\end{array}$

$\begin{array}{lll}1.2 & 11 & 4.5\end{array}$

$\begin{array}{lll}30 & 2.2 & 12\end{array}$

$8.1 \quad 4.6 \quad 8.6$

$\begin{array}{lll}43 & 38 & 50\end{array}$

$\begin{array}{lll}37 & 28 & 26 \\ 29 & 11 & 16\end{array}$

$\begin{array}{lll}66 & 29 & 26\end{array}$

$\begin{array}{lll}91 & 30 & 41\end{array}$

$\begin{array}{lll}17 & 6 & 9.3\end{array}$

$\begin{array}{lll}17 & 19 & 14\end{array}$

$10 \quad 28$

$\begin{array}{lll}35 & 14 & 18\end{array}$

$\begin{array}{lll}0.7 & 0.3 & 0.6\end{array}$

$\begin{array}{lll}7 & 7 & 5.9 \\ 7 & 7 & 6.3\end{array}$

$\begin{array}{lll}7 & 7 & 6.3\end{array}$

4.8

$82 \quad 46$

$\begin{array}{lll}2.7 & 2.7 & 2.7\end{array}$

$\begin{array}{lll}13 & 10 & 9.5\end{array}$

$\begin{array}{lll}75 & 24 & 35\end{array}$

$56 \quad 46 \quad 30$

$\begin{array}{lll}7.2 & 7.2 & 7.2\end{array}$

$\begin{array}{lll}0.4 & 0.4 & 0.4\end{array}$

31

0.3

$\begin{array}{lll}31 & 31 & 16\end{array}$

$\begin{array}{lll}0.5 & 0.5 & 0.4\end{array}$

$\begin{array}{rrr}0 & 2 & 0.5\end{array}$

$\begin{array}{lll}30 & 20 & 9.9\end{array}$

$\begin{array}{lll}132 & 132 & 66\end{array}$

$\begin{array}{lll}0.4 & 0.4 & 0.4\end{array}$

$\begin{array}{rrr}51 & 45 & 36 \\ 13 & 13 & 7.9\end{array}$

$\begin{array}{lll}5.4 & 6.3 & 9.2\end{array}$

$\begin{array}{lll}68 & 55 & 46\end{array}$

$\begin{array}{lll}0.5 & 0.5 & 0.5\end{array}$

$\begin{array}{rrr}8 & 9 & 6.1\end{array}$

$\begin{array}{lll}6 & 10 & 6.5\end{array}$

$\begin{array}{lll}1.5 & 1.5 & 1.5\end{array}$

$\begin{array}{lll}14 & 14 & 16\end{array}$

$\begin{array}{lll}2.7 & 2.7 & 2.7\end{array}$

$14 \quad 14 \quad 14$

$\begin{array}{rrr}0 & 7 & 5.6 \\ 16 & 16 & 18\end{array}$

$\begin{array}{lll}14 & 13 & 15\end{array}$

$\begin{array}{lll}9.3 & 4.3 & 4.3\end{array}$

$\begin{array}{rrr}10 & 8 & 9.4\end{array}$

$\begin{array}{rrr}0.7 & 0.7 & 0.7 \\ 23 & 6 & 12\end{array}$

$\begin{array}{lll}3.8 & 3.8 & 3.5\end{array}$

$\begin{array}{lll}9.5 & 7.7 & 6.2\end{array}$ 
Table A-4: Regional Independent Hydro Projects

Continued

PNW Loads and Resources Study

2013 - 2014 Operating Year

1937 Water Year

2003 White Book

Average Energy in Megawatts

Aug1 Aug16

Oct Nov Dec Jan

Mar Apr1 Apr16

May Jun

Jul Avg

72 Other Entities

$\begin{array}{rr}0 & 0 \\ 1049 & 1076\end{array}$

0

$\begin{array}{rr}0 & \\ 903 & 970\end{array}$

$\begin{array}{rrrrrrrrrr}0 & 0 & 0 & 0 & 0 & 0 & 0 & 0 & 0 & 0 \\ 970 & 783 & 798 & 945 & 1213 & 1268 & 1555 & 1605 & 1161 & 1085\end{array}$


Table A-5: Regional Imports

PNW Loads and Resources Study

2004 - 2005 Operating Year

Dec

Jan Feb Mar Apr1 Apr16 May Jun Jul Avg

-Imports From East of Continental Divide-

1 BEPC To NWE PwrS

2 NWE To NWE PWrS

3 PPL To BPA PwrS

4 PPL To PPL PwrS

5 PPLM To IPC PwrS

6 Total Imports From ECD

\begin{tabular}{rrrrrrrrrrrrrrr}
0 & 0 & 0 & 0 & 84 & 84 & 84 & 84 & 84 & 84 & 84 & 0 & 0 & 0 & 42 \\
0 & 0 & 0 & 0 & 0 & 0 & 0 & 0 & 0 & 0 & 0 & 0 & 0 & 0 & 0 \\
13 & 13 & 42 & 108 & 161 & 215 & 189 & 145 & 117 & 90 & 90 & 0.1 & 18 & 34 & 94 \\
206 & 206 & 124 & 149 & 163 & 241 & 236 & 226 & 184 & 189 & 189 & 199 & 208 & 224 & 196 \\
14 & 14 & 0 & 0 & 0 & 0 & 0 & 0 & 0 & 0 & 0 & 0 & 46 & 43 & 8.6 \\
\hline 33 & $\mathbf{2 3 3}$ & $\mathbf{1 6 6}$ & $\mathbf{2 5 7}$ & $\mathbf{4 0 8}$ & $\mathbf{5 4 0}$ & $\mathbf{5 0 9}$ & $\mathbf{4 5 5}$ & $\mathbf{3 8 5}$ & $\mathbf{3 6 3}$ & $\mathbf{3 6 3}$ & $\mathbf{1 9 9}$ & $\mathbf{2 7 2}$ & $\mathbf{3 0 1}$ & $\mathbf{3 4 1}$
\end{tabular}

-Imports From Pacific Southwest-

7 ANHM To BPA PwrX

8 BURB To BPA XchgNrg

9 BURB To BPA PkRepl

10 GLEN To BPA XchgNrg

11 GLEN To BPA PkRepl

12 GLEN To PGE S/Pwr/X

13 NCPA To SCL S/Pwr/X

14 PASA To BPA XchgNrg

15 PASA To BPA PkRepl

16 PASA To BPA XchgNrg

17 PASA To BPA S/N/X

18 PASA To BPA PkRepl

19 PG\&E To PSE S/Pwr/X

20 RVSD To BPA XchgNrg

21 RVSD To BPA PkRepl

22 RVSD To BPA PkRepl

23 RVSD To BPA XchgNrg

24 RVSD To BPA S/Pwr/X

25 RVSD To BPA PkRepl

26 Total Imports From PSW

\begin{tabular}{rrrrrrrrrrrrrrr}
0 & 0 & 0 & 20 & 20 & 20 & 20 & 20 & 20 & 0 & 0 & 0 & 0 & 0 & 10 \\
2.6 & 2.6 & 2.6 & 2.6 & 3.8 & 5 & 5 & 5 & 5 & 5 & 2.6 & 2.6 & 2.6 & 2.6 & 3.6 \\
5.4 & 5.4 & 5.4 & 4 & 2.7 & 2.7 & 2.7 & 2.7 & 2.7 & 2.7 & 2.7 & 4 & 5.4 & 5.4 & 3.8 \\
2.9 & 2.9 & 2.9 & 2.9 & 4.2 & 5.6 & 5.6 & 5.6 & 5.6 & 5.6 & 2.9 & 2.9 & 2.9 & 2.9 & 4 \\
6.0 & 6.0 & 6.0 & 4.5 & 3.0 & 3.0 & 3.0 & 3.0 & 3.0 & 3.0 & 3.0 & 4.5 & 6.0 & 6.0 & 4.2 \\
0 & 0 & 0 & 0 & 8.9 & 8.9 & 8.9 & 8.9 & 0 & 0 & 0 & 0 & 0 & 0 & 3.0 \\
0 & 0 & 0 & 0 & 24 & 30 & 30 & 30 & 30 & 6.2 & 6.2 & 0 & 0 & 0 & 12 \\
1.7 & 1.7 & 1.7 & 1.7 & 2.5 & 3.4 & 3.4 & 3.4 & 3.4 & 3.4 & 1.7 & 1.7 & 1.7 & 1.7 & 2.4 \\
3.6 & 3.6 & 3.6 & 2.7 & 1.8 & 1.8 & 1.8 & 1.8 & 1.8 & 1.8 & 1.8 & 2.7 & 3.6 & 3.6 & 2.5 \\
0 & 0 & 3.5 & 3.5 & 3.5 & 3.5 & 3.5 & 3.5 & 1.7 & 0 & 0 & 0 & 0 & 0 & 1.9 \\
0 & 0 & 0.7 & 0.7 & 0.7 & 0.7 & 0.7 & 0.7 & 0.3 & 0 & 0 & 0 & 0 & 0 & 0.4 \\
4.5 & 4.5 & 4.5 & 0 & 0 & 0 & 0 & 0 & 0 & 0 & 0 & 0 & 0 & 4.5 & 1.1 \\
0 & 0 & 0 & 0 & 134 & 195 & 161 & 61 & 0 & 0 & 0 & 0 & 0 & 0 & 46 \\
0 & 0 & 0 & 5.8 & 5.8 & 5.8 & 5.8 & 5.8 & 5.8 & 5.8 & 0 & 0 & 0 & 0 & 3.1 \\
0 & 0 & 0 & 0 & 4.8 & 4.8 & 4.8 & 4.8 & 4.8 & 4.8 & 4.8 & 6.8 & 0 & 0 & 3.0 \\
6.8 & 6.8 & 6.8 & 6.8 & 0 & 0 & 0 & 0 & 0 & 0 & 0 & 0 & 6.8 & 6.8 & 2.8 \\
0 & 0 & 0 & 0 & 16 & 16 & 16 & 16 & 16 & 16 & 0 & 0 & 0 & 0 & 7.3 \\
0 & 0 & 0 & 0 & 9.2 & 9.2 & 9.2 & 9.2 & 9.2 & 9.2 & 0 & 0 & 0 & 0 & 4.2 \\
11 & 11 & 11 & 11 & 2.7 & 2.7 & 2.7 & 2.7 & 2.7 & 2.7 & 2.7 & 0 & 0 & 11 & 4.9 \\
\hline 44 & $\mathbf{4 4}$ & $\mathbf{4 8}$ & 66 & $\mathbf{2 4 7}$ & $\mathbf{3 1 8}$ & $\mathbf{2 8 4}$ & $\mathbf{1 8 4}$ & $\mathbf{1 1 2}$ & $\mathbf{6 6}$ & $\mathbf{2 8}$ & $\mathbf{2 5}$ & $\mathbf{2 9}$ & $\mathbf{4 4}$ & $\mathbf{1 2 1}$
\end{tabular}

-Imports From Inland Southwest-

27 Other Entities To BPA

28 SPP To BPA PwrS

29 WAPA To GLEC ARP

30 Total Imports From ISW

\begin{tabular}{rrrrrrrrrrrrrrr}
50 & 50 & 50 & 50 & 50 & 50 & 50 & 50 & 50 & 50 & 50 & 0 & 0 & 50 & 42 \\
$\mathbf{7 5}$ & 75 & 75 & 45 & 45 & 45 & 45 & 45 & 45 & 75 & 75 & 75 & 75 & 75 & 60 \\
3.3 & 3.3 & 3.1 & 2.9 & 3.3 & 3.7 & 3.8 & 4 & 3.3 & 3.3 & 3.3 & 2.8 & 3.1 & 3.3 & 3.3 \\
\hline $\mathbf{1 2 8}$ & $\mathbf{1 2 8}$ & $\mathbf{1 2 8}$ & $\mathbf{9 8}$ & $\mathbf{9 8}$ & $\mathbf{9 9}$ & $\mathbf{9 9}$ & $\mathbf{9 9}$ & $\mathbf{9 8}$ & $\mathbf{1 2 8}$ & $\mathbf{1 2 8}$ & $\mathbf{7 8}$ & $\mathbf{7 8}$ & $\mathbf{1 2 8}$ & $\mathbf{1 0 5}$
\end{tabular}

-Imports From Canada-

$31 \mathrm{BCHP}$ To BPA PwrS

32 BCHP To SCL PwrS

33 Total Imports From Canada

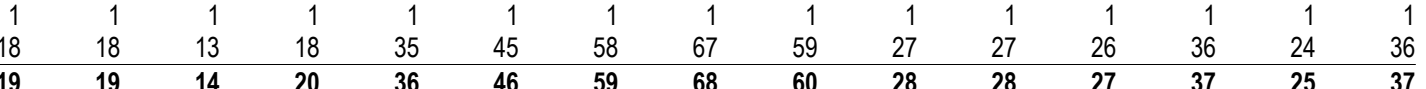

-Imports From Other Entities-

34 Total Imports From Other Entities

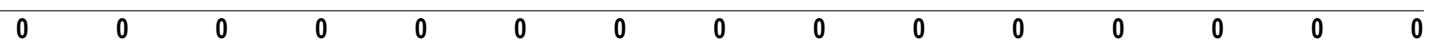

-Total Imports-

35 Federal System

36 Public Entities

37 Investor-Owned Entities

38 Other Entities

\begin{tabular}{|c|c|c|c|c|c|c|c|c|c|c|c|c|c|c|}
\hline 183 & 183 & 216 & 270 & 338 & 395 & 369 & 325 & 295 & 276 & 238 & 101 & 123 & 204 & 256 \\
\hline 21 & 21 & 16 & 21 & 62 & 79 & 92 & 101 & 93 & 37 & 37 & 28 & 39 & 27 & 51 \\
\hline 220 & 220 & 124 & 149 & 389 & 529 & 490 & 380 & 268 & 273 & 273 & 199 & 254 & 268 & 295 \\
\hline 0 & 0 & 0 & 0 & 0 & 0 & 0 & 0 & 0 & 0 & 0 & 0 & 0 & 0 & 0 \\
\hline
\end{tabular}


Table A-5: Regional Imports

PNW Loads and Resources Study

2005 - 2006 Operating Year

Nov Dec Jan F Jan Feb Mar Apr1 Apr16 May Jun Jul Avg -Imports From East of Continental Divide1 BEPC To NWE PwrS 2 NWE To NWE PWrS

3 PPL To BPA PwrS

4 PPL To PPL PwrS

5 PPLM To IPC PwrS

6 Total Imports From ECD

Sep Oct

-Imports From Pacific Southwest-

7 BURB To BPA XchgNrg

8 BURB To BPA PkRepl

9 GLEN To BPA XchgNrg

10 GLEN To BPA PkRepl

11 GLEN To PGE S/Pwr/X

12 NCPA To SCL S/Pwr/X

13 PASA To BPA XchgNrg

14 PASA To BPA PkRepl

15 PASA To BPA XchgNrg

16 PASA To BPA S/N/X

17 PASA To BPA PkRepl

18 PG\&E To PSE S/Pwr/X

19 RVSD To BPA XchgNrg

20 RVSD To BPA PkRepl

21 RVSD To BPA PkRepl

22 RVSD To BPA XchgNrg

23 RVSD To BPA S/Pwr/X

24 RVSD To BPA PkRepl

25 Total Imports From PSW

\begin{tabular}{rrrrrrrrrrrrrrr}
0 & 0 & 0 & 0 & 84 & 84 & 84 & 84 & 84 & 84 & 84 & 0 & 0 & 0 & 42 \\
0 & 0 & 0 & 0 & 0 & 0 & 0 & 0 & 0 & 0 & 0 & 0 & 0 & 0 & 0 \\
13 & 13 & 42 & 108 & 161 & 215 & 189 & 145 & 117 & 90 & 90 & 0.1 & 18 & 34 & 94 \\
215 & 215 & 193 & 191 & 224 & 233 & 231 & 218 & 162 & 172 & 172 & 121 & 206 & 202 & 197 \\
46 & 46 & 0 & 0 & 0 & 0 & 0 & 0 & 0 & 0 & 0 & 0 & 46 & 43 & 11 \\
\hline $\mathbf{2 7 5}$ & $\mathbf{2 7 5}$ & $\mathbf{2 3 5}$ & $\mathbf{2 9 9}$ & $\mathbf{4 6 9}$ & $\mathbf{5 3 2}$ & $\mathbf{5 0 4}$ & $\mathbf{4 4 7}$ & $\mathbf{3 6 2}$ & $\mathbf{3 4 5}$ & $\mathbf{3 4 5}$ & $\mathbf{1 2 1}$ & $\mathbf{2 7 0}$ & $\mathbf{2 7 9}$ & $\mathbf{3 4 5}$
\end{tabular}

-Imports From Inland Southwest-

26 Other Entities To BPA

27 SPP To BPA PwrS

28 WAPA TO GLEC ARP

29 Total Imports From ISW

\begin{tabular}{|c|c|c|c|c|c|c|c|c|c|c|c|c|c|c|}
\hline 2.6 & 2.6 & 2.6 & 2.6 & 3.8 & 5 & 5 & 5 & 5 & 5 & 2.6 & 2.6 & 2.6 & 2.6 & 3.6 \\
\hline 5.4 & 5.4 & 5.4 & 4 & 2.7 & 2.7 & 2.7 & 2.7 & 2.7 & 2.7 & 2.7 & 4 & 5.4 & 5.4 & 3.8 \\
\hline 2.9 & 2.9 & 2.9 & 2.9 & 4.2 & 5.6 & 5.6 & 5.6 & 5.6 & 5.6 & 2.9 & 2.9 & 2.9 & 2.9 & 4 \\
\hline 6.0 & 6.0 & 6.0 & 4.5 & 3.0 & 3.0 & 3.0 & 3.0 & 3.0 & 3.0 & 3.0 & 4.5 & 6.0 & 6.0 & 4.2 \\
\hline 0 & 0 & 0 & 0 & 8.9 & 8.9 & 8.9 & 8.9 & 0 & 0 & 0 & 0 & 0 & 0 & 3.0 \\
\hline 0 & 0 & 0 & 0 & 24 & 30 & 30 & 30 & 30 & 6.2 & 6.2 & 0 & 0 & 0 & 12 \\
\hline 1.7 & 1.7 & 1.7 & 1.7 & 2.5 & 3.4 & 3.4 & 3.4 & 3.4 & 3.4 & 1.7 & 1.7 & 1.7 & 1.7 & 2.4 \\
\hline 3.6 & 3.6 & 3.6 & 2.7 & 1.8 & 1.8 & 1.8 & 1.8 & 1.8 & 1.8 & 1.8 & 2.7 & 3.6 & 3.6 & 2.5 \\
\hline 0 & 0 & 3.5 & 3.5 & 3.5 & 3.5 & 3.5 & 3.5 & 1.7 & 0 & 0 & 0 & 0 & 0 & 1.9 \\
\hline 0 & 0 & 0.7 & 0.7 & 0.7 & 0.7 & 0.7 & 0.7 & 0.3 & 0 & 0 & 0 & 0 & 0 & 0.4 \\
\hline 4.5 & 4.5 & 4.5 & 0 & 0 & 0 & 0 & 0 & 0 & 0 & 0 & 0 & 0 & 4.5 & 1.1 \\
\hline 0 & 0 & 0 & 0 & 134 & 195 & 161 & 61 & 0 & 0 & 0 & 0 & 0 & 0 & 46 \\
\hline 0 & 0 & 0 & 5.8 & 5.8 & 5.8 & 5.8 & 5.8 & 5.8 & 5.8 & 0 & 0 & 0 & 0 & 3.1 \\
\hline 0 & 0 & 0 & 0 & 4.8 & 4.8 & 4.8 & 4.8 & 4.8 & 4.8 & 4.8 & 6.8 & 0 & 0 & 3.0 \\
\hline 6.8 & 6.8 & 6.8 & 6.8 & 0 & 0 & 0 & 0 & 0 & 0 & 0 & 0 & 6.8 & 6.8 & 2.8 \\
\hline 0 & 0 & 0 & 0 & 16 & 16 & 16 & 16 & 16 & 16 & 0 & 0 & 0 & 0 & 7.3 \\
\hline 0 & 0 & 0 & 0 & 9.2 & 9.2 & 9.2 & 9.2 & 9.2 & 9.2 & 0 & 0 & 0 & 0 & 4.2 \\
\hline 11 & 11 & 11 & 11 & 2.7 & 2.7 & 2.7 & 2.7 & 2.7 & 2.7 & 2.7 & 0 & 0 & 11 & 4.9 \\
\hline 44 & 44 & 48 & 46 & 227 & 298 & 264 & 164 & 92 & 66 & 28 & 25 & 29 & 44 & 111 \\
\hline
\end{tabular}

-Imports From Canada-

30 BCHP To BPA PwrS

31 BCHP To SCL PwrS

32 Total Imports From Canada

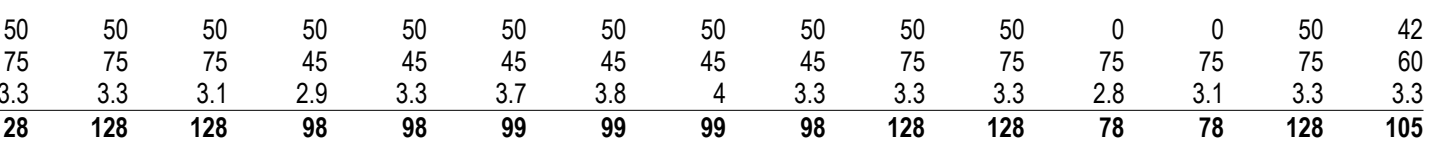

-Imports From Other Entities-

33 Total Imports From Other Entities

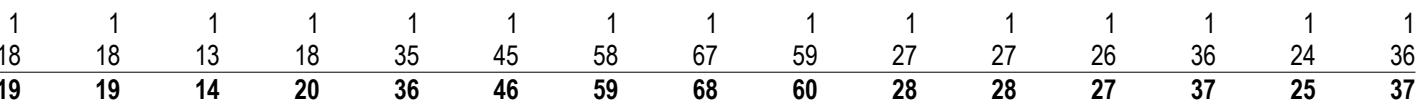

-Total Imports.

34 Federal System

35 Public Entities

36 Investor-Owned Entities

37 Other Entities

38 Total Imports.

\begin{tabular}{|c|c|c|c|c|c|c|c|c|c|c|c|c|c|c|}
\hline 0 & 0 & 0 & 0 & 0 & 0 & 0 & 0 & 0 & 0 & 0 & 0 & 0 & 0 & $\overline{0}$ \\
\hline 183 & 183 & 216 & 250 & 318 & 375 & 349 & 305 & 275 & 276 & 238 & 101 & 123 & 204 & 246 \\
\hline 21 & 21 & 16 & 21 & 62 & 79 & 92 & 101 & 93 & 37 & 37 & 28 & 39 & 27 & 51 \\
\hline 262 & 262 & 193 & 191 & 450 & 521 & 485 & 372 & 246 & 256 & 256 & 121 & 252 & 245 & 300 \\
\hline 0 & 0 & 0 & 0 & 0 & 0 & 0 & 0 & 0 & 0 & 0 & 0 & 0 & 0 & 0 \\
\hline 466 & 466 & 426 & 462 & 830 & 976 & 926 & 778 & 613 & 568 & 530 & 251 & 415 & 476 & 597 \\
\hline
\end{tabular}


Table A-5: Regional Imports

PNW Loads and Resources Study

2007 - 2008 Operating Year 2003 White Book

Dec

Jan Feb Mar Apr1 Apr16 May Jun Jul Avg

-Imports From East of Continental Divide-

1 BEPC To NWE PwrS

2 NWE To NWE PwrS

3 PPL To BPA PwrS

4 PPL To PPL PwrS

5 PPLM To IPC PwrS

6 Total Imports From ECD

$\begin{array}{rrrrrrrrrrrrrrr}0 & 0 & 0 & 0 & 84 & 84 & 84 & 84 & 84 & 84 & 84 & 0 & 0 & 0 & 42 \\ 0 & 0 & 0 & 0 & 0 & 0 & 0 & 0 & 0 & 0 & 0 & 0 & 0 & 0 & 0 \\ 13 & 13 & 42 & 108 & 161 & 215 & 189 & 145 & 117 & 90 & 90 & 0.1 & 18 & 34 & 94 \\ 160 & 160 & 98 & 136 & 152 & 200 & 140 & 182 & 144 & 146 & 146 & 135 & 169 & 178 & 153 \\ 46 & 46 & 0 & 0 & 0 & 0 & 0 & 0 & 0 & 0 & 0 & 0 & 44 & 45 & 11 \\ \mathbf{2 2 0} & \mathbf{2 2 0} & \mathbf{1 4 0} & \mathbf{2 4 4} & \mathbf{3 9 7} & \mathbf{4 9 9} & \mathbf{4 1 3} & \mathbf{4 1 1} & \mathbf{3 4 5} & \mathbf{3 2 0} & \mathbf{3 2 0} & \mathbf{1 3 5} & \mathbf{2 3 2} & \mathbf{2 5 7} & \mathbf{3 0 1}\end{array}$

-Imports From Pacific Southwest-

7 BURB To BPA XchgNrg

8 BURB To BPA PkRepl

9 GLEN To BPA XchgNrg

10 GLEN To BPA PkRepl

11 GLEN To PGE S/Pwr/X

12 NCPA To SCL S/Pwr/X

13 PASA To BPA XchgNrg

14 PASA To BPA PkRepl

15 PASA To BPA XchgNrg

16 PASA To BPA S/N/X

17 PASA To BPA PkRepl

18 PG\&E To PSE S/Pwr/X

19 RVSD To BPA XchgNrg

20 RVSD To BPA PkRepl

21 RVSD To BPA PkRepl

22 RVSD To BPA XchgNrg

23 RVSD To BPA S/Pwr/X

24 RVSD To BPA PkRepl

25 Total Imports From PSW

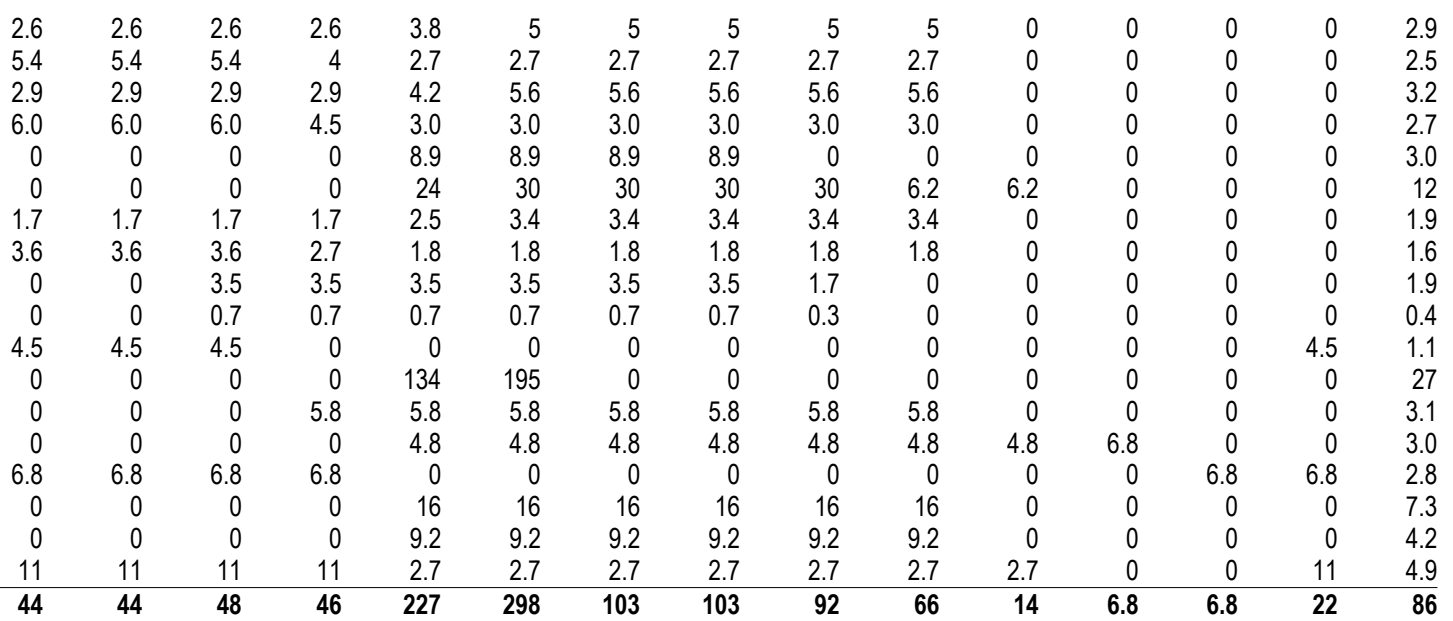

-Imports From Inland Southwest-

26 SPP To BPA PwrS

27 Total Imports From ISW

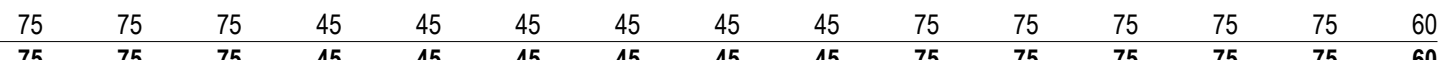

-Imports From Canada-

28 BCHP To BPA PwrS

29 BCHP To SCL PwrS

30 Total Imports From Canada

\begin{tabular}{|c|c|c|c|c|c|c|c|c|c|c|c|c|c|}
\hline 1 & 1 & 1 & 1 & 1 & 1 & 1 & 1 & 1 & 1 & 1 & 1 & 1 & 1 \\
\hline 18 & 13 & 18 & 35 & 45 & 58 & 67 & 59 & 27 & 27 & 26 & 36 & 24 & 36 \\
\hline 19 & 14 & 20 & 36 & 46 & 59 & 68 & 60 & 28 & 28 & 27 & 37 & 25 & 37 \\
\hline
\end{tabular}

-Imports From Other Entities-

31 Total Imports From Other Entities

$\begin{array}{lllllllllllllll}0 & 0 & 0 & 0 & 0 & 0 & 0 & 0 & 0 & 0 & 0 & 0 & 0 & 0 & 0\end{array}$

-Total Imports-

32 Federal System

33 Public Entities

34 Investor-Owned Entities

35 Other Entities

36 Total Imports.

\begin{tabular}{|c|c|c|c|c|c|c|c|c|c|c|c|c|c|c|}
\hline 133 & 133 & 166 & 200 & 268 & 325 & 299 & 255 & 225 & 226 & 173 & 83 & 101 & 132 & 199 \\
\hline 18 & 18 & 13 & 18 & 58 & 75 & 88 & 97 & 89 & 34 & 34 & 26 & 36 & 24 & 48 \\
\hline 207 & 207 & 98 & 136 & 378 & 488 & 233 & 275 & 228 & 230 & 230 & 135 & 213 & 223 & 237 \\
\hline 0 & 0 & 0 & 0 & 0 & 0 & 0 & 0 & 0 & 0 & 0 & 0 & 0 & 0 & \\
\hline 357 & 357 & 277 & 355 & 704 & 889 & 620 & 627 & 543 & 489 & 437 & 243 & 351 & 379 & 84 \\
\hline
\end{tabular}


2 NWE To NWE PwrS

3 PPL To BPA PwrS

4 PPL To PPL PwrS

5 PPLM To IPC PwrS

6 Total Imports From ECD

$\begin{array}{rrrrrrrrrrrrrrr}0 & 0 & 0 & 0 & 84 & 84 & 84 & 84 & 84 & 84 & 84 & 0 & 0 & 0 & 42 \\ 0 & 0 & 0 & 0 & 0 & 0 & 0 & 0 & 0 & 0 & 0 & 0 & 0 & 0 & 0 \\ 13 & 13 & 42 & 108 & 161 & 215 & 189 & 145 & 117 & 90 & 90 & 0.1 & 18 & 34 & 94 \\ 0 & 0 & 0 & 0 & 0 & 0 & 0 & 0 & 0 & 0 & 0 & 0 & 0 & 0 & 0 \\ 45 & 45 & 0 & 0 & 0 & 0 & 0 & 0 & 0 & 0 & 0 & 0 & 46 & 45 & 11 \\ \mathbf{5 8} & \mathbf{5 8} & \mathbf{4 2} & \mathbf{1 0 8} & \mathbf{2 4 5} & \mathbf{2 9 9} & \mathbf{2 7 3} & \mathbf{2 2 9} & \mathbf{2 0 1} & \mathbf{1 7 4} & \mathbf{1 7 4} & \mathbf{0 . 1} & \mathbf{6 4} & \mathbf{7 8} & \mathbf{1 4 8}\end{array}$

-Imports From Pacific Southwest-

7 GLEN To PGE S/Pwr/X

8 NCPA To SCL S/Pwr/X

9 PASA To BPA XchgNrg

10 PASA To BPA S/N/X

11 PASA To BPA PkRepl

12 PG\&E TO PSE S/Pwr/X

13 RVSD To BPA XchgNrg

14 RVSD To BPA PkRepl

15 RVSD To BPA PkRepl

16 RVSD To BPA XchgNrg

17 RVSD To BPA S/Pwr/X

18 RVSD To BPA PkRepl

19 Total Imports From PSW

$\begin{array}{rrrrrrrrrrrrrrr}0 & 0 & 0 & 0 & 8.9 & 8.9 & 8.9 & 8.9 & 0 & 0 & 0 & 0 & 0 & 0 & 3.0 \\ 0 & 0 & 0 & 0 & 24 & 30 & 30 & 30 & 30 & 6.2 & 6.2 & 0 & 0 & 0 & 12 \\ 0 & 0 & 3.5 & 3.5 & 3.5 & 3.5 & 3.5 & 3.5 & 1.7 & 0 & 0 & 0 & 0 & 0 & 1.9 \\ 0 & 0 & 0.7 & 0.7 & 0.7 & 0.7 & 0.7 & 0.7 & 0.3 & 0 & 0 & 0 & 0 & 0 & 0.4 \\ 4.5 & 4.5 & 4.5 & 0 & 0 & 0 & 0 & 0 & 0 & 0 & 0 & 0 & 0 & 4.5 & 1.1 \\ 0 & 0 & 0 & 0 & 0 & 0 & 0 & 0 & 0 & 0 & 0 & 0 & 0 & 0 & 0 \\ 0 & 0 & 0 & 5.8 & 5.8 & 5.8 & 5.8 & 5.8 & 5.8 & 5.8 & 0 & 0 & 0 & 0 & 3.1 \\ 0 & 0 & 0 & 0 & 4.8 & 4.8 & 4.8 & 4.8 & 4.8 & 4.8 & 4.8 & 6.8 & 0 & 0 & 3.0 \\ 6.8 & 6.8 & 6.8 & 6.8 & 0 & 0 & 0 & 0 & 0 & 0 & 0 & 0 & 6.8 & 6.8 & 2.8 \\ 0 & 0 & 0 & 0 & 16 & 16 & 16 & 16 & 16 & 16 & 0 & 0 & 0 & 0 & 7.3 \\ 0 & 0 & 0 & 0 & 9.2 & 9.2 & 9.2 & 9.2 & 9.2 & 9.2 & 0 & 0 & 0 & 0 & 4.2 \\ 11 & 11 & 11 & 11 & 2.7 & 2.7 & 2.7 & 2.7 & 2.7 & 2.7 & 2.7 & 0 & 0 & 11 & 4.9 \\ \mathbf{2 2} & \mathbf{2 2} & \mathbf{2 6} & \mathbf{2 7} & \mathbf{7 5} & \mathbf{8 2} & \mathbf{8 2} & \mathbf{8 2} & \mathbf{7 1} & \mathbf{4 5} & \mathbf{1 4} & \mathbf{6 . 8} & \mathbf{6 . 8} & \mathbf{2 2} & \mathbf{4 4}\end{array}$

-Imports From Inland Southwest-

20 SPP To BPA PwrS

21 Total Imports From ISW

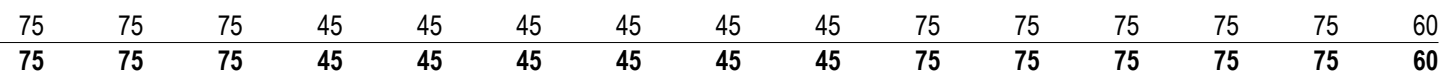

-Imports From Canada-

22 BCHP To BPA PwrS

23 BCHP To SCL PwrS

24 Total Imports From Canada

-Imports From Other Entities-

25 Total Imports From Other Entities

\begin{tabular}{|c|c|c|c|c|c|c|c|c|c|c|c|c|c|}
\hline 1 & 1 & 1 & 1 & 1 & 1 & 1 & 1 & 1 & 1 & 1 & 1 & 1 & 1 \\
\hline 18 & 13 & 18 & 35 & 45 & 58 & 67 & 59 & 27 & 27 & 26 & 36 & 24 & 36 \\
\hline 19 & 14 & 20 & 36 & 46 & 59 & 68 & 60 & 28 & 28 & 27 & 37 & 25 & 37 \\
\hline
\end{tabular}

-Total Imports.

26 Federal System

27 Public Entities

28 Investor-Owned Entities

29 Other Entities

30 Total Imports.

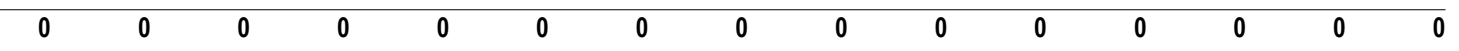

\begin{tabular}{|c|c|c|c|c|c|c|c|c|c|c|c|c|c|}
\hline 111 & 144 & 182 & 249 & 304 & 277 & 234 & 203 & 204 & 173 & 83 & 101 & 132 & 184 \\
\hline 18 & 13 & 18 & 58 & 75 & 88 & 97 & 89 & 34 & 34 & 26 & 36 & 24 & 48 \\
\hline 45 & 0 & 0 & 93 & 93 & 93 & 93 & 84 & 84 & 84 & 0 & 46 & 45 & 56 \\
\hline 0 & 0 & 0 & 0 & 0 & 0 & 0 & 0 & 0 & 0 & 0 & 0 & 0 & 0 \\
\hline 173 & 157 & 200 & 401 & 472 & 458 & 424 & 377 & 322 & 291 & 109 & 184 & 200 & 288 \\
\hline
\end{tabular}



Dec Jan $F e$ Feb Mar Apr1 Apr16 May Jun Jul Avg

-Imports From East of Continental Divide-

1 BEPC To NWE PwrS

2 NWE To NWE PwrS

3 PPL To BPA PwrS

4 PPLM To IPC PwrS

5 Total Imports From ECD

\begin{tabular}{|c|c|c|c|c|c|c|c|c|c|c|c|c|c|}
\hline 0 & 0 & 0 & 84 & 84 & 84 & 84 & 84 & 84 & 84 & 0 & 0 & 0 & 42 \\
\hline 0 & 0 & 0 & 0 & 0 & 0 & 0 & 0 & 0 & 0 & 0 & 0 & 0 & 0 \\
\hline 13 & 42 & 108 & 161 & 215 & 189 & 145 & 117 & 90 & 90 & 0.1 & 18 & 34 & 94 \\
\hline 45 & 0 & 0 & 0 & 0 & 0 & 0 & 0 & 0 & 0 & 0 & 0 & 0 & 3.7 \\
\hline 58 & 42 & 108 & 245 & 299 & 273 & 229 & 201 & 174 & 174 & 0.1 & 18 & 34 & 140 \\
\hline
\end{tabular}

-Imports From Pacific Southwest-

6 GLEN To PGE S/Pwr/X

7 NCPA To SCL S/Pwr/X

8 PASA To BPA XchgNrg

9 PASA TO BPA S/N/X

10 PASA To BPA PkRepl

11 RVSD To BPA XchgNrg

12 RVSD To BPA PkRepl

13 RVSD To BPA PkRepl

14 RVSD To BPA XchgNrg

15 RVSD To BPA S/Pwr/X

16 RVSD To BPA PkRepl

17 Total Imports From PSW

$\begin{array}{rrrrrrrrrrrrrrr}0 & 0 & 0 & 0 & 8.9 & 8.9 & 8.9 & 8.9 & 0 & 0 & 0 & 0 & 0 & 0 & 3.0 \\ 0 & 0 & 0 & 0 & 24 & 30 & 30 & 30 & 30 & 6.2 & 6.2 & 0 & 0 & 0 & 12 \\ 0 & 0 & 3.5 & 3.5 & 3.5 & 3.5 & 3.5 & 3.5 & 1.7 & 0 & 0 & 0 & 0 & 0 & 1.9 \\ 0 & 0 & 0.7 & 0.7 & 0.7 & 0.7 & 0.7 & 0.7 & 0.3 & 0 & 0 & 0 & 0 & 0 & 0.4 \\ 4.5 & 4.5 & 4.5 & 0 & 0 & 0 & 0 & 0 & 0 & 0 & 0 & 0 & 0 & 4.5 & 1.1 \\ 0 & 0 & 0 & 5.8 & 5.8 & 5.8 & 5.8 & 5.8 & 5.8 & 5.8 & 0 & 0 & 0 & 0 & 3.1 \\ 0 & 0 & 0 & 0 & 4.8 & 4.8 & 4.8 & 4.8 & 4.8 & 4.8 & 4.8 & 6.8 & 0 & 0 & 3.0 \\ 6.8 & 6.8 & 6.8 & 6.8 & 0 & 0 & 0 & 0 & 0 & 0 & 0 & 0 & 0 & 0 & 1.7 \\ 0 & 0 & 0 & 0 & 16 & 16 & 16 & 16 & 16 & 16 & 0 & 0 & 0 & 0 & 7.3 \\ 0 & 0 & 0 & 0 & 9.2 & 9.2 & 9.2 & 9.2 & 9.2 & 9.2 & 0 & 0 & 0 & 0 & 4.2 \\ 11 & 11 & 11 & 11 & 2.7 & 2.7 & 2.7 & 2.7 & 2.7 & 2.7 & 2.7 & 0 & 0 & 11 & 4.9 \\ \mathbf{2 2} & \mathbf{2 2} & \mathbf{2 6} & \mathbf{2 7} & \mathbf{7 5} & \mathbf{8 2} & \mathbf{8 2} & \mathbf{8 2} & \mathbf{7 1} & \mathbf{4 5} & \mathbf{1 4} & \mathbf{6 . 8} & \mathbf{0} & \mathbf{1 5} & \mathbf{4 3}\end{array}$

-Imports From Inland Southwest-

19 Total Imports From ISW

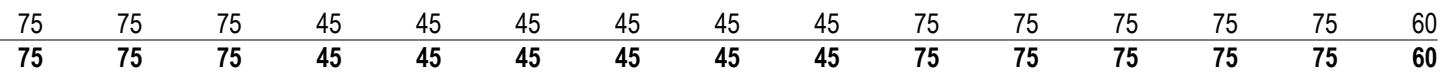

-Imports From Canada-

20 BCHP To BPA PwrS

21 BCHP To SCL PwrS

22 Total Imports From Canada

\begin{tabular}{|c|c|c|c|c|c|c|c|c|c|c|c|c|c|}
\hline 1 & 1 & 1 & 1 & 1 & 1 & 1 & 1 & 1 & 1 & 1 & 1 & 1 & 1 \\
\hline 18 & 13 & 18 & 35 & 45 & 58 & 67 & 59 & 27 & 27 & 26 & 36 & 24 & 36 \\
\hline 19 & 14 & 20 & 36 & 46 & 59 & 68 & 60 & 28 & 28 & 27 & 37 & 25 & 37 \\
\hline
\end{tabular}

-Imports From Other Entities-

23 Total Imports From Other Entities

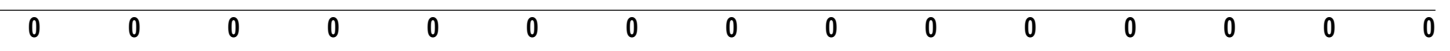

-Total Imports-

24 Federal System

25 Public Entities

26 Investor-Owned Entities

27 Other Entities

28 Total Imports.

\begin{tabular}{|c|c|c|c|c|c|c|c|c|c|c|c|c|c|c|}
\hline 111 & 111 & 144 & 182 & 249 & 304 & 277 & 234 & 203 & 204 & 173 & 83 & 94 & 125 & 183 \\
\hline 18 & 18 & 13 & 18 & 58 & 75 & 88 & 97 & 89 & 34 & 34 & 26 & 36 & 24 & 48 \\
\hline 45 & 45 & 0 & 0 & 93 & 93 & 93 & 93 & 84 & 84 & 84 & 0 & 0 & 0 & 49 \\
\hline 0 & 0 & 0 & 0 & 0 & 0 & 0 & 0 & 0 & 0 & 0 & 0 & 0 & 0 & 0 \\
\hline 173 & 173 & 157 & 200 & 401 & 472 & 458 & 424 & 377 & 322 & 291 & 109 & 130 & 149 & 280 \\
\hline
\end{tabular}



2003 White Book

-Imports From East of Continental Divide-

1 NWE To NWE PwrS

2 PPL To BPA PwrS

3 Total Imports From ECD

May Jun Jul Avg

-Imports From Pacific Southwest-

4 GLEN To PGE S/Pwr/X

5 NCPA To SCL S/Pwr/X

6 PASA To BPA XchgNrg

7 PASA TO BPA S/N/X

8 PASA To BPA PkRepl

9 RVSD To BPA XchgNrg

10 RVSD To BPA S/Pwr/X

11 RVSD To BPA PkRepl

12 Total Imports From PSW

\begin{tabular}{|c|c|c|c|c|c|c|c|c|c|c|c|c|c|}
\hline 0 & 0 & 0 & 0 & 0 & 0 & 0 & 0 & 0 & 0 & 0 & 0 & 0 & 0 \\
\hline 13 & 42 & 108 & 161 & 215 & 189 & 145 & 117 & 90 & 90 & 0.1 & 18 & 34 & 94 \\
\hline 13 & 42 & 108 & 161 & 215 & 189 & 145 & 117 & 90 & 90 & 0.1 & 18 & 34 & 94 \\
\hline
\end{tabular}

-Imports From Inland Southwest-

13 SPP To BPA PwrS

14 Total Imports From ISW

$\begin{array}{rr}0 & 0 \\ 0 & 0 \\ 0 & 0 \\ 0 & 0 \\ 4.5 & 4.5 \\ 0 & 0 \\ 0 & 0 \\ 11 & 11 \\ 15 & 15\end{array}$

$\begin{array}{rr}0 & 0 \\ 0 & 0 \\ 3.5 & 3.5 \\ 0.7 & 0.7 \\ 4.5 & 0 \\ 0 & 0 \\ 0 & 0 \\ 11 & 11 \\ 19 & 15\end{array}$

$\begin{array}{rrrr}8.9 & 8.9 & 8.9 & 8.9 \\ 24 & 30 & 30 & \\ 3.5 & 3.5 & 3.5 & 3.5 \\ 0.7 & 0.7 & 0.7 & 0.7 \\ 0 & 0 & 0 & \\ 16 & 16 & 16 & \\ 9.2 & 9.2 & 9.2 & 9.2 \\ 2.7 & 2.7 & 2.7 & 2.7 \\ 65 & 71 & 71 & 71\end{array}$

\begin{tabular}{rrrrrrrr}
8.9 & 0 & 0 & 0 & 0 & 0 & 0 & 3.0 \\
30 & 30 & 6.2 & 6.2 & 0 & 0 & 0 & 12 \\
3.5 & 1.7 & 0 & 0 & 0 & 0 & 0 & 1.9 \\
0.7 & 0.3 & 0 & 0 & 0 & 0 & 0 & 0.4 \\
0 & 0 & 0 & 0 & 0 & 0 & 4.5 & 1.1 \\
16 & 16 & 16 & 0 & 0 & 0 & 0 & 7.3 \\
9.2 & 9.2 & 9.2 & 0 & 0 & 0 & 0 & 4.2 \\
2.7 & 2.7 & 2.7 & 2.7 & 0 & 0 & 11 & 4.9 \\
\hline 71 & $\mathbf{6 0}$ & $\mathbf{3 4}$ & $\mathbf{8 . 9}$ & $\mathbf{0}$ & $\mathbf{0}$ & $\mathbf{1 5}$ & $\mathbf{3 5}$
\end{tabular}

-Imports From Canada-

15 BCHP To BPA PwrS

16 BCHP To SCL PwrS

17 Total Imports From Canada

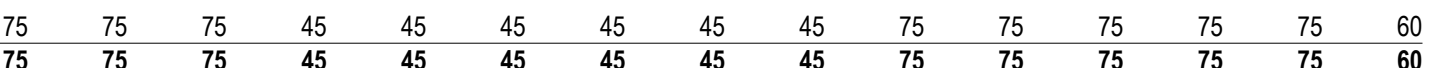

-Imports From Other Entities-

18 Total Imports From Other Entities

-Total Imports

19 Federal System

20 Public Entities

21 Investor-Owned Entities

22 Other Entities

23 Total Imports.

\begin{tabular}{|c|c|c|c|c|c|c|c|c|c|c|c|c|c|}
\hline 1 & 1 & 1 & 1 & 1 & 1 & 1 & 1 & 1 & 1 & 1 & 1 & 1 & 1 \\
\hline 18 & 13 & 18 & 35 & 45 & 58 & 67 & 59 & 27 & 27 & 26 & 36 & 24 & 36 \\
\hline 19 & 14 & 20 & 36 & 46 & 59 & 68 & 60 & 28 & 28 & 27 & 37 & 25 & 37 \\
\hline
\end{tabular}

$\begin{array}{lllllllllllllll}0 & 0 & 0 & 0 & 0 & 0 & 0 & 0 & 0 & 0 & 0 & 0 & 0 & 0 & 0\end{array}$

\begin{tabular}{|c|c|c|c|c|c|c|c|c|c|c|c|c|c|c|}
\hline 104 & 104 & 137 & 169 & 239 & 293 & 267 & 223 & 193 & 194 & 168 & 76 & 94 & 125 & 175 \\
\hline 18 & 18 & 13 & 18 & 58 & 75 & 88 & 97 & 89 & 34 & 34 & 26 & 36 & 24 & 48 \\
\hline 0 & 0 & 0 & 0 & 8.9 & 8.9 & 8.9 & 8.9 & 0 & 0 & 0 & 0 & 0 & 0 & 3.0 \\
\hline 0 & 0 & 0 & 0 & 0 & 0 & 0 & 0 & 0 & 0 & 0 & 0 & 0 & 0 & 0 \\
\hline
\end{tabular}



2003 White Book

-Imports From East of Continental Divide-

1 NWE To NWE PwrS

2 PPL To BPA PwrS

3 Total Imports From ECD

May Jun Jul Avg

-Imports From Pacific Southwest-

4 GLEN To PGE S/Pwr/X

5 NCPA To SCL S/Pwr/X

6 PASA To BPA XchgNrg

7 PASA TO BPA S/N/X

8 PASA To BPA PkRepl

9 RVSD To BPA XchgNrg

10 RVSD To BPA S/Pwr/X

11 RVSD To BPA PkRepl

12 Total Imports From PSW

\begin{tabular}{|c|c|c|c|c|c|c|c|c|c|c|c|c|c|}
\hline 0 & 0 & 0 & 0 & 0 & 0 & 0 & 0 & 0 & 0 & 0 & 0 & 0 & 0 \\
\hline 13 & 42 & 108 & 161 & 215 & 189 & 145 & 117 & 90 & 90 & 0.1 & 18 & 34 & 94 \\
\hline 13 & 42 & 108 & 161 & 215 & 189 & 145 & 117 & 90 & 90 & 0.1 & 18 & 34 & 94 \\
\hline
\end{tabular}

-Imports From Inland Southwest-

13 SPP To BPA PwrS

14 Total Imports From ISW

$\begin{array}{rr}0 & 0 \\ 0 & 0 \\ 0 & 0 \\ 0 & 0 \\ 4.5 & 4.5 \\ 0 & 0 \\ 0 & 0 \\ 11 & 11 \\ 15 & 15\end{array}$

$\begin{array}{rr}0 & 0 \\ 0 & 0 \\ 3.5 & 3.5 \\ 0.7 & 0.7 \\ 4.5 & 0 \\ 0 & 0 \\ 0 & 0 \\ 11 & 11 \\ 19 & 15\end{array}$

$\begin{array}{rrrr}8.9 & 8.9 & 8.9 & 8.9 \\ 24 & 30 & 30 & \\ 3.5 & 3.5 & 3.5 & 3.5 \\ 0.7 & 0.7 & 0.7 & 0.7 \\ 0 & 0 & 0 & \\ 16 & 16 & 16 & \\ 9.2 & 9.2 & 9.2 & 9.2 \\ 2.7 & 2.7 & 2.7 & 2.7 \\ 65 & 71 & 71 & 71\end{array}$

\begin{tabular}{rrrrrrrr}
8.9 & 0 & 0 & 0 & 0 & 0 & 0 & 3.0 \\
30 & 30 & 6.2 & 6.2 & 0 & 0 & 0 & 12 \\
3.5 & 1.7 & 0 & 0 & 0 & 0 & 0 & 1.9 \\
0.7 & 0.3 & 0 & 0 & 0 & 0 & 0 & 0.4 \\
0 & 0 & 0 & 0 & 0 & 0 & 4.5 & 1.1 \\
16 & 16 & 16 & 0 & 0 & 0 & 0 & 7.3 \\
9.2 & 9.2 & 9.2 & 0 & 0 & 0 & 0 & 4.2 \\
2.7 & 2.7 & 2.7 & 2.7 & 0 & 0 & 11 & 4.9 \\
\hline 71 & $\mathbf{6 0}$ & $\mathbf{3 4}$ & $\mathbf{8 . 9}$ & $\mathbf{0}$ & $\mathbf{0}$ & $\mathbf{1 5}$ & $\mathbf{3 5}$
\end{tabular}

-Imports From Canada-

15 BCHP To BPA PwrS

16 BCHP To SCL PwrS

17 Total Imports From Canada

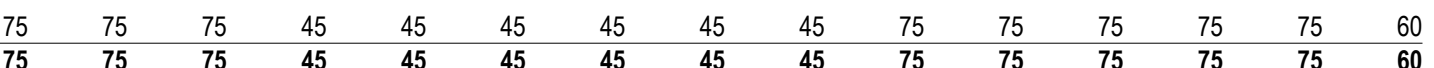

-Imports From Other Entities-

18 Total Imports From Other Entities

-Total Imports

19 Federal System

20 Public Entities

21 Investor-Owned Entities

22 Other Entities

23 Total Imports.

\begin{tabular}{|c|c|c|c|c|c|c|c|c|c|c|c|c|c|}
\hline 1 & 1 & 1 & 1 & 1 & 1 & 1 & 1 & 1 & 1 & 1 & 1 & 1 & 1 \\
\hline 18 & 13 & 18 & 35 & 45 & 58 & 67 & 59 & 27 & 27 & 26 & 36 & 24 & 36 \\
\hline 19 & 14 & 20 & 36 & 46 & 59 & 68 & 60 & 28 & 28 & 27 & 37 & 25 & 37 \\
\hline
\end{tabular}

$\begin{array}{lllllllllllllll}0 & 0 & 0 & 0 & 0 & 0 & 0 & 0 & 0 & 0 & 0 & 0 & 0 & 0 & 0\end{array}$

\begin{tabular}{|c|c|c|c|c|c|c|c|c|c|c|c|c|c|c|}
\hline 104 & 104 & 137 & 169 & 239 & 293 & 267 & 223 & 193 & 194 & 168 & 76 & 94 & 125 & 175 \\
\hline 18 & 18 & 13 & 18 & 58 & 75 & 88 & 97 & 89 & 34 & 34 & 26 & 36 & 24 & 48 \\
\hline 0 & 0 & 0 & 0 & 8.9 & 8.9 & 8.9 & 8.9 & 0 & 0 & 0 & 0 & 0 & 0 & 3.0 \\
\hline 0 & 0 & 0 & 0 & 0 & 0 & 0 & 0 & 0 & 0 & 0 & 0 & 0 & 0 & 0 \\
\hline
\end{tabular}



2003 White Book

Aug1 Aug16 Sep Oct Nov Dec Jan Feb Mar Apr1 Apr16 May Jun Jul Avg

-Imports From East of Continental Divide-

1 NWE To NWE PwrS

2 PPL To BPA PwrS

3 Total Imports From ECD

\begin{tabular}{|c|c|c|c|c|c|c|c|c|c|c|c|c|c|}
\hline 0 & 0 & 0 & 0 & 0 & 0 & 0 & 0 & 0 & 0 & 0 & 0 & 0 & 0 \\
\hline 13 & 42 & 108 & 161 & 215 & 189 & 145 & 117 & 90 & 90 & 0.1 & 18 & 34 & 94 \\
\hline 13 & 42 & 108 & 161 & 215 & 189 & 145 & 117 & 90 & 90 & 0.1 & 18 & 34 & 94 \\
\hline
\end{tabular}

-Imports From Pacific Southwest-

4 NCPA To SCL S/Pwr/X

5 PASA To BPA XchgNrg

6 PASA To BPA S/N/X

7 PASA To BPA PkRepl

8 RVSD To BPA XchgNrg

9 RVSD To BPA S/Pwr/X

10 RVSD To BPA PkRepl

11 Total Imports From PSW

$\begin{array}{rrrrrrrrrrrrrrr}0 & 0 & 0 & 0 & 24 & 30 & 30 & 30 & 30 & 6.2 & 6.2 & 0 & 0 & 0 & 12 \\ 0 & 0 & 3.5 & 3.5 & 3.5 & 3.5 & 3.5 & 3.5 & 1.7 & 0 & 0 & 0 & 0 & 0 & 1.9 \\ 0 & 0 & 0.7 & 0.7 & 0.7 & 0.7 & 0.7 & 0.7 & 0.3 & 0 & 0 & 0 & 0 & 0 & 0.4 \\ 4.5 & 4.5 & 4.5 & 0 & 0 & 0 & 0 & 0 & 0 & 0 & 0 & 0 & 0 & 4.5 & 1.1 \\ 0 & 0 & 0 & 0 & 16 & 16 & 16 & 16 & 16 & 16 & 0 & 0 & 0 & 0 & 7.3 \\ 0 & 0 & 0 & 0 & 9.2 & 9.2 & 9.2 & 9.2 & 9.2 & 9.2 & 0 & 0 & 0 & 0 & 4.2 \\ 11 & 11 & 11 & 11 & 2.7 & 2.7 & 2.7 & 2.7 & 2.7 & 2.7 & 2.7 & 0 & 0 & 11 & 4.9 \\ 15 & \mathbf{1 5} & \mathbf{1 9} & \mathbf{1 5} & \mathbf{5 6} & \mathbf{6 2} & \mathbf{6 2} & \mathbf{6 2} & \mathbf{6 0} & \mathbf{3 4} & \mathbf{8 . 9} & \mathbf{0} & \mathbf{0} & \mathbf{1 5} & \mathbf{3 2}\end{array}$

-Imports From Inland Southwest-

12 SPP To BPA PwrS

13 Total Imports From ISW

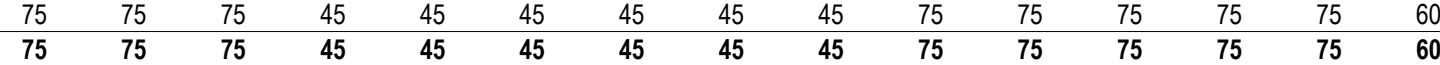

-Imports From Canada-

14 BCHP To BPA PwrS

15 BCHP To SCL PwrS

16 Total Imports From Canada

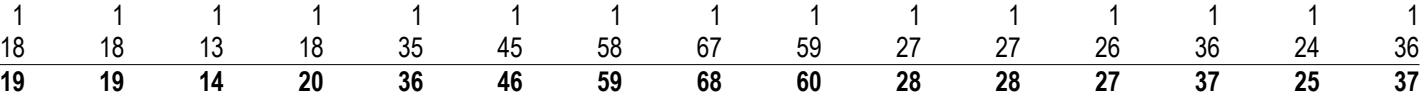

-Imports From Other Entities-

17 Total Imports From Other Entities

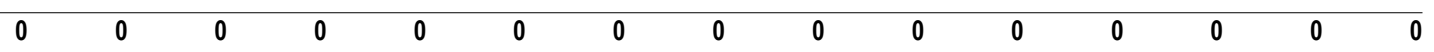

-Total Imports-

18 Federal System

19 Public Entities

20 Investor-Owned Entities

21 Other Entities

22 Total Imports

\begin{tabular}{|c|c|c|c|c|c|c|c|c|c|c|c|c|c|c|}
\hline 104 & 104 & 137 & 169 & 239 & 293 & 267 & 223 & 193 & 194 & 168 & 76 & 94 & 125 & 175 \\
\hline 18 & 18 & 13 & 18 & 58 & 75 & 88 & 97 & 89 & 34 & 34 & 26 & 36 & 24 & 48 \\
\hline 0 & 0 & 0 & 0 & 0 & 0 & 0 & 0 & 0 & 0 & 0 & 0 & 0 & 0 & 0 \\
\hline 0 & 0 & 0 & 0 & 0 & 0 & 0 & 0 & 0 & 0 & 0 & 0 & 0 & 0 & 0 \\
\hline
\end{tabular}



2003 White Book

-Imports From East of Continental Divide-

1 NWE To NWE PwrS

2 PPL To BPA PwrS

3 Total Imports From ECD

May Jun Jul Avg

-Imports From Pacific Southwest-

4 NCPA To SCL S/Pwr/X

5 PASA To BPA XchgNrg

6 PASA To BPA S/N/X

7 PASA To BPA PkRepl

8 RVSD To BPA XchgNrg

9 RVSD To BPA S/Pwr/X

10 RVSD To BPA PkRepl

11 Total Imports From PSW

\begin{tabular}{|c|c|c|c|c|c|c|c|c|c|c|c|c|c|}
\hline 0 & 0 & 0 & 0 & 0 & 0 & 0 & 0 & 0 & 0 & 0 & 0 & 0 & 0 \\
\hline 13 & 42 & 108 & 161 & 215 & 189 & 145 & 117 & 90 & 90 & 0.1 & 18 & 34 & 94 \\
\hline 13 & 42 & 108 & 161 & 215 & 189 & 145 & 117 & 90 & 90 & 0.1 & 18 & 34 & 94 \\
\hline
\end{tabular}

-Imports From Inland Southwest-

12 SPP To BPA PwrS

13 Total Imports From ISW

$\begin{array}{rrrrrrrrrrrrrrr}0 & 0 & 0 & 0 & 24 & 30 & 30 & 30 & 30 & 6.2 & 6.2 & 0 & 0 & 0 & 12 \\ 0 & 0 & 3.5 & 3.5 & 3.5 & 3.5 & 3.5 & 3.5 & 1.7 & 0 & 0 & 0 & 0 & 0 & 1.9 \\ 0 & 0 & 0.7 & 0.7 & 0.7 & 0.7 & 0.7 & 0.7 & 0.3 & 0 & 0 & 0 & 0 & 0 & 0.4 \\ 4.5 & 4.5 & 4.5 & 0 & 0 & 0 & 0 & 0 & 0 & 0 & 0 & 0 & 0 & 4.5 & 1.1 \\ 0 & 0 & 0 & 0 & 16 & 16 & 16 & 16 & 16 & 16 & 0 & 0 & 0 & 0 & 7.3 \\ 0 & 0 & 0 & 0 & 9.2 & 9.2 & 9.2 & 9.2 & 9.2 & 9.2 & 0 & 0 & 0 & 0 & 4.2 \\ 11 & 11 & 11 & 11 & 2.7 & 2.7 & 2.7 & 2.7 & 2.7 & 2.7 & 2.7 & 0 & 0 & 11 & 4.9 \\ \mathbf{1 5} & \mathbf{1 5} & \mathbf{1 9} & \mathbf{1 5} & \mathbf{5 6} & \mathbf{6 2} & \mathbf{6 2} & \mathbf{6 2} & \mathbf{6 0} & \mathbf{3 4} & \mathbf{8 . 9} & \mathbf{0} & \mathbf{0} & \mathbf{1 5} & \mathbf{3 2}\end{array}$

-Imports From Canada-

14 BCHP To BPA PwrS

15 BCHP To SCL PwrS

16 Total Imports From Canada

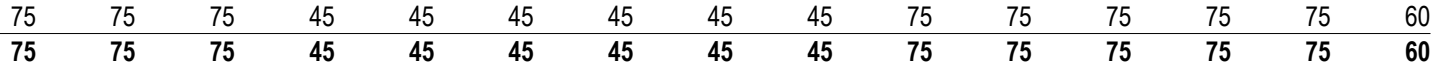

-Imports From Other Entities-

17 Total Imports From Other Entities

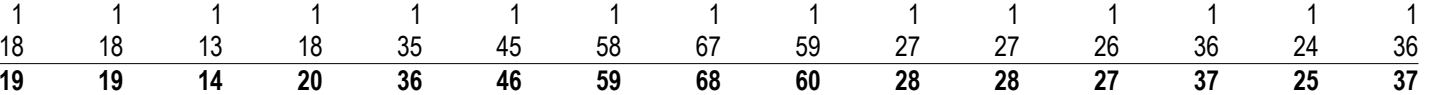

-Total Imports-

18 Federal System

19 Public Entities

20 Investor-Owned Entities

21 Other Entities

22 Total Imports

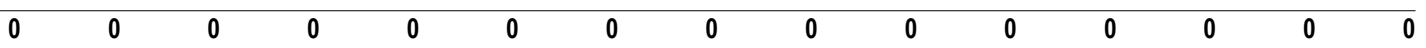

$\begin{array}{rrrrrrrrrrrrrrr}104 & 104 & 137 & 169 & 239 & 293 & 267 & 223 & 193 & 194 & 168 & 76 & 94 & 125 & 175 \\ 18 & 18 & 13 & 18 & 58 & 75 & 88 & 97 & 89 & 34 & 34 & 26 & 36 & 24 & 48 \\ 0 & 0 & 0 & 0 & 0 & 0 & 0 & 0 & 0 & 0 & 0 & 0 & 0 & 0 & 0 \\ 0 & 0 & 0 & 0 & 0 & 0 & 0 & 0 & 0 & 0 & 0 & 0 & 0 & 0 & 0 \\ \mathbf{1 2 2} & \mathbf{1 2 2} & \mathbf{1 5 0} & \mathbf{1 8 8} & \mathbf{2 9 7} & \mathbf{3 6 9} & \mathbf{3 5 5} & \mathbf{3 2 0} & \mathbf{2 8 2} & \mathbf{2 2 7} & \mathbf{2 0 2} & \mathbf{1 0 2} & \mathbf{1 3 0} & \mathbf{1 4 9} & \mathbf{2 2 3}\end{array}$


Table A-6: Regional Small Thermal and Miscellaneous

PNW Loads and Resources Study

2004 - 2005 Operating Year

$3 / 31 / 2004$ 2003 White Book

Average Energy in Megawatts Aug Aug16 Sep Oct Nov Dec Jan Feb Mar Apr1 Apr16 May Jun Jul Avg

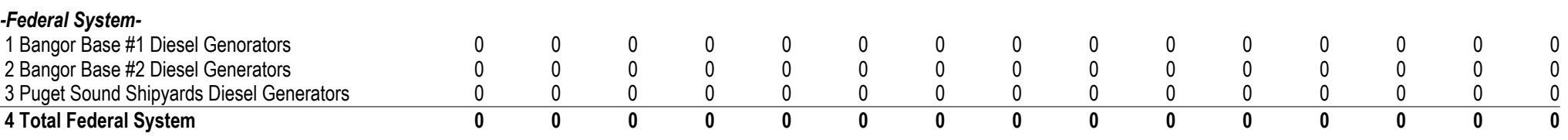

-Public Entities-

5 Total Public Entities

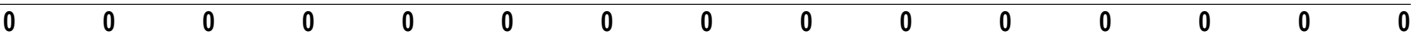

-Investor-Owned Entities-

6 Crystal Mountain

7 Energy Mgmt Systems

8 Total Investor-Owned Entities

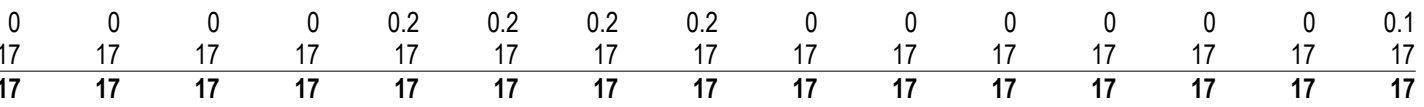

-Other Entities-

9 Total Other Entities

$0 \quad 0$

-Small Thermal and Miscellaneous-

10 Federal System

11 Public Entities

12 Investor-Owned Entities

13 Other Entities

14 Total Small Termal \& Misc.

$\begin{array}{rrrrrrrrrrrrrrr}0 & 0 & 0 & 0 & 0 & 0 & 0 & 0 & 0 & 0 & 0 & 0 & 0 & 0 & 0 \\ 0 & 0 & 0 & 0 & 0 & 0 & 0 & 0 & 0 & 0 & 0 & 0 & 0 & 0 & 0 \\ 17 & 17 & 17 & 17 & 17 & 17 & 17 & 17 & 17 & 17 & 17 & 17 & 17 & 17 & 17 \\ 0 & 0 & 0 & 0 & 0 & 0 & 0 & 0 & 0 & 0 & 0 & 0 & 0 & 0 & 0 \\ 17 & 17 & 17 & 17 & 17 & 17 & 17 & 17 & \mathbf{1 7} & \mathbf{1 7} & \mathbf{1 7} & \mathbf{1 7} & \mathbf{1 7} & \mathbf{1 7} & \mathbf{1 7}\end{array}$


Table A-6: Regional Small Thermal and Miscellaneous

PNW Loads and Resources Study

2005 - 2006 Operating Year

$3 / 31 / 2004$ 2003 White Book

Average Energy in Megawatts Aug Aug16 Sep Oct Nov Dec Jan Feb Mar Apr1 Apr16 May Jun Jul Avg

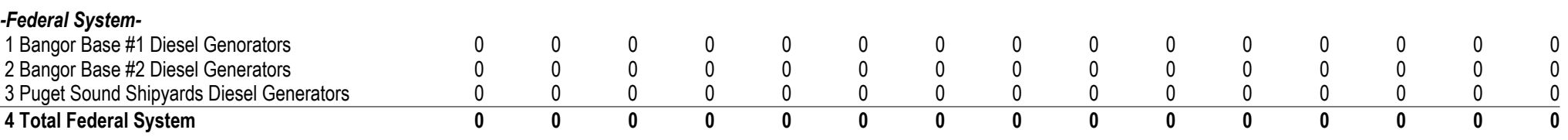

-Public Entities-

5 Total Public Entities

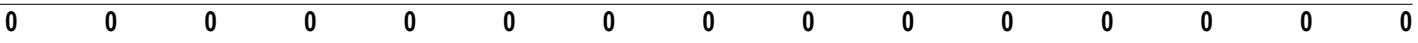

-Investor-Owned Entities-

6 Crystal Mountain

7 Energy Mgmt Systems

8 Total Investor-Owned Entities

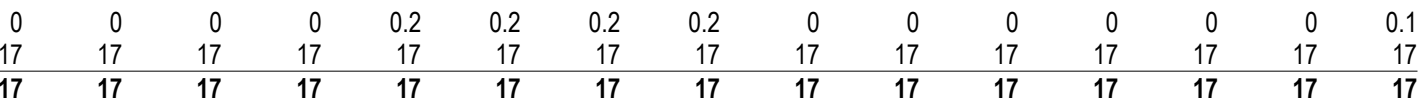

-Other Entities-

9 Total Other Entities

$0 \quad 0$

-Small Thermal and Miscellaneous-

10 Federal System

11 Public Entities

12 Investor-Owned Entities

13 Other Entities

14 Total Small Termal \& Misc.

$\begin{array}{rrrrrrrrrrrrrrr}0 & 0 & 0 & 0 & 0 & 0 & 0 & 0 & 0 & 0 & 0 & 0 & 0 & 0 & 0 \\ 0 & 0 & 0 & 0 & 0 & 0 & 0 & 0 & 0 & 0 & 0 & 0 & 0 & 0 & 0 \\ 17 & 17 & 17 & 17 & 17 & 17 & 17 & 17 & 17 & 17 & 17 & 17 & 17 & 17 & 17 \\ 0 & 0 & 0 & 0 & 0 & 0 & 0 & 0 & 0 & 0 & 0 & 0 & 0 & 0 & 0 \\ 17 & 17 & 17 & 17 & 17 & 17 & 17 & 17 & \mathbf{1 7} & \mathbf{1 7} & \mathbf{1 7} & \mathbf{1 7} & \mathbf{1 7} & \mathbf{1 7} & \mathbf{1 7}\end{array}$


Table A-6: Regional Small Thermal and Miscellaneous

PNW Loads and Resources Study

2006 - 2007 Operating Year

$3 / 31 / 2004$ 2003 White Book

Average Energy in Megawatts Aug Aug16 Sep Oct Nov Dec Jan Feb Mar Apr1 Apr16 May Jun Jul Avg

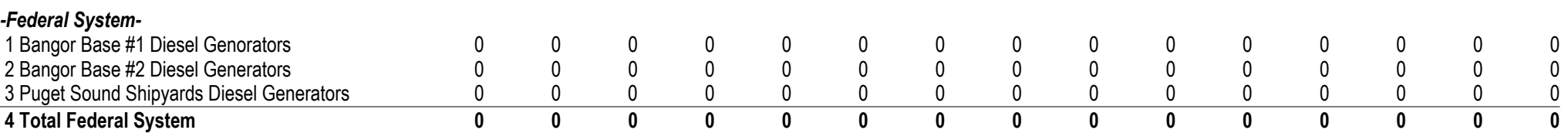

Public Entities-

5 Total Public Entities

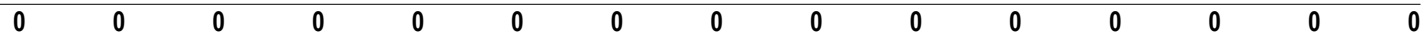

-Investor-Owned Entities-

6 Crystal Mountain

7 Energy Mgmt Systems

8 Total Investor-Owned Entities

\begin{tabular}{rrrrrrrrrrrrrrr}
0 & 0 & 0 & 0 & 0.2 & 0.2 & 0.2 & 0.2 & 0 & 0 & 0 & 0 & 0 & 0 & 0.1 \\
17 & 17 & 17 & 17 & 17 & 17 & 17 & 17 & 17 & 17 & 17 & 17 & 17 & 17 & 17 \\
\hline 17 & 17 & 17 & 17 & 17 & 17 & 17 & 17 & 17 & 17 & 17 & 17 & 17 & 17 & 17
\end{tabular}

-Other Entities-

9 Total Other Entities

$0 \quad 0$

-Small Thermal and Miscellaneous-

10 Federal System

11 Public Entities

12 Investor-Owned Entities

13 Other Entities

14 Total Small Termal \& Misc.

$\begin{array}{rrrrrrrrrrrrrrr}0 & 0 & 0 & 0 & 0 & 0 & 0 & 0 & 0 & 0 & 0 & 0 & 0 & 0 & 0 \\ 0 & 0 & 0 & 0 & 0 & 0 & 0 & 0 & 0 & 0 & 0 & 0 & 0 & 0 & 0 \\ 17 & 17 & 17 & 17 & 17 & 17 & 17 & 17 & 17 & 17 & 17 & 17 & 17 & 17 & 17 \\ 0 & 0 & 0 & 0 & 0 & 0 & 0 & 0 & 0 & 0 & 0 & 0 & 0 & 0 & 0 \\ 17 & 17 & 17 & 17 & 17 & 17 & 17 & 17 & \mathbf{1 7} & \mathbf{1 7} & \mathbf{1 7} & \mathbf{1 7} & \mathbf{1 7} & \mathbf{1 7} & \mathbf{1 7}\end{array}$


Table A-6: Regional Small Thermal and Miscellaneous

PNW Loads and Resources Study

2007 - 2008 Operating Year

$3 / 31 / 2004$ 2003 White Book

Average Energy in Megawatts Aug Aug16 Sep Oct Nov Dec Jan Feb Mar Apr1 Apr16 May Jun Jul Avg

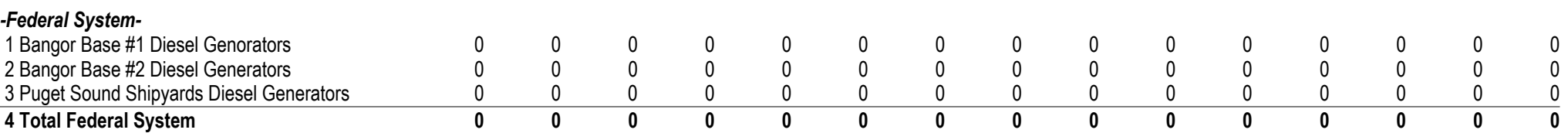

Public Entities-

5 Total Public Entities

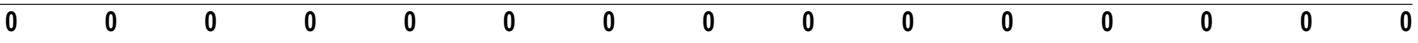

-Investor-Owned Entities-

6 Crystal Mountain

7 Energy Mgmt Systems

8 Total Investor-Owned Entities

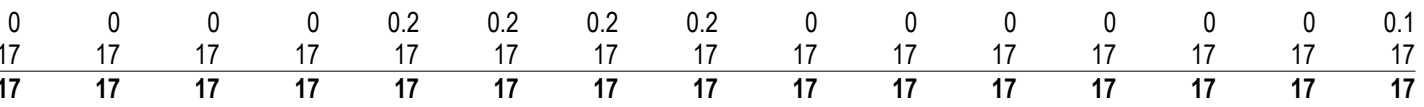

-Other Entities-

9 Total Other Entities

$0 \quad 0$

-Small Thermal and Miscellaneous-

10 Federal System

11 Public Entities

12 Investor-Owned Entities

13 Other Entities

14 Total Small Termal \& Misc.

$\begin{array}{rrrrrrrrrrrrrrr}0 & 0 & 0 & 0 & 0 & 0 & 0 & 0 & 0 & 0 & 0 & 0 & 0 & 0 & 0 \\ 0 & 0 & 0 & 0 & 0 & 0 & 0 & 0 & 0 & 0 & 0 & 0 & 0 & 0 & 0 \\ 17 & 17 & 17 & 17 & 17 & 17 & 17 & 17 & 17 & 17 & 17 & 17 & 17 & 17 & 17 \\ 0 & 0 & 0 & 0 & 0 & 0 & 0 & 0 & 0 & 0 & 0 & 0 & 0 & 0 & 0 \\ 17 & 17 & 17 & 17 & 17 & 17 & 17 & 17 & \mathbf{1 7} & \mathbf{1 7} & \mathbf{1 7} & \mathbf{1 7} & \mathbf{1 7} & \mathbf{1 7} & \mathbf{1 7}\end{array}$


Table A-6: Regional Small Thermal and Miscellaneous

PNW Loads and Resources Study

2008 - 2009 Operating Year

$3 / 31 / 2004$ 2003 White Book

Average Energy in Megawatts Aug Aug16 Sep Oct Nov Dec Jan Feb Mar Apr1 Apr16 May Jun Jul Avg

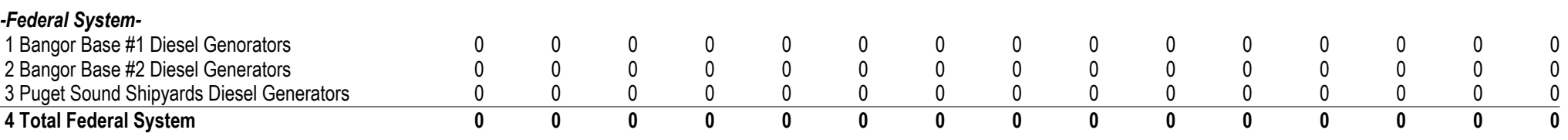

Public Entities-

5 Total Public Entities

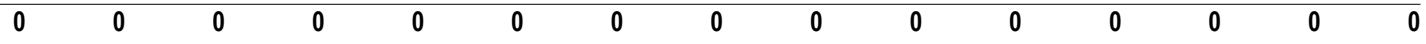

-Investor-Owned Entities-

6 Crystal Mountain

7 Energy Mgmt Systems

8 Total Investor-Owned Entities

\begin{tabular}{rrrrrrrrrrrrrrr}
0 & 0 & 0 & 0 & 0.2 & 0.2 & 0.2 & 0.2 & 0 & 0 & 0 & 0 & 0 & 0 & 0.1 \\
17 & 17 & 17 & 17 & 17 & 17 & 17 & 17 & 17 & 17 & 17 & 17 & 17 & 17 & 17 \\
\hline 17 & 17 & 17 & 17 & 17 & 17 & 17 & 17 & 17 & 17 & 17 & 17 & 17 & 17 & 17
\end{tabular}

-Other Entities-

9 Total Other Entities

$0 \quad 0$

-Small Thermal and Miscellaneous-

10 Federal System

11 Public Entities

12 Investor-Owned Entities

13 Other Entities

14 Total Small Termal \& Misc.

$\begin{array}{rrrrrrrrrrrrrrr}0 & 0 & 0 & 0 & 0 & 0 & 0 & 0 & 0 & 0 & 0 & 0 & 0 & 0 & 0 \\ 0 & 0 & 0 & 0 & 0 & 0 & 0 & 0 & 0 & 0 & 0 & 0 & 0 & 0 & 0 \\ 17 & 17 & 17 & 17 & 17 & 17 & 17 & 17 & 17 & 17 & 17 & 17 & 17 & 17 & 17 \\ 0 & 0 & 0 & 0 & 0 & 0 & 0 & 0 & 0 & 0 & 0 & 0 & 0 & 0 & 0 \\ 17 & 17 & 17 & 17 & 17 & 17 & 17 & 17 & \mathbf{1 7} & \mathbf{1 7} & \mathbf{1 7} & \mathbf{1 7} & \mathbf{1 7} & \mathbf{1 7} & \mathbf{1 7}\end{array}$


Table A-6: Regional Small Thermal and Miscellaneous

PNW Loads and Resources Study

2009 - 2010 Operating Year

$3 / 31 / 2004$ 2003 White Book

Average Energy in Megawatts Aug Aug16 Sep Oct Nov Dec Jan Feb Mar Apr1 Apr16 May Jun Jul Avg

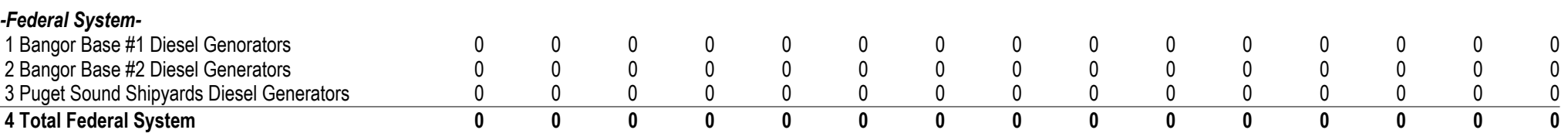

-Public Entities-

5 Total Public Entities

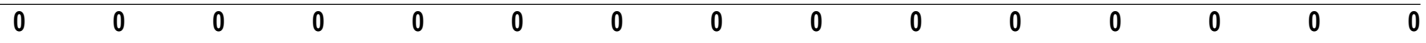

-Investor-Owned Entities-

6 Crystal Mountain

7 Energy Mgmt Systems

8 Total Investor-Owned Entities

\begin{tabular}{rrrrrrrrrrrrrrr}
0 & 0 & 0 & 0 & 0.2 & 0.2 & 0.2 & 0.2 & 0 & 0 & 0 & 0 & 0 & 0 & 0.1 \\
17 & 17 & 17 & 17 & 17 & 17 & 17 & 17 & 17 & 17 & 17 & 17 & 17 & 17 & 17 \\
\hline 17 & 17 & 17 & 17 & 17 & 17 & 17 & 17 & 17 & 17 & 17 & 17 & 17 & 17 & 17
\end{tabular}

-Other Entities-

9 Total Other Entities

$0 \quad 0$

-Small Thermal and Miscellaneous-

10 Federal System

11 Public Entities

12 Investor-Owned Entities

13 Other Entities

14 Total Small Termal \& Misc.

$\begin{array}{rrrrrrrrrrrrrrr}0 & 0 & 0 & 0 & 0 & 0 & 0 & 0 & 0 & 0 & 0 & 0 & 0 & 0 & 0 \\ 0 & 0 & 0 & 0 & 0 & 0 & 0 & 0 & 0 & 0 & 0 & 0 & 0 & 0 & 0 \\ 17 & 17 & 17 & 17 & 17 & 17 & 17 & 17 & 17 & 17 & 17 & 17 & 17 & 17 & 17 \\ 0 & 0 & 0 & 0 & 0 & 0 & 0 & 0 & 0 & 0 & 0 & 0 & 0 & 0 & 0 \\ 17 & 17 & 17 & 17 & 17 & 17 & 17 & 17 & \mathbf{1 7} & \mathbf{1 7} & \mathbf{1 7} & \mathbf{1 7} & \mathbf{1 7} & \mathbf{1 7} & \mathbf{1 7}\end{array}$


Table A-6: Regional Small Thermal and Miscellaneous

PNW Loads and Resources Study

2010 - 2011 Operating Year

$3 / 31 / 2004$ 2003 White Book

Average Energy in Megawatts Aug Aug16 Sep Oct Nov Dec Jan Feb Mar Apr1 Apr16 May Jun Jul Avg

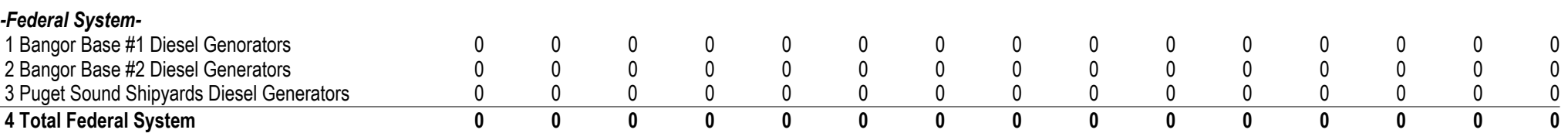

Public Entities-

5 Total Public Entities

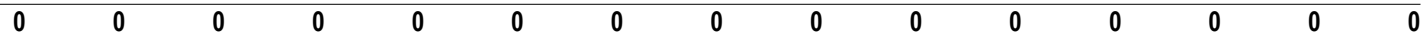

-Investor-Owned Entities-

6 Crystal Mountain

7 Energy Mgmt Systems

8 Total Investor-Owned Entities

\begin{tabular}{rrrrrrrrrrrrrrr}
0 & 0 & 0 & 0 & 0.2 & 0.2 & 0.2 & 0.2 & 0 & 0 & 0 & 0 & 0 & 0 & 0.1 \\
17 & 17 & 17 & 17 & 17 & 17 & 17 & 17 & 17 & 17 & 17 & 17 & 17 & 17 & 17 \\
\hline 17 & 17 & 17 & 17 & 17 & 17 & 17 & 17 & 17 & 17 & 17 & 17 & 17 & 17 & 17
\end{tabular}

-Other Entities-

9 Total Other Entities

$0 \quad 0$

-Small Thermal and Miscellaneous-

10 Federal System

11 Public Entities

12 Investor-Owned Entities

13 Other Entities

14 Total Small Termal \& Misc.

$\begin{array}{rrrrrrrrrrrrrrr}0 & 0 & 0 & 0 & 0 & 0 & 0 & 0 & 0 & 0 & 0 & 0 & 0 & 0 & 0 \\ 0 & 0 & 0 & 0 & 0 & 0 & 0 & 0 & 0 & 0 & 0 & 0 & 0 & 0 & 0 \\ 17 & 17 & 17 & 17 & 17 & 17 & 17 & 17 & 17 & 17 & 17 & 17 & 17 & 17 & 17 \\ 0 & 0 & 0 & 0 & 0 & 0 & 0 & 0 & 0 & 0 & 0 & 0 & 0 & 0 & 0 \\ 17 & 17 & 17 & 17 & 17 & 17 & 17 & 17 & \mathbf{1 7} & \mathbf{1 7} & \mathbf{1 7} & \mathbf{1 7} & \mathbf{1 7} & \mathbf{1 7} & \mathbf{1 7}\end{array}$


Table A-6: Regional Small Thermal and Miscellaneous

PNW Loads and Resources Study

2011 - 2012 Operating Year

$3 / 31 / 2004$ 2003 White Book

Average Energy in Megawatts Aug Aug16 Sep Oct Nov Dec Jan Feb Mar Apr1 Apr16 May Jun Jul Avg

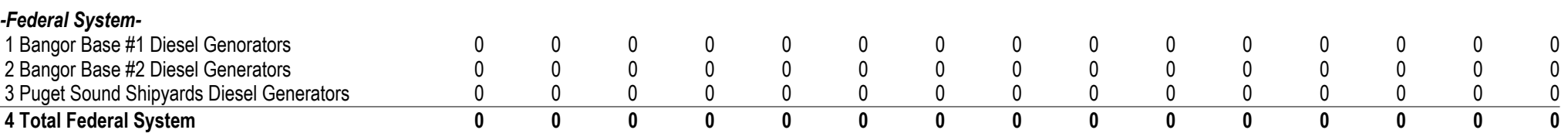

Public Entities-

5 Total Public Entities

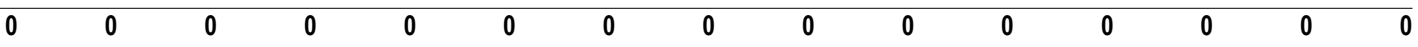

-Investor-Owned Entities-

6 Crystal Mountain

7 Energy Mgmt Systems

8 Total Investor-Owned Entities

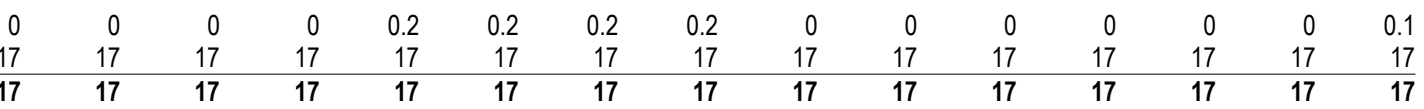

-Other Entities-

9 Total Other Entities

$0 \quad 0$

-Small Thermal and Miscellaneous-

10 Federal System

11 Public Entities

12 Investor-Owned Entities

13 Other Entities

14 Total Small Termal \& Misc.

$\begin{array}{rrrrrrrrrrrrrrr}0 & 0 & 0 & 0 & 0 & 0 & 0 & 0 & 0 & 0 & 0 & 0 & 0 & 0 & 0 \\ 0 & 0 & 0 & 0 & 0 & 0 & 0 & 0 & 0 & 0 & 0 & 0 & 0 & 0 & 0 \\ 17 & 17 & 17 & 17 & 17 & 17 & 17 & 17 & 17 & 17 & 17 & 17 & 17 & 17 & 17 \\ 0 & 0 & 0 & 0 & 0 & 0 & 0 & 0 & 0 & 0 & 0 & 0 & 0 & 0 & 0 \\ 17 & 17 & 17 & 17 & 17 & 17 & 17 & 17 & \mathbf{1 7} & \mathbf{1 7} & \mathbf{1 7} & \mathbf{1 7} & \mathbf{1 7} & \mathbf{1 7} & \mathbf{1 7}\end{array}$


Table A-6: Regional Small Thermal and Miscellaneous

PNW Loads and Resources Study

2012 - 2013 Operating Year

$3 / 31 / 2004$ 2003 White Book

Average Energy in Megawatts Aug Aug16 Sep Oct Nov Dec Jan Feb Mar Apr1 Apr16 May Jun Jul Avg

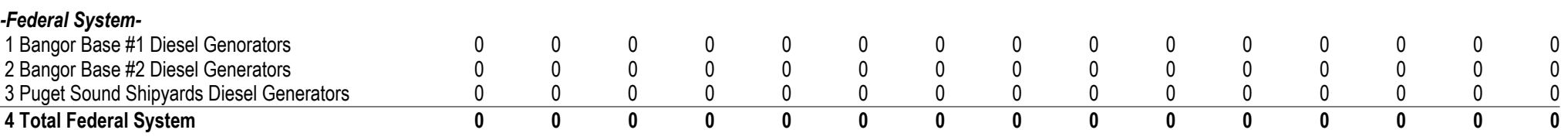

Public Entities-

5 Total Public Entities

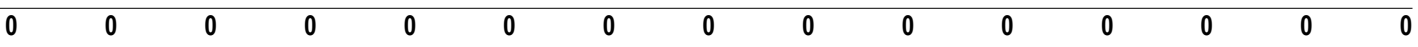

-Investor-Owned Entities-

6 Crystal Mountain

7 Energy Mgmt Systems

8 Total Investor-Owned Entities

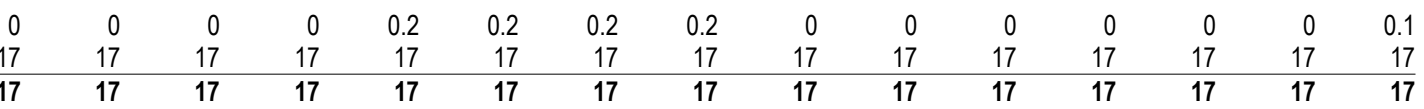

-Other Entities-

9 Total Other Entities

$0 \quad 0$

-Small Thermal and Miscellaneous-

10 Federal System

11 Public Entities

12 Investor-Owned Entities

13 Other Entities

14 Total Small Termal \& Misc.

$\begin{array}{rrrrrrrrrrrrrrr}0 & 0 & 0 & 0 & 0 & 0 & 0 & 0 & 0 & 0 & 0 & 0 & 0 & 0 & 0 \\ 0 & 0 & 0 & 0 & 0 & 0 & 0 & 0 & 0 & 0 & 0 & 0 & 0 & 0 & 0 \\ 17 & 17 & 17 & 17 & 17 & 17 & 17 & 17 & 17 & 17 & 17 & 17 & 17 & 17 & 17 \\ 0 & 0 & 0 & 0 & 0 & 0 & 0 & 0 & 0 & 0 & 0 & 0 & 0 & 0 & 0 \\ 17 & 17 & 17 & 17 & 17 & 17 & 17 & 17 & \mathbf{1 7} & \mathbf{1 7} & \mathbf{1 7} & \mathbf{1 7} & \mathbf{1 7} & \mathbf{1 7} & \mathbf{1 7}\end{array}$


Table A-6: Regional Small Thermal and Miscellaneous

PNW Loads and Resources Study

2013 - 2014 Operating Year

$3 / 31 / 2004$ 2003 White Book

Average Energy in Megawatts Aug Aug16 Sep Oct Nov Dec Jan Feb Mar Apr1 Apr16 May Jun Jul Avg

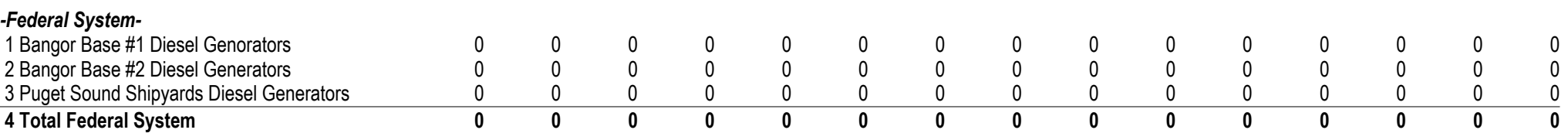

Public Entities-

5 Total Public Entities

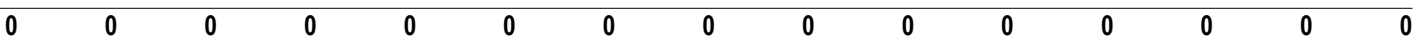

-Investor-Owned Entities-

6 Crystal Mountain

7 Energy Mgmt Systems

8 Total Investor-Owned Entities

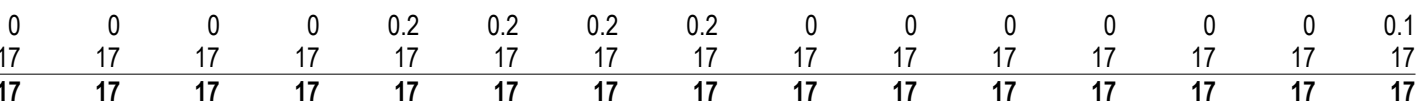

-Other Entities-

9 Total Other Entities

$0 \quad 0$

-Small Thermal and Miscellaneous-

10 Federal System

11 Public Entities

12 Investor-Owned Entities

13 Other Entities

14 Total Small Termal \& Misc.

$\begin{array}{rrrrrrrrrrrrrrr}0 & 0 & 0 & 0 & 0 & 0 & 0 & 0 & 0 & 0 & 0 & 0 & 0 & 0 & 0 \\ 0 & 0 & 0 & 0 & 0 & 0 & 0 & 0 & 0 & 0 & 0 & 0 & 0 & 0 & 0 \\ 17 & 17 & 17 & 17 & 17 & 17 & 17 & 17 & 17 & 17 & 17 & 17 & 17 & 17 & 17 \\ 0 & 0 & 0 & 0 & 0 & 0 & 0 & 0 & 0 & 0 & 0 & 0 & 0 & 0 & 0 \\ 17 & 17 & 17 & 17 & 17 & 17 & 17 & 17 & \mathbf{1 7} & \mathbf{1 7} & \mathbf{1 7} & \mathbf{1 7} & \mathbf{1 7} & \mathbf{1 7} & \mathbf{1 7}\end{array}$


Table A-7: Regional Combustion Turbines

PNW Loads and Resources Study

2004 - 2005 Operating Year

2003 White Book

Aug1 Aug16

Sep Oct Nov

Dec Jan Fe

Mar Apr1 Apr16 May Jun Jul Avg

-Federal System-

1 Total Federal System

-Public Entities-

2 Benton CT

3 Frederickson CT

4 Hoquiam Diesels

5 Olympic View Generating Station

6 Pasco Harbor Generating Station

7 Randolph Road Diesel Farm

8 River Road

9 Roosevelt Landfill

10 Springfield Gen Farm

11 Wauna Peaking Project

12 Total Public Entities

$0 \quad 0 \quad 0$

0

0

$\begin{array}{rrrrrrrrrrrrrrr}0 & 0 & 0 & 1 & 1.5 & 2.5 & 3 & 2 & 0 & 0 & 0 & 0 & 0 & 0 & 0.8 \\ 125 & 125 & 125 & 125 & 125 & 125 & 125 & 0 & 0 & 0 & 0 & 0 & 0 & 125 & 73 \\ 0 & 0 & 0 & 0 & 0 & 0 & 0 & 0 & 0 & 0 & 0 & 0 & 0 & 0 & 0 \\ 0 & 0 & 0 & 0 & 0 & 0 & 0 & 0 & 0 & 0 & 0 & 0 & 0 & 0 & 0 \\ 4.4 & 4.4 & 4.4 & 4.4 & 4.4 & 4.4 & 4.4 & 4.4 & 4.4 & 4.4 & 4.4 & 0 & 4.4 & 4.4 & 4 \\ 0 & 0 & 0 & 0 & 0 & 0 & 0 & 0 & 0 & 0 & 0 & 0 & 0 & 0 & 0 \\ 233 & 233 & 236 & 241 & 243 & 246 & 248 & 246 & 243 & 244 & 244 & 0 & 242 & 233 & 221 \\ 8 & 8 & 8 & 8 & 8 & 8 & 8 & 8 & 8 & 8 & 8 & 8 & 8 & 8 & 8 \\ 0 & 0 & 0 & 0 & 0 & 0 & 0 & 0 & 0 & 0 & 0 & 0 & 0 & 0 & 0 \\ 2.5 & 2.5 & 2.5 & 2.5 & 2.5 & 2.5 & 2.5 & 2.5 & 2.5 & 2.5 & 2.5 & 0 & 2.5 & 2.5 & 2.3 \\ 373 & 373 & 376 & 382 & 385 & 388 & 391 & \mathbf{2 6 2} & \mathbf{2 5 8} & \mathbf{2 5 9} & \mathbf{2 5 9} & \mathbf{8} & \mathbf{2 5 7} & \mathbf{3 7 3} & \mathbf{3 0 9}\end{array}$

-Investor-Owned Entities-

13 Basin Creek Project

14 Beaver

15 Beaver 8

16 Big Hanaford CCCT

17 Boulder Park

18 Danskin

19 Encogen

20 Frederickson CT

21 Fredonia $1 \& 2$

22 Fredonia $3 \& 4$

23 Fredrickson $1 \& 2$

24 Kettle Falls CT

25 Northeastern $1 \& 2$

26 Whitehorn 2 \& 3

27 Total Investor-Owned Entities

-Other Enities-

28 Chehalis Generation Facility

29 Frederickson CT

30 Klamath Peaking Unit

31 Lancaster Power Project (Rathdrum)

32 Total Other Enities

\begin{tabular}{|c|c|c|c|c|c|c|c|c|c|c|c|c|c|c|}
\hline 0 & 0 & 0 & 4 & 4 & 4 & 4 & 4 & 4 & 4 & 4 & 4 & 4 & 4 & 3.3 \\
\hline 459 & 459 & 453 & 475 & 471 & 435 & 478 & 488 & 362 & 423 & 423 & 472 & 466 & 459 & 453 \\
\hline 0 & 0 & 0 & 0 & 0 & 0 & 0 & 0 & 0 & 0 & 0 & 0 & 0 & 0 & 0 \\
\hline 244 & 244 & 244 & 244 & 244 & 244 & 244 & 244 & 244 & 244 & 244 & 0 & 244 & 244 & 224 \\
\hline 4 & 4 & 4 & 4 & 4 & 4 & 4 & 4 & 4 & 4 & 4 & 4 & 4 & 4 & 4 \\
\hline 140 & 140 & 159 & 115 & 83 & 69 & 66 & 66 & 58 & 64 & 64 & 53 & 50 & 100 & 85 \\
\hline 125 & 125 & 125 & 125 & 125 & 125 & 125 & 0 & 0 & 0 & 0 & 0 & 0 & 125 & 73 \\
\hline 44 & 44 & 82 & 25 & 0 & 0 & 7 & 0 & 0 & 0 & 0 & 0 & 0 & 5 & 14 \\
\hline 0 & 0 & 9 & 0 & 0 & 0 & 0 & 0 & 0 & 0 & 0 & 0 & 0 & 0 & 0.8 \\
\hline 7.2 & 7.2 & 7.2 & 7.2 & 7.2 & 7.2 & 7.2 & 7.2 & 7.2 & 7.2 & 7.2 & 3.4 & 7.2 & 7.2 & 6.9 \\
\hline 48 & 48 & 48 & 48 & 48 & 48 & 48 & 48 & 48 & 48 & 48 & 0 & 48 & 48 & 44 \\
\hline 0 & 0 & 0 & 0 & 0 & 0 & 0 & 0 & 0 & 0 & 0 & 0 & 0 & 0 & 0 \\
\hline
\end{tabular}

-Combustion Turbines-

33 Federal System

34 Public Entities

35 Investor-Owned Entities

36 Other Entities

37 Total Combustion Turbines

\begin{tabular}{|c|c|c|c|c|c|c|c|c|c|c|c|c|c|}
\hline 455 & 455 & 455 & 455 & 455 & 455 & 455 & 455 & 455 & 455 & 455 & 0 & 455 & 417 \\
\hline 0.4 & 0.4 & 0.4 & 0.4 & 0.4 & 0.4 & 0 & 0 & 0 & 0 & 0 & 0 & 0.4 & 0.2 \\
\hline 15 & 15 & 15 & 20 & 25 & 25 & 25 & 15 & 0 & 0 & 0 & 0 & 15 & 14 \\
\hline 267 & 267 & 267 & 267 & 267 & 267 & 267 & 267 & 0 & 0 & 267 & 267 & 267 & 244 \\
\hline
\end{tabular}

\begin{tabular}{lrrrrrrrrrrrrrrrr} 
& 0 & 0 & 0 & 0 & 0 & 0 & 0 & 0 & 0 & 0 & 0 & 0 & 0 & 0 & 0 & 25 \\
& 373 & 373 & 376 & 382 & 385 & 388 & 391 & 262 & 258 & 259 & 259 & 8 & 257 & 373 & 309 \\
& 1096 & 1096 & 1156 & 1072 & 1011 & 984 & 1115 & 993 & 847 & 881 & 828 & 536 & 848 & 1021 & 961 \\
& 737 & 737 & 737 & 737 & 742 & 747 & 747 & 746 & 736 & 455 & 455 & 721 & 267 & 737 & 676 \\
\hline Turbines & $\mathbf{2 2 0 6}$ & $\mathbf{2 2 0 6}$ & $\mathbf{2 2 6 9}$ & $\mathbf{2 1 9 1}$ & $\mathbf{2 1 3 7}$ & $\mathbf{2 1 1 9}$ & $\mathbf{2 2 5 2}$ & $\mathbf{2 0 0 2}$ & $\mathbf{1 8 4 1}$ & $\mathbf{1 5 9 4}$ & $\mathbf{1 5 4 1}$ & $\mathbf{1 2 6 6}$ & $\mathbf{1 3 7 2}$ & $\mathbf{2 1 3 1}$ & $\mathbf{1 9 4 6}$
\end{tabular}


Table A-7: Regional Combustion Turbines

PNW Loads and Resources Study

2005 - 2006 Operating Year 2003 White Book

$\begin{array}{llll}0 & 0 & 0 & 0\end{array}$

-Public Entities-

2 Benton CT

3 Frederickson CT

4 Hoquiam Diesels

5 Olympic View Generating Station

6 Pasco Harbor Generating Station

7 Randolph Road Diesel Farm

8 River Road

9 Roosevelt Landfill

10 Springfield Gen Farm

11 Wauna Peaking Project

12 Total Public Entities

\begin{tabular}{|c|c|c|c|c|c|c|c|c|c|c|c|c|c|}
\hline 0 & 0 & 1 & 1.5 & 2.5 & 3 & 2 & 0 & 0 & 0 & 0 & 0 & 0 & 0.8 \\
\hline 125 & 125 & 125 & 125 & 125 & 125 & 0 & 0 & 0 & 0 & 0 & 0 & 125 & 73 \\
\hline 0 & 0 & 0 & 0 & 0 & 0 & 0 & 0 & 0 & 0 & 0 & 0 & 0 & 0 \\
\hline 0 & 0 & 0 & 0 & 0 & 0 & 0 & 0 & 0 & 0 & 0 & 0 & 0 & 0 \\
\hline 4.4 & 4.4 & 4.4 & 4.4 & 4.4 & 4.4 & 4.4 & 4.4 & 4.4 & 4.4 & 0 & 4.4 & 4.4 & 4 \\
\hline 0 & 0 & 0 & 0 & 0 & 0 & 0 & 0 & 0 & 0 & 0 & 0 & 0 & 0 \\
\hline 233 & 236 & 241 & 243 & 246 & 248 & 246 & 243 & 244 & 244 & 165 & 242 & 233 & 235 \\
\hline 8 & 8 & 8 & 8 & 8 & 8 & 8 & 8 & 8 & 8 & 8 & 8 & 8 & 8 \\
\hline 0 & 0 & 0 & 0 & 0 & 0 & 0 & 0 & 0 & 0 & 0 & 0 & 0 & 0 \\
\hline 2.5 & 2.5 & 2.5 & 2.5 & 2.5 & 2.5 & 2.5 & 2.5 & 2.5 & 2.5 & 0 & 2.5 & 2.5 & 2.3 \\
\hline 373 & 376 & 2 & 5 & 8 & 1 & 2 & 88 & 9 & 9 & 3 & 7 & 373 & 323 \\
\hline
\end{tabular}

-Investor-Owned Entities-

13 Basin Creek Project

14 Beaver

15 Beaver 8

16 Big Hanaford CCCT

17 Boulder Park

18 Danskin

19 Encogen

20 Frederickson $\mathrm{CT}$

21 Fredonia $1 \& 2$

22 Fredonia $3 \& 4$

23 Fredrickson $1 \& 2$

24 Kettle Falls CT

25 Northeastern $1 \& 2$

26 Whitehorn $2 \& 3$

27 Total Investor-Owned Entities

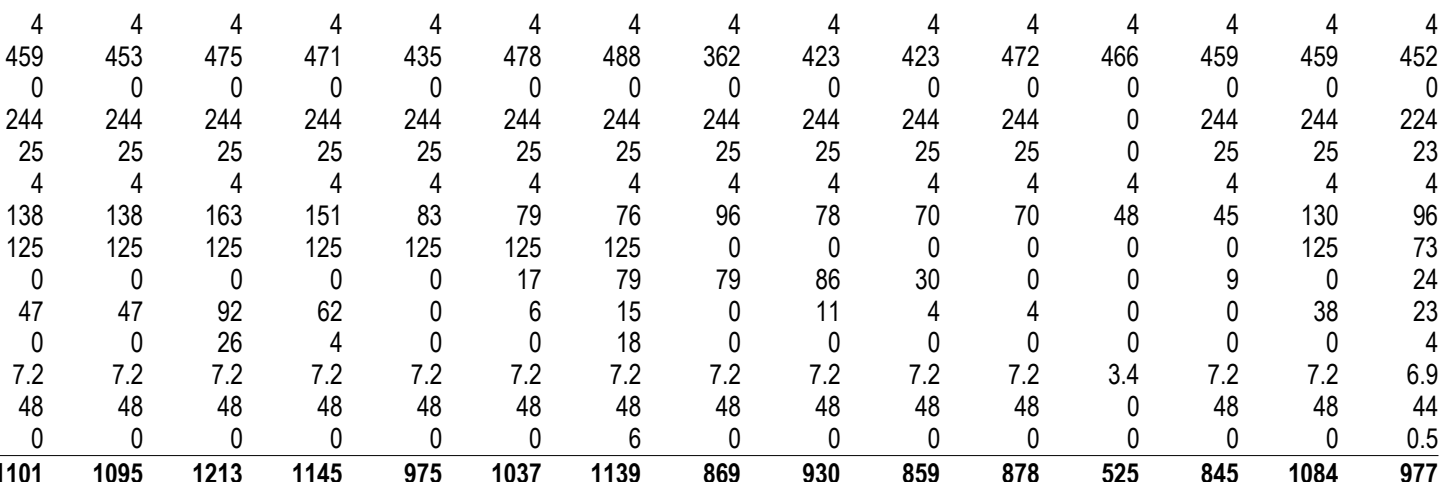

-Other Enities-

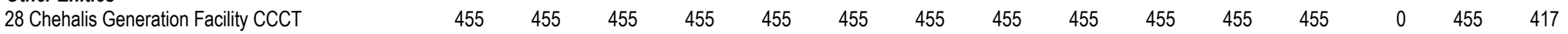

29 Frederickson CT

30 Klamath Peaking Unit

31 Lancaster Power Project (Rathdrum)

32 Total Other Enities

\begin{tabular}{rrrrrrrrrrrrrrr}
0.4 & 0.4 & 0.4 & 0.4 & 0.4 & 0.4 & 0.4 & 0 & 0 & 0 & 0 & 0 & 0 & 0.4 & 0.2 \\
15 & 15 & 15 & 15 & 20 & 25 & 25 & 25 & 15 & 0 & 0 & 0 & 0 & 15 & 14 \\
267 & 267 & 267 & 267 & 267 & 267 & 267 & 267 & 267 & 0 & 0 & 267 & 267 & 267 & 244 \\
\hline 737 & 737 & 737 & 737 & 742 & $\mathbf{7 4 7}$ & $\mathbf{7 4 7}$ & $\mathbf{7 4 6}$ & $\mathbf{7 3 6}$ & $\mathbf{4 5 5}$ & $\mathbf{4 5 5}$ & $\mathbf{7 2 1}$ & $\mathbf{2 6 7}$ & $\mathbf{7 3 7}$ & $\mathbf{6 7 6}$
\end{tabular}

-Combustion Turbines-

33 Federal System

34 Public Entities

35 Investor-Owned Entities

36 Other Entities

\begin{tabular}{|c|c|c|c|c|c|c|c|c|c|c|c|c|c|}
\hline 0 & 0 & 0 & 0 & 0 & 0 & 0 & 0 & 0 & 0 & 0 & 0 & 0 & 0 \\
\hline 373 & 376 & 382 & 385 & 388 & 391 & 262 & 258 & 259 & 259 & 173 & 257 & 373 & 323 \\
\hline 1095 & 1213 & 1145 & 975 & 1037 & 1139 & 869 & 930 & 859 & 878 & 525 & 845 & 1084 & 977 \\
\hline 737 & 737 & 737 & 742 & 747 & 747 & 746 & 736 & 455 & 455 & 721 & 267 & 737 & 676 \\
\hline 2205 & 2326 & 2264 & 21 & 2172 & 2276 & 1878 & 1924 & 1572 & 1591 & 1419 & 1369 & 2194 & 1976 \\
\hline
\end{tabular}


Table A-7: Regional Combustion Turbines

PNW Loads and Resources Study

2006 - 2007 Operating Year 2003 White Book

Aug1 Aug16 Sep Oct Nov Dec Jan Feb Mar Apr1 Apr16

May Jun Jul Avg

-Federal System-

1 Total Federal System

$\begin{array}{lll}0 & 0 & 0\end{array}$

-Public Entities-

2 Benton CT

3 Frederickson CT

4 Hoquiam Diesels

5 Olympic View Generating Station

6 Pasco Harbor Generating Station

7 Randolph Road Diesel Farm

8 River Road

9 Roosevelt Landfill

10 Springfield Gen Farm

11 Wauna Peaking Project

12 Total Public Entities

\begin{tabular}{|c|c|c|c|c|c|c|c|c|c|c|c|c|c|c|}
\hline 0 & 0 & 0 & 1 & 1.5 & 2.5 & 3 & 2 & 0 & 0 & 0 & 0 & 0 & 0 & 0.8 \\
\hline 125 & 125 & 125 & 125 & 125 & 125 & 125 & 0 & 0 & 0 & 0 & 0 & 0 & 125 & 73 \\
\hline 0 & 0 & 0 & 0 & 0 & 0 & 0 & 0 & 0 & 0 & 0 & 0 & 0 & 0 & 0 \\
\hline 0 & 0 & 0 & 0 & 0 & 0 & 0 & 0 & 0 & 0 & 0 & 0 & 0 & 0 & 0 \\
\hline 4.4 & 4.4 & 4.4 & 4.4 & 4.4 & 4.4 & 4.4 & 4.4 & 4.4 & 4.4 & 4.4 & 0 & 4.4 & 4.4 & 4 \\
\hline 0 & 0 & 0 & 0 & 0 & 0 & 0 & 0 & 0 & 0 & 0 & 0 & 0 & 0 & 0 \\
\hline 233 & 233 & 236 & 180 & 180 & 180 & 180 & 180 & 180 & 180 & 180 & 165 & 180 & 180 & 188 \\
\hline 8 & 8 & 8 & 8 & 8 & 8 & 8 & 8 & 8 & 8 & 8 & 8 & 8 & 8 & 8 \\
\hline 0 & 0 & 0 & 0 & 0 & 0 & 0 & 0 & 0 & 0 & 0 & 0 & 0 & 0 & 0 \\
\hline 2.5 & 2.5 & 2.5 & 2.5 & 2.5 & 2.5 & 2.5 & 2.5 & 2.5 & 2.5 & 2.5 & 0 & 2.5 & 2.5 & 2.3 \\
\hline 73 & 37 & 376 & 321 & 2 & 3 & 323 & 197 & 5 & 195 & 5 & 173 & 195 & 320 & 276 \\
\hline
\end{tabular}

-Investor-Owned Entities-

13 Basin Creek Project

14 Beaver

15 Beaver 8

16 Big Hanaford CCCT

17 Boulder Park

18 Danskin

19 Encogen

20 Frederickson $\mathrm{CT}$

21 Fredonia $1 \& 2$

22 Fredonia $3 \& 4$

23 Fredrickson $1 \& 2$

24 Kettle Falls CT

25 Northeastern $1 \& 2$

26 Whitehorn $2 \& 3$

27 Total Investor-Owned Entities

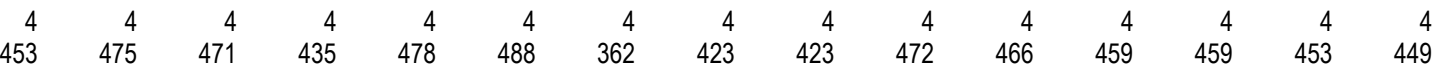

-Other Enities-

28 Chehalis Generation Facility $\mathrm{CCC}$

29 Frederickson CT

30 Klamath Peaking Unit

31 Lancaster Power Project (Rathdrum)

32 Total Other Enities

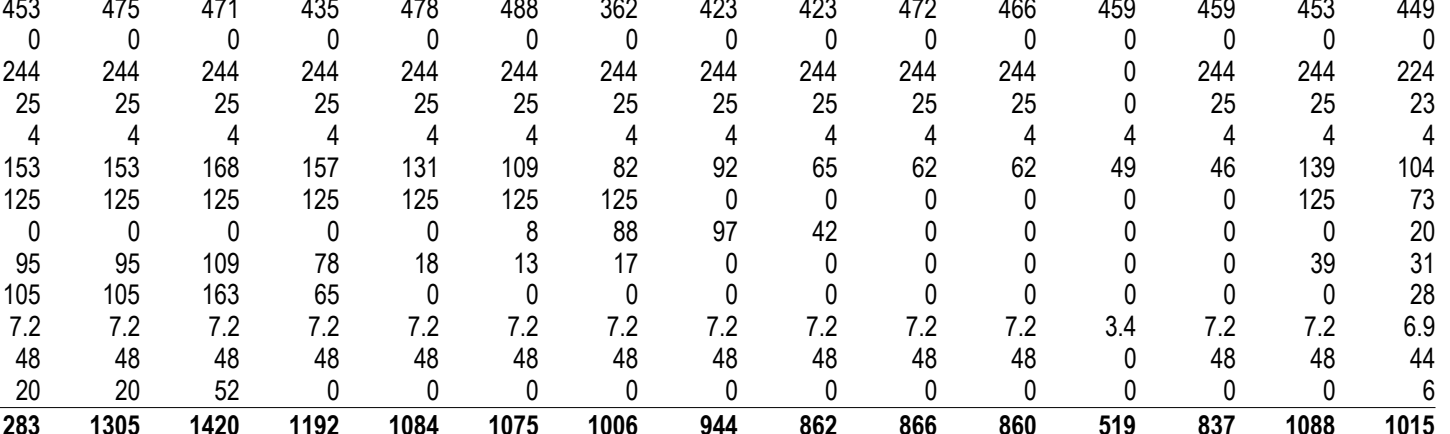

-Combustion Turbines-

33 Federal System

34 Public Entities

35 Investor-Owned Entities

36 Other Entities

\begin{tabular}{|c|c|c|c|c|c|c|c|c|c|c|c|c|c|}
\hline 455 & 455 & 455 & 455 & 455 & 455 & 455 & 455 & 455 & 455 & 455 & 0 & 455 & 417 \\
\hline 0.4 & 0.4 & 0.4 & 0.4 & 0.4 & 0.4 & 0 & 0 & 0 & 0 & 0 & 0 & 0.4 & 0.2 \\
\hline 15 & 15 & 15 & 20 & 25 & 25 & 25 & 15 & 0 & 0 & 0 & 0 & 15 & 14 \\
\hline 267 & 267 & 267 & 267 & 267 & 267 & 267 & 267 & 0 & 0 & 267 & 267 & 267 & 244 \\
\hline 737 & 737 & 737 & 742 & 747 & 747 & 746 & 736 & 455 & 455 & 721 & 267 & 737 & 676 \\
\hline
\end{tabular}

37 Total Combustion Turbines

\begin{tabular}{|c|c|c|c|c|c|c|c|c|c|c|c|c|c|}
\hline 0 & 0 & 0 & 0 & 0 & 0 & 0 & 0 & 0 & 0 & 0 & 0 & 0 & 0 \\
\hline 373 & 376 & 321 & 322 & 323 & 323 & 197 & 195 & 195 & 195 & 173 & 195 & 320 & 276 \\
\hline 1305 & 1420 & 1192 & 1084 & 1075 & 1006 & 944 & 862 & 866 & 860 & 519 & 837 & 1088 & 1015 \\
\hline 737 & 737 & 737 & 742 & 747 & 747 & 746 & 736 & 455 & 455 & 721 & 267 & 737 & 676 \\
\hline 241 & 2533 & 2250 & 2147 & 2144 & 2076 & 1887 & 1793 & 1516 & 1510 & 1413 & 1299 & 2145 & 1967 \\
\hline
\end{tabular}


Table A-7: Regional Combustion Turbines

PNW Loads and Resources Study

2007 - 2008 Operating Year 2003 White Book

Aug1 Aug16 Sep Oct Nov Dec Jan Feb Mar Apr1 Apr16

May Jun Jul Avg

-Federal System-

1 Total Federal System

$\begin{array}{lll}0 & 0 & 0\end{array}$

-Public Entities-

2 Benton CT

3 Frederickson CT

4 Hoquiam Diesels

5 Olympic View Generating Station

6 Pasco Harbor Generating Station

7 Randolph Road Diesel Farm

8 River Road

9 Roosevelt Landfill

10 Springfield Gen Farm

11 Wauna Peaking Project

12 Total Public Entities

\begin{tabular}{|c|c|c|c|c|c|c|c|c|c|c|c|c|c|c|}
\hline 0 & 0 & 0 & 1 & 1.5 & 2.5 & 3 & 2 & 0 & 0 & 0 & 0 & 0 & 0 & 0.8 \\
\hline 125 & 125 & 125 & 125 & 125 & 125 & 125 & 0 & 0 & 0 & 0 & 0 & 0 & 125 & 73 \\
\hline 0 & 0 & 0 & 0 & 0 & 0 & 0 & 0 & 0 & 0 & 0 & 0 & 0 & 0 & 0 \\
\hline 0 & 0 & 0 & 0 & 0 & 0 & 0 & 0 & 0 & 0 & 0 & 0 & 0 & 0 & 0 \\
\hline 4.4 & 4.4 & 4.4 & 4.4 & 4.4 & 4.4 & 4.4 & 4.4 & 4.4 & 4.4 & 4.4 & 0 & 4.4 & 4.4 & 4 \\
\hline 0 & 0 & 0 & 0 & 0 & 0 & 0 & 0 & 0 & 0 & 0 & 0 & 0 & 0 & 0 \\
\hline 180 & 180 & 180 & 180 & 180 & 180 & 180 & 180 & 180 & 180 & 180 & 78 & 180 & 180 & 172 \\
\hline 8 & 8 & 8 & 8 & 8 & 8 & 8 & 8 & 8 & 8 & 8 & 8 & 8 & 8 & 8 \\
\hline 0 & 0 & 0 & 0 & 0 & 0 & 0 & 0 & 0 & 0 & 0 & 0 & 0 & 0 & 0 \\
\hline 2.5 & 2.5 & 2.5 & 2.5 & 2.5 & 2.5 & 2.5 & 2.5 & 2.5 & 2.5 & 2.5 & 0 & 2.5 & 2.5 & 2.3 \\
\hline 20 & 320 & 320 & 321 & 322 & 3 & 323 & 197 & 5 & 195 & 5 & 6 & 195 & 320 & 260 \\
\hline
\end{tabular}

-Investor-Owned Entities-

13 Basin Creek Project

14 Beaver

15 Beaver 8

16 Big Hanaford CCCT

17 Boulder Park

18 Danskin

19 Encogen

20 Frederickson $\mathrm{CT}$

21 Fredonia $1 \& 2$

22 Fredonia $3 \& 4$

23 Fredrickson $1 \& 2$

24 Kettle Falls CT

25 Northeastern $1 \& 2$

26 Whitehorn $2 \& 3$

27 Total Investor-Owned Entities

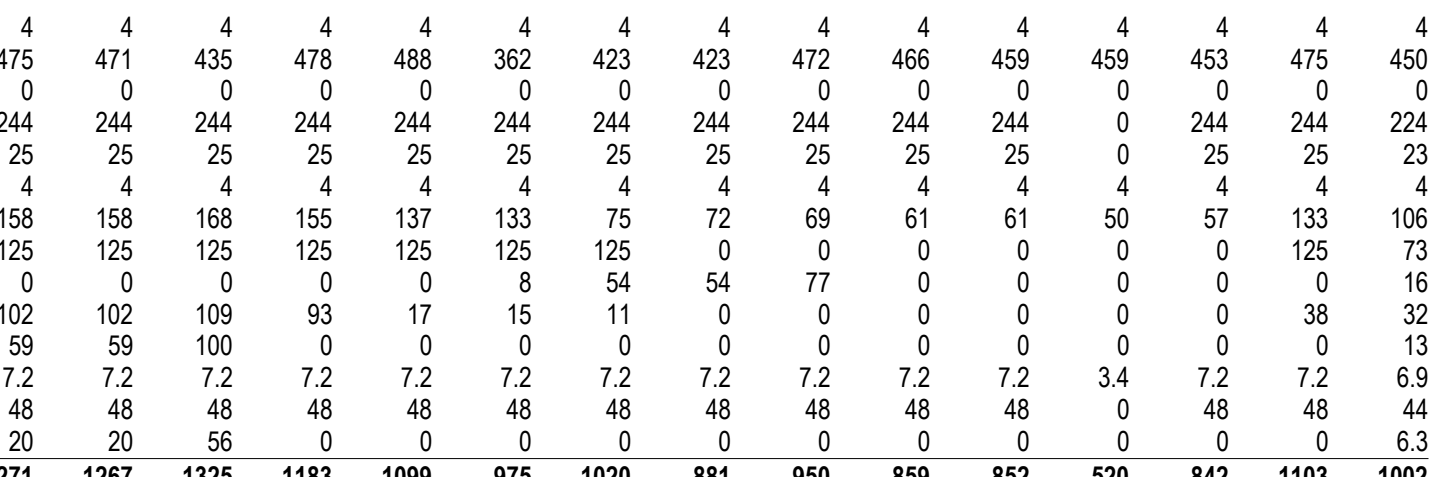

-Other Enities-

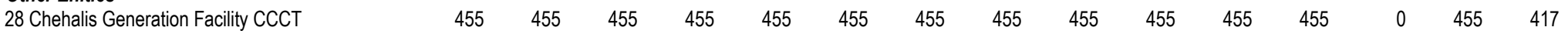

29 Frederickson CT

30 Klamath Peaking Unit

31 Lancaster Power Project (Rathdrum)

32 Total Other Enities

\begin{tabular}{rrrrrrrrrrrrrrr}
0.4 & 0.4 & 0.4 & 0.4 & 0.4 & 0.4 & 0.4 & 0 & 0 & 0 & 0 & 0 & 0 & 0.4 & 0.2 \\
15 & 15 & 15 & 15 & 20 & 25 & 25 & 25 & 15 & 0 & 0 & 0 & 0 & 15 & 14 \\
267 & 267 & 267 & 267 & 267 & 267 & 267 & 267 & 267 & 0 & 0 & 267 & 267 & 267 & 244 \\
\hline 737 & 737 & 737 & 737 & 742 & $\mathbf{7 4 7}$ & $\mathbf{7 4 7}$ & $\mathbf{7 4 6}$ & $\mathbf{7 3 6}$ & $\mathbf{4 5 5}$ & $\mathbf{4 5 5}$ & $\mathbf{7 2 1}$ & $\mathbf{2 6 7}$ & $\mathbf{7 3 7}$ & $\mathbf{6 7 6}$
\end{tabular}

-Combustion Turbines-

33 Federal System

34 Public Entities

35 Investor-Owned Entities

36 Other Entities

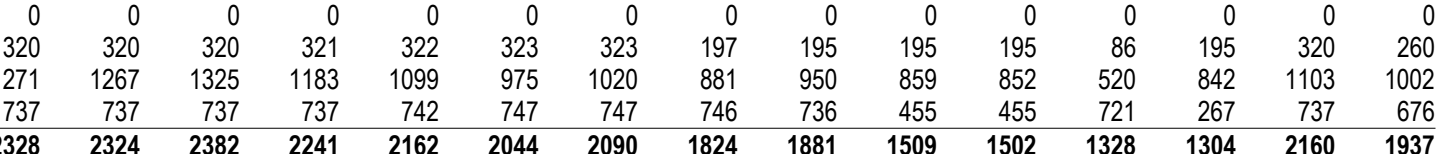


Table A-7: Regional Combustion Turbines

PNW Loads and Resources Study

2008 - 2009 Operating Year 2003 White Book

Aug1 Aug16 Sep Oct Nov Dec Jan Feb Mar Apr1 Apr16

May Jun Jul Avg

-Federal System-

1 Total Federal System

$\begin{array}{lll}0 & 0 & 0\end{array}$

-Public Entities-

2 Benton CT

3 Frederickson CT

4 Hoquiam Diesels

5 Olympic View Generating Station

6 Pasco Harbor Generating Station

7 Randolph Road Diesel Farm

8 River Road

9 Roosevelt Landfill

10 Springfield Gen Farm

11 Wauna Peaking Project

12 Total Public Entities

\begin{tabular}{|c|c|c|c|c|c|c|c|c|c|c|c|c|c|c|}
\hline 0 & 0 & 0 & 1 & 1.5 & 2.5 & 3 & 2 & 0 & 0 & 0 & 0 & 0 & 0 & 0.8 \\
\hline 125 & 125 & 125 & 125 & 125 & 125 & 125 & 0 & 0 & 0 & 0 & 0 & 0 & 125 & 73 \\
\hline 0 & 0 & 0 & 0 & 0 & 0 & 0 & 0 & 0 & 0 & 0 & 0 & 0 & 0 & 0 \\
\hline 0 & 0 & 0 & 0 & 0 & 0 & 0 & 0 & 0 & 0 & 0 & 0 & 0 & 0 & 0 \\
\hline 4.4 & 4.4 & 4.4 & 4.4 & 4.4 & 4.4 & 4.4 & 4.4 & 4.4 & 4.4 & 4.4 & 0 & 4.4 & 4.4 & 4 \\
\hline 0 & 0 & 0 & 0 & 0 & 0 & 0 & 0 & 0 & 0 & 0 & 0 & 0 & 0 & 0 \\
\hline 180 & 180 & 180 & 180 & 180 & 180 & 180 & 180 & 180 & 180 & 180 & 165 & 180 & 180 & 179 \\
\hline 8 & 8 & 8 & 8 & 8 & 8 & 8 & 8 & 8 & 8 & 8 & 8 & 8 & 8 & 8 \\
\hline 0 & 0 & 0 & 0 & 0 & 0 & 0 & 0 & 0 & 0 & 0 & 0 & 0 & 0 & 0 \\
\hline 2.5 & 2.5 & 2.5 & 2.5 & 2.5 & 2.5 & 2.5 & 2.5 & 2.5 & 2.5 & 2.5 & 0 & 2.5 & 2.5 & 2.3 \\
\hline & 320 & 320 & 321 & 322 & 3 & 323 & 197 & 5 & 195 & 195 & 173 & 195 & 320 & 267 \\
\hline
\end{tabular}

-Investor-Owned Entities-

13 Basin Creek Project

14 Beaver

15 Beaver 8

16 Big Hanaford CCCT

17 Boulder Park

18 Danskin

19 Encogen

20 Frederickson $\mathrm{CT}$

21 Fredonia $1 \& 2$

22 Fredonia $3 \& 4$

23 Fredrickson $1 \& 2$

24 Kettle Falls CT

25 Northeastern $1 \& 2$

26 Whitehorn $2 \& 3$

27 Total Investor-Owned Entities

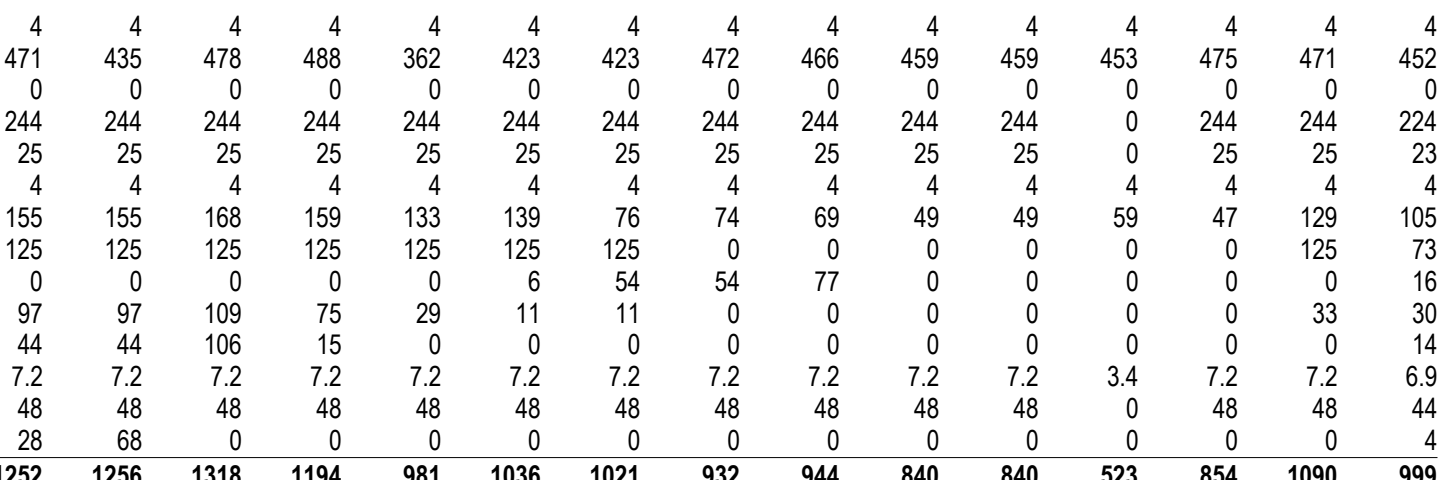

-Other Enities-

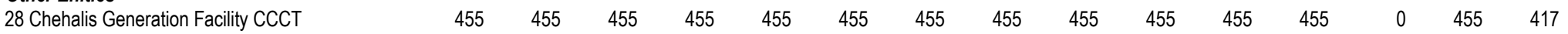

29 Frederickson CT

30 Klamath Peaking Unit

31 Lancaster Power Project (Rathdrum)

32 Total Other Enities

\begin{tabular}{rrrrrrrrrrrrrrr}
0.4 & 0.4 & 0.4 & 0.4 & 0.4 & 0.4 & 0.4 & 0 & 0 & 0 & 0 & 0 & 0 & 0.4 & 0.2 \\
15 & 15 & 15 & 15 & 20 & 25 & 25 & 25 & 15 & 0 & 0 & 0 & 0 & 15 & 14 \\
267 & 267 & 267 & 267 & 267 & 267 & 267 & 267 & 267 & 0 & 0 & 267 & 267 & 267 & 244 \\
\hline 737 & 737 & 737 & 737 & 742 & $\mathbf{7 4 7}$ & $\mathbf{7 4 7}$ & $\mathbf{7 4 6}$ & $\mathbf{7 3 6}$ & $\mathbf{4 5 5}$ & $\mathbf{4 5 5}$ & $\mathbf{7 2 1}$ & $\mathbf{2 6 7}$ & $\mathbf{7 3 7}$ & $\mathbf{6 7 6}$
\end{tabular}

-Combustion Turbines-

33 Federal System

34 Public Entities

35 Investor-Owned Entities

36 Other Entities

\begin{tabular}{|c|c|c|c|c|c|c|c|c|c|c|c|c|c|}
\hline 0 & 0 & 0 & 0 & 0 & 0 & 0 & 0 & 0 & 0 & 0 & 0 & 0 & 0 \\
\hline 320 & 320 & 321 & 322 & 323 & 323 & 197 & 195 & 195 & 195 & 173 & 195 & 320 & 267 \\
\hline 1256 & 1318 & 1194 & 981 & 1036 & 1021 & 932 & 944 & 840 & 840 & 523 & 854 & 1090 & 999 \\
\hline 737 & 737 & 737 & 742 & 747 & 747 & 746 & 736 & 455 & 455 & 721 & 267 & 737 & 676 \\
\hline 31 & 2375 & 2252 & 2044 & 2105 & 2091 & 1875 & 1875 & 1490 & 1490 & 1417 & 1316 & 2147 & $\overline{941}$ \\
\hline
\end{tabular}


Table A-7: Regional Combustion Turbines

PNW Loads and Resources Study

2009 - 2010 Operating Year

2003 White Book

Aug1 Aug16 Sep Oct Nov

Dec Jan Feb Mar Apr1 Apr16

May

Jun Jul Avg

-Federal System-

1 Total Federal System

$\begin{array}{lll}0 & 0 & 0\end{array}$

-Public Entities-

2 Benton CT

3 Frederickson CT

4 Hoquiam Diesels

5 Olympic View Generating Station

6 Pasco Harbor Generating Station

7 Randolph Road Diesel Farm

8 River Road

9 Roosevelt Landfill

10 Springfield Gen Farm

11 Wauna Peaking Project

12 Total Public Entities

\begin{tabular}{|c|c|c|c|c|c|c|c|c|c|c|c|c|c|c|}
\hline 0 & 0 & 0 & 1 & 1.5 & 2.5 & 3 & 2 & 0 & 0 & 0 & 0 & 0 & 0 & 0.8 \\
\hline 125 & 125 & 125 & 125 & 125 & 125 & 125 & 0 & 0 & 0 & 0 & 0 & 0 & 125 & 73 \\
\hline 0 & 0 & 0 & 0 & 0 & 0 & 0 & 0 & 0 & 0 & 0 & 0 & 0 & 0 & 0 \\
\hline 0 & 0 & 0 & 0 & 0 & 0 & 0 & 0 & 0 & 0 & 0 & 0 & 0 & 0 & 0 \\
\hline 4.4 & 4.4 & 4.4 & 4.4 & 4.4 & 4.4 & 4.4 & 4.4 & 4.4 & 4.4 & 4.4 & 0 & 4.4 & 4.4 & 4 \\
\hline 0 & 0 & 0 & 0 & 0 & 0 & 0 & 0 & 0 & 0 & 0 & 0 & 0 & 0 & 0 \\
\hline 180 & 180 & 180 & 180 & 180 & 180 & 180 & 180 & 180 & 180 & 180 & 165 & 180 & 180 & 179 \\
\hline 8 & 8 & 8 & 8 & 8 & 8 & 8 & 8 & 8 & 8 & 8 & 8 & 8 & 8 & 8 \\
\hline 0 & 0 & 0 & 0 & 0 & 0 & 0 & 0 & 0 & 0 & 0 & 0 & 0 & 0 & 0 \\
\hline 2.5 & 2.5 & 2.5 & 2.5 & 2.5 & 2.5 & 2.5 & 2.5 & 2.5 & 2.5 & 2.5 & 0 & 2.5 & 2.5 & 2.3 \\
\hline & 320 & 320 & 321 & 322 & 3 & 323 & 197 & 5 & 195 & 195 & 173 & 195 & 320 & 267 \\
\hline
\end{tabular}

-Investor-Owned Entities-

13 Basin Creek Project

14 Beaver

15 Beaver 8

16 Big Hanaford CCCT

17 Boulder Park

18 Danskin

19 Encogen

20 Frederickson $\mathrm{CT}$

21 Fredonia $1 \& 2$

22 Fredonia $3 \& 4$

23 Fredrickson $1 \& 2$

24 Kettle Falls CT

25 Northeastern $1 \& 2$

26 Whitehorn $2 \& 3$

27 Total Investor-Owned Entities

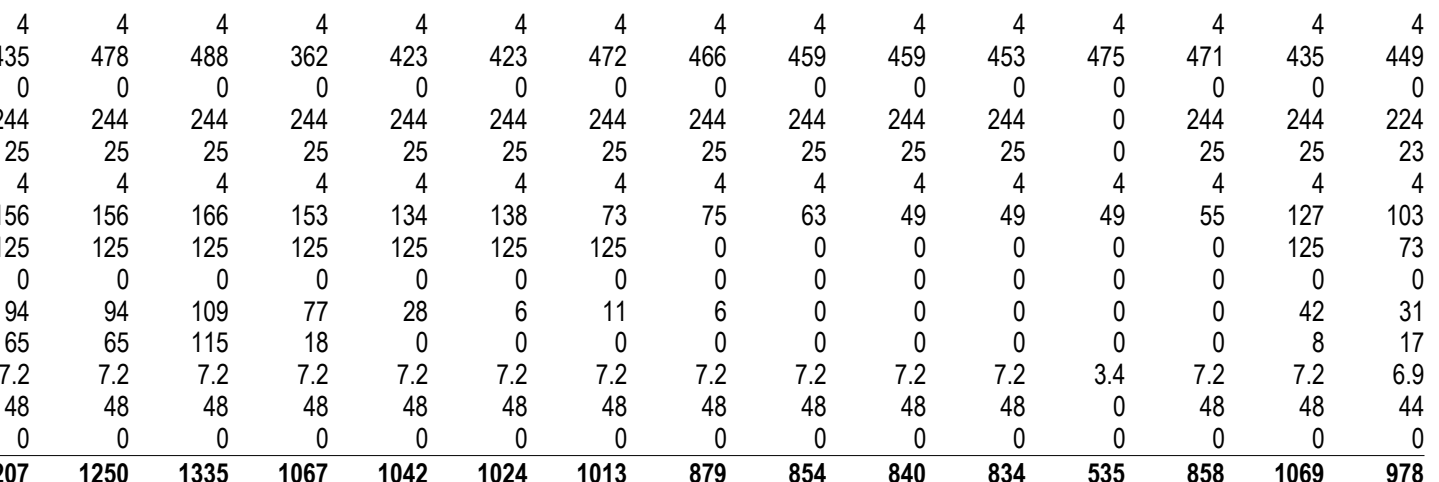

-Other Enities-

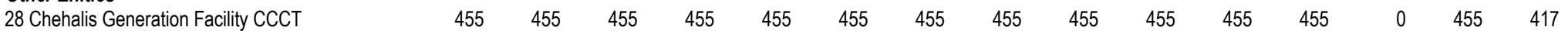

29 Frederickson CT

30 Klamath Peaking Unit

31 Lancaster Power Project (Rathdrum)

32 Total Other Enities

\begin{tabular}{|c|c|c|c|c|c|c|c|c|c|c|c|c|c|c|}
\hline 0.4 & 0.4 & 0.4 & 0.4 & 0.4 & 0.4 & 0.4 & 0 & 0 & 0 & 0 & 0 & 0 & 0.4 & 0.2 \\
\hline 15 & 15 & 15 & 15 & 20 & 25 & 25 & 25 & 15 & 0 & 0 & 0 & 0 & 15 & 14 \\
\hline 267 & 267 & 267 & 267 & 267 & 267 & 267 & 267 & 267 & 0 & 0 & 267 & 267 & 267 & 244 \\
\hline 737 & 707 & & & & & & & 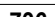 & E & $\boldsymbol{\tau}$ & 70 & 7 & 727 & 76 \\
\hline
\end{tabular}

-Combustion Turbines-

33 Federal System

34 Public Entities

35 Investor-Owned Entities

36 Other Entities

\begin{tabular}{|c|c|c|c|c|c|c|c|c|c|c|c|c|c|}
\hline 0 & 0 & 0 & 0 & 0 & 0 & 0 & 0 & 0 & 0 & 0 & 0 & 0 & 0 \\
\hline 320 & 320 & 321 & 322 & 323 & 323 & 197 & 195 & 195 & 195 & 173 & 195 & 320 & 267 \\
\hline 1250 & 1335 & 1067 & 1042 & 1024 & 1013 & 879 & 854 & 840 & 834 & 535 & 858 & 1069 & 978 \\
\hline 737 & 737 & 737 & 742 & 747 & 747 & 746 & 736 & 455 & 455 & 721 & 267 & 737 & 676 \\
\hline 230 & 2392 & 25 & 0 & 093 & 083 & 32 & 785 & 490 & 484 & 1170 & & & \\
\hline
\end{tabular}


Table A-7: Regional Combustion Turbines

PNW Loads and Resources Study

2010 - 2011 Operating Year 2003 White Book

Aug1 Aug16 Sep Oct Nov

Dec Jan Feb Mar Apr1 Apr16

May

Jun Jul Avg

-Federal System-

1 Total Federal System

$\begin{array}{lll}0 & 0 & 0\end{array}$

-Public Entities-

2 Benton CT

3 Frederickson CT

4 Hoquiam Diesels

5 Olympic View Generating Station

6 Pasco Harbor Generating Station

7 Randolph Road Diesel Farm

8 River Road

9 Roosevelt Landfill

10 Springfield Gen Farm

11 Wauna Peaking Project

12 Total Public Entities

\begin{tabular}{|c|c|c|c|c|c|c|c|c|c|c|c|c|c|}
\hline 0 & 0 & 1 & 1.5 & 2.5 & 3 & 2 & 0 & 0 & 0 & 0 & 0 & 0 & 0.8 \\
\hline 125 & 125 & 125 & 125 & 125 & 125 & 0 & 0 & 0 & 0 & 0 & 0 & 125 & 73 \\
\hline 0 & 0 & 0 & 0 & 0 & 0 & 0 & 0 & 0 & 0 & 0 & 0 & 0 & 0 \\
\hline 0 & 0 & 0 & 0 & 0 & 0 & 0 & 0 & 0 & 0 & 0 & 0 & 0 & 0 \\
\hline 4.4 & 4.4 & 4.4 & 4.4 & 4.4 & 4.4 & 4.4 & 4.4 & 4.4 & 4.4 & 0 & 4.4 & 4.4 & 4 \\
\hline 0 & 0 & 0 & 0 & 0 & 0 & 0 & 0 & 0 & 0 & 0 & 0 & 0 & 0 \\
\hline 188 & 188 & 188 & 188 & 188 & 188 & 188 & 188 & 188 & 188 & 0 & 188 & 188 & 172 \\
\hline 8 & 8 & 8 & 8 & 8 & 8 & 8 & 8 & 8 & 8 & 8 & 8 & 8 & 8 \\
\hline 0 & 0 & 0 & 0 & 0 & 0 & 0 & 0 & 0 & 0 & 0 & 0 & 0 & 0 \\
\hline 2.5 & 2.5 & 2.5 & 2.5 & 2.5 & 2.5 & 2.5 & 2.5 & 2.5 & 2.5 & 0 & 2.5 & 2.5 & 2.3 \\
\hline 328 & 328 & 329 & 9 & 0 & 331 & 204 & 202 & 202 & 2 & 8 & 202 & 328 & 260 \\
\hline
\end{tabular}

-Investor-Owned Entities-

13 Basin Creek Project

14 Beaver

15 Beaver 8

16 Big Hanaford CCCT

17 Boulder Park

18 Danskin

19 Encogen

20 Frederickson $\mathrm{CT}$

21 Fredonia $1 \& 2$

22 Fredonia $3 \& 4$

23 Fredrickson $1 \& 2$

24 Kettle Falls CT

25 Northeastern $1 \& 2$

26 Whitehorn $2 \& 3$

27 Total Investor-Owned Entities

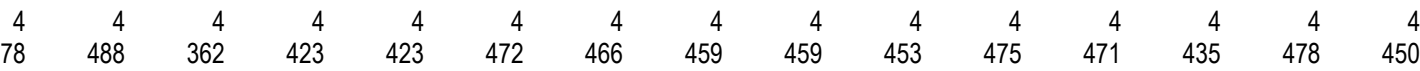

-Other Enities-

28 Chehalis Generation Facility

29 Frederickson CT

30 Klamath Peaking Unit

31 Lancaster Power Project (Rathdrum)

32 Total Other Enities

\begin{tabular}{|c|c|c|c|c|c|c|c|c|c|c|c|c|c|c|}
\hline & 4 & 4 & & & & T & 4 & 4 & 4 & 4 & 4 & 4 & 4 & \\
\hline 78 & 488 & 362 & 423 & 423 & 472 & 466 & 459 & 459 & 453 & 475 & 471 & 435 & 478 & 450 \\
\hline 0 & 0 & 0 & 0 & 0 & 0 & 0 & 0 & 0 & 0 & 0 & 0 & 0 & 0 & 0 \\
\hline 244 & 244 & 244 & 244 & 244 & 244 & 244 & 244 & 244 & 244 & 244 & 0 & 244 & 244 & 224 \\
\hline 25 & 25 & 25 & 25 & 25 & 25 & 25 & 25 & 25 & 25 & 25 & 0 & 25 & 25 & 23 \\
\hline 4 & 4 & 4 & 4 & 4 & 4 & 4 & 4 & 4 & 4 & 4 & 4 & 4 & 4 & 4 \\
\hline 155 & 155 & 168 & 156 & 113 & 117 & 53 & 75 & 58 & 51 & 51 & 49 & 53 & 116 & 97 \\
\hline 125 & 125 & 125 & 125 & 125 & 125 & 125 & 0 & 0 & 0 & 0 & 0 & 0 & 125 & 73 \\
\hline 0 & 0 & 0 & 0 & 0 & 6 & 54 & 54 & 77 & 0 & 0 & 0 & 0 & 0 & 16 \\
\hline 94 & 94 & 109 & 70 & 18 & 19 & 6 & 0 & 0 & 0 & 0 & 0 & 0 & 0 & 26 \\
\hline 54 & 54 & 63 & 18 & 0 & 0 & 0 & 0 & 0 & 0 & 0 & 0 & 0 & 0 & 11 \\
\hline 7.2 & 7.2 & 7.2 & 7.2 & 7.2 & 7.2 & 7.2 & 7.2 & 7.2 & 7.2 & 7.2 & 3.4 & 7.2 & 7.2 & 6.9 \\
\hline 48 & 48 & 48 & 48 & 48 & 48 & 48 & 48 & 48 & 48 & 48 & 0 & 48 & 48 & 44 \\
\hline 0 & 0 & 0 & 0 & 0 & 0 & 0 & 0 & 0 & 0 & 0 & 0 & 0 & 0 & \\
\hline 1238 & 1248 & 1159 & 1124 & 1011 & 1071 & 1036 & 920 & 926 & 836 & 858 & 531 & 820 & 1051 & 3 \\
\hline
\end{tabular}

-Combustion Turbines-

33 Federal System

34 Public Entities

35 Investor-Owned Entities

36 Other Entities

\begin{tabular}{rrrrrrrrrrrrrrr}
455 & 455 & 455 & 455 & 455 & 455 & 455 & 455 & 455 & 455 & 455 & 455 & 0 & 455 & 417 \\
0.4 & 0.4 & 0.4 & 0.4 & 0.4 & 0.4 & 0.4 & 0 & 0 & 0 & 0 & 0 & 0 & 0.4 & 0.2 \\
15 & 15 & 15 & 15 & 20 & 25 & 25 & 25 & 15 & 0 & 0 & 0 & 0 & 15 & 14 \\
267 & 267 & 267 & 267 & 267 & 267 & 267 & 267 & 267 & 0 & 0 & 267 & 267 & 267 & 244 \\
\hline $\mathbf{7 3 7}$ & $\mathbf{7 3 7}$ & $\mathbf{7 3 7}$ & $\mathbf{7 3 7}$ & $\mathbf{7 4 2}$ & $\mathbf{7 4 7}$ & $\mathbf{7 4 7}$ & $\mathbf{7 4 6}$ & $\mathbf{7 3 6}$ & $\mathbf{4 5 5}$ & $\mathbf{4 5 5}$ & $\mathbf{7 2 1}$ & $\mathbf{2 6 7}$ & $\mathbf{7 3 7}$ & $\mathbf{6 7 6}$
\end{tabular}

37 Total Combustion Turbines

\begin{tabular}{|c|c|c|c|c|c|c|c|c|c|c|c|c|c|}
\hline 0 & 0 & 0 & 0 & 0 & 0 & 0 & 0 & 0 & $c$ & 0 & c & 0 & 0 \\
\hline 328 & 328 & 329 & 329 & 330 & 331 & 204 & 202 & 202 & 202 & 8 & 202 & 328 & 260 \\
\hline 1248 & 1159 & 1124 & 1011 & 1071 & 1036 & 920 & 926 & 836 & 858 & 531 & 82 & 1051 & 978 \\
\hline 737 & 737 & 737 & 742 & 747 & 747 & 746 & 736 & 455 & 455 & 721 & 26 & 737 & 676 \\
\hline 231 & 2223 & 2189 & 208 & 2148 & 2113 & 1871 & 1865 & 1493 & 1515 & 1261 & 128 & 2115 & 014 \\
\hline
\end{tabular}


Table A-7: Regional Combustion Turbines

PNW Loads and Resources Study

2011 - 2012 Operating Year 2003 White Book

Aug1 Aug16 Sep Oct Nov Dec Jan Feb Mar Apr1 Apr16

May Jun Jul Avg

-Federal System-

1 Total Federal System

$\begin{array}{lll}0 & 0 & 0\end{array}$

-Public Entities-

2 Benton CT

3 Frederickson CT

4 Hoquiam Diesels

5 Olympic View Generating Station

6 Pasco Harbor Generating Station

7 Randolph Road Diesel Farm

8 River Road

9 Roosevelt Landfill

10 Springfield Gen Farm

11 Wauna Peaking Project

12 Total Public Entities

\begin{tabular}{|c|c|c|c|c|c|c|c|c|c|c|c|c|c|c|}
\hline 0 & 0 & 0 & 1 & 1.5 & 2.5 & 3 & 2 & 0 & 0 & 0 & 0 & 0 & 0 & 0.8 \\
\hline 125 & 125 & 125 & 125 & 125 & 125 & 125 & 0 & 0 & 0 & 0 & 0 & 0 & 125 & 73 \\
\hline 0 & 0 & 0 & 0 & 0 & 0 & 0 & 0 & 0 & 0 & 0 & 0 & 0 & 0 & 0 \\
\hline 0 & 0 & 0 & 0 & 0 & 0 & 0 & 0 & 0 & 0 & 0 & 0 & 0 & 0 & 0 \\
\hline 4.4 & 4.4 & 4.4 & 4.4 & 4.4 & 4.4 & 4.4 & 4.4 & 4.4 & 4.4 & 4.4 & 0 & 4.4 & 4.4 & 4 \\
\hline 0 & 0 & 0 & 0 & 0 & 0 & 0 & 0 & 0 & 0 & 0 & 0 & 0 & 0 & 0 \\
\hline 196 & 196 & 196 & 196 & 196 & 196 & 196 & 196 & 196 & 196 & 196 & 0 & 196 & 196 & 180 \\
\hline 8 & 8 & 8 & 8 & 8 & 8 & 8 & 8 & 8 & 8 & 8 & 8 & 8 & 8 & 8 \\
\hline 0 & 0 & 0 & 0 & 0 & 0 & 0 & 0 & 0 & 0 & 0 & 0 & 0 & 0 & 0 \\
\hline 2.5 & 2.5 & 2.5 & 2.5 & 2.5 & 2.5 & 2.5 & 2.5 & 2.5 & 2.5 & 2.5 & 0 & 2.5 & 2.5 & 2.3 \\
\hline 36 & 336 & 336 & 337 & 338 & 339 & 339 & 213 & 1 & 211 & 211 & 8 & 211 & 336 & 268 \\
\hline
\end{tabular}

-Investor-Owned Entities-

13 Basin Creek Project

14 Beaver

15 Beaver 8

16 Big Hanaford CCCT

17 Boulder Park

18 Danskin

19 Encogen

20 Frederickson $\mathrm{CT}$

21 Fredonia $1 \& 2$

22 Fredonia $3 \& 4$

23 Fredrickson $1 \& 2$

24 Kettle Falls CT

25 Northeastern $1 \& 2$

26 Whitehorn $2 \& 3$

27 Total Investor-Owned Entities

\begin{tabular}{|c|c|c|c|c|c|c|c|c|c|c|c|c|c|}
\hline 4 & 4 & 4 & 4 & 4 & 4 & 4 & 4 & 4 & 4 & 4 & 4 & 4 & 4 \\
\hline 362 & 423 & 423 & 472 & 466 & 459 & 459 & 453 & 475 & 471 & 435 & 478 & 488 & 454 \\
\hline 0 & 0 & 0 & 0 & 0 & 0 & 0 & 0 & 0 & 0 & 0 & 0 & 0 & 0 \\
\hline 244 & 244 & 244 & 244 & 244 & 244 & 244 & 244 & 244 & 244 & 0 & 244 & 244 & 224 \\
\hline 25 & 25 & 25 & 25 & 25 & 25 & 25 & 25 & 25 & 25 & 0 & 25 & 25 & 23 \\
\hline 4 & 4 & 4 & 4 & 4 & 4 & 4 & 4 & 4 & 4 & 4 & 4 & 4 & 4 \\
\hline 152 & 164 & 141 & 103 & 121 & 65 & 65 & 54 & 47 & 47 & 43 & 51 & 116 & 94 \\
\hline 125 & 125 & 125 & 125 & 125 & 125 & 0 & 0 & 0 & 0 & 0 & 0 & 125 & 73 \\
\hline 0 & 0 & 0 & 0 & 6 & 54 & 54 & 77 & 0 & 0 & 0 & 0 & 0 & 16 \\
\hline 40 & 90 & 100 & 53 & 11 & 0 & 6 & 0 & 0 & 0 & 0 & 0 & 27 & 29 \\
\hline 50 & 47 & 4 & 0 & 0 & 0 & 0 & 0 & 0 & 0 & 0 & 0 & 0 & 8.4 \\
\hline 7.2 & 7.2 & 7.2 & 7.2 & 7.2 & 7.2 & 7.2 & 7.2 & 7.2 & 7.2 & 3.4 & 7.2 & 7.2 & 6.9 \\
\hline 48 & 48 & 48 & 48 & 48 & 48 & 48 & 48 & 48 & 48 & 0 & 48 & 48 & 44 \\
\hline 0 & 0 & 0 & 0 & 0 & 0 & 0 & 0 & 0 & 0 & 0 & 0 & 0 & 0 \\
\hline 1111 & 1181 & 1125 & 1085 & 1061 & 1035 & 916 & 916 & 854 & 850 & 489 & 861 & 1088 & 980 \\
\hline
\end{tabular}

-Other Enities-

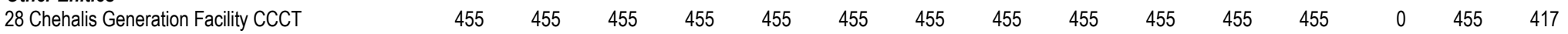

29 Frederickson CT

30 Klamath Peaking Unit

31 Lancaster Power Project (Rathdrum)

32 Total Other Enities

\begin{tabular}{rrrrrrrrrrrrrrr}
0.4 & 0.4 & 0.4 & 0.4 & 0.4 & 0.4 & 0.4 & 0 & 0 & 0 & 0 & 0 & 0 & 0.4 & 0.2 \\
15 & 15 & 15 & 15 & 20 & 25 & 25 & 25 & 15 & 0 & 0 & 0 & 0 & 15 & 14 \\
267 & 267 & 267 & 267 & 267 & 267 & 267 & 267 & 267 & 0 & 0 & 267 & 267 & 267 & 244 \\
\hline 737 & 737 & 737 & 737 & 742 & $\mathbf{7 4 7}$ & $\mathbf{7 4 7}$ & $\mathbf{7 4 6}$ & $\mathbf{7 3 6}$ & $\mathbf{4 5 5}$ & $\mathbf{4 5 5}$ & $\mathbf{7 2 1}$ & $\mathbf{2 6 7}$ & $\mathbf{7 3 7}$ & $\mathbf{6 7 6}$
\end{tabular}

-Combustion Turbines-

33 Federal System

34 Public Entities

35 Investor-Owned Entities

36 Other Entities

\begin{tabular}{|c|c|c|c|c|c|c|c|c|c|c|c|c|c|}
\hline 0 & 0 & 0 & 0 & 0 & 0 & 0 & 0 & 0 & 0 & 0 & 0 & 0 & 0 \\
\hline 336 & 336 & 337 & 338 & 339 & 339 & 213 & 211 & 211 & 211 & 8 & 211 & 336 & 268 \\
\hline 1111 & 1181 & 1125 & 1085 & 1061 & 1035 & 916 & 916 & 854 & 850 & 489 & 861 & 1088 & 980 \\
\hline 737 & 737 & 737 & 742 & 747 & 747 & 746 & 736 & 455 & 455 & 721 & 267 & 737 & 676 \\
\hline 18 & 2254 & 2199 & 2164 & 2146 & 2121 & 1875 & 1863 & 1520 & 1516 & 1219 & 39 & 2161 & 1923 \\
\hline
\end{tabular}


Table A-7: Regional Combustion Turbines

PNW Loads and Resources Study

2012 - 2013 Operating Year 2003 White Book

Aug1 Aug16 Sep Oct Nov Dec Jan Feb Mar Apr1 Apr16

May Jun Jul Avg

-Federal System-

1 Total Federal System

$\begin{array}{lll}0 & 0 & 0\end{array}$

-Public Entities-

2 Benton CT

3 Frederickson CT

4 Hoquiam Diesels

5 Olympic View Generating Station

6 Pasco Harbor Generating Station

7 Randolph Road Diesel Farm

8 River Road

9 Roosevelt Landfill

10 Springfield Gen Farm

11 Wauna Peaking Project

12 Total Public Entities

\begin{tabular}{|c|c|c|c|c|c|c|c|c|c|c|c|c|c|c|}
\hline 0 & 0 & 0 & 1 & 1.5 & 2.5 & 3 & 2 & 0 & 0 & 0 & 0 & 0 & 0 & 0.8 \\
\hline 125 & 125 & 125 & 125 & 125 & 125 & 125 & 0 & 0 & 0 & 0 & 0 & 0 & 125 & 73 \\
\hline 0 & 0 & 0 & 0 & 0 & 0 & 0 & 0 & 0 & 0 & 0 & 0 & 0 & 0 & 0 \\
\hline 0 & 0 & 0 & 0 & 0 & 0 & 0 & 0 & 0 & 0 & 0 & 0 & 0 & 0 & 0 \\
\hline 4.4 & 4.4 & 4.4 & 4.4 & 4.4 & 4.4 & 4.4 & 4.4 & 4.4 & 4.4 & 4.4 & 0 & 4.4 & 4.4 & 4 \\
\hline 0 & 0 & 0 & 0 & 0 & 0 & 0 & 0 & 0 & 0 & 0 & 0 & 0 & 0 & 0 \\
\hline 201 & 201 & 201 & 201 & 201 & 201 & 201 & 201 & 201 & 201 & 201 & 78 & 201 & 201 & 191 \\
\hline 8 & 8 & 8 & 8 & 8 & 8 & 8 & 8 & 8 & 8 & 8 & 8 & 8 & 8 & 8 \\
\hline 0 & 0 & 0 & 0 & 0 & 0 & 0 & 0 & 0 & 0 & 0 & 0 & 0 & 0 & 0 \\
\hline 2.5 & 2.5 & 2.5 & 2.5 & 2.5 & 2.5 & 2.5 & 2.5 & 2.5 & 2.5 & 2.5 & 0 & 2.5 & 2.5 & 2.3 \\
\hline 41 & 341 & 341 & 342 & 343 & 344 & 344 & 8 & 6 & 216 & 216 & 6 & 6 & 341 & 279 \\
\hline
\end{tabular}

-Investor-Owned Entities-

13 Basin Creek Project

14 Beaver

15 Beaver 8

16 Big Hanaford CCCT

17 Boulder Park

18 Danskin

19 Encogen

20 Frederickson $\mathrm{CT}$

21 Fredonia $1 \& 2$

22 Fredonia $3 \& 4$

23 Fredrickson $1 \& 2$

24 Kettle Falls CT

25 Northeastern $1 \& 2$

26 Whitehorn $2 \& 3$

27 Total Investor-Owned Entities

341

-Other Enities-

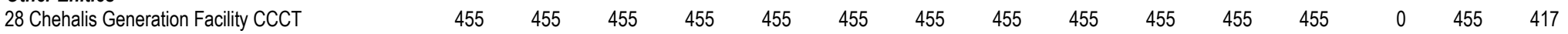

29 Frederickson CT

30 Klamath Peaking Unit

31 Lancaster Power Project (Rathdrum)

32 Total Other Enities

\begin{tabular}{|c|c|c|c|c|c|c|c|c|c|c|c|c|c|c|}
\hline 4 & 4 & 4 & 4 & 4 & 4 & 4 & 4 & 4 & 4 & 4 & 4 & 4 & 4 & 4 \\
\hline 362 & 423 & 423 & 472 & 466 & 459 & 459 & 453 & 475 & 471 & 435 & 478 & 488 & 362 & 448 \\
\hline 244 & 244 & 244 & 244 & 244 & 244 & 244 & 244 & 244 & 244 & 244 & 0 & 244 & 244 & 224 \\
\hline 4 & 4 & 4 & 4 & 4 & 4 & 4 & 4 & 4 & 4 & 4 & 4 & 4 & 4 & 4 \\
\hline 151 & 151 & 159 & 130 & 101 & 105 & 64 & 64 & 57 & 48 & 48 & 41 & 41 & 105 & 89 \\
\hline 125 & 125 & 125 & 125 & 125 & 125 & 125 & 0 & 0 & 0 & 0 & 0 & 0 & 125 & 73 \\
\hline 81 & 81 & 109 & 53 & 0 & 6 & 0 & 0 & 0 & 0 & 0 & 0 & 0 & 25 & 23 \\
\hline 46 & 46 & 55 & 4 & 0 & 0 & 0 & 0 & 0 & 0 & 0 & 0 & 0 & 6 & 9.3 \\
\hline 7.2 & 7.2 & 7.2 & 7.2 & 7.2 & 7.2 & 7.2 & 7.2 & 7.2 & 7.2 & 7.2 & 3.4 & 7.2 & 7.2 & 6.9 \\
\hline 48 & 48 & 48 & 48 & 48 & 48 & 48 & 48 & 48 & 48 & 48 & 0 & 48 & 48 & 44 \\
\hline 0 & 0 & 0 & 0 & 0 & 0 & 0 & 0 & 0 & 0 & 0 & 0 & 0 & 0 & 0 \\
\hline 097 & 1158 & 1203 & 1116 & 1024 & 1033 & 1034 & 903 & 941 & 851 & 815 & 530 & 861 & 955 & 963 \\
\hline
\end{tabular}

-Combustion Turbines-

33 Federal System

34 Public Entities

35 Investor-Owned Entities

36 Other Entities

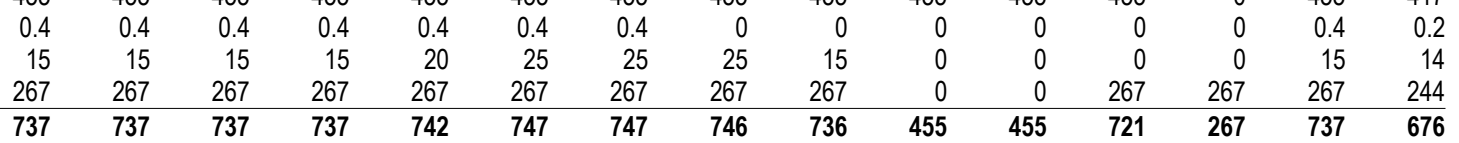

37 Total Combustion Turbines

\begin{tabular}{rrrrrrrrrrrrrrr}
0 & 0 & 0 & 0 & 0 & 0 & 0 & 0 & 0 & 0 & 0 & 0 & 0 & 0 & 0 \\
341 & 341 & 341 & 342 & 343 & 344 & 344 & 218 & 216 & 216 & 216 & 86 & 216 & 341 & 279 \\
1097 & 1158 & 1203 & 1116 & 1024 & 1033 & 1034 & 903 & 941 & 851 & 815 & 530 & 861 & 955 & 963 \\
737 & 737 & 737 & 737 & 742 & 747 & 747 & 746 & 736 & 455 & 455 & 721 & 267 & 737 & 676 \\
\hline $\mathbf{2 1 7 5}$ & $\mathbf{2 2 3 6}$ & $\mathbf{2 2 8 1}$ & $\mathbf{2 1 9 5}$ & $\mathbf{2 1 0 8}$ & $\mathbf{2 1 2 3}$ & $\mathbf{2 1 2 5}$ & $\mathbf{1 8 6 7}$ & $\mathbf{1 8 9 3}$ & $\mathbf{1 5 2 2}$ & $\mathbf{1 4 8 6}$ & $\mathbf{1 3 3 8}$ & $\mathbf{1 3 4 4}$ & $\mathbf{2 0 3 3}$ & $\mathbf{1 9 1 8}$
\end{tabular}


Table A-7: Regional Combustion Turbines

PNW Loads and Resources Study

2013 - 2014 Operating Year 2003 White Book

Aug1 Aug16

Sep Oct Nov

Dec Jan Feb Mar Apr1 Apr16

May Jun Jul Avg

-Federal System-

1 Total Federal System

$\begin{array}{lll}0 & 0 & 0\end{array}$

-Public Entities-

2 Benton CT

3 Frederickson CT

4 Hoquiam Diesels

5 Olympic View Generating Station

6 Pasco Harbor Generating Station

7 Randolph Road Diesel Farm

8 River Road

9 Roosevelt Landfill

10 Springfield Gen Farm

11 Wauna Peaking Project

12 Total Public Entities

\begin{tabular}{|c|c|c|c|c|c|c|c|c|c|c|c|c|c|c|}
\hline 0 & 0 & 0 & 1 & 1.5 & 2.5 & 3 & 2 & 0 & 0 & 0 & 0 & 0 & 0 & 0.8 \\
\hline 125 & 125 & 125 & 125 & 125 & 125 & 125 & 0 & 0 & 0 & 0 & 0 & 0 & 125 & 73 \\
\hline 0 & 0 & 0 & 0 & 0 & 0 & 0 & 0 & 0 & 0 & 0 & 0 & 0 & 0 & 0 \\
\hline 0 & 0 & 0 & 0 & 0 & 0 & 0 & 0 & 0 & 0 & 0 & 0 & 0 & 0 & 0 \\
\hline 4.4 & 4.4 & 4.4 & 4.4 & 4.4 & 4.4 & 4.4 & 4.4 & 4.4 & 4.4 & 4.4 & 0 & 4.4 & 4.4 & 4 \\
\hline 0 & 0 & 0 & 0 & 0 & 0 & 0 & 0 & 0 & 0 & 0 & 0 & 0 & 0 & 0 \\
\hline 212 & 212 & 212 & 212 & 212 & 212 & 212 & 212 & 212 & 212 & 212 & 78 & 212 & 212 & 201 \\
\hline 8 & 8 & 8 & 8 & 8 & 8 & 8 & 8 & 8 & 8 & 8 & 8 & 8 & 8 & 8 \\
\hline 0 & 0 & 0 & 0 & 0 & 0 & 0 & 0 & 0 & 0 & 0 & 0 & 0 & 0 & 0 \\
\hline 2.5 & 2.5 & 2.5 & 2.5 & 2.5 & 2.5 & 2.5 & 2.5 & 2.5 & 2.5 & 2.5 & 0 & 2.5 & 2.5 & 2.3 \\
\hline 52 & 352 & 2 & 353 & 353 & 354 & 355 & 229 & 77 & 227 & 7 & 6 & 227 & 352 & 289 \\
\hline
\end{tabular}

-Investor-Owned Entities-

13 Basin Creek Project

14 Beaver

15 Beaver 8

16 Big Hanaford CCCT

17 Boulder Park

18 Danskin

19 Encogen

20 Frederickson $\mathrm{CT}$

21 Fredonia $1 \& 2$

22 Fredonia $3 \& 4$

23 Fredrickson $1 \& 2$

24 Kettle Falls CT

25 Northeastern $1 \& 2$

26 Whitehorn $2 \& 3$

27 Total Investor-Owned Entities

352

-Other Enities-

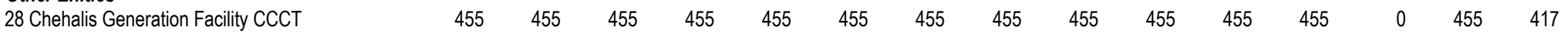

29 Frederickson CT

30 Klamath Peaking Unit

31 Lancaster Power Project (Rathdrum)

32 Total Other Enities

\begin{tabular}{|c|c|c|c|c|c|c|c|c|c|c|c|c|c|c|}
\hline 4 & 4 & 4 & 4 & 4 & 4 & 4 & 4 & 4 & 4 & 4 & 4 & 4 & 4 & 4 \\
\hline 423 & 423 & 472 & 466 & 459 & 459 & 453 & 475 & 471 & 435 & 478 & 488 & 362 & 423 & 451 \\
\hline 0 & 0 & 0 & 0 & 0 & 0 & 0 & 0 & 0 & 0 & 0 & 0 & 0 & 0 & 0 \\
\hline 244 & 244 & 244 & 244 & 244 & 244 & 244 & 244 & 244 & 244 & 244 & 0 & 244 & 244 & 224 \\
\hline 4 & 4 & 4 & 4 & 4 & 4 & 4 & 4 & 4 & 4 & 4 & 4 & 4 & 4 & 4 \\
\hline 151 & 151 & 159 & 130 & 101 & 105 & 64 & 64 & 57 & 48 & 48 & 41 & 41 & 105 & 89 \\
\hline 125 & 125 & 125 & 125 & 125 & 125 & 125 & 0 & 0 & 0 & 0 & 0 & 0 & 125 & 73 \\
\hline 64 & 64 & 94 & 34 & 6 & 0 & 0 & 0 & 0 & 0 & 0 & 0 & 0 & 18 & 18 \\
\hline 28 & 28 & 47 & 0 & 0 & 0 & 0 & 0 & 0 & 0 & 0 & 0 & 0 & 0 & 6.3 \\
\hline 7.2 & 7.2 & 7.2 & 7.2 & 7.2 & 7.2 & 7.2 & 7.2 & 7.2 & 7.2 & 7.2 & 3.4 & 7.2 & 7.2 & 6.9 \\
\hline 48 & 48 & 48 & 48 & 48 & 48 & 48 & 48 & 48 & 48 & 48 & 0 & 48 & 48 & 44 \\
\hline 0 & 0 & 0 & 0 & 0 & 0 & 0 & 0 & 0 & 0 & 0 & 0 & 0 & 0 & 0 \\
\hline & 1123 & 1229 & 1087 & 1023 & 1021 & 1028 & 925 & 937 & 815 & 858 & 540 & 735 & 1003 & 957 \\
\hline
\end{tabular}

-Combustion Turbines-

33 Federal System

34 Public Entities

35 Investor-Owned Entities

36 Other Entities

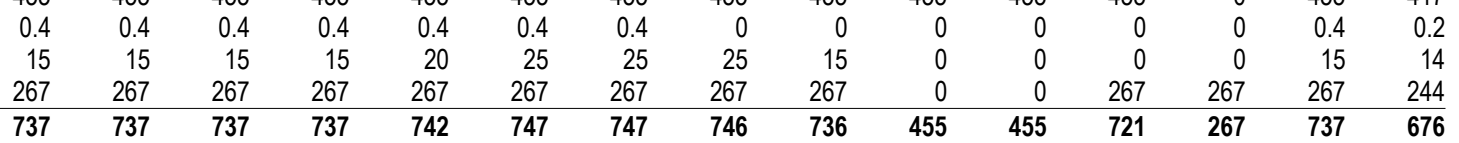

37 Total Combustion Turbines

\begin{tabular}{rrrrrrrrrrrrrrr}
0 & 0 & 0 & 0 & 0 & 0 & 0 & 0 & 0 & 0 & 0 & 0 & 0 & 0 & 0 \\
352 & 352 & 352 & 353 & 353 & 354 & 355 & 229 & 227 & 227 & 227 & 86 & 227 & 352 & 289 \\
123 & 1123 & 1229 & 1087 & 1023 & 1021 & 1028 & 925 & 937 & 815 & 858 & 540 & 735 & 1003 & 957 \\
737 & 737 & 737 & 737 & 742 & 747 & 747 & 746 & 736 & 455 & 455 & 721 & 267 & 737 & 676 \\
\hline 2211 & $\mathbf{2 2 1 1}$ & $\mathbf{2 3 1 7}$ & $\mathbf{2 1 7 6}$ & $\mathbf{2 1 1 8}$ & $\mathbf{2 1 2 2}$ & $\mathbf{2 1 2 9}$ & $\mathbf{1 9 0 0}$ & $\mathbf{1 9 0 0}$ & $\mathbf{1 4 9 6}$ & $\mathbf{1 5 3 9}$ & $\mathbf{1 3 4 8}$ & $\mathbf{1 2 2 8}$ & $\mathbf{2 0 9 1}$ & $\mathbf{1 9 2 2}$
\end{tabular}


Table A-8: Regional Renewable Resources

PNW Loads and Resources Study

2004 - 2005 Operating Year

$3 / 31 / 2004$ 2003 White Book

Average Energy in Megawatts Aug Aug16 Sep Oct Nov Dec Jan Feb Mar Apr1 Apr16 May Jun Jul Avg

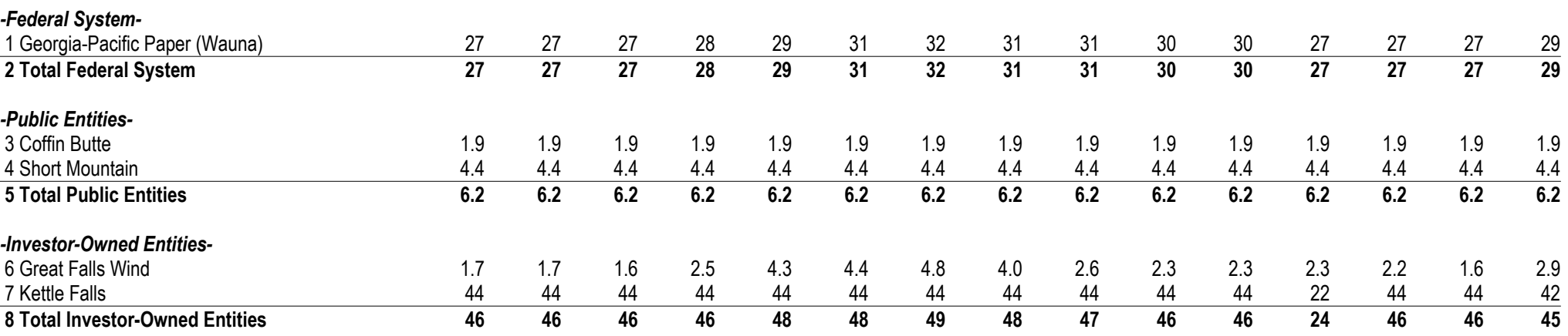

-Other Entities-

9 Total Other Entities

-Renewable Resources-

10 Federal System

11 Public Entities

12 Investor-Owned Entities

13 Other Entities

14 Total Renewable Resources

$\begin{array}{lll}0 & 0 & 0\end{array}$

0

$\begin{array}{llll}0 & 0 & 0 & 0\end{array}$

0

0

0

0

$\begin{array}{rrrrrrrrrrrrrrr}27 & 27 & 27 & 28 & 29 & 31 & 32 & 31 & 31 & 30 & 30 & 27 & 27 & 27 & 29 \\ 6.2 & 6.2 & 6.2 & 6.2 & 6.2 & 6.2 & 6.2 & 6.2 & 6.2 & 6.2 & 6.2 & 6.2 & 6.2 & 6.2 & 6.2 \\ 46 & 46 & 46 & 46 & 48 & 48 & 49 & 48 & 47 & 46 & 46 & 24 & 46 & 46 & 45 \\ 0 & 0 & 0 & 0 & 0 & 0 & 0 & 0 & 0 & 0 & 0 & 0 & 0 & 0 & 0 \\ \mathbf{7 9} & \mathbf{7 9} & \mathbf{7 9} & \mathbf{8 1} & \mathbf{8 4} & \mathbf{8 6} & \mathbf{8 7} & \mathbf{8 5} & \mathbf{8 4} & \mathbf{8 2} & \mathbf{8 2} & \mathbf{5 7} & \mathbf{7 9} & \mathbf{7 9} & \mathbf{8 0}\end{array}$


Table A-8: Regional Renewable Resources

PNW Loads and Resources Study

2005 - 2006 Operating Year

$3 / 31 / 2004$ 2003 White Book

Average Energy in Megawatts Aug Aug16 Sep Oct Nov Dec Jan Feb Mar Apr1 Apr16 May Jun Jul Avg

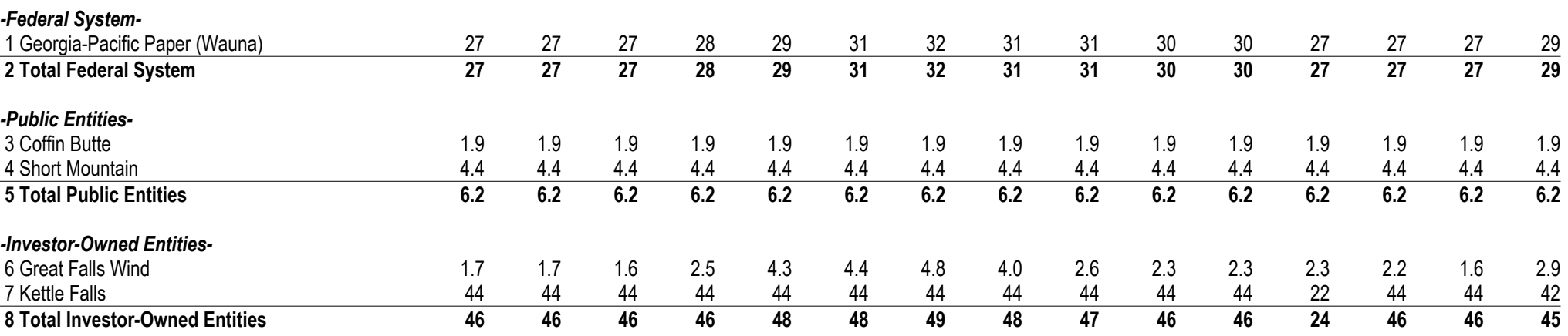

-Other Entities-

9 Total Other Entities

-Renewable Resources-

10 Federal System

11 Public Entities

12 Investor-Owned Entities

13 Other Entities

14 Total Renewable Resources

$\begin{array}{lll}0 & 0 & 0\end{array}$

0

$\begin{array}{llll}0 & 0 & 0 & 0\end{array}$

0

0

0

0

$\begin{array}{rrrrrrrrrrrrrrr}27 & 27 & 27 & 28 & 29 & 31 & 32 & 31 & 31 & 30 & 30 & 27 & 27 & 27 & 29 \\ 6.2 & 6.2 & 6.2 & 6.2 & 6.2 & 6.2 & 6.2 & 6.2 & 6.2 & 6.2 & 6.2 & 6.2 & 6.2 & 6.2 & 6.2 \\ 46 & 46 & 46 & 46 & 48 & 48 & 49 & 48 & 47 & 46 & 46 & 24 & 46 & 46 & 45 \\ 0 & 0 & 0 & 0 & 0 & 0 & 0 & 0 & 0 & 0 & 0 & 0 & 0 & 0 & 0 \\ \mathbf{7 9} & \mathbf{7 9} & \mathbf{7 9} & \mathbf{8 1} & \mathbf{8 4} & \mathbf{8 6} & \mathbf{8 7} & \mathbf{8 5} & \mathbf{8 4} & \mathbf{8 2} & \mathbf{8 2} & \mathbf{5 7} & \mathbf{7 9} & \mathbf{7 9} & \mathbf{8 0}\end{array}$


Table A-8: Regional Renewable Resources

PNW Loads and Resources Study

2006 - 2007 Operating Year

$3 / 31 / 2004$ 2003 White Book

Average Energy in Megawatts Aug Aug16 Sep Oct Nov Dec Jan Feb Mar Apr1 Apr16 May Jun Jul Avg

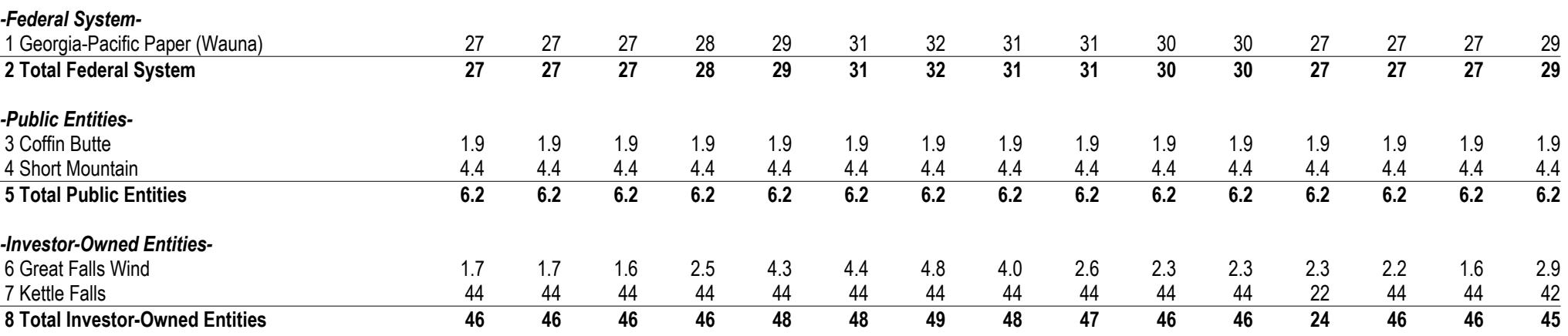

-Other Entities-

9 Total Other Entities

-Renewable Resources-

10 Federal System

11 Public Entities

12 Investor-Owned Entities

13 Other Entities

14 Total Renewable Resources

$\begin{array}{lll}0 & 0 & 0\end{array}$

0

$\begin{array}{llll}0 & 0 & 0 & 0\end{array}$

0

0

0

0

$\begin{array}{rrrrrrrrrrrrrrr}27 & 27 & 27 & 28 & 29 & 31 & 32 & 31 & 31 & 30 & 30 & 27 & 27 & 27 & 29 \\ 6.2 & 6.2 & 6.2 & 6.2 & 6.2 & 6.2 & 6.2 & 6.2 & 6.2 & 6.2 & 6.2 & 6.2 & 6.2 & 6.2 & 6.2 \\ 46 & 46 & 46 & 46 & 48 & 48 & 49 & 48 & 47 & 46 & 46 & 24 & 46 & 46 & 45 \\ 0 & 0 & 0 & 0 & 0 & 0 & 0 & 0 & 0 & 0 & 0 & 0 & 0 & 0 & 0 \\ \mathbf{7 9} & \mathbf{7 9} & \mathbf{7 9} & \mathbf{8 1} & \mathbf{8 4} & \mathbf{8 6} & \mathbf{8 7} & \mathbf{8 5} & \mathbf{8 4} & \mathbf{8 2} & \mathbf{8 2} & \mathbf{5 7} & \mathbf{7 9} & \mathbf{7 9} & \mathbf{8 0}\end{array}$


Table A-8: Regional Renewable Resources

PNW Loads and Resources Study

2007 - 2008 Operating Year

$3 / 31 / 2004$ 2003 White Book

Average Energy in Megawatts Aug Aug16 Sep Oct Nov Dec Jan Feb Mar Apr1 Apr16 May Jun Jul Avg

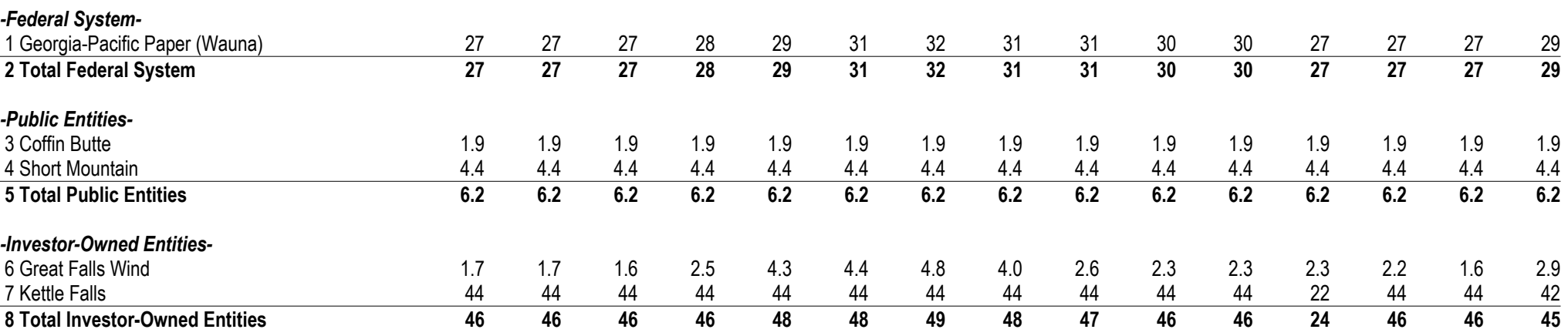

-Other Entities-

9 Total Other Entities

-Renewable Resources-

10 Federal System

11 Public Entities

12 Investor-Owned Entities

13 Other Entities

14 Total Renewable Resources

$0 \quad 0 \quad 0$

0

$\begin{array}{llll}0 & 0 & 0 & 0\end{array}$

0

0

0

0

$\begin{array}{rrrrrrrrrrrrrrr}27 & 27 & 27 & 28 & 29 & 31 & 32 & 31 & 31 & 30 & 30 & 27 & 27 & 27 & 29 \\ 6.2 & 6.2 & 6.2 & 6.2 & 6.2 & 6.2 & 6.2 & 6.2 & 6.2 & 6.2 & 6.2 & 6.2 & 6.2 & 6.2 & 6.2 \\ 46 & 46 & 46 & 46 & 48 & 48 & 49 & 48 & 47 & 46 & 46 & 24 & 46 & 46 & 45 \\ 0 & 0 & 0 & 0 & 0 & 0 & 0 & 0 & 0 & 0 & 0 & 0 & 0 & 0 & 0 \\ \mathbf{7 9} & \mathbf{7 9} & \mathbf{7 9} & \mathbf{8 1} & \mathbf{8 4} & \mathbf{8 6} & \mathbf{8 7} & \mathbf{8 5} & \mathbf{8 4} & \mathbf{8 2} & \mathbf{8 2} & \mathbf{5 7} & \mathbf{7 9} & \mathbf{7 9} & \mathbf{8 0}\end{array}$


Table A-8: Regional Renewable Resources

PNW Loads and Resources Study

2008 - 2009 Operating Year

$3 / 31 / 2004$ 2003 White Book

Average Energy in Megawatts Aug Aug16 Sep Oct Nov Dec Jan Feb Mar Apr1 Apr16 May Jun Jul Avg

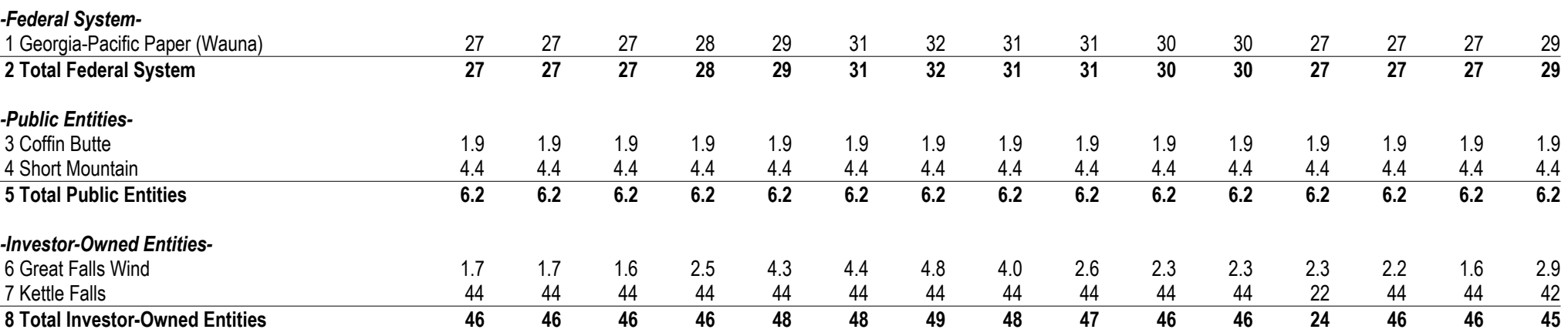

-Other Entities-

9 Total Other Entities

-Renewable Resources-

10 Federal System

11 Public Entities

12 Investor-Owned Entities

13 Other Entities

14 Total Renewable Resources

$\begin{array}{lll}0 & 0 & 0\end{array}$

0

$\begin{array}{llll}0 & 0 & 0 & 0\end{array}$

0

0

0

0

$\begin{array}{rrrrrrrrrrrrrrr}27 & 27 & 27 & 28 & 29 & 31 & 32 & 31 & 31 & 30 & 30 & 27 & 27 & 27 & 29 \\ 6.2 & 6.2 & 6.2 & 6.2 & 6.2 & 6.2 & 6.2 & 6.2 & 6.2 & 6.2 & 6.2 & 6.2 & 6.2 & 6.2 & 6.2 \\ 46 & 46 & 46 & 46 & 48 & 48 & 49 & 48 & 47 & 46 & 46 & 24 & 46 & 46 & 45 \\ 0 & 0 & 0 & 0 & 0 & 0 & 0 & 0 & 0 & 0 & 0 & 0 & 0 & 0 & 0 \\ \mathbf{7 9} & \mathbf{7 9} & \mathbf{7 9} & \mathbf{8 1} & \mathbf{8 4} & \mathbf{8 6} & \mathbf{8 7} & \mathbf{8 5} & \mathbf{8 4} & \mathbf{8 2} & \mathbf{8 2} & \mathbf{5 7} & \mathbf{7 9} & \mathbf{7 9} & \mathbf{8 0}\end{array}$


Table A-8: Regional Renewable Resources

PNW Loads and Resources Study

2009 - 2010 Operating Year

$3 / 31 / 2004$ 2003 White Book

Average Energy in Megawatts Aug Aug16 Sep Oct Nov Dec Jan Feb Mar Apr1 Apr16 May Jun Jul Avg

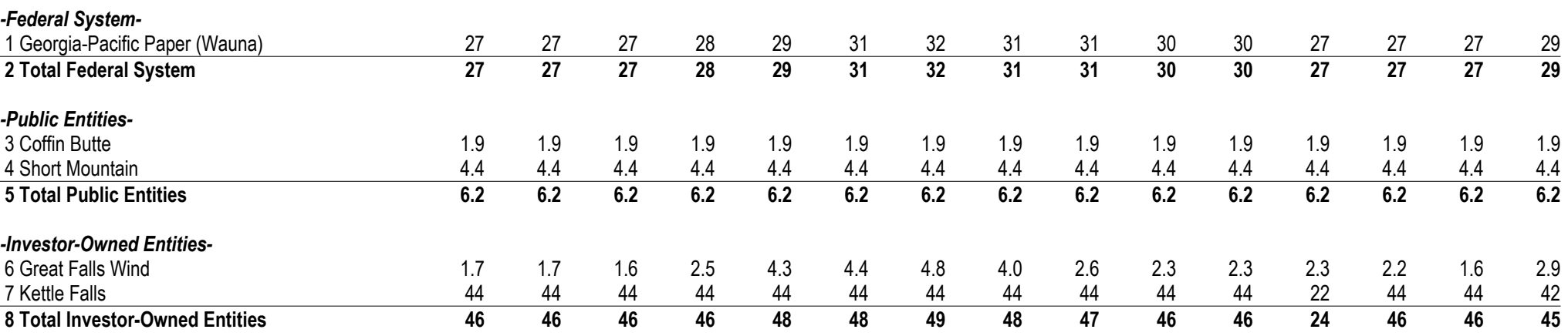

-Other Entities-

9 Total Other Entities

-Renewable Resources-

10 Federal System

11 Public Entities

12 Investor-Owned Entities

13 Other Entities

14 Total Renewable Resources

$0 \quad 0 \quad 0$

0

$\begin{array}{llll}0 & 0 & 0 & 0\end{array}$

0

0

0

0

$\begin{array}{rrrrrrrrrrrrrrr}27 & 27 & 27 & 28 & 29 & 31 & 32 & 31 & 31 & 30 & 30 & 27 & 27 & 27 & 29 \\ 6.2 & 6.2 & 6.2 & 6.2 & 6.2 & 6.2 & 6.2 & 6.2 & 6.2 & 6.2 & 6.2 & 6.2 & 6.2 & 6.2 & 6.2 \\ 46 & 46 & 46 & 46 & 48 & 48 & 49 & 48 & 47 & 46 & 46 & 24 & 46 & 46 & 45 \\ 0 & 0 & 0 & 0 & 0 & 0 & 0 & 0 & 0 & 0 & 0 & 0 & 0 & 0 & 0 \\ \mathbf{7 9} & \mathbf{7 9} & \mathbf{7 9} & \mathbf{8 1} & \mathbf{8 4} & \mathbf{8 6} & \mathbf{8 7} & \mathbf{8 5} & \mathbf{8 4} & \mathbf{8 2} & \mathbf{8 2} & \mathbf{5 7} & \mathbf{7 9} & \mathbf{7 9} & \mathbf{8 0}\end{array}$


Table A-8: Regional Renewable Resources

PNW Loads and Resources Study

2010 - 2011 Operating Year

$3 / 31 / 2004$ 2003 White Book

Average Energy in Megawatts Aug Aug16 Sep Oct Nov Dec Jan Feb Mar Apr1 Apr16 May Jun Jul Avg

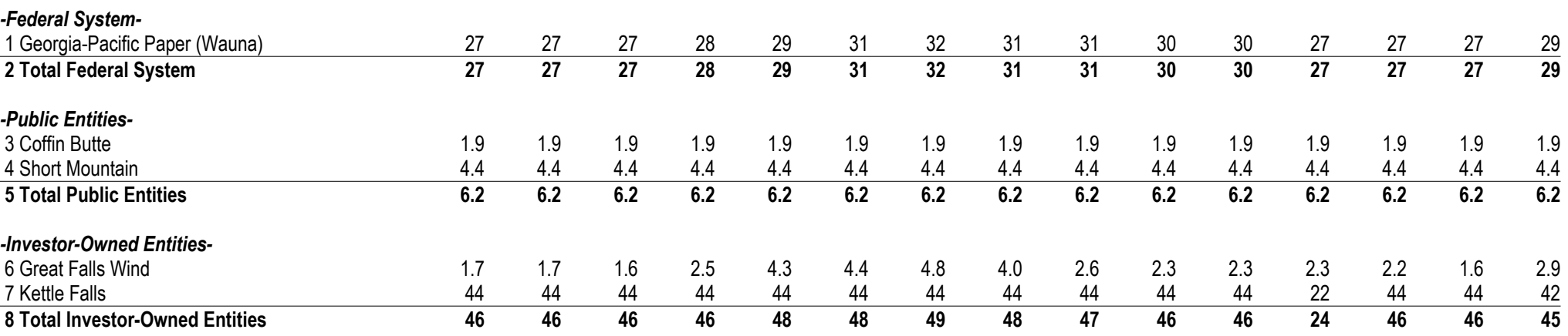

-Other Entities-

9 Total Other Entities

-Renewable Resources-

10 Federal System

11 Public Entities

12 Investor-Owned Entities

13 Other Entities

14 Total Renewable Resources

$\begin{array}{lll}0 & 0 & 0\end{array}$

0

$\begin{array}{llll}0 & 0 & 0 & 0\end{array}$

0

0

0

0

$\begin{array}{rrrrrrrrrrrrrrr}27 & 27 & 27 & 28 & 29 & 31 & 32 & 31 & 31 & 30 & 30 & 27 & 27 & 27 & 29 \\ 6.2 & 6.2 & 6.2 & 6.2 & 6.2 & 6.2 & 6.2 & 6.2 & 6.2 & 6.2 & 6.2 & 6.2 & 6.2 & 6.2 & 6.2 \\ 46 & 46 & 46 & 46 & 48 & 48 & 49 & 48 & 47 & 46 & 46 & 24 & 46 & 46 & 45 \\ 0 & 0 & 0 & 0 & 0 & 0 & 0 & 0 & 0 & 0 & 0 & 0 & 0 & 0 & 0 \\ \mathbf{7 9} & \mathbf{7 9} & \mathbf{7 9} & \mathbf{8 1} & \mathbf{8 4} & \mathbf{8 6} & \mathbf{8 7} & \mathbf{8 5} & \mathbf{8 4} & \mathbf{8 2} & \mathbf{8 2} & \mathbf{5 7} & \mathbf{7 9} & \mathbf{7 9} & \mathbf{8 0}\end{array}$


Table A-8: Regional Renewable Resources

PNW Loads and Resources Study

2011 - 2012 Operating Year

$3 / 31 / 2004$ 2003 White Book

Average Energy in Megawatts Aug Aug16 Sep Oct Nov Dec Jan Feb Mar Apr1 Apr16 May Jun Jul Avg

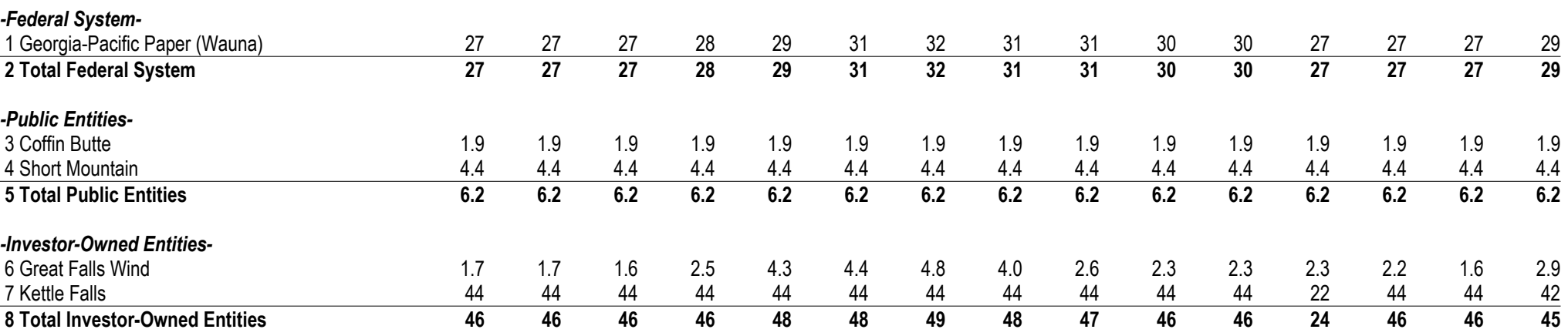

-Other Entities-

9 Total Other Entities

-Renewable Resources-

10 Federal System

11 Public Entities

12 Investor-Owned Entities

13 Other Entities

14 Total Renewable Resources

$\begin{array}{lll}0 & 0 & 0\end{array}$

0

$\begin{array}{llll}0 & 0 & 0 & 0\end{array}$

0

0

0

0

$\begin{array}{rrrrrrrrrrrrrrr}27 & 27 & 27 & 28 & 29 & 31 & 32 & 31 & 31 & 30 & 30 & 27 & 27 & 27 & 29 \\ 6.2 & 6.2 & 6.2 & 6.2 & 6.2 & 6.2 & 6.2 & 6.2 & 6.2 & 6.2 & 6.2 & 6.2 & 6.2 & 6.2 & 6.2 \\ 46 & 46 & 46 & 46 & 48 & 48 & 49 & 48 & 47 & 46 & 46 & 24 & 46 & 46 & 45 \\ 0 & 0 & 0 & 0 & 0 & 0 & 0 & 0 & 0 & 0 & 0 & 0 & 0 & 0 & 0 \\ \mathbf{7 9} & \mathbf{7 9} & \mathbf{7 9} & \mathbf{8 1} & \mathbf{8 4} & \mathbf{8 6} & \mathbf{8 7} & \mathbf{8 5} & \mathbf{8 4} & \mathbf{8 2} & \mathbf{8 2} & \mathbf{5 7} & \mathbf{7 9} & \mathbf{7 9} & \mathbf{8 0}\end{array}$


Table A-8: Regional Renewable Resources

PNW Loads and Resources Study

2012 - 2013 Operating Year

$3 / 31 / 2004$ 2003 White Book

Average Energy in Megawatts Aug Aug16 Sep Oct Nov Dec Jan Feb Mar Apr1 Apr16 May Jun Jul Avg

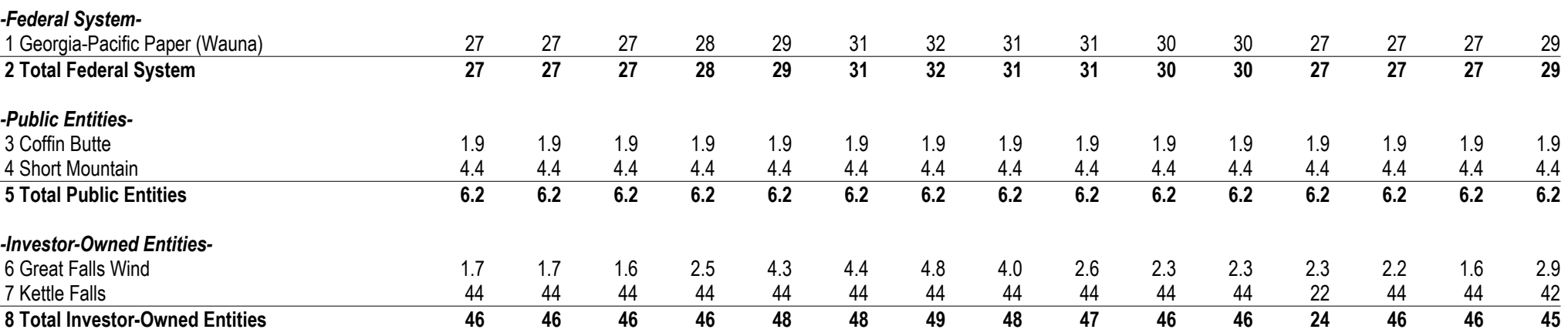

-Other Entities-

9 Total Other Entities

-Renewable Resources-

10 Federal System

11 Public Entities

12 Investor-Owned Entities

13 Other Entities

14 Total Renewable Resources

$\begin{array}{lll}0 & 0 & 0\end{array}$

0

$\begin{array}{llll}0 & 0 & 0 & 0\end{array}$

0

0

0

0

$\begin{array}{rrrrrrrrrrrrrrr}27 & 27 & 27 & 28 & 29 & 31 & 32 & 31 & 31 & 30 & 30 & 27 & 27 & 27 & 29 \\ 6.2 & 6.2 & 6.2 & 6.2 & 6.2 & 6.2 & 6.2 & 6.2 & 6.2 & 6.2 & 6.2 & 6.2 & 6.2 & 6.2 & 6.2 \\ 46 & 46 & 46 & 46 & 48 & 48 & 49 & 48 & 47 & 46 & 46 & 24 & 46 & 46 & 45 \\ 0 & 0 & 0 & 0 & 0 & 0 & 0 & 0 & 0 & 0 & 0 & 0 & 0 & 0 & 0 \\ \mathbf{7 9} & \mathbf{7 9} & \mathbf{7 9} & \mathbf{8 1} & \mathbf{8 4} & \mathbf{8 6} & \mathbf{8 7} & \mathbf{8 5} & \mathbf{8 4} & \mathbf{8 2} & \mathbf{8 2} & \mathbf{5 7} & \mathbf{7 9} & \mathbf{7 9} & \mathbf{8 0}\end{array}$


Table A-8: Regional Renewable Resources

PNW Loads and Resources Study

2013 - 2014 Operating Year

$3 / 31 / 2004$ 2003 White Book

Average Energy in Megawatts Aug Aug16 Sep Oct Nov Dec Jan Feb Mar Apr1 Apr16 May Jun Jul Avg

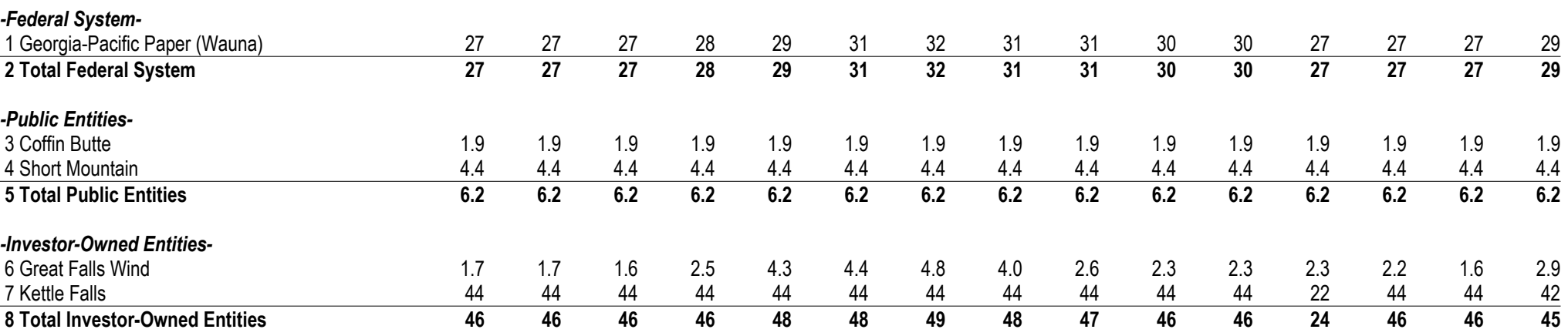

-Other Entities-

9 Total Other Entities

-Renewable Resources-

10 Federal System

11 Public Entities

12 Investor-Owned Entities

13 Other Entities

14 Total Renewable Resources

$\begin{array}{lll}0 & 0 & 0\end{array}$

0

$\begin{array}{llll}0 & 0 & 0 & 0\end{array}$

0

0

0

0

$\begin{array}{rrrrrrrrrrrrrrr}27 & 27 & 27 & 28 & 29 & 31 & 32 & 31 & 31 & 30 & 30 & 27 & 27 & 27 & 29 \\ 6.2 & 6.2 & 6.2 & 6.2 & 6.2 & 6.2 & 6.2 & 6.2 & 6.2 & 6.2 & 6.2 & 6.2 & 6.2 & 6.2 & 6.2 \\ 46 & 46 & 46 & 46 & 48 & 48 & 49 & 48 & 47 & 46 & 46 & 24 & 46 & 46 & 45 \\ 0 & 0 & 0 & 0 & 0 & 0 & 0 & 0 & 0 & 0 & 0 & 0 & 0 & 0 & 0 \\ \mathbf{7 9} & \mathbf{7 9} & \mathbf{7 9} & \mathbf{8 1} & \mathbf{8 4} & \mathbf{8 6} & \mathbf{8 7} & \mathbf{8 5} & \mathbf{8 4} & \mathbf{8 2} & \mathbf{8 2} & \mathbf{5 7} & \mathbf{7 9} & \mathbf{7 9} & \mathbf{8 0}\end{array}$



2003 White Book

May Jun Jul Avg

-Federal System-

1 Total Federal System Dec Jan Feb Mar Apr1 Apr16

-Public Entities-

2 WEYCO Energy Center

3 Total Public Entities

0

0

-Investor_Owned Entities-

4 Coyote Springs \#1

5 Coyote Springs \#2

6 Georgia-Pacific Paper (Camas)

7 Hermiston Cogeneration

8 Thompson River Co-Gen

9 Total Investor-Owned Entities

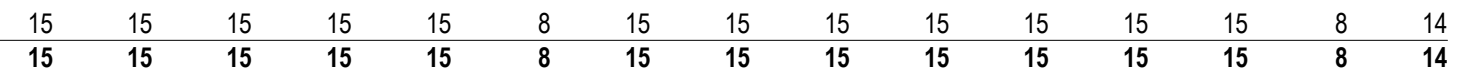

$0 \quad 0$

-Other Entities-

10 Coyote Springs \#2

11 Hermiston Power Project

12 Klamath Cogneration Project

13 Longview Fibre

14 Total Other Entities

-Cogeneration-

15 Federal System

16 Public Entities

17 Investor-Owned Entities

18 Other Entities

\begin{tabular}{|c|c|c|c|c|c|c|c|c|c|c|c|c|c|}
\hline 48 & 48 & 48 & 48 & 48 & 48 & 48 & 48 & 48 & 48 & 0 & 48 & 48 & 44 \\
\hline 9.6 & 9.6 & 9.6 & 9.6 & 9.6 & 9.6 & 9.6 & 9.6 & 9.6 & 9.6 & 9.6 & 9.6 & 9.6 & 9.6 \\
\hline 863 & 867 & 869 & 876 & 880 & 881 & 878 & 874 & 870 & 870 & 240 & 865 & 863 & 819 \\
\hline
\end{tabular}

19 Total Cogeneration

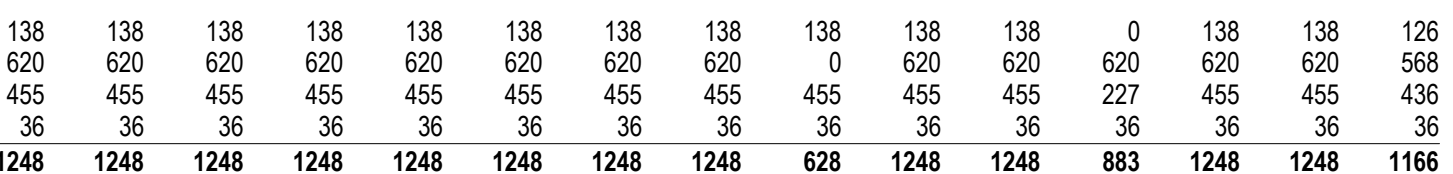



2003 White Book

May Jun Jul Avg

-Federal System-

1 Total Federal System Dec Jan Feb Mar Apr1 Apr16

-Public Entities-

2 WEYCO Energy Center

3 Total Public Entities

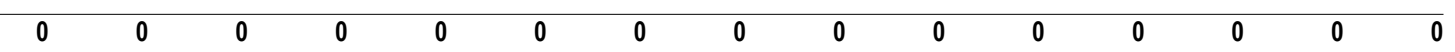

-Investor_Owned Entities-

4 Coyote Springs \#1

5 Coyote Springs \#2

6 Georgia-Pacific Paper (Camas)

7 Hermiston Cogeneration

8 Thompson River Co-Gen

9 Total Investor-Owned Entities

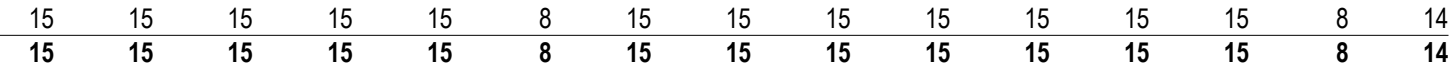

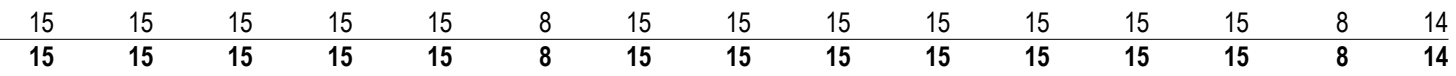

$\begin{array}{lllllllllllllll}15 & 15 & 15 & 15 & 15 & 8 & 15 & 15 & 15 & 15 & 15 & 15 & 15 & 8 & 14 \\ 15 & \mathbf{1 5} & \mathbf{1 5} & \mathbf{1 5} & \mathbf{1 5} & \mathbf{8} & \mathbf{1 5} & \mathbf{1 5} & \mathbf{1 5} & \mathbf{1 5} & \mathbf{1 5} & \mathbf{1 5} & \mathbf{1 5} & \mathbf{8} & \mathbf{1 5}\end{array}$

-Other Entities-

10 Coyote Springs \#2

11 Hermiston Power Project

12 Klamath Cogneration Project

13 Longview Fibre

14 Total Other Entities

-Cogeneration-

15 Federal System

16 Public Entities

17 Investor-Owned Entities

18 Other Entities

\begin{tabular}{|c|c|c|c|c|c|c|c|c|c|c|c|c|c|c|}
\hline 225 & 225 & 229 & 231 & 238 & 242 & 243 & 240 & 236 & 232 & 232 & 230 & 227 & 225 & 233 \\
\hline 138 & 138 & 138 & 138 & 138 & 138 & 138 & 138 & 138 & 138 & 138 & 0 & 138 & 138 & 126 \\
\hline 48 & 48 & 48 & 48 & 48 & 48 & 48 & 48 & 48 & 48 & 48 & 0 & 48 & 48 & 44 \\
\hline 443 & 443 & 443 & 443 & 443 & 443 & 443 & 443 & 443 & 443 & 443 & 0 & 443 & 443 & 406 \\
\hline 9.6 & 9.6 & 9.6 & 9.6 & 9.6 & 9.6 & 9.6 & 9.6 & 9.6 & 9.6 & 9.6 & 9.6 & 9.6 & 9.6 & 9.6 \\
\hline 863 & 863 & 867 & 869 & 876 & 880 & 881 & 878 & 874 & 870 & 870 & 240 & 865 & 863 & 819 \\
\hline
\end{tabular}

19 Total Cogeneration

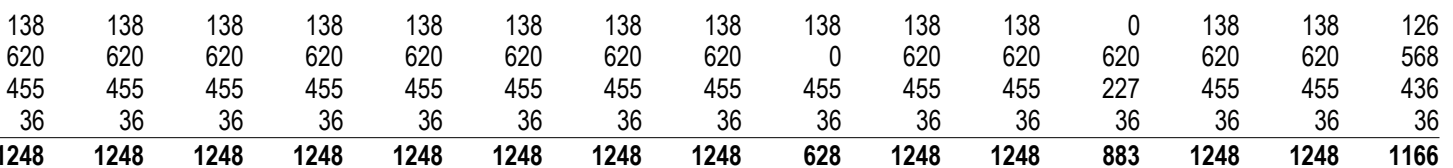



2003 White Book

May Jun Jul Avg

-Federal System-

1 Total Federal System Dec Jan Feb Mar Apr1 Apr16

-Public Entities-

2 WEYCO Energy Center

3 Total Public Entities

$\begin{array}{llllllllllllll}0 & 0 & 0 & 0 & 0 & 0 & 0 & 0 & 0 & 0 & 0 & 0 & 0 & 0\end{array}$

-Investor_Owned Entities-

4 Coyote Springs \#1

5 Coyote Springs \#2

6 Georgia-Pacific Paper (Camas)

7 Hermiston Cogeneration

8 Thompson River Co-Gen

9 Total Investor-Owned Entities

$\begin{array}{lllllllllllllll}15 & 15 & 15 & 15 & 15 & 8 & 15 & 15 & 15 & 15 & 15 & 15 & 15 & 8 & 14 \\ 15 & \mathbf{1 5} & \mathbf{1 5} & \mathbf{1 5} & \mathbf{1 5} & \mathbf{8} & \mathbf{1 5} & \mathbf{1 5} & \mathbf{1 5} & \mathbf{1 5} & \mathbf{1 5} & \mathbf{1 5} & \mathbf{1 5} & \mathbf{8} & \mathbf{1 4}\end{array}$

$15 \quad 15 \quad 15$

-Other Entities-

10 Coyote Springs \#2

11 Hermiston Power Project

12 Klamath Cogneration Project

13 Longview Fibre

14 Total Other Entities

-Cogeneration-

15 Federal System

16 Public Entities

17 Investor-Owned Entities

18 Other Entities

\begin{tabular}{|c|c|c|c|c|c|c|c|c|c|c|c|c|c|c|}
\hline 225 & 225 & 229 & 231 & 238 & 242 & 243 & 240 & 236 & 232 & 232 & 230 & 227 & 225 & 233 \\
\hline 138 & 138 & 138 & 138 & 138 & 138 & 138 & 138 & 138 & 138 & 138 & 0 & 138 & 138 & 126 \\
\hline 48 & 48 & 48 & 48 & 48 & 48 & 48 & 48 & 48 & 48 & 48 & 0 & 48 & 48 & 44 \\
\hline 443 & 443 & 443 & 443 & 443 & 443 & 443 & 443 & 443 & 443 & 443 & 0 & 443 & 443 & 406 \\
\hline 9.6 & 9.6 & 9.6 & 9.6 & 9.6 & 9.6 & 9.6 & 9.6 & 9.6 & 9.6 & 9.6 & 9.6 & 9.6 & 9.6 & 9.6 \\
\hline 863 & 863 & 867 & 869 & 876 & 880 & 881 & 878 & 874 & 870 & 870 & 240 & 865 & 863 & 819 \\
\hline
\end{tabular}

19 Total Cogeneration

\begin{tabular}{|c|c|c|c|c|c|c|c|c|c|c|c|c|c|}
\hline 138 & 138 & 138 & 138 & 138 & 138 & 138 & 138 & 138 & 138 & 0 & 138 & 138 & 126 \\
\hline 620 & 620 & 620 & 620 & 620 & 620 & 620 & 0 & 620 & 620 & 620 & 620 & 620 & 568 \\
\hline 36 & 36 & 36 & 36 & 36 & 36 & 36 & 36 & 36 & 36 & 36 & 36 & 36 & \\
\hline
\end{tabular}

\begin{tabular}{rrrrrrrrrrrrrrr}
0 & 0 & 0 & 0 & 0 & 0 & 0 & 0 & 0 & 0 & 0 & 0 & 0 & 0 & 0 \\
15 & 15 & 15 & 15 & 15 & 8 & 15 & 15 & 15 & 15 & 15 & 15 & 15 & 8 & 14 \\
863 & 863 & 867 & 869 & 876 & 880 & 881 & 878 & 874 & 870 & 870 & 240 & 865 & 863 & 819 \\
1248 & 1248 & 1248 & 1248 & 1248 & 1248 & 1248 & 1248 & 628 & 1248 & 1248 & 883 & 1248 & 1248 & 1166 \\
\hline 2126 & 2126 & 2130 & 2132 & 2139 & 2136 & $\mathbf{2 1 4 4}$ & $\mathbf{2 1 4 1}$ & $\mathbf{1 5 1 8}$ & $\mathbf{2 1 3 3}$ & $\mathbf{2 1 3 3}$ & $\mathbf{1 1 3 7}$ & $\mathbf{2 1 2 8}$ & $\mathbf{2 1 1 9}$ & $\mathbf{1 9 9 9}$
\end{tabular}



2003 White Book

May Jun Jul Avg

-Federal System-

1 Total Federal System Dec Jan Feb Mar Apr1 Apr16

-Public Entities-

2 WEYCO Energy Center

3 Total Public Entities

$\begin{array}{llllllllllllll}0 & 0 & 0 & 0 & 0 & 0 & 0 & 0 & 0 & 0 & 0 & 0 & 0 & 0\end{array}$

-Investor_Owned Entities-

4 Coyote Springs \#1

5 Coyote Springs \#2

6 Georgia-Pacific Paper (Camas)

7 Hermiston Cogeneration

8 Thompson River Co-Gen

9 Total Investor-Owned Entities

$\begin{array}{lllllllllllllll}15 & 15 & 15 & 15 & 15 & 8 & 15 & 15 & 15 & 15 & 15 & 15 & 15 & 8 & 14 \\ 15 & \mathbf{1 5} & \mathbf{1 5} & \mathbf{1 5} & \mathbf{1 5} & \mathbf{8} & \mathbf{1 5} & \mathbf{1 5} & \mathbf{1 5} & \mathbf{1 5} & \mathbf{1 5} & \mathbf{1 5} & \mathbf{1 5} & \mathbf{8} & \mathbf{1 4}\end{array}$

$15 \quad 15 \quad 15$

-Other Entities-

10 Coyote Springs \#2

11 Hermiston Power Project

12 Klamath Cogneration Project

13 Longview Fibre

14 Total Other Entities

-Cogeneration-

15 Federal System

16 Public Entities

17 Investor-Owned Entities

18 Other Entities

\begin{tabular}{|c|c|c|c|c|c|c|c|c|c|c|c|c|c|c|}
\hline 225 & 225 & 229 & 231 & 238 & 242 & 243 & 240 & 236 & 232 & 232 & 230 & 227 & 225 & 233 \\
\hline 138 & 138 & 138 & 138 & 138 & 138 & 138 & 138 & 138 & 138 & 138 & 0 & 138 & 138 & 126 \\
\hline 48 & 48 & 48 & 48 & 48 & 48 & 48 & 48 & 48 & 48 & 48 & 0 & 48 & 48 & 44 \\
\hline 443 & 443 & 443 & 443 & 443 & 443 & 443 & 443 & 443 & 443 & 443 & 0 & 443 & 443 & 406 \\
\hline 9.6 & 9.6 & 9.6 & 9.6 & 9.6 & 9.6 & 9.6 & 9.6 & 9.6 & 9.6 & 9.6 & 9.6 & 9.6 & 9.6 & 9.6 \\
\hline 863 & 863 & 867 & 869 & 876 & 880 & 881 & 878 & 874 & 870 & 870 & 240 & 865 & 863 & 819 \\
\hline
\end{tabular}

19 Total Cogeneration

\begin{tabular}{|c|c|c|c|c|c|c|c|c|c|c|c|c|c|}
\hline 138 & 138 & 138 & 138 & 138 & 138 & 138 & 138 & 138 & 138 & 0 & 138 & 138 & 126 \\
\hline 620 & 620 & 620 & 620 & 620 & 620 & 620 & 0 & 620 & 620 & 620 & 620 & 620 & 568 \\
\hline 36 & 36 & 36 & 36 & 36 & 36 & 36 & 36 & 36 & 36 & 36 & 36 & 36 & \\
\hline
\end{tabular}

\begin{tabular}{rrrrrrrrrrrrrrr}
0 & 0 & 0 & 0 & 0 & 0 & 0 & 0 & 0 & 0 & 0 & 0 & 0 & 0 & 0 \\
15 & 15 & 15 & 15 & 15 & 8 & 15 & 15 & 15 & 15 & 15 & 15 & 15 & 8 & 14 \\
863 & 863 & 867 & 869 & 876 & 880 & 881 & 878 & 874 & 870 & 870 & 240 & 865 & 863 & 819 \\
1248 & 1248 & 1248 & 1248 & 1248 & 1248 & 1248 & 1248 & 628 & 1248 & 1248 & 883 & 1248 & 1248 & 1166 \\
\hline 2126 & 2126 & 2130 & 2132 & 2139 & 2136 & $\mathbf{2 1 4 4}$ & $\mathbf{2 1 4 1}$ & $\mathbf{1 5 1 8}$ & $\mathbf{2 1 3 3}$ & $\mathbf{2 1 3 3}$ & $\mathbf{1 1 3 7}$ & $\mathbf{2 1 2 8}$ & $\mathbf{2 1 1 9}$ & $\mathbf{1 9 9 9}$
\end{tabular}



2003 White Book

May Jun Jul Avg

-Federal System-

1 Total Federal System Dec Jan Feb Mar Apr1 Apr16

-Public Entities-

2 WEYCO Energy Center

3 Total Public Entities

$\begin{array}{llllllllllllll}0 & 0 & 0 & 0 & 0 & 0 & 0 & 0 & 0 & 0 & 0 & 0 & 0 & 0\end{array}$

-Investor_Owned Entities-

4 Coyote Springs \#1

5 Coyote Springs \#2

6 Georgia-Pacific Paper (Camas)

7 Hermiston Cogeneration

8 Thompson River Co-Gen

9 Total Investor-Owned Entities

$\begin{array}{lllllllllllllll}15 & 15 & 15 & 15 & 15 & 8 & 15 & 15 & 15 & 15 & 15 & 15 & 15 & 8 & 14 \\ 15 & \mathbf{1 5} & \mathbf{1 5} & \mathbf{1 5} & \mathbf{1 5} & \mathbf{8} & \mathbf{1 5} & \mathbf{1 5} & \mathbf{1 5} & \mathbf{1 5} & \mathbf{1 5} & \mathbf{1 5} & \mathbf{1 5} & \mathbf{8} & \mathbf{1 4}\end{array}$

$15 \quad 15 \quad 15$

-Other Entities-

10 Coyote Springs \#2

11 Hermiston Power Project

12 Klamath Cogneration Project

13 Longview Fibre

14 Total Other Entities

-Cogeneration-

15 Federal System

16 Public Entities

17 Investor-Owned Entities

18 Other Entities

\begin{tabular}{|c|c|c|c|c|c|c|c|c|c|c|c|c|c|c|}
\hline 225 & 225 & 229 & 231 & 238 & 242 & 243 & 240 & 236 & 232 & 232 & 230 & 227 & 225 & 233 \\
\hline 138 & 138 & 138 & 138 & 138 & 138 & 138 & 138 & 138 & 138 & 138 & 0 & 138 & 138 & 126 \\
\hline 48 & 48 & 48 & 48 & 48 & 48 & 48 & 48 & 48 & 48 & 48 & 0 & 48 & 48 & 44 \\
\hline 443 & 443 & 443 & 443 & 443 & 443 & 443 & 443 & 443 & 443 & 443 & 0 & 443 & 443 & 406 \\
\hline 9.6 & 9.6 & 9.6 & 9.6 & 9.6 & 9.6 & 9.6 & 9.6 & 9.6 & 9.6 & 9.6 & 9.6 & 9.6 & 9.6 & 9.6 \\
\hline 863 & 863 & 867 & 869 & 876 & 880 & 881 & 878 & 874 & 870 & 870 & 240 & 865 & 863 & 819 \\
\hline
\end{tabular}

19 Total Cogeneration

\begin{tabular}{|c|c|c|c|c|c|c|c|c|c|c|c|c|c|}
\hline 138 & 138 & 138 & 138 & 138 & 138 & 138 & 138 & 138 & 138 & 0 & 138 & 138 & 126 \\
\hline 620 & 620 & 620 & 620 & 620 & 620 & 620 & 0 & 620 & 620 & 620 & 620 & 620 & 568 \\
\hline 36 & 36 & 36 & 36 & 36 & 36 & 36 & 36 & 36 & 36 & 36 & 36 & 36 & \\
\hline
\end{tabular}

\begin{tabular}{rrrrrrrrrrrrrrr}
0 & 0 & 0 & 0 & 0 & 0 & 0 & 0 & 0 & 0 & 0 & 0 & 0 & 0 & 0 \\
15 & 15 & 15 & 15 & 15 & 8 & 15 & 15 & 15 & 15 & 15 & 15 & 15 & 8 & 14 \\
863 & 863 & 867 & 869 & 876 & 880 & 881 & 878 & 874 & 870 & 870 & 240 & 865 & 863 & 819 \\
1248 & 1248 & 1248 & 1248 & 1248 & 1248 & 1248 & 1248 & 628 & 1248 & 1248 & 883 & 1248 & 1248 & 1166 \\
\hline 2126 & 2126 & 2130 & 2132 & 2139 & 2136 & $\mathbf{2 1 4 4}$ & $\mathbf{2 1 4 1}$ & $\mathbf{1 5 1 8}$ & $\mathbf{2 1 3 3}$ & $\mathbf{2 1 3 3}$ & $\mathbf{1 1 3 7}$ & $\mathbf{2 1 2 8}$ & $\mathbf{2 1 1 9}$ & $\mathbf{1 9 9 9}$
\end{tabular}



2003 White Book

May Jun Jul Avg

-Federal System-

1 Total Federal System Dec Jan Feb Mar Apr1 Apr16

-Public Entities-

2 WEYCO Energy Center

3 Total Public Entities

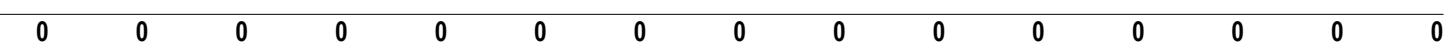

-Investor_Owned Entities-

4 Coyote Springs \#1

5 Coyote Springs \#2

6 Georgia-Pacific Paper (Camas)

7 Hermiston Cogeneration

8 Thompson River Co-Gen

9 Total Investor-Owned Entities

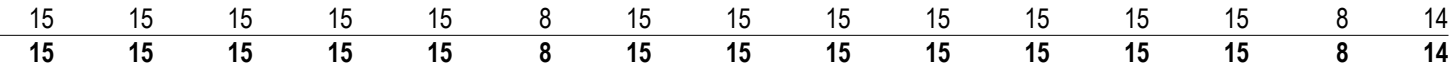

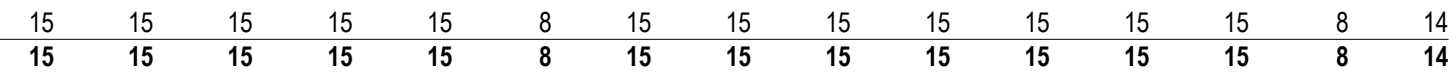

$\begin{array}{lllllllllllllll}15 & 15 & 15 & 15 & 15 & 8 & 15 & 15 & 15 & 15 & 15 & 15 & 15 & 8 & 14 \\ 15 & \mathbf{1 5} & \mathbf{1 5} & \mathbf{1 5} & \mathbf{1 5} & \mathbf{8} & \mathbf{1 5} & \mathbf{1 5} & \mathbf{1 5} & \mathbf{1 5} & \mathbf{1 5} & \mathbf{1 5} & \mathbf{1 5} & \mathbf{8} & \mathbf{1 5}\end{array}$

-Other Entities-

10 Coyote Springs \#2

11 Hermiston Power Project

12 Klamath Cogneration Project

13 Longview Fibre

14 Total Other Entities

-Cogeneration-

15 Federal System

16 Public Entities

17 Investor-Owned Entities

18 Other Entities

\begin{tabular}{|c|c|c|c|c|c|c|c|c|c|c|c|c|c|c|}
\hline 225 & 225 & 229 & 231 & 238 & 242 & 243 & 240 & 236 & 232 & 232 & 230 & 227 & 225 & 233 \\
\hline 138 & 138 & 138 & 138 & 138 & 138 & 138 & 138 & 138 & 138 & 138 & 0 & 138 & 138 & 126 \\
\hline 48 & 48 & 48 & 48 & 48 & 48 & 48 & 48 & 48 & 48 & 48 & 0 & 48 & 48 & 44 \\
\hline 443 & 443 & 443 & 443 & 443 & 443 & 443 & 443 & 443 & 443 & 443 & 0 & 443 & 443 & 406 \\
\hline 9.6 & 9.6 & 9.6 & 9.6 & 9.6 & 9.6 & 9.6 & 9.6 & 9.6 & 9.6 & 9.6 & 9.6 & 9.6 & 9.6 & 9.6 \\
\hline 863 & 863 & 867 & 869 & 876 & 880 & 881 & 878 & 874 & 870 & 870 & 240 & 865 & 863 & 819 \\
\hline
\end{tabular}

19 Total Cogeneration

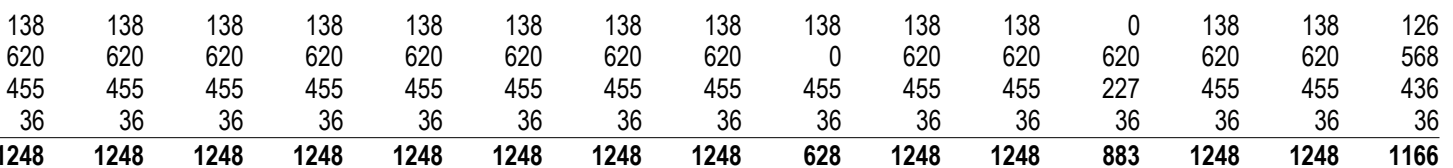



2003 White Book

May Jun Jul Avg

-Federal System-

1 Total Federal System Dec Jan Feb Mar Apr1 Apr16

-Public Entities-

2 WEYCO Energy Center

3 Total Public Entities

0

0

$\begin{array}{lll}0 & 0\end{array}$

$\begin{array}{llll}15 & 15 & 15 & 15 \\ 15 & \mathbf{1 5} & \mathbf{1 5} & 15\end{array}$

-Investor_Owned Entities-

4 Coyote Springs \#1

5 Coyote Springs \#2

6 Georgia-Pacific Paper (Camas)

7 Hermiston Cogeneration

8 Thompson River Co-Gen

9 Total Investor-Owned Entities

$15 \quad 15$

0

0

0

$0 \quad 0$

0

0

$0 \quad 0$

-Other Entities-

10 Coyote Springs \#2

11 Hermiston Power Project

12 Klamath Cogneration Project

13 Longview Fibre

14 Total Other Entities

\begin{tabular}{|c|c|c|c|c|c|c|c|c|c|c|c|c|c|c|}
\hline 48 & 48 & 48 & 48 & 48 & 48 & 48 & 48 & 48 & 48 & 48 & 0 & 48 & 48 & 44 \\
\hline 9.6 & 9.6 & 9.6 & 9.6 & 9.6 & 9.6 & 9.6 & 9.6 & 9.6 & 9.6 & 9.6 & 9.6 & 9.6 & 9.6 & 9.6 \\
\hline 863 & 863 & 867 & 869 & 876 & 880 & 881 & 878 & 874 & 870 & 870 & 240 & 865 & 863 & 819 \\
\hline
\end{tabular}

-Cogeneration-

15 Federal System

16 Public Entities

17 Investor-Owned Entities

18 Other Entities

\begin{tabular}{|c|c|c|c|c|c|c|c|c|c|c|c|c|c|}
\hline 455 & 455 & 455 & 455 & 455 & 455 & 455 & 455 & 455 & 455 & 227 & 455 & 455 & 436 \\
\hline 1248 & 1248 & 1248 & 1248 & 1248 & 1248 & 1248 & 628 & 1248 & 1248 & 883 & 1248 & 1248 & 1166 \\
\hline
\end{tabular}

19 Total Cogeneration

\begin{tabular}{rrrrrrrrrrrrrrr}
0 & 0 & 0 & 0 & 0 & 0 & 0 & 0 & 0 & 0 & 0 & 0 & 0 & 0 & 0 \\
15 & 15 & 15 & 15 & 15 & 8 & 15 & 15 & 15 & 15 & 15 & 15 & 15 & 8 & 14 \\
863 & 863 & 867 & 869 & 876 & 880 & 881 & 878 & 874 & 870 & 870 & 240 & 865 & 863 & 819 \\
1248 & 1248 & 1248 & 1248 & 1248 & 1248 & 1248 & 1248 & 628 & 1248 & 1248 & 883 & 1248 & 1248 & 1166 \\
\hline 2126 & 2126 & 2130 & 2132 & 2139 & 2136 & $\mathbf{2 1 4 4}$ & $\mathbf{2 1 4 1}$ & $\mathbf{1 5 1 8}$ & $\mathbf{2 1 3 3}$ & $\mathbf{2 1 3 3}$ & $\mathbf{1 1 3 7}$ & $\mathbf{2 1 2 8}$ & $\mathbf{2 1 1 9}$ & $\mathbf{1 9 9 9}$
\end{tabular}



2003 White Book

May Jun Jul Avg

-Federal System-

1 Total Federal System Dec Jan Feb Mar Apr1 Apr16

-Public Entities-

2 WEYCO Energy Center

3 Total Public Entities

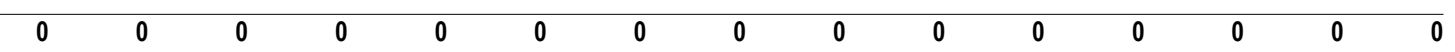

-Investor_Owned Entities-

4 Coyote Springs \#1

5 Coyote Springs \#2

6 Georgia-Pacific Paper (Camas)

7 Hermiston Cogeneration

8 Thompson River Co-Gen

9 Total Investor-Owned Entities

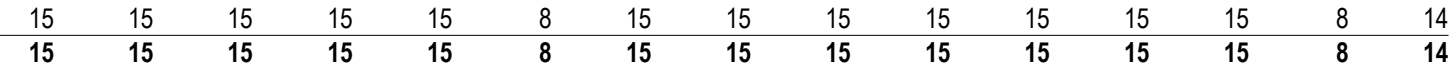

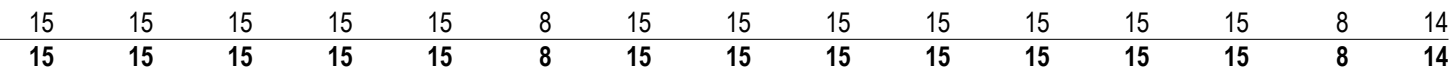

$\begin{array}{lllllllllllllll}15 & 15 & 15 & 15 & 15 & 8 & 15 & 15 & 15 & 15 & 15 & 15 & 15 & 8 & 14 \\ 15 & \mathbf{1 5} & \mathbf{1 5} & \mathbf{1 5} & \mathbf{1 5} & \mathbf{8} & \mathbf{1 5} & \mathbf{1 5} & \mathbf{1 5} & \mathbf{1 5} & \mathbf{1 5} & \mathbf{1 5} & \mathbf{1 5} & \mathbf{8} & \mathbf{1 5}\end{array}$

-Other Entities-

10 Coyote Springs \#2

11 Hermiston Power Project

12 Klamath Cogneration Project

13 Longview Fibre

14 Total Other Entities

-Cogeneration-

15 Federal System

16 Public Entities

17 Investor-Owned Entities

18 Other Entities

\begin{tabular}{|c|c|c|c|c|c|c|c|c|c|c|c|c|c|c|}
\hline 225 & 225 & 229 & 231 & 238 & 242 & 243 & 240 & 236 & 232 & 232 & 230 & 227 & 225 & 233 \\
\hline 138 & 138 & 138 & 138 & 138 & 138 & 138 & 138 & 138 & 138 & 138 & 0 & 138 & 138 & 126 \\
\hline 48 & 48 & 48 & 48 & 48 & 48 & 48 & 48 & 48 & 48 & 48 & 0 & 48 & 48 & 44 \\
\hline 443 & 443 & 443 & 443 & 443 & 443 & 443 & 443 & 443 & 443 & 443 & 0 & 443 & 443 & 406 \\
\hline 9.6 & 9.6 & 9.6 & 9.6 & 9.6 & 9.6 & 9.6 & 9.6 & 9.6 & 9.6 & 9.6 & 9.6 & 9.6 & 9.6 & 9.6 \\
\hline 863 & 863 & 867 & 869 & 876 & 880 & 881 & 878 & 874 & 870 & 870 & 240 & 865 & 863 & 819 \\
\hline
\end{tabular}

19 Total Cogeneration

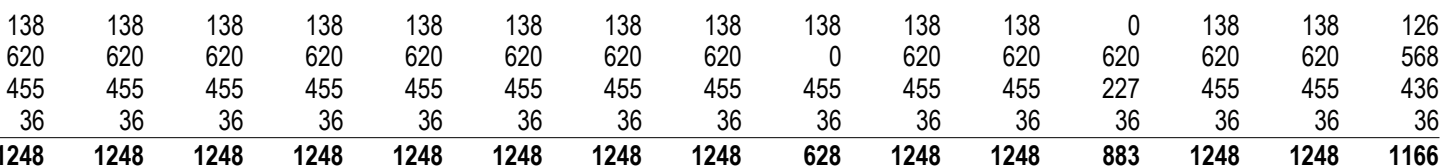



2003 White Book

May Jun Jul Avg

-Federal System-

1 Total Federal System Dec Jan Feb Mar Apr1 Apr16

-Public Entities-

2 WEYCO Energy Center

3 Total Public Entities

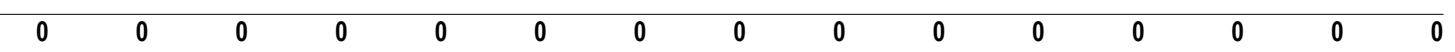

-Investor_Owned Entities-

4 Coyote Springs \#1

5 Coyote Springs \#2

6 Georgia-Pacific Paper (Camas)

7 Hermiston Cogeneration

8 Thompson River Co-Gen

9 Total Investor-Owned Entities

$\begin{array}{lllllllllllllll}15 & 15 & 15 & 15 & 15 & 8 & 15 & 15 & 15 & 15 & 15 & 15 & 15 & 8 & 14 \\ 15 & \mathbf{1 5} & \mathbf{1 5} & \mathbf{1 5} & \mathbf{1 5} & \mathbf{8} & \mathbf{1 5} & \mathbf{1 5} & \mathbf{1 5} & \mathbf{1 5} & \mathbf{1 5} & \mathbf{1 5} & \mathbf{1 5} & \mathbf{8} & \mathbf{1 4}\end{array}$

$15 \quad 15 \quad 15$

-Other Entities-

10 Coyote Springs \#2

11 Hermiston Power Project

12 Klamath Cogneration Project

13 Longview Fibre

14 Total Other Entities

-Cogeneration-

15 Federal System

16 Public Entities

17 Investor-Owned Entities

18 Other Entities

\begin{tabular}{|c|c|c|c|c|c|c|c|c|c|c|c|c|c|c|}
\hline 48 & 48 & 48 & 48 & 48 & 48 & 48 & 48 & 48 & 48 & 48 & 0 & 48 & 48 & 44 \\
\hline 9.6 & 9.6 & 9.6 & 9.6 & 9.6 & 9.6 & 9.6 & 9.6 & 9.6 & 9.6 & 9.6 & 9.6 & 9.6 & 9.6 & 9.6 \\
\hline 863 & 863 & 867 & 869 & 876 & 880 & 881 & 878 & 874 & 870 & 870 & 240 & 865 & 863 & 819 \\
\hline
\end{tabular}

19 Total Cogeneration

\begin{tabular}{|c|c|c|c|c|c|c|c|c|c|c|c|c|c|}
\hline 138 & 138 & 138 & 138 & 138 & 138 & 138 & 138 & 138 & 138 & 0 & 138 & 138 & 126 \\
\hline 620 & 620 & 620 & 620 & 620 & 620 & 620 & 0 & 620 & 620 & 620 & 620 & 620 & 568 \\
\hline 36 & 36 & 36 & 36 & 36 & 36 & 36 & 36 & 36 & 36 & 36 & 36 & 36 & 6 \\
\hline
\end{tabular}

\begin{tabular}{rrrrrrrrrrrrrrr}
0 & 0 & 0 & 0 & 0 & 0 & 0 & 0 & 0 & 0 & 0 & 0 & 0 & 0 & 0 \\
15 & 15 & 15 & 15 & 15 & 8 & 15 & 15 & 15 & 15 & 15 & 15 & 15 & 8 & 14 \\
863 & 863 & 867 & 869 & 876 & 880 & 881 & 878 & 874 & 870 & 870 & 240 & 865 & 863 & 819 \\
1248 & 1248 & 1248 & 1248 & 1248 & 1248 & 1248 & 1248 & 628 & 1248 & 1248 & 883 & 1248 & 1248 & 1166 \\
\hline 2126 & 2126 & 2130 & 2132 & 2139 & 2136 & $\mathbf{2 1 4 4}$ & $\mathbf{2 1 4 1}$ & $\mathbf{1 5 1 8}$ & $\mathbf{2 1 3 3}$ & $\mathbf{2 1 3 3}$ & $\mathbf{1 1 3 7}$ & $\mathbf{2 1 2 8}$ & $\mathbf{2 1 1 9}$ & $\mathbf{1 9 9 9}$
\end{tabular}



2003 White Book

May Jun Jul Avg

-Federal System-

1 Total Federal System Dec Jan Feb Mar Apr1 Apr16

-Public Entities-

2 WEYCO Energy Center

3 Total Public Entities

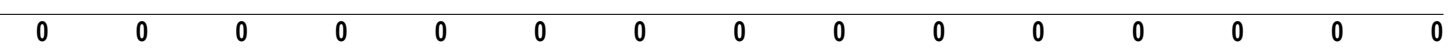

-Investor_Owned Entities-

4 Coyote Springs \#1

5 Coyote Springs \#2

6 Georgia-Pacific Paper (Camas)

7 Hermiston Cogeneration

8 Thompson River Co-Gen

9 Total Investor-Owned Entities

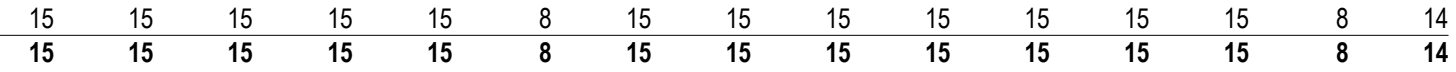

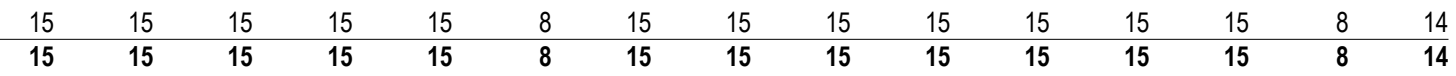

$\begin{array}{lllllllllllllll}15 & 15 & 15 & 15 & 15 & 8 & 15 & 15 & 15 & 15 & 15 & 15 & 15 & 8 & 14 \\ 15 & \mathbf{1 5} & \mathbf{1 5} & \mathbf{1 5} & \mathbf{1 5} & \mathbf{8} & \mathbf{1 5} & \mathbf{1 5} & \mathbf{1 5} & \mathbf{1 5} & \mathbf{1 5} & \mathbf{1 5} & \mathbf{1 5} & \mathbf{8} & \mathbf{1 5}\end{array}$

-Other Entities-

10 Coyote Springs \#2

11 Hermiston Power Project

12 Klamath Cogneration Project

13 Longview Fibre

14 Total Other Entities

-Cogeneration-

15 Federal System

16 Public Entities

17 Investor-Owned Entities

18 Other Entities

\begin{tabular}{|c|c|c|c|c|c|c|c|c|c|c|c|c|c|c|}
\hline 225 & 225 & 229 & 231 & 238 & 242 & 243 & 240 & 236 & 232 & 232 & 230 & 227 & 225 & 233 \\
\hline 138 & 138 & 138 & 138 & 138 & 138 & 138 & 138 & 138 & 138 & 138 & 0 & 138 & 138 & 126 \\
\hline 48 & 48 & 48 & 48 & 48 & 48 & 48 & 48 & 48 & 48 & 48 & 0 & 48 & 48 & 44 \\
\hline 443 & 443 & 443 & 443 & 443 & 443 & 443 & 443 & 443 & 443 & 443 & 0 & 443 & 443 & 406 \\
\hline 9.6 & 9.6 & 9.6 & 9.6 & 9.6 & 9.6 & 9.6 & 9.6 & 9.6 & 9.6 & 9.6 & 9.6 & 9.6 & 9.6 & 9.6 \\
\hline 863 & 863 & 867 & 869 & 876 & 880 & 881 & 878 & 874 & 870 & 870 & 240 & 865 & 863 & 819 \\
\hline
\end{tabular}

19 Total Cogeneration

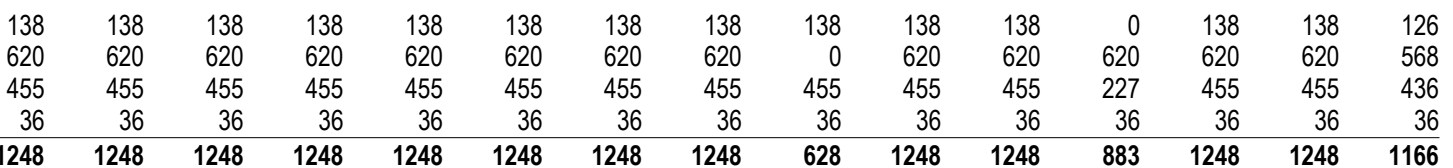



2003 White Book

Aug1 Aug16 Sep Oct Nov Dec Jan Feb Mar Apr1 Apr16 May Jun Jul Avg

-Boardman: Coal-

1 Idaho Power Company

2 Portland General Electric

3 San Diego Gas \& Electric

4 Turlock Irrigation District

5 Boardman: Regional Total

-Centralia \#1: Coal-

6 Trans Alta Utilities Corp

7 Centralia \#1: Regional Total

-Centralia \#2: Coal-

8 Trans Alta Utilities Corp

9 Centralia \#2: Regional Total

-Colstrip \#1: Coal-

10 Puget Sound Energy

11 Colstrip \#1: Regional Total

-Colstrip \#2: Coal-

12 Puget Sound Energy

13 Colstrip \#2: Regional Total

-Colstrip \#3: Coal-

14 Avista Corp (WWP Division)

15 Pacific Power

16 Portland General Electric

17 Puget Sound Energy

18 Colstrip \#3: Regional Total

-Colstrip \#4: Coal-

19 Avista Corp (WWP Division)

20 Northwestern Energy LLC (MPC)

21 Pacific Power

22 Portland General Electric

23 Puget Sound Energy

24 Colstrip \#4: Regional Total

-Columbia Generating Station: Uranium.

25 BPA - Power Business

26 Columbia Generating Station: Regional Total

-Jim Bridger \#1: Coal-

27 Idaho Power Company

28 Pacific Power

29 Jim Bridger \#1: Regional Total

-Jim Bridger \#2: Coal-

30 Idaho Power Company

31 Pacific Power

32 Jim Bridger \#2: Regional Total

-Jim Bridger \#3: Coal-

33 Idaho Power Company

34 Pacific Power

35 Jim Bridger \#3: Regional Total

-Jim Bridger \#4: Coal.

36 Idaho Power Company

37 Pacific Power

38 Jim Bridger \#4: Regional Total

-Valmy \#1: Coal-

39 Idaho Power Company

40 Valmy \#1: Regional Total

-Valmy \#2: Coal-

41 Idaho Power Company

42 Valmy \#2: Regional Total

-Total Large Thermal-

43 Federal System

44 Public Entities

45 Investor-Owned Entities

46 Other Entities

\begin{tabular}{|c|c|c|c|c|c|c|c|c|c|c|c|c|}
\hline 52 & 52 & 52 & 52 & 52 & 52 & 52 & 52 & 3.4 & 3.4 & 0 & 52 & 52 \\
\hline 337 & 337 & 337 & 337 & 337 & 337 & 337 & 337 & 22 & 22 & 0 & 337 & 337 \\
\hline 77 & 77 & 77 & 77 & 77 & 77 & 77 & 77 & 5 & 5 & 0 & 77 & 77 \\
\hline 52 & 52 & 52 & 52 & 52 & 52 & 52 & 52 & 3.4 & 3.4 & 0 & 52 & 52 \\
\hline 517 & 517 & 517 & 517 & 517 & 517 & 517 & 517 & 34 & 34 & 0 & 517 & 517 \\
\hline
\end{tabular}

$\begin{array}{lllllllllllllll}626 & 626 & 626 & 626 & 626 & 626 & 626 & 626 & 626 & 626 & 626 & 626 & 626 & 626 & 626 \\ \mathbf{6 2 6} & \mathbf{6 2 6} & \mathbf{6 2 6} & \mathbf{6 2 6} & \mathbf{6 2 6} & \mathbf{6 2 6} & \mathbf{6 2 6} & \mathbf{6 2 6} & \mathbf{6 2 6} & \mathbf{6 2 6} & \mathbf{6 2 6} & \mathbf{6 2 6} & \mathbf{6 2 6} & \mathbf{6 2 6} & \mathbf{6 2 6}\end{array}$

$\begin{array}{lllllllllllllll}626 & 626 & 626 & 626 & 626 & 626 & 626 & 626 & 566 & 0 & 0 & 445 & 626 & 626 & 554 \\ \mathbf{6 2 6} & \mathbf{6 2 6} & \mathbf{6 2 6} & \mathbf{6 2 6} & \mathbf{6 2 6} & \mathbf{6 2 6} & \mathbf{6 2 6} & \mathbf{6 2 6} & \mathbf{5 6 6} & \mathbf{0} & \mathbf{0} & \mathbf{4 4 5} & \mathbf{6 2 6} & \mathbf{6 2 6} & \mathbf{5 5 4}\end{array}$

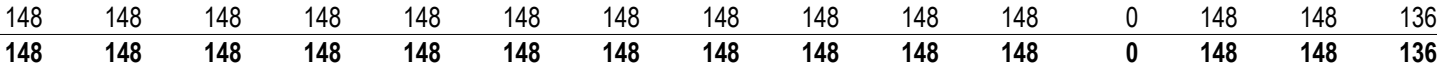

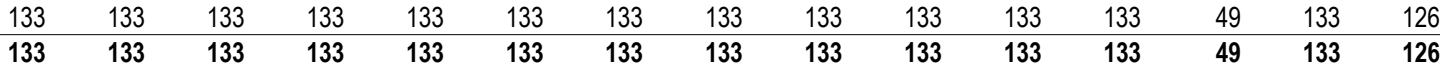

\begin{tabular}{rrrrrrrrrrrrrrr}
99 & 99 & 99 & 99 & 99 & 99 & 99 & 99 & 99 & 99 & 99 & 99 & 99 & 99 & 99 \\
66 & 66 & 66 & 66 & 66 & 66 & 66 & 66 & 66 & 66 & 66 & 66 & 66 & 66 & 66 \\
132 & 132 & 132 & 132 & 132 & 132 & 132 & 132 & 132 & 132 & 132 & 132 & 132 & 132 & 132 \\
165 & 165 & 165 & 165 & 165 & 165 & 165 & 165 & 165 & 165 & 165 & 165 & 165 & 165 & 165 \\
\hline $\mathbf{4 6 2}$ & $\mathbf{4 6 2}$ & $\mathbf{4 6 2}$ & $\mathbf{4 6 2}$ & $\mathbf{4 6 2}$ & $\mathbf{4 6 2}$ & $\mathbf{4 6 2}$ & $\mathbf{4 6 2}$ & $\mathbf{4 6 2}$ & $\mathbf{4 6 2}$ & $\mathbf{4 6 2}$ & $\mathbf{4 6 2}$ & $\mathbf{4 6 2}$ & $\mathbf{4 6 2}$ & $\mathbf{4 6 2}$
\end{tabular}

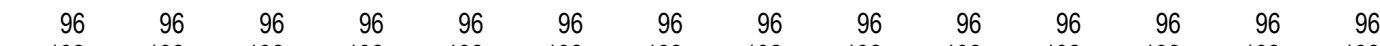

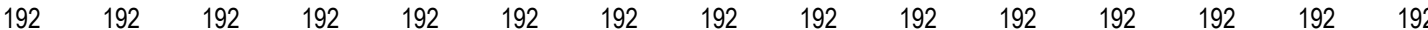

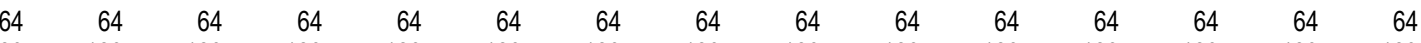

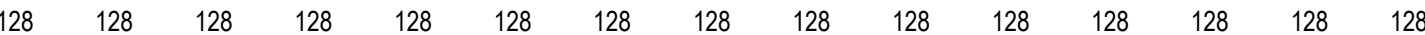

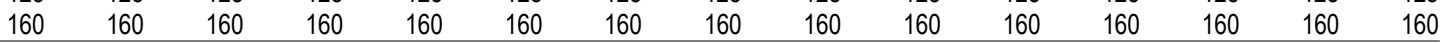

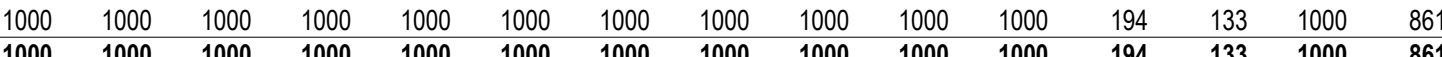

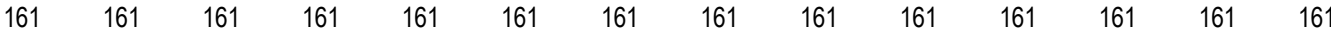

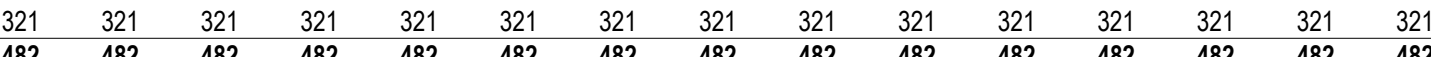

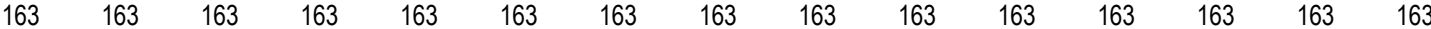

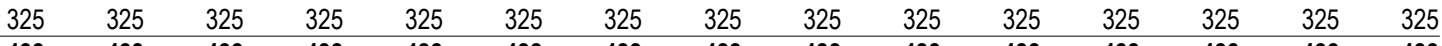

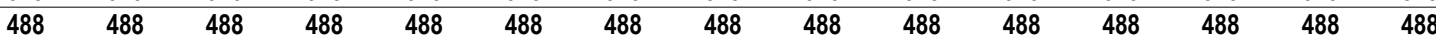

\begin{tabular}{|c|c|c|c|c|c|c|c|c|c|c|c|c|c|}
\hline 18 & 318 & 318 & 318 & 318 & 318 & 318 & 318 & 318 & 318 & 318 & 318 & 223 & 318 \\
\hline 477 & 477 & 477 & 477 & 477 & 477 & 477 & 477 & 477 & 477 & 477 & 477 & 334 & 477 \\
\hline
\end{tabular}

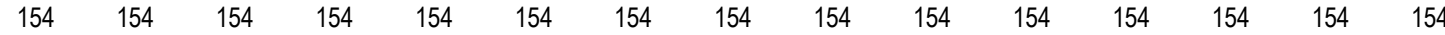

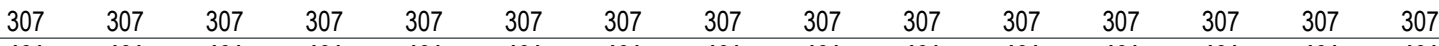

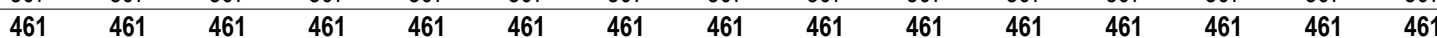

$\begin{array}{lllllllllllllll}103 & 103 & 103 & 103 & 103 & 103 & 103 & 103 & 103 & 0 & 69 & 103 & 103 & 103 & 98 \\ 103 & 103 & 103 & 103 & 103 & 103 & 103 & 103 & 103 & \mathbf{0} & \mathbf{6 9} & \mathbf{1 0 3} & \mathbf{1 0 3} & \mathbf{1 0 3} & \mathbf{9 8}\end{array}$

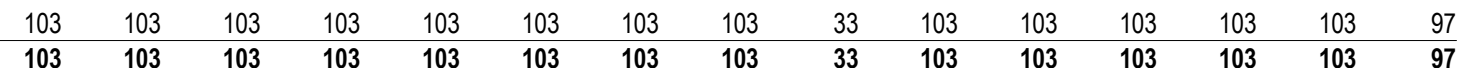

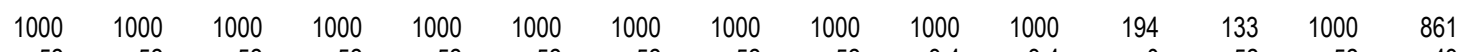

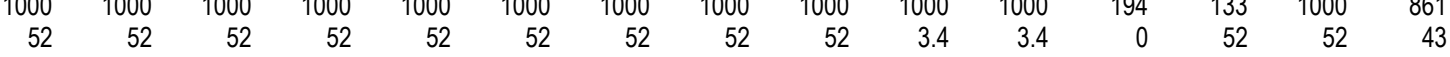

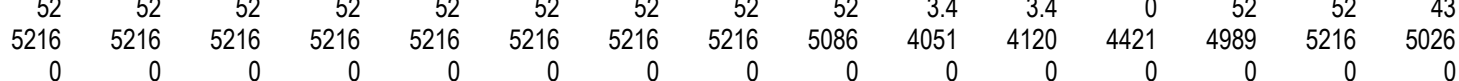


2003 White Book Dec Jan $\mathrm{Fe}$ Mar Apr1 Apr16

May

Jun
Jul Avg

$\begin{array}{rrrrrrrrrrr}0 & 0 & 0 & 0 & 0 & 0 & 0 & 0 & 0 & 0 & 0 \\ 6268 & 6268 & 6268 & 6268 & 6268 & 6268 & 6268 & 6268 & 6137 & 5055 & 5124\end{array}$

$\begin{array}{rrrr}0 & 0 & 0 & 0 \\ 4615 & 5174 & 6268 & 5930\end{array}$



2003 White Book

Aug1 Aug16 Sep Oct Nov Dec Jan Feb Mar Apr1 Apr16 May Jun Jul Avg

-Boardman: Coal-

1 Idaho Power Company

2 Portland General Electric

3 San Diego Gas \& Electric

4 Turlock Irrigation District

5 Boardman: Regional Total

-Centralia \#1: Coal-

6 Trans Alta Utilities Corp

7 Centralia \#1: Regional Total

-Centralia \#2: Coal-

8 Trans Alta Utilities Corp

9 Centralia \#2: Regional Total

-Colstrip \#1: Coal-

10 Puget Sound Energy

11 Colstrip \#1: Regional Total

-Colstrip \#2: Coal-

12 Puget Sound Energy

13 Colstrip \#2: Regional Total

-Colstrip \#3: Coal-

14 Avista Corp (WWP Division)

15 Pacific Power

16 Portland General Electric

17 Puget Sound Energy

18 Colstrip \#3: Regional Total

-Colstrip \#4: Coal-

19 Avista Corp (WWP Division)

20 Northwestern Energy LLC (MPC)

21 Pacific Power

22 Portland General Electric

23 Puget Sound Energy

24 Colstrip \#4: Regional Total

-Columbia Generating Station: Uranium.

25 BPA - Power Business

26 Columbia Generating Station: Regional Total

-Jim Bridger \#1: Coal-

27 Idaho Power Company

28 Pacific Power

29 Jim Bridger \#1: Regional Total

-Jim Bridger \#2: Coal-

30 Idaho Power Company

31 Pacific Power

32 Jim Bridger \#2: Regional Total

-Jim Bridger \#3: Coal-

33 Idaho Power Company

34 Pacific Power

35 Jim Bridger \#3: Regional Total

-Jim Bridger \#4: Coal.

36 Idaho Power Company

37 Pacific Power

38 Jim Bridger \#4: Regional Total

-Valmy \#1: Coal-

39 Idaho Power Company

40 Valmy \#1: Regional Total

-Valmy \#2: Coal-

41 Idaho Power Company

42 Valmy \#2: Regional Total

-Total Large Thermal-

43 Federal System

44 Public Entities

45 Investor-Owned Entities

46 Other Entities

\begin{tabular}{|c|c|c|c|c|c|c|c|c|c|c|c|c|}
\hline 52 & 52 & 52 & 52 & 52 & 52 & 52 & 52 & 3.4 & 3.4 & 0 & 52 & 52 \\
\hline 337 & 337 & 337 & 337 & 337 & 337 & 337 & 337 & 22 & 22 & 0 & 337 & 337 \\
\hline 77 & 77 & 77 & 77 & 77 & 77 & 77 & 77 & 5 & 5 & 0 & 77 & 77 \\
\hline 52 & 52 & 52 & 52 & 52 & 52 & 52 & 52 & 3.4 & 3.4 & 0 & 52 & 52 \\
\hline 517 & 517 & 517 & 517 & 517 & 517 & 517 & 517 & 34 & 34 & 0 & 517 & 517 \\
\hline
\end{tabular}

\begin{tabular}{lllllllllllllll}
626 & 626 & 626 & 626 & 626 & 626 & 626 & 626 & 626 & 626 & 626 & 40 & 626 & 626 & 577 \\
\hline $\mathbf{6 2 6}$ & $\mathbf{6 2 6}$ & $\mathbf{6 2 6}$ & $\mathbf{6 2 6}$ & $\mathbf{6 2 6}$ & $\mathbf{6 2 6}$ & $\mathbf{6 2 6}$ & $\mathbf{6 2 6}$ & $\mathbf{6 2 6}$ & $\mathbf{6 2 6}$ & $\mathbf{6 2 6}$ & $\mathbf{4 0}$ & $\mathbf{6 2 6}$ & $\mathbf{6 2 6}$ & $\mathbf{5 7 7}$
\end{tabular}

$\begin{array}{lllllllllllllll}626 & 626 & 626 & 626 & 626 & 626 & 626 & 626 & 626 & 626 & 626 & 626 & 626 & 626 & 626 \\ \mathbf{6 2 6} & \mathbf{6 2 6} & \mathbf{6 2 6} & \mathbf{6 2 6} & \mathbf{6 2 6} & \mathbf{6 2 6} & \mathbf{6 2 6} & \mathbf{6 2 6} & \mathbf{6 2 6} & \mathbf{6 2 6} & \mathbf{6 2 6} & \mathbf{6 2 6} & \mathbf{6 2 6} & \mathbf{6 2 6} & \mathbf{6 2 6}\end{array}$

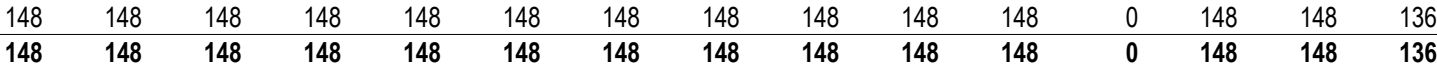

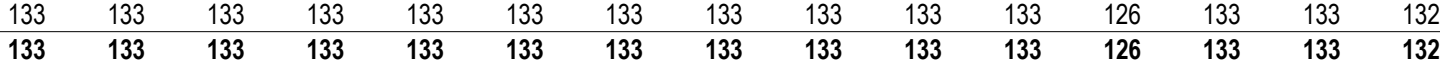

\begin{tabular}{rrrrrrrrrrrrrrr}
99 & 99 & 99 & 99 & 99 & 99 & 99 & 99 & 99 & 99 & 99 & 68 & 99 & 99 & 96 \\
66 & 66 & 66 & 66 & 66 & 66 & 66 & 66 & 66 & 66 & 66 & 46 & 66 & 66 & 64 \\
132 & 132 & 132 & 132 & 132 & 132 & 132 & 132 & 132 & 132 & 132 & 91 & 132 & 132 & 129 \\
165 & 165 & 165 & 165 & 165 & 165 & 165 & 165 & 165 & 165 & 165 & 114 & 165 & 165 & 161 \\
\hline $\mathbf{4 6 2}$ & $\mathbf{4 6 2}$ & $\mathbf{4 6 2}$ & $\mathbf{4 6 2}$ & $\mathbf{4 6 2}$ & $\mathbf{4 6 2}$ & $\mathbf{4 6 2}$ & $\mathbf{4 6 2}$ & $\mathbf{4 6 2}$ & $\mathbf{4 6 2}$ & $\mathbf{4 6 2}$ & $\mathbf{3 1 9}$ & $\mathbf{4 6 2}$ & $\mathbf{4 6 2}$ & $\mathbf{4 5 0}$
\end{tabular}

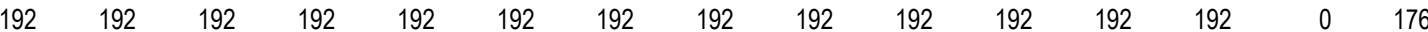

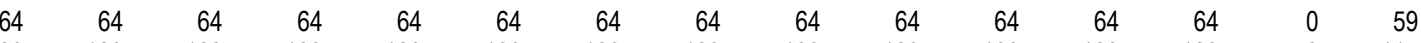

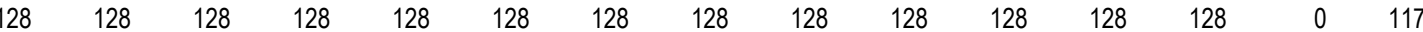

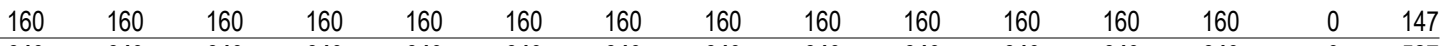

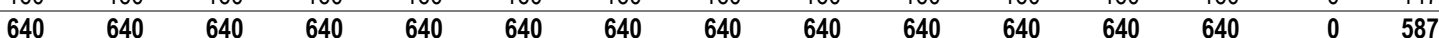

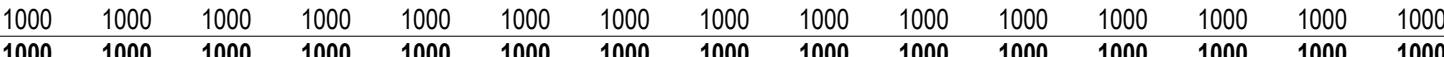

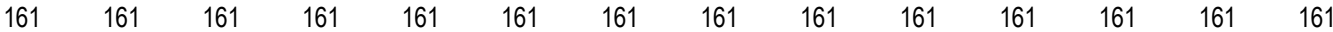

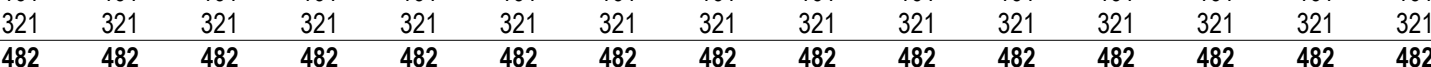

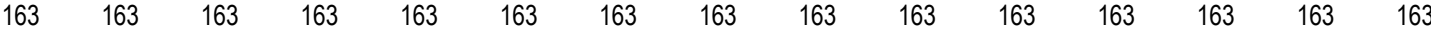

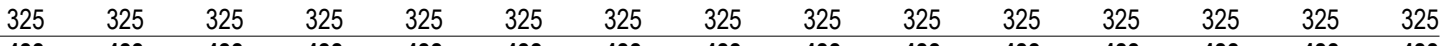

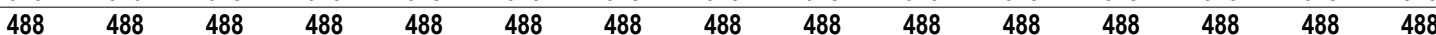

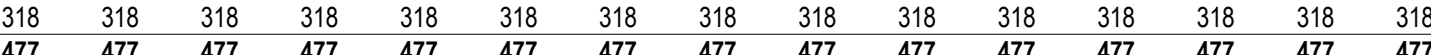

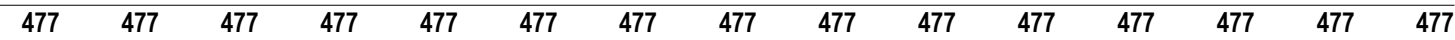

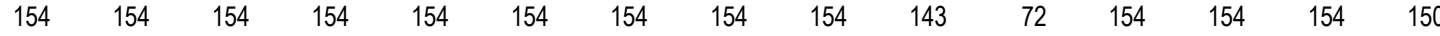

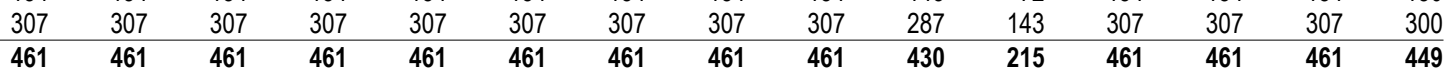

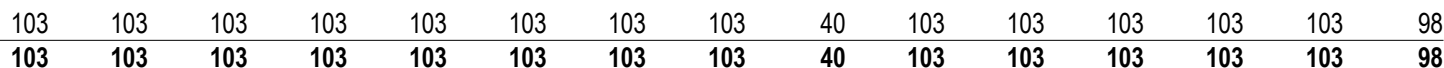

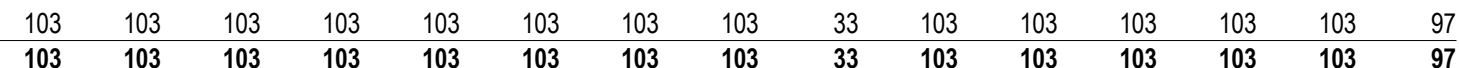

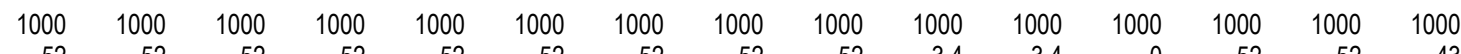

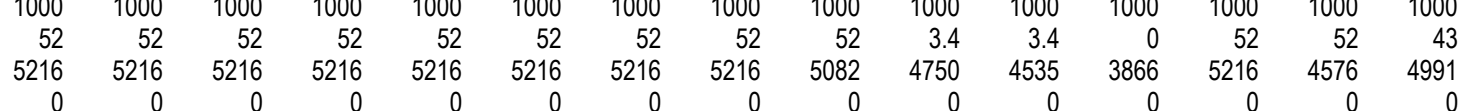



Dec Jan $\mathrm{Fe}$ Mar Apr1 Apr16

May Jun Jul Avg

$\begin{array}{rrrrrrrrrrr}0 & 0 & 0 & 0 & 0 & 0 & 0 & 0 & 0 & 0 & 0 \\ 6267 & 6267 & 6267 & 6267 & 6267 & 6267 & 6267 & 6267 & 6134 & 5753 & 5538\end{array}$

$\begin{array}{rrrr}0 & 0 & 0 & 0 \\ 4866 & 6267 & 5627 & 6034\end{array}$



2003 White Book

Aug1 Aug16 Sep Oct Nov Dec Jan Feb Mar Apr1 Apr16 May Jun Jul Avg

-Boardman: Coal-

1 Idaho Power Company

2 Portland General Electric

3 San Diego Gas \& Electric

4 Turlock Irrigation District

5 Boardman: Regional Total

-Centralia \#1: Coal-

6 Trans Alta Utilities Corp

7 Centralia \#1: Regional Total

-Centralia \#2: Coal-

8 Trans Alta Utilities Corp

9 Centralia \#2: Regional Total

-Colstrip \#1: Coal-

10 Puget Sound Energy

11 Colstrip \#1: Regional Total

-Colstrip \#2: Coal-

12 Puget Sound Energy

13 Colstrip \#2: Regional Total

-Colstrip \#3: Coal-

14 Avista Corp (WWP Division)

15 Pacific Power

16 Portland General Electric

17 Puget Sound Energy

18 Colstrip \#3: Regional Total

-Colstrip \#4: Coal-

19 Avista Corp (WWP Division)

20 Northwestern Energy LLC (MPC)

21 Pacific Power

22 Portland General Electric

23 Puget Sound Energy

24 Colstrip \#4: Regional Total

-Columbia Generating Station: Uranium.

25 BPA - Power Business

26 Columbia Generating Station: Regional Total

-Jim Bridger \#1: Coal-

27 Idaho Power Company

28 Pacific Power

29 Jim Bridger \#1: Regional Total

-Jim Bridger \#2: Coal-

30 Idaho Power Company

31 Pacific Power

32 Jim Bridger \#2: Regional Total

-Jim Bridger \#3: Coal-

33 Idaho Power Company

34 Pacific Power

35 Jim Bridger \#3: Regional Total

-Jim Bridger \#4: Coal.

36 Idaho Power Company

37 Pacific Power

38 Jim Bridger \#4: Regional Total

-Valmy \#1: Coal-

39 Idaho Power Company

40 Valmy \#1: Regional Total

-Valmy \#2: Coal-

41 Idaho Power Company

42 Valmy \#2: Regional Total

-Total Large Thermal-

43 Federal System

44 Public Entities

45 Investor-Owned Entities

46 Other Entities

$\begin{array}{rrrrrrrrrrrrrrr}52 & 52 & 52 & 52 & 52 & 52 & 52 & 52 & 52 & 3.4 & 3.4 & 0 & 52 & 52 & 43 \\ 337 & 337 & 337 & 337 & 337 & 337 & 337 & 337 & 337 & 22 & 22 & 0 & 337 & 337 & 283 \\ 77 & 77 & 77 & 77 & 77 & 77 & 77 & 77 & 77 & \mathbf{5} & \mathbf{5} & 0 & 77 & 77 & 64 \\ 52 & 52 & 52 & 52 & 52 & 52 & 52 & 52 & 52 & 3.4 & 3.4 & 0 & 52 & \mathbf{5 2} & 43 \\ \mathbf{5 1 7} & \mathbf{5 1 7} & \mathbf{5 1 7} & \mathbf{5 1 7} & \mathbf{5 1 7} & \mathbf{5 1 7} & \mathbf{5 1 7} & \mathbf{5 1 7} & \mathbf{5 1 7} & \mathbf{3 4} & \mathbf{3 4} & \mathbf{0} & \mathbf{5 1 7} & \mathbf{5 1 7} & \mathbf{4 3 4}\end{array}$

$\begin{array}{lllllllllllllll}626 & 626 & 626 & 626 & 626 & 626 & 626 & 626 & 626 & 626 & 626 & 626 & 626 & 626 & 626 \\ \mathbf{6 2 6} & \mathbf{6 2 6} & \mathbf{6 2 6} & \mathbf{6 2 6} & \mathbf{6 2 6} & \mathbf{6 2 6} & \mathbf{6 2 6} & \mathbf{6 2 6} & \mathbf{6 2 6} & \mathbf{6 2 6} & \mathbf{6 2 6} & \mathbf{6 2 6} & \mathbf{6 2 6} & \mathbf{6 2 6} & \mathbf{6 2 6}\end{array}$

$\begin{array}{lllllllllllllll}626 & 626 & 626 & 626 & 626 & 626 & 626 & 626 & 626 & 626 & 626 & 40 & 626 & 626 & 577 \\ \mathbf{6 2 6} & \mathbf{6 2 6} & \mathbf{6 2 6} & \mathbf{6 2 6} & \mathbf{6 2 6} & \mathbf{6 2 6} & \mathbf{6 2 6} & \mathbf{6 2 6} & \mathbf{6 2 6} & \mathbf{6 2 6} & \mathbf{6 2 6} & \mathbf{4 0} & \mathbf{6 2 6} & \mathbf{6 2 6} & \mathbf{5 7 7}\end{array}$

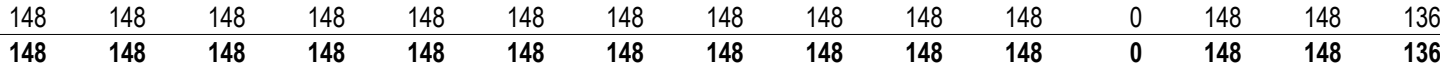

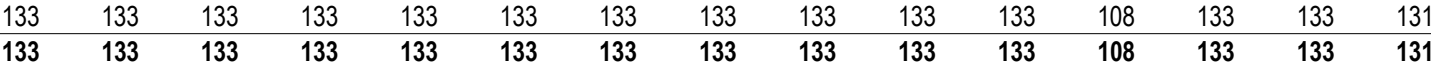

\begin{tabular}{rrrrrrrrrrrrrrr}
99 & 99 & 99 & 99 & 99 & 99 & 99 & 99 & 99 & 19 & 19 & 34 & 99 & 99 & 87 \\
66 & 66 & 66 & 66 & 66 & 66 & 66 & 66 & 66 & 13 & 13 & 23 & 66 & 66 & 58 \\
132 & 132 & 132 & 132 & 132 & 132 & 132 & 132 & 132 & 26 & 26 & 46 & 132 & 132 & 116 \\
165 & 165 & 165 & 165 & 165 & 165 & 165 & 165 & 165 & 32 & 32 & 57 & 165 & 165 & 145 \\
\hline $\mathbf{4 6 2}$ & $\mathbf{4 6 2}$ & $\mathbf{4 6 2}$ & $\mathbf{4 6 2}$ & $\mathbf{4 6 2}$ & $\mathbf{4 6 2}$ & $\mathbf{4 6 2}$ & $\mathbf{4 6 2}$ & $\mathbf{4 6 2}$ & $\mathbf{9 0}$ & $\mathbf{9 0}$ & $\mathbf{1 6 0}$ & $\mathbf{4 6 2}$ & $\mathbf{4 6 2}$ & $\mathbf{4 0 6}$
\end{tabular}

\begin{tabular}{rrrrrrrrrrrrrrr}
0 & 96 & 96 & 96 & 96 & 96 & 96 & 96 & 96 & 96 & 96 & 96 & 96 & 96 & 92 \\
0 & 192 & 192 & 192 & 192 & 192 & 192 & 192 & 192 & 192 & 192 & 192 & 192 & 192 & 184 \\
0 & 64 & 64 & 64 & 64 & 64 & 64 & 64 & 64 & 64 & 64 & 64 & 64 & 64 & 61 \\
0 & 128 & 128 & 128 & 128 & 128 & 128 & 128 & 128 & 128 & 128 & 128 & 128 & 128 & 123 \\
0 & 160 & 160 & 160 & 160 & 160 & 160 & 160 & 160 & 160 & 160 & 160 & 160 & 160 & 153 \\
\hline $\mathbf{0}$ & $\mathbf{6 4 0}$ & $\mathbf{6 4 0}$ & $\mathbf{6 4 0}$ & $\mathbf{6 4 0}$ & $\mathbf{6 4 0}$ & $\mathbf{6 4 0}$ & $\mathbf{6 4 0}$ & $\mathbf{6 4 0}$ & $\mathbf{6 4 0}$ & $\mathbf{6 4 0}$ & $\mathbf{6 4 0}$ & $\mathbf{6 4 0}$ & $\mathbf{6 4 0}$ & $\mathbf{6 1 3}$
\end{tabular}

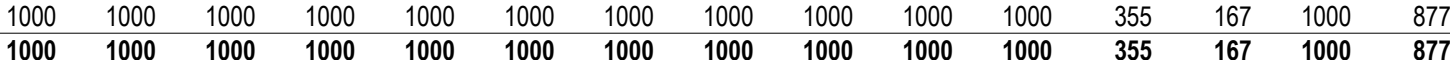

\begin{tabular}{|c|c|c|c|c|c|c|c|c|c|c|c|c|c|c|}
\hline 161 & 161 & 161 & 161 & 161 & 161 & 161 & 161 & 161 & 161 & 161 & 5.3 & 32 & 161 & 137 \\
\hline 321 & 321 & 321 & 321 & 321 & 321 & 321 & 321 & 321 & 321 & 321 & 11 & 64 & 321 & 274 \\
\hline 482 & 482 & 482 & 482 & 482 & 482 & 482 & 482 & 482 & 482 & 482 & 16 & 96 & 482 & 41 \\
\hline
\end{tabular}

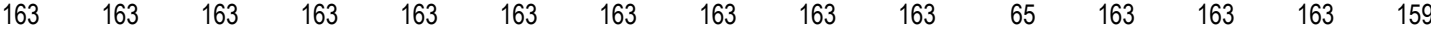

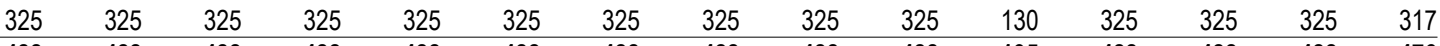

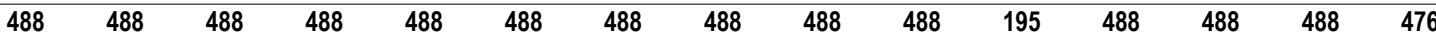

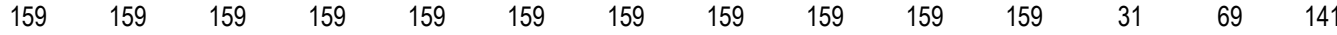

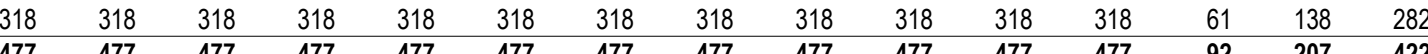

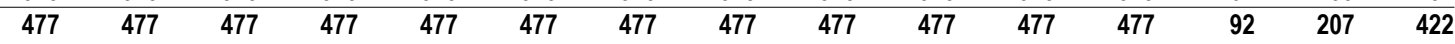

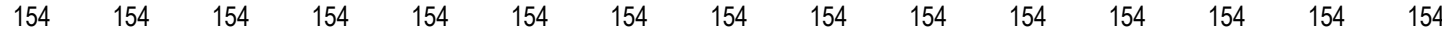

\begin{tabular}{|c|c|c|c|c|c|c|c|c|c|c|c|c|c|}
\hline 07 & 307 & 307 & 307 & 307 & 307 & 307 & 307 & 307 & 307 & 307 & 307 & 307 & 307 \\
\hline 4 & 461 & 461 & 461 & 461 & 461 & 461 & 461 & 461 & 461 & 461 & 461 & 461 & \\
\hline
\end{tabular}

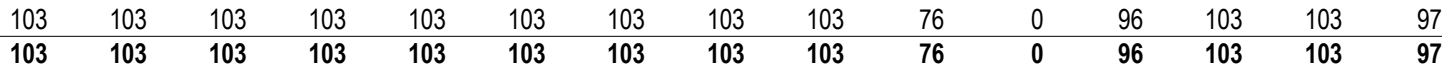

$\begin{array}{lllllllllllllll}103 & 103 & 103 & 103 & 103 & 103 & 103 & 103 & 47 & 96 & 103 & 103 & 103 & 103 & 98 \\ 103 & 103 & 103 & 103 & 103 & 103 & 103 & 103 & \mathbf{4 7} & \mathbf{9 6} & \mathbf{1 0 3} & \mathbf{1 0 3} & \mathbf{1 0 3} & \mathbf{1 0 3} & \mathbf{9 8}\end{array}$

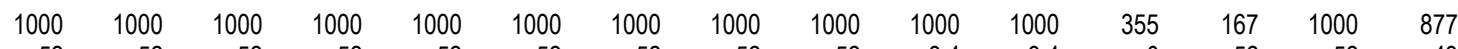

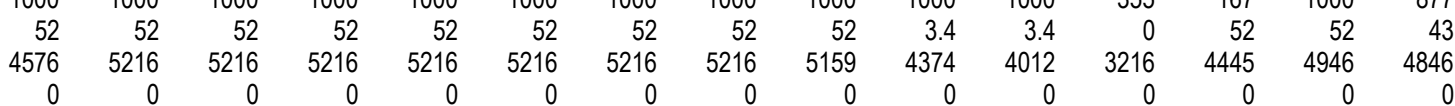


2003 White Book Dec Jan $\mathrm{Fe}$ Mar Apr1 Apr16

May

Jun
Jul Avg

$\begin{array}{rrrrrrrrrrr}0 & 0 & 0 & 0 & 0 & 0 & 0 & 0 & 0 & 0 & 0 \\ 5627 & 6267 & 6267 & 6267 & 6267 & 6267 & 6267 & 6267 & 6211 & 5377 & 5016\end{array}$

$\begin{array}{rrrr}0 & 0 & 0 & 0 \\ 3571 & 4663 & 5997 & 5766\end{array}$



2003 White Book

Aug1 Aug16 Sep Oct Nov Dec Jan Feb Mar Apr1 Apr16 May Jun Jul Avg

-Boardman: Coal-

1 Idaho Power Company

2 Portland General Electric

3 San Diego Gas \& Electric

4 Turlock Irrigation District

5 Boardman: Regional Total

-Centralia \#1: Coal-

6 Trans Alta Utilities Corp

7 Centralia \#1: Regional Total

-Centralia \#2: Coal-

8 Trans Alta Utilities Corp

9 Centralia \#2: Regional Total

-Colstrip \#1: Coal-

10 Puget Sound Energy

11 Colstrip \#1: Regional Total

-Colstrip \#2: Coal-

12 Puget Sound Energy

13 Colstrip \#2: Regional Total

-Colstrip \#3: Coal-

14 Avista Corp (WWP Division)

15 Pacific Power

16 Portland General Electric

17 Puget Sound Energy

18 Colstrip \#3: Regional Total

-Colstrip \#4: Coal-

19 Avista Corp (WWP Division)

20 Northwestern Energy LLC (MPC)

21 Pacific Power

22 Portland General Electric

23 Puget Sound Energy

24 Colstrip \#4: Regional Total

-Columbia Generating Station: Uranium.

25 BPA - Power Business

26 Columbia Generating Station: Regional Total

-Jim Bridger \#1: Coal-

27 Idaho Power Company

28 Pacific Power

29 Jim Bridger \#1: Regional Total

-Jim Bridger \#2: Coal-

30 Idaho Power Company

31 Pacific Power

32 Jim Bridger \#2: Regional Total

-Jim Bridger \#3: Coal-

33 Idaho Power Company

34 Pacific Power

35 Jim Bridger \#3: Regional Total

-Jim Bridger \#4: Coal-

36 Idaho Power Company

37 Pacific Power

38 Jim Bridger \#4: Regional Total

-Valmy \#1: Coal-

39 Idaho Power Company

40 Valmy \#1: Regional Total

-Valmy \#2: Coal-

41 Idaho Power Company

42 Valmy \#2: Regional Total

-Total Large Thermal-

43 Federal System

44 Public Entities

45 Investor-Owned Entities

46 Other Entities

$\begin{array}{rrrrrrrrrrrrrrr}52 & 52 & 52 & 52 & 52 & 52 & 52 & 52 & 52 & 3.4 & 3.4 & 0 & 52 & 52 & 43 \\ 337 & 337 & 337 & 337 & 337 & 337 & 337 & 337 & 337 & 22 & 22 & 0 & 337 & 337 & 283 \\ 77 & 77 & 77 & 77 & 77 & 77 & 77 & 77 & 77 & \mathbf{5} & \mathbf{5} & 0 & 77 & 77 & 64 \\ 52 & 52 & 52 & 52 & 52 & 52 & 52 & 52 & 52 & 3.4 & 3.4 & 0 & 52 & \mathbf{5 2} & 43 \\ \mathbf{5 1 7} & \mathbf{5 1 7} & \mathbf{5 1 7} & \mathbf{5 1 7} & \mathbf{5 1 7} & \mathbf{5 1 7} & \mathbf{5 1 7} & \mathbf{5 1 7} & \mathbf{5 1 7} & \mathbf{3 4} & \mathbf{3 4} & \mathbf{0} & \mathbf{5 1 7} & \mathbf{5 1 7} & \mathbf{4 3 4}\end{array}$

\begin{tabular}{lllllllllllllll}
626 & 626 & 626 & 626 & 626 & 626 & 626 & 626 & 626 & 626 & 626 & 40 & 626 & 626 & 577 \\
\hline $\mathbf{6 2 6}$ & $\mathbf{6 2 6}$ & $\mathbf{6 2 6}$ & $\mathbf{6 2 6}$ & $\mathbf{6 2 6}$ & $\mathbf{6 2 6}$ & $\mathbf{6 2 6}$ & $\mathbf{6 2 6}$ & $\mathbf{6 2 6}$ & $\mathbf{6 2 6}$ & $\mathbf{6 2 6}$ & $\mathbf{4 0}$ & $\mathbf{6 2 6}$ & $\mathbf{6 2 6}$ & $\mathbf{5 7 7}$
\end{tabular}

$\begin{array}{lllllllllllllll}626 & 626 & 626 & 626 & 626 & 626 & 626 & 626 & 626 & 626 & 626 & 626 & 626 & 626 & 626 \\ \mathbf{6 2 6} & \mathbf{6 2 6} & \mathbf{6 2 6} & \mathbf{6 2 6} & \mathbf{6 2 6} & \mathbf{6 2 6} & \mathbf{6 2 6} & \mathbf{6 2 6} & \mathbf{6 2 6} & \mathbf{6 2 6} & \mathbf{6 2 6} & \mathbf{6 2 6} & \mathbf{6 2 6} & \mathbf{6 2 6} & \mathbf{6 2 6}\end{array}$

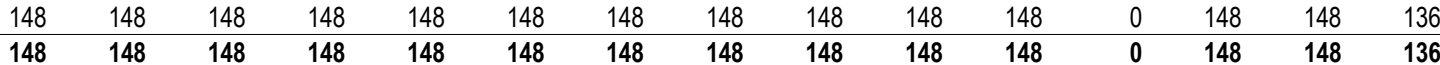

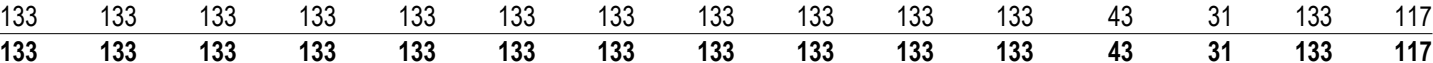

\begin{tabular}{rrrrrrrrrrrrrrr}
99 & 99 & 99 & 99 & 99 & 99 & 99 & 99 & 99 & 99 & 99 & 99 & 99 & 99 & 99 \\
66 & 66 & 66 & 66 & 66 & 66 & 66 & 66 & 66 & 66 & 66 & 66 & 66 & 66 & 66 \\
132 & 132 & 132 & 132 & 132 & 132 & 132 & 132 & 132 & 132 & 132 & 132 & 132 & 132 & 132 \\
165 & 165 & 165 & 165 & 165 & 165 & 165 & 165 & 165 & 165 & 165 & 165 & 165 & 165 & 165 \\
\hline $\mathbf{4 6 2}$ & $\mathbf{4 6 2}$ & $\mathbf{4 6 2}$ & $\mathbf{4 6 2}$ & $\mathbf{4 6 2}$ & $\mathbf{4 6 2}$ & $\mathbf{4 6 2}$ & $\mathbf{4 6 2}$ & $\mathbf{4 6 2}$ & $\mathbf{4 6 2}$ & $\mathbf{4 6 2}$ & $\mathbf{4 6 2}$ & $\mathbf{4 6 2}$ & $\mathbf{4 6 2}$ & $\mathbf{4 6 2}$
\end{tabular}

\begin{tabular}{|c|c|c|c|c|c|c|c|c|c|c|c|c|c|c|}
\hline 96 & 96 & 96 & 96 & 96 & 96 & 96 & 96 & 96 & 96 & 96 & 96 & 67 & 96 & 94 \\
\hline 192 & 192 & 192 & 192 & 192 & 192 & 192 & 192 & 192 & 192 & 192 & 192 & 134 & 192 & 187 \\
\hline 64 & 64 & 64 & 64 & 64 & 64 & 64 & 64 & 64 & 64 & 64 & 64 & 45 & 64 & 62 \\
\hline 128 & 128 & 128 & 128 & 128 & 128 & 128 & 128 & 128 & 128 & 128 & 128 & 90 & 128 & 125 \\
\hline 160 & 160 & 160 & 160 & 160 & 160 & 160 & 160 & 160 & 160 & 160 & 160 & 112 & 160 & 156 \\
\hline 40 & 640 & 640 & 640 & 640 & 640 & 640 & 640 & 640 & 640 & 640 & 640 & 448 & 640 & 624 \\
\hline
\end{tabular}

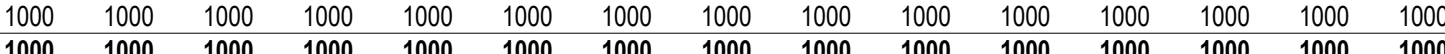

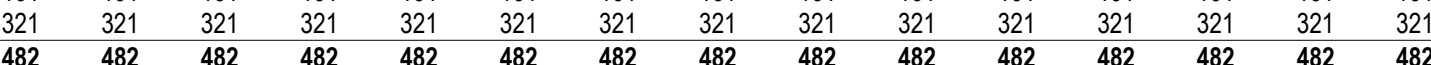

\begin{tabular}{lllllllllllllll}
163 & 163 & 163 & 163 & 163 & 163 & 163 & 163 & 163 & 163 & 163 & 163 & 163 & 163 & 163 \\
325 & 325 & 325 & 325 & 325 & 325 & 325 & 325 & 325 & 325 & 325 & 325 & 325 & 325 & 325 \\
\hline $\mathbf{4 8 8}$ & $\mathbf{4 8 8}$ & $\mathbf{4 8 8}$ & $\mathbf{4 8 8}$ & $\mathbf{4 8 8}$ & $\mathbf{4 8 8}$ & $\mathbf{4 8 8}$ & $\mathbf{4 8 8}$ & $\mathbf{4 8 8}$ & $\mathbf{4 8 8}$ & $\mathbf{4 8 8}$ & $\mathbf{4 8 8}$ & $\mathbf{4 8 8}$ & $\mathbf{4 8 8}$ & $\mathbf{4 8 8}$
\end{tabular}

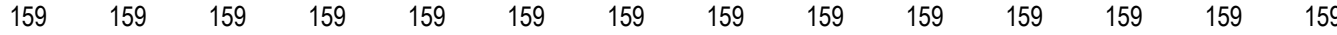

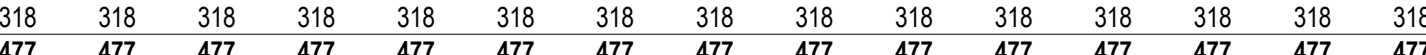

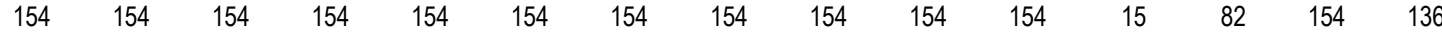

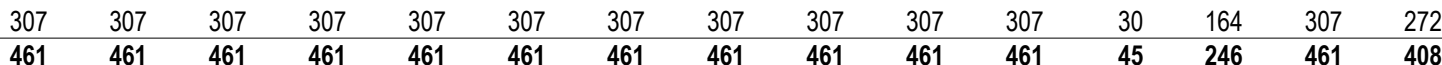

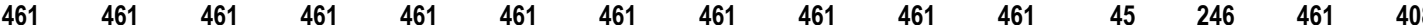

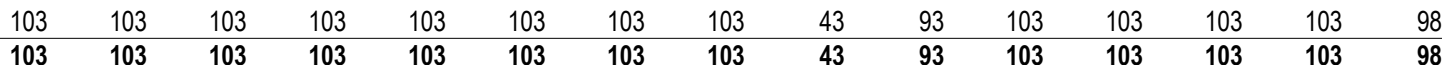

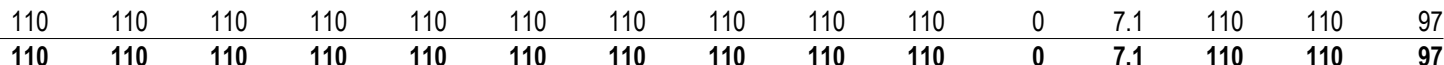

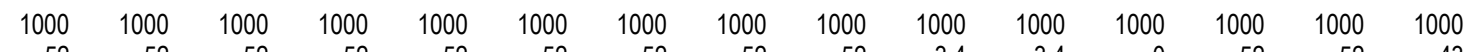

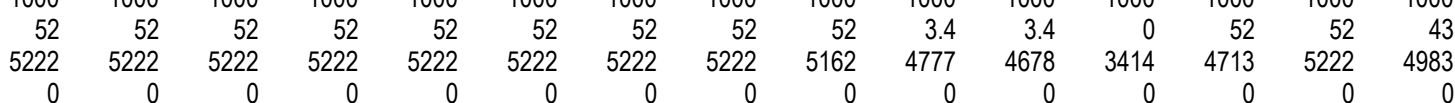




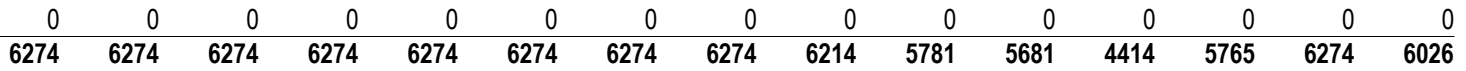



2003 White Book

Aug1 Aug16 Sep Oct Nov Dec Jan Feb Mar Apr1 Apr16 May Jun Jul Avg

-Boardman: Coal-

1 Idaho Power Company

2 Portland General Electric

3 San Diego Gas \& Electric

4 Turlock Irrigation District

5 Boardman: Regional Total

-Centralia \#1: Coal-

6 Trans Alta Utilities Corp

7 Centralia \#1: Regional Total

-Centralia \#2: Coal-

8 Trans Alta Utilities Corp

9 Centralia \#2: Regional Total

-Colstrip \#1: Coal-

10 Puget Sound Energy

11 Colstrip \#1: Regional Total

-Colstrip \#2: Coal-

12 Puget Sound Energy

13 Colstrip \#2: Regional Total

-Colstrip \#3: Coal-

14 Avista Corp (WWP Division)

15 Pacific Power

16 Portland General Electric

17 Puget Sound Energy

18 Colstrip \#3: Regional Total

-Colstrip \#4: Coal-

19 Avista Corp (WWP Division)

20 Northwestern Energy LLC (MPC)

21 Pacific Power

22 Portland General Electric

23 Puget Sound Energy

24 Colstrip \#4: Regional Total

-Columbia Generating Station: Uranium.

25 BPA - Power Business

26 Columbia Generating Station: Regional Total

-Jim Bridger \#1: Coal-

27 Idaho Power Company

28 Pacific Power

29 Jim Bridger \#1: Regional Total

-Jim Bridger \#2: Coal-

30 Idaho Power Company

31 Pacific Power

32 Jim Bridger \#2: Regional Total

-Jim Bridger \#3: Coal-

33 Idaho Power Company

34 Pacific Power

35 Jim Bridger \#3: Regional Total

-Jim Bridger \#4: Coal-

36 Idaho Power Company

37 Pacific Power

38 Jim Bridger \#4: Regional Total

-Valmy \#1: Coal-

39 Idaho Power Company

40 Valmy \#1: Regional Total

-Valmy \#2: Coal-

41 Idaho Power Company

42 Valmy \#2: Regional Total

-Total Large Thermal-

43 Federal System

44 Public Entities

45 Investor-Owned Entities

46 Other Entities

\begin{tabular}{|c|c|c|c|c|c|c|c|c|c|c|c|c|}
\hline 52 & 52 & 52 & 52 & 52 & 52 & 52 & 52 & 3.4 & 3.4 & 0 & 52 & 52 \\
\hline 337 & 337 & 337 & 337 & 337 & 337 & 337 & 337 & 22 & 22 & 0 & 337 & 337 \\
\hline 77 & 77 & 77 & 77 & 77 & 77 & 77 & 77 & 5 & 5 & 0 & 77 & 77 \\
\hline 52 & 52 & 52 & 52 & 52 & 52 & 52 & 52 & 3.4 & 3.4 & 0 & 52 & 52 \\
\hline 517 & 517 & 517 & 517 & 517 & 517 & 517 & 517 & 34 & 34 & 0 & 517 & 517 \\
\hline
\end{tabular}

$\begin{array}{lllllllllllllll}626 & 626 & 626 & 626 & 626 & 626 & 626 & 626 & 626 & 626 & 626 & 626 & 626 & 626 & 626 \\ \mathbf{6 2 6} & \mathbf{6 2 6} & \mathbf{6 2 6} & \mathbf{6 2 6} & \mathbf{6 2 6} & \mathbf{6 2 6} & \mathbf{6 2 6} & \mathbf{6 2 6} & \mathbf{6 2 6} & \mathbf{6 2 6} & \mathbf{6 2 6} & \mathbf{6 2 6} & \mathbf{6 2 6} & \mathbf{6 2 6} & \mathbf{6 2 6}\end{array}$

$\begin{array}{lllllllllllllll}626 & 626 & 626 & 626 & 626 & 626 & 626 & 626 & 626 & 626 & 626 & 40 & 626 & 626 & 577 \\ \mathbf{6 2 6} & \mathbf{6 2 6} & \mathbf{6 2 6} & \mathbf{6 2 6} & \mathbf{6 2 6} & \mathbf{6 2 6} & \mathbf{6 2 6} & \mathbf{6 2 6} & \mathbf{6 2 6} & \mathbf{6 2 6} & \mathbf{6 2 6} & \mathbf{4 0} & \mathbf{6 2 6} & \mathbf{6 2 6} & \mathbf{5 7 7}\end{array}$

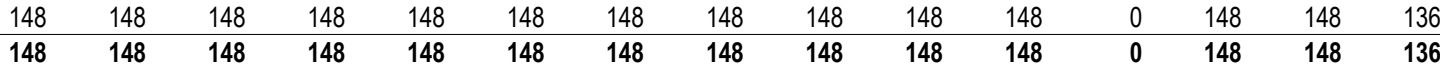

\begin{tabular}{|c|c|c|c|c|c|c|c|c|c|c|c|c|}
\hline \multirow{2}{*}{$\begin{array}{ll}33 & 133 \\
33 & 133\end{array}$} & 133 & 133 & 133 & 133 & 133 & 133 & 133 & 133 & 133 & 13 & 4 & \multirow[t]{2}{*}{133} \\
\hline & 133 & 133 & 133 & 133 & 133 & 133 & 133 & 133 & 20 & & & \\
\hline
\end{tabular}

\begin{tabular}{rrrrrrrrrrrrrrr}
99 & 99 & 99 & 99 & 99 & 99 & 99 & 99 & 99 & 99 & 99 & 24 & 99 & 99 & 93 \\
66 & 66 & 66 & 66 & 66 & 66 & 66 & 66 & 66 & 66 & 66 & 16 & 66 & 66 & 62 \\
132 & 132 & 132 & 132 & 132 & 132 & 132 & 132 & 132 & 132 & 132 & 32 & 132 & 132 & 124 \\
165 & 165 & 165 & 165 & 165 & 165 & 165 & 165 & 165 & 165 & 165 & 40 & 165 & 165 & 155 \\
\hline $\mathbf{4 6 2}$ & $\mathbf{4 6 2}$ & $\mathbf{4 6 2}$ & $\mathbf{4 6 2}$ & $\mathbf{4 6 2}$ & $\mathbf{4 6 2}$ & $\mathbf{4 6 2}$ & $\mathbf{4 6 2}$ & $\mathbf{4 6 2}$ & $\mathbf{4 6 2}$ & $\mathbf{4 6 2}$ & $\mathbf{1 1 3}$ & $\mathbf{4 6 2}$ & $\mathbf{4 6 2}$ & $\mathbf{4 3 3}$
\end{tabular}

$\begin{array}{rrrrrrrrrrrrrrr}96 & 96 & 96 & 96 & 96 & 96 & 96 & 96 & 96 & 96 & 96 & 96 & 47 & 96 & 92 \\ 192 & 192 & 192 & 192 & 192 & 192 & 192 & 192 & 192 & 192 & 192 & 192 & 94 & 192 & 184 \\ 64 & 64 & 64 & 64 & 64 & 64 & 64 & 64 & 64 & 64 & 64 & 64 & 31 & 64 & 61 \\ 128 & 128 & 128 & 128 & 128 & 128 & 128 & 128 & 128 & 128 & 128 & 128 & 62 & 128 & 123 \\ 160 & 160 & 160 & 160 & 160 & 160 & 160 & 160 & 160 & 160 & 160 & 160 & 78 & 160 & 153 \\ \mathbf{6 4 0} & \mathbf{6 4 0} & \mathbf{6 4 0} & \mathbf{6 4 0} & \mathbf{6 4 0} & \mathbf{6 4 0} & \mathbf{6 4 0} & \mathbf{6 4 0} & \mathbf{6 4 0} & \mathbf{6 4 0} & \mathbf{6 4 0} & \mathbf{6 4 0} & \mathbf{3 1 2} & \mathbf{6 4 0} & \mathbf{6 1 3}\end{array}$

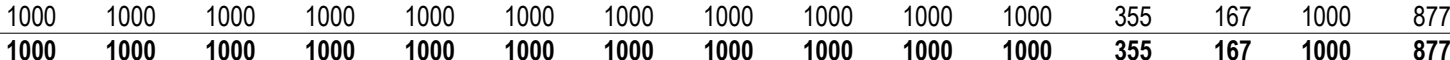

\begin{tabular}{|c|c|c|c|c|c|c|c|c|c|c|c|c|c|}
\hline 161 & 161 & 161 & 161 & 161 & 161 & 161 & 161 & 161 & 161 & 64 & 161 & 161 & 161 \\
\hline 321 & 321 & 321 & 321 & 321 & 321 & 321 & 321 & 321 & 321 & 129 & 321 & 321 & 321 \\
\hline 482 & 482 & 482 & 482 & 482 & 482 & 482 & 482 & 482 & 482 & 193 & 482 & 482 & 482 \\
\hline
\end{tabular}

\begin{tabular}{lllllllllllllll}
163 & 163 & 163 & 163 & 163 & 163 & 163 & 163 & 163 & 163 & 163 & 5.3 & 163 & 163 & 150 \\
325 & 325 & 325 & 325 & 325 & 325 & 325 & 325 & 325 & 325 & 325 & 11 & 325 & 325 & 299 \\
\hline $\mathbf{4 8 8}$ & $\mathbf{4 8 8}$ & $\mathbf{4 8 8}$ & $\mathbf{4 8 8}$ & $\mathbf{4 8 8}$ & $\mathbf{4 8 8}$ & $\mathbf{4 8 8}$ & $\mathbf{4 8 8}$ & $\mathbf{4 8 8}$ & $\mathbf{4 8 8}$ & $\mathbf{4 8 8}$ & $\mathbf{1 6}$ & $\mathbf{4 8 8}$ & $\mathbf{4 8 8}$ & $\mathbf{4 4 9}$
\end{tabular}

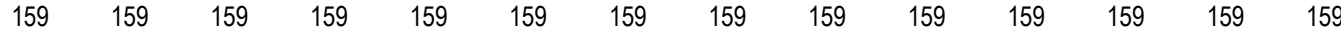

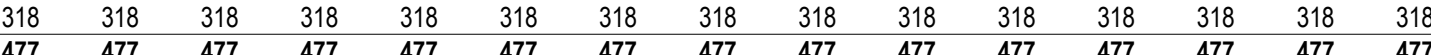

$\begin{array}{lllllllllllllll}154 & 154 & 154 & 154 & 154 & 154 & 154 & 154 & 154 & 154 & 154 & 154 & 154 & 154 & 154\end{array}$

\begin{tabular}{|c|c|c|c|c|c|c|c|c|c|c|c|c|c|}
\hline 07 & 307 & 307 & 307 & 307 & 307 & 307 & 307 & 307 & 307 & 307 & 307 & 307 & 307 \\
\hline 4 & 461 & 461 & 461 & 461 & 461 & 461 & 461 & 461 & 461 & 461 & 461 & 461 & \\
\hline
\end{tabular}

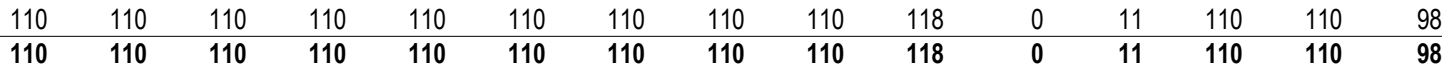

$\begin{array}{lllllllllllllll}103 & 103 & 103 & 103 & 103 & 103 & 103 & 103 & 40 & 89 & 103 & 103 & 103 & 103 & 97 \\ 103 & 103 & 103 & 103 & 103 & 103 & 103 & 103 & 40 & 89 & 103 & 103 & 103 & 103 & 97\end{array}$

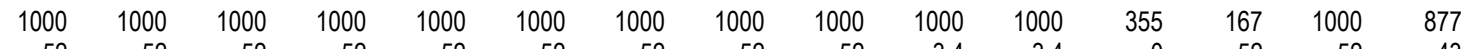

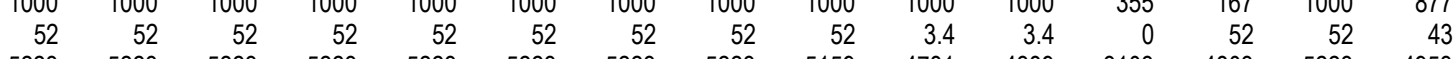

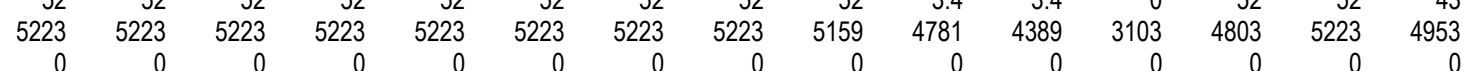


2003 White Book Dec Jan Fe Mar Apr1 Apr16

May

Jun
Jul Avg

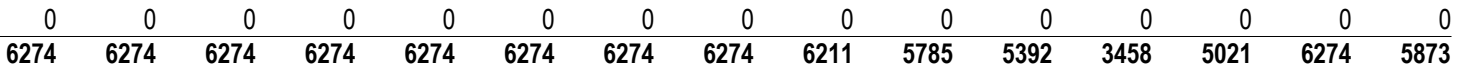



2003 White Book

Aug1 Aug16 Sep Oct Nov Dec Jan Feb Mar Apr1 Apr16 May Jun Jul Avg

-Boardman: Coal-

1 Idaho Power Company

2 Portland General Electric

3 San Diego Gas \& Electric

4 Turlock Irrigation District

5 Boardman: Regional Total

-Centralia \#1: Coal-

6 Trans Alta Utilities Corp

7 Centralia \#1: Regional Total

-Centralia \#2: Coal-

8 Trans Alta Utilities Corp

9 Centralia \#2: Regional Total

-Colstrip \#1: Coal-

10 Puget Sound Energy

11 Colstrip \#1: Regional Total

-Colstrip \#2: Coal-

12 Puget Sound Energy

13 Colstrip \#2: Regional Total

-Colstrip \#3: Coal-

14 Avista Corp (WWP Division)

15 Pacific Power

16 Portland General Electric

17 Puget Sound Energy

18 Colstrip \#3: Regional Total

-Colstrip \#4: Coal-

19 Avista Corp (WWP Division)

20 Northwestern Energy LLC (MPC)

21 Pacific Power

22 Portland General Electric

23 Puget Sound Energy

24 Colstrip \#4: Regional Total

-Columbia Generating Station: Uranium.

25 BPA - Power Business

26 Columbia Generating Station: Regional Total

-Jim Bridger \#1: Coal-

27 Idaho Power Company

28 Pacific Power

29 Jim Bridger \#1: Regional Total

-Jim Bridger \#2: Coal-

30 Idaho Power Company

31 Pacific Power

32 Jim Bridger \#2: Regional Total

-Jim Bridger \#3: Coal-

33 Idaho Power Company

34 Pacific Power

35 Jim Bridger \#3: Regional Total

-Jim Bridger \#4: Coal.

36 Idaho Power Company

37 Pacific Power

38 Jim Bridger \#4: Regional Total

-Valmy \#1: Coal-

39 Idaho Power Company

40 Valmy \#1: Regional Total

-Valmy \#2: Coal-

41 Idaho Power Company

42 Valmy \#2: Regional Total

-Total Large Thermal-

43 Federal System

44 Public Entities

45 Investor-Owned Entities

46 Other Entities

\begin{tabular}{|c|c|c|c|c|c|c|c|c|c|c|c|c|}
\hline 52 & 52 & 52 & 52 & 52 & 52 & 52 & 52 & 3.4 & 3.4 & 0 & 52 & 52 \\
\hline 337 & 337 & 337 & 337 & 337 & 337 & 337 & 337 & 22 & 22 & 0 & 337 & 337 \\
\hline 77 & 77 & 77 & 77 & 77 & 77 & 77 & 77 & 5 & 5 & 0 & 77 & 77 \\
\hline 52 & 52 & 52 & 52 & 52 & 52 & 52 & 52 & 3.4 & 3.4 & 0 & 52 & 52 \\
\hline 517 & 517 & 517 & 517 & 517 & 517 & 517 & 517 & 34 & 34 & 0 & 517 & 517 \\
\hline
\end{tabular}

$\begin{array}{lllllllllllllll}626 & 626 & 626 & 626 & 626 & 626 & 626 & 626 & 566 & 0 & 0 & 445 & 626 & 626 & 554 \\ \mathbf{6 2 6} & \mathbf{6 2 6} & \mathbf{6 2 6} & \mathbf{6 2 6} & \mathbf{6 2 6} & \mathbf{6 2 6} & \mathbf{6 2 6} & \mathbf{6 2 6} & \mathbf{5 6 6} & \mathbf{0} & \mathbf{0} & \mathbf{4 4 5} & \mathbf{6 2 6} & \mathbf{6 2 6} & \mathbf{5 5 4}\end{array}$

$\begin{array}{lllllllllllllll}626 & 626 & 626 & 626 & 626 & 626 & 626 & 626 & 626 & 626 & 626 & 626 & 626 & 626 & 626 \\ \mathbf{6 2 6} & \mathbf{6 2 6} & \mathbf{6 2 6} & \mathbf{6 2 6} & \mathbf{6 2 6} & \mathbf{6 2 6} & \mathbf{6 2 6} & \mathbf{6 2 6} & \mathbf{6 2 6} & \mathbf{6 2 6} & \mathbf{6 2 6} & \mathbf{6 2 6} & \mathbf{6 2 6} & \mathbf{6 2 6} & \mathbf{6 2 6}\end{array}$

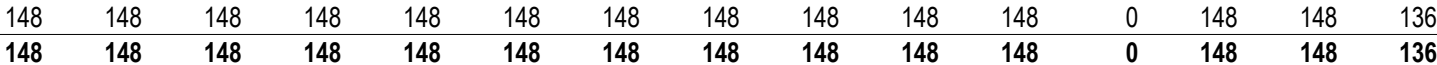

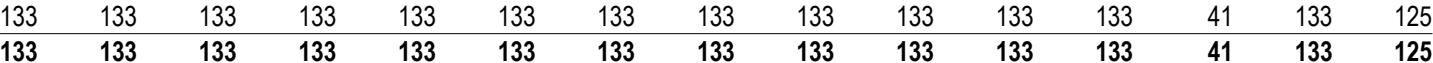

\begin{tabular}{rrrrrrrrrrrrrrr}
99 & 99 & 99 & 99 & 99 & 99 & 99 & 99 & 99 & 99 & 99 & 24 & 99 & 99 & 93 \\
66 & 66 & 66 & 66 & 66 & 66 & 66 & 66 & 66 & 66 & 66 & 16 & 66 & 66 & 62 \\
132 & 132 & 132 & 132 & 132 & 132 & 132 & 132 & 132 & 132 & 132 & 32 & 132 & 132 & 124 \\
165 & 165 & 165 & 165 & 165 & 165 & 165 & 165 & 165 & 165 & 165 & $\mathbf{4 0}$ & 165 & 165 & 155 \\
\hline $\mathbf{4 6 2}$ & $\mathbf{4 6 2}$ & $\mathbf{4 6 2}$ & $\mathbf{4 6 2}$ & $\mathbf{4 6 2}$ & $\mathbf{4 6 2}$ & $\mathbf{4 6 2}$ & $\mathbf{4 6 2}$ & $\mathbf{4 6 2}$ & $\mathbf{4 6 2}$ & $\mathbf{4 6 2}$ & $\mathbf{1 1 3}$ & $\mathbf{4 6 2}$ & $\mathbf{4 6 2}$ & $\mathbf{4 3 3}$
\end{tabular}

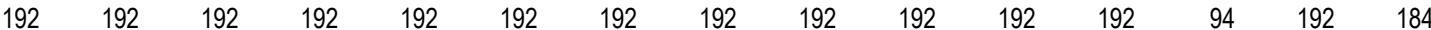

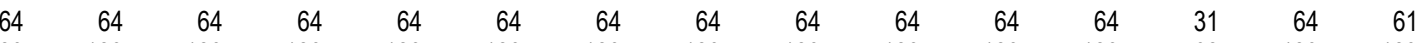

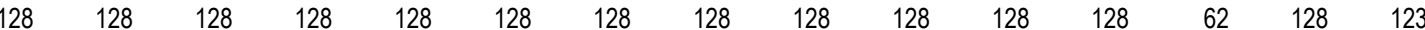

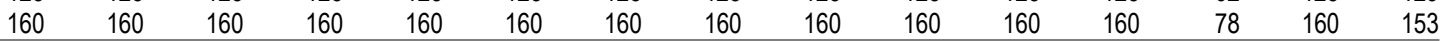

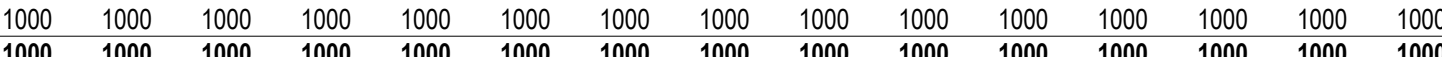

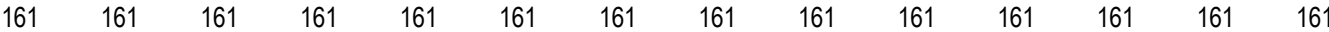

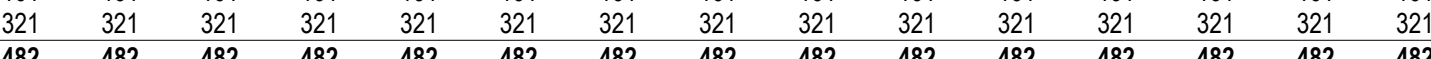

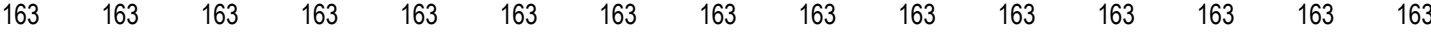

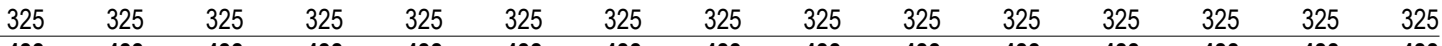

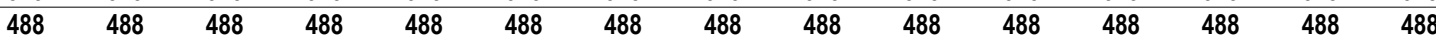

$\begin{array}{lllllllllllllll}318 & 318 & 318 & 318 & 318 & 318 & 318 & 318 & 318 & 318 & 318 & 287 & 32 & 318 & 292 \\ \mathbf{4 7 7} & \mathbf{4 7 7} & \mathbf{4 7 7} & \mathbf{4 7 7} & \mathbf{4 7 7} & \mathbf{4 7 7} & \mathbf{4 7 7} & \mathbf{4 7 7} & \mathbf{4 7 7} & \mathbf{4 7 7} & \mathbf{4 7 7} & \mathbf{4 3 1} & \mathbf{4 8} & \mathbf{4 7 7} & \mathbf{4 3 7}\end{array}$

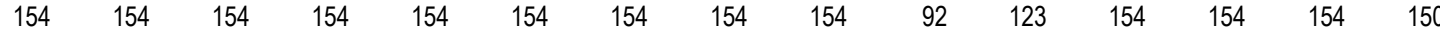

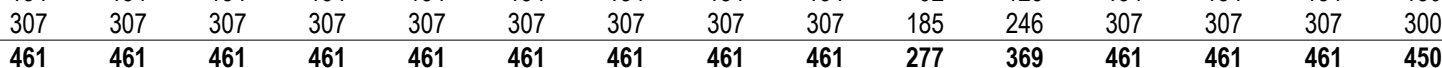

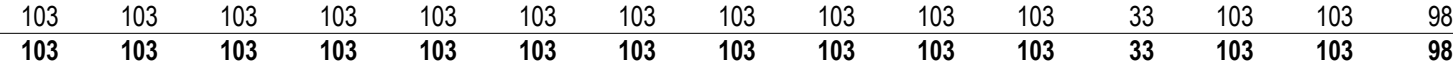

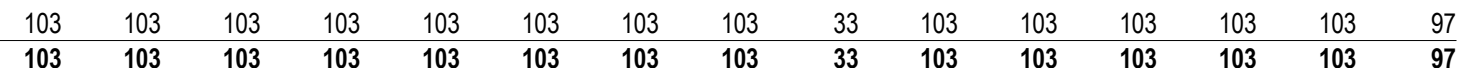

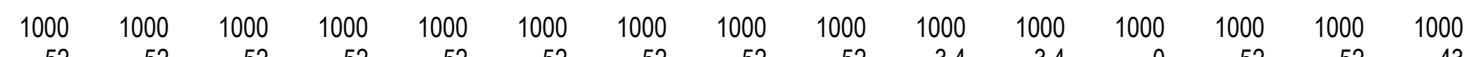

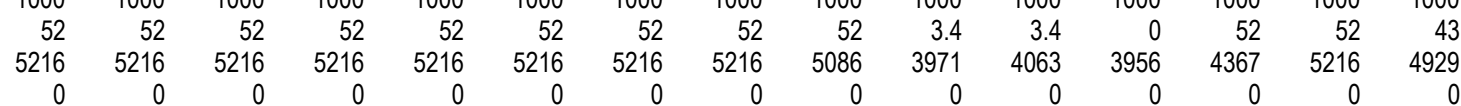


2003 White Book Sep

Oct Nov Dec Jan Fe Mar Apr1 Apr16

May

Jun Jul Avg

$\begin{array}{rrrrrrrrrrr}0 & 0 & 0 & 0 & 0 & 0 & 0 & 0 & 0 & 0 & 0 \\ 6268 & 6268 & 6268 & 6268 & 6268 & 6268 & 6268 & 6268 & 6137 & 4974 & 5066\end{array}$

$\begin{array}{rrrr}0 & 0 & 0 & 0 \\ 4956 & 5419 & 6268 & 5973\end{array}$



2003 White Book

Aug1 Aug16 Sep Oct Nov Dec Jan Feb Mar Apr1 Apr16 May Jun Jul Avg

-Boardman: Coal-

1 Idaho Power Company

2 Portland General Electric

3 San Diego Gas \& Electric

4 Turlock Irrigation District

5 Boardman: Regional Total

-Centralia \#1: Coal-

6 Trans Alta Utilities Corp

7 Centralia \#1: Regional Total

-Centralia \#2: Coal-

8 Trans Alta Utilities Corp

9 Centralia \#2: Regional Total

-Colstrip \#1: Coal-

10 Puget Sound Energy

11 Colstrip \#1: Regional Total

-Colstrip \#2: Coal-

12 Puget Sound Energy

13 Colstrip \#2: Regional Total

-Colstrip \#3: Coal-

14 Avista Corp (WWP Division)

15 Pacific Power

16 Portland General Electric

17 Puget Sound Energy

18 Colstrip \#3: Regional Total

-Colstrip \#4: Coal-

19 Avista Corp (WWP Division)

20 Northwestern Energy LLC (MPC)

21 Pacific Power

22 Portland General Electric

23 Puget Sound Energy

24 Colstrip \#4: Regional Total

-Columbia Generating Station: Uranium.

25 BPA - Power Business

26 Columbia Generating Station: Regional Total

-Jim Bridger \#1: Coal-

27 Idaho Power Company

28 Pacific Power

29 Jim Bridger \#1: Regional Total

-Jim Bridger \#2: Coal-

30 Idaho Power Company

31 Pacific Power

32 Jim Bridger \#2: Regional Total

-Jim Bridger \#3: Coal-

33 Idaho Power Company

34 Pacific Power

35 Jim Bridger \#3: Regional Total

-Jim Bridger \#4: Coal-

36 Idaho Power Company

37 Pacific Power

38 Jim Bridger \#4: Regional Total

-Valmy \#1: Coal-

39 Idaho Power Company

40 Valmy \#1: Regional Total

-Valmy \#2: Coal-

41 Idaho Power Company

42 Valmy \#2: Regional Total

-Total Large Thermal-

43 Federal System

44 Public Entities

45 Investor-Owned Entities

46 Other Entities

$\begin{array}{rrrrrrrrrrrrrrr}52 & 52 & 52 & 52 & 52 & 52 & 52 & 52 & 52 & 3.4 & 3.4 & 0 & 52 & 52 & 43 \\ 337 & 337 & 337 & 337 & 337 & 337 & 337 & 337 & 337 & 22 & 22 & 0 & 337 & 337 & 283 \\ 77 & 77 & 77 & 77 & 77 & 77 & 77 & 77 & 77 & \mathbf{5} & \mathbf{5} & 0 & 77 & 77 & 64 \\ 52 & 52 & 52 & 52 & 52 & 52 & 52 & 52 & 52 & 3.4 & 3.4 & 0 & 52 & \mathbf{5 2} & 43 \\ \mathbf{5 1 7} & \mathbf{5 1 7} & \mathbf{5 1 7} & \mathbf{5 1 7} & \mathbf{5 1 7} & \mathbf{5 1 7} & \mathbf{5 1 7} & \mathbf{5 1 7} & \mathbf{5 1 7} & \mathbf{3 4} & \mathbf{3 4} & \mathbf{0} & \mathbf{5 1 7} & \mathbf{5 1 7} & \mathbf{4 3 4}\end{array}$

$\begin{array}{lllllllllllllll}626 & 626 & 626 & 626 & 626 & 626 & 626 & 626 & 626 & 626 & 626 & 626 & 626 & 626 & 626 \\ \mathbf{6 2 6} & \mathbf{6 2 6} & \mathbf{6 2 6} & \mathbf{6 2 6} & \mathbf{6 2 6} & \mathbf{6 2 6} & \mathbf{6 2 6} & \mathbf{6 2 6} & \mathbf{6 2 6} & \mathbf{6 2 6} & \mathbf{6 2 6} & \mathbf{6 2 6} & \mathbf{6 2 6} & \mathbf{6 2 6} & \mathbf{6 2 6}\end{array}$

$\begin{array}{lllllllllllllll}626 & 626 & 626 & 626 & 626 & 626 & 626 & 626 & 566 & 0 & 0 & 445 & 626 & 626 & 554 \\ \mathbf{6 2 6} & \mathbf{6 2 6} & \mathbf{6 2 6} & \mathbf{6 2 6} & \mathbf{6 2 6} & \mathbf{6 2 6} & \mathbf{6 2 6} & \mathbf{6 2 6} & \mathbf{5 6 6} & \mathbf{0} & \mathbf{0} & \mathbf{4 4 5} & \mathbf{6 2 6} & \mathbf{6 2 6} & \mathbf{5 5 4}\end{array}$

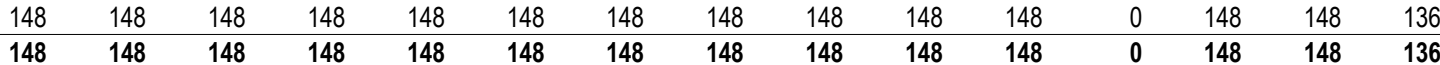

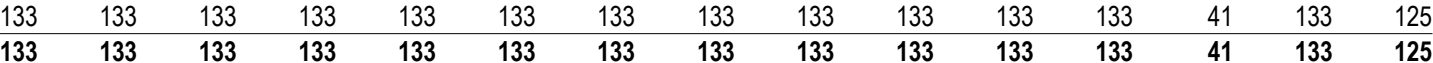

\begin{tabular}{rrrrrrrrrrrrrrr}
99 & 99 & 99 & 99 & 99 & 99 & 99 & 99 & 99 & 99 & 99 & 99 & 99 & 99 & 99 \\
66 & 66 & 66 & 66 & 66 & 66 & 66 & 66 & 66 & 66 & 66 & 66 & 66 & 66 & 66 \\
132 & 132 & 132 & 132 & 132 & 132 & 132 & 132 & 132 & 132 & 132 & 132 & 132 & 132 & 132 \\
165 & 165 & 165 & 165 & 165 & 165 & 165 & 165 & 165 & 165 & 165 & 165 & 165 & 165 & 165 \\
\hline $\mathbf{4 6 2}$ & $\mathbf{4 6 2}$ & $\mathbf{4 6 2}$ & $\mathbf{4 6 2}$ & $\mathbf{4 6 2}$ & $\mathbf{4 6 2}$ & $\mathbf{4 6 2}$ & $\mathbf{4 6 2}$ & $\mathbf{4 6 2}$ & $\mathbf{4 6 2}$ & $\mathbf{4 6 2}$ & $\mathbf{4 6 2}$ & $\mathbf{4 6 2}$ & $\mathbf{4 6 2}$ & $\mathbf{4 6 2}$
\end{tabular}

$\begin{array}{rrrrrrrrrrrrrrr}96 & 96 & 96 & 96 & 96 & 96 & 96 & 96 & 96 & 96 & 96 & 96 & 47 & 96 & 92 \\ 192 & 192 & 192 & 192 & 192 & 192 & 192 & 192 & 192 & 192 & 192 & 192 & 94 & 192 & 184 \\ 64 & 64 & 64 & 64 & 64 & 64 & 64 & 64 & 64 & 64 & 64 & 64 & 31 & 64 & 61 \\ 128 & 128 & 128 & 128 & 128 & 128 & 128 & 128 & 128 & 128 & 128 & 128 & 62 & 128 & 123 \\ 160 & 160 & 160 & 160 & 160 & 160 & 160 & 160 & 160 & 160 & 160 & 160 & 78 & 160 & 153 \\ \mathbf{6 4 0} & \mathbf{6 4 0} & \mathbf{6 4 0} & \mathbf{6 4 0} & \mathbf{6 4 0} & \mathbf{6 4 0} & \mathbf{6 4 0} & \mathbf{6 4 0} & \mathbf{6 4 0} & \mathbf{6 4 0} & \mathbf{6 4 0} & \mathbf{6 4 0} & \mathbf{3 1 2} & \mathbf{6 4 0} & \mathbf{6 1 3}\end{array}$

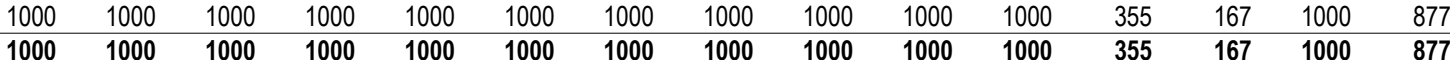

\begin{tabular}{|c|c|c|c|c|c|c|c|c|c|c|c|c|c|c|}
\hline 161 & 161 & 161 & 161 & 161 & 161 & 161 & 161 & 161 & 161 & 86 & 41 & 161 & 161 & 148 \\
\hline 321 & 321 & 321 & 321 & 321 & 321 & 321 & 321 & 321 & 321 & 171 & 83 & 321 & 321 & 295 \\
\hline 482 & 482 & 482 & 482 & 482 & 482 & 482 & 482 & 482 & 482 & 257 & 124 & 482 & 482 & 443 \\
\hline
\end{tabular}

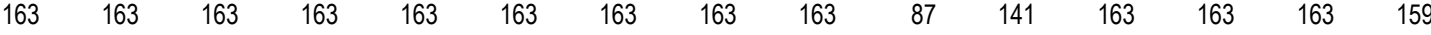

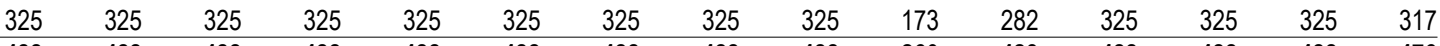

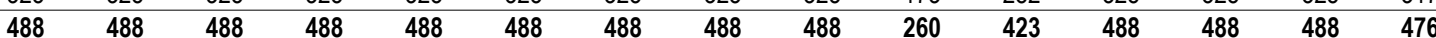

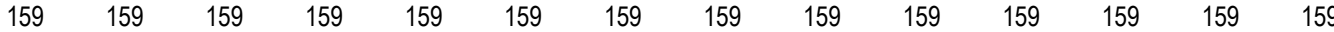

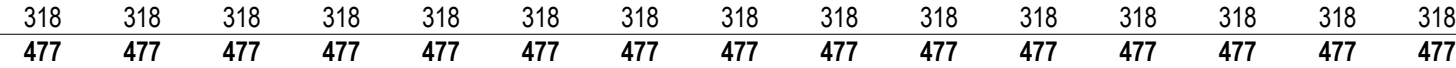

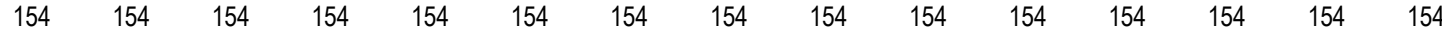

\begin{tabular}{|c|c|c|c|c|c|c|c|c|c|c|c|c|c|}
\hline 07 & 307 & 307 & 307 & 307 & 307 & 307 & 307 & 307 & 307 & 307 & 307 & 307 & 307 \\
\hline 4 & 461 & 461 & 461 & 461 & 461 & 461 & 461 & 461 & 461 & 461 & 461 & 461 & \\
\hline
\end{tabular}

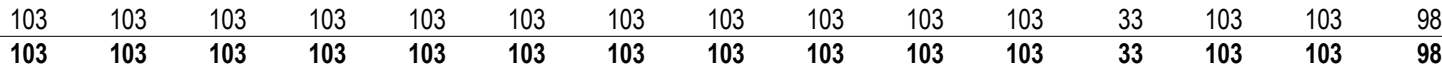

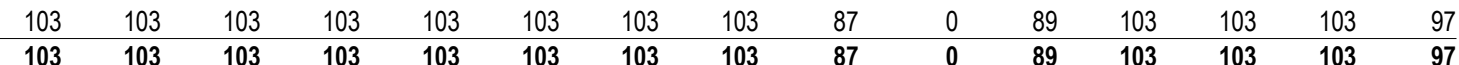

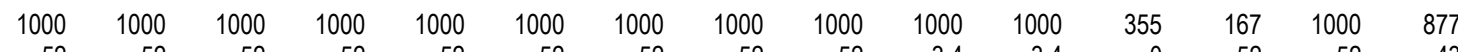

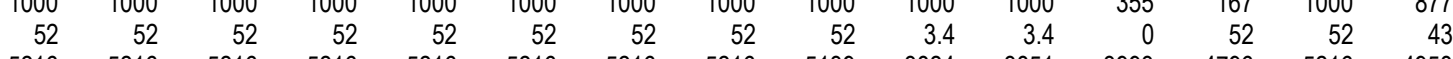

$\begin{array}{rrrrrrrrrrrrrrr}5216 & 5216 & 5216 & 5216 & 5216 & 5216 & 5216 & 5216 & 5139 & 3824 & 3851 & 3993 & 4796 & 5216 & 4958 \\ 0 & 0 & 0 & 0 & 0 & 0 & 0 & 0 & 0 & 0 & 0 & 0 & 0 & 0\end{array}$ 
2003 White Book

Oct Nov Dec Jan Fe Mar Apr1 Apr16

May Jun Jul Avg

$\begin{array}{rrr}0 & 0 & 0\end{array}$

$\begin{array}{rrrrrrrr}0 & 0 & 0 & 0 & 0 & 0 & 0 & 0 \\ 6268 & 6268 & 6268 & 6268 & 6268 & 6191 & 4827 & 4855\end{array}$

$\begin{array}{rrrr}0 & 0 & 0 & 0 \\ 4348 & 5015 & 6268 & 5878\end{array}$



2003 White Book

Aug1 Aug16 Sep Oct Nov Dec Jan Feb Mar Apr1 Apr16 May Jun Jul Avg

-Boardman: Coal-

1 Idaho Power Company

2 Portland General Electric

3 San Diego Gas \& Electric

4 Turlock Irrigation District

5 Boardman: Regional Total

-Centralia \#1: Coal-

6 Trans Alta Utilities Corp

7 Centralia \#1: Regional Total

-Centralia \#2: Coal-

8 Trans Alta Utilities Corp

9 Centralia \#2: Regional Total

-Colstrip \#1: Coal-

10 Puget Sound Energy

11 Colstrip \#1: Regional Total

-Colstrip \#2: Coal-

12 Puget Sound Energy

13 Colstrip \#2: Regional Total

-Colstrip \#3: Coal-

14 Avista Corp (WWP Division)

15 Pacific Power

16 Portland General Electric

17 Puget Sound Energy

18 Colstrip \#3: Regional Total

-Colstrip \#4: Coal-

19 Avista Corp (WWP Division)

20 Northwestern Energy LLC (MPC)

21 Pacific Power

22 Portland General Electric

23 Puget Sound Energy

24 Colstrip \#4: Regional Total

-Columbia Generating Station: Uranium

25 BPA - Power Business

$\begin{array}{rrrrrrrrrrrrrrr}52 & 52 & 52 & 52 & 52 & 52 & 52 & 52 & 52 & 3.4 & 3.4 & 0 & 52 & 52 & 43 \\ 337 & 337 & 337 & 337 & 337 & 337 & 337 & 337 & 337 & 22 & 22 & 0 & 337 & 337 & 283 \\ 77 & 77 & 77 & 77 & 77 & 77 & 77 & 77 & 77 & \mathbf{5} & \mathbf{5} & 0 & 77 & 77 & 64 \\ 52 & 52 & 52 & 52 & 52 & 52 & 52 & 52 & 52 & 3.4 & 3.4 & 0 & 52 & \mathbf{5 2} & 43 \\ \mathbf{5 1 7} & \mathbf{5 1 7} & \mathbf{5 1 7} & \mathbf{5 1 7} & \mathbf{5 1 7} & \mathbf{5 1 7} & \mathbf{5 1 7} & \mathbf{5 1 7} & \mathbf{5 1 7} & \mathbf{3 4} & \mathbf{3 4} & \mathbf{0} & \mathbf{5 1 7} & \mathbf{5 1 7} & \mathbf{4 3 4}\end{array}$

26 Columbia Generating Station: Regional Total

-Jim Bridger \#1: Coal-

27 Idaho Power Company

28 Pacific Power

29 Jim Bridger \#1: Regional Total

-Jim Bridger \#2: Coal-

30 Idaho Power Company

31 Pacific Power

32 Jim Bridger \#2: Regional Total

-Jim Bridger \#3: Coal-

33 Idaho Power Company

34 Pacific Power

35 Jim Bridger \#3: Regional Total

-Jim Bridger \#4: Coal-

36 Idaho Power Company

37 Pacific Power

38 Jim Bridger \#4: Regional Total

-Valmy \#1: Coal-

39 Idaho Power Company

40 Valmy \#1: Regional Total

-Valmy \#2: Coal-

41 Idaho Power Company

42 Valmy \#2: Regional Total

-Total Large Thermal-

43 Federal System

44 Public Entities

45 Investor-Owned Entities

46 Other Entities

\begin{tabular}{lllllllllllllll}
626 & 626 & 626 & 626 & 626 & 626 & 626 & 626 & 626 & 626 & 626 & 40 & 626 & 626 & 577 \\
\hline $\mathbf{6 2 6}$ & $\mathbf{6 2 6}$ & $\mathbf{6 2 6}$ & $\mathbf{6 2 6}$ & $\mathbf{6 2 6}$ & $\mathbf{6 2 6}$ & $\mathbf{6 2 6}$ & $\mathbf{6 2 6}$ & $\mathbf{6 2 6}$ & $\mathbf{6 2 6}$ & $\mathbf{6 2 6}$ & $\mathbf{4 0}$ & $\mathbf{6 2 6}$ & $\mathbf{6 2 6}$ & $\mathbf{5 7 7 7}$
\end{tabular}

$\begin{array}{lllllllllllllll}626 & 626 & 626 & 626 & 626 & 626 & 626 & 626 & 626 & 626 & 626 & 626 & 626 & 626 & 626 \\ \mathbf{6 2 6} & \mathbf{6 2 6} & \mathbf{6 2 6} & \mathbf{6 2 6} & \mathbf{6 2 6} & \mathbf{6 2 6} & \mathbf{6 2 6} & \mathbf{6 2 6} & \mathbf{6 2 6} & \mathbf{6 2 6} & \mathbf{6 2 6} & \mathbf{6 2 6} & \mathbf{6 2 6} & \mathbf{6 2 6} & \mathbf{6 2 6}\end{array}$

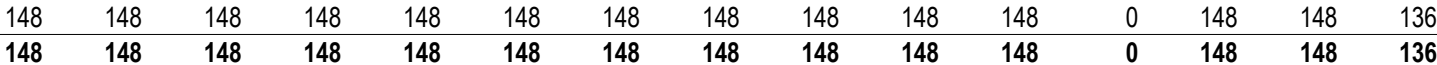

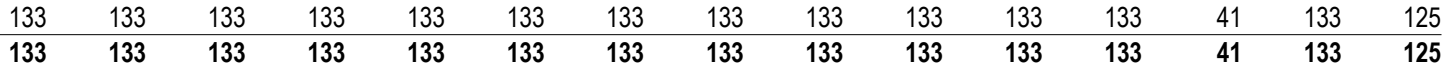

$\begin{array}{rrrrrrrrrrrrrrr}99 & 99 & 99 & 99 & 99 & 99 & 99 & 99 & 99 & 99 & 99 & 24 & 99 & 99 & 93 \\ 66 & 66 & 66 & 66 & 66 & 66 & 66 & 66 & 66 & 66 & 66 & 16 & 66 & 66 & 62 \\ 132 & 132 & 132 & 132 & 132 & 132 & 132 & 132 & 132 & 132 & 132 & 32 & 132 & 132 & 124 \\ 165 & 165 & 165 & 165 & 165 & 165 & 165 & 165 & 165 & 165 & 165 & \mathbf{4 0} & 165 & 165 & 155 \\ \mathbf{4 6 2} & \mathbf{4 6 2} & \mathbf{4 6 2} & \mathbf{4 6 2} & \mathbf{4 6 2} & \mathbf{4 6 2} & \mathbf{4 6 2} & \mathbf{4 6 2} & \mathbf{4 6 2} & \mathbf{4 6 2} & \mathbf{4 6 2} & \mathbf{1 1 3} & \mathbf{4 6 2} & \mathbf{4 6 2} & \mathbf{4 3 3}\end{array}$

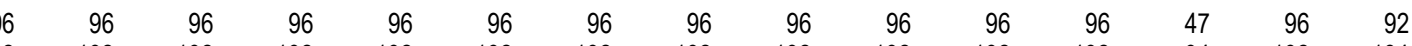

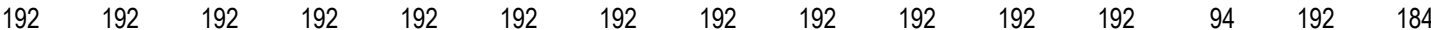

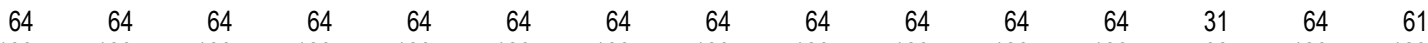

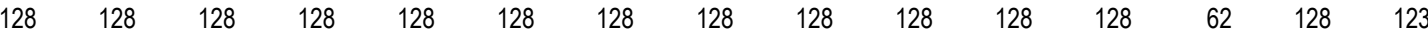

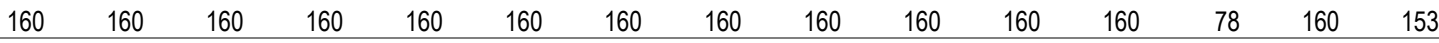

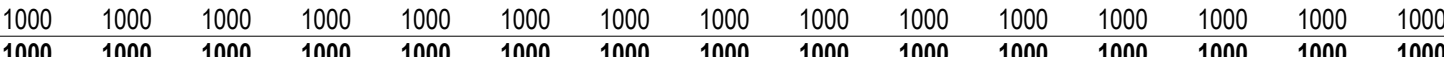

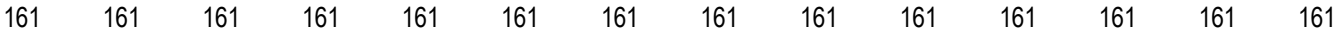

\begin{tabular}{|c|c|c|c|c|c|c|c|c|c|c|c|c|}
\hline 321 & 321 & 321 & 321 & 321 & 321 & 321 & 321 & 321 & 321 & 32 & 32 & 321 \\
\hline 482 & $\frac{011}{48 ?}$ & $48 ?$ & UL1 & $\frac{0<1}{482}$ & UL1 & $\begin{array}{l}481 \\
187\end{array}$ & UL1 & 482 & 487 & 4 & 1 & 021 \\
\hline
\end{tabular}

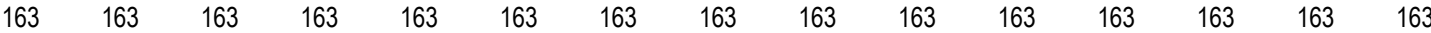

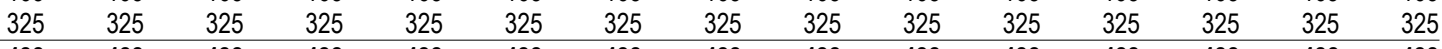

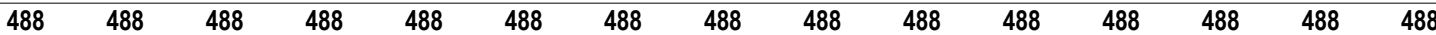

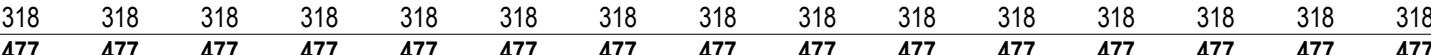

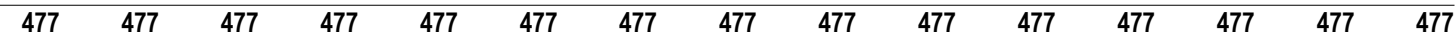

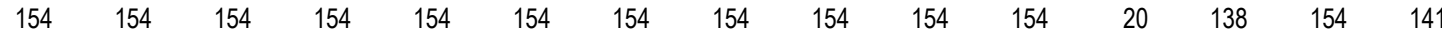

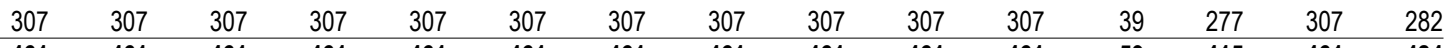

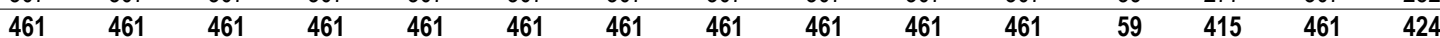

$\begin{array}{lllllllllllllll}103 & 103 & 103 & 103 & 103 & 103 & 103 & 103 & 103 & 69 & 0 & 100 & 103 & 103 & 97 \\ 103 & 103 & 103 & 103 & 103 & 103 & 103 & 103 & 103 & \mathbf{6 9} & \mathbf{0} & \mathbf{1 0 0} & \mathbf{1 0 3} & \mathbf{1 0 3} & \mathbf{9 7}\end{array}$

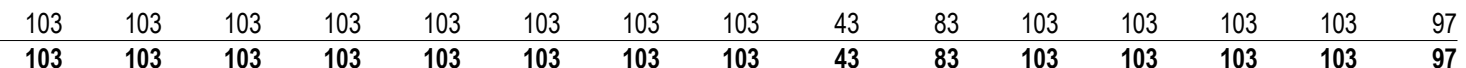

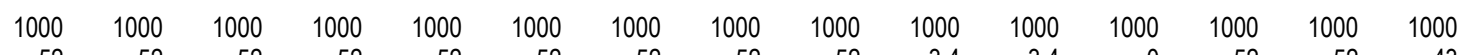

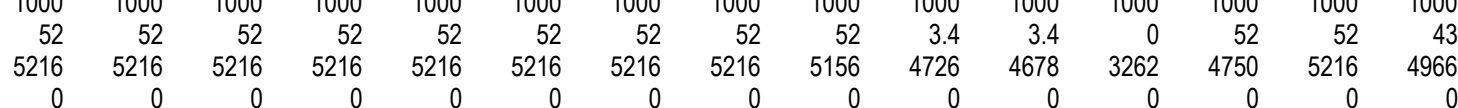


Table A-10: Regional Large Thermal

PNW Loads and Resources Study

2011 - 2012 Operating Year

2003 White Book

Oct Nov Dec Jan $\mathrm{Fe}$ Mar Apr1 Apr16

May

Jun Jul Avg

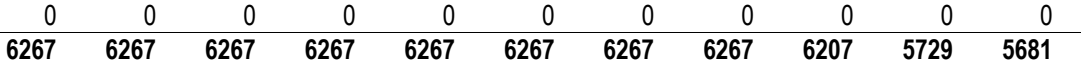

$\begin{array}{rrrr}0 & 0 & 0 & 0 \\ 4262 & 5801 & 6267 & 6009\end{array}$



2003 White Book

Aug1 Aug16 Sep Oct Nov Dec Jan Feb Mar Apr1 Apr16 May Jun Jul Avg

-Boardman: Coal-

1 Idaho Power Company

2 Portland General Electric

3 San Diego Gas \& Electric

4 Turlock Irrigation District

5 Boardman: Regional Total

-Centralia \#1: Coal-

6 Trans Alta Utilities Corp

7 Centralia \#1: Regional Total

-Centralia \#2: Coal-

8 Trans Alta Utilities Corp

9 Centralia \#2: Regional Total

-Colstrip \#1: Coal-

10 Puget Sound Energy

11 Colstrip \#1: Regional Total

-Colstrip \#2: Coal-

12 Puget Sound Energy

13 Colstrip \#2: Regional Total

-Colstrip \#3: Coal-

14 Avista Corp (WWP Division)

15 Pacific Power

16 Portland General Electric

17 Puget Sound Energy

18 Colstrip \#3: Regional Total

-Colstrip \#4: Coal-

19 Avista Corp (WWP Division)

20 Northwestern Energy LLC (MPC)

21 Pacific Power

22 Portland General Electric

23 Puget Sound Energy

24 Colstrip \#4: Regional Total

-Columbia Generating Station: Uranium.

25 BPA - Power Business

26 Columbia Generating Station: Regional Total

-Jim Bridger \#1: Coal-

27 Idaho Power Company

28 Pacific Power

29 Jim Bridger \#1: Regional Total

-Jim Bridger \#2: Coal-

30 Idaho Power Company

31 Pacific Power

32 Jim Bridger \#2: Regional Total

-Jim Bridger \#3: Coal-

33 Idaho Power Company

34 Pacific Power

35 Jim Bridger \#3: Regional Total

-Jim Bridger \#4: Coal.

36 Idaho Power Company

37 Pacific Power

38 Jim Bridger \#4: Regional Total

-Valmy \#1: Coal-

39 Idaho Power Company

40 Valmy \#1: Regional Total

-Valmy \#2: Coal-

41 Idaho Power Company

42 Valmy \#2: Regional Total

-Total Large Thermal-

43 Federal System

44 Public Entities

45 Investor-Owned Entities

46 Other Entities

\begin{tabular}{|c|c|c|c|c|c|c|c|c|c|c|c|c|}
\hline 52 & 52 & 52 & 52 & 52 & 52 & 52 & 52 & 3.4 & 3.4 & 0 & 52 & 52 \\
\hline 337 & 337 & 337 & 337 & 337 & 337 & 337 & 337 & 22 & 22 & 0 & 337 & 337 \\
\hline 77 & 77 & 77 & 77 & 77 & 77 & 77 & 77 & 5 & 5 & 0 & 77 & 77 \\
\hline 52 & 52 & 52 & 52 & 52 & 52 & 52 & 52 & 3.4 & 3.4 & 0 & 52 & 52 \\
\hline 517 & 517 & 517 & 517 & 517 & 517 & 517 & 517 & 34 & 34 & 0 & 517 & 517 \\
\hline
\end{tabular}

$\begin{array}{lllllllllllllll}626 & 626 & 626 & 626 & 626 & 626 & 626 & 626 & 626 & 626 & 626 & 626 & 626 & 626 & 626 \\ \mathbf{6 2 6} & \mathbf{6 2 6} & \mathbf{6 2 6} & \mathbf{6 2 6} & \mathbf{6 2 6} & \mathbf{6 2 6} & \mathbf{6 2 6} & \mathbf{6 2 6} & \mathbf{6 2 6} & \mathbf{6 2 6} & \mathbf{6 2 6} & \mathbf{6 2 6} & \mathbf{6 2 6} & \mathbf{6 2 6} & \mathbf{6 2 6}\end{array}$

$\begin{array}{lllllllllllllll}626 & 626 & 626 & 626 & 626 & 626 & 626 & 626 & 626 & 626 & 626 & 40 & 626 & 626 & 577 \\ \mathbf{6 2 6} & \mathbf{6 2 6} & \mathbf{6 2 6} & \mathbf{6 2 6} & \mathbf{6 2 6} & \mathbf{6 2 6} & \mathbf{6 2 6} & \mathbf{6 2 6} & \mathbf{6 2 6} & \mathbf{6 2 6} & \mathbf{6 2 6} & \mathbf{4 0} & \mathbf{6 2 6} & \mathbf{6 2 6} & \mathbf{5 7 7}\end{array}$

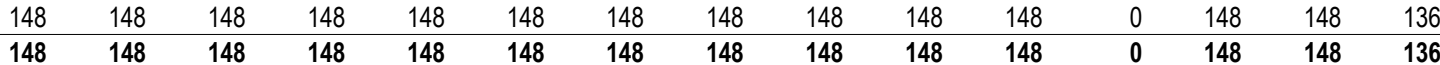

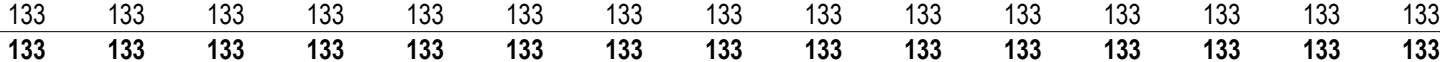

\begin{tabular}{rrrrrrrrrrrrrrr}
99 & 99 & 99 & 99 & 99 & 99 & 99 & 99 & 99 & 99 & 99 & 99 & 99 & 99 & 99 \\
66 & 66 & 66 & 66 & 66 & 66 & 66 & 66 & 66 & 66 & 66 & 66 & 66 & 66 & 66 \\
132 & 132 & 132 & 132 & 132 & 132 & 132 & 132 & 132 & 132 & 132 & 132 & 132 & 132 & 132 \\
165 & 165 & 165 & 165 & 165 & 165 & 165 & 165 & 165 & 165 & 165 & 165 & 165 & 165 & 165 \\
\hline $\mathbf{4 6 2}$ & $\mathbf{4 6 2}$ & $\mathbf{4 6 2}$ & $\mathbf{4 6 2}$ & $\mathbf{4 6 2}$ & $\mathbf{4 6 2}$ & $\mathbf{4 6 2}$ & $\mathbf{4 6 2}$ & $\mathbf{4 6 2}$ & $\mathbf{4 6 2}$ & $\mathbf{4 6 2}$ & $\mathbf{4 6 2}$ & $\mathbf{4 6 2}$ & $\mathbf{4 6 2}$ & $\mathbf{4 6 2}$
\end{tabular}

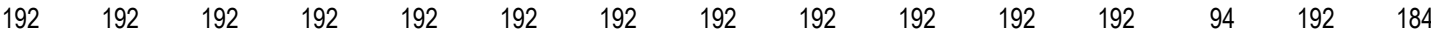

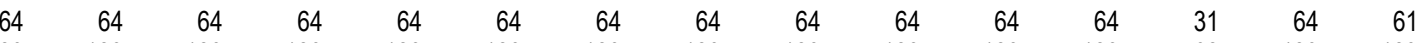

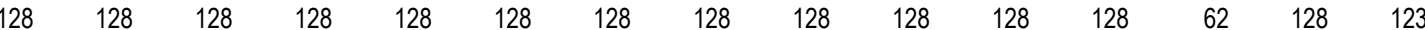

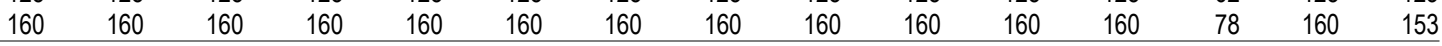

$\begin{array}{lllllllllllllll}1000 & 1000 & 1000 & 1000 & 1000 & 1000 & 1000 & 1000 & 1000 & 1000 & 1000 & 355 & 167 & 1000 & 877 \\ 1000 & 1000 & 1000 & 1000 & 1000 & 1000 & 1000 & 1000 & 1000 & 1000 & 1000 & 355 & 167 & 1000 & 877\end{array}$

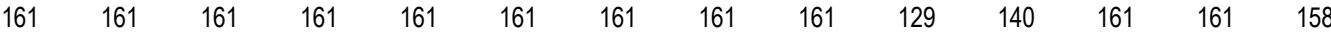

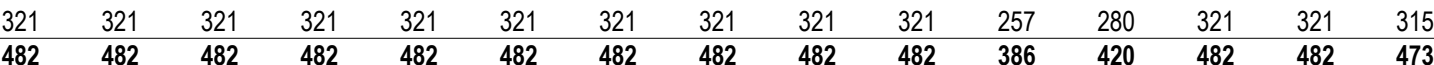

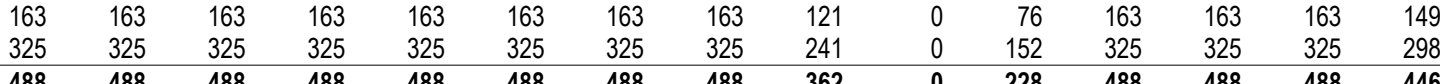

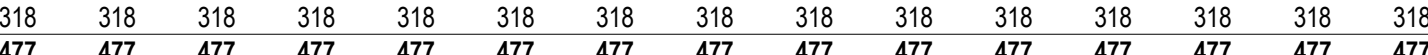

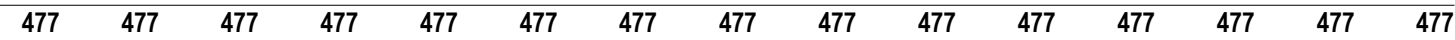

$\begin{array}{lllllllllllllll}154 & 154 & 154 & 154 & 154 & 154 & 154 & 154 & 154 & 154 & 154 & 154 & 154 & 154 & 154\end{array}$

\begin{tabular}{|c|c|c|c|c|c|c|c|c|c|c|c|c|c|}
\hline 07 & 307 & 307 & 307 & 307 & 307 & 307 & 307 & 307 & 307 & 307 & 307 & 307 & 307 \\
\hline 4 & 461 & 461 & 461 & 461 & 461 & 461 & 461 & 461 & 461 & 461 & 461 & 461 & \\
\hline
\end{tabular}

$\begin{array}{lllllllllllllll}103 & 103 & 103 & 103 & 103 & 103 & 103 & 103 & 40 & 96 & 103 & 103 & 103 & 103 & 98 \\ 103 & 103 & 103 & 103 & 103 & 103 & 103 & 103 & \mathbf{4 0} & \mathbf{9 6} & \mathbf{1 0 3} & \mathbf{1 0 3} & \mathbf{1 0 3} & \mathbf{1 0 3} & \mathbf{9 8}\end{array}$

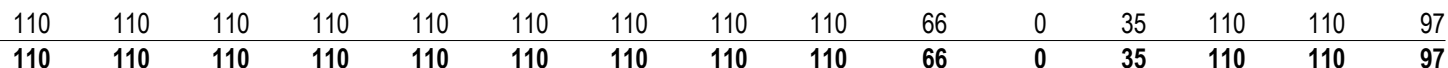

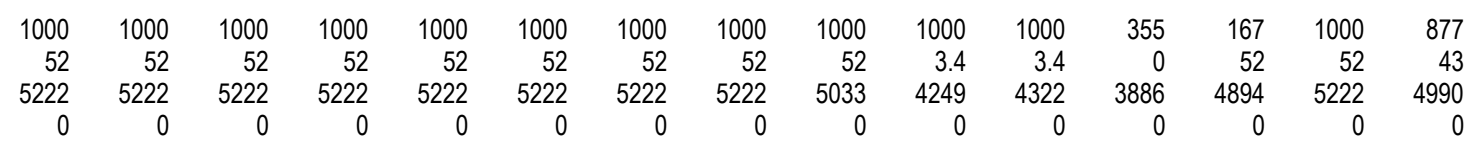


2003 White Book

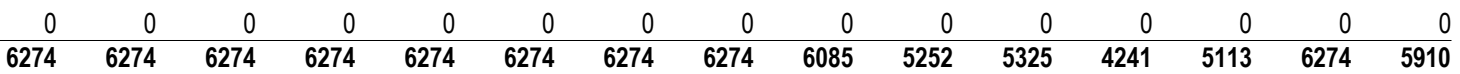



2003 White Book

Aug1 Aug16 Sep Oct Nov Dec Jan Feb Mar Apr1 Apr16 May Jun Jul Avg

-Boardman: Coal-

1 Idaho Power Company

2 Portland General Electric

3 San Diego Gas \& Electric

4 Turlock Irrigation District

5 Boardman: Regional Total

-Centralia \#1: Coal-

6 Trans Alta Utilities Corp

7 Centralia \#1: Regional Total

-Centralia \#2: Coal-

8 Trans Alta Utilities Corp

9 Centralia \#2: Regional Total

-Colstrip \#1: Coal-

10 Puget Sound Energy

11 Colstrip \#1: Regional Total

-Colstrip \#2: Coal-

12 Puget Sound Energy

13 Colstrip \#2: Regional Total

-Colstrip \#3: Coal-

14 Avista Corp (WWP Division)

15 Pacific Power

16 Portland General Electric

17 Puget Sound Energy

18 Colstrip \#3: Regional Total

-Colstrip \#4: Coal-

19 Avista Corp (WWP Division)

20 Northwestern Energy LLC (MPC)

21 Pacific Power

22 Portland General Electric

23 Puget Sound Energy

24 Colstrip \#4: Regional Total

-Columbia Generating Station: Uranium.

25 BPA - Power Business

26 Columbia Generating Station: Regional Total

-Jim Bridger \#1: Coal-

27 Idaho Power Company

28 Pacific Power

29 Jim Bridger \#1: Regional Total

-Jim Bridger \#2: Coal-

30 Idaho Power Company

31 Pacific Power

32 Jim Bridger \#2: Regional Total

-Jim Bridger \#3: Coal-

33 Idaho Power Company

34 Pacific Power

35 Jim Bridger \#3: Regional Total

-Jim Bridger \#4: Coal-

36 Idaho Power Company

37 Pacific Power

38 Jim Bridger \#4: Regional Total

-Valmy \#1: Coal-

39 Idaho Power Company

40 Valmy \#1: Regional Total

-Valmy \#2: Coal-

41 Idaho Power Company

42 Valmy \#2: Regional Total

-Total Large Thermal-

43 Federal System

44 Public Entities

45 Investor-Owned Entities

46 Other Entities

\begin{tabular}{|c|c|c|c|c|c|c|c|c|c|c|c|c|}
\hline 52 & 52 & 52 & 52 & 52 & 52 & 52 & 52 & 3.4 & 3.4 & 0 & 52 & 52 \\
\hline 337 & 337 & 337 & 337 & 337 & 337 & 337 & 337 & 22 & 22 & 0 & 337 & 337 \\
\hline 77 & 77 & 77 & 77 & 77 & 77 & 77 & 77 & 5 & 5 & 0 & 77 & 77 \\
\hline 52 & 52 & 52 & 52 & 52 & 52 & 52 & 52 & 3.4 & 3.4 & 0 & 52 & 52 \\
\hline 517 & 517 & 517 & 517 & 517 & 517 & 517 & 517 & 34 & 34 & 0 & 517 & 517 \\
\hline
\end{tabular}

\begin{tabular}{lllllllllllllll}
626 & 626 & 626 & 626 & 626 & 626 & 626 & 626 & 626 & 626 & 626 & 40 & 626 & 626 & 577 \\
\hline $\mathbf{6 2 6}$ & $\mathbf{6 2 6}$ & $\mathbf{6 2 6}$ & $\mathbf{6 2 6}$ & $\mathbf{6 2 6}$ & $\mathbf{6 2 6}$ & $\mathbf{6 2 6}$ & $\mathbf{6 2 6}$ & $\mathbf{6 2 6}$ & $\mathbf{6 2 6}$ & $\mathbf{6 2 6}$ & $\mathbf{4 0}$ & $\mathbf{6 2 6}$ & $\mathbf{6 2 6}$ & $\mathbf{5 7 7}$
\end{tabular}

$\begin{array}{lllllllllllllll}626 & 626 & 626 & 626 & 626 & 626 & 626 & 626 & 626 & 626 & 626 & 626 & 626 & 626 & 626 \\ \mathbf{6 2 6} & \mathbf{6 2 6} & \mathbf{6 2 6} & \mathbf{6 2 6} & \mathbf{6 2 6} & \mathbf{6 2 6} & \mathbf{6 2 6} & \mathbf{6 2 6} & \mathbf{6 2 6} & \mathbf{6 2 6} & \mathbf{6 2 6} & \mathbf{6 2 6} & \mathbf{6 2 6} & \mathbf{6 2 6} & \mathbf{6 2 6}\end{array}$

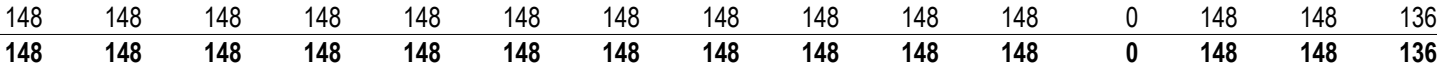

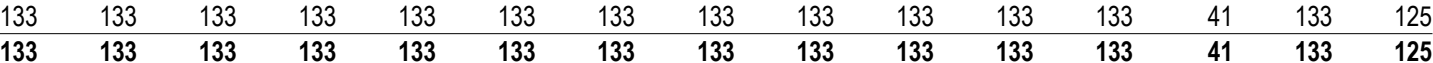

$\begin{array}{rrrrrrrrrrrrrrr}99 & 99 & 99 & 99 & 99 & 99 & 99 & 99 & 99 & 99 & 99 & 24 & 99 & 99 & 93 \\ 66 & 66 & 66 & 66 & 66 & 66 & 66 & 66 & 66 & 66 & 66 & 16 & 66 & 66 & 62 \\ 132 & 132 & 132 & 132 & 132 & 132 & 132 & 132 & 132 & 132 & 132 & 32 & 132 & 132 & 124 \\ 165 & 165 & 165 & 165 & 165 & 165 & 165 & 165 & 165 & 165 & 165 & \mathbf{4 0} & 165 & 165 & 155 \\ \mathbf{4 6 2} & \mathbf{4 6 2} & \mathbf{4 6 2} & \mathbf{4 6 2} & \mathbf{4 6 2} & \mathbf{4 6 2} & \mathbf{4 6 2} & \mathbf{4 6 2} & \mathbf{4 6 2} & \mathbf{4 6 2} & \mathbf{4 6 2} & \mathbf{1 1 3} & \mathbf{4 6 2} & \mathbf{4 6 2} & \mathbf{4 3 3}\end{array}$

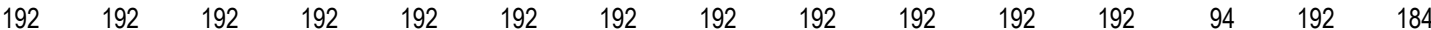

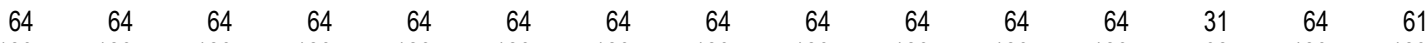

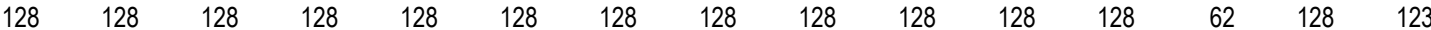

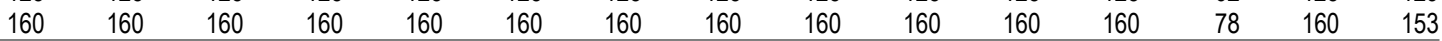

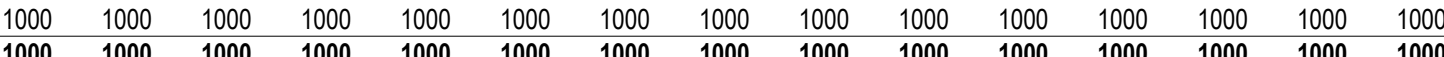

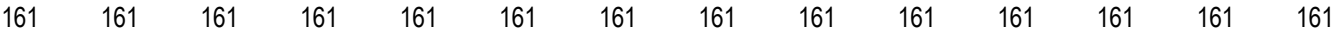

\begin{tabular}{|c|c|c|c|c|c|c|c|c|c|c|c|c|}
\hline 321 & 321 & 321 & 321 & 321 & 321 & 321 & 321 & 321 & 321 & 32 & 32 & 321 \\
\hline 18? & $\frac{5<1}{182}$ & D21 & $\frac{0<1}{182}$ & $\frac{3<1}{182}$ & $\frac{3<1}{182}$ & $\frac{3<1}{182}$ & $\begin{array}{l}0<1 \\
182\end{array}$ & $\begin{array}{l}0<1 \\
182\end{array}$ & $\frac{021}{189}$ & 0 & 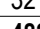 & $0 \angle 1$ \\
\hline
\end{tabular}

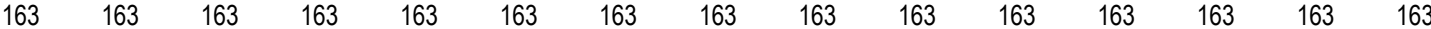

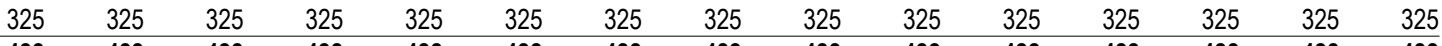

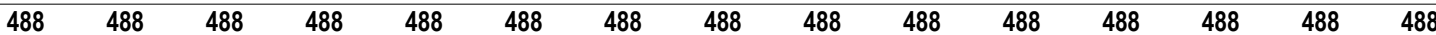

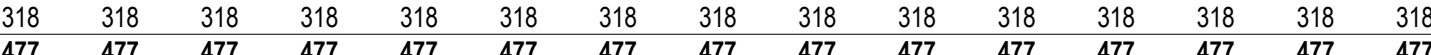

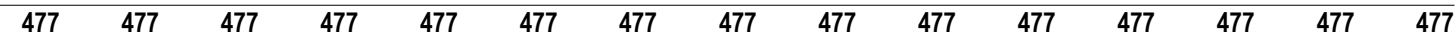

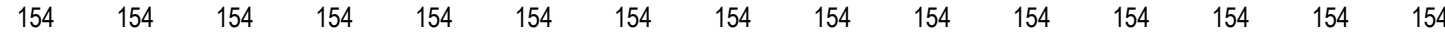

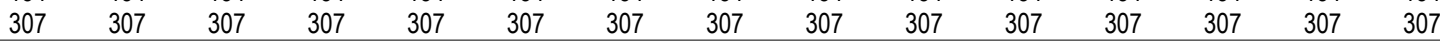

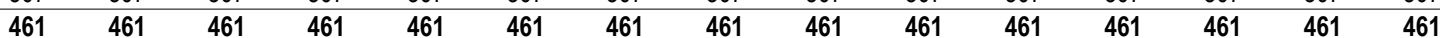

$\begin{array}{lllllllllllllll}103 & 103 & 103 & 103 & 103 & 103 & 103 & 103 & 40 & 89 & 103 & 103 & 103 & 103 & 97 \\ 103 & 103 & 103 & 103 & 103 & \mathbf{1 0 3} & \mathbf{1 0 3} & \mathbf{1 0 3} & \mathbf{4 0} & \mathbf{8 9} & \mathbf{1 0 3} & \mathbf{1 0 3} & \mathbf{1 0 3} & \mathbf{1 0 3} & \mathbf{9 7}\end{array}$

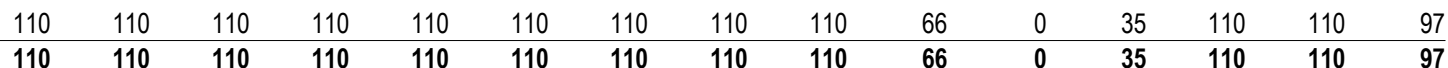

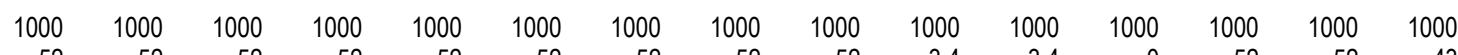

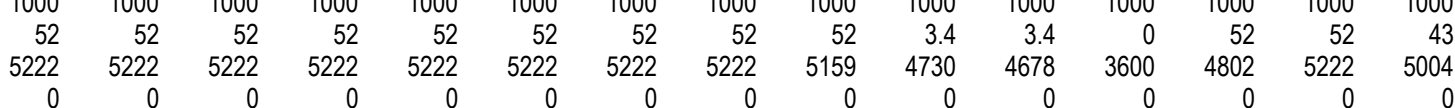


2003 White Book Dec Jan $\mathrm{Fe}$ Mar Apr1 Apr16

May

Jun
Jul Avg

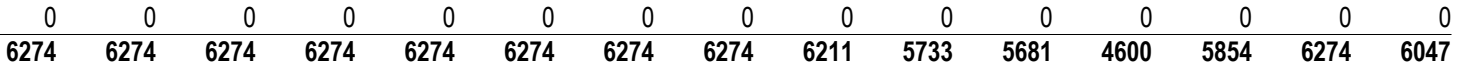



2003 White Book

-Federal System-

Aug1 Aug16 Sep Oct Nov Dec Jan Feb Mar Apr1 Apr16 May Jun Jul Avg

1 Total Federal System

$\begin{array}{lllllllllllllll}0 & 0 & 0 & 0 & 0 & 0 & 0 & 0 & 0 & 0 & 0 & 0 & 0 & 0 & 0\end{array}$

-Public Entities-

2 Total Public Entities

0

0

-Investor-Owned Entities-

3 Total Investor-Owned Entities

\begin{tabular}{llllllllllllll}
0 & 0 & 0 & 0 & 0 & 0 & 0 & 0 & 0 & 0 & 0 & 0 & 0 & 0 \\
\hline
\end{tabular}

-Other Entities-

4 Total Other Entities

0

0

-Restoration-

5 Federal System

6 Public Entities

7 Investor-Owned Entities

8 Other Entities

9 Total Restoration

\begin{tabular}{lllllllllllllll}
0 & 0 & 0 & 0 & 0 & 0 & 0 & 0 & 0 & 0 & 0 & 0 & 0 & 0 & 0 \\
0 & 0 & 0 & 0 & 0 & 0 & 0 & 0 & 0 & 0 & 0 & 0 & 0 & 0 & 0 \\
0 & 0 & 0 & 0 & 0 & 0 & 0 & 0 & 0 & 0 & 0 & 0 & 0 & 0 & 0 \\
0 & 0 & 0 & 0 & 0 & 0 & 0 & 0 & 0 & 0 & 0 & 0 & 0 & 0 & 0 \\
\hline & 0 & 0 & 0 & 0 & 0 & 0 & 0 & 0 & 0 & 0 & 0 & 0 & 0 & 0
\end{tabular}



2003 White Book

-Federal System

Aug1 Aug16 Sep Oct Nov Dec Jan Feb Mar Apr1 Apr16 May Jun Jul Avg

1 Total Federal System

$\begin{array}{lllllllllllllll}0 & 0 & 0 & 0 & 0 & 0 & 0 & 0 & 0 & 0 & 0 & 0 & 0 & 0 & 0\end{array}$

-Public Entities-

2 Total Public Entities

0

0

-Investor-Owned Entities-

3 Total Investor-Owned Entities

\begin{tabular}{llllllllllllll}
0 & 0 & 0 & 0 & 0 & 0 & 0 & 0 & 0 & 0 & 0 & 0 & 0 & 0 \\
\hline
\end{tabular}

-Other Entities-

4 Total Other Entities

0

0

-Restoration-

5 Federal System

6 Public Entities

7 Investor-Owned Entities

8 Other Entities

9 Total Restoration

\begin{tabular}{lllllllllllllll}
0 & 0 & 0 & 0 & 0 & 0 & 0 & 0 & 0 & 0 & 0 & 0 & 0 & 0 & 0 \\
0 & 0 & 0 & 0 & 0 & 0 & 0 & 0 & 0 & 0 & 0 & 0 & 0 & 0 & 0 \\
0 & 0 & 0 & 0 & 0 & 0 & 0 & 0 & 0 & 0 & 0 & 0 & 0 & 0 & 0 \\
0 & 0 & 0 & 0 & 0 & 0 & 0 & 0 & 0 & 0 & 0 & 0 & 0 & 0 & 0 \\
\hline & 0 & 0 & 0 & 0 & 0 & 0 & 0 & 0 & 0 & 0 & 0 & 0 & 0 & 0
\end{tabular}




$0 \quad 0 \quad 0 \quad 0$

-Investor-Owned Entities-

3 Total Investor-Owned Entities

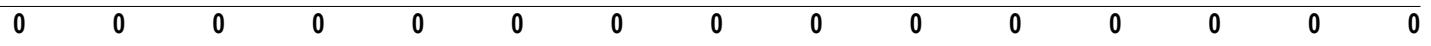

-Other Entities-

4 Total Other Entities

0

-Restoration-

5 Federal System

6 Public Entities

7 Investor-Owned Entities

8 Other Entities

9 Total Restoration

0

$\begin{array}{llllll}0 & 0 & 0 & 0 & 0 & 0\end{array}$

0

0

$\begin{array}{lllllllllllllll}0 & 0 & 0 & 0 & 0 & 0 & 0 & 0 & 0 & 0 & 0 & 0 & 0 & 0 & 0 \\ 0 & 0 & 0 & 0 & 0 & 0 & 0 & 0 & 0 & 0 & 0 & 0 & 0 & 0 & 0 \\ 0 & 0 & 0 & 0 & 0 & 0 & 0 & 0 & 0 & 0 & 0 & 0 & 0 & 0 & 0 \\ 0 & 0 & 0 & 0 & 0 & 0 & 0 & 0 & 0 & 0 & 0 & 0 & 0 & 0 & 0 \\ 0 & \mathbf{0} & \mathbf{0} & \mathbf{0} & \mathbf{0} & \mathbf{0} & \mathbf{0} & \mathbf{0} & \mathbf{0} & \mathbf{0} & \mathbf{0} & \mathbf{0} & \mathbf{0} & \mathbf{0} & \mathbf{0}\end{array}$



2003 White Book

-Federal System

1 Total Federal System

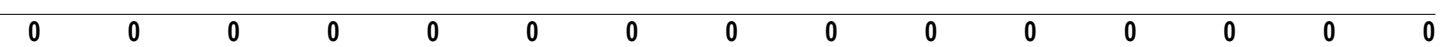

-Public Entities-

2 Total Public Entities

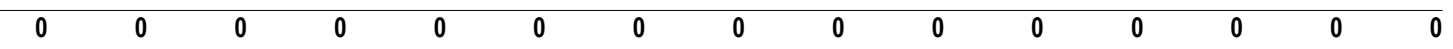

-Investor-Owned Entities-

3 Total Investor-Owned Entities

\begin{tabular}{llllllllllllll}
0 & 0 & 0 & 0 & 0 & 0 & 0 & 0 & 0 & 0 & 0 & 0 & 0 & 0 \\
\hline
\end{tabular}

-Other Entities-

4 Total Other Entities

0

0

-Restoration-

5 Federal System

6 Public Entities

7 Investor-Owned Entities

8 Other Entities

9 Total Restoration

$\begin{array}{lllllllllllllll}0 & 0 & 0 & 0 & 0 & 0 & 0 & 0 & 0 & 0 & 0 & 0 & 0 & 0 & 0 \\ 0 & 0 & 0 & 0 & 0 & 0 & 0 & 0 & 0 & 0 & 0 & 0 & 0 & 0 & 0 \\ 0 & 0 & 0 & 0 & 0 & 0 & 0 & 0 & 0 & 0 & 0 & 0 & 0 & 0 & 0 \\ 0 & 0 & 0 & 0 & 0 & 0 & 0 & 0 & 0 & 0 & 0 & 0 & 0 & 0 & 0 \\ 0 & 0 & 0 & 0 & 0 & 0 & 0 & 0 & 0 & 0 & 0 & 0 & 0 & 0 & 0\end{array}$



2003 White Book

-Federal System

Aug1 Aug16 Sep Oct Nov Dec Jan Feb Mar Apr1 Apr16 May Jun Jul Avg

1 Total Federal System

$\begin{array}{lllllllllllllll}0 & 0 & 0 & 0 & 0 & 0 & 0 & 0 & 0 & 0 & 0 & 0 & 0 & 0 & 0\end{array}$

-Public Entities-

2 Total Public Entities

0

0

-Investor-Owned Entities-

3 Total Investor-Owned Entities

\begin{tabular}{llllllllllllll}
0 & 0 & 0 & 0 & 0 & 0 & 0 & 0 & 0 & 0 & 0 & 0 & 0 & 0 \\
\hline
\end{tabular}

-Other Entities-

4 Total Other Entities

0

0

-Restoration-

5 Federal System

6 Public Entities

7 Investor-Owned Entities

8 Other Entities

9 Total Restoration

\begin{tabular}{lllllllllllllll}
0 & 0 & 0 & 0 & 0 & 0 & 0 & 0 & 0 & 0 & 0 & 0 & 0 & 0 & 0 \\
0 & 0 & 0 & 0 & 0 & 0 & 0 & 0 & 0 & 0 & 0 & 0 & 0 & 0 & 0 \\
0 & 0 & 0 & 0 & 0 & 0 & 0 & 0 & 0 & 0 & 0 & 0 & 0 & 0 & 0 \\
0 & 0 & 0 & 0 & 0 & 0 & 0 & 0 & 0 & 0 & 0 & 0 & 0 & 0 & 0 \\
\hline & 0 & 0 & 0 & 0 & 0 & 0 & 0 & 0 & 0 & 0 & 0 & 0 & 0 & 0
\end{tabular}




$0 \quad 0 \quad 0$

-Public Entities-

2 Total Public Entities

0

0

-Investor-Owned Entities-

3 Total Investor-Owned Entities

0

-Other Entities-

4 Total Other Entities

0

0

$\begin{array}{lll}0 & 0 & 0\end{array}$

-Restoration-

5 Federal System

6 Public Entities

7 Investor-Owned Entities

8 Other Entities

9 Total Restoration

$\begin{array}{lllllllllllllll}0 & 0 & 0 & 0 & 0 & 0 & 0 & 0 & 0 & 0 & 0 & 0 & 0 & 0 & 0 \\ 0 & 0 & 0 & 0 & 0 & 0 & 0 & 0 & 0 & 0 & 0 & 0 & 0 & 0 & 0 \\ 0 & 0 & 0 & 0 & 0 & 0 & 0 & 0 & 0 & 0 & 0 & 0 & 0 & 0 & 0 \\ 0 & 0 & 0 & 0 & 0 & 0 & 0 & 0 & 0 & 0 & 0 & 0 & 0 & 0 & 0 \\ 0 & 0 & 0 & 0 & 0 & 0 & 0 & 0 & 0 & 0 & 0 & 0 & 0 & 0 & 0\end{array}$



2003 White Book

-Federal System-

Aug1 Aug16 Sep Oct Nov Dec Jan Feb Mar Apr1 Apr16 May Jun Jul Avg

1 Total Federal System

$\begin{array}{lllllllllllllll}0 & 0 & 0 & 0 & 0 & 0 & 0 & 0 & 0 & 0 & 0 & 0 & 0 & 0 & 0\end{array}$

-Public Entities-

2 Total Public Entities

0

0

-Investor-Owned Entities-

3 Total Investor-Owned Entities

\begin{tabular}{llllllllllllll}
0 & 0 & 0 & 0 & 0 & 0 & 0 & 0 & 0 & 0 & 0 & 0 & 0 & 0 \\
\hline
\end{tabular}

-Other Entities-

4 Total Other Entities

0

0

-Restoration-

5 Federal System

6 Public Entities

7 Investor-Owned Entities

8 Other Entities

9 Total Restoration

\begin{tabular}{lllllllllllllll}
0 & 0 & 0 & 0 & 0 & 0 & 0 & 0 & 0 & 0 & 0 & 0 & 0 & 0 & 0 \\
0 & 0 & 0 & 0 & 0 & 0 & 0 & 0 & 0 & 0 & 0 & 0 & 0 & 0 & 0 \\
0 & 0 & 0 & 0 & 0 & 0 & 0 & 0 & 0 & 0 & 0 & 0 & 0 & 0 & 0 \\
0 & 0 & 0 & 0 & 0 & 0 & 0 & 0 & 0 & 0 & 0 & 0 & 0 & 0 & 0 \\
\hline & 0 & 0 & 0 & 0 & 0 & 0 & 0 & 0 & 0 & 0 & 0 & 0 & 0 & 0
\end{tabular}



2003 White Book

-Federal System-

Aug1 Aug16 Sep Oct Nov Dec Jan Feb Mar Apr1 Apr16 May Jun Jul Avg

\begin{tabular}{llllllllllllll}
0 & 0 & 0 & 0 & 0 & 0 & 0 & 0 & 0 & 0 & 0 & 0 & 0 & 0 \\
\hline
\end{tabular}

-Public Entities-

2 Total Public Entities

0

0

-Investor-Owned Entities-

3 Total Investor-Owned Entities

\begin{tabular}{llllllllllllll}
0 & 0 & 0 & 0 & 0 & 0 & 0 & 0 & 0 & 0 & 0 & 0 & 0 & 0 \\
\hline
\end{tabular}

-Other Entities-

4 Total Other Entities

0

0

-Restoration-

5 Federal System

6 Public Entities

7 Investor-Owned Entities

8 Other Entities

9 Total Restoration

\begin{tabular}{lllllllllllllll}
0 & 0 & 0 & 0 & 0 & 0 & 0 & 0 & 0 & 0 & 0 & 0 & 0 & 0 & 0 \\
0 & 0 & 0 & 0 & 0 & 0 & 0 & 0 & 0 & 0 & 0 & 0 & 0 & 0 & 0 \\
0 & 0 & 0 & 0 & 0 & 0 & 0 & 0 & 0 & 0 & 0 & 0 & 0 & 0 & 0 \\
0 & 0 & 0 & 0 & 0 & 0 & 0 & 0 & 0 & 0 & 0 & 0 & 0 & 0 & 0 \\
\hline & 0 & 0 & 0 & 0 & 0 & 0 & 0 & 0 & 0 & 0 & 0 & 0 & 0 & 0
\end{tabular}



2003 White Book

-Federal System

Aug1 Aug16 Sep Oct Nov Dec Jan Feb Mar Apr1 Apr16 May Jun Jul Avg

\begin{tabular}{llllllllllllll}
0 & 0 & 0 & 0 & 0 & 0 & 0 & 0 & 0 & 0 & 0 & 0 & 0 & 0 \\
\hline
\end{tabular}

-Public Entities-

2 Total Public Entities

0

$\begin{array}{lll}0 & 0 & 0 \\ 0 & 0 & 0\end{array}$

-Investor-Owned Entities-

3 Total Investor-Owned Entities

\begin{tabular}{llllllllllllll}
0 & 0 & 0 & 0 & 0 & 0 & 0 & 0 & 0 & 0 & 0 & 0 & 0 & 0 \\
\hline
\end{tabular}

-Other Entities-

4 Total Other Entities

0

0

-Restoration-

5 Federal System

6 Public Entities

7 Investor-Owned Entities

8 Other Entities

9 Total Restoration

\begin{tabular}{lllllllllllllll}
0 & 0 & 0 & 0 & 0 & 0 & 0 & 0 & 0 & 0 & 0 & 0 & 0 & 0 & 0 \\
0 & 0 & 0 & 0 & 0 & 0 & 0 & 0 & 0 & 0 & 0 & 0 & 0 & 0 & 0 \\
0 & 0 & 0 & 0 & 0 & 0 & 0 & 0 & 0 & 0 & 0 & 0 & 0 & 0 & 0 \\
0 & 0 & 0 & 0 & 0 & 0 & 0 & 0 & 0 & 0 & 0 & 0 & 0 & 0 & 0 \\
\hline & 0 & 0 & 0 & 0 & 0 & 0 & 0 & 0 & 0 & 0 & 0 & 0 & 0 & 0
\end{tabular}




$0 \quad 0 \quad 0 \quad 0$

-Investor-Owned Entities-

3 Total Investor-Owned Entities

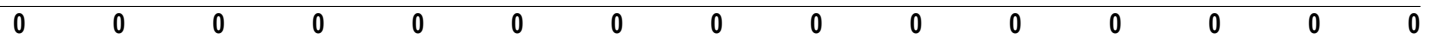

-Other Entities-

4 Total Other Entities

0

-Restoration-

5 Federal System

6 Public Entities

7 Investor-Owned Entities

8 Other Entities

9 Total Restoration

0

$\begin{array}{llllll}0 & 0 & 0 & 0 & 0 & 0\end{array}$

0

0

$\begin{array}{lllllllllllllll}0 & 0 & 0 & 0 & 0 & 0 & 0 & 0 & 0 & 0 & 0 & 0 & 0 & 0 & 0 \\ 0 & 0 & 0 & 0 & 0 & 0 & 0 & 0 & 0 & 0 & 0 & 0 & 0 & 0 & 0 \\ 0 & 0 & 0 & 0 & 0 & 0 & 0 & 0 & 0 & 0 & 0 & 0 & 0 & 0 & 0 \\ 0 & 0 & 0 & 0 & 0 & 0 & 0 & 0 & 0 & 0 & 0 & 0 & 0 & 0 & 0 \\ 0 & \mathbf{0} & \mathbf{0} & \mathbf{0} & \mathbf{0} & \mathbf{0} & \mathbf{0} & \mathbf{0} & \mathbf{0} & \mathbf{0} & \mathbf{0} & \mathbf{0} & \mathbf{0} & \mathbf{0} & \mathbf{0}\end{array}$




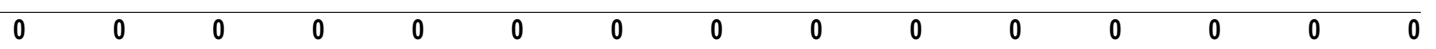

-Priest Rapids CER for CSPE-

2 Priest Rapids: Regional Totals

$\begin{array}{llllllllllllll}0 & 0 & 0 & 0 & 0 & 0 & 0 & 0 & 0 & 0 & 0 & 0 & 0 & 0\end{array}$

-Wanapum CER for CSPE.

3 Wanapum: Regional Totals

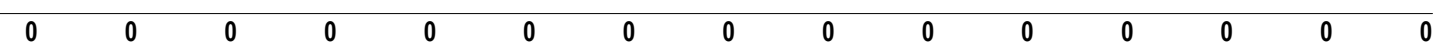

-Rock Island P.H. \#1 CER for CSPE-

4 Rock Island P.H. \#1: Regional Totals

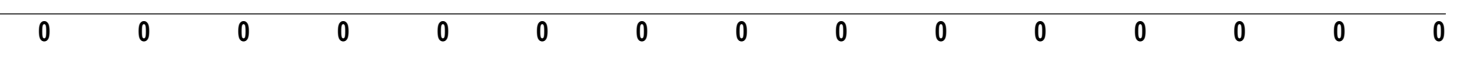

-Rock Island P.H. \#2 CER for CSPE-

5 Rock Island P.H. \#2: Regional Totals

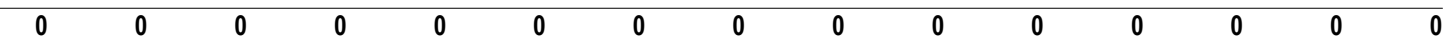

-Rocky Reach CER for CSPE-

6 Rocky Reach: Regional Totals

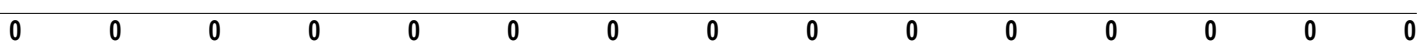

-Wells CER for CSPE-

7 Wells: Regional Totals

-Total CER For CSPE-

8 Federal System

9 Public Agencies

10 Investor-Owned Utilities

11 Other Entities

12 Non-NW Utilities

13 Total CER For CSPE

\begin{tabular}{|c|c|c|c|c|c|c|c|c|c|c|c|c|c|c|}
\hline 0 & 0 & 0 & 0 & 0 & 0 & 0 & 0 & 0 & 0 & 0 & 0 & 0 & 0 & 0 \\
\hline 0 & 0 & 0 & 0 & 0 & 0 & 0 & 0 & 0 & 0 & 0 & 0 & 0 & 0 & 0 \\
\hline 0 & 0 & 0 & 0 & 0 & 0 & 0 & 0 & 0 & 0 & 0 & 0 & 0 & 0 & 0 \\
\hline 0 & 0 & 0 & 0 & 0 & 0 & 0 & 0 & 0 & 0 & 0 & 0 & 0 & 0 & 0 \\
\hline 0 & 0 & 0 & 0 & 0 & 0 & 0 & 0 & 0 & 0 & 0 & 0 & 0 & 0 & 0 \\
\hline
\end{tabular}




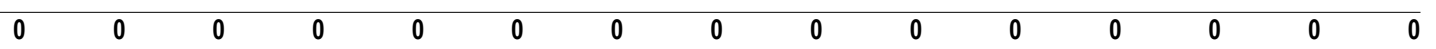

-Priest Rapids CER for CSPE-

2 Priest Rapids: Regional Totals

$\begin{array}{llllllllllllll}0 & 0 & 0 & 0 & 0 & 0 & 0 & 0 & 0 & 0 & 0 & 0 & 0 & 0\end{array}$

-Wanapum CER for CSPE.

3 Wanapum: Regional Totals

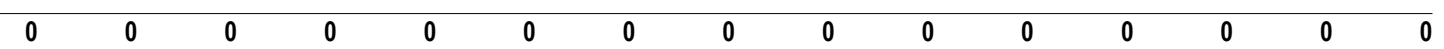

-Rock Island P.H. \#1 CER for CSPE-

4 Rock Island P.H. \#1: Regional Totals

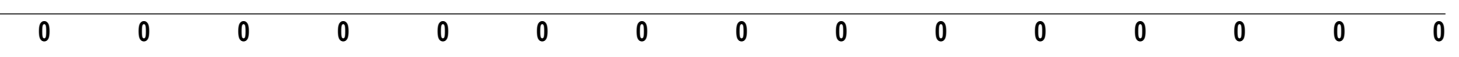

-Rock Island P.H. \#2 CER for CSPE-

5 Rock Island P.H. \#2: Regional Totals

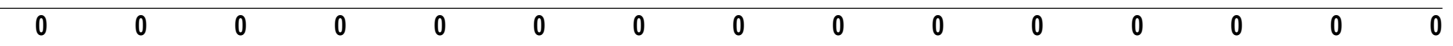

-Rocky Reach CER for CSPE-

6 Rocky Reach: Regional Totals

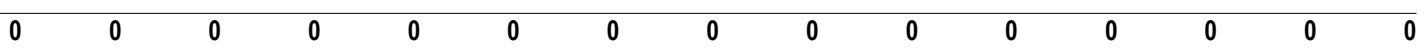

-Wells CER for CSPE-

7 Wells: Regional Totals

-Total CER For CSPE-

8 Federal System

9 Public Agencies

10 Investor-Owned Utilities

11 Other Entities

12 Non-NW Utilities

13 Total CER For CSPE

$\begin{array}{llllllllllllllllll}\mathbf{0} & \mathbf{0} & \mathbf{0} & \mathbf{0} & \mathbf{0} & \mathbf{0} & \mathbf{0} & \mathbf{0} & \mathbf{0} & \mathbf{0} & \mathbf{0} & \mathbf{0} & \mathbf{0} & \mathbf{0} & \mathbf{0} \\ & & & & & & & & & & & & & & & & & \\ 0 & 0 & 0 & 0 & 0 & 0 & 0 & 0 & 0 & 0 & 0 & 0 & 0 & 0 & 0 \\ 0 & 0 & 0 & 0 & 0 & 0 & 0 & 0 & 0 & 0 & 0 & 0 & 0 & 0 & 0 \\ 0 & 0 & 0 & 0 & 0 & 0 & 0 & 0 & 0 & 0 & 0 & 0 & 0 & 0 & 0 \\ 0 & 0 & 0 & 0 & 0 & 0 & 0 & 0 & 0 & 0 & 0 & 0 & 0 & 0 & 0 \\ 0 & 0 & 0 & 0 & 0 & 0 & 0 & 0 & 0 & 0 & 0 & 0 & 0 & 0 & 0 \\ \mathbf{0} & \mathbf{0} & \mathbf{0} & \mathbf{0} & \mathbf{0} & \mathbf{0} & \mathbf{0} & \mathbf{0} & \mathbf{0} & \mathbf{0} & \mathbf{0} & \mathbf{0} & \mathbf{0} & \mathbf{0} & \mathbf{0}\end{array}$




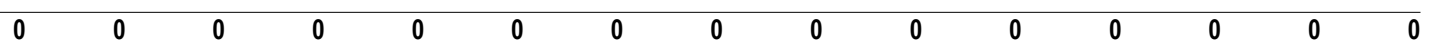

-Priest Rapids CER for CSPE-

2 Priest Rapids: Regional Totals

$\begin{array}{llllllllllllll}0 & 0 & 0 & 0 & 0 & 0 & 0 & 0 & 0 & 0 & 0 & 0 & 0 & 0\end{array}$

-Wanapum CER for CSPE.

3 Wanapum: Regional Totals

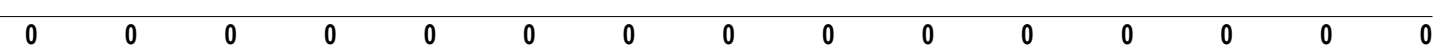

-Rock Island P.H. \#1 CER for CSPE-

4 Rock Island P.H. \#1: Regional Totals

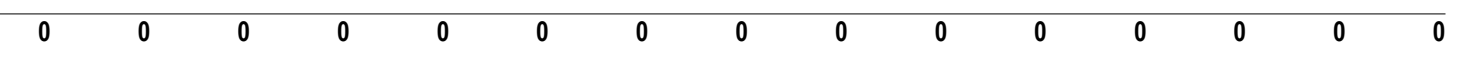

-Rock Island P.H. \#2 CER for CSPE-

5 Rock Island P.H. \#2: Regional Totals

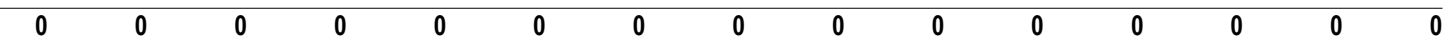

-Rocky Reach CER for CSPE-

6 Rocky Reach: Regional Totals

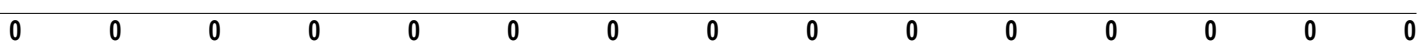

-Wells CER for CSPE-

7 Wells: Regional Totals

-Total CER For CSPE-

8 Federal System

9 Public Agencies

10 Investor-Owned Utilities

11 Other Entities

12 Non-NW Utilities

13 Total CER For CSPE

\begin{tabular}{|c|c|c|c|c|c|c|c|c|c|c|c|c|c|c|}
\hline 0 & 0 & 0 & 0 & 0 & 0 & 0 & 0 & 0 & 0 & 0 & 0 & 0 & 0 & 0 \\
\hline 0 & 0 & 0 & 0 & 0 & 0 & 0 & 0 & 0 & 0 & 0 & 0 & 0 & 0 & 0 \\
\hline 0 & 0 & 0 & 0 & 0 & 0 & 0 & 0 & 0 & 0 & 0 & 0 & 0 & 0 & 0 \\
\hline 0 & 0 & 0 & 0 & 0 & 0 & 0 & 0 & 0 & 0 & 0 & 0 & 0 & 0 & 0 \\
\hline 0 & 0 & 0 & 0 & 0 & 0 & 0 & 0 & 0 & 0 & 0 & 0 & 0 & 0 & 0 \\
\hline
\end{tabular}



2003 White Book

-BPA CER for CSPE-

1 BPA: Regional Totals

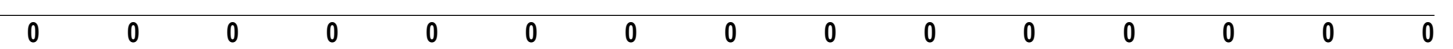

Priest Rapids CER for CSPE-

2 Priest Rapids: Regional Totals

$\begin{array}{llllllllllllll}0 & 0 & 0 & 0 & 0 & 0 & 0 & 0 & 0 & 0 & 0 & 0 & 0 & 0\end{array}$

-Wanapum CER for CSPE.

3 Wanapum: Regional Totals

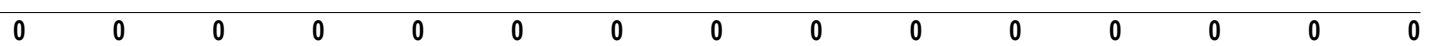

-Rock Island P.H. \#1 CER for CSPE-

4 Rock Island P.H. \#1: Regional Totals

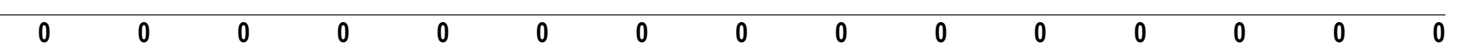

-Rock Island P.H. \#2 CER for CSPE-

5 Rock Island P.H. \#2: Regional Totals

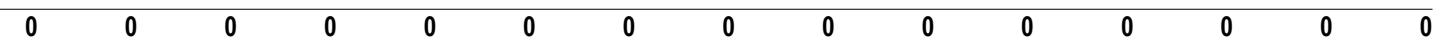

-Rocky Reach CER for CSPE-

6 Rocky Reach: Regional Totals

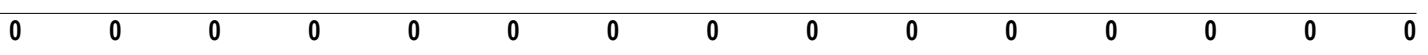

-Wells CER for CSPE-

7 Wells: Regional Totals

-Total CER For CSPE-

8 Federal System

9 Public Agencies

10 Investor-Owned Utilities

11 Other Entities

12 Non-NW Utilities

13 Total CER For CSPE

$\begin{array}{lllllllllllllll}\mathbf{0} & \mathbf{0} & \mathbf{0} & \mathbf{0} & \mathbf{0} & \mathbf{0} & \mathbf{0} & \mathbf{0} & \mathbf{0} & \mathbf{0} & \mathbf{0} & \mathbf{0} & \mathbf{0} & \mathbf{0} & \mathbf{0} \\ & & & & & & & & & & & & & & \\ 0 & 0 & 0 & 0 & 0 & 0 & 0 & 0 & 0 & 0 & 0 & 0 & 0 & 0 & 0 \\ 0 & 0 & 0 & 0 & 0 & 0 & 0 & 0 & 0 & 0 & 0 & 0 & 0 & 0 & 0 \\ 0 & 0 & 0 & 0 & 0 & 0 & 0 & 0 & 0 & 0 & 0 & 0 & 0 & 0 & 0 \\ 0 & 0 & 0 & 0 & 0 & 0 & 0 & 0 & 0 & 0 & 0 & 0 & 0 & 0 & 0 \\ 0 & 0 & 0 & 0 & 0 & 0 & 0 & 0 & 0 & 0 & 0 & 0 & 0 & 0 & 0 \\ \mathbf{0} & \mathbf{0} & \mathbf{0} & \mathbf{0} & \mathbf{0} & \mathbf{0} & \mathbf{0} & \mathbf{0} & \mathbf{0} & \mathbf{0} & \mathbf{0} & \mathbf{0} & \mathbf{0} & \mathbf{0} & \mathbf{0}\end{array}$




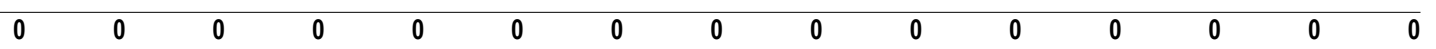

-Priest Rapids CER for CSPE-

2 Priest Rapids: Regional Totals

$\begin{array}{llllllllllllll}0 & 0 & 0 & 0 & 0 & 0 & 0 & 0 & 0 & 0 & 0 & 0 & 0 & 0\end{array}$

-Wanapum CER for CSPE.

3 Wanapum: Regional Totals

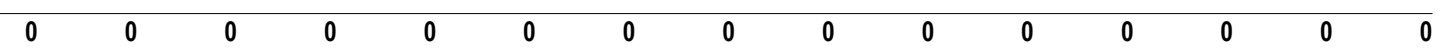

-Rock Island P.H. \#1 CER for CSPE-

4 Rock Island P.H. \#1: Regional Totals

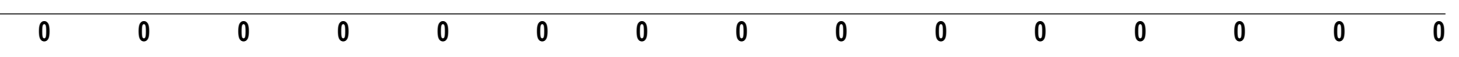

-Rock Island P.H. \#2 CER for CSPE-

5 Rock Island P.H. \#2: Regional Totals

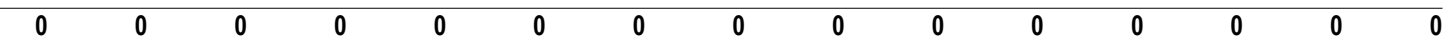

-Rocky Reach CER for CSPE-

6 Rocky Reach: Regional Totals

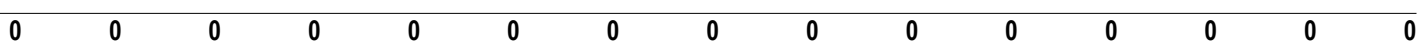

-Wells CER for CSPE-

7 Wells: Regional Totals

-Total CER For CSPE-

8 Federal System

9 Public Agencies

10 Investor-Owned Utilities

11 Other Entities

12 Non-NW Utilities

13 Total CER For CSPE

$\begin{array}{llllllllllllllllll}\mathbf{0} & \mathbf{0} & \mathbf{0} & \mathbf{0} & \mathbf{0} & \mathbf{0} & \mathbf{0} & \mathbf{0} & \mathbf{0} & \mathbf{0} & \mathbf{0} & \mathbf{0} & \mathbf{0} & \mathbf{0} & \mathbf{0} \\ & & & & & & & & & & & & & & & & & \\ 0 & 0 & 0 & 0 & 0 & 0 & 0 & 0 & 0 & 0 & 0 & 0 & 0 & 0 & 0 \\ 0 & 0 & 0 & 0 & 0 & 0 & 0 & 0 & 0 & 0 & 0 & 0 & 0 & 0 & 0 \\ 0 & 0 & 0 & 0 & 0 & 0 & 0 & 0 & 0 & 0 & 0 & 0 & 0 & 0 & 0 \\ 0 & 0 & 0 & 0 & 0 & 0 & 0 & 0 & 0 & 0 & 0 & 0 & 0 & 0 & 0 \\ 0 & 0 & 0 & 0 & 0 & 0 & 0 & 0 & 0 & 0 & 0 & 0 & 0 & 0 & 0 \\ \mathbf{0} & \mathbf{0} & \mathbf{0} & \mathbf{0} & \mathbf{0} & \mathbf{0} & \mathbf{0} & \mathbf{0} & \mathbf{0} & \mathbf{0} & \mathbf{0} & \mathbf{0} & \mathbf{0} & \mathbf{0} & \mathbf{0}\end{array}$




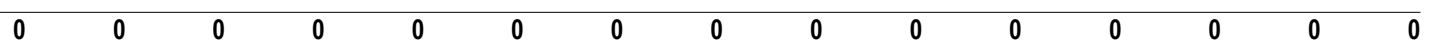

-Priest Rapids CER for CSPE-

2 Priest Rapids: Regional Totals

$\begin{array}{llllllllllllll}0 & 0 & 0 & 0 & 0 & 0 & 0 & 0 & 0 & 0 & 0 & 0 & 0 & 0\end{array}$

-Wanapum CER for CSPE.

3 Wanapum: Regional Totals

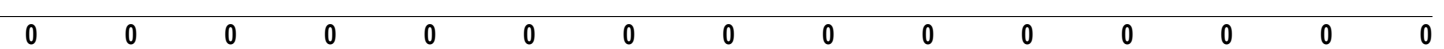

-Rock Island P.H. \#1 CER for CSPE-

4 Rock Island P.H. \#1: Regional Totals

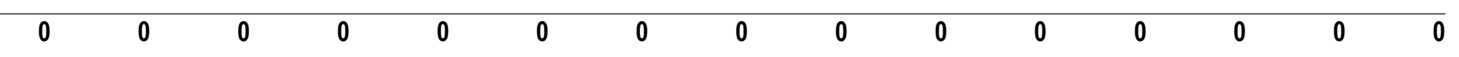

-Rock Island P.H. \#2 CER for CSPE-

5 Rock Island P.H. \#2: Regional Totals

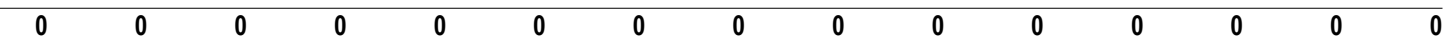

-Rocky Reach CER for CSPE-

6 Rocky Reach: Regional Totals

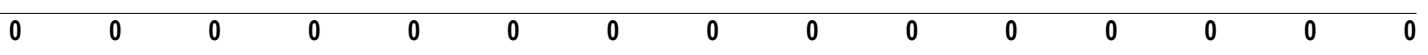

-Wells CER for CSPE-

7 Wells: Regional Totals

-Total CER For CSPE-

8 Federal System

9 Public Agencies

10 Investor-Owned Utilities

11 Other Entities

12 Non-NW Utilities

13 Total CER For CSPE

\begin{tabular}{|c|c|c|c|c|c|c|c|c|c|c|c|c|c|c|}
\hline 0 & 0 & 0 & 0 & 0 & 0 & 0 & 0 & 0 & 0 & 0 & 0 & 0 & 0 & 0 \\
\hline 0 & 0 & 0 & 0 & 0 & 0 & 0 & 0 & 0 & 0 & 0 & 0 & 0 & 0 & 0 \\
\hline 0 & 0 & 0 & 0 & 0 & 0 & 0 & 0 & 0 & 0 & 0 & 0 & 0 & 0 & 0 \\
\hline 0 & 0 & 0 & 0 & 0 & 0 & 0 & 0 & 0 & 0 & 0 & 0 & 0 & 0 & 0 \\
\hline 0 & 0 & 0 & 0 & 0 & 0 & 0 & 0 & 0 & 0 & 0 & 0 & 0 & 0 & 0 \\
\hline
\end{tabular}




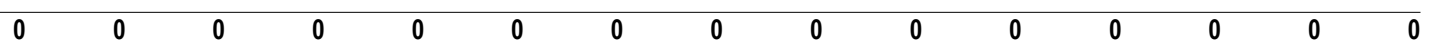

-Priest Rapids CER for CSPE-

2 Priest Rapids: Regional Totals

$\begin{array}{llllllllllllll}0 & 0 & 0 & 0 & 0 & 0 & 0 & 0 & 0 & 0 & 0 & 0 & 0 & 0\end{array}$

-Wanapum CER for CSPE.

3 Wanapum: Regional Totals

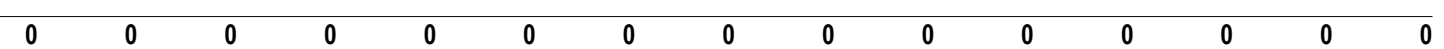

-Rock Island P.H. \#1 CER for CSPE-

4 Rock Island P.H. \#1: Regional Totals

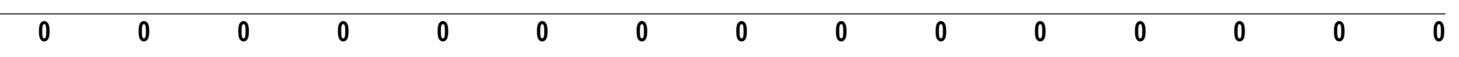

-Rock Island P.H. \#2 CER for CSPE-

5 Rock Island P.H. \#2: Regional Totals

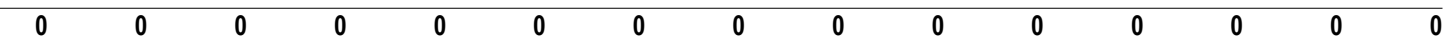

-Rocky Reach CER for CSPE-

6 Rocky Reach: Regional Totals

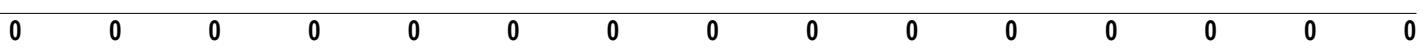

-Wells CER for CSPE-

7 Wells: Regional Totals

-Total CER For CSPE-

8 Federal System

9 Public Agencies

10 Investor-Owned Utilities

11 Other Entities

12 Non-NW Utilities

13 Total CER For CSPE

\begin{tabular}{|c|c|c|c|c|c|c|c|c|c|c|c|c|c|c|}
\hline 0 & 0 & 0 & 0 & 0 & 0 & 0 & 0 & 0 & 0 & 0 & 0 & 0 & 0 & 0 \\
\hline 0 & 0 & 0 & 0 & 0 & 0 & 0 & 0 & 0 & 0 & 0 & 0 & 0 & 0 & 0 \\
\hline 0 & 0 & 0 & 0 & 0 & 0 & 0 & 0 & 0 & 0 & 0 & 0 & 0 & 0 & 0 \\
\hline 0 & 0 & 0 & 0 & 0 & 0 & 0 & 0 & 0 & 0 & 0 & 0 & 0 & 0 & 0 \\
\hline 0 & 0 & 0 & 0 & 0 & 0 & 0 & 0 & 0 & 0 & 0 & 0 & 0 & 0 & 0 \\
\hline
\end{tabular}




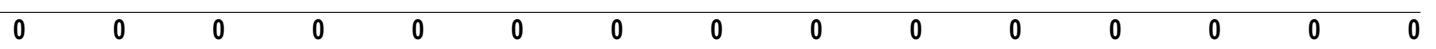

-Priest Rapids CER for CSPE-

2 Priest Rapids: Regional Totals

$\begin{array}{llllllllllllll}0 & 0 & 0 & 0 & 0 & 0 & 0 & 0 & 0 & 0 & 0 & 0 & 0 & 0\end{array}$

-Wanapum CER for CSPE.

3 Wanapum: Regional Totals

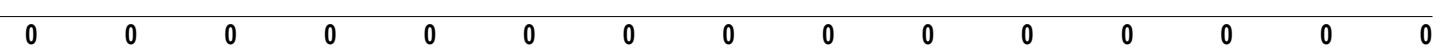

-Rock Island P.H. \#1 CER for CSPE-

4 Rock Island P.H. \#1: Regional Totals

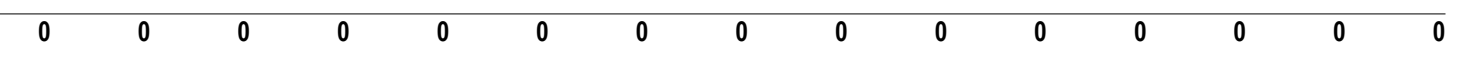

-Rock Island P.H. \#2 CER for CSPE-

5 Rock Island P.H. \#2: Regional Totals

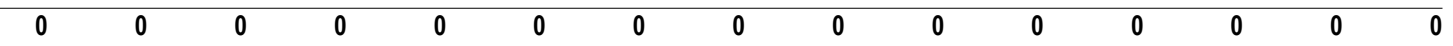

-Rocky Reach CER for CSPE-

6 Rocky Reach: Regional Totals

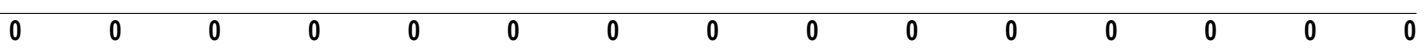

-Wells CER for CSPE-

7 Wells: Regional Totals

-Total CER For CSPE-

8 Federal System

9 Public Agencies

10 Investor-Owned Utilities

11 Other Entities

12 Non-NW Utilities

13 Total CER For CSPE

\begin{tabular}{|c|c|c|c|c|c|c|c|c|c|c|c|c|c|c|}
\hline 0 & 0 & 0 & 0 & 0 & 0 & 0 & 0 & 0 & 0 & 0 & 0 & 0 & 0 & 0 \\
\hline 0 & 0 & 0 & 0 & 0 & 0 & 0 & 0 & 0 & 0 & 0 & 0 & 0 & 0 & 0 \\
\hline 0 & 0 & 0 & 0 & 0 & 0 & 0 & 0 & 0 & 0 & 0 & 0 & 0 & 0 & 0 \\
\hline 0 & 0 & 0 & 0 & 0 & 0 & 0 & 0 & 0 & 0 & 0 & 0 & 0 & 0 & 0 \\
\hline 0 & 0 & 0 & 0 & 0 & 0 & 0 & 0 & 0 & 0 & 0 & 0 & 0 & 0 & 0 \\
\hline
\end{tabular}




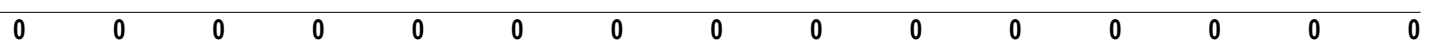

-Priest Rapids CER for CSPE-

2 Priest Rapids: Regional Totals

$\begin{array}{llllllllllllll}0 & 0 & 0 & 0 & 0 & 0 & 0 & 0 & 0 & 0 & 0 & 0 & 0 & 0\end{array}$

-Wanapum CER for CSPE.

3 Wanapum: Regional Totals

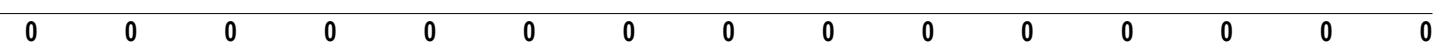

-Rock Island P.H. \#1 CER for CSPE-

4 Rock Island P.H. \#1: Regional Totals

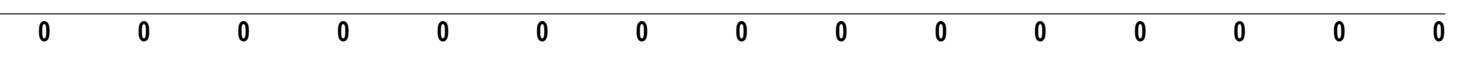

-Rock Island P.H. \#2 CER for CSPE-

5 Rock Island P.H. \#2: Regional Totals

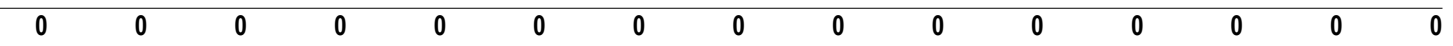

-Rocky Reach CER for CSPE-

6 Rocky Reach: Regional Totals

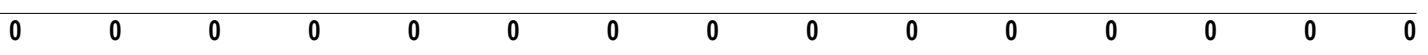

-Wells CER for CSPE-

7 Wells: Regional Totals

-Total CER For CSPE-

8 Federal System

9 Public Agencies

10 Investor-Owned Utilities

11 Other Entities

12 Non-NW Utilities

13 Total CER For CSPE

$\begin{array}{llllllllllllllllll}\mathbf{0} & \mathbf{0} & \mathbf{0} & \mathbf{0} & \mathbf{0} & \mathbf{0} & \mathbf{0} & \mathbf{0} & \mathbf{0} & \mathbf{0} & \mathbf{0} & \mathbf{0} & \mathbf{0} & \mathbf{0} & \mathbf{0} \\ & & & & & & & & & & & & & & & & & \\ 0 & 0 & 0 & 0 & 0 & 0 & 0 & 0 & 0 & 0 & 0 & 0 & 0 & 0 & 0 \\ 0 & 0 & 0 & 0 & 0 & 0 & 0 & 0 & 0 & 0 & 0 & 0 & 0 & 0 & 0 \\ 0 & 0 & 0 & 0 & 0 & 0 & 0 & 0 & 0 & 0 & 0 & 0 & 0 & 0 & 0 \\ 0 & 0 & 0 & 0 & 0 & 0 & 0 & 0 & 0 & 0 & 0 & 0 & 0 & 0 & 0 \\ 0 & 0 & 0 & 0 & 0 & 0 & 0 & 0 & 0 & 0 & 0 & 0 & 0 & 0 & 0 \\ \mathbf{0} & \mathbf{0} & \mathbf{0} & \mathbf{0} & \mathbf{0} & \mathbf{0} & \mathbf{0} & \mathbf{0} & \mathbf{0} & \mathbf{0} & \mathbf{0} & \mathbf{0} & \mathbf{0} & \mathbf{0} & \mathbf{0}\end{array}$




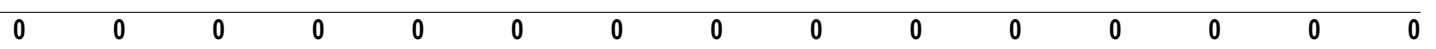

-Priest Rapids CER for CSPE-

2 Priest Rapids: Regional Totals

$\begin{array}{llllllllllllll}0 & 0 & 0 & 0 & 0 & 0 & 0 & 0 & 0 & 0 & 0 & 0 & 0 & 0\end{array}$

-Wanapum CER for CSPE.

3 Wanapum: Regional Totals

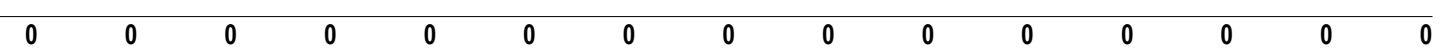

-Rock Island P.H. \#1 CER for CSPE-

4 Rock Island P.H. \#1: Regional Totals

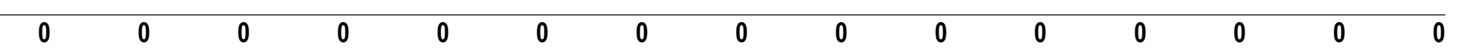

-Rock Island P.H. \#2 CER for CSPE-

5 Rock Island P.H. \#2: Regional Totals

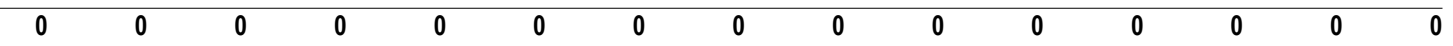

-Rocky Reach CER for CSPE-

6 Rocky Reach: Regional Totals

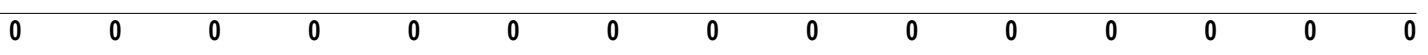

-Wells CER for CSPE-

7 Wells: Regional Totals

-Total CER For CSPE-

8 Federal System

9 Public Agencies

10 Investor-Owned Utilities

11 Other Entities

12 Non-NW Utilities

13 Total CER For CSPE

\begin{tabular}{|c|c|c|c|c|c|c|c|c|c|c|c|c|c|c|}
\hline 0 & 0 & 0 & 0 & 0 & 0 & 0 & 0 & 0 & 0 & 0 & 0 & 0 & 0 & 0 \\
\hline 0 & 0 & 0 & 0 & 0 & 0 & 0 & 0 & 0 & 0 & 0 & 0 & 0 & 0 & 0 \\
\hline 0 & 0 & 0 & 0 & 0 & 0 & 0 & 0 & 0 & 0 & 0 & 0 & 0 & 0 & 0 \\
\hline 0 & 0 & 0 & 0 & 0 & 0 & 0 & 0 & 0 & 0 & 0 & 0 & 0 & 0 & 0 \\
\hline 0 & 0 & 0 & 0 & 0 & 0 & 0 & 0 & 0 & 0 & 0 & 0 & 0 & 0 & 0 \\
\hline
\end{tabular}


Table A-13: Columbia Storage Power Exchange Purchase (CSPE)

PNW Loads and Resources Study

2004 - 2005 Operating Year

-Generating Public Entities-

1 BPA to GPE

2 Total Generating Public Entities

Oct Nov

Dec Jan Feb Mar Apr1 Apr16

May Jun Jul Avg

Small \& Non-Gen Public Entities-

3 BPA to S\&NGE

4 Total Small \& Non-Gen Public Entities

$\begin{array}{lllllllllllllll}0 & 0 & 0 & 0 & 0 & 0 & 0 & 0 & 0 & 0 & 0 & 0 & 0 & 0 & 0 \\ 0 & 0 & 0 & 0 & 0 & 0 & 0 & 0 & 0 & 0 & 0 & 0 & 0 & 0 & 0\end{array}$

-Investor-Owned Entities-

5 BPA to IOU

6 Total Investor-Owned Entities

\begin{tabular}{|c|c|c|c|c|c|c|c|c|c|c|c|c|c|}
\hline 0 & 0 & 0 & 0 & 0 & 0 & 0 & 0 & 0 & 0 & 0 & 0 & 0 & 0 \\
\hline 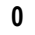 & 0 & 0 & 0 & 0 & 0 & 0 & 0 & 0 & 0 & 0 & 0 & 0 & 0 \\
\hline
\end{tabular}

-Other Entities-

7 BPA to OTHER

8 Total Other Entities

$\begin{array}{lll}0 & 0 & 0 \\ 0 & 0 & 0\end{array}$

-CSPE-

9 Generating Public Agencies

10 Small \& Non-Gen Public Agencies

11 Investor-Owned Utilities

12 Other Entities

13 Total CSPE

\begin{tabular}{|c|c|c|c|c|c|c|c|c|c|c|c|c|c|}
\hline 0 & 0 & 0 & 0 & 0 & 0 & 0 & 0 & 0 & 0 & 0 & 0 & 0 & 0 \\
\hline 0 & 0 & 0 & 0 & 0 & 0 & 0 & 0 & 0 & 0 & 0 & 0 & 0 & 0 \\
\hline
\end{tabular}

$\begin{array}{lllllllllllllll}0 & 0 & 0 & 0 & 0 & 0 & 0 & 0 & 0 & 0 & 0 & 0 & 0 & 0 & 0 \\ 0 & 0 & 0 & 0 & 0 & 0 & 0 & 0 & 0 & 0 & 0 & 0 & 0 & 0 & 0 \\ 0 & 0 & 0 & 0 & 0 & 0 & 0 & 0 & 0 & 0 & 0 & 0 & 0 & 0 & 0 \\ 0 & 0 & 0 & 0 & 0 & 0 & 0 & 0 & 0 & 0 & 0 & 0 & 0 & 0 & 0 \\ 0 & 0 & 0 & 0 & 0 & 0 & 0 & 0 & 0 & \mathbf{0} & \mathbf{0} & \mathbf{0} & \mathbf{0} & \mathbf{0} & \mathbf{0}\end{array}$


Table A-13: Columbia Storage Power Exchange Purchase (CSPE)

PNW Loads and Resources Study

2005 - 2006 Operating Year

-Generating Public Entities-

1 BPA to GPE

2 Total Generating Public Entities

Oct Nov Dec Jan Feb Mar Apr1 Apr16

May Jun Jul Avg

-Small \& Non-Gen Public Entities-

3 BPA to S\&NGE

4 Total Small \& Non-Gen Public Entities

$\begin{array}{lllllllllllllll}0 & 0 & 0 & 0 & 0 & 0 & 0 & 0 & 0 & 0 & 0 & 0 & 0 & 0 & 0 \\ 0 & 0 & 0 & 0 & 0 & 0 & 0 & 0 & 0 & 0 & 0 & 0 & 0 & 0 & 0\end{array}$

-Investor-Owned Entities-

5 BPA to IOU

6 Total Investor-Owned Entities

\begin{tabular}{|c|c|c|c|c|c|c|c|c|c|c|c|c|c|}
\hline 0 & 0 & 0 & 0 & 0 & 0 & 0 & 0 & 0 & 0 & 0 & 0 & 0 & 0 \\
\hline 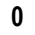 & 0 & 0 & 0 & 0 & 0 & 0 & 0 & 0 & 0 & 0 & 0 & 0 & 0 \\
\hline
\end{tabular}

-Other Entities-

7 BPA to OTHER

8 Total Other Entities

$\begin{array}{lll}0 & 0 & 0 \\ 0 & 0 & 0\end{array}$

-CSPE-

9 Generating Public Agencies

10 Small \& Non-Gen Public Agencies

11 Investor-Owned Utilities

12 Other Entities

13 Total CSPE

\begin{tabular}{|c|c|c|c|c|c|c|c|c|c|c|c|c|c|}
\hline 0 & 0 & 0 & 0 & 0 & 0 & 0 & 0 & 0 & 0 & 0 & 0 & 0 & 0 \\
\hline 0 & 0 & 0 & 0 & 0 & 0 & 0 & 0 & 0 & 0 & 0 & 0 & 0 & 0 \\
\hline
\end{tabular}

$\begin{array}{lllllllllllllll}0 & 0 & 0 & 0 & 0 & 0 & 0 & 0 & 0 & 0 & 0 & 0 & 0 & 0 & 0 \\ 0 & 0 & 0 & 0 & 0 & 0 & 0 & 0 & 0 & 0 & 0 & 0 & 0 & 0 & 0 \\ 0 & 0 & 0 & 0 & 0 & 0 & 0 & 0 & 0 & 0 & 0 & 0 & 0 & 0 & 0 \\ 0 & 0 & 0 & 0 & 0 & 0 & 0 & 0 & 0 & 0 & 0 & 0 & 0 & 0 & 0 \\ 0 & 0 & 0 & 0 & 0 & 0 & \mathbf{0} & \mathbf{0} & \mathbf{0} & \mathbf{0} & \mathbf{0} & \mathbf{0} & \mathbf{0} & \mathbf{0} & \mathbf{0}\end{array}$


Table A-13: Columbia Storage Power Exchange Purchase (CSPE)

PNW Loads and Resources Study

2006 - 2007 Operating Year

-Generating Public Entities-

1 BPA to GPE

2 Total Generating Public Entities

Oct Nov Dec Jan Feb Mar Apr1 Apr16

May Jun Jul Avg

-Small \& Non-Gen Public Entities-

3 BPA to S\&NGE

4 Total Small \& Non-Gen Public Entities

$\begin{array}{lllllllllllllll}0 & 0 & 0 & 0 & 0 & 0 & 0 & 0 & 0 & 0 & 0 & 0 & 0 & 0 & 0 \\ 0 & 0 & 0 & 0 & 0 & 0 & 0 & 0 & 0 & 0 & 0 & 0 & 0 & 0 & 0\end{array}$

-Investor-Owned Entities-

5 BPA to IOU

6 Total Investor-Owned Entities

$\begin{array}{lll}0 & 0 & 0 \\ 0 & 0 & 0\end{array}$

-Other Entities-

7 BPA to OTHER

8 Total Other Entities

$\begin{array}{lll}0 & 0 & 0 \\ 0 & 0 & 0\end{array}$

-CSPE-

9 Generating Public Agencies

10 Small \& Non-Gen Public Agencies

11 Investor-Owned Utilities

12 Other Entities

13 Total CSPE

\begin{tabular}{|c|c|c|c|c|c|c|c|c|c|c|c|c|c|}
\hline 0 & 0 & 0 & 0 & 0 & 0 & 0 & 0 & 0 & 0 & 0 & 0 & 0 & 0 \\
\hline 0 & 0 & 0 & 0 & 0 & 0 & 0 & 0 & 0 & 0 & 0 & 0 & 0 & 0 \\
\hline
\end{tabular}

$\begin{array}{lllllllllllllll}0 & 0 & 0 & 0 & 0 & 0 & 0 & 0 & 0 & 0 & 0 & 0 & 0 & 0 & 0 \\ 0 & 0 & 0 & 0 & 0 & 0 & 0 & 0 & 0 & 0 & 0 & 0 & 0 & 0 & 0 \\ 0 & 0 & 0 & 0 & 0 & 0 & 0 & 0 & 0 & 0 & 0 & 0 & 0 & 0 & 0 \\ 0 & 0 & 0 & 0 & 0 & 0 & 0 & 0 & 0 & 0 & 0 & 0 & 0 & 0 & 0 \\ 0 & 0 & 0 & 0 & 0 & 0 & \mathbf{0} & \mathbf{0} & \mathbf{0} & \mathbf{0} & \mathbf{0} & \mathbf{0} & \mathbf{0} & \mathbf{0} & \mathbf{0}\end{array}$


Table A-13: Columbia Storage Power Exchange Purchase (CSPE)

PNW Loads and Resources Study

2007 - 2008 Operating Year 2003 White Book

-Generating Public Entities-

1 BPA to GPE

2 Total Generating Public Entities

$\begin{array}{lllllllllllllll}0 & 0 & 0 & 0 & 0 & 0 & 0 & 0 & 0 & 0 & 0 & 0 & 0 & 0 & 0 \\ 0 & 0 & 0 & 0 & 0 & 0 & 0 & 0 & 0 & 0 & 0 & 0 & 0 & 0 & 0\end{array}$

-Small \& Non-Gen Public Entities-

3 BPA to S\&NGE

4 Total Small \& Non-Gen Public Entities

$\begin{array}{llll}0 & 0 & 0 & 0 \\ 0 & 0 & 0 & 0\end{array}$

Investor-Owned Entities-

5 BPA to IOU

6 Total Investor-Owned Entities

$\begin{array}{lll}0 & 0 & 0 \\ 0 & 0 & 0\end{array}$

-Other Entities-

7 BPA to OTHER

8 Total Other Entities

$\begin{array}{lll}0 & 0 & 0 \\ 0 & 0 & 0\end{array}$

-CSPE-

9 Generating Public Agencies

10 Small \& Non-Gen Public Agencies

11 Investor-Owned Utilities

12 Other Entities

13 Total CSPE

$\begin{array}{lllllllllllllll}0 & 0 & 0 & 0 & 0 & 0 & 0 & 0 & 0 & 0 & 0 & 0 & 0 & 0 & 0 \\ 0 & 0 & 0 & 0 & 0 & 0 & 0 & 0 & 0 & 0 & 0 & 0 & 0 & 0 & 0 \\ 0 & 0 & 0 & 0 & 0 & 0 & 0 & 0 & 0 & 0 & 0 & 0 & 0 & 0 & 0 \\ 0 & 0 & 0 & 0 & 0 & 0 & 0 & 0 & 0 & 0 & 0 & 0 & 0 & 0 & 0 \\ 0 & \mathbf{0} & \mathbf{0} & \mathbf{0} & \mathbf{0} & \mathbf{0} & \mathbf{0} & \mathbf{0} & \mathbf{0} & \mathbf{0} & \mathbf{0} & \mathbf{0} & \mathbf{0} & \mathbf{0} & \mathbf{0}\end{array}$


Table A-13: Columbia Storage Power Exchange Purchase (CSPE)

PNW Loads and Resources Study

2008 - 2009 Operating Year

-Generating Public Entities-

1 BPA to GPE

2 Total Generating Public Entities

Oct Nov

Dec Jan Feb Mar Apr1 Apr16

May Jun Jul Avg

Small \& Non-Gen Public Entities-

3 BPA to S\&NGE

4 Total Small \& Non-Gen Public Entities

$\begin{array}{lllllllllllllll}0 & 0 & 0 & 0 & 0 & 0 & 0 & 0 & 0 & 0 & 0 & 0 & 0 & 0 & 0 \\ 0 & 0 & 0 & 0 & 0 & 0 & 0 & 0 & 0 & 0 & 0 & 0 & 0 & 0 & 0\end{array}$

-Investor-Owned Entities-

5 BPA to IOU

6 Total Investor-Owned Entities

\begin{tabular}{|c|c|c|c|c|c|c|c|c|c|c|c|c|c|}
\hline 0 & 0 & 0 & 0 & 0 & 0 & 0 & 0 & 0 & 0 & 0 & 0 & 0 & 0 \\
\hline 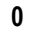 & 0 & 0 & 0 & 0 & 0 & 0 & 0 & 0 & 0 & 0 & 0 & 0 & 0 \\
\hline
\end{tabular}

-Other Entities-

7 BPA to OTHER

8 Total Other Entities

$\begin{array}{lll}0 & 0 & 0 \\ 0 & 0 & 0\end{array}$

-CSPE-

9 Generating Public Agencies

10 Small \& Non-Gen Public Agencies

11 Investor-Owned Utilities

12 Other Entities

13 Total CSPE

\begin{tabular}{|c|c|c|c|c|c|c|c|c|c|c|c|c|c|}
\hline 0 & 0 & 0 & 0 & 0 & 0 & 0 & 0 & 0 & 0 & 0 & 0 & 0 & 0 \\
\hline 0 & 0 & 0 & 0 & 0 & 0 & 0 & 0 & 0 & 0 & 0 & 0 & 0 & 0 \\
\hline
\end{tabular}

$\begin{array}{lllllllllllllll}0 & 0 & 0 & 0 & 0 & 0 & 0 & 0 & 0 & 0 & 0 & 0 & 0 & 0 & 0 \\ 0 & 0 & 0 & 0 & 0 & 0 & 0 & 0 & 0 & 0 & 0 & 0 & 0 & 0 & 0 \\ 0 & 0 & 0 & 0 & 0 & 0 & 0 & 0 & 0 & 0 & 0 & 0 & 0 & 0 & 0 \\ 0 & 0 & 0 & 0 & 0 & 0 & 0 & 0 & 0 & 0 & 0 & 0 & 0 & 0 & 0 \\ 0 & 0 & 0 & 0 & 0 & 0 & \mathbf{0} & \mathbf{0} & \mathbf{0} & \mathbf{0} & \mathbf{0} & \mathbf{0} & \mathbf{0} & \mathbf{0} & \mathbf{0}\end{array}$


Table A-13: Columbia Storage Power Exchange Purchase (CSPE)

PNW Loads and Resources Study

2009 - 2010 Operating Year

2003 White Book

-Generating Public Entities-

1 BPA to GPE

2 Total Generating Public Entities

\begin{tabular}{|c|c|c|c|c|c|c|c|c|c|c|c|}
\hline 0 & 0 & 0 & 0 & 0 & 0 & 0 & 0 & 0 & 0 & 0 & ) \\
\hline 0 & 0 & 0 & 0 & 0 & 0 & 0 & 0 & 0 & 0 & 0 & \\
\hline
\end{tabular}

-Small \& Non-Gen Public Entities-

3 BPA to S\&NGE

4 Total Small \& Non-Gen Public Entities

$\begin{array}{lll}0 & 0 & 0 \\ 0 & 0 & 0\end{array}$

Investor-Owned Entities-

5 BPA to IOU

6 Total Investor-Owned Entities

$\begin{array}{lll}0 & 0 & 0 \\ 0 & 0 & 0\end{array}$

-Other Entities-

7 BPA to OTHER

8 Total Other Entities

$\begin{array}{lll}0 & 0 & 0 \\ 0 & 0 & 0\end{array}$

-CSPE-

9 Generating Public Agencies

10 Small \& Non-Gen Public Agencies

11 Investor-Owned Utilities

12 Other Entities

13 Total CSPE

$\begin{array}{lllllllllllllll}0 & 0 & 0 & 0 & 0 & 0 & 0 & 0 & 0 & 0 & 0 & 0 & 0 & 0 & 0 \\ 0 & 0 & 0 & 0 & 0 & 0 & 0 & 0 & 0 & 0 & 0 & 0 & 0 & 0 & 0 \\ 0 & 0 & 0 & 0 & 0 & 0 & 0 & 0 & 0 & 0 & 0 & 0 & 0 & 0 & 0 \\ 0 & 0 & 0 & 0 & 0 & 0 & 0 & 0 & 0 & 0 & 0 & 0 & 0 & 0 & 0 \\ 0 & \mathbf{0} & \mathbf{0} & \mathbf{0} & \mathbf{0} & \mathbf{0} & \mathbf{0} & \mathbf{0} & \mathbf{0} & \mathbf{0} & \mathbf{0} & \mathbf{0} & \mathbf{0} & \mathbf{0} & \mathbf{0}\end{array}$


Table A-13: Columbia Storage Power Exchange Purchase (CSPE)

PNW Loads and Resources Study

2010 - 2011 Operating Year

2003 White Book

-Generating Public Entities-

1 BPA to GPE

2 Total Generating Public Entities

\begin{tabular}{|c|c|c|c|c|c|c|c|c|c|c|c|}
\hline 0 & 0 & 0 & 0 & 0 & 0 & 0 & 0 & 0 & 0 & 0 & ) \\
\hline 0 & 0 & 0 & 0 & 0 & 0 & 0 & 0 & 0 & 0 & 0 & \\
\hline
\end{tabular}

-Small \& Non-Gen Public Entities-

3 BPA to S\&NGE

4 Total Small \& Non-Gen Public Entities

$\begin{array}{lll}0 & 0 & 0 \\ 0 & 0 & 0\end{array}$

Investor-Owned Entities-

5 BPA to IOU

6 Total Investor-Owned Entities

$\begin{array}{lll}0 & 0 & 0 \\ 0 & 0 & 0\end{array}$

-Other Entities-

7 BPA to OTHER

8 Total Other Entities

$\begin{array}{lll}0 & 0 & 0 \\ 0 & 0 & 0\end{array}$

-CSPE-

9 Generating Public Agencies

10 Small \& Non-Gen Public Agencies

11 Investor-Owned Utilities

12 Other Entities

13 Total CSPE

$\begin{array}{lllllllllllllll}0 & 0 & 0 & 0 & 0 & 0 & 0 & 0 & 0 & 0 & 0 & 0 & 0 & 0 & 0 \\ 0 & 0 & 0 & 0 & 0 & 0 & 0 & 0 & 0 & 0 & 0 & 0 & 0 & 0 & 0 \\ 0 & 0 & 0 & 0 & 0 & 0 & 0 & 0 & 0 & 0 & 0 & 0 & 0 & 0 & 0 \\ 0 & 0 & 0 & 0 & 0 & 0 & 0 & 0 & 0 & 0 & 0 & 0 & 0 & 0 & 0 \\ 0 & \mathbf{0} & \mathbf{0} & \mathbf{0} & \mathbf{0} & \mathbf{0} & \mathbf{0} & \mathbf{0} & \mathbf{0} & \mathbf{0} & \mathbf{0} & \mathbf{0} & \mathbf{0} & \mathbf{0} & \mathbf{0}\end{array}$


-Generating Public Entities-

$1 \mathrm{BPA}$ to GPE

2 Total Generating Public Entities

Oct Nov Dec Jan Feb Mar Apr1 Apr16

May Jun Jul Avg

-Small \& Non-Gen Public Entities-

3 BPA to S\&NGE

4 Total Small \& Non-Gen Public Entities

$\begin{array}{lllllllllllllll}0 & 0 & 0 & 0 & 0 & 0 & 0 & 0 & 0 & 0 & 0 & 0 & 0 & 0 & 0 \\ 0 & 0 & 0 & 0 & 0 & 0 & 0 & 0 & 0 & 0 & 0 & 0 & 0 & 0 & 0\end{array}$

Investor-Owned Entities-

5 BPA to IOU

6 Total Investor-Owned Entities

$\begin{array}{lll}0 & 0 & 0 \\ 0 & 0 & 0\end{array}$

-Other Entities-

7 BPA to OTHER

8 Total Other Entities

$\begin{array}{lll}0 & 0 & 0 \\ 0 & 0 & 0\end{array}$

-CSPE-

9 Generating Public Agencies

10 Small \& Non-Gen Public Agencies

11 Investor-Owned Utilities

12 Other Entities

13 Total CSPE

\begin{tabular}{|c|c|c|c|c|c|c|c|c|c|c|c|c|c|}
\hline 0 & 0 & 0 & 0 & 0 & 0 & 0 & 0 & 0 & 0 & 0 & 0 & 0 & 0 \\
\hline 0 & 0 & 0 & 0 & 0 & 0 & 0 & 0 & 0 & 0 & 0 & 0 & 0 & 0 \\
\hline
\end{tabular}

$\begin{array}{lllllllllllllll}0 & 0 & 0 & 0 & 0 & 0 & 0 & 0 & 0 & 0 & 0 & 0 & 0 & 0 & 0 \\ 0 & 0 & 0 & 0 & 0 & 0 & 0 & 0 & 0 & 0 & 0 & 0 & 0 & 0 & 0 \\ 0 & 0 & 0 & 0 & 0 & 0 & 0 & 0 & 0 & 0 & 0 & 0 & 0 & 0 & 0 \\ 0 & 0 & 0 & 0 & 0 & 0 & 0 & 0 & 0 & 0 & 0 & 0 & 0 & 0 & 0 \\ 0 & 0 & 0 & 0 & 0 & 0 & \mathbf{0} & \mathbf{0} & \mathbf{0} & \mathbf{0} & \mathbf{0} & \mathbf{0} & \mathbf{0} & \mathbf{0} & \mathbf{0}\end{array}$


-Generating Public Entities-

$1 \mathrm{BPA}$ to GPE

2 Total Generating Public Entities

Oct Nov Dec Jan Feb Mar Apr1 Apr16

May Jun Jul Avg

-Small \& Non-Gen Public Entities-

3 BPA to S\&NGE

4 Total Small \& Non-Gen Public Entities

$\begin{array}{lllllllllllllll}0 & 0 & 0 & 0 & 0 & 0 & 0 & 0 & 0 & 0 & 0 & 0 & 0 & 0 & 0 \\ 0 & 0 & 0 & 0 & 0 & 0 & 0 & 0 & 0 & 0 & 0 & 0 & 0 & 0 & 0\end{array}$

-Investor-Owned Entities-

5 BPA to IOU

6 Total Investor-Owned Entities

$\begin{array}{lll}0 & 0 & 0 \\ 0 & 0 & 0\end{array}$

-Other Entities-

7 BPA to OTHER

8 Total Other Entities

$\begin{array}{lll}0 & 0 & 0 \\ 0 & 0 & 0\end{array}$

-CSPE-

9 Generating Public Agencies

10 Small \& Non-Gen Public Agencies

11 Investor-Owned Utilities

12 Other Entities

13 Total CSPE

$\begin{array}{lll}0 & 0 & 0 \\ 0 & 0 & 0\end{array}$

$\begin{array}{lllllllllllllll}0 & 0 & 0 & 0 & 0 & 0 & 0 & 0 & 0 & 0 & 0 & 0 & 0 & 0 & 0 \\ 0 & 0 & 0 & 0 & 0 & 0 & 0 & 0 & 0 & 0 & 0 & 0 & 0 & 0 & 0 \\ 0 & 0 & 0 & 0 & 0 & 0 & 0 & 0 & 0 & 0 & 0 & 0 & 0 & 0 & 0 \\ 0 & 0 & 0 & 0 & 0 & 0 & 0 & 0 & 0 & 0 & 0 & 0 & 0 & 0 & 0 \\ 0 & \mathbf{0} & \mathbf{0} & \mathbf{0} & \mathbf{0} & \mathbf{0} & \mathbf{0} & \mathbf{0} & \mathbf{0} & \mathbf{0} & \mathbf{0} & \mathbf{0} & \mathbf{0} & \mathbf{0} & \mathbf{0}\end{array}$


-Generating Public Entities-

$1 \mathrm{BPA}$ to GPE

2 Total Generating Public Entities

Oct Nov Dec Jan Feb Mar Apr1 Apr16

May Jun Jul Avg

-Small \& Non-Gen Public Entities-

3 BPA to S\&NGE

4 Total Small \& Non-Gen Public Entities

$\begin{array}{lllllllllllllll}0 & 0 & 0 & 0 & 0 & 0 & 0 & 0 & 0 & 0 & 0 & 0 & 0 & 0 & 0 \\ 0 & 0 & 0 & 0 & 0 & 0 & 0 & 0 & 0 & 0 & 0 & 0 & 0 & 0 & 0\end{array}$

-Investor-Owned Entities-

5 BPA to IOU

6 Total Investor-Owned Entities

$\begin{array}{lll}0 & 0 & 0 \\ 0 & 0 & 0\end{array}$

-Other Entities-

7 BPA to OTHER

8 Total Other Entities

$\begin{array}{lll}0 & 0 & 0 \\ 0 & 0 & 0\end{array}$

-CSPE-

9 Generating Public Agencies

10 Small \& Non-Gen Public Agencies

11 Investor-Owned Utilities

12 Other Entities

13 Total CSPE

\begin{tabular}{|c|c|c|c|c|c|c|c|c|c|c|c|c|c|}
\hline 0 & 0 & 0 & 0 & 0 & 0 & 0 & 0 & 0 & 0 & 0 & 0 & 0 & 0 \\
\hline 0 & 0 & 0 & 0 & 0 & 0 & 0 & 0 & 0 & 0 & 0 & 0 & 0 & 0 \\
\hline
\end{tabular}

$\begin{array}{lllllllllllllll}0 & 0 & 0 & 0 & 0 & 0 & 0 & 0 & 0 & 0 & 0 & 0 & 0 & 0 & 0 \\ 0 & 0 & 0 & 0 & 0 & 0 & 0 & 0 & 0 & 0 & 0 & 0 & 0 & 0 & 0 \\ 0 & 0 & 0 & 0 & 0 & 0 & 0 & 0 & 0 & 0 & 0 & 0 & 0 & 0 & 0 \\ 0 & 0 & 0 & 0 & 0 & 0 & 0 & 0 & 0 & 0 & 0 & 0 & 0 & 0 & 0 \\ 0 & 0 & 0 & 0 & 0 & 0 & \mathbf{0} & \mathbf{0} & \mathbf{0} & \mathbf{0} & \mathbf{0} & \mathbf{0} & \mathbf{0} & \mathbf{0} & \mathbf{0}\end{array}$


Table A-14: Supplemental \& Entitlement Capacity

PNW Loads and Resources Study

2004 - 2005 Operating Year

2003 White Book

Average Energy in Megawatts

Aug1 Aug16

Sep

Oct Nov Dec Jan Feb Mar Apr1 Apr16

May Jun Jul Avg Supplemental \& Entitlement Capacity

-Deliveries-

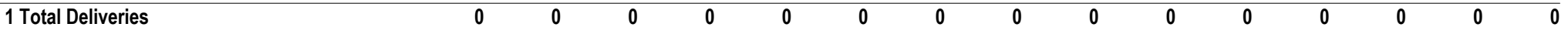

-Replacement-

2 Total Replacement

0

$0 \quad 0 \quad 0$

$\begin{array}{llllllllllllllll}\mathbf{0} & \mathbf{0} & \mathbf{0} & \mathbf{0} & \mathbf{0} & \mathbf{0} & \mathbf{0} & \mathbf{0} & \mathbf{0} & \mathbf{0} & \mathbf{0} & \mathbf{0} & \mathbf{0} & \mathbf{0} & \mathbf{0} \\ 0 & 0 & 0 & 0 & 0 & 0 & 0 & 0 & 0 & 0 & 0 & 0 & 0 & 0 & 0 \\ 0 & 0 & 0 & 0 & 0 & 0 & 0 & 0 & 0 & 0 & 0 & 0 & 0 & 0 & 0 \\ 0 & 0 & 0 & 0 & 0 & 0 & 0 & 0 & 0 & 0 & 0 & 0 & 0 & 0 & 0 \\ 0 & 0 & 0 & 0 & 0 & 0 & 0 & 0 & 0 & 0 & 0 & 0 & 0 & 0 & 0 \\ \mathbf{0} & \mathbf{0} & \mathbf{0} & \mathbf{0} & \mathbf{0} & \mathbf{0} & \mathbf{0} & \mathbf{0} & \mathbf{0} & \mathbf{0} & \mathbf{0} & \mathbf{0} & \mathbf{0} & \mathbf{0} & \mathbf{0}\end{array}$

$\begin{array}{lllllll}0 & 0 & 0 & 0 & 0 & 0 & 0\end{array}$

-Total Deliveries-

3 Federal Entities

4 Public Entities

5 Investor-Owned Entities

6 Other Entities

7 Total Deliveries

-Total Replacement-

8 Federal Entities

9 Public Entities

10 Investor-Owned Entities

11 Other Entities

12 Total Replacement

\begin{tabular}{lllllllllllllll}
0 & 0 & 0 & 0 & 0 & 0 & 0 & 0 & 0 & 0 & 0 & 0 & 0 & 0 & 0 \\
0 & 0 & 0 & 0 & 0 & 0 & 0 & 0 & 0 & 0 & 0 & 0 & 0 & 0 & 0 \\
0 & 0 & 0 & 0 & 0 & 0 & 0 & 0 & 0 & 0 & 0 & 0 & 0 & 0 & 0 \\
0 & 0 & 0 & 0 & 0 & 0 & 0 & 0 & 0 & 0 & 0 & 0 & 0 & 0 & 0 \\
\hline $\mathbf{0}$ & $\mathbf{0}$ & $\mathbf{0}$ & $\mathbf{0}$ & $\mathbf{0}$ & $\mathbf{0}$ & $\mathbf{0}$ & $\mathbf{0}$ & $\mathbf{0}$ & $\mathbf{0}$ & $\mathbf{0}$ & $\mathbf{0}$ & $\mathbf{0}$ & $\mathbf{0}$ & $\mathbf{0}$
\end{tabular}



2003 White Book

Supplemental \& Entitlement Capacity

-Deliveries-

May Jun Jul Avg

1 Total Delive

-Replacement-

2 Total Replacement

Oct Nov Dec Jan Feb Mar Apr1 Apr16

-Total Deliveries-

3 Federal Entities

4 Public Entities

5 Investor-Owned Entities

6 Other Entities

7 Total Deliveries

$0 \quad 0$

$0 \quad 0$

0

$0 \quad 0 \quad 0$

$0 \quad 0 \quad 0$

\begin{tabular}{|c|c|c|c|c|c|c|c|c|c|c|c|c|c|}
\hline 0 & 0 & 0 & 0 & 0 & 0 & 0 & 0 & 0 & 0 & 0 & 0 & 0 & 0 \\
\hline 0 & 0 & 0 & 0 & 0 & 0 & 0 & 0 & 0 & 0 & 0 & 0 & 0 & 0 \\
\hline 0 & 0 & 0 & 0 & 0 & 0 & 0 & 0 & 0 & 0 & 0 & 0 & 0 & 0 \\
\hline 0 & 0 & 0 & 0 & 0 & 0 & 0 & 0 & 0 & 0 & 0 & 0 & 0 & 0 \\
\hline 0 & 0 & 0 & 0 & 0 & 0 & 0 & 0 & 0 & 0 & 0 & 0 & 0 & 0 \\
\hline 0 & 0 & 0 & 0 & 0 & 0 & 0 & 0 & 0 & 0 & 0 & 0 & 0 & 0 \\
\hline
\end{tabular}

$\begin{array}{lllllll}0 & 0 & 0 & 0 & 0 & 0 & 0\end{array}$

-Total Replacement-

8 Federal Entities

9 Public Entities

10 Investor-Owned Entities

11 Other Entities

12 Total Replacement 
Table A-14: Supplemental \& Entitlement Capacity

PNW Loads and Resources Study

2006 - 2007 Operating Year

2003 White Book

Average Energy in Megawatts

Aug1 Aug16

Sep

Oct Nov Dec Jan Feb Mar Apr1 Apr16

May Jun Jul Avg Supplemental \& Entitlement Capacity

-Deliveries-

1 Total Deliveries

$0 \quad 0$

$0 \quad 0 \quad 0$

0

-Replacement

2 Total Replacement

0

$0 \quad 0$

$0 \quad 0$

0

0

0

-Total Deliveries-

3 Federal Entities

4 Public Entities

5 Investor-Owned Entities

6 Other Entities

7 Total Deliveries

$\begin{array}{lll}0 & 0 & 0 \\ 0 & 0 & 0 \\ 0 & 0 & 0 \\ 0 & 0 & 0 \\ 0 & 0 & 0\end{array}$

$\begin{array}{ll}0 & 0 \\ 0 & 0 \\ 0 & 0 \\ 0 & 0 \\ 0 & 0\end{array}$

$\begin{array}{lll}0 & 0 & 0 \\ 0 & 0 & 0 \\ 0 & 0 & 0 \\ 0 & 0 & 0 \\ 0 & 0 & 0\end{array}$

$\begin{array}{lll}0 & 0 & 0 \\ 0 & 0 & 0 \\ 0 & 0 & 0 \\ 0 & 0 & 0 \\ 0 & 0 & 0\end{array}$

-Total Replacement-

8 Federal Entities

9 Public Entities

10 Investor-Owned Entities

11 Other Entities

12 Total Replacement

\begin{tabular}{lllllllllllllll}
0 & 0 & 0 & 0 & 0 & 0 & 0 & 0 & 0 & 0 & 0 & 0 & 0 & 0 & 0 \\
0 & 0 & 0 & 0 & 0 & 0 & 0 & 0 & 0 & 0 & 0 & 0 & 0 & 0 & 0 \\
0 & 0 & 0 & 0 & 0 & 0 & 0 & 0 & 0 & 0 & 0 & 0 & 0 & 0 & 0 \\
0 & 0 & 0 & 0 & 0 & 0 & 0 & 0 & 0 & 0 & 0 & 0 & 0 & 0 & 0 \\
\hline $\mathbf{0}$ & $\mathbf{0}$ & $\mathbf{0}$ & $\mathbf{0}$ & $\mathbf{0}$ & $\mathbf{0}$ & $\mathbf{0}$ & $\mathbf{0}$ & $\mathbf{0}$ & $\mathbf{0}$ & $\mathbf{0}$ & $\mathbf{0}$ & $\mathbf{0}$ & $\mathbf{0}$ & $\mathbf{0}$
\end{tabular}



2003 White Book

Supplemental \& Entitlement Capacity

-Deliveries-

1 Total Deliveries

-Replacement-

2 Total Replacement

-Total Deliveries-

3 Federal Entities

4 Public Entities

5 Investor-Owned Entities

6 Other Entities

7 Total Deliveries

-Total Replacement-

8 Federal Entities

9 Public Entities

10 Investor-Owned Entities

11 Other Entities

12 Total Replacement

Oct Nov Dec Jan Feb Mar Apr1 Apr16

May Jun Jul Avg

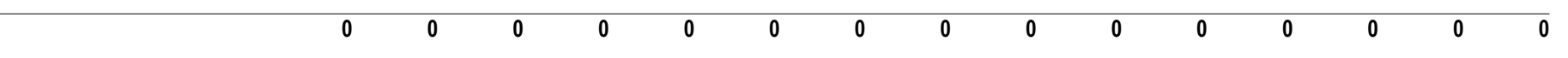

\begin{tabular}{|c|c|c|c|c|c|c|c|c|c|c|c|c|c|c|c|}
\hline \multirow{5}{*}{ tities } & 0 & 0 & 0 & 0 & 0 & 0 & 0 & 0 & 0 & 0 & 0 & 0 & 0 & 0 & 0 \\
\hline & 0 & 0 & 0 & 0 & 0 & 0 & 0 & 0 & 0 & 0 & 0 & 0 & 0 & 0 & 0 \\
\hline & 0 & 0 & 0 & 0 & 0 & 0 & 0 & 0 & 0 & 0 & 0 & 0 & 0 & 0 & 0 \\
\hline & 0 & 0 & 0 & 0 & 0 & 0 & 0 & 0 & 0 & 0 & 0 & 0 & 0 & 0 & 0 \\
\hline & 0 & 0 & 0 & 0 & 0 & 0 & 0 & 0 & 0 & 0 & 0 & 0 & 0 & 0 & 0 \\
\hline
\end{tabular}

\begin{tabular}{|c|c|c|c|c|c|c|c|c|c|c|c|c|c|c|c|}
\hline & 0 & 0 & 0 & 0 & 0 & 0 & 0 & 0 & 0 & 0 & 0 & 0 & 0 & 0 & 0 \\
\hline \multirow{3}{*}{ Entities } & 0 & 0 & 0 & 0 & 0 & 0 & 0 & 0 & 0 & 0 & 0 & 0 & 0 & 0 & 0 \\
\hline & 0 & 0 & 0 & 0 & 0 & 0 & 0 & 0 & 0 & 0 & 0 & 0 & 0 & 0 & 0 \\
\hline & 0 & 0 & 0 & 0 & 0 & 0 & 0 & 0 & 0 & 0 & 0 & 0 & 0 & 0 & 0 \\
\hline & 0 & 0 & 0 & 0 & 0 & 0 & 0 & 0 & 0 & 0 & 0 & 0 & 0 & 0 & 0 \\
\hline
\end{tabular}


Table A-14: Supplemental \& Entitlement Capacity

PNW Loads and Resources Study

2008 - 2009 Operating Year

2003 White Book

Average Energy in Megawatts

Aug1 Aug16

Sep

Oct Nov Dec Jan Feb Mar Apr1 Apr16

May Jun Jul Avg Supplemental \& Entitlement Capacity

-Deliveries-

1 Total Deliveries

$0 \quad 0$

$0 \quad 0 \quad 0$

0

-Replacement

2 Total Replacement

0

$0 \quad 0$

$0 \quad 0$

0

0

0

-Total Deliveries-

3 Federal Entities

4 Public Entities

5 Investor-Owned Entities

6 Other Entities

7 Total Deliveries

$\begin{array}{lll}0 & 0 & 0 \\ 0 & 0 & 0 \\ 0 & 0 & 0 \\ 0 & 0 & 0 \\ 0 & 0 & 0\end{array}$

$\begin{array}{ll}0 & 0 \\ 0 & 0 \\ 0 & 0 \\ 0 & 0 \\ 0 & 0\end{array}$

$\begin{array}{llll}0 & 0 & 0 & 0 \\ 0 & 0 & 0 & 0 \\ 0 & 0 & 0 & 0 \\ 0 & 0 & 0 & 0 \\ 0 & 0 & 0 & 0\end{array}$

$\begin{array}{lll}\mathbf{0} & \mathbf{0} & \mathbf{0} \\ & & \\ 0 & 0 & \\ 0 & 0 & 0 \\ 0 & 0 & 0 \\ 0 & 0 & 0 \\ \mathbf{0} & \mathbf{0} & 0\end{array}$

-Total Replacement-

8 Federal Entities

9 Public Entities

10 Investor-Owned Entities

11 Other Entities

12 Total Replacement

\begin{tabular}{lllllllllllllll}
0 & 0 & 0 & 0 & 0 & 0 & 0 & 0 & 0 & 0 & 0 & 0 & 0 & 0 & 0 \\
0 & 0 & 0 & 0 & 0 & 0 & 0 & 0 & 0 & 0 & 0 & 0 & 0 & 0 & 0 \\
0 & 0 & 0 & 0 & 0 & 0 & 0 & 0 & 0 & 0 & 0 & 0 & 0 & 0 & 0 \\
0 & 0 & 0 & 0 & 0 & 0 & 0 & 0 & 0 & 0 & 0 & 0 & 0 & 0 & 0 \\
\hline $\mathbf{0}$ & $\mathbf{0}$ & $\mathbf{0}$ & $\mathbf{0}$ & $\mathbf{0}$ & $\mathbf{0}$ & $\mathbf{0}$ & $\mathbf{0}$ & $\mathbf{0}$ & $\mathbf{0}$ & $\mathbf{0}$ & $\mathbf{0}$ & $\mathbf{0}$ & $\mathbf{0}$ & $\mathbf{0}$
\end{tabular}


Supplemental \& Entitlement Capacity

-Deliveries-

Oct Nov Dec Jan Feb Mar Apr1 Apr16 May Jun Jul Avg

1 Total Deliveries

$0 \quad 0$

$0 \quad 0$

0

0

eplacement-

2 Total Replacement

$0 \quad 0$

$0 \quad 0 \quad 0$

$\begin{array}{llllllllllllllll}\mathbf{0} & \mathbf{0} & \mathbf{0} & \mathbf{0} & \mathbf{0} & \mathbf{0} & \mathbf{0} & \mathbf{0} & \mathbf{0} & \mathbf{0} & \mathbf{0} & \mathbf{0} & \mathbf{0} & \mathbf{0} & \mathbf{0} \\ 0 & 0 & 0 & 0 & 0 & 0 & 0 & 0 & 0 & 0 & 0 & 0 & 0 & 0 & 0 \\ 0 & 0 & 0 & 0 & 0 & 0 & 0 & 0 & 0 & 0 & 0 & 0 & 0 & 0 & 0 \\ 0 & 0 & 0 & 0 & 0 & 0 & 0 & 0 & 0 & 0 & 0 & 0 & 0 & 0 & 0 \\ 0 & 0 & 0 & 0 & 0 & 0 & 0 & 0 & 0 & 0 & 0 & 0 & 0 & 0 & 0 \\ \mathbf{0} & \mathbf{0} & \mathbf{0} & \mathbf{0} & \mathbf{0} & \mathbf{0} & \mathbf{0} & \mathbf{0} & \mathbf{0} & \mathbf{0} & \mathbf{0} & \mathbf{0} & \mathbf{0} & \mathbf{0} & \mathbf{0}\end{array}$

$\begin{array}{lllllll}0 & 0 & 0 & 0 & 0 & 0 & 0\end{array}$

-Total Deliveries-

3 Federal Entities

4 Public Entities

5 Investor-Owned Entities

6 Other Entities

7 Total Deliveries

-Total Replacement-

8 Federal Entities

9 Public Entities

10 Investor-Owned Entities

11 Other Entities

12 Total Replacement

\begin{tabular}{lllllllllllllll}
0 & 0 & 0 & 0 & 0 & 0 & 0 & 0 & 0 & 0 & 0 & 0 & 0 & 0 & 0 \\
0 & 0 & 0 & 0 & 0 & 0 & 0 & 0 & 0 & 0 & 0 & 0 & 0 & 0 & 0 \\
0 & 0 & 0 & 0 & 0 & 0 & 0 & 0 & 0 & 0 & 0 & 0 & 0 & 0 & 0 \\
0 & 0 & 0 & 0 & 0 & 0 & 0 & 0 & 0 & 0 & 0 & 0 & 0 & 0 & 0 \\
\hline $\mathbf{0}$ & $\mathbf{0}$ & $\mathbf{0}$ & $\mathbf{0}$ & $\mathbf{0}$ & $\mathbf{0}$ & $\mathbf{0}$ & $\mathbf{0}$ & $\mathbf{0}$ & $\mathbf{0}$ & $\mathbf{0}$ & $\mathbf{0}$ & $\mathbf{0}$ & $\mathbf{0}$ & $\mathbf{0}$
\end{tabular}


Supplemental \& Entitlement Capacity

-Deliveries-

Oct Nov Dec Jan Feb Mar Apr1 Apr16 May Jun Jul Avg

1 Total Deliveries

-Replacement

2 Total Replacement

-Total Deliveries-

3 Federal Entities

4 Public Entities

5 Investor-Owned Entities

6 Other Entities

7 Total Deliveries

-Total Replacement-

8 Federal Entities

9 Public Entities

10 Investor-Owned Entities

11 Other Entities

12 Total Replacement

$\begin{array}{llllllllllllllll}0 & 0 & 0 & 0 & 0 & 0 & 0 & 0 & 0 & 0 & 0 & 0 & 0 & 0 & 0\end{array}$

\begin{tabular}{llllllllllllll}
0 & 0 & 0 & 0 & 0 & 0 & 0 & 0 & 0 & 0 & 0 & 0 & 0 & 0 \\
\hline
\end{tabular}

\begin{tabular}{lllllllllllllll}
0 & 0 & 0 & 0 & 0 & 0 & 0 & 0 & 0 & 0 & 0 & 0 & 0 & 0 & 0 \\
0 & 0 & 0 & 0 & 0 & 0 & 0 & 0 & 0 & 0 & 0 & 0 & 0 & 0 & 0 \\
0 & 0 & 0 & 0 & 0 & 0 & 0 & 0 & 0 & 0 & 0 & 0 & 0 & 0 & 0 \\
0 & 0 & 0 & 0 & 0 & 0 & 0 & 0 & 0 & 0 & 0 & 0 & 0 & 0 & 0 \\
\hline & 0 & 0 & 0 & 0 & 0 & 0 & 0 & 0 & 0 & 0 & 0 & 0 & 0 & 0
\end{tabular}

\begin{tabular}{|c|c|c|c|c|c|c|c|c|c|c|c|c|c|c|c|}
\hline & 0 & 0 & 0 & 0 & 0 & 0 & 0 & 0 & 0 & 0 & 0 & 0 & 0 & 0 & 0 \\
\hline & 0 & 0 & 0 & 0 & 0 & 0 & 0 & 0 & 0 & 0 & 0 & 0 & 0 & 0 & 0 \\
\hline ies & 0 & 0 & 0 & 0 & 0 & 0 & 0 & 0 & 0 & 0 & 0 & 0 & 0 & 0 & 0 \\
\hline & 0 & 0 & 0 & 0 & 0 & 0 & 0 & 0 & 0 & 0 & 0 & 0 & 0 & 0 & 0 \\
\hline & 0 & 0 & 0 & 0 & 0 & 0 & 0 & 0 & 0 & 0 & 0 & 0 & 0 & 0 & 0 \\
\hline
\end{tabular}



2003 White Book

Supplemental \& Entitlement Capacity

-Deliveries-

May Jun Jul Avg

1 Total Delive

-Replacement-

2 Total Replacement

Oct Nov Dec Jan Feb Mar Apr1 Apr16

-Total Deliveries-

3 Federal Entities

4 Public Entities

5 Investor-Owned Entities

6 Other Entities

7 Total Deliveries

$0 \quad 0$

$0 \quad 0$

0

$0 \quad 0 \quad 0$

$0 \quad 0 \quad 0$

\begin{tabular}{|c|c|c|c|c|c|c|c|c|c|c|c|c|c|}
\hline 0 & 0 & 0 & 0 & 0 & 0 & 0 & 0 & 0 & 0 & 0 & 0 & 0 & 0 \\
\hline 0 & 0 & 0 & 0 & 0 & 0 & 0 & 0 & 0 & 0 & 0 & 0 & 0 & 0 \\
\hline 0 & 0 & 0 & 0 & 0 & 0 & 0 & 0 & 0 & 0 & 0 & 0 & 0 & 0 \\
\hline 0 & 0 & 0 & 0 & 0 & 0 & 0 & 0 & 0 & 0 & 0 & 0 & 0 & 0 \\
\hline 0 & 0 & 0 & 0 & 0 & 0 & 0 & 0 & 0 & 0 & 0 & 0 & 0 & 0 \\
\hline 0 & 0 & 0 & 0 & 0 & 0 & 0 & 0 & 0 & 0 & 0 & 0 & 0 & 0 \\
\hline
\end{tabular}

$\begin{array}{lllllll}0 & 0 & 0 & 0 & 0 & 0 & 0\end{array}$

-Total Replacement-

8 Federal Entities

9 Public Entities

10 Investor-Owned Entities

11 Other Entities

12 Total Replacement 
Supplemental \& Entitlement Capacity

-Deliveries-

Oct Nov Dec Jan Feb Mar Apr1 Apr16 May Jun Jul Avg

1 Total Deliveries

-Replacement

2 Total Replacement

-Total Deliveries-

3 Federal Entities

4 Public Entities

5 Investor-Owned Entities

6 Other Entities

7 Total Deliveries

-Total Replacement-

8 Federal Entities

9 Public Entities

10 Investor-Owned Entities

11 Other Entities

12 Total Replacement

$\begin{array}{llllllllllllllll}0 & 0 & 0 & 0 & 0 & 0 & 0 & 0 & 0 & 0 & 0 & 0 & 0 & 0 & 0\end{array}$

\begin{tabular}{llllllllllllll}
0 & 0 & 0 & 0 & 0 & 0 & 0 & 0 & 0 & 0 & 0 & 0 & 0 & 0 \\
\hline
\end{tabular}

\begin{tabular}{lllllllllllllll}
0 & 0 & 0 & 0 & 0 & 0 & 0 & 0 & 0 & 0 & 0 & 0 & 0 & 0 & 0 \\
0 & 0 & 0 & 0 & 0 & 0 & 0 & 0 & 0 & 0 & 0 & 0 & 0 & 0 & 0 \\
0 & 0 & 0 & 0 & 0 & 0 & 0 & 0 & 0 & 0 & 0 & 0 & 0 & 0 & 0 \\
0 & 0 & 0 & 0 & 0 & 0 & 0 & 0 & 0 & 0 & 0 & 0 & 0 & 0 & 0 \\
\hline & 0 & 0 & 0 & 0 & 0 & 0 & 0 & 0 & 0 & 0 & 0 & 0 & 0 & 0
\end{tabular}

\begin{tabular}{|c|c|c|c|c|c|c|c|c|c|c|c|c|c|c|c|}
\hline & 0 & 0 & 0 & 0 & 0 & 0 & 0 & 0 & 0 & 0 & 0 & 0 & 0 & 0 & 0 \\
\hline & 0 & 0 & 0 & 0 & 0 & 0 & 0 & 0 & 0 & 0 & 0 & 0 & 0 & 0 & 0 \\
\hline ies & 0 & 0 & 0 & 0 & 0 & 0 & 0 & 0 & 0 & 0 & 0 & 0 & 0 & 0 & 0 \\
\hline & 0 & 0 & 0 & 0 & 0 & 0 & 0 & 0 & 0 & 0 & 0 & 0 & 0 & 0 & 0 \\
\hline & 0 & 0 & 0 & 0 & 0 & 0 & 0 & 0 & 0 & 0 & 0 & 0 & 0 & 0 & 0 \\
\hline
\end{tabular}


Supplemental \& Entitlement Capacity

-Deliveries-

Oct Nov Dec Jan Feb Mar Apr1 Apr16 May Jun Jul Avg

1 Total Deliveries

-Replacement

2 Total Replacement

-Total Deliveries-

3 Federal Entities

4 Public Entities

5 Investor-Owned Entities

6 Other Entities

7 Total Deliveries

-Total Replacement-

8 Federal Entities

9 Public Entities

10 Investor-Owned Entities

11 Other Entities

12 Total Replacement

$\begin{array}{llllllllllllllll}0 & 0 & 0 & 0 & 0 & 0 & 0 & 0 & 0 & 0 & 0 & 0 & 0 & 0 & 0\end{array}$

\begin{tabular}{llllllllllllll}
0 & 0 & 0 & 0 & 0 & 0 & 0 & 0 & 0 & 0 & 0 & 0 & 0 & 0 \\
\hline
\end{tabular}

\begin{tabular}{lllllllllllllll}
0 & 0 & 0 & 0 & 0 & 0 & 0 & 0 & 0 & 0 & 0 & 0 & 0 & 0 & 0 \\
0 & 0 & 0 & 0 & 0 & 0 & 0 & 0 & 0 & 0 & 0 & 0 & 0 & 0 & 0 \\
0 & 0 & 0 & 0 & 0 & 0 & 0 & 0 & 0 & 0 & 0 & 0 & 0 & 0 & 0 \\
0 & 0 & 0 & 0 & 0 & 0 & 0 & 0 & 0 & 0 & 0 & 0 & 0 & 0 & 0 \\
\hline & 0 & 0 & 0 & 0 & 0 & 0 & 0 & 0 & 0 & 0 & 0 & 0 & 0 & 0
\end{tabular}

\begin{tabular}{|c|c|c|c|c|c|c|c|c|c|c|c|c|c|c|c|}
\hline & 0 & 0 & 0 & 0 & 0 & 0 & 0 & 0 & 0 & 0 & 0 & 0 & 0 & 0 & 0 \\
\hline & 0 & 0 & 0 & 0 & 0 & 0 & 0 & 0 & 0 & 0 & 0 & 0 & 0 & 0 & 0 \\
\hline ies & 0 & 0 & 0 & 0 & 0 & 0 & 0 & 0 & 0 & 0 & 0 & 0 & 0 & 0 & 0 \\
\hline & 0 & 0 & 0 & 0 & 0 & 0 & 0 & 0 & 0 & 0 & 0 & 0 & 0 & 0 & 0 \\
\hline & 0 & 0 & 0 & 0 & 0 & 0 & 0 & 0 & 0 & 0 & 0 & 0 & 0 & 0 & 0 \\
\hline
\end{tabular}




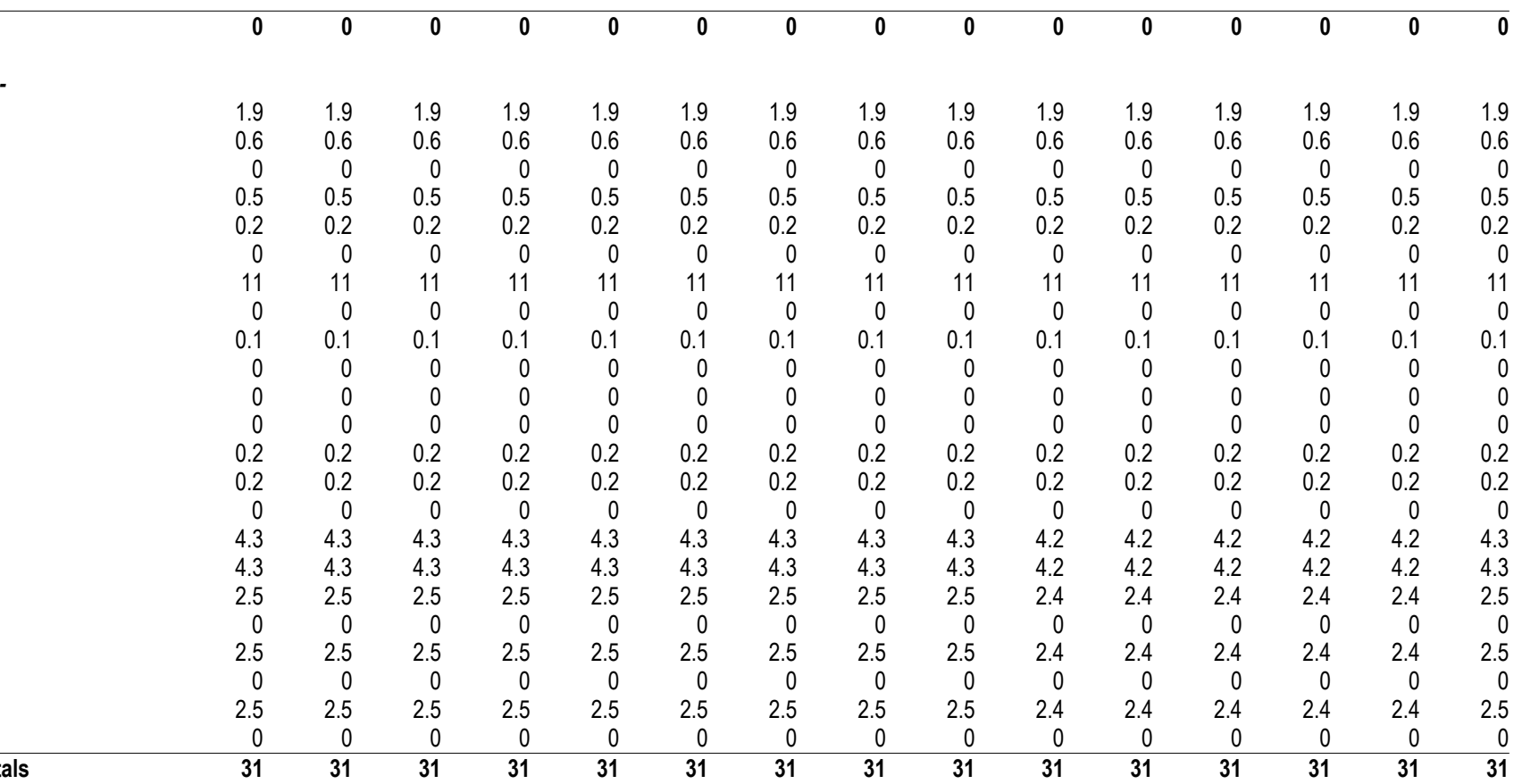

-Priest Rapids CER for Canada-

2 AVWP to BPA

3 COPD to BPA

4 CWPC to BPA

5 EWEB to BPA

6 FGRV to BPA

7 FREC to BPA

8 GCPD to BPA

9 ICLP to BPA

$10 \mathrm{KITT}$ to BPA

$11 \mathrm{KOOT}$ to BPA

12 LREC to BPA

13 LVE to BPA

$14 \mathrm{MCMN}$ to BPA

15 MTFR to BPA

16 NLEC to BPA

17 PGE to BPA

$18 \mathrm{PPL}$ to BPA

19 PSE to BPA

20 RREC to BPA

$21 \mathrm{SCL}$ to BPA

22 SLEC to BPA

23 TPU to BPA

24 UNEC to BPA

25 Priest Rapids: Regional Totals

-Wanapum CER for Canada-

26 AVWP to BPA

27 COPD to BPA

28 CWPC to BPA

29 EWEB to BPA

$30 \mathrm{FGRV}$ to BPA

31 FREC to BPA

32 GCPD to BPA

$33 \mathrm{ICLP}$ to BPA

$34 \mathrm{KITT}$ to BPA

$35 \mathrm{KOOT}$ to BPA

36 LREC to BPA

37 LVE to BPA

38 MCMN to BPA

39 MTFR to BPA

40 NLEC to BPA

41 PGE to BPA

$42 \mathrm{PPL}$ to BPA

43 PSE to BPA

44 RREC to BPA

$45 \mathrm{SCL}$ to BPA

46 SLEC to BPA

47 TPU to BPA

48 UNEC to BPA

49 Wanapum: Regional Totals

$\begin{array}{rrrrrrrrrrrrrrr}2.4 & 2.4 & 2.4 & 2.4 & 2.4 & 2.4 & 2.4 & 2.4 & 2.4 & 2.4 & 2.4 & 2.4 & 2.4 & 2.4 & 2.4 \\ 0.8 & 0.8 & 0.8 & 0.8 & 0.8 & 0.8 & 0.8 & 0.8 & 0.8 & 0.8 & 0.8 & 0.8 & 0.8 & 0.8 & 0.8 \\ 0 & 0 & 0 & 0 & 0 & 0 & 0 & 0 & 0 & 0 & 0 & 0 & 0 & 0 & 0 \\ 0.7 & 0.7 & 0.7 & 0.7 & 0.7 & 0.7 & 0.7 & 0.7 & 0.7 & 0.7 & 0.7 & 0.7 & 0.7 & 0.7 & 0.7 \\ 0.2 & 0.2 & 0.2 & 0.2 & 0.2 & 0.2 & 0.2 & 0.2 & 0.2 & 0.2 & 0.2 & 0.2 & 0.2 & 0.2 & 0.2 \\ 0 & 0 & 0 & 0 & 0 & 0 & 0 & 0 & 0 & 0 & 0 & 0 & 0 & 0 & 0 \\ 11 & 11 & 11 & 11 & 11 & 11 & 11 & 11 & 11 & 11 & 11 & 11 & 11 & 11 & 11 \\ 0 & 0 & 0 & 0 & 0 & 0 & 0 & 0 & 0 & 0 & 0 & 0 & 0 & 0 & 0 \\ 0 & 0 & 0 & 0 & 0 & 0 & 0 & 0 & 0 & 0 & 0 & 0 & 0 & 0 & 0 \\ 0 & 0 & 0 & 0 & 0 & 0 & 0 & 0 & 0 & 0 & 0 & 0 & 0 & 0 & 0 \\ 0 & 0 & 0 & 0 & 0 & 0 & 0 & 0 & 0 & 0 & 0 & 0 & 0 & 0 & 0 \\ 0 & 0 & 0 & 0 & 0 & 0 & 0 & 0 & 0 & 0 & 0 & 0 & 0 & 0 & 0 \\ 0.2 & 0.2 & 0.2 & 0.2 & 0.2 & 0.2 & 0.2 & 0.2 & 0.2 & 0.2 & 0.2 & 0.2 & 0.2 & 0.2 & 0.2 \\ 0.2 & 0.2 & 0.2 & 0.2 & 0.2 & 0.2 & 0.2 & 0.2 & 0.2 & 0.2 & 0.2 & 0.2 & 0.2 & 0.2 & 0.2 \\ 0 & 0 & 0 & 0 & 0 & 0 & 0 & 0 & 0 & 0 & 0 & 0 & 0 & 0 & 0 \\ 5.5 & 5.5 & 5.5 & 5.5 & 5.5 & 5.5 & 5.5 & 5.5 & 5.5 & 5.5 & 5.5 & 5.5 & 5.5 & 5.5 & 5.5 \\ 5.5 & 5.5 & 5.5 & 5.5 & 5.5 & 5.5 & 5.5 & 5.5 & 5.5 & 5.5 & 5.5 & 5.5 & 5.5 & 5.5 & 5.5 \\ 3.2 & 3.2 & 3.2 & 3.2 & 3.2 & 3.2 & 3.2 & 3.2 & 3.2 & 3.2 & 3.2 & 3.2 & 3.2 & 3.2 & 3.2 \\ 0 & 0 & 0 & 0 & 0 & 0 & 0 & 0 & 0 & 0 & 0 & 0 & 0 & 0 & 0 \\ 0 & 0 & 0 & 0 & 0 & 0 & 0 & 0 & 0 & 0 & 0 & 0 & 0 & 0 & 0 \\ 0 & 0 & 0 & 0 & 0 & 0 & 0 & 0 & 0 & 0 & 0 & 0 & 0 & 0 & 0 \\ 0 & 0 & 0 & 0 & 0 & 0 & 0 & 0 & 0 & 0 & 0 & 0 & 0 & 0 & 0 \\ 0 & 0 & 0 & 0 & 0 & 0 & 0 & 0 & 0 & 0 & 0 & 0 & 0 & 0 & 0 \\ 30 & \mathbf{3 0} & \mathbf{3 0} & \mathbf{3 0} & \mathbf{3 0} & \mathbf{3 0} & \mathbf{3 0} & \mathbf{3 0} & \mathbf{3 0} & \mathbf{2 9} & \mathbf{2 9} & \mathbf{2 9} & \mathbf{2 9} & \mathbf{2 9} & \mathbf{2 9}\end{array}$

-Rock Island P.H. \#1 CER for Canada-

$50 \mathrm{CHPD}$ to BPA

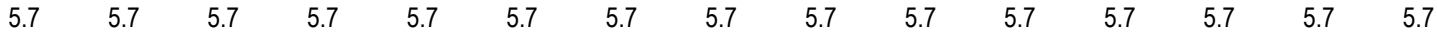
51 PSE to BPA

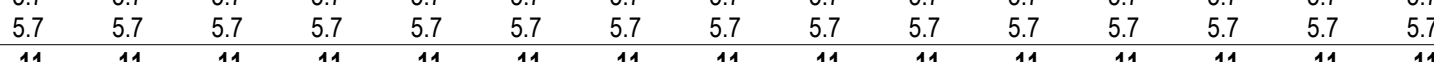

-Rock Island P.H. \#2 CER for Canada-

53 CHPD to BPA

54 PSE to BPA

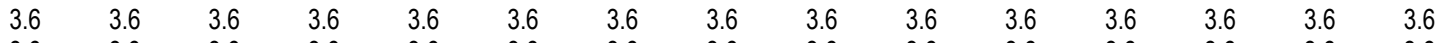

55 Rock Island P.H. \#2: Regional Totals

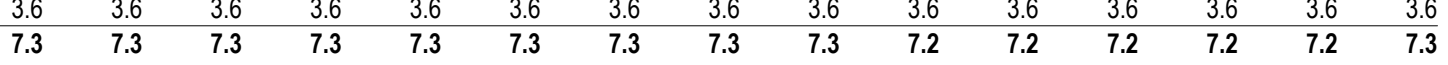

-Rocky Reach CER for Canada-

56 AVWP to BPA

57 CHPD to BPA

58 CLKM to BPA

59 DOPD to BPA

60 PGE to BPA

$61 \mathrm{PPL}$ to BPA

62 PSE to BPA

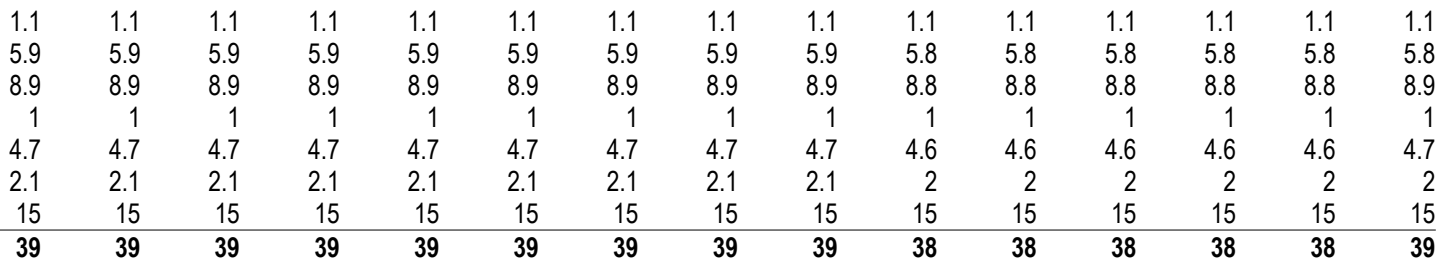


Table A-15: Canadian Entitlement Return For Canada

PNW Loads and Resources Study

2004 - 2005 Operating Year

2003 White Book Jul Avg

Average Energy in Megawatts

Aug1 Aug16

Sep

Oct Nov Dec Jan Feb Mar Apr1 Apr16

May Jun 64 AVWP to BPA 65 DOPD to BPA 66 OKPD to BPA 67 PGE to BPA $68 \mathrm{PPL}$ to BPA 69 PSE to BPA

70 Wells: Regional Totals

$\begin{array}{rrrrrrrrrrrrrrr}0.9 & 0.9 & 0.9 & 0.9 & 0.9 & 0.9 & 0.9 & 0.9 & 0.9 & 0.9 & 0.9 & 0.9 & 0.9 & 0.9 & 0.9 \\ 7.5 & 7.5 & 7.5 & 7.5 & 7.5 & 7.5 & 7.5 & 7.5 & 7.5 & 7.4 & 7.4 & 7.4 & 7.4 & 7.4 & 7.5 \\ 2 & 2 & 2 & 2 & 2 & 2 & 2 & 2 & 2 & 2.0 & 2.0 & 2.0 & 2.0 & 2.0 & 2 \\ 5.1 & 5.1 & 5.1 & 5.1 & 5.1 & 5.1 & 5.1 & 5.1 & 5.1 & 5 & 5 & 5 & 5 & 5 & 5.1 \\ 1.7 & 1.7 & 1.7 & 1.7 & 1.7 & 1.7 & 1.7 & 1.7 & 1.7 & 1.7 & 1.7 & 1.7 & 1.7 & 1.7 & 1.7 \\ 7.9 & 7.9 & 7.9 & 7.9 & 7.9 & 7.9 & 7.9 & 7.9 & 7.9 & 7.8 & 7.8 & 7.8 & 7.8 & 7.8 & 7.8 \\ \mathbf{2 5} & \mathbf{2 5} & \mathbf{2 5} & \mathbf{2 5} & \mathbf{2 5} & \mathbf{2 5} & \mathbf{2 5} & \mathbf{2 5} & \mathbf{2 5} & \mathbf{2 5} & \mathbf{2 5} & \mathbf{2 5} & \mathbf{2 5} & \mathbf{2 5} & \mathbf{2 5}\end{array}$

\section{-Total CER For Canada-}

71 Federal System

72 Public Entities

73 Investor-Owned Entities

74 Other Entities

75 Non-NW Entities

76 Total CER For Canada

\begin{tabular}{|c|c|c|c|c|c|c|c|c|c|c|c|c|c|}
\hline 0 & 0 & 0 & 0 & 0 & 0 & 0 & 0 & 0 & 0 & 0 & 0 & 0 & 0 \\
\hline 57 & 57 & 57 & 57 & 57 & 57 & 57 & 57 & 56 & 56 & 56 & 56 & 56 & 56 \\
\hline 77 & 77 & 77 & 77 & 77 & 77 & 77 & 77 & 77 & 77 & 77 & 77 & 77 & 77 \\
\hline 8.9 & 8.9 & 8.9 & 8.9 & 8.9 & 8.9 & 8.9 & 8.9 & 8.8 & 8.8 & 8.8 & 8.8 & 8.8 & 8.9 \\
\hline 0 & 0 & 0 & 0 & 0 & 0 & 0 & 0 & 0 & 0 & 0 & 0 & 0 & 0 \\
\hline 143 & 143 & 143 & 143 & 143 & 143 & 143 & 143 & 141 & 141 & 141 & 141 & 141 & 42 \\
\hline
\end{tabular}



2003 White Book

$\begin{array}{rrrrrrrrrrrrrrr}\mathbf{0} & \mathbf{0} & \mathbf{0} & \mathbf{0} & \mathbf{0} & \mathbf{0} & \mathbf{0} & \mathbf{0} & \mathbf{0} & \mathbf{0} & \mathbf{0} & \mathbf{0} & \mathbf{0} & \mathbf{0} & \mathbf{0} \\ & & & & & & & & & & & & & & \\ 1.9 & 1.9 & 1.9 & 1.9 & 0.9 & 0.9 & 0.9 & 0.9 & 0.9 & 0.8 & 0.8 & 0.8 & 0.8 & 0.8 & 1.1 \\ 0.6 & 0.6 & 0.6 & 0.6 & 0.3 & 0.3 & 0.3 & 0.3 & 0.3 & 0.3 & 0.3 & 0.3 & 0.3 & 0.3 & 0.4 \\ 0 & 0 & 0 & 0 & 0 & 0 & 0 & 0 & 0 & 0 & 0 & 0 & 0 & 0 & 0 \\ 0.5 & 0.5 & 0.5 & 0.5 & 0.2 & 0.2 & 0.2 & 0.2 & 0.2 & 0.2 & 0.2 & 0.2 & 0.2 & 0.2 & 0.3 \\ 0.2 & 0.2 & 0.2 & 0.2 & 0.1 & 0.1 & 0.1 & 0.1 & 0.1 & 0.1 & 0.1 & 0.1 & 0.1 & 0.1 & 0.1 \\ 0 & 0 & 0 & 0 & 0 & 0 & 0 & 0 & 0 & 0 & 0 & 0 & 0 & 0 & 0 \\ 11 & 11 & 11 & 11 & 21 & 21 & 21 & 21 & 21 & 21 & 21 & 21 & 21 & 21 & 19 \\ 0 & 0 & 0 & 0 & 0 & 0 & 0 & 0 & 0 & 0 & 0 & 0 & 0 & 0 & 0 \\ 0.1 & 0.1 & 0.1 & 0.1 & 0.1 & 0.1 & 0.1 & 0.1 & 0.1 & 0.1 & 0.1 & 0.1 & 0.1 & 0.1 & 0.1 \\ 0 & 0 & 0 & 0 & 0 & 0 & 0 & 0 & 0 & 0 & 0 & 0 & 0 & 0 & 0 \\ 0 & 0 & 0 & 0 & 0 & 0 & 0 & 0 & 0 & 0 & 0 & 0 & 0 & 0 & 0 \\ 0 & 0 & 0 & 0 & 0 & 0 & 0 & 0 & 0 & 0 & 0 & 0 & 0 & 0 & 0 \\ 0.2 & 0.2 & 0.2 & 0.2 & 0.1 & 0.1 & 0.1 & 0.1 & 0.1 & 0.1 & 0.1 & 0.1 & 0.1 & 0.1 & 0.1 \\ 0.2 & 0.2 & 0.2 & 0.2 & 0.1 & 0.1 & 0.1 & 0.1 & 0.1 & 0.1 & 0.1 & 0.1 & 0.1 & 0.1 & 0.1 \\ 0 & 0 & 0 & 0 & 0 & 0 & 0 & 0 & 0 & 0 & 0 & 0 & 0 & 0 & 0 \\ 4.2 & 4.2 & 4.2 & 4.2 & 2.0 & 2.0 & 2.0 & 2.0 & 2.0 & 1.9 & 1.9 & 1.9 & 1.9 & 1.9 & 2.5 \\ 4.2 & 4.2 & 4.2 & 4.2 & 2.0 & 2.0 & 2.0 & 2.0 & 2.0 & 1.9 & 1.9 & 1.9 & 1.9 & 1.9 & 2.5 \\ 2.4 & 2.4 & 2.4 & 2.4 & 1.2 & 1.2 & 1.2 & 1.2 & 1.2 & 1.1 & 1.1 & 1.1 & 1.1 & 1.1 & 1.5 \\ 0 & 0 & 0 & 0 & 0 & 0 & 0 & 0 & 0 & 0 & 0 & 0 & 0 & 0 & 0 \\ 2.4 & 2.4 & 2.4 & 2.4 & 1.1 & 1.1 & 1.1 & 1.1 & 1.1 & 1 & 1 & 1 & 1 & 1 & 1.4 \\ 0 & 0 & 0 & 0 & 0 & 0 & 0 & 0 & 0 & 0 & 0 & 0 & 0 & 0 & 0 \\ 2.4 & 2.4 & 2.4 & 2.4 & 1.1 & 1.1 & 1.1 & 1.1 & 1.1 & 1 & 1 & 1 & 1 & 1 & 1.4 \\ 0 & 0 & 0 & 0 & 0 & 0 & 0 & 0 & 0 & 0 & 0 & 0 & 0 & 0 & 0 \\ \mathbf{3 1} & \mathbf{3 1} & \mathbf{3 1} & \mathbf{3 1} & \mathbf{3 1} & \mathbf{3 1} & \mathbf{3 1} & \mathbf{3 1} & \mathbf{3 1} & \mathbf{2 9} & \mathbf{2 9} & \mathbf{2 9} & \mathbf{2 9} & \mathbf{2 9} & \mathbf{3 0}\end{array}$

-Priest Rapids CER for Canada-

2 AVWP to BPA

3 COPD to BPA

4 CWPC to BPA

5 EWEB to BPA

6 FGRV to BPA

7 FREC to BPA

8 GCPD to BPA

9 ICLP to BPA

$10 \mathrm{KITT}$ to BPA

$11 \mathrm{KOOT}$ to BPA

12 LREC to BPA

13 LVE to BPA

14 MCMN to BPA

15 MTFR to BPA

16 NLEC to BPA

17 PGE to BPA

$18 \mathrm{PPL}$ to $\mathrm{BPA}$

19 PSE to BPA

20 RREC to BPA

$21 \mathrm{SCL}$ to BPA

22 SLEC to BPA

23 TPU to BPA

24 UNEC to BPA

25 Priest Rapids: Regional Totals

$\begin{array}{crrrr} & 1.9 & 1.9 & 1.9 & 1.9 \\ & 0.6 & 0.6 & 0.6 & 0.0 \\ & 0 & 0 & 0 & \\ & 0.5 & 0.5 & 0.5 & 0.5 \\ & 0.2 & 0.2 & 0.2 & 0.2 \\ & 0 & 0 & 0 & \\ & 11 & 11 & 11 & 11 \\ & 0 & 0 & 0 & \\ & 0.1 & 0.1 & 0.1 & 0.1 \\ & 0 & 0 & 0 & \\ & 0 & 0 & 0 & \\ & 0 & 0 & 0 & \\ & 0.2 & 0.2 & 0.2 & 0.2 \\ & 0.2 & 0.2 & 0.2 & 0.2 \\ & 0 & 0 & 0 & \\ & 4.2 & 4.2 & 4.2 & 4.2 \\ & 4.2 & 4.2 & 4.2 & 4.2 \\ & 2.4 & 2.4 & 2.4 & 2.4 \\ & 0 & 0 & 0 & \\ & 2.4 & 2.4 & 2.4 & 2.4 \\ & 0 & 0 & 0 & \\ & 2.4 & 2.4 & 2.4 & 2.4 \\ & 0 & 0 & 0 & \\ & 31 & 31 & 31 & 31\end{array}$

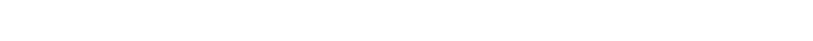

\begin{tabular}{rrrr}
2.4 & 2.4 & 2.4 & 2.4 \\
0.8 & 0.8 & 0.8 & 0.8 \\
0 & 0 & 0 & 0 \\
0.7 & 0.7 & 0.7 & 0.7 \\
0.2 & 0.2 & 0.2 & 0.2 \\
0 & 0 & 0 & 0 \\
11 & 11 & 11 & 11 \\
0 & 0 & 0 & \\
0 & 0 & 0 & \\
0 & 0 & 0 & \\
0 & 0 & 0 & \\
0 & 0 & 0 & 0 \\
0.2 & 0.2 & 0.2 & 0.2 \\
0.2 & 0.2 & 0.2 & 0.2 \\
0 & 0 & 0 & 0 \\
5.5 & 5.5 & 5.5 & 5.5 \\
5.5 & 5.5 & 5.5 & 5.5 \\
3.2 & 3.2 & 3.2 & 3.2 \\
0 & 0 & 0 & 0 \\
0 & 0 & 0 & 0 \\
0 & 0 & 0 & 0 \\
0 & 0 & 0 & 0 \\
0 & 0 & 0 & 0 \\
\hline
\end{tabular}

-Wanapum CER for Canada-

26 AVWP to BPA

27 COPD to BPA

28 CWPC to BPA

29 EWEB to BPA

$30 \mathrm{FGRV}$ to BPA

$31 \mathrm{FREC}$ to BPA

32 GCPD to BPA

33 ICLP to BPA

35 KOOT to BPA

36 LREC to BPA

37 LVE to BPA

$38 \mathrm{MCMN}$ to BPA

39 MTFR to BPA

40 NLEC to BPA

41 PGE to BPA

$42 \mathrm{PPL}$ to $\mathrm{BPA}$

44 RREC to BPA

$45 \mathrm{SCL}$ to BPA

46 SLEC to BPA

47 TPU to BPA

48 UNEC to BPA

49 Wanapum: Regional Totals

$\begin{array}{rrr}2.4 & 2.4 & 2.4 \\ 0.8 & 0.8 & 0.8 \\ 0 & 0 & 0 \\ 0.7 & 0.7 & 0.7 \\ 0.2 & 0.2 & 0.2 \\ 0 & 0 & 0 \\ 11 & 11 & 11 \\ 0 & 0 & 0 \\ 0 & 0 & 0 \\ 0 & 0 & 0 \\ 0 & 0 & 0 \\ 0 & 0 & 0 \\ 0.2 & 0.2 & 0.2 \\ 0.2 & 0.2 & 0.2 \\ 0 & 0 & 0 \\ 5.5 & 5.5 & 5.5 \\ 5.5 & 5.5 & 5.5 \\ 3.2 & 3.2 & 3.2\end{array}$

$\begin{array}{rrrrrrrr}2.4 & 2.4 & 2.3 & 2.3 & 2.3 & 2.3 & 2.3 & 2.4 \\ 0.8 & 0.8 & 0.8 & 0.8 & 0.8 & 0.8 & 0.8 & 0.8 \\ 0 & 0 & 0 & 0 & 0 & 0 & 0 & 0 \\ 0.7 & 0.7 & 0.6 & 0.6 & 0.6 & 0.6 & 0.6 & 0.7 \\ 0.2 & 0.2 & 0.2 & 0.2 & 0.2 & 0.2 & 0.2 & 0.2 \\ 0 & 0 & 0 & 0 & 0 & 0 & 0 & 0 \\ 11 & 11 & 10 & 10 & 10 & 10 & 10 & 11 \\ 0 & 0 & 0 & 0 & 0 & 0 & 0 & 0 \\ 0 & 0 & 0 & 0 & 0 & 0 & 0 & 0 \\ 0 & 0 & 0 & 0 & 0 & 0 & 0 & 0 \\ 0 & 0 & 0 & 0 & 0 & 0 & 0 & 0 \\ 0 & 0 & 0 & 0 & 0 & 0 & 0 & 0 \\ 0.2 & 0.2 & 0.2 & 0.2 & 0.2 & 0.2 & 0.2 & 0.2 \\ 0.2 & 0.2 & 0.2 & 0.2 & 0.2 & 0.2 & 0.2 & 0.2 \\ 0 & 0 & 0 & 0 & 0 & 0 & 0 & 0 \\ 5.5 & 5.5 & 5.2 & 5.2 & 5.2 & 5.2 & 5.2 & 5.4 \\ 5.5 & 5.5 & 5.2 & 5.2 & 5.2 & 5.2 & 5.2 & 5.4 \\ 3.2 & 3.2 & 3 & 3 & 3 & 3 & 3 & 3.1 \\ 0 & 0 & 0 & 0 & 0 & 0 & 0 & 0 \\ 0 & 0 & 0 & 0 & 0 & 0 & 0 & 0 \\ 0 & 0 & 0 & 0 & 0 & 0 & 0 & 0 \\ 0 & 0 & 0 & 0 & 0 & 0 & 0 & 0 \\ 0 & 0 & 0 & 0 & 0 & 0 & 0 & 0 \\ \mathbf{2 9} & \mathbf{2 9} & \mathbf{2 8} & \mathbf{2 8} & \mathbf{2 8} & \mathbf{2 8} & \mathbf{2 8} & \mathbf{2 8}\end{array}$

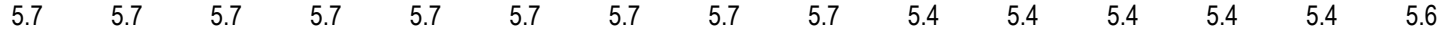

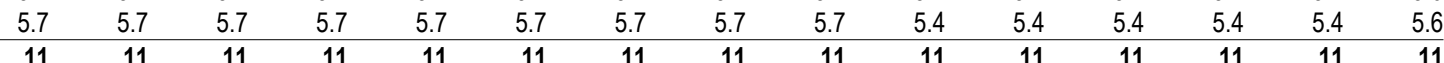

\begin{tabular}{|c|c|c|c|c|c|c|c|c|c|c|c|c|c|c|}
\hline 3.6 & 3.6 & 3.6 & 3.6 & 3.6 & 3.6 & 3.6 & 3.6 & 3.6 & 3.5 & 3.5 & 3.5 & 3.5 & 3.5 & 3.6 \\
\hline 3 & 3.6 & 3.6 & 3.6 & 3.6 & 3.6 & 3.6 & 3.6 & 3.6 & 3.5 & 3.5 & 3.5 & 3.5 & 3.5 & 3.6 \\
\hline 7.2 & 7.2 & 7.2 & 7.2 & 7.2 & 7.2 & 7.2 & 7.2 & 7.2 & 6.9 & 6.9 & 6.9 & 6.9 & 6.9 & 1 \\
\hline
\end{tabular}

-Rock Island P.H. \#1 CER for Canada-

$50 \mathrm{CHPD}$ to BPA

51 PSE to BPA

52 Rock Island P.H. \#1: Regional Totals

-Rock Island P.H. \#2 CER for Canada-

53 CHPD to BPA

54 PSE to BPA

55 Rock Island P.H. \#2: Regional Totals

-Rocky Reach CER for Canada-

56 AVWP to BPA

$57 \mathrm{CHPD}$ to BPA

58 CLKM to BPA

59 DOPD to BPA

60 PGE to BPA

$61 \mathrm{PPL}$ to BPA

62 PSE to BPA

63 Rocky Reach: Regional Totals 
Table A-15: Canadian Entitlement Return For Canada

PNW Loads and Resources Study

2005 - 2006 Operating Year

2003 White Book

Average Energy in Megawatts

Aug1 Aug1

Sep Oct Nov

Dec Jan Feb Mar Apr1 Apr16

May Jun Jul Avg

64 AVWP to BPA

65 DOPD to BPA

66 OKPD to BPA

67 PGE to BPA

$68 \mathrm{PPL}$ to BPA

69 PSE to BPA

70 Wells: Regional Totals

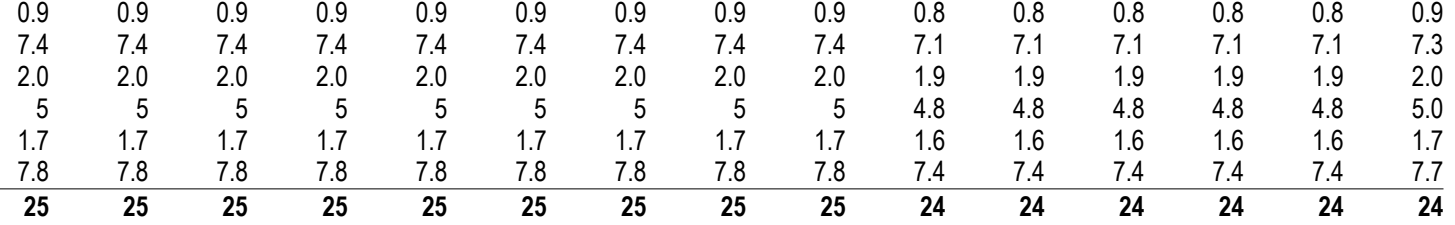

-Total CER For Canada-

71 Federal System

72 Public Entities

73 Investor-Owned Entities

74 Other Entities

75 Non-NW Entities

76 Total CER For Canada

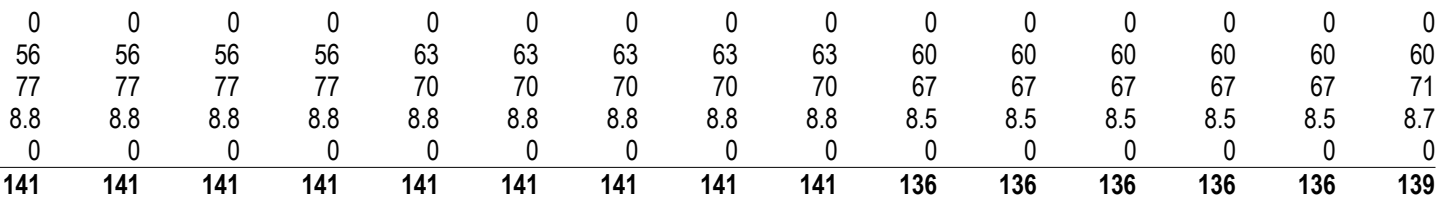



2003 White Book

Dec

Jan Feb Mar Apr1 Apr16

May

Jun Jul Avg

-BPA Canada-

1 BPA: Regional Totals

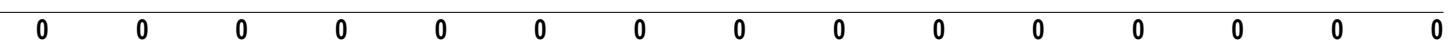

-Priest Rapids CER for Canada-

2 AVWP to BPA

3 COPD to BPA

4 CWPC to BPA

5 EWEB to BPA

6 FGRV to BPA

7 FREC to BPA

8 GCPD to BPA

9 ICLP to BPA

$10 \mathrm{KITT}$ to BPA

$11 \mathrm{KOOT}$ to BPA

12 LREC to BPA

13 LVE to BPA

14 MCMN to BPA

15 MTFR to BPA

16 NLEC to BPA

17 PGE to BPA

$18 \mathrm{PPL}$ to BPA

19 PSE to BPA

20 RREC to BPA

$21 \mathrm{SCL}$ to BPA

22 SLEC to BPA

23 TPU to BPA

24 UNEC to BPA

25 Priest Rapids: Regional Totals

$\begin{array}{rrrrrrrrrrrrrrr}0.8 & 0.8 & 0.8 & 0.8 & 0.8 & 0.8 & 0.8 & 0.8 & 0.8 & 0.8 & 0.8 & 0.8 & 0.8 & 0.8 & 0.8 \\ 0.3 & 0.3 & 0.3 & 0.3 & 0.3 & 0.3 & 0.3 & 0.3 & 0.3 & 0.3 & 0.3 & 0.3 & 0.3 & 0.3 & 0.3 \\ 0 & 0 & 0 & 0 & 0 & 0 & 0 & 0 & 0 & 0 & 0 & 0 & 0 & 0 & 0 \\ 0.2 & 0.2 & 0.2 & 0.2 & 0.2 & 0.2 & 0.2 & 0.2 & 0.2 & 0.2 & 0.2 & 0.2 & 0.2 & 0.2 & 0.2 \\ 0.1 & 0.1 & 0.1 & 0.1 & 0.1 & 0.1 & 0.1 & 0.1 & 0.1 & 0.1 & 0.1 & 0.1 & 0.1 & 0.1 & 0.1 \\ 0 & 0 & 0 & 0 & 0 & 0 & 0 & 0 & 0 & 0 & 0 & 0 & 0 & 0 & 0 \\ 21 & 21 & 21 & 21 & 21 & 21 & 21 & 21 & 21 & 20 & 20 & 20 & 20 & 20 & 20 \\ 0 & 0 & 0 & 0 & 0 & 0 & 0 & 0 & 0 & 0 & 0 & 0 & 0 & 0 & 0 \\ 0.1 & 0.1 & 0.1 & 0.1 & 0.1 & 0.1 & 0.1 & 0.1 & 0.1 & 0 & 0 & 0 & 0 & 0 & 0 \\ 0 & 0 & 0 & 0 & 0 & 0 & 0 & 0 & 0 & 0 & 0 & 0 & 0 & 0 & 0 \\ 0 & 0 & 0 & 0 & 0 & 0 & 0 & 0 & 0 & 0 & 0 & 0 & 0 & 0 & 0 \\ 0 & 0 & 0 & 0 & 0 & 0 & 0 & 0 & 0 & 0 & 0 & 0 & 0 & 0 & 0 \\ 0.1 & 0.1 & 0.1 & 0.1 & 0.1 & 0.1 & 0.1 & 0.1 & 0.1 & 0.1 & 0.1 & 0.1 & 0.1 & 0.1 & 0.1 \\ 0.1 & 0.1 & 0.1 & 0.1 & 0.1 & 0.1 & 0.1 & 0.1 & 0.1 & 0.1 & 0.1 & 0.1 & 0.1 & 0.1 & 0.1 \\ 0 & 0 & 0 & 0 & 0 & 0 & 0 & 0 & 0 & 0 & 0 & 0 & 0 & 0 & 0 \\ 1.9 & 1.9 & 1.9 & 1.9 & 1.9 & 1.9 & 1.9 & 1.9 & 1.9 & 1.9 & 1.9 & 1.9 & 1.9 & 1.9 & 1.9 \\ 1.9 & 1.9 & 1.9 & 1.9 & 1.9 & 1.9 & 1.9 & 1.9 & 1.9 & 1.9 & 1.9 & 1.9 & 1.9 & 1.9 & 1.9 \\ 1.1 & 1.1 & 1.1 & 1.1 & 1.1 & 1.1 & 1.1 & 1.1 & 1.1 & 1.1 & 1.1 & 1.1 & 1.1 & 1.1 & 1.1 \\ 0 & 0 & 0 & 0 & 0 & 0 & 0 & 0 & 0 & 0 & 0 & 0 & 0 & 0 & 0 \\ 1 & 1 & 1 & 1 & 1 & 1 & 1 & 1 & 1 & 1.0 & 1.0 & 1.0 & 1.0 & 1.0 & 1 \\ 0 & 0 & 0 & 0 & 0 & 0 & 0 & 0 & 0 & 0 & 0 & 0 & 0 & 0 & 0 \\ 1 & 1 & 1 & 1 & 1 & 1 & 1 & 1 & 1 & 1.0 & 1.0 & 1.0 & 1.0 & 1.0 & 1 \\ 0 & 0 & 0 & 0 & 0 & 0 & 0 & 0 & 0 & 0 & 0 & 0 & 0 & 0 & 0 \\ \mathbf{2 9} & \mathbf{2 9} & \mathbf{2 9} & \mathbf{2 9} & \mathbf{2 9} & \mathbf{2 9} & \mathbf{2 9} & \mathbf{2 9} & \mathbf{2 9} & \mathbf{2 9} & \mathbf{2 9} & \mathbf{2 8} & \mathbf{2 8} & \mathbf{2 8} & \mathbf{2 9}\end{array}$

-Wanapum CER for Canada-

26 AVWP to BPA

27 COPD to BPA

28 CWPC to BPA

29 EWEB to BPA

$30 \mathrm{FGRV}$ to BPA

31 FREC to BPA

32 GCPD to BPA

33 ICLP to BPA

$34 \mathrm{KITT}$ to BPA

$35 \mathrm{KOOT}$ to BPA

36 LREC to BPA

37 LVE to BPA

$38 \mathrm{MCMN}$ to BPA

39 MTFR to BPA

40 NLEC to BPA

41 PGE to BPA

$42 \mathrm{PPL}$ to BPA

43 PSE to BPA

44 RREC to BPA

$45 \mathrm{SCL}$ to BPA

46 SLEC to BPA

47 TPU to BPA

48 UNEC to BPA

49 Wanapum: Regional Totals

$\begin{array}{rrrrrrrrrrrrrrr}2.3 & 2.3 & 2.3 & 2.3 & 2.3 & 2.3 & 2.3 & 2.3 & 2.3 & 2.2 & 2.2 & 2.2 & 2.2 & 2.2 & 2.3 \\ 0.8 & 0.8 & 0.8 & 0.8 & 0.8 & 0.8 & 0.8 & 0.8 & 0.8 & 0.7 & 0.7 & 0.7 & 0.7 & 0.7 & 0.7 \\ 0 & 0 & 0 & 0 & 0 & 0 & 0 & 0 & 0 & 0 & 0 & 0 & 0 & 0 & 0 \\ 0.6 & 0.6 & 0.6 & 0.6 & 0.6 & 0.6 & 0.6 & 0.6 & 0.6 & 0.6 & 0.6 & 0.6 & 0.6 & 0.6 & 0.6 \\ 0.2 & 0.2 & 0.2 & 0.2 & 0.2 & 0.2 & 0.2 & 0.2 & 0.2 & 0.2 & 0.2 & 0.2 & 0.2 & 0.2 & 0.2 \\ 0 & 0 & 0 & 0 & 0 & 0 & 0 & 0 & 0 & 0 & 0 & 0 & 0 & 0 & 0 \\ 10 & 10 & 10 & 10 & 10 & 10 & 10 & 10 & 10 & 9.9 & 9.9 & 9.9 & 9.9 & 9.9 & 10 \\ 0 & 0 & 0 & 0 & 0 & 0 & 0 & 0 & 0 & 0 & 0 & 0 & 0 & 0 & 0 \\ 0 & 0 & 0 & 0 & 0 & 0 & 0 & 0 & 0 & 0 & 0 & 0 & 0 & 0 & 0 \\ 0 & 0 & 0 & 0 & 0 & 0 & 0 & 0 & 0 & 0 & 0 & 0 & 0 & 0 & 0 \\ 0 & 0 & 0 & 0 & 0 & 0 & 0 & 0 & 0 & 0 & 0 & 0 & 0 & 0 & 0 \\ 0 & 0 & 0 & 0 & 0 & 0 & 0 & 0 & 0 & 0 & 0 & 0 & 0 & 0 & 0 \\ 0.2 & 0.2 & 0.2 & 0.2 & 0.2 & 0.2 & 0.2 & 0.2 & 0.2 & 0.2 & 0.2 & 0.2 & 0.2 & 0.2 & 0.2 \\ 0.2 & 0.2 & 0.2 & 0.2 & 0.2 & 0.2 & 0.2 & 0.2 & 0.2 & 0.2 & 0.2 & 0.2 & 0.2 & 0.2 & 0.2 \\ 0 & 0 & 0 & 0 & 0 & 0 & 0 & 0 & 0 & 0 & 0 & 0 & 0 & 0 & 0 \\ 5.2 & 5.2 & 5.2 & 5.2 & 5.2 & 5.2 & 5.2 & 5.2 & 5.2 & 5.1 & 5.1 & 5.1 & 5.1 & 5.1 & 5.2 \\ 5.2 & 5.2 & 5.2 & 5.2 & 5.2 & 5.2 & 5.2 & 5.2 & 5.2 & 5.1 & 5.1 & 5.1 & 5.1 & 5.1 & 5.2 \\ 3 & 3 & 3 & 3 & 3 & 3 & 3 & 3 & 3 & 2.9 & 2.9 & 2.9 & 2.9 & 2.9 & 3.0 \\ 0 & 0 & 0 & 0 & 0 & 0 & 0 & 0 & 0 & 0 & 0 & 0 & 0 & 0 & 0 \\ 0 & 0 & 0 & 0 & 0 & 0 & 0 & 0 & 0 & 0 & 0 & 0 & 0 & 0 & 0 \\ 0 & 0 & 0 & 0 & 0 & 0 & 0 & 0 & 0 & 0 & 0 & 0 & 0 & 0 & 0 \\ 0 & 0 & 0 & 0 & 0 & 0 & 0 & 0 & 0 & 0 & 0 & 0 & 0 & 0 & 0 \\ 0 & 0 & 0 & 0 & 0 & 0 & 0 & 0 & 0 & 0 & 0 & 0 & 0 & 0 & 0 \\ \mathbf{2 8} & \mathbf{2 8} & \mathbf{2 8} & \mathbf{2 8} & \mathbf{2 8} & \mathbf{2 8} & \mathbf{2 8} & \mathbf{2 8} & \mathbf{2 8} & \mathbf{2 8} & \mathbf{2 7} & \mathbf{2 7} & \mathbf{2 7} & \mathbf{2 7} & \mathbf{2 7}\end{array}$

-Rock Island P.H. \#1 CER for Canada-

$50 \mathrm{CHPD}$ to BPA

51 PSE to BPA

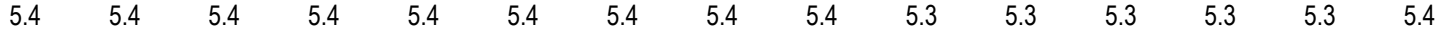

52 Rock Island P.H. \#1: Regional Totals

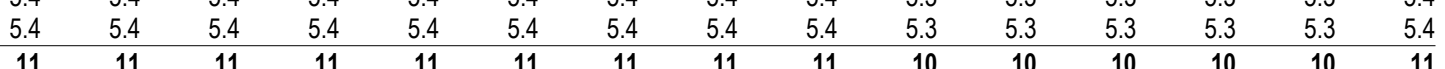

-Rock Island P.H. \#2 CER for Canada-

53 CHPD to BPA

54 PSE to BPA

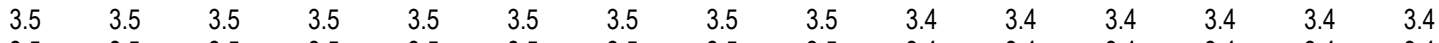

55 Rock Island P.H. \#2: Regional Totals

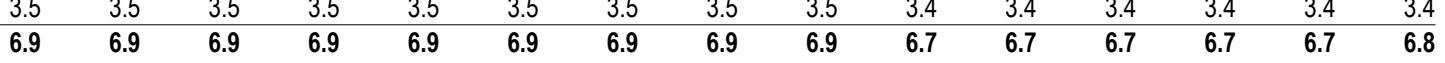

-Rocky Reach CER for Canada-

56 AVWP to BPA

$57 \mathrm{CHPD}$ to BPA

58 CLKM to BPA

59 DOPD to BPA

60 PGE to BPA

$61 \mathrm{PPL}$ to BPA

62 PSE to BPA

\begin{tabular}{|c|c|c|c|c|c|c|c|c|c|c|c|c|c|}
\hline 1.1 & 1.1 & 1.1 & 1.1 & 1.1 & 1.1 & 1.1 & 1.1 & 1 & 1 & 1 & 1 & 1 & 1.1 \\
\hline 5.6 & 5.6 & 5.6 & 5.6 & 5.6 & 5.6 & 5.6 & 5.6 & 5.4 & 5.4 & 5.4 & 5.4 & 5.4 & 5.5 \\
\hline 8.5 & 8.5 & 8.5 & 8.5 & 8.5 & 8.5 & 8.5 & 8.5 & 8.2 & 8.2 & 8.2 & 8.2 & 8.2 & 8.4 \\
\hline 1.0 & 1.0 & 1.0 & 1.0 & 1.0 & 1.0 & 1.0 & 1.0 & 1.0 & 1.0 & 1.0 & 1.0 & 1.0 & 1.0 \\
\hline 4.5 & 4.5 & 4.5 & 4.5 & 4.5 & 4.5 & 4.5 & 4.5 & 4.3 & 4.3 & 4.3 & 4.3 & 4.3 & 4.4 \\
\hline 2.0 & 2.0 & 2.0 & 2.0 & 2.0 & 2.0 & 2.0 & 2.0 & 1.9 & 1.9 & 1.9 & 1.9 & 1.9 & 1.9 \\
\hline 14 & 14 & 14 & 14 & 14 & 14 & 14 & 14 & 14 & 14 & 14 & 14 & 14 & 14 \\
\hline 37 & 37 & 37 & 37 & 37 & 37 & 37 & 37 & 36 & 36 & 36 & 36 & 36 & 36 \\
\hline
\end{tabular}


Table A-15: Canadian Entitlement Return For Canada

PNW Loads and Resources Study

2006 - 2007 Operating Year

2003 White Book

Jul Avg

Average Energy in Megawatts

Aug1 Aug16

Sep Oct Nov Dec Jan Feb Mar Apr1 Apr16

May Jun

64 AVWP to BPA

65 DOPD to BPA

66 OKPD to BPA

$\begin{array}{llllllllll}0.8 & 0.8 & 0.8 & 0.8 & 0.8 & 0.8 & 0.8 & 0.8 & 0.8 & 0.8\end{array}$

67 PGE to BPA

$68 \mathrm{PPL}$ to BPA

69 PSE to BPA

70 Wells: Regional Totals

$\begin{array}{lll}7.1 & 7.1 & 7.1\end{array}$

$\begin{array}{ll}0.8 & 0.8 \\ 1.1 & 7.1\end{array}$

7.1

7.1

0.8
7.1

7.1

6.9

0.8
6.9

0.8

0.8

6.9

6.9

0.8

6.9

0.8

$\begin{array}{lll}.9 & 1.9 & 1.9 \\ 4.8 & 4.8 & 4.8\end{array}$

1.6

$1.6 \quad 1.6$

$4.8 \quad 4.8$

4.8

4.8

4.8

4.8

4.8

4.8
1.6

4.7

1.8

4.7

1.8
4.7

1.8

4.7

1.8
4.7

\begin{tabular}{ll}
7.4 & 7.4 \\
\hline 24 & 24
\end{tabular}

\begin{tabular}{rr}
7.4 & 7.4 \\
\hline 24 & 24
\end{tabular}

7.4
24

$1.6 \quad 1.6$

-Total CER For Canada-

71 Federal System

72 Public Entities

73 Investor-Owned Entities

74 Other Entities

75 Non-NW Entities

76 Total CER For Canada

\begin{tabular}{|c|c|c|c|c|c|c|c|c|c|c|c|c|c|c|}
\hline 0 & 0 & 0 & 0 & 0 & 0 & 0 & 0 & 0 & 0 & 0 & 0 & 0 & 0 & 0 \\
\hline 60 & 60 & 60 & 60 & 60 & 60 & 60 & 60 & 60 & 58 & 58 & 58 & 58 & 58 & 60 \\
\hline 67 & 67 & 67 & 67 & 67 & 67 & 67 & 67 & 67 & 65 & 65 & 65 & 65 & 65 & 66 \\
\hline 8.5 & 8.5 & 8.5 & 8.5 & 8.5 & 8.5 & 8.5 & 8.5 & 8.5 & 8.2 & 8.2 & 8.2 & 8.2 & 8.2 & 8.4 \\
\hline 136 & 136 & 136 & 136 & 136 & 136 & 136 & 136 & 136 & 132 & 132 & 132 & 132 & 132 & 134 \\
\hline
\end{tabular}



2003 White Book

Dec

Jan Feb Mar Apr1 Apr16

May

Jun Jul Avg

-BPA Canada-

1 BPA: Regional Totals

$\begin{array}{llllllllllllll}0 & 0 & 0 & 0 & 0 & 0 & 0 & 0 & 0 & 0 & 0 & 0 & 0 & 0\end{array}$

-Priest Rapids CER for Canada-

2 AVWP to BPA

3 COPD to BPA

4 CWPC to BPA

5 EWEB to BPA

6 FGRV to BPA

7 FREC to BPA

8 GCPD to BPA

9 ICLP to BPA

$10 \mathrm{KITT}$ to BPA

$11 \mathrm{KOOT}$ to BPA

12 LREC to BPA

13 LVE to BPA

14 MCMN to BPA

15 MTFR to BPA

16 NLEC to BPA

17 PGE to BPA

18 PPL to BPA

19 PSE to BPA

20 RREC to BPA

$21 \mathrm{SCL}$ to BPA

22 SLEC to BPA

23 TPU to BPA

24 UNEC to BPA

\begin{tabular}{|c|c|c|c|c|c|c|c|c|c|c|c|c|c|}
\hline 0.8 & 0.8 & 0.8 & 0.8 & 0.8 & 0.8 & 0.8 & 0.8 & 0.8 & 0.8 & 0.8 & 0.8 & 0.8 & 0.8 \\
\hline 0.3 & 0.3 & 0.3 & 0.3 & 0.3 & 0.3 & 0.3 & 0.3 & 0.3 & 0.3 & 0.3 & 0.3 & 0.3 & 0.3 \\
\hline 0 & 0 & 0 & 0 & 0 & 0 & 0 & 0 & 0 & 0 & 0 & 0 & 0 & 0 \\
\hline 0.2 & 0.2 & 0.2 & 0.2 & 0.2 & 0.2 & 0.2 & 0.2 & 0.2 & 0.2 & 0.2 & 0.2 & 0.2 & 0.2 \\
\hline 0.1 & 0.1 & 0.1 & 0.1 & 0.1 & 0.1 & 0.1 & 0.1 & 0.1 & 0.1 & 0.1 & 0.1 & 0.1 & 0.1 \\
\hline 0 & 0 & 0 & 0 & 0 & 0 & 0 & 0 & 0 & 0 & 0 & 0 & 0 & 0 \\
\hline 20 & 20 & 20 & 20 & 20 & 20 & 20 & 20 & 20 & 20 & 20 & 20 & 20 & 20 \\
\hline 0 & 0 & 0 & 0 & 0 & 0 & 0 & 0 & 0 & 0 & 0 & 0 & 0 & 0 \\
\hline 0 & 0 & 0 & 0 & 0 & 0 & 0 & 0 & 0 & 0 & 0 & 0 & 0 & 0 \\
\hline 0 & 0 & 0 & 0 & 0 & 0 & 0 & 0 & 0 & 0 & 0 & 0 & 0 & 0 \\
\hline 0 & 0 & 0 & 0 & 0 & 0 & 0 & 0 & 0 & 0 & 0 & 0 & 0 & 0 \\
\hline 0 & 0 & 0 & 0 & 0 & 0 & 0 & 0 & 0 & 0 & 0 & 0 & 0 & 0 \\
\hline 0.1 & 0.1 & 0.1 & 0.1 & 0.1 & 0.1 & 0.1 & 0.1 & 0.1 & 0.1 & 0.1 & 0.1 & 0.1 & 0.1 \\
\hline 0.1 & 0.1 & 0.1 & 0.1 & 0.1 & 0.1 & 0.1 & 0.1 & 0.1 & 0.1 & 0.1 & 0.1 & 0.1 & 0.1 \\
\hline 0 & 0 & 0 & 0 & 0 & 0 & 0 & 0 & 0 & 0 & 0 & 0 & 0 & 0 \\
\hline 1.9 & 1.9 & 1.9 & 1.9 & 1.9 & 1.9 & 1.9 & 1.9 & 1.8 & 1.8 & 1.8 & 1.8 & 1.8 & 1.9 \\
\hline 1.9 & 1.9 & 1.9 & 1.9 & 1.9 & 1.9 & 1.9 & 1.9 & 1.8 & 1.8 & 1.8 & 1.8 & 1.8 & 1.9 \\
\hline 1.1 & 1.1 & 1.1 & 1.1 & 1.1 & 1.1 & 1.1 & 1.1 & 1.1 & 1.1 & 1.1 & 1.1 & 1.1 & 1.1 \\
\hline 0 & 0 & 0 & 0 & 0 & 0 & 0 & 0 & 0 & 0 & 0 & 0 & 0 & 0 \\
\hline 1.0 & 1.0 & 1.0 & 1.0 & 1.0 & 1.0 & 1.0 & 1.0 & 1.0 & 1.0 & 1.0 & 1.0 & 1.0 & 1.0 \\
\hline 0 & 0 & 0 & 0 & 0 & 0 & 0 & 0 & 0 & 0 & 0 & 0 & 0 & 0 \\
\hline 1.0 & 1.0 & 1.0 & 1.0 & 1.0 & 1.0 & 1.0 & 1.0 & 1.0 & 1.0 & 1.0 & 1.0 & 1.0 & 1.0 \\
\hline 0 & 0 & 0 & 0 & 0 & 0 & 0 & 0 & 0 & 0 & 0 & 0 & 0 & 0 \\
\hline 28 & 28 & 28 & 28 & 28 & 28 & 28 & 28 & 28 & 28 & 28 & 28 & 28 & 28 \\
\hline
\end{tabular}

-Wanapum CER for Canada-

26 AVWP to BPA

27 COPD to BPA

28 CWPC to BPA

29 EWEB to BPA

$30 \mathrm{FGRV}$ to BPA

31 FREC to BPA

32 GCPD to BPA

$33 \mathrm{ICLP}$ to BPA

$34 \mathrm{KITT}$ to BPA

$35 \mathrm{KOOT}$ to BPA

36 LREC to BPA

37 LVE to BPA

$38 \mathrm{MCMN}$ to BPA

39 MTFR to BPA

40 NLEC to BPA

41 PGE to BPA

$42 \mathrm{PPL}$ to BPA

43 PSE to BPA

44 RREC to BPA

$45 \mathrm{SCL}$ to BPA

46 SLEC to BPA

47 TPU to BPA

48 UNEC to BPA

49 Wanapum: Regional Totals

$\begin{array}{rrrrrrrrrrrrrrr}2.2 & 2.2 & 2.2 & 2.2 & 2.2 & 2.2 & 2.2 & 2.2 & 2.2 & 2.2 & 2.2 & 2.2 & 2.2 & 2.2 & 2.2 \\ 0.7 & 0.7 & 0.7 & 0.7 & 0.7 & 0.7 & 0.7 & 0.7 & 0.7 & 0.7 & 0.7 & 0.7 & 0.7 & 0.7 & 0.7 \\ 0 & 0 & 0 & 0 & 0 & 0 & 0 & 0 & 0 & 0 & 0 & 0 & 0 & 0 & 0 \\ 0.6 & 0.6 & 0.6 & 0.6 & 0.6 & 0.6 & 0.6 & 0.6 & 0.6 & 0.6 & 0.6 & 0.6 & 0.6 & 0.6 & 0.6 \\ 0.2 & 0.2 & 0.2 & 0.2 & 0.2 & 0.2 & 0.2 & 0.2 & 0.2 & 0.2 & 0.2 & 0.2 & 0.2 & 0.2 & 0.2 \\ 0 & 0 & 0 & 0 & 0 & 0 & 0 & 0 & 0 & 0 & 0 & 0 & 0 & 0 & 0 \\ 9.9 & 9.9 & 9.9 & 9.9 & 9.9 & 9.9 & 9.9 & 9.9 & 9.9 & 9.8 & 9.8 & 9.8 & 9.8 & 9.8 & 9.9 \\ 0 & 0 & 0 & 0 & 0 & 0 & 0 & 0 & 0 & 0 & 0 & 0 & 0 & 0 & 0 \\ 0 & 0 & 0 & 0 & 0 & 0 & 0 & 0 & 0 & 0 & 0 & 0 & 0 & 0 & 0 \\ 0 & 0 & 0 & 0 & 0 & 0 & 0 & 0 & 0 & 0 & 0 & 0 & 0 & 0 & 0 \\ 0 & 0 & 0 & 0 & 0 & 0 & 0 & 0 & 0 & 0 & 0 & 0 & 0 & 0 & 0 \\ 0 & 0 & 0 & 0 & 0 & 0 & 0 & 0 & 0 & 0 & 0 & 0 & 0 & 0 & 0 \\ 0.2 & 0.2 & 0.2 & 0.2 & 0.2 & 0.2 & 0.2 & 0.2 & 0.2 & 0.2 & 0.2 & 0.2 & 0.2 & 0.2 & 0.2 \\ 0.2 & 0.2 & 0.2 & 0.2 & 0.2 & 0.2 & 0.2 & 0.2 & 0.2 & 0.2 & 0.2 & 0.2 & 0.2 & 0.2 & 0.2 \\ 0 & 0 & 0 & 0 & 0 & 0 & 0 & 0 & 0 & 0 & 0 & 0 & 0 & 0 & 0 \\ 5.1 & 5.1 & 5.1 & 5.1 & 5.1 & 5.1 & 5.1 & 5.1 & 5.1 & 5 & 5 & 5 & 5 & 5 & 5.1 \\ 5.1 & 5.1 & 5.1 & 5.1 & 5.1 & 5.1 & 5.1 & 5.1 & 5.1 & 5 & 5 & 5 & 5 & 5 & 5.1 \\ 2.9 & 2.9 & 2.9 & 2.9 & 2.9 & 2.9 & 2.9 & 2.9 & 2.9 & 2.9 & 2.9 & 2.9 & 2.9 & 2.9 & 2.9 \\ 0 & 0 & 0 & 0 & 0 & 0 & 0 & 0 & 0 & 0 & 0 & 0 & 0 & 0 & 0 \\ 0 & 0 & 0 & 0 & 0 & 0 & 0 & 0 & 0 & 0 & 0 & 0 & 0 & 0 & 0 \\ 0 & 0 & 0 & 0 & 0 & 0 & 0 & 0 & 0 & 0 & 0 & 0 & 0 & 0 & 0 \\ 0 & 0 & 0 & 0 & 0 & 0 & 0 & 0 & 0 & 0 & 0 & 0 & 0 & 0 & 0 \\ 0 & 0 & 0 & 0 & 0 & 0 & 0 & 0 & 0 & 0 & 0 & 0 & 0 & 0 & 0 \\ \mathbf{2 7} & \mathbf{2 7} & \mathbf{2 7} & \mathbf{2 7} & \mathbf{2 7} & \mathbf{2 7} & \mathbf{2 7} & \mathbf{2 7} & \mathbf{2 7} & \mathbf{2 7} & \mathbf{2 7} & \mathbf{2 7} & \mathbf{2 7} & \mathbf{2 7} & \mathbf{2 7}\end{array}$

-Rock Island P.H. \#1 CER for Canada-

$50 \mathrm{CHPD}$ to BPA

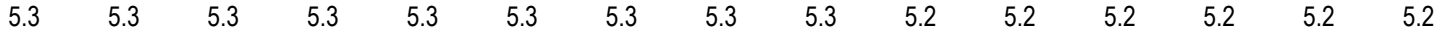

51 PSE to BPA

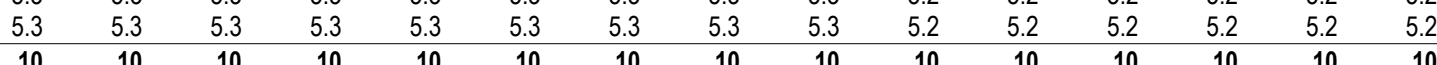

-Rock Island P.H. \#2 CER for Canada-

53 CHPD to BPA

54 PSE to BPA

55 Rock Island P.H. \#2: Regional Totals

$\begin{array}{lllllllllllllll}3.4 & 3.4 & 3.4 & 3.4 & 3.4 & 3.4 & 3.4 & 3.4 & 3.4 & 3.3 & 3.3 & 3.3 & 3.3 & 3.3 & 3.3 \\ 3.4 & 3.4 & 3.4 & 3.4 & 3.4 & 3.4 & 3.4 & 3.4 & 3.4 & 3.3 & 3.3 & 3.3 & 3.3 & 3.3 & 3.3\end{array}$

-Rocky Reach CER for Canada-

56 AVWP to BPA

57 CHPD to BPA

58 CLKM to BPA

59 DOPD to BPA

60 PGE to BPA

$61 \mathrm{PPL}$ to BPA

62 PSE to BPA

$\begin{array}{llll}6.7 & 6.7 & 6.7 & 6.7\end{array}$

63 Rocky Reach: Regional Totals

\begin{tabular}{|c|c|c|c|c|c|c|c|c|c|c|c|c|c|c|}
\hline 1 & 1 & 1 & 1 & 1 & 1 & 1 & 1 & 1 & 1 & 1 & 1 & 1 & 1 & 1 \\
\hline 5.4 & 5.4 & 5.4 & 5.4 & 5.4 & 5.4 & 5.4 & 5.4 & 5.4 & 5.3 & 5.3 & 5.3 & 5.3 & 5.3 & 5.4 \\
\hline 8.2 & 8.2 & 8.2 & 8.2 & 8.2 & 8.2 & 8.2 & 8.2 & 8.2 & 8.1 & 8.1 & 8.1 & 8.1 & 8.1 & 8.2 \\
\hline 1.0 & 1.0 & 1.0 & 1.0 & 1.0 & 1.0 & 1.0 & 1.0 & 1.0 & 1.0 & 1.0 & 1.0 & 1.0 & 1.0 & 1.0 \\
\hline 4.3 & 4.3 & 4.3 & 4.3 & 4.3 & 4.3 & 4.3 & 4.3 & 4.3 & 4.3 & 4.3 & 4.3 & 4.3 & 4.3 & 4.3 \\
\hline 1.9 & 1.9 & 1.9 & 1.9 & 1.9 & 1.9 & 1.9 & 1.9 & 1.9 & 1.9 & 1.9 & 1.9 & 1.9 & 1.9 & 1.9 \\
\hline 14 & 14 & 14 & 14 & 14 & 14 & 14 & 14 & 14 & 14 & 14 & 14 & 14 & 14 & 14 \\
\hline 36 & 36 & 36 & 36 & 36 & 36 & 36 & 36 & 36 & 35 & 35 & 35 & 35 & 35 & 36 \\
\hline
\end{tabular}


Table A-15: Canadian Entitlement Return For Canada

PNW Loads and Resources Study

2007 - 2008 Operating Year

2003 White Book

Average Energy in Megawatts Aug1 Aug16 Sep Oct Nov Dec Jan Feb Mar Apr1 Apr16 May Jun Jul Avg

64 AVWP to BPA

65 DOPD to BPA

66 OKPD to BPA

67 PGE to BPA

$68 \mathrm{PPL}$ to $\mathrm{BPA}$

69 PSE to BPA

70 Wells: Regional Totals

$0.8=0.8$

$\begin{array}{lll}0.8 & 0.8 & 0.8 \\ 6.9 & 6.9 & 6.9 \\ 1.8 & 1.8 & 1.8 \\ 4.7 & 4.7 & 4.7 \\ 1.6 & 1.6 & 1.6 \\ 7.2 & 7.2 & 7.2 \\ \mathbf{2 3} & \mathbf{2 3} & 23\end{array}$

\begin{tabular}{cc}
0.8 & 0.8 \\
6.9 & 6.9 \\
1.8 & 1.8 \\
4.7 & 4.7 \\
1.6 & 1.6 \\
7.2 & 7.2 \\
\hline 23 & 23
\end{tabular}

$\begin{array}{rrr} & & \\ 0.8 & 0.8 & 0.8 \\ 6.9 & 6.9 & 6.9 \\ 1.8 & 1.8 & 1.8 \\ 4.7 & 4.7 & 4.7 \\ 1.6 & 1.6 & 1.6 \\ 7.2 & 7.2 & 7.2 \\ \mathbf{2 3} & \mathbf{2 3} & \mathbf{2 3}\end{array}$

\section{-Total CER For Canada-}

71 Federal System

72 Public Entities

73 Investor-Owned Entities

74 Other Entities

75 Non-NW Entities

76 Total CER For Canada

\begin{tabular}{|c|c|c|c|c|c|c|c|c|c|c|c|c|c|}
\hline 0 & 0 & 0 & 0 & 0 & 0 & 0 & 0 & 0 & 0 & 0 & 0 & 0 & 0 \\
\hline 58 & 58 & 58 & 58 & 58 & 58 & 58 & 58 & 58 & 58 & 58 & 58 & 58 & 58 \\
\hline 65 & 65 & 65 & 65 & 65 & 65 & 65 & 65 & 64 & 64 & 64 & 64 & 64 & 65 \\
\hline 8.2 & 8.2 & 8.2 & 8.2 & 8.2 & 8.2 & 8.2 & 8.2 & 8.1 & 8.1 & 8.1 & 8.1 & 8.1 & 8.2 \\
\hline 0 & 0 & 0 & 0 & 0 & 0 & 0 & 0 & 0 & 0 & 0 & 0 & 0 & 0 \\
\hline 132 & 132 & 132 & 132 & 132 & 132 & 132 & 132 & 130 & 130 & 130 & 130 & 130 & 131 \\
\hline
\end{tabular}




\begin{tabular}{|c|c|c|c|c|c|c|c|c|c|c|c|c|c|c|}
\hline 0 & 0 & 0 & 0 & 0 & 0 & 0 & 0 & 0 & 0 & 0 & 0 & 0 & 0 & 0 \\
\hline 0.8 & 0.8 & 0.8 & 0.8 & 0.8 & 0.8 & 0.8 & 0.8 & 0.8 & 0.8 & 0.8 & 0.8 & 0.8 & 0.8 & 0.8 \\
\hline 0.3 & 0.3 & 0.3 & 0.3 & 0.3 & 0.3 & 0.3 & 0.3 & 0.3 & 0.3 & 0.3 & 0.3 & 0.3 & 0.3 & 0.3 \\
\hline 0 & 0 & 0 & 0 & 0 & 0 & 0 & 0 & 0 & 0 & 0 & 0 & 0 & 0 & 0 \\
\hline 0.2 & 0.2 & 0.2 & 0.2 & 0.2 & 0.2 & 0.2 & 0.2 & 0.2 & 0.2 & 0.2 & 0.2 & 0.2 & 0.2 & 0.2 \\
\hline 0.1 & 0.1 & 0.1 & 0.1 & 0.1 & 0.1 & 0.1 & 0.1 & 0.1 & 0.1 & 0.1 & 0.1 & 0.1 & 0.1 & 0.1 \\
\hline 0 & 0 & 0 & 0 & 0 & 0 & 0 & 0 & 0 & 0 & 0 & 0 & 0 & 0 & 0 \\
\hline 20 & 20 & 20 & 20 & 20 & 20 & 20 & 20 & 20 & 20 & 20 & 20 & 20 & 20 & 20 \\
\hline 0 & 0 & 0 & 0 & 0 & 0 & 0 & 0 & 0 & 0 & 0 & 0 & 0 & 0 & 0 \\
\hline 0 & 0 & 0 & 0 & 0 & 0 & 0 & 0 & 0 & 0 & 0 & 0 & 0 & 0 & 0 \\
\hline 0 & 0 & 0 & 0 & 0 & 0 & 0 & 0 & 0 & 0 & 0 & 0 & 0 & 0 & 0 \\
\hline 0 & 0 & 0 & 0 & 0 & 0 & 0 & 0 & 0 & 0 & 0 & 0 & 0 & 0 & 0 \\
\hline 0 & 0 & 0 & 0 & 0 & 0 & 0 & 0 & 0 & 0 & 0 & 0 & 0 & 0 & 0 \\
\hline 0.1 & 0.1 & 0.1 & 0.1 & 0.1 & 0.1 & 0.1 & 0.1 & 0.1 & 0.1 & 0.1 & 0.1 & 0.1 & 0.1 & 0.1 \\
\hline 0.1 & 0.1 & 0.1 & 0.1 & 0.1 & 0.1 & 0.1 & 0.1 & 0.1 & 0.1 & 0.1 & 0.1 & 0.1 & 0.1 & 0.1 \\
\hline 0 & 0 & 0 & 0 & 0 & 0 & 0 & 0 & 0 & 0 & 0 & 0 & 0 & 0 & 0 \\
\hline 1.8 & 1.8 & 1.8 & 1.8 & 1.8 & 1.8 & 1.8 & 1.8 & 1.8 & 1.8 & 1.8 & 1.8 & 1.8 & 1.8 & 1.8 \\
\hline 1.8 & 1.8 & 1.8 & 1.8 & 1.8 & 1.8 & 1.8 & 1.8 & 1.8 & 1.8 & 1.8 & 1.8 & 1.8 & 1.8 & 1.8 \\
\hline 1.1 & 1.1 & 1.1 & 1.1 & 1.1 & 1.1 & 1.1 & 1.1 & 1.1 & 1.1 & 1.1 & 1.1 & 1.1 & 1.1 & 1.1 \\
\hline 0 & 0 & 0 & 0 & 0 & 0 & 0 & 0 & 0 & 0 & 0 & 0 & 0 & 0 & 0 \\
\hline 1.0 & 1.0 & 1.0 & 1.0 & 1.0 & 1.0 & 1.0 & 1.0 & 1.0 & 1.0 & 1.0 & 1.0 & 1.0 & 1.0 & 1.0 \\
\hline 0 & 0 & 0 & 0 & 0 & 0 & 0 & 0 & 0 & 0 & 0 & 0 & 0 & 0 & 0 \\
\hline 1.0 & 1.0 & 1.0 & 1.0 & 1.0 & 1.0 & 1.0 & 1.0 & 1.0 & 1.0 & 1.0 & 1.0 & 1.0 & 1.0 & 1.0 \\
\hline 0 & 0 & 0 & 0 & 0 & 0 & 0 & 0 & 0 & 0 & 0 & 0 & 0 & 0 & 0 \\
\hline 28 & 28 & 28 & 28 & 28 & 28 & 28 & 28 & 28 & 28 & 28 & 28 & 28 & 28 & 28 \\
\hline
\end{tabular}

-Priest Rapids CER for Canada-

2 AVWP to BPA

3 COPD to BPA

4 CWPC to BPA

5 EWEB to BPA

6 FGRV to BPA

7 FREC to BPA

8 GCPD to BPA

9 ICLP to BPA

$10 \mathrm{KITT}$ to BPA

$11 \mathrm{KOOT}$ to BPA

12 LREC to BPA

13 LVE to BPA

14 MCMN to BPA

15 MTFR to BPA

16 NLEC to BPA

17 PGE to BPA

$18 \mathrm{PPL}$ to BPA

19 PSE to BPA

20 RREC to BPA

$21 \mathrm{SCL}$ to BPA

22 SLEC to BPA

23 TPU to BPA

24 UNEC to BPA

25 Priest Rapids: Regional Totals

-Wanapum CER for Canada-

26 AVWP to BPA

27 COPD to BPA

28 CWPC to BPA

29 EWEB to BPA

$30 \mathrm{FGRV}$ to BPA

31 FREC to BPA

32 GCPD to BPA

$33 \mathrm{ICLP}$ to BPA

$34 \mathrm{KITT}$ to BPA

$35 \mathrm{KOOT}$ to BPA

36 LREC to BPA

37 LVE to BPA

$38 \mathrm{MCMN}$ to BPA

39 MTFR to BPA

40 NLEC to BPA

41 PGE to BPA

$42 \mathrm{PPL}$ to $\mathrm{BPA}$

43 PSE to BPA

44 RREC to BPA

$45 \mathrm{SCL}$ to BPA

46 SLEC to BPA

47 TPU to BPA

48 UNEC to BPA

49 Wanapum: Regional Totals

\begin{tabular}{|c|c|c|c|c|c|c|c|c|c|c|c|c|c|c|}
\hline 2.2 & 2.2 & 2.2 & 2.2 & 2.2 & 2.2 & 2.2 & 2.2 & 2.2 & 2.2 & 2.2 & 2.2 & 2.2 & 2.2 & 2.2 \\
\hline 0.7 & 0.7 & 0.7 & 0.7 & 0.7 & 0.7 & 0.7 & 0.7 & 0.7 & 0.7 & 0.7 & 0.7 & 0.7 & 0.7 & 0.7 \\
\hline 0 & 0 & 0 & 0 & 0 & 0 & 0 & 0 & 0 & 0 & 0 & 0 & 0 & 0 & 0 \\
\hline 0.6 & 0.6 & 0.6 & 0.6 & 0.6 & 0.6 & 0.6 & 0.6 & 0.6 & 0.6 & 0.6 & 0.6 & 0.6 & 0.6 & 0.6 \\
\hline 0.2 & 0.2 & 0.2 & 0.2 & 0.2 & 0.2 & 0.2 & 0.2 & 0.2 & 0.2 & 0.2 & 0.2 & 0.2 & 0.2 & 0.2 \\
\hline 0 & 0 & 0 & 0 & 0 & 0 & 0 & 0 & 0 & 0 & 0 & 0 & 0 & 0 & 0 \\
\hline 9.8 & 9.8 & 9.8 & 9.8 & 9.8 & 9.8 & 9.8 & 9.8 & 9.8 & 9.7 & 9.7 & 9.7 & 9.7 & 9.7 & 9.8 \\
\hline 0 & 0 & 0 & 0 & 0 & 0 & 0 & 0 & 0 & 0 & 0 & 0 & 0 & 0 & 0 \\
\hline 0 & 0 & 0 & 0 & 0 & 0 & 0 & 0 & 0 & 0 & 0 & 0 & 0 & 0 & 0 \\
\hline 0 & 0 & 0 & 0 & 0 & 0 & 0 & 0 & 0 & 0 & 0 & 0 & 0 & 0 & 0 \\
\hline 0 & 0 & 0 & 0 & 0 & 0 & 0 & 0 & 0 & 0 & 0 & 0 & 0 & 0 & 0 \\
\hline 0 & 0 & 0 & 0 & 0 & 0 & 0 & 0 & 0 & 0 & 0 & 0 & 0 & 0 & 0 \\
\hline 0.2 & 0.2 & 0.2 & 0.2 & 0.2 & 0.2 & 0.2 & 0.2 & 0.2 & 0.2 & 0.2 & 0.2 & 0.2 & 0.2 & 0.2 \\
\hline 0.2 & 0.2 & 0.2 & 0.2 & 0.2 & 0.2 & 0.2 & 0.2 & 0.2 & 0.2 & 0.2 & 0.2 & 0.2 & 0.2 & 0.2 \\
\hline 0 & 0 & 0 & 0 & 0 & 0 & 0 & 0 & 0 & 0 & 0 & 0 & 0 & 0 & 0 \\
\hline 5 & 5 & 5 & 5 & 5 & 5 & 5 & 5 & 5 & 5.0 & 5.0 & 5.0 & 5.0 & 5.0 & 5 \\
\hline 5 & 5 & 5 & 5 & 5 & 5 & 5 & 5 & 5 & 5.0 & 5.0 & 5.0 & 5.0 & 5.0 & 5 \\
\hline 2.9 & 2.9 & 2.9 & 2.9 & 2.9 & 2.9 & 2.9 & 2.9 & 2.9 & 2.9 & 2.9 & 2.9 & 2.9 & 2.9 & 2.9 \\
\hline 0 & 0 & 0 & 0 & 0 & 0 & 0 & 0 & 0 & 0 & 0 & 0 & 0 & 0 & 0 \\
\hline 0 & 0 & 0 & 0 & 0 & 0 & 0 & 0 & 0 & 0 & 0 & 0 & 0 & 0 & 0 \\
\hline 0 & 0 & 0 & 0 & 0 & 0 & 0 & 0 & 0 & 0 & 0 & 0 & 0 & 0 & 0 \\
\hline 0 & 0 & 0 & 0 & 0 & 0 & 0 & 0 & 0 & 0 & 0 & 0 & 0 & 0 & 0 \\
\hline 0 & 0 & 0 & 0 & 0 & 0 & 0 & 0 & 0 & 0 & 0 & 0 & 0 & 0 & 0 \\
\hline 27 & 27 & 27 & 27 & 27 & 27 & 27 & 27 & 27 & 27 & 27 & 27 & 27 & 27 & 27 \\
\hline
\end{tabular}

-Rock Island P.H. \#1 CER for Canada-

$50 \mathrm{CHPD}$ to BPA

51 PSE to BPA

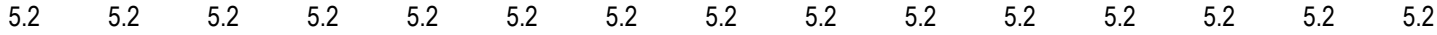

52 Rock Island P.H. \#1: Regional Totals

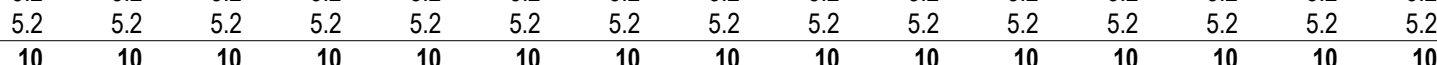

-Rock Island P.H. \#2 CER for Canada-

53 CHPD to BPA

54 PSE to BPA

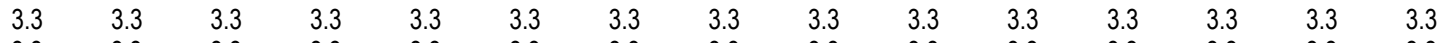

55 Rock Island P.H. \#2: Regional Totals

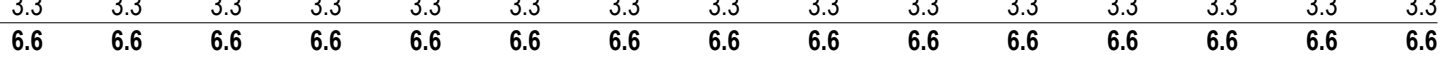

-Rocky Reach CER for Canada-

56 AVWP to BPA

$57 \mathrm{CHPD}$ to BPA

58 CLKM to BPA

59 DOPD to BPA

60 PGE to BPA

$61 \mathrm{PPL}$ to BPA

62 PSE to BPA

63 Rocky Reach: Regional Totals

\begin{tabular}{|c|c|c|c|c|c|c|c|c|c|c|c|c|c|}
\hline 1 & 1 & 1 & 1 & 1 & 1 & 1 & 1 & 1 & 1 & 1 & 1 & 1 & 1 \\
\hline 5.3 & 5.3 & 5.3 & 5.3 & 5.3 & 5.3 & 5.3 & 5.3 & 5.3 & 5.3 & 5.3 & 5.3 & 5.3 & 5.3 \\
\hline 8.1 & 8.1 & 8.1 & 8.1 & 8.1 & 8.1 & 8.1 & 8.1 & 8.1 & 8.1 & 8.1 & 8.1 & 8.1 & 8.1 \\
\hline 1.0 & 1.0 & 1.0 & 1.0 & 1.0 & 1.0 & 1.0 & 1.0 & 0.9 & 0.9 & 0.9 & 0.9 & 0.9 & 1.0 \\
\hline 4.3 & 4.3 & 4.3 & 4.3 & 4.3 & 4.3 & 4.3 & 4.3 & 4.2 & 4.2 & 4.2 & 4.2 & 4.2 & 4.3 \\
\hline 1.9 & 1.9 & 1.9 & 1.9 & 1.9 & 1.9 & 1.9 & 1.9 & 1.9 & 1.9 & 1.9 & 1.9 & 1.9 & 1.9 \\
\hline 14 & 14 & 14 & 14 & 14 & 14 & 14 & 14 & 14 & 14 & 14 & 14 & 14 & 14 \\
\hline 35 & 35 & 35 & 35 & 35 & 35 & 35 & 35 & 35 & 35 & 35 & 35 & 35 & 35 \\
\hline
\end{tabular}


Table A-15: Canadian Entitlement Return For Canada

PNW Loads and Resources Study

2008 - 2009 Operating Year

2003 White Book

Avg

Average Energy in Megawatts

Aug1 Aug16

Sep

Oct Nov

Dec
Jan $F$

64 AVWP to BPA

65 DOPD to BPA

66 OKPD to BPA

67 PGE to BPA

$68 \mathrm{PPL}$ to BPA

69 PSE to BPA

70 Wells: Regional Totals

$\begin{array}{lll}0.8 & 0.8 & 0.8 \\ 6.8 & 6.8 & 6.8\end{array}$

$\begin{array}{lll}6.8 & 6.8 & 6.8\end{array}$

$\begin{array}{lll}1.8 & 1.8 & 1.8\end{array}$

$\begin{array}{lll}4.6 & 4.6 & 4.6\end{array}$

$1.6 \quad 1.6 \quad 1.6$

7.1

$7.1-7.1-1.6$

$\begin{array}{ll}0.8 & 0.8 \\ 6.8 & 6.8 \\ 1.8 & 1.8\end{array}$

0.8

6.8
1.8

-Total CER For Canada-

71 Federal System

72 Public Entities

73 Investor-Owned Entities

74 Other Entities

75 Non-NW Entities

76 Total CER For Canada

\begin{tabular}{|c|c|c|c|c|c|c|c|c|c|c|c|c|c|}
\hline 0 & 0 & 0 & 0 & 0 & 0 & 0 & 0 & 0 & 0 & 0 & 0 & 0 & 0 \\
\hline 58 & 58 & 58 & 58 & 58 & 58 & 58 & 58 & 57 & 57 & 57 & 57 & 57 & 58 \\
\hline 64 & 64 & 64 & 64 & 64 & 64 & 64 & 64 & 64 & 64 & 64 & 64 & 64 & 64 \\
\hline 8.1 & 8.1 & 8.1 & 8.1 & 8.1 & 8.1 & 8.1 & 8.1 & 8.1 & 8.1 & 8.1 & 8.1 & 8.1 & 8.1 \\
\hline 0 & 0 & 0 & 0 & 0 & 0 & 0 & 0 & 0 & 0 & 0 & 0 & 0 & 0 \\
\hline 130 & 130 & 130 & 130 & 130 & 130 & 130 & 130 & 129 & 129 & 129 & 129 & 129 & 130 \\
\hline
\end{tabular}



2003 White Book

Dec Jan Feb Mar Apr1 Apr16

$\begin{array}{llllllllllllll}0 & 0 & 0 & 0 & 0 & 0 & 0 & 0 & 0 & 0 & 0 & 0 & 0 & 0\end{array}$

-Priest Rapids CER for Canada-

2 AVWP to BPA

3 COPD to BPA

4 CWPC to BPA

5 EWEB to BPA

6 FGRV to BPA

7 FREC to BPA

8 GCPD to BPA

9 ICLP to BPA

$10 \mathrm{KITT}$ to BPA

$11 \mathrm{KOOT}$ to BPA

12 LREC to BPA

13 LVE to BPA

14 MCMN to BPA

15 MTFR to BPA

16 NLEC to BPA

17 PGE to BPA

$18 \mathrm{PPL}$ to BPA

19 PSE to BPA

20 RREC to BPA

$21 \mathrm{SCL}$ to BPA

22 SLEC to BPA

23 TPU to BPA

24 UNEC to BPA

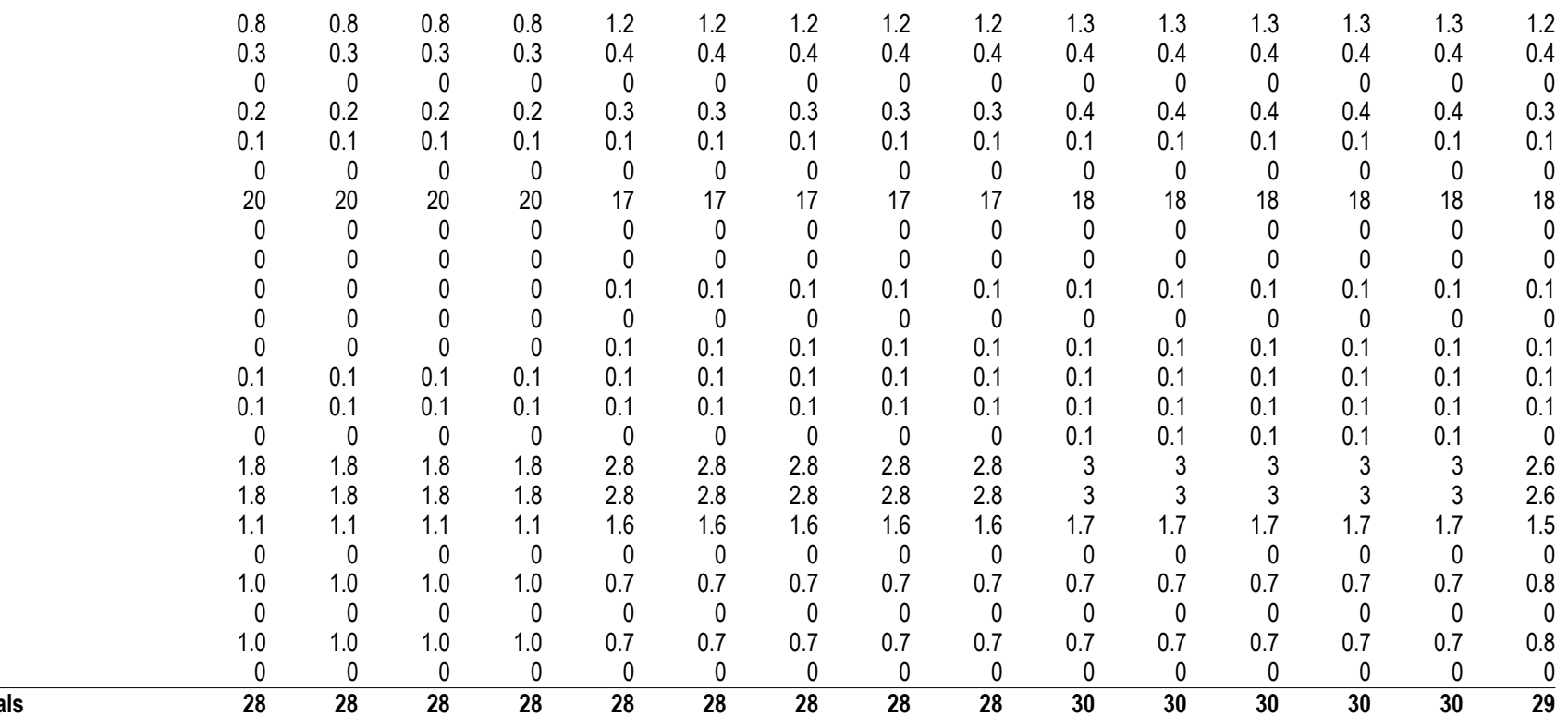

-Wanapum CER for Canada-

26 AVWP to BPA

27 COPD to BPA

28 CWPC to BPA

29 EWEB to BPA

$30 \mathrm{FGRV}$ to BPA

31 FREC to BPA

32 GCPD to BPA

$33 \mathrm{ICLP}$ to BPA

$34 \mathrm{KITT}$ to BPA

$35 \mathrm{KOOT}$ to BPA

36 LREC to BPA

37 LVE to BPA

$38 \mathrm{MCMN}$ to BPA

39 MTFR to BPA

40 NLEC to BPA

41 PGE to BPA

$42 \mathrm{PPL}$ to BPA

43 PSE to BPA

44 RREC to BPA

$45 \mathrm{SCL}$ to BPA

46 SLEC to BPA

47 TPU to BPA

48 UNEC to BPA

49 Wanapum: Regional Totals

$\begin{array}{rrrrrrrrrrrrrrr}2.2 & 2.2 & 2.2 & 2.2 & 1.2 & 1.2 & 1.2 & 1.2 & 1.2 & 1.3 & 1.3 & 1.3 & 1.3 & 1.3 & 1.5 \\ 0.7 & 0.7 & 0.7 & 0.7 & 0.4 & 0.4 & 0.4 & 0.4 & 0.4 & 0.4 & 0.4 & 0.4 & 0.4 & 0.4 & 0.5 \\ 0 & 0 & 0 & 0 & 0 & 0 & 0 & 0 & 0 & 0 & 0 & 0 & 0 & 0 & 0 \\ 0.6 & 0.6 & 0.6 & 0.6 & 0.3 & 0.3 & 0.3 & 0.3 & 0.3 & 0.4 & 0.4 & 0.4 & 0.4 & 0.4 & 0.4 \\ 0.2 & 0.2 & 0.2 & 0.2 & 0.1 & 0.1 & 0.1 & 0.1 & 0.1 & 0.1 & 0.1 & 0.1 & 0.1 & 0.1 & 0.1 \\ 0 & 0 & 0 & 0 & 0 & 0 & 0 & 0 & 0 & 0 & 0 & 0 & 0 & 0 & 0 \\ 9.7 & 9.7 & 9.7 & 9.7 & 16 & 16 & 16 & 16 & 16 & 17 & 17 & 17 & 17 & 17 & 15 \\ 0 & 0 & 0 & 0 & 0 & 0 & 0 & 0 & 0 & 0 & 0 & 0 & 0 & 0 & 0 \\ 0 & 0 & 0 & 0 & 0 & 0 & 0 & 0 & 0 & 0 & 0 & 0 & 0 & 0 & 0 \\ 0 & 0 & 0 & 0 & 0.1 & 0.1 & 0.1 & 0.1 & 0.1 & 0.1 & 0.1 & 0.1 & 0.1 & 0.1 & 0 \\ 0 & 0 & 0 & 0 & 0 & 0 & 0 & 0 & 0 & 0 & 0 & 0 & 0 & 0 & 0 \\ 0 & 0 & 0 & 0 & 0.1 & 0.1 & 0.1 & 0.1 & 0.1 & 0.1 & 0.1 & 0.1 & 0.1 & 0.1 & 0 \\ 0.2 & 0.2 & 0.2 & 0.2 & 0.1 & 0.1 & 0.1 & 0.1 & 0.1 & 0.1 & 0.1 & 0.1 & 0.1 & 0.1 & 0.1 \\ 0.2 & 0.2 & 0.2 & 0.2 & 0.1 & 0.1 & 0.1 & 0.1 & 0.1 & 0.1 & 0.1 & 0.1 & 0.1 & 0.1 & 0.1 \\ 0 & 0 & 0 & 0 & 0 & 0 & 0 & 0 & 0 & 0 & 0 & 0 & 0 & 0 & 0 \\ 5.0 & 5.0 & 5.0 & 5.0 & 2.7 & 2.7 & 2.7 & 2.7 & 2.7 & 2.9 & 2.9 & 2.9 & 2.9 & 2.9 & 3.3 \\ 5.0 & 5.0 & 5.0 & 5.0 & 2.7 & 2.7 & 2.7 & 2.7 & 2.7 & 2.9 & 2.9 & 2.9 & 2.9 & 2.9 & 3.3 \\ 2.9 & 2.9 & 2.9 & 2.9 & 1.6 & 1.6 & 1.6 & 1.6 & 1.6 & 1.7 & 1.7 & 1.7 & 1.7 & 1.7 & 1.9 \\ 0 & 0 & 0 & 0 & 0 & 0 & 0 & 0 & 0 & 0 & 0 & 0 & 0 & 0 & 0 \\ 0 & 0 & 0 & 0 & 0.7 & 0.7 & 0.7 & 0.7 & 0.7 & 0.7 & 0.7 & 0.7 & 0.7 & 0.7 & 0.5 \\ 0 & 0 & 0 & 0 & 0 & 0 & 0 & 0 & 0 & 0 & 0 & 0 & 0 & 0 & 0 \\ 0 & 0 & 0 & 0 & 0.7 & 0.7 & 0.7 & 0.7 & 0.7 & 0.7 & 0.7 & 0.7 & 0.7 & 0.7 & 0.5 \\ 0 & 0 & 0 & 0 & 0 & 0 & 0 & 0 & 0 & 0 & 0 & 0 & 0 & 0 & 0 \\ \mathbf{2 7} & \mathbf{2 7} & \mathbf{2 7} & \mathbf{2 7} & \mathbf{2 7} & \mathbf{2 7} & \mathbf{2 7} & \mathbf{2 7} & \mathbf{2 7} & \mathbf{2 9} & \mathbf{2 9} & \mathbf{2 9} & \mathbf{2 9} & \mathbf{2 9} & \mathbf{2 7}\end{array}$

-Rock Island P.H. \#1 CER for Canada-

$50 \mathrm{CHPD}$ to BPA

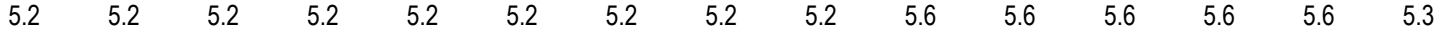

51 PSE to BPA

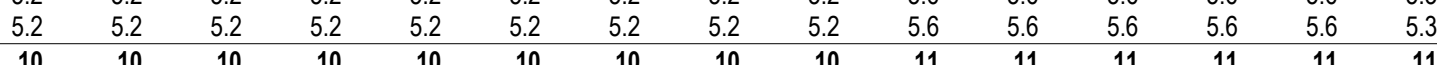

-Rock Island P.H. \#2 CER for Canada-

53 CHPD to BPA

54 PSE to BPA

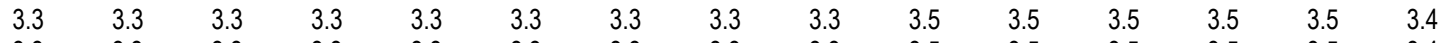

55 Rock Island P.H. \#2: Regional Totals

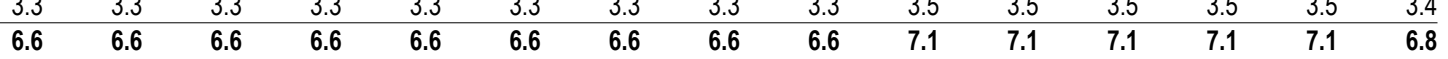

-Rocky Reach CER for Canada-

56 AVWP to BPA

$57 \mathrm{CHPD}$ to BPA

58 CLKM to BPA

59 DOPD to BPA

60 PGE to BPA

$61 \mathrm{PPL}$ to BPA

62 PSE to BPA

63 Rocky Reach: Regional Totals

\begin{tabular}{|c|c|c|c|c|c|c|c|c|c|c|c|c|c|}
\hline 1 & 1 & 1 & 1 & 1 & 1 & 1 & 1 & 1.1 & 1.1 & 1.1 & 1.1 & 1.1 & 1 \\
\hline 5.3 & 5.3 & 5.3 & 5.3 & 5.3 & 5.3 & 5.3 & 5.3 & 5.7 & 5.7 & 5.7 & 5.7 & 5.7 & 5.4 \\
\hline 8.1 & 8.1 & 8.1 & 8.1 & 8.1 & 8.1 & 8.1 & 8.1 & 8.7 & 8.7 & 8.7 & 8.7 & 8.7 & 8.3 \\
\hline 0.9 & 0.9 & 0.9 & 0.9 & 0.9 & 0.9 & 0.9 & 0.9 & 1 & 1 & 1 & 1 & 1 & 1.0 \\
\hline 4.2 & 4.2 & 4.2 & 4.2 & 4.2 & 4.2 & 4.2 & 4.2 & 4.6 & 4.6 & 4.6 & 4.6 & 4.6 & 4.4 \\
\hline 1.9 & 1.9 & 1.9 & 1.9 & 1.9 & 1.9 & 1.9 & 1.9 & 2 & 2 & 2 & 2 & 2 & 1.9 \\
\hline 14 & 14 & 14 & 14 & 14 & 14 & 14 & 14 & 15 & 15 & 15 & 15 & 15 & 14 \\
\hline 35 & 35 & 35 & 35 & 35 & 35 & 35 & 35 & 38 & 38 & 38 & 38 & 38 & 36 \\
\hline
\end{tabular}


Table A-15: Canadian Entitlement Return For Canada

PNW Loads and Resources Study

2009 - 2010 Operating Year

2003 White Book Jul Avg

Average Energy in Megawatts

Aug1 Aug16

Sep

Oct Nov Dec Jan Feb Mar Apr1 Apr16

May Jun 64 AVWP to BPA 65 DOPD to BPA 66 OKPD to BPA 67 PGE to BPA $68 \mathrm{PPL}$ to BPA 69 PSE to BPA

70 Wells: Regional Totals

$\begin{array}{lll}0.8 & 0.8 & 0.8 \\ 6.8 & 6.8 & 6.8\end{array}$

6.8

$0.8 \quad 0.8$

0.8
6.8

0.8
6.8

0.8
6.8

0.8

6.8

0.9
7.3

7.3
2.0

0.9
7.3

0.9

0.9

7.3

7.3

0.9

7.3

7.3

0.8

$\begin{array}{lll}4.6 & 4.6 & 4.6\end{array}$

$\begin{array}{llll}1.6 & 1.6 & 16 & 1.6\end{array}$

$\begin{array}{lll}4.6 & 4.6 & 4.6\end{array}$

$4.6 \quad 4.6$

5.0
1.7

5.0

5.0

2.0

5.0

$1.7 \quad 1.7$

$23 \quad 23$

\begin{tabular}{rrrr}
7.6 & 7.6 & 7.6 & 7.3 \\
\hline $\mathbf{2 4}$ & $\mathbf{2 4}$ & $\mathbf{2 4}$ & $\mathbf{2 3}$
\end{tabular}

-Total CER For Canada-

71 Federal System

72 Public Entities

73 Investor-Owned Entities

74 Other Entities

75 Non-NW Entities

76 Total CER For Canada

$\begin{array}{rrrrrrrrrrrrrrr}0 & 0 & 0 & 0 & 0 & 0 & 0 & 0 & 0 & 0 & 0 & 0 & 0 & 0 & 0 \\ 57 & 57 & 57 & 57 & 61 & 61 & 61 & 61 & 61 & 66 & 66 & 66 & 66 & 66 & 62 \\ 64 & 64 & 64 & 64 & 60 & 60 & 60 & 60 & 60 & 64 & 64 & 64 & 64 & 64 & 62 \\ 8.1 & 8.1 & 8.1 & 8.1 & 8.1 & 8.1 & 8.1 & 8.1 & 8.1 & 8.7 & 8.7 & 8.7 & 8.7 & 8.7 & 8.3 \\ 0 & 0 & 0 & 0 & 0 & 0 & 0 & 0 & 0 & 0 & 0 & 0 & 0 & 0 & 0 \\ 129 & 129 & 129 & 129 & 129 & 129 & 129 & 129 & 129 & 139 & 139 & 139 & 139 & 139 & 133\end{array}$


Dec

Jan Feb Mar Apr1 Apr16

May

Jun Jul Avg

-BPA Canada-

1 BPA: Regional Totals

$\begin{array}{llllllllllllll}0 & 0 & 0 & 0 & 0 & 0 & 0 & 0 & 0 & 0 & 0 & 0 & 0 & 0\end{array}$

-Priest Rapids CER for Canada-

2 AVWP to BPA

3 COPD to BPA

4 CWPC to BPA

5 EWEB to BPA

6 FGRV to BPA

7 FREC to BPA

8 GCPD to BPA

9 ICLP to BPA

$10 \mathrm{KITT}$ to BPA

$11 \mathrm{KOOT}$ to BPA

12 LREC to BPA

13 LVE to BPA

$14 \mathrm{MCMN}$ to BPA

15 MTFR to BPA

16 NLEC to BPA

17 PGE to BPA

$18 \mathrm{PPL}$ to BPA

19 PSE to BPA

20 RREC to BPA

$21 \mathrm{SCL}$ to BPA

22 SLEC to BPA

23 TPU to BPA

24 UNEC to BPA

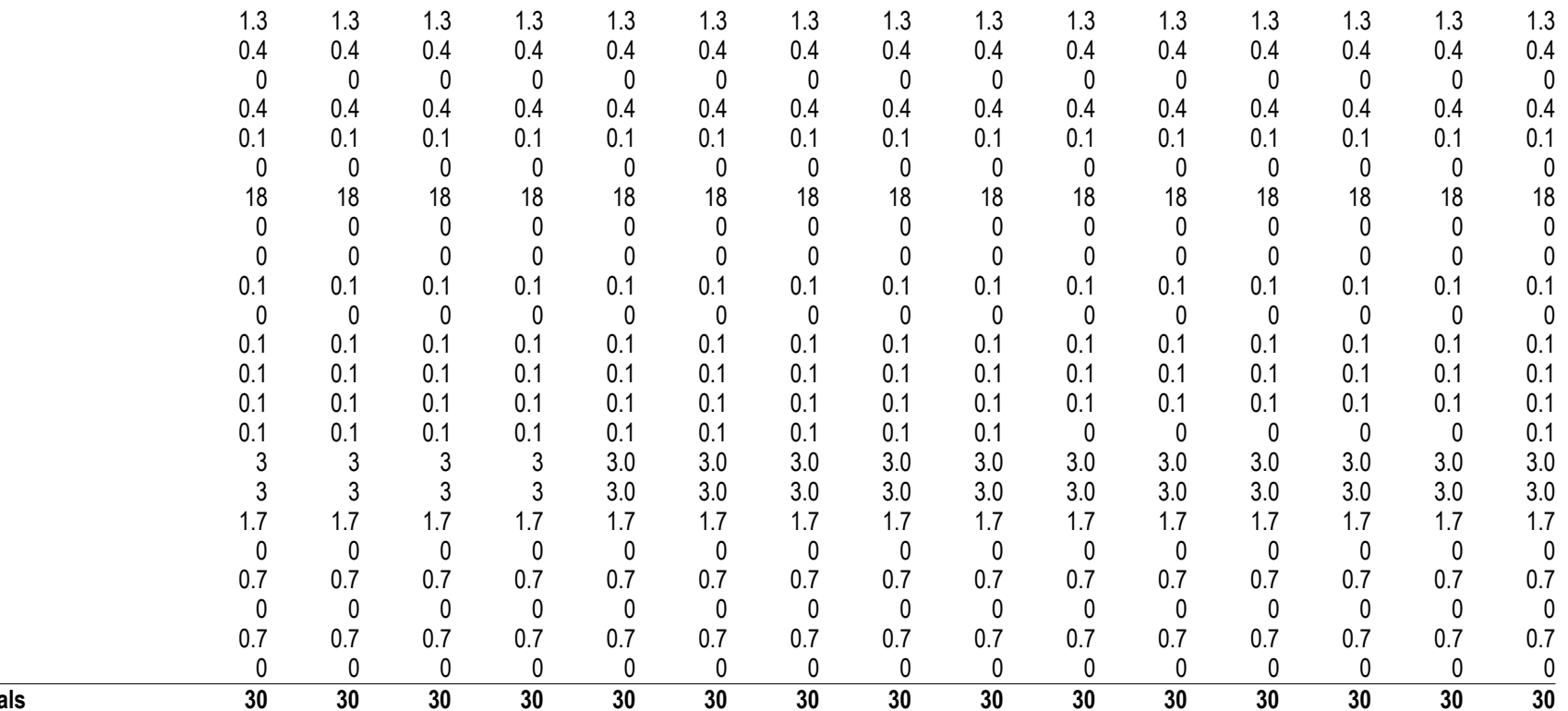

-Wanapum CER for Canada-

26 AVWP to BPA

27 COPD to BPA

28 CWPC to BPA

29 EWEB to BPA

$30 \mathrm{FGRV}$ to BPA

31 FREC to BPA

32 GCPD to BPA

33 ICLP to BPA

$34 \mathrm{KITT}$ to BPA

$35 \mathrm{KOOT}$ to BPA

36 LREC to BPA

37 LVE to BPA

$38 \mathrm{MCMN}$ to BPA

39 MTFR to BPA

40 NLEC to BPA

41 PGE to BPA

$42 \mathrm{PPL}$ to BPA

43 PSE to BPA

44 RREC to BPA

$45 \mathrm{SCL}$ to BPA

46 SLEC to BPA

47 TPU to BPA

48 UNEC to BPA

49 Wanapum: Regional Totals

\begin{tabular}{|c|c|c|c|c|c|c|c|c|c|c|c|c|c|c|}
\hline 1.3 & 1.3 & 1.3 & 1.3 & 1.3 & 1.3 & 1.3 & 1.3 & 1.3 & 1.2 & 1.2 & 1.2 & 1.2 & 1.2 & 1.3 \\
\hline 0.4 & 0.4 & 0.4 & 0.4 & 0.4 & 0.4 & 0.4 & 0.4 & 0.4 & 0.4 & 0.4 & 0.4 & 0.4 & 0.4 & 0.4 \\
\hline 0 & 0 & 0 & 0 & 0 & 0 & 0 & 0 & 0 & 0 & 0 & 0 & 0 & 0 & 0 \\
\hline 0.4 & 0.4 & 0.4 & 0.4 & 0.4 & 0.4 & 0.4 & 0.4 & 0.4 & 0.3 & 0.3 & 0.3 & 0.3 & 0.3 & 0.4 \\
\hline 0.1 & 0.1 & 0.1 & 0.1 & 0.1 & 0.1 & 0.1 & 0.1 & 0.1 & 0.1 & 0.1 & 0.1 & 0.1 & 0.1 & 0.1 \\
\hline 0 & 0 & 0 & 0 & 0 & 0 & 0 & 0 & 0 & 0 & 0 & 0 & 0 & 0 & 0 \\
\hline 17 & 17 & 17 & 17 & 17 & 17 & 17 & 17 & 17 & 17 & 17 & 17 & 17 & 17 & 17 \\
\hline 0 & 0 & 0 & 0 & 0 & 0 & 0 & 0 & 0 & 0 & 0 & 0 & 0 & 0 & 0 \\
\hline 0 & 0 & 0 & 0 & 0 & 0 & 0 & 0 & 0 & 0 & 0 & 0 & 0 & 0 & 0 \\
\hline 0.1 & 0.1 & 0.1 & 0.1 & 0.1 & 0.1 & 0.1 & 0.1 & 0.1 & 0.1 & 0.1 & 0.1 & 0.1 & 0.1 & 0.1 \\
\hline 0 & 0 & 0 & 0 & 0 & 0 & 0 & 0 & 0 & 0 & 0 & 0 & 0 & 0 & 0 \\
\hline 0.1 & 0.1 & 0.1 & 0.1 & 0.1 & 0.1 & 0.1 & 0.1 & 0.1 & 0.1 & 0.1 & 0.1 & 0.1 & 0.1 & 0.1 \\
\hline 0.1 & 0.1 & 0.1 & 0.1 & 0.1 & 0.1 & 0.1 & 0.1 & 0.1 & 0.1 & 0.1 & 0.1 & 0.1 & 0.1 & 0.1 \\
\hline 0.1 & 0.1 & 0.1 & 0.1 & 0.1 & 0.1 & 0.1 & 0.1 & 0.1 & 0.1 & 0.1 & 0.1 & 0.1 & 0.1 & 0.1 \\
\hline 0 & 0 & 0 & 0 & 0 & 0 & 0 & 0 & 0 & 0 & 0 & 0 & 0 & 0 & 0 \\
\hline 2.9 & 2.9 & 2.9 & 2.9 & 2.9 & 2.9 & 2.9 & 2.9 & 2.9 & 2.8 & 2.8 & 2.8 & 2.8 & 2.8 & 2.9 \\
\hline 2.9 & 2.9 & 2.9 & 2.9 & 2.9 & 2.9 & 2.9 & 2.9 & 2.9 & 2.8 & 2.8 & 2.8 & 2.8 & 2.8 & 2.9 \\
\hline 1.7 & 1.7 & 1.7 & 1.7 & 1.6 & 1.6 & 1.6 & 1.6 & 1.6 & 1.6 & 1.6 & 1.6 & 1.6 & 1.6 & 1.6 \\
\hline 0 & 0 & 0 & 0 & 0 & 0 & 0 & 0 & 0 & 0 & 0 & 0 & 0 & 0 & 0 \\
\hline 0.7 & 0.7 & 0.7 & 0.7 & 0.7 & 0.7 & 0.7 & 0.7 & 0.7 & 0.7 & 0.7 & 0.7 & 0.7 & 0.7 & 0.7 \\
\hline 0 & 0 & 0 & 0 & 0 & 0 & 0 & 0 & 0 & 0 & 0 & 0 & 0 & 0 & 0 \\
\hline 0.7 & 0.7 & 0.7 & 0.7 & 0.7 & 0.7 & 0.7 & 0.7 & 0.7 & 0.7 & 0.7 & 0.7 & 0.7 & 0.7 & 0.7 \\
\hline 0 & 0 & 0 & 0 & 0 & 0 & 0 & 0 & 0 & 0 & 0 & 0 & 0 & 0 & 0 \\
\hline 29 & 29 & 29 & 29 & 29 & 29 & 29 & 29 & 29 & 28 & 28 & 28 & 28 & 28 & 29 \\
\hline
\end{tabular}

-Rock Island P.H. \#1 CER for Canada-

$\begin{array}{lllllllllllllll}50 & \mathrm{CHPD} & & \end{array}$

51 PSE to BPA

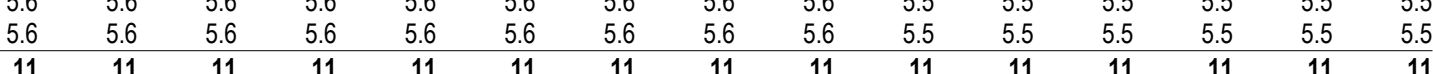

52 Rock Island P.H. \#1: Regional Totals

-Rock Island P.H. \#2 CER for Canada-

53 CHPD to BPA

54 PSE to BPA

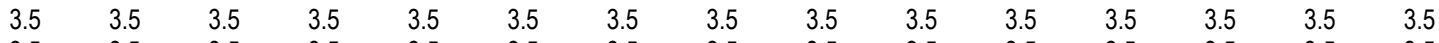

55 Rock Island P.H. \#2: Regional Totals

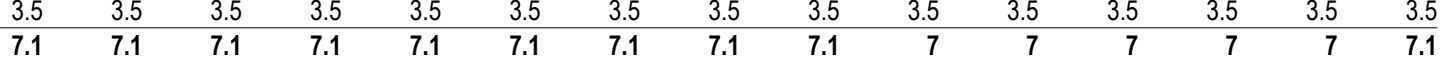

-Rocky Reach CER for Canada-

56 AVWP to BPA

$57 \mathrm{CHPD}$ to BPA

58 CLKM to BPA

59 DOPD to BPA

60 PGE to BPA

$61 \mathrm{PPL}$ to BPA

62 PSE to BPA

63 Rocky Reach: Regional Totals

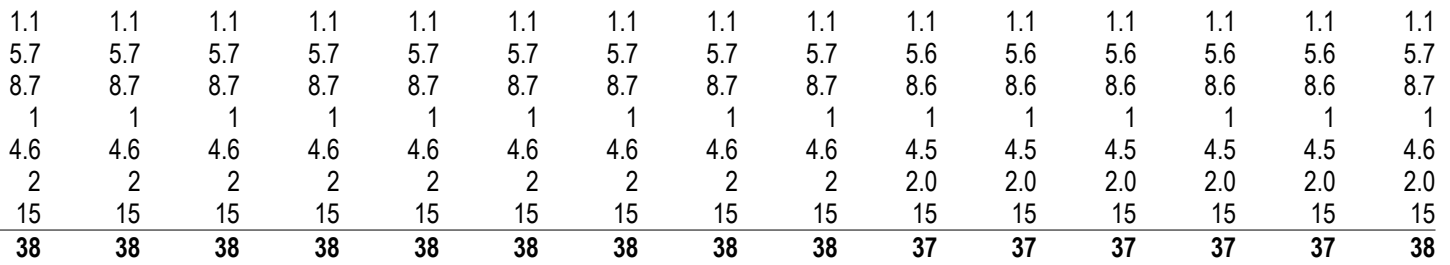


Table A-15: Canadian Entitlement Return For Canada

PNW Loads and Resources Study

2010 - 2011 Operating Year

2003 White Book Jul Avg

Average Energy in Megawatts

Aug1 Aug16

Sep Oct Nov Dec Jan Feb Mar Apr1 Apr16

May Jun 64 AVWP to BPA 65 DOPD to BPA 66 OKPD to BPA 67 PGE to BPA $68 \mathrm{PPL}$ to BPA 69 PSE to BPA

70 Wells: Regional Totals

$\begin{array}{rrrrrrrrrrrrrrr}0.9 & 0.9 & 0.9 & 0.9 & 0.9 & 0.9 & 0.9 & 0.9 & 0.9 & 0.8 & 0.8 & 0.8 & 0.8 & 0.8 & 0.9 \\ 7.3 & 7.3 & 7.3 & 7.3 & 7.3 & 7.3 & 7.3 & 7.3 & 7.3 & 7.2 & 7.2 & 7.2 & 7.2 & 7.2 & 7.3 \\ 2.0 & 2.0 & 2.0 & 2.0 & 2.0 & 2.0 & 2.0 & 2.0 & 2.0 & 1.9 & 1.9 & 1.9 & 1.9 & 1.9 & 1.9 \\ 5.0 & 5.0 & 5.0 & 5.0 & 5.0 & 5.0 & 5.0 & 5.0 & 5.0 & 4.9 & 4.9 & 4.9 & 4.9 & 4.9 & 4.9 \\ 1.7 & 1.7 & 1.7 & 1.7 & 1.7 & 1.7 & 1.7 & 1.7 & 1.7 & 1.7 & 1.7 & 1.7 & 1.7 & 1.7 & 1.7 \\ 7.6 & \mathbf{7 . 6} & \mathbf{7 . 6} & \mathbf{7 . 6} & \mathbf{7 . 6} & \mathbf{7 . 6} & \mathbf{7 . 6} & \mathbf{7 . 6} & \mathbf{7 . 6} & \mathbf{7 . 5} & 7.5 & 7.5 & 7.5 & 7.5 & 7.6 \\ \mathbf{2 4} & \mathbf{2 4} & \mathbf{2 4} & \mathbf{2 4} & \mathbf{2 4} & \mathbf{2 4} & \mathbf{2 4} & \mathbf{2 4} & \mathbf{2 4} & \mathbf{2 4} & \mathbf{2 4} & \mathbf{2 4} & \mathbf{2 4} & \mathbf{2 4} & \mathbf{2 4}\end{array}$

-Total CER For Canada-

71 Federal System

72 Public Entities

73 Investor-Owned Entities

74 Other Entities

75 Non-NW Entities

76 Total CER For Canada

$\begin{array}{rrrrrrrrrrrrrrr}0 & 0 & 0 & 0 & 0 & 0 & 0 & 0 & 0 & 0 & 0 & 0 & 0 & 0 & 0 \\ 66 & 66 & 66 & 66 & 66 & 66 & 66 & 66 & 66 & 65 & 65 & 65 & 65 & 65 & 66 \\ 64 & 64 & 64 & 64 & 64 & 64 & 64 & 64 & 64 & 63 & 63 & 63 & 63 & 63 & 64 \\ 8.7 & 8.7 & 8.7 & 8.7 & 8.7 & 8.7 & 8.7 & 8.7 & 8.7 & 8.6 & 8.6 & 8.6 & 8.6 & 8.6 & 8.7 \\ 0 & 0 & 0 & 0 & 0 & 0 & 0 & 0 & 0 & 0 & 0 & 0 & 0 & 0 & 0 \\ 139 & 139 & 139 & 139 & 139 & 139 & 139 & 139 & 139 & 138 & 138 & 138 & 138 & 138 & 139\end{array}$



2003 White Book

Dec

Jan Feb Mar Apr1 Apr16

May

Jun Jul Avg

-BPA Canada-

1 BPA: Regional Totals

$\begin{array}{llllllllllllll}0 & 0 & 0 & 0 & 0 & 0 & 0 & 0 & 0 & 0 & 0 & 0 & 0 & 0\end{array}$

-Priest Rapids CER for Canada-

2 AVWP to BPA

3 COPD to BPA

4 CWPC to BPA

5 EWEB to BPA

6 FGRV to BPA

7 FREC to BPA

8 GCPD to BPA

9 ICLP to BPA

$10 \mathrm{KITT}$ to BPA

$11 \mathrm{KOOT}$ to BPA

12 LREC to BPA

13 LVE to BPA

14 MCMN to BPA

15 MTFR to BPA

16 NLEC to BPA

17 PGE to BPA

$18 \mathrm{PPL}$ to BPA

19 PSE to BPA

20 RREC to BPA

$21 \mathrm{SCL}$ to BPA

22 SLEC to BPA

23 TPU to BPA

24 UNEC to BPA

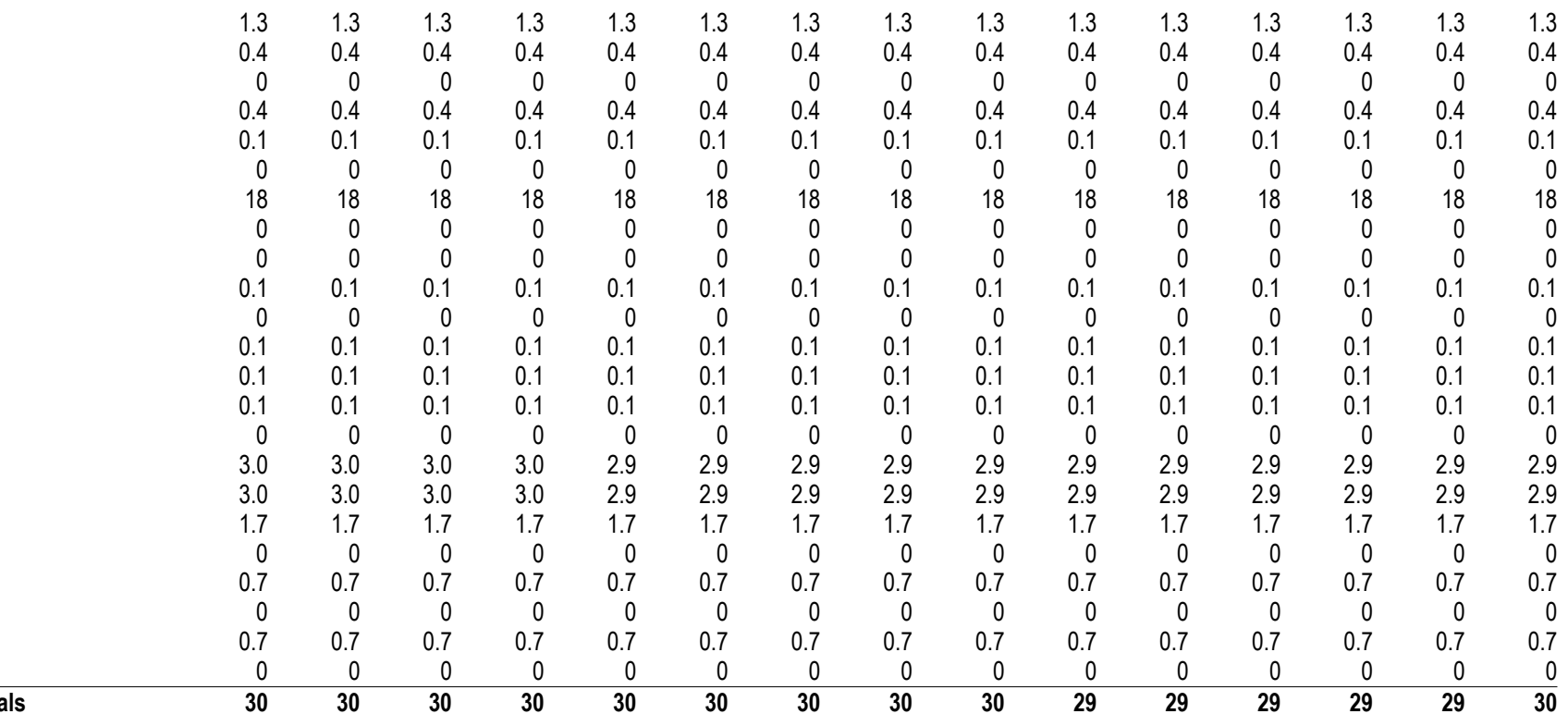

-Wanapum CER for Canada-

26 AVWP to BPA

27 COPD to BPA

28 CWPC to BPA

29 EWEB to BPA

30 FGRV to BPA

31 FREC to BPA

32 GCPD to BPA

$33 \mathrm{ICLP}$ to BPA

$34 \mathrm{KITT}$ to BPA

$35 \mathrm{KOOT}$ to BPA

36 LREC to BPA

37 LVE to BPA

38 MCMN to BPA

39 MTFR to BPA

40 NLEC to BPA

41 PGE to BPA

$42 \mathrm{PPL}$ to BPA

43 PSE to BPA

44 RREC to BPA

$45 \mathrm{SCL}$ to BPA

46 SLEC to BPA

47 TPU to BPA

48 UNEC to BPA

49 Wanapum: Regional Totals

\begin{tabular}{|c|c|c|c|c|c|c|c|c|c|c|c|c|c|c|}
\hline 1.2 & 1.2 & 1.2 & 1.2 & 1.2 & 1.2 & 1.2 & 1.2 & 1.2 & 1.2 & 1.2 & 1.2 & 1.2 & 1.2 & 1.2 \\
\hline 0.4 & 0.4 & 0.4 & 0.4 & 0.4 & 0.4 & 0.4 & 0.4 & 0.4 & 0.4 & 0.4 & 0.4 & 0.4 & 0.4 & 0.4 \\
\hline 0 & 0 & 0 & 0 & 0 & 0 & 0 & 0 & 0 & 0 & 0 & 0 & 0 & 0 & 0 \\
\hline 0.3 & 0.3 & 0.3 & 0.3 & 0.3 & 0.3 & 0.3 & 0.3 & 0.3 & 0.3 & 0.3 & 0.3 & 0.3 & 0.3 & 0.3 \\
\hline 0.1 & 0.1 & 0.1 & 0.1 & 0.1 & 0.1 & 0.1 & 0.1 & 0.1 & 0.1 & 0.1 & 0.1 & 0.1 & 0.1 & 0.1 \\
\hline 0 & 0 & 0 & 0 & 0 & 0 & 0 & 0 & 0 & 0 & 0 & 0 & 0 & 0 & 0 \\
\hline 17 & 17 & 17 & 17 & 17 & 17 & 17 & 17 & 17 & 17 & 17 & 17 & 17 & 17 & 17 \\
\hline 0 & 0 & 0 & 0 & 0 & 0 & 0 & 0 & 0 & 0 & 0 & 0 & 0 & 0 & 0 \\
\hline 0 & 0 & 0 & 0 & 0 & 0 & 0 & 0 & 0 & 0 & 0 & 0 & 0 & 0 & 0 \\
\hline 0.1 & 0.1 & 0.1 & 0.1 & 0.1 & 0.1 & 0.1 & 0.1 & 0.1 & 0.1 & 0.1 & 0.1 & 0.1 & 0.1 & 0.1 \\
\hline 0 & 0 & 0 & 0 & 0 & 0 & 0 & 0 & 0 & 0 & 0 & 0 & 0 & 0 & 0 \\
\hline 0.1 & 0.1 & 0.1 & 0.1 & 0.1 & 0.1 & 0.1 & 0.1 & 0.1 & 0.1 & 0.1 & 0.1 & 0.1 & 0.1 & 0.1 \\
\hline 0.1 & 0.1 & 0.1 & 0.1 & 0.1 & 0.1 & 0.1 & 0.1 & 0.1 & 0.1 & 0.1 & 0.1 & 0.1 & 0.1 & 0.1 \\
\hline 0.1 & 0.1 & 0.1 & 0.1 & 0.1 & 0.1 & 0.1 & 0.1 & 0.1 & 0.1 & 0.1 & 0.1 & 0.1 & 0.1 & 0.1 \\
\hline 0 & 0 & 0 & 0 & 0 & 0 & 0 & 0 & 0 & 0 & 0 & 0 & 0 & 0 & 0 \\
\hline 2.8 & 2.8 & 2.8 & 2.8 & 2.8 & 2.8 & 2.8 & 2.8 & 2.8 & 2.7 & 2.7 & 2.7 & 2.7 & 2.7 & 2.8 \\
\hline 2.8 & 2.8 & 2.8 & 2.8 & 2.8 & 2.8 & 2.8 & 2.8 & 2.8 & 2.7 & 2.7 & 2.7 & 2.7 & 2.7 & 2.8 \\
\hline 1.6 & 1.6 & 1.6 & 1.6 & 1.6 & 1.6 & 1.6 & 1.6 & 1.6 & 1.6 & 1.6 & 1.6 & 1.6 & 1.6 & 1.6 \\
\hline 0 & 0 & 0 & 0 & 0 & 0 & 0 & 0 & 0 & 0 & 0 & 0 & 0 & 0 & 0 \\
\hline 0.7 & 0.7 & 0.7 & 0.7 & 0.7 & 0.7 & 0.7 & 0.7 & 0.7 & 0.7 & 0.7 & 0.7 & 0.7 & 0.7 & 0.7 \\
\hline 0 & 0 & 0 & 0 & 0 & 0 & 0 & 0 & 0 & 0 & 0 & 0 & 0 & 0 & 0 \\
\hline 0.7 & 0.7 & 0.7 & 0.7 & 0.7 & 0.7 & 0.7 & 0.7 & 0.7 & 0.7 & 0.7 & 0.7 & 0.7 & 0.7 & 0.7 \\
\hline 0 & 0 & 0 & 0 & 0 & 0 & 0 & 0 & 0 & 0 & 0 & 0 & 0 & 0 & 0 \\
\hline 28 & 28 & 28 & 28 & 28 & 28 & 28 & 28 & 28 & 28 & 28 & 28 & 28 & 28 & 28 \\
\hline
\end{tabular}

-Rock Island P.H. \#1 CER for Canada-

$50 \mathrm{CHPD}$ to BPA

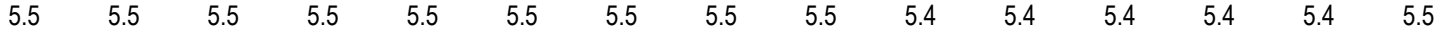

51 PSE to BPA

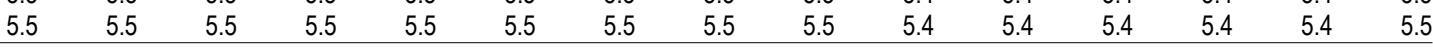

52 Rock Island P.H. \#1: Regional Totals

-Rock Island P.H. \#2 CER for Canada-

53 CHPD to BPA

54 PSE to BPA

55 Rock Island P.H. \#2: Regional Totals

$\begin{array}{llllll}3.5 & 3.5 & 3.5 & 3.5 & 3.5 & 3.5 \\ 3.5 & 3.5 & 3.5 & 3.5 & 3.5 & 3.5\end{array}$

-Rocky Reach CER for Canada-

56 AVWP to BPA

$57 \mathrm{CHPD}$ to BPA

58 CLKM to BPA

59 DOPD to BPA

60 PGE to BPA

$61 \mathrm{PPL}$ to BPA

62 PSE to BPA

63 Rocky Reach: Regional Totals

\begin{tabular}{|c|c|c|c|c|c|c|c|c|c|c|c|c|c|c|}
\hline 3.5 & 3.5 & 3.5 & 3.5 & 3.5 & 3.5 & 3.5 & 3.5 & 3.5 & 3.5 & 3.5 & 3.5 & 3.5 & 3.5 & 3.5 \\
\hline 3.5 & 3.5 & 3.5 & 3.5 & 3.5 & 3.5 & 3.5 & 3.5 & 3.5 & 3.5 & 3.5 & 3.5 & 3.5 & 3.5 & .5 \\
\hline 7 & 7 & 7 & 7 & 7 & 7 & 7 & 7 & 7 & 6.9 & 6.9 & 6.9 & 6.9 & 6.9 & 7.0 \\
\hline
\end{tabular}

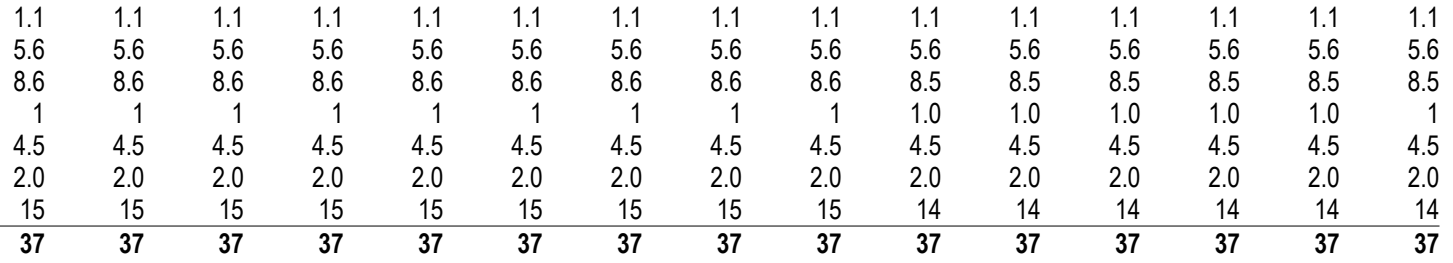


Table A-15: Canadian Entitlement Return For Canada

PNW Loads and Resources Study

2011 - 2012 Operating Year

2003 White Book Jul Avg

Average Energy in Megawatts

Aug1 Aug16

Sep Oct Nov Dec Jan Feb Mar Apr1 Apr16

May Jun 64 AVWP to BPA 65 DOPD to BPA 66 OKPD to BPA 67 PGE to BPA $68 \mathrm{PPL}$ to BPA 69 PSE to BPA

70 Wells: Regional Totals

\begin{tabular}{lllllllllllllll}
0.8 & 0.8 & 0.8 & 0.8 & 0.8 & 0.8 & 0.8 & 0.8 & 0.8 & 0.8 & 0.8 & 0.8 & 0.8 & 0.8 & 0.8 \\
7.2 & 7.2 & 7.2 & 7.2 & 7.2 & 7.2 & 7.2 & 7.2 & 7.2 & 7.1 & 7.1 & 7.1 & 7.1 & 7.1 & 7.2 \\
1.9 & 1.9 & 1.9 & 1.9 & 1.9 & 1.9 & 1.9 & 1.9 & 1.9 & 1.9 & 1.9 & 1.9 & 1.9 & 1.9 & 1.9 \\
4.9 & 4.9 & 4.9 & 4.9 & 4.9 & 4.9 & 4.9 & 4.9 & 4.9 & 4.8 & 4.8 & 4.8 & 4.8 & 4.8 & 4.9 \\
1.7 & 1.7 & 1.7 & 1.7 & 1.7 & 1.7 & 1.7 & 1.7 & 1.7 & 1.6 & 1.6 & 1.6 & 1.6 & 1.6 & 1.7 \\
7.5 & 7.5 & 7.5 & 7.5 & 7.5 & 7.5 & 7.5 & 7.5 & 7.5 & 7.4 & 7.4 & 7.4 & 7.4 & 7.4 & 7.5 \\
\hline $\mathbf{2 4}$ & $\mathbf{2 4}$ & $\mathbf{2 4}$ & $\mathbf{2 4}$ & $\mathbf{2 4}$ & $\mathbf{2 4}$ & $\mathbf{2 4}$ & $\mathbf{2 4}$ & $\mathbf{2 4}$ & $\mathbf{2 4}$ & $\mathbf{2 4}$ & $\mathbf{2 4}$ & $\mathbf{2 4}$ & $\mathbf{2 4}$ & $\mathbf{2 4}$
\end{tabular}

\section{-Total CER For Canada-}

71 Federal System

72 Public Entities

73 Investor-Owned Entities

74 Other Entities

75 Non-NW Entities

76 Total CER For Canada

$\begin{array}{rrrrrrrrrrrrrrr}0 & 0 & 0 & 0 & 0 & 0 & 0 & 0 & 0 & 0 & 0 & 0 & 0 & 0 & 0 \\ 65 & 65 & 65 & 65 & 66 & 66 & 66 & 66 & 66 & 65 & 65 & 65 & 65 & 65 & 65 \\ 63 & 63 & 63 & 63 & 63 & 63 & 63 & 63 & 63 & 62 & 62 & 62 & 62 & 62 & 63 \\ 8.6 & 8.6 & 8.6 & 8.6 & 8.6 & 8.6 & 8.6 & 8.6 & 8.6 & 8.5 & 8.5 & 8.5 & 8.5 & 8.5 & 8.5 \\ 0 & 0 & 0 & 0 & 0 & 0 & 0 & 0 & 0 & 0 & 0 & 0 & 0 & 0 & 0 \\ 138 & 138 & 138 & 138 & 138 & 138 & 138 & 138 & 138 & 135 & 135 & 135 & 135 & 135 & 137\end{array}$



2003 White Book

Dec

Jan Feb Mar Apr1 Apr16

May

Jun Jul Avg

-BPA Canada-

1 BPA: Regional Totals

$\begin{array}{rrrrrrrrrrrrrrr}\mathbf{0} & \mathbf{0} & \mathbf{0} & \mathbf{0} & \mathbf{0} & \mathbf{0} & \mathbf{0} & \mathbf{0} & \mathbf{0} & \mathbf{0} & \mathbf{0} & \mathbf{0} & \mathbf{0} & \mathbf{0} & \mathbf{0} \\ & & & & & & & & & & & & & & \\ 1.3 & 1.3 & 1.3 & 1.3 & 1.2 & 1.2 & 1.2 & 1.2 & 1.2 & 1.2 & 1.2 & 1.2 & 1.2 & 1.2 & 1.2 \\ 0.4 & 0.4 & 0.4 & 0.4 & 0.4 & 0.4 & 0.4 & 0.4 & 0.4 & 0.4 & 0.4 & 0.4 & 0.4 & 0.4 & 0.4 \\ 0 & 0 & 0 & 0 & 0 & 0 & 0 & 0 & 0 & 0 & 0 & 0 & 0 & 0 & 0 \\ 0.4 & 0.4 & 0.4 & 0.4 & 0.3 & 0.3 & 0.3 & 0.3 & 0.3 & 0.3 & 0.3 & 0.3 & 0.3 & 0.3 & 0.3 \\ 0.1 & 0.1 & 0.1 & 0.1 & 0.1 & 0.1 & 0.1 & 0.1 & 0.1 & 0.1 & 0.1 & 0.1 & 0.1 & 0.1 & 0.1 \\ 0 & 0 & 0 & 0 & 0 & 0 & 0 & 0 & 0 & 0 & 0 & 0 & 0 & 0 & 0 \\ 18 & 18 & 18 & 18 & 18 & 18 & 18 & 18 & 18 & 18 & 18 & 18 & 18 & 18 & 18 \\ 0 & 0 & 0 & 0 & 0 & 0 & 0 & 0 & 0 & 0 & 0 & 0 & 0 & 0 & 0 \\ 0 & 0 & 0 & 0 & 0 & 0 & 0 & 0 & 0 & 0 & 0 & 0 & 0 & 0 & 0 \\ 0.1 & 0.1 & 0.1 & 0.1 & 0.1 & 0.1 & 0.1 & 0.1 & 0.1 & 0.1 & 0.1 & 0.1 & 0.1 & 0.1 & 0.1 \\ 0 & 0 & 0 & 0 & 0 & 0 & 0 & 0 & 0 & 0 & 0 & 0 & 0 & 0 & 0 \\ 0.1 & 0.1 & 0.1 & 0.1 & 0.1 & 0.1 & 0.1 & 0.1 & 0.1 & 0.1 & 0.1 & 0.1 & 0.1 & 0.1 & 0.1 \\ 0.1 & 0.1 & 0.1 & 0.1 & 0.1 & 0.1 & 0.1 & 0.1 & 0.1 & 0.1 & 0.1 & 0.1 & 0.1 & 0.1 & 0.1 \\ 0.1 & 0.1 & 0.1 & 0.1 & 0.1 & 0.1 & 0.1 & 0.1 & 0.1 & 0.1 & 0.1 & 0.1 & 0.1 & 0.1 & 0.1 \\ 0 & 0 & 0 & 0 & 0 & 0 & 0 & 0 & 0 & 0 & 0 & 0 & 0 & 0 & 0 \\ 2.9 & 2.9 & 2.9 & 2.9 & 2.8 & 2.8 & 2.8 & 2.8 & 2.8 & 2.8 & 2.8 & 2.8 & 2.8 & 2.8 & 2.8 \\ 2.9 & 2.9 & 2.9 & 2.9 & 2.8 & 2.8 & 2.8 & 2.8 & 2.8 & 2.8 & 2.8 & 2.8 & 2.8 & 2.8 & 2.8 \\ 1.7 & 1.7 & 1.7 & 1.7 & 1.6 & 1.6 & 1.6 & 1.6 & 1.6 & 1.6 & 1.6 & 1.6 & 1.6 & 1.6 & 1.6 \\ 0 & 0 & 0 & 0 & 0 & 0 & 0 & 0 & 0 & 0 & 0 & 0 & 0 & 0 & 0 \\ 0.7 & 0.7 & 0.7 & 0.7 & 0.7 & 0.7 & 0.7 & 0.7 & 0.7 & 0.7 & 0.7 & 0.7 & 0.7 & 0.7 & 0.7 \\ 0 & 0 & 0 & 0 & 0 & 0 & 0 & 0 & 0 & 0 & 0 & 0 & 0 & 0 & 0 \\ 0.7 & 0.7 & 0.7 & 0.7 & 0.7 & 0.7 & 0.7 & 0.7 & 0.7 & 0.7 & 0.7 & 0.7 & 0.7 & 0.7 & 0.7 \\ 0 & 0 & 0 & 0 & 0 & 0 & 0 & 0 & 0 & 0 & 0 & 0 & 0 & 0 & 0 \\ \mathbf{2 9} & \mathbf{2 9} & \mathbf{2 9} & \mathbf{2 9} & \mathbf{2 9} & \mathbf{2 9} & \mathbf{2 9} & \mathbf{2 9} & \mathbf{2 9} & \mathbf{2 9} & \mathbf{2 9} & \mathbf{2 9} & \mathbf{2 9} & \mathbf{2 9} & \mathbf{2 9}\end{array}$

-Priest Rapids CER for Canada-

2 AVWP to BPA

3 COPD to BPA

4 CWPC to BPA

5 EWEB to BPA

6 FGRV to BPA

7 FREC to BPA

8 GCPD to BPA

9 ICLP to BPA

$10 \mathrm{KITT}$ to BPA

$11 \mathrm{KOOT}$ to BPA

12 LREC to BPA

13 LVE to BPA

14 MCMN to BPA

15 MTFR to BPA

16 NLEC to BPA

17 PGE to BPA

$18 \mathrm{PPL}$ to BPA

19 PSE to BPA

20 RREC to BPA

$21 \mathrm{SCL}$ to BPA

22 SLEC to BPA

23 TPU to BPA

24 UNEC to BPA

25 Priest Rapids: Regional Totals

-Wanapum CER for Canada-

26 AVWP to BPA

27 COPD to BPA

28 CWPC to BPA

29 EWEB to BPA

30 FGRV to BPA

31 FREC to BPA

32 GCPD to BPA

$33 \mathrm{ICLP}$ to BPA

$34 \mathrm{KITT}$ to BPA

$35 \mathrm{KOOT}$ to BPA

36 LREC to BPA

37 LVE to BPA

38 MCMN to BPA

39 MTFR to BPA

40 NLEC to BPA

41 PGE to BPA

$42 \mathrm{PPL}$ to BPA

43 PSE to BPA

44 RREC to BPA

$45 \mathrm{SCL}$ to BPA

46 SLEC to BPA

47 TPU to BPA

48 UNEC to BPA

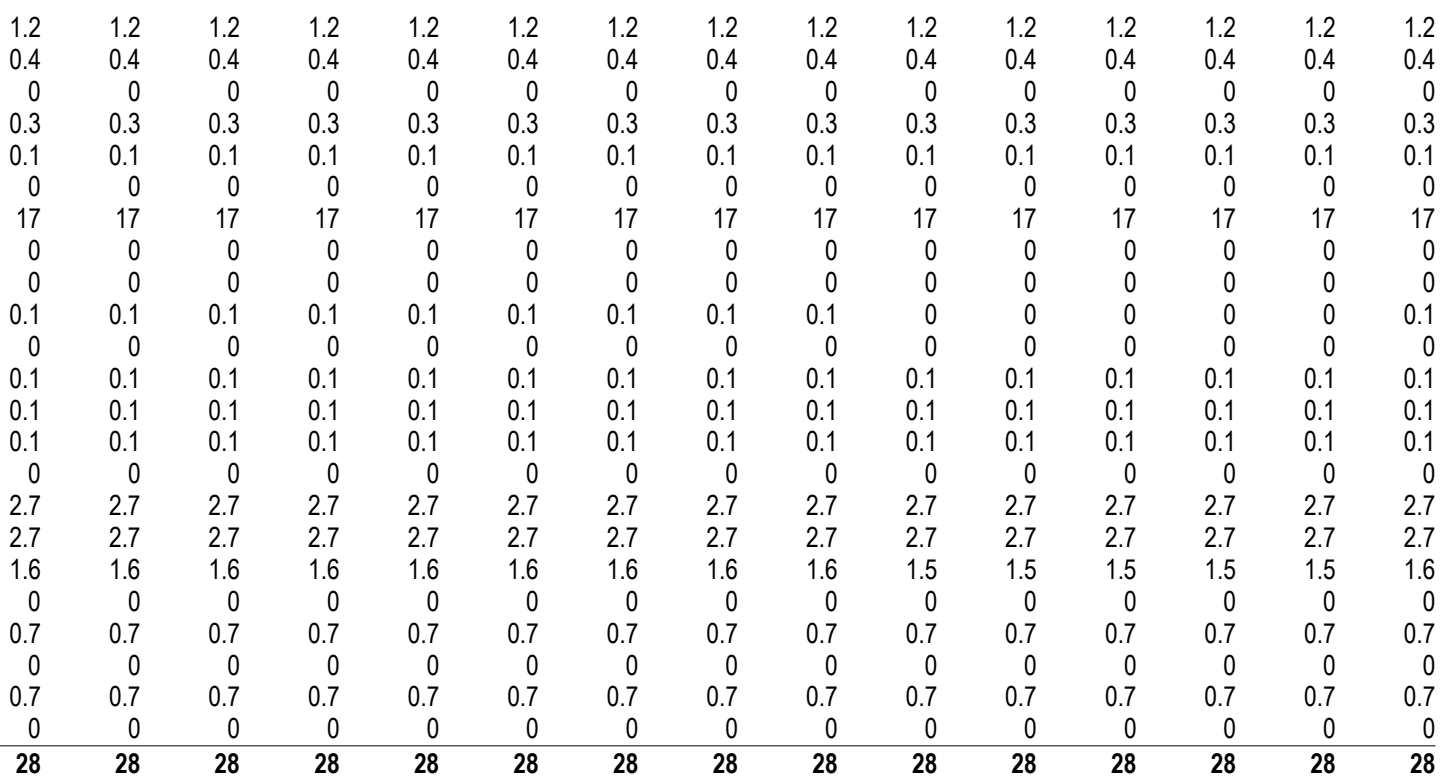

-Rock Island P.H. \#1 CER for Canada-

$50 \mathrm{CHPD}$ to BPA

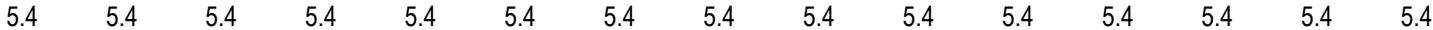
51 PSE to BPA

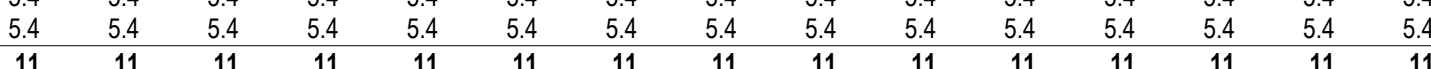

-Rock Island P.H. \#2 CER for Canada-

53 CHPD to BPA

54 PSE to BPA

55 Rock Island P.H. \#2: Regional Totals

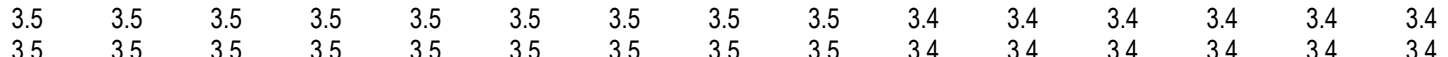

-Rocky Reach CER for Canada-

56 AVWP to BPA

$57 \mathrm{CHPD}$ to BPA

58 CLKM to BPA

59 DOPD to BPA

60 PGE to BPA

$61 \mathrm{PPL}$ to BPA

62 PSE to BPA

$\begin{array}{llll}6.9 & 6.9 & 6.9 & 6.9\end{array}$

63 Rocky Reach: Regional Totals

\begin{tabular}{|c|c|c|c|c|c|c|c|c|c|c|c|c|c|c|}
\hline 1.1 & 1.1 & 1.1 & 1.1 & 1.1 & 1.1 & 1.1 & 1.1 & 1.1 & 1.1 & 1.1 & 1.1 & 1.1 & 1.1 & 1.1 \\
\hline 5.6 & 5.6 & 5.6 & 5.6 & 5.6 & 5.6 & 5.6 & 5.6 & 5.6 & 5.5 & 5.5 & 5.5 & 5.5 & 5.5 & 5.5 \\
\hline 3.5 & 8.5 & 8.5 & 8.5 & 8.5 & 8.5 & 8.5 & 8.5 & 8.5 & 8.3 & 8.3 & 8.3 & 8.3 & 8.3 & 8.4 \\
\hline .0 & 1.0 & 1.0 & 1.0 & 1.0 & 1.0 & 1.0 & 1.0 & 1.0 & 1.0 & 1.0 & 1.0 & 1.0 & 1.0 & 1.0 \\
\hline 4.5 & 4.5 & 4.5 & 4.5 & 4.5 & 4.5 & 4.5 & 4.5 & 4.5 & 4.4 & 4.4 & 4.4 & 4.4 & 4.4 & 4.4 \\
\hline 2.0 & 2.0 & 2.0 & 2.0 & 2.0 & 2.0 & 2.0 & 2.0 & 2.0 & 1.9 & 1.9 & 1.9 & 1.9 & 1.9 & 1.9 \\
\hline 14 & 14 & 14 & 14 & 14 & 14 & 14 & 14 & 14 & 14 & 14 & 14 & 14 & 14 & 14 \\
\hline & 37 & 37 & 37 & 37 & 37 & 37 & 37 & 37 & 36 & 36 & 36 & 36 & 36 & 37 \\
\hline
\end{tabular}


Table A-15: Canadian Entitlement Return For Canada

PNW Loads and Resources Study

2012 - 2013 Operating Year

2003 White Book

May Jun Jul Avg

Average Energy in Megawatts

Aug1 Aug16

Sep

Oct Nov Dec Jan Feb Mar Apr1 Apr16

64 AVWP to BPA

65 DOPD to BPA

66 OKPD to BPA

67 PGE to BPA

$68 \mathrm{PPL}$ to BPA

69 PSE to BPA

70 Wells: Regional Totals

$\begin{array}{lllllllll}0.8 & 0.8 & 0.8 & 0.8 & 0.8 & 0.8 & 0.8 & 0.8 & 0.8\end{array}$

$\begin{array}{lll}0.8 & 0.8 & 0.8 \\ 1.9 & 7.1 & 7.1\end{array}$

$1.9 \quad 1.9$

7.1

7.1

7.1

0.8
7.1
1.9

7.1

0.8
7.1

7.1

0.8

0.8
7

0.8

$\begin{array}{llll}0.8 & 0.8 & 0.8\end{array}$

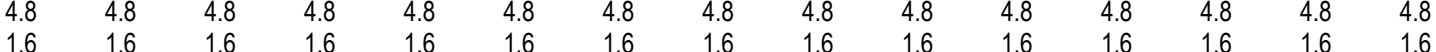

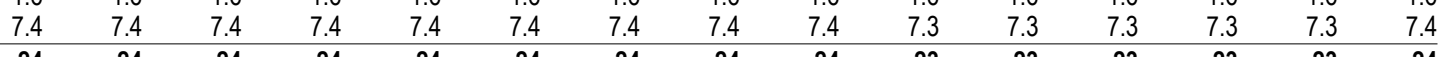

-Total CER For Canada-

71 Federal System

72 Public Entities

73 Investor-Owned Entities

74 Other Entities

75 Non-NW Entities

76 Total CER For Canada

$\begin{array}{rrrrrrrrrrrrrrr}0 & 0 & 0 & 0 & 0 & 0 & 0 & 0 & 0 & 0 & 0 & 0 & 0 & 0 & 0 \\ 65 & 65 & 65 & 65 & 65 & 65 & 65 & 65 & 65 & 64 & 64 & 64 & 64 & 64 & 65 \\ 62 & 62 & 62 & 62 & 62 & 62 & 62 & 62 & 62 & 61 & 61 & 61 & 61 & 61 & 62 \\ 8.5 & 8.5 & 8.5 & 8.5 & 8.5 & 8.5 & 8.5 & 8.5 & 8.5 & 8.3 & 8.3 & 8.3 & 8.3 & 8.3 & 8.4 \\ 0 & 0 & 0 & 0 & 0 & 0 & 0 & 0 & 0 & 0 & 0 & 0 & 0 & 0 & 0 \\ 135 & 135 & 135 & 135 & 135 & 135 & 135 & 135 & 135 & 134 & 134 & 134 & 134 & 134 & 135\end{array}$



2003 White Book

-Priest Rapids CER for Canada-

2 AVWP to BPA

3 COPD to BPA

4 CWPC to BPA

5 EWEB to BPA

6 FGRV to BPA

7 FREC to BPA

8 GCPD to BPA

9 ICLP to BPA

$10 \mathrm{KITT}$ to BPA

$11 \mathrm{KOOT}$ to BPA

12 LREC to BPA

13 LVE to BPA

14 MCMN to BPA

15 MTFR to BPA

16 NLEC to BPA

17 PGE to BPA

$18 \mathrm{PPL}$ to $\mathrm{BPA}$

19 PSE to BPA

20 RREC to BPA

$21 \mathrm{SCL}$ to BPA

22 SLEC to BPA

23 TPU to BPA

24 UNEC to BPA

25 Priest Rapids: Regional Totals

-Wanapum CER for Canada-

26 AVWP to BPA

27 COPD to BPA

28 CWPC to BPA

29 EWEB to BPA

30 FGRV to BPA

31 FREC to BPA

32 GCPD to BPA

33 ICLP to BPA

$34 \mathrm{KITT}$ to BPA

$35 \mathrm{KOOT}$ to BPA

36 LREC to BPA

37 LVE to BPA

38 MCMN to BPA

39 MTFR to BPA

40 NLEC to BPA

41 PGE to BPA

$42 \mathrm{PPL}$ to BPA

43 PSE to BPA

44 RREC to BPA

$45 \mathrm{SCL}$ to BPA

46 SLEC to BPA

47 TPU to BPA

48 UNEC to BPA

49 Wanapum: Regional Totals

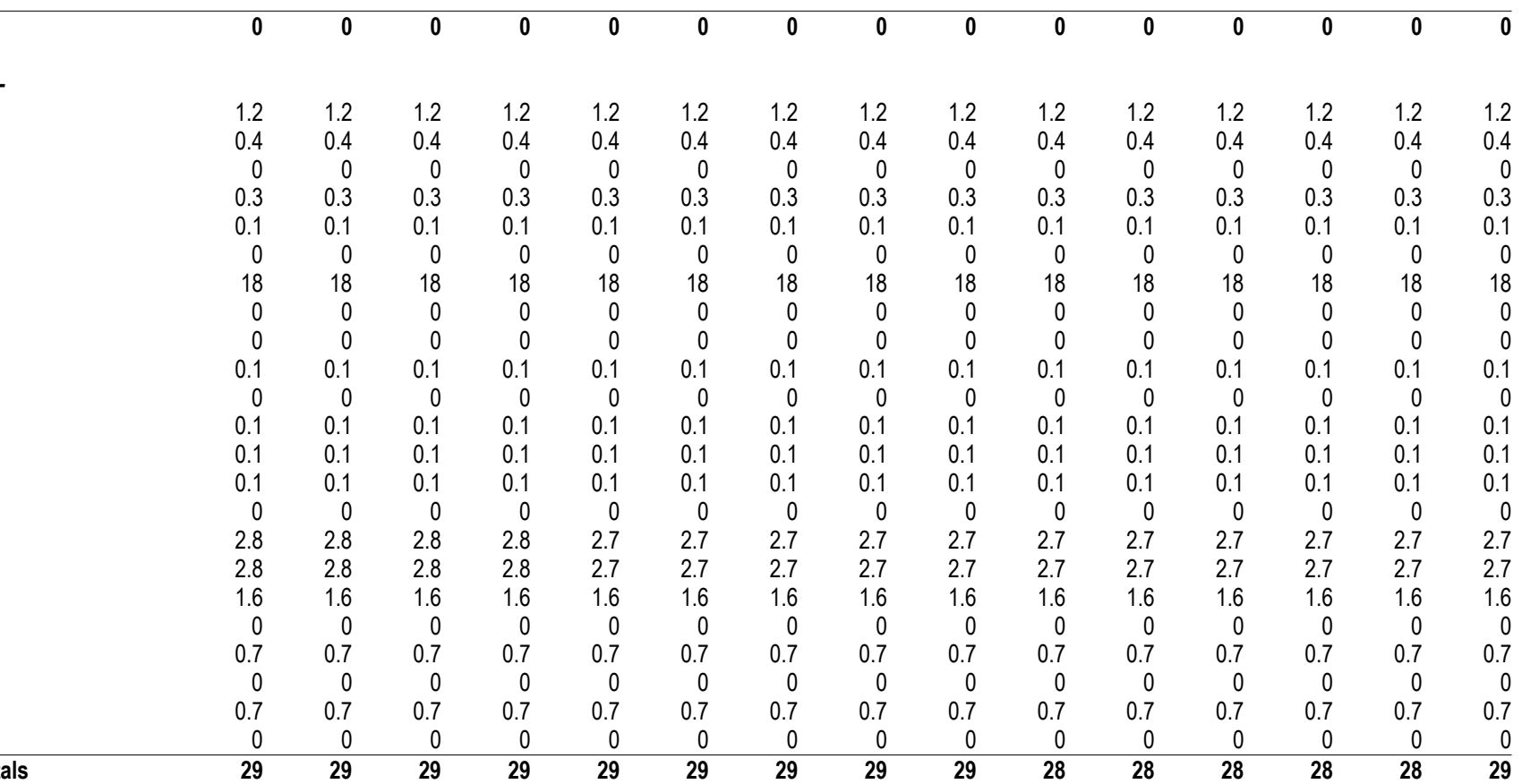

\begin{tabular}{rrrrrrr}
1.2 & 1.2 & 1.2 & 1.2 & 1.1 & 1.1 & 1.1 \\
0.4 & 0.4 & 0.4 & 0.4 & 0.4 & 0.4 & 0.4 \\
0 & 0 & 0 & 0 & 0 & 0 & 0 \\
0.3 & 0.3 & 0.3 & 0.3 & 0.3 & 0.3 & 0.3 \\
0.1 & 0.1 & 0.1 & 0.1 & 0.1 & 0.1 & 0.1 \\
0 & 0 & 0 & 0 & 0 & 0 & 0 \\
17 & 17 & 17 & 17 & 17 & 17 & 17 \\
0 & 0 & 0 & 0 & 0 & 0 & 0 \\
0 & 0 & 0 & 0 & 0 & 0 & 0 \\
0 & 0 & 0 & 0 & 0 & 0 & 0 \\
0 & 0 & 0 & 0 & 0 & 0 & 0 \\
0.1 & 0.1 & 0.1 & 0.1 & 0.1 & 0.1 & 0.1 \\
0.1 & 0.1 & 0.1 & 0.1 & 0.1 & 0.1 & 0.1 \\
0.1 & 0.1 & 0.1 & 0.1 & 0.1 & 0.1 & 0.1 \\
0 & 0 & 0 & 0 & 0 & 0 & 0 \\
2.7 & 2.7 & 2.7 & 2.7 & 2.6 & 2.6 & 2.6 \\
2.7 & 2.7 & 2.7 & 2.7 & 2.6 & 2.6 & 2.6 \\
1.5 & 1.5 & 1.5 & 1.5 & 1.5 & 1.5 & 1.5 \\
0 & 0 & 0 & 0 & 0 & 0 & 0 \\
0.7 & 0.7 & 0.7 & 0.7 & 0.6 & 0.6 & 0.6 \\
0 & 0 & 0 & 0 & 0 & 0 & 0 \\
0.7 & 0.7 & 0.7 & 0.7 & 0.6 & 0.6 & 0.6 \\
0 & 0 & 0 & 0 & 0 & 0 & 0 \\
\hline 28 & 28 & 28 & 28 & 27 & 27 & 0.17
\end{tabular}

$\begin{array}{rrrrrrrr}1.1 & 1.1 & 1.1 & 1.1 & 1.1 & 1.1 & 1.1 & 1.1 \\ 0.4 & 0.4 & 0.4 & 0.4 & 0.4 & 0.4 & 0.4 & 0.4 \\ 0 & 0 & 0 & 0 & 0 & 0 & 0 & 0 \\ 0.3 & 0.3 & 0.3 & 0.3 & 0.3 & 0.3 & 0.3 & 0.3 \\ 0.1 & 0.1 & 0.1 & 0.1 & 0.1 & 0.1 & 0.1 & 0.1 \\ 0 & 0 & 0 & 0 & 0 & 0 & 0 & 0 \\ 17 & 17 & 17 & 17 & 17 & 17 & 17 & 17 \\ 0 & 0 & 0 & 0 & 0 & 0 & 0 & 0 \\ 0 & 0 & 0 & 0 & 0 & 0 & 0 & 0 \\ 0 & 0 & 0 & 0 & 0 & 0 & 0 & 0 \\ 0 & 0 & 0 & 0 & 0 & 0 & 0 & 0 \\ 0.1 & 0.1 & 0.1 & 0.1 & 0.1 & 0.1 & 0.1 & 0.1 \\ 0.1 & 0.1 & 0.1 & 0.1 & 0.1 & 0.1 & 0.1 & 0.1 \\ 0.1 & 0.1 & 0.1 & 0.1 & 0.1 & 0.1 & 0.1 & 0.1 \\ 0 & 0 & 0 & 0 & 0 & 0 & 0 & 0 \\ 2.6 & 2.6 & 2.6 & 2.6 & 2.6 & 2.6 & 2.6 & 2.6 \\ 2.6 & 2.6 & 2.6 & 2.6 & 2.6 & 2.6 & 2.6 & 2.6 \\ 1.5 & 1.5 & 1.5 & 1.5 & 1.5 & 1.5 & 1.5 & 1.5 \\ 0 & 0 & 0 & 0 & 0 & 0 & 0 & 0 \\ 0.6 & 0.6 & 0.6 & 0.6 & 0.6 & 0.6 & 0.6 & 0.6 \\ 0 & 0 & 0 & 0 & 0 & 0 & 0 & 0 \\ 0.6 & 0.6 & 0.6 & 0.6 & 0.6 & 0.6 & 0.6 & 0.6 \\ 0 & 0 & 0 & 0 & 0 & 0 & 0 & 0 \\ \mathbf{2 7} & \mathbf{2 7} & \mathbf{2 7} & \mathbf{2 7} & \mathbf{2 7} & \mathbf{2 7} & \mathbf{2 7} & \mathbf{2 7}\end{array}$

-Rock Island P.H. \#1 CER for Canada-

$\begin{array}{llllllllllllllll}50 & \mathrm{CHPD} & & \end{array}$ 51 PSE to BPA

\begin{tabular}{rrrrrrrrrrrrrrr}
5.4 & 5.4 & 5.4 & 5.4 & 5.4 & 5.4 & 5.4 & 5.4 & 5.4 & 5.3 & 5.3 & 5.3 & 5.3 & 5.3 & 5.3 \\
5.4 & 5.4 & 5.4 & 5.4 & 5.4 & 5.4 & 5.4 & 5.4 & 5.4 & 5.3 & 5.3 & 5.3 & 5.3 & 5.3 & 5.3 \\
\hline 11 & 11 & 11 & 11 & 11 & 11 & 11 & 11 & 11 & 11 & 11 & 11 & 11 & 11 & 11
\end{tabular}

-Rock Island P.H. \#2 CER for Canada-

53 CHPD to BPA

54 PSE to BPA

55 Rock Island P.H. \#2: Regional Totals

\begin{tabular}{lllllllllllllll}
3.4 & 3.4 & 3.4 & 3.4 & 3.4 & 3.4 & 3.4 & 3.4 & 3.4 & 3.4 & 3.4 & 3.4 & 3.4 & 3.4 & 3.4 \\
3.4 & 3.4 & 3.4 & 3.4 & 3.4 & 3.4 & 3.4 & 3.4 & 3.4 & 3.4 & 3.4 & 3.4 & 3.4 & 3.4 & 3.4 \\
\hline $\mathbf{6 . 8}$ & $\mathbf{6 . 8}$ & $\mathbf{6 . 8}$ & $\mathbf{6 . 8}$ & $\mathbf{6 . 8}$ & $\mathbf{6 . 8}$ & $\mathbf{6 . 8}$ & $\mathbf{6 . 8}$ & $\mathbf{6 . 8}$ & $\mathbf{6 . 7}$ & $\mathbf{6 . 7}$ & $\mathbf{6 . 7}$ & $\mathbf{6 . 7}$ & $\mathbf{6 . 7}$ & $\mathbf{6 . 8}$
\end{tabular}

-Rocky Reach CER for Canada-

56 AVWP to BPA

$57 \mathrm{CHPD}$ to BPA

58 CLKM to BPA

59 DOPD to BPA

60 PGE to BPA

$61 \mathrm{PPL}$ to BPA

62 PSE to BPA

63 Rocky Reach: Regional Totals

\begin{tabular}{|c|c|c|c|c|c|c|c|c|c|c|c|c|c|}
\hline 1.1 & 1.1 & 1.1 & 1.1 & 1.1 & 1.1 & 1.1 & 1.1 & 1 & 1 & 1 & 1 & 1 & 1 \\
\hline 5.5 & 5.5 & 5.5 & 5.5 & 5.5 & 5.5 & 5.5 & 5.5 & 5.4 & 5.4 & 5.4 & 5.4 & 5.4 & 5.5 \\
\hline 8.3 & 8.3 & 8.3 & 8.3 & 8.3 & 8.3 & 8.3 & 8.3 & 8.3 & 8.3 & 8.3 & 8.3 & 8.3 & 8.3 \\
\hline 1.0 & 1.0 & 1.0 & 1.0 & 1.0 & 1.0 & 1.0 & 1.0 & 1.0 & 1.0 & 1.0 & 1.0 & 1.0 & 1.0 \\
\hline 4.4 & 4.4 & 4.4 & 4.4 & 4.4 & 4.4 & 4.4 & 4.4 & 4.3 & 4.3 & 4.3 & 4.3 & 4.3 & 4.4 \\
\hline 1.9 & 1.9 & 1.9 & 1.9 & 1.9 & 1.9 & 1.9 & 1.9 & 1.9 & 1.9 & 1.9 & 1.9 & 1.9 & 1.9 \\
\hline 14 & 14 & 14 & 14 & 14 & 14 & 14 & 14 & 14 & 14 & 14 & 14 & 14 & 14 \\
\hline 36 & 36 & 36 & 36 & 36 & 36 & 36 & 36 & 36 & 36 & 36 & 36 & 36 & 36 \\
\hline
\end{tabular}


Table A-15: Canadian Entitlement Return For Canada

PNW Loads and Resources Study

2013 - 2014 Operating Year

2003 White Book Jul Avg

Average Energy in Megawatts

Aug1 Aug16

Sep Oct Nov Dec Jan Feb Mar Apr1 Apr16

May Jun 64 AVWP to BPA 65 DOPD to BPA 66 OKPD to BPA 67 PGE to BPA $68 \mathrm{PPL}$ to BPA 69 PSE to BPA

70 Wells: Regional Totals

$\begin{array}{rrrr}0.8 & 0.8 & 0.8 & 0.8 \\ 7 & 7 & 7 & \\ 1.9 & 1.9 & 1.9 & 1.9 \\ 4.8 & 4.8 & 4.8 & 4.8 \\ 1.6 & 1.6 & 1.6 & 1.6 \\ 7.3 & 7.3 & 7.3 & 7.3 \\ \mathbf{2 3} & \mathbf{2 3} & \mathbf{2 3} & \mathbf{2 3}\end{array}$

\begin{tabular}{rr}
0.8 & 0.8 \\
7 & \\
1.9 & 1.9 \\
4.8 & 4.8 \\
1.6 & 1.6 \\
7.3 & 7.3 \\
\hline 23 & 23
\end{tabular}

\begin{tabular}{rr}
0.8 & 0.8 \\
7 & 7 \\
1.9 & 1.9 \\
4.8 & 4.8 \\
1.6 & 1.6 \\
7.3 & 7.3 \\
\hline 23 & 23
\end{tabular}

\begin{tabular}{rr}
0.8 & 0.8 \\
7 & \\
1.9 & 1.9 \\
4.8 & 4.8 \\
1.6 & 1.6 \\
7.3 & 7.3 \\
\hline 23 & $\mathbf{2 3}$
\end{tabular}

\begin{tabular}{ll}
0.8 & 0.8 \\
7 & 7.0 \\
.9 & 1.9 \\
.8 & 4.7 \\
.6 & 1.6 \\
.3 & 7.3 \\
\hline 23 & 23
\end{tabular}

\begin{tabular}{rr}
0.8 & 0.8 \\
7.0 & 7.0 \\
1.9 & 1.9 \\
4.7 & 4.7 \\
1.6 & 1.6 \\
7.3 & 7.3 \\
\hline 23 & $\mathbf{2 3}$
\end{tabular}

$\begin{array}{rrrrr}0.8 & 0.8 & 0.8 & 0.8 & 0.8 \\ 7.0 & 7.0 & 7.0 & 7.0 & 7 \\ 1.9 & 1.9 & 1.9 & 1.9 & 1.9 \\ 4.7 & 4.7 & 4.7 & 4.7 & 4.7 \\ 1.6 & 1.6 & 1.6 & 1.6 & 1.6 \\ 7.3 & 7.3 & 7.3 & 7.3 & 7.3 \\ \mathbf{2 3} & \mathbf{2 3} & \mathbf{2 3} & \mathbf{2 3} & \mathbf{2 3}\end{array}$

\section{Total CER For Canada-}

71 Federal System

72 Public Entities

73 Investor-Owned Entities

74 Other Entities

75 Non-NW Entities

76 Total CER For Canada

\begin{tabular}{|c|c|c|c|c|c|c|c|c|c|c|c|c|c|}
\hline 0 & 0 & 0 & 0 & 0 & 0 & 0 & 0 & 0 & 0 & 0 & 0 & 0 & 0 \\
\hline 64 & 64 & 64 & 64 & 64 & 64 & 64 & 64 & 64 & 64 & 64 & 64 & 64 & 64 \\
\hline 8.3 & 8.3 & 8.3 & 8.3 & 8.3 & 8.3 & 8.3 & 8.3 & 8.3 & 8.3 & 8.3 & 8.3 & 8.3 & 8.3 \\
\hline 134 & 134 & 134 & 134 & 134 & 134 & 134 & 134 & 132 & 132 & 132 & 132 & 132 & 133 \\
\hline
\end{tabular}


Table A-16: Regional Intra-Regional Transfers

PNW Loads and Resources Study

2004 - 2005 Operating Year

Sep

Oct Nov

Dec

Jan Feb Mar Apr1 Apr16

May Jun Jul Avg

-Intra-Regional Transfers1 AVWP To BPA WP3Set 2 AVWP To PGE CapS 3 BPA To AVWP WP3Set 4 BPA To EWEB PwrS 5 BPA To Other Entities 6 BPA To PPL S/Pwr/X 7 BPA To PPL S/N/X 8 BPA To PPL CapS 9 BPA To PPL PwrS 10 BPA To PSE WP3Set 11 BPA TO PSE PwrS 12 CHPD To PGE $\mathrm{C} / \mathrm{N} / \mathrm{X}$ 13 CIDF To UPL PwrS 14 FPLEV To PGE PwrS 15 GCPD To PPL PwrS 16 NWCP To PSE PwrS 17 NWE To MVP PwrS 18 NWE To PSE PwrS 19 Other Entities To AVWP 20 Other Entities To BPA 21 Other Entities To GHPD 22 PGE To AVWP PkRepl 23 PGE To CHPD PkRepl 24 PGE To CHPD XchgNrg 25 PGE To PPL Cove Repl 26 PKE To SCL PwrS 27 POPD To SCL PwrS 28 PPL To AVWP S/Pwr/X 29 PPL To BPA S/N/X 30 PPL To BPA PkRepl 31 PPL To BPA S/Pwr/X 32 PPL To FLHD PwrS 33 PPL To SUB PwrS 34 PSE To BPA WP3Set 35 SCL To POPD PwrS 36 SHPD To PSE PwrS 37 TAUC To AVWP PwrS 38 TPU To BPA PwrX 39 WSPE To PGE PwrS 40 Total Contracts

-Total Contracts Out-

41 Federal System

42 Public Entities

43 Investor-Owned Entities

44 Other Entities

45 Total Contracts Out

-Total Contracts In-

46 Federal System

47 Public Entities

48 Investor-Owned Entities

49 Other Entities

\begin{tabular}{|c|c|c|c|c|c|c|c|c|c|c|c|c|c|c|}
\hline 0 & 0 & 56 & 56 & 56 & 56 & 56 & 56 & 56 & 56 & 56 & 0 & 56 & 0 & 42 \\
\hline 45 & 45 & 45 & 45 & 45 & 45 & 45 & 45 & 45 & 45 & 45 & 45 & 45 & 45 & 45 \\
\hline 0 & 0 & 0 & 0 & 101 & 101 & 101 & 101 & 50 & 49 & 50 & 0 & 0 & 0 & 42 \\
\hline 2 & 2 & 2 & 2 & 2 & 2 & 2 & 2 & 2 & 2 & 2 & 2 & 2 & 2 & 2 \\
\hline 1050 & 1077 & 223 & 54 & 54 & 54 & 54 & 54 & 54 & 3.5 & 3.5 & 3.5 & 3.5 & 54 & 139 \\
\hline 0 & 0 & 0 & 0 & 0 & 0 & 0 & 0 & 0 & 0 & 0 & 0 & 35 & 34 & 5.7 \\
\hline 0 & 0 & 0 & 0 & 0 & 0 & 0 & 0 & 0 & 0 & 0 & 0 & 0 & 0 & 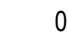 \\
\hline 184 & 184 & 194 & 183 & 229 & 187 & 211 & 199 & 177 & 173 & 173 & 167 & 174 & 159 & 186 \\
\hline 13 & 13 & 42 & 108 & 161 & 215 & 189 & 145 & 117 & 90 & 90 & 0.1 & 18 & 34 & 94 \\
\hline 0 & 0 & 0 & 0 & 101 & 101 & 101 & 101 & 50 & 49 & 50 & 0 & 0 & 0 & 42 \\
\hline 0 & 0 & 0 & 0 & 0 & 0 & 0 & 0 & 0 & 0 & 0 & 0 & 0 & 0 & 0 \\
\hline 24 & 24 & 24 & 24 & 0 & 0 & 0 & 0 & 0 & 0 & 0 & 0 & 24 & 24 & 10 \\
\hline 15 & 15 & 0 & 0 & 0 & 0 & 0 & 0 & 0 & 0 & 0 & 0 & 17 & 19 & 4.3 \\
\hline 7.4 & 7.4 & 7.4 & 7.4 & 7.4 & 7.4 & 7.4 & 7.4 & 7.4 & 7.4 & 7.4 & 7.4 & 7.4 & 7.4 & 7.4 \\
\hline 13 & 13 & 9.9 & 7.9 & 6.6 & 8.2 & 8.6 & 7.4 & 7.8 & 10 & 10 & 13 & 14 & 14 & 10.0 \\
\hline 4.8 & 4.8 & 4.9 & 5 & 4.8 & 2.8 & 3.7 & 3.7 & 4.5 & 4.6 & 4.6 & 4.6 & 4.7 & 4.8 & 4.4 \\
\hline 11 & 11 & 11 & 11 & 7.5 & 7.5 & 7.5 & 7.5 & 7.5 & 11 & 11 & 11 & 11 & 11 & 9.6 \\
\hline 89 & 89 & 89 & 89 & 89 & 89 & 89 & 89 & 89 & 89 & 89 & 89 & 89 & 89 & 89 \\
\hline 100 & 100 & 100 & 100 & 100 & 100 & 100 & 100 & 100 & 100 & 100 & 100 & 100 & 100 & 100 \\
\hline 1230 & 1232 & 1259 & 1203 & 1203 & 1203 & 1203 & 1203 & 1204 & 953 & 553 & 552 & 554 & 1202 & 1064 \\
\hline 15 & 15 & 15 & 15 & 15 & 15 & 15 & 15 & 15 & 15 & 15 & 15 & 15 & 15 & 15 \\
\hline 45 & 45 & 45 & 45 & 45 & 45 & 45 & 45 & 45 & 45 & 45 & 45 & 45 & 45 & 45 \\
\hline 18 & 18 & 18 & 18 & 0 & 0 & 0 & 0 & 0 & 0 & 0 & 0 & 18 & 17 & 7.4 \\
\hline 0 & 0 & 0 & 0 & 25 & 25 & 25 & 25 & 0 & 0 & 0 & 0 & 25 & 25 & 12 \\
\hline 1 & 1 & 1 & 1 & 1 & 1 & 1 & 1 & 1 & 1 & 1 & 1 & 1 & 1 & \\
\hline 95 & 95 & 95 & 95 & 95 & 95 & 95 & 95 & 95 & 95 & 95 & 95 & 95 & 95 & 95 \\
\hline 9 & 9 & 9 & 9 & 9 & 9 & 9 & 9 & 9 & 9 & 9 & 9 & 9 & 9 & \\
\hline 0 & 0 & 0 & 0 & 0 & 0 & 0 & 0 & 0 & 0 & 0 & 0 & 0 & 0 & 0 \\
\hline 0 & 0 & 0 & 0 & 0 & 0 & 0 & 0 & 0 & 0 & 0 & 0 & 0 & 0 & 0 \\
\hline 184 & 184 & 194 & 183 & 229 & 187 & 211 & 199 & 177 & 173 & 173 & 167 & 174 & 159 & 186 \\
\hline 0 & 0 & 23 & 23 & 23 & 0 & 0 & 0 & 0 & 0 & 0 & 0 & 0 & 0 & 5.8 \\
\hline 16 & 16 & 16 & 16 & 16 & 16 & 16 & 16 & 16 & 16 & 16 & 16 & 16 & 16 & 16 \\
\hline 34 & 34 & 30 & 30 & 34 & 34 & 30 & 27 & 27 & 27 & 27 & 24 & 24 & 24 & 28 \\
\hline 0 & 0 & 56 & 56 & 56 & 56 & 56 & 56 & 56 & 56 & 56 & 0 & 56 & 0 & 42 \\
\hline 32 & 32 & 32 & 32 & 32 & 32 & 32 & 32 & 32 & 32 & 32 & 32 & 32 & 32 & 32 \\
\hline 7.4 & 7.4 & 8.3 & 9.2 & 12 & 16 & 15 & 14 & 12 & 10 & 10 & 8.7 & 7.9 & 7.3 & 11 \\
\hline 188 & 188 & 188 & 188 & 188 & 188 & 188 & 188 & 188 & 179 & 94 & 94 & 161 & 188 & 174 \\
\hline 35 & 35 & 35 & 0 & 0 & 0 & 35 & 35 & 35 & 35 & 35 & 35 & 35 & 35 & 0 \\
\hline 42 & 41 & 41 & 35 & 36 & 40 & 44 & 40 & 40 & 38 & 64 & 42 & 46 & 41 & 41 \\
\hline 3508 & 3538 & 2873 & 2650 & 2981 & 2938 & 2996 & 2917 & 2719 & 2374 & 1916 & 1577 & 1915 & 2506 & 2645 \\
\hline
\end{tabular}

\begin{tabular}{rrrrrrrrrrrrrrr}
1248 & 1277 & 461 & 346 & 647 & 660 & 658 & 602 & 450 & 367 & 368 & 172 & 232 & 282 & 512 \\
140 & 140 & 123 & 88 & 64 & 68 & 104 & 101 & 101 & 102 & 102 & 102 & 144 & 145 & 107 \\
837 & 839 & 965 & 953 & 1006 & 942 & 963 & 947 & 900 & 890 & 805 & 684 & 913 & 812 & 898 \\
1283 & 1283 & 1324 & 1263 & 1263 & 1267 & 1271 & 1267 & 1269 & 1015 & 641 & 618 & 625 & 1268 & 1129 \\
\hline $\mathbf{3 5 0 8}$ & $\mathbf{3 5 3 8}$ & $\mathbf{2 8 7 3}$ & $\mathbf{2 6 5 0}$ & $\mathbf{2 9 8 1}$ & $\mathbf{2 9 3 8}$ & $\mathbf{2 9 9 6}$ & $\mathbf{2 9 1 7}$ & $\mathbf{2 7 1 9}$ & $\mathbf{2 3 7 4}$ & $\mathbf{1 9 1 6}$ & $\mathbf{1 5 7 7}$ & $\mathbf{1 9 1 5}$ & $\mathbf{2 5 0 6}$ & $\mathbf{2 6 4 5}$
\end{tabular}

\begin{tabular}{|c|c|c|c|c|c|c|c|c|c|c|c|c|c|}
\hline 1463 & 1634 & 1532 & 1575 & 1510 & 1569 & 1557 & 1536 & 1284 & 884 & 765 & 887 & 1407 & 1376 \\
\hline 253 & 217 & 217 & 228 & 228 & 225 & 221 & 196 & 196 & 196 & 193 & 236 & 235 & 221 \\
\hline 895 & 841 & 851 & 1129 & 1150 & 1152 & 1089 & 937 & 893 & 836 & 619 & 792 & 815 & 928 \\
\hline 928 & 181 & 50 & 50 & 50 & 50 & 50 & 50 & 0 & 0 & 0 & 0 & 50 & 120 \\
\hline 3538 & 2873 & 2650 & 2981 & 2938 & 2996 & 2917 & 2719 & 2374 & 1916 & 1577 & 915 & 2506 & 2645 \\
\hline
\end{tabular}



2003 White Book

-Intra-Regional Transfers1 AVWP To BPA WP3Set 2 AVWP To PGE CapS 3 BPA To AVWP WP3Set 4 BPA To EWEB PwrS 5 BPA To Other Entities 6 BPA To PPL PwrS 7 BPA To PPL CapS 8 BPA To PPL S/Pwr/X 9 BPA To PPL S/N/X 10 BPA To PSE WP3Set 11 BPA TO PSE PwrS 12 CHPD TO PGE C/N/X 13 CIDF To UPL PwrS 14 FPLEV TO PGE PwrS 15 GCPD To AVWP PwrS 16 GCPD To COPD PwrS 17 GCPD To EWEB PwrS 18 GCPD To FGRV PwrS 19 GCPD To FREC PwrS 20 GCPD To KITT PwrS 21 GCPD To KOOT PwrS 22 GCPD To LVE PwrS 23 GCPD To LVE PwrS 24 GCPD To MCMN PwrS 25 GCPD To MTFR PwrS 26 GCPD To PGE PwrS 27 GCPD To PPL PwrS 28 GCPD To PPL PwrS 29 GCPD To PSE PwrS 30 GCPD To TPU PwrS 31 NWCP To PSE PwrS 32 NWE TO MVP PwrS 33 NWE TO PSE PWrS 34 Other Entities To AVWP 35 Other Entities To BPA 36 Other Entities To GHPD 37 PGE To AVWP PkRepl 38 PGE To CHPD PkRepl 39 PGE To CHPD XchgNrg 40 PGE To PPL Cove Repl 41 PKE To SCL PwrS 42 PPL To BPA S/Pwr/X 43 PPL To BPA S/N/X 44 PPL To BPA PkRepl 45 PPL To FLHD PwrS 46 PPL To SUB PwrS 47 PSE To BPA WP3Set 48 SCL To POPD PwrS 49 SHPD To PSE PwrS 50 TAUC To AVWP PwrS 51 TPU To BPA PwrX 52 WSPE To PGE PwrS 53 Total Contracts

\begin{tabular}{|c|c|c|c|c|c|c|c|c|c|c|c|c|c|c|}
\hline 0 & 0 & 56 & 56 & 56 & 56 & 56 & 56 & 56 & 56 & 56 & 0 & 56 & 0 & 42 \\
\hline 45 & 45 & 45 & 45 & 45 & 45 & 45 & 45 & 45 & 45 & 45 & 45 & 45 & 45 & 45 \\
\hline 0 & 0 & 0 & 0 & 101 & 101 & 101 & 101 & 50 & 49 & 50 & 0 & 0 & 0 & 42 \\
\hline 2 & 2 & 2 & 2 & 2 & 2 & 2 & 2 & 2 & 2 & 2 & 2 & 2 & 2 & 2 \\
\hline 13 & 13 & 42 & 108 & 161 & 215 & 189 & 145 & 117 & 90 & 90 & 0.1 & 18 & 34 & 94 \\
\hline 184 & 184 & 194 & 183 & 229 & 187 & 211 & 199 & 177 & 173 & 173 & 167 & 174 & 159 & 186 \\
\hline 0 & 0 & 0 & 0 & 0 & 0 & 0 & 0 & 0 & 0 & 0 & 0 & 35 & 34 & 5.7 \\
\hline 0 & 0 & 0 & 0 & 101 & 101 & 101 & 101 & 50 & 49 & 50 & 0 & 0 & 0 & 42 \\
\hline 0 & 0 & 0 & 0 & 0 & 0 & 0 & 0 & 0 & 0 & 0 & 0 & 0 & 0 & 0 \\
\hline 24 & 24 & 24 & 24 & 0 & 0 & 0 & 0 & 0 & 0 & 0 & 0 & 24 & 24 & 10 \\
\hline 15 & 15 & 0 & 0 & 0 & 0 & 0 & 0 & 0 & 0 & 0 & 0 & 17 & 19 & 4.3 \\
\hline 7.4 & 7.4 & 7.4 & 7.4 & 7.4 & 7.4 & 7.4 & 7.4 & 7.4 & 7.4 & 7.4 & 7.4 & 7.4 & 7.4 & 7.4 \\
\hline 0 & 0 & 0 & 0 & 15 & 15 & 15 & 15 & 14 & 23 & 23 & 29 & 30 & 26 & 15 \\
\hline 0 & 0 & 0 & 0 & 0 & 0 & 0 & 0 & 0 & 1 & 1 & 1 & 1 & 1 & 0.3 \\
\hline 0 & 0 & 0 & 0 & 1 & 1 & 1 & 1 & 1 & 1 & 1 & 2 & 2 & 1 & 0.9 \\
\hline 0 & 0 & 0 & 0 & 1 & 1 & 1 & 1 & 1 & 1 & 1 & 1 & 1 & 1 & 0.8 \\
\hline 0 & 0 & 0 & 0 & 1 & 1 & 1 & 1 & 1 & 1 & 1 & 1 & 1 & 1 & 0.8 \\
\hline 0 & 0 & 0 & 0 & 1 & 1 & 1 & 1 & 1 & 1 & 1 & 1 & 1 & 1 & 0.8 \\
\hline 0 & 0 & 0 & 0 & 1 & 1 & 1 & 1 & 1 & 2 & 2 & 2 & 3 & 2 & 1.2 \\
\hline 0 & 0 & 0 & 0 & 1 & 1 & 1 & 1 & 1 & 2 & 2 & 2 & 3 & 2 & 1.2 \\
\hline 0 & 0 & 0 & 0 & 33 & 33 & 33 & 33 & 33 & 53 & 53 & 67 & 68 & 59 & 34 \\
\hline 13 & 13 & 9.9 & 7.9 & 6.6 & 8.2 & 8.6 & 7.4 & 7.8 & 10 & 10 & 13 & 14 & 14 & 10.0 \\
\hline 0 & 0 & 0 & 0 & 33 & 33 & 33 & 33 & 33 & 53 & 53 & 67 & 68 & 59 & 34 \\
\hline 0 & 0 & 0 & 0 & 19 & 19 & 19 & 19 & 19 & 31 & 31 & 39 & 39 & 34 & 20 \\
\hline 18 & 18 & 18 & 18 & 0 & 0 & 0 & 0 & 0 & 0 & 0 & 0 & 18 & 17 & 7.4 \\
\hline 0 & 0 & 0 & 0 & 25 & 25 & 25 & 25 & 0 & 0 & 0 & 0 & 25 & 25 & 12 \\
\hline 1 & 1 & 1 & 1 & 1 & 1 & 1 & 1 & 1 & 1 & 1 & 1 & 1 & 1 & 1 \\
\hline 95 & 95 & 95 & 95 & 95 & 95 & 95 & 95 & 95 & 95 & 95 & 95 & 95 & 95 & 95 \\
\hline 0 & 0 & 23 & 23 & 23 & 0 & 0 & 0 & 0 & 0 & 0 & 0 & 0 & 0 & 5.8 \\
\hline 0 & 0 & 0 & 0 & 0 & 0 & 0 & 0 & 0 & 0 & 0 & 0 & 0 & 0 & 0 \\
\hline 184 & 184 & 194 & 183 & 229 & 187 & 211 & 199 & 177 & 173 & 173 & 167 & 174 & 159 & 186 \\
\hline 16 & 16 & 16 & 16 & 16 & 16 & 16 & 16 & 16 & 16 & 16 & 16 & 16 & 16 & 16 \\
\hline 34 & 34 & 30 & 30 & 34 & 34 & 30 & 27 & 27 & 27 & 27 & 24 & 24 & 24 & 28 \\
\hline 0 & 0 & 56 & 56 & 56 & 56 & 56 & 56 & 56 & 56 & 56 & 0 & 56 & 0 & 42 \\
\hline 43 & 43 & 43 & 43 & 43 & 43 & 43 & 43 & 43 & 43 & 43 & 43 & 43 & 43 & 43 \\
\hline 7.4 & 7.4 & 8.3 & 9.2 & 12 & 16 & 15 & 14 & 12 & 10 & 10 & 8.7 & 7.9 & 7.3 & 11 \\
\hline 188 & 188 & 188 & 188 & 188 & 188 & 188 & 188 & 188 & 188 & 188 & 100 & 188 & 188 & 181 \\
\hline 35 & 35 & 35 & 0 & 0 & 0 & 35 & 35 & 35 & 35 & 35 & 35 & 35 & 35 & 26 \\
\hline 42 & 41 & 41 & 35 & 36 & 40 & 44 & 40 & 40 & 38 & 64 & 42 & 46 & 41 & 41 \\
\hline 2488 & 2489 & 2650 & 2650 & 3115 & 3071 & 2728 & 2652 & 2450 & 2194 & 2220 & 1850 & 2122 & 2340 & 2527 \\
\hline
\end{tabular}

-Total Contracts Out-

54 Federal System

55 Public Entities

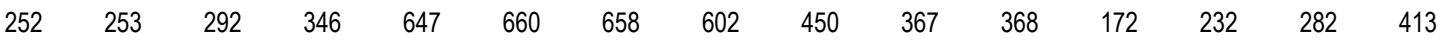
56 Investor-Owned Entities

57 Other Entities

$142 \quad 142$

125

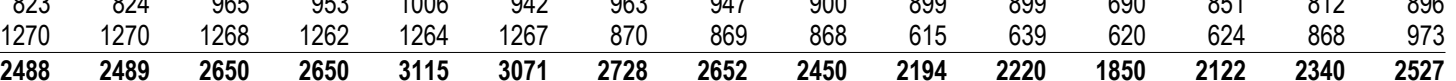

-Total Contracts In-

59 Federal System

60 Public Entities

61 Investor-Owned Entities

62 Other Entities

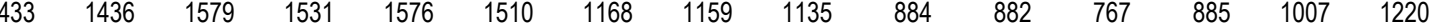

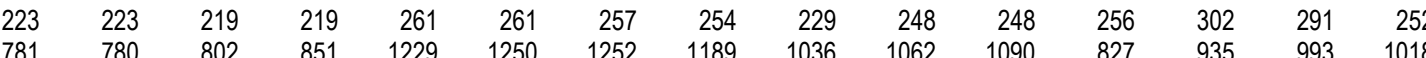

63 Total Contracts In

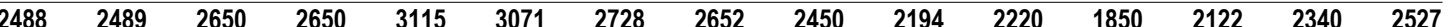



2003 White Book

Sep

Oct Nov

Dec

Jan Feb Mar Apr1 Apr16

May

Jun

Jul Avg

-Intra-Regional Transfers.

1 AVWP To BPA WP3Set

2 AVWP To PGE CapS

3 BPA To AVWP WP3Set

4 BPA To EWEB PwrS

5 BPA To Other Entities

6 BPA To PPL S/N/X

7 BPA To PPL PwrS

8 BPA To PPL CapS

9 BPA To PPL S/Pwr/X

10 BPA To PSE WP3Set

11 BPA TO PSE PwrS

13 CIDF To UPL PwrS

14 FPLEV To PGE PwrS

15 GCPD To AVWP PwrS

16 GCPD To COPD PwrS

17 GCPD To EWEB PwrS

18 GCPD To FGRV PwrS

19 GCPD To FREC PwrS

20 GCPD To KITT PwrS

21 GCPD To KOOT PwrS

22 GCPD To LVE PwrS

23 GCPD To LVE PwrS

24 GCPD To MCMN PwrS

25 GCPD To MTFR PwrS

26 GCPD To PGE PwrS

27 GCPD To PPL PwrS

28 GCPD To PPL PwrS

29 GCPD To PSE PwrS

30 GCPD To TPU PwrS

31 NWCP To PSE PwrS

32 NWE TO MVP PwrS

33 NWE TO PSE PwrS

34 Other Entities To AVWP

35 Other Entities To BPA

36 Other Entities To GHPD

37 PGE To AVWP PkRepl

38 PGE To CHPD PkRepl

39 PGE To CHPD XchgNrg

40 PGE To PPL Cove Repl

41 PKE To SCL PwrS

42 PPL To BPA S/Pwr/X

43 PPL To BPA PkRepl

44 PPL To BPA S/N/X

45 PPL To FLHD PwrS

46 PPL To SUB PwrS

47 PSE To BPA WP3Set

48 SCL To POPD PwrS

49 SHPD To PSE PwrS

50 TAUC TO AVWP PwrS

51 TPU To BPA PwrX

52 WSPE To PGE PwrS

53 Total Contracts
12 CHPD To PGE C/N/X

\begin{tabular}{|c|c|c|c|c|c|c|c|c|c|c|c|c|c|c|}
\hline 0 & 0 & 56 & 56 & 56 & 56 & 56 & 56 & 56 & 56 & 56 & 0 & 56 & 0 & 42 \\
\hline 45 & 45 & 45 & 45 & 45 & 45 & 45 & 45 & 45 & 45 & 45 & 45 & 45 & 45 & 45 \\
\hline 0 & 0 & 0 & 0 & 101 & 101 & 101 & 101 & 50 & 49 & 50 & 0 & 0 & 0 & 42 \\
\hline 2 & 2 & 2 & 0 & 0 & 0 & 0 & 0 & 0 & 0 & 0 & 0 & 0 & 0 & 0.3 \\
\hline 54 & 54 & 54 & 0 & 0 & 0 & 0 & 0 & 0 & 0 & 0 & 0 & 0 & 0 & 8.9 \\
\hline 0 & 0 & 0 & 0 & 0 & 0 & 0 & 0 & 0 & 0 & 0 & 0 & 0 & 0 & 0 \\
\hline 13 & 13 & 42 & 108 & 161 & 215 & 189 & 145 & 117 & 90 & 90 & 0.1 & 18 & 34 & 94 \\
\hline 184 & 184 & 194 & 183 & 229 & 187 & 211 & 199 & 177 & 173 & 173 & 167 & 174 & 159 & 186 \\
\hline 0 & 0 & 0 & 0 & 0 & 0 & 0 & 0 & 0 & 0 & 0 & 0 & 35 & 34 & 5.7 \\
\hline 0 & 0 & 0 & 0 & 101 & 101 & 101 & 101 & 50 & 49 & 50 & 0 & 0 & 0 & 42 \\
\hline 0 & 0 & 0 & 0 & 0 & 0 & 0 & 0 & 0 & 0 & 0 & 0 & 0 & 0 & 0 \\
\hline 24 & 24 & 24 & 24 & 0 & 0 & 0 & 0 & 0 & 0 & 0 & 0 & 24 & 24 & 10 \\
\hline 15 & 15 & 0 & 0 & 0 & 0 & 0 & 0 & 0 & 0 & 0 & 0 & 17 & 19 & 4.3 \\
\hline 7.4 & 7.4 & 7.4 & 7.4 & 7.4 & 7.4 & 7.4 & 7.4 & 7.4 & 7.4 & 7.4 & 7.4 & 7.4 & 7.4 & 7.4 \\
\hline 18 & 18 & 16 & 19 & 19 & 19 & 17 & 18 & 18 & 26 & 26 & 32 & 32 & 28 & 22 \\
\hline 6 & 6 & 5 & 6 & 6 & 6 & 6 & 6 & 6 & 9 & 9 & 11 & 10 & 9 & 7.2 \\
\hline 5 & 5 & 5 & 5 & 5 & 5 & 5 & 5 & 5 & 7 & 7 & 9 & 9 & 8 & 6.1 \\
\hline 2 & 2 & 1 & 2 & 2 & 2 & 1 & 1 & 2 & 2 & 2 & 3 & 3 & 2 & 1.9 \\
\hline 0 & 0 & 0 & 0 & 1 & 1 & 0 & 0 & 0 & 1 & 1 & 1 & 1 & 1 & 0.5 \\
\hline 1 & 1 & 1 & 1 & 1 & 1 & 1 & 1 & 1 & 1 & 1 & 2 & 2 & 1 & 1.2 \\
\hline 1 & 1 & 1 & 1 & 1 & 1 & 1 & 1 & 1 & 1 & 1 & 1 & 1 & 1 & 1 \\
\hline 1 & 1 & 1 & 1 & 1 & 1 & 1 & 1 & 1 & 1 & 1 & 1 & 1 & 1 & 1 \\
\hline 1 & 1 & 1 & 1 & 1 & 1 & 1 & 1 & 1 & 1 & 1 & 1 & 1 & 1 & 1 \\
\hline 2 & 2 & 1 & 2 & 2 & 2 & 1 & 1 & 2 & 2 & 2 & 3 & 3 & 2 & 1.9 \\
\hline 2 & 2 & 1 & 2 & 2 & 2 & 1 & 1 & 2 & 2 & 2 & 3 & 3 & 2 & 1.9 \\
\hline 41 & 41 & 37 & 43 & 44 & 44 & 40 & 40 & 40 & 60 & 60 & 73 & 72 & 63 & 50 \\
\hline 41 & 41 & 37 & 43 & 44 & 44 & 40 & 40 & 40 & 60 & 60 & 73 & 72 & 63 & 50 \\
\hline 13 & 13 & 9.9 & 7.9 & 6.6 & 8.2 & 8.6 & 7.4 & 7.8 & 10 & 10 & 13 & 14 & 14 & 10.0 \\
\hline 23 & 23 & 21 & 25 & 25 & 25 & 23 & 23 & 23 & 34 & 34 & 42 & 42 & 36 & 28 \\
\hline 19 & 19 & 17 & 20 & 20 & 20 & 18 & 18 & 18 & 27 & 27 & 33 & 33 & 29 & 23 \\
\hline 4.8 & 4.8 & 4.9 & 5 & 4.8 & 2.8 & 3.7 & 3.7 & 4.5 & 4.6 & 4.6 & 4.6 & 4.7 & 4.8 & 4.4 \\
\hline 11 & 11 & 11 & 11 & 7.5 & 7.5 & 7.5 & 7.5 & 7.5 & 11 & 11 & 11 & 11 & 11 & 9.6 \\
\hline 89 & 89 & 89 & 89 & 89 & 89 & 89 & 89 & 89 & 89 & 89 & 89 & 89 & 89 & 89 \\
\hline 100 & 100 & 100 & 100 & 100 & 100 & 100 & 100 & 100 & 100 & 100 & 100 & 100 & 100 & 100 \\
\hline 805 & 803 & 803 & 375 & 375 & 374 & 0 & 0 & 0 & 0 & 0 & 0 & 0 & 0 & 228 \\
\hline 15 & 15 & 15 & 0 & 0 & 0 & 0 & 0 & 0 & 0 & 0 & 0 & 0 & 0 & 2.5 \\
\hline 45 & 45 & 45 & 45 & 45 & 45 & 45 & 45 & 45 & 45 & 45 & 45 & 45 & 45 & 45 \\
\hline 18 & 18 & 18 & 18 & 0 & 0 & 0 & 0 & 0 & 0 & 0 & 0 & 18 & 17 & 7.4 \\
\hline 0 & 0 & 0 & 0 & 25 & 25 & 25 & 25 & 0 & 0 & 0 & 0 & 25 & 25 & 12 \\
\hline 1 & 1 & 1 & 1 & 1 & 1 & 1 & 1 & 1 & 1 & 1 & 1 & 1 & 1 & 1 \\
\hline 95 & 95 & 95 & 95 & 95 & 95 & 0 & 0 & 0 & 0 & 0 & 0 & 0 & 0 & 40 \\
\hline 0 & 0 & 23 & 23 & 23 & 0 & 0 & 0 & 0 & 0 & 0 & 0 & 0 & 0 & 5.8 \\
\hline 184 & 184 & 194 & 183 & 229 & 187 & 211 & 199 & 177 & 173 & 173 & 167 & 174 & 159 & 186 \\
\hline 0 & 0 & 0 & 0 & 0 & 0 & 0 & 0 & 0 & 0 & 0 & 0 & 0 & 0 & 0 \\
\hline 16 & 16 & 16 & 0 & 0 & 0 & 0 & 0 & 0 & 0 & 0 & 0 & 0 & 0 & 2.7 \\
\hline 34 & 34 & 30 & 30 & 34 & 34 & 30 & 27 & 27 & 27 & 27 & 24 & 24 & 24 & 28 \\
\hline 0 & 0 & 56 & 56 & 56 & 56 & 56 & 56 & 56 & 56 & 56 & 0 & 56 & 0 & 42 \\
\hline 43 & 43 & 43 & 43 & 43 & 43 & 43 & 43 & 43 & 43 & 43 & 43 & 43 & 43 & 43 \\
\hline 7.4 & 7.4 & 8.3 & 9.2 & 12 & 16 & 15 & 14 & 12 & 10 & 10 & 8.7 & 7.9 & 7.3 & 11 \\
\hline 188 & 188 & 188 & 188 & 188 & 188 & 188 & 188 & 188 & 188 & 188 & 188 & 100 & 188 & 181 \\
\hline 35 & 35 & 35 & 0 & 0 & 0 & 0 & 0 & 0 & 0 & 0 & 0 & 0 & 0 & 5.8 \\
\hline 42 & 41 & 41 & 35 & 36 & 40 & 44 & 40 & 40 & 38 & 64 & 42 & 46 & 41 & 41 \\
\hline 253 & 2250 & 2395 & 1908 & 2242 & 2198 & 1734 & 1657 & 1461 & 1499 & 1526 & 1242 & 1420 & 1337 & 1780 \\
\hline
\end{tabular}

-Total Contracts Out-

54 Federal System

55 Public Entities

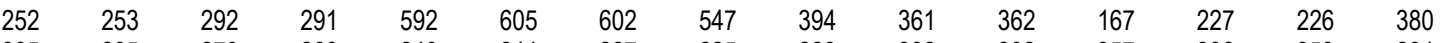

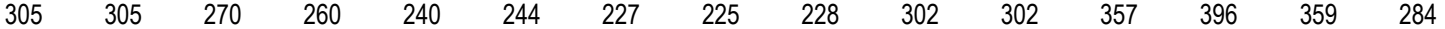

56 Investor-Owned Entities

57 Other Entities

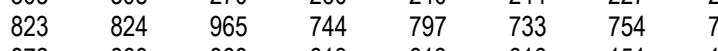

2253

$2250 \quad 2395$

$2395 \quad 1908$

$908 \quad 2242 \quad 2198$

$\begin{array}{rrrr}2198 & 1734 & 1657 & 1461\end{array}$

-

59 Federal System

60 Public Entities

61 Investor-Owned Entities

62 Other Entities

$\begin{array}{rrrrrrrrrrrrrrr}1035 & 1034 & 1179 & 704 & 747 & 681 & 331 & 319 & 297 & 296 & 296 & 178 & 297 & 170 & 519 \\ 263 & 263 & 253 & 227 & 239 & 239 & 134 & 131 & 109 & 124 & 124 & 135 & 177 & 166 & 183 \\ 904 & 903 & 913 & 977 & 1257 & 1278 & 1269 & 1207 & 1055 & 1079 & 1106 & 929 & 946 & 1001 & 1069 \\ 50 & 50 & 50 & 0 & 0 & 0 & 0 & 0 & 0 & 0 & 0 & 0 & 0 & 0 & 8.3 \\ \mathbf{2 2 5 3} & \mathbf{2 2 5 0} & \mathbf{2 3 9 5} & \mathbf{1 9 0 8} & \mathbf{2 2 4 2} & \mathbf{2 1 9 8} & \mathbf{1 7 3 4} & \mathbf{1 6 5 7} & \mathbf{1 4 6 1} & \mathbf{1 4 9 9} & \mathbf{1 5 2 6} & \mathbf{1 2 4 2} & \mathbf{1 4 2 0} & \mathbf{1 3 3 7} & \mathbf{1 7 8 0}\end{array}$


Sep

Oct Nov Dec Jan Feb Mar Apr1 Apr16

-Intra-Regional Transfers1 AVWP To BPA WP3Set 2 AVWP To PGE CapS 3 BPA To AVWP WP3Set 4 BPA To PPL PwrS 5 BPA To PPL CapS 6 BPA To PPL S/Pwr/X 7 BPA TO PPL S/N/X 8 BPA To PSE WP3Set 9 CHPD TO PGE C/N/X 10 CIDF To UPL PwrS 11 FPLEV To PGE PwrS 12 GCPD To AVWP PwrS 13 GCPD To COPD PwrS 14 GCPD To EWEB PwrS 15 GCPD To FGRV PwrS 16 GCPD To FREC PwrS 17 GCPD To KITT PwrS 18 GCPD To KOOT PwrS 19 GCPD To LVE PwrS 20 GCPD To LVE PwrS 21 GCPD To MCMN PwrS 22 GCPD To MTFR PwrS 23 GCPD To PGE PwrS 24 GCPD To PPL PwrS 25 GCPD To PPL PwrS 26 GCPD To PSE PwrS 27 GCPD To TPU PwrS 28 NWCP To PSE PwrS 29 NWE To MVP PwrS

30 NWE To PSE PwrS

31 Other Entities To AVWP 32 PGE To AVWP PkRepl 33 PGE To CHPD XchgNrg 34 PGE To CHPD PkRepl 35 PGE To PPL Cove Repl 36 PPL To BPA S/N/X 37 PPL To BPA PkRepl 38 PPL To BPA S/Pwr/X 39 PPL To SUB PwrS 40 PSE To BPA WP3Set 41 SCL To POPD PwrS 42 SHPD To PSE PwrS 43 TAUC To AVWP PwrS 44 WSPE To PGE PwrS 45 Total Contracts

-Total Contracts Out-

46 Federal System

47 Public Entities

48 Investor-Owned Entities

49 Other Entities

\begin{tabular}{|c|c|c|c|c|c|c|c|c|c|c|c|c|c|c|}
\hline 0 & 0 & 56 & 56 & 56 & 56 & 56 & 56 & 56 & 56 & 56 & 0 & 56 & 0 & 42 \\
\hline 45 & 45 & 45 & 45 & 45 & 45 & 45 & 45 & 45 & 45 & 45 & 45 & 45 & 45 & 45 \\
\hline 0 & 0 & 0 & 0 & 101 & 101 & 101 & 101 & 50 & 49 & 50 & 0 & 0 & 0 & 42 \\
\hline 13 & 13 & 42 & 108 & 161 & 215 & 189 & 145 & 117 & 90 & 90 & 0.1 & 18 & 34 & 94 \\
\hline 184 & 184 & 194 & 183 & 229 & 187 & 211 & 192 & 177 & 173 & 173 & 167 & 174 & 159 & 186 \\
\hline 0 & 0 & 0 & 0 & 0 & 0 & 0 & 0 & 0 & 0 & 0 & 0 & 35 & 34 & 5.7 \\
\hline 0 & 0 & 0 & 0 & 0 & 0 & 0 & 0 & 0 & 0 & 0 & 0 & 0 & 0 & 0 \\
\hline 0 & 0 & 0 & 0 & 101 & 101 & 101 & 101 & 50 & 49 & 50 & 0 & 0 & 0 & 42 \\
\hline 24 & 24 & 24 & 24 & 0 & 0 & 0 & 0 & 0 & 0 & 0 & 0 & 24 & 24 & 10 \\
\hline 15 & 15 & 0 & 0 & 0 & 0 & 0 & 0 & 0 & 0 & 0 & 0 & 17 & 19 & 4.3 \\
\hline 7.4 & 7.4 & 7.4 & 7.4 & 7.4 & 7.4 & 7.4 & 7.4 & 7.4 & 7.4 & 7.4 & 7.4 & 7.4 & 7.4 & 7.4 \\
\hline 20 & 20 & 19 & 19 & 19 & 19 & 17 & 18 & 18 & 26 & 26 & 32 & 32 & 28 & 22 \\
\hline 6 & 6 & 6 & 6 & 6 & 6 & 6 & 6 & 6 & 9 & 9 & 11 & 10 & 9 & .3 \\
\hline 6 & 6 & 5 & 5 & 5 & 5 & 5 & 5 & 5 & 7 & 7 & 9 & 9 & 8 & 6.2 \\
\hline 2 & 2 & 2 & 2 & 2 & 1 & 1 & 1 & 2 & 2 & 2 & 3 & 3 & 2 & 1.9 \\
\hline 1 & 1 & 1 & 0 & 1 & 1 & 0 & 0 & 0 & 1 & 1 & 1 & 1 & 1 & 0.7 \\
\hline 1 & 1 & 1 & 1 & 1 & 1 & 1 & 1 & 1 & 1 & 1 & 2 & 2 & 1 & 1.2 \\
\hline 1 & 1 & 1 & 1 & 1 & 1 & 1 & 1 & 1 & 1 & 1 & 1 & 1 & 1 & \\
\hline 1 & 1 & 1 & 1 & 1 & 1 & 1 & 1 & 1 & 1 & 1 & 1 & 1 & 1 & \\
\hline 1 & 1 & 1 & 1 & 1 & 1 & 1 & 1 & 1 & 1 & 1 & 1 & 1 & 1 & \\
\hline 2 & 2 & 2 & 2 & 2 & 1 & 1 & 1 & 2 & 2 & 2 & 3 & 3 & 2 & 1.9 \\
\hline 2 & 2 & 2 & 2 & 2 & 1 & 1 & 1 & 2 & 2 & 2 & 3 & 3 & 2 & 1.9 \\
\hline 45 & 45 & 43 & 43 & 44 & 44 & 40 & 40 & 40 & 60 & 60 & 73 & 72 & 63 & 51 \\
\hline 13 & 13 & 9.9 & 7.9 & 6.6 & 8.2 & 8.6 & 7.4 & 7.8 & 10 & 10 & 13 & 14 & 14 & 10.0 \\
\hline 45 & 45 & 43 & 43 & 44 & 44 & 40 & 40 & 40 & 60 & 60 & 73 & 72 & 63 & 51 \\
\hline 26 & 26 & 25 & 25 & 25 & 25 & 23 & 23 & 23 & 34 & 34 & 42 & 42 & 36 & 29 \\
\hline 21 & 21 & 20 & 20 & 20 & 20 & 18 & 18 & 18 & 27 & 27 & 33 & 33 & 29 & 23 \\
\hline 4.8 & 4.8 & 4.9 & 5 & 4.8 & 2.8 & 3.7 & 3.7 & 4.5 & 4.6 & 4.6 & 4.6 & 4.7 & 4.8 & 4.4 \\
\hline 11 & 11 & 11 & 11 & 7.5 & 7.5 & 7.5 & 7.5 & 7.5 & 11 & 11 & 11 & 11 & 11 & 9. \\
\hline 89 & 89 & 89 & 89 & 89 & 89 & 89 & 89 & 89 & 89 & 89 & 89 & 89 & 89 & 89 \\
\hline 100 & 100 & 100 & 100 & 100 & 100 & 100 & 100 & 100 & 100 & 100 & 100 & 100 & 100 & 100 \\
\hline 45 & 45 & 45 & 45 & 45 & 45 & 45 & 45 & 45 & 45 & 45 & 45 & 45 & 45 & 45 \\
\hline 0 & 0 & 0 & 0 & 25 & 25 & 25 & 25 & 0 & 0 & 0 & 0 & 25 & 25 & 12 \\
\hline 18 & 18 & 18 & 18 & 0 & 0 & 0 & 0 & 0 & 0 & 0 & 0 & 18 & 17 & 7.4 \\
\hline 1 & 1 & 1 & 1 & 1 & 1 & 1 & 1 & 1 & 1 & 1 & 1 & 1 & 1 & \\
\hline 0 & 0 & 0 & 0 & 0 & 0 & 0 & 0 & 0 & 0 & 0 & 0 & 0 & 0 & 0 \\
\hline 184 & 184 & 194 & 183 & 229 & 187 & 211 & 192 & 177 & 173 & 173 & 167 & 174 & 159 & 186 \\
\hline 0 & 0 & 23 & 23 & 23 & 0 & 0 & 0 & 0 & 0 & 0 & 0 & 0 & 0 & \\
\hline 34 & 34 & 30 & 30 & 34 & 34 & 30 & 27 & 27 & 27 & 27 & 24 & 24 & 24 & 28 \\
\hline 0 & 0 & 56 & 56 & 56 & 56 & 56 & 56 & 56 & 56 & 56 & 0 & 56 & 0 & 42 \\
\hline 43 & 43 & 43 & 43 & 43 & 43 & 43 & 43 & 43 & 43 & 43 & 43 & 43 & 43 & 43 \\
\hline 7.4 & 7.4 & 8.3 & 9.2 & 12 & 16 & 15 & 14 & 12 & 10 & 10 & 8.7 & 7.9 & 7.3 & 11 \\
\hline 188 & 188 & 188 & 188 & 188 & 188 & 188 & 188 & 188 & 188 & 188 & 100 & 188 & 188 & 181 \\
\hline 42 & 41 & 41 & 35 & 36 & 40 & 44 & 40 & 40 & 38 & 64 & 42 & 46 & 41 & \\
\hline 1248 & 1248 & 1402 & 1438 & 1772 & 1726 & 1734 & 1643 & 1461 & 1499 & 1526 & 1154 & 1508 & 1337 & \\
\hline
\end{tabular}

50 Total Contracts Out

-Total Contracts In-

51 Federal System

52 Public Entities

53 Investor-Owned Entities

54 Other Entities

\begin{tabular}{rrrrrrrrrrrrrrr}
197 & 197 & 236 & 291 & 592 & 605 & 602 & 540 & 394 & 361 & 362 & 167 & 227 & 226 & 370 \\
287 & 287 & 262 & 260 & 240 & 241 & 227 & 225 & 228 & 302 & 302 & 357 & 396 & 359 & 282 \\
614 & 615 & 756 & 744 & 797 & 733 & 754 & 731 & 691 & 690 & 690 & 481 & 732 & 603 & 694 \\
150 & 148 & 148 & 143 & 143 & 147 & 151 & 147 & 148 & 145 & 171 & 149 & 154 & 149 & 149 \\
\hline $\mathbf{1 2 4 8}$ & $\mathbf{1 2 4 8}$ & $\mathbf{1 4 0 2}$ & $\mathbf{1 4 3 8}$ & $\mathbf{1 7 7 2}$ & $\mathbf{1 7 2 6}$ & $\mathbf{1 7 3 4}$ & $\mathbf{1 6 4 3}$ & $\mathbf{1 4 6 1}$ & $\mathbf{1 4 9 9}$ & $\mathbf{1 5 2 6}$ & $\mathbf{1 1 5 4}$ & $\mathbf{1 5 0 8}$ & $\mathbf{1 3 3 7}$ & $\mathbf{1 4 9 5}$
\end{tabular}

55 Total Contracts In

\begin{tabular}{|c|c|c|c|c|c|c|c|c|c|c|c|c|c|}
\hline 196 & 341 & 329 & 372 & 307 & 331 & 312 & 297 & 296 & 296 & 178 & 297 & 170 & 285 \\
\hline 139 & 133 & 132 & 144 & 141 & 134 & 131 & 109 & 124 & 124 & 135 & 177 & 166 & 139 \\
\hline 913 & 929 & 977 & 1257 & 1278 & 1269 & 1200 & 1055 & 1079 & 1106 & 841 & 1033 & 1001 & 1071 \\
\hline 0 & 0 & 0 & 0 & 0 & 0 & 0 & 0 & 0 & 0 & 0 & 0 & 0 & 0 \\
\hline 48 & 402 & 1438 & 1772 & 1726 & 1734 & 1643 & 1461 & 1499 & 1526 & 1154 & 1508 & 1337 & 1495 \\
\hline
\end{tabular}



2003 White Book

Sep

Oct Nov

Dec

Jan Feb Mar Apr1 Apr16

May Jun Jul Avg

-Intra-Regional Transfers1 AVWP To BPA WP3Set 2 AVWP To PGE CapS 3 BPA To AVWP WP3Set 4 BPA To PPL PwrS 5 BPA To PPL CapS 6 BPA To PPL S/Pwr/X 7 BPA TO PPL S/N/X 8 BPA To PSE WP3Set 9 CHPD TO PGE C/N/X 10 CIDF To UPL PwrS 11 FPLEV To PGE PwrS 12 GCPD To AVWP PwrS 13 GCPD To COPD PwrS 14 GCPD To EWEB PwrS 15 GCPD To FGRV PwrS 16 GCPD To FREC PwrS 17 GCPD To KITT PwrS 18 GCPD To KOOT PwrS 19 GCPD To LVE PwrS 20 GCPD To LVE PwrS 21 GCPD To MCMN PwrS 22 GCPD To MTFR PwrS 23 GCPD To PGE PwrS 24 GCPD To PPL PwrS 25 GCPD To PPL PwrS 26 GCPD To PSE PwrS 27 GCPD To TPU PwrS 28 NWCP To PSE PwrS 29 NWE To MVP PwrS 30 NWE To PSE PwrS 31 Other Entities To AVWP 32 PGE To AVWP PkRepl 33 PGE To CHPD PkRepl 34 PGE To CHPD XchgNrg 35 PGE To PPL Cove Repl $36 \mathrm{PPL}$ To BPA S/N/X 37 PPL To BPA PkRepl 38 PPL To BPA S/Pwr/X 39 PPL To SUB PwrS 40 PSE To BPA WP3Set 41 SCL To POPD PwrS 42 SHPD To PSE PwrS 43 TAUC To AVWP PwrS 44 WSPE To PGE PwrS 45 Total Contracts

-Total Contracts Out-

46 Federal System

47 Public Entities

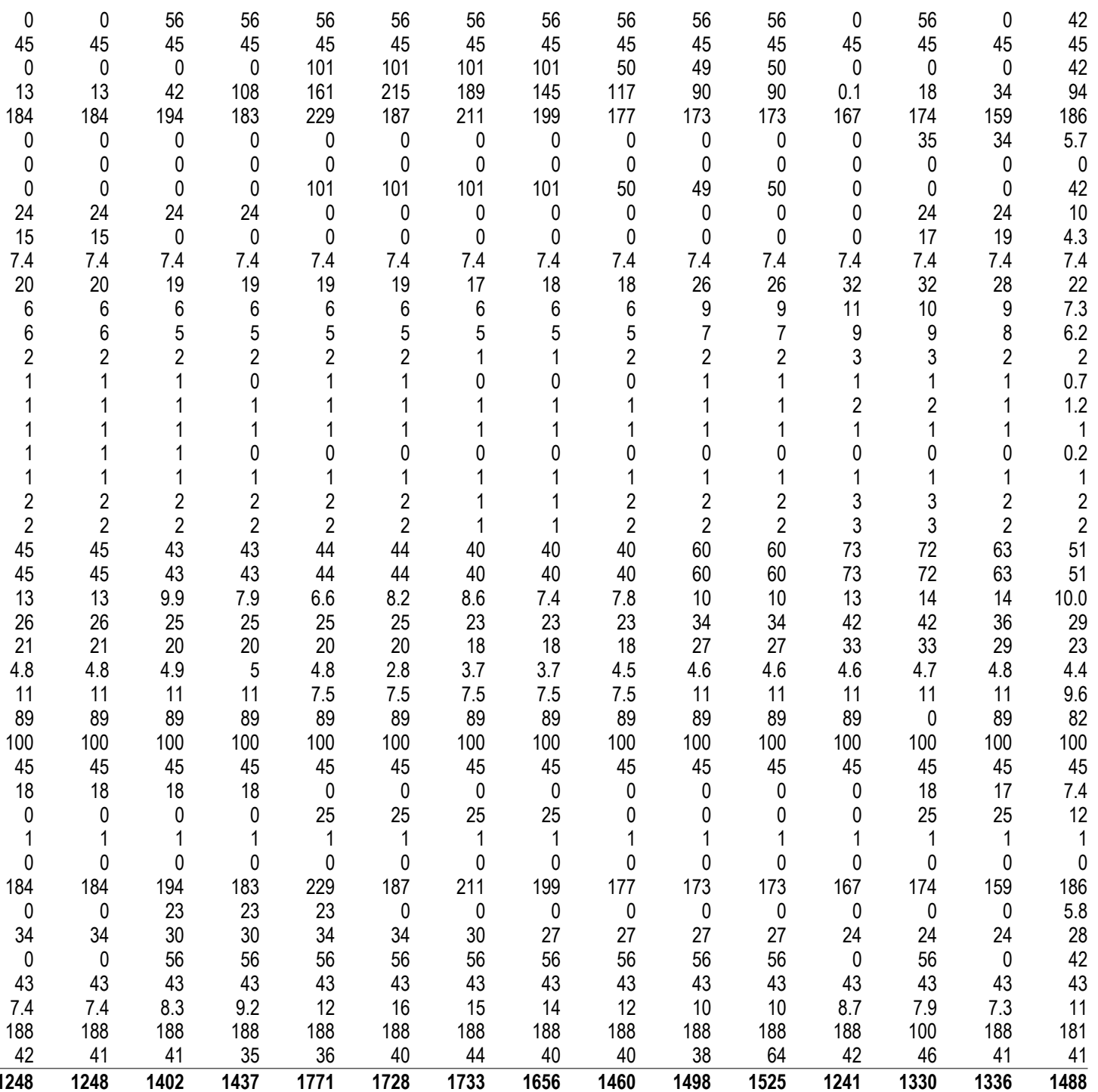

48 Investor-Owned Entities

49 Other Entities

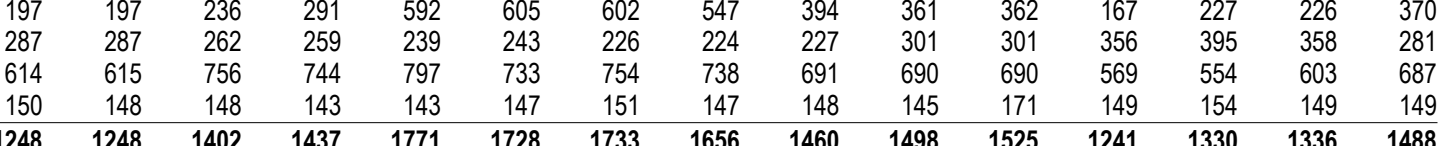

-Total Contracts In-

51 Federal System

52 Public Entities

53 Investor-Owned Entities

54 Other Entities

55 Total Contracts In

$\begin{array}{rrrrrrrrrrrrrrr}195 & 196 & 341 & 329 & 372 & 307 & 331 & 319 & 297 & 296 & 296 & 178 & 297 & 170 & 286 \\ 139 & 139 & 133 & 131 & 143 & 143 & 133 & 130 & 108 & 123 & 123 & 134 & 176 & 165 & 138 \\ 914 & 913 & 929 & 977 & 1257 & 1278 & 1269 & 1207 & 1055 & 1079 & 1106 & 929 & 856 & 1001 & 1064 \\ 0 & 0 & 0 & 0 & 0 & 0 & 0 & 0 & 0 & 0 & 0 & 0 & 0 & 0 & 0 \\ 1248 & \mathbf{1 2 4 8} & \mathbf{1 4 0 2} & \mathbf{1 4 3 7} & \mathbf{1 7 7 1} & \mathbf{1 7 2 8} & \mathbf{1 7 3 3} & \mathbf{1 6 5 6} & \mathbf{1 4 6 0} & \mathbf{1 4 9 8} & \mathbf{1 5 2 5} & \mathbf{1 2 4 1} & \mathbf{1 3 3 0} & \mathbf{1 3 3 6} & \mathbf{1 4 8 8}\end{array}$


Sep

Oct Nov

Dec

Jan Feb Mar Apr1 Apr16

May Jun Jul Avg

-Intra-Regional Transfers1 AVWP To BPA WP3Set 2 AVWP To PGE CapS 3 BPA To AVWP WP3Set 4 BPA To PPL PwrS 5 BPA To PPL CapS 6 BPA To PPL S/Pwr/X 7 BPA TO PPL S/N/X 8 BPA To PSE WP3Set 9 CHPD TO PGE C/N/X 10 CIDF To UPL PwrS 11 FPLEV To PGE PwrS 12 GCPD To AVWP PwrS 13 GCPD To COPD PwrS 14 GCPD To EWEB PwrS 15 GCPD To FGRV PwrS 16 GCPD To FREC PwrS 17 GCPD To KITT PwrS 18 GCPD To KOOT PwrS 19 GCPD To LVE PwrS 20 GCPD To LVE PwrS 21 GCPD To MCMN PwrS 22 GCPD To MTFR PwrS 23 GCPD To PGE PwrS 24 GCPD To PPL PwrS 25 GCPD To PPL PwrS 26 GCPD To PSE PwrS 27 GCPD To TPU PwrS 28 NWCP To PSE PwrS 29 NWE To MVP PwrS 30 NWE To PSE PwrS 31 Other Entities To AVWP 32 PGE To AVWP PkRepl 33 PGE To CHPD PkRepl 34 PGE To CHPD XchgNrg 35 PGE To PPL Cove Repl $36 \mathrm{PPL}$ To BPA S/N/X 37 PPL To BPA PkRepl 38 PPL To BPA S/Pwr/X 39 PPL To SUB PwrS 40 PSE To BPA WP3Set 41 SCL To POPD PwrS 42 SHPD To PSE PwrS 43 TAUC To AVWP PwrS 44 WSPE To PGE PwrS 45 Total Contracts

-Total Contracts Out-

46 Federal System

47 Public Entities

48 Investor-Owned Entities

49 Other Entities

50 Total Contracts Out

-Total Contracts In-

51 Federal System

52 Public Entities

53 Investor-Owned Entities

54 Other Entities

55 Total Contracts In

\begin{tabular}{|c|c|c|c|c|c|c|c|c|c|c|c|c|c|}
\hline 0 & 56 & 56 & 56 & 56 & 56 & 56 & 56 & 56 & 56 & 0 & 56 & 0 & 42 \\
\hline 45 & 45 & 45 & 45 & 45 & 45 & 45 & 45 & 45 & 45 & 45 & 45 & 45 & 45 \\
\hline 0 & 0 & 0 & 101 & 101 & 101 & 101 & 50 & 49 & 50 & 0 & 0 & 0 & 42 \\
\hline 13 & 42 & 108 & 161 & 215 & 189 & 145 & 117 & 90 & 90 & 0.1 & 18 & 34 & 94 \\
\hline 184 & 194 & 183 & 229 & 187 & 211 & 199 & 177 & 173 & 173 & 167 & 174 & 159 & 186 \\
\hline 0 & 0 & 0 & 0 & 0 & 0 & 0 & 0 & 0 & 0 & 0 & 35 & 34 & 5.7 \\
\hline 0 & 0 & 0 & 0 & 0 & 0 & 0 & 0 & 0 & 0 & 0 & 0 & 0 & 0 \\
\hline 0 & 0 & 0 & 101 & 101 & 101 & 101 & 50 & 49 & 50 & 0 & 0 & 0 & 42 \\
\hline 24 & 24 & 24 & 0 & 0 & 0 & 0 & 0 & 0 & 0 & 0 & 24 & 24 & 10 \\
\hline 15 & 0 & 0 & 0 & 0 & 0 & 0 & 0 & 0 & 0 & 0 & 17 & 19 & 4.3 \\
\hline 7.4 & 7.4 & 7.4 & 7.4 & 7.4 & 7.4 & 7.4 & 7.4 & 7.4 & 7.4 & 7.4 & 7.4 & 7.4 & 7.4 \\
\hline 20 & 19 & 19 & 18 & 18 & 16 & 16 & 16 & 24 & 24 & 30 & 29 & 26 & 21 \\
\hline 6 & 6 & 6 & 6 & 6 & 5 & 5 & 5 & 8 & 8 & 10 & 10 & 8 & 6.8 \\
\hline 6 & 5 & 5 & 5 & 5 & 5 & 5 & 5 & 7 & 7 & 8 & 8 & 7 & 5.9 \\
\hline 2 & 2 & 2 & 1 & 1 & 1 & 1 & 1 & 2 & 2 & 2 & 2 & 2 & 1.6 \\
\hline 1 & 1 & 0 & 1 & 1 & 0 & 0 & 0 & 1 & 1 & 1 & 1 & 1 & 0.7 \\
\hline 1 & 1 & 1 & 1 & 1 & 0 & 0 & 0 & 1 & 1 & 1 & 1 & 1 & 0.8 \\
\hline 1 & 1 & 1 & 1 & 1 & 1 & 1 & 1 & 1 & 1 & 1 & 1 & 1 & 1 \\
\hline 1 & 1 & 1 & 1 & 1 & 1 & 1 & 1 & 1 & 1 & 1 & 1 & 1 & 1 \\
\hline 0 & 0 & 0 & 0 & 0 & 0 & 0 & 0 & 0 & 0 & 0 & 0 & 0 & 0 \\
\hline 2 & 2 & 2 & 1 & 1 & 1 & 1 & 1 & 2 & 2 & 2 & 2 & 2 & 1.6 \\
\hline 2 & 2 & 2 & 1 & 1 & 1 & 1 & 1 & 2 & 2 & 2 & 2 & 2 & 1.6 \\
\hline 45 & 43 & 43 & 41 & 41 & 37 & 37 & 38 & 56 & 56 & 68 & 67 & 59 & 48 \\
\hline 13 & 9.9 & 7.9 & 6.6 & 8.2 & 8.6 & 7.4 & 7.8 & 10 & 10 & 13 & 14 & 14 & 10.0 \\
\hline 45 & 43 & 43 & 41 & 41 & 37 & 37 & 38 & 56 & 56 & 68 & 67 & 59 & 48 \\
\hline 26 & 25 & 25 & 24 & 24 & 21 & 22 & 22 & 32 & 32 & 39 & 39 & 34 & 28 \\
\hline 21 & 20 & 20 & 10 & 10 & 9 & 9 & 9 & 14 & 14 & 17 & 16 & 14 & 14 \\
\hline 4.8 & 4.9 & 5 & 4.8 & 2.8 & 3.7 & 3.7 & 4.5 & 4.6 & 4.6 & 4.6 & 4.7 & 4.8 & 4.4 \\
\hline 11 & 11 & 11 & 7.5 & 7.5 & 7.5 & 7.5 & 7.5 & 11 & 11 & 11 & 11 & 11 & 9.6 \\
\hline 89 & 89 & 89 & 89 & 89 & 89 & 89 & 89 & 89 & 89 & 89 & 89 & 89 & 89 \\
\hline 100 & 100 & 100 & 100 & 100 & 100 & 100 & 100 & 100 & 100 & 100 & 100 & 100 & 100 \\
\hline 45 & 45 & 45 & 45 & 45 & 45 & 45 & 45 & 45 & 45 & 45 & 45 & 45 & 45 \\
\hline 18 & 18 & 18 & 0 & 0 & 0 & 0 & 0 & 0 & 0 & 0 & 18 & 17 & 7.4 \\
\hline 0 & 0 & 0 & 25 & 25 & 25 & 25 & 0 & 0 & 0 & 0 & 25 & 25 & 12 \\
\hline 1 & 1 & 1 & 1 & 1 & 1 & 1 & 1 & 1 & 1 & 1 & 1 & 0 & 0.9 \\
\hline 0 & 0 & 0 & 0 & 0 & 0 & 0 & 0 & 0 & 0 & 0 & 0 & 0 & 0 \\
\hline 184 & 194 & 183 & 229 & 187 & 211 & 199 & 177 & 173 & 173 & 167 & 174 & 159 & 186 \\
\hline 0 & 23 & 23 & 23 & 0 & 0 & 0 & 0 & 0 & 0 & 0 & 0 & 0 & 5.8 \\
\hline 34 & 30 & 30 & 34 & 34 & 30 & 27 & 27 & 27 & 27 & 24 & 24 & 24 & 28 \\
\hline 0 & 56 & 56 & 56 & 56 & 56 & 56 & 56 & 56 & 56 & 0 & 56 & 0 & 42 \\
\hline 43 & 43 & 43 & 43 & 43 & 43 & 43 & 43 & 43 & 43 & 43 & 43 & 43 & 43 \\
\hline 7.4 & 8.3 & 9.2 & 12 & 16 & 15 & 14 & 0 & 0 & 0 & 0 & 0 & 0 & 6.8 \\
\hline 188 & 188 & 188 & 188 & 188 & 188 & 188 & 179 & 94 & 94 & 161 & 188 & 188 & 177 \\
\hline 42 & 41 & 35 & 36 & 40 & 44 & 40 & 40 & 38 & 64 & 42 & 46 & 41 & 41 \\
\hline 1247 & 1401 & 1437 & 1750 & 1707 & 1713 & 1636 & 1417 & 1368 & 1395 & 1168 & 1461 & 1299 & 1468 \\
\hline
\end{tabular}

$\begin{array}{rrrrrrrrrrrrrrr}197 & 197 & 236 & 291 & 592 & 605 & 602 & 547 & 394 & 361 & 362 & 167 & 227 & 226 & 370 \\ 286 & 286 & 261 & 259 & 218 & 222 & 206 & 204 & 194 & 265 & 265 & 310 & 349 & 322 & 258 \\ 614 & 615 & 756 & 744 & 797 & 733 & 754 & 738 & 682 & 597 & 596 & 542 & 732 & 602 & 691 \\ 150 & 148 & 148 & 143 & 143 & 147 & 151 & 147 & 148 & 145 & 171 & 149 & 154 & 149 & 149 \\ 1247 & \mathbf{1 2 4 7} & \mathbf{1 4 0 1} & \mathbf{1 4 3 7} & \mathbf{1 7 5 0} & \mathbf{1 7 0 7} & \mathbf{1 7 1 3} & \mathbf{1 6 3 6} & \mathbf{1 4 1 7} & \mathbf{1 3 6 8} & \mathbf{1 3 9 5} & \mathbf{1 1 6 8} & \mathbf{1 4 6 1} & \mathbf{1 2 9 9} & \mathbf{1 4 6 8}\end{array}$

\begin{tabular}{|c|c|c|c|c|c|c|c|c|c|c|c|c|c|}
\hline 196 & 341 & 329 & 372 & 307 & 331 & 319 & 297 & 296 & 296 & 178 & 297 & 170 & 286 \\
\hline 138 & 132 & 131 & 130 & 130 & 122 & 119 & 94 & 109 & 109 & 112 & 154 & 148 & 127 \\
\hline 913 & 929 & 977 & 1249 & 1270 & 1260 & 1198 & 1026 & 962 & 990 & 878 & 1010 & 981 & 1056 \\
\hline 0 & 0 & 0 & 0 & 0 & 0 & 0 & 0 & 0 & 0 & 0 & 0 & 0 & 0 \\
\hline 1247 & 1401 & 1437 & 1750 & 1707 & 1713 & 1636 & 1417 & 1368 & 1395 & 1168 & 1461 & 1299 & \\
\hline
\end{tabular}


2003 White Book

Sep

Oct Nov

Dec

Jan Feb Mar Apr1 Apr16

May Jun Jul Avg

-Intra-Regional Transfers1 AVWP To BPA WP3Set 2 AVWP To PGE CapS 3 BPA To AVWP WP3Set 4 BPA To PPL PwrS 5 BPA To PPL CapS 6 BPA To PPL S/Pwr/X 7 BPA TO PPL S/N/X 8 BPA To PSE WP3Set 9 CHPD TO PGE C/N/X 10 CIDF To UPL PwrS 11 FPLEV To PGE PwrS 12 GCPD To AVWP PwrS 13 GCPD To COPD PwrS 14 GCPD To EWEB PwrS 15 GCPD To FGRV PwrS 16 GCPD To FREC PwrS 17 GCPD To KITT PwrS 18 GCPD To KOOT PwrS 19 GCPD To LVE PwrS 20 GCPD To LVE PwrS 21 GCPD To MCMN PwrS 22 GCPD To MTFR PwrS 23 GCPD To PGE PwrS 24 GCPD To PPL PwrS 25 GCPD To PPL PwrS 26 GCPD To PSE PwrS 27 GCPD To TPU PwrS 28 NWCP To PSE PwrS 29 NWE To MVP PwrS 30 NWE TO PSE PwrS 31 Other Entities To AVWP 32 PGE To AVWP PkRepl 33 PGE To CHPD PkRepl 34 PGE To CHPD XchgNrg 35 PPL To BPA S/N/X 36 PPL To BPA PkRepl 37 PPL To BPA S/Pwr/X 38 PPL To SUB PwrS 39 PSE To BPA WP3Set 40 SCL To POPD PwrS 41 TAUC To AVWP PwrS 42 WSPE To PGE PwrS 43 Total Contracts

-Total Contracts Out-

44 Federal System

45 Public Entities

46 Investor-Owned Entities

47 Other Entities

\begin{tabular}{|c|c|c|c|c|c|c|c|c|c|c|c|c|c|c|}
\hline 0 & 0 & 56 & 56 & 56 & 56 & 56 & 56 & 56 & 56 & 56 & 0 & 56 & 0 & 42 \\
\hline 45 & 45 & 45 & 45 & 45 & 45 & 45 & 45 & 45 & 45 & 45 & 45 & 45 & 45 & 45 \\
\hline 0 & 0 & 0 & 0 & 101 & 101 & 101 & 101 & 50 & 49 & 50 & 0 & 0 & 0 & 42 \\
\hline 13 & 13 & 42 & 108 & 161 & 215 & 189 & 145 & 117 & 90 & 90 & 0.1 & 18 & 34 & 94 \\
\hline 184 & 184 & 194 & 183 & 229 & 187 & 211 & 199 & 177 & 173 & 173 & 167 & 174 & 159 & 186 \\
\hline 0 & 0 & 0 & 0 & 0 & 0 & 0 & 0 & 0 & 0 & 0 & 0 & 35 & 34 & 5.7 \\
\hline 0 & 0 & 0 & 0 & 0 & 0 & 0 & 0 & 0 & 0 & 0 & 0 & 0 & 0 & 0 \\
\hline 0 & 0 & 0 & 0 & 101 & 101 & 101 & 101 & 50 & 49 & 50 & 0 & 0 & 0 & 42 \\
\hline 24 & 24 & 24 & 24 & 0 & 0 & 0 & 0 & 0 & 0 & 0 & 0 & 0 & 0 & \\
\hline 15 & 15 & 0 & 0 & 0 & 0 & 0 & 0 & 0 & 0 & 0 & 0 & 17 & 19 & 4.3 \\
\hline 7.4 & 7.4 & 7.4 & 7.4 & 7.4 & 7.4 & 7.4 & 7.4 & 7.4 & 7.4 & 7.4 & 7.4 & 7.4 & 7.4 & 7.4 \\
\hline 18 & 18 & 18 & 17 & 18 & 18 & 16 & 16 & 16 & 24 & 24 & 30 & 29 & 26 & 20 \\
\hline 6 & 6 & 6 & 6 & 6 & 6 & 5 & 5 & 5 & 8 & 8 & 10 & 10 & 8 & 6.8 \\
\hline 5 & 5 & 5 & 5 & 5 & 5 & 5 & 5 & 5 & 7 & 7 & 8 & 8 & 7 & 8.8 \\
\hline 2 & 2 & 1 & 1 & 1 & 1 & 1 & 1 & 1 & 2 & 2 & 2 & 2 & 2 & 1.4 \\
\hline 1 & 1 & 1 & 0 & 1 & 1 & 0 & 0 & 0 & 1 & 1 & 1 & 1 & 1 & 0.7 \\
\hline 1 & 1 & 0 & 0 & 1 & 1 & 0 & 0 & 0 & 1 & 1 & 1 & 1 & 1 & 0.6 \\
\hline 1 & 1 & 1 & 1 & 1 & 1 & 1 & 1 & 1 & 1 & 1 & 1 & 1 & 1 & \\
\hline 1 & 1 & 1 & 1 & 1 & 1 & 1 & 1 & 1 & 1 & 1 & 1 & 1 & 1 & \\
\hline 0 & 0 & 0 & 0 & 0 & 0 & 0 & 0 & 0 & 0 & 0 & 0 & 0 & 0 & \\
\hline 2 & 2 & 1 & 1 & 1 & 1 & 1 & 1 & 1 & 2 & 2 & 2 & 2 & 2 & 1.4 \\
\hline 2 & 2 & 1 & 1 & 1 & 1 & 1 & 1 & 1 & 2 & 2 & 2 & 2 & 2 & 1.4 \\
\hline 42 & 42 & 40 & 40 & 41 & 41 & 37 & 37 & 38 & 56 & 56 & 68 & 67 & 59 & 47 \\
\hline 13 & 13 & 9.9 & 7.9 & 6.6 & 8.2 & 8.6 & 7.4 & 7.8 & 10 & 10 & 13 & 14 & 14 & 10.0 \\
\hline 42 & 42 & 40 & 40 & 41 & 41 & 37 & 37 & 38 & 56 & 56 & 68 & 67 & 59 & 47 \\
\hline 24 & 24 & 23 & 23 & 24 & 24 & 21 & 22 & 22 & 32 & 32 & 39 & 39 & 34 & 27 \\
\hline 10 & 10 & 10 & 10 & 10 & 10 & 9 & 9 & 9 & 14 & 14 & 17 & 16 & 14 & 12 \\
\hline 4.8 & 4.8 & 4.9 & 5 & 4.8 & 2.8 & 3.7 & 3.7 & 4.5 & 4.6 & 4.6 & 4.6 & 4.7 & 4.8 & 4.4 \\
\hline 11 & 11 & 11 & 11 & 7.5 & 7.5 & 7.5 & 7.5 & 7.5 & 11 & 11 & 11 & 11 & 11 & 9.6 \\
\hline 89 & 89 & 89 & 89 & 89 & 89 & 0 & 0 & 0 & 0 & 0 & 0 & 0 & 0 & 37 \\
\hline 100 & 100 & 100 & 100 & 100 & 100 & 0 & 0 & 0 & 0 & 0 & 0 & 0 & 0 & 42 \\
\hline 45 & 45 & 45 & 45 & 45 & 45 & 45 & 45 & 45 & 45 & 45 & 45 & 45 & 45 & 45 \\
\hline 18 & 18 & 18 & 18 & 0 & 0 & 0 & 0 & 0 & 0 & 0 & 0 & 0 & 0 & 4.5 \\
\hline 0 & 0 & 0 & 0 & 25 & 25 & 25 & 25 & 0 & 0 & 0 & 0 & 0 & 0 & 8.3 \\
\hline 0 & 0 & 0 & 0 & 0 & 0 & 0 & 0 & 0 & 0 & 0 & 0 & 0 & 0 & 0 \\
\hline 184 & 184 & 194 & 183 & 229 & 187 & 211 & 199 & 177 & 173 & 173 & 167 & 174 & 159 & 186 \\
\hline 0 & 0 & 23 & 23 & 23 & 0 & 0 & 0 & 0 & 0 & 0 & 0 & 0 & 0 & 5.8 \\
\hline 34 & 34 & 30 & 30 & 34 & 34 & 30 & 27 & 27 & 27 & 27 & 24 & 24 & 24 & $\angle 0$ \\
\hline 0 & 0 & 56 & 56 & 56 & 56 & 56 & 56 & 56 & 56 & 56 & 0 & 56 & 0 & 42 \\
\hline 43 & 43 & 43 & 43 & 43 & 43 & 43 & 43 & 43 & 43 & 43 & 43 & 43 & 43 & 43 \\
\hline 188 & 188 & 188 & 188 & 188 & 188 & 188 & 188 & 188 & 179 & 94 & 94 & 161 & 188 & 174 \\
\hline 42 & 41 & 41 & 35 & 36 & 40 & 44 & 40 & 40 & 38 & 64 & 42 & 46 & 41 & 41 \\
\hline 1217 & 1216 & 1369 & 1403 & 1738 & 1690 & 1508 & 1432 & 1236 & 1262 & 1205 & 911 & 1176 & 1043 & \\
\hline
\end{tabular}

48 Total Contracts Out

\begin{tabular}{|c|c|c|c|c|c|c|c|c|c|c|c|c|c|}
\hline 197 & 236 & 291 & 592 & 605 & 602 & 547 & 394 & 361 & 362 & 167 & 227 & 226 & 370 \\
\hline 257 & 230 & 226 & 207 & 206 & 190 & 190 & 194 & 265 & 265 & 310 & 325 & 298 & 242 \\
\hline 614 & 755 & 743 & 796 & 732 & 663 & 648 & 601 & 591 & 506 & 385 & 571 & 471 & 627 \\
\hline 148 & 148 & 143 & 143 & 147 & 51 & 47 & 48 & 45 & 71 & 49 & 54 & 49 & 91 \\
\hline 216 & 1369 & 1403 & 1738 & 1690 & 1508 & 1432 & 1236 & 1262 & 1205 & 911 & 1176 & 1043 & 330 \\
\hline
\end{tabular}

-Total Contracts In-

49 Federal System

50 Public Entities

51 Investor-Owned Entities

52 Other Entities

53 Total Contracts In

\begin{tabular}{|c|c|c|c|c|c|c|c|c|c|c|c|c|c|}
\hline 196 & 341 & 329 & 372 & 307 & 331 & 319 & 297 & 296 & 296 & 178 & 297 & 170 & 286 \\
\hline 126 & 118 & 117 & 130 & 130 & 122 & 119 & 94 & 109 & 109 & 112 & 111 & 106 & 116 \\
\hline 894 & 910 & 957 & 1237 & 1253 & 1054 & 994 & 845 & 857 & 800 & 621 & 768 & 768 & 928 \\
\hline 0 & 0 & 0 & 0 & 0 & 0 & 0 & 0 & 0 & 0 & 0 & 0 & 0 & 0 \\
\hline 216 & 1369 & 1403 & 1738 & 1690 & 1508 & 1432 & 1236 & 1262 & 1205 & 911 & 1176 & 1043 & 1330 \\
\hline
\end{tabular}


Table A-16: Regional Intra-Regional Transfers

PNW Loads and Resources Study

2011 - 2012 Operating Year

2003 White Book

Aug1 Aug16 Sep Oct Nov

Dec

Jan Feb Mar Apr1 Apr16

May

Jun Jul Avg

-Intra-Regional Transfers-

1 AVWP To BPA WP3Set

2 AVWP To PGE CapS

3 BPA To AVWP WP3Set

4 BPA To PPL PwrS

5 BPA To PPL CapS

6 BPA To PPL S/Pwr/X

7 BPA TO PPL S/N/X

8 BPA To PSE WP3Set

9 CIDF To UPL PwrS

10 FPLEV To PGE PwrS

11 GCPD To AVWP PwrS

12 GCPD To COPD PwrS

13 GCPD To EWEB PwrS

14 GCPD To FGRV PwrS

15 GCPD To FREC PwrS

16 GCPD To KITT PwrS

17 GCPD To KOOT PwrS

18 GCPD To LVE PwrS

19 GCPD To LVE PwrS

20 GCPD To MCMN PwrS

21 GCPD To MTFR PwrS

22 GCPD To PGE PwrS

23 GCPD To PPL PwrS

24 GCPD To PPL PwrS

25 GCPD To PSE PwrS

26 GCPD To TPU PwrS

27 NWCP To PSE PwrS

28 NWE To MVP PwrS

29 PGE To AVWP PkRepl

$30 \mathrm{PPL}$ To BPA S/N/X

31 PPL To BPA PkRepl

32 PPL To BPA S/Pwr/X

33 PPL TO SUB PwrS

34 PSE To BPA WP3Set

35 TAUC To AVWP PwrS

36 WSPE To PGE PwrS

37 Total Contracts

-Total Contracts Out-

38 Federal System

39 Public Entities

40 Investor-Owned Entities

41 Other Entities

42 Total Contracts Out

Total Contracts In-

43 Federal System

44 Public Entities

45 Investor-Owned Entities

46 Other Entities

47 Total Contracts In

\begin{tabular}{|c|c|c|c|c|c|c|c|c|c|c|c|c|c|}
\hline 0 & 56 & 56 & 56 & 56 & 56 & 56 & 56 & 56 & 56 & 0 & 56 & 0 & 42 \\
\hline 45 & 45 & 45 & 45 & 45 & 45 & 45 & 45 & 45 & 45 & 45 & 45 & 45 & 45 \\
\hline 0 & 0 & 0 & 101 & 101 & 101 & 101 & 50 & 49 & 50 & 0 & 0 & 0 & 42 \\
\hline 13 & 42 & 108 & 161 & 215 & 189 & 145 & 117 & 90 & 90 & 0.1 & 18 & 34 & 94 \\
\hline 184 & 0 & 0 & 0 & 0 & 0 & 0 & 0 & 0 & 0 & 0 & 0 & 0 & 15 \\
\hline 0 & 0 & 0 & 0 & 0 & 0 & 0 & 0 & 0 & 0 & 0 & 35 & 34 & 5.7 \\
\hline 0 & 0 & 0 & 0 & 0 & 0 & 0 & 0 & 0 & 0 & 0 & 0 & 0 & 0 \\
\hline 0 & 0 & 0 & 101 & 101 & 101 & 101 & 50 & 49 & 50 & 0 & 0 & 0 & 42 \\
\hline 15 & 0 & 0 & 0 & 0 & 0 & 0 & 0 & 0 & 0 & 0 & 17 & 19 & 4.3 \\
\hline 7.4 & 7.4 & 7.4 & 7.4 & 7.4 & 7.4 & 7.4 & 7.4 & 7.4 & 7.4 & 7.4 & 7.4 & 7.4 & 7.4 \\
\hline 18 & 18 & 0 & 0 & 0 & 0 & 0 & 0 & 0 & 0 & 0 & 0 & 0 & 3 \\
\hline 6 & 6 & 0 & 0 & 0 & 0 & 0 & 0 & 0 & 0 & 0 & 0 & 0 & 1 \\
\hline 5 & 5 & 0 & 0 & 0 & 0 & 0 & 0 & 0 & 0 & 0 & 0 & 0 & 0.8 \\
\hline 2 & 1 & 0 & 0 & 0 & 0 & 0 & 0 & 0 & 0 & 0 & 0 & 0 & 0.3 \\
\hline 1 & 1 & 0 & 0 & 0 & 0 & 0 & 0 & 0 & 0 & 0 & 0 & 0 & 0.2 \\
\hline 1 & 0 & 0 & 0 & 0 & 0 & 0 & 0 & 0 & 0 & 0 & 0 & 0 & 0.1 \\
\hline 1 & 1 & 0 & 0 & 0 & 0 & 0 & 0 & 0 & 0 & 0 & 0 & 0 & 0.2 \\
\hline 1 & 1 & 0 & 0 & 0 & 0 & 0 & 0 & 0 & 0 & 0 & 0 & 0 & 0.2 \\
\hline 0 & 0 & 0 & 0 & 0 & 0 & 0 & 0 & 0 & 0 & 0 & 0 & 0 & 0 \\
\hline 2 & 1 & 0 & 0 & 0 & 0 & 0 & 0 & 0 & 0 & 0 & 0 & 0 & 0.3 \\
\hline 2 & 1 & 0 & 0 & 0 & 0 & 0 & 0 & 0 & 0 & 0 & 0 & 0 & 0.3 \\
\hline 42 & 40 & 0 & 0 & 0 & 0 & 0 & 0 & 0 & 0 & 0 & 0 & 0 & 6.8 \\
\hline 42 & 40 & 0 & 0 & 0 & 0 & 0 & 0 & 0 & 0 & 0 & 0 & 0 & 6.8 \\
\hline 13 & 9.9 & 7.9 & 6.6 & 8.2 & 8.6 & 7.4 & 7.8 & 10 & 10 & 13 & 14 & 14 & 10.0 \\
\hline 24 & 23 & 0 & 0 & 0 & 0 & 0 & 0 & 0 & 0 & 0 & 0 & 0 & 3.9 \\
\hline 10 & 10 & 0 & 0 & 0 & 0 & 0 & 0 & 0 & 0 & 0 & 0 & 0 & 1.7 \\
\hline 4.8 & 4.9 & 5 & 4.8 & 2.8 & 3.7 & 3.7 & 4.5 & 4.6 & 4.6 & 4.6 & 4.7 & 4.8 & 4.4 \\
\hline 11 & 11 & 11 & 7.5 & 7.5 & 7.5 & 7.5 & 7.5 & 11 & 11 & 11 & 11 & 11 & 9.6 \\
\hline 45 & 45 & 45 & 45 & 45 & 45 & 45 & 45 & 45 & 45 & 45 & 45 & 45 & 45 \\
\hline 0 & 0 & 0 & 0 & 0 & 0 & 0 & 0 & 0 & 0 & 0 & 0 & 0 & 0 \\
\hline 184 & 0 & 0 & 0 & 0 & 0 & 0 & 0 & 0 & 0 & 0 & 0 & 0 & 15 \\
\hline 0 & 23 & 23 & 23 & 0 & 0 & 0 & 0 & 0 & 0 & 0 & 0 & 0 & 5.8 \\
\hline 34 & 30 & 30 & 34 & 34 & 30 & 27 & 27 & 27 & 27 & 24 & 24 & 24 & 28 \\
\hline 0 & 56 & 56 & 56 & 56 & 56 & 56 & 56 & 56 & 56 & 0 & 56 & 0 & 42 \\
\hline 188 & 188 & 188 & 188 & 188 & 188 & 188 & 188 & 188 & 188 & 100 & 188 & 188 & 181 \\
\hline 42 & 41 & 35 & 36 & 40 & 44 & 40 & 40 & 38 & 64 & 42 & 46 & 41 & 41 \\
\hline 942 & 707 & 617 & 871 & 906 & 882 & 829 & 700 & 675 & 703 & 290 & 566 & 466 & 705 \\
\hline
\end{tabular}

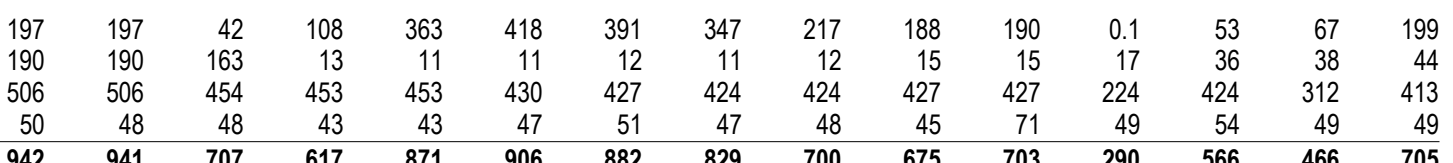

\begin{tabular}{|c|c|c|c|c|c|c|c|c|c|c|c|c|c|c|}
\hline 195 & 196 & 147 & 146 & 143 & 120 & 120 & 120 & 120 & 123 & 123 & 11 & 123 & 11 & 115 \\
\hline 64 & 64 & 57 & 30 & 34 & 34 & 30 & 27 & 27 & 27 & 27 & 24 & 24 & 24 & 33 \\
\hline 682 & 681 & 503 & 441 & 695 & 753 & 732 & 683 & 554 & 525 & 553 & 256 & 419 & 431 & 557 \\
\hline 0 & 0 & 0 & 0 & 0 & 0 & 0 & 0 & 0 & 0 & 0 & 0 & 0 & 0 & 0 \\
\hline 942 & 941 & 707 & 617 & 871 & 906 & 882 & 829 & 700 & 675 & 703 & 290 & 566 & 466 & 705 \\
\hline
\end{tabular}



2003 White Book Jul Avg
-Intra-Regional Transfers. 1 AVWP To BPA WP3Set 2 AVWP To PGE CapS

3 BPA To AVWP WP3Set

4 BPA To PPL PwrS

5 BPA To PPL S/Pwr/X

6 BPA To PPL S/N/X

7 BPA To PSE WP3Set

8 CIDF To UPL PwrS

9 FPLEV To PGE PwrS

10 GCPD To PPL PwrS

11 NWCP To PSE PwrS

12 NWE To MVP PwrS

13 PGE To AVWP PkRepl

14 PPL To BPA S/N/X

$15 \mathrm{PPL}$ To BPA S/Pwr/X

16 PPL To SUB PwrS

17 PSE To BPA WP3Set

18 TAUC To AVWP PwrS

19 WSPE To PGE PwrS

20 Total Contracts
Aug1 Aug16 Sep

Oct Nov Dec Jan Feb Mar Apr1 Apr16 May Jun

-Total Contracts Out-

21 Federal System

22 Public Entities

23 Investor-Owned Entities

24 Other Entities

25 Total Contracts Out

\begin{tabular}{|c|c|c|c|c|c|c|c|c|c|c|c|c|c|}
\hline 0 & 56 & 56 & 56 & 56 & 56 & 56 & 56 & 56 & 56 & 0 & 56 & 0 & 42 \\
\hline 45 & 45 & 45 & 45 & 45 & 45 & 45 & 45 & 45 & 45 & 45 & 45 & 45 & 45 \\
\hline 0 & 0 & 0 & 101 & 101 & 101 & 101 & 50 & 49 & 50 & 0 & 0 & 0 & 42 \\
\hline 13 & 42 & 108 & 161 & 215 & 189 & 145 & 117 & 90 & 90 & 0.1 & 18 & 34 & 94 \\
\hline 0 & 0 & 0 & 0 & 0 & 0 & 0 & 0 & 0 & 0 & 0 & 35 & 34 & 5.7 \\
\hline 0 & 0 & 0 & 0 & 0 & 0 & 0 & 0 & 0 & 0 & 0 & 0 & 0 & 0 \\
\hline 0 & 0 & 0 & 101 & 101 & 101 & 101 & 50 & 49 & 50 & 0 & 0 & 0 & 42 \\
\hline 15 & 0 & 0 & 0 & 0 & 0 & 0 & 0 & 0 & 0 & 0 & 17 & 19 & 4.3 \\
\hline 7.4 & 7.4 & 7.4 & 7.4 & 7.4 & 7.4 & 7.4 & 7.4 & 7.4 & 7.4 & 7.4 & 7.4 & 7.4 & 7.4 \\
\hline 13 & 9.9 & 7.9 & 6.6 & 8.2 & 8.6 & 7.4 & 7.8 & 10 & 10 & 13 & 14 & 14 & 10.0 \\
\hline 4.8 & 4.9 & 5 & 4.8 & 2.8 & 0 & 0 & 0 & 0 & 0 & 0 & 0 & 0 & 1.9 \\
\hline 11 & 11 & 11 & 7.5 & 7.5 & 7.5 & 7.5 & 7.5 & 11 & 11 & 11 & 11 & 11 & 9.6 \\
\hline 45 & 45 & 45 & 45 & 45 & 45 & 45 & 45 & 45 & 45 & 45 & 45 & 45 & 45 \\
\hline 0 & 0 & 0 & 0 & 0 & 0 & 0 & 0 & 0 & 0 & 0 & 0 & 0 & 0 \\
\hline 0 & 23 & 23 & 23 & 0 & 0 & 0 & 0 & 0 & 0 & 0 & 0 & 0 & 5.8 \\
\hline 34 & 30 & 30 & 34 & 34 & 30 & 27 & 27 & 27 & 27 & 24 & 24 & 24 & 28 \\
\hline 0 & 56 & 56 & 56 & 56 & 56 & 56 & 56 & 56 & 56 & 0 & 56 & 0 & 42 \\
\hline 188 & 188 & 188 & 188 & 188 & 188 & 188 & 188 & 188 & 188 & 188 & 100 & 188 & 181 \\
\hline 41 & 41 & 35 & 36 & 40 & 44 & 40 & 40 & 38 & 64 & 42 & 46 & 41 & 41 \\
\hline 415 & 559 & 617 & 871 & 906 & 878 & 825 & 696 & 671 & 699 & 374 & 474 & 461 & 647 \\
\hline
\end{tabular}

-Total Contracts In-

26 Federal System

27 Public Entities

28 Investor-Owned Entities

29 Other Entities

30 Total Contracts In

\begin{tabular}{|c|c|c|c|c|c|c|c|c|c|c|c|c|c|c|}
\hline 13 & 13 & 42 & 108 & 363 & 418 & 391 & 347 & 217 & 188 & 190 & 0.1 & 53 & 67 & 184 \\
\hline 33 & 33 & 15 & 13 & 11 & 11 & 8.6 & 7.4 & 7.8 & 10 & 10 & 13 & 31 & 33 & 16 \\
\hline 322 & 322 & 454 & 453 & 453 & 430 & 427 & 424 & 424 & 427 & 427 & 312 & 336 & 312 & 398 \\
\hline 50 & 48 & 48 & 43 & 43 & 47 & 51 & 47 & 48 & 45 & 71 & 49 & 54 & 49 & 49 \\
\hline 117 & 415 & 559 & 617 & 871 & 906 & 878 & 825 & 696 & 671 & 699 & 374 & 474 & 461 & 647 \\
\hline
\end{tabular}

\begin{tabular}{|c|c|c|c|c|c|c|c|c|c|c|c|c|c|c|}
\hline 11 & 11 & 147 & 146 & 143 & 120 & 120 & 120 & 120 & 123 & 123 & 11 & 123 & 11 & 100 \\
\hline 34 & 34 & 30 & 30 & 34 & 34 & 30 & 27 & 27 & 27 & 27 & 24 & 24 & 24 & 28 \\
\hline 372 & 371 & 382 & 441 & 695 & 753 & 728 & 679 & 549 & 521 & 548 & 339 & 327 & 426 & 519 \\
\hline 0 & 0 & 0 & 0 & 0 & 0 & 0 & 0 & 0 & 0 & 0 & 0 & 0 & 0 & 0 \\
\hline 417 & 415 & 559 & 617 & 871 & 906 & 878 & 825 & 696 & 671 & 699 & 374 & 474 & 461 & 647 \\
\hline
\end{tabular}


Table A-16: Regional Intra-Regional Transfers

PNW Loads and Resources Study

2013 - 2014 Operating Year

2003 White Book

Aug1 Aug16 Sep Oct Nov Dec Jan Feb Mar Apr1 Apr16

May Jun Jul Avg

-Intra-Regional Transfers

1 AVWP To BPA WP3Set

2 AVWP To PGE CapS

3 BPA To AVWP WP3Set

4 BPA To PPL PwrS

5 BPA To PPL S/Pwr/X

6 BPA To PPL S/N/X

7 BPA To PSE WP3Set

8 CIDF To UPL PwrS

9 FPLEV TO PGE PwrS

10 GCPD To PPL PwrS

11 NWE To MVP PwrS

12 PGE To AVWP PkRepl

$13 \mathrm{PPL}$ To BPA S/N/X

14 PPL To BPA S/Pwr/X

15 PPL To SUB PwrS

16 PSE To BPA WP3Set

17 TAUC To AVWP PwrS

18 WSPE To PGE PwrS

19 Total Contracts

$\begin{array}{rrrrrrrrrrrrrrr}0 & 0 & 56 & 56 & 56 & 56 & 56 & 56 & 56 & 56 & 56 & 0 & 56 & 0 & 42 \\ 45 & 45 & 45 & 45 & 45 & 45 & 45 & 45 & 45 & 45 & 45 & 45 & 45 & 45 & 45 \\ 0 & 0 & 0 & 0 & 101 & 101 & 101 & 101 & 50 & 49 & 50 & 0 & 0 & 0 & 42 \\ 13 & 13 & 42 & 108 & 161 & 215 & 189 & 145 & 117 & 90 & 90 & 0.1 & 18 & 34 & 94 \\ 0 & 0 & 0 & 0 & 0 & 0 & 0 & 0 & 0 & 0 & 0 & 0 & 0 & 0 & 0 \\ 0 & 0 & 0 & 0 & 0 & 0 & 0 & 0 & 0 & 0 & 0 & 0 & 0 & 0 & 0 \\ 0 & 0 & 0 & 0 & 101 & 101 & 101 & 101 & 50 & 49 & 50 & 0 & 0 & 0 & 42 \\ 15 & 15 & 0 & 0 & 0 & 0 & 0 & 0 & 0 & 0 & 0 & 0 & 17 & 19 & 4.3 \\ 7.4 & 7.4 & 7.4 & 7.4 & 7.4 & 7.4 & 7.4 & 7.4 & 7.4 & 7.4 & 7.4 & 7.4 & 7.4 & 7.4 & 7.4 \\ 13 & 13 & 9.9 & 7.9 & 6.6 & 8.2 & 8.6 & 7.4 & 7.8 & 10 & 10 & 13 & 14 & 14 & 10.0 \\ 11 & 11 & 11 & 11 & 7.5 & 7.5 & 7.5 & 7.5 & 7.5 & 11 & 11 & 11 & 11 & 11 & 9.6 \\ 45 & 45 & 45 & 45 & 45 & 45 & 45 & 45 & 45 & 45 & 45 & 45 & 45 & 45 & 45 \\ 0 & 0 & 0 & 0 & 0 & 0 & 0 & 0 & 0 & 0 & 0 & 0 & 0 & 0 & 0 \\ 0 & 0 & 23 & 23 & 23 & 0 & 0 & 0 & 0 & 0 & 0 & 0 & 0 & 0 & 5.8 \\ 34 & 34 & 30 & 30 & 34 & 34 & 30 & 27 & 27 & 27 & 27 & 24 & 24 & 24 & 28 \\ 0 & 0 & 56 & 56 & 56 & 56 & 56 & 56 & 56 & 56 & 56 & 0 & 56 & 0 & 42 \\ 188 & 188 & 188 & 188 & 188 & 188 & 188 & 188 & 188 & 188 & 188 & 100 & 188 & 188 & 181 \\ 42 & 41 & 41 & 35 & 36 & 40 & 44 & 40 & 40 & 38 & 64 & 42 & 46 & 41 & 41 \\ 412 & 411 & \mathbf{5 5 4} & \mathbf{6 1 2} & \mathbf{8 6 6} & \mathbf{9 0 3} & \mathbf{8 7 8} & \mathbf{8 2 5} & \mathbf{6 9 6} & \mathbf{6 7 1} & \mathbf{6 9 9} & \mathbf{2 8 6} & \mathbf{5 2 7} & \mathbf{4 2 7} & \mathbf{6 3 9}\end{array}$

-Total Contracts Out-

20 Federal System

21 Public Entities

\begin{tabular}{|c|c|c|c|c|c|c|c|c|c|c|c|c|c|c|}
\hline 13 & 13 & 42 & 108 & 363 & 418 & 391 & 347 & 217 & 188 & 190 & 0.1 & 18 & 34 & 178 \\
\hline 28 & 28 & 9.9 & 7.9 & 6.6 & 8.2 & 8.6 & 7.4 & 7.8 & 10 & 10 & 13 & 31 & 33 & 14 \\
\hline 322 & 322 & 454 & 453 & 453 & 430 & 427 & 424 & 424 & 427 & 427 & 224 & 424 & 312 & 398 \\
\hline 50 & 48 & 48 & 43 & 43 & 47 & 51 & 47 & 48 & 45 & 71 & 49 & 54 & 49 & 49 \\
\hline 412 & 411 & 554 & 612 & 866 & 903 & 878 & 825 & 696 & 671 & 699 & 286 & 527 & 427 & 639 \\
\hline
\end{tabular}

23 Other Entities

4

-Total Contracts In-

25 Federal System

26 Public Entities

27 Investor-Owned Entities

28 Other Entities

29 Total Contracts In

\begin{tabular}{|c|c|c|c|c|c|c|c|c|c|c|c|c|c|c|}
\hline 11 & 11 & 147 & 146 & 143 & 120 & 120 & 120 & 120 & 123 & 123 & 11 & 123 & 11 & 100 \\
\hline 34 & 34 & 30 & 30 & 34 & 34 & 30 & 27 & 27 & 27 & 27 & 24 & 24 & 24 & 28 \\
\hline 0 & 0 & 0 & 0 & 0 & 0 & 0 & 0 & 0 & 0 & 0 & 0 & 0 & 0 & 0 \\
\hline
\end{tabular}


Table A-17: Regional Capacity Reserves

PNW Loads and Resources Study

2004 - 2005 Operating Year

1937 Water Year

2003 White Book

Oct Nov

Dec Jan Fe

Feb Mar Apr1 Apr16 May Jun Jul Avg

Hydro Reserves

$1 \mathrm{BPA}$ - Power Business

2 Total Federal Entities

$\begin{array}{lllllllllllllll}0 & 0 & 0 & 0 & 0 & 0 & 0 & 0 & 0 & 0 & 0 & 0 & 0 & 0 & 0 \\ 0 & 0 & 0 & 0 & 0 & 0 & 0 & 0 & 0 & 0 & 0 & 0 & 0 & 0 & 0\end{array}$

-Generating Public Entities-

3 Chelan County PUD \#1

4 Clark County PUD \#1

5 Cowlitz County PUD \#1

6 Douglas County PUD \#1

7 Eugene Water \& Electric Board

8 Grant County PUD \#2

9 Grays Harbor PUD \#1

10 Okanogan County PUD \#1

11 Pend Oreille County PUD \#1

12 Seattle City Light

13 Snohomish County PUD \#1

14 Tacoma Public Utilities

15 Total Generating Public Entities

$\begin{array}{lllllllllllllll}0 & 0 & 0 & 0 & 0 & 0 & 0 & 0 & 0 & 0 & 0 & 0 & 0 & 0 & 0 \\ 0 & 0 & 0 & 0 & 0 & 0 & 0 & 0 & 0 & 0 & 0 & 0 & 0 & 0 & 0 \\ 0 & 0 & 0 & 0 & 0 & 0 & 0 & 0 & 0 & 0 & 0 & 0 & 0 & 0 & 0 \\ 0 & 0 & 0 & 0 & 0 & 0 & 0 & 0 & 0 & 0 & 0 & 0 & 0 & 0 & 0 \\ 0 & 0 & 0 & 0 & 0 & 0 & 0 & 0 & 0 & 0 & 0 & 0 & 0 & 0 & 0 \\ 0 & 0 & 0 & 0 & 0 & 0 & 0 & 0 & 0 & 0 & 0 & 0 & 0 & 0 & 0 \\ 0 & 0 & 0 & 0 & 0 & 0 & 0 & 0 & 0 & 0 & 0 & 0 & 0 & 0 & 0 \\ 0 & 0 & 0 & 0 & 0 & 0 & 0 & 0 & 0 & 0 & 0 & 0 & 0 & 0 & 0 \\ 0 & 0 & 0 & 0 & 0 & 0 & 0 & 0 & 0 & 0 & 0 & 0 & 0 & 0 & 0 \\ 0 & 0 & 0 & 0 & 0 & 0 & 0 & 0 & 0 & 0 & 0 & 0 & 0 & 0 & 0 \\ 0 & 0 & 0 & 0 & 0 & 0 & 0 & 0 & 0 & 0 & 0 & 0 & 0 & 0 & 0 \\ 0 & 0 & 0 & 0 & 0 & 0 & 0 & 0 & 0 & 0 & 0 & 0 & 0 & 0 & 0 \\ 0 & 0 & \mathbf{0} & \mathbf{0} & \mathbf{0} & \mathbf{0} & \mathbf{0} & \mathbf{0} & \mathbf{0} & \mathbf{0} & \mathbf{0} & \mathbf{0} & \mathbf{0} & \mathbf{0} & \mathbf{0}\end{array}$

-Non-Generating Public Entities-

16 Ashland, City of

17 Benton County PUD \#1

18 Bonners Ferry, City of

19 Centralia, City of

20 Clallam County PUD \#1

21 Clearwater Power

22 Consumers Power

23 Emerald County PUD

24 Energy Northwest

25 Fall River Elec Coop

26 Forest Grove, City of

27 Franklin County PUD \#1

28 Idaho County L \& P

29 Idaho Falls, City of

30 Kittitas County PUD \#1

31 Klickitat County PUD \#1

32 Kootenai Electric Coop

33 Lewis County PUD \#1

34 Lost River Elec Coop

35 Lower Valley Energy

36 Mason County PUD \#1

37 McMinnville, City of

38 Milton Freewater, City of

39 Northern Lights

40 Northern Wasco County PUD

41 Oregon Trail Coop

42 Port Angeles, City of

43 Raft River Elec Coop

44 Salmon River Elec Coop

45 Soda Springs, City of

46 Tanner Elec Coop

47 Tillamook PUD \#1

48 United Electric Coop

49 Total Non-Generating Public Entities

\begin{tabular}{|c|c|c|c|c|c|c|c|c|c|c|c|c|c|}
\hline 0 & 0 & 0 & 0 & 0 & 0 & 0 & 0 & 0 & 0 & 0 & 0 & 0 & 0 \\
\hline 0 & 0 & 0 & 0 & 0 & 0 & 0 & 0 & 0 & 0 & 0 & 0 & 0 & 0 \\
\hline 0 & 0 & 0 & 0 & 0 & 0 & 0 & 0 & 0 & 0 & 0 & 0 & 0 & 0 \\
\hline 0 & 0 & 0 & 0 & 0 & 0 & 0 & 0 & 0 & 0 & 0 & 0 & 0 & 0 \\
\hline 0 & 0 & 0 & 0 & 0 & 0 & 0 & 0 & 0 & 0 & 0 & 0 & 0 & 0 \\
\hline 0 & 0 & 0 & 0 & 0 & 0 & 0 & 0 & 0 & 0 & 0 & 0 & 0 & 0 \\
\hline 0 & 0 & 0 & 0 & 0 & 0 & 0 & 0 & 0 & 0 & 0 & 0 & 0 & 0 \\
\hline 0 & 0 & 0 & 0 & 0 & 0 & 0 & 0 & 0 & 0 & 0 & 0 & 0 & 0 \\
\hline 0 & 0 & 0 & 0 & 0 & 0 & 0 & 0 & 0 & 0 & 0 & 0 & 0 & 0 \\
\hline 0 & 0 & 0 & 0 & 0 & 0 & 0 & 0 & 0 & 0 & 0 & 0 & 0 & 0 \\
\hline 0 & 0 & 0 & 0 & 0 & 0 & 0 & 0 & 0 & 0 & 0 & 0 & 0 & 0 \\
\hline 0 & 0 & 0 & 0 & 0 & 0 & 0 & 0 & 0 & 0 & 0 & 0 & 0 & 0 \\
\hline 0 & 0 & 0 & 0 & 0 & 0 & 0 & 0 & 0 & 0 & 0 & 0 & 0 & 0 \\
\hline 0 & 0 & 0 & 0 & 0 & 0 & 0 & 0 & 0 & 0 & 0 & 0 & 0 & 0 \\
\hline 0 & 0 & 0 & 0 & 0 & 0 & 0 & 0 & 0 & 0 & 0 & 0 & 0 & 0 \\
\hline 0 & 0 & 0 & 0 & 0 & 0 & 0 & 0 & 0 & 0 & 0 & 0 & 0 & 0 \\
\hline 0 & 0 & 0 & 0 & 0 & 0 & 0 & 0 & 0 & 0 & 0 & 0 & 0 & 0 \\
\hline 0 & 0 & 0 & 0 & 0 & 0 & 0 & 0 & 0 & 0 & 0 & 0 & 0 & 0 \\
\hline 0 & 0 & 0 & 0 & 0 & 0 & 0 & 0 & 0 & 0 & 0 & 0 & 0 & 0 \\
\hline 0 & 0 & 0 & 0 & 0 & 0 & 0 & 0 & 0 & 0 & 0 & 0 & 0 & 0 \\
\hline 0 & 0 & 0 & 0 & 0 & 0 & 0 & 0 & 0 & 0 & 0 & 0 & 0 & 0 \\
\hline 0 & 0 & 0 & 0 & 0 & 0 & 0 & 0 & 0 & 0 & 0 & 0 & 0 & 0 \\
\hline 0 & 0 & 0 & 0 & 0 & 0 & 0 & 0 & 0 & 0 & 0 & 0 & 0 & 0 \\
\hline 0 & 0 & 0 & 0 & 0 & 0 & 0 & 0 & 0 & 0 & 0 & 0 & 0 & 0 \\
\hline 0 & 0 & 0 & 0 & 0 & 0 & 0 & 0 & 0 & 0 & 0 & 0 & 0 & 0 \\
\hline 0 & 0 & 0 & 0 & 0 & 0 & 0 & 0 & 0 & 0 & 0 & 0 & 0 & 0 \\
\hline 0 & 0 & 0 & 0 & 0 & 0 & 0 & 0 & 0 & 0 & 0 & 0 & 0 & 0 \\
\hline 0 & 0 & 0 & 0 & 0 & 0 & 0 & 0 & 0 & 0 & 0 & 0 & 0 & 0 \\
\hline 0 & 0 & 0 & 0 & 0 & 0 & 0 & 0 & 0 & 0 & 0 & 0 & 0 & 0 \\
\hline 0 & 0 & 0 & 0 & 0 & 0 & 0 & 0 & 0 & 0 & 0 & 0 & 0 & 0 \\
\hline 0 & 0 & 0 & 0 & 0 & 0 & 0 & 0 & 0 & 0 & 0 & 0 & 0 & 0 \\
\hline 0 & 0 & 0 & 0 & 0 & 0 & 0 & 0 & 0 & 0 & 0 & 0 & 0 & 0 \\
\hline 0 & 0 & 0 & 0 & 0 & 0 & 0 & 0 & 0 & 0 & 0 & 0 & 0 & 0 \\
\hline 0 & 0 & 0 & 0 & 0 & 0 & 0 & 0 & 0 & 0 & 0 & 0 & 0 & 0 \\
\hline
\end{tabular}

-Investor-Owned Entities-

50 Avista Corp (WWP Division)

51 Idaho Power Company

52 Northwestern Energy LLC (MPC)

53 Pacific Power

54 Portland General Electric

55 Puget Sound Energy

56 Total Investor-Owned Entities

-Other Entities-

57 Colockum Transmission Company

58 Longview Fibre

59 Warm Springs Power Enterprise

60 Total Other Entities

$\begin{array}{lllllllllllllll}0 & 0 & 0 & 0 & 0 & 0 & 0 & 0 & 0 & 0 & 0 & 0 & 0 & 0 & 0 \\ 0 & 0 & 0 & 0 & 0 & 0 & 0 & 0 & 0 & 0 & 0 & 0 & 0 & 0 & 0 \\ 0 & 0 & 0 & 0 & 0 & 0 & 0 & 0 & 0 & 0 & 0 & 0 & 0 & 0 & 0 \\ 0 & 0 & 0 & 0 & 0 & 0 & 0 & 0 & 0 & 0 & 0 & 0 & 0 & 0 & 0 \\ 0 & 0 & 0 & 0 & 0 & 0 & 0 & 0 & 0 & 0 & 0 & 0 & 0 & 0 & 0 \\ 0 & 0 & 0 & 0 & 0 & 0 & 0 & 0 & 0 & 0 & 0 & 0 & 0 & 0 & 0 \\ 0 & \mathbf{0} & \mathbf{0} & \mathbf{0} & \mathbf{0} & \mathbf{0} & \mathbf{0} & \mathbf{0} & \mathbf{0} & \mathbf{0} & \mathbf{0} & \mathbf{0} & \mathbf{0} & \mathbf{0} & \mathbf{0}\end{array}$

Small Thermal \& Misc. Reserves

-Federal Entities-

61 BPA - Power Business

62 U.S. Naval Station, Everett (Jim Creek)

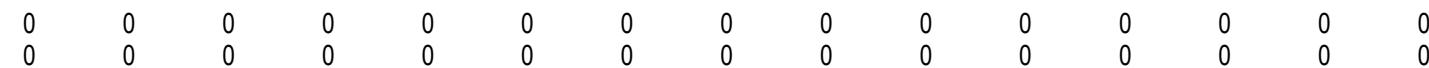




$\begin{array}{lllllllllllllll}0 & 0 & 0 & 0 & 0 & 0 & 0 & 0 & 0 & 0 & 0 & 0 & 0 & 0 & 0 \\ 0 & 0 & 0 & 0 & 0 & 0 & 0 & 0 & 0 & 0 & 0 & 0 & 0 & 0 & 0\end{array}$

-Generating Public Entities-

65 Chelan County PUD \#1

66 Clark County PUD \#1

67 Cowlitz County PUD \#1

68 Douglas County PUD \#1

69 Eugene Water \& Electric Board

70 Grant County PUD \#2

71 Grays Harbor PUD \#1

72 Okanogan County PUD \#1

73 Seattle City Light

74 Snohomish County PUD \#1

75 Springfield Utility Board

76 Tacoma Public Utilities

77 Total Generating Public Entities

$\begin{array}{lllllllllllllll}0 & 0 & 0 & 0 & 0 & 0 & 0 & 0 & 0 & 0 & 0 & 0 & 0 & 0 & 0 \\ 0 & 0 & 0 & 0 & 0 & 0 & 0 & 0 & 0 & 0 & 0 & 0 & 0 & 0 & 0 \\ 0 & 0 & 0 & 0 & 0 & 0 & 0 & 0 & 0 & 0 & 0 & 0 & 0 & 0 & 0 \\ 0 & 0 & 0 & 0 & 0 & 0 & 0 & 0 & 0 & 0 & 0 & 0 & 0 & 0 & 0 \\ 0 & 0 & 0 & 0 & 0 & 0 & 0 & 0 & 0 & 0 & 0 & 0 & 0 & 0 & 0 \\ 0 & 0 & 0 & 0 & 0 & 0 & 0 & 0 & 0 & 0 & 0 & 0 & 0 & 0 & 0 \\ 0 & 0 & 0 & 0 & 0 & 0 & 0 & 0 & 0 & 0 & 0 & 0 & 0 & 0 & 0 \\ 0 & 0 & 0 & 0 & 0 & 0 & 0 & 0 & 0 & 0 & 0 & 0 & 0 & 0 & 0 \\ 0 & 0 & 0 & 0 & 0 & 0 & 0 & 0 & 0 & 0 & 0 & 0 & 0 & 0 & 0 \\ 0 & 0 & 0 & 0 & 0 & 0 & 0 & 0 & 0 & 0 & 0 & 0 & 0 & 0 & 0 \\ 0 & 0 & 0 & 0 & 0 & 0 & 0 & 0 & 0 & 0 & 0 & 0 & 0 & 0 & 0 \\ 0 & 0 & 0 & 0 & 0 & 0 & 0 & 0 & 0 & 0 & 0 & 0 & 0 & 0 \\ 0 & 0 & \mathbf{0} & \mathbf{0} & \mathbf{0} & \mathbf{0} & \mathbf{0} & \mathbf{0} & \mathbf{0} & \mathbf{0} & \mathbf{0} & \mathbf{0} & \mathbf{0} & \mathbf{0} & \mathbf{0}\end{array}$

-Non-Generating Public Entities-

78 Ashland, City of

79 Benton County PUD \#1

80 Clallam County PUD \#1

81 Clatskanie PUD

82 Consumers Power

83 Emerald County PUD

84 Energy Northwest

85 Fall River Elec Coop

86 Franklin County PUD \#1

87 Klickitat County PUD \#1

88 Lewis County PUD \#1

89 Mason County PUD \#1

90 Mason County PUD \#3

91 Oregon Trail Coop

92 Tanner Elec Coop

93 Tillamook PUD \#1

94 Total Non-Generating Public Entities

$\begin{array}{lllllllllllllll}0 & 0 & 0 & 0 & 0 & 0 & 0 & 0 & 0 & 0 & 0 & 0 & 0 & 0 & 0 \\ 0 & 0 & 0 & 0 & 0 & 0 & 0 & 0 & 0 & 0 & 0 & 0 & 0 & 0 & 0 \\ 0 & 0 & 0 & 0 & 0 & 0 & 0 & 0 & 0 & 0 & 0 & 0 & 0 & 0 & 0 \\ 0 & 0 & 0 & 0 & 0 & 0 & 0 & 0 & 0 & 0 & 0 & 0 & 0 & 0 & 0 \\ 0 & 0 & 0 & 0 & 0 & 0 & 0 & 0 & 0 & 0 & 0 & 0 & 0 & 0 & 0 \\ 0 & 0 & 0 & 0 & 0 & 0 & 0 & 0 & 0 & 0 & 0 & 0 & 0 & 0 & 0 \\ 0 & 0 & 0 & 0 & 0 & 0 & 0 & 0 & 0 & 0 & 0 & 0 & 0 & 0 & 0 \\ 0 & 0 & 0 & 0 & 0 & 0 & 0 & 0 & 0 & 0 & 0 & 0 & 0 & 0 & 0 \\ 0 & 0 & 0 & 0 & 0 & 0 & 0 & 0 & 0 & 0 & 0 & 0 & 0 & 0 & 0 \\ 0 & 0 & 0 & 0 & 0 & 0 & 0 & 0 & 0 & 0 & 0 & 0 & 0 & 0 & 0 \\ 0 & 0 & 0 & 0 & 0 & 0 & 0 & 0 & 0 & 0 & 0 & 0 & 0 & 0 & 0 \\ 0 & 0 & 0 & 0 & 0 & 0 & 0 & 0 & 0 & 0 & 0 & 0 & 0 & 0 & 0 \\ 0 & 0 & 0 & 0 & 0 & 0 & 0 & 0 & 0 & 0 & 0 & 0 & 0 & 0 & 0 \\ 0 & 0 & 0 & 0 & 0 & 0 & 0 & 0 & 0 & 0 & 0 & 0 & 0 & 0 & 0 \\ 0 & 0 & 0 & 0 & 0 & 0 & 0 & 0 & 0 & 0 & 0 & 0 & 0 & 0 & 0 \\ 0 & 0 & 0 & 0 & 0 & 0 & 0 & 0 & 0 & 0 & 0 & 0 & 0 & 0 & 0 \\ 0 & \mathbf{0} & \mathbf{0} & \mathbf{0} & \mathbf{0} & \mathbf{0} & \mathbf{0} & \mathbf{0} & \mathbf{0} & \mathbf{0} & \mathbf{0} & \mathbf{0} & \mathbf{0} & \mathbf{0} & \mathbf{0}\end{array}$

-Investor-Owned Entities-

95 Avista Corp (WWP Division)

96 Idaho Power Company

97 Northwestern Energy LLC (MPC)

98 Pacific Power

99 Portland General Electric

100 Puget Sound Energy

101 Trans Alta Utilities Corp

102 Utah Power

103 Total Investor-Owned Entities

\begin{tabular}{lllllllllllllll}
0 & 0 & 0 & 0 & 0 & 0 & 0 & 0 & 0 & 0 & 0 & 0 & 0 & 0 & 0 \\
0 & 0 & 0 & 0 & 0 & 0 & 0 & 0 & 0 & 0 & 0 & 0 & 0 & 0 & 0 \\
0 & 0 & 0 & 0 & 0 & 0 & 0 & 0 & 0 & 0 & 0 & 0 & 0 & 0 & 0 \\
0 & 0 & 0 & 0 & 0 & 0 & 0 & 0 & 0 & 0 & 0 & 0 & 0 & 0 & 0 \\
0 & 0 & 0 & 0 & 0 & 0 & 0 & 0 & 0 & 0 & 0 & 0 & 0 & 0 & 0 \\
0 & 0 & 0 & 0 & 0 & 0 & 0 & 0 & 0 & 0 & 0 & 0 & 0 & 0 & 0 \\
0 & 0 & 0 & 0 & 0 & 0 & 0 & 0 & 0 & 0 & 0 & 0 & 0 & 0 & 0 \\
0 & 0 & 0 & 0 & 0 & 0 & 0 & 0 & 0 & 0 & 0 & 0 & 0 & 0 & 0 \\
\hline $\mathbf{0}$ & $\mathbf{0}$ & $\mathbf{0}$ & $\mathbf{0}$ & $\mathbf{0}$ & $\mathbf{0}$ & $\mathbf{0}$ & $\mathbf{0}$ & $\mathbf{0}$ & $\mathbf{0}$ & $\mathbf{0}$ & $\mathbf{0}$ & $\mathbf{0}$ & $\mathbf{0}$ & $\mathbf{0}$
\end{tabular}

-Other Entities-

104 Avista Energy Inc.

105 Chehalis Power Inc.

106 FPL Energy Vansycle LLC

107 Frederickson Power LP,

108 Hermiston Power Partnership

109 Klamath Pacific Energy

110 Longview Fibre

111 Mirant Americas Energy Marketing, L.P.

112 PPM Energy

113 SP Newsprint

114 Total Other Entities

Large Thermal Reserves

-Federal Entities-

115 BPA - Power Business 116 Total Federal Entities

$\begin{array}{lllllllllllllll}0 & 0 & 0 & 0 & 0 & 0 & 0 & 0 & 0 & 0 & 0 & 0 & 0 & 0 & 0 \\ 0 & 0 & 0 & 0 & 0 & 0 & 0 & 0 & 0 & 0 & 0 & 0 & 0 & 0 & 0 \\ 0 & 0 & 0 & 0 & 0 & 0 & 0 & 0 & 0 & 0 & 0 & 0 & 0 & 0 & 0 \\ 0 & 0 & 0 & 0 & 0 & 0 & 0 & 0 & 0 & 0 & 0 & 0 & 0 & 0 & 0 \\ 0 & 0 & 0 & 0 & 0 & 0 & 0 & 0 & 0 & 0 & 0 & 0 & 0 & 0 & 0 \\ 0 & 0 & 0 & 0 & 0 & 0 & 0 & 0 & 0 & 0 & 0 & 0 & 0 & 0 & 0 \\ 0 & 0 & 0 & 0 & 0 & 0 & 0 & 0 & 0 & 0 & 0 & 0 & 0 & 0 & 0 \\ 0 & 0 & 0 & 0 & 0 & 0 & 0 & 0 & 0 & 0 & 0 & 0 & 0 & 0 & 0 \\ 0 & 0 & 0 & 0 & 0 & 0 & 0 & 0 & 0 & 0 & 0 & 0 & 0 & 0 & 0 \\ 0 & 0 & 0 & 0 & 0 & 0 & 0 & 0 & 0 & 0 & 0 & 0 & 0 & 0 & 0 \\ 0 & \mathbf{0} & \mathbf{0} & \mathbf{0} & \mathbf{0} & \mathbf{0} & \mathbf{0} & \mathbf{0} & \mathbf{0} & \mathbf{0} & \mathbf{0} & \mathbf{0} & \mathbf{0} & \mathbf{0} & \mathbf{0}\end{array}$

-Generating Public Entities-

117 Turlock Irrigation District

118 Total Generating Public Entities

-Non-Generating Public Entities-

119 Total Non-Generating Public Entities 
Average Energy in Megawatts

120 Avista Corp (WWP Division)

121 Idaho Power Company

122 Northwestern Energy LLC (MPC)

123 Pacific Power

124 Portland General Electric

125 Puget Sound Energy

126 San Diego Gas \& Electric

127 Trans Alta Utilities Corp

128 Total Investor-Owned Entities

\section{Water Year}

2003 White Book

-Other Entities-

129 Total Other Entities

\section{Aug1 Aug16}

Sep

Oct Nov Dec Jan Feb

Mar Apr1 Apr1

May Jun Jul Avg

Contract Reserves

-Federal Entities-

130 Total Federal Entities

$\begin{array}{llll}0 & 0 & 0 & 0 \\ 0 & 0 & 0 & 0 \\ 0 & 0 & 0 & 0 \\ 0 & 0 & 0 & 0 \\ 0 & 0 & 0 & 0 \\ 0 & 0 & 0 & 0 \\ 0 & 0 & 0 & 0 \\ 0 & 0 & 0 & 0 \\ 0 & 0 & 0 & 0\end{array}$

$\begin{array}{ll}0 & 0 \\ 0 & 0 \\ 0 & 0 \\ 0 & 0 \\ 0 & 0 \\ 0 & 0 \\ 0 & 0 \\ 0 & 0 \\ 0 & 0\end{array}$

$\begin{array}{lll}0 & 0 & 0 \\ 0 & 0 & 0 \\ 0 & 0 & 0 \\ 0 & 0 & 0 \\ 0 & 0 & 0 \\ 0 & 0 & \\ 0 & 0 & \\ 0 & 0 & 0 \\ 0 & 0\end{array}$

0
0
0
0
0
0
0
0
0

$\begin{array}{ll}0 & 0 \\ 0 & 0 \\ 0 & 0 \\ 0 & 0 \\ 0 & 0 \\ 0 & 0 \\ 0 & 0 \\ 0 & 0 \\ 0 & 0\end{array}$

$\begin{array}{lllllll}0 & 0 & 0 & 0 & 0 & 0 & 0 \\ 0 & 0 & 0 & 0 & 0 & 0 & 0 \\ 0 & 0 & 0 & 0 & 0 & 0 & 0 \\ 0 & 0 & 0 & 0 & 0 & 0 & 0 \\ 0 & 0 & 0 & 0 & 0 & 0 & 0 \\ 0 & 0 & 0 & 0 & 0 & 0 & 0 \\ 0 & 0 & 0 & 0 & 0 & 0 & 0 \\ 0 & 0 & 0 & 0 & 0 & 0 & 0 \\ 0 & 0 & 0 & 0 & 0 & 0 & 0\end{array}$

-Generating Public Entities-

131 Total Generating Public Entities

$0 \quad 0$

0

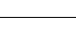

-Non-Generating Public Entities-

132 Total Non-Generating Public Entities

-Investor-Owned Entities-

133 Northwestern Energy LLC (MPC)

134 Pacific Power

135 Total Investor-Owned Entities

$\begin{array}{lllllllllllllll}0 & 0 & 0 & 0 & 0 & 0 & 0 & 0 & 0 & 0 & 0 & 0 & 0 & 0 & 0 \\ 0 & 0 & 0 & 0 & 0 & 0 & 0 & 0 & 0 & 0 & 0 & 0 & 0 & 0 & 0 \\ 0 & 0 & 0 & 0 & 0 & 0 & 0 & 0 & 0 & 0 & 0 & 0 & 0 & 0 & 0\end{array}$

-Other Entities-

136 Total Other Entities

-Total Capacity Reserves-

137 Total Hydro Reserves

138 Total Small Thermal \& Misc Reserves

139 Total Large Thermal Reserves

140 Total Contract Reserves

141 Total Capacity Reserves

$\begin{array}{lllllllllllllll}0 & 0 & 0 & 0 & 0 & 0 & 0 & 0 & 0 & 0 & 0 & 0 & 0 & 0 & 0 \\ 0 & 0 & 0 & 0 & 0 & 0 & 0 & 0 & 0 & 0 & 0 & 0 & 0 & 0 & 0 \\ 0 & 0 & 0 & 0 & 0 & 0 & 0 & 0 & 0 & 0 & 0 & 0 & 0 & 0 & 0 \\ 0 & 0 & 0 & 0 & 0 & 0 & 0 & 0 & 0 & 0 & 0 & 0 & 0 & 0 & 0 \\ 0 & 0 & 0 & 0 & 0 & 0 & 0 & 0 & 0 & 0 & 0 & 0 & 0 & 0 & 0\end{array}$


Table A-17: Regional Capacity Reserves

PNW Loads and Resources Study

2005 - 2006 Operating Year

1937 Water Year

2003 White Book

Oct Nov

Dec Jan Fe

Feb Mar Apr1 Apr16 May Jun Jul Avg

Hydro Reserves

$1 \mathrm{BPA}$ - Power Business

2 Total Federal Entities

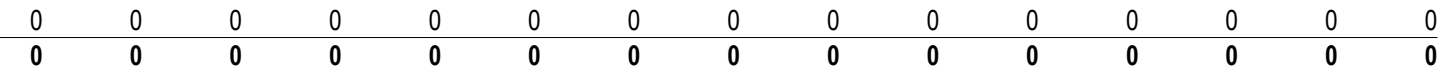

-Generating Public Entities-

3 Chelan County PUD \#1

4 Clark County PUD \#1

5 Cowlitz County PUD \#1

6 Douglas County PUD \#1

7 Eugene Water \& Electric Board

8 Grant County PUD \#2

9 Grays Harbor PUD \#1

10 Okanogan County PUD \#1

11 Pend Oreille County PUD \#1

12 Seattle City Light

13 Snohomish County PUD \#1

14 Tacoma Public Utilities

15 Total Generating Public Entities

$\begin{array}{lllllllllllllll}0 & 0 & 0 & 0 & 0 & 0 & 0 & 0 & 0 & 0 & 0 & 0 & 0 & 0 & 0 \\ 0 & 0 & 0 & 0 & 0 & 0 & 0 & 0 & 0 & 0 & 0 & 0 & 0 & 0 & 0 \\ 0 & 0 & 0 & 0 & 0 & 0 & 0 & 0 & 0 & 0 & 0 & 0 & 0 & 0 & 0 \\ 0 & 0 & 0 & 0 & 0 & 0 & 0 & 0 & 0 & 0 & 0 & 0 & 0 & 0 & 0 \\ 0 & 0 & 0 & 0 & 0 & 0 & 0 & 0 & 0 & 0 & 0 & 0 & 0 & 0 & 0 \\ 0 & 0 & 0 & 0 & 0 & 0 & 0 & 0 & 0 & 0 & 0 & 0 & 0 & 0 & 0 \\ 0 & 0 & 0 & 0 & 0 & 0 & 0 & 0 & 0 & 0 & 0 & 0 & 0 & 0 & 0 \\ 0 & 0 & 0 & 0 & 0 & 0 & 0 & 0 & 0 & 0 & 0 & 0 & 0 & 0 & 0 \\ 0 & 0 & 0 & 0 & 0 & 0 & 0 & 0 & 0 & 0 & 0 & 0 & 0 & 0 & 0 \\ 0 & 0 & 0 & 0 & 0 & 0 & 0 & 0 & 0 & 0 & 0 & 0 & 0 & 0 & 0 \\ 0 & 0 & 0 & 0 & 0 & 0 & 0 & 0 & 0 & 0 & 0 & 0 & 0 & 0 & 0 \\ 0 & 0 & 0 & 0 & 0 & 0 & 0 & 0 & 0 & 0 & 0 & 0 & 0 & 0 & 0 \\ 0 & 0 & \mathbf{0} & \mathbf{0} & \mathbf{0} & \mathbf{0} & \mathbf{0} & \mathbf{0} & \mathbf{0} & \mathbf{0} & \mathbf{0} & \mathbf{0} & \mathbf{0} & \mathbf{0} & \mathbf{0}\end{array}$

-Non-Generating Public Entities-

16 Ashland, City of

17 Benton County PUD \#1

18 Bonners Ferry, City of

19 Centralia, City of

20 Clallam County PUD \#1

21 Clearwater Power

22 Consumers Power

23 Emerald County PUD

24 Energy Northwest

25 Fall River Elec Coop

26 Forest Grove, City of

27 Franklin County PUD \#1

28 Idaho County L \& P

29 Idaho Falls, City of

30 Kittitas County PUD \#1

31 Klickitat County PUD \#1

32 Kootenai Electric Coop

33 Lewis County PUD \#1

34 Lost River Elec Coop

35 Lower Valley Energy

36 Mason County PUD \#1

37 McMinnville, City of

38 Milton Freewater, City of

39 Northern Lights

40 Northern Wasco County PUD

41 Oregon Trail Coop

42 Port Angeles, City of

43 Raft River Elec Coop

44 Salmon River Elec Coop

45 Soda Springs, City of

46 Tanner Elec Coop

47 Tillamook PUD \#1

48 United Electric Coop

49 Total Non-Generating Public Entities

\begin{tabular}{|c|c|c|c|c|c|c|c|c|c|c|c|c|c|}
\hline 0 & 0 & 0 & 0 & 0 & 0 & 0 & 0 & 0 & 0 & 0 & 0 & 0 & 0 \\
\hline 0 & 0 & 0 & 0 & 0 & 0 & 0 & 0 & 0 & 0 & 0 & 0 & 0 & 0 \\
\hline 0 & 0 & 0 & 0 & 0 & 0 & 0 & 0 & 0 & 0 & 0 & 0 & 0 & 0 \\
\hline 0 & 0 & 0 & 0 & 0 & 0 & 0 & 0 & 0 & 0 & 0 & 0 & 0 & 0 \\
\hline 0 & 0 & 0 & 0 & 0 & 0 & 0 & 0 & 0 & 0 & 0 & 0 & 0 & 0 \\
\hline 0 & 0 & 0 & 0 & 0 & 0 & 0 & 0 & 0 & 0 & 0 & 0 & 0 & 0 \\
\hline 0 & 0 & 0 & 0 & 0 & 0 & 0 & 0 & 0 & 0 & 0 & 0 & 0 & 0 \\
\hline 0 & 0 & 0 & 0 & 0 & 0 & 0 & 0 & 0 & 0 & 0 & 0 & 0 & 0 \\
\hline 0 & 0 & 0 & 0 & 0 & 0 & 0 & 0 & 0 & 0 & 0 & 0 & 0 & 0 \\
\hline 0 & 0 & 0 & 0 & 0 & 0 & 0 & 0 & 0 & 0 & 0 & 0 & 0 & 0 \\
\hline 0 & 0 & 0 & 0 & 0 & 0 & 0 & 0 & 0 & 0 & 0 & 0 & 0 & 0 \\
\hline 0 & 0 & 0 & 0 & 0 & 0 & 0 & 0 & 0 & 0 & 0 & 0 & 0 & 0 \\
\hline 0 & 0 & 0 & 0 & 0 & 0 & 0 & 0 & 0 & 0 & 0 & 0 & 0 & 0 \\
\hline 0 & 0 & 0 & 0 & 0 & 0 & 0 & 0 & 0 & 0 & 0 & 0 & 0 & 0 \\
\hline 0 & 0 & 0 & 0 & 0 & 0 & 0 & 0 & 0 & 0 & 0 & 0 & 0 & 0 \\
\hline 0 & 0 & 0 & 0 & 0 & 0 & 0 & 0 & 0 & 0 & 0 & 0 & 0 & 0 \\
\hline 0 & 0 & 0 & 0 & 0 & 0 & 0 & 0 & 0 & 0 & 0 & 0 & 0 & 0 \\
\hline 0 & 0 & 0 & 0 & 0 & 0 & 0 & 0 & 0 & 0 & 0 & 0 & 0 & 0 \\
\hline 0 & 0 & 0 & 0 & 0 & 0 & 0 & 0 & 0 & 0 & 0 & 0 & 0 & 0 \\
\hline 0 & 0 & 0 & 0 & 0 & 0 & 0 & 0 & 0 & 0 & 0 & 0 & 0 & 0 \\
\hline 0 & 0 & 0 & 0 & 0 & 0 & 0 & 0 & 0 & 0 & 0 & 0 & 0 & 0 \\
\hline 0 & 0 & 0 & 0 & 0 & 0 & 0 & 0 & 0 & 0 & 0 & 0 & 0 & 0 \\
\hline 0 & 0 & 0 & 0 & 0 & 0 & 0 & 0 & 0 & 0 & 0 & 0 & 0 & 0 \\
\hline 0 & 0 & 0 & 0 & 0 & 0 & 0 & 0 & 0 & 0 & 0 & 0 & 0 & 0 \\
\hline 0 & 0 & 0 & 0 & 0 & 0 & 0 & 0 & 0 & 0 & 0 & 0 & 0 & 0 \\
\hline 0 & 0 & 0 & 0 & 0 & 0 & 0 & 0 & 0 & 0 & 0 & 0 & 0 & 0 \\
\hline 0 & 0 & 0 & 0 & 0 & 0 & 0 & 0 & 0 & 0 & 0 & 0 & 0 & 0 \\
\hline 0 & 0 & 0 & 0 & 0 & 0 & 0 & 0 & 0 & 0 & 0 & 0 & 0 & 0 \\
\hline 0 & 0 & 0 & 0 & 0 & 0 & 0 & 0 & 0 & 0 & 0 & 0 & 0 & 0 \\
\hline 0 & 0 & 0 & 0 & 0 & 0 & 0 & 0 & 0 & 0 & 0 & 0 & 0 & 0 \\
\hline 0 & 0 & 0 & 0 & 0 & 0 & 0 & 0 & 0 & 0 & 0 & 0 & 0 & 0 \\
\hline 0 & 0 & 0 & 0 & 0 & 0 & 0 & 0 & 0 & 0 & 0 & 0 & 0 & 0 \\
\hline 0 & 0 & 0 & 0 & 0 & 0 & 0 & 0 & 0 & 0 & 0 & 0 & 0 & 0 \\
\hline 0 & 0 & 0 & 0 & 0 & 0 & 0 & 0 & 0 & 0 & 0 & 0 & 0 & 0 \\
\hline
\end{tabular}

-Investor-Owned Entities-

50 Avista Corp (WWP Division)

51 Idaho Power Company

52 Northwestern Energy LLC (MPC)

53 Pacific Power

54 Portland General Electric

55 Puget Sound Energy

56 Total Investor-Owned Entities

-Other Entities-

57 Colockum Transmission Company

58 Longview Fibre

59 Warm Springs Power Enterprise

60 Total Other Entities

$\begin{array}{lllllllllllllll}0 & 0 & 0 & 0 & 0 & 0 & 0 & 0 & 0 & 0 & 0 & 0 & 0 & 0 & 0 \\ 0 & 0 & 0 & 0 & 0 & 0 & 0 & 0 & 0 & 0 & 0 & 0 & 0 & 0 & 0 \\ 0 & 0 & 0 & 0 & 0 & 0 & 0 & 0 & 0 & 0 & 0 & 0 & 0 & 0 & 0 \\ 0 & 0 & 0 & 0 & 0 & 0 & 0 & 0 & 0 & 0 & 0 & 0 & 0 & 0 & 0 \\ 0 & 0 & 0 & 0 & 0 & 0 & 0 & 0 & 0 & 0 & 0 & 0 & 0 & 0 & 0 \\ 0 & 0 & 0 & 0 & 0 & 0 & 0 & 0 & 0 & 0 & 0 & 0 & 0 & 0 & 0 \\ 0 & \mathbf{0} & \mathbf{0} & \mathbf{0} & \mathbf{0} & \mathbf{0} & \mathbf{0} & \mathbf{0} & \mathbf{0} & \mathbf{0} & \mathbf{0} & \mathbf{0} & \mathbf{0} & \mathbf{0} & \mathbf{0}\end{array}$

Small Thermal \& Misc. Reserves

-Federal Entities-

61 BPA - Power Business

62 U.S. Naval Station, Everett (Jim Creek)

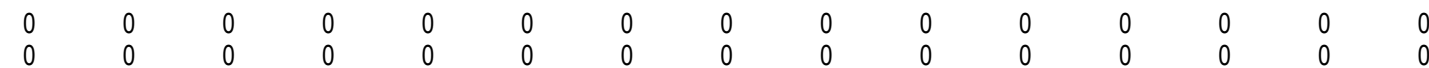




$\begin{array}{lllllllllllllll}0 & 0 & 0 & 0 & 0 & 0 & 0 & 0 & 0 & 0 & 0 & 0 & 0 & 0 & 0 \\ 0 & 0 & 0 & 0 & 0 & 0 & 0 & 0 & 0 & 0 & 0 & 0 & 0 & 0 & 0\end{array}$

-Generating Public Entities-

65 Chelan County PUD \#1

66 Clark County PUD \#1

67 Cowlitz County PUD \#1

68 Douglas County PUD \#1

69 Eugene Water \& Electric Board

70 Grant County PUD \#2

71 Grays Harbor PUD \#1

72 Okanogan County PUD \#1

73 Seattle City Light

74 Snohomish County PUD \#1

75 Springfield Utility Board

76 Tacoma Public Utilities

77 Total Generating Public Entities

$\begin{array}{lllllllllllllll}0 & 0 & 0 & 0 & 0 & 0 & 0 & 0 & 0 & 0 & 0 & 0 & 0 & 0 & 0 \\ 0 & 0 & 0 & 0 & 0 & 0 & 0 & 0 & 0 & 0 & 0 & 0 & 0 & 0 & 0 \\ 0 & 0 & 0 & 0 & 0 & 0 & 0 & 0 & 0 & 0 & 0 & 0 & 0 & 0 & 0 \\ 0 & 0 & 0 & 0 & 0 & 0 & 0 & 0 & 0 & 0 & 0 & 0 & 0 & 0 & 0 \\ 0 & 0 & 0 & 0 & 0 & 0 & 0 & 0 & 0 & 0 & 0 & 0 & 0 & 0 & 0 \\ 0 & 0 & 0 & 0 & 0 & 0 & 0 & 0 & 0 & 0 & 0 & 0 & 0 & 0 & 0 \\ 0 & 0 & 0 & 0 & 0 & 0 & 0 & 0 & 0 & 0 & 0 & 0 & 0 & 0 & 0 \\ 0 & 0 & 0 & 0 & 0 & 0 & 0 & 0 & 0 & 0 & 0 & 0 & 0 & 0 & 0 \\ 0 & 0 & 0 & 0 & 0 & 0 & 0 & 0 & 0 & 0 & 0 & 0 & 0 & 0 & 0 \\ 0 & 0 & 0 & 0 & 0 & 0 & 0 & 0 & 0 & 0 & 0 & 0 & 0 & 0 & 0 \\ 0 & 0 & 0 & 0 & 0 & 0 & 0 & 0 & 0 & 0 & 0 & 0 & 0 & 0 & 0 \\ 0 & 0 & 0 & 0 & 0 & 0 & 0 & 0 & 0 & 0 & 0 & 0 & 0 & 0 \\ 0 & 0 & \mathbf{0} & \mathbf{0} & \mathbf{0} & \mathbf{0} & \mathbf{0} & \mathbf{0} & \mathbf{0} & \mathbf{0} & \mathbf{0} & \mathbf{0} & \mathbf{0} & \mathbf{0} & \mathbf{0}\end{array}$

-Non-Generating Public Entities-

78 Ashland, City of

79 Benton County PUD \#1

80 Clallam County PUD \#1

81 Clatskanie PUD

82 Consumers Power

83 Emerald County PUD

84 Energy Northwest

85 Fall River Elec Coop

86 Franklin County PUD \#1

87 Klickitat County PUD \#1

88 Lewis County PUD \#1

89 Mason County PUD \#1

90 Mason County PUD \#3

91 Oregon Trail Coop

92 Tanner Elec Coop

93 Tillamook PUD \#1

94 Total Non-Generating Public Entities

$\begin{array}{lllllllllllllll}0 & 0 & 0 & 0 & 0 & 0 & 0 & 0 & 0 & 0 & 0 & 0 & 0 & 0 & 0 \\ 0 & 0 & 0 & 0 & 0 & 0 & 0 & 0 & 0 & 0 & 0 & 0 & 0 & 0 & 0 \\ 0 & 0 & 0 & 0 & 0 & 0 & 0 & 0 & 0 & 0 & 0 & 0 & 0 & 0 & 0 \\ 0 & 0 & 0 & 0 & 0 & 0 & 0 & 0 & 0 & 0 & 0 & 0 & 0 & 0 & 0 \\ 0 & 0 & 0 & 0 & 0 & 0 & 0 & 0 & 0 & 0 & 0 & 0 & 0 & 0 & 0 \\ 0 & 0 & 0 & 0 & 0 & 0 & 0 & 0 & 0 & 0 & 0 & 0 & 0 & 0 & 0 \\ 0 & 0 & 0 & 0 & 0 & 0 & 0 & 0 & 0 & 0 & 0 & 0 & 0 & 0 & 0 \\ 0 & 0 & 0 & 0 & 0 & 0 & 0 & 0 & 0 & 0 & 0 & 0 & 0 & 0 & 0 \\ 0 & 0 & 0 & 0 & 0 & 0 & 0 & 0 & 0 & 0 & 0 & 0 & 0 & 0 & 0 \\ 0 & 0 & 0 & 0 & 0 & 0 & 0 & 0 & 0 & 0 & 0 & 0 & 0 & 0 & 0 \\ 0 & 0 & 0 & 0 & 0 & 0 & 0 & 0 & 0 & 0 & 0 & 0 & 0 & 0 & 0 \\ 0 & 0 & 0 & 0 & 0 & 0 & 0 & 0 & 0 & 0 & 0 & 0 & 0 & 0 & 0 \\ 0 & 0 & 0 & 0 & 0 & 0 & 0 & 0 & 0 & 0 & 0 & 0 & 0 & 0 & 0 \\ 0 & 0 & 0 & 0 & 0 & 0 & 0 & 0 & 0 & 0 & 0 & 0 & 0 & 0 & 0 \\ 0 & 0 & 0 & 0 & 0 & 0 & 0 & 0 & 0 & 0 & 0 & 0 & 0 & 0 & 0 \\ 0 & 0 & 0 & 0 & 0 & 0 & 0 & 0 & 0 & 0 & 0 & 0 & 0 & 0 & 0 \\ 0 & \mathbf{0} & \mathbf{0} & \mathbf{0} & \mathbf{0} & \mathbf{0} & \mathbf{0} & \mathbf{0} & \mathbf{0} & \mathbf{0} & \mathbf{0} & \mathbf{0} & \mathbf{0} & \mathbf{0} & \mathbf{0}\end{array}$

-Investor-Owned Entities-

95 Avista Corp (WWP Division)

96 Idaho Power Company

97 Northwestern Energy LLC (MPC)

98 Pacific Power

99 Portland General Electric

100 Puget Sound Energy

101 Trans Alta Utilities Corp

102 Utah Power

103 Total Investor-Owned Entities

\begin{tabular}{lllllllllllllll}
0 & 0 & 0 & 0 & 0 & 0 & 0 & 0 & 0 & 0 & 0 & 0 & 0 & 0 & 0 \\
0 & 0 & 0 & 0 & 0 & 0 & 0 & 0 & 0 & 0 & 0 & 0 & 0 & 0 & 0 \\
0 & 0 & 0 & 0 & 0 & 0 & 0 & 0 & 0 & 0 & 0 & 0 & 0 & 0 & 0 \\
0 & 0 & 0 & 0 & 0 & 0 & 0 & 0 & 0 & 0 & 0 & 0 & 0 & 0 & 0 \\
0 & 0 & 0 & 0 & 0 & 0 & 0 & 0 & 0 & 0 & 0 & 0 & 0 & 0 & 0 \\
0 & 0 & 0 & 0 & 0 & 0 & 0 & 0 & 0 & 0 & 0 & 0 & 0 & 0 & 0 \\
0 & 0 & 0 & 0 & 0 & 0 & 0 & 0 & 0 & 0 & 0 & 0 & 0 & 0 & 0 \\
0 & 0 & 0 & 0 & 0 & 0 & 0 & 0 & 0 & 0 & 0 & 0 & 0 & 0 & 0 \\
\hline $\mathbf{0}$ & $\mathbf{0}$ & $\mathbf{0}$ & $\mathbf{0}$ & $\mathbf{0}$ & $\mathbf{0}$ & $\mathbf{0}$ & $\mathbf{0}$ & $\mathbf{0}$ & $\mathbf{0}$ & $\mathbf{0}$ & $\mathbf{0}$ & $\mathbf{0}$ & $\mathbf{0}$ & $\mathbf{0}$
\end{tabular}

-Other Entities-

104 Avista Energy Inc.

105 Chehalis Power Inc.

106 FPL Energy Vansycle LLC

107 Frederickson Power LP,

108 Hermiston Power Partnership

109 Klamath Pacific Energy

110 Longview Fibre

111 Mirant Americas Energy Marketing, L.P.

112 PPM Energy

113 SP Newsprint

114 Total Other Entities

Large Thermal Reserves

-Federal Entities-

115 BPA - Power Business 116 Total Federal Entities

$\begin{array}{lllllllllllllll}0 & 0 & 0 & 0 & 0 & 0 & 0 & 0 & 0 & 0 & 0 & 0 & 0 & 0 & 0 \\ 0 & 0 & 0 & 0 & 0 & 0 & 0 & 0 & 0 & 0 & 0 & 0 & 0 & 0 & 0 \\ 0 & 0 & 0 & 0 & 0 & 0 & 0 & 0 & 0 & 0 & 0 & 0 & 0 & 0 & 0 \\ 0 & 0 & 0 & 0 & 0 & 0 & 0 & 0 & 0 & 0 & 0 & 0 & 0 & 0 & 0 \\ 0 & 0 & 0 & 0 & 0 & 0 & 0 & 0 & 0 & 0 & 0 & 0 & 0 & 0 & 0 \\ 0 & 0 & 0 & 0 & 0 & 0 & 0 & 0 & 0 & 0 & 0 & 0 & 0 & 0 & 0 \\ 0 & 0 & 0 & 0 & 0 & 0 & 0 & 0 & 0 & 0 & 0 & 0 & 0 & 0 & 0 \\ 0 & 0 & 0 & 0 & 0 & 0 & 0 & 0 & 0 & 0 & 0 & 0 & 0 & 0 & 0 \\ 0 & 0 & 0 & 0 & 0 & 0 & 0 & 0 & 0 & 0 & 0 & 0 & 0 & 0 & 0 \\ 0 & 0 & 0 & 0 & 0 & 0 & 0 & 0 & 0 & 0 & 0 & 0 & 0 & 0 & 0 \\ 0 & \mathbf{0} & \mathbf{0} & \mathbf{0} & \mathbf{0} & \mathbf{0} & \mathbf{0} & \mathbf{0} & \mathbf{0} & \mathbf{0} & \mathbf{0} & \mathbf{0} & \mathbf{0} & \mathbf{0} & \mathbf{0}\end{array}$

-Generating Public Entities-

117 Turlock Irrigation District

118 Total Generating Public Entities

-Non-Generating Public Entities-

119 Total Non-Generating Public Entities 
Average Energy in Megawatts

120 Avista Corp (WWP Division)

121 Idaho Power Company

122 Northwestern Energy LLC (MPC)

123 Pacific Power

124 Portland General Electric

125 Puget Sound Energy

126 San Diego Gas \& Electric

127 Trans Alta Utilities Corp

128 Total Investor-Owned Entities

\section{Water Year}

2003 White Book

-Other Entities-

129 Total Other Entities

\section{Aug1 Aug16}

Sep

Oct Nov Dec Jan Feb

Mar Apr1 Apr1

May Jun Jul Avg

Contract Reserves

-Federal Entities-

130 Total Federal Entities

$\begin{array}{llll}0 & 0 & 0 & 0 \\ 0 & 0 & 0 & 0 \\ 0 & 0 & 0 & 0 \\ 0 & 0 & 0 & 0 \\ 0 & 0 & 0 & 0 \\ 0 & 0 & 0 & 0 \\ 0 & 0 & 0 & 0 \\ 0 & 0 & 0 & 0 \\ 0 & 0 & 0 & 0\end{array}$

$\begin{array}{ll}0 & 0 \\ 0 & 0 \\ 0 & 0 \\ 0 & 0 \\ 0 & 0 \\ 0 & 0 \\ 0 & 0 \\ 0 & 0 \\ 0 & 0\end{array}$

$\begin{array}{lll}0 & 0 & 0 \\ 0 & 0 & 0 \\ 0 & 0 & 0 \\ 0 & 0 & 0 \\ 0 & 0 & 0 \\ 0 & 0 & \\ 0 & 0 & \\ 0 & 0 & 0 \\ 0 & 0\end{array}$

0
0
0
0
0
0
0
0
0

$\begin{array}{ll}0 & 0 \\ 0 & 0 \\ 0 & 0 \\ 0 & 0 \\ 0 & 0 \\ 0 & 0 \\ 0 & 0 \\ 0 & 0 \\ 0\end{array}$

$\begin{array}{lllllll}0 & 0 & 0 & 0 & 0 & 0 & 0 \\ 0 & 0 & 0 & 0 & 0 & 0 & 0 \\ 0 & 0 & 0 & 0 & 0 & 0 & 0 \\ 0 & 0 & 0 & 0 & 0 & 0 & 0 \\ 0 & 0 & 0 & 0 & 0 & 0 & 0 \\ 0 & 0 & 0 & 0 & 0 & 0 & 0 \\ 0 & 0 & 0 & 0 & 0 & 0 & 0 \\ 0 & 0 & 0 & 0 & 0 & 0 & 0 \\ 0 & 0 & 0 & 0 & 0 & 0 & 0\end{array}$

-Generating Public Entities-

131 Total Generating Public Entities

0

0

0

0

0

0

0

$\begin{array}{llll}0 & 0 & 0 & 0\end{array}$

-Non-Generating Public Entities-

132 Total Non-Generating Public Entities

-Investor-Owned Entities-

133 Northwestern Energy LLC (MPC)

134 Pacific Power

135 Total Investor-Owned Entities

$\begin{array}{llllllllllllllll}0 & 0 & 0 & 0 & 0 & 0 & 0 & 0 & 0 & 0 & 0 & 0 & 0 & 0\end{array}$

-Other Entities-

136 Total Other Entities

-Total Capacity Reserves-

137 Total Hydro Reserves

138 Total Small Thermal \& Misc Reserves

139 Total Large Thermal Reserves

140 Total Contract Reserves

141 Total Capacity Reserves

$\begin{array}{lllllllllllllll}0 & 0 & 0 & 0 & 0 & 0 & 0 & 0 & 0 & 0 & 0 & 0 & 0 & 0 & 0\end{array}$


Table A-17: Regional Capacity Reserves

PNW Loads and Resources Study

2006 - 2007 Operating Year

1937 Water Year

2003 White Book

Oct Nov

Dec Jan Fe

Feb Mar Apr1 Apr16 May Jun Jul Avg

Hydro Reserves

$1 \mathrm{BPA}$ - Power Business

2 Total Federal Entities

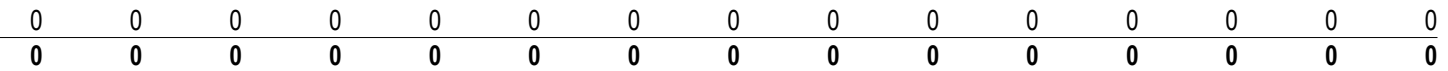

-Generating Public Entities-

3 Chelan County PUD \#1

4 Clark County PUD \#1

5 Cowlitz County PUD \#1

6 Douglas County PUD \#1

7 Eugene Water \& Electric Board

8 Grant County PUD \#2

9 Grays Harbor PUD \#1

10 Okanogan County PUD \#1

11 Pend Oreille County PUD \#1

12 Seattle City Light

13 Snohomish County PUD \#1

14 Tacoma Public Utilities

15 Total Generating Public Entities

$\begin{array}{lllllllllllllll}0 & 0 & 0 & 0 & 0 & 0 & 0 & 0 & 0 & 0 & 0 & 0 & 0 & 0 & 0 \\ 0 & 0 & 0 & 0 & 0 & 0 & 0 & 0 & 0 & 0 & 0 & 0 & 0 & 0 & 0 \\ 0 & 0 & 0 & 0 & 0 & 0 & 0 & 0 & 0 & 0 & 0 & 0 & 0 & 0 & 0 \\ 0 & 0 & 0 & 0 & 0 & 0 & 0 & 0 & 0 & 0 & 0 & 0 & 0 & 0 & 0 \\ 0 & 0 & 0 & 0 & 0 & 0 & 0 & 0 & 0 & 0 & 0 & 0 & 0 & 0 & 0 \\ 0 & 0 & 0 & 0 & 0 & 0 & 0 & 0 & 0 & 0 & 0 & 0 & 0 & 0 & 0 \\ 0 & 0 & 0 & 0 & 0 & 0 & 0 & 0 & 0 & 0 & 0 & 0 & 0 & 0 & 0 \\ 0 & 0 & 0 & 0 & 0 & 0 & 0 & 0 & 0 & 0 & 0 & 0 & 0 & 0 & 0 \\ 0 & 0 & 0 & 0 & 0 & 0 & 0 & 0 & 0 & 0 & 0 & 0 & 0 & 0 & 0 \\ 0 & 0 & 0 & 0 & 0 & 0 & 0 & 0 & 0 & 0 & 0 & 0 & 0 & 0 & 0 \\ 0 & 0 & 0 & 0 & 0 & 0 & 0 & 0 & 0 & 0 & 0 & 0 & 0 & 0 & 0 \\ 0 & 0 & 0 & 0 & 0 & 0 & 0 & 0 & 0 & 0 & 0 & 0 & 0 & 0 & 0 \\ 0 & 0 & \mathbf{0} & \mathbf{0} & \mathbf{0} & \mathbf{0} & \mathbf{0} & \mathbf{0} & \mathbf{0} & \mathbf{0} & \mathbf{0} & \mathbf{0} & \mathbf{0} & \mathbf{0} & \mathbf{0}\end{array}$

-Non-Generating Public Entities-

16 Ashland, City of

17 Benton County PUD \#1

18 Bonners Ferry, City of

19 Centralia, City of

20 Clallam County PUD \#1

21 Clearwater Power

22 Consumers Power

23 Emerald County PUD

24 Energy Northwest

25 Fall River Elec Coop

26 Forest Grove, City of

27 Franklin County PUD \#1

28 Idaho County L \& P

29 Idaho Falls, City of

30 Kittitas County PUD \#1

31 Klickitat County PUD \#1

32 Kootenai Electric Coop

33 Lewis County PUD \#1

34 Lost River Elec Coop

35 Lower Valley Energy

36 Mason County PUD \#1

37 McMinnville, City of

38 Milton Freewater, City of

39 Northern Lights

40 Northern Wasco County PUD

41 Oregon Trail Coop

42 Port Angeles, City of

43 Raft River Elec Coop

44 Salmon River Elec Coop

45 Soda Springs, City of

46 Tanner Elec Coop

47 Tillamook PUD \#1

48 United Electric Coop

49 Total Non-Generating Public Entities

\begin{tabular}{|c|c|c|c|c|c|c|c|c|c|c|c|c|c|}
\hline 0 & 0 & 0 & 0 & 0 & 0 & 0 & 0 & 0 & 0 & 0 & 0 & 0 & 0 \\
\hline 0 & 0 & 0 & 0 & 0 & 0 & 0 & 0 & 0 & 0 & 0 & 0 & 0 & 0 \\
\hline 0 & 0 & 0 & 0 & 0 & 0 & 0 & 0 & 0 & 0 & 0 & 0 & 0 & 0 \\
\hline 0 & 0 & 0 & 0 & 0 & 0 & 0 & 0 & 0 & 0 & 0 & 0 & 0 & 0 \\
\hline 0 & 0 & 0 & 0 & 0 & 0 & 0 & 0 & 0 & 0 & 0 & 0 & 0 & 0 \\
\hline 0 & 0 & 0 & 0 & 0 & 0 & 0 & 0 & 0 & 0 & 0 & 0 & 0 & 0 \\
\hline 0 & 0 & 0 & 0 & 0 & 0 & 0 & 0 & 0 & 0 & 0 & 0 & 0 & 0 \\
\hline 0 & 0 & 0 & 0 & 0 & 0 & 0 & 0 & 0 & 0 & 0 & 0 & 0 & 0 \\
\hline 0 & 0 & 0 & 0 & 0 & 0 & 0 & 0 & 0 & 0 & 0 & 0 & 0 & 0 \\
\hline 0 & 0 & 0 & 0 & 0 & 0 & 0 & 0 & 0 & 0 & 0 & 0 & 0 & 0 \\
\hline 0 & 0 & 0 & 0 & 0 & 0 & 0 & 0 & 0 & 0 & 0 & 0 & 0 & 0 \\
\hline 0 & 0 & 0 & 0 & 0 & 0 & 0 & 0 & 0 & 0 & 0 & 0 & 0 & 0 \\
\hline 0 & 0 & 0 & 0 & 0 & 0 & 0 & 0 & 0 & 0 & 0 & 0 & 0 & 0 \\
\hline 0 & 0 & 0 & 0 & 0 & 0 & 0 & 0 & 0 & 0 & 0 & 0 & 0 & 0 \\
\hline 0 & 0 & 0 & 0 & 0 & 0 & 0 & 0 & 0 & 0 & 0 & 0 & 0 & 0 \\
\hline 0 & 0 & 0 & 0 & 0 & 0 & 0 & 0 & 0 & 0 & 0 & 0 & 0 & 0 \\
\hline 0 & 0 & 0 & 0 & 0 & 0 & 0 & 0 & 0 & 0 & 0 & 0 & 0 & 0 \\
\hline 0 & 0 & 0 & 0 & 0 & 0 & 0 & 0 & 0 & 0 & 0 & 0 & 0 & 0 \\
\hline 0 & 0 & 0 & 0 & 0 & 0 & 0 & 0 & 0 & 0 & 0 & 0 & 0 & 0 \\
\hline 0 & 0 & 0 & 0 & 0 & 0 & 0 & 0 & 0 & 0 & 0 & 0 & 0 & 0 \\
\hline 0 & 0 & 0 & 0 & 0 & 0 & 0 & 0 & 0 & 0 & 0 & 0 & 0 & 0 \\
\hline 0 & 0 & 0 & 0 & 0 & 0 & 0 & 0 & 0 & 0 & 0 & 0 & 0 & 0 \\
\hline 0 & 0 & 0 & 0 & 0 & 0 & 0 & 0 & 0 & 0 & 0 & 0 & 0 & 0 \\
\hline 0 & 0 & 0 & 0 & 0 & 0 & 0 & 0 & 0 & 0 & 0 & 0 & 0 & 0 \\
\hline 0 & 0 & 0 & 0 & 0 & 0 & 0 & 0 & 0 & 0 & 0 & 0 & 0 & 0 \\
\hline 0 & 0 & 0 & 0 & 0 & 0 & 0 & 0 & 0 & 0 & 0 & 0 & 0 & 0 \\
\hline 0 & 0 & 0 & 0 & 0 & 0 & 0 & 0 & 0 & 0 & 0 & 0 & 0 & 0 \\
\hline 0 & 0 & 0 & 0 & 0 & 0 & 0 & 0 & 0 & 0 & 0 & 0 & 0 & 0 \\
\hline 0 & 0 & 0 & 0 & 0 & 0 & 0 & 0 & 0 & 0 & 0 & 0 & 0 & 0 \\
\hline 0 & 0 & 0 & 0 & 0 & 0 & 0 & 0 & 0 & 0 & 0 & 0 & 0 & 0 \\
\hline 0 & 0 & 0 & 0 & 0 & 0 & 0 & 0 & 0 & 0 & 0 & 0 & 0 & 0 \\
\hline 0 & 0 & 0 & 0 & 0 & 0 & 0 & 0 & 0 & 0 & 0 & 0 & 0 & 0 \\
\hline 0 & 0 & 0 & 0 & 0 & 0 & 0 & 0 & 0 & 0 & 0 & 0 & 0 & 0 \\
\hline 0 & 0 & 0 & 0 & 0 & 0 & 0 & 0 & 0 & 0 & 0 & 0 & 0 & 0 \\
\hline
\end{tabular}

-Investor-Owned Entities-

50 Avista Corp (WWP Division)

51 Idaho Power Company

52 Northwestern Energy LLC (MPC)

53 Pacific Power

54 Portland General Electric

55 Puget Sound Energy

56 Total Investor-Owned Entities

-Other Entities-

57 Colockum Transmission Company

58 Longview Fibre

59 Warm Springs Power Enterprise

60 Total Other Entities

$\begin{array}{lllllllllllllll}0 & 0 & 0 & 0 & 0 & 0 & 0 & 0 & 0 & 0 & 0 & 0 & 0 & 0 & 0 \\ 0 & 0 & 0 & 0 & 0 & 0 & 0 & 0 & 0 & 0 & 0 & 0 & 0 & 0 & 0 \\ 0 & 0 & 0 & 0 & 0 & 0 & 0 & 0 & 0 & 0 & 0 & 0 & 0 & 0 & 0 \\ 0 & 0 & 0 & 0 & 0 & 0 & 0 & 0 & 0 & 0 & 0 & 0 & 0 & 0 & 0 \\ 0 & 0 & 0 & 0 & 0 & 0 & 0 & 0 & 0 & 0 & 0 & 0 & 0 & 0 & 0 \\ 0 & 0 & 0 & 0 & 0 & 0 & 0 & 0 & 0 & 0 & 0 & 0 & 0 & 0 & 0 \\ 0 & \mathbf{0} & \mathbf{0} & \mathbf{0} & \mathbf{0} & \mathbf{0} & \mathbf{0} & \mathbf{0} & \mathbf{0} & \mathbf{0} & \mathbf{0} & \mathbf{0} & \mathbf{0} & \mathbf{0} & \mathbf{0}\end{array}$

Small Thermal \& Misc. Reserves

-Federal Entities-

61 BPA - Power Business

62 U.S. Naval Station, Everett (Jim Creek)

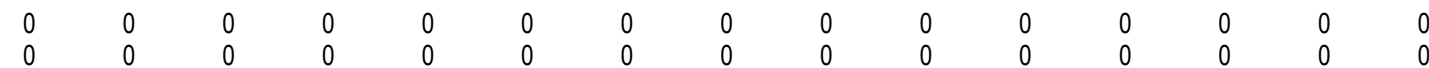




$\begin{array}{lllllllllllllll}0 & 0 & 0 & 0 & 0 & 0 & 0 & 0 & 0 & 0 & 0 & 0 & 0 & 0 & 0 \\ 0 & 0 & 0 & 0 & 0 & 0 & 0 & 0 & 0 & 0 & 0 & 0 & 0 & 0 & 0\end{array}$

-Generating Public Entities-

65 Chelan County PUD \#1

66 Clark County PUD \#1

67 Cowlitz County PUD \#1

68 Douglas County PUD \#1

69 Eugene Water \& Electric Board

70 Grant County PUD \#2

71 Grays Harbor PUD \#1

72 Okanogan County PUD \#1

73 Seattle City Light

74 Snohomish County PUD \#1

75 Springfield Utility Board

76 Tacoma Public Utilities

77 Total Generating Public Entities

$\begin{array}{lllllllllllllll}0 & 0 & 0 & 0 & 0 & 0 & 0 & 0 & 0 & 0 & 0 & 0 & 0 & 0 & 0 \\ 0 & 0 & 0 & 0 & 0 & 0 & 0 & 0 & 0 & 0 & 0 & 0 & 0 & 0 & 0 \\ 0 & 0 & 0 & 0 & 0 & 0 & 0 & 0 & 0 & 0 & 0 & 0 & 0 & 0 & 0 \\ 0 & 0 & 0 & 0 & 0 & 0 & 0 & 0 & 0 & 0 & 0 & 0 & 0 & 0 & 0 \\ 0 & 0 & 0 & 0 & 0 & 0 & 0 & 0 & 0 & 0 & 0 & 0 & 0 & 0 & 0 \\ 0 & 0 & 0 & 0 & 0 & 0 & 0 & 0 & 0 & 0 & 0 & 0 & 0 & 0 & 0 \\ 0 & 0 & 0 & 0 & 0 & 0 & 0 & 0 & 0 & 0 & 0 & 0 & 0 & 0 & 0 \\ 0 & 0 & 0 & 0 & 0 & 0 & 0 & 0 & 0 & 0 & 0 & 0 & 0 & 0 & 0 \\ 0 & 0 & 0 & 0 & 0 & 0 & 0 & 0 & 0 & 0 & 0 & 0 & 0 & 0 & 0 \\ 0 & 0 & 0 & 0 & 0 & 0 & 0 & 0 & 0 & 0 & 0 & 0 & 0 & 0 & 0 \\ 0 & 0 & 0 & 0 & 0 & 0 & 0 & 0 & 0 & 0 & 0 & 0 & 0 & 0 & 0 \\ 0 & 0 & 0 & 0 & 0 & 0 & 0 & 0 & 0 & 0 & 0 & 0 & 0 & 0 \\ 0 & 0 & \mathbf{0} & \mathbf{0} & \mathbf{0} & \mathbf{0} & \mathbf{0} & \mathbf{0} & \mathbf{0} & \mathbf{0} & \mathbf{0} & \mathbf{0} & \mathbf{0} & \mathbf{0} & \mathbf{0}\end{array}$

-Non-Generating Public Entities-

78 Ashland, City of

79 Benton County PUD \#1

80 Clallam County PUD \#1

81 Clatskanie PUD

82 Consumers Power

83 Emerald County PUD

84 Energy Northwest

85 Fall River Elec Coop

86 Franklin County PUD \#1

87 Klickitat County PUD \#1

88 Lewis County PUD \#1

89 Mason County PUD \#1

90 Mason County PUD \#3

91 Oregon Trail Coop

92 Tanner Elec Coop

93 Tillamook PUD \#1

94 Total Non-Generating Public Entities

$\begin{array}{lllllllllllllll}0 & 0 & 0 & 0 & 0 & 0 & 0 & 0 & 0 & 0 & 0 & 0 & 0 & 0 & 0 \\ 0 & 0 & 0 & 0 & 0 & 0 & 0 & 0 & 0 & 0 & 0 & 0 & 0 & 0 & 0 \\ 0 & 0 & 0 & 0 & 0 & 0 & 0 & 0 & 0 & 0 & 0 & 0 & 0 & 0 & 0 \\ 0 & 0 & 0 & 0 & 0 & 0 & 0 & 0 & 0 & 0 & 0 & 0 & 0 & 0 & 0 \\ 0 & 0 & 0 & 0 & 0 & 0 & 0 & 0 & 0 & 0 & 0 & 0 & 0 & 0 & 0 \\ 0 & 0 & 0 & 0 & 0 & 0 & 0 & 0 & 0 & 0 & 0 & 0 & 0 & 0 & 0 \\ 0 & 0 & 0 & 0 & 0 & 0 & 0 & 0 & 0 & 0 & 0 & 0 & 0 & 0 & 0 \\ 0 & 0 & 0 & 0 & 0 & 0 & 0 & 0 & 0 & 0 & 0 & 0 & 0 & 0 & 0 \\ 0 & 0 & 0 & 0 & 0 & 0 & 0 & 0 & 0 & 0 & 0 & 0 & 0 & 0 & 0 \\ 0 & 0 & 0 & 0 & 0 & 0 & 0 & 0 & 0 & 0 & 0 & 0 & 0 & 0 & 0 \\ 0 & 0 & 0 & 0 & 0 & 0 & 0 & 0 & 0 & 0 & 0 & 0 & 0 & 0 & 0 \\ 0 & 0 & 0 & 0 & 0 & 0 & 0 & 0 & 0 & 0 & 0 & 0 & 0 & 0 & 0 \\ 0 & 0 & 0 & 0 & 0 & 0 & 0 & 0 & 0 & 0 & 0 & 0 & 0 & 0 & 0 \\ 0 & 0 & 0 & 0 & 0 & 0 & 0 & 0 & 0 & 0 & 0 & 0 & 0 & 0 & 0 \\ 0 & 0 & 0 & 0 & 0 & 0 & 0 & 0 & 0 & 0 & 0 & 0 & 0 & 0 & 0 \\ 0 & 0 & 0 & 0 & 0 & 0 & 0 & 0 & 0 & 0 & 0 & 0 & 0 & 0 & 0 \\ 0 & \mathbf{0} & \mathbf{0} & \mathbf{0} & \mathbf{0} & \mathbf{0} & \mathbf{0} & \mathbf{0} & \mathbf{0} & \mathbf{0} & \mathbf{0} & \mathbf{0} & \mathbf{0} & \mathbf{0} & \mathbf{0}\end{array}$

-Investor-Owned Entities-

95 Avista Corp (WWP Division)

96 Idaho Power Company

97 Northwestern Energy LLC (MPC)

98 Pacific Power

99 Portland General Electric

100 Puget Sound Energy

101 Trans Alta Utilities Corp

102 Utah Power

103 Total Investor-Owned Entities

\begin{tabular}{lllllllllllllll}
0 & 0 & 0 & 0 & 0 & 0 & 0 & 0 & 0 & 0 & 0 & 0 & 0 & 0 & 0 \\
0 & 0 & 0 & 0 & 0 & 0 & 0 & 0 & 0 & 0 & 0 & 0 & 0 & 0 & 0 \\
0 & 0 & 0 & 0 & 0 & 0 & 0 & 0 & 0 & 0 & 0 & 0 & 0 & 0 & 0 \\
0 & 0 & 0 & 0 & 0 & 0 & 0 & 0 & 0 & 0 & 0 & 0 & 0 & 0 & 0 \\
0 & 0 & 0 & 0 & 0 & 0 & 0 & 0 & 0 & 0 & 0 & 0 & 0 & 0 & 0 \\
0 & 0 & 0 & 0 & 0 & 0 & 0 & 0 & 0 & 0 & 0 & 0 & 0 & 0 & 0 \\
0 & 0 & 0 & 0 & 0 & 0 & 0 & 0 & 0 & 0 & 0 & 0 & 0 & 0 & 0 \\
0 & 0 & 0 & 0 & 0 & 0 & 0 & 0 & 0 & 0 & 0 & 0 & 0 & 0 & 0 \\
\hline $\mathbf{0}$ & $\mathbf{0}$ & $\mathbf{0}$ & $\mathbf{0}$ & $\mathbf{0}$ & $\mathbf{0}$ & $\mathbf{0}$ & $\mathbf{0}$ & $\mathbf{0}$ & $\mathbf{0}$ & $\mathbf{0}$ & $\mathbf{0}$ & $\mathbf{0}$ & $\mathbf{0}$ & $\mathbf{0}$
\end{tabular}

-Other Entities-

104 Avista Energy Inc.

105 Chehalis Power Inc.

106 FPL Energy Vansycle LLC

107 Frederickson Power LP,

108 Hermiston Power Partnership

109 Klamath Pacific Energy

110 Longview Fibre

111 Mirant Americas Energy Marketing, L.P.

112 PPM Energy

113 SP Newsprint

114 Total Other Entities

Large Thermal Reserves

-Federal Entities-

115 BPA - Power Business 116 Total Federal Entities

$\begin{array}{lllllllllllllll}0 & 0 & 0 & 0 & 0 & 0 & 0 & 0 & 0 & 0 & 0 & 0 & 0 & 0 & 0 \\ 0 & 0 & 0 & 0 & 0 & 0 & 0 & 0 & 0 & 0 & 0 & 0 & 0 & 0 & 0 \\ 0 & 0 & 0 & 0 & 0 & 0 & 0 & 0 & 0 & 0 & 0 & 0 & 0 & 0 & 0 \\ 0 & 0 & 0 & 0 & 0 & 0 & 0 & 0 & 0 & 0 & 0 & 0 & 0 & 0 & 0 \\ 0 & 0 & 0 & 0 & 0 & 0 & 0 & 0 & 0 & 0 & 0 & 0 & 0 & 0 & 0 \\ 0 & 0 & 0 & 0 & 0 & 0 & 0 & 0 & 0 & 0 & 0 & 0 & 0 & 0 & 0 \\ 0 & 0 & 0 & 0 & 0 & 0 & 0 & 0 & 0 & 0 & 0 & 0 & 0 & 0 & 0 \\ 0 & 0 & 0 & 0 & 0 & 0 & 0 & 0 & 0 & 0 & 0 & 0 & 0 & 0 & 0 \\ 0 & 0 & 0 & 0 & 0 & 0 & 0 & 0 & 0 & 0 & 0 & 0 & 0 & 0 & 0 \\ 0 & 0 & 0 & 0 & 0 & 0 & 0 & 0 & 0 & 0 & 0 & 0 & 0 & 0 & 0 \\ 0 & \mathbf{0} & \mathbf{0} & \mathbf{0} & \mathbf{0} & \mathbf{0} & \mathbf{0} & \mathbf{0} & \mathbf{0} & \mathbf{0} & \mathbf{0} & \mathbf{0} & \mathbf{0} & \mathbf{0} & \mathbf{0}\end{array}$

-Generating Public Entities-

117 Turlock Irrigation District

118 Total Generating Public Entities

-Non-Generating Public Entities-

119 Total Non-Generating Public Entities 
Average Energy in Megawatts

120 Avista Corp (WWP Division)

121 Idaho Power Company

122 Northwestern Energy LLC (MPC)

123 Pacific Power

124 Portland General Electric

125 Puget Sound Energy

126 San Diego Gas \& Electric

127 Trans Alta Utilities Corp

128 Total Investor-Owned Entities

\section{Water Year}

2003 White Book

-Other Entities-

129 Total Other Entities

\section{Aug1 Aug16}

Sep

Oct Nov Dec Jan Feb

Mar Apr1 Apr1

May Jun Jul Avg

Contract Reserves

-Federal Entities-

130 Total Federal Entities

$\begin{array}{llll}0 & 0 & 0 & 0 \\ 0 & 0 & 0 & 0 \\ 0 & 0 & 0 & 0 \\ 0 & 0 & 0 & 0 \\ 0 & 0 & 0 & 0 \\ 0 & 0 & 0 & 0 \\ 0 & 0 & 0 & 0 \\ 0 & 0 & 0 & 0 \\ 0 & 0 & 0 & 0\end{array}$

$\begin{array}{ll}0 & 0 \\ 0 & 0 \\ 0 & 0 \\ 0 & 0 \\ 0 & 0 \\ 0 & 0 \\ 0 & 0 \\ 0 & 0 \\ 0 & 0\end{array}$

$\begin{array}{ll}0 & 0 \\ 0 & 0 \\ 0 & 0 \\ 0 & 0 \\ 0 & 0 \\ 0 & 0 \\ 0 & 0 \\ 0 & 0 \\ 0 & 0\end{array}$

$\begin{array}{ll}0 & 0 \\ 0 & 0 \\ 0 & 0 \\ 0 & 0 \\ 0 & 0 \\ 0 & 0 \\ 0 & 0 \\ 0 & 0 \\ 0 & 0\end{array}$

$\begin{array}{ll}0 & 0 \\ 0 & \\ 0 & \\ 0 & 0 \\ 0 & 0 \\ 0 & 0 \\ 0 & 0 \\ 0 & 0 \\ 0 & 0\end{array}$

0
0
0
0
0
0
0
0
0

$\begin{array}{lll}0 & 0 & 0 \\ 0 & 0 & 0 \\ 0 & 0 & 0 \\ 0 & 0 & 0 \\ 0 & 0 & 0 \\ 0 & 0 & 0 \\ 0 & 0 & 0 \\ 0 & 0 & 0 \\ 0 & 0 & 0\end{array}$

$\begin{array}{lllll}0 & 0 & 0 & 0 & 0 \\ 0 & 0 & 0 & 0 & 0 \\ 0 & 0 & 0 & 0 & 0 \\ 0 & 0 & 0 & 0 & 0 \\ 0 & 0 & 0 & 0 & 0 \\ 0 & 0 & 0 & 0 & 0 \\ 0 & 0 & 0 & 0 & 0 \\ 0 & 0 & 0 & 0 & 0 \\ 0 & 0 & 0 & 0 & 0\end{array}$

-Generating Public Entities-

131 Total Generating Public Entities

0

0

0

-Non-Generating Public Entities-

132 Total Non-Generating Public Entities

-Investor-Owned Entities-

133 Northwestern Energy LLC (MPC)

134 Pacific Power

135 Total Investor-Owned Entities

$\begin{array}{lllllllllllllll}0 & 0 & 0 & 0 & 0 & 0 & 0 & 0 & 0 & 0 & 0 & 0 & 0 & 0 & 0 \\ 0 & 0 & 0 & 0 & 0 & 0 & 0 & 0 & 0 & 0 & 0 & 0 & 0 & 0 & 0 \\ 0 & 0 & 0 & 0 & 0 & 0 & 0 & 0 & 0 & 0 & 0 & 0 & 0 & 0 & 0\end{array}$

-Other Entities-

136 Total Other Entities

-Total Capacity Reserves-

137 Total Hydro Reserves

138 Total Small Thermal \& Misc Reserves

139 Total Large Thermal Reserves

140 Total Contract Reserves

141 Total Capacity Reserves

$\begin{array}{lllllllllllllll}0 & 0 & 0 & 0 & 0 & 0 & 0 & 0 & 0 & 0 & 0 & 0 & 0 & 0 & 0 \\ 0 & 0 & 0 & 0 & 0 & 0 & 0 & 0 & 0 & 0 & 0 & 0 & 0 & 0 & 0 \\ 0 & 0 & 0 & 0 & 0 & 0 & 0 & 0 & 0 & 0 & 0 & 0 & 0 & 0 & 0 \\ 0 & 0 & 0 & 0 & 0 & 0 & 0 & 0 & 0 & 0 & 0 & 0 & 0 & 0 & 0 \\ 0 & 0 & 0 & 0 & 0 & 0 & 0 & 0 & 0 & 0 & 0 & 0 & 0 & 0 & 0\end{array}$


Table A-17: Regional Capacity Reserves

PNW Loads and Resources Study

2007 - 2008 Operating Year

1937 Water Year

2003 White Book

Oct Nov

Dec Jan Feb

Mar Apr1 Apr16 May Jun

Jul Avg

Hydro Reserves

-Federal Entities-

$1 \mathrm{BPA}$ - Power Business

2 Total Federal Entities

-Generating Public Entities-

3 Chelan County PUD \#1

4 Clark County PUD \#1

5 Cowlitz County PUD \#1

6 Douglas County PUD \#1

7 Eugene Water \& Electric Board

8 Grant County PUD \#2

9 Grays Harbor PUD \#1

10 Okanogan County PUD \#1

11 Pend Oreille County PUD \#1

12 Seattle City Light

13 Snohomish County PUD \#1

14 Tacoma Public Utilities

15 Total Generating Public Entities

-Non-Generating Public Entities-

16 Ashland, City of

17 Benton County PUD \#1

18 Bonners Ferry, City of

19 Centralia, City of

20 Clallam County PUD \#1

21 Clearwater Power

22 Consumers Power

23 Emerald County PUD

24 Energy Northwest

25 Fall River Elec Coop

26 Forest Grove, City of

27 Franklin County PUD \#1

28 Idaho County L \& P

29 Idaho Falls, City of

30 Kittitas County PUD \#1

31 Klickitat County PUD \#1

32 Kootenai Electric Coop

33 Lewis County PUD \#1

34 Lost River Elec Coop

35 Lower Valley Energy

36 Mason County PUD \#1

37 McMinnville, City of

38 Milton Freewater, City of

39 Northern Lights

40 Northern Wasco County PUD

41 Oregon Trail Coop

42 Port Angeles, City of

43 Raft River Elec Coop

44 Salmon River Elec Coop

45 Soda Springs, City of

46 Tanner Elec Coop

47 Tillamook PUD \#1

48 United Electric Coop

49 Total Non-Generating Public Entities

$\begin{array}{lllllllllllllll}0 & 0 & 0 & 0 & 0 & 0 & 0 & 0 & 0 & 0 & 0 & 0 & 0 & 0 & 0 \\ 0 & 0 & 0 & 0 & 0 & 0 & 0 & 0 & 0 & 0 & 0 & 0 & 0 & 0 & 0 \\ 0 & 0 & 0 & 0 & 0 & 0 & 0 & 0 & 0 & 0 & 0 & 0 & 0 & 0 & 0 \\ 0 & 0 & 0 & 0 & 0 & 0 & 0 & 0 & 0 & 0 & 0 & 0 & 0 & 0 & 0 \\ 0 & 0 & 0 & 0 & 0 & 0 & 0 & 0 & 0 & 0 & 0 & 0 & 0 & 0 & 0 \\ 0 & 0 & 0 & 0 & 0 & 0 & 0 & 0 & 0 & 0 & 0 & 0 & 0 & 0 & 0 \\ 0 & 0 & 0 & 0 & 0 & 0 & 0 & 0 & 0 & 0 & 0 & 0 & 0 & 0 & 0 \\ 0 & 0 & 0 & 0 & 0 & 0 & 0 & 0 & 0 & 0 & 0 & 0 & 0 & 0 & 0 \\ 0 & 0 & 0 & 0 & 0 & 0 & 0 & 0 & 0 & 0 & 0 & 0 & 0 & 0 & 0 \\ 0 & 0 & 0 & 0 & 0 & 0 & 0 & 0 & 0 & 0 & 0 & 0 & 0 & 0 & 0 \\ 0 & 0 & 0 & 0 & 0 & 0 & 0 & 0 & 0 & 0 & 0 & 0 & 0 & 0 & 0 \\ 0 & 0 & 0 & 0 & 0 & 0 & 0 & 0 & 0 & 0 & 0 & 0 & 0 & 0 & 0 \\ 0 & \mathbf{0} & \mathbf{0} & \mathbf{0} & \mathbf{0} & \mathbf{0} & \mathbf{0} & \mathbf{0} & \mathbf{0} & \mathbf{0} & \mathbf{0} & \mathbf{0} & \mathbf{0} & \mathbf{0} & \mathbf{0}\end{array}$

-Investor-Owned Entities-

50 Avista Corp (WWP Division)

51 Idaho Power Company

52 Northwestern Energy LLC (MPC)

53 Pacific Power

54 Portland General Electric

\begin{tabular}{|c|c|c|c|c|c|c|c|c|c|c|c|c|c|}
\hline 0 & 0 & 0 & 0 & 0 & 0 & 0 & 0 & 0 & 0 & 0 & 0 & 0 & 0 \\
\hline 0 & 0 & 0 & 0 & 0 & 0 & 0 & 0 & 0 & 0 & 0 & 0 & 0 & 0 \\
\hline 0 & 0 & 0 & 0 & 0 & 0 & 0 & 0 & 0 & 0 & 0 & 0 & 0 & 0 \\
\hline 0 & 0 & 0 & 0 & 0 & 0 & 0 & 0 & 0 & 0 & 0 & 0 & 0 & 0 \\
\hline 0 & 0 & 0 & 0 & 0 & 0 & 0 & 0 & 0 & 0 & 0 & 0 & 0 & 0 \\
\hline 0 & 0 & 0 & 0 & 0 & 0 & 0 & 0 & 0 & 0 & 0 & 0 & 0 & 0 \\
\hline 0 & 0 & 0 & 0 & 0 & 0 & 0 & 0 & 0 & 0 & 0 & 0 & 0 & 0 \\
\hline 0 & 0 & 0 & 0 & 0 & 0 & 0 & 0 & 0 & 0 & 0 & 0 & 0 & 0 \\
\hline 0 & 0 & 0 & 0 & 0 & 0 & 0 & 0 & 0 & 0 & 0 & 0 & 0 & 0 \\
\hline 0 & 0 & 0 & 0 & 0 & 0 & 0 & 0 & 0 & 0 & 0 & 0 & 0 & 0 \\
\hline 0 & 0 & 0 & 0 & 0 & 0 & 0 & 0 & 0 & 0 & 0 & 0 & 0 & 0 \\
\hline 0 & 0 & 0 & 0 & 0 & 0 & 0 & 0 & 0 & 0 & 0 & 0 & 0 & 0 \\
\hline 0 & 0 & 0 & 0 & 0 & 0 & 0 & 0 & 0 & 0 & 0 & 0 & 0 & 0 \\
\hline 0 & 0 & 0 & 0 & 0 & 0 & 0 & 0 & 0 & 0 & 0 & 0 & 0 & 0 \\
\hline 0 & 0 & 0 & 0 & 0 & 0 & 0 & 0 & 0 & 0 & 0 & 0 & 0 & 0 \\
\hline 0 & 0 & 0 & 0 & 0 & 0 & 0 & 0 & 0 & 0 & 0 & 0 & 0 & 0 \\
\hline 0 & 0 & 0 & 0 & 0 & 0 & 0 & 0 & 0 & 0 & 0 & 0 & 0 & 0 \\
\hline 0 & 0 & 0 & 0 & 0 & 0 & 0 & 0 & 0 & 0 & 0 & 0 & 0 & 0 \\
\hline 0 & 0 & 0 & 0 & 0 & 0 & 0 & 0 & 0 & 0 & 0 & 0 & 0 & 0 \\
\hline 0 & 0 & 0 & 0 & 0 & 0 & 0 & 0 & 0 & 0 & 0 & 0 & 0 & 0 \\
\hline 0 & 0 & 0 & 0 & 0 & 0 & 0 & 0 & 0 & 0 & 0 & 0 & 0 & 0 \\
\hline 0 & 0 & 0 & 0 & 0 & 0 & 0 & 0 & 0 & 0 & 0 & 0 & 0 & 0 \\
\hline 0 & 0 & 0 & 0 & 0 & 0 & 0 & 0 & 0 & 0 & 0 & 0 & 0 & 0 \\
\hline 0 & 0 & 0 & 0 & 0 & 0 & 0 & 0 & 0 & 0 & 0 & 0 & 0 & 0 \\
\hline 0 & 0 & 0 & 0 & 0 & 0 & 0 & 0 & 0 & 0 & 0 & 0 & 0 & 0 \\
\hline 0 & 0 & 0 & 0 & 0 & 0 & 0 & 0 & 0 & 0 & 0 & 0 & 0 & 0 \\
\hline 0 & 0 & 0 & 0 & 0 & 0 & 0 & 0 & 0 & 0 & 0 & 0 & 0 & 0 \\
\hline 0 & 0 & 0 & 0 & 0 & 0 & 0 & 0 & 0 & 0 & 0 & 0 & 0 & 0 \\
\hline 0 & 0 & 0 & 0 & 0 & 0 & 0 & 0 & 0 & 0 & 0 & 0 & 0 & 0 \\
\hline 0 & 0 & 0 & 0 & 0 & 0 & 0 & 0 & 0 & 0 & 0 & 0 & 0 & 0 \\
\hline 0 & 0 & 0 & 0 & 0 & 0 & 0 & 0 & 0 & 0 & 0 & 0 & 0 & 0 \\
\hline 0 & 0 & 0 & 0 & 0 & 0 & 0 & 0 & 0 & 0 & 0 & 0 & 0 & 0 \\
\hline 0 & 0 & 0 & 0 & 0 & 0 & 0 & 0 & 0 & 0 & 0 & 0 & 0 & 0 \\
\hline 0 & 0 & 0 & 0 & 0 & 0 & 0 & 0 & 0 & 0 & 0 & 0 & 0 & 0 \\
\hline
\end{tabular}

55 Puget Sound Energy

56 Total Investor-Owned Entities

-Other Entities-

57 Colockum Transmission Company

58 Longview Fibre

59 Warm Springs Power Enterprise

60 Total Other Entities

$\begin{array}{lllllllllllllll}0 & 0 & 0 & 0 & 0 & 0 & 0 & 0 & 0 & 0 & 0 & 0 & 0 & 0 & 0 \\ 0 & 0 & 0 & 0 & 0 & 0 & 0 & 0 & 0 & 0 & 0 & 0 & 0 & 0 & 0 \\ 0 & 0 & 0 & 0 & 0 & 0 & 0 & 0 & 0 & 0 & 0 & 0 & 0 & 0 & 0 \\ 0 & 0 & 0 & 0 & 0 & 0 & 0 & 0 & 0 & 0 & 0 & 0 & 0 & 0 & 0 \\ 0 & 0 & 0 & 0 & 0 & 0 & 0 & 0 & 0 & 0 & 0 & 0 & 0 & 0 & 0 \\ 0 & 0 & 0 & 0 & 0 & 0 & 0 & 0 & 0 & 0 & 0 & 0 & 0 & 0 & 0 \\ 0 & \mathbf{0} & \mathbf{0} & \mathbf{0} & \mathbf{0} & \mathbf{0} & \mathbf{0} & \mathbf{0} & \mathbf{0} & \mathbf{0} & \mathbf{0} & \mathbf{0} & \mathbf{0} & \mathbf{0} & \mathbf{0}\end{array}$

Small Thermal \& Misc. Reserves

-Federal Entities-

61 BPA - Power Business

62 U.S. Naval Station, Everett (Jim Creek)

$\begin{array}{llllll}0 & 0 & 0 & 0 & 0 & 0 \\ 0 & 0 & 0 & 0 & 0 & 0\end{array}$

0

0
0

0
0

0
0

0
0

0
0

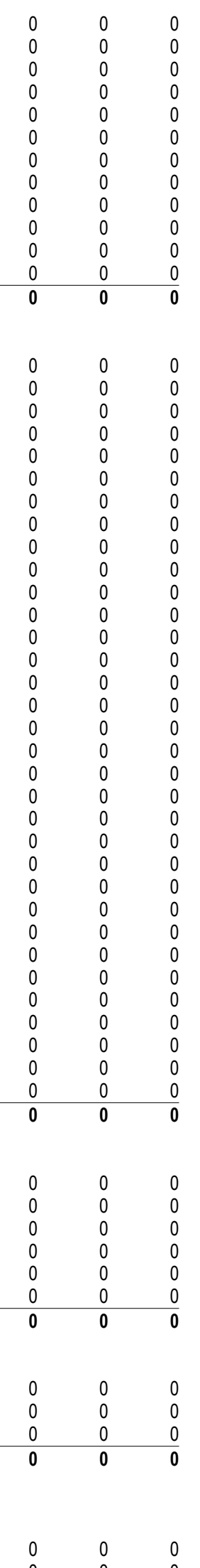




$\begin{array}{lllllllllllllll}0 & 0 & 0 & 0 & 0 & 0 & 0 & 0 & 0 & 0 & 0 & 0 & 0 & 0 & 0 \\ 0 & 0 & 0 & 0 & 0 & 0 & 0 & 0 & 0 & 0 & 0 & 0 & 0 & 0 & 0\end{array}$

-Generating Public Entities-

65 Chelan County PUD \#1

66 Clark County PUD \#1

67 Cowlitz County PUD \#1

68 Douglas County PUD \#1

69 Eugene Water \& Electric Board

70 Grant County PUD \#2

71 Grays Harbor PUD \#1

72 Okanogan County PUD \#1

73 Seattle City Light

74 Snohomish County PUD \#1

75 Springfield Utility Board

76 Tacoma Public Utilities

77 Total Generating Public Entities

$\begin{array}{lllllllllllllll}0 & 0 & 0 & 0 & 0 & 0 & 0 & 0 & 0 & 0 & 0 & 0 & 0 & 0 & 0 \\ 0 & 0 & 0 & 0 & 0 & 0 & 0 & 0 & 0 & 0 & 0 & 0 & 0 & 0 & 0 \\ 0 & 0 & 0 & 0 & 0 & 0 & 0 & 0 & 0 & 0 & 0 & 0 & 0 & 0 & 0 \\ 0 & 0 & 0 & 0 & 0 & 0 & 0 & 0 & 0 & 0 & 0 & 0 & 0 & 0 & 0 \\ 0 & 0 & 0 & 0 & 0 & 0 & 0 & 0 & 0 & 0 & 0 & 0 & 0 & 0 & 0 \\ 0 & 0 & 0 & 0 & 0 & 0 & 0 & 0 & 0 & 0 & 0 & 0 & 0 & 0 & 0 \\ 0 & 0 & 0 & 0 & 0 & 0 & 0 & 0 & 0 & 0 & 0 & 0 & 0 & 0 & 0 \\ 0 & 0 & 0 & 0 & 0 & 0 & 0 & 0 & 0 & 0 & 0 & 0 & 0 & 0 & 0 \\ 0 & 0 & 0 & 0 & 0 & 0 & 0 & 0 & 0 & 0 & 0 & 0 & 0 & 0 & 0 \\ 0 & 0 & 0 & 0 & 0 & 0 & 0 & 0 & 0 & 0 & 0 & 0 & 0 & 0 & 0 \\ 0 & 0 & 0 & 0 & 0 & 0 & 0 & 0 & 0 & 0 & 0 & 0 & 0 & 0 & 0 \\ 0 & 0 & 0 & 0 & 0 & 0 & 0 & 0 & 0 & 0 & 0 & 0 & 0 & 0 \\ 0 & 0 & \mathbf{0} & \mathbf{0} & \mathbf{0} & \mathbf{0} & \mathbf{0} & \mathbf{0} & \mathbf{0} & \mathbf{0} & \mathbf{0} & \mathbf{0} & \mathbf{0} & \mathbf{0} & \mathbf{0}\end{array}$

-Non-Generating Public Entities-

78 Ashland, City of

79 Benton County PUD \#1

80 Clallam County PUD \#1

81 Clatskanie PUD

82 Consumers Power

83 Emerald County PUD

84 Energy Northwest

85 Fall River Elec Coop

86 Franklin County PUD \#1

87 Klickitat County PUD \#1

88 Lewis County PUD \#1

89 Mason County PUD \#1

90 Mason County PUD \#3

91 Oregon Trail Coop

92 Tanner Elec Coop

93 Tillamook PUD \#1

94 Total Non-Generating Public Entities

$\begin{array}{lllllllllllllll}0 & 0 & 0 & 0 & 0 & 0 & 0 & 0 & 0 & 0 & 0 & 0 & 0 & 0 & 0 \\ 0 & 0 & 0 & 0 & 0 & 0 & 0 & 0 & 0 & 0 & 0 & 0 & 0 & 0 & 0 \\ 0 & 0 & 0 & 0 & 0 & 0 & 0 & 0 & 0 & 0 & 0 & 0 & 0 & 0 & 0 \\ 0 & 0 & 0 & 0 & 0 & 0 & 0 & 0 & 0 & 0 & 0 & 0 & 0 & 0 & 0 \\ 0 & 0 & 0 & 0 & 0 & 0 & 0 & 0 & 0 & 0 & 0 & 0 & 0 & 0 & 0 \\ 0 & 0 & 0 & 0 & 0 & 0 & 0 & 0 & 0 & 0 & 0 & 0 & 0 & 0 & 0 \\ 0 & 0 & 0 & 0 & 0 & 0 & 0 & 0 & 0 & 0 & 0 & 0 & 0 & 0 & 0 \\ 0 & 0 & 0 & 0 & 0 & 0 & 0 & 0 & 0 & 0 & 0 & 0 & 0 & 0 & 0 \\ 0 & 0 & 0 & 0 & 0 & 0 & 0 & 0 & 0 & 0 & 0 & 0 & 0 & 0 & 0 \\ 0 & 0 & 0 & 0 & 0 & 0 & 0 & 0 & 0 & 0 & 0 & 0 & 0 & 0 & 0 \\ 0 & 0 & 0 & 0 & 0 & 0 & 0 & 0 & 0 & 0 & 0 & 0 & 0 & 0 & 0 \\ 0 & 0 & 0 & 0 & 0 & 0 & 0 & 0 & 0 & 0 & 0 & 0 & 0 & 0 & 0 \\ 0 & 0 & 0 & 0 & 0 & 0 & 0 & 0 & 0 & 0 & 0 & 0 & 0 & 0 & 0 \\ 0 & 0 & 0 & 0 & 0 & 0 & 0 & 0 & 0 & 0 & 0 & 0 & 0 & 0 & 0 \\ 0 & 0 & 0 & 0 & 0 & 0 & 0 & 0 & 0 & 0 & 0 & 0 & 0 & 0 & 0 \\ 0 & 0 & 0 & 0 & 0 & 0 & 0 & 0 & 0 & 0 & 0 & 0 & 0 & 0 & 0 \\ 0 & \mathbf{0} & \mathbf{0} & \mathbf{0} & \mathbf{0} & \mathbf{0} & \mathbf{0} & \mathbf{0} & \mathbf{0} & \mathbf{0} & \mathbf{0} & \mathbf{0} & \mathbf{0} & \mathbf{0} & \mathbf{0}\end{array}$

-Investor-Owned Entities-

95 Avista Corp (WWP Division)

96 Idaho Power Company

97 Northwestern Energy LLC (MPC)

98 Pacific Power

99 Portland General Electric

100 Puget Sound Energy

101 Trans Alta Utilities Corp

102 Utah Power

103 Total Investor-Owned Entities

\begin{tabular}{lllllllllllllll}
0 & 0 & 0 & 0 & 0 & 0 & 0 & 0 & 0 & 0 & 0 & 0 & 0 & 0 & 0 \\
0 & 0 & 0 & 0 & 0 & 0 & 0 & 0 & 0 & 0 & 0 & 0 & 0 & 0 & 0 \\
0 & 0 & 0 & 0 & 0 & 0 & 0 & 0 & 0 & 0 & 0 & 0 & 0 & 0 & 0 \\
0 & 0 & 0 & 0 & 0 & 0 & 0 & 0 & 0 & 0 & 0 & 0 & 0 & 0 & 0 \\
0 & 0 & 0 & 0 & 0 & 0 & 0 & 0 & 0 & 0 & 0 & 0 & 0 & 0 & 0 \\
0 & 0 & 0 & 0 & 0 & 0 & 0 & 0 & 0 & 0 & 0 & 0 & 0 & 0 & 0 \\
0 & 0 & 0 & 0 & 0 & 0 & 0 & 0 & 0 & 0 & 0 & 0 & 0 & 0 & 0 \\
0 & 0 & 0 & 0 & 0 & 0 & 0 & 0 & 0 & 0 & 0 & 0 & 0 & 0 & 0 \\
\hline $\mathbf{0}$ & $\mathbf{0}$ & $\mathbf{0}$ & $\mathbf{0}$ & $\mathbf{0}$ & $\mathbf{0}$ & $\mathbf{0}$ & $\mathbf{0}$ & $\mathbf{0}$ & $\mathbf{0}$ & $\mathbf{0}$ & $\mathbf{0}$ & $\mathbf{0}$ & $\mathbf{0}$ & $\mathbf{0}$
\end{tabular}

-Other Entities-

104 Avista Energy Inc.

105 Chehalis Power Inc.

106 FPL Energy Vansycle LLC

107 Frederickson Power LP,

108 Hermiston Power Partnership

109 Klamath Pacific Energy

110 Longview Fibre

111 Mirant Americas Energy Marketing, L.P.

112 PPM Energy

113 SP Newsprint

114 Total Other Entities

Large Thermal Reserves

-Federal Entities-

115 BPA - Power Business 116 Total Federal Entities

$\begin{array}{lllllllllllllll}0 & 0 & 0 & 0 & 0 & 0 & 0 & 0 & 0 & 0 & 0 & 0 & 0 & 0 & 0 \\ 0 & 0 & 0 & 0 & 0 & 0 & 0 & 0 & 0 & 0 & 0 & 0 & 0 & 0 & 0 \\ 0 & 0 & 0 & 0 & 0 & 0 & 0 & 0 & 0 & 0 & 0 & 0 & 0 & 0 & 0 \\ 0 & 0 & 0 & 0 & 0 & 0 & 0 & 0 & 0 & 0 & 0 & 0 & 0 & 0 & 0 \\ 0 & 0 & 0 & 0 & 0 & 0 & 0 & 0 & 0 & 0 & 0 & 0 & 0 & 0 & 0 \\ 0 & 0 & 0 & 0 & 0 & 0 & 0 & 0 & 0 & 0 & 0 & 0 & 0 & 0 & 0 \\ 0 & 0 & 0 & 0 & 0 & 0 & 0 & 0 & 0 & 0 & 0 & 0 & 0 & 0 & 0 \\ 0 & 0 & 0 & 0 & 0 & 0 & 0 & 0 & 0 & 0 & 0 & 0 & 0 & 0 & 0 \\ 0 & 0 & 0 & 0 & 0 & 0 & 0 & 0 & 0 & 0 & 0 & 0 & 0 & 0 & 0 \\ 0 & 0 & 0 & 0 & 0 & 0 & 0 & 0 & 0 & 0 & 0 & 0 & 0 & 0 & 0 \\ 0 & \mathbf{0} & \mathbf{0} & \mathbf{0} & \mathbf{0} & \mathbf{0} & \mathbf{0} & \mathbf{0} & \mathbf{0} & \mathbf{0} & \mathbf{0} & \mathbf{0} & \mathbf{0} & \mathbf{0} & \mathbf{0}\end{array}$

-Generating Public Entities-

117 Turlock Irrigation District

118 Total Generating Public Entities

-Non-Generating Public Entities-

119 Total Non-Generating Public Entities 
Average Energy in Megawatts

120 Avista Corp (WWP Division)

121 Idaho Power Company

122 Northwestern Energy LLC (MPC)

123 Pacific Power

124 Portland General Electric

125 Puget Sound Energy

126 San Diego Gas \& Electric

127 Trans Alta Utilities Corp

128 Total Investor-Owned Entities

\section{Water Year}

2003 White Book

-Other Entities-

129 Total Other Entities

\section{Aug1 Aug16}

Sep

Oct Nov Dec Jan Feb

Mar Apr1 Apr1

May Jun Jul Avg

Contract Reserves

-Federal Entities-

130 Total Federal Entities

$\begin{array}{llll}0 & 0 & 0 & 0 \\ 0 & 0 & 0 & 0 \\ 0 & 0 & 0 & 0 \\ 0 & 0 & 0 & 0 \\ 0 & 0 & 0 & 0 \\ 0 & 0 & 0 & 0 \\ 0 & 0 & 0 & 0 \\ 0 & 0 & 0 & 0 \\ 0 & 0 & 0 & 0\end{array}$

$\begin{array}{ll}0 & 0 \\ 0 & 0 \\ 0 & 0 \\ 0 & 0 \\ 0 & 0 \\ 0 & 0 \\ 0 & 0 \\ 0 & 0 \\ 0 & 0\end{array}$

$\begin{array}{ll}0 & 0 \\ 0 & 0 \\ 0 & 0 \\ 0 & 0 \\ 0 & 0 \\ 0 & 0 \\ 0 & 0 \\ 0 & 0 \\ 0 & 0\end{array}$

$\begin{array}{ll}0 & 0 \\ 0 & 0 \\ 0 & 0 \\ 0 & 0 \\ 0 & 0 \\ 0 & 0 \\ 0 & 0 \\ 0 & 0 \\ 0 & 0\end{array}$

0
0
0
0
0
0
0
0

0
0
0
0
0
0
0
0
0

$\begin{array}{lll}0 & 0 & 0 \\ 0 & 0 & 0 \\ 0 & 0 & 0 \\ 0 & 0 & 0 \\ 0 & 0 & 0 \\ 0 & 0 & 0 \\ 0 & 0 & 0 \\ 0 & 0 & 0 \\ 0 & 0 & 0\end{array}$

$\begin{array}{lllll}0 & 0 & 0 & 0 & 0 \\ 0 & 0 & 0 & 0 & 0 \\ 0 & 0 & 0 & 0 & 0 \\ 0 & 0 & 0 & 0 & 0 \\ 0 & 0 & 0 & 0 & 0 \\ 0 & 0 & 0 & 0 & 0 \\ 0 & 0 & 0 & 0 & 0 \\ 0 & 0 & 0 & 0 & 0 \\ 0 & 0 & 0 & 0 & 0\end{array}$

-Generating Public Entities-

131 Total Generating Public Entities

0

0

0

-Non-Generating Public Entities-

132 Total Non-Generating Public Entities

-Investor-Owned Entities-

133 Northwestern Energy LLC (MPC)

134 Pacific Power

135 Total Investor-Owned Entities

$\begin{array}{lllllllllllllll}0 & 0 & 0 & 0 & 0 & 0 & 0 & 0 & 0 & 0 & 0 & 0 & 0 & 0 & 0 \\ 0 & 0 & 0 & 0 & 0 & 0 & 0 & 0 & 0 & 0 & 0 & 0 & 0 & 0 & 0 \\ 0 & 0 & 0 & 0 & 0 & 0 & 0 & 0 & 0 & 0 & 0 & 0 & 0 & 0 & 0\end{array}$

-Other Entities-

136 Total Other Entities

-Total Capacity Reserves-

137 Total Hydro Reserves

138 Total Small Thermal \& Misc Reserves

139 Total Large Thermal Reserves

140 Total Contract Reserves

141 Total Capacity Reserves

$\begin{array}{lllllllllllllll}0 & 0 & 0 & 0 & 0 & 0 & 0 & 0 & 0 & 0 & 0 & 0 & 0 & 0 & 0 \\ 0 & 0 & 0 & 0 & 0 & 0 & 0 & 0 & 0 & 0 & 0 & 0 & 0 & 0 & 0 \\ 0 & 0 & 0 & 0 & 0 & 0 & 0 & 0 & 0 & 0 & 0 & 0 & 0 & 0 & 0 \\ 0 & 0 & 0 & 0 & 0 & 0 & 0 & 0 & 0 & 0 & 0 & 0 & 0 & 0 & 0 \\ 0 & 0 & 0 & 0 & 0 & 0 & 0 & 0 & 0 & 0 & 0 & 0 & 0 & 0 & 0\end{array}$


Table A-17: Regional Capacity Reserves

PNW Loads and Resources Study

2008 - 2009 Operating Year

1937 Water Year

2003 White Book

Oct Nov

Dec Jan Fe

Feb Mar Apr1 Apr16 May Jun Jul Avg

Hydro Reserves

$1 \mathrm{BPA}$ - Power Business

2 Total Federal Entities

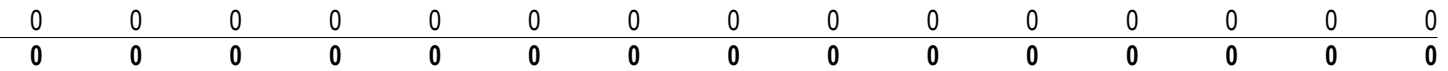

-Generating Public Entities-

3 Chelan County PUD \#1

4 Clark County PUD \#1

5 Cowlitz County PUD \#1

6 Douglas County PUD \#1

7 Eugene Water \& Electric Board

8 Grant County PUD \#2

9 Grays Harbor PUD \#1

10 Okanogan County PUD \#1

11 Pend Oreille County PUD \#1

12 Seattle City Light

13 Snohomish County PUD \#1

14 Tacoma Public Utilities

15 Total Generating Public Entities

$\begin{array}{lllllllllllllll}0 & 0 & 0 & 0 & 0 & 0 & 0 & 0 & 0 & 0 & 0 & 0 & 0 & 0 & 0 \\ 0 & 0 & 0 & 0 & 0 & 0 & 0 & 0 & 0 & 0 & 0 & 0 & 0 & 0 & 0 \\ 0 & 0 & 0 & 0 & 0 & 0 & 0 & 0 & 0 & 0 & 0 & 0 & 0 & 0 & 0 \\ 0 & 0 & 0 & 0 & 0 & 0 & 0 & 0 & 0 & 0 & 0 & 0 & 0 & 0 & 0 \\ 0 & 0 & 0 & 0 & 0 & 0 & 0 & 0 & 0 & 0 & 0 & 0 & 0 & 0 & 0 \\ 0 & 0 & 0 & 0 & 0 & 0 & 0 & 0 & 0 & 0 & 0 & 0 & 0 & 0 & 0 \\ 0 & 0 & 0 & 0 & 0 & 0 & 0 & 0 & 0 & 0 & 0 & 0 & 0 & 0 & 0 \\ 0 & 0 & 0 & 0 & 0 & 0 & 0 & 0 & 0 & 0 & 0 & 0 & 0 & 0 & 0 \\ 0 & 0 & 0 & 0 & 0 & 0 & 0 & 0 & 0 & 0 & 0 & 0 & 0 & 0 & 0 \\ 0 & 0 & 0 & 0 & 0 & 0 & 0 & 0 & 0 & 0 & 0 & 0 & 0 & 0 & 0 \\ 0 & 0 & 0 & 0 & 0 & 0 & 0 & 0 & 0 & 0 & 0 & 0 & 0 & 0 & 0 \\ 0 & 0 & 0 & 0 & 0 & 0 & 0 & 0 & 0 & 0 & 0 & 0 & 0 & 0 & 0 \\ 0 & 0 & \mathbf{0} & \mathbf{0} & \mathbf{0} & \mathbf{0} & \mathbf{0} & \mathbf{0} & \mathbf{0} & \mathbf{0} & \mathbf{0} & \mathbf{0} & \mathbf{0} & \mathbf{0} & \mathbf{0}\end{array}$

-Non-Generating Public Entities-

16 Ashland, City of

17 Benton County PUD \#1

18 Bonners Ferry, City of

19 Centralia, City of

20 Clallam County PUD \#1

21 Clearwater Power

22 Consumers Power

23 Emerald County PUD

24 Energy Northwest

25 Fall River Elec Coop

26 Forest Grove, City of

27 Franklin County PUD \#1

28 Idaho County L \& P

29 Idaho Falls, City of

30 Kittitas County PUD \#1

31 Klickitat County PUD \#1

32 Kootenai Electric Coop

33 Lewis County PUD \#1

34 Lost River Elec Coop

35 Lower Valley Energy

36 Mason County PUD \#1

37 McMinnville, City of

38 Milton Freewater, City of

39 Northern Lights

40 Northern Wasco County PUD

41 Oregon Trail Coop

42 Port Angeles, City of

43 Raft River Elec Coop

44 Salmon River Elec Coop

45 Soda Springs, City of

46 Tanner Elec Coop

47 Tillamook PUD \#1

48 United Electric Coop

49 Total Non-Generating Public Entities

\begin{tabular}{|c|c|c|c|c|c|c|c|c|c|c|c|c|c|}
\hline 0 & 0 & 0 & 0 & 0 & 0 & 0 & 0 & 0 & 0 & 0 & 0 & 0 & 0 \\
\hline 0 & 0 & 0 & 0 & 0 & 0 & 0 & 0 & 0 & 0 & 0 & 0 & 0 & 0 \\
\hline 0 & 0 & 0 & 0 & 0 & 0 & 0 & 0 & 0 & 0 & 0 & 0 & 0 & 0 \\
\hline 0 & 0 & 0 & 0 & 0 & 0 & 0 & 0 & 0 & 0 & 0 & 0 & 0 & 0 \\
\hline 0 & 0 & 0 & 0 & 0 & 0 & 0 & 0 & 0 & 0 & 0 & 0 & 0 & 0 \\
\hline 0 & 0 & 0 & 0 & 0 & 0 & 0 & 0 & 0 & 0 & 0 & 0 & 0 & 0 \\
\hline 0 & 0 & 0 & 0 & 0 & 0 & 0 & 0 & 0 & 0 & 0 & 0 & 0 & 0 \\
\hline 0 & 0 & 0 & 0 & 0 & 0 & 0 & 0 & 0 & 0 & 0 & 0 & 0 & 0 \\
\hline 0 & 0 & 0 & 0 & 0 & 0 & 0 & 0 & 0 & 0 & 0 & 0 & 0 & 0 \\
\hline 0 & 0 & 0 & 0 & 0 & 0 & 0 & 0 & 0 & 0 & 0 & 0 & 0 & 0 \\
\hline 0 & 0 & 0 & 0 & 0 & 0 & 0 & 0 & 0 & 0 & 0 & 0 & 0 & 0 \\
\hline 0 & 0 & 0 & 0 & 0 & 0 & 0 & 0 & 0 & 0 & 0 & 0 & 0 & 0 \\
\hline 0 & 0 & 0 & 0 & 0 & 0 & 0 & 0 & 0 & 0 & 0 & 0 & 0 & 0 \\
\hline 0 & 0 & 0 & 0 & 0 & 0 & 0 & 0 & 0 & 0 & 0 & 0 & 0 & 0 \\
\hline 0 & 0 & 0 & 0 & 0 & 0 & 0 & 0 & 0 & 0 & 0 & 0 & 0 & 0 \\
\hline 0 & 0 & 0 & 0 & 0 & 0 & 0 & 0 & 0 & 0 & 0 & 0 & 0 & 0 \\
\hline 0 & 0 & 0 & 0 & 0 & 0 & 0 & 0 & 0 & 0 & 0 & 0 & 0 & 0 \\
\hline 0 & 0 & 0 & 0 & 0 & 0 & 0 & 0 & 0 & 0 & 0 & 0 & 0 & 0 \\
\hline 0 & 0 & 0 & 0 & 0 & 0 & 0 & 0 & 0 & 0 & 0 & 0 & 0 & 0 \\
\hline 0 & 0 & 0 & 0 & 0 & 0 & 0 & 0 & 0 & 0 & 0 & 0 & 0 & 0 \\
\hline 0 & 0 & 0 & 0 & 0 & 0 & 0 & 0 & 0 & 0 & 0 & 0 & 0 & 0 \\
\hline 0 & 0 & 0 & 0 & 0 & 0 & 0 & 0 & 0 & 0 & 0 & 0 & 0 & 0 \\
\hline 0 & 0 & 0 & 0 & 0 & 0 & 0 & 0 & 0 & 0 & 0 & 0 & 0 & 0 \\
\hline 0 & 0 & 0 & 0 & 0 & 0 & 0 & 0 & 0 & 0 & 0 & 0 & 0 & 0 \\
\hline 0 & 0 & 0 & 0 & 0 & 0 & 0 & 0 & 0 & 0 & 0 & 0 & 0 & 0 \\
\hline 0 & 0 & 0 & 0 & 0 & 0 & 0 & 0 & 0 & 0 & 0 & 0 & 0 & 0 \\
\hline 0 & 0 & 0 & 0 & 0 & 0 & 0 & 0 & 0 & 0 & 0 & 0 & 0 & 0 \\
\hline 0 & 0 & 0 & 0 & 0 & 0 & 0 & 0 & 0 & 0 & 0 & 0 & 0 & 0 \\
\hline 0 & 0 & 0 & 0 & 0 & 0 & 0 & 0 & 0 & 0 & 0 & 0 & 0 & 0 \\
\hline 0 & 0 & 0 & 0 & 0 & 0 & 0 & 0 & 0 & 0 & 0 & 0 & 0 & 0 \\
\hline 0 & 0 & 0 & 0 & 0 & 0 & 0 & 0 & 0 & 0 & 0 & 0 & 0 & 0 \\
\hline 0 & 0 & 0 & 0 & 0 & 0 & 0 & 0 & 0 & 0 & 0 & 0 & 0 & 0 \\
\hline 0 & 0 & 0 & 0 & 0 & 0 & 0 & 0 & 0 & 0 & 0 & 0 & 0 & 0 \\
\hline 0 & 0 & 0 & 0 & 0 & 0 & 0 & 0 & 0 & 0 & 0 & 0 & 0 & 0 \\
\hline
\end{tabular}

-Investor-Owned Entities-

50 Avista Corp (WWP Division)

51 Idaho Power Company

52 Northwestern Energy LLC (MPC)

53 Pacific Power

54 Portland General Electric

55 Puget Sound Energy

56 Total Investor-Owned Entities

-Other Entities-

57 Colockum Transmission Company

58 Longview Fibre

59 Warm Springs Power Enterprise

60 Total Other Entities

$\begin{array}{lllllllllllllll}0 & 0 & 0 & 0 & 0 & 0 & 0 & 0 & 0 & 0 & 0 & 0 & 0 & 0 & 0 \\ 0 & 0 & 0 & 0 & 0 & 0 & 0 & 0 & 0 & 0 & 0 & 0 & 0 & 0 & 0 \\ 0 & 0 & 0 & 0 & 0 & 0 & 0 & 0 & 0 & 0 & 0 & 0 & 0 & 0 & 0 \\ 0 & 0 & 0 & 0 & 0 & 0 & 0 & 0 & 0 & 0 & 0 & 0 & 0 & 0 & 0 \\ 0 & 0 & 0 & 0 & 0 & 0 & 0 & 0 & 0 & 0 & 0 & 0 & 0 & 0 & 0 \\ 0 & 0 & 0 & 0 & 0 & 0 & 0 & 0 & 0 & 0 & 0 & 0 & 0 & 0 & 0 \\ 0 & \mathbf{0} & \mathbf{0} & \mathbf{0} & \mathbf{0} & \mathbf{0} & \mathbf{0} & \mathbf{0} & \mathbf{0} & \mathbf{0} & \mathbf{0} & \mathbf{0} & \mathbf{0} & \mathbf{0} & \mathbf{0}\end{array}$

Small Thermal \& Misc. Reserves

-Federal Entities-

61 BPA - Power Business

62 U.S. Naval Station, Everett (Jim Creek)

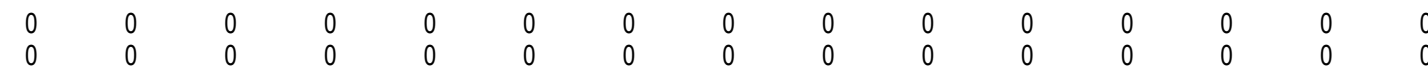




$\begin{array}{lllllllllllllll}0 & 0 & 0 & 0 & 0 & 0 & 0 & 0 & 0 & 0 & 0 & 0 & 0 & 0 & 0 \\ 0 & 0 & 0 & 0 & 0 & 0 & 0 & 0 & 0 & 0 & 0 & 0 & 0 & 0 & 0\end{array}$

-Generating Public Entities-

65 Chelan County PUD \#1

66 Clark County PUD \#1

67 Cowlitz County PUD \#1

68 Douglas County PUD \#1

69 Eugene Water \& Electric Board

70 Grant County PUD \#2

71 Grays Harbor PUD \#1

72 Okanogan County PUD \#1

73 Seattle City Light

74 Snohomish County PUD \#1

75 Springfield Utility Board

76 Tacoma Public Utilities

77 Total Generating Public Entities

$\begin{array}{lllllllllllllll}0 & 0 & 0 & 0 & 0 & 0 & 0 & 0 & 0 & 0 & 0 & 0 & 0 & 0 & 0 \\ 0 & 0 & 0 & 0 & 0 & 0 & 0 & 0 & 0 & 0 & 0 & 0 & 0 & 0 & 0 \\ 0 & 0 & 0 & 0 & 0 & 0 & 0 & 0 & 0 & 0 & 0 & 0 & 0 & 0 & 0 \\ 0 & 0 & 0 & 0 & 0 & 0 & 0 & 0 & 0 & 0 & 0 & 0 & 0 & 0 & 0 \\ 0 & 0 & 0 & 0 & 0 & 0 & 0 & 0 & 0 & 0 & 0 & 0 & 0 & 0 & 0 \\ 0 & 0 & 0 & 0 & 0 & 0 & 0 & 0 & 0 & 0 & 0 & 0 & 0 & 0 & 0 \\ 0 & 0 & 0 & 0 & 0 & 0 & 0 & 0 & 0 & 0 & 0 & 0 & 0 & 0 & 0 \\ 0 & 0 & 0 & 0 & 0 & 0 & 0 & 0 & 0 & 0 & 0 & 0 & 0 & 0 & 0 \\ 0 & 0 & 0 & 0 & 0 & 0 & 0 & 0 & 0 & 0 & 0 & 0 & 0 & 0 & 0 \\ 0 & 0 & 0 & 0 & 0 & 0 & 0 & 0 & 0 & 0 & 0 & 0 & 0 & 0 & 0 \\ 0 & 0 & 0 & 0 & 0 & 0 & 0 & 0 & 0 & 0 & 0 & 0 & 0 & 0 & 0 \\ 0 & 0 & 0 & 0 & 0 & 0 & 0 & 0 & 0 & 0 & 0 & 0 & 0 & 0 \\ 0 & 0 & \mathbf{0} & \mathbf{0} & \mathbf{0} & \mathbf{0} & \mathbf{0} & \mathbf{0} & \mathbf{0} & \mathbf{0} & \mathbf{0} & \mathbf{0} & \mathbf{0} & \mathbf{0} & \mathbf{0}\end{array}$

-Non-Generating Public Entities-

78 Ashland, City of

79 Benton County PUD \#1

80 Clallam County PUD \#1

81 Clatskanie PUD

82 Consumers Power

83 Emerald County PUD

84 Energy Northwest

85 Fall River Elec Coop

86 Franklin County PUD \#1

87 Klickitat County PUD \#1

88 Lewis County PUD \#1

89 Mason County PUD \#1

90 Mason County PUD \#3

91 Oregon Trail Coop

92 Tanner Elec Coop

93 Tillamook PUD \#1

94 Total Non-Generating Public Entities

$\begin{array}{lllllllllllllll}0 & 0 & 0 & 0 & 0 & 0 & 0 & 0 & 0 & 0 & 0 & 0 & 0 & 0 & 0 \\ 0 & 0 & 0 & 0 & 0 & 0 & 0 & 0 & 0 & 0 & 0 & 0 & 0 & 0 & 0 \\ 0 & 0 & 0 & 0 & 0 & 0 & 0 & 0 & 0 & 0 & 0 & 0 & 0 & 0 & 0 \\ 0 & 0 & 0 & 0 & 0 & 0 & 0 & 0 & 0 & 0 & 0 & 0 & 0 & 0 & 0 \\ 0 & 0 & 0 & 0 & 0 & 0 & 0 & 0 & 0 & 0 & 0 & 0 & 0 & 0 & 0 \\ 0 & 0 & 0 & 0 & 0 & 0 & 0 & 0 & 0 & 0 & 0 & 0 & 0 & 0 & 0 \\ 0 & 0 & 0 & 0 & 0 & 0 & 0 & 0 & 0 & 0 & 0 & 0 & 0 & 0 & 0 \\ 0 & 0 & 0 & 0 & 0 & 0 & 0 & 0 & 0 & 0 & 0 & 0 & 0 & 0 & 0 \\ 0 & 0 & 0 & 0 & 0 & 0 & 0 & 0 & 0 & 0 & 0 & 0 & 0 & 0 & 0 \\ 0 & 0 & 0 & 0 & 0 & 0 & 0 & 0 & 0 & 0 & 0 & 0 & 0 & 0 & 0 \\ 0 & 0 & 0 & 0 & 0 & 0 & 0 & 0 & 0 & 0 & 0 & 0 & 0 & 0 & 0 \\ 0 & 0 & 0 & 0 & 0 & 0 & 0 & 0 & 0 & 0 & 0 & 0 & 0 & 0 & 0 \\ 0 & 0 & 0 & 0 & 0 & 0 & 0 & 0 & 0 & 0 & 0 & 0 & 0 & 0 & 0 \\ 0 & 0 & 0 & 0 & 0 & 0 & 0 & 0 & 0 & 0 & 0 & 0 & 0 & 0 & 0 \\ 0 & 0 & 0 & 0 & 0 & 0 & 0 & 0 & 0 & 0 & 0 & 0 & 0 & 0 & 0 \\ 0 & 0 & 0 & 0 & 0 & 0 & 0 & 0 & 0 & 0 & 0 & 0 & 0 & 0 & 0 \\ 0 & \mathbf{0} & \mathbf{0} & \mathbf{0} & \mathbf{0} & \mathbf{0} & \mathbf{0} & \mathbf{0} & \mathbf{0} & \mathbf{0} & \mathbf{0} & \mathbf{0} & \mathbf{0} & \mathbf{0} & \mathbf{0}\end{array}$

-Investor-Owned Entities-

95 Avista Corp (WWP Division)

96 Idaho Power Company

97 Northwestern Energy LLC (MPC)

98 Pacific Power

99 Portland General Electric

100 Puget Sound Energy

101 Trans Alta Utilities Corp

102 Utah Power

103 Total Investor-Owned Entities

\begin{tabular}{lllllllllllllll}
0 & 0 & 0 & 0 & 0 & 0 & 0 & 0 & 0 & 0 & 0 & 0 & 0 & 0 & 0 \\
0 & 0 & 0 & 0 & 0 & 0 & 0 & 0 & 0 & 0 & 0 & 0 & 0 & 0 & 0 \\
0 & 0 & 0 & 0 & 0 & 0 & 0 & 0 & 0 & 0 & 0 & 0 & 0 & 0 & 0 \\
0 & 0 & 0 & 0 & 0 & 0 & 0 & 0 & 0 & 0 & 0 & 0 & 0 & 0 & 0 \\
0 & 0 & 0 & 0 & 0 & 0 & 0 & 0 & 0 & 0 & 0 & 0 & 0 & 0 & 0 \\
0 & 0 & 0 & 0 & 0 & 0 & 0 & 0 & 0 & 0 & 0 & 0 & 0 & 0 & 0 \\
0 & 0 & 0 & 0 & 0 & 0 & 0 & 0 & 0 & 0 & 0 & 0 & 0 & 0 & 0 \\
0 & 0 & 0 & 0 & 0 & 0 & 0 & 0 & 0 & 0 & 0 & 0 & 0 & 0 & 0 \\
\hline $\mathbf{0}$ & $\mathbf{0}$ & $\mathbf{0}$ & $\mathbf{0}$ & $\mathbf{0}$ & $\mathbf{0}$ & $\mathbf{0}$ & $\mathbf{0}$ & $\mathbf{0}$ & $\mathbf{0}$ & $\mathbf{0}$ & $\mathbf{0}$ & $\mathbf{0}$ & $\mathbf{0}$ & $\mathbf{0}$
\end{tabular}

-Other Entities-

104 Avista Energy Inc.

105 Chehalis Power Inc.

106 FPL Energy Vansycle LLC

107 Frederickson Power LP,

108 Hermiston Power Partnership

109 Klamath Pacific Energy

110 Longview Fibre

111 Mirant Americas Energy Marketing, L.P.

112 PPM Energy

113 SP Newsprint

114 Total Other Entities

Large Thermal Reserves

-Federal Entities-

115 BPA - Power Business 116 Total Federal Entities

$\begin{array}{lllllllllllllll}0 & 0 & 0 & 0 & 0 & 0 & 0 & 0 & 0 & 0 & 0 & 0 & 0 & 0 & 0 \\ 0 & 0 & 0 & 0 & 0 & 0 & 0 & 0 & 0 & 0 & 0 & 0 & 0 & 0 & 0 \\ 0 & 0 & 0 & 0 & 0 & 0 & 0 & 0 & 0 & 0 & 0 & 0 & 0 & 0 & 0 \\ 0 & 0 & 0 & 0 & 0 & 0 & 0 & 0 & 0 & 0 & 0 & 0 & 0 & 0 & 0 \\ 0 & 0 & 0 & 0 & 0 & 0 & 0 & 0 & 0 & 0 & 0 & 0 & 0 & 0 & 0 \\ 0 & 0 & 0 & 0 & 0 & 0 & 0 & 0 & 0 & 0 & 0 & 0 & 0 & 0 & 0 \\ 0 & 0 & 0 & 0 & 0 & 0 & 0 & 0 & 0 & 0 & 0 & 0 & 0 & 0 & 0 \\ 0 & 0 & 0 & 0 & 0 & 0 & 0 & 0 & 0 & 0 & 0 & 0 & 0 & 0 & 0 \\ 0 & 0 & 0 & 0 & 0 & 0 & 0 & 0 & 0 & 0 & 0 & 0 & 0 & 0 & 0 \\ 0 & 0 & 0 & 0 & 0 & 0 & 0 & 0 & 0 & 0 & 0 & 0 & 0 & 0 & 0 \\ 0 & \mathbf{0} & \mathbf{0} & \mathbf{0} & \mathbf{0} & \mathbf{0} & \mathbf{0} & \mathbf{0} & \mathbf{0} & \mathbf{0} & \mathbf{0} & \mathbf{0} & \mathbf{0} & \mathbf{0} & \mathbf{0}\end{array}$

-Generating Public Entities-

117 Turlock Irrigation District

118 Total Generating Public Entities

-Non-Generating Public Entities-

119 Total Non-Generating Public Entities 
Average Energy in Megawatts

120 Avista Corp (WWP Division)

121 Idaho Power Company

122 Northwestern Energy LLC (MPC)

123 Pacific Power

124 Portland General Electric

125 Puget Sound Energy

126 San Diego Gas \& Electric

127 Trans Alta Utilities Corp

128 Total Investor-Owned Entities

\section{Water Year}

2003 White Book

-Other Entities-

129 Total Other Entities

\section{Aug1 Aug16}

Sep

Oct Nov Dec Jan Feb

Mar Apr1 Apr1

May Jun Jul Avg

Contract Reserves

-Federal Entities-

130 Total Federal Entities

$\begin{array}{llll}0 & 0 & 0 & 0 \\ 0 & 0 & 0 & 0 \\ 0 & 0 & 0 & 0 \\ 0 & 0 & 0 & 0 \\ 0 & 0 & 0 & 0 \\ 0 & 0 & 0 & 0 \\ 0 & 0 & 0 & 0 \\ 0 & 0 & 0 & 0 \\ 0 & 0 & 0 & 0\end{array}$

$\begin{array}{ll}0 & 0 \\ 0 & 0 \\ 0 & 0 \\ 0 & 0 \\ 0 & 0 \\ 0 & 0 \\ 0 & 0 \\ 0 & 0 \\ 0 & 0\end{array}$

$\begin{array}{lll}0 & 0 & 0 \\ 0 & 0 & 0 \\ 0 & 0 & 0 \\ 0 & 0 & 0 \\ 0 & 0 & 0 \\ 0 & 0 & 0 \\ 0 & 0 & 0 \\ 0 & 0 & 0 \\ 0 & 0 & 0\end{array}$

0
0
0
0
0
0
0
0
0

$\begin{array}{ll}0 & 0 \\ 0 & 0 \\ 0 & 0 \\ 0 & 0 \\ 0 & 0 \\ 0 & 0 \\ 0 & 0 \\ 0 & 0 \\ 0 & 0\end{array}$

$\begin{array}{lllllll}0 & 0 & 0 & 0 & 0 & 0 & 0 \\ 0 & 0 & 0 & 0 & 0 & 0 & 0 \\ 0 & 0 & 0 & 0 & 0 & 0 & 0 \\ 0 & 0 & 0 & 0 & 0 & 0 & 0 \\ 0 & 0 & 0 & 0 & 0 & 0 & 0 \\ 0 & 0 & 0 & 0 & 0 & 0 & 0 \\ 0 & 0 & 0 & 0 & 0 & 0 & 0 \\ 0 & 0 & 0 & 0 & 0 & 0 & 0 \\ 0 & 0 & 0 & 0 & 0 & 0 & 0\end{array}$

-Generating Public Entities-

131 Total Generating Public Entities

0

0

0

$\begin{array}{lllllllllllllll}0 & 0 & 0 & 0 & 0 & 0 & 0 & 0 & 0 & 0 & 0 & 0 & 0 & 0 & 0\end{array}$

-Non-Generating Public Entities-

132 Total Non-Generating Public Entities

$0 \quad 0 \quad 0$

0

nvestor-Owned Entities-

133 Northwestern Energy LLC (MPC)

134 Pacific Power

135 Total Investor-Owned Entities

$\begin{array}{lllllllllllllll}0 & 0 & 0 & 0 & 0 & 0 & 0 & 0 & 0 & 0 & 0 & 0 & 0 & 0 & 0 \\ 0 & 0 & 0 & 0 & 0 & 0 & 0 & 0 & 0 & 0 & 0 & 0 & 0 & 0 & 0 \\ 0 & 0 & 0 & 0 & 0 & 0 & 0 & 0 & 0 & 0 & 0 & 0 & 0 & 0 & 0\end{array}$

-Other Entities-

136 Total Other Entities

-Total Capacity Reserves-

137 Total Hydro Reserves

138 Total Small Thermal \& Misc Reserves

139 Total Large Thermal Reserves

140 Total Contract Reserves

141 Total Capacity Reserves 
Table A-17: Regional Capacity Reserves

PNW Loads and Resources Study

2009 - 2010 Operating Year

1937 Water Year

2003 White Book

Oct Nov

Dec Jan Fe

Feb

Mar Apr1 Apr16 May Jun Jul Avg

Hydro Reserves

1 BPA - Power Business

2 Total Federal Entities

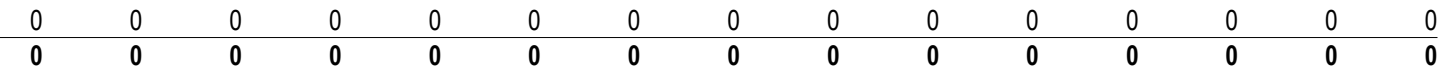

-Generating Public Entities-

3 Chelan County PUD \#1

4 Clark County PUD \#1

5 Cowlitz County PUD \#1

6 Douglas County PUD \#1

7 Eugene Water \& Electric Board

8 Grant County PUD \#2

9 Grays Harbor PUD \#1

10 Okanogan County PUD \#1

11 Pend Oreille County PUD \#1

12 Seattle City Light

13 Snohomish County PUD \#1

14 Tacoma Public Utilities

15 Total Generating Public Entities

$\begin{array}{lllllllllllllll}0 & 0 & 0 & 0 & 0 & 0 & 0 & 0 & 0 & 0 & 0 & 0 & 0 & 0 & 0 \\ 0 & 0 & 0 & 0 & 0 & 0 & 0 & 0 & 0 & 0 & 0 & 0 & 0 & 0 & 0 \\ 0 & 0 & 0 & 0 & 0 & 0 & 0 & 0 & 0 & 0 & 0 & 0 & 0 & 0 & 0 \\ 0 & 0 & 0 & 0 & 0 & 0 & 0 & 0 & 0 & 0 & 0 & 0 & 0 & 0 & 0 \\ 0 & 0 & 0 & 0 & 0 & 0 & 0 & 0 & 0 & 0 & 0 & 0 & 0 & 0 & 0 \\ 0 & 0 & 0 & 0 & 0 & 0 & 0 & 0 & 0 & 0 & 0 & 0 & 0 & 0 & 0 \\ 0 & 0 & 0 & 0 & 0 & 0 & 0 & 0 & 0 & 0 & 0 & 0 & 0 & 0 & 0 \\ 0 & 0 & 0 & 0 & 0 & 0 & 0 & 0 & 0 & 0 & 0 & 0 & 0 & 0 & 0 \\ 0 & 0 & 0 & 0 & 0 & 0 & 0 & 0 & 0 & 0 & 0 & 0 & 0 & 0 & 0 \\ 0 & 0 & 0 & 0 & 0 & 0 & 0 & 0 & 0 & 0 & 0 & 0 & 0 & 0 & 0 \\ 0 & 0 & 0 & 0 & 0 & 0 & 0 & 0 & 0 & 0 & 0 & 0 & 0 & 0 & 0 \\ 0 & 0 & 0 & 0 & 0 & 0 & 0 & 0 & 0 & 0 & 0 & 0 & 0 & 0 & 0 \\ 0 & 0 & \mathbf{0} & \mathbf{0} & \mathbf{0} & \mathbf{0} & \mathbf{0} & \mathbf{0} & \mathbf{0} & \mathbf{0} & \mathbf{0} & \mathbf{0} & \mathbf{0} & \mathbf{0} & \mathbf{0}\end{array}$

-Non-Generating Public Entities-

16 Ashland, City of

17 Benton County PUD \#1

18 Bonners Ferry, City of

19 Centralia, City of

20 Clallam County PUD \#1

21 Clearwater Power

22 Consumers Power

23 Emerald County PUD

24 Energy Northwest

25 Fall River Elec Coop

26 Forest Grove, City of

27 Franklin County PUD \#1

28 Idaho County L \& P

29 Idaho Falls, City of

30 Kittitas County PUD \#1

31 Klickitat County PUD \#1

32 Kootenai Electric Coop

33 Lewis County PUD \#1

34 Lost River Elec Coop

35 Lower Valley Energy

36 Mason County PUD \#1

37 McMinnville, City of

38 Milton Freewater, City of

39 Northern Lights

40 Northern Wasco County PUD

41 Oregon Trail Coop

42 Port Angeles, City of

43 Raft River Elec Coop

44 Salmon River Elec Coop

45 Soda Springs, City of

46 Tanner Elec Coop

47 Tillamook PUD \#1

48 United Electric Coop

49 Total Non-Generating Public Entities

\begin{tabular}{|c|c|c|c|c|c|c|c|c|c|c|c|c|c|}
\hline 0 & 0 & 0 & 0 & 0 & 0 & 0 & 0 & 0 & 0 & 0 & 0 & 0 & 0 \\
\hline 0 & 0 & 0 & 0 & 0 & 0 & 0 & 0 & 0 & 0 & 0 & 0 & 0 & 0 \\
\hline 0 & 0 & 0 & 0 & 0 & 0 & 0 & 0 & 0 & 0 & 0 & 0 & 0 & 0 \\
\hline 0 & 0 & 0 & 0 & 0 & 0 & 0 & 0 & 0 & 0 & 0 & 0 & 0 & 0 \\
\hline 0 & 0 & 0 & 0 & 0 & 0 & 0 & 0 & 0 & 0 & 0 & 0 & 0 & 0 \\
\hline 0 & 0 & 0 & 0 & 0 & 0 & 0 & 0 & 0 & 0 & 0 & 0 & 0 & 0 \\
\hline 0 & 0 & 0 & 0 & 0 & 0 & 0 & 0 & 0 & 0 & 0 & 0 & 0 & 0 \\
\hline 0 & 0 & 0 & 0 & 0 & 0 & 0 & 0 & 0 & 0 & 0 & 0 & 0 & 0 \\
\hline 0 & 0 & 0 & 0 & 0 & 0 & 0 & 0 & 0 & 0 & 0 & 0 & 0 & 0 \\
\hline 0 & 0 & 0 & 0 & 0 & 0 & 0 & 0 & 0 & 0 & 0 & 0 & 0 & 0 \\
\hline 0 & 0 & 0 & 0 & 0 & 0 & 0 & 0 & 0 & 0 & 0 & 0 & 0 & 0 \\
\hline 0 & 0 & 0 & 0 & 0 & 0 & 0 & 0 & 0 & 0 & 0 & 0 & 0 & 0 \\
\hline 0 & 0 & 0 & 0 & 0 & 0 & 0 & 0 & 0 & 0 & 0 & 0 & 0 & 0 \\
\hline 0 & 0 & 0 & 0 & 0 & 0 & 0 & 0 & 0 & 0 & 0 & 0 & 0 & 0 \\
\hline 0 & 0 & 0 & 0 & 0 & 0 & 0 & 0 & 0 & 0 & 0 & 0 & 0 & 0 \\
\hline 0 & 0 & 0 & 0 & 0 & 0 & 0 & 0 & 0 & 0 & 0 & 0 & 0 & 0 \\
\hline 0 & 0 & 0 & 0 & 0 & 0 & 0 & 0 & 0 & 0 & 0 & 0 & 0 & 0 \\
\hline 0 & 0 & 0 & 0 & 0 & 0 & 0 & 0 & 0 & 0 & 0 & 0 & 0 & 0 \\
\hline 0 & 0 & 0 & 0 & 0 & 0 & 0 & 0 & 0 & 0 & 0 & 0 & 0 & 0 \\
\hline 0 & 0 & 0 & 0 & 0 & 0 & 0 & 0 & 0 & 0 & 0 & 0 & 0 & 0 \\
\hline 0 & 0 & 0 & 0 & 0 & 0 & 0 & 0 & 0 & 0 & 0 & 0 & 0 & 0 \\
\hline 0 & 0 & 0 & 0 & 0 & 0 & 0 & 0 & 0 & 0 & 0 & 0 & 0 & 0 \\
\hline 0 & 0 & 0 & 0 & 0 & 0 & 0 & 0 & 0 & 0 & 0 & 0 & 0 & 0 \\
\hline 0 & 0 & 0 & 0 & 0 & 0 & 0 & 0 & 0 & 0 & 0 & 0 & 0 & 0 \\
\hline 0 & 0 & 0 & 0 & 0 & 0 & 0 & 0 & 0 & 0 & 0 & 0 & 0 & 0 \\
\hline 0 & 0 & 0 & 0 & 0 & 0 & 0 & 0 & 0 & 0 & 0 & 0 & 0 & 0 \\
\hline 0 & 0 & 0 & 0 & 0 & 0 & 0 & 0 & 0 & 0 & 0 & 0 & 0 & 0 \\
\hline 0 & 0 & 0 & 0 & 0 & 0 & 0 & 0 & 0 & 0 & 0 & 0 & 0 & 0 \\
\hline 0 & 0 & 0 & 0 & 0 & 0 & 0 & 0 & 0 & 0 & 0 & 0 & 0 & 0 \\
\hline 0 & 0 & 0 & 0 & 0 & 0 & 0 & 0 & 0 & 0 & 0 & 0 & 0 & 0 \\
\hline 0 & 0 & 0 & 0 & 0 & 0 & 0 & 0 & 0 & 0 & 0 & 0 & 0 & 0 \\
\hline 0 & 0 & 0 & 0 & 0 & 0 & 0 & 0 & 0 & 0 & 0 & 0 & 0 & 0 \\
\hline 0 & 0 & 0 & 0 & 0 & 0 & 0 & 0 & 0 & 0 & 0 & 0 & 0 & 0 \\
\hline 0 & 0 & 0 & 0 & 0 & 0 & 0 & 0 & 0 & 0 & 0 & 0 & 0 & 0 \\
\hline
\end{tabular}

-Investor-Owned Entities-

50 Avista Corp (WWP Division)

51 Idaho Power Company

52 Northwestern Energy LLC (MPC)

53 Pacific Power

54 Portland General Electric

55 Puget Sound Energy

56 Total Investor-Owned Entities

-Other Entities-

57 Colockum Transmission Company

58 Longview Fibre

59 Warm Springs Power Enterprise

60 Total Other Entities

$\begin{array}{lllllllllllllll}0 & 0 & 0 & 0 & 0 & 0 & 0 & 0 & 0 & 0 & 0 & 0 & 0 & 0 & 0 \\ 0 & 0 & 0 & 0 & 0 & 0 & 0 & 0 & 0 & 0 & 0 & 0 & 0 & 0 & 0 \\ 0 & 0 & 0 & 0 & 0 & 0 & 0 & 0 & 0 & 0 & 0 & 0 & 0 & 0 & 0 \\ 0 & 0 & 0 & 0 & 0 & 0 & 0 & 0 & 0 & 0 & 0 & 0 & 0 & 0 & 0 \\ 0 & 0 & 0 & 0 & 0 & 0 & 0 & 0 & 0 & 0 & 0 & 0 & 0 & 0 & 0 \\ 0 & 0 & 0 & 0 & 0 & 0 & 0 & 0 & 0 & 0 & 0 & 0 & 0 & 0 & 0 \\ 0 & \mathbf{0} & \mathbf{0} & \mathbf{0} & \mathbf{0} & \mathbf{0} & \mathbf{0} & \mathbf{0} & \mathbf{0} & \mathbf{0} & \mathbf{0} & \mathbf{0} & \mathbf{0} & \mathbf{0} & \mathbf{0}\end{array}$

Small Thermal \& Misc. Reserves

-Federal Entities-

61 BPA - Power Business

62 U.S. Naval Station, Everett (Jim Creek)

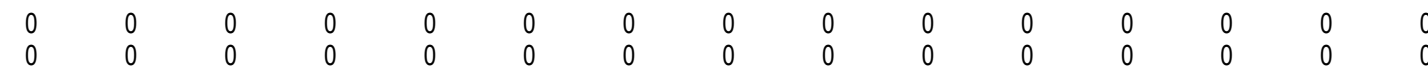




$\begin{array}{lllllllllllllll}0 & 0 & 0 & 0 & 0 & 0 & 0 & 0 & 0 & 0 & 0 & 0 & 0 & 0 & 0 \\ 0 & 0 & 0 & 0 & 0 & 0 & 0 & 0 & 0 & 0 & 0 & 0 & 0 & 0 & 0\end{array}$

-Generating Public Entities-

65 Chelan County PUD \#1

66 Clark County PUD \#1

67 Cowlitz County PUD \#1

68 Douglas County PUD \#1

69 Eugene Water \& Electric Board

70 Grant County PUD \#2

71 Grays Harbor PUD \#1

72 Okanogan County PUD \#1

73 Seattle City Light

74 Snohomish County PUD \#1

75 Springfield Utility Board

76 Tacoma Public Utilities

77 Total Generating Public Entities

$\begin{array}{lllllllllllllll}0 & 0 & 0 & 0 & 0 & 0 & 0 & 0 & 0 & 0 & 0 & 0 & 0 & 0 & 0 \\ 0 & 0 & 0 & 0 & 0 & 0 & 0 & 0 & 0 & 0 & 0 & 0 & 0 & 0 & 0 \\ 0 & 0 & 0 & 0 & 0 & 0 & 0 & 0 & 0 & 0 & 0 & 0 & 0 & 0 & 0 \\ 0 & 0 & 0 & 0 & 0 & 0 & 0 & 0 & 0 & 0 & 0 & 0 & 0 & 0 & 0 \\ 0 & 0 & 0 & 0 & 0 & 0 & 0 & 0 & 0 & 0 & 0 & 0 & 0 & 0 & 0 \\ 0 & 0 & 0 & 0 & 0 & 0 & 0 & 0 & 0 & 0 & 0 & 0 & 0 & 0 & 0 \\ 0 & 0 & 0 & 0 & 0 & 0 & 0 & 0 & 0 & 0 & 0 & 0 & 0 & 0 & 0 \\ 0 & 0 & 0 & 0 & 0 & 0 & 0 & 0 & 0 & 0 & 0 & 0 & 0 & 0 & 0 \\ 0 & 0 & 0 & 0 & 0 & 0 & 0 & 0 & 0 & 0 & 0 & 0 & 0 & 0 & 0 \\ 0 & 0 & 0 & 0 & 0 & 0 & 0 & 0 & 0 & 0 & 0 & 0 & 0 & 0 & 0 \\ 0 & 0 & 0 & 0 & 0 & 0 & 0 & 0 & 0 & 0 & 0 & 0 & 0 & 0 & 0 \\ 0 & 0 & 0 & 0 & 0 & 0 & 0 & 0 & 0 & 0 & 0 & 0 & 0 & 0 \\ 0 & 0 & \mathbf{0} & \mathbf{0} & \mathbf{0} & \mathbf{0} & \mathbf{0} & \mathbf{0} & \mathbf{0} & \mathbf{0} & \mathbf{0} & \mathbf{0} & \mathbf{0} & \mathbf{0} & \mathbf{0}\end{array}$

-Non-Generating Public Entities-

78 Ashland, City of

79 Benton County PUD \#1

80 Clallam County PUD \#1

81 Clatskanie PUD

82 Consumers Power

83 Emerald County PUD

84 Energy Northwest

85 Fall River Elec Coop

86 Franklin County PUD \#1

87 Klickitat County PUD \#1

88 Lewis County PUD \#1

89 Mason County PUD \#1

90 Mason County PUD \#3

91 Oregon Trail Coop

92 Tanner Elec Coop

93 Tillamook PUD \#1

94 Total Non-Generating Public Entities

$\begin{array}{lllllllllllllll}0 & 0 & 0 & 0 & 0 & 0 & 0 & 0 & 0 & 0 & 0 & 0 & 0 & 0 & 0 \\ 0 & 0 & 0 & 0 & 0 & 0 & 0 & 0 & 0 & 0 & 0 & 0 & 0 & 0 & 0 \\ 0 & 0 & 0 & 0 & 0 & 0 & 0 & 0 & 0 & 0 & 0 & 0 & 0 & 0 & 0 \\ 0 & 0 & 0 & 0 & 0 & 0 & 0 & 0 & 0 & 0 & 0 & 0 & 0 & 0 & 0 \\ 0 & 0 & 0 & 0 & 0 & 0 & 0 & 0 & 0 & 0 & 0 & 0 & 0 & 0 & 0 \\ 0 & 0 & 0 & 0 & 0 & 0 & 0 & 0 & 0 & 0 & 0 & 0 & 0 & 0 & 0 \\ 0 & 0 & 0 & 0 & 0 & 0 & 0 & 0 & 0 & 0 & 0 & 0 & 0 & 0 & 0 \\ 0 & 0 & 0 & 0 & 0 & 0 & 0 & 0 & 0 & 0 & 0 & 0 & 0 & 0 & 0 \\ 0 & 0 & 0 & 0 & 0 & 0 & 0 & 0 & 0 & 0 & 0 & 0 & 0 & 0 & 0 \\ 0 & 0 & 0 & 0 & 0 & 0 & 0 & 0 & 0 & 0 & 0 & 0 & 0 & 0 & 0 \\ 0 & 0 & 0 & 0 & 0 & 0 & 0 & 0 & 0 & 0 & 0 & 0 & 0 & 0 & 0 \\ 0 & 0 & 0 & 0 & 0 & 0 & 0 & 0 & 0 & 0 & 0 & 0 & 0 & 0 & 0 \\ 0 & 0 & 0 & 0 & 0 & 0 & 0 & 0 & 0 & 0 & 0 & 0 & 0 & 0 & 0 \\ 0 & 0 & 0 & 0 & 0 & 0 & 0 & 0 & 0 & 0 & 0 & 0 & 0 & 0 & 0 \\ 0 & 0 & 0 & 0 & 0 & 0 & 0 & 0 & 0 & 0 & 0 & 0 & 0 & 0 & 0 \\ 0 & 0 & 0 & 0 & 0 & 0 & 0 & 0 & 0 & 0 & 0 & 0 & 0 & 0 & 0 \\ 0 & \mathbf{0} & \mathbf{0} & \mathbf{0} & \mathbf{0} & \mathbf{0} & \mathbf{0} & \mathbf{0} & \mathbf{0} & \mathbf{0} & \mathbf{0} & \mathbf{0} & \mathbf{0} & \mathbf{0} & \mathbf{0}\end{array}$

-Investor-Owned Entities-

95 Avista Corp (WWP Division)

96 Idaho Power Company

97 Northwestern Energy LLC (MPC)

98 Pacific Power

99 Portland General Electric

100 Puget Sound Energy

101 Trans Alta Utilities Corp

102 Utah Power

103 Total Investor-Owned Entities

\begin{tabular}{lllllllllllllll}
0 & 0 & 0 & 0 & 0 & 0 & 0 & 0 & 0 & 0 & 0 & 0 & 0 & 0 & 0 \\
0 & 0 & 0 & 0 & 0 & 0 & 0 & 0 & 0 & 0 & 0 & 0 & 0 & 0 & 0 \\
0 & 0 & 0 & 0 & 0 & 0 & 0 & 0 & 0 & 0 & 0 & 0 & 0 & 0 & 0 \\
0 & 0 & 0 & 0 & 0 & 0 & 0 & 0 & 0 & 0 & 0 & 0 & 0 & 0 & 0 \\
0 & 0 & 0 & 0 & 0 & 0 & 0 & 0 & 0 & 0 & 0 & 0 & 0 & 0 & 0 \\
0 & 0 & 0 & 0 & 0 & 0 & 0 & 0 & 0 & 0 & 0 & 0 & 0 & 0 & 0 \\
0 & 0 & 0 & 0 & 0 & 0 & 0 & 0 & 0 & 0 & 0 & 0 & 0 & 0 & 0 \\
0 & 0 & 0 & 0 & 0 & 0 & 0 & 0 & 0 & 0 & 0 & 0 & 0 & 0 & 0 \\
\hline $\mathbf{0}$ & $\mathbf{0}$ & $\mathbf{0}$ & $\mathbf{0}$ & $\mathbf{0}$ & $\mathbf{0}$ & $\mathbf{0}$ & $\mathbf{0}$ & $\mathbf{0}$ & $\mathbf{0}$ & $\mathbf{0}$ & $\mathbf{0}$ & $\mathbf{0}$ & $\mathbf{0}$ & $\mathbf{0}$
\end{tabular}

-Other Entities-

104 Avista Energy Inc.

105 Chehalis Power Inc.

106 FPL Energy Vansycle LLC

107 Frederickson Power LP,

108 Hermiston Power Partnership

109 Klamath Pacific Energy

110 Longview Fibre

111 Mirant Americas Energy Marketing, L.P.

112 PPM Energy

113 SP Newsprint

114 Total Other Entities

Large Thermal Reserves

-Federal Entities-

115 BPA - Power Business 116 Total Federal Entities

$\begin{array}{lllllllllllllll}0 & 0 & 0 & 0 & 0 & 0 & 0 & 0 & 0 & 0 & 0 & 0 & 0 & 0 & 0 \\ 0 & 0 & 0 & 0 & 0 & 0 & 0 & 0 & 0 & 0 & 0 & 0 & 0 & 0 & 0 \\ 0 & 0 & 0 & 0 & 0 & 0 & 0 & 0 & 0 & 0 & 0 & 0 & 0 & 0 & 0 \\ 0 & 0 & 0 & 0 & 0 & 0 & 0 & 0 & 0 & 0 & 0 & 0 & 0 & 0 & 0 \\ 0 & 0 & 0 & 0 & 0 & 0 & 0 & 0 & 0 & 0 & 0 & 0 & 0 & 0 & 0 \\ 0 & 0 & 0 & 0 & 0 & 0 & 0 & 0 & 0 & 0 & 0 & 0 & 0 & 0 & 0 \\ 0 & 0 & 0 & 0 & 0 & 0 & 0 & 0 & 0 & 0 & 0 & 0 & 0 & 0 & 0 \\ 0 & 0 & 0 & 0 & 0 & 0 & 0 & 0 & 0 & 0 & 0 & 0 & 0 & 0 & 0 \\ 0 & 0 & 0 & 0 & 0 & 0 & 0 & 0 & 0 & 0 & 0 & 0 & 0 & 0 & 0 \\ 0 & 0 & 0 & 0 & 0 & 0 & 0 & 0 & 0 & 0 & 0 & 0 & 0 & 0 & 0 \\ 0 & \mathbf{0} & \mathbf{0} & \mathbf{0} & \mathbf{0} & \mathbf{0} & \mathbf{0} & \mathbf{0} & \mathbf{0} & \mathbf{0} & \mathbf{0} & \mathbf{0} & \mathbf{0} & \mathbf{0} & \mathbf{0}\end{array}$

-Generating Public Entities-

117 Turlock Irrigation District

118 Total Generating Public Entities

-Non-Generating Public Entities-

119 Total Non-Generating Public Entities 
Average Energy in Megawatts

120 Avista Corp (WWP Division)

121 Idaho Power Company

122 Northwestern Energy LLC (MPC)

123 Pacific Power

124 Portland General Electric

125 Puget Sound Energy

126 San Diego Gas \& Electric

127 Trans Alta Utilities Corp

128 Total Investor-Owned Entities

\section{Water Year}

2003 White Book

-Other Entities-

129 Total Other Entities

\title{
Aug1 Aug16
}

Sep

Oct Nov Dec Jan Feb

Mar Apr1 Apr1

May Jun Jul Avg

Contract Reserves

-Federal Entities-

130 Total Federal Entities

$\begin{array}{llll}0 & 0 & 0 & 0 \\ 0 & 0 & 0 & 0 \\ 0 & 0 & 0 & 0 \\ 0 & 0 & 0 & 0 \\ 0 & 0 & 0 & 0 \\ 0 & 0 & 0 & 0 \\ 0 & 0 & 0 & 0 \\ 0 & 0 & 0 & 0 \\ 0 & 0 & 0 & 0\end{array}$

$\begin{array}{ll}0 & 0 \\ 0 & 0 \\ 0 & 0 \\ 0 & 0 \\ 0 & 0 \\ 0 & 0 \\ 0 & 0 \\ 0 & 0 \\ 0 & 0\end{array}$

$\begin{array}{lll}0 & 0 & 0 \\ 0 & 0 & 0 \\ 0 & 0 & 0 \\ 0 & 0 & 0 \\ 0 & 0 & 0 \\ 0 & 0 & \\ 0 & 0 & \\ 0 & 0 & 0 \\ 0 & 0\end{array}$

0
0
0
0
0
0
0
0
0

0
0
0
0
0
0
0
0
0

$\begin{array}{lllllll}0 & 0 & 0 & 0 & 0 & 0 & 0 \\ 0 & 0 & 0 & 0 & 0 & 0 & 0 \\ 0 & 0 & 0 & 0 & 0 & 0 & 0 \\ 0 & 0 & 0 & 0 & 0 & 0 & 0 \\ 0 & 0 & 0 & 0 & 0 & 0 & 0 \\ 0 & 0 & 0 & 0 & 0 & 0 & 0 \\ 0 & 0 & 0 & 0 & 0 & 0 & 0 \\ 0 & 0 & 0 & 0 & 0 & 0 & 0 \\ 0 & 0 & 0 & 0 & 0 & 0 & 0\end{array}$

-Generating Public Entities-

131 Total Generating Public Entities

$0 \quad 0$

0

(n)

-Non-Generating Public Entities-

132 Total Non-Generating Public Entities

-Investor-Owned Entities-

133 Northwestern Energy LLC (MPC)

134 Total Investor-Owned Entities

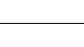

0

0

$0 \quad 0 \quad 0$

10

0

0

0

\begin{abstract}
0
\end{abstract}

10

-Other Entities-

135 Total Other Entities

0

0

-Total Capacity Reserves-

136 Total Hydro Reserves

137 Total Small Thermal \& Misc Reserves

138 Total Large Thermal Reserves

139 Total Contract Reserves

140 Total Capacity Reserves

\begin{tabular}{lllllllllllllll}
0 & 0 & 0 & 0 & 0 & 0 & 0 & 0 & 0 & 0 & 0 & 0 & 0 & 0 & 0 \\
0 & 0 & 0 & 0 & 0 & 0 & 0 & 0 & 0 & 0 & 0 & 0 & 0 & 0 & 0 \\
0 & 0 & 0 & 0 & 0 & 0 & 0 & 0 & 0 & 0 & 0 & 0 & 0 & 0 & 0 \\
0 & 0 & 0 & 0 & 0 & 0 & 0 & 0 & 0 & 0 & 0 & 0 & 0 & 0 \\
\hline $\mathbf{0}$ & $\mathbf{0}$ & $\mathbf{0}$ & $\mathbf{0}$ & $\mathbf{0}$ & $\mathbf{0}$ & $\mathbf{0}$ & $\mathbf{0}$ & $\mathbf{0}$ & $\mathbf{0}$ & $\mathbf{0}$ & $\mathbf{0}$ & $\mathbf{0}$ & $\mathbf{0}$ & $\mathbf{0}$
\end{tabular}


Table A-17: Regional Capacity Reserves

PNW Loads and Resources Study

2010 - 2011 Operating Year

1937 Water Year

2003 White Book

Oct Nov

Dec Jan Fe

Feb

Mar Apr1 Apr16 May Jun Jul Avg

Hydro Reserves

1 BPA - Power Business

2 Total Federal Entities

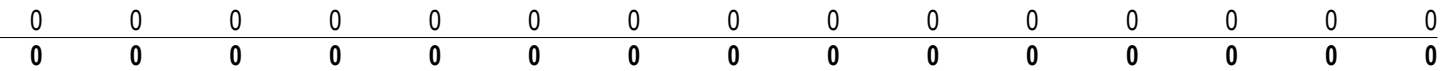

-Generating Public Entities-

3 Chelan County PUD \#1

4 Clark County PUD \#1

5 Cowlitz County PUD \#1

6 Douglas County PUD \#1

7 Eugene Water \& Electric Board

8 Grant County PUD \#2

9 Grays Harbor PUD \#1

10 Okanogan County PUD \#1

11 Pend Oreille County PUD \#1

12 Seattle City Light

13 Snohomish County PUD \#1

14 Tacoma Public Utilities

15 Total Generating Public Entities

$\begin{array}{lllllllllllllll}0 & 0 & 0 & 0 & 0 & 0 & 0 & 0 & 0 & 0 & 0 & 0 & 0 & 0 & 0 \\ 0 & 0 & 0 & 0 & 0 & 0 & 0 & 0 & 0 & 0 & 0 & 0 & 0 & 0 & 0 \\ 0 & 0 & 0 & 0 & 0 & 0 & 0 & 0 & 0 & 0 & 0 & 0 & 0 & 0 & 0 \\ 0 & 0 & 0 & 0 & 0 & 0 & 0 & 0 & 0 & 0 & 0 & 0 & 0 & 0 & 0 \\ 0 & 0 & 0 & 0 & 0 & 0 & 0 & 0 & 0 & 0 & 0 & 0 & 0 & 0 & 0 \\ 0 & 0 & 0 & 0 & 0 & 0 & 0 & 0 & 0 & 0 & 0 & 0 & 0 & 0 & 0 \\ 0 & 0 & 0 & 0 & 0 & 0 & 0 & 0 & 0 & 0 & 0 & 0 & 0 & 0 & 0 \\ 0 & 0 & 0 & 0 & 0 & 0 & 0 & 0 & 0 & 0 & 0 & 0 & 0 & 0 & 0 \\ 0 & 0 & 0 & 0 & 0 & 0 & 0 & 0 & 0 & 0 & 0 & 0 & 0 & 0 & 0 \\ 0 & 0 & 0 & 0 & 0 & 0 & 0 & 0 & 0 & 0 & 0 & 0 & 0 & 0 & 0 \\ 0 & 0 & 0 & 0 & 0 & 0 & 0 & 0 & 0 & 0 & 0 & 0 & 0 & 0 & 0 \\ 0 & 0 & 0 & 0 & 0 & 0 & 0 & 0 & 0 & 0 & 0 & 0 & 0 & 0 & 0 \\ 0 & 0 & \mathbf{0} & \mathbf{0} & \mathbf{0} & \mathbf{0} & \mathbf{0} & \mathbf{0} & \mathbf{0} & \mathbf{0} & \mathbf{0} & \mathbf{0} & \mathbf{0} & \mathbf{0} & \mathbf{0}\end{array}$

-Non-Generating Public Entities-

16 Ashland, City of

17 Benton County PUD \#1

18 Bonners Ferry, City of

19 Centralia, City of

20 Clallam County PUD \#1

21 Clearwater Power

22 Consumers Power

23 Emerald County PUD

24 Energy Northwest

25 Fall River Elec Coop

26 Forest Grove, City of

27 Franklin County PUD \#1

28 Idaho County L \& P

29 Idaho Falls, City of

30 Kittitas County PUD \#1

31 Klickitat County PUD \#1

32 Kootenai Electric Coop

33 Lewis County PUD \#1

34 Lost River Elec Coop

35 Lower Valley Energy

36 Mason County PUD \#1

37 McMinnville, City of

38 Milton Freewater, City of

39 Northern Lights

40 Northern Wasco County PUD

41 Oregon Trail Coop

42 Port Angeles, City of

43 Raft River Elec Coop

44 Salmon River Elec Coop

45 Soda Springs, City of

46 Tanner Elec Coop

47 Tillamook PUD \#1

48 United Electric Coop

49 Total Non-Generating Public Entities

\begin{tabular}{|c|c|c|c|c|c|c|c|c|c|c|c|c|c|}
\hline 0 & 0 & 0 & 0 & 0 & 0 & 0 & 0 & 0 & 0 & 0 & 0 & 0 & 0 \\
\hline 0 & 0 & 0 & 0 & 0 & 0 & 0 & 0 & 0 & 0 & 0 & 0 & 0 & 0 \\
\hline 0 & 0 & 0 & 0 & 0 & 0 & 0 & 0 & 0 & 0 & 0 & 0 & 0 & 0 \\
\hline 0 & 0 & 0 & 0 & 0 & 0 & 0 & 0 & 0 & 0 & 0 & 0 & 0 & 0 \\
\hline 0 & 0 & 0 & 0 & 0 & 0 & 0 & 0 & 0 & 0 & 0 & 0 & 0 & 0 \\
\hline 0 & 0 & 0 & 0 & 0 & 0 & 0 & 0 & 0 & 0 & 0 & 0 & 0 & 0 \\
\hline 0 & 0 & 0 & 0 & 0 & 0 & 0 & 0 & 0 & 0 & 0 & 0 & 0 & 0 \\
\hline 0 & 0 & 0 & 0 & 0 & 0 & 0 & 0 & 0 & 0 & 0 & 0 & 0 & 0 \\
\hline 0 & 0 & 0 & 0 & 0 & 0 & 0 & 0 & 0 & 0 & 0 & 0 & 0 & 0 \\
\hline 0 & 0 & 0 & 0 & 0 & 0 & 0 & 0 & 0 & 0 & 0 & 0 & 0 & 0 \\
\hline 0 & 0 & 0 & 0 & 0 & 0 & 0 & 0 & 0 & 0 & 0 & 0 & 0 & 0 \\
\hline 0 & 0 & 0 & 0 & 0 & 0 & 0 & 0 & 0 & 0 & 0 & 0 & 0 & 0 \\
\hline 0 & 0 & 0 & 0 & 0 & 0 & 0 & 0 & 0 & 0 & 0 & 0 & 0 & 0 \\
\hline 0 & 0 & 0 & 0 & 0 & 0 & 0 & 0 & 0 & 0 & 0 & 0 & 0 & 0 \\
\hline 0 & 0 & 0 & 0 & 0 & 0 & 0 & 0 & 0 & 0 & 0 & 0 & 0 & 0 \\
\hline 0 & 0 & 0 & 0 & 0 & 0 & 0 & 0 & 0 & 0 & 0 & 0 & 0 & 0 \\
\hline 0 & 0 & 0 & 0 & 0 & 0 & 0 & 0 & 0 & 0 & 0 & 0 & 0 & 0 \\
\hline 0 & 0 & 0 & 0 & 0 & 0 & 0 & 0 & 0 & 0 & 0 & 0 & 0 & 0 \\
\hline 0 & 0 & 0 & 0 & 0 & 0 & 0 & 0 & 0 & 0 & 0 & 0 & 0 & 0 \\
\hline 0 & 0 & 0 & 0 & 0 & 0 & 0 & 0 & 0 & 0 & 0 & 0 & 0 & 0 \\
\hline 0 & 0 & 0 & 0 & 0 & 0 & 0 & 0 & 0 & 0 & 0 & 0 & 0 & 0 \\
\hline 0 & 0 & 0 & 0 & 0 & 0 & 0 & 0 & 0 & 0 & 0 & 0 & 0 & 0 \\
\hline 0 & 0 & 0 & 0 & 0 & 0 & 0 & 0 & 0 & 0 & 0 & 0 & 0 & 0 \\
\hline 0 & 0 & 0 & 0 & 0 & 0 & 0 & 0 & 0 & 0 & 0 & 0 & 0 & 0 \\
\hline 0 & 0 & 0 & 0 & 0 & 0 & 0 & 0 & 0 & 0 & 0 & 0 & 0 & 0 \\
\hline 0 & 0 & 0 & 0 & 0 & 0 & 0 & 0 & 0 & 0 & 0 & 0 & 0 & 0 \\
\hline 0 & 0 & 0 & 0 & 0 & 0 & 0 & 0 & 0 & 0 & 0 & 0 & 0 & 0 \\
\hline 0 & 0 & 0 & 0 & 0 & 0 & 0 & 0 & 0 & 0 & 0 & 0 & 0 & 0 \\
\hline 0 & 0 & 0 & 0 & 0 & 0 & 0 & 0 & 0 & 0 & 0 & 0 & 0 & 0 \\
\hline 0 & 0 & 0 & 0 & 0 & 0 & 0 & 0 & 0 & 0 & 0 & 0 & 0 & 0 \\
\hline 0 & 0 & 0 & 0 & 0 & 0 & 0 & 0 & 0 & 0 & 0 & 0 & 0 & 0 \\
\hline 0 & 0 & 0 & 0 & 0 & 0 & 0 & 0 & 0 & 0 & 0 & 0 & 0 & 0 \\
\hline 0 & 0 & 0 & 0 & 0 & 0 & 0 & 0 & 0 & 0 & 0 & 0 & 0 & 0 \\
\hline 0 & 0 & 0 & 0 & 0 & 0 & 0 & 0 & 0 & 0 & 0 & 0 & 0 & 0 \\
\hline
\end{tabular}

-Investor-Owned Entities-

50 Avista Corp (WWP Division)

51 Idaho Power Company

52 Northwestern Energy LLC (MPC)

53 Pacific Power

54 Portland General Electric

55 Puget Sound Energy

56 Total Investor-Owned Entities

-Other Entities-

57 Colockum Transmission Company

58 Longview Fibre

59 Warm Springs Power Enterprise

60 Total Other Entities

$\begin{array}{lllllllllllllll}0 & 0 & 0 & 0 & 0 & 0 & 0 & 0 & 0 & 0 & 0 & 0 & 0 & 0 & 0 \\ 0 & 0 & 0 & 0 & 0 & 0 & 0 & 0 & 0 & 0 & 0 & 0 & 0 & 0 & 0 \\ 0 & 0 & 0 & 0 & 0 & 0 & 0 & 0 & 0 & 0 & 0 & 0 & 0 & 0 & 0 \\ 0 & 0 & 0 & 0 & 0 & 0 & 0 & 0 & 0 & 0 & 0 & 0 & 0 & 0 & 0 \\ 0 & 0 & 0 & 0 & 0 & 0 & 0 & 0 & 0 & 0 & 0 & 0 & 0 & 0 & 0 \\ 0 & 0 & 0 & 0 & 0 & 0 & 0 & 0 & 0 & 0 & 0 & 0 & 0 & 0 & 0 \\ 0 & \mathbf{0} & \mathbf{0} & \mathbf{0} & \mathbf{0} & \mathbf{0} & \mathbf{0} & \mathbf{0} & \mathbf{0} & \mathbf{0} & \mathbf{0} & \mathbf{0} & \mathbf{0} & \mathbf{0} & \mathbf{0}\end{array}$

Small Thermal \& Misc. Reserves

-Federal Entities-

61 BPA - Power Business

62 U.S. Naval Station, Everett (Jim Creek)

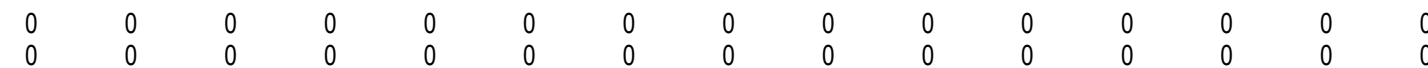




$\begin{array}{lllllllllllllll}0 & 0 & 0 & 0 & 0 & 0 & 0 & 0 & 0 & 0 & 0 & 0 & 0 & 0 & 0 \\ 0 & 0 & 0 & 0 & 0 & 0 & 0 & 0 & 0 & 0 & 0 & 0 & 0 & 0 & 0\end{array}$

-Generating Public Entities-

65 Chelan County PUD \#1

66 Clark County PUD \#1

67 Cowlitz County PUD \#1

68 Douglas County PUD \#1

69 Eugene Water \& Electric Board

70 Grant County PUD \#2

71 Grays Harbor PUD \#1

72 Okanogan County PUD \#1

73 Seattle City Light

74 Snohomish County PUD \#1

75 Springfield Utility Board

76 Tacoma Public Utilities

77 Total Generating Public Entities

$\begin{array}{lllllllllllllll}0 & 0 & 0 & 0 & 0 & 0 & 0 & 0 & 0 & 0 & 0 & 0 & 0 & 0 & 0 \\ 0 & 0 & 0 & 0 & 0 & 0 & 0 & 0 & 0 & 0 & 0 & 0 & 0 & 0 & 0 \\ 0 & 0 & 0 & 0 & 0 & 0 & 0 & 0 & 0 & 0 & 0 & 0 & 0 & 0 & 0 \\ 0 & 0 & 0 & 0 & 0 & 0 & 0 & 0 & 0 & 0 & 0 & 0 & 0 & 0 & 0 \\ 0 & 0 & 0 & 0 & 0 & 0 & 0 & 0 & 0 & 0 & 0 & 0 & 0 & 0 & 0 \\ 0 & 0 & 0 & 0 & 0 & 0 & 0 & 0 & 0 & 0 & 0 & 0 & 0 & 0 & 0 \\ 0 & 0 & 0 & 0 & 0 & 0 & 0 & 0 & 0 & 0 & 0 & 0 & 0 & 0 & 0 \\ 0 & 0 & 0 & 0 & 0 & 0 & 0 & 0 & 0 & 0 & 0 & 0 & 0 & 0 & 0 \\ 0 & 0 & 0 & 0 & 0 & 0 & 0 & 0 & 0 & 0 & 0 & 0 & 0 & 0 & 0 \\ 0 & 0 & 0 & 0 & 0 & 0 & 0 & 0 & 0 & 0 & 0 & 0 & 0 & 0 & 0 \\ 0 & 0 & 0 & 0 & 0 & 0 & 0 & 0 & 0 & 0 & 0 & 0 & 0 & 0 & 0 \\ 0 & 0 & 0 & 0 & 0 & 0 & 0 & 0 & 0 & 0 & 0 & 0 & 0 & 0 \\ 0 & 0 & \mathbf{0} & \mathbf{0} & \mathbf{0} & \mathbf{0} & \mathbf{0} & \mathbf{0} & \mathbf{0} & \mathbf{0} & \mathbf{0} & \mathbf{0} & \mathbf{0} & \mathbf{0} & \mathbf{0}\end{array}$

-Non-Generating Public Entities-

78 Ashland, City of

79 Benton County PUD \#1

80 Clallam County PUD \#1

81 Clatskanie PUD

82 Consumers Power

83 Emerald County PUD

84 Energy Northwest

85 Fall River Elec Coop

86 Franklin County PUD \#1

87 Klickitat County PUD \#1

88 Lewis County PUD \#1

89 Mason County PUD \#1

90 Mason County PUD \#3

91 Oregon Trail Coop

92 Tanner Elec Coop

93 Tillamook PUD \#1

94 Total Non-Generating Public Entities

$\begin{array}{lllllllllllllll}0 & 0 & 0 & 0 & 0 & 0 & 0 & 0 & 0 & 0 & 0 & 0 & 0 & 0 & 0 \\ 0 & 0 & 0 & 0 & 0 & 0 & 0 & 0 & 0 & 0 & 0 & 0 & 0 & 0 & 0 \\ 0 & 0 & 0 & 0 & 0 & 0 & 0 & 0 & 0 & 0 & 0 & 0 & 0 & 0 & 0 \\ 0 & 0 & 0 & 0 & 0 & 0 & 0 & 0 & 0 & 0 & 0 & 0 & 0 & 0 & 0 \\ 0 & 0 & 0 & 0 & 0 & 0 & 0 & 0 & 0 & 0 & 0 & 0 & 0 & 0 & 0 \\ 0 & 0 & 0 & 0 & 0 & 0 & 0 & 0 & 0 & 0 & 0 & 0 & 0 & 0 & 0 \\ 0 & 0 & 0 & 0 & 0 & 0 & 0 & 0 & 0 & 0 & 0 & 0 & 0 & 0 & 0 \\ 0 & 0 & 0 & 0 & 0 & 0 & 0 & 0 & 0 & 0 & 0 & 0 & 0 & 0 & 0 \\ 0 & 0 & 0 & 0 & 0 & 0 & 0 & 0 & 0 & 0 & 0 & 0 & 0 & 0 & 0 \\ 0 & 0 & 0 & 0 & 0 & 0 & 0 & 0 & 0 & 0 & 0 & 0 & 0 & 0 & 0 \\ 0 & 0 & 0 & 0 & 0 & 0 & 0 & 0 & 0 & 0 & 0 & 0 & 0 & 0 & 0 \\ 0 & 0 & 0 & 0 & 0 & 0 & 0 & 0 & 0 & 0 & 0 & 0 & 0 & 0 & 0 \\ 0 & 0 & 0 & 0 & 0 & 0 & 0 & 0 & 0 & 0 & 0 & 0 & 0 & 0 & 0 \\ 0 & 0 & 0 & 0 & 0 & 0 & 0 & 0 & 0 & 0 & 0 & 0 & 0 & 0 & 0 \\ 0 & 0 & 0 & 0 & 0 & 0 & 0 & 0 & 0 & 0 & 0 & 0 & 0 & 0 & 0 \\ 0 & 0 & 0 & 0 & 0 & 0 & 0 & 0 & 0 & 0 & 0 & 0 & 0 & 0 & 0 \\ 0 & \mathbf{0} & \mathbf{0} & \mathbf{0} & \mathbf{0} & \mathbf{0} & \mathbf{0} & \mathbf{0} & \mathbf{0} & \mathbf{0} & \mathbf{0} & \mathbf{0} & \mathbf{0} & \mathbf{0} & \mathbf{0}\end{array}$

-Investor-Owned Entities-

95 Avista Corp (WWP Division)

96 Idaho Power Company

97 Northwestern Energy LLC (MPC)

98 Pacific Power

99 Portland General Electric

100 Puget Sound Energy

101 Trans Alta Utilities Corp

102 Utah Power

103 Total Investor-Owned Entities

\begin{tabular}{lllllllllllllll}
0 & 0 & 0 & 0 & 0 & 0 & 0 & 0 & 0 & 0 & 0 & 0 & 0 & 0 & 0 \\
0 & 0 & 0 & 0 & 0 & 0 & 0 & 0 & 0 & 0 & 0 & 0 & 0 & 0 & 0 \\
0 & 0 & 0 & 0 & 0 & 0 & 0 & 0 & 0 & 0 & 0 & 0 & 0 & 0 & 0 \\
0 & 0 & 0 & 0 & 0 & 0 & 0 & 0 & 0 & 0 & 0 & 0 & 0 & 0 & 0 \\
0 & 0 & 0 & 0 & 0 & 0 & 0 & 0 & 0 & 0 & 0 & 0 & 0 & 0 & 0 \\
0 & 0 & 0 & 0 & 0 & 0 & 0 & 0 & 0 & 0 & 0 & 0 & 0 & 0 & 0 \\
0 & 0 & 0 & 0 & 0 & 0 & 0 & 0 & 0 & 0 & 0 & 0 & 0 & 0 & 0 \\
0 & 0 & 0 & 0 & 0 & 0 & 0 & 0 & 0 & 0 & 0 & 0 & 0 & 0 & 0 \\
\hline $\mathbf{0}$ & $\mathbf{0}$ & $\mathbf{0}$ & $\mathbf{0}$ & $\mathbf{0}$ & $\mathbf{0}$ & $\mathbf{0}$ & $\mathbf{0}$ & $\mathbf{0}$ & $\mathbf{0}$ & $\mathbf{0}$ & $\mathbf{0}$ & $\mathbf{0}$ & $\mathbf{0}$ & $\mathbf{0}$
\end{tabular}

-Other Entities-

104 Avista Energy Inc.

105 Chehalis Power Inc.

106 FPL Energy Vansycle LLC

107 Frederickson Power LP,

108 Hermiston Power Partnership

109 Klamath Pacific Energy

110 Longview Fibre

111 Mirant Americas Energy Marketing, L.P.

112 PPM Energy

113 SP Newsprint

114 Total Other Entities

Large Thermal Reserves

-Federal Entities-

115 BPA - Power Business 116 Total Federal Entities

$\begin{array}{lllllllllllllll}0 & 0 & 0 & 0 & 0 & 0 & 0 & 0 & 0 & 0 & 0 & 0 & 0 & 0 & 0 \\ 0 & 0 & 0 & 0 & 0 & 0 & 0 & 0 & 0 & 0 & 0 & 0 & 0 & 0 & 0 \\ 0 & 0 & 0 & 0 & 0 & 0 & 0 & 0 & 0 & 0 & 0 & 0 & 0 & 0 & 0 \\ 0 & 0 & 0 & 0 & 0 & 0 & 0 & 0 & 0 & 0 & 0 & 0 & 0 & 0 & 0 \\ 0 & 0 & 0 & 0 & 0 & 0 & 0 & 0 & 0 & 0 & 0 & 0 & 0 & 0 & 0 \\ 0 & 0 & 0 & 0 & 0 & 0 & 0 & 0 & 0 & 0 & 0 & 0 & 0 & 0 & 0 \\ 0 & 0 & 0 & 0 & 0 & 0 & 0 & 0 & 0 & 0 & 0 & 0 & 0 & 0 & 0 \\ 0 & 0 & 0 & 0 & 0 & 0 & 0 & 0 & 0 & 0 & 0 & 0 & 0 & 0 & 0 \\ 0 & 0 & 0 & 0 & 0 & 0 & 0 & 0 & 0 & 0 & 0 & 0 & 0 & 0 & 0 \\ 0 & 0 & 0 & 0 & 0 & 0 & 0 & 0 & 0 & 0 & 0 & 0 & 0 & 0 & 0 \\ 0 & \mathbf{0} & \mathbf{0} & \mathbf{0} & \mathbf{0} & \mathbf{0} & \mathbf{0} & \mathbf{0} & \mathbf{0} & \mathbf{0} & \mathbf{0} & \mathbf{0} & \mathbf{0} & \mathbf{0} & \mathbf{0}\end{array}$

-Generating Public Entities-

117 Turlock Irrigation District

118 Total Generating Public Entities

-Non-Generating Public Entities-

119 Total Non-Generating Public Entities 
Average Energy in Megawatts

120 Avista Corp (WWP Division)

121 Idaho Power Company

122 Northwestern Energy LLC (MPC)

123 Pacific Power

124 Portland General Electric

125 Puget Sound Energy

126 San Diego Gas \& Electric

127 Trans Alta Utilities Corp

128 Total Investor-Owned Entities

\section{Water Year}

2003 White Book

-Other Entities-

129 Total Other Entities

\title{
Aug1 Aug16
}

Sep

Oct Nov Dec Jan Feb

Mar Apr1 Apr1

May Jun Jul Avg

Contract Reserves

-Federal Entities-

130 Total Federal Entities

$\begin{array}{llll}0 & 0 & 0 & 0 \\ 0 & 0 & 0 & 0 \\ 0 & 0 & 0 & 0 \\ 0 & 0 & 0 & 0 \\ 0 & 0 & 0 & 0 \\ 0 & 0 & 0 & 0 \\ 0 & 0 & 0 & 0 \\ 0 & 0 & 0 & 0 \\ 0 & 0 & 0 & 0\end{array}$

$\begin{array}{ll}0 & 0 \\ 0 & 0 \\ 0 & 0 \\ 0 & 0 \\ 0 & 0 \\ 0 & 0 \\ 0 & 0 \\ 0 & 0 \\ 0 & 0\end{array}$

$\begin{array}{lll}0 & 0 & 0 \\ 0 & 0 & 0 \\ 0 & 0 & 0 \\ 0 & 0 & 0 \\ 0 & 0 & 0 \\ 0 & 0 & \\ 0 & 0 & \\ 0 & 0 & 0 \\ 0 & 0\end{array}$

0
0
0
0
0
0
0
0
0

$\begin{array}{ll}0 & 0 \\ 0 & 0 \\ 0 & 0 \\ 0 & 0 \\ 0 & 0 \\ 0 & \\ 0 & \\ 0 & \\ 0 & \end{array}$

$\begin{array}{lllllll}0 & 0 & 0 & 0 & 0 & 0 & 0 \\ 0 & 0 & 0 & 0 & 0 & 0 & 0 \\ 0 & 0 & 0 & 0 & 0 & 0 & 0 \\ 0 & 0 & 0 & 0 & 0 & 0 & 0 \\ 0 & 0 & 0 & 0 & 0 & 0 & 0 \\ 0 & 0 & 0 & 0 & 0 & 0 & 0 \\ 0 & 0 & 0 & 0 & 0 & 0 & 0 \\ 0 & 0 & 0 & 0 & 0 & 0 & 0 \\ 0 & 0 & 0 & 0 & 0 & 0 & 0\end{array}$

-Generating Public Entities-

131 Total Generating Public Entities

$0 \quad 0$

\begin{abstract}
0
\end{abstract}
0

0

0

0

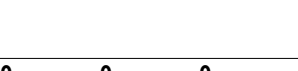

$\begin{array}{llll}0 & 0 & 0 & 0\end{array}$

-Non-Generating Public Entities-

132 Total Non-Generating Public Entities

-Investor-Owned Entities-

133 Northwestern Energy LLC (MPC)

134 Total Investor-Owned Entities

$\begin{array}{lllllllllllllll}0 & 0 & 0 & 0 & 0 & 0 & 0 & 0 & 0 & 0 & 0 & 0 & 0 & 0 & 0\end{array}$

-Other Entities-

135 Total Other Entities

0

0

0

\section{-Total Capacity Reserves-}

136 Total Hydro Reserves

137 Total Small Thermal \& Misc Reserves

138 Total Large Thermal Reserves

139 Total Contract Reserves

140 Total Capacity Reserves

$\begin{array}{llllllllllllll}0 & 0 & 0 & 0 & 0 & 0 & 0 & 0 & 0 & 0 & 0 & 0 & 0 & 0 \\ 0\end{array}$


Table A-17: Regional Capacity Reserves

PNW Loads and Resources Study

2011 - 2012 Operating Year

1937 Water Year

2003 White Book

Oct Nov

Dec Jan Fe

Feb Mar Apr1 Apr16 May Jun Jul Avg

Hydro Reserves

$1 \mathrm{BPA}$ - Power Business

2 Total Federal Entities

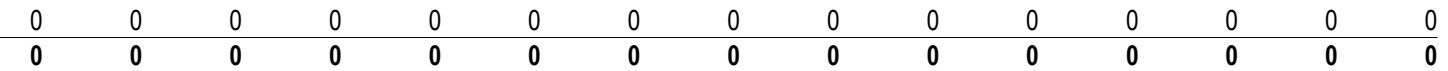

-Generating Public Entities-

3 Chelan County PUD \#1

4 Clark County PUD \#1

5 Cowlitz County PUD \#1

6 Douglas County PUD \#1

7 Eugene Water \& Electric Board

8 Grant County PUD \#2

9 Grays Harbor PUD \#1

10 Okanogan County PUD \#1

11 Pend Oreille County PUD \#1

12 Seattle City Light

13 Snohomish County PUD \#1

14 Tacoma Public Utilities

15 Total Generating Public Entities

$\begin{array}{lllllllllllllll}0 & 0 & 0 & 0 & 0 & 0 & 0 & 0 & 0 & 0 & 0 & 0 & 0 & 0 & 0 \\ 0 & 0 & 0 & 0 & 0 & 0 & 0 & 0 & 0 & 0 & 0 & 0 & 0 & 0 & 0 \\ 0 & 0 & 0 & 0 & 0 & 0 & 0 & 0 & 0 & 0 & 0 & 0 & 0 & 0 & 0 \\ 0 & 0 & 0 & 0 & 0 & 0 & 0 & 0 & 0 & 0 & 0 & 0 & 0 & 0 & 0 \\ 0 & 0 & 0 & 0 & 0 & 0 & 0 & 0 & 0 & 0 & 0 & 0 & 0 & 0 & 0 \\ 0 & 0 & 0 & 0 & 0 & 0 & 0 & 0 & 0 & 0 & 0 & 0 & 0 & 0 & 0 \\ 0 & 0 & 0 & 0 & 0 & 0 & 0 & 0 & 0 & 0 & 0 & 0 & 0 & 0 & 0 \\ 0 & 0 & 0 & 0 & 0 & 0 & 0 & 0 & 0 & 0 & 0 & 0 & 0 & 0 & 0 \\ 0 & 0 & 0 & 0 & 0 & 0 & 0 & 0 & 0 & 0 & 0 & 0 & 0 & 0 & 0 \\ 0 & 0 & 0 & 0 & 0 & 0 & 0 & 0 & 0 & 0 & 0 & 0 & 0 & 0 & 0 \\ 0 & 0 & 0 & 0 & 0 & 0 & 0 & 0 & 0 & 0 & 0 & 0 & 0 & 0 & 0 \\ 0 & 0 & 0 & 0 & 0 & 0 & 0 & 0 & 0 & 0 & 0 & 0 & 0 & 0 & 0 \\ 0 & 0 & \mathbf{0} & \mathbf{0} & \mathbf{0} & \mathbf{0} & \mathbf{0} & \mathbf{0} & \mathbf{0} & \mathbf{0} & \mathbf{0} & \mathbf{0} & \mathbf{0} & \mathbf{0} & \mathbf{0}\end{array}$

-Non-Generating Public Entities-

16 Ashland, City of

17 Benton County PUD \#1

18 Bonners Ferry, City of

19 Centralia, City of

20 Clallam County PUD \#1

21 Clearwater Power

22 Consumers Power

23 Emerald County PUD

24 Energy Northwest

25 Fall River Elec Coop

26 Forest Grove, City of

27 Franklin County PUD \#1

28 Idaho County L \& P

29 Idaho Falls, City of

30 Kittitas County PUD \#1

31 Klickitat County PUD \#1

32 Kootenai Electric Coop

33 Lewis County PUD \#1

34 Lost River Elec Coop

35 Lower Valley Energy

36 Mason County PUD \#1

37 McMinnville, City of

38 Milton Freewater, City of

39 Northern Lights

40 Northern Wasco County PUD

41 Oregon Trail Coop

42 Port Angeles, City of

43 Raft River Elec Coop

44 Salmon River Elec Coop

45 Soda Springs, City of

46 Tanner Elec Coop

47 Tillamook PUD \#1

48 United Electric Coop

49 Total Non-Generating Public Entities

\begin{tabular}{|c|c|c|c|c|c|c|c|c|c|c|c|c|c|}
\hline 0 & 0 & 0 & 0 & 0 & 0 & 0 & 0 & 0 & 0 & 0 & 0 & 0 & 0 \\
\hline 0 & 0 & 0 & 0 & 0 & 0 & 0 & 0 & 0 & 0 & 0 & 0 & 0 & 0 \\
\hline 0 & 0 & 0 & 0 & 0 & 0 & 0 & 0 & 0 & 0 & 0 & 0 & 0 & 0 \\
\hline 0 & 0 & 0 & 0 & 0 & 0 & 0 & 0 & 0 & 0 & 0 & 0 & 0 & 0 \\
\hline 0 & 0 & 0 & 0 & 0 & 0 & 0 & 0 & 0 & 0 & 0 & 0 & 0 & 0 \\
\hline 0 & 0 & 0 & 0 & 0 & 0 & 0 & 0 & 0 & 0 & 0 & 0 & 0 & 0 \\
\hline 0 & 0 & 0 & 0 & 0 & 0 & 0 & 0 & 0 & 0 & 0 & 0 & 0 & 0 \\
\hline 0 & 0 & 0 & 0 & 0 & 0 & 0 & 0 & 0 & 0 & 0 & 0 & 0 & 0 \\
\hline 0 & 0 & 0 & 0 & 0 & 0 & 0 & 0 & 0 & 0 & 0 & 0 & 0 & 0 \\
\hline 0 & 0 & 0 & 0 & 0 & 0 & 0 & 0 & 0 & 0 & 0 & 0 & 0 & 0 \\
\hline 0 & 0 & 0 & 0 & 0 & 0 & 0 & 0 & 0 & 0 & 0 & 0 & 0 & 0 \\
\hline 0 & 0 & 0 & 0 & 0 & 0 & 0 & 0 & 0 & 0 & 0 & 0 & 0 & 0 \\
\hline 0 & 0 & 0 & 0 & 0 & 0 & 0 & 0 & 0 & 0 & 0 & 0 & 0 & 0 \\
\hline 0 & 0 & 0 & 0 & 0 & 0 & 0 & 0 & 0 & 0 & 0 & 0 & 0 & 0 \\
\hline 0 & 0 & 0 & 0 & 0 & 0 & 0 & 0 & 0 & 0 & 0 & 0 & 0 & 0 \\
\hline 0 & 0 & 0 & 0 & 0 & 0 & 0 & 0 & 0 & 0 & 0 & 0 & 0 & 0 \\
\hline 0 & 0 & 0 & 0 & 0 & 0 & 0 & 0 & 0 & 0 & 0 & 0 & 0 & 0 \\
\hline 0 & 0 & 0 & 0 & 0 & 0 & 0 & 0 & 0 & 0 & 0 & 0 & 0 & 0 \\
\hline 0 & 0 & 0 & 0 & 0 & 0 & 0 & 0 & 0 & 0 & 0 & 0 & 0 & 0 \\
\hline 0 & 0 & 0 & 0 & 0 & 0 & 0 & 0 & 0 & 0 & 0 & 0 & 0 & 0 \\
\hline 0 & 0 & 0 & 0 & 0 & 0 & 0 & 0 & 0 & 0 & 0 & 0 & 0 & 0 \\
\hline 0 & 0 & 0 & 0 & 0 & 0 & 0 & 0 & 0 & 0 & 0 & 0 & 0 & 0 \\
\hline 0 & 0 & 0 & 0 & 0 & 0 & 0 & 0 & 0 & 0 & 0 & 0 & 0 & 0 \\
\hline 0 & 0 & 0 & 0 & 0 & 0 & 0 & 0 & 0 & 0 & 0 & 0 & 0 & 0 \\
\hline 0 & 0 & 0 & 0 & 0 & 0 & 0 & 0 & 0 & 0 & 0 & 0 & 0 & 0 \\
\hline 0 & 0 & 0 & 0 & 0 & 0 & 0 & 0 & 0 & 0 & 0 & 0 & 0 & 0 \\
\hline 0 & 0 & 0 & 0 & 0 & 0 & 0 & 0 & 0 & 0 & 0 & 0 & 0 & 0 \\
\hline 0 & 0 & 0 & 0 & 0 & 0 & 0 & 0 & 0 & 0 & 0 & 0 & 0 & 0 \\
\hline 0 & 0 & 0 & 0 & 0 & 0 & 0 & 0 & 0 & 0 & 0 & 0 & 0 & 0 \\
\hline 0 & 0 & 0 & 0 & 0 & 0 & 0 & 0 & 0 & 0 & 0 & 0 & 0 & 0 \\
\hline 0 & 0 & 0 & 0 & 0 & 0 & 0 & 0 & 0 & 0 & 0 & 0 & 0 & 0 \\
\hline 0 & 0 & 0 & 0 & 0 & 0 & 0 & 0 & 0 & 0 & 0 & 0 & 0 & 0 \\
\hline 0 & 0 & 0 & 0 & 0 & 0 & 0 & 0 & 0 & 0 & 0 & 0 & 0 & 0 \\
\hline 0 & 0 & 0 & 0 & 0 & 0 & 0 & 0 & 0 & 0 & 0 & 0 & 0 & 0 \\
\hline
\end{tabular}

-Investor-Owned Entities-

50 Avista Corp (WWP Division)

51 Idaho Power Company

52 Northwestern Energy LLC (MPC)

53 Pacific Power

54 Portland General Electric

55 Puget Sound Energy

56 Total Investor-Owned Entities

-Other Entities-

57 Colockum Transmission Company

58 Longview Fibre

59 Warm Springs Power Enterprise

60 Total Other Entities

$\begin{array}{lllllllllllllll}0 & 0 & 0 & 0 & 0 & 0 & 0 & 0 & 0 & 0 & 0 & 0 & 0 & 0 & 0 \\ 0 & 0 & 0 & 0 & 0 & 0 & 0 & 0 & 0 & 0 & 0 & 0 & 0 & 0 & 0 \\ 0 & 0 & 0 & 0 & 0 & 0 & 0 & 0 & 0 & 0 & 0 & 0 & 0 & 0 & 0 \\ 0 & 0 & 0 & 0 & 0 & 0 & 0 & 0 & 0 & 0 & 0 & 0 & 0 & 0 & 0 \\ 0 & 0 & 0 & 0 & 0 & 0 & 0 & 0 & 0 & 0 & 0 & 0 & 0 & 0 & 0 \\ 0 & 0 & 0 & 0 & 0 & 0 & 0 & 0 & 0 & 0 & 0 & 0 & 0 & 0 & 0 \\ 0 & \mathbf{0} & \mathbf{0} & \mathbf{0} & \mathbf{0} & \mathbf{0} & \mathbf{0} & \mathbf{0} & \mathbf{0} & \mathbf{0} & \mathbf{0} & \mathbf{0} & \mathbf{0} & \mathbf{0} & \mathbf{0}\end{array}$

Small Thermal \& Misc. Reserves

-Federal Entities-

61 BPA - Power Business

62 U.S. Naval Station, Everett (Jim Creek)

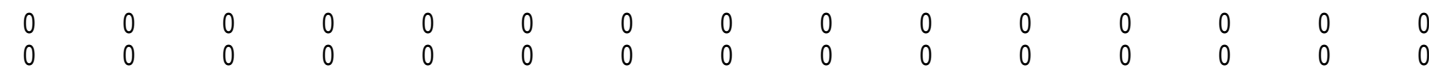




$\begin{array}{lllllllllllllll}0 & 0 & 0 & 0 & 0 & 0 & 0 & 0 & 0 & 0 & 0 & 0 & 0 & 0 & 0 \\ 0 & 0 & 0 & 0 & 0 & 0 & 0 & 0 & 0 & 0 & 0 & 0 & 0 & 0 & 0\end{array}$

-Generating Public Entities-

65 Chelan County PUD \#1

66 Clark County PUD \#1

67 Cowlitz County PUD \#1

68 Douglas County PUD \#1

69 Eugene Water \& Electric Board

70 Grant County PUD \#2

71 Grays Harbor PUD \#1

72 Okanogan County PUD \#1

73 Seattle City Light

74 Snohomish County PUD \#1

75 Springfield Utility Board

76 Tacoma Public Utilities

77 Total Generating Public Entities

$\begin{array}{lllllllllllllll}0 & 0 & 0 & 0 & 0 & 0 & 0 & 0 & 0 & 0 & 0 & 0 & 0 & 0 & 0 \\ 0 & 0 & 0 & 0 & 0 & 0 & 0 & 0 & 0 & 0 & 0 & 0 & 0 & 0 & 0 \\ 0 & 0 & 0 & 0 & 0 & 0 & 0 & 0 & 0 & 0 & 0 & 0 & 0 & 0 & 0 \\ 0 & 0 & 0 & 0 & 0 & 0 & 0 & 0 & 0 & 0 & 0 & 0 & 0 & 0 & 0 \\ 0 & 0 & 0 & 0 & 0 & 0 & 0 & 0 & 0 & 0 & 0 & 0 & 0 & 0 & 0 \\ 0 & 0 & 0 & 0 & 0 & 0 & 0 & 0 & 0 & 0 & 0 & 0 & 0 & 0 & 0 \\ 0 & 0 & 0 & 0 & 0 & 0 & 0 & 0 & 0 & 0 & 0 & 0 & 0 & 0 & 0 \\ 0 & 0 & 0 & 0 & 0 & 0 & 0 & 0 & 0 & 0 & 0 & 0 & 0 & 0 & 0 \\ 0 & 0 & 0 & 0 & 0 & 0 & 0 & 0 & 0 & 0 & 0 & 0 & 0 & 0 & 0 \\ 0 & 0 & 0 & 0 & 0 & 0 & 0 & 0 & 0 & 0 & 0 & 0 & 0 & 0 & 0 \\ 0 & 0 & 0 & 0 & 0 & 0 & 0 & 0 & 0 & 0 & 0 & 0 & 0 & 0 & 0 \\ 0 & 0 & 0 & 0 & 0 & 0 & 0 & 0 & 0 & 0 & 0 & 0 & 0 & 0 \\ 0 & 0 & \mathbf{0} & \mathbf{0} & \mathbf{0} & \mathbf{0} & \mathbf{0} & \mathbf{0} & \mathbf{0} & \mathbf{0} & \mathbf{0} & \mathbf{0} & \mathbf{0} & \mathbf{0} & \mathbf{0}\end{array}$

-Non-Generating Public Entities-

78 Ashland, City of

79 Benton County PUD \#1

80 Clallam County PUD \#1

81 Clatskanie PUD

82 Consumers Power

83 Emerald County PUD

84 Energy Northwest

85 Fall River Elec Coop

86 Franklin County PUD \#1

87 Klickitat County PUD \#1

88 Lewis County PUD \#1

89 Mason County PUD \#1

90 Mason County PUD \#3

91 Oregon Trail Coop

92 Tanner Elec Coop

93 Tillamook PUD \#1

94 Total Non-Generating Public Entities

$\begin{array}{lllllllllllllll}0 & 0 & 0 & 0 & 0 & 0 & 0 & 0 & 0 & 0 & 0 & 0 & 0 & 0 & 0 \\ 0 & 0 & 0 & 0 & 0 & 0 & 0 & 0 & 0 & 0 & 0 & 0 & 0 & 0 & 0 \\ 0 & 0 & 0 & 0 & 0 & 0 & 0 & 0 & 0 & 0 & 0 & 0 & 0 & 0 & 0 \\ 0 & 0 & 0 & 0 & 0 & 0 & 0 & 0 & 0 & 0 & 0 & 0 & 0 & 0 & 0 \\ 0 & 0 & 0 & 0 & 0 & 0 & 0 & 0 & 0 & 0 & 0 & 0 & 0 & 0 & 0 \\ 0 & 0 & 0 & 0 & 0 & 0 & 0 & 0 & 0 & 0 & 0 & 0 & 0 & 0 & 0 \\ 0 & 0 & 0 & 0 & 0 & 0 & 0 & 0 & 0 & 0 & 0 & 0 & 0 & 0 & 0 \\ 0 & 0 & 0 & 0 & 0 & 0 & 0 & 0 & 0 & 0 & 0 & 0 & 0 & 0 & 0 \\ 0 & 0 & 0 & 0 & 0 & 0 & 0 & 0 & 0 & 0 & 0 & 0 & 0 & 0 & 0 \\ 0 & 0 & 0 & 0 & 0 & 0 & 0 & 0 & 0 & 0 & 0 & 0 & 0 & 0 & 0 \\ 0 & 0 & 0 & 0 & 0 & 0 & 0 & 0 & 0 & 0 & 0 & 0 & 0 & 0 & 0 \\ 0 & 0 & 0 & 0 & 0 & 0 & 0 & 0 & 0 & 0 & 0 & 0 & 0 & 0 & 0 \\ 0 & 0 & 0 & 0 & 0 & 0 & 0 & 0 & 0 & 0 & 0 & 0 & 0 & 0 & 0 \\ 0 & 0 & 0 & 0 & 0 & 0 & 0 & 0 & 0 & 0 & 0 & 0 & 0 & 0 & 0 \\ 0 & 0 & 0 & 0 & 0 & 0 & 0 & 0 & 0 & 0 & 0 & 0 & 0 & 0 & 0 \\ 0 & 0 & 0 & 0 & 0 & 0 & 0 & 0 & 0 & 0 & 0 & 0 & 0 & 0 & 0 \\ 0 & \mathbf{0} & \mathbf{0} & \mathbf{0} & \mathbf{0} & \mathbf{0} & \mathbf{0} & \mathbf{0} & \mathbf{0} & \mathbf{0} & \mathbf{0} & \mathbf{0} & \mathbf{0} & \mathbf{0} & \mathbf{0}\end{array}$

-Investor-Owned Entities-

95 Avista Corp (WWP Division)

96 Idaho Power Company

97 Northwestern Energy LLC (MPC)

98 Pacific Power

99 Portland General Electric

100 Puget Sound Energy

101 Trans Alta Utilities Corp

102 Utah Power

103 Total Investor-Owned Entities

\begin{tabular}{lllllllllllllll}
0 & 0 & 0 & 0 & 0 & 0 & 0 & 0 & 0 & 0 & 0 & 0 & 0 & 0 & 0 \\
0 & 0 & 0 & 0 & 0 & 0 & 0 & 0 & 0 & 0 & 0 & 0 & 0 & 0 & 0 \\
0 & 0 & 0 & 0 & 0 & 0 & 0 & 0 & 0 & 0 & 0 & 0 & 0 & 0 & 0 \\
0 & 0 & 0 & 0 & 0 & 0 & 0 & 0 & 0 & 0 & 0 & 0 & 0 & 0 & 0 \\
0 & 0 & 0 & 0 & 0 & 0 & 0 & 0 & 0 & 0 & 0 & 0 & 0 & 0 & 0 \\
0 & 0 & 0 & 0 & 0 & 0 & 0 & 0 & 0 & 0 & 0 & 0 & 0 & 0 & 0 \\
0 & 0 & 0 & 0 & 0 & 0 & 0 & 0 & 0 & 0 & 0 & 0 & 0 & 0 & 0 \\
0 & 0 & 0 & 0 & 0 & 0 & 0 & 0 & 0 & 0 & 0 & 0 & 0 & 0 & 0 \\
\hline $\mathbf{0}$ & $\mathbf{0}$ & $\mathbf{0}$ & $\mathbf{0}$ & $\mathbf{0}$ & $\mathbf{0}$ & $\mathbf{0}$ & $\mathbf{0}$ & $\mathbf{0}$ & $\mathbf{0}$ & $\mathbf{0}$ & $\mathbf{0}$ & $\mathbf{0}$ & $\mathbf{0}$ & $\mathbf{0}$
\end{tabular}

-Other Entities-

104 Avista Energy Inc.

105 Chehalis Power Inc.

106 FPL Energy Vansycle LLC

107 Frederickson Power LP,

108 Hermiston Power Partnership

109 Klamath Pacific Energy

110 Longview Fibre

111 Mirant Americas Energy Marketing, L.P.

112 PPM Energy

113 SP Newsprint

114 Total Other Entities

Large Thermal Reserves

-Federal Entities-

115 BPA - Power Business 116 Total Federal Entities

$\begin{array}{lllllllllllllll}0 & 0 & 0 & 0 & 0 & 0 & 0 & 0 & 0 & 0 & 0 & 0 & 0 & 0 & 0 \\ 0 & 0 & 0 & 0 & 0 & 0 & 0 & 0 & 0 & 0 & 0 & 0 & 0 & 0 & 0 \\ 0 & 0 & 0 & 0 & 0 & 0 & 0 & 0 & 0 & 0 & 0 & 0 & 0 & 0 & 0 \\ 0 & 0 & 0 & 0 & 0 & 0 & 0 & 0 & 0 & 0 & 0 & 0 & 0 & 0 & 0 \\ 0 & 0 & 0 & 0 & 0 & 0 & 0 & 0 & 0 & 0 & 0 & 0 & 0 & 0 & 0 \\ 0 & 0 & 0 & 0 & 0 & 0 & 0 & 0 & 0 & 0 & 0 & 0 & 0 & 0 & 0 \\ 0 & 0 & 0 & 0 & 0 & 0 & 0 & 0 & 0 & 0 & 0 & 0 & 0 & 0 & 0 \\ 0 & 0 & 0 & 0 & 0 & 0 & 0 & 0 & 0 & 0 & 0 & 0 & 0 & 0 & 0 \\ 0 & 0 & 0 & 0 & 0 & 0 & 0 & 0 & 0 & 0 & 0 & 0 & 0 & 0 & 0 \\ 0 & 0 & 0 & 0 & 0 & 0 & 0 & 0 & 0 & 0 & 0 & 0 & 0 & 0 & 0 \\ 0 & \mathbf{0} & \mathbf{0} & \mathbf{0} & \mathbf{0} & \mathbf{0} & \mathbf{0} & \mathbf{0} & \mathbf{0} & \mathbf{0} & \mathbf{0} & \mathbf{0} & \mathbf{0} & \mathbf{0} & \mathbf{0}\end{array}$

-Generating Public Entities-

117 Turlock Irrigation District

118 Total Generating Public Entities

-Non-Generating Public Entities-

119 Total Non-Generating Public Entities 
Average Energy in Megawatts

120 Avista Corp (WWP Division)

121 Idaho Power Company

122 Northwestern Energy LLC (MPC)

123 Pacific Power

124 Portland General Electric

125 Puget Sound Energy

126 San Diego Gas \& Electric

127 Trans Alta Utilities Corp

128 Total Investor-Owned Entities

\section{Water Year}

2003 White Book

-Other Entities-

129 Total Other Entities

\section{Aug1 Aug16}

Sep

Oct Nov Dec Jan Feb

Mar Apr1 Apr1

May Jun Jul Avg

Contract Reserves

-Federal Entities-

130 Total Federal Entities

$\begin{array}{llll}0 & 0 & 0 & 0 \\ 0 & 0 & 0 & 0 \\ 0 & 0 & 0 & 0 \\ 0 & 0 & 0 & 0 \\ 0 & 0 & 0 & 0 \\ 0 & 0 & 0 & 0 \\ 0 & 0 & 0 & 0 \\ 0 & 0 & 0 & 0 \\ 0 & 0 & 0 & 0\end{array}$

$\begin{array}{ll}0 & 0 \\ 0 & 0 \\ 0 & 0 \\ 0 & 0 \\ 0 & 0 \\ 0 & 0 \\ 0 & 0 \\ 0 & 0 \\ 0 & 0\end{array}$

$\begin{array}{lll}0 & 0 & 0 \\ 0 & 0 & 0 \\ 0 & 0 & 0 \\ 0 & 0 & 0 \\ 0 & 0 & 0 \\ 0 & 0 & \\ 0 & 0 & \\ 0 & 0 & 0 \\ 0 & 0\end{array}$

0
0
0
0
0
0
0
0
0

-Generating Public Entities-

131 Total Generating Public Entities

0

0

$\begin{array}{llllllll}0 & 0 & 0 & 0 & 0 & 0 & 0 & 0 \\ 0 & 0 & 0 & 0 & 0 & 0 & 0 & 0 \\ 0 & 0 & 0 & 0 & 0 & 0 & 0 & 0 \\ 0 & 0 & 0 & 0 & 0 & 0 & 0 & 0 \\ 0 & 0 & 0 & 0 & 0 & 0 & 0 & 0 \\ 0 & 0 & 0 & 0 & 0 & 0 & 0 & 0 \\ 0 & 0 & 0 & 0 & 0 & 0 & 0 & 0 \\ 0 & 0 & 0 & 0 & 0 & 0 & 0 & 0 \\ 0 & 0 & 0 & 0 & 0 & 0 & 0 & 0\end{array}$

-Non-Generating Public Entities-

132 Total Non-Generating Public Entities

-Investor-Owned Entities-

133 Northwestern Energy LLC (MPC)

134 Total Investor-Owned Entities

$\begin{array}{lllllllllllllll}0 & 0 & 0 & 0 & 0 & 0 & 0 & 0 & 0 & 0 & 0 & 0 & 0 & 0 & 0 \\ 0 & 0 & 0 & 0 & 0 & 0 & 0 & 0 & 0 & 0 & 0 & 0 & 0 & 0 & 0\end{array}$

-Other Entities-

135 Total Other Entities

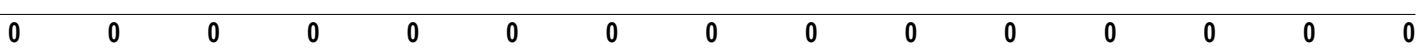

-Total Capacity Reserves-

136 Total Hydro Reserves

137 Total Small Thermal \& Misc Reserves

138 Total Large Thermal Reserves

139 Total Contract Reserves

140 Total Capacity Reserves

$\begin{array}{lllllllllllllll}0 & 0 & 0 & 0 & 0 & 0 & 0 & 0 & 0 & 0 & 0 & 0 & 0 & 0 & 0 \\ 0 & 0 & 0 & 0 & 0 & 0 & 0 & 0 & 0 & 0 & 0 & 0 & 0 & 0 & 0 \\ 0 & 0 & 0 & 0 & 0 & 0 & 0 & 0 & 0 & 0 & 0 & 0 & 0 & 0 & 0 \\ 0 & 0 & 0 & 0 & 0 & 0 & 0 & 0 & 0 & 0 & 0 & 0 & 0 & 0 & 0 \\ 0 & \mathbf{0} & \mathbf{0} & \mathbf{0} & \mathbf{0} & \mathbf{0} & \mathbf{0} & \mathbf{0} & \mathbf{0} & \mathbf{0} & \mathbf{0} & \mathbf{0} & \mathbf{0} & \mathbf{0} & \mathbf{0}\end{array}$


Table A-17: Regional Capacity Reserves

PNW Loads and Resources Study

2012 - 2013 Operating Year

1937 Water Year

2003 White Book

Oct Nov

Dec Jan Fe

Feb

Mar Apr1 Apr16 May Jun Jul Avg

Hydro Reserves

1 BPA - Power Business

2 Total Federal Entities

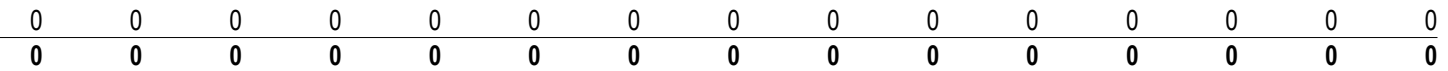

-Generating Public Entities-

3 Chelan County PUD \#1

4 Clark County PUD \#1

5 Cowlitz County PUD \#1

6 Douglas County PUD \#1

7 Eugene Water \& Electric Board

8 Grant County PUD \#2

9 Grays Harbor PUD \#1

10 Okanogan County PUD \#1

11 Pend Oreille County PUD \#1

12 Seattle City Light

13 Snohomish County PUD \#1

14 Tacoma Public Utilities

15 Total Generating Public Entities

$\begin{array}{lllllllllllllll}0 & 0 & 0 & 0 & 0 & 0 & 0 & 0 & 0 & 0 & 0 & 0 & 0 & 0 & 0 \\ 0 & 0 & 0 & 0 & 0 & 0 & 0 & 0 & 0 & 0 & 0 & 0 & 0 & 0 & 0 \\ 0 & 0 & 0 & 0 & 0 & 0 & 0 & 0 & 0 & 0 & 0 & 0 & 0 & 0 & 0 \\ 0 & 0 & 0 & 0 & 0 & 0 & 0 & 0 & 0 & 0 & 0 & 0 & 0 & 0 & 0 \\ 0 & 0 & 0 & 0 & 0 & 0 & 0 & 0 & 0 & 0 & 0 & 0 & 0 & 0 & 0 \\ 0 & 0 & 0 & 0 & 0 & 0 & 0 & 0 & 0 & 0 & 0 & 0 & 0 & 0 & 0 \\ 0 & 0 & 0 & 0 & 0 & 0 & 0 & 0 & 0 & 0 & 0 & 0 & 0 & 0 & 0 \\ 0 & 0 & 0 & 0 & 0 & 0 & 0 & 0 & 0 & 0 & 0 & 0 & 0 & 0 & 0 \\ 0 & 0 & 0 & 0 & 0 & 0 & 0 & 0 & 0 & 0 & 0 & 0 & 0 & 0 & 0 \\ 0 & 0 & 0 & 0 & 0 & 0 & 0 & 0 & 0 & 0 & 0 & 0 & 0 & 0 & 0 \\ 0 & 0 & 0 & 0 & 0 & 0 & 0 & 0 & 0 & 0 & 0 & 0 & 0 & 0 & 0 \\ 0 & 0 & 0 & 0 & 0 & 0 & 0 & 0 & 0 & 0 & 0 & 0 & 0 & 0 & 0 \\ 0 & 0 & \mathbf{0} & \mathbf{0} & \mathbf{0} & \mathbf{0} & \mathbf{0} & \mathbf{0} & \mathbf{0} & \mathbf{0} & \mathbf{0} & \mathbf{0} & \mathbf{0} & \mathbf{0} & \mathbf{0}\end{array}$

-Non-Generating Public Entities-

16 Ashland, City of

17 Benton County PUD \#1

18 Bonners Ferry, City of

19 Centralia, City of

20 Clallam County PUD \#1

21 Clearwater Power

22 Consumers Power

23 Emerald County PUD

24 Energy Northwest

25 Fall River Elec Coop

26 Forest Grove, City of

27 Franklin County PUD \#1

28 Idaho County L \& P

29 Idaho Falls, City of

30 Kittitas County PUD \#1

31 Klickitat County PUD \#1

32 Kootenai Electric Coop

33 Lewis County PUD \#1

34 Lost River Elec Coop

35 Lower Valley Energy

36 Mason County PUD \#1

37 McMinnville, City of

38 Milton Freewater, City of

39 Northern Lights

40 Northern Wasco County PUD

41 Oregon Trail Coop

42 Port Angeles, City of

43 Raft River Elec Coop

44 Salmon River Elec Coop

45 Soda Springs, City of

46 Tanner Elec Coop

47 Tillamook PUD \#1

48 United Electric Coop

49 Total Non-Generating Public Entities

\begin{tabular}{|c|c|c|c|c|c|c|c|c|c|c|c|c|c|}
\hline 0 & 0 & 0 & 0 & 0 & 0 & 0 & 0 & 0 & 0 & 0 & 0 & 0 & 0 \\
\hline 0 & 0 & 0 & 0 & 0 & 0 & 0 & 0 & 0 & 0 & 0 & 0 & 0 & 0 \\
\hline 0 & 0 & 0 & 0 & 0 & 0 & 0 & 0 & 0 & 0 & 0 & 0 & 0 & 0 \\
\hline 0 & 0 & 0 & 0 & 0 & 0 & 0 & 0 & 0 & 0 & 0 & 0 & 0 & 0 \\
\hline 0 & 0 & 0 & 0 & 0 & 0 & 0 & 0 & 0 & 0 & 0 & 0 & 0 & 0 \\
\hline 0 & 0 & 0 & 0 & 0 & 0 & 0 & 0 & 0 & 0 & 0 & 0 & 0 & 0 \\
\hline 0 & 0 & 0 & 0 & 0 & 0 & 0 & 0 & 0 & 0 & 0 & 0 & 0 & 0 \\
\hline 0 & 0 & 0 & 0 & 0 & 0 & 0 & 0 & 0 & 0 & 0 & 0 & 0 & 0 \\
\hline 0 & 0 & 0 & 0 & 0 & 0 & 0 & 0 & 0 & 0 & 0 & 0 & 0 & 0 \\
\hline 0 & 0 & 0 & 0 & 0 & 0 & 0 & 0 & 0 & 0 & 0 & 0 & 0 & 0 \\
\hline 0 & 0 & 0 & 0 & 0 & 0 & 0 & 0 & 0 & 0 & 0 & 0 & 0 & 0 \\
\hline 0 & 0 & 0 & 0 & 0 & 0 & 0 & 0 & 0 & 0 & 0 & 0 & 0 & 0 \\
\hline 0 & 0 & 0 & 0 & 0 & 0 & 0 & 0 & 0 & 0 & 0 & 0 & 0 & 0 \\
\hline 0 & 0 & 0 & 0 & 0 & 0 & 0 & 0 & 0 & 0 & 0 & 0 & 0 & 0 \\
\hline 0 & 0 & 0 & 0 & 0 & 0 & 0 & 0 & 0 & 0 & 0 & 0 & 0 & 0 \\
\hline 0 & 0 & 0 & 0 & 0 & 0 & 0 & 0 & 0 & 0 & 0 & 0 & 0 & 0 \\
\hline 0 & 0 & 0 & 0 & 0 & 0 & 0 & 0 & 0 & 0 & 0 & 0 & 0 & 0 \\
\hline 0 & 0 & 0 & 0 & 0 & 0 & 0 & 0 & 0 & 0 & 0 & 0 & 0 & 0 \\
\hline 0 & 0 & 0 & 0 & 0 & 0 & 0 & 0 & 0 & 0 & 0 & 0 & 0 & 0 \\
\hline 0 & 0 & 0 & 0 & 0 & 0 & 0 & 0 & 0 & 0 & 0 & 0 & 0 & 0 \\
\hline 0 & 0 & 0 & 0 & 0 & 0 & 0 & 0 & 0 & 0 & 0 & 0 & 0 & 0 \\
\hline 0 & 0 & 0 & 0 & 0 & 0 & 0 & 0 & 0 & 0 & 0 & 0 & 0 & 0 \\
\hline 0 & 0 & 0 & 0 & 0 & 0 & 0 & 0 & 0 & 0 & 0 & 0 & 0 & 0 \\
\hline 0 & 0 & 0 & 0 & 0 & 0 & 0 & 0 & 0 & 0 & 0 & 0 & 0 & 0 \\
\hline 0 & 0 & 0 & 0 & 0 & 0 & 0 & 0 & 0 & 0 & 0 & 0 & 0 & 0 \\
\hline 0 & 0 & 0 & 0 & 0 & 0 & 0 & 0 & 0 & 0 & 0 & 0 & 0 & 0 \\
\hline 0 & 0 & 0 & 0 & 0 & 0 & 0 & 0 & 0 & 0 & 0 & 0 & 0 & 0 \\
\hline 0 & 0 & 0 & 0 & 0 & 0 & 0 & 0 & 0 & 0 & 0 & 0 & 0 & 0 \\
\hline 0 & 0 & 0 & 0 & 0 & 0 & 0 & 0 & 0 & 0 & 0 & 0 & 0 & 0 \\
\hline 0 & 0 & 0 & 0 & 0 & 0 & 0 & 0 & 0 & 0 & 0 & 0 & 0 & 0 \\
\hline 0 & 0 & 0 & 0 & 0 & 0 & 0 & 0 & 0 & 0 & 0 & 0 & 0 & 0 \\
\hline 0 & 0 & 0 & 0 & 0 & 0 & 0 & 0 & 0 & 0 & 0 & 0 & 0 & 0 \\
\hline 0 & 0 & 0 & 0 & 0 & 0 & 0 & 0 & 0 & 0 & 0 & 0 & 0 & 0 \\
\hline 0 & 0 & 0 & 0 & 0 & 0 & 0 & 0 & 0 & 0 & 0 & 0 & 0 & 0 \\
\hline
\end{tabular}

-Investor-Owned Entities-

50 Avista Corp (WWP Division)

51 Idaho Power Company

52 Northwestern Energy LLC (MPC)

53 Pacific Power

54 Portland General Electric

55 Puget Sound Energy

56 Total Investor-Owned Entities

-Other Entities-

57 Colockum Transmission Company

58 Longview Fibre

59 Warm Springs Power Enterprise

60 Total Other Entities

$\begin{array}{lllllllllllllll}0 & 0 & 0 & 0 & 0 & 0 & 0 & 0 & 0 & 0 & 0 & 0 & 0 & 0 & 0 \\ 0 & 0 & 0 & 0 & 0 & 0 & 0 & 0 & 0 & 0 & 0 & 0 & 0 & 0 & 0 \\ 0 & 0 & 0 & 0 & 0 & 0 & 0 & 0 & 0 & 0 & 0 & 0 & 0 & 0 & 0 \\ 0 & 0 & 0 & 0 & 0 & 0 & 0 & 0 & 0 & 0 & 0 & 0 & 0 & 0 & 0 \\ 0 & 0 & 0 & 0 & 0 & 0 & 0 & 0 & 0 & 0 & 0 & 0 & 0 & 0 & 0 \\ 0 & 0 & 0 & 0 & 0 & 0 & 0 & 0 & 0 & 0 & 0 & 0 & 0 & 0 & 0 \\ 0 & \mathbf{0} & \mathbf{0} & \mathbf{0} & \mathbf{0} & \mathbf{0} & \mathbf{0} & \mathbf{0} & \mathbf{0} & \mathbf{0} & \mathbf{0} & \mathbf{0} & \mathbf{0} & \mathbf{0} & \mathbf{0}\end{array}$

Small Thermal \& Misc. Reserves

-Federal Entities-

61 BPA - Power Business

62 U.S. Naval Station, Everett (Jim Creek)

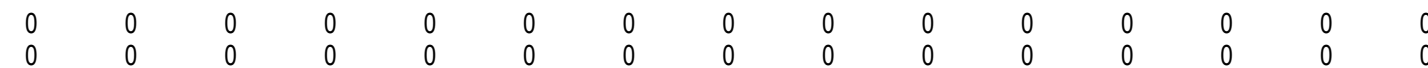




$\begin{array}{lllllllllllllll}0 & 0 & 0 & 0 & 0 & 0 & 0 & 0 & 0 & 0 & 0 & 0 & 0 & 0 & 0 \\ 0 & 0 & 0 & 0 & 0 & 0 & 0 & 0 & 0 & 0 & 0 & 0 & 0 & 0 & 0\end{array}$

-Generating Public Entities-

65 Chelan County PUD \#1

66 Clark County PUD \#1

67 Cowlitz County PUD \#1

68 Douglas County PUD \#1

69 Eugene Water \& Electric Board

70 Grant County PUD \#2

71 Grays Harbor PUD \#1

72 Okanogan County PUD \#1

73 Seattle City Light

74 Snohomish County PUD \#1

75 Springfield Utility Board

76 Tacoma Public Utilities

77 Total Generating Public Entities

$\begin{array}{lllllllllllllll}0 & 0 & 0 & 0 & 0 & 0 & 0 & 0 & 0 & 0 & 0 & 0 & 0 & 0 & 0 \\ 0 & 0 & 0 & 0 & 0 & 0 & 0 & 0 & 0 & 0 & 0 & 0 & 0 & 0 & 0 \\ 0 & 0 & 0 & 0 & 0 & 0 & 0 & 0 & 0 & 0 & 0 & 0 & 0 & 0 & 0 \\ 0 & 0 & 0 & 0 & 0 & 0 & 0 & 0 & 0 & 0 & 0 & 0 & 0 & 0 & 0 \\ 0 & 0 & 0 & 0 & 0 & 0 & 0 & 0 & 0 & 0 & 0 & 0 & 0 & 0 & 0 \\ 0 & 0 & 0 & 0 & 0 & 0 & 0 & 0 & 0 & 0 & 0 & 0 & 0 & 0 & 0 \\ 0 & 0 & 0 & 0 & 0 & 0 & 0 & 0 & 0 & 0 & 0 & 0 & 0 & 0 & 0 \\ 0 & 0 & 0 & 0 & 0 & 0 & 0 & 0 & 0 & 0 & 0 & 0 & 0 & 0 & 0 \\ 0 & 0 & 0 & 0 & 0 & 0 & 0 & 0 & 0 & 0 & 0 & 0 & 0 & 0 & 0 \\ 0 & 0 & 0 & 0 & 0 & 0 & 0 & 0 & 0 & 0 & 0 & 0 & 0 & 0 & 0 \\ 0 & 0 & 0 & 0 & 0 & 0 & 0 & 0 & 0 & 0 & 0 & 0 & 0 & 0 & 0 \\ 0 & 0 & 0 & 0 & 0 & 0 & 0 & 0 & 0 & 0 & 0 & 0 & 0 & 0 \\ 0 & 0 & \mathbf{0} & \mathbf{0} & \mathbf{0} & \mathbf{0} & \mathbf{0} & \mathbf{0} & \mathbf{0} & \mathbf{0} & \mathbf{0} & \mathbf{0} & \mathbf{0} & \mathbf{0} & \mathbf{0}\end{array}$

-Non-Generating Public Entities-

78 Ashland, City of

79 Benton County PUD \#1

80 Clallam County PUD \#1

81 Clatskanie PUD

82 Consumers Power

83 Emerald County PUD

84 Energy Northwest

85 Fall River Elec Coop

86 Franklin County PUD \#1

87 Klickitat County PUD \#1

88 Lewis County PUD \#1

89 Mason County PUD \#1

90 Mason County PUD \#3

91 Oregon Trail Coop

92 Tanner Elec Coop

93 Tillamook PUD \#1

94 Total Non-Generating Public Entities

$\begin{array}{lllllllllllllll}0 & 0 & 0 & 0 & 0 & 0 & 0 & 0 & 0 & 0 & 0 & 0 & 0 & 0 & 0 \\ 0 & 0 & 0 & 0 & 0 & 0 & 0 & 0 & 0 & 0 & 0 & 0 & 0 & 0 & 0 \\ 0 & 0 & 0 & 0 & 0 & 0 & 0 & 0 & 0 & 0 & 0 & 0 & 0 & 0 & 0 \\ 0 & 0 & 0 & 0 & 0 & 0 & 0 & 0 & 0 & 0 & 0 & 0 & 0 & 0 & 0 \\ 0 & 0 & 0 & 0 & 0 & 0 & 0 & 0 & 0 & 0 & 0 & 0 & 0 & 0 & 0 \\ 0 & 0 & 0 & 0 & 0 & 0 & 0 & 0 & 0 & 0 & 0 & 0 & 0 & 0 & 0 \\ 0 & 0 & 0 & 0 & 0 & 0 & 0 & 0 & 0 & 0 & 0 & 0 & 0 & 0 & 0 \\ 0 & 0 & 0 & 0 & 0 & 0 & 0 & 0 & 0 & 0 & 0 & 0 & 0 & 0 & 0 \\ 0 & 0 & 0 & 0 & 0 & 0 & 0 & 0 & 0 & 0 & 0 & 0 & 0 & 0 & 0 \\ 0 & 0 & 0 & 0 & 0 & 0 & 0 & 0 & 0 & 0 & 0 & 0 & 0 & 0 & 0 \\ 0 & 0 & 0 & 0 & 0 & 0 & 0 & 0 & 0 & 0 & 0 & 0 & 0 & 0 & 0 \\ 0 & 0 & 0 & 0 & 0 & 0 & 0 & 0 & 0 & 0 & 0 & 0 & 0 & 0 & 0 \\ 0 & 0 & 0 & 0 & 0 & 0 & 0 & 0 & 0 & 0 & 0 & 0 & 0 & 0 & 0 \\ 0 & 0 & 0 & 0 & 0 & 0 & 0 & 0 & 0 & 0 & 0 & 0 & 0 & 0 & 0 \\ 0 & 0 & 0 & 0 & 0 & 0 & 0 & 0 & 0 & 0 & 0 & 0 & 0 & 0 & 0 \\ 0 & 0 & 0 & 0 & 0 & 0 & 0 & 0 & 0 & 0 & 0 & 0 & 0 & 0 & 0 \\ 0 & \mathbf{0} & \mathbf{0} & \mathbf{0} & \mathbf{0} & \mathbf{0} & \mathbf{0} & \mathbf{0} & \mathbf{0} & \mathbf{0} & \mathbf{0} & \mathbf{0} & \mathbf{0} & \mathbf{0} & \mathbf{0}\end{array}$

-Investor-Owned Entities-

95 Avista Corp (WWP Division)

96 Idaho Power Company

97 Northwestern Energy LLC (MPC)

98 Pacific Power

99 Portland General Electric

100 Puget Sound Energy

101 Trans Alta Utilities Corp

102 Utah Power

103 Total Investor-Owned Entities

\begin{tabular}{lllllllllllllll}
0 & 0 & 0 & 0 & 0 & 0 & 0 & 0 & 0 & 0 & 0 & 0 & 0 & 0 & 0 \\
0 & 0 & 0 & 0 & 0 & 0 & 0 & 0 & 0 & 0 & 0 & 0 & 0 & 0 & 0 \\
0 & 0 & 0 & 0 & 0 & 0 & 0 & 0 & 0 & 0 & 0 & 0 & 0 & 0 & 0 \\
0 & 0 & 0 & 0 & 0 & 0 & 0 & 0 & 0 & 0 & 0 & 0 & 0 & 0 & 0 \\
0 & 0 & 0 & 0 & 0 & 0 & 0 & 0 & 0 & 0 & 0 & 0 & 0 & 0 & 0 \\
0 & 0 & 0 & 0 & 0 & 0 & 0 & 0 & 0 & 0 & 0 & 0 & 0 & 0 & 0 \\
0 & 0 & 0 & 0 & 0 & 0 & 0 & 0 & 0 & 0 & 0 & 0 & 0 & 0 & 0 \\
0 & 0 & 0 & 0 & 0 & 0 & 0 & 0 & 0 & 0 & 0 & 0 & 0 & 0 & 0 \\
\hline $\mathbf{0}$ & $\mathbf{0}$ & $\mathbf{0}$ & $\mathbf{0}$ & $\mathbf{0}$ & $\mathbf{0}$ & $\mathbf{0}$ & $\mathbf{0}$ & $\mathbf{0}$ & $\mathbf{0}$ & $\mathbf{0}$ & $\mathbf{0}$ & $\mathbf{0}$ & $\mathbf{0}$ & $\mathbf{0}$
\end{tabular}

-Other Entities-

104 Avista Energy Inc.

105 Chehalis Power Inc.

106 FPL Energy Vansycle LLC

107 Frederickson Power LP,

108 Hermiston Power Partnership

109 Klamath Pacific Energy

110 Longview Fibre

111 Mirant Americas Energy Marketing, L.P.

112 PPM Energy

113 SP Newsprint

114 Total Other Entities

Large Thermal Reserves

-Federal Entities-

115 BPA - Power Business 116 Total Federal Entities

$\begin{array}{lllllllllllllll}0 & 0 & 0 & 0 & 0 & 0 & 0 & 0 & 0 & 0 & 0 & 0 & 0 & 0 & 0 \\ 0 & 0 & 0 & 0 & 0 & 0 & 0 & 0 & 0 & 0 & 0 & 0 & 0 & 0 & 0 \\ 0 & 0 & 0 & 0 & 0 & 0 & 0 & 0 & 0 & 0 & 0 & 0 & 0 & 0 & 0 \\ 0 & 0 & 0 & 0 & 0 & 0 & 0 & 0 & 0 & 0 & 0 & 0 & 0 & 0 & 0 \\ 0 & 0 & 0 & 0 & 0 & 0 & 0 & 0 & 0 & 0 & 0 & 0 & 0 & 0 & 0 \\ 0 & 0 & 0 & 0 & 0 & 0 & 0 & 0 & 0 & 0 & 0 & 0 & 0 & 0 & 0 \\ 0 & 0 & 0 & 0 & 0 & 0 & 0 & 0 & 0 & 0 & 0 & 0 & 0 & 0 & 0 \\ 0 & 0 & 0 & 0 & 0 & 0 & 0 & 0 & 0 & 0 & 0 & 0 & 0 & 0 & 0 \\ 0 & 0 & 0 & 0 & 0 & 0 & 0 & 0 & 0 & 0 & 0 & 0 & 0 & 0 & 0 \\ 0 & 0 & 0 & 0 & 0 & 0 & 0 & 0 & 0 & 0 & 0 & 0 & 0 & 0 & 0 \\ 0 & \mathbf{0} & \mathbf{0} & \mathbf{0} & \mathbf{0} & \mathbf{0} & \mathbf{0} & \mathbf{0} & \mathbf{0} & \mathbf{0} & \mathbf{0} & \mathbf{0} & \mathbf{0} & \mathbf{0} & \mathbf{0}\end{array}$

-Generating Public Entities-

117 Turlock Irrigation District

118 Total Generating Public Entities

-Non-Generating Public Entities-

119 Total Non-Generating Public Entities 
Average Energy in Megawatts

120 Avista Corp (WWP Division)

121 Idaho Power Company

122 Northwestern Energy LLC (MPC)

123 Pacific Power

124 Portland General Electric

125 Puget Sound Energy

126 San Diego Gas \& Electric

127 Trans Alta Utilities Corp

128 Total Investor-Owned Entities

\section{Water Year}

2003 White Book

-Other Entities-

129 Total Other Entities

\section{Aug1 Aug16}

Sep

Oct Nov Dec Jan Feb

Mar Apr1 Apr1

May Jun Jul Avg

Contract Reserves

-Federal Entities-

130 Total Federal Entities

$\begin{array}{llll}0 & 0 & 0 & 0 \\ 0 & 0 & 0 & 0 \\ 0 & 0 & 0 & 0 \\ 0 & 0 & 0 & 0 \\ 0 & 0 & 0 & 0 \\ 0 & 0 & 0 & 0 \\ 0 & 0 & 0 & 0 \\ 0 & 0 & 0 & 0 \\ 0 & 0 & 0 & 0\end{array}$

$\begin{array}{ll}0 & 0 \\ 0 & 0 \\ 0 & 0 \\ 0 & 0 \\ 0 & 0 \\ 0 & 0 \\ 0 & 0 \\ 0 & 0 \\ 0 & 0\end{array}$

$\begin{array}{lll}0 & 0 & 0 \\ 0 & 0 & 0 \\ 0 & 0 & 0 \\ 0 & 0 & 0 \\ 0 & 0 & 0 \\ 0 & 0 & \\ 0 & 0 & \\ 0 & 0 & 0 \\ 0 & 0\end{array}$

0
0
0
0
0
0
0
0
0

$\begin{array}{ll}0 & 0 \\ 0 & 0 \\ 0 & 0 \\ 0 & 0 \\ 0 & 0 \\ 0 & \\ 0 & \\ 0 & \\ 0 & \end{array}$

$\begin{array}{lllllll}0 & 0 & 0 & 0 & 0 & 0 & 0 \\ 0 & 0 & 0 & 0 & 0 & 0 & 0 \\ 0 & 0 & 0 & 0 & 0 & 0 & 0 \\ 0 & 0 & 0 & 0 & 0 & 0 & 0 \\ 0 & 0 & 0 & 0 & 0 & 0 & 0 \\ 0 & 0 & 0 & 0 & 0 & 0 & 0 \\ 0 & 0 & 0 & 0 & 0 & 0 & 0 \\ 0 & 0 & 0 & 0 & 0 & 0 & 0 \\ 0 & 0 & 0 & 0 & 0 & 0 & 0\end{array}$

-Generating Public Entities-

131 Total Generating Public Entities

$0 \quad 0$

0

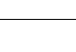

-Non-Generating Public Entities-

132 Total Non-Generating Public Entities

-Investor-Owned Entities-

133 Northwestern Energy LLC (MPC)

134 Total Investor-Owned Entities

$\begin{array}{llllllllllllll}0 & 0 & 0 & 0 & 0 & 0 & 0 & 0 & 0 & 0 & 0 & 0 & 0 & 0\end{array}$

-Other Entities-

135 Total Other Entities

$0 \quad 0$

-Total Capacity Reserves-

136 Total Hydro Reserves

137 Total Small Thermal \& Misc Reserves

138 Total Large Thermal Reserves

139 Total Contract Reserves

140 Total Capacity Reserves

$\begin{array}{lllllllllllllll}0 & 0 & 0 & 0 & 0 & 0 & 0 & 0 & 0 & 0 & 0 & 0 & 0 & 0 & 0 \\ 0 & 0 & 0 & 0 & 0 & 0 & 0 & 0 & 0 & 0 & 0 & 0 & 0 & 0 & 0 \\ 0 & 0 & 0 & 0 & 0 & 0 & 0 & 0 & 0 & 0 & 0 & 0 & 0 & 0 & 0 \\ 0 & 0 & 0 & 0 & 0 & 0 & 0 & 0 & 0 & 0 & 0 & 0 & 0 & 0 \\ 0 & 0 & 0 & 0 & 0 & 0 & 0 & 0 & 0 & 0 & 0 & 0 & 0 & 0\end{array}$


Table A-17: Regional Capacity Reserves

PNW Loads and Resources Study

2013 - 2014 Operating Year

1937 Water Year

2003 White Book

Oct Nov

Dec Jan Fe

Feb

Mar Apr1 Apr16 May Jun Jul Avg

Hydro Reserves

1 BPA - Power Business

2 Total Federal Entities

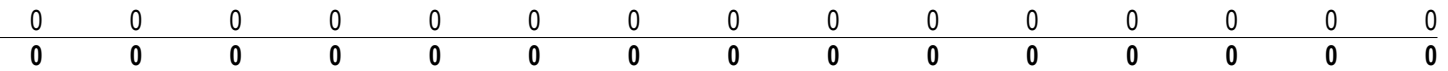

-Generating Public Entities-

3 Chelan County PUD \#1

4 Clark County PUD \#1

5 Cowlitz County PUD \#1

6 Douglas County PUD \#1

7 Eugene Water \& Electric Board

8 Grant County PUD \#2

9 Grays Harbor PUD \#1

10 Okanogan County PUD \#1

11 Pend Oreille County PUD \#1

12 Seattle City Light

13 Snohomish County PUD \#1

14 Tacoma Public Utilities

15 Total Generating Public Entities

$\begin{array}{lllllllllllllll}0 & 0 & 0 & 0 & 0 & 0 & 0 & 0 & 0 & 0 & 0 & 0 & 0 & 0 & 0 \\ 0 & 0 & 0 & 0 & 0 & 0 & 0 & 0 & 0 & 0 & 0 & 0 & 0 & 0 & 0 \\ 0 & 0 & 0 & 0 & 0 & 0 & 0 & 0 & 0 & 0 & 0 & 0 & 0 & 0 & 0 \\ 0 & 0 & 0 & 0 & 0 & 0 & 0 & 0 & 0 & 0 & 0 & 0 & 0 & 0 & 0 \\ 0 & 0 & 0 & 0 & 0 & 0 & 0 & 0 & 0 & 0 & 0 & 0 & 0 & 0 & 0 \\ 0 & 0 & 0 & 0 & 0 & 0 & 0 & 0 & 0 & 0 & 0 & 0 & 0 & 0 & 0 \\ 0 & 0 & 0 & 0 & 0 & 0 & 0 & 0 & 0 & 0 & 0 & 0 & 0 & 0 & 0 \\ 0 & 0 & 0 & 0 & 0 & 0 & 0 & 0 & 0 & 0 & 0 & 0 & 0 & 0 & 0 \\ 0 & 0 & 0 & 0 & 0 & 0 & 0 & 0 & 0 & 0 & 0 & 0 & 0 & 0 & 0 \\ 0 & 0 & 0 & 0 & 0 & 0 & 0 & 0 & 0 & 0 & 0 & 0 & 0 & 0 & 0 \\ 0 & 0 & 0 & 0 & 0 & 0 & 0 & 0 & 0 & 0 & 0 & 0 & 0 & 0 & 0 \\ 0 & 0 & 0 & 0 & 0 & 0 & 0 & 0 & 0 & 0 & 0 & 0 & 0 & 0 & 0 \\ 0 & 0 & \mathbf{0} & \mathbf{0} & \mathbf{0} & \mathbf{0} & \mathbf{0} & \mathbf{0} & \mathbf{0} & \mathbf{0} & \mathbf{0} & \mathbf{0} & \mathbf{0} & \mathbf{0} & \mathbf{0}\end{array}$

-Non-Generating Public Entities-

16 Ashland, City of

17 Benton County PUD \#1

18 Bonners Ferry, City of

19 Centralia, City of

20 Clallam County PUD \#1

21 Clearwater Power

22 Consumers Power

23 Emerald County PUD

24 Energy Northwest

25 Fall River Elec Coop

26 Forest Grove, City of

27 Franklin County PUD \#1

28 Idaho County L \& P

29 Idaho Falls, City of

30 Kittitas County PUD \#1

31 Klickitat County PUD \#1

32 Kootenai Electric Coop

33 Lewis County PUD \#1

34 Lost River Elec Coop

35 Lower Valley Energy

36 Mason County PUD \#1

37 McMinnville, City of

38 Milton Freewater, City of

39 Northern Lights

40 Northern Wasco County PUD

41 Oregon Trail Coop

42 Port Angeles, City of

43 Raft River Elec Coop

44 Salmon River Elec Coop

45 Soda Springs, City of

46 Tanner Elec Coop

47 Tillamook PUD \#1

48 United Electric Coop

49 Total Non-Generating Public Entities

\begin{tabular}{|c|c|c|c|c|c|c|c|c|c|c|c|c|c|}
\hline 0 & 0 & 0 & 0 & 0 & 0 & 0 & 0 & 0 & 0 & 0 & 0 & 0 & 0 \\
\hline 0 & 0 & 0 & 0 & 0 & 0 & 0 & 0 & 0 & 0 & 0 & 0 & 0 & 0 \\
\hline 0 & 0 & 0 & 0 & 0 & 0 & 0 & 0 & 0 & 0 & 0 & 0 & 0 & 0 \\
\hline 0 & 0 & 0 & 0 & 0 & 0 & 0 & 0 & 0 & 0 & 0 & 0 & 0 & 0 \\
\hline 0 & 0 & 0 & 0 & 0 & 0 & 0 & 0 & 0 & 0 & 0 & 0 & 0 & 0 \\
\hline 0 & 0 & 0 & 0 & 0 & 0 & 0 & 0 & 0 & 0 & 0 & 0 & 0 & 0 \\
\hline 0 & 0 & 0 & 0 & 0 & 0 & 0 & 0 & 0 & 0 & 0 & 0 & 0 & 0 \\
\hline 0 & 0 & 0 & 0 & 0 & 0 & 0 & 0 & 0 & 0 & 0 & 0 & 0 & 0 \\
\hline 0 & 0 & 0 & 0 & 0 & 0 & 0 & 0 & 0 & 0 & 0 & 0 & 0 & 0 \\
\hline 0 & 0 & 0 & 0 & 0 & 0 & 0 & 0 & 0 & 0 & 0 & 0 & 0 & 0 \\
\hline 0 & 0 & 0 & 0 & 0 & 0 & 0 & 0 & 0 & 0 & 0 & 0 & 0 & 0 \\
\hline 0 & 0 & 0 & 0 & 0 & 0 & 0 & 0 & 0 & 0 & 0 & 0 & 0 & 0 \\
\hline 0 & 0 & 0 & 0 & 0 & 0 & 0 & 0 & 0 & 0 & 0 & 0 & 0 & 0 \\
\hline 0 & 0 & 0 & 0 & 0 & 0 & 0 & 0 & 0 & 0 & 0 & 0 & 0 & 0 \\
\hline 0 & 0 & 0 & 0 & 0 & 0 & 0 & 0 & 0 & 0 & 0 & 0 & 0 & 0 \\
\hline 0 & 0 & 0 & 0 & 0 & 0 & 0 & 0 & 0 & 0 & 0 & 0 & 0 & 0 \\
\hline 0 & 0 & 0 & 0 & 0 & 0 & 0 & 0 & 0 & 0 & 0 & 0 & 0 & 0 \\
\hline 0 & 0 & 0 & 0 & 0 & 0 & 0 & 0 & 0 & 0 & 0 & 0 & 0 & 0 \\
\hline 0 & 0 & 0 & 0 & 0 & 0 & 0 & 0 & 0 & 0 & 0 & 0 & 0 & 0 \\
\hline 0 & 0 & 0 & 0 & 0 & 0 & 0 & 0 & 0 & 0 & 0 & 0 & 0 & 0 \\
\hline 0 & 0 & 0 & 0 & 0 & 0 & 0 & 0 & 0 & 0 & 0 & 0 & 0 & 0 \\
\hline 0 & 0 & 0 & 0 & 0 & 0 & 0 & 0 & 0 & 0 & 0 & 0 & 0 & 0 \\
\hline 0 & 0 & 0 & 0 & 0 & 0 & 0 & 0 & 0 & 0 & 0 & 0 & 0 & 0 \\
\hline 0 & 0 & 0 & 0 & 0 & 0 & 0 & 0 & 0 & 0 & 0 & 0 & 0 & 0 \\
\hline 0 & 0 & 0 & 0 & 0 & 0 & 0 & 0 & 0 & 0 & 0 & 0 & 0 & 0 \\
\hline 0 & 0 & 0 & 0 & 0 & 0 & 0 & 0 & 0 & 0 & 0 & 0 & 0 & 0 \\
\hline 0 & 0 & 0 & 0 & 0 & 0 & 0 & 0 & 0 & 0 & 0 & 0 & 0 & 0 \\
\hline 0 & 0 & 0 & 0 & 0 & 0 & 0 & 0 & 0 & 0 & 0 & 0 & 0 & 0 \\
\hline 0 & 0 & 0 & 0 & 0 & 0 & 0 & 0 & 0 & 0 & 0 & 0 & 0 & 0 \\
\hline 0 & 0 & 0 & 0 & 0 & 0 & 0 & 0 & 0 & 0 & 0 & 0 & 0 & 0 \\
\hline 0 & 0 & 0 & 0 & 0 & 0 & 0 & 0 & 0 & 0 & 0 & 0 & 0 & 0 \\
\hline 0 & 0 & 0 & 0 & 0 & 0 & 0 & 0 & 0 & 0 & 0 & 0 & 0 & 0 \\
\hline 0 & 0 & 0 & 0 & 0 & 0 & 0 & 0 & 0 & 0 & 0 & 0 & 0 & 0 \\
\hline 0 & 0 & 0 & 0 & 0 & 0 & 0 & 0 & 0 & 0 & 0 & 0 & 0 & 0 \\
\hline
\end{tabular}

-Investor-Owned Entities-

50 Avista Corp (WWP Division)

51 Idaho Power Company

52 Northwestern Energy LLC (MPC)

53 Pacific Power

54 Portland General Electric

55 Puget Sound Energy

56 Total Investor-Owned Entities

-Other Entities-

57 Colockum Transmission Company

58 Longview Fibre

59 Warm Springs Power Enterprise

60 Total Other Entities

$\begin{array}{lllllllllllllll}0 & 0 & 0 & 0 & 0 & 0 & 0 & 0 & 0 & 0 & 0 & 0 & 0 & 0 & 0 \\ 0 & 0 & 0 & 0 & 0 & 0 & 0 & 0 & 0 & 0 & 0 & 0 & 0 & 0 & 0 \\ 0 & 0 & 0 & 0 & 0 & 0 & 0 & 0 & 0 & 0 & 0 & 0 & 0 & 0 & 0 \\ 0 & 0 & 0 & 0 & 0 & 0 & 0 & 0 & 0 & 0 & 0 & 0 & 0 & 0 & 0 \\ 0 & 0 & 0 & 0 & 0 & 0 & 0 & 0 & 0 & 0 & 0 & 0 & 0 & 0 & 0 \\ 0 & 0 & 0 & 0 & 0 & 0 & 0 & 0 & 0 & 0 & 0 & 0 & 0 & 0 & 0 \\ 0 & \mathbf{0} & \mathbf{0} & \mathbf{0} & \mathbf{0} & \mathbf{0} & \mathbf{0} & \mathbf{0} & \mathbf{0} & \mathbf{0} & \mathbf{0} & \mathbf{0} & \mathbf{0} & \mathbf{0} & \mathbf{0}\end{array}$

Small Thermal \& Misc. Reserves

-Federal Entities-

61 BPA - Power Business

62 U.S. Naval Station, Everett (Jim Creek)

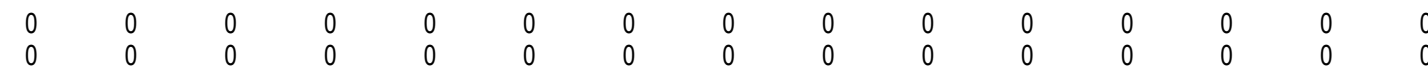




$\begin{array}{lllllllllllllll}0 & 0 & 0 & 0 & 0 & 0 & 0 & 0 & 0 & 0 & 0 & 0 & 0 & 0 & 0 \\ 0 & 0 & 0 & 0 & 0 & 0 & 0 & 0 & 0 & 0 & 0 & 0 & 0 & 0 & 0\end{array}$

-Generating Public Entities-

65 Chelan County PUD \#1

66 Clark County PUD \#1

67 Cowlitz County PUD \#1

68 Douglas County PUD \#1

69 Eugene Water \& Electric Board

70 Grant County PUD \#2

71 Grays Harbor PUD \#1

72 Okanogan County PUD \#1

73 Seattle City Light

74 Snohomish County PUD \#1

75 Springfield Utility Board

76 Tacoma Public Utilities

77 Total Generating Public Entities

$\begin{array}{lllllllllllllll}0 & 0 & 0 & 0 & 0 & 0 & 0 & 0 & 0 & 0 & 0 & 0 & 0 & 0 & 0 \\ 0 & 0 & 0 & 0 & 0 & 0 & 0 & 0 & 0 & 0 & 0 & 0 & 0 & 0 & 0 \\ 0 & 0 & 0 & 0 & 0 & 0 & 0 & 0 & 0 & 0 & 0 & 0 & 0 & 0 & 0 \\ 0 & 0 & 0 & 0 & 0 & 0 & 0 & 0 & 0 & 0 & 0 & 0 & 0 & 0 & 0 \\ 0 & 0 & 0 & 0 & 0 & 0 & 0 & 0 & 0 & 0 & 0 & 0 & 0 & 0 & 0 \\ 0 & 0 & 0 & 0 & 0 & 0 & 0 & 0 & 0 & 0 & 0 & 0 & 0 & 0 & 0 \\ 0 & 0 & 0 & 0 & 0 & 0 & 0 & 0 & 0 & 0 & 0 & 0 & 0 & 0 & 0 \\ 0 & 0 & 0 & 0 & 0 & 0 & 0 & 0 & 0 & 0 & 0 & 0 & 0 & 0 & 0 \\ 0 & 0 & 0 & 0 & 0 & 0 & 0 & 0 & 0 & 0 & 0 & 0 & 0 & 0 & 0 \\ 0 & 0 & 0 & 0 & 0 & 0 & 0 & 0 & 0 & 0 & 0 & 0 & 0 & 0 & 0 \\ 0 & 0 & 0 & 0 & 0 & 0 & 0 & 0 & 0 & 0 & 0 & 0 & 0 & 0 & 0 \\ 0 & 0 & 0 & 0 & 0 & 0 & 0 & 0 & 0 & 0 & 0 & 0 & 0 & 0 \\ 0 & 0 & \mathbf{0} & \mathbf{0} & \mathbf{0} & \mathbf{0} & \mathbf{0} & \mathbf{0} & \mathbf{0} & \mathbf{0} & \mathbf{0} & \mathbf{0} & \mathbf{0} & \mathbf{0} & \mathbf{0}\end{array}$

-Non-Generating Public Entities-

78 Ashland, City of

79 Benton County PUD \#1

80 Clallam County PUD \#1

81 Clatskanie PUD

82 Consumers Power

83 Emerald County PUD

84 Energy Northwest

85 Fall River Elec Coop

86 Franklin County PUD \#1

87 Klickitat County PUD \#1

88 Lewis County PUD \#1

89 Mason County PUD \#1

90 Mason County PUD \#3

91 Oregon Trail Coop

92 Tanner Elec Coop

93 Tillamook PUD \#1

94 Total Non-Generating Public Entities

$\begin{array}{lllllllllllllll}0 & 0 & 0 & 0 & 0 & 0 & 0 & 0 & 0 & 0 & 0 & 0 & 0 & 0 & 0 \\ 0 & 0 & 0 & 0 & 0 & 0 & 0 & 0 & 0 & 0 & 0 & 0 & 0 & 0 & 0 \\ 0 & 0 & 0 & 0 & 0 & 0 & 0 & 0 & 0 & 0 & 0 & 0 & 0 & 0 & 0 \\ 0 & 0 & 0 & 0 & 0 & 0 & 0 & 0 & 0 & 0 & 0 & 0 & 0 & 0 & 0 \\ 0 & 0 & 0 & 0 & 0 & 0 & 0 & 0 & 0 & 0 & 0 & 0 & 0 & 0 & 0 \\ 0 & 0 & 0 & 0 & 0 & 0 & 0 & 0 & 0 & 0 & 0 & 0 & 0 & 0 & 0 \\ 0 & 0 & 0 & 0 & 0 & 0 & 0 & 0 & 0 & 0 & 0 & 0 & 0 & 0 & 0 \\ 0 & 0 & 0 & 0 & 0 & 0 & 0 & 0 & 0 & 0 & 0 & 0 & 0 & 0 & 0 \\ 0 & 0 & 0 & 0 & 0 & 0 & 0 & 0 & 0 & 0 & 0 & 0 & 0 & 0 & 0 \\ 0 & 0 & 0 & 0 & 0 & 0 & 0 & 0 & 0 & 0 & 0 & 0 & 0 & 0 & 0 \\ 0 & 0 & 0 & 0 & 0 & 0 & 0 & 0 & 0 & 0 & 0 & 0 & 0 & 0 & 0 \\ 0 & 0 & 0 & 0 & 0 & 0 & 0 & 0 & 0 & 0 & 0 & 0 & 0 & 0 & 0 \\ 0 & 0 & 0 & 0 & 0 & 0 & 0 & 0 & 0 & 0 & 0 & 0 & 0 & 0 & 0 \\ 0 & 0 & 0 & 0 & 0 & 0 & 0 & 0 & 0 & 0 & 0 & 0 & 0 & 0 & 0 \\ 0 & 0 & 0 & 0 & 0 & 0 & 0 & 0 & 0 & 0 & 0 & 0 & 0 & 0 & 0 \\ 0 & 0 & 0 & 0 & 0 & 0 & 0 & 0 & 0 & 0 & 0 & 0 & 0 & 0 & 0 \\ 0 & \mathbf{0} & \mathbf{0} & \mathbf{0} & \mathbf{0} & \mathbf{0} & \mathbf{0} & \mathbf{0} & \mathbf{0} & \mathbf{0} & \mathbf{0} & \mathbf{0} & \mathbf{0} & \mathbf{0} & \mathbf{0}\end{array}$

-Investor-Owned Entities-

95 Avista Corp (WWP Division)

96 Idaho Power Company

97 Northwestern Energy LLC (MPC)

98 Pacific Power

99 Portland General Electric

100 Puget Sound Energy

101 Trans Alta Utilities Corp

102 Utah Power

103 Total Investor-Owned Entities

\begin{tabular}{lllllllllllllll}
0 & 0 & 0 & 0 & 0 & 0 & 0 & 0 & 0 & 0 & 0 & 0 & 0 & 0 & 0 \\
0 & 0 & 0 & 0 & 0 & 0 & 0 & 0 & 0 & 0 & 0 & 0 & 0 & 0 & 0 \\
0 & 0 & 0 & 0 & 0 & 0 & 0 & 0 & 0 & 0 & 0 & 0 & 0 & 0 & 0 \\
0 & 0 & 0 & 0 & 0 & 0 & 0 & 0 & 0 & 0 & 0 & 0 & 0 & 0 & 0 \\
0 & 0 & 0 & 0 & 0 & 0 & 0 & 0 & 0 & 0 & 0 & 0 & 0 & 0 & 0 \\
0 & 0 & 0 & 0 & 0 & 0 & 0 & 0 & 0 & 0 & 0 & 0 & 0 & 0 & 0 \\
0 & 0 & 0 & 0 & 0 & 0 & 0 & 0 & 0 & 0 & 0 & 0 & 0 & 0 & 0 \\
0 & 0 & 0 & 0 & 0 & 0 & 0 & 0 & 0 & 0 & 0 & 0 & 0 & 0 & 0 \\
\hline $\mathbf{0}$ & $\mathbf{0}$ & $\mathbf{0}$ & $\mathbf{0}$ & $\mathbf{0}$ & $\mathbf{0}$ & $\mathbf{0}$ & $\mathbf{0}$ & $\mathbf{0}$ & $\mathbf{0}$ & $\mathbf{0}$ & $\mathbf{0}$ & $\mathbf{0}$ & $\mathbf{0}$ & $\mathbf{0}$
\end{tabular}

-Other Entities-

104 Avista Energy Inc.

105 Chehalis Power Inc.

106 FPL Energy Vansycle LLC

107 Frederickson Power LP,

108 Hermiston Power Partnership

109 Klamath Pacific Energy

110 Longview Fibre

111 Mirant Americas Energy Marketing, L.P.

112 PPM Energy

113 SP Newsprint

114 Total Other Entities

Large Thermal Reserves

-Federal Entities-

115 BPA - Power Business 116 Total Federal Entities

$\begin{array}{lllllllllllllll}0 & 0 & 0 & 0 & 0 & 0 & 0 & 0 & 0 & 0 & 0 & 0 & 0 & 0 & 0 \\ 0 & 0 & 0 & 0 & 0 & 0 & 0 & 0 & 0 & 0 & 0 & 0 & 0 & 0 & 0 \\ 0 & 0 & 0 & 0 & 0 & 0 & 0 & 0 & 0 & 0 & 0 & 0 & 0 & 0 & 0 \\ 0 & 0 & 0 & 0 & 0 & 0 & 0 & 0 & 0 & 0 & 0 & 0 & 0 & 0 & 0 \\ 0 & 0 & 0 & 0 & 0 & 0 & 0 & 0 & 0 & 0 & 0 & 0 & 0 & 0 & 0 \\ 0 & 0 & 0 & 0 & 0 & 0 & 0 & 0 & 0 & 0 & 0 & 0 & 0 & 0 & 0 \\ 0 & 0 & 0 & 0 & 0 & 0 & 0 & 0 & 0 & 0 & 0 & 0 & 0 & 0 & 0 \\ 0 & 0 & 0 & 0 & 0 & 0 & 0 & 0 & 0 & 0 & 0 & 0 & 0 & 0 & 0 \\ 0 & 0 & 0 & 0 & 0 & 0 & 0 & 0 & 0 & 0 & 0 & 0 & 0 & 0 & 0 \\ 0 & 0 & 0 & 0 & 0 & 0 & 0 & 0 & 0 & 0 & 0 & 0 & 0 & 0 & 0 \\ 0 & \mathbf{0} & \mathbf{0} & \mathbf{0} & \mathbf{0} & \mathbf{0} & \mathbf{0} & \mathbf{0} & \mathbf{0} & \mathbf{0} & \mathbf{0} & \mathbf{0} & \mathbf{0} & \mathbf{0} & \mathbf{0}\end{array}$

-Generating Public Entities-

117 Turlock Irrigation District

118 Total Generating Public Entities

-Non-Generating Public Entities-

119 Total Non-Generating Public Entities 
Average Energy in Megawatts

120 Avista Corp (WWP Division)

121 Idaho Power Company

122 Northwestern Energy LLC (MPC)

123 Pacific Power

124 Portland General Electric

125 Puget Sound Energy

126 San Diego Gas \& Electric

127 Trans Alta Utilities Corp

128 Total Investor-Owned Entities

\section{Water Year}

2003 White Book

-Other Entities-

129 Total Other Entities

\title{
Aug1 Aug16
}

Sep

Oct Nov Dec Jan Feb

Mar Apr1 Apr1

May Jun Jul Avg

Contract Reserves

-Federal Entities-

130 Total Federal Entities

$\begin{array}{llll}0 & 0 & 0 & 0 \\ 0 & 0 & 0 & 0 \\ 0 & 0 & 0 & 0 \\ 0 & 0 & 0 & 0 \\ 0 & 0 & 0 & 0 \\ 0 & 0 & 0 & 0 \\ 0 & 0 & 0 & 0 \\ 0 & 0 & 0 & 0 \\ 0 & 0 & 0 & 0\end{array}$

$\begin{array}{ll}0 & 0 \\ 0 & 0 \\ 0 & 0 \\ 0 & 0 \\ 0 & 0 \\ 0 & 0 \\ 0 & 0 \\ 0 & 0 \\ 0 & 0\end{array}$

$\begin{array}{lll}0 & 0 & 0 \\ 0 & 0 & 0 \\ 0 & 0 & 0 \\ 0 & 0 & 0 \\ 0 & 0 & 0 \\ 0 & 0 & \\ 0 & 0 & \\ 0 & 0 & 0 \\ 0 & 0\end{array}$

0
0
0
0
0
0
0
0
0

$\begin{array}{ll}0 & 0 \\ 0 & 0 \\ 0 & 0 \\ 0 & 0 \\ 0 & 0 \\ 0 & \\ 0 & \\ 0 & \\ 0 & \end{array}$

$\begin{array}{lllllll}0 & 0 & 0 & 0 & 0 & 0 & 0 \\ 0 & 0 & 0 & 0 & 0 & 0 & 0 \\ 0 & 0 & 0 & 0 & 0 & 0 & 0 \\ 0 & 0 & 0 & 0 & 0 & 0 & 0 \\ 0 & 0 & 0 & 0 & 0 & 0 & 0 \\ 0 & 0 & 0 & 0 & 0 & 0 & 0 \\ 0 & 0 & 0 & 0 & 0 & 0 & 0 \\ 0 & 0 & 0 & 0 & 0 & 0 & 0 \\ 0 & 0 & 0 & 0 & 0 & 0 & 0\end{array}$

-Generating Public Entities-

131 Total Generating Public Entities

$0 \quad 0$

\begin{abstract}
0
\end{abstract}
0

0

0

-Non-Generating Public Entities-

132 Total Non-Generating Public Entities

-Investor-Owned Entities-

133 Northwestern Energy LLC (MPC)

134 Total Investor-Owned Entities

$\begin{array}{lllllllllllllll}0 & 0 & 0 & 0 & 0 & 0 & 0 & 0 & 0 & 0 & 0 & 0 & 0 & 0 & 0\end{array}$

-Other Entities-

135 Total Other Entities

\begin{tabular}{llllllllllllll}
0 & 0 & 0 & 0 & 0 & 0 & 0 & 0 & 0 & 0 & 0 & 0 & 0 & 0 \\
\hline
\end{tabular}

-Total Capacity Reserves-

136 Total Hydro Reserves

137 Total Small Thermal \& Misc Reserves

138 Total Large Thermal Reserves

139 Total Contract Reserves

140 Total Capacity Reserves

$\begin{array}{lllllllllllllll}0 & 0 & 0 & 0 & 0 & 0 & 0 & 0 & 0 & 0 & 0 & 0 & 0 & 0 & 0 \\ 0 & 0 & 0 & 0 & 0 & 0 & 0 & 0 & 0 & 0 & 0 & 0 & 0 & 0 & 0 \\ 0 & 0 & 0 & 0 & 0 & 0 & 0 & 0 & 0 & 0 & 0 & 0 & 0 & 0 & 0 \\ 0 & 0 & 0 & 0 & 0 & 0 & 0 & 0 & 0 & 0 & 0 & 0 & 0 & 0 & 0 \\ 0 & 0 & 0 & 0 & 0 & 0 & 0 & 0 & 0 & 0 & 0 & 0 & 0 & 0 & 0\end{array}$


Table A-18: Wells and Chelan Allocation

PNW Loads and Resources Study

2004 - 2005 Operating Year

1937 Water Year

2003 White Book

Average Energy in Megawatts Aug1 Aug16 Sep Oct Nov Dec Jan Feb Mar Apr1 Apr16 May Jun Jul Avg

-Wells-

1 Wells

2 Wells Before Enc

$\begin{array}{lll}591 & 478 & 3 \\ 591 & 478 & 3\end{array}$

$310 \quad 391$

$\begin{array}{llll}467 & 459 & 359 & 339 \\ 467 & 459 & 359 & 339\end{array}$

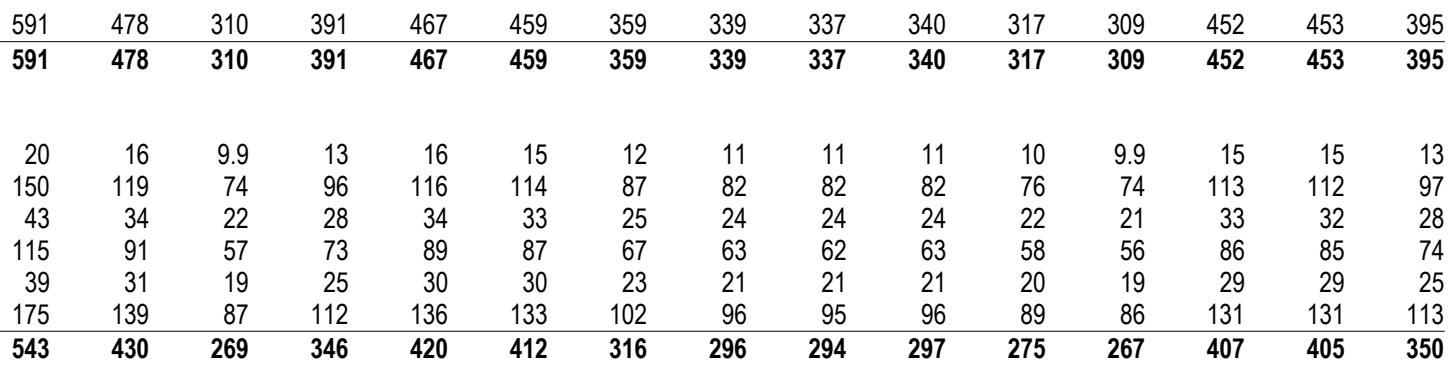

$\begin{array}{llllll}340 & 317 & 309 & 452 & 453 & 395 \\ 340 & 317 & 309 & 452 & 453 & 395\end{array}$

3 AVWP Share

4 DOPD Share

5 OKPD Share

6 PGE Share

7 PPL Share

8 PSE Share

9 Wells After Enc

conter

10 Chelan

11 Chelan Before Enc

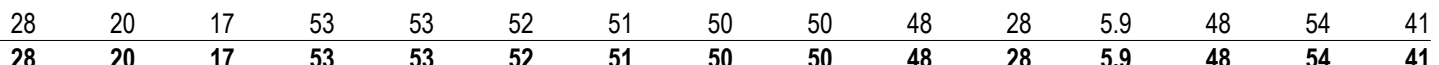

-Chelan-

12 CHPD Share

13 Chelan After Enc

\begin{tabular}{|c|c|c|c|c|c|c|c|c|c|c|c|c|c|}
\hline 31 & 23 & 20 & 55 & 55 & 55 & 54 & 54 & 54 & 52 & 32 & 9.9 & 51 & 57 \\
\hline 31 & 23 & 20 & 55 & 55 & 55 & 54 & 54 & 54 & 52 & 32 & 9.9 & 51 & 57 \\
\hline
\end{tabular}


2003 White Book

Average Energy in Megawatts Aug1 Aug16 Sep Oct Nov Dec Jan Feb Mar Apr1 Apr16 May Jun Jul Avg -Wells-

1 Wells

2 Wells Before Enc

$\begin{array}{lll}591 & 478 & 3 \\ 591 & 478 & 3\end{array}$

$\begin{array}{llllll}310 & 391 & 467 & 459 & 359 & 339 \\ 310 & 391 & 467 & 459 & 359 & 339\end{array}$

-Wells-

3 AVWP Share

4 DOPD Share

5 OKPD Share

6 PGE Share

7 PPL Share

8 PSE Share

9 Wells After Enc

\begin{tabular}{|c|c|c|c|c|c|c|c|c|c|c|c|c|c|c|}
\hline 20 & 16 & 9.9 & 13 & 16 & 15 & 12 & 11 & 11 & 11 & 10 & 9.9 & 15 & 15 & 13 \\
\hline 150 & 119 & 74 & 96 & 116 & 114 & 87 & 82 & 82 & 82 & 76 & 74 & 113 & 112 & 97 \\
\hline 43 & 34 & 22 & 28 & 34 & 33 & 25 & 24 & 24 & 24 & 22 & 21 & 33 & 32 & 28 \\
\hline 115 & 91 & 57 & 73 & 89 & 87 & 67 & 63 & 62 & 63 & 58 & 56 & 86 & 85 & 74 \\
\hline 39 & 31 & 19 & 25 & 30 & 30 & 23 & 21 & 21 & 21 & 20 & 19 & 29 & 29 & 25 \\
\hline 175 & 139 & 87 & 112 & 136 & 133 & 102 & 96 & 95 & 96 & 89 & 86 & 131 & 131 & 113 \\
\hline 543 & 430 & 269 & 346 & 420 & 412 & 316 & 296 & 294 & 297 & 275 & 267 & 407 & 405 & 350 \\
\hline
\end{tabular}

-Chelan-

10 Chelan

11 Chelan Before Enc

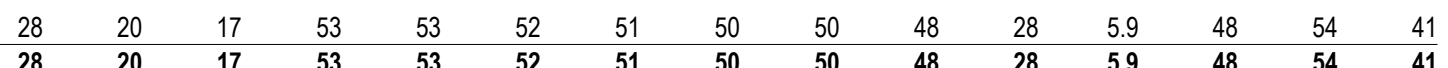

-Chelan-

12 CHPD Share

13 Chelan After Enc

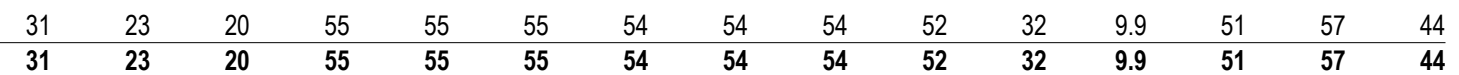


1937 Water Year

2003 White Book

Average Energy in Megawatts Aug1 Aug16 Sep Oct Nov Dec Jan Feb Mar Apr1 Apr16 May Jun Jul Avg

-Wells-

1 Wells

2 Wells Before Enc

$\begin{array}{lll}591 & 478 & 3 \\ 591 & 478 & 3\end{array}$

$310 \quad 391$

$\begin{array}{llll}467 & 459 & 359 & 339 \\ 467 & 459 & 359 & 339\end{array}$

\begin{tabular}{|c|c|c|c|c|c|c|c|c|c|c|c|c|c|c|}
\hline 591 & 478 & 310 & 391 & 467 & 459 & 359 & 339 & 337 & 340 & 317 & 309 & 452 & 453 & 395 \\
\hline 591 & 478 & 310 & 391 & 467 & 459 & 359 & 339 & 337 & 340 & 317 & 309 & 452 & 453 & 395 \\
\hline 150 & 119 & 74 & 96 & 116 & 114 & 87 & 82 & 82 & 82 & 76 & 74 & 113 & 112 & 97 \\
\hline 43 & 34 & 22 & 28 & 34 & 33 & 25 & 24 & 24 & 24 & 22 & 21 & 33 & 32 & 28 \\
\hline 115 & 91 & 57 & 73 & 89 & 87 & 67 & 63 & 62 & 63 & 58 & 56 & 86 & 85 & 74 \\
\hline 175 & 139 & 87 & 112 & 136 & 133 & 102 & 96 & 95 & 96 & 89 & 86 & 131 & 131 & 113 \\
\hline 543 & 430 & 269 & 346 & 420 & 412 & 316 & 296 & 294 & 297 & 275 & 267 & 407 & 405 & 350 \\
\hline
\end{tabular}

\begin{tabular}{|c|c|c|c|}
\hline 317 & 309 & 452 & 453 \\
\hline 317 & 309 & 452 & 453 \\
\hline
\end{tabular}

3 AVWP Share

4 DOPD Share

5 OKPD Share

6 PGE Share

7 PPL Share

8 PSE Share

9 Wells After Enc

conter

10 Chelan

11 Chelan Before Enc

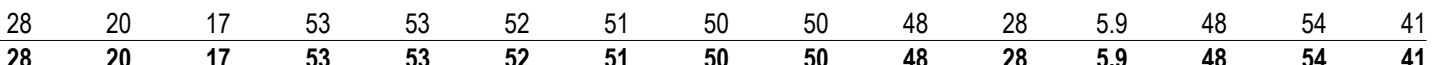

-Chelan-

12 CHPD Share

13 Chelan After Enc

\begin{tabular}{|c|c|c|c|c|c|c|c|c|c|c|c|c|c|}
\hline 31 & 23 & 20 & 55 & 55 & 55 & 54 & 54 & 54 & 52 & 32 & 9.9 & 51 & 57 \\
\hline 31 & 23 & 20 & 55 & 55 & 55 & 54 & 54 & 54 & 52 & 32 & 9.9 & 51 & 57 \\
\hline
\end{tabular}


2003 White Book

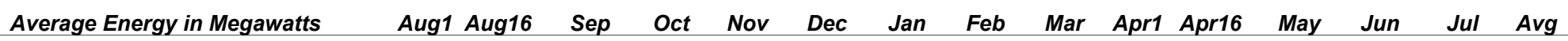

-Wells-

1 Wells

2 Wells Before Enc

$\begin{array}{lll}591 & 478 & 3 \\ 591 & 478 & 3\end{array}$

$310 \quad 391$

$\begin{array}{llll}467 & 459 & 359 & 339 \\ 467 & 459 & 359 & 339\end{array}$

\begin{tabular}{|c|c|c|c|c|c|c|c|c|c|c|c|c|c|c|}
\hline 591 & 478 & 310 & 391 & 467 & 459 & 359 & 339 & 337 & 340 & 317 & 309 & 452 & 453 & 395 \\
\hline 591 & 478 & 310 & 391 & 467 & 459 & 359 & 339 & 337 & 340 & 317 & 309 & 452 & 453 & 395 \\
\hline 150 & 119 & 74 & 96 & 116 & 114 & 87 & 82 & 82 & 82 & 76 & 74 & 113 & 112 & 97 \\
\hline 43 & 34 & 22 & 28 & 34 & 33 & 25 & 24 & 24 & 24 & 22 & 21 & 33 & 32 & 28 \\
\hline 115 & 91 & 57 & 73 & 89 & 87 & 67 & 63 & 62 & 63 & 58 & 56 & 86 & 85 & 74 \\
\hline 175 & 139 & 87 & 112 & 136 & 133 & 102 & 96 & 95 & 96 & 89 & 86 & 131 & 131 & 113 \\
\hline 543 & 430 & 269 & 346 & 420 & 412 & 316 & 296 & 294 & 297 & 275 & 267 & 407 & 405 & 350 \\
\hline
\end{tabular}

\begin{tabular}{|c|c|c|c|}
\hline 317 & 309 & 452 & 453 \\
\hline 317 & 309 & 452 & 453 \\
\hline
\end{tabular}

3 AVWP Share

4 DOPD Share

5 OKPD Share

6 PGE Share

7 PPL Share

8 PSE Share

9 Wells After Enc

conter

10 Chelan

11 Chelan Before Enc

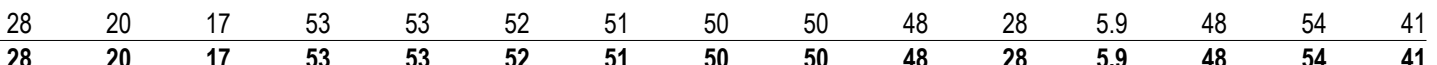

-Chelan-

12 CHPD Share

13 Chelan After Enc

\begin{tabular}{|c|c|c|c|c|c|c|c|c|c|c|c|c|c|}
\hline 31 & 23 & 20 & 55 & 55 & 55 & 54 & 54 & 54 & 52 & 32 & 9.9 & 51 & 57 \\
\hline 31 & 23 & 20 & 55 & 55 & 55 & 54 & 54 & 54 & 52 & 32 & 9.9 & 51 & 57 \\
\hline
\end{tabular}


1937 Water Year

2003 White Book

Average Energy in Megawatts Aug1 Aug16 Sep Oct Nov Dec Jan Feb Mar Apr1 Apr16 May Jun Jul Avg

-Wells-

1 Wells

2 Wells Before Enc

$\begin{array}{lll}591 & 478 & 3 \\ 591 & 478 & 3\end{array}$

$310 \quad 391$

$\begin{array}{llll}467 & 459 & 359 & 339 \\ 467 & 459 & 359 & 339\end{array}$

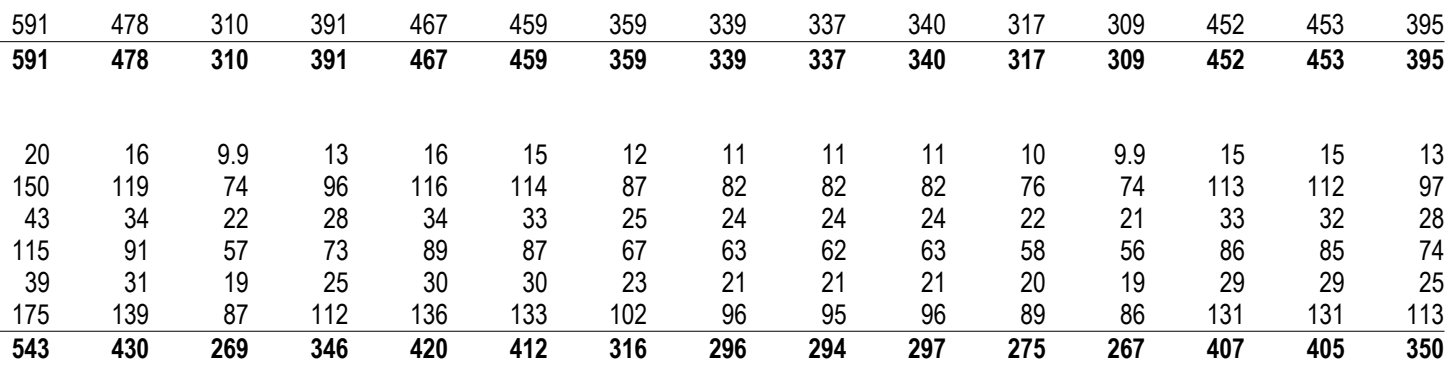

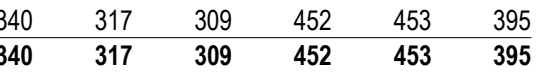

3 AVWP Share

4 DOPD Share

5 OKPD Share

6 PGE Share

7 PPL Share

8 PSE Share

9 Wells After Enc

chaterer-

10 Chelan

11 Chelan Before Enc

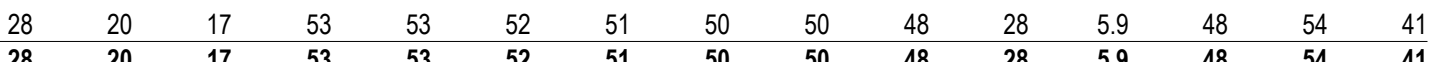

-Chelan-

12 CHPD Share

13 Chelan After Enc

\begin{tabular}{|c|c|c|c|c|c|c|c|c|c|c|c|c|c|}
\hline 31 & 23 & 20 & 55 & 55 & 55 & 54 & 54 & 54 & 52 & 32 & 9.9 & 51 & 57 \\
\hline 31 & 23 & 20 & 55 & 55 & 55 & 54 & 54 & 54 & 52 & 32 & 9.9 & 51 & 57 \\
\hline
\end{tabular}


2003 White Book

Average Energy in Megawatts Aug1 Aug16 Sep Oct Nov Dec Jan Feb Mar Apr1 Apr16 May Jun Jul Avg -Wells-

1 Wells

2 Wells Before Enc

$\begin{array}{lll}591 & 478 & 3 \\ 591 & 478 & 3\end{array}$

$\begin{array}{llllll}310 & 391 & 467 & 459 & 359 & 339 \\ 310 & 391 & 467 & 459 & 359 & 339\end{array}$

-Wells-

3 AVWP Share

4 DOPD Share

5 OKPD Share

6 PGE Share

7 PPL Share

8 PSE Share

9 Wells After Enc

\begin{tabular}{|c|c|c|c|c|c|c|c|c|c|c|c|c|c|c|}
\hline 20 & 16 & 9.9 & 13 & 16 & 15 & 12 & 11 & 11 & 11 & 10 & 9.9 & 15 & 15 & 13 \\
\hline 150 & 119 & 74 & 96 & 116 & 114 & 87 & 82 & 82 & 82 & 76 & 74 & 113 & 112 & 97 \\
\hline 43 & 34 & 22 & 28 & 34 & 33 & 25 & 24 & 24 & 24 & 22 & 21 & 33 & 32 & 28 \\
\hline 115 & 91 & 57 & 73 & 89 & 87 & 67 & 63 & 62 & 63 & 58 & 56 & 86 & 85 & 74 \\
\hline 39 & 31 & 19 & 25 & 30 & 30 & 23 & 21 & 21 & 21 & 20 & 19 & 29 & 29 & 25 \\
\hline 175 & 139 & 87 & 112 & 136 & 133 & 102 & 96 & 95 & 96 & 89 & 86 & 131 & 131 & 113 \\
\hline 543 & 430 & 269 & 346 & 420 & 412 & 316 & 296 & 294 & 297 & 275 & 267 & 407 & 405 & 350 \\
\hline
\end{tabular}

-Chelan-

10 Chelan

11 Chelan Before Enc

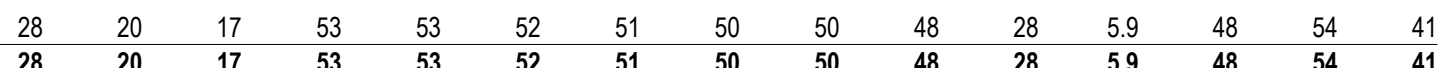

-Chelan-

12 CHPD Share

13 Chelan After Enc

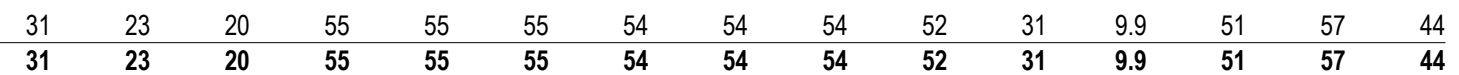


2003 White Book

Average Energy in Megawatts Aug1 Aug16 Sep Oct Nov Dec Jan Feb Mar Apr1 Apr16 May Jun Jul Avg -Wells-

1 Wells

2 Wells Before Enc

$\begin{array}{lll}591 & 478 & 3 \\ 591 & 478 & 3\end{array}$

$\begin{array}{llllll}310 & 391 & 467 & 459 & 359 & 339 \\ 310 & 391 & 467 & 459 & 359 & 339\end{array}$

-Wells-

3 AVWP Share

4 DOPD Share

5 OKPD Share

6 PGE Share

7 PPL Share

8 PSE Share

9 Wells After Enc

\begin{tabular}{|c|c|c|c|c|c|c|c|c|c|c|c|c|c|c|}
\hline 20 & 16 & 9.9 & 13 & 16 & 15 & 12 & 11 & 11 & 11 & 10 & 9.9 & 15 & 15 & 13 \\
\hline 150 & 119 & 74 & 96 & 116 & 114 & 87 & 82 & 82 & 82 & 76 & 74 & 113 & 112 & 97 \\
\hline 43 & 34 & 22 & 28 & 34 & 33 & 25 & 24 & 24 & 24 & 22 & 21 & 33 & 32 & 28 \\
\hline 115 & 91 & 57 & 73 & 89 & 87 & 67 & 63 & 62 & 63 & 58 & 56 & 86 & 85 & 74 \\
\hline 39 & 31 & 19 & 25 & 30 & 30 & 23 & 21 & 21 & 21 & 20 & 19 & 29 & 29 & 25 \\
\hline 175 & 139 & 87 & 112 & 136 & 133 & 102 & 96 & 95 & 96 & 89 & 86 & 131 & 131 & 113 \\
\hline 543 & 430 & 269 & 346 & 420 & 412 & 316 & 296 & 294 & 297 & 275 & 267 & 407 & 405 & 350 \\
\hline
\end{tabular}

-Chelan-

10 Chelan

11 Chelan Before Enc

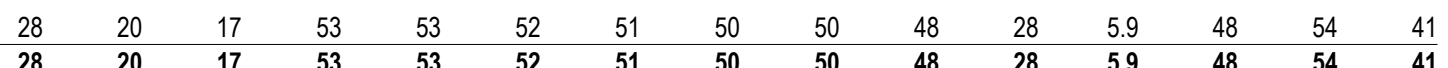

-Chelan-

12 CHPD Share

13 Chelan After Enc

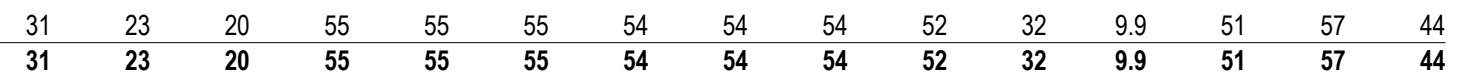


2003 White Book

Average Energy in Megawatts Aug1 Aug16 Sep Oct Nov Dec Jan Feb Mar Apr1 Apr16 May Jun Jul Avg -Wells-

1 Wells

2 Wells Before Enc

$\begin{array}{lll}591 & 478 & 3 \\ 591 & 478 & 3\end{array}$

$\begin{array}{llllll}310 & 391 & 467 & 459 & 359 & 339 \\ 310 & 391 & 467 & 459 & 359 & 339\end{array}$

-Wells-

3 AVWP Share

4 DOPD Share

5 OKPD Share

6 PGE Share

7 PPL Share

8 PSE Share

9 Wells After Enc

\begin{tabular}{|c|c|c|c|c|c|c|c|c|c|c|c|c|c|c|}
\hline 20 & 16 & 9.9 & 13 & 16 & 15 & 12 & 11 & 11 & 11 & 10 & 9.9 & 15 & 15 & 13 \\
\hline 150 & 119 & 74 & 96 & 116 & 114 & 87 & 82 & 82 & 82 & 76 & 74 & 113 & 112 & 97 \\
\hline 43 & 34 & 22 & 28 & 34 & 33 & 25 & 24 & 24 & 24 & 22 & 21 & 33 & 32 & 28 \\
\hline 115 & 91 & 57 & 73 & 89 & 87 & 67 & 63 & 62 & 63 & 58 & 56 & 86 & 85 & 74 \\
\hline 39 & 31 & 19 & 25 & 30 & 30 & 23 & 21 & 21 & 21 & 20 & 19 & 29 & 29 & 25 \\
\hline 175 & 139 & 87 & 112 & 136 & 133 & 102 & 96 & 95 & 96 & 89 & 86 & 131 & 131 & 113 \\
\hline 543 & 430 & 269 & 346 & 420 & 412 & 316 & 296 & 294 & 297 & 275 & 267 & 407 & 405 & 350 \\
\hline
\end{tabular}

-Chelan-

10 Chelan

11 Chelan Before Enc

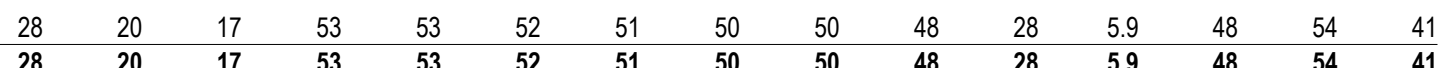

-Chelan-

12 CHPD Share

13 Chelan After Enc

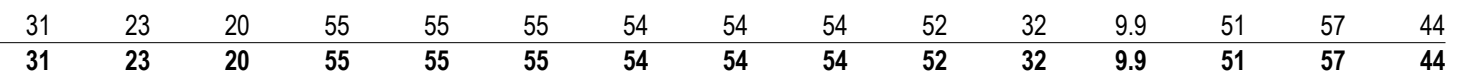


2003 White Book

Average Energy in Megawatts Aug1 Aug16 Sep Oct Nov Dec Jan Feb Mar Apr1 Apr16 May Jun Jul Avg -Wells-

1 Wells

2 Wells Before Enc

$\begin{array}{lll}591 & 478 & 3 \\ 591 & 478 & 3\end{array}$

$\begin{array}{llllll}310 & 391 & 467 & 459 & 359 & 339 \\ 310 & 391 & 467 & 459 & 359 & 339\end{array}$

-Wells-

3 AVWP Share

4 DOPD Share

5 OKPD Share

6 PGE Share

7 PPL Share

8 PSE Share

9 Wells After Enc

\begin{tabular}{|c|c|c|c|c|c|c|c|c|c|c|c|c|c|c|}
\hline 20 & 16 & 9.9 & 13 & 16 & 15 & 12 & 11 & 11 & 11 & 10 & 9.9 & 15 & 15 & 13 \\
\hline 150 & 119 & 74 & 96 & 116 & 114 & 87 & 82 & 82 & 82 & 76 & 74 & 113 & 112 & 97 \\
\hline 43 & 34 & 22 & 28 & 34 & 33 & 25 & 24 & 24 & 24 & 22 & 21 & 33 & 32 & 28 \\
\hline 115 & 91 & 57 & 73 & 89 & 87 & 67 & 63 & 62 & 63 & 58 & 56 & 86 & 85 & 74 \\
\hline 39 & 31 & 19 & 25 & 30 & 30 & 23 & 21 & 21 & 21 & 20 & 19 & 29 & 29 & 25 \\
\hline 175 & 139 & 87 & 112 & 136 & 133 & 102 & 96 & 95 & 96 & 89 & 86 & 131 & 131 & 113 \\
\hline 543 & 430 & 269 & 346 & 420 & 412 & 316 & 296 & 294 & 297 & 275 & 267 & 407 & 405 & 350 \\
\hline
\end{tabular}

-Chelan-

10 Chelan

11 Chelan Before Enc

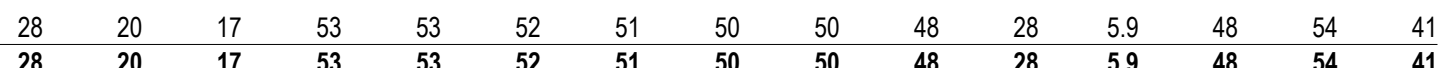

-Chelan-

12 CHPD Share

13 Chelan After Enc

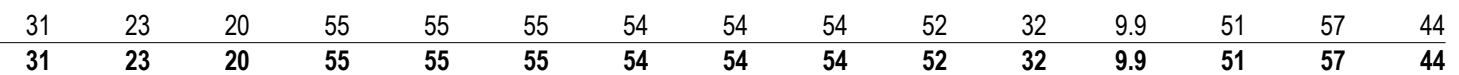


2003 White Book

Average Energy in Megawatts Aug1 Aug16 Sep Oct Nov Dec Jan Feb Mar Apr1 Apr16 May Jun Jul Avg -Wells-

1 Wells

2 Wells Before Enc

$\begin{array}{lll}591 & 478 & 3 \\ 591 & 478 & 3\end{array}$

$\begin{array}{llllll}310 & 391 & 467 & 459 & 359 & 339 \\ 310 & 391 & 467 & 459 & 359 & 339\end{array}$

-Wells-

3 AVWP Share

4 DOPD Share

5 OKPD Share

6 PGE Share

7 PPL Share

8 PSE Share

9 Wells After Enc

\begin{tabular}{|c|c|c|c|c|c|c|c|c|c|c|c|c|c|}
\hline 16 & 9.9 & 13 & 16 & 15 & 12 & 11 & 11 & 11 & 10 & 9.9 & 15 & 15 & 13 \\
\hline 119 & 74 & 96 & 116 & 114 & 87 & 82 & 82 & 82 & 76 & 74 & 113 & 112 & 97 \\
\hline 43 & 22 & 28 & 34 & 33 & 25 & 24 & 24 & 24 & 22 & 21 & 33 & 32 & 28 \\
\hline 115 & 57 & 73 & 89 & 87 & 67 & 63 & 62 & 63 & 58 & 56 & 86 & 85 & 74 \\
\hline 31 & 19 & 25 & 30 & 30 & 23 & 21 & 21 & 21 & 20 & 19 & 29 & 29 & 25 \\
\hline 139 & 87 & 112 & 136 & 133 & 102 & 96 & 95 & 96 & 89 & 86 & 131 & 131 & 113 \\
\hline 430 & 269 & 346 & 420 & 412 & 316 & 296 & 294 & 297 & 275 & 267 & 407 & 405 & 350 \\
\hline
\end{tabular}

-Chelan-

10 Chelan

11 Chelan Before Enc

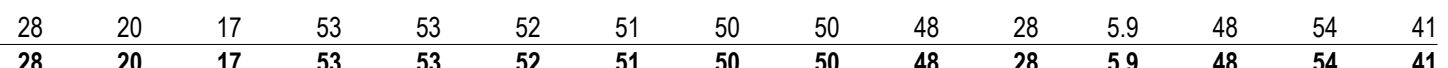

-Chelan-

12 CHPD Share

13 Chelan After Enc

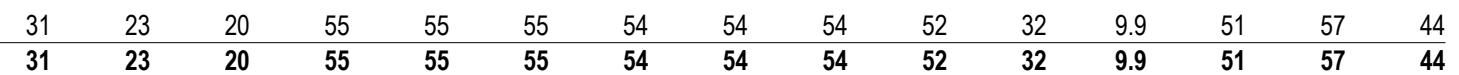


-Rocky Reach-

1 Rocky Reach

2 Rocky Reach Before Enc

Aug1 Aug16 S

Oct Nov Dec Jan Feb

$\begin{array}{lll}808 & 641 & 4 \\ 808 & 641 & 403\end{array}$

-Rocky Reach-

3 AVWP Share

4 CHPD Share

5 CLKM Share

6 DOPD Share

7 PGE Share

8 PPL Share

9 PSE Share

10 Rocky Reach After Enc

\begin{tabular}{|c|c|c|c|c|c|c|c|c|c|c|c|c|c|}
\hline 24 & 12 & 16 & 19 & 19 & 15 & 14 & 14 & 14 & 12 & 11 & 19 & 18 & 16 \\
\hline 100 & 64 & 83 & 99 & 98 & 76 & 72 & 72 & 72 & 63 & 60 & 97 & 95 & 83 \\
\hline 152 & 97 & 126 & 151 & 149 & 116 & 109 & 109 & 109 & 96 & 91 & 148 & 144 & 126 \\
\hline 18 & 12 & 15 & 18 & 18 & 14 & 13 & 13 & 13 & 12 & 11 & 18 & 17 & 15 \\
\hline 79 & 50 & 66 & 79 & 78 & 60 & 57 & 57 & 57 & 50 & 47 & 77 & 75 & 66 \\
\hline 35 & 22 & 29 & 35 & 34 & 27 & 25 & 25 & 25 & 22 & 21 & 34 & 33 & 29 \\
\hline 257 & 163 & 213 & 255 & 251 & 196 & 185 & 185 & 185 & 162 & 153 & 250 & 244 & 213 \\
\hline 660 & 420 & 547 & 656 & 646 & 504 & 474 & 475 & 476 & 416 & 394 & 642 & 627 & 548 \\
\hline
\end{tabular}

-Rock Island PH\#1-

11 Rock Island PH\#1

12 Rock Island PH\#1 Before Enc

$142 \quad 148 \quad 108$

-Rock Island PH\#1-

13 CHPD Share

14 PSE Share

15 Rock Island PH\#1 After Enc

$637 \quad 628$

$487 \quad 458$

Mar Apr1 Apr16 May Jun Jul Avg

-Rock Island PH\#2-

16 Rock Island PH\#2

17 Rock Island PH\#2 Before Enc

142148

132

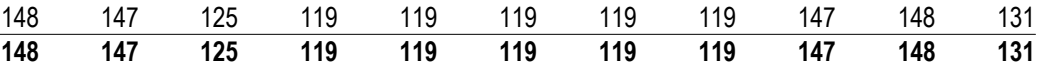

-Rock Island PH\#2-

18 CHPD Share

19 PSE Share

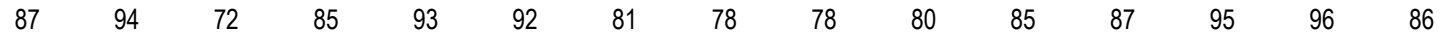

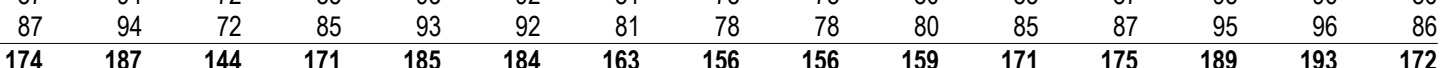

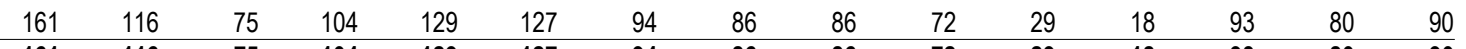

$\begin{array}{lllllllllllll}161 & 116 & 75 & 104 & 129 & 127 & 94 & 86 & 86 & 72 & 29 & 18 & 93\end{array}$

\begin{tabular}{|c|c|c|c|c|c|c|c|c|c|c|c|c|c|c|}
\hline 99 & 73 & 50 & 67 & 81 & 80 & 61 & 57 & 57 & 48 & 21 & 13 & 60 & 52 & 58 \\
\hline 99 & 73 & 50 & 67 & 81 & 80 & 61 & 57 & 57 & 48 & 21 & 13 & 60 & 52 & 58 \\
\hline
\end{tabular}


2003 White Book

-Rocky Reach-

1 Rocky Reach

2 Rocky Reach Before Enc

Aug1 Aug16 S

Oct Nov Dec Jan Feb

$808 \quad 641 \quad 403$

$808 \quad 641 \quad 403$

$528 \quad 637$

\begin{tabular}{lllllllllll}
637 & 628 & 487 & 458 & 459 & 460 & 400 & 378 & 624 & 609 & 531 \\
\hline 637 & $\mathbf{6 2 8}$ & $\mathbf{4 8 7}$ & $\mathbf{4 5 8}$ & $\mathbf{4 5 9}$ & $\mathbf{4 6 0}$ & $\mathbf{4 0 0}$ & $\mathbf{3 7 8}$ & $\mathbf{6 2 4}$ & $\mathbf{6 0 9}$ & $\mathbf{5 3 1}$
\end{tabular}

-Rocky Reach-

3 AVWP Share

4 CHPD Share

5 CLKM Share

6 DOPD Share

7 PGE Share

8 PPL Share

9 PSE Share

10 Rocky Reach After Enc

$\begin{array}{rrrrrrrrrrrrrrr}24 & 19 & 12 & 16 & 19 & 19 & 15 & 14 & 14 & 14 & 12 & 11 & 19 & 18 & 16 \\ 125 & 100 & 64 & 83 & 99 & 98 & 76 & 72 & 72 & 72 & 63 & 60 & 97 & 95 & 83 \\ 190 & 152 & 97 & 126 & 151 & 149 & 116 & 109 & 109 & 109 & 96 & 91 & 148 & 144 & 126 \\ 23 & 18 & 12 & 15 & 18 & 18 & 14 & 13 & 13 & 13 & 12 & 11 & 18 & 17 & 15 \\ 99 & 79 & 50 & 66 & 79 & 78 & 60 & 57 & 57 & 57 & 50 & 47 & 77 & 75 & 66 \\ 44 & 35 & 22 & 29 & 35 & 34 & 27 & 25 & 25 & 25 & 22 & 21 & 34 & 33 & 29 \\ 322 & 257 & 163 & 213 & 255 & 251 & 196 & 185 & 185 & 185 & 162 & 153 & 250 & 244 & 213 \\ \mathbf{8 2 7} & \mathbf{6 6 0} & \mathbf{4 2 0} & \mathbf{5 4 7} & \mathbf{6 5 6} & \mathbf{6 4 6} & \mathbf{5 0 4} & \mathbf{4 7 4} & \mathbf{4 7 5} & \mathbf{4 7 6} & \mathbf{4 1 6} & \mathbf{3 9 4} & \mathbf{6 4 2} & \mathbf{6 2 7} & \mathbf{5 4 8}\end{array}$

-Rock Island PH\#1-

11 Rock Island PH\#1

12 Rock Island PH\#1 Before Enc

$142 \quad 148 \quad 108$

-Rock Island PH\#1-

13 CHPD Share

14 PSE Share

15 Rock Island PH\#1 After Enc

142148

$108 \quad 132$

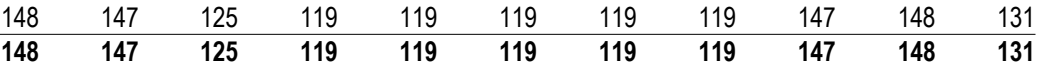

-Rock Island PH\#2-

16 Rock Island PH\#2

17 Rock Island PH\#2 Before Enc

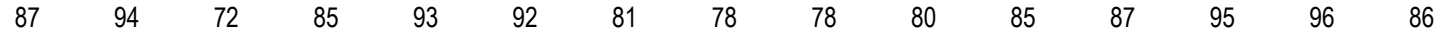

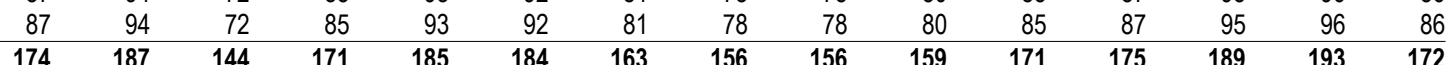

-Rock Island PH\#2-

18 CHPD Share

19 PSE Share

20 Rock Island PH\#2 After Enc

\begin{tabular}{lllllllllllllll}
161 & 116 & 75 & 104 & 129 & 127 & 94 & 86 & 86 & 72 & 29 & 18 & 93 & 80 & 90 \\
\hline
\end{tabular}

$\begin{array}{lllllllllllll}161 & 116 & 75 & 104 & 129 & 127 & 94 & 86 & 86 & 72 & 29 & 18 & 93\end{array}$

\begin{tabular}{|c|c|c|c|c|c|c|c|c|c|c|c|c|c|c|}
\hline 99 & 73 & 50 & 67 & 81 & 80 & 61 & 57 & 57 & 48 & 21 & 13 & 60 & 52 & 58 \\
\hline 99 & 73 & 50 & 67 & 81 & 80 & 61 & 57 & 57 & 48 & 21 & 13 & 60 & 52 & 58 \\
\hline
\end{tabular}


2003 White Book

-Rocky Reach-

1 Rocky Reach

2 Rocky Reach Before Enc

Aug1 Aug16 S

Oct Nov Dec Jan Feb

$\begin{array}{lll}808 & 641 & 403 \\ 808 & 641 & 403\end{array}$

$528 \quad 637$

\begin{tabular}{lllllllllll}
637 & 628 & 487 & 458 & 459 & 460 & 400 & 378 & 624 & 609 & 531 \\
\hline 637 & $\mathbf{6 2 8}$ & $\mathbf{4 8 7}$ & $\mathbf{4 5 8}$ & $\mathbf{4 5 9}$ & $\mathbf{4 6 0}$ & $\mathbf{4 0 0}$ & $\mathbf{3 7 8}$ & $\mathbf{6 2 4}$ & $\mathbf{6 0 9}$ & $\mathbf{5 3 1}$
\end{tabular}

-Rocky Reach-

3 AVWP Share

4 CHPD Share

5 CLKM Share

6 DOPD Share

7 PGE Share

8 PPL Share

9 PSE Share

10 Rocky Reach After Enc

$\begin{array}{rrrrrrrrrrrrrrr}24 & 19 & 12 & 16 & 19 & 19 & 15 & 14 & 14 & 14 & 12 & 11 & 19 & 18 & 16 \\ 125 & 100 & 64 & 83 & 99 & 98 & 76 & 72 & 72 & 72 & 63 & 60 & 97 & 95 & 83 \\ 190 & 152 & 97 & 126 & 151 & 149 & 116 & 109 & 109 & 109 & 96 & 91 & 148 & 144 & 126 \\ 23 & 18 & 12 & 15 & 18 & 18 & 14 & 13 & 13 & 13 & 12 & 11 & 18 & 17 & 15 \\ 99 & 79 & 50 & 66 & 79 & 78 & 60 & 57 & 57 & 57 & 50 & 47 & 77 & 75 & 66 \\ 44 & 35 & 22 & 29 & 35 & 34 & 27 & 25 & 25 & 25 & 22 & 21 & 34 & 33 & 29 \\ 322 & 257 & 163 & 213 & 255 & 251 & 196 & 185 & 185 & 185 & 162 & 153 & 250 & 244 & 213 \\ \mathbf{8 2 7} & \mathbf{6 6 0} & \mathbf{4 2 0} & \mathbf{5 4 7} & \mathbf{6 5 6} & \mathbf{6 4 6} & \mathbf{5 0 4} & \mathbf{4 7 4} & \mathbf{4 7 5} & \mathbf{4 7 6} & \mathbf{4 1 6} & \mathbf{3 9 4} & \mathbf{6 4 2} & \mathbf{6 2 7} & \mathbf{5 4 8}\end{array}$

-Rock Island PH\#1-

11 Rock Island PH\#1

12 Rock Island PH\#1 Before Enc

$142 \quad 148 \quad 108$

-Rock Island PH\#1-

13 CHPD Share

14 PSE Share

15 Rock Island PH\#1 After Enc

142148

$108 \quad 132$

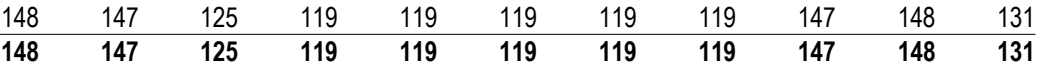

-Rock Island PH\#2-

16 Rock Island PH\#2

17 Rock Island PH\#2 Before Enc

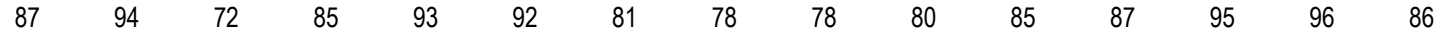

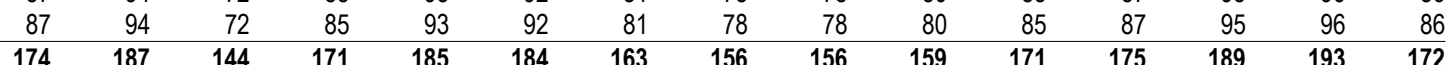

-Rock Island PH\#2-

18 CHPD Share

19 PSE Share

20 Rock Island PH\#2 After Enc

\begin{tabular}{lllllllllllllll}
161 & 116 & 75 & 104 & 129 & 127 & 94 & 86 & 86 & 72 & 29 & 18 & 93 & 80 & 90 \\
\hline
\end{tabular}

$\begin{array}{lllllllllllll}161 & 116 & 75 & 104 & 129 & 127 & 94 & 86 & 86 & 72 & 29 & 18 & 93\end{array}$

\begin{tabular}{|c|c|c|c|c|c|c|c|c|c|c|c|c|c|c|}
\hline 99 & 73 & 50 & 67 & 81 & 80 & 61 & 57 & 57 & 48 & 21 & 13 & 60 & 52 & 58 \\
\hline 99 & 73 & 50 & 67 & 81 & 80 & 61 & 57 & 57 & 48 & 21 & 13 & 60 & 52 & 58 \\
\hline
\end{tabular}




\section{White \\ 2003 White Book}

-Rocky Reach-

1 Rocky Reach

2 Rocky Reach Before Enc

Aug1 Aug16 S

Oct Nov Dec Jan Feb

eb Ma

Apr1 Apr16 May Jun Jul Avg

-Rocky Reach-

3 AVWP Share

4 CHPD Share

5 CLKM Share

6 DOPD Share

7 PGE Share

8 PPL Share

9 PSE Share

$808 \quad 641 \quad 403$

$808 \quad 641 \quad 403$

$528 \quad 637$

$\begin{array}{lllllllllll}637 & 628 & 487 & 458 & 459 & 460 & 400 & 378 & 624 & 609 & 531 \\ \mathbf{6 3 7} & \mathbf{6 2 8} & \mathbf{4 8 7} & \mathbf{4 5 8} & \mathbf{4 5 9} & \mathbf{4 6 0} & \mathbf{4 0 0} & \mathbf{3 7 8} & \mathbf{6 2 4} & \mathbf{6 0 9} & \mathbf{5 3 1}\end{array}$

10 Rocky Reach After Enc

\begin{tabular}{rrrrrrrrrrrrrrr}
24 & 19 & 12 & 16 & 19 & 19 & 15 & 14 & 14 & 14 & 12 & 11 & 19 & 18 & 16 \\
125 & 100 & 64 & 83 & 99 & 98 & 76 & 72 & 72 & 72 & 63 & 60 & 97 & 95 & 83 \\
190 & 152 & 97 & 126 & 151 & 149 & 116 & 109 & 109 & 109 & 96 & 91 & 148 & 144 & 126 \\
23 & 18 & 12 & 15 & 18 & 18 & 14 & 13 & 13 & 13 & 12 & 11 & 18 & 17 & 15 \\
99 & 79 & 50 & 66 & 79 & 78 & 60 & 57 & 57 & 57 & 50 & 47 & 77 & 75 & 66 \\
44 & 35 & 22 & 29 & 35 & 34 & 27 & 25 & 25 & 25 & 22 & 21 & 34 & 33 & 29 \\
322 & 257 & 163 & 213 & 255 & 251 & 196 & 185 & 185 & 185 & 162 & 153 & 250 & 244 & 213 \\
\hline $\mathbf{8 2 7}$ & $\mathbf{6 6 0}$ & $\mathbf{4 2 0}$ & $\mathbf{5 4 7}$ & $\mathbf{6 5 6}$ & $\mathbf{6 4 6}$ & $\mathbf{5 0 4}$ & $\mathbf{4 7 4}$ & $\mathbf{4 7 5}$ & $\mathbf{4 7 6}$ & $\mathbf{4 1 6}$ & $\mathbf{3 9 4}$ & $\mathbf{6 4 2}$ & $\mathbf{6 2 7}$ & $\mathbf{5 4 8}$
\end{tabular}

-Rock Island PH\#1-

11 Rock Island PH\#1

12 Rock Island PH\#1 Before Enc

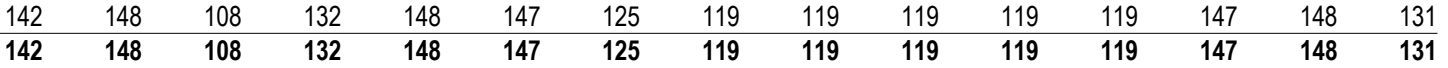

-Rock Island PH\#1-

13 CHPD Share

14 PSE Share

15 Rock Island PH\#1 After Enc

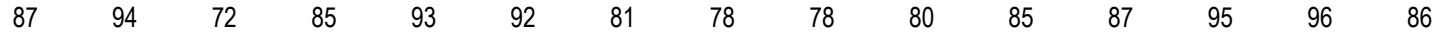

-Rock Island PH\#2-

16 Rock Island PH\#2

17 Rock Island PH\#2 Before Enc

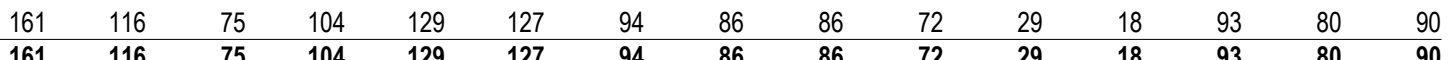

-Rock Island PH\#2-

18 CHPD Share

19 PSE Share

20 Rock Island PH\#2 After Enc

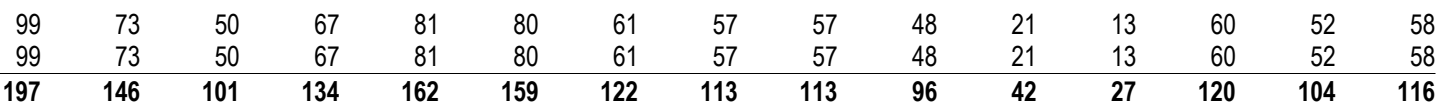




\section{White Book \\ 2003 White Book}

-Rocky Reach-

1 Rocky Reach

2 Rocky Reach Before Enc

Aug1 Aug16 S

Oct Nov Dec Jan Feb

$\begin{array}{lll}808 & 641 & 403 \\ 808 & 641 & 403\end{array}$

$\begin{array}{lll}403 & 528 & 637 \\ 403 & 528 & 637\end{array}$

$637 \quad 628$

$487 \quad 458$

$\begin{array}{llllllll}458 & 459 & 460 & 400 & 378 & 624 & 609 & 531 \\ \mathbf{4 5 8} & \mathbf{4 5 9} & \mathbf{4 6 0} & \mathbf{4 0 0} & \mathbf{3 7 8} & \mathbf{6 2 4} & \mathbf{6 0 9} & \mathbf{5 3 1}\end{array}$

-Rocky Reach-

3 AVWP Share

4 CHPD Share

sachs suma

6 DOPD Share

7 PGE Share

8 PPL Share

9 PSE Share

\begin{tabular}{|c|c|c|c|c|c|c|c|c|c|c|c|c|c|}
\hline 19 & 12 & 16 & 19 & 19 & 15 & 14 & 14 & 14 & 12 & 11 & 19 & 18 & 16 \\
\hline 100 & 64 & 83 & 99 & 98 & 76 & 72 & 72 & 72 & 63 & 60 & 97 & 95 & 83 \\
\hline 152 & 97 & 126 & 151 & 149 & 116 & 109 & 109 & 109 & 96 & 91 & 148 & 144 & 126 \\
\hline 18 & 12 & 15 & 18 & 18 & 14 & 13 & 13 & 13 & 12 & 11 & 18 & 17 & 15 \\
\hline 79 & 50 & 66 & 79 & 78 & 60 & 57 & 57 & 57 & 50 & 47 & 77 & 75 & 66 \\
\hline 35 & 22 & 29 & 35 & 34 & 27 & 25 & 25 & 25 & 22 & 21 & 34 & 33 & 29 \\
\hline 257 & 163 & 213 & 255 & 251 & 196 & 185 & 185 & 185 & 162 & 153 & 250 & 244 & 213 \\
\hline
\end{tabular}

-Rock Island PH\#1-

11 Rock Island PH\#1

12 Rock Island PH\#1 Before Enc

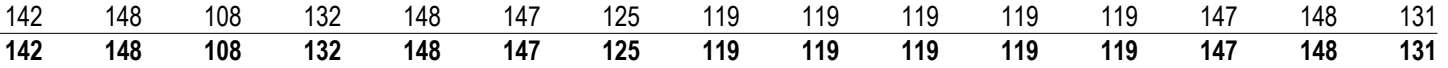

-Rock Island PH\#1-

13 CHPD Share

14 PSE Share

15 Rock Island PH\#1 After Enc

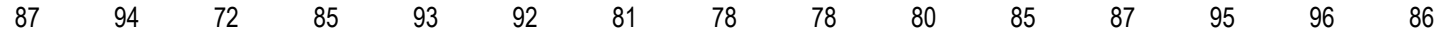

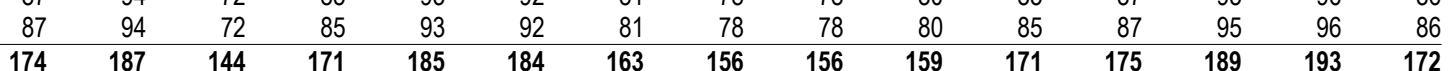

-Rock Island PH\#2-

16 Rock Island PH\#2

17 Rock Island PH\#2 Before Enc

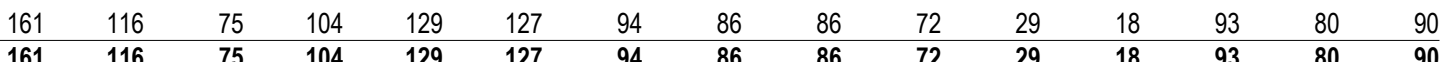

-Rock Island PH\#2-

18 CHPD Share

19 PSE Share

20 Rock Island PH\#2 After Enc

\begin{tabular}{|c|c|c|c|c|c|c|c|c|c|c|c|c|c|}
\hline 99 & 73 & 50 & 67 & 81 & 80 & 61 & 57 & 57 & 48 & 21 & 13 & 60 & 52 \\
\hline 99 & 73 & 50 & 67 & 81 & 80 & 61 & 57 & 57 & 48 & 21 & 13 & 60 & 52 \\
\hline 97 & 146 & 101 & 134 & 162 & 159 & 122 & 113 & 113 & 96 & 42 & 27 & 120 & 104 \\
\hline
\end{tabular}


2003 White Book

-Rocky Reach-

1 Rocky Reach

2 Rocky Reach Before Enc

Aug1 Aug16 S

Oct Nov Dec Jan Feb

$\begin{array}{lll}808 & 641 & 403 \\ 808 & 641 & 403\end{array}$

$528 \quad 637$

$\begin{array}{lll}628 & 487 & 458\end{array}$

Mar Apr1 Apr16 May Jun Jul Avg

-Rocky Reach-

3 AVWP Share

4 CHPD Share

5 CLKM Share

6 DOPD Share

7 PGE Share

8 PPL Share

9 PSE Share

10 Rocky Reach After Enc

\begin{tabular}{|c|c|c|c|c|c|c|c|c|c|c|c|c|c|c|}
\hline 24 & 19 & 12 & 16 & 19 & 19 & 15 & 14 & 14 & 14 & 12 & 11 & 19 & 18 & 16 \\
\hline 125 & 100 & 64 & 83 & 99 & 98 & 76 & 72 & 72 & 72 & 63 & 60 & 97 & 95 & 83 \\
\hline 190 & 152 & 97 & 126 & 151 & 149 & 116 & 109 & 109 & 109 & 96 & 91 & 148 & 144 & 126 \\
\hline 23 & 18 & 12 & 15 & 18 & 18 & 14 & 13 & 13 & 13 & 12 & 11 & 18 & 17 & 15 \\
\hline 99 & 79 & 50 & 66 & 79 & 78 & 60 & 57 & 57 & 57 & 50 & 47 & 77 & 75 & 66 \\
\hline 44 & 35 & 22 & 29 & 35 & 34 & 27 & 25 & 25 & 25 & 22 & 21 & 34 & 33 & 29 \\
\hline 322 & 257 & 163 & 213 & 255 & 251 & 196 & 185 & 185 & 185 & 162 & 153 & 250 & 244 & 213 \\
\hline 827 & 660 & 420 & 547 & 656 & 646 & 504 & 474 & 475 & 476 & 416 & 394 & 642 & 627 & 548 \\
\hline
\end{tabular}

-Rock Island PH\#1-

11 Rock Island PH\#1

12 Rock Island PH\#1 Before Enc

$142 \quad 148 \quad 108$

-Rock Island PH\#1-

13 CHPD Share

14 PSE Share

15 Rock Island PH\#1 After Enc

142148

132

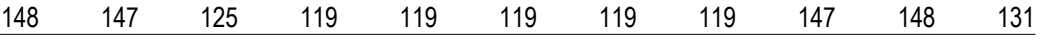

-Rock Island PH\#2-

16 Rock Island PH\#2

17 Rock Island PH\#2 Before Enc

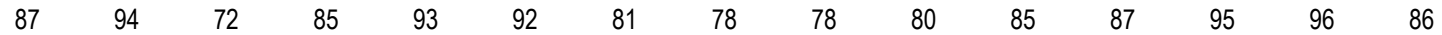

\begin{tabular}{|c|c|c|c|c|c|c|c|c|c|c|c|c|c|c|}
\hline 87 & 94 & 72 & 85 & 93 & 92 & 81 & 78 & 78 & 80 & 85 & 87 & 95 & 96 & 86 \\
\hline 174 & 187 & 111 & 174 & 105 & 100 & 10 & 450 & 450 & & & & & & \\
\hline
\end{tabular}

-Rock Island PH\#2-

18 CHPD Share

19 PSE Share

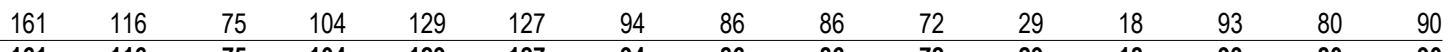

20 Rock Island PH\#2 After Enc

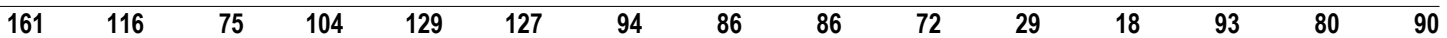

\begin{tabular}{|c|c|c|c|c|c|c|c|c|c|c|c|c|c|c|}
\hline 99 & 73 & 50 & 67 & 81 & 80 & 61 & 57 & 57 & 48 & 21 & 13 & 60 & 52 & 58 \\
\hline 99 & 73 & 50 & 67 & 81 & 80 & 61 & 57 & 57 & 48 & 21 & 13 & 60 & 52 & 58 \\
\hline
\end{tabular}


-Rocky Reach-

1 Rocky Reach

2 Rocky Reach Before Enc

Aug1 Aug16 S

Oct Nov Dec Jan Feb

$\begin{array}{lll}808 & 641 & 4 \\ 808 & 641 & 403\end{array}$

-Rocky Reach-

3 AVWP Share

4 CHPD Share

5 CLKM Share

6 DOPD Share

7 PGE Share

8 PPL Share

9 PSE Share

10 Rocky Reach After Enc

\begin{tabular}{|c|c|c|c|c|c|c|c|c|c|c|c|c|c|}
\hline 19 & 12 & 16 & 19 & 19 & 15 & 14 & 14 & 14 & 12 & 11 & 19 & 18 & 16 \\
\hline 100 & 64 & 83 & 99 & 98 & 76 & 72 & 72 & 72 & 63 & 60 & 97 & 95 & 83 \\
\hline 18 & 12 & 15 & 18 & 18 & 14 & 13 & 13 & 13 & 12 & 11 & 18 & 17 & 15 \\
\hline 35 & 22 & 29 & 35 & 34 & 27 & 25 & 25 & 25 & 22 & 21 & 34 & 33 & 29 \\
\hline 257 & 163 & 213 & 255 & 251 & 196 & 185 & 185 & 185 & 162 & 153 & 250 & 244 & 213 \\
\hline 660 & 420 & 547 & 656 & 646 & 504 & 474 & 475 & 476 & 416 & 394 & 642 & 627 & 548 \\
\hline
\end{tabular}

-Rock Island PH\#1-

11 Rock Island PH\#1

12 Rock Island PH\#1 Before Enc

$142 \quad 148 \quad 108$

-Rock Island PH\#1-

13 CHPD Share

14 PSE Share

15 Rock Island PH\#1 After Enc

$637 \quad 628$

$487 \quad 458$

\begin{tabular}{llllllll}
458 & 459 & 460 & 400 & 378 & 624 & 609 & 531 \\
\hline 458 & $\mathbf{4 5 9}$ & $\mathbf{4 6 0}$ & $\mathbf{4 0 0}$ & $\mathbf{3 7 8}$ & $\mathbf{6 2 4}$ & $\mathbf{6 0 9}$ & $\mathbf{5 3 1}$
\end{tabular}

-Rock Island PH\#2-

16 Rock Island PH\#2

17 Rock Island PH\#2 Before Enc

$142 \quad 148$

$\begin{array}{llll}108 & 132 & 148 & 147 \\ 108 & 132 & 148 & 147\end{array}$

$25 \quad 119$

$\begin{array}{lllllllllllllll}87 & 94 & 72 & 85 & 93 & 92 & 81 & 78 & 78 & 80 & 85 & 87 & 95 & 96 & 86\end{array}$

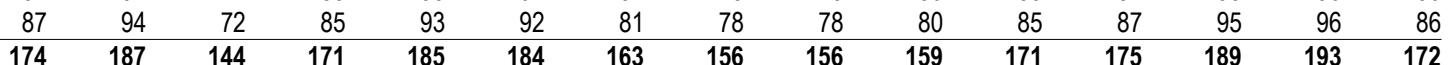

-Rock Island PH\#2-

18 CHPD Share

19 PSE Share

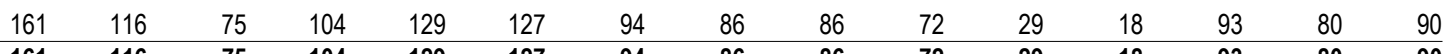

20 Rock Island PH\#2 After Enc

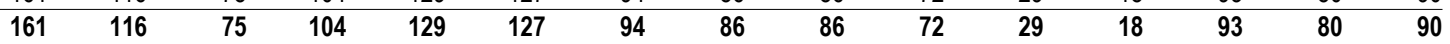

\begin{tabular}{|c|c|c|c|c|c|c|c|c|c|c|c|c|c|c|}
\hline 99 & 73 & 50 & 67 & 81 & 80 & 61 & 57 & 57 & 48 & 21 & 13 & 60 & 52 & 58 \\
\hline 99 & 73 & 50 & 67 & 81 & 80 & 61 & 57 & 57 & 48 & 21 & 13 & 60 & 52 & 58 \\
\hline
\end{tabular}


-Rocky Reach-

1 Rocky Reach

2 Rocky Reach Before Enc

Aug1 Aug16 S

Oct Nov Dec Jan Feb

$\begin{array}{lll}808 & 641 & 4 \\ 808 & 641 & 403\end{array}$

-Rocky Reach-

3 AVWP Share

4 CHPD Share

5 CLKM Share

6 DOPD Share

7 PGE Share

8 PPL Share

9 PSE Share

10 Rocky Reach After Enc

\begin{tabular}{|c|c|c|c|c|c|c|c|c|c|c|c|c|c|}
\hline 24 & 12 & 16 & 19 & 19 & 15 & 14 & 14 & 14 & 12 & 11 & 19 & 18 & 16 \\
\hline 100 & 64 & 83 & 99 & 98 & 76 & 72 & 72 & 72 & 63 & 60 & 97 & 95 & 83 \\
\hline 152 & 97 & 126 & 151 & 149 & 116 & 109 & 109 & 109 & 96 & 91 & 148 & 144 & 126 \\
\hline 18 & 12 & 15 & 18 & 18 & 14 & 13 & 13 & 13 & 12 & 11 & 18 & 17 & 15 \\
\hline 79 & 50 & 66 & 79 & 78 & 60 & 57 & 57 & 57 & 50 & 47 & 77 & 75 & 66 \\
\hline 35 & 22 & 29 & 35 & 34 & 27 & 25 & 25 & 25 & 22 & 21 & 34 & 33 & 29 \\
\hline 257 & 163 & 213 & 255 & 251 & 196 & 185 & 185 & 185 & 162 & 153 & 250 & 244 & 213 \\
\hline 660 & 420 & 547 & 656 & 646 & 504 & 474 & 475 & 476 & 416 & 394 & 642 & 627 & 548 \\
\hline
\end{tabular}

-Rock Island PH\#1-

11 Rock Island PH\#1

12 Rock Island PH\#1 Before Enc

$142 \quad 148 \quad 108$

-Rock Island PH\#1-

13 CHPD Share

14 PSE Share

15 Rock Island PH\#1 After Enc

$637 \quad 628$

$487 \quad 458$

Mar Apr1 Apr16 May Jun Jul Avg

-Rock Island PH\#2-

16 Rock Island PH\#2

17 Rock Island PH\#2 Before Enc

142148

132

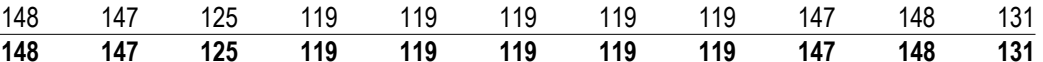

-Rock Island PH\#2-

18 CHPD Share

19 PSE Share

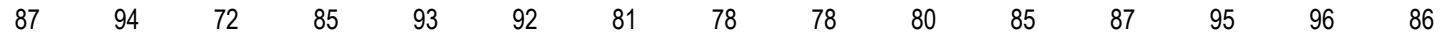

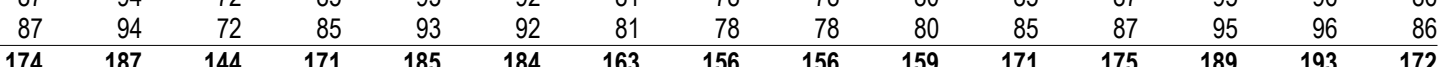

\begin{tabular}{|c|c|c|c|c|c|c|c|c|c|c|c|c|c|c|}
\hline 161 & 116 & 75 & 104 & 129 & 127 & 94 & 86 & 86 & 72 & 29 & 18 & 0 & 8 & 90 \\
\hline
\end{tabular}

$\begin{array}{lllllllllllll}161 & 116 & 75 & 104 & 129 & 127 & 94 & 86 & 86 & 72 & 29 & 18 & 93\end{array}$

\begin{tabular}{|c|c|c|c|c|c|c|c|c|c|c|c|c|c|c|}
\hline 99 & 73 & 50 & 67 & 81 & 80 & 61 & 57 & 57 & 48 & 21 & 13 & 60 & 52 & 58 \\
\hline 99 & 73 & 50 & 67 & 81 & 80 & 61 & 57 & 57 & 48 & 21 & 13 & 60 & 52 & 58 \\
\hline
\end{tabular}


-Rocky Reach-

1 Rocky Reach

2 Rocky Reach Before Enc

Aug1 Aug16 S

2003 White Book

-Rocky Reach-

3 AVWP Share

4 CHPD Share

5 CLKM Share

6 DOPD Share

7 PGE Share

8 PPL Share

9 PSE Share

10 Rocky Reach After Enc

$808 \quad 641 \quad 403$

$808 \quad 641 \quad 403$

Oct Nov Dec Jan Fel

Mar Apr1 Apr16 May Jun Jul Avg

-Rock Island PH\#1-

11 Rock Island PH\#1

12 Rock Island PH\#1 Before Enc

$\begin{array}{rrrrrrrrrrrrrrr}24 & 19 & 12 & 16 & 19 & 19 & 15 & 14 & 14 & 14 & 12 & 11 & 19 & 18 & 16 \\ 125 & 100 & 64 & 83 & 99 & 98 & 76 & 72 & 72 & 72 & 63 & 60 & 97 & 95 & 83 \\ 190 & 152 & 97 & 126 & 151 & 149 & 116 & 109 & 109 & 109 & 96 & 91 & 148 & 144 & 126 \\ 23 & 18 & 12 & 15 & 18 & 18 & 14 & 13 & 13 & 13 & 12 & 11 & 18 & 17 & 15 \\ 99 & 79 & 50 & 66 & 79 & 78 & 60 & 57 & 57 & 57 & 50 & 47 & 77 & 75 & 66 \\ 44 & 35 & 22 & 29 & 35 & 34 & 27 & 25 & 25 & 25 & 22 & 21 & 34 & 33 & 29 \\ 322 & 257 & 163 & 213 & 255 & 251 & 196 & 185 & 185 & 185 & 162 & 153 & 250 & 244 & 213 \\ \mathbf{8 2 7} & \mathbf{6 6 0} & \mathbf{4 2 0} & \mathbf{5 4 7} & \mathbf{6 5 6} & \mathbf{6 4 6} & \mathbf{5 0 4} & \mathbf{4 7 4} & \mathbf{4 7 5} & \mathbf{4 7 6} & \mathbf{4 1 6} & \mathbf{3 9 4} & \mathbf{6 4 2} & \mathbf{6 2 7} & \mathbf{5 4 8}\end{array}$

-Rock Island PH\#1-

13 CHPD Share

14 PSE Share

15 Rock Island PH\#1 After Enc

$637 \quad 628$

$487 \quad 458$

$458 \quad 459$

$\begin{array}{llllll}460 & 400 & 378 & 624 & 609 & 531\end{array}$

-Rock Island PH\#2-

16 Rock Island PH\#2

17 Rock Island PH\#2 Before Enc

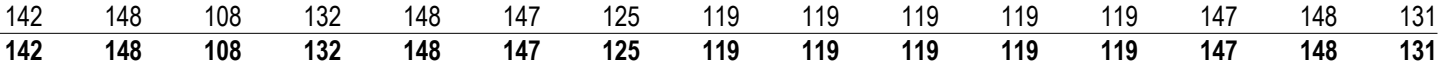

-Rock Island PH\#2-

18 CHPD Share

19 PSE Share

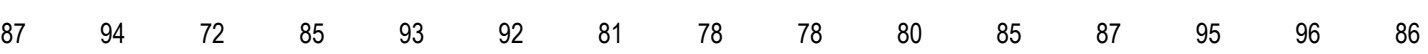

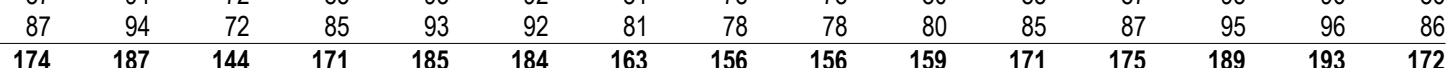

20 Rock Island PH\#2 After Enc

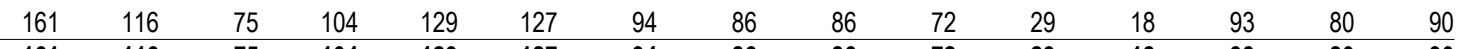

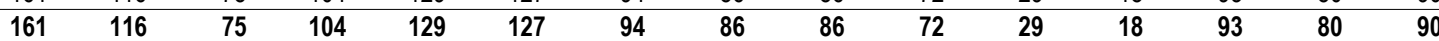

\begin{tabular}{|c|c|c|c|c|c|c|c|c|c|c|c|c|c|c|}
\hline 99 & 73 & 50 & 67 & 81 & 80 & 61 & 57 & 57 & 48 & 21 & 13 & 60 & 52 & 58 \\
\hline 99 & 73 & 50 & 67 & 81 & 80 & 61 & 57 & 57 & 48 & 21 & 13 & 60 & 52 & 58 \\
\hline 197 & 146 & 101 & 134 & 162 & 159 & 122 & 113 & 113 & 96 & 42 & 27 & 120 & 104 & 116 \\
\hline
\end{tabular}


Water Year

Average Energy in Megawatts Aug1 Aug16 Sep Oct Nov Dec Jan Feb Mar Apr1 Apr16 May Jun Jul Avg -Wanapum-

1 Wanapum

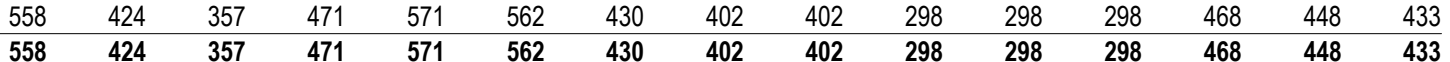

-Wanapum-

3 AVWP Share

4 COPD Share

5 CWPC Share

6 EWEB Share

7 FGRV Share

8 FREC Share

9 GCPD Share

10 ICLP Share

$11 \mathrm{KITT}$ Share

12 KOOT Share

13 LREC Share

14 LVE Share

15 MCMN Share

16 MTFR Share

17 NLEC Share

18 PGE Share

19 PPL Share

20 PSE Share

21 RREC Share

22 SCL Share

23 SLEC Share

24 TPU Share

25 UNEC Share

$\begin{array}{rrrrrrrrrrrrrrr}40 & 29 & 24 & 33 & 41 & 40 & 29 & 27 & 27 & 19 & 19 & 19 & 32 & 31 & 30 \\ 13 & 9.5 & 7.8 & 11 & 13 & 13 & 9.7 & 9.0 & 9.0 & 6.2 & 6.2 & 6.2 & 11 & 10 & 9.8 \\ 0 & 0 & 0 & 0 & 0 & 0 & 0 & 0 & 0 & 0 & 0 & 0 & 0 & 0 & 0 \\ 11 & 8.1 & 6.7 & 9.2 & 11 & 11 & 8.3 & 7.7 & 7.7 & 5.3 & 5.3 & 5.3 & 9.1 & 8.6 & 8.3 \\ 3.4 & 2.5 & 2 & 2.8 & 3.5 & 3.4 & 2.5 & 2.3 & 2.3 & 1.6 & 1.6 & 1.6 & 2.8 & 2.6 & 2.5 \\ 0 & 0 & 0 & 0 & 0 & 0 & 0 & 0 & 0 & 0 & 0 & 0 & 0 & 0 & 0 \\ 177 & 128 & 106 & 145 & 181 & 178 & 131 & 121 & 121 & 84 & 84 & 83 & 144 & 137 & 132 \\ 0 & 0 & 0 & 0 & 0 & 0 & 0 & 0 & 0 & 0 & 0 & 0 & 0 & 0 & 0 \\ 0 & 0 & 0 & 0 & 0 & 0 & 0 & 0 & 0 & 0 & 0 & 0 & 0 & 0 & 0 \\ 0 & 0 & 0 & 0 & 0 & 0 & 0 & 0 & 0 & 0 & 0 & 0 & 0 & 0 & 0 \\ 0 & 0 & 0 & 0 & 0 & 0 & 0 & 0 & 0 & 0 & 0 & 0 & 0 & 0 & 0 \\ 0 & 0 & 0 & 0 & 0 & 0 & 0 & 0 & 0 & 0 & 0 & 0 & 0 & 0 & 0 \\ 3.4 & 2.5 & 2 & 2.8 & 3.5 & 3.4 & 2.5 & 2.3 & 2.3 & 1.6 & 1.6 & 1.6 & 2.8 & 2.6 & 2.5 \\ 3.4 & 2.5 & 2 & 2.8 & 3.5 & 3.4 & 2.5 & 2.3 & 2.3 & 1.6 & 1.6 & 1.6 & 2.8 & 2.6 & 2.5 \\ 0 & 0 & 0 & 0 & 0 & 0 & 0 & 0 & 0 & 0 & 0 & 0 & 0 & 0 & 0 \\ 90 & 66 & 54 & 74 & 93 & 91 & 67 & 62 & 62 & 43 & 43 & 43 & 74 & 70 & 68 \\ 90 & 66 & 54 & 74 & 93 & 91 & 67 & 62 & 62 & 43 & 43 & 43 & 74 & 70 & 68 \\ 52 & 38 & 31 & 43 & 54 & 53 & 39 & 36 & 36 & 25 & 25 & 25 & 43 & 41 & 39 \\ 0 & 0 & 0 & 0 & 0 & 0 & 0 & 0 & 0 & 0 & 0 & 0 & 0 & 0 & 0 \\ 0 & 0 & 0 & 0 & 0 & 0 & 0 & 0 & 0 & 0 & 0 & 0 & 0 & 0 & 0 \\ 0 & 0 & 0 & 0 & 0 & 0 & 0 & 0 & 0 & 0 & 0 & 0 & 0 & 0 & 0 \\ 0 & 0 & 0 & 0 & 0 & 0 & 0 & 0 & 0 & 0 & 0 & 0 & 0 & 0 & 0 \\ 0 & 0 & 0 & 0 & 0 & 0 & 0 & 0 & 0 & 0 & 0 & 0 & 0 & 0 & 0 \\ 484 & 351 & 290 & 398 & 497 & 488 & 359 & 333 & 333 & \mathbf{2 2 9} & \mathbf{2 2 9} & \mathbf{2 2 9} & \mathbf{3 9 5} & \mathbf{3 7 5} & \mathbf{3 6 2}\end{array}$

-Priest Rapids.

27 Priest Rapids

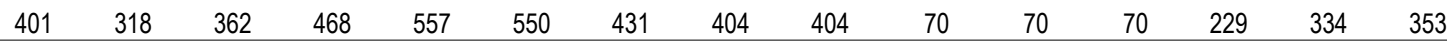

28 Priest Rapids Before Enc

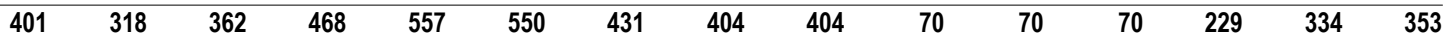

-Priest Rapids-

29 AVWP Share

30 COPD Share

31 CWPC Share

32 EWEB Share

33 FGRV Share

34 FREC Share

35 GCPD Share

36 ICLP Share

37 KITT Share

38 KOOT Share

39 LREC Share

40 LVE Share

41 MCMN Share

42 MTFR Share

43 NLEC Share

44 PGE Share

45 PPL Share

46 PSE Share

47 RREC Share

48 SCL Share

49 SLEC Share

50 TPU Share

51 UNEC Share

$\begin{array}{rrrrrrrrrrrrrrr}\mathbf{4 0 1} & \mathbf{3 1 8} & \mathbf{3 6 8} & \mathbf{5 5} & \mathbf{5 5} & \mathbf{4 3 1} & \mathbf{4 0 4} & \mathbf{4 0 4} & \mathbf{7 0} & \mathbf{7 0} & \mathbf{7 0} & \mathbf{2 2 9} & \mathbf{3 3 4} & \mathbf{3 5 3} \\ & & & & & & & & & & & & & \\ 23 & 18 & 21 & 28 & 33 & 33 & 25 & 24 & 24 & 3.3 & 3.3 & 3.3 & 13 & 19 & 21 \\ 7.7 & 6 & 6.9 & 9 & 11 & 11 & 8.3 & 7.8 & 7.8 & 1.1 & 1.1 & 1.1 & 4.2 & 6.3 & 6.7 \\ 0 & 0 & 0 & 0 & 0 & 0 & 0 & 0 & 0 & 0 & 0 & 0 & 0 & 0 & 0 \\ 6.5 & 5.1 & 5.9 & 7.7 & 9.2 & 9.1 & 7.1 & 6.6 & 6.6 & 0.9 & 0.9 & 0.9 & 3.6 & 5.4 & 5.7 \\ 1.9 & 1.5 & 1.7 & 2.3 & 2.7 & 2.7 & 2.1 & 1.9 & 1.9 & 0.3 & 0.3 & 0.3 & 1.1 & 1.6 & 1.7 \\ 0 & 0 & 0 & 0 & 0 & 0 & 0 & 0 & 0 & 0 & 0 & 0 & 0 & 0 & 0 \\ 140 & 110 & 127 & 165 & 197 & 195 & 152 & 142 & 142 & 20 & 20 & 20 & 77 & 116 & 123 \\ 0 & 0 & 0 & 0 & 0 & 0 & 0 & 0 & 0 & 0 & 0 & 0 & 0 & 0 & 0 \\ 1.5 & 1.2 & 1.4 & 1.8 & 2.2 & 2.1 & 1.7 & 1.6 & 1.6 & 0.2 & 0.2 & 0.2 & 0.8 & 1.3 & 1.3 \\ 0 & 0 & 0 & 0 & 0 & 0 & 0 & 0 & 0 & 0 & 0 & 0 & 0 & 0 & 0 \\ 0 & 0 & 0 & 0 & 0 & 0 & 0 & 0 & 0 & 0 & 0 & 0 & 0 & 0 & 0 \\ 0 & 0 & 0 & 0 & 0 & 0 & 0 & 0 & 0 & 0 & 0 & 0 & 0 & 0 & 0 \\ 1.9 & 1.5 & 1.7 & 2.3 & 2.7 & 2.7 & 2.1 & 1.9 & 1.9 & 0.3 & 0.3 & 0.3 & 1.1 & 1.6 & 1.7 \\ 1.9 & 1.5 & 1.7 & 2.3 & 2.7 & 2.7 & 2.1 & 1.9 & 1.9 & 0.3 & 0.3 & 0.3 & 1.1 & 1.6 & 1.7 \\ 0 & 0 & 0 & 0 & 0 & 0 & 0 & 0 & 0 & 0 & 0 & 0 & 0 & 0 & 0 \\ 53 & 42 & 48 & 63 & 75 & 74 & 58 & 54 & 54 & 7.6 & 7.6 & 7.6 & 29 & 44 & 47 \\ 53 & 42 & 48 & 63 & 75 & 74 & 58 & 54 & 54 & 7.6 & 7.6 & 7.6 & 29 & 44 & 47 \\ 31 & 24 & 28 & 36 & 43 & 43 & 33 & 31 & 31 & 4.4 & 4.4 & 4.4 & 17 & 25 & 27 \\ 0 & 0 & 0 & 0 & 0 & 0 & 0 & 0 & 0 & 0 & 0 & 0 & 0 & 0 & 0 \\ 31 & 24 & 28 & 36 & 43 & 43 & 33 & 31 & 31 & 4.4 & 4.4 & 4.4 & 17 & 25 & 27 \\ 0 & 0 & 0 & 0 & 0 & 0 & 0 & 0 & 0 & 0 & 0 & 0 & 0 & 0 & 0 \\ 31 & 24 & 28 & 36 & 43 & 43 & 33 & 31 & 31 & 4.4 & 4.4 & 4.4 & 17 & 25 & 27 \\ 0 & 0 & 0 & 0 & 0 & 0 & 0 & 0 & 0 & 0 & 0 & 0 & 0 & 0 & 0 \\ 385 & 301 & 347 & 452 & \mathbf{5 4 1} & \mathbf{5 3 3} & \mathbf{4 1 6} & \mathbf{3 8 9} & \mathbf{3 8 9} & \mathbf{5 5} & \mathbf{5 5} & \mathbf{5 5} & \mathbf{2 1 2} & \mathbf{3 1 7} & \mathbf{3 3 7}\end{array}$


Water Year

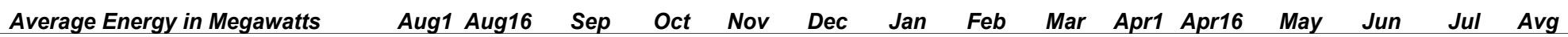
-Wanapum-

1 Wanapum

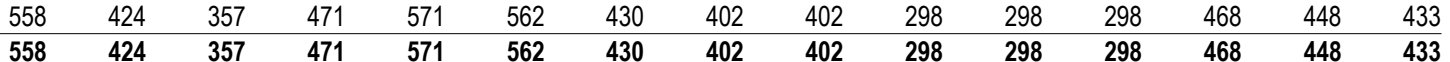

-Wanapum-

3 AVWP Share

4 COPD Share

5 CWPC Share

6 EWEB Share

7 FGRV Share

8 FREC Share

9 GCPD Share

10 ICLP Share

$11 \mathrm{KITT}$ Share

12 KOOT Share

13 LREC Share

14 LVE Share

15 MCMN Share

16 MTFR Share

17 NLEC Share

18 PGE Share

19 PPL Share

20 PSE Share

21 RREC Share

22 SCL Share

23 SLEC Share

24 TPU Share

25 UNEC Share

$\begin{array}{rrrrrrrrrrrrrrr}40 & 29 & 24 & 33 & 41 & 40 & 29 & 27 & 27 & 19 & 19 & 19 & 32 & 31 & 30 \\ 13 & 9.5 & 7.8 & 11 & 13 & 13 & 9.7 & 9.0 & 9.0 & 6.2 & 6.2 & 6.2 & 11 & 10 & 9.8 \\ 0 & 0 & 0 & 0 & 0 & 0 & 0 & 0 & 0 & 0 & 0 & 0 & 0 & 0 & 0 \\ 11 & 8.1 & 6.7 & 9.2 & 11 & 11 & 8.3 & 7.7 & 7.7 & 5.3 & 5.3 & 5.3 & 9.1 & 8.6 & 8.3 \\ 3.4 & 2.5 & 2 & 2.8 & 3.5 & 3.4 & 2.5 & 2.3 & 2.3 & 1.6 & 1.6 & 1.6 & 2.8 & 2.6 & 2.5 \\ 0 & 0 & 0 & 0 & 0 & 0 & 0 & 0 & 0 & 0 & 0 & 0 & 0 & 0 & 0 \\ 177 & 128 & 106 & 145 & 181 & 178 & 131 & 121 & 121 & 84 & 84 & 83 & 144 & 137 & 132 \\ 0 & 0 & 0 & 0 & 0 & 0 & 0 & 0 & 0 & 0 & 0 & 0 & 0 & 0 & 0 \\ 0 & 0 & 0 & 0 & 0 & 0 & 0 & 0 & 0 & 0 & 0 & 0 & 0 & 0 & 0 \\ 0 & 0 & 0 & 0 & 0 & 0 & 0 & 0 & 0 & 0 & 0 & 0 & 0 & 0 & 0 \\ 0 & 0 & 0 & 0 & 0 & 0 & 0 & 0 & 0 & 0 & 0 & 0 & 0 & 0 & 0 \\ 0 & 0 & 0 & 0 & 0 & 0 & 0 & 0 & 0 & 0 & 0 & 0 & 0 & 0 & 0 \\ 3.4 & 2.5 & 2 & 2.8 & 3.5 & 3.4 & 2.5 & 2.3 & 2.3 & 1.6 & 1.6 & 1.6 & 2.8 & 2.6 & 2.5 \\ 3.4 & 2.5 & 2 & 2.8 & 3.5 & 3.4 & 2.5 & 2.3 & 2.3 & 1.6 & 1.6 & 1.6 & 2.8 & 2.6 & 2.5 \\ 0 & 0 & 0 & 0 & 0 & 0 & 0 & 0 & 0 & 0 & 0 & 0 & 0 & 0 & 0 \\ 90 & 66 & 54 & 74 & 93 & 91 & 67 & 62 & 62 & 43 & 43 & 43 & 74 & 70 & 68 \\ 90 & 66 & 54 & 74 & 93 & 91 & 67 & 62 & 62 & 43 & 43 & 43 & 74 & 70 & 68 \\ 52 & 38 & 31 & 43 & 54 & 53 & 39 & 36 & 36 & 25 & 25 & 25 & 43 & 41 & 39 \\ 0 & 0 & 0 & 0 & 0 & 0 & 0 & 0 & 0 & 0 & 0 & 0 & 0 & 0 & 0 \\ 0 & 0 & 0 & 0 & 0 & 0 & 0 & 0 & 0 & 0 & 0 & 0 & 0 & 0 & 0 \\ 0 & 0 & 0 & 0 & 0 & 0 & 0 & 0 & 0 & 0 & 0 & 0 & 0 & 0 & 0 \\ 0 & 0 & 0 & 0 & 0 & 0 & 0 & 0 & 0 & 0 & 0 & 0 & 0 & 0 & 0 \\ 0 & 0 & 0 & 0 & 0 & 0 & 0 & 0 & 0 & 0 & 0 & 0 & 0 & 0 & 0 \\ 484 & 351 & 290 & 398 & 497 & 488 & 359 & 333 & 333 & \mathbf{2 2 9} & \mathbf{2 2 9} & \mathbf{2 2 9} & \mathbf{3 9 5} & \mathbf{3 7 5} & \mathbf{3 6 2}\end{array}$

-Priest Rapids-

27 Priest Rapids

28 Priest Rapids Before Enc

\begin{tabular}{lllllllllllllll}
401 & 318 & 362 & 468 & 557 & 550 & 431 & 404 & 404 & 70 & 70 & 70 & 229 & 334 & 353 \\
\hline $\mathbf{4 0 1}$ & $\mathbf{3 1 8}$ & $\mathbf{3 6 2}$ & $\mathbf{4 6 8}$ & $\mathbf{5 5 7}$ & $\mathbf{5 5 0}$ & $\mathbf{4 3 1}$ & $\mathbf{4 0 4}$ & $\mathbf{4 0 4}$ & $\mathbf{7 0}$ & $\mathbf{7 0}$ & $\mathbf{7 0}$ & $\mathbf{2 2 9}$ & $\mathbf{3 3 4}$ & $\mathbf{3 5 3}$
\end{tabular}

-Priest Rapids-

29 AVWP Share

30 COPD Share

31 CWPC Share

32 EWEB Share

33 FGRV Share

34 FREC Share

35 GCPD Share

36 ICLP Share

$37 \mathrm{KITT}$ Share

38 KOOT Share

39 LREC Share

40 LVE Share

41 MCMN Share

42 MTFR Share

43 NLEC Share

44 PGE Share

45 PPL Share

46 PSE Share

47 RREC Share

$48 \mathrm{SCL}$ Share

49 SLEC Share

50 TPU Share

51 UNEC Share

52 Priest Rapids After Enc

\begin{tabular}{|c|c|c|c|c|c|c|c|c|c|c|c|c|c|c|}
\hline 23 & 18 & 21 & 28 & 16 & 15 & 12 & 11 & 11 & 1.6 & 1.6 & 1.6 & 6.1 & 9.1 & 13 \\
\hline 7.7 & 6 & 6.9 & 9 & 5.1 & 5 & 3.9 & 3.7 & 3.7 & 0.5 & 0.5 & 0.5 & 2 & 3.0 & 4.2 \\
\hline 0 & 0 & 0 & 0 & 0.4 & 0.4 & 0.3 & 0.3 & 0.3 & 0 & 0 & 0 & 0.2 & 0.2 & 0.2 \\
\hline 6.5 & 5.1 & 5.9 & 7.7 & 4.3 & 4.3 & 3.3 & 3.1 & 3.1 & 0.4 & 0.4 & 0.4 & 1.7 & 2.5 & 3.6 \\
\hline 1.9 & 1.5 & 1.7 & 2.3 & 1.3 & 1.3 & 1.0 & 0.9 & 0.9 & 0.1 & 0.1 & 0.1 & 0.5 & 0.8 & 1 \\
\hline 0 & 0 & 0 & 0 & 0.5 & 0.5 & 0.4 & 0.3 & 0.3 & 0 & 0 & 0 & 0.2 & 0.3 & 0.2 \\
\hline 140 & 110 & 127 & 165 & 378 & 373 & 291 & 272 & 272 & 38 & 38 & 38 & 149 & 222 & 204 \\
\hline 0 & 0 & 0 & 0 & 0.1 & 0.1 & 0.1 & 0.1 & 0.1 & 0 & 0 & 0 & 0.1 & 0.1 & 0.1 \\
\hline 1.5 & 1.2 & 1.4 & 1.8 & 0.9 & 0.9 & 0.7 & 0.7 & 0.7 & 0.1 & 0.1 & 0.1 & 0.4 & 0.5 & 0.8 \\
\hline 0 & 0 & 0 & 0 & 0.7 & 0.7 & 0.6 & 0.5 & 0.5 & 0.1 & 0.1 & 0.1 & 0.3 & 0.4 & 0.3 \\
\hline 0 & 0 & 0 & 0 & 0.1 & 0.1 & 0.1 & 0.1 & 0.1 & 0 & 0 & 0 & 0 & 0.1 & 0 \\
\hline 0 & 0 & 0 & 0 & 0.8 & 0.8 & 0.6 & 0.6 & 0.6 & 0.1 & 0.1 & 0.1 & 0.3 & 0.5 & 0.4 \\
\hline 1.9 & 1.5 & 1.7 & 2.3 & 1.3 & 1.3 & 1.0 & 0.9 & 0.9 & 0.1 & 0.1 & 0.1 & 0.5 & 0.8 & 1 \\
\hline 1.9 & 1.5 & 1.7 & 2.3 & 1.3 & 1.3 & 1.0 & 0.9 & 0.9 & 0.1 & 0.1 & 0.1 & 0.5 & 0.8 & 1 \\
\hline 0 & 0 & 0 & 0 & 0.6 & 0.6 & 0.5 & 0.5 & 0.5 & 0.1 & 0.1 & 0.1 & 0.3 & 0.4 & 0.3 \\
\hline 53 & 42 & 48 & 63 & 35 & 35 & 27 & 25 & 25 & 3.6 & 3.6 & 3.6 & 14 & 21 & 29 \\
\hline 53 & 42 & 48 & 63 & 35 & 35 & 27 & 25 & 25 & 3.6 & 3.6 & 3.6 & 14 & 21 & 29 \\
\hline 31 & 24 & 28 & 36 & 20 & 20 & 16 & 15 & 15 & 2.1 & 2.1 & 2.1 & 8 & 12 & 17 \\
\hline 0 & 0 & 0 & 0 & 0.1 & 0.1 & 0.1 & 0.1 & 0.1 & 0 & 0 & 0 & 0.1 & 0.1 & 0.1 \\
\hline 31 & 24 & 28 & 36 & 19 & 18 & 14 & 13 & 13 & 1.9 & 1.9 & 1.9 & 7.4 & 11 & 16 \\
\hline 0 & 0 & 0 & 0 & 0.1 & 0.1 & 0.1 & 0.1 & 0.1 & 0 & 0 & 0 & 0 & 0.1 & 0.1 \\
\hline 31 & 24 & 28 & 36 & 19 & 18 & 14 & 13 & 13 & 1.9 & 1.9 & 1.9 & 7.4 & 11 & 16 \\
\hline 0 & 0 & 0 & 0 & 0.2 & 0.2 & 0.2 & 0.2 & 0.2 & 0 & 0 & 0 & 0.1 & 0.1 & 0.1 \\
\hline 385 & 301 & 347 & 452 & 541 & 533 & 416 & 389 & 389 & 55 & 55 & 55 & 212 & 317 & 337 \\
\hline
\end{tabular}


Water Year

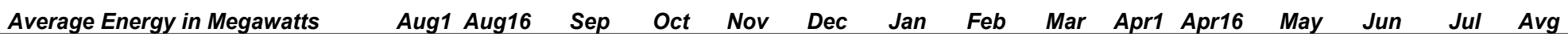
-Wanapum-

1 Wanapum

2 Wanapum Before Enc

558 $471 \quad 571$

$571 \quad 562$

$\begin{array}{llllllllll}562 & 430 & 402 & 402 & 298 & 298 & 298 & 468 & 448 & 433 \\ \mathbf{5 6 2} & \mathbf{4 3 0} & \mathbf{4 0 2} & \mathbf{4 0 2} & \mathbf{2 9 8} & \mathbf{2 9 8} & \mathbf{2 9 8} & \mathbf{4 6 8} & \mathbf{4 4 8} & \mathbf{4 3 3}\end{array}$

-Wanapum-

3 AVWP Share

4 COPD Share

5 CWPC Share

6 EWEB Share

7 FGRV Share

8 FREC Share

9 GCPD Share

10 ICLP Share

$11 \mathrm{KITT}$ Share

12 KOOT Share

13 LREC Share

14 LVE Share

15 MCMN Share

16 MTFR Share

17 NLEC Share

18 PGE Share

19 PPL Share

20 PSE Share

21 RREC Share

22 SCL Share

23 SLEC Share

24 TPU Share

25 UNEC Share

\begin{tabular}{|c|c|c|c|c|c|c|c|c|c|c|c|c|c|c|}
\hline 40 & 29 & 24 & 33 & 41 & 40 & 29 & 27 & 27 & 19 & 19 & 19 & 32 & 31 & 30 \\
\hline 13 & 9.5 & 7.8 & 11 & 13 & 13 & 9.7 & 9.0 & 9.0 & 6.2 & 6.2 & 6.2 & 11 & 10 & 9.8 \\
\hline 0 & 0 & 0 & 0 & 0 & 0 & 0 & 0 & 0 & 0 & 0 & 0 & 0 & 0 & 0 \\
\hline 11 & 8.1 & 6.7 & 9.2 & 11 & 11 & 8.3 & 7.7 & 7.7 & 5.3 & 5.3 & 5.3 & 9.1 & 8.6 & 8.3 \\
\hline 3.4 & 2.5 & 2 & 2.8 & 3.5 & 3.4 & 2.5 & 2.3 & 2.3 & 1.6 & 1.6 & 1.6 & 2.8 & 2.6 & 2.5 \\
\hline 0 & 0 & 0 & 0 & 0 & 0 & 0 & 0 & 0 & 0 & 0 & 0 & 0 & 0 & 0 \\
\hline 177 & 128 & 106 & 145 & 181 & 178 & 131 & 121 & 121 & 84 & 84 & 83 & 144 & 137 & 132 \\
\hline 0 & 0 & 0 & 0 & 0 & 0 & 0 & 0 & 0 & 0 & 0 & 0 & 0 & 0 & 0 \\
\hline 0 & 0 & 0 & 0 & 0 & 0 & 0 & 0 & 0 & 0 & 0 & 0 & 0 & 0 & 0 \\
\hline 0 & 0 & 0 & 0 & 0 & 0 & 0 & 0 & 0 & 0 & 0 & 0 & 0 & 0 & 0 \\
\hline 0 & 0 & 0 & 0 & 0 & 0 & 0 & 0 & 0 & 0 & 0 & 0 & 0 & 0 & 0 \\
\hline 0 & 0 & 0 & 0 & 0 & 0 & 0 & 0 & 0 & 0 & 0 & 0 & 0 & 0 & 0 \\
\hline 3.4 & 2.5 & 2 & 2.8 & 3.5 & 3.4 & 2.5 & 2.3 & 2.3 & 1.6 & 1.6 & 1.6 & 2.8 & 2.6 & 2.5 \\
\hline 3.4 & 2.5 & 2 & 2.8 & 3.5 & 3.4 & 2.5 & 2.3 & 2.3 & 1.6 & 1.6 & 1.6 & 2.8 & 2.6 & 2.5 \\
\hline 0 & 0 & 0 & 0 & 0 & 0 & 0 & 0 & 0 & 0 & 0 & 0 & 0 & 0 & 0 \\
\hline 90 & 66 & 54 & 74 & 93 & 91 & 67 & 62 & 62 & 43 & 43 & 43 & 74 & 70 & 68 \\
\hline 90 & 66 & 54 & 74 & 93 & 91 & 67 & 62 & 62 & 43 & 43 & 43 & 74 & 70 & 68 \\
\hline 52 & 38 & 31 & 43 & 54 & 53 & 39 & 36 & 36 & 25 & 25 & 25 & 43 & 41 & 39 \\
\hline 0 & 0 & 0 & 0 & 0 & 0 & 0 & 0 & 0 & 0 & 0 & 0 & 0 & 0 & 0 \\
\hline 0 & 0 & 0 & 0 & 0 & 0 & 0 & 0 & 0 & 0 & 0 & 0 & 0 & 0 & 0 \\
\hline 0 & 0 & 0 & 0 & 0 & 0 & 0 & 0 & 0 & 0 & 0 & 0 & 0 & 0 & 0 \\
\hline 0 & 0 & 0 & 0 & 0 & 0 & 0 & 0 & 0 & 0 & 0 & 0 & 0 & 0 & 0 \\
\hline 0 & 0 & 0 & 0 & 0 & 0 & 0 & 0 & 0 & 0 & 0 & 0 & 0 & 0 & 0 \\
\hline 484 & 351 & 290 & 398 & 497 & 488 & 359 & 333 & 333 & 229 & 229 & 229 & 395 & 375 & 362 \\
\hline
\end{tabular}

-Priest Rapids-

27 Priest Rapids

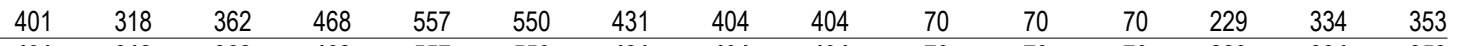

28 Priest Rapids Before Enc

$\begin{array}{lllllllllllllll}401 & 318 & 362 & 468 & 557 & 550 & 431 & 404 & 404 & 70 & 70 & 70 & 229 & 334 & 353\end{array}$

-Priest Rapids-

29 AVWP Share

30 COPD Share

31 CWPC Share

32 EWEB Share

33 FGRV Share

34 FREC Share

35 GCPD Share

36 ICLP Share

37 KITT Share

38 KOOT Share

39 LREC Share

40 LVE Share

41 MCMN Share

42 MTFR Share

43 NLEC Share

44 PGE Share

45 PPL Share

46 PSE Share

47 RREC Share

$48 \mathrm{SCL}$ Share

49 SLEC Share

50 TPU Share

51 UNEC Share

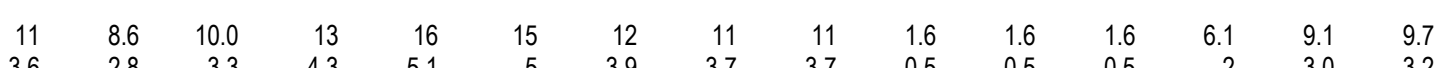

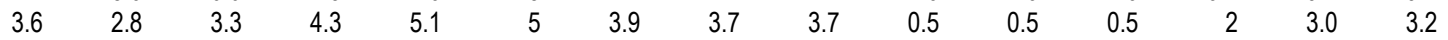

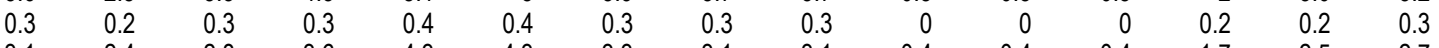

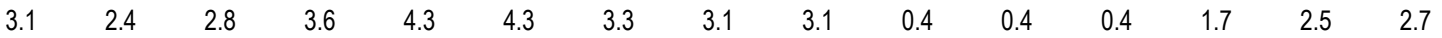

52 Priest Rapids After Enc

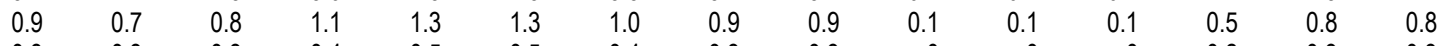

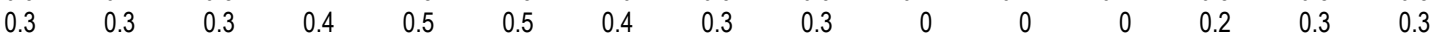

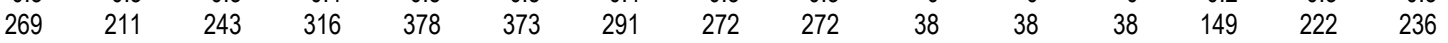

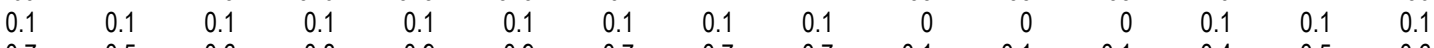

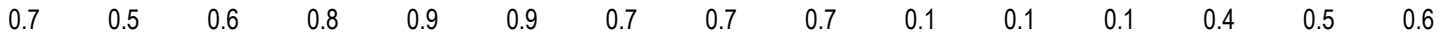

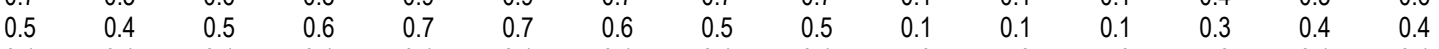

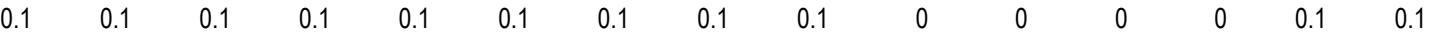

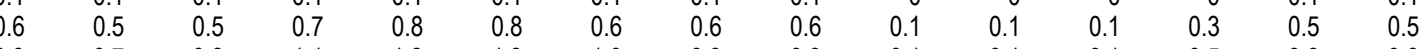

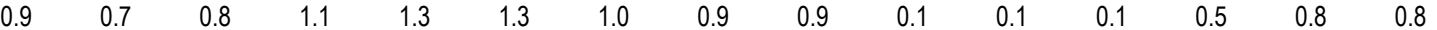

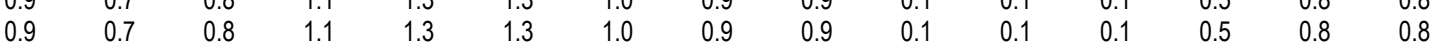

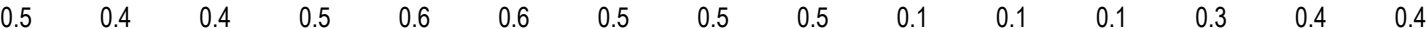

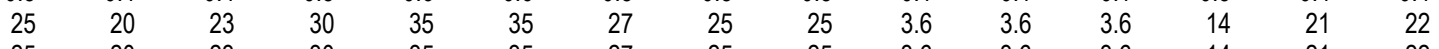

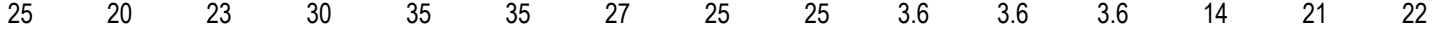

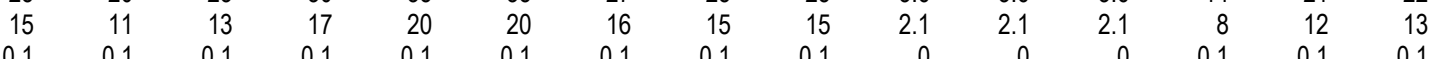

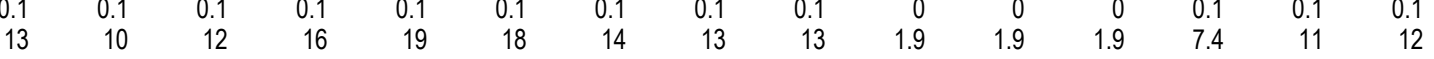

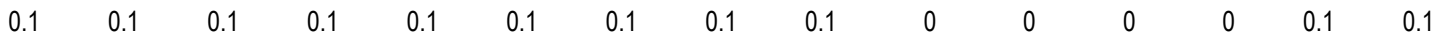

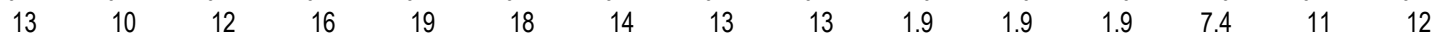

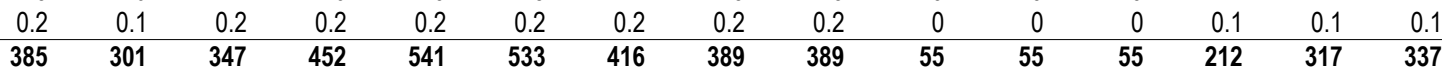


558

558

424

$357 \quad 471 \quad 571$

\begin{tabular}{|c|c|c|c|c|c|c|c|c|c|c|c|c|c|c|}
\hline 40 & 29 & 24 & 33 & 41 & 40 & 29 & 27 & 27 & 19 & 19 & 19 & 32 & 31 & 30 \\
\hline 13 & 9.5 & 7.8 & 11 & 13 & 13 & 9.7 & 9.0 & 9.0 & 6.2 & 6.2 & 6.2 & 11 & 10 & 9.8 \\
\hline 0 & 0 & 0 & 0 & 0 & 0 & 0 & 0 & 0 & 0 & 0 & 0 & 0 & 0 & 0 \\
\hline 11 & 8.1 & 6.7 & 9.2 & 11 & 11 & 8.3 & 7.7 & 7.7 & 5.3 & 5.3 & 5.3 & 9.1 & 8.6 & 8.3 \\
\hline 0 & 0 & 0 & 0 & 0 & 0 & 0 & 0 & 0 & 0 & 0 & 0 & 0 & 0 & 0 \\
\hline 177 & 128 & 106 & 145 & 181 & 178 & 131 & 121 & 121 & 84 & 84 & 83 & 144 & 137 & 132 \\
\hline 0 & 0 & 0 & 0 & 0 & 0 & 0 & 0 & 0 & 0 & 0 & 0 & 0 & 0 & 0 \\
\hline 0 & 0 & 0 & 0 & 0 & 0 & 0 & 0 & 0 & 0 & 0 & 0 & 0 & 0 & 0 \\
\hline 0 & 0 & 0 & 0 & 0 & 0 & 0 & 0 & 0 & 0 & 0 & 0 & 0 & 0 & 0 \\
\hline 0 & 0 & 0 & 0 & 0 & 0 & 0 & 0 & 0 & 0 & 0 & 0 & 0 & 0 & 0 \\
\hline 3.4 & 2.5 & 2 & 2.8 & 3.5 & 3.4 & 2.5 & 2.3 & 2.3 & 1.6 & 1.6 & 1.6 & 2.8 & 2.6 & 2.5 \\
\hline 3.4 & 2.5 & 2 & 2.8 & 3.5 & 3.4 & 2.5 & 2.3 & 2.3 & 1.6 & 1.6 & 1.6 & 2.8 & 2.6 & 2.5 \\
\hline 0 & 0 & 0 & 0 & 0 & 0 & 0 & 0 & 0 & 0 & 0 & 0 & 0 & 0 & 0 \\
\hline 0 & 0 & 0 & 0 & 0 & 0 & 0 & 0 & 0 & 0 & 0 & 0 & 0 & 0 & 0 \\
\hline 0 & 0 & 0 & 0 & 0 & 0 & 0 & 0 & 0 & 0 & 0 & 0 & 0 & 0 & 0 \\
\hline 0 & 0 & 0 & 0 & 0 & 0 & 0 & 0 & 0 & 0 & 0 & 0 & 0 & 0 & 0 \\
\hline 0 & 0 & 0 & 0 & 0 & 0 & 0 & 0 & 0 & 0 & 0 & 0 & 0 & 0 & 0 \\
\hline 0 & 0 & 0 & 0 & 0 & 0 & 0 & 0 & 0 & 0 & 0 & 0 & 0 & 0 & 0 \\
\hline 484 & 351 & 290 & 398 & 497 & 488 & 359 & 333 & 333 & 229 & 229 & 229 & 395 & 375 & 362 \\
\hline
\end{tabular}

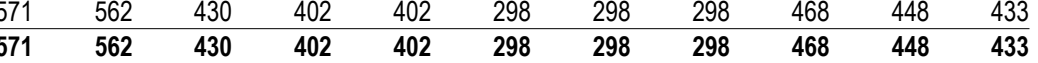

-Wanapum-

3 AVWP Share

4 COPD Share

5 CWPC Share

7 FGRV Share

8 FREC Share

9 GCPD Share

10 ICLP Share

$11 \mathrm{KITT}$ Share

12 KOOT Share

13 LREC Share

14 LVE Share

15 MCMN Share

16 MTFR Share

17 NLEC Share

18 PGE Share

19 PPL Share

20 PSE Share

21 RREC Share

22 SCL Share

23 SLEC Share

24 TPU Share

25 UNEC Share

26 Wanapum After Enc
6 EWEB Share

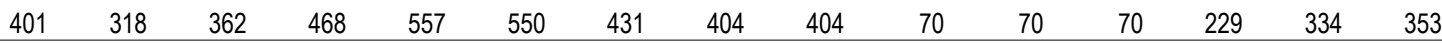

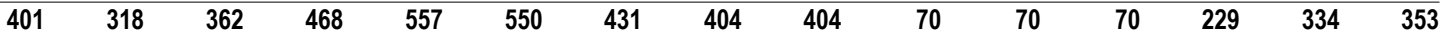

Priest Rapids-

29 AVWP Share

30 COPD Share

31 CWPC Share

32 EWEB Share

33 FGRV Share

34 FREC Share

35 GCPD Share

36 ICLP Share

37 KITT Share

38 KOOT Share

39 LREC Share

40 LVE Share

41 MCMN Share

42 MTFR Share

43 NLEC Share

44 PGE Share

45 PPL Share

46 PSE Share

47 RREC Share

48 SCL Share

49 SLEC Share

50 TPU Share

51 UNEC Share

52 Priest Rapids After Enc

\begin{tabular}{|c|c|c|c|c|c|c|c|c|c|c|c|c|c|}
\hline 8.6 & 10.0 & 13 & 16 & 15 & 12 & 11 & 11 & 1.6 & 1.6 & 1.6 & 6.1 & 9.1 & 9.7 \\
\hline 3.6 & 3.3 & 4.3 & 5.1 & 5 & 3.9 & 3.7 & 3.7 & 0.5 & 0.5 & 0.5 & 2 & 3.0 & 3.2 \\
\hline 0.3 & 0.3 & 0.3 & 0.4 & 0.4 & 0.3 & 0.3 & 0.3 & 0 & 0 & 0 & 0.2 & 0.2 & 0.3 \\
\hline 3.1 & 2.8 & 3.6 & 4.3 & 4.3 & 3.3 & 3.1 & 3.1 & 0.4 & 0.4 & 0.4 & 1.7 & 2.5 & 2.7 \\
\hline 0.7 & 0.8 & 1.1 & 1.3 & 1.3 & 1.0 & 0.9 & 0.9 & 0.1 & 0.1 & 0.1 & 0.5 & 0.8 & 0.8 \\
\hline 0.3 & 0.3 & 0.4 & 0.5 & 0.5 & 0.4 & 0.3 & 0.3 & 0 & 0 & 0 & 0.2 & 0.3 & 0.3 \\
\hline 211 & 243 & 316 & 378 & 373 & 291 & 272 & 272 & 38 & 38 & 38 & 149 & 222 & 236 \\
\hline 0.1 & 0.1 & 0.1 & 0.1 & 0.1 & 0.1 & 0.1 & 0.1 & 0 & 0 & 0 & 0.1 & 0.1 & 0.1 \\
\hline 0.7 & 0.6 & 0.8 & 0.9 & 0.9 & 0.7 & 0.7 & 0.7 & 0.1 & 0.1 & 0.1 & 0.4 & 0.5 & 0.6 \\
\hline 0.5 & 0.5 & 0.6 & 0.7 & 0.7 & 0.6 & 0.5 & 0.5 & 0.1 & 0.1 & 0.1 & 0.3 & 0.4 & 0.4 \\
\hline 0.1 & 0.1 & 0.1 & 0.1 & 0.1 & 0.1 & 0.1 & 0.1 & 0 & 0 & 0 & 0 & 0.1 & 0.1 \\
\hline 0.5 & 0.5 & 0.7 & 0.8 & 0.8 & 0.6 & 0.6 & 0.6 & 0.1 & 0.1 & 0.1 & 0.3 & 0.5 & 0.5 \\
\hline 0.7 & 0.8 & 1.1 & 1.3 & 1.3 & 1.0 & 0.9 & 0.9 & 0.1 & 0.1 & 0.1 & 0.5 & 0.8 & 0.8 \\
\hline 0.7 & 0.8 & 1.1 & 1.3 & 1.3 & 1.0 & 0.9 & 0.9 & 0.1 & 0.1 & 0.1 & 0.5 & 0.8 & 0.8 \\
\hline 0.4 & 0.4 & 0.5 & 0.6 & 0.6 & 0.5 & 0.5 & 0.5 & 0.1 & 0.1 & 0.1 & 0.3 & 0.4 & 0.4 \\
\hline 20 & 23 & 30 & 35 & 35 & 27 & 25 & 25 & 3.6 & 3.6 & 3.6 & 14 & 21 & 22 \\
\hline 20 & 23 & 30 & 35 & 35 & 27 & 25 & 25 & 3.6 & 3.6 & 3.6 & 14 & 21 & 22 \\
\hline 11 & 13 & 17 & 20 & 20 & 16 & 15 & 15 & 2.1 & 2.1 & 2.1 & 8 & 12 & 13 \\
\hline 0.1 & 0.1 & 0.1 & 0.1 & 0.1 & 0.1 & 0.1 & 0.1 & 0 & 0 & 0 & 0.1 & 0.1 & 0.1 \\
\hline 10 & 12 & 16 & 19 & 18 & 14 & 13 & 13 & 1.9 & 1.9 & 1.9 & 7.4 & 11 & 12 \\
\hline 0.1 & 0.1 & 0.1 & 0.1 & 0.1 & 0.1 & 0.1 & 0.1 & 0 & 0 & 0 & 0 & 0.1 & 0.1 \\
\hline 10 & 12 & 16 & 19 & 18 & 14 & 13 & 13 & 1.9 & 1.9 & 1.9 & 7.4 & 11 & 12 \\
\hline 0.2 & 0.2 & 0.2 & 0.2 & 0.2 & 0.2 & 0.2 & 0.2 & 0 & 0 & 0 & 0.1 & 0.1 & 0.1 \\
\hline 301 & 347 & 452 & 541 & 533 & 416 & 389 & 389 & 55 & 55 & 55 & 212 & 317 & 337 \\
\hline
\end{tabular}


558

558

424

$357 \quad 471 \quad 571$

\begin{tabular}{|c|c|c|c|c|c|c|c|c|c|c|c|c|c|c|}
\hline 40 & 29 & 24 & 33 & 41 & 40 & 29 & 27 & 27 & 19 & 19 & 19 & 32 & 31 & 30 \\
\hline 13 & 9.5 & 7.8 & 11 & 13 & 13 & 9.7 & 9.0 & 9.0 & 6.2 & 6.2 & 6.2 & 11 & 10 & 9.8 \\
\hline 0 & 0 & 0 & 0 & 0 & 0 & 0 & 0 & 0 & 0 & 0 & 0 & 0 & 0 & 0 \\
\hline 11 & 8.1 & 6.7 & 9.2 & 11 & 11 & 8.3 & 7.7 & 7.7 & 5.3 & 5.3 & 5.3 & 9.1 & 8.6 & 8.3 \\
\hline 0 & 0 & 0 & 0 & 0 & 0 & 0 & 0 & 0 & 0 & 0 & 0 & 0 & 0 & 0 \\
\hline 177 & 128 & 106 & 145 & 181 & 178 & 131 & 121 & 121 & 84 & 84 & 83 & 144 & 137 & 132 \\
\hline 0 & 0 & 0 & 0 & 0 & 0 & 0 & 0 & 0 & 0 & 0 & 0 & 0 & 0 & 0 \\
\hline 0 & 0 & 0 & 0 & 0 & 0 & 0 & 0 & 0 & 0 & 0 & 0 & 0 & 0 & 0 \\
\hline 0 & 0 & 0 & 0 & 0 & 0 & 0 & 0 & 0 & 0 & 0 & 0 & 0 & 0 & 0 \\
\hline 0 & 0 & 0 & 0 & 0 & 0 & 0 & 0 & 0 & 0 & 0 & 0 & 0 & 0 & 0 \\
\hline 3.4 & 2.5 & 2 & 2.8 & 3.5 & 3.4 & 2.5 & 2.3 & 2.3 & 1.6 & 1.6 & 1.6 & 2.8 & 2.6 & 2.5 \\
\hline 3.4 & 2.5 & 2 & 2.8 & 3.5 & 3.4 & 2.5 & 2.3 & 2.3 & 1.6 & 1.6 & 1.6 & 2.8 & 2.6 & 2.5 \\
\hline 0 & 0 & 0 & 0 & 0 & 0 & 0 & 0 & 0 & 0 & 0 & 0 & 0 & 0 & 0 \\
\hline 0 & 0 & 0 & 0 & 0 & 0 & 0 & 0 & 0 & 0 & 0 & 0 & 0 & 0 & 0 \\
\hline 0 & 0 & 0 & 0 & 0 & 0 & 0 & 0 & 0 & 0 & 0 & 0 & 0 & 0 & 0 \\
\hline 0 & 0 & 0 & 0 & 0 & 0 & 0 & 0 & 0 & 0 & 0 & 0 & 0 & 0 & 0 \\
\hline 0 & 0 & 0 & 0 & 0 & 0 & 0 & 0 & 0 & 0 & 0 & 0 & 0 & 0 & 0 \\
\hline 0 & 0 & 0 & 0 & 0 & 0 & 0 & 0 & 0 & 0 & 0 & 0 & 0 & 0 & 0 \\
\hline 484 & 351 & 290 & 398 & 497 & 488 & 359 & 333 & 333 & 229 & 229 & 229 & 395 & 375 & 362 \\
\hline
\end{tabular}

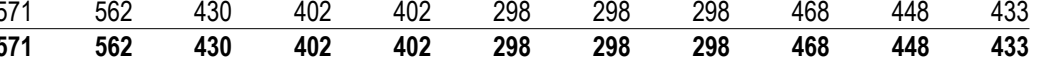

-Wanapum-

3 AVWP Share

4 COPD Share

5 CWPC Share

7 FGRV Share

8 FREC Share

9 GCPD Share

10 ICLP Share

$11 \mathrm{KITT}$ Share

12 KOOT Share

13 LREC Share

14 LVE Share

15 MCMN Share

16 MTFR Share

17 NLEC Share

18 PGE Share

19 PPL Share

20 PSE Share

21 RREC Share

22 SCL Share

23 SLEC Share

24 TPU Share

25 UNEC Share

26 Wanapum After Enc
6 EWEB Share

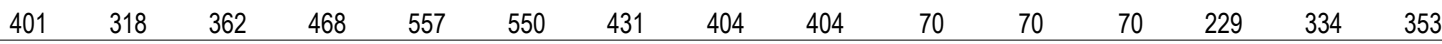

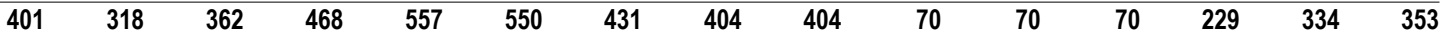

Priest Rapids-

29 AVWP Share

30 COPD Share

31 CWPC Share

32 EWEB Share

33 FGRV Share

34 FREC Share

35 GCPD Share

36 ICLP Share

37 KITT Share

38 KOOT Share

39 LREC Share

40 LVE Share

41 MCMN Share

42 MTFR Share

43 NLEC Share

44 PGE Share

45 PPL Share

46 PSE Share

47 RREC Share

48 SCL Share

49 SLEC Share

50 TPU Share

51 UNEC Share

52 Priest Rapids After Enc

\begin{tabular}{|c|c|c|c|c|c|c|c|c|c|c|c|c|c|}
\hline 8.6 & 10.0 & 13 & 16 & 15 & 12 & 11 & 11 & 1.6 & 1.6 & 1.6 & 6.1 & 9.1 & 9.7 \\
\hline 3.6 & 3.3 & 4.3 & 5.1 & 5 & 3.9 & 3.7 & 3.7 & 0.5 & 0.5 & 0.5 & 2 & 3.0 & 3.2 \\
\hline 0.3 & 0.3 & 0.3 & 0.4 & 0.4 & 0.3 & 0.3 & 0.3 & 0 & 0 & 0 & 0.2 & 0.2 & 0.3 \\
\hline 2.4 & 2.8 & 3.6 & 4.3 & 4.3 & 3.3 & 3.1 & 3.1 & 0.4 & 0.4 & 0.4 & 1.7 & 2.5 & 2.7 \\
\hline 0.7 & 0.8 & 1.1 & 1.3 & 1.3 & 1.0 & 0.9 & 0.9 & 0.1 & 0.1 & 0.1 & 0.5 & 0.8 & 0.8 \\
\hline 0.3 & 0.3 & 0.4 & 0.5 & 0.5 & 0.4 & 0.3 & 0.3 & 0 & 0 & 0 & 0.2 & 0.3 & 0.3 \\
\hline 211 & 243 & 316 & 378 & 373 & 291 & 272 & 272 & 38 & 38 & 38 & 149 & 222 & 236 \\
\hline 0.1 & 0.1 & 0.1 & 0.1 & 0.1 & 0.1 & 0.1 & 0.1 & 0 & 0 & 0 & 0.1 & 0.1 & 0.1 \\
\hline 0.5 & 0.6 & 0.8 & 0.9 & 0.9 & 0.7 & 0.7 & 0.7 & 0.1 & 0.1 & 0.1 & 0.4 & 0.5 & 0.6 \\
\hline 0.4 & 0.5 & 0.6 & 0.7 & 0.7 & 0.6 & 0.5 & 0.5 & 0.1 & 0.1 & 0.1 & 0.3 & 0.4 & 0.4 \\
\hline 0.1 & 0.1 & 0.1 & 0.1 & 0.1 & 0.1 & 0.1 & 0.1 & 0 & 0 & 0 & 0 & 0.1 & 0.1 \\
\hline 0.5 & 0.5 & 0.7 & 0.8 & 0.8 & 0.6 & 0.6 & 0.6 & 0.1 & 0.1 & 0.1 & 0.3 & 0.5 & 0.5 \\
\hline 0.7 & 0.8 & 1.1 & 1.3 & 1.3 & 1.0 & 0.9 & 0.9 & 0.1 & 0.1 & 0.1 & 0.5 & 0.8 & 0.8 \\
\hline 0.7 & 0.8 & 1.1 & 1.3 & 1.3 & 1.0 & 0.9 & 0.9 & 0.1 & 0.1 & 0.1 & 0.5 & 0.8 & 0.8 \\
\hline 0.4 & 0.4 & 0.5 & 0.6 & 0.6 & 0.5 & 0.5 & 0.5 & 0.1 & 0.1 & 0.1 & 0.3 & 0.4 & 0.4 \\
\hline 20 & 23 & 30 & 35 & 35 & 27 & 25 & 25 & 3.6 & 3.6 & 3.6 & 14 & 21 & 22 \\
\hline 20 & 23 & 30 & 35 & 35 & 27 & 25 & 25 & 3.6 & 3.6 & 3.6 & 14 & 21 & 22 \\
\hline 11 & 13 & 17 & 20 & 20 & 16 & 15 & 15 & 2.1 & 2.1 & 2.1 & 8 & 12 & 13 \\
\hline 0.1 & 0.1 & 0.1 & 0.1 & 0.1 & 0.1 & 0.1 & 0.1 & 0 & 0 & 0 & 0.1 & 0.1 & 0.1 \\
\hline 10 & 12 & 16 & 19 & 18 & 14 & 13 & 13 & 1.9 & 1.9 & 1.9 & 7.4 & 11 & 12 \\
\hline 0.1 & 0.1 & 0.1 & 0.1 & 0.1 & 0.1 & 0.1 & 0.1 & 0 & 0 & 0 & 0 & 0.1 & 0.1 \\
\hline 10 & 12 & 16 & 19 & 18 & 14 & 13 & 13 & 1.9 & 1.9 & 1.9 & 7.4 & 11 & 12 \\
\hline 0.1 & 0.2 & 0.2 & 0.2 & 0.2 & 0.2 & 0.2 & 0.2 & 0 & 0 & 0 & 0.1 & 0.1 & 0.1 \\
\hline 301 & 347 & 452 & 541 & 533 & 416 & 389 & 389 & 55 & 55 & 55 & 212 & 317 & 337 \\
\hline
\end{tabular}


Table A-20: Wanapum and Priest Rapids Allocation
PNW Loads and Resources Study

2009 - 2010 Operating Year

1937 Water Year

2003 White Book

1 Wanapum

2 Wanapum Before Enc

558

$558 \quad 424 \quad 357 \quad 471$

3 AVWP Share

4 COPD Share

5 CWPC Share

6 EWEB Share

7 FGRV Share

8 FREC Share

9 GCPD Share

10 ICLP Share

$11 \mathrm{KITT}$ Share

12 KOOT Share

13 LREC Share

14 LVE Share

$15 \mathrm{MCMN}$ Share

16 MTFR Share

17 NLEC Share

18 PGE Share

19 PPL Share

20 PSE Share

21 RREC Share

22 SCL Share

23 SLEC Share

24 TPU Share

25 UNEC Share

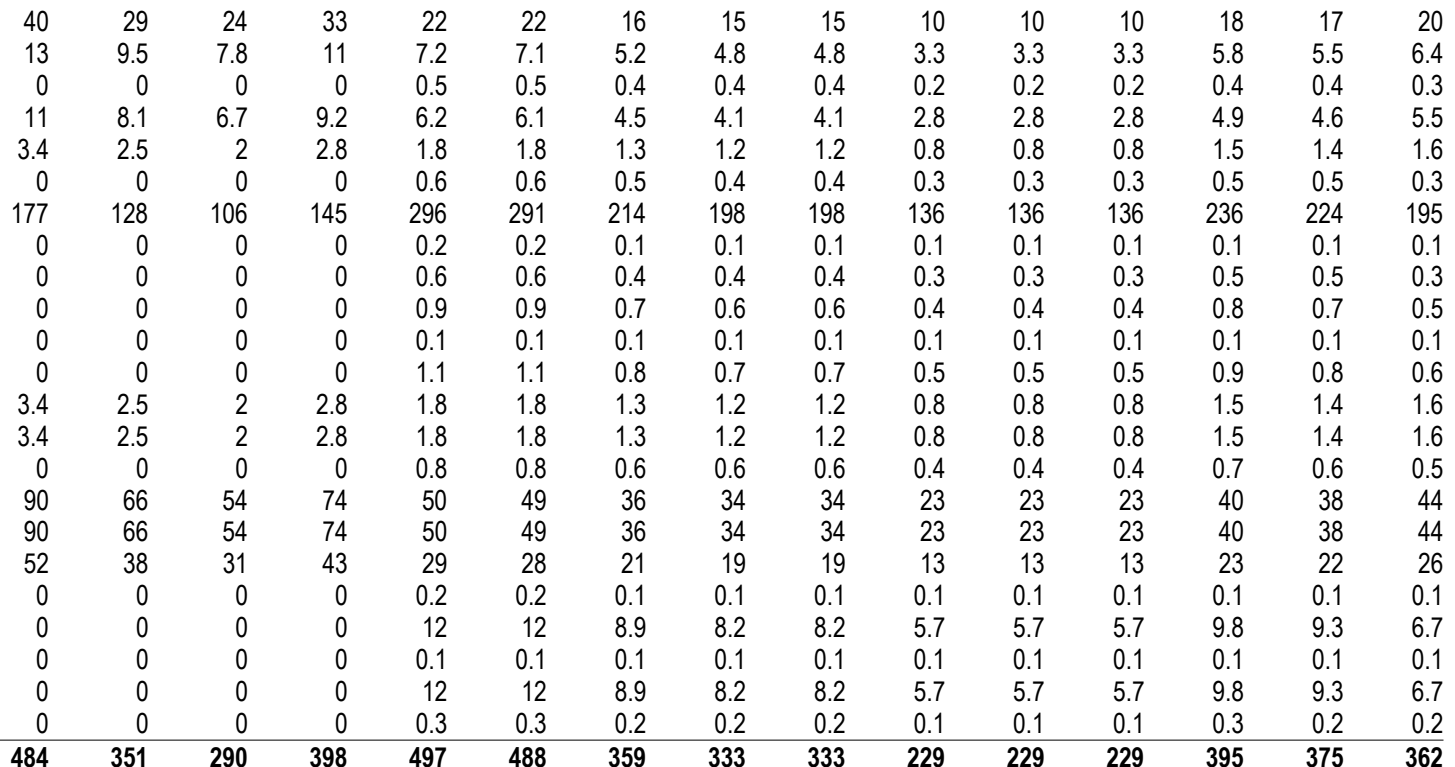

-Priest Rapids-

27 Priest Rapids

28 Priest Rapids Before Enc

$362 \quad 468 \quad 557$

$557 \quad 550 \quad 431$

$431 \quad 404$

$404 \quad 404$

404

\begin{tabular}{llllll}
70 & 70 & 70 & 229 & 334 & 353 \\
\hline 70 & $\mathbf{7 0}$ & $\mathbf{7 0}$ & $\mathbf{2 2 9}$ & $\mathbf{3 3 4}$ & $\mathbf{3 5 3}$
\end{tabular}

-Priest Rapids-

29 AVWP Share

30 COPD Share

31 CWPC Share

32 EWEB Share

33 FGRV Share

34 FREC Share

35 GCPD Share

36 ICLP Share

37 KITT Share

38 KOOT Share

39 LREC Share

SOLVES Strat

41 MCMN Share

42 MTFR Share

asmecosina

44 PGE Share

45 PPL Share

46 PSE Share

47 RREC Share

48 SCL Share

49 SLEC Share

50 TPU Share

51 UNEC Share

52 Priest Rapids After Enc $\begin{array}{llllllllllllll}401 & 318 & 362 & 468 & 557 & 550 & 431 & 404 & 404 & 70 & 70 & 70 & 229 & 334\end{array}$

\begin{tabular}{|c|c|c|c|c|c|c|c|c|c|c|c|c|}
\hline 8.6 & 10.0 & 13 & 24 & 24 & 18 & 17 & 17 & 2.4 & 2.4 & 2.4 & 9.4 & 14 \\
\hline 2.8 & 3.3 & 4.3 & 7.9 & 7.8 & 6.1 & 5.7 & 5.7 & 0.8 & 0.8 & 0.8 & 3.1 & 4.6 \\
\hline 0.2 & 0.3 & 0.3 & 0.6 & 0.6 & 0.5 & 0.4 & 0.4 & 0.1 & 0.1 & 0.1 & 0.2 & 0.3 \\
\hline 2.4 & 2.8 & 3.6 & 6.7 & 6.6 & 5.1 & 4.8 & 4.8 & 0.7 & 0.7 & 0.7 & 2.6 & 3.9 \\
\hline 0.7 & 0.8 & 1.1 & 2 & 2.0 & 1.5 & 1.4 & 1.4 & 0.2 & 0.2 & 0.2 & 0.8 & 1.2 \\
\hline 0.3 & 0.3 & 0.4 & 0.7 & 0.7 & 0.5 & 0.5 & 0.5 & 0.1 & 0.1 & 0.1 & 0.3 & 0.4 \\
\hline 211 & 243 & 316 & 322 & 318 & 248 & 232 & 232 & 33 & 33 & 33 & 127 & 189 \\
\hline 0.1 & 0.1 & 0.1 & 0.2 & 0.2 & 0.1 & 0.1 & 0.1 & 0 & 0 & 0 & 0.1 & 0.1 \\
\hline 0.5 & 0.6 & 0.8 & 0.7 & 0.7 & 0.5 & 0.5 & 0.5 & 0.1 & 0.1 & 0.1 & 0.3 & 0.4 \\
\hline 0.4 & 0.5 & 0.6 & 1 & 1 & 0.8 & 0.7 & 0.7 & 0.1 & 0.1 & 0.1 & 0.4 & 0.6 \\
\hline 0.1 & 0.1 & 0.1 & 0.1 & 0.1 & 0.1 & 0.1 & 0.1 & 0 & 0 & 0 & 0.1 & 0.1 \\
\hline 0.5 & 0.5 & 0.7 & 1.2 & 1.2 & 0.9 & 0.9 & 0.9 & 0.1 & 0.1 & 0.1 & 0.5 & 0.7 \\
\hline 0.7 & 0.8 & 1.1 & 2 & 2.0 & 1.5 & 1.4 & 1.4 & 0.2 & 0.2 & 0.2 & 0.8 & 1.2 \\
\hline 0.7 & 0.8 & 1.1 & 2 & 2.0 & 1.5 & 1.4 & 1.4 & 0.2 & 0.2 & 0.2 & 0.8 & 1.2 \\
\hline 0.4 & 0.4 & 0.5 & 0.9 & 0.9 & 0.7 & 0.7 & 0.7 & 0.1 & 0.1 & 0.1 & 0.4 & 0.5 \\
\hline 20 & 23 & 30 & 55 & 54 & 42 & 39 & 39 & 5.5 & 5.5 & 5.5 & 21 & 32 \\
\hline 20 & 23 & 30 & 55 & 54 & 42 & 39 & 39 & 5.5 & 5.5 & 5.5 & 21 & 32 \\
\hline 11 & 13 & 17 & 31 & 31 & 24 & 23 & 23 & 3.2 & 3.2 & 3.2 & 12 & 18 \\
\hline 0.1 & 0.1 & 0.1 & 0.2 & 0.2 & 0.1 & 0.1 & 0.1 & 0 & 0 & 0 & 0.1 & 0.1 \\
\hline 10 & 12 & 16 & 13 & 13 & 10 & 9.6 & 9.6 & 1.4 & 1.4 & 1.4 & 5.3 & 7.8 \\
\hline 0.1 & 0.1 & 0.1 & 0.2 & 0.2 & 0.1 & 0.1 & 0.1 & 0 & 0 & 0 & 0.1 & 0.1 \\
\hline 10 & 12 & 16 & 13 & 13 & 10 & 9.6 & 9.6 & 1.4 & 1.4 & 1.4 & 5.3 & 7.8 \\
\hline 0.1 & 0.2 & 0.2 & 0.3 & 0.3 & 0.3 & 0.2 & 0.2 & 0 & 0 & 0 & 0.1 & 0.2 \\
\hline 301 & 347 & 452 & 541 & 533 & 416 & 389 & 389 & 55 & 55 & 55 & 212 & 317 \\
\hline
\end{tabular}


Table A-20: Wanapum and Priest Rapids Allocation
PNW Loads and Resources Study

2010 - 2011 Operating Year

1937 Water Year

2003 White Book

1 Wanapum

558 6 Sep

Oct Nov Dec Jan Feb Mar Apr1 Apr16 May Jun Jul Avg

2 Wanapum Before Enc

558

$\begin{array}{ll}424 & 357 \\ 424 & 357\end{array}$

$\begin{array}{ll}357 & 471 \\ 357 & 471\end{array}$

471
471

$\begin{array}{ll}571 & 562 \\ \mathbf{5 7 1} & \mathbf{5 6}\end{array}$

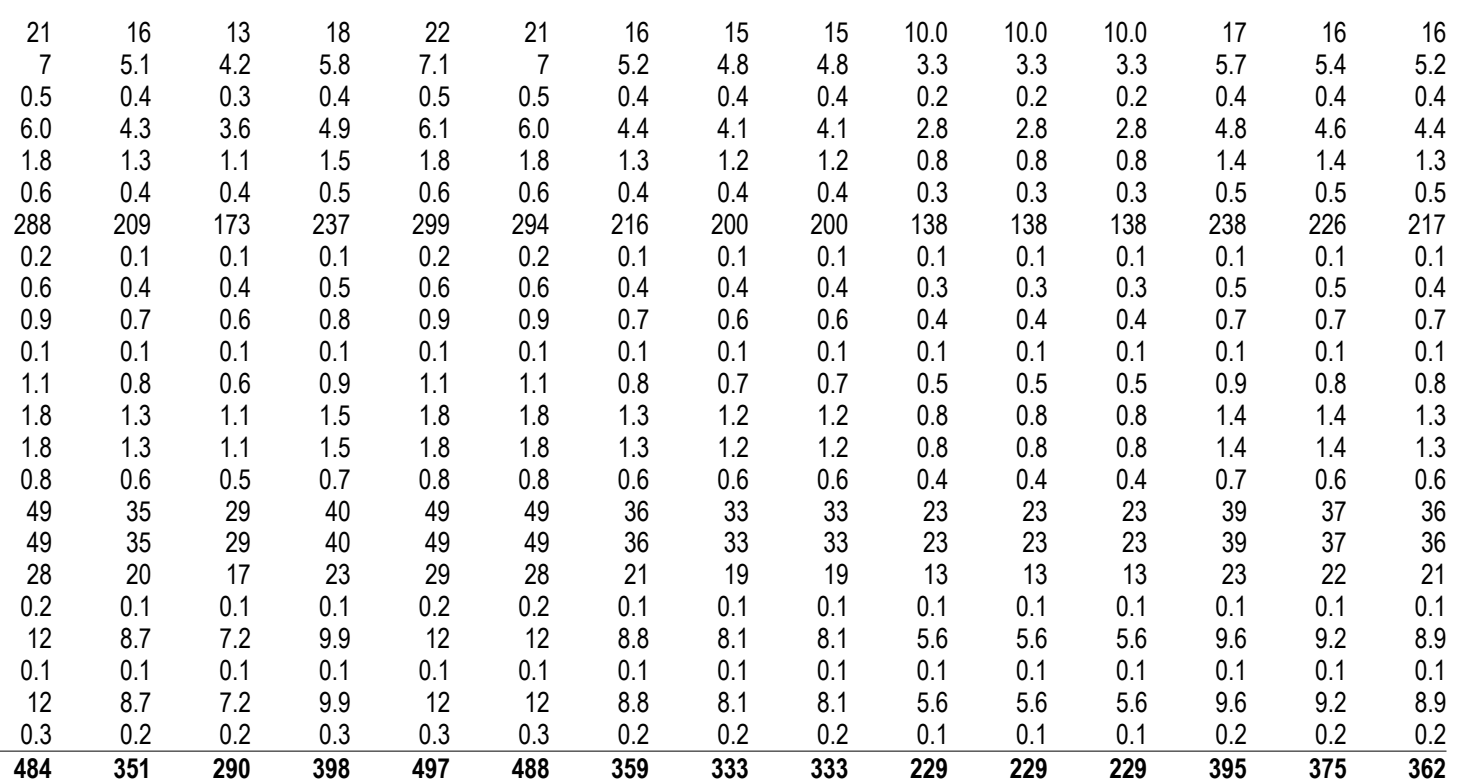

-Priest Rapids

27 Priest Rapids 40 $318 \quad 362$ $468 \quad 557$ $550 \quad 43$ 404 404

\begin{tabular}{llllll}
70 & 70 & 70 & 229 & 334 & 353 \\
\hline 70 & 70 & 70 & $\mathbf{2 2 9}$ & $\mathbf{3 3 4}$ & $\mathbf{3 5 3}$
\end{tabular}

-Priest Rapids. 29 AVWP Share 30 COPD Share 31 CWPC Share 32 EWEB Share 33 FGRV Share 34 FREC Share 35 GCPD Share 36 ICLP Share

37 KITT Share 38 KOOT Share 3⿻上丨ecsiare SOLVES Strat 41 MCMN Share 42 MTFR Share asmecstare 44 PGE Share 45 PPL Share 46 PSE Share 47 RREC Share 48 SCL Share 49 SLEC Share 50 TPU Share 51 UNEC Share 52 Priest Rapids After Enc

\begin{tabular}{|c|c|c|c|c|c|c|c|c|c|c|c|c|c|c|}
\hline 17 & 13 & 15 & 20 & 24 & 23 & 18 & 17 & 17 & 2.4 & 2.4 & 2.4 & 9.3 & 14 & 15 \\
\hline 5.6 & 4.4 & 5.1 & 6.6 & 7.8 & 7.6 & 6.0 & 5.6 & 5.6 & 0.8 & 0.8 & 0.8 & 3 & 4.5 & 4.9 \\
\hline 0.4 & 0.3 & 0.4 & 0.5 & 0.6 & 0.6 & 0.4 & 0.4 & 0.4 & 0.1 & 0.1 & 0.1 & 0.2 & 0.3 & 0.4 \\
\hline 4.8 & 3.7 & 4.3 & 5.6 & 6.6 & 6.5 & 5.1 & 4.7 & 4.7 & 0.7 & 0.7 & 0.7 & 2.6 & 3.9 & 4.1 \\
\hline 0.5 & 0.4 & 0.4 & 0.6 & 0.7 & 0.7 & 0.5 & 0.5 & 0.5 & 0.1 & 0.1 & 0.1 & 0.3 & 0.4 & 0.4 \\
\hline 229 & 180 & 207 & 270 & 326 & 321 & 250 & 234 & 234 & 33 & 33 & 33 & 128 & 191 & 203 \\
\hline 0.1 & 0.1 & 0.1 & 0.2 & 0.2 & 0.2 & 0.1 & 0.1 & 0.1 & 0 & 0 & 0 & 0.1 & 0.1 & 0.1 \\
\hline 0.7 & 0.6 & 0.7 & 0.9 & 1 & 1.0 & 0.8 & 0.7 & 0.7 & 0.1 & 0.1 & 0.1 & 0.4 & 0.6 & 0.6 \\
\hline 0.1 & 0.1 & 0.1 & 0.1 & 0.1 & 0.1 & 0.1 & 0.1 & 0.1 & 0 & 0 & 0 & 0.1 & 0.1 & 0.1 \\
\hline 0.9 & 0.7 & 0.8 & 1.0 & 1.2 & 1.2 & 0.9 & 0.8 & 0.8 & 0.1 & 0.1 & 0.1 & 0.5 & 0.7 & 0.7 \\
\hline 1.4 & 1.1 & 1.3 & 1.7 & 2.0 & 2.0 & 1.5 & 1.4 & 1.4 & 0.2 & 0.2 & 0.2 & 0.8 & 1.2 & 1.2 \\
\hline 1.4 & 1.1 & 1.3 & 1.7 & 2.0 & 2.0 & 1.5 & 1.4 & 1.4 & 0.2 & 0.2 & 0.2 & 0.8 & 1.2 & 1.2 \\
\hline 0.7 & 0.5 & 0.6 & 0.8 & 0.9 & 0.9 & 0.7 & 0.6 & 0.6 & 0.1 & 0.1 & 0.1 & 0.4 & 0.5 & 0.6 \\
\hline 0.1 & 0.1 & 0.1 & 0.1 & 0.2 & 0.2 & 0.1 & 0.1 & 0.1 & 0 & 0 & 0 & 0.1 & 0.1 & 0.1 \\
\hline 9.5 & 7.5 & 8.6 & 11 & 13 & 13 & 10 & 9.5 & 9.5 & 1.3 & 1.3 & 1.3 & 5.2 & 7.7 & 8.3 \\
\hline 0.1 & 0.1 & 0.1 & 0.1 & 0.2 & 0.2 & 0.1 & 0.1 & 0.1 & 0 & 0 & 0 & 0.1 & 0.1 & 0.1 \\
\hline 9.5 & 7.5 & 8.6 & 11 & 13 & 13 & 10 & 9.5 & 9.5 & 1.3 & 1.3 & 1.3 & 5.2 & 7.7 & 8.3 \\
\hline 0.2 & 0.2 & 0.2 & 0.3 & 0.3 & 0.3 & 0.3 & 0.2 & 0.2 & 0 & 0 & 0 & 0.1 & 0.2 & 0.2 \\
\hline 385 & 301 & 347 & 452 & 541 & 533 & 416 & 389 & 389 & 55 & 55 & 55 & 212 & 317 & 337 \\
\hline
\end{tabular}


Table A-20: Wanapum and Priest Rapids Allocation
PNW Loads and Resources Study

2011 - 2012 Operating Year

1937 Water Year

2003 White Book

1 Wanapum

558

$424 \quad 357$

Sep Oct Nov

Dec Jan

Feb Mar Apr1 Apr16 May Jun Jul Avg

2 Wanapum Before Enc

558

424

$357 \quad 471$

$571 \quad 562$

\begin{tabular}{|c|c|c|c|c|c|c|c|c|c|c|c|c|c|c|}
\hline 21 & 15 & 13 & 17 & 21 & 21 & 16 & 14 & 14 & 9.9 & 9.9 & 9.9 & 17 & 16 & 16 \\
\hline 6.9 & 5 & 4.2 & 5.7 & 7.1 & 6.9 & 5.1 & 4.7 & 4.7 & 3.2 & 3.2 & 3.2 & 5.6 & 5.3 & 5.2 \\
\hline 5.9 & 4.3 & 3.5 & 4.9 & 6.0 & 5.9 & 4.3 & 4 & 4 & 2.8 & 2.8 & 2.8 & 4.8 & 4.5 & 4.4 \\
\hline 0.6 & 0.4 & 0.4 & 0.5 & 0.6 & 0.6 & 0.4 & 0.4 & 0.4 & 0.3 & 0.3 & 0.3 & 0.5 & 0.5 & 0.4 \\
\hline 291 & 211 & 175 & 240 & 302 & 296 & 218 & 202 & 202 & 139 & 139 & 139 & 240 & 228 & 219 \\
\hline 0.2 & 0.1 & 0.1 & 0.1 & 0.2 & 0.2 & 0.1 & 0.1 & 0.1 & 0.1 & 0.1 & 0.1 & 0.1 & 0.1 & 0.1 \\
\hline 0.9 & 0.7 & 0.5 & 0.7 & 0.9 & 0.9 & 0.7 & 0.6 & 0.6 & 0.4 & 0.4 & 0.4 & 0.7 & 0.7 & 0.7 \\
\hline 0.1 & 0.1 & 0.1 & 0.1 & 0.1 & 0.1 & 0.1 & 0.1 & 0.1 & 0.1 & 0.1 & 0.1 & 0.1 & 0.1 & 0.1 \\
\hline 1.1 & 0.8 & 0.6 & 0.9 & 1.1 & 1.1 & 0.8 & 0.7 & 0.7 & 0.5 & 0.5 & 0.5 & 0.8 & 0.8 & 0.8 \\
\hline 1.8 & 1.3 & 1.1 & 1.5 & 1.8 & 1.8 & 1.3 & 1.2 & 1.2 & 0.8 & 0.8 & 0.8 & 1.4 & 1.4 & 1.3 \\
\hline 1.8 & 1.3 & 1.1 & 1.5 & 1.8 & 1.8 & 1.3 & 1.2 & 1.2 & 0.8 & 0.8 & 0.8 & 1.4 & 1.4 & 1.3 \\
\hline 0.8 & 0.6 & 0.5 & 0.7 & 0.8 & 0.8 & 0.6 & 0.5 & 0.5 & 0.4 & 0.4 & 0.4 & 0.7 & 0.6 & 0.6 \\
\hline 0.2 & 0.1 & 0.1 & 0.1 & 0.2 & 0.2 & 0.1 & 0.1 & 0.1 & 0.1 & 0.1 & 0.1 & 0.1 & 0.1 & 0.1 \\
\hline 12 & 8.6 & 7.1 & 9.7 & 12 & 12 & 8.7 & 8 & 8 & 5.5 & 5.5 & 5.5 & 9.5 & 9.1 & 8.8 \\
\hline 0.1 & 0.1 & 0.1 & 0.1 & 0.1 & 0.1 & 0.1 & 0.1 & 0.1 & 0.1 & 0.1 & 0.1 & 0.1 & 0.1 & 0.1 \\
\hline 12 & 8.6 & 7.1 & 9.7 & 12 & 12 & 8.7 & 8 & 8 & 5.5 & 5.5 & 5.5 & 9.5 & 9.1 & 8.8 \\
\hline 0.3 & 0.2 & 0.2 & 0.3 & 0.3 & 0.3 & 0.2 & 0.2 & 0.2 & 0.1 & 0.1 & 0.1 & 0.2 & 0.2 & 0.2 \\
\hline 484 & 351 & 290 & 398 & 497 & 488 & 359 & 333 & 333 & 229 & 229 & 229 & 395 & 375 & 362 \\
\hline
\end{tabular}

3 AVWP Sh

4 COPD Share

5 CWPC Share

6 EWEB Share

7 FGRV Share

8 FREC Share

9 GCPD Share

10 ICLP Share

11 KITT Share

12 KOOT Share

13 LREC Share

14 LVE Share

15 MCMN Share

16 MTFR Share

17 NLEC Share

18 PGE Share

19 PPL Share

20 PSE Share

21 RREC Share

22 SCL Share

23 SLEC Share

24 TPU Share
25 UNEC Share

\begin{tabular}{llllllllll}
62 & 430 & 402 & 402 & 298 & 298 & 298 & 468 & 448 & 433 \\
\hline 62 & 430 & 402 & 402 & 298 & 298 & 298 & 468 & 448 & 433
\end{tabular}

26 Wanapum After Enc

484

-Priest Rapids-

27 Priest Rapids

$\begin{array}{lllllllllllllll}401 & 318 & 362 & 468 & 557 & 550 & 431 & 404 & 404 & 70 & 70 & 70 & 229 & 334 & 353 \\ \mathbf{4 0 1} & \mathbf{3 1 8} & \mathbf{3 6 2} & \mathbf{4 6 8} & \mathbf{5 5 7} & \mathbf{5 5 0} & \mathbf{4 3 1} & \mathbf{4 0 4} & \mathbf{4 0 4} & \mathbf{7 0} & \mathbf{7 0} & \mathbf{7 0} & \mathbf{2 2 9} & \mathbf{3 3 4} & \mathbf{3 5 3}\end{array}$

28 Priest Rapids Before Enc

-Priest Rapids-

29 AVWP Share

30 COPD Share

31 CWPC Share

32 EWEB Share

33 FGRV Share

34 FREC Share

35 GCPD Share

36 ICLP Share

37 KITT Share

38 KOOT Share

39 LREC Share

40 LVE Share

41 MCMN Share

42 MTFR Share

43 NLEC Share

44 PGE Share

45 PPL Share

46 PSE Share

47 RREC Share

$48 \mathrm{SCL}$ Share

49 SLEC Share

50 TPU Share

51 UNEC Share

52 Priest Rapids After Enc

\begin{tabular}{|c|c|c|c|c|c|c|c|c|c|c|c|c|c|}
\hline 17 & 15 & 20 & 23 & 23 & 18 & 17 & 17 & 2.4 & 2.4 & 2.4 & 9.2 & 14 & 15 \\
\hline 5.5 & 5.0 & 6.5 & 7.7 & 7.6 & 5.9 & 5.5 & 5.5 & 0.8 & 0.8 & 0.8 & 3 & 4.5 & 4.8 \\
\hline 0.4 & 0.4 & 0.5 & 0.6 & 0.6 & 0.4 & 0.4 & 0.4 & 0.1 & 0.1 & 0.1 & 0.2 & 0.3 & 0.4 \\
\hline 4.7 & 4.2 & 5.5 & 6.5 & 6.4 & 5 & 4.7 & 4.7 & 0.7 & 0.7 & 0.7 & 2.6 & 3.8 & 4.1 \\
\hline 1.4 & 1.3 & 1.7 & 2.0 & 1.9 & 1.5 & 1.4 & 1.4 & 0.2 & 0.2 & 0.2 & 0.8 & 1.1 & 1.2 \\
\hline 0.5 & 0.4 & 0.6 & 0.7 & 0.7 & 0.5 & 0.5 & 0.5 & 0.1 & 0.1 & 0.1 & 0.3 & 0.4 & 0.4 \\
\hline 182 & 209 & 272 & 328 & 323 & 252 & 236 & 236 & 33 & 33 & 33 & 129 & 192 & 204 \\
\hline 0.1 & 0.1 & 0.2 & 0.2 & 0.2 & 0.1 & 0.1 & 0.1 & 0 & 0 & 0 & 0.1 & 0.1 & 0.1 \\
\hline 0.5 & 0.4 & 0.6 & 0.7 & 0.7 & 0.5 & 0.5 & 0.5 & 0.1 & 0.1 & 0.1 & 0.3 & 0.4 & 0.4 \\
\hline 0.7 & 0.6 & 0.8 & 1 & 1.0 & 0.8 & 0.7 & 0.7 & 0.1 & 0.1 & 0.1 & 0.4 & 0.6 & 0.6 \\
\hline 0.1 & 0.1 & 0.1 & 0.1 & 0.1 & 0.1 & 0.1 & 0.1 & 0 & 0 & 0 & 0.1 & 0.1 & 0.1 \\
\hline 0.7 & 0.8 & 1.0 & 1.2 & 1.1 & 0.9 & 0.8 & 0.8 & 0.1 & 0.1 & 0.1 & 0.5 & 0.7 & 0.7 \\
\hline 1.4 & 1.3 & 1.7 & 2.0 & 1.9 & 1.5 & 1.4 & 1.4 & 0.2 & 0.2 & 0.2 & 0.8 & 1.1 & 1.2 \\
\hline 1.4 & 1.3 & 1.7 & 2.0 & 1.9 & 1.5 & 1.4 & 1.4 & 0.2 & 0.2 & 0.2 & 0.8 & 1.1 & 1.2 \\
\hline 0.6 & 0.6 & 0.8 & 0.9 & 0.9 & 0.7 & 0.6 & 0.6 & 0.1 & 0.1 & 0.1 & 0.4 & 0.5 & 0.6 \\
\hline 30 & 35 & 45 & 53 & 52 & 41 & 38 & 38 & 5.4 & 5.4 & 5.4 & 21 & 31 & 33 \\
\hline 30 & 35 & 45 & 53 & 52 & 41 & 38 & 38 & 5.4 & 5.4 & 5.4 & 21 & 31 & 33 \\
\hline 17 & 20 & 26 & 31 & 30 & 24 & 22 & 22 & 3.1 & 3.1 & 3.1 & 12 & 18 & 19 \\
\hline 0.1 & 0.1 & 0.1 & 0.2 & 0.2 & 0.1 & 0.1 & 0.1 & 0 & 0 & 0 & 0.1 & 0.1 & 0.1 \\
\hline 9.4 & 8.5 & 11 & 13 & 13 & 10 & 9.4 & 9.4 & 1.3 & 1.3 & 1.3 & 5.1 & 7.6 & 8.2 \\
\hline 0.1 & 0.1 & 0.1 & 0.2 & 0.1 & 0.1 & 0.1 & 0.1 & 0 & 0 & 0 & 0.1 & 0.1 & 0.1 \\
\hline 9.4 & 8.5 & 11 & 13 & 13 & 10 & 9.4 & 9.4 & 1.3 & 1.3 & 1.3 & 5.1 & 7.6 & 8.2 \\
\hline 0.2 & 0.2 & 0.3 & 0.3 & 0.3 & 0.3 & 0.2 & 0.2 & 0 & 0 & 0 & 0.1 & 0.2 & 0.2 \\
\hline 301 & 347 & 452 & 541 & 533 & 416 & 389 & 389 & 55 & 55 & 55 & 212 & 317 & 337 \\
\hline
\end{tabular}


Table A-20: Wanapum and Priest Rapids Allocation

PNW Loads and Resources Study

2012 - 2013 Operating Year

1937 Water Year

2003 White Book

1 Wanapum

558

$424 \quad 357$

Sep Oct Nov

Dec Jan

Feb Mar Apr1 Apr16 May Jun Jul Avg

2 Wanapum Before Enc

558

424

$357 \quad 471$

571

562
562

430
430

$\begin{array}{ll}402 & 402\end{array}$

$402 \quad 298$

$298 \quad 298$

\begin{tabular}{lllll}
298 & 298 & 468 & 448 & 433 \\
\hline 298 & 298 & 468 & 448 & 433
\end{tabular}

-

3 AVWP Share

4 COPD Share

5 CWPC Share

6 EWEB Share

8 FREC Share

9 GCPD Share

10 ICLP Share

11 KITT Share

12 KOOT Share

13 LREC Share

14 LVE Share

15 MCMN Share

16 MTFR Share

17 NLEC Share

18 PGE Share

19 PPL Share

20 PSE Share

21 RREC Share

22 SCL Share

23 SLEC Share

24 TPU Share

25 UNEC Share

\begin{tabular}{|c|c|c|c|c|c|c|c|c|c|c|c|c|c|c|}
\hline 21 & 15 & 13 & 17 & 21 & 21 & 15 & 14 & 14 & 9.7 & 9.7 & 9.7 & 17 & 16 & 15 \\
\hline 6.9 & 5.0 & 4.1 & 5.7 & 6.9 & 6.8 & 5.0 & 4.6 & 4.6 & 3.2 & 3.2 & 3.2 & 5.5 & 5.2 & 5.1 \\
\hline 0.5 & 0.4 & 0.3 & 0.4 & 0.5 & 0.5 & 0.4 & 0.3 & 0.3 & 0.2 & 0.2 & 0.2 & 0.4 & 0.4 & 0.4 \\
\hline 5.8 & 4.2 & 3.5 & 4.8 & 5.9 & 5.8 & 4.3 & 3.9 & 3.9 & 2.7 & 2.7 & 2.7 & 4.7 & 4.4 & 4.3 \\
\hline 0.6 & 0.4 & 0.4 & 0.5 & 0.6 & 0.6 & 0.4 & 0.4 & 0.4 & 0.3 & 0.3 & 0.3 & 0.5 & 0.5 & 0.4 \\
\hline 293 & 213 & 176 & 241 & 305 & 300 & 221 & 204 & 204 & 141 & 141 & 140 & 243 & 231 & 222 \\
\hline 0.2 & 0.1 & 0.1 & 0.1 & 0.2 & 0.2 & 0.1 & 0.1 & 0.1 & 0.1 & 0.1 & 0.1 & 0.1 & 0.1 & 0.1 \\
\hline 0.9 & 0.6 & 0.5 & 0.7 & 0.9 & 0.9 & 0.7 & 0.6 & 0.6 & 0.4 & 0.4 & 0.4 & 0.7 & 0.7 & 0.7 \\
\hline 0.1 & 0.1 & 0.1 & 0.1 & 0.1 & 0.1 & 0.1 & 0.1 & 0.1 & 0.1 & 0.1 & 0.1 & 0.1 & 0.1 & 0.1 \\
\hline 1 & 0.8 & 0.6 & 0.9 & 1 & 1 & 0.8 & 0.7 & 0.7 & 0.5 & 0.5 & 0.5 & 0.8 & 0.8 & 0.8 \\
\hline 1.8 & 1.3 & 1.1 & 1.4 & 1.8 & 1.7 & 1.3 & 1.2 & 1.2 & 0.8 & 0.8 & 0.8 & 1.4 & 1.3 & 1.3 \\
\hline 1.8 & 1.3 & 1.1 & 1.4 & 1.8 & 1.7 & 1.3 & 1.2 & 1.2 & 0.8 & 0.8 & 0.8 & 1.4 & 1.3 & 1.3 \\
\hline 0.8 & 0.6 & 0.5 & 0.7 & 0.8 & 0.8 & 0.6 & 0.5 & 0.5 & 0.4 & 0.4 & 0.4 & 0.6 & 0.6 & 0.6 \\
\hline 0.2 & 0.1 & 0.1 & 0.1 & 0.2 & 0.2 & 0.1 & 0.1 & 0.1 & 0.1 & 0.1 & 0.1 & 0.1 & 0.1 & 0.1 \\
\hline 12 & 8.5 & 7 & 9.6 & 12 & 12 & 8.5 & 7.9 & 7.9 & 5.4 & 5.4 & 5.4 & 9.3 & 8.9 & 8.6 \\
\hline 0.1 & 0.1 & 0.1 & 0.1 & 0.1 & 0.1 & 0.1 & 0.1 & 0.1 & 0.1 & 0.1 & 0.1 & 0.1 & 0.1 & 0.1 \\
\hline 12 & 8.5 & 7 & 9.6 & 12 & 12 & 8.5 & 7.9 & 7.9 & 5.4 & 5.4 & 5.4 & 9.3 & 8.9 & 8.6 \\
\hline 0.3 & 0.2 & 0.2 & 0.2 & 0.3 & 0.3 & 0.2 & 0.2 & 0.2 & 0.1 & 0.1 & 0.1 & 0.2 & 0.2 & 0.2 \\
\hline 484 & 351 & 290 & 398 & 497 & 488 & 359 & 333 & 333 & 229 & 229 & 229 & 395 & 375 & 362 \\
\hline
\end{tabular}

-Priest Rapids-

27 Priest Rapids

28 Priest Rapids Before Enc

-Priest Rapids-

29 AVWP Share

30 COPD Share

31 CWPC Share

32 EWEB Share

33 FGRV Share

34 FREC Share

35 GCPD Share

36 ICLP Share

37 KITT Share

38 KOOT Share

39 LREC Share

40 LVE Share

41 MCMN Share

42 MTFR Share

as MEE Shana

44 PGE Share

45 PPL Share

46 PSE Share

47 RREC Share

48 SCL Share

49 SLEC Share

50 TPU Share

51 UNEC Share

\begin{tabular}{|c|c|c|c|c|c|c|c|c|c|c|c|}
\hline 401 & 318 & 362 & 468 & 557 & 550 & 431 & 404 & 404 & 70 & 70 & 353 \\
\hline 401 & 318 & 362 & 468 & 557 & 550 & 431 & 404 & 404 & 70 & 70 & \\
\hline
\end{tabular}

52 Priest Rapids After Enc

\begin{tabular}{|c|c|c|c|c|c|c|c|c|c|c|c|c|c|}
\hline 13 & 15 & 20 & 23 & 23 & 18 & 16 & 16 & 2.3 & 2.3 & 2.3 & 9.0 & 13 & 14 \\
\hline 4.3 & 4.9 & 6.4 & 7.5 & 7.4 & 5.8 & 5.4 & 5.4 & 0.8 & 0.8 & 0.8 & 3.0 & 4.4 & 4.7 \\
\hline 3.6 & 4.2 & 5.5 & 6.4 & 6.3 & 4.9 & 4.6 & 4.6 & 0.6 & 0.6 & 0.6 & 2.5 & 3.7 & 4 \\
\hline 0.4 & 0.4 & 0.6 & 0.6 & 0.6 & 0.5 & 0.5 & 0.5 & 0.1 & 0.1 & 0.1 & 0.3 & 0.4 & 0.4 \\
\hline 183 & 210 & 274 & 332 & 328 & 255 & 239 & 239 & 34 & 34 & 34 & 130 & 195 & 207 \\
\hline 0.1 & 0.1 & 0.2 & 0.2 & 0.2 & 0.1 & 0.1 & 0.1 & 0 & 0 & 0 & 0.1 & 0.1 & 0.1 \\
\hline 0.6 & 0.6 & 0.8 & 1.0 & 1.0 & 0.8 & 0.7 & 0.7 & 0.1 & 0.1 & 0.1 & 0.4 & 0.6 & 0.6 \\
\hline 0.1 & 0.1 & 0.1 & 0.1 & 0.1 & 0.1 & 0.1 & 0.1 & 0 & 0 & 0 & 0.1 & 0.1 & 0.1 \\
\hline 0.6 & 0.7 & 1.0 & 1.1 & 1.1 & 0.9 & 0.8 & 0.8 & 0.1 & 0.1 & 0.1 & 0.4 & 0.7 & 0.7 \\
\hline 1.1 & 1.3 & 1.6 & 1.9 & 1.9 & 1.5 & 1.4 & 1.4 & 0.2 & 0.2 & 0.2 & 0.8 & 1.1 & 1.2 \\
\hline 1.1 & 1.3 & 1.6 & 1.9 & 1.9 & 1.5 & 1.4 & 1.4 & 0.2 & 0.2 & 0.2 & 0.8 & 1.1 & 1.2 \\
\hline 0.5 & 0.6 & 0.7 & 0.9 & 0.9 & 0.7 & 0.6 & 0.6 & 0.1 & 0.1 & 0.1 & 0.3 & 0.5 & 0.5 \\
\hline 0.1 & 0.1 & 0.1 & 0.2 & 0.2 & 0.1 & 0.1 & 0.1 & 0 & 0 & 0 & 0.1 & 0.1 & 0.1 \\
\hline 7.3 & 8.4 & 11 & 13 & 13 & 9.8 & 9.2 & 9.2 & 1.3 & 1.3 & 1.3 & 5 & 7.5 & 8 \\
\hline 0.1 & 0.1 & 0.1 & 0.2 & 0.1 & 0.1 & 0.1 & 0.1 & 0 & 0 & 0 & 0.1 & 0.1 & 0.1 \\
\hline 7.3 & 8.4 & 11 & 13 & 13 & 9.8 & 9.2 & 9.2 & 1.3 & 1.3 & 1.3 & 5 & 7.5 & 8 \\
\hline 0.2 & 0.2 & 0.3 & 0.3 & 0.3 & 0.3 & 0.2 & 0.2 & 0 & 0 & 0 & 0.1 & 0.2 & 0.2 \\
\hline 301 & 347 & 452 & 541 & 533 & 416 & 389 & 389 & 55 & 55 & 55 & 212 & 317 & 337 \\
\hline
\end{tabular}


Table A-20: Wanapum and Priest Rapids Allocation

PNW Loads and Resources Study

2013 - 2014 Operating Year

1937 Water Year

2003 White Book

558

Sep

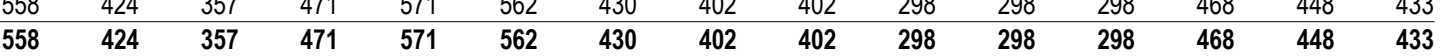

-Wanapum-

3 AVWP Share

4 COPD Share

5 CWPC Share

6 EWEB Share

7 FGRV Share

8 FREC Share

9 GCPD Share

10 ICLP Share

$11 \mathrm{KITT}$ Share

12 KOOT Share

13 LREC Share

14 LVE Share

15 MCMN Share

16 MTFR Share

17 NLEC Share

18 PGE Share

19 PPL Share

20 PSE Share

21 RREC Share

22 SCL Share

23 SLEC Share

24 TPU Share

25 UNEC Share

\begin{tabular}{|c|c|c|c|c|c|c|c|c|c|c|c|c|c|c|}
\hline 20 & 15 & 12 & 17 & 21 & 20 & 15 & 14 & 14 & 9.5 & 9.5 & 9.5 & 16 & 16 & 15 \\
\hline 6.7 & 4.9 & 4 & 5.5 & 6.8 & 6.7 & 4.9 & 4.6 & 4.6 & 3.1 & 3.1 & 3.1 & 5.4 & 5.1 & 5.0 \\
\hline 0.5 & 0.4 & 0.3 & 0.4 & 0.5 & 0.5 & 0.4 & 0.3 & 0.3 & 0.2 & 0.2 & 0.2 & 0.4 & 0.4 & 0.4 \\
\hline 5.7 & 4.1 & 3.4 & 4.7 & 5.8 & 5.7 & 4.2 & 3.9 & 3.9 & 2.7 & 2.7 & 2.7 & 4.6 & 4.4 & 4.2 \\
\hline 1.7 & 1.2 & 1 & 1.4 & 1.7 & 1.7 & 1.3 & 1.2 & 1.2 & 0.8 & 0.8 & 0.8 & 1.4 & 1.3 & 1.3 \\
\hline 0.6 & 0.4 & 0.3 & 0.5 & 0.6 & 0.6 & 0.4 & 0.4 & 0.4 & 0.3 & 0.3 & 0.3 & 0.5 & 0.4 & 0.4 \\
\hline 297 & 216 & 178 & 245 & 309 & 303 & 223 & 207 & 207 & 142 & 142 & 142 & 245 & 233 & 224 \\
\hline 0.2 & 0.1 & 0.1 & 0.1 & 0.2 & 0.2 & 0.1 & 0.1 & 0.1 & 0.1 & 0.1 & 0.1 & 0.1 & 0.1 & 0.1 \\
\hline 0.6 & 0.4 & 0.3 & 0.5 & 0.6 & 0.6 & 0.4 & 0.4 & 0.4 & 0.3 & 0.3 & 0.3 & 0.5 & 0.4 & 0.4 \\
\hline 0.9 & 0.6 & 0.5 & 0.7 & 0.9 & 0.9 & 0.6 & 0.6 & 0.6 & 0.4 & 0.4 & 0.4 & 0.7 & 0.7 & 0.6 \\
\hline 0.1 & 0.1 & 0.1 & 0.1 & 0.1 & 0.1 & 0.1 & 0.1 & 0.1 & 0.1 & 0.1 & 0.1 & 0.1 & 0.1 & 0.1 \\
\hline 1 & 0.7 & 0.6 & 0.8 & 1 & 1 & 0.7 & 0.7 & 0.7 & 0.5 & 0.5 & 0.5 & 0.8 & 0.8 & 0.8 \\
\hline 1.7 & 1.2 & 1 & 1.4 & 1.7 & 1.7 & 1.3 & 1.2 & 1.2 & 0.8 & 0.8 & 0.8 & 1.4 & 1.3 & 1.3 \\
\hline 1.7 & 1.2 & 1 & 1.4 & 1.7 & 1.7 & 1.3 & 1.2 & 1.2 & 0.8 & 0.8 & 0.8 & 1.4 & 1.3 & 1.3 \\
\hline 0.8 & 0.6 & 0.5 & 0.6 & 0.8 & 0.8 & 0.6 & 0.5 & 0.5 & 0.4 & 0.4 & 0.4 & 0.6 & 0.6 & 0.6 \\
\hline 47 & 34 & 28 & 38 & 47 & 46 & 34 & 32 & 32 & 22 & 22 & 22 & 37 & 36 & 34 \\
\hline 47 & 34 & 28 & 38 & 47 & 46 & 34 & 32 & 32 & 22 & 22 & 22 & 38 & 36 & 35 \\
\hline 27 & 20 & 16 & 22 & 27 & 27 & 20 & 18 & 18 & 13 & 13 & 13 & 22 & 21 & 20 \\
\hline 0.2 & 0.1 & 0.1 & 0.1 & 0.2 & 0.2 & 0.1 & 0.1 & 0.1 & 0.1 & 0.1 & 0.1 & 0.1 & 0.1 & 0.1 \\
\hline 11 & 8.3 & 6.9 & 9.4 & 12 & 11 & 8.4 & 7.7 & 7.7 & 5.3 & 5.3 & 5.3 & 9.2 & 8.7 & 8.5 \\
\hline 0.1 & 0.1 & 0.1 & 0.1 & 0.1 & 0.1 & 0.1 & 0.1 & 0.1 & 0.1 & 0.1 & 0.1 & 0.1 & 0.1 & 0.1 \\
\hline 11 & 8.3 & 6.9 & 9.4 & 12 & 11 & 8.4 & 7.7 & 7.7 & 5.3 & 5.3 & 5.3 & 9.2 & 8.7 & 8.5 \\
\hline 0.3 & 0.2 & 0.2 & 0.2 & 0.3 & 0.3 & 0.2 & 0.2 & 0.2 & 0.1 & 0.1 & 0.1 & 0.2 & 0.2 & 0.2 \\
\hline 484 & 351 & 290 & 398 & 497 & 488 & 359 & 333 & 333 & 229 & 229 & 229 & 395 & 375 & 362 \\
\hline
\end{tabular}

-Priest Rapids-

27 Priest Rapids

28 Priest Rapids Before Enc

$318 \quad 36$

$362 \quad 468 \quad 557$

$557 \quad 550 \quad 431 \quad 404$

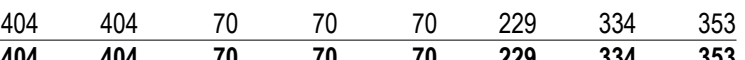

-Priest Rapids-

29 AVWP Share 30 COPD Share

31 CWPC Share

32 EWEB Share

33 FGRV Share

34 FREC Share

35 GCPD Share

36 ICLP Share

37 KITT Share

38 KOOT Share

39 LREC Share

40 LVE Share

41 MCMN Share

42 MTFR Share

43 NLEC Share

44 PGE Share

45 PPL Share

46 PSE Share

47 RREC Share

$48 \mathrm{SCL}$ Share

49 SLEC Share

50 TPU Share

51 UNEC Share

\begin{tabular}{|c|c|c|c|c|c|c|c|c|c|c|c|c|c|}
\hline 16 & 15 & 19 & 23 & 22 & 17 & 16 & 16 & 2.3 & 2.3 & 2.3 & 8.8 & 13 & 14 \\
\hline 5.4 & 4.8 & 6.3 & 7.4 & 7.3 & 5.7 & 5.3 & 5.3 & 0.8 & 0.8 & 0.8 & 2.9 & 4.3 & 4.6 \\
\hline 0.4 & 0.4 & 0.5 & 0.6 & 0.5 & 0.4 & 0.4 & 0.4 & 0.1 & 0.1 & 0.1 & 0.2 & 0.3 & 0.3 \\
\hline 4.6 & 4.1 & 5.3 & 6.3 & 6.2 & 4.8 & 4.5 & 4.5 & 0.6 & 0.6 & 0.6 & 2.5 & 3.7 & 3.9 \\
\hline 1.4 & 1.2 & 1.6 & 1.9 & 1.9 & 1.5 & 1.4 & 1.4 & 0.2 & 0.2 & 0.2 & 0.7 & 1.1 & 1.2 \\
\hline 0.5 & 0.4 & 0.5 & 0.6 & 0.6 & 0.5 & 0.5 & 0.5 & 0.1 & 0.1 & 0.1 & 0.3 & 0.4 & 0.4 \\
\hline 185 & 213 & 278 & 336 & 331 & 258 & 241 & 241 & 34 & 34 & 34 & 132 & 197 & 209 \\
\hline 0.1 & 0.1 & 0.2 & 0.2 & 0.2 & 0.1 & 0.1 & 0.1 & 0 & 0 & 0 & 0.1 & 0.1 & 0.1 \\
\hline 0.5 & 0.4 & 0.5 & 0.6 & 0.6 & 0.5 & 0.5 & 0.5 & 0.1 & 0.1 & 0.1 & 0.3 & 0.4 & 0.4 \\
\hline 0.7 & 0.6 & 0.8 & 1.0 & 0.9 & 0.7 & 0.7 & 0.7 & 0.1 & 0.1 & 0.1 & 0.4 & 0.6 & 0.6 \\
\hline 0.1 & 0.1 & 0.1 & 0.1 & 0.1 & 0.1 & 0.1 & 0.1 & 0 & 0 & 0 & 0.1 & 0.1 & 0.1 \\
\hline 0.8 & 0.7 & 1.0 & 1.1 & 1.1 & 0.9 & 0.8 & 0.8 & 0.1 & 0.1 & 0.1 & 0.4 & 0.7 & 0.7 \\
\hline 1.4 & 1.2 & 1.6 & 1.9 & 1.9 & 1.5 & 1.4 & 1.4 & 0.2 & 0.2 & 0.2 & 0.7 & 1.1 & 1.2 \\
\hline 1.4 & 1.2 & 1.6 & 1.9 & 1.9 & 1.5 & 1.4 & 1.4 & 0.2 & 0.2 & 0.2 & 0.7 & 1.1 & 1.2 \\
\hline 0.6 & 0.6 & 0.7 & 0.9 & 0.8 & 0.7 & 0.6 & 0.6 & 0.1 & 0.1 & 0.1 & 0.3 & 0.5 & 0.5 \\
\hline 37 & 33 & 44 & 51 & 51 & 39 & 37 & 37 & 5.2 & 5.2 & 5.2 & 20 & 30 & 32 \\
\hline 37 & 33 & 44 & 51 & 51 & 39 & 37 & 37 & 5.2 & 5.2 & 5.2 & 20 & 30 & 32 \\
\hline 21 & 19 & 25 & 30 & 29 & 23 & 21 & 21 & 3.0 & 3.0 & 3.0 & 12 & 17 & 19 \\
\hline 0.1 & 0.1 & 0.1 & 0.2 & 0.2 & 0.1 & 0.1 & 0.1 & 0 & 0 & 0 & 0.1 & 0.1 & 0.1 \\
\hline 9.1 & 8.2 & 11 & 13 & 12 & 9.7 & 9.1 & 9.1 & 1.3 & 1.3 & 1.3 & 4.9 & 7.4 & 7.9 \\
\hline 0.1 & 0.1 & 0.1 & 0.2 & 0.1 & 0.1 & 0.1 & 0.1 & 0 & 0 & 0 & 0.1 & 0.1 & 0.1 \\
\hline 9.1 & 8.2 & 11 & 13 & 12 & 9.7 & 9.1 & 9.1 & 1.3 & 1.3 & 1.3 & 4.9 & 7.4 & 7.9 \\
\hline 0.2 & 0.2 & 0.3 & 0.3 & 0.3 & 0.2 & 0.2 & 0.2 & 0 & 0 & 0 & 0.1 & 0.2 & 0.2 \\
\hline 301 & 347 & 452 & 541 & 533 & 416 & 389 & 389 & 55 & 55 & 55 & 212 & 317 & 337 \\
\hline
\end{tabular}


Table A-21: Swift \#1 and Swift \#2 Allocation

PNW Loads and Resources Study

2004 - 2005 Operating Year

1937 Water Year

2003 White Book

Average Energy in Megawatts Aug1 Aug16 Sep Oct Nov Dec Jan Feb Mar Apr1 Apr16 May Jun Jul Avg

-Swift 1-

1 Swift 1

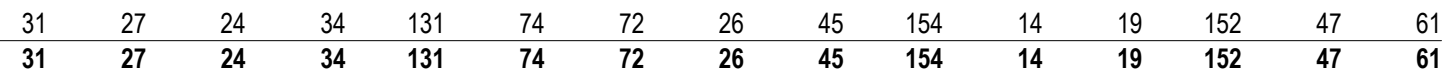

2 Swift 1 Before Enc

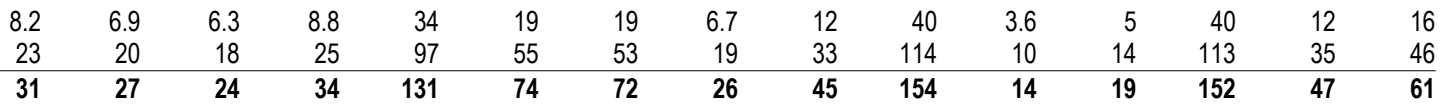

4 PPL Share

5 Swift 1 After Enc

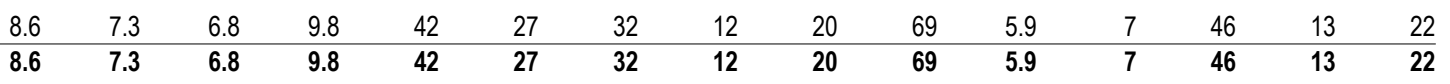

-Swift 2-

6 Swift 2

7 Swift 2 Before Enc

\begin{tabular}{|c|c|c|c|c|c|c|c|c|c|c|c|c|c|c|}
\hline 2.2 & 1.9 & 1.8 & 2.5 & 11 & 6.9 & 8.2 & 3.2 & 5.3 & 18 & 1.5 & 1.8 & 12 & 3.5 & 5.7 \\
\hline 6.4 & 5.4 & 5 & 7.3 & 31 & 20 & 23 & 9.1 & 15 & 51 & 4.4 & 5.2 & 34 & 9.9 & 16 \\
\hline 8.6 & 7.3 & 6.8 & 9.8 & 42 & 27 & 32 & 12 & 20 & 69 & 5.9 & 7 & 46 & 13 & 22 \\
\hline
\end{tabular}

-Swift 2-

8 COPD Share

9 PPL Share 
Table A-21: Swift \#1 and Swift \#2 Allocation

PNW Loads and Resources Study

2005 - 2006 Operating Year

1937 Water Year

2003 White Book

Average Energy in Megawatts Aug1 Aug16 Sep Oct Nov Dec Jan Feb Mar Apr1 Apr16 May Jun Jul Avg

-Swift 1-

1 Swift 1

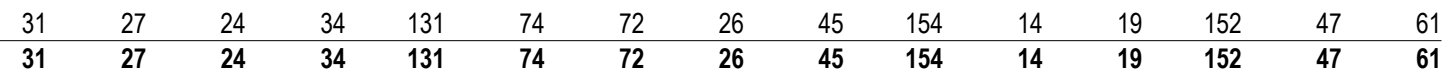

2 Swift 1 Before Enc

\begin{tabular}{|c|c|c|c|c|c|c|c|c|c|c|c|c|c|}
\hline 8.2 & $\begin{array}{r}6.9 \\
20\end{array}$ & $\begin{array}{r}6.3 \\
18\end{array}$ & $\begin{array}{r}8.8 \\
25\end{array}$ & $\begin{array}{l}34 \\
97\end{array}$ & $\begin{array}{l}19 \\
55\end{array}$ & $\begin{array}{l}19 \\
53\end{array}$ & $\begin{array}{r}6.7 \\
19\end{array}$ & $\begin{array}{l}12 \\
33\end{array}$ & $\begin{array}{r}40 \\
114\end{array}$ & $\begin{array}{r}3.6 \\
10\end{array}$ & $\begin{array}{r}5 \\
14\end{array}$ & $\begin{array}{r}40 \\
113\end{array}$ & $\begin{array}{l}12 \\
35\end{array}$ \\
\hline 31 & 27 & 24 & 34 & 131 & 74 & 72 & 26 & 45 & 154 & 14 & 19 & 152 & 47 \\
\hline
\end{tabular}

3 COPD Shar

4 PPL Share

5 Swift 1 After Enc

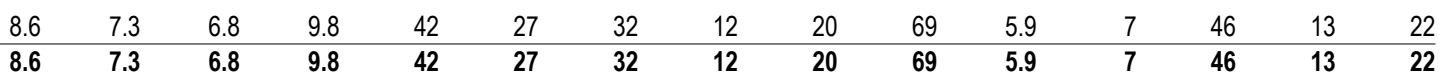

-Swift 2-

6 Swift 2

7 Swift 2 Before Enc

\begin{tabular}{|c|c|c|c|c|c|c|c|c|c|c|c|c|c|c|}
\hline 2.2 & 1.9 & 1.8 & 2.5 & 11 & 6.9 & 8.2 & 3.2 & 5.3 & 18 & 1.5 & 1.8 & 12 & 3.5 & 5.7 \\
\hline 6.4 & 5.4 & 5 & 7.3 & 31 & 20 & 23 & 9.1 & 15 & 51 & 4.4 & 5.2 & 34 & 9.9 & 16 \\
\hline 8.6 & 7.3 & 6.8 & 9.8 & 42 & 27 & 32 & 12 & 20 & 69 & 5.9 & 7 & 46 & 13 & 22 \\
\hline
\end{tabular}

-Swift 2-

8 COPD Share

9 PPL Share 
Table A-21: Swift \#1 and Swift \#2 Allocation

PNW Loads and Resources Study

2006 - 2007 Operating Year

1937 Water Year

2003 White Book

Average Energy in Megawatts Aug1 Aug16 Sep Oct Nov Dec Jan Feb Mar Apr1 Apr16 May Jun Jul Avg

-Swift 1-

1 Swift 1

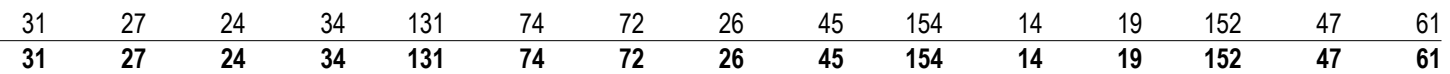

2 Swift 1 Before Enc

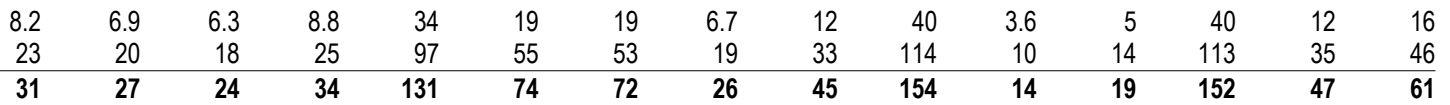

4 PPL Share

5 Swift 1 After Enc

\begin{tabular}{|c|c|c|c|c|c|c|c|c|c|c|c|c|c|c|}
\hline 8.6 & 7.3 & 6.8 & 9.8 & 42 & 27 & 32 & 12 & 20 & 69 & 5.9 & 7 & 46 & 13 & 22 \\
\hline 8 & 7.3 & 6.8 & 9.8 & 42 & 27 & 32 & 12 & 20 & 69 & 5.9 & 7 & 46 & 13 & 22 \\
\hline
\end{tabular}

-Swift 2-

6 Swift 2

7 Swift 2 Before Enc

\begin{tabular}{|c|c|c|c|c|c|c|c|c|c|c|c|c|c|c|}
\hline 2.2 & 1.9 & 1.8 & 2.5 & 11 & 6.9 & 8.2 & 3.2 & 5.3 & 18 & 1.5 & 1.8 & 12 & 3.5 & 5.7 \\
\hline 6.4 & 5.4 & 5 & 7.3 & 31 & 20 & 23 & 9.1 & 15 & 51 & 4.4 & 5.2 & 34 & 9.9 & 16 \\
\hline 8.6 & 7.3 & 6.8 & 9.8 & 42 & 27 & 32 & 12 & 20 & 69 & 5.9 & 7 & 46 & 13 & 22 \\
\hline
\end{tabular}

-Swift 2-

8 COPD Share

9 PPL Share

10 Swift 2 After Enc 
Table A-21: Swift \#1 and Swift \#2 Allocation

PNW Loads and Resources Study

2007 - 2008 Operating Year

1937 Water Year

2003 White Book

Average Energy in Megawatts Aug1 Aug16 Sep Oct Nov Dec Jan Feb Mar Apr1 Apr16 May Jun Jul Avg

-Swift 1-

1 Swift 1

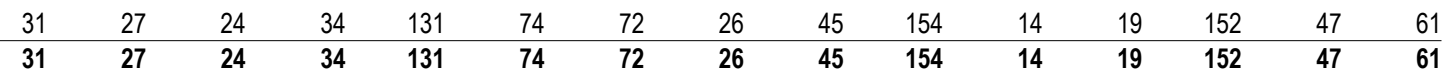

2 Swift 1 Before Enc

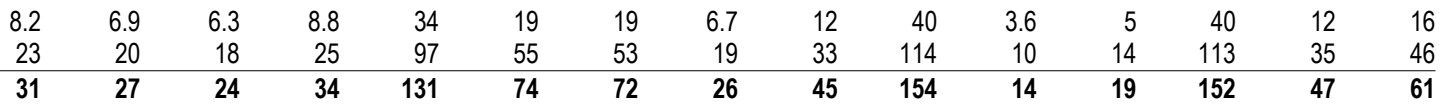

4 PPL Share

5 Swift 1 After Enc

\begin{tabular}{|c|c|c|c|c|c|c|c|c|c|c|c|c|c|c|}
\hline 8.6 & 7.3 & 6.8 & 9.8 & 42 & 27 & 32 & 12 & 20 & 69 & 5.9 & 7 & 46 & 13 & 22 \\
\hline 8 & 7.3 & 6.8 & 9.8 & 42 & 27 & 32 & 12 & 20 & 69 & 5.9 & 7 & 46 & 13 & 22 \\
\hline
\end{tabular}

6 Swift 2

7 Swift 2 Before Enc

\begin{tabular}{|c|c|c|c|c|c|c|c|c|c|c|c|c|c|c|}
\hline 2.2 & 1.9 & 1.8 & 2.5 & 11 & 6.9 & 8.2 & 3.2 & 5.3 & 18 & 1.5 & 1.8 & 12 & 3.5 & 5.7 \\
\hline 6.4 & 5.4 & 5 & 7.3 & 31 & 20 & 23 & 9.1 & 15 & 51 & 4.4 & 5.2 & 34 & 9.9 & 16 \\
\hline 8.6 & 7.3 & 6.8 & 9.8 & 42 & 27 & 32 & 12 & 20 & 69 & 5.9 & 7 & 46 & 13 & 22 \\
\hline
\end{tabular}

-Swift 2-

8 COPD Share

9 PPL Share 
Table A-21: Swift \#1 and Swift \#2 Allocation

PNW Loads and Resources Study

2008 - 2009 Operating Year

1937 Water Year

2003 White Book

Average Energy in Megawatts Aug1 Aug16 Sep Oct Nov Dec Jan Feb Mar Apr1 Apr16 May Jun Jul Avg

-Swift 1-

1 Swift 1

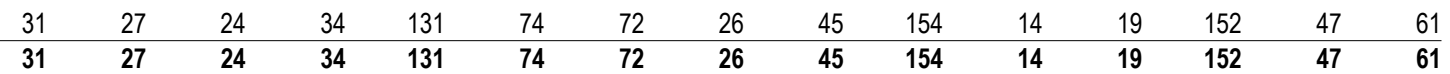

2 Swift 1 Before Enc

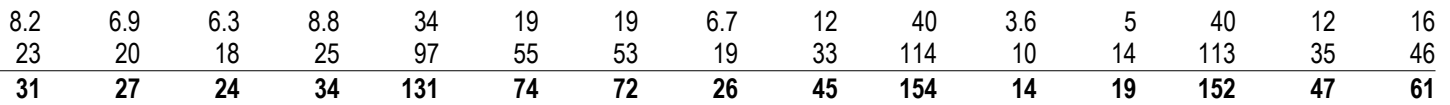

3 COPD Shar

4 PPL Share

5 Swift 1 After Enc

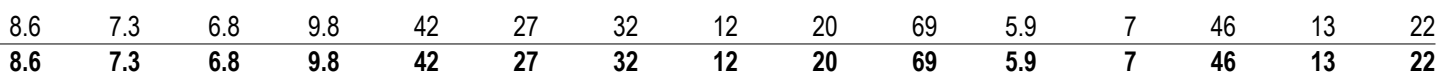

-Swift 2-

6 Swift 2

7 Swift 2 Before Enc

\begin{tabular}{|c|c|c|c|c|c|c|c|c|c|c|c|c|c|c|}
\hline 2.2 & 1.9 & 1.8 & 2.5 & 11 & 6.9 & 8.2 & 3.2 & 5.3 & 18 & 1.5 & 1.8 & 12 & 3.5 & 5.7 \\
\hline 6.4 & 5.4 & 5 & 7.3 & 31 & 20 & 23 & 9.1 & 15 & 51 & 4.4 & 5.2 & 34 & 9.9 & 16 \\
\hline 8.6 & 7.3 & 6.8 & 9.8 & 42 & 27 & 32 & 12 & 20 & 69 & 5.9 & 7 & 46 & 13 & 22 \\
\hline
\end{tabular}

-Swift 2-

8 COPD Share

9 PPL Share 
Table A-21: Swift \#1 and Swift \#2 Allocation

PNW Loads and Resources Study

2009 - 2010 Operating Year

1937 Water Year

2003 White Book

Average Energy in Megawatts Aug1 Aug16 Sep Oct Nov Dec Jan Feb Mar Apr1 Apr16 May Jun Jul Avg

-Swift 1-

1 Swift 1

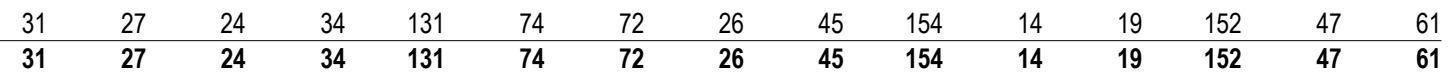

2 Swift 1 Before Enc

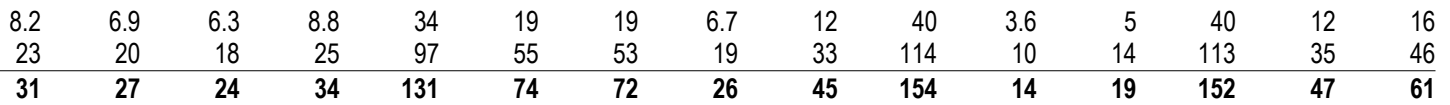

3 COPD Shar

4 PPL Share

5 Swift 1 After Enc

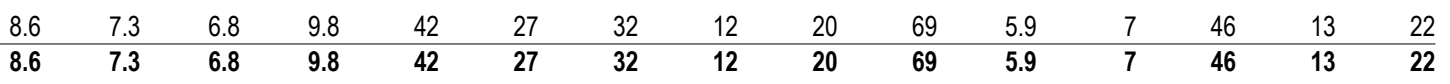

-Swift 2-

6 Swift 2

7 Swift 2 Before Enc

\begin{tabular}{|c|c|c|c|c|c|c|c|c|c|c|c|c|c|c|}
\hline 2.2 & 1.9 & 1.8 & 2.5 & 11 & 6.9 & 8.2 & 3.2 & 5.3 & 18 & 1.5 & 1.8 & 12 & 3.5 & 5.7 \\
\hline 6.4 & 5.4 & 5 & 7.3 & 31 & 20 & 23 & 9.1 & 15 & 51 & 4.4 & 5.2 & 34 & 9.9 & 16 \\
\hline 8.6 & 7.3 & 6.8 & 9.8 & 42 & 27 & 32 & 12 & 20 & 69 & 5.9 & 7 & 46 & 13 & 22 \\
\hline
\end{tabular}

-Swift 2-

8 COPD Share

9 PPL Share 
Table A-21: Swift \#1 and Swift \#2 Allocation

PNW Loads and Resources Study

2010 - 2011 Operating Year

1937 Water Year

2003 White Book

Average Energy in Megawatts Aug1 Aug16 Sep Oct Nov Dec Jan Feb Mar Apr1 Apr16 May Jun Jul Avg

-Swift 1-

1 Swift 1

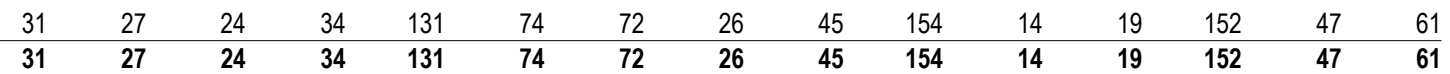

2 Swift 1 Before Enc

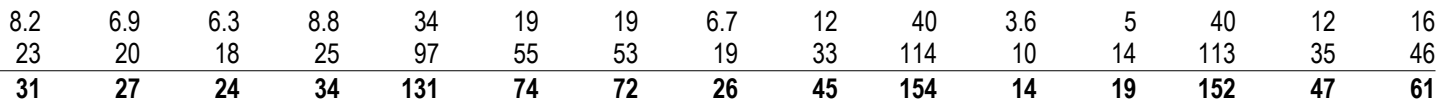

4 PPL Share

5 Swift 1 After Enc

\begin{tabular}{|c|c|c|c|c|c|c|c|c|c|c|c|c|c|c|}
\hline 8.6 & 7.3 & 6.8 & 9.8 & 42 & 27 & 32 & 12 & 20 & 69 & 5.9 & 7 & 46 & 13 & 22 \\
\hline 8 & 7.3 & 6.8 & 9.8 & 42 & 27 & 32 & 12 & 20 & 69 & 5.9 & 7 & 46 & 13 & 22 \\
\hline
\end{tabular}

6 Swift 2

7 Swift 2 Before Enc

\begin{tabular}{|c|c|c|c|c|c|c|c|c|c|c|c|c|c|c|}
\hline 2.2 & 1.9 & 1.8 & 2.5 & 11 & 6.9 & 8.2 & 3.2 & 5.3 & 18 & 1.5 & 1.8 & 12 & 3.5 & 5.7 \\
\hline 6.4 & 5.4 & 5 & 7.3 & 31 & 20 & 23 & 9.1 & 15 & 51 & 4.4 & 5.2 & 34 & 9.9 & 16 \\
\hline 8.6 & 7.3 & 6.8 & 9.8 & 42 & 27 & 32 & 12 & 20 & 69 & 5.9 & 7 & 46 & 13 & 22 \\
\hline
\end{tabular}

-Swift 2-

8 COPD Share

9 PPL Share 
Table A-21: Swift \#1 and Swift \#2 Allocation

PNW Loads and Resources Study

2011 - 2012 Operating Year

1937 Water Year

2003 White Book

Average Energy in Megawatts Aug1 Aug16 Sep Oct Nov Dec Jan Feb Mar Apr1 Apr16 May Jun Jul Avg

-Swift 1-

1 Swift 1

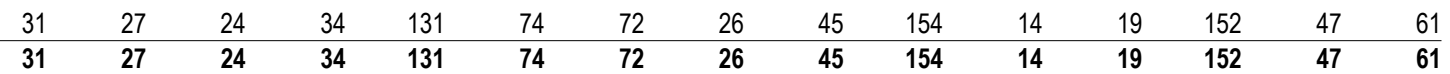

2 Swift 1 Before Enc

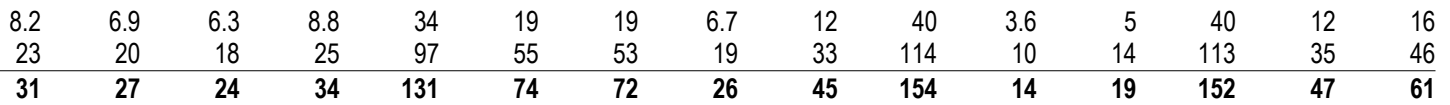

4 PPL Share

5 Swift 1 After Enc

\begin{tabular}{|c|c|c|c|c|c|c|c|c|c|c|c|c|c|c|}
\hline 8.6 & 7.3 & 6.8 & 9.8 & 42 & 27 & 32 & 12 & 20 & 69 & 5.9 & 7 & 46 & 13 & 22 \\
\hline 8 & 7.3 & 6.8 & 9.8 & 42 & 27 & 32 & 12 & 20 & 69 & 5.9 & 7 & 46 & 13 & 22 \\
\hline
\end{tabular}

-Swift 2-

6 Swift 2

7 Swift 2 Before Enc

\begin{tabular}{|c|c|c|c|c|c|c|c|c|c|c|c|c|c|c|}
\hline 2.2 & 1.9 & 1.8 & 2.5 & 11 & 6.9 & 8.2 & 3.2 & 5.3 & 18 & 1.5 & 1.8 & 12 & 3.5 & 5.7 \\
\hline 6.4 & 5.4 & 5 & 7.3 & 31 & 20 & 23 & 9.1 & 15 & 51 & 4.4 & 5.2 & 34 & 9.9 & 16 \\
\hline 8.6 & 7.3 & 6.8 & 9.8 & 42 & 27 & 32 & 12 & 20 & 69 & 5.9 & 7 & 46 & 13 & 22 \\
\hline
\end{tabular}

-Swift 2-

8 COPD Share

9 PPL Share

10 Swift 2 After Enc 
Table A-21: Swift \#1 and Swift \#2 Allocation

PNW Loads and Resources Study

2012 - 2013 Operating Year

1937 Water Year

2003 White Book

Average Energy in Megawatts Aug1 Aug16 Sep Oct Nov Dec Jan Feb Mar Apr1 Apr16 May Jun Jul Avg

-Swift 1-

1 Swift 1

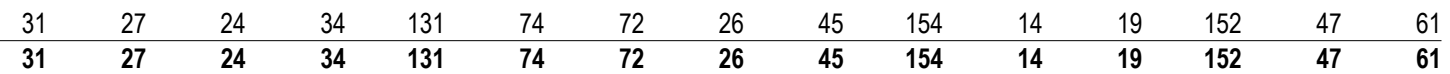

2 Swift 1 Before Enc

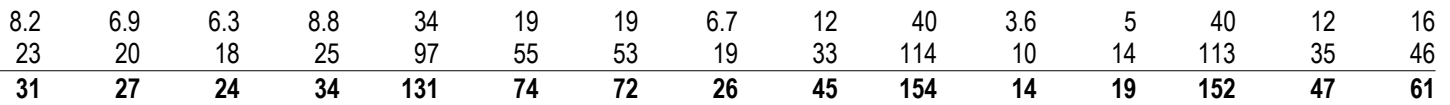

3 COPD Shar

4 PPL Share

5 Swift 1 After Enc

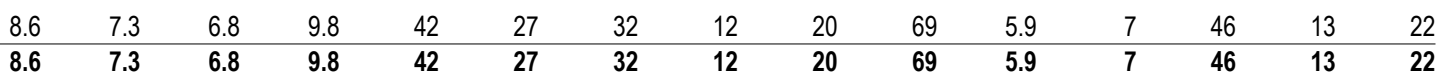

-Swift 2-

6 Swift 2

7 Swift 2 Before Enc

$\begin{array}{rrrrrrrrrrrrrrr}2.2 & 1.9 & 1.8 & 2.5 & 11 & 6.9 & 8.2 & 3.2 & 5.3 & 18 & 1.5 & 1.8 & 12 & 3.5 & 5.7 \\ 6.4 & 5.4 & 5 & 7.3 & 31 & 20 & 23 & 9.1 & 15 & 51 & 4.4 & 5.2 & 34 & 9.9 & 16 \\ \mathbf{8 . 6} & \mathbf{7 . 3} & \mathbf{6 . 8} & \mathbf{9 . 8} & \mathbf{4 2} & \mathbf{2 7} & \mathbf{3 2} & \mathbf{1 2} & \mathbf{2 0} & \mathbf{6 9} & \mathbf{5 . 9} & \mathbf{7} & \mathbf{4 6} & \mathbf{1 3} & \mathbf{2 2}\end{array}$

-Swift 2-

8 COPD Share

9 PPL Share 
Table A-21: Swift \#1 and Swift \#2 Allocation

PNW Loads and Resources Study

2013 - 2014 Operating Year

1937 Water Year

2003 White Book

Average Energy in Megawatts Aug1 Aug16 Sep Oct Nov Dec Jan Feb Mar Apr1 Apr16 May Jun Jul Avg

-Swift 1-

1 Swift 1

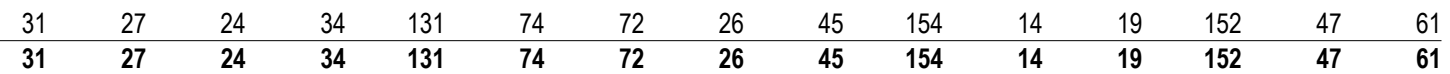

2 Swift 1 Before Enc

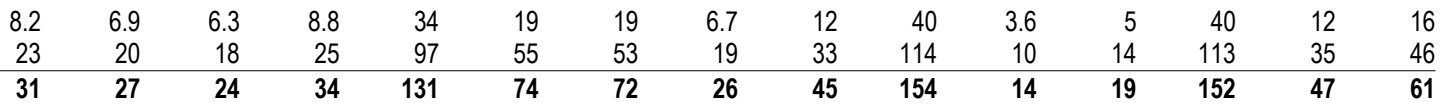

3 COPD Shar

4 PPL Share

5 Swift 1 After Enc

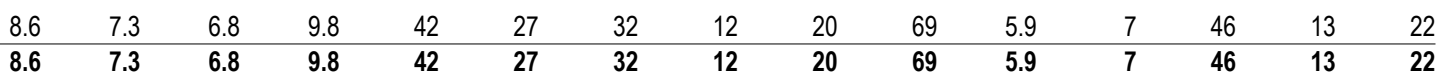

-Swift 2-

6 Swift 2

7 Swift 2 Before Enc

\begin{tabular}{|c|c|c|c|c|c|c|c|c|c|c|c|c|c|c|}
\hline 2.2 & 1.9 & 1.8 & 2.5 & 11 & 6.9 & 8.2 & 3.2 & 5.3 & 18 & 1.5 & 1.8 & 12 & 3.5 & 5.7 \\
\hline 6.4 & 5.4 & 5 & 7.3 & 31 & 20 & 23 & 9.1 & 15 & 51 & 4.4 & 5.2 & 34 & 9.9 & 16 \\
\hline 8.6 & 7.3 & 6.8 & 9.8 & 42 & 27 & 32 & 12 & 20 & 69 & 5.9 & 7 & 46 & 13 & 22 \\
\hline
\end{tabular}

-Swift 2-

8 COPD Share

9 PPL Share 
Table A-22: BPA Power Sale Contracts

PNW Loads and Resources Study

2004 - 2005 Operating Year

1937 Water Year

2003 White Book

Average Energy in Megawatts Aug1 Aug16 Sep Oct Nov Dec Jan Feb Mar Apr1 Apr16 May Jun Jul Avg

-Federal Entities-

1 Federal Entities

2 Total Federal Entities

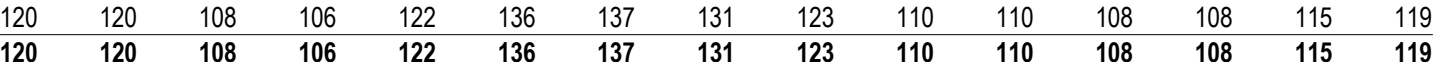

-U.S. Bureau of Reclamation-

3 U.S. Bureau of Reclamation

$\begin{array}{lllllllllllllll}309 & 275 & 166 & 129 & 0 & 0 & 0 & 1.0 & 23 & 252 & 315 & 268 & 298 & 332 & 149 \\ \mathbf{3 0 9} & \mathbf{2 7 5} & \mathbf{1 6 6} & \mathbf{1 2 9} & \mathbf{0} & \mathbf{0} & \mathbf{0} & \mathbf{1 . 0} & \mathbf{2 3} & \mathbf{2 5 2} & \mathbf{3 1 5} & \mathbf{2 6 8} & \mathbf{2 9 8} & \mathbf{3 3 2} & \mathbf{1 4 9}\end{array}$

-Direct Service Industry-

5 Direct Service Industry

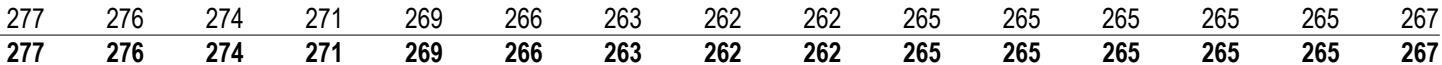

6 Total Direct Service Industry

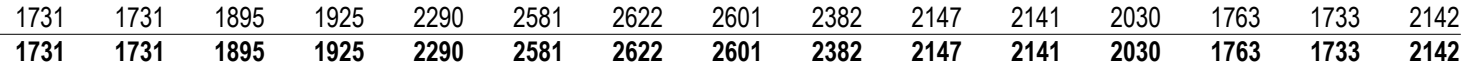

-Generating Public Entities.

7 Generating Public Entities

-Generating Public Entities (Slice)-

9 Generating Public Entities (Slic

10 Total Generating Public Entities (Slice

$\begin{array}{lllllllllllllll}1399 & 1074 & 831 & 1014 & 1108 & 1117 & 915 & 873 & 951 & 903 & 870 & 927 & 1063 & 1206 & 1011 \\ 1399 & \mathbf{1 0 7 4} & \mathbf{8 3 1} & \mathbf{1 0 1 4} & \mathbf{1 1 0 8} & \mathbf{1 1 1 7} & \mathbf{9 1 5} & \mathbf{8 7 3} & \mathbf{9 5 1} & \mathbf{9 0 3} & \mathbf{8 7 0} & \mathbf{9 2 7} & \mathbf{1 0 6 3} & \mathbf{1 2 0 6} & \mathbf{1 0 1 1}\end{array}$

-Non-Generating Public Entities-

11 Non-Generating Public Entities

12 Total Non-Generating Public Entities

\begin{tabular}{lllllllllllllll}
2618 & 2618 & 2399 & 2386 & 2678 & 3076 & 3175 & 3035 & 2765 & 2602 & 2597 & 2540 & 2600 & 2717 & 2716 \\
\hline $\mathbf{2 6 1 8}$ & $\mathbf{2 6 1 8}$ & $\mathbf{2 3 9 9}$ & $\mathbf{2 3 8 6}$ & $\mathbf{2 6 7 8}$ & $\mathbf{3 0 7 6}$ & $\mathbf{3 1 7 5}$ & $\mathbf{3 0 3 5}$ & $\mathbf{2 7 6 5}$ & $\mathbf{2 6 0 2}$ & $\mathbf{2 5 9 7}$ & $\mathbf{2 5 4 0}$ & $\mathbf{2 6 0 0}$ & $\mathbf{2 7 1 7}$ & $\mathbf{2 7 1 6}$
\end{tabular}

-Non-Generating Public Entities (Slice)-

13 Non-Generating Public Entities (Slice)

14 Total Non-Generating Public Entities (Slice)

$\begin{array}{lllllllllllllll}840 & 645 & 499 & 609 & 665 & 670 & 549 & 524 & 571 & 542 & 523 & 556 & 638 & 724 & 607 \\ \mathbf{8 4 0} & \mathbf{6 4 5} & \mathbf{4 9 9} & \mathbf{6 0 9} & \mathbf{6 6 5} & \mathbf{6 7 0} & \mathbf{5 4 9} & \mathbf{5 2 4} & \mathbf{5 7 1} & \mathbf{5 4 2} & \mathbf{5 2 3} & \mathbf{5 5 6} & \mathbf{6 3 8} & \mathbf{7 2 4} & \mathbf{6 0 7}\end{array}$

-Investor-Owned Entities-

15 Investor-Owned Entities

16 Total Investor-Owned Entities

$\begin{array}{lllllllllllllll}382 & 382 & 382 & 382 & 382 & 382 & 382 & 382 & 382 & 382 & 382 & 382 & 382 & 382 & 382 \\ \mathbf{3 8 2} & \mathbf{3 8 2} & \mathbf{3 8 2} & \mathbf{3 8 2} & \mathbf{3 8 2} & \mathbf{3 8 2} & \mathbf{3 8 2} & \mathbf{3 8 2} & \mathbf{3 8 2} & \mathbf{3 8 2} & \mathbf{3 8 2} & \mathbf{3 8 2} & \mathbf{3 8 2} & \mathbf{3 8 2} & \mathbf{3 8 2}\end{array}$

-Other Entities-

17 Other PNW Entities

18 Total Other Entities

0

0

0

0

0

Power Sales Contracts-

19 Federal Entities

20 U.S. Bureau of Reclamation

21 Direct Service Industry

22 Generating Public Entities

23 Non-Generating Public Entities

24 Investor-Owned Entities

25 Other Entities

26 Total Power Sales Contracts

$\begin{array}{rrrrrr}120 & 120 & 108 & 106 & 122 & 136 \\ 309 & 275 & 166 & 129 & 0 & \\ 277 & 276 & 274 & 271 & 269 & 266 \\ 3130 & 2805 & 2726 & 2940 & 3398 & 3697 \\ 3458 & 3262 & 2897 & 2995 & 3343 & 37 \\ 382 & 382 & 382 & 382 & 382 & 382 \\ 0 & 0 & 0 & 0 & 0 & \\ \mathbf{7 6 7 5} & \mathbf{7 1 2 0} & \mathbf{6 5 5 3} & \mathbf{6 8 2 2} & \mathbf{7 5 1 4} & \mathbf{8 2}\end{array}$

0

0


Table A-22: BPA Power Sale Contracts

PNW Loads and Resources Study

2005 - 2006 Operating Year

1937 Water Year

2003 White Book

Average Energy in Megawatts Aug1 Aug16 Sep Oct Nov Dec Jan Feb Mar Apr1 Apr16 May Jun Jul Avg

-Federal Entities-

1 Federal Entities

2 Total Federal Entities

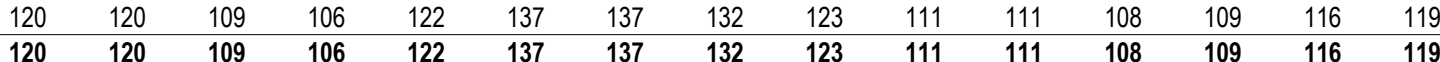

-U.S. Bureau of Reclamation-

3 U.S. Bureau of Reclamation

$\begin{array}{lllllllllllllll}309 & 275 & 166 & 129 & 0 & 0 & 0 & 1.0 & 23 & 252 & 315 & 268 & 298 & 332 & 149 \\ \mathbf{3 0 9} & \mathbf{2 7 5} & \mathbf{1 6 6} & \mathbf{1 2 9} & \mathbf{0} & \mathbf{0} & \mathbf{0} & \mathbf{1 . 0} & \mathbf{2 3} & \mathbf{2 5 2} & \mathbf{3 1 5} & \mathbf{2 6 8} & \mathbf{2 9 8} & \mathbf{3 3 2} & \mathbf{1 4 9}\end{array}$

-Direct Service Industry-

5 Direct Service Industry

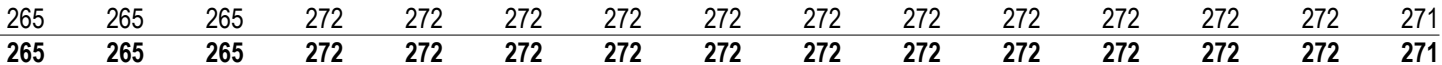

6 Total Direct Service Industry

$\begin{array}{lllllllllllllll}1723 & 1723 & 1897 & 1935 & 2299 & 2585 & 2626 & 2580 & 2388 & 2101 & 2095 & 1980 & 1715 & 1685 & 2126 \\ 1 \mathbf{1 7 2 3} & \mathbf{1 7 2 3} & \mathbf{1 8 9 7} & \mathbf{1 9 3 5} & \mathbf{2 2 9 9} & \mathbf{2 5 8 5} & \mathbf{2 6 2 6} & \mathbf{2 5 8 0} & \mathbf{2 3 8 8} & \mathbf{2 1 0 1} & \mathbf{2 0 9 5} & \mathbf{1 9 8 0} & \mathbf{1 7 1 5} & \mathbf{1 6 8 5} & \mathbf{2 1 2 6}\end{array}$

-Generating Public Entities.

7 Generating Public Entities

-Generating Public Entities (Slice)-

9 Generating Public Entities (Slice)

$\begin{array}{lllllllllllllll}1410 & 1084 & 840 & 1021 & 1116 & 1124 & 921 & 879 & 957 & 909 & 875 & 1015 & 1188 & 1210 & 1034 \\ 1410 & 1084 & \mathbf{8 4 0} & \mathbf{1 0 2 1} & \mathbf{1 1 1 6} & \mathbf{1 1 2 4} & \mathbf{9 2 1} & \mathbf{8 7 9} & \mathbf{9 5 7} & \mathbf{9 0 9} & \mathbf{8 7 5} & \mathbf{1 0 1 5} & \mathbf{1 1 8 8} & \mathbf{1 2 1 0} & \mathbf{1 0 3 4}\end{array}$

-Non-Generating Public Entities-

11 Non-Generating Public Entities

12 Total Non-Generating Public Entities

$\begin{array}{lllllllllllllll}2654 & 2654 & 2432 & 2416 & 2712 & 3116 & 3223 & 3082 & 2808 & 2644 & 2637 & 2580 & 2640 & 2759 & 2755 \\ \mathbf{2 6 5 4} & \mathbf{2 6 5 4} & \mathbf{2 4 3 2} & \mathbf{2 4 1 6} & \mathbf{2 7 1 2} & \mathbf{3 1 1 6} & \mathbf{3 2 2 3} & \mathbf{3 0 8 2} & \mathbf{2 8 0 8} & \mathbf{2 6 4 4} & \mathbf{2 6 3 7} & \mathbf{2 5 8 0} & \mathbf{2 6 4 0} & \mathbf{2 7 5 9} & \mathbf{2 7 5 5}\end{array}$

-Non-Generating Public Entities (Slice)-

13 Non-Generating Public Entities (Slice)

14 Total Non-Generating Public Entities (Slice

\begin{tabular}{lllllllllllllll}
846 & 651 & 504 & 613 & 670 & 675 & 553 & 528 & 574 & 546 & 526 & 609 & 713 & 726 & 621 \\
\hline $\mathbf{8 4 6}$ & $\mathbf{6 5 1}$ & $\mathbf{5 0 4}$ & $\mathbf{6 1 3}$ & $\mathbf{6 7 0}$ & $\mathbf{6 7 5}$ & $\mathbf{5 5 3}$ & $\mathbf{5 2 8}$ & $\mathbf{5 7 4}$ & $\mathbf{5 4 6}$ & $\mathbf{5 2 6}$ & $\mathbf{6 0 9}$ & $\mathbf{7 1 3}$ & $\mathbf{7 2 6}$ & $\mathbf{6 2 1}$
\end{tabular}

-Investor-Owned Entities-

15 Investor-Owned Entities

16 Total Investor-Owned Entities

$\begin{array}{lllllllllllllll}382 & 382 & 382 & 382 & 382 & 382 & 382 & 382 & 382 & 382 & 382 & 382 & 382 & 382 & 382 \\ \mathbf{3 8 2} & \mathbf{3 8 2} & \mathbf{3 8 2} & \mathbf{3 8 2} & \mathbf{3 8 2} & \mathbf{3 8 2} & \mathbf{3 8 2} & \mathbf{3 8 2} & \mathbf{3 8 2} & \mathbf{3 8 2} & \mathbf{3 8 2} & \mathbf{3 8 2} & \mathbf{3 8 2} & \mathbf{3 8 2} & \mathbf{3 8 2}\end{array}$

-Other Entities-

17 Other PNW Entities

18 Total Other Entities

0

0

0

$\begin{array}{ll}0 & 0 \\ 0 & 0\end{array}$

0

0

19 Federal Entities

20 U.S. Bureau of Reclamation

21 Direct Service Industry

22 Generating Public Entities

23 Non-Generating Public Entities

24 Investor-Owned Entities

25 Other Entities

26 Total Power Sales Contracts

$\begin{array}{rrrr}120 & 120 & 109 & 106 \\ 309 & 275 & 166 & 129 \\ 265 & 265 & 265 & 272 \\ 3133 & 2807 & 2737 & 2956 \\ 3500 & 3304 & 2936 & 3029 \\ 382 & 382 & 382 & 382 \\ 0 & 0 & 0 & 0 \\ 7709 & \mathbf{7 1 5 4} & \mathbf{6 5 9 5} & \mathbf{6 8 7 5}\end{array}$

\begin{tabular}{rr}
122 & 137 \\
0 & 0 \\
272 & 272 \\
3415 & 3709 \\
3382 & 3791 \\
382 & 382 \\
0 & 0 \\
\hline 7573 & $\mathbf{8 2 9 0}$
\end{tabular}

$\begin{array}{rrr}137 & 132 & 123 \\ 0 & 1.0 & 23 \\ 272 & 272 & 27 \\ 3547 & 3459 & 334 \\ 3777 & 3609 & 338 \\ 382 & 382 & 38 \\ 0 & 0 & \\ \mathbf{8 1 1 5} & \mathbf{7 8 5 5} & \mathbf{7 5 2}\end{array}$

0

0

0

$\begin{array}{rrr}0 & 0 & 0 \\ 0 & 0 & 0 \\ & & \\ 109 & 116 & 119 \\ 298 & 332 & 149 \\ 272 & 272 & 271 \\ 903 & 2895 & 3160 \\ 354 & 3485 & 3376 \\ 382 & 382 & 382 \\ 0 & 0 & 0 \\ 317 & 7482 & 7458\end{array}$


Table A-22: BPA Power Sale Contracts

PNW Loads and Resources Study

2006 - 2007 Operating Year

1937 Water Year

2003 White Book

Average Energy in Megawatts Aug1 Aug16 Sep Oct Nov Dec Jan Feb Mar Apr1 Apr16 May Jun Jul Avg

-Federal Entities-

1 Federal Entities

2 Total Federal Entities

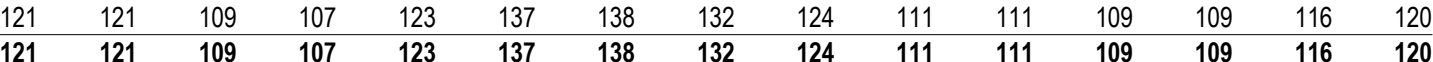

-U.S. Bureau of Reclamation-

3 U.S. Bureau of Reclamation

$\begin{array}{lllllllllllllll}309 & 275 & 166 & 129 & 0 & 0 & 0 & 1.0 & 23 & 252 & 315 & 268 & 298 & 332 & 149 \\ \mathbf{3 0 9} & \mathbf{2 7 5} & \mathbf{1 6 6} & \mathbf{1 2 9} & \mathbf{0} & \mathbf{0} & \mathbf{0} & \mathbf{1 . 0} & \mathbf{2 3} & \mathbf{2 5 2} & \mathbf{3 1 5} & \mathbf{2 6 8} & \mathbf{2 9 8} & \mathbf{3 3 2} & \mathbf{1 4 9}\end{array}$

-Direct Service Industry-

5 Direct Service Industry

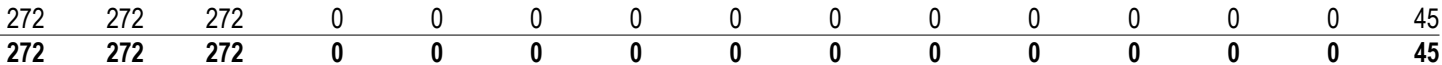

-Generating Public Entities-

7 Generating Public Entities

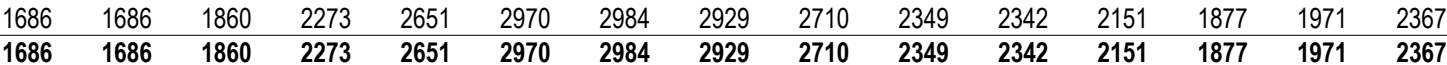

8 Total Generating Public Entities

1686

-Generating Public Entities (Slice)-

9 Generating Public Entities (Slice)

\begin{tabular}{lllllllllllllll}
1417 & 1091 & 846 & 1025 & 1120 & 1129 & 925 & 882 & 960 & 914 & 879 & 921 & 1088 & 1215 & 1022 \\
\hline 1417 & $\mathbf{1 0 9 1}$ & $\mathbf{8 4 6}$ & $\mathbf{1 0 2 5}$ & $\mathbf{1 1 2 0}$ & $\mathbf{1 1 2 9}$ & $\mathbf{9 2 5}$ & $\mathbf{8 8 2}$ & $\mathbf{9 6 0}$ & $\mathbf{9 1 4}$ & $\mathbf{8 7 9}$ & $\mathbf{9 2 1}$ & $\mathbf{1 0 8 8}$ & $\mathbf{1 2 1 5}$ & $\mathbf{1 0 2 2}$
\end{tabular}

-Non-Generating Public Entities-

11 Non-Generating Public Entities

12 Total Non-Generating Public Entities

$\begin{array}{lllllllllllllll}2695 & 2695 & 2480 & 2580 & 2906 & 3270 & 3419 & 3274 & 2967 & 2817 & 2809 & 2772 & 2856 & 2972 & 2917 \\ \mathbf{2 6 9 5} & \mathbf{2 6 9 5} & \mathbf{2 4 8 0} & \mathbf{2 5 8 0} & \mathbf{2 9 0 6} & \mathbf{3 2 7 0} & \mathbf{3 4 1 9} & \mathbf{3 2 7 4} & \mathbf{2 9 6 7} & \mathbf{2 8 1 7} & \mathbf{2 8 0 9} & \mathbf{2 7 7 2} & \mathbf{2 8 5 6} & \mathbf{2 9 7 2} & \mathbf{2 9 1 7}\end{array}$

-Non-Generating Public Entities (Slice)-

13 Non-Generating Public Entities (Slice)

14 Total Non-Generating Public Entities (Slice)

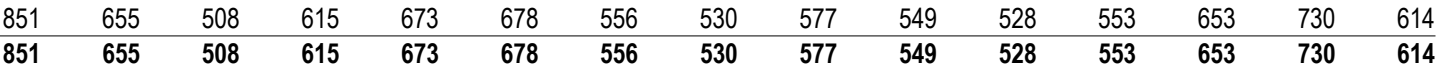

-Investor-Owned Entities-

15 Investor-Owned Entities

16 Total Investor-Owned Entities

\begin{tabular}{|c|c|c|c|c|c|c|c|c|c|}
\hline \multirow{2}{*}{$\frac{38}{38}$} & 382 & 0 & 0 & 0 & 0 & 0 & 0 & 0 & 0 \\
\hline & 382 & 0 & 0 & 0 & 0 & 0 & 0 & 0 & \\
\hline
\end{tabular}

-Other Entities-

17 Other PNW Entities

18 Total Other Entities

0

0

0

-Power Sales Contracts-

19 Federal Entities

20 U.S. Bureau of Reclamation

21 Direct Service Industry

22 Generating Public Entities

23 Non-Generating Public Entities

24 Investor-Owned Entities

25 Other Entities

26 Total Power Sales Contracts

\begin{tabular}{|c|c|c|c|c|c|c|c|c|c|c|c|c|c|c|}
\hline 121 & 121 & 109 & 107 & 123 & 137 & 138 & 132 & 124 & 111 & 111 & 109 & 109 & 116 & 12 \\
\hline 309 & 275 & 166 & 129 & 0 & 0 & 0 & 1.0 & 23 & 252 & 315 & 268 & 298 & 332 & 14 \\
\hline 272 & 272 & 272 & 0 & 0 & 0 & 0 & 0 & 0 & 0 & 0 & 0 & 0 & 0 & \\
\hline 3103 & 2776 & 2706 & 3298 & 3771 & 4098 & 3910 & 3812 & 3670 & 3262 & 3221 & 3073 & 2965 & 3186 & 48 \\
\hline 3546 & 3350 & 2988 & 3195 & 3579 & 3948 & 3975 & 3803 & 3544 & 3365 & 3336 & 3325 & 3509 & 3701 & \\
\hline 382 & 382 & 382 & 0 & 0 & 0 & 0 & 0 & 0 & 0 & 0 & 0 & 0 & 0 & \\
\hline 0 & 0 & 0 & 0 & 0 & 0 & 0 & 0 & 0 & 0 & 0 & 0 & 0 & 0 & \\
\hline 7732 & 7176 & 6624 & 6730 & 7473 & 8184 & 8022 & 7749 & 7360 & 6991 & 6984 & 6774 & 3881 & 7335 & \\
\hline
\end{tabular}


Table A-22: BPA Power Sale Contracts

PNW Loads and Resources Study

2007 - 2008 Operating Year

1937 Water Year

2003 White Book

Average Energy in Megawatts Aug1 Aug16 Sep Oct Nov Dec Jan Feb Mar Apr1 Apr16 May Jun Jul Avg

-Federal Entities-

1 Federal Entities

2 Total Federal Entities

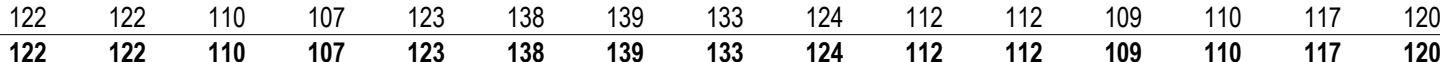

-U.S. Bureau of Reclamation-

3 U.S. Bureau of Reclamation

$\begin{array}{lllllllllllllll}309 & 275 & 166 & 129 & 0 & 0 & 0 & 1.0 & 23 & 252 & 315 & 268 & 298 & 332 & 149 \\ \mathbf{3 0 9} & \mathbf{2 7 5} & \mathbf{1 6 6} & \mathbf{1 2 9} & \mathbf{0} & \mathbf{0} & \mathbf{0} & \mathbf{1 . 0} & \mathbf{2 3} & \mathbf{2 5 2} & \mathbf{3 1 5} & \mathbf{2 6 8} & \mathbf{2 9 8} & \mathbf{3 3 2} & \mathbf{1 4 9}\end{array}$

-Direct Service Industry-

5 Direct Service Industry

6 Total Direct Service Industry

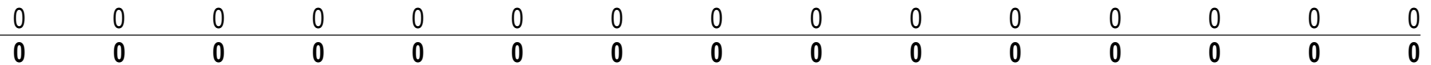

-Generating Public Entities-

7 Generating Public Entities

$\begin{array}{lllllllllllllll}2039 & 2039 & 2180 & 2282 & 2663 & 2983 & 2997 & 2923 & 2723 & 2357 & 2350 & 2161 & 1887 & 1979 & 2431 \\ \mathbf{2 0 3 9} & \mathbf{2 0 3 9} & \mathbf{2 1 8 0} & \mathbf{2 2 8 2} & \mathbf{2 6 6 3} & \mathbf{2 9 8 3} & \mathbf{2 9 9 7} & \mathbf{2 9 2 3} & \mathbf{2 7 2 3} & \mathbf{2 3 5 7} & \mathbf{2 3 5 0} & \mathbf{2 1 6 1} & \mathbf{1 8 8 7} & \mathbf{1 9 7 9} & \mathbf{2 4 3 1}\end{array}$

8 Total Generating Public Entities

-Generating Public Entities (Slice)-

9 Generating Public Entities (Slice)

\begin{tabular}{lllllllllllllll}
1425 & 1097 & 850 & 1030 & 1127 & 1136 & 930 & 887 & 964 & 918 & 883 & 1021 & 1198 & 1222 & 1044 \\
\hline 1425 & 1097 & $\mathbf{8 5 0}$ & $\mathbf{1 0 3 0}$ & $\mathbf{1 1 2 7}$ & $\mathbf{1 1 3 6}$ & $\mathbf{9 3 0}$ & $\mathbf{8 8 7}$ & $\mathbf{9 6 4}$ & $\mathbf{9 1 8}$ & $\mathbf{8 8 3}$ & $\mathbf{1 0 2 1}$ & $\mathbf{1 1 9 8}$ & $\mathbf{1 2 2 2}$ & $\mathbf{1 0 4 4}$
\end{tabular}

-Non-Generating Public Entities-

11 Non-Generating Public Entities

12 Total Non-Generating Public Entities

$\begin{array}{lllllllllllllll}2924 & 2924 & 2649 & 2607 & 2937 & 3306 & 3457 & 3275 & 3000 & 2847 & 2839 & 2802 & 2886 & 3003 & 2974 \\ \mathbf{2 9 2 4} & \mathbf{2 9 2 4} & \mathbf{2 6 4 9} & \mathbf{2 6 0 7} & \mathbf{2 9 3 7} & \mathbf{3 3 0 6} & \mathbf{3 4 5 7} & \mathbf{3 2 7 5} & \mathbf{3 0 0 0} & \mathbf{2 8 4 7} & \mathbf{2 8 3 9} & \mathbf{2 8 0 2} & \mathbf{2 8 8 6} & \mathbf{3 0 0 3} & \mathbf{2 9 7 4}\end{array}$

-Non-Generating Public Entities (Slice)-

13 Non-Generating Public Entities (Slice)

14 Total Non-Generating Public Entities (Slice)

$\begin{array}{lllllllllllllll}855 & 658 & 511 & 619 & 677 & 682 & 559 & 533 & 579 & 551 & 530 & 613 & 719 & 734 & 627 \\ \mathbf{8 5 5} & \mathbf{6 5 8} & \mathbf{5 1 1} & \mathbf{6 1 9} & \mathbf{6 7 7} & \mathbf{6 8 2} & \mathbf{5 5 9} & \mathbf{5 3 3} & \mathbf{5 7 9} & \mathbf{5 5 1} & \mathbf{5 3 0} & \mathbf{6 1 3} & \mathbf{7 1 9} & \mathbf{7 3 4} & \mathbf{6 2 7}\end{array}$

-Investor-Owned Entities-

15 Investor-Owned Entities

16 Total Investor-Owned Entities

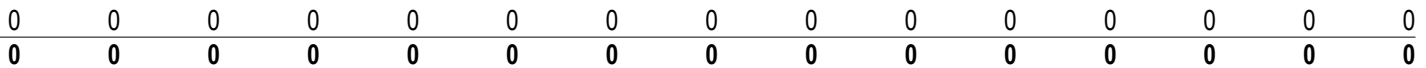

-Other Entities-

17 Other PNW Entities

18 Total Other Entities

0

0

0

0

ower Sales Contracts-

19 Federal Entities

20 U.S. Bureau of Reclamation

21 Direct Service Industry

22 Generating Public Entities

23 Non-Generating Public Entities

24 Investor-Owned Entities

25 Other Entities

26 Total Power Sales Contracts

$\begin{array}{rrrr}122 & 122 & 110 & 107 \\ 309 & 275 & 166 & 129 \\ 0 & 0 & 0 & \\ 3464 & 3135 & 3030 & 3313 \\ 3780 & 3583 & 3159 & 3226 \\ 0 & 0 & 0 & \\ 0 & 0 & 0 & \\ 7674 & 7115 & 6466 & 6775\end{array}$

$\begin{array}{rrr}107 & 123 & 138 \\ 129 & 0 & 0 \\ 0 & 0 & 0 \\ 3313 & 3790 & 4119 \\ 3226 & 3614 & 3988 \\ 0 & 0 & 0 \\ 0 & 0 & 0 \\ \mathbf{6 7 7 5} & \mathbf{7 5 2 8} & \mathbf{8 2 4 5}\end{array}$

$\begin{array}{rr}139 & 133 \\ 0 & \\ 0 & \\ 3928 & 3810 \\ 4015 & 3808 \\ 0 & \\ 0 & \\ 8081 & 7752\end{array}$

$\begin{array}{rrrrrrrr}0 & 0 & 0 & 0 & 0 & 0 & 0 & 0 \\ \mathbf{0} & \mathbf{0} & \mathbf{0} & \mathbf{0} & \mathbf{0} & \mathbf{0} & \mathbf{0} & \mathbf{0} \\ & & & & & & & \\ 133 & 124 & 112 & 112 & 109 & 110 & 117 & 120 \\ 1.0 & 23 & 252 & 315 & 268 & 298 & 332 & 149 \\ 0 & 0 & 0 & 0 & 0 & 0 & 0 & 0 \\ 3810 & 3687 & 3275 & 3233 & 3182 & 3085 & 3201 & 3475 \\ 3808 & 3579 & 3398 & 3369 & 3415 & 3605 & 3737 & 3601 \\ 0 & 0 & 0 & 0 & 0 & 0 & 0 & 0 \\ 0 & 0 & 0 & 0 & 0 & 0 & 0 & 0 \\ 7752 & \mathbf{7 4 1 4} & \mathbf{7 0 3 6} & \mathbf{7 0 2 9} & \mathbf{6 9 7 5} & \mathbf{7 0 9 8} & \mathbf{7 3 8 7} & \mathbf{7 3 4 6}\end{array}$


Table A-22: BPA Power Sale Contracts

PNW Loads and Resources Study

2008 - 2009 Operating Year

1937 Water Year

2003 White Book

Average Energy in Megawatts Aug1 Aug16 Sep Oct Nov Dec Jan Feb Mar Apr1 Apr16 May Jun Jul Avg

-Federal Entities-

1 Federal Entities

2 Total Federal Entities

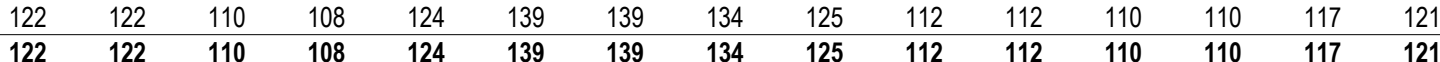

-U.S. Bureau of Reclamation-

3 U.S. Bureau of Reclamation

$\begin{array}{lllllllllllllll}309 & 275 & 166 & 129 & 0 & 0 & 0 & 1.0 & 23 & 252 & 315 & 268 & 298 & 332 & 149 \\ \mathbf{3 0 9} & \mathbf{2 7 5} & \mathbf{1 6 6} & \mathbf{1 2 9} & \mathbf{0} & \mathbf{0} & \mathbf{0} & \mathbf{1 . 0} & \mathbf{2 3} & \mathbf{2 5 2} & \mathbf{3 1 5} & \mathbf{2 6 8} & \mathbf{2 9 8} & \mathbf{3 3 2} & \mathbf{1 4 9}\end{array}$

-Direct Service Industry-

5 Direct Service Industry

6 Total Direct Service Industry

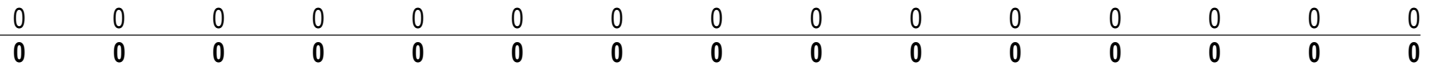

-Generating Public Entities-

7 Generating Public Entities

$\begin{array}{lllllllllllllll}2049 & 2049 & 2188 & 2292 & 2675 & 2994 & 3011 & 2954 & 2734 & 2367 & 2360 & 2172 & 1894 & 1987 & 2443 \\ \mathbf{2 0 4 9} & \mathbf{2 0 4 9} & \mathbf{2 1 8 8} & \mathbf{2 2 9 2} & \mathbf{2 6 7 5} & \mathbf{2 9 9 4} & \mathbf{3 0 1 1} & \mathbf{2 9 5 4} & \mathbf{2 7 3 4} & \mathbf{2 3 6 7} & \mathbf{2 3 6 0} & \mathbf{2 1 7 2} & \mathbf{1 8 9 4} & \mathbf{1 9 8 7} & \mathbf{2 4 4 3}\end{array}$

8 Total Generating Public Entities

-Generating Public Entities (Slice)-

9 Generating Public Entities (Slice)

\begin{tabular}{lllllllllllllll}
1428 & 1099 & 853 & 1033 & 1130 & 1139 & 933 & 889 & 967 & 920 & 885 & 926 & 1095 & 1225 & 1030 \\
\hline 1428 & 1099 & $\mathbf{8 5 3}$ & 1033 & 1130 & $\mathbf{1 1 3 9}$ & $\mathbf{9 3 3}$ & $\mathbf{8 8 9}$ & $\mathbf{9 6 7}$ & $\mathbf{9 2 0}$ & $\mathbf{8 8 5}$ & $\mathbf{9 2 6}$ & $\mathbf{1 0 9 5}$ & $\mathbf{1 2 2 5}$ & $\mathbf{1 0 3 0}$
\end{tabular}

-Non-Generating Public Entities-

11 Non-Generating Public Entities

12 Total Non-Generating Public Entities

\begin{tabular}{lllllllllllllll}
2955 & 2955 & 2677 & 2634 & 2969 & 3343 & 3494 & 3348 & 3034 & 2878 & 2871 & 2832 & 2916 & 3034 & 3009 \\
\hline $\mathbf{2 9 5 5}$ & $\mathbf{2 9 5 5}$ & $\mathbf{2 6 7 7}$ & $\mathbf{2 6 3 4}$ & $\mathbf{2 9 6 9}$ & $\mathbf{3 3 4 3}$ & $\mathbf{3 4 9 4}$ & $\mathbf{3 3 4 8}$ & $\mathbf{3 0 3 4}$ & $\mathbf{2 8 7 8}$ & $\mathbf{2 8 7 1}$ & $\mathbf{2 8 3 2}$ & $\mathbf{2 9 1 6}$ & $\mathbf{3 0 3 4}$ & $\mathbf{3 0 0 9}$
\end{tabular}

-Non-Generating Public Entities (Slice)-

13 Non-Generating Public Entities (Slice)

14 Total Non-Generating Public Entities (Slice)

$\begin{array}{lllllllllllllll}858 & 660 & 512 & 620 & 679 & 684 & 560 & 534 & 581 & 553 & 531 & 556 & 657 & \mathbf{7 3 6} & 618 \\ \mathbf{8 5 8} & \mathbf{6 6 0} & \mathbf{5 1 2} & \mathbf{6 2 0} & \mathbf{6 7 9} & \mathbf{6 8 4} & \mathbf{5 6 0} & \mathbf{5 3 4} & \mathbf{5 8 1} & \mathbf{5 5 3} & \mathbf{5 3 1} & \mathbf{5 5 6} & \mathbf{6 5 7} & \mathbf{7 3 6} & \mathbf{6 1 8}\end{array}$

-Investor-Owned Entities-

15 Investor-Owned Entities

16 Total Investor-Owned Entities

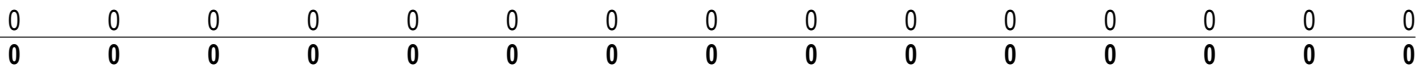

-Other Entities-

17 Other PNW Entities

18 Total Other Entities

0

0

0

0

Power Sales Contracts-

19 Federal Entities

20 U.S. Bureau of Reclamation

21 Direct Service Industry

22 Generating Public Entities

23 Non-Generating Public Entities

24 Investor-Owned Entities

25 Other Entities

26 Total Power Sales Contracts

\begin{tabular}{|c|c|c|c|c|c|c|c|c|c|c|c|c|c|c|}
\hline 122 & 122 & 110 & 108 & 124 & 139 & 139 & 134 & 125 & 112 & 112 & 110 & 110 & 117 & 121 \\
\hline 309 & 275 & 166 & 129 & 0 & 0 & 0 & 1.0 & 23 & 252 & 315 & 268 & 298 & 332 & 149 \\
\hline 0 & 0 & 0 & 0 & 0 & 0 & 0 & 0 & 0 & 0 & 0 & 0 & 0 & 0 & 0 \\
\hline 3477 & 3148 & 3041 & 3325 & 3805 & 4133 & 3943 & 3844 & 3701 & 3287 & 3245 & 3097 & 2989 & 3212 & 3472 \\
\hline 3812 & 3615 & 3189 & 3255 & 3647 & 4026 & 4054 & 3882 & 3614 & 3431 & 3402 & 3388 & 3574 & 3770 & 3627 \\
\hline 0 & 0 & 0 & 0 & 0 & 0 & 0 & 0 & 0 & 0 & 0 & 0 & 0 & 0 & \\
\hline 0 & 0 & 0 & 0 & 0 & 0 & 0 & 0 & 0 & 0 & 0 & 0 & 0 & 0 & \\
\hline 20 & 7160 & 6506 & 6816 & 7577 & 8297 & 8137 & 7860 & 7463 & 7083 & 7074 & 6863 & 6971 & 31 & \\
\hline
\end{tabular}


Table A-22: BPA Power Sale Contracts

PNW Loads and Resources Study

2009 - 2010 Operating Year

1937 Water Year

2003 White Book

-Federal Entities-

1 Federal Entities

2 Total Federal Entities

Aug1 Aug16 Sep Oct Nov Dec Jan Feb Mar Apr1 Apr16 May Jun Jul Avg

U.S. Bureau of Reclamation-

3 U.S. Bureau of Reclamation

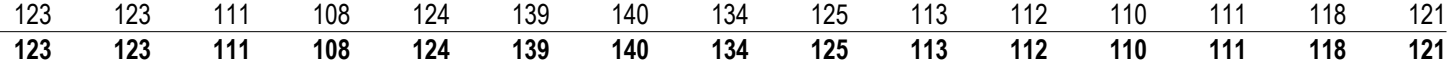

4 Total U.S. Bureau of Reclamation

$309 \quad 275 \quad 166$

-Direct Service Industry-

5 Direct Service Industry

6 Total Direct Service Industry

\begin{tabular}{lllllllllllllll}
0 & 0 & 0 & 0 & 0 & 0 & 0 & 0 & 0 & 0 & 0 & 0 & 0 & 0 & 0 \\
\hline & 0 & 0 & 0 & 0 & 0 & 0 & 0 & 0 & 0 & 0 & 0 & 0 & 0 & 0
\end{tabular}

-Generating Public Entities-

7 Generating Public Entities

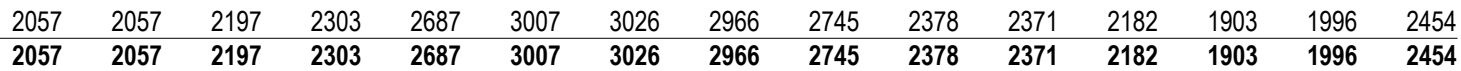

-Generating Public Entities (Slice)-

9 Generating Public Entities (Slice)

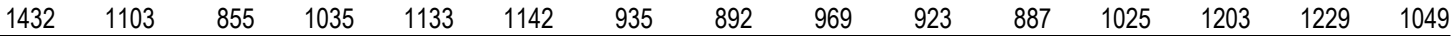

10 Total Generating Public Entities (Slice

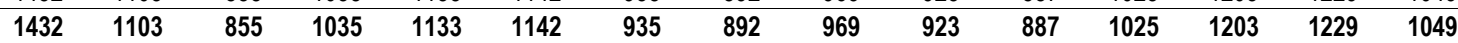

-Non-Generating Public Entities-

11 Non-Generating Public Entities

12 Total Non-Generating Public Entities

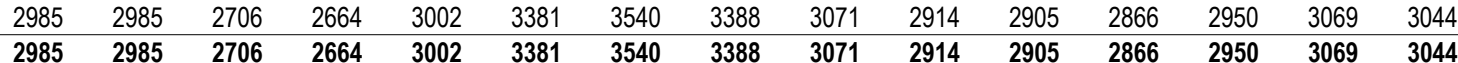

-Non-Generating Public Entities (Slice)-

13 Non-Generating Public Entities (Slice)

14 Total Non-Generating Public Entities (Slice)

$860 \quad 662 \quad 513$

-Investor-Owned Entities-

15 Investor-Owned Entities

16 Total Investor-Owned Entities

$\begin{array}{llllllllllllll}0 & 0 & 0 & 0 & 0 & 0 & 0 & 0 & 0 & 0 & 0 & 0 & 0 & 0 \\ 0 & 0 & 0 & 0 & 0 & 0 & 0 & 0 & 0 & 0 & 0 & 0 & 0 & 0\end{array}$

-Other Entities-

17 Other PNW Entities

18 Total Other Entities

$\begin{array}{lllllllllllllll}0 & 0 & 0 & 0 & 0 & 0 & 0 & 0 & 0 & 0 & 0 & 0 & 0 & 0 & 0 \\ 0 & 0 & 0 & 0 & 0 & 0 & 0 & 0 & 0 & 0 & 0 & 0 & 0 & 0 & 0\end{array}$

-Power Sales Contracts-

19 Federal Entities

20 U.S. Bureau of Reclamation

21 Direct Service Industry

22 Generating Public Entities

23 Non-Generating Public Entities

24 Investor-Owned Entities

25 Other Entities

26 Total Power Sales Contracts

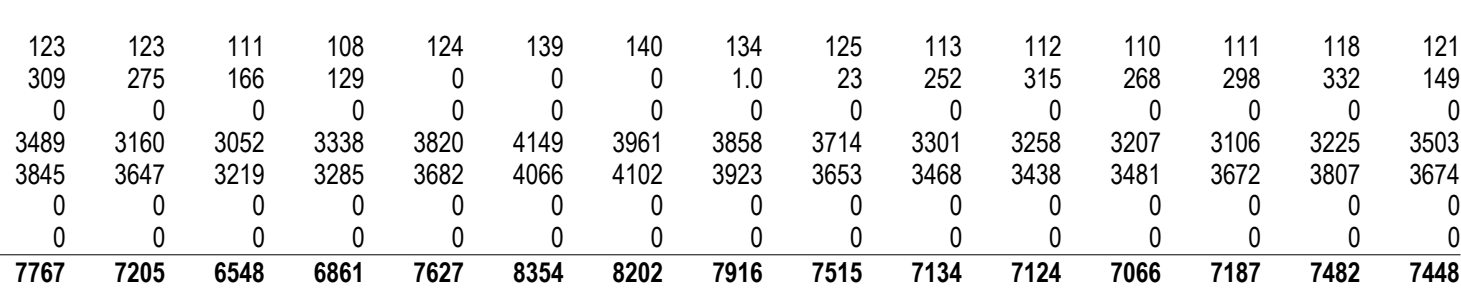


Table A-22: BPA Power Sale Contracts

PNW Loads and Resources Study

2010 - 2011 Operating Year

1937 Water Year

2003 White Book

-Federal Entities-

1 Federal Entities

2 Total Federal Entities

Aug1 Aug16 Sep Oct Nov Dec Jan Feb Mar Apr1 Apr16 May Jun Jul Avg

-U.S. Bureau of Reclamation-

3 U.S. Bureau of Reclamation

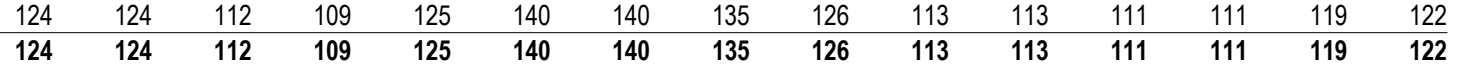

4 Total U.S. Bureau of Reclamation

$309 \quad 275 \quad 166$

-Direct Service Industry-

5 Direct Service Industry

6 Total Direct Service Industry

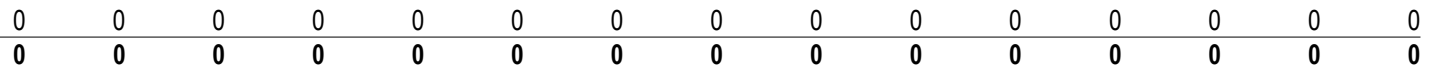

-Generating Public Entities-

7 Generating Public Entities

$\begin{array}{lllllllllllllll}2065 & 2065 & 2205 & 2313 & 2696 & 3019 & 3039 & 2978 & 2756 & 2387 & 2381 & 2191 & 1911 & 2004 & 2463 \\ \mathbf{2 0 6 5} & \mathbf{2 0 6 5} & \mathbf{2 2 0 5} & \mathbf{2 3 1 3} & \mathbf{2 6 9 6} & \mathbf{3 0 1 9} & \mathbf{3 0 3 9} & \mathbf{2 9 7 8} & \mathbf{2 7 5 6} & \mathbf{2 3 8 7} & \mathbf{2 3 8 1} & \mathbf{2 1 9 1} & \mathbf{1 9 1 1} & \mathbf{2 0 0 4} & \mathbf{2 4 6 3}\end{array}$

8 Total Generating Public Entities

-Generating Public Entities (Slice)-

9 Generating Public Entities (Slice)

\begin{tabular}{|c|c|c|c|c|c|c|c|c|c|c|c|c|}
\hline 1105 & 856 & 1037 & 1135 & 1144 & 937 & 893 & 971 & 925 & 888 & 929 & 1100 & 1232 \\
\hline 1105 & 856 & 1037 & 1135 & 1144 & 937 & 893 & 971 & 925 & 888 & 929 & 1100 & 23 \\
\hline
\end{tabular}

-Non-Generating Public Entities-

11 Non-Generating Public Entities

12 Total Non-Generating Public Entities

$\begin{array}{lllllllllllllll}3020 & 3020 & 2739 & 2696 & 3038 & 3421 & 3578 & 3424 & 3103 & 2944 & 2936 & 2896 & 2980 & 3101 & 3078 \\ \mathbf{3 0 2 0} & \mathbf{3 0 2 0} & \mathbf{2 7 3 9} & \mathbf{2 6 9 6} & \mathbf{3 0 3 8} & \mathbf{3 4 2 1} & \mathbf{3 5 7 8} & \mathbf{3 4 2 4} & \mathbf{3 1 0 3} & \mathbf{2 9 4 4} & \mathbf{2 9 3 6} & \mathbf{2 8 9 6} & \mathbf{2 9 8 0} & \mathbf{3 1 0 1} & \mathbf{3 0 7 8}\end{array}$

-Non-Generating Public Entities (Slice)-

13 Non-Generating Public Entities (Slice)

14 Total Non-Generating Public Entities (Slice)

$862 \quad 663 \quad 514$

-Investor-Owned Entities-

15 Investor-Owned Entities

16 Total Investor-Owned Entities

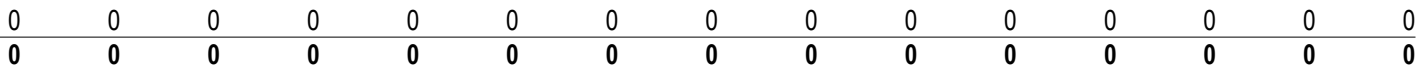

-Other Entities-

17 Other PNW Entities

18 Total Other Entities

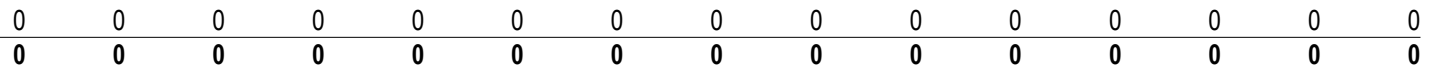

-Power Sales Contracts-

19 Federal Entities

20 U.S. Bureau of Reclamation

21 Direct Service Industry

22 Generating Public Entities

23 Non-Generating Public Entities

24 Investor-Owned Entities

25 Other Entities

26 Total Power Sales Contracts

\begin{tabular}{|c|c|c|c|c|c|c|c|c|c|c|c|c|c|}
\hline 124 & 124 & 112 & 109 & 125 & 140 & 140 & 135 & 126 & 113 & 113 & 111 & 111 & 119 \\
\hline 309 & 275 & 166 & 129 & 0 & 0 & 0 & 1.0 & 23 & 252 & 315 & 268 & 298 & 332 \\
\hline 0 & 0 & 0 & 0 & 0 & 0 & 0 & 0 & 0 & 0 & 0 & 0 & 0 & 0 \\
\hline 3500 & 3170 & 3061 & 3350 & 3832 & 4163 & 3976 & 3871 & 3727 & 3313 & 3269 & 3120 & 3010 & 3236 \\
\hline 3882 & 3684 & 3253 & 3318 & 3719 & 4108 & 4140 & 3960 & 3687 & 3499 & 3469 & 3453 & 3640 & 3840 \\
\hline 0 & 0 & 0 & 0 & 0 & 0 & 0 & 0 & 0 & 0 & 0 & 0 & 0 & 0 \\
\hline 0 & 0 & 0 & 0 & 0 & 0 & 0 & 0 & 0 & 0 & 0 & 0 & 0 & 0 \\
\hline 315 & 7252 & 6592 & 6906 & 7676 & 8411 & 8257 & 7967 & 7562 & 7177 & 7166 & 6952 & 7060 & 7527 \\
\hline
\end{tabular}


Table A-22: BPA Power Sale Contracts

PNW Loads and Resources Study

2011 - 2012 Operating Year

1937 Water Year

2003 White Book

-Federal Entities-

1 Federal Entities

Aug1 Aug16 Sep Oct Nov Dec Jan Feb Mar Apr1 Apr16 May Jun Jul Avg

2 Total Federal Entities

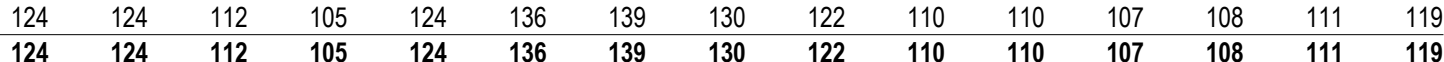

-U.S. Bureau of Reclamation-

3 U.S. Bureau of Reclamation

\begin{tabular}{|c|c|c|c|c|c|c|c|c|c|c|c|c|}
\hline 275 & 166 & 129 & 0 & 0 & 0 & 1.0 & 23 & 252 & 315 & 268 & 298 & 332 \\
\hline 275 & 166 & 129 & 0 & 0 & 0 & 1.0 & 23 & 252 & 315 & 268 & 298 & 332 \\
\hline
\end{tabular}

-Direct Service Industry-

5 Direct Service Industry

6 Total Direct Service Industry

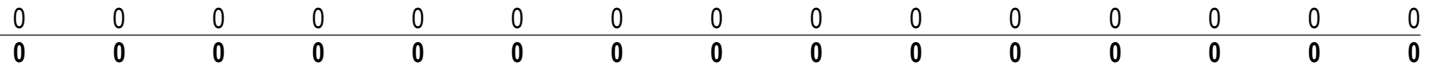

-Generating Public Entities-

7 Generating Public Entities

\begin{tabular}{lllllllllllllll}
2072 & 2072 & 2213 & 2352 & 2754 & 3086 & 3087 & 2940 & 2800 & 2426 & 2419 & 2229 & 1948 & 2030 & 2494 \\
\hline 2072 & $\mathbf{2 0 7 2}$ & $\mathbf{2 2 1 3}$ & $\mathbf{2 3 5 2}$ & $\mathbf{2 7 5 4}$ & $\mathbf{3 0 8 6}$ & $\mathbf{3 0 8 7}$ & $\mathbf{2 9 4 0}$ & $\mathbf{2 8 0 0}$ & $\mathbf{2 4 2 6}$ & $\mathbf{2 4 1 9}$ & $\mathbf{2 2 2 9}$ & $\mathbf{1 9 4 8}$ & $\mathbf{2 0 3 0}$ & $\mathbf{2 4 9 4}$
\end{tabular}

8 Total Generating Public Entities

-Generating Public Entities (Slice)-

9 Generating Public Entities (Slice)

$\begin{array}{lllllllllllllll}1437 & 1107 & 858 & 1039 & 1137 & 1146 & 938 & 895 & 973 & 927 & 889 & 1028 & 1206 & 1234 & 1053 \\ 1437 & \mathbf{1 1 0 7} & \mathbf{8 5 8} & \mathbf{1 0 3 9} & \mathbf{1 1 3 7} & \mathbf{1 1 4 6} & \mathbf{9 3 8} & \mathbf{8 9 5} & \mathbf{9 7 3} & \mathbf{9 2 7} & \mathbf{8 8 9} & \mathbf{1 0 2 8} & \mathbf{1 2 0 6} & \mathbf{1 2 3 4} & \mathbf{1 0 5 3}\end{array}$

-Non-Generating Public Entities-

11 Non-Generating Public Entities

12 Total Non-Generating Public Entities

\begin{tabular}{lllllllllllllll}
3047 & 3047 & 2763 & 2745 & 3099 & 3498 & 3567 & 3403 & 3106 & 2942 & 2934 & 2913 & 3023 & 3141 & 3103 \\
\hline 3047 & $\mathbf{3 0 4 7}$ & $\mathbf{2 7 6 3}$ & $\mathbf{2 7 4 5}$ & $\mathbf{3 0 9 9}$ & $\mathbf{3 4 9 8}$ & $\mathbf{3 5 6 7}$ & $\mathbf{3 4 0 3}$ & $\mathbf{3 1 0 6}$ & $\mathbf{2 9 4 2}$ & $\mathbf{2 9 3 4}$ & $\mathbf{2 9 1 3}$ & $\mathbf{3 0 2 3}$ & $\mathbf{3 1 4 1}$ & $\mathbf{3 1 0 3}$
\end{tabular}

-Non-Generating Public Entities (Slice)-

13 Non-Generating Public Entities (Slice)

14 Total Non-Generating Public Entities (Slice)

$863 \quad 664 \quad 515$

-Investor-Owned Entities-

15 Investor-Owned Entities

16 Total Investor-Owned Entities

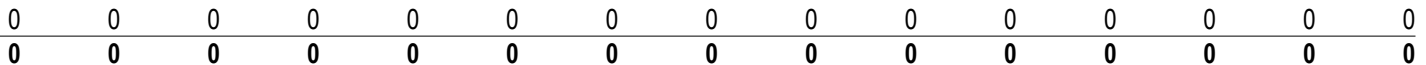

-Other Entities-

17 Other PNW Entities

18 Total Other Entities

\begin{tabular}{|c|c|c|c|c|c|c|c|c|c|}
\hline 0 & 0 & 0 & 0 & 0 & 0 & 0 & 0 & & \multirow[t]{2}{*}{0} \\
\hline 0 & 0 & 0 & 0 & 0 & 0 & 0 & 0 & & \\
\hline
\end{tabular}

-Power Sales Contracts-

19 Federal Entities

20 U.S. Bureau of Reclamation

21 Direct Service Industry

22 Generating Public Entities

23 Non-Generating Public Entities

24 Investor-Owned Entities

25 Other Entities

26 Total Power Sales Contracts

\begin{tabular}{|c|c|c|c|c|c|c|c|c|c|c|c|c|c|c|}
\hline 124 & 124 & 112 & 105 & 124 & 136 & 139 & 130 & 122 & 110 & 110 & 107 & 108 & 111 & 119 \\
\hline 309 & 275 & 166 & 129 & 0 & 0 & 0 & 1.0 & 23 & 252 & 315 & 268 & 298 & 332 & 149 \\
\hline 0 & 0 & 0 & 0 & 0 & 0 & 0 & 0 & 0 & 0 & 0 & 0 & 0 & 0 & \\
\hline 3509 & 3179 & 3070 & 3390 & 3891 & 4232 & 4026 & 3835 & 3773 & 3353 & 3309 & 3257 & 3155 & 3263 & 3547 \\
\hline 3910 & 3712 & 3278 & 3369 & 3781 & 4186 & 4130 & 3940 & 3690 & 3499 & 3468 & 3530 & 3747 & 3881 & 3735 \\
\hline 0 & 0 & 0 & 0 & 0 & 0 & 0 & 0 & 0 & 0 & 0 & 0 & 0 & 0 & \\
\hline 0 & 0 & 0 & 0 & 0 & 0 & 0 & 0 & 0 & 0 & 0 & 0 & 0 & 0 & \\
\hline 352 & 7289 & 6626 & 6993 & 7797 & 8554 & 8295 & 7905 & 7607 & 7214 & 7201 & 7161 & 7308 & 7588 & 75 \\
\hline
\end{tabular}


Table A-22: BPA Power Sale Contracts

PNW Loads and Resources Study

2012 - 2013 Operating Year

1937 Water Year

2003 White Book

Average Energy in Megawatts Aug1 Aug16 Sep Oct Nov Dec Jan Feb Mar Apr1 Apr16 May Jun Jul Avg

-Federal Entities-

1 Federal Entities

2 Total Federal Entities

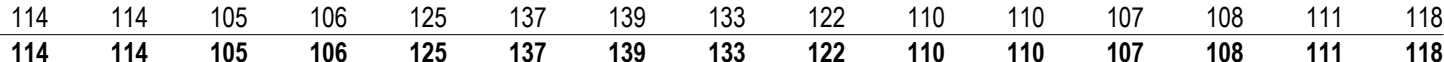

-U.S. Bureau of Reclamation-

3 U.S. Bureau of Reclamation

$\begin{array}{lllllllllllllll}309 & 275 & 166 & 129 & 0 & 0 & 0 & 1.0 & 23 & 252 & 315 & 268 & 298 & 332 & 149 \\ \mathbf{3 0 9} & \mathbf{2 7 5} & \mathbf{1 6 6} & \mathbf{1 2 9} & \mathbf{0} & \mathbf{0} & \mathbf{0} & \mathbf{1 . 0} & \mathbf{2 3} & \mathbf{2 5 2} & \mathbf{3 1 5} & \mathbf{2 6 8} & \mathbf{2 9 8} & \mathbf{3 3 2} & \mathbf{1 4 9}\end{array}$

-Direct Service Industry-

5 Direct Service Industry

6 Total Direct Service Industry

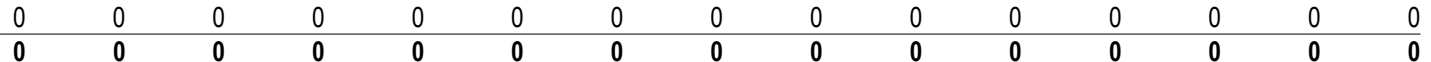

-Generating Public Entities-

7 Generating Public Entities

\begin{tabular}{lllllllllllllll}
2092 & 2092 & 2232 & 2356 & 2760 & 3092 & 3094 & 3048 & 2806 & 2432 & 2425 & 2235 & 1954 & 2035 & 2511 \\
\hline 2092 & $\mathbf{2 0 9 2}$ & $\mathbf{2 2 3 2}$ & $\mathbf{2 3 5 6}$ & $\mathbf{2 7 6 0}$ & $\mathbf{3 0 9 2}$ & $\mathbf{3 0 9 4}$ & $\mathbf{3 0 4 8}$ & $\mathbf{2 8 0 6}$ & $\mathbf{2 4 3 2}$ & $\mathbf{2 4 2 5}$ & $\mathbf{2 2 3 5}$ & $\mathbf{1 9 5 4}$ & $\mathbf{2 0 3 5}$ & $\mathbf{2 5 1 1}$
\end{tabular}

8 Total Generating Public Entities

-Generating Public Entities (Slice)-

9 Generating Public Entities (Slice)

$\begin{array}{lllllllllllllll}1440 & 1109 & 859 & 1041 & 1139 & 1148 & 940 & 896 & 975 & 929 & 891 & 931 & 1103 & 1236 & 1038 \\ 1440 & 1109 & \mathbf{8 5 9} & 1041 & 1139 & 1148 & \mathbf{9 4 0} & \mathbf{8 9 6} & \mathbf{9 7 5} & \mathbf{9 2 9} & \mathbf{8 9 1} & \mathbf{9 3 1} & \mathbf{1 1 0 3} & \mathbf{1 2 3 6} & \mathbf{1 0 3 8}\end{array}$

-Non-Generating Public Entities-

11 Non-Generating Public Entities

12 Total Non-Generating Public Entities

\begin{tabular}{lllllllllllllll}
3092 & 3092 & 2790 & 2761 & 3118 & 3518 & 3603 & 3492 & 3137 & 2972 & 2964 & 2942 & 3052 & 3172 & 3137 \\
\hline $\mathbf{3 0 9 2}$ & $\mathbf{3 0 9 2}$ & $\mathbf{2 7 9 0}$ & $\mathbf{2 7 6 1}$ & $\mathbf{3 1 1 8}$ & $\mathbf{3 5 1 8}$ & $\mathbf{3 6 0 3}$ & $\mathbf{3 4 9 2}$ & $\mathbf{3 1 3 7}$ & $\mathbf{2 9 7 2}$ & $\mathbf{2 9 6 4}$ & $\mathbf{2 9 4 2}$ & $\mathbf{3 0 5 2}$ & $\mathbf{3 1 7 2}$ & $\mathbf{3 1 3 7}$
\end{tabular}

-Non-Generating Public Entities (Slice)-

13 Non-Generating Public Entities (Slice)

14 Total Non-Generating Public Entities (Slice)

$864 \quad 666 \quad 516$

-Investor-Owned Entities-

15 Investor-Owned Entities

16 Total Investor-Owned Entities

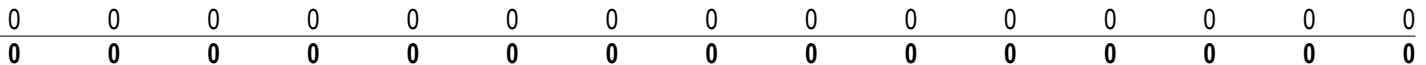

-Other Entities-

17 Other PNW Entities

18 Total Other Entities

\begin{tabular}{|c|c|c|c|c|c|c|c|c|c|}
\hline 0 & 0 & 0 & 0 & 0 & 0 & 0 & 0 & & \multirow[t]{2}{*}{0} \\
\hline 0 & 0 & 0 & 0 & 0 & 0 & 0 & 0 & & \\
\hline
\end{tabular}

-Power Sales Contracts-

19 Federal Entities

20 U.S. Bureau of Reclamation

21 Direct Service Industry

22 Generating Public Entities

23 Non-Generating Public Entities

24 Investor-Owned Entities

25 Other Entities

26 Total Power Sales Contracts

\begin{tabular}{|c|c|c|c|c|c|c|c|c|c|c|c|c|c|c|}
\hline 114 & 114 & 105 & 106 & 125 & 137 & 139 & 133 & 122 & 110 & 110 & 107 & 108 & 111 & 118 \\
\hline 309 & 275 & 166 & 129 & 0 & 0 & 0 & 1.0 & 23 & 252 & 315 & 268 & 298 & 332 & 149 \\
\hline 0 & 0 & 0 & 0 & 0 & 0 & 0 & 0 & 0 & 0 & 0 & 0 & 0 & 0 & 0 \\
\hline 3532 & 3201 & 3091 & 3397 & 3899 & 4240 & 4035 & 3945 & 3781 & 3361 & 3316 & 3167 & 3057 & 3272 & 3549 \\
\hline 3956 & 3757 & 3306 & 3386 & 3802 & 4208 & 4168 & 4030 & 3723 & 3530 & 3499 & 3502 & 3714 & 3914 & 3760 \\
\hline 0 & 0 & 0 & 0 & 0 & 0 & 0 & 0 & 0 & 0 & 0 & 0 & 0 & 0 & \\
\hline 0 & 0 & 0 & 0 & 0 & 0 & 0 & 0 & 0 & 0 & 0 & 0 & 0 & 0 & 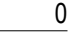 \\
\hline 11 & 7347 & 6668 & 7018 & 7826 & 8585 & 8341 & 8109 & 7648 & 7253 & 7240 & 7043 & 7177 & 29 & 75 \\
\hline
\end{tabular}


Table A-22: BPA Power Sale Contracts

PNW Loads and Resources Study

2013 - 2014 Operating Year

1937 Water Year

2003 White Book

Average Energy in Megawatts Aug1 Aug16 Sep Oct Nov Dec Jan Feb Mar Apr1 Apr16 May Jun Jul Avg

-Federal Entities-

1 Federal Entities

2 Total Federal Entities

$\begin{array}{llr}114 & 114 & 105 \\ 114 & 114 & 105\end{array}$

$105-107$

$\begin{array}{lll}309 & 275 & 166 \\ 309 & 275 & 166\end{array}$

S. Bureau of Reclamation

4 Total U.S. Bureau of Reclamation

-Direct Service Industry-

5 Direct Service Industry

6 Total Direct Service Industry

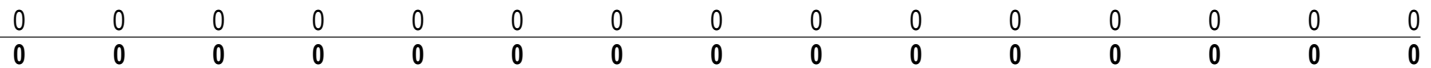

-Generating Public Entities-

7 Generating Public Entities

$\begin{array}{lllllllllllllll}2098 & 2098 & 2238 & 2363 & 2767 & 3099 & 3102 & 3056 & 2813 & 2439 & 2432 & 2241 & 1960 & 2041 & 2518 \\ \mathbf{2 0 9 8} & \mathbf{2} 098 & \mathbf{2 2 3 8} & \mathbf{2 3 6 3} & \mathbf{2 7 6 7} & \mathbf{3 0 9 9} & \mathbf{3 1 0 2} & \mathbf{3 0 5 6} & \mathbf{2 8 1 3} & \mathbf{2 4 3 9} & \mathbf{2 4 3 2} & \mathbf{2 2 4 1} & \mathbf{1 9 6 0} & \mathbf{2 0 4 1} & \mathbf{2 5 1 8}\end{array}$

8 Total Generating Public Entities

-Generating Public Entities (Slice)-

9 Generating Public Entities (Slice)

$\begin{array}{lllllllllllllll}1442 & 1110 & 860 & 1042 & 1141 & 1150 & 942 & 898 & 976 & 930 & 892 & 1030 & 1209 & 1238 & 1056 \\ 1442 & \mathbf{1 1 1 0} & \mathbf{8 6 0} & \mathbf{1 0 4 2} & \mathbf{1 1 4 1} & \mathbf{1 1 5 0} & \mathbf{9 4 2} & \mathbf{8 9 8} & \mathbf{9 7 6} & \mathbf{9 3 0} & \mathbf{8 9 2} & \mathbf{1 0 3 0} & \mathbf{1 2 0 9} & \mathbf{1 2 3 8} & \mathbf{1 0 5 6}\end{array}$

-Non-Generating Public Entities-

11 Non-Generating Public Entities

12 Total Non-Generating Public Entities

$\begin{array}{lllllllllllllll}3122 & 3122 & 2819 & 2789 & 3151 & 3555 & 3640 & 3527 & 3171 & 3002 & 2994 & 2971 & 3082 & 3202 & 3169 \\ \mathbf{3 1 2 2} & \mathbf{3 1 2 2} & \mathbf{2 8 1 9} & \mathbf{2 7 8 9} & \mathbf{3 1 5 1} & \mathbf{3 5 5 5} & \mathbf{3 6 4 0} & \mathbf{3 5 2 7} & \mathbf{3 1 7 1} & \mathbf{3 0 0 2} & \mathbf{2 9 9 4} & \mathbf{2 9 7 1} & \mathbf{3 0 8 2} & \mathbf{3 2 0 2} & \mathbf{3 1 6 9}\end{array}$

-Non-Generating Public Entities (Slice)-

13 Non-Generating Public Entities (Slice)

14 Total Non-Generating Public Entities (Slice)

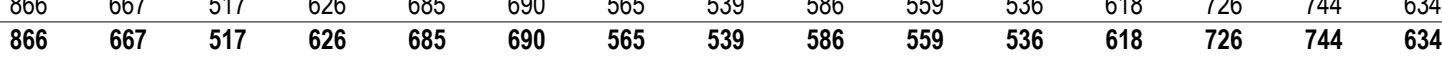

-Investor-Owned Entities-

15 Investor-Owned Entities

16 Total Investor-Owned Entities

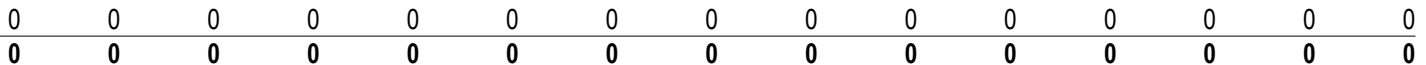

-Other Entities-

17 Other PNW Entities

18 Total Other Entities

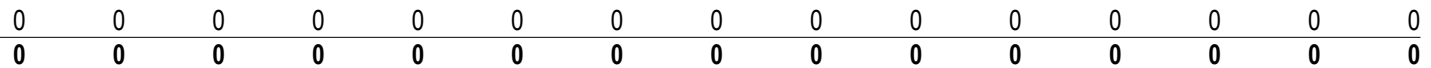

-Power Sales Contracts-

19 Federal Entities

20 U.S. Bureau of Reclamation

21 Direct Service Industry

22 Generating Public Entities

23 Non-Generating Public Entities

24 Investor-Owned Entities

25 Other Entities

26 Total Power Sales Contracts

\begin{tabular}{|c|c|c|c|c|c|c|c|c|c|c|c|c|c|c|}
\hline 114 & 114 & 105 & 107 & 126 & 137 & 139 & 134 & 122 & 110 & 110 & 107 & 108 & 112 & 118 \\
\hline 309 & 275 & 166 & 129 & 0 & 0 & 0 & 1.0 & 23 & 252 & 315 & 268 & 298 & 332 & 149 \\
\hline 0 & 0 & 0 & 0 & 0 & 0 & 0 & 0 & 0 & 0 & 0 & 0 & 0 & 0 & 0 \\
\hline 3540 & 3208 & 3098 & 3405 & 3907 & 4249 & 4043 & 3953 & 3789 & 3369 & 3324 & 3271 & 3169 & 3279 & 3574 \\
\hline 3987 & 3788 & 3335 & 3414 & 3835 & 4246 & 4205 & 4066 & 3757 & 3561 & 3530 & 3589 & 3808 & 3946 & 3803 \\
\hline 0 & 0 & 0 & 0 & 0 & 0 & 0 & 0 & 0 & 0 & 0 & 0 & 0 & 0 & \\
\hline 0 & 0 & 0 & 0 & 0 & 0 & 0 & 0 & 0 & 0 & 0 & 0 & 0 & 0 & \\
\hline 7950 & 7386 & 6704 & 7055 & 7869 & 8632 & 8387 & 8154 & 7691 & 7292 & 7279 & 7236 & 7384 & 7669 & $\theta$ \\
\hline
\end{tabular}


-Federal Entities-

1 Federal Entities

Aug1 Aug16 Sep Oct Nov Dec Jan Feb Mar Apr1 Apr16 May Jun Jul Avg

2 Total Federal Entities

\begin{tabular}{|c|c|c|c|c|c|c|c|c|c|c|c|c|c|c|}
\hline 83 & 81 & 58 & 76 & 101 & 104 & 107 & 98 & 91 & 113 & 113 & 107 & 94 & 75 & 92 \\
\hline 83 & 81 & 58 & 76 & 101 & 104 & 107 & 98 & 91 & 113 & 113 & 107 & 94 & 75 & 92 \\
\hline
\end{tabular}

-Generating Public Entities-

3 Generating Public Entities

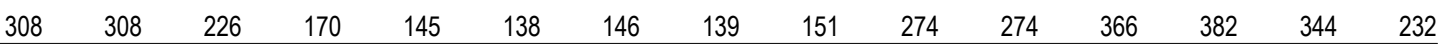

4 Total Generating Public Entities

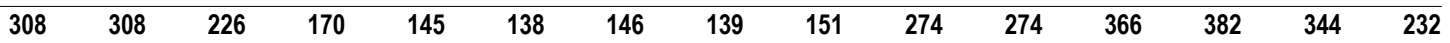

-Non-Generating Public Entities-

5 Non-Generating Public Entities

6 Total Non-Generating Public Entities

\begin{tabular}{lllllllllllllll}
9.1 & 9.1 & 9 & 9.7 & 11 & 11 & 12 & 11 & 9.9 & 9.5 & 9.5 & 9.5 & 9.4 & 8.9 & 10 \\
\hline $\mathbf{9 . 1}$ & $\mathbf{9 . 1}$ & $\mathbf{9}$ & $\mathbf{9 . 7}$ & $\mathbf{1 1}$ & $\mathbf{1 1}$ & $\mathbf{1 2}$ & $\mathbf{1 1}$ & $\mathbf{9 . 9}$ & $\mathbf{9 . 5}$ & $\mathbf{9 . 5}$ & $\mathbf{9 . 5}$ & $\mathbf{9 . 4}$ & $\mathbf{8 . 9}$ & $\mathbf{1 0}$
\end{tabular}

-Investor-Owned Entities-

7 Investor-Owned Entities

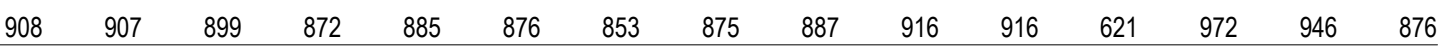

8 Total Investor-owned Entities

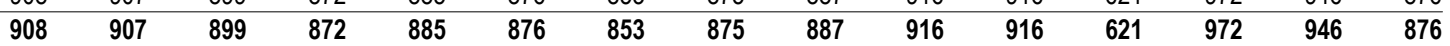

-Other Entities-

9 Other Entities

10 Total Other Entities

$\begin{array}{lllllllllllllll}84 & 84 & 73 & 85 & 90 & 83 & 87 & 83 & 83 & 89 & 89 & 90 & 92 & 85 & 85 \\ \mathbf{8 4} & \mathbf{8 4} & \mathbf{7 3} & \mathbf{8 5} & \mathbf{9 0} & \mathbf{8 3} & \mathbf{8 7} & \mathbf{8 3} & \mathbf{8 3} & \mathbf{8 9} & \mathbf{8 9} & \mathbf{9 0} & \mathbf{9 2} & \mathbf{8 5} & \mathbf{8 5}\end{array}$

-Total Non-Utility Generating Resources-

11 Federal Entities

12 Public Entities

13 Investor-Owned Entities

14 Other Entities

15 Total Non-Utility Generation

$\begin{array}{rrrrrrrrrrrrrrr}83 & 81 & 58 & 76 & 101 & 104 & 107 & 98 & 91 & 113 & 113 & 107 & 94 & 75 & 92 \\ 317 & 317 & 235 & 180 & 156 & 149 & 157 & 150 & 161 & 284 & 284 & 375 & 391 & 353 & 242 \\ 908 & 907 & 899 & 872 & 885 & 876 & 853 & 875 & 887 & 916 & 916 & 621 & 972 & 946 & 876 \\ 84 & 84 & 73 & 85 & 90 & 83 & 87 & 83 & 83 & 89 & 89 & 90 & 92 & 85 & 85 \\ 1392 & \mathbf{1 3 9 0} & \mathbf{1 2 6 5} & \mathbf{1 2 1 3} & \mathbf{1 2 3 2} & \mathbf{1 2 1 2} & \mathbf{1 2 0 4} & \mathbf{1 2 0 6} & \mathbf{1 2 2 3} & \mathbf{1 4 0 2} & \mathbf{1 4 0 2} & \mathbf{1 1 9 2} & \mathbf{1 5 5 0} & \mathbf{1 4 5 9} & \mathbf{1 2 9 6}\end{array}$


-Federal Entities-

1 Federal Entities

Aug1 Aug16 Sep Oct Nov Dec Jan Feb Mar Apr1 Apr16 May Jun Jul Avg

2 Total Federal Entities

\begin{tabular}{|c|c|c|c|c|c|c|c|c|c|c|c|c|c|c|}
\hline 83 & 81 & 58 & 76 & 101 & 104 & 107 & 98 & 91 & 113 & 113 & 107 & 94 & 75 & 92 \\
\hline 83 & 81 & 58 & 76 & 101 & 104 & 107 & 98 & 91 & 113 & 113 & 107 & 94 & 75 & 92 \\
\hline
\end{tabular}

-Generating Public Entities-

3 Generating Public Entities

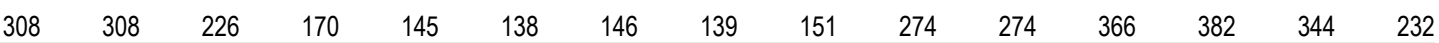

4 Total Generating Public Entities

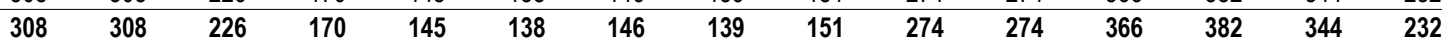

-Non-Generating Public Entities-

5 Non-Generating Public Entities

6 Total Non-Generating Public Entities

\begin{tabular}{lllllllllllllll}
9.1 & 9.1 & 9 & 9.7 & 11 & 11 & 6.1 & 5.4 & 4.2 & 3.9 & 3.9 & 3.9 & 3.8 & 3.3 & 6.8 \\
\hline $\mathbf{9 . 1}$ & $\mathbf{9 . 1}$ & $\mathbf{9}$ & $\mathbf{9 . 7}$ & $\mathbf{1 1}$ & $\mathbf{1 1}$ & $\mathbf{6 . 1}$ & $\mathbf{5 . 4}$ & $\mathbf{4 . 2}$ & $\mathbf{3 . 9}$ & $\mathbf{3 . 9}$ & $\mathbf{3 . 9}$ & $\mathbf{3 . 8}$ & $\mathbf{3 . 3}$ & $\mathbf{6 . 8}$
\end{tabular}

-Investor-Owned Entities-

7 Investor-Owned Entities

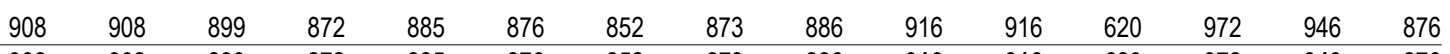

8 Total Investor-owned Entities

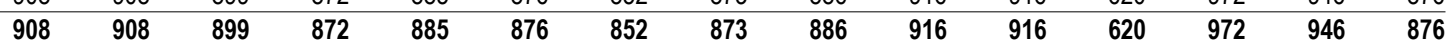

-Other Entities-

9 Other Entities

10 Total Other Entities

$\begin{array}{lllllllllllllll}84 & 84 & 73 & 85 & 90 & 83 & 87 & 83 & 83 & 89 & 89 & 90 & 92 & 85 & 85 \\ \mathbf{8 4} & \mathbf{8 4} & \mathbf{7 3} & \mathbf{8 5} & \mathbf{9 0} & \mathbf{8 3} & \mathbf{8 7} & \mathbf{8 3} & \mathbf{8 3} & \mathbf{8 9} & \mathbf{8 9} & \mathbf{9 0} & \mathbf{9 2} & \mathbf{8 5} & \mathbf{8 5}\end{array}$

-Total Non-Utility Generating Resources-

11 Federal Entities

12 Public Entities

13 Investor-Owned Entities

14 Other Entities

15 Total Non-Utility Generation

$\begin{array}{rrrrrrrrrrrrrrr}83 & 81 & 58 & 76 & 101 & 104 & 107 & 98 & 91 & 113 & 113 & 107 & 94 & 75 & 92 \\ 317 & 317 & 235 & 180 & 156 & 149 & 152 & 144 & 156 & 278 & 278 & 370 & 386 & 348 & 239 \\ 908 & 908 & 899 & 872 & 885 & 876 & 852 & 873 & 886 & 916 & 916 & 620 & 972 & 946 & 876 \\ 84 & 84 & 73 & 85 & 90 & 83 & 87 & 83 & 83 & 89 & 89 & 90 & 92 & 85 & 85 \\ 1392 & \mathbf{1 3 9 1} & \mathbf{1 2 6 6} & \mathbf{1 2 1 3} & \mathbf{1 2 3 2} & \mathbf{1 2 1 3} & \mathbf{1 1 9 8} & \mathbf{1 1 9 9} & \mathbf{1 2 1 6} & \mathbf{1 3 9 7} & \mathbf{1 3 9 6} & \mathbf{1 1 8 7} & \mathbf{1 5 4 4} & \mathbf{1 4 5 4} & \mathbf{1 2 9 2}\end{array}$


-Federal Entities-

1 Federal Entities

Aug1 Aug16 Sep Oct Nov Dec Jan Feb Mar Apr1 Apr16 May Jun Jul Avg

2 Total Federal Entities

\begin{tabular}{|c|c|c|c|c|c|c|c|c|c|c|c|c|c|c|}
\hline 59 & 59 & 52 & 116 & 127 & 127 & 130 & 125 & 119 & 121 & 121 & 118 & 120 & 109 & 110 \\
\hline 59 & 59 & 52 & 116 & 127 & 127 & 130 & 125 & 119 & 121 & 121 & 118 & 120 & 109 & 110 \\
\hline
\end{tabular}

-Generating Public Entities-

3 Generating Public Entities

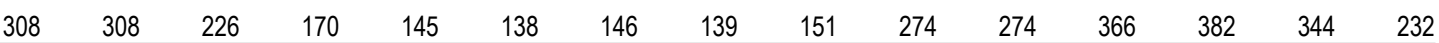

4 Total Generating Public Entities

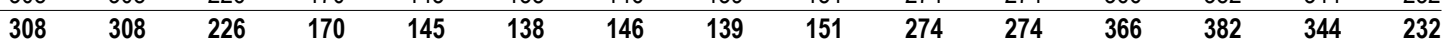

-Non-Generating Public Entities-

5 Non-Generating Public Entities

6 Total Non-Generating Public Entities

\begin{tabular}{|c|c|c|c|c|c|c|c|c|c|c|c|c|c|c|}
\hline 3.4 & 3.4 & 3.4 & 4.1 & 5.7 & 5.8 & 6.1 & 5.4 & 4.2 & 3.9 & 3.9 & 3.9 & 3.8 & 3.3 & 4.4 \\
\hline 34 & 3.4 & 3.4 & 4.1 & 5.7 & 5.8 & 6.1 & 5.4 & 4.2 & 3.9 & 3.9 & 3.9 & 3.8 & 3.3 & 4.4 \\
\hline
\end{tabular}

-Investor-Owned Entities-

7 Investor-Owned Entities

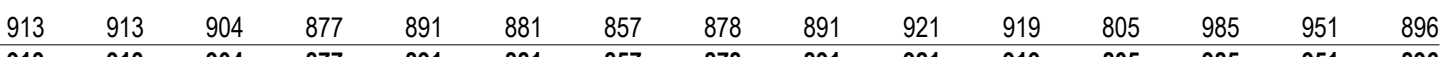

8 Total Investor-owned Entities

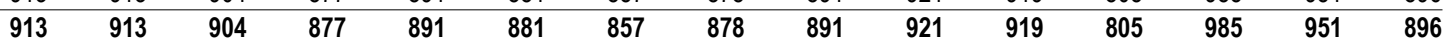

-Other Entities-

9 Other Entities

10 Total Other Entities

$\begin{array}{lllllllllllllll}84 & 84 & 73 & 85 & 90 & 83 & 87 & 83 & 83 & 89 & 89 & 90 & 92 & 85 & 85 \\ \mathbf{8 4} & \mathbf{8 4} & \mathbf{7 3} & \mathbf{8 5} & \mathbf{9 0} & \mathbf{8 3} & \mathbf{8 7} & \mathbf{8 3} & \mathbf{8 3} & \mathbf{8 9} & \mathbf{8 9} & \mathbf{9 0} & \mathbf{9 2} & \mathbf{8 5} & \mathbf{8 5}\end{array}$

-Total Non-Utility Generating Resources-

11 Federal Entities

12 Public Entities

13 Investor-Owned Entities

14 Other Entities

15 Total Non-Utility Generation

\begin{tabular}{|c|c|c|c|c|c|c|c|c|c|c|c|c|c|c|}
\hline 59 & 59 & 52 & 116 & 127 & 127 & 130 & 125 & 119 & 121 & 121 & 118 & 120 & 109 & 110 \\
\hline 312 & 312 & 229 & 174 & 150 & 143 & 152 & 144 & 156 & 278 & 278 & 370 & 386 & 348 & 237 \\
\hline 913 & 913 & 904 & 877 & 891 & 881 & 857 & 878 & 891 & 921 & 919 & 805 & 985 & 951 & 896 \\
\hline 84 & 84 & 73 & 85 & 90 & 83 & 87 & 83 & 83 & 89 & 89 & 90 & 92 & 85 & 85 \\
\hline 68 & 1368 & 1259 & 1252 & 1258 & 1235 & 1226 & 1231 & 1249 & 1409 & 1408 & 1382 & 1583 & 1493 & \\
\hline
\end{tabular}



2003 White Book

\begin{tabular}{|c|c|c|c|c|c|c|c|c|c|c|c|c|c|c|}
\hline 09 & 109 & 102 & 116 & 127 & 127 & 130 & 125 & 119 & 121 & 121 & 118 & 120 & 109 & 119 \\
\hline 09 & 109 & 102 & 116 & 127 & 127 & 130 & 125 & 119 & 121 & 121 & 118 & 120 & 109 & 119 \\
\hline
\end{tabular}

2 Total Federal Entities

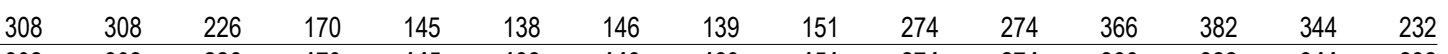

-Generating Public Entities-

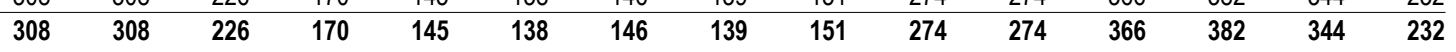

-Non-Generating Public Entities-

5 Non-Generating Public Entities

6 Total Non-Generating Public Entities

\begin{tabular}{|c|c|c|c|c|c|c|c|c|c|c|c|c|c|c|}
\hline 3.4 & 3.4 & 3.4 & 4.1 & 5.7 & 5.8 & 6.1 & 5.4 & 4.2 & 3.9 & 3.9 & 3.9 & 3.8 & 3.3 & 4.4 \\
\hline 34 & 3.4 & 3.4 & 4.1 & 5.7 & 5.8 & 6.1 & 5.4 & 4.2 & 3.9 & 3.9 & 3.9 & 3.8 & 3.3 & 4.4 \\
\hline
\end{tabular}

-Investor-Owned Entities-

7 Investor-Owned Entities

$\begin{array}{lllllllllllllll}\mathbf{9 1 3} & \mathbf{9 1 4} & \mathbf{9 0 5} & \mathbf{8 7 8} & 891 & \mathbf{8 8 2} & \mathbf{8 5 8} & \mathbf{8 8 0} & 892 & \mathbf{9 2 1} & \mathbf{9 1 9} & \mathbf{8 0 5} & \mathbf{9 7 7} & \mathbf{9 5 0} & 896 \\ \mathbf{9 1 3} & \mathbf{9 1 4} & \mathbf{9 0 5} & \mathbf{8 7 8} & \mathbf{8 9 1} & \mathbf{8 8 2} & \mathbf{8 5 8} & \mathbf{8 8 0} & \mathbf{8 9 2} & \mathbf{9 2 1} & \mathbf{9 1 9} & \mathbf{8 0 5} & \mathbf{9 7 7} & \mathbf{9 5 0} & \mathbf{8 9 6}\end{array}$

-Other Entities-

9 Other Entities

10 Total Other Entities

$\begin{array}{lllllllllllllll}84 & 84 & 73 & 85 & 90 & 83 & 87 & 83 & 83 & 89 & 89 & 90 & 92 & 85 & 85 \\ \mathbf{8 4} & \mathbf{8 4} & \mathbf{7 3} & \mathbf{8 5} & \mathbf{9 0} & \mathbf{8 3} & \mathbf{8 7} & \mathbf{8 3} & \mathbf{8 3} & \mathbf{8 9} & \mathbf{8 9} & \mathbf{9 0} & \mathbf{9 2} & \mathbf{8 5} & \mathbf{8 5}\end{array}$

-Total Non-Utility Generating Resources-

11 Federal Entities

12 Public Entities

13 Investor-Owned Entities

14 Other Entities

15 Total Non-Utility Generation

\begin{tabular}{|c|c|c|c|c|c|c|c|c|c|c|c|c|c|}
\hline 109 & 102 & 116 & 127 & 127 & 130 & 125 & 119 & 121 & 121 & 118 & 120 & 109 & 119 \\
\hline 312 & 229 & 174 & 150 & 143 & 152 & 144 & 156 & 278 & 278 & 370 & 386 & 348 & 237 \\
\hline 914 & 905 & 878 & 891 & 882 & 858 & 880 & 892 & 921 & 919 & 805 & 977 & 950 & 896 \\
\hline 84 & 73 & 85 & 90 & 83 & 87 & 83 & 83 & 89 & 89 & 90 & 92 & 85 & 85 \\
\hline 1418 & 1309 & 1253 & 1259 & 1235 & 1226 & 1232 & 1250 & 1409 & 1408 & 1383 & 1575 & 1491 & 13 \\
\hline
\end{tabular}


-Federal Entities-

1 Federal Entities

Aug1 Aug16 Sep Oct Nov Dec Jan Feb Mar Apr1 Apr16 May Jun Jul Avg

2 Total Federal Entities

\begin{tabular}{|c|c|c|c|c|c|c|c|c|c|c|c|c|c|c|}
\hline 09 & 109 & 102 & 116 & 127 & 127 & 130 & 125 & 119 & 121 & 121 & 118 & 120 & 109 & 119 \\
\hline 00 & 109 & 102 & 116 & 127 & 127 & 130 & 125 & 119 & 121 & 121 & 118 & 120 & 109 & 119 \\
\hline
\end{tabular}

-Generating Public Entities-

3 Generating Public Entities

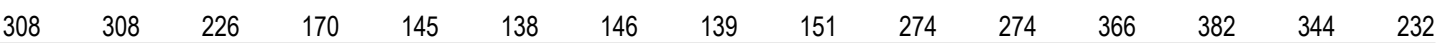

4 Total Generating Public Entities

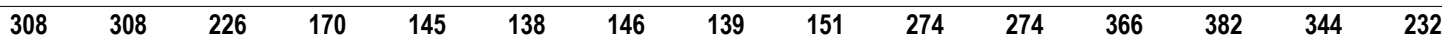

-Non-Generating Public Entities-

5 Non-Generating Public Entities

6 Total Non-Generating Public Entities

\begin{tabular}{|c|c|c|c|c|c|c|c|c|c|c|c|c|c|c|}
\hline 3.4 & 3.4 & 3.4 & 4.1 & 5.7 & 5.8 & 6.1 & 5.4 & 4.2 & 3.9 & 3.9 & 3.9 & 3.8 & 3.3 & 4.4 \\
\hline 34 & 3.4 & 3.4 & 4.1 & 5.7 & 5.8 & 6.1 & 5.4 & 4.2 & 3.9 & 3.9 & 3.9 & 3.8 & 3.3 & 4.4 \\
\hline
\end{tabular}

-Investor-Owned Entities-

7 Investor-Owned Entities

$\begin{array}{lllllllllllllll}\mathbf{9 1 3} & \mathbf{9 1 4} & \mathbf{9 0 5} & \mathbf{8 7 8} & 892 & 882 & 858 & 880 & 892 & 921 & \mathbf{9 2 1} & \mathbf{8 0 6} & \mathbf{9 7 8} & \mathbf{9 5 0} & 896 \\ \mathbf{9 1 3} & \mathbf{9 1 4} & \mathbf{9 0 5} & \mathbf{8 7 8} & \mathbf{8 9 2} & \mathbf{8 8 2} & \mathbf{8 5 8} & \mathbf{8 8 0} & \mathbf{8 9 2} & \mathbf{9 2 1} & \mathbf{9 2 1} & \mathbf{8 0 6} & \mathbf{9 7 8} & \mathbf{9 5 0} & \mathbf{8 9 6}\end{array}$

8 Total Investor-owned Entities

-Other Entities-

9 Other Entities

10 Total Other Entities

$\begin{array}{lllllllllllllll}84 & 84 & 73 & 85 & 90 & 83 & 87 & 83 & 83 & 89 & 89 & 90 & 92 & 85 & 85 \\ \mathbf{8 4} & \mathbf{8 4} & \mathbf{7 3} & \mathbf{8 5} & \mathbf{9 0} & \mathbf{8 3} & \mathbf{8 7} & \mathbf{8 3} & \mathbf{8 3} & \mathbf{8 9} & \mathbf{8 9} & \mathbf{9 0} & \mathbf{9 2} & \mathbf{8 5} & \mathbf{8 5}\end{array}$

-Total Non-Utility Generating Resources-

11 Federal Entities

12 Public Entities

13 Investor-Owned Entities

14 Other Entities

15 Total Non-Utility Generation

\begin{tabular}{|c|c|c|c|c|c|c|c|c|c|c|c|c|c|}
\hline 109 & 102 & 116 & 127 & 127 & 130 & 125 & 119 & 121 & 121 & 118 & 120 & 109 & 119 \\
\hline 312 & 229 & 174 & 150 & 143 & 152 & 144 & 156 & 278 & 278 & 370 & 386 & 348 & 237 \\
\hline 914 & 905 & 878 & 892 & 882 & 858 & 880 & 892 & 921 & 921 & 806 & 978 & 950 & 896 \\
\hline 84 & 73 & 85 & 90 & 83 & 87 & 83 & 83 & 89 & 89 & 90 & 92 & 85 & 85 \\
\hline 1419 & 1309 & 1253 & 1259 & 1236 & 1227 & 1232 & 1250 & 1409 & 1409 & 1383 & 1575 & 1492 & 13 \\
\hline
\end{tabular}


Aug1 Aug16 Sep Oct Nov Dec Jan Feb Mar Apr1 Apr16 May Jun Jul Avg

-Federal Entities-

1 Federal Entities

2 Total Federal Entities

\begin{tabular}{|c|c|c|c|c|c|c|c|c|c|c|c|c|c|c|}
\hline 09 & 109 & 102 & 116 & 127 & 127 & 130 & 125 & 119 & 121 & 121 & 118 & 120 & 109 & 119 \\
\hline 0 & 109 & 102 & 116 & 127 & 127 & 130 & 125 & 119 & 121 & 121 & 118 & 120 & 109 & 119 \\
\hline
\end{tabular}

-Generating Public Entities-

3 Generating Public Entities

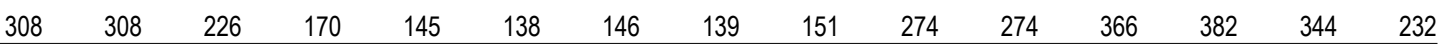

4 Total Generating Public Entities

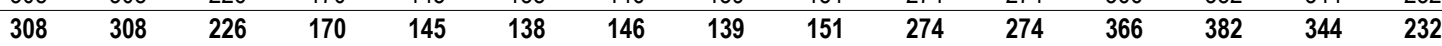

-Non-Generating Public Entities-

5 Non-Generating Public Entities

6 Total Non-Generating Public Entities

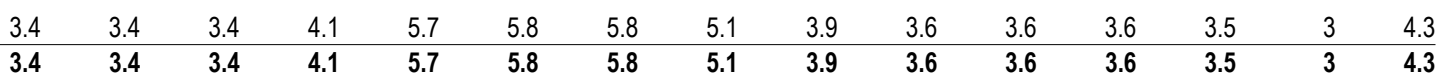

-Investor-Owned Entities-

7 Investor-Owned Entities

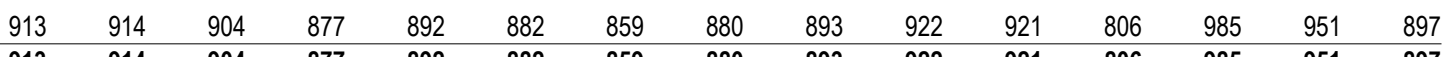

8 Total Investor-owned Entities

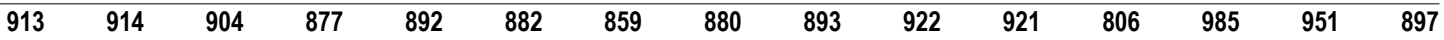

-Other Entities-

9 Other Entities

10 Total Other Entities

$\begin{array}{lllllllllllllll}84 & 84 & 73 & 85 & 90 & 83 & 87 & 83 & 83 & 89 & 89 & 90 & 92 & 85 & 85 \\ \mathbf{8 4} & \mathbf{8 4} & \mathbf{7 3} & \mathbf{8 5} & \mathbf{9 0} & \mathbf{8 3} & \mathbf{8 7} & \mathbf{8 3} & \mathbf{8 3} & \mathbf{8 9} & \mathbf{8 9} & \mathbf{9 0} & \mathbf{9 2} & \mathbf{8 5} & \mathbf{8 5}\end{array}$

-Total Non-Utility Generating Resources-

11 Federal Entities

12 Public Entities

13 Investor-Owned Entities

14 Other Entities

15 Total Non-Utility Generation

\begin{tabular}{|c|c|c|c|c|c|c|c|c|c|c|c|c|c|}
\hline 109 & 102 & 116 & 127 & 127 & 130 & 125 & 119 & 121 & 121 & 118 & 120 & 109 & 119 \\
\hline 312 & 229 & 174 & 150 & 143 & 151 & 144 & 155 & 278 & 278 & 370 & 385 & 347 & 237 \\
\hline 914 & 904 & 877 & 892 & 882 & 859 & 880 & 893 & 922 & 921 & 806 & 985 & 951 & 897 \\
\hline 84 & 73 & 85 & 90 & 83 & 87 & 83 & 83 & 89 & 89 & 90 & 92 & 85 & 85 \\
\hline 1418 & 1308 & 1252 & 1259 & 1236 & 1227 & 1232 & 1250 & 410 & 1409 & 1383 & 1583 & 1493 & 38 \\
\hline
\end{tabular}


-Federal Entities-

1 Federal Entities

Aug1 Aug16 Sep Oct Nov Dec Jan Feb Mar Apr1 Apr16 May Jun Jul Avg

2 Total Federal Entities

\begin{tabular}{|c|c|c|c|c|c|c|c|c|c|c|c|c|c|c|}
\hline 09 & 109 & 102 & 116 & 127 & 127 & 130 & 125 & 119 & 121 & 121 & 118 & 120 & 109 & 119 \\
\hline 00 & 109 & 102 & 116 & 127 & 127 & 130 & 125 & 119 & 121 & 121 & 118 & 120 & 109 & 119 \\
\hline
\end{tabular}

-Generating Public Entities-

3 Generating Public Entities

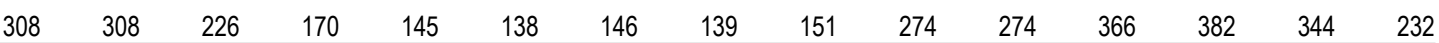

4 Total Generating Public Entities

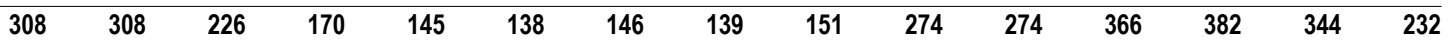

-Non-Generating Public Entities-

5 Non-Generating Public Entities

6 Total Non-Generating Public Entities

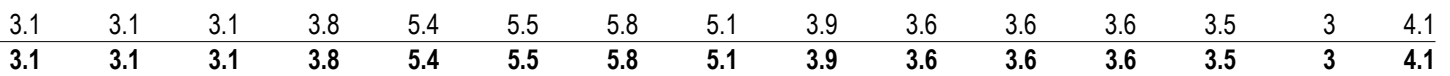

-Investor-Owned Entities-

7 Investor-Owned Entities

$\begin{array}{lllllllllllllll}\mathbf{9 1 4} & \mathbf{9 1 4} & \mathbf{9 0 4} & 877 & 899 & 888 & 865 & 888 & \mathbf{9 0 0} & \mathbf{9 2 8} & \mathbf{9 2 7} & 807 & \mathbf{9 7 8} & \mathbf{9 5 1} & \mathbf{9 0 0} \\ \mathbf{9 1 4} & \mathbf{9 1 4} & \mathbf{9 0 4} & \mathbf{8 7 7} & \mathbf{8 9 9} & \mathbf{8 8 8} & \mathbf{8 6 5} & \mathbf{8 8 8} & \mathbf{9 0 0} & \mathbf{9 2 8} & \mathbf{9 2 7} & \mathbf{8 0 7} & \mathbf{9 7 8} & \mathbf{9 5 1} & \mathbf{9 0 0}\end{array}$

-Other Entities-

9 Other Entities

10 Total Other Entities

$\begin{array}{lllllllllllllll}84 & 84 & 73 & 85 & 90 & 83 & 87 & 83 & 83 & 89 & 89 & 90 & 92 & 85 & 85 \\ \mathbf{8 4} & \mathbf{8 4} & \mathbf{7 3} & \mathbf{8 5} & \mathbf{9 0} & \mathbf{8 3} & \mathbf{8 7} & \mathbf{8 3} & \mathbf{8 3} & \mathbf{8 9} & \mathbf{8 9} & \mathbf{9 0} & \mathbf{9 2} & \mathbf{8 5} & \mathbf{8 5}\end{array}$

-Total Non-Utility Generating Resources-

11 Federal Entities

12 Public Entities

13 Investor-Owned Entities

14 Other Entities

15 Total Non-Utility Generation

\begin{tabular}{|c|c|c|c|c|c|c|c|c|c|c|c|c|c|}
\hline 109 & 102 & 116 & 127 & 127 & 130 & 125 & 119 & 121 & 121 & 118 & 120 & 109 & 119 \\
\hline 311 & 229 & 174 & 150 & 143 & 151 & 144 & 155 & 278 & 278 & 370 & 385 & 347 & 237 \\
\hline 914 & 904 & 877 & 899 & 888 & 865 & 888 & 900 & 928 & 927 & 807 & 978 & 951 & 900 \\
\hline 84 & 73 & 85 & 90 & 83 & 87 & 83 & 83 & 89 & 89 & 90 & 92 & 85 & 85 \\
\hline 1418 & 1308 & 1253 & 1266 & 1241 & 1233 & 1240 & 1258 & 1416 & 1416 & 1384 & 1576 & 1493 & 10 \\
\hline
\end{tabular}



2003 White Book

-Federal Entities-

1 Federal Entities

Aug1 Aug16 Sep Oct Nov Dec Jan Feb Mar Apr1 Apr16 May Jun Jul Avg

2 Total Federal Entities

\begin{tabular}{|c|c|c|c|c|c|c|c|c|c|c|c|c|c|c|}
\hline 109 & 109 & 102 & 116 & 127 & 127 & 130 & 125 & 119 & 121 & 121 & 118 & 120 & 109 & 119 \\
\hline 109 & 109 & 102 & 116 & 127 & 127 & 130 & 125 & 119 & 121 & 121 & 118 & 120 & 109 & 119 \\
\hline
\end{tabular}

-Generating Public Entities-

3 Generating Public Entities

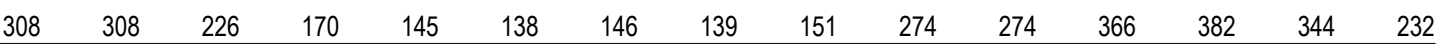

4 Total Generating Public Entities

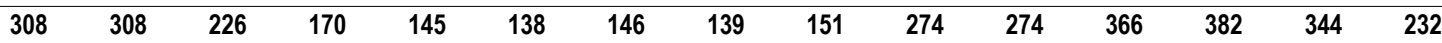

-Non-Generating Public Entities

5 Non-Generating Public Entities

6 Total Non-Generating Public Entities

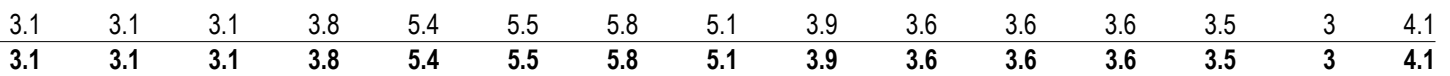

-Investor-Owned Entities-

7 Investor-Owned Entities

$\begin{array}{lllllllllllllll}\mathbf{9 1 4} & \mathbf{9 1 4} & \mathbf{9 0 5} & \mathbf{8 7 8} & \mathbf{8 9 9} & \mathbf{8 8 8} & \mathbf{8 6 6} & \mathbf{8 8 8} & 899 & \mathbf{9 2 7} & \mathbf{9 2 8} & 807 & \mathbf{9 7 9} & \mathbf{9 5 2} & \mathbf{9 0 0} \\ \mathbf{9 1 4} & \mathbf{9 1 4} & \mathbf{9 0 5} & \mathbf{8 7 8} & \mathbf{8 9 9} & \mathbf{8 8 8} & \mathbf{8 6 6} & \mathbf{8 8 8} & \mathbf{8 9 9} & \mathbf{9 2 7} & \mathbf{9 2 8} & \mathbf{8 0 7} & \mathbf{9 7 9} & \mathbf{9 5 2} & \mathbf{9 0 0}\end{array}$

-Other Entities-

9 Other Entities

10 Total Other Entities

$\begin{array}{lllllllllllllll}84 & 84 & 73 & 85 & 90 & 83 & 87 & 83 & 83 & 89 & 89 & 90 & 92 & 85 & 85 \\ \mathbf{8 4} & \mathbf{8 4} & \mathbf{7 3} & \mathbf{8 5} & \mathbf{9 0} & \mathbf{8 3} & \mathbf{8 7} & \mathbf{8 3} & \mathbf{8 3} & \mathbf{8 9} & \mathbf{8 9} & \mathbf{9 0} & \mathbf{9 2} & \mathbf{8 5} & \mathbf{8 5}\end{array}$

-Total Non-Utility Generating Resources-

11 Federal Entities

12 Public Entities

13 Investor-Owned Entities

14 Other Entities

15 Total Non-Utility Generation

\begin{tabular}{|c|c|c|c|c|c|c|c|c|c|c|c|c|c|}
\hline 109 & 102 & 116 & 127 & 127 & 130 & 125 & 119 & 121 & 121 & 118 & 120 & 109 & 119 \\
\hline 311 & 229 & 174 & 150 & 143 & 151 & 144 & 155 & 278 & 278 & 370 & 385 & 347 & 237 \\
\hline 914 & 905 & 878 & 899 & 888 & 866 & 888 & 899 & 927 & 928 & 807 & 979 & 952 & 900 \\
\hline 84 & 73 & 85 & 90 & 83 & 87 & 83 & 83 & 89 & 89 & 90 & 92 & 85 & 85 \\
\hline 1418 & 1309 & 1253 & 1266 & 1241 & 1234 & 1240 & 1256 & 1416 & 1416 & 1384 & 1576 & 1493 & 10 \\
\hline
\end{tabular}



2003 White Book

-Federal Entities-

1 Federal Entities

Aug1 Aug16 Sep Oct Nov Dec Jan Feb Mar Apr1 Apr16 May Jun Jul Avg

2 Total Federal Entities

\begin{tabular}{|c|c|c|c|c|c|c|c|c|c|c|c|c|c|c|}
\hline 109 & 109 & 102 & 116 & 127 & 127 & 130 & 125 & 119 & 121 & 121 & 118 & 120 & 109 & 119 \\
\hline 109 & 109 & 102 & 116 & 127 & 127 & 130 & 125 & 119 & 121 & 121 & 118 & 120 & 109 & 119 \\
\hline
\end{tabular}

-Generating Public Entities-

3 Generating Public Entities

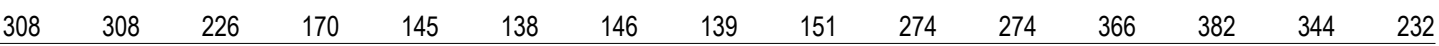

4 Total Generating Public Entities

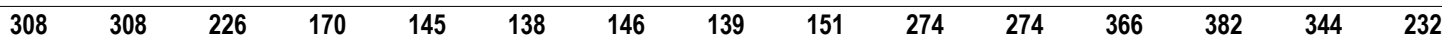

-Non-Generating Public Entities-

5 Non-Generating Public Entities

6 Total Non-Generating Public Entities

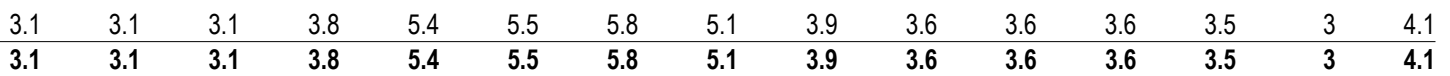

-Investor-Owned Entities-

7 Investor-Owned Entities

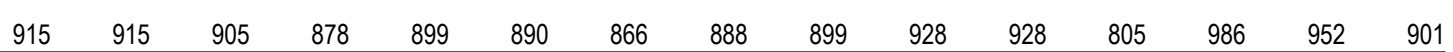

8 Total Investor-owned Entities

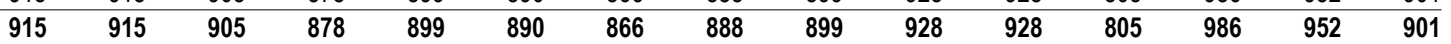

-Other Entities-

9 Other Entities

10 Total Other Entities

$\begin{array}{lllllllllllllll}84 & 84 & 73 & 85 & 90 & 83 & 87 & 83 & 83 & 89 & 89 & 90 & 92 & 85 & 85 \\ \mathbf{8 4} & \mathbf{8 4} & \mathbf{7 3} & \mathbf{8 5} & \mathbf{9 0} & \mathbf{8 3} & \mathbf{8 7} & \mathbf{8 3} & \mathbf{8 3} & \mathbf{8 9} & \mathbf{8 9} & \mathbf{9 0} & \mathbf{9 2} & \mathbf{8 5} & \mathbf{8 5}\end{array}$

-Total Non-Utility Generating Resources-

11 Federal Entities

12 Public Entities

13 Investor-Owned Entities

14 Other Entities

15 Total Non-Utility Generation

\begin{tabular}{|c|c|c|c|c|c|c|c|c|c|c|c|c|c|}
\hline 109 & 102 & 116 & 127 & 127 & 130 & 125 & 119 & 121 & 121 & 118 & 120 & 109 & 119 \\
\hline 311 & 229 & 174 & 150 & 143 & 151 & 144 & 155 & 278 & 278 & 370 & 385 & 347 & 237 \\
\hline 915 & 905 & 878 & 899 & 890 & 866 & 888 & 899 & 928 & 928 & 805 & 986 & 952 & 901 \\
\hline 84 & 73 & 85 & 90 & 83 & 87 & 83 & 83 & 89 & 89 & 90 & 92 & 85 & 85 \\
\hline 1419 & 1309 & 1253 & 1266 & 1243 & 1234 & 1240 & 1257 & 416 & 1416 & 1383 & 1583 & 1494 & 41 \\
\hline
\end{tabular}


-Federal Entities-

1 Federal Entities

Aug1 Aug16 Sep Oct Nov Dec Jan Feb Mar Apr1 Apr16 May Jun Jul Avg

2 Total Federal Entities

\begin{tabular}{|c|c|c|c|c|c|c|c|c|c|c|c|c|c|c|}
\hline 09 & 109 & 102 & 116 & 127 & 127 & 130 & 125 & 119 & 121 & 121 & 118 & 120 & 109 & 119 \\
\hline 00 & 109 & 102 & 116 & 127 & 127 & 130 & 125 & 119 & 121 & 121 & 118 & 120 & 109 & 119 \\
\hline
\end{tabular}

-Generating Public Entities-

3 Generating Public Entities

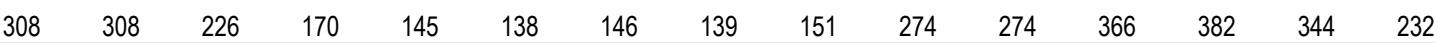

4 Total Generating Public Entities

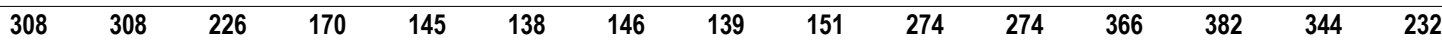

-Non-Generating Public Entities-

5 Non-Generating Public Entities

6 Total Non-Generating Public Entities

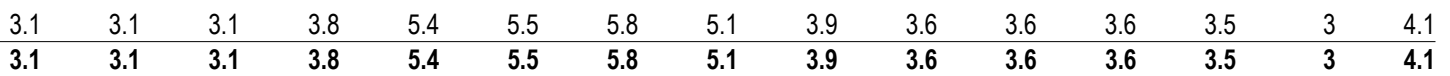

-Investor-Owned Entities-

7 Investor-Owned Entities

$\begin{array}{lllllllllllllll}\mathbf{9 1 4} & \mathbf{9 1 5} & \mathbf{9 0 6} & \mathbf{8 7 9} & \mathbf{9 0 0} & \mathbf{8 9 0} & \mathbf{8 6 7} & \mathbf{8 8 8} & \mathbf{9 0 1} & \mathbf{9 2 9} & \mathbf{9 2 8} & \mathbf{8 0 6} & \mathbf{9 7 9} & \mathbf{9 5 2} & \mathbf{9 0 1} \\ \mathbf{9 1 4} & \mathbf{9 1 5} & \mathbf{9 0 6} & \mathbf{8 7 9} & \mathbf{9 0 0} & \mathbf{8 9 0} & \mathbf{8 6 7} & \mathbf{8 8 8} & \mathbf{9 0 1} & \mathbf{9 2 9} & \mathbf{9 2 8} & \mathbf{8 0 6} & \mathbf{9 7 9} & \mathbf{9 5 2} & \mathbf{9 0 1}\end{array}$

-Other Entities-

9 Other Entities

10 Total Other Entities

$\begin{array}{lllllllllllllll}84 & 84 & 73 & 85 & 90 & 83 & 87 & 83 & 83 & 89 & 89 & 90 & 92 & 85 & 85 \\ \mathbf{8 4} & \mathbf{8 4} & \mathbf{7 3} & \mathbf{8 5} & \mathbf{9 0} & \mathbf{8 3} & \mathbf{8 7} & \mathbf{8 3} & \mathbf{8 3} & \mathbf{8 9} & \mathbf{8 9} & \mathbf{9 0} & \mathbf{9 2} & \mathbf{8 5} & \mathbf{8 5}\end{array}$

-Total Non-Utility Generating Resources-

11 Federal Entities

12 Public Entities

13 Investor-Owned Entities

14 Other Entities

15 Total Non-Utility Generation

\begin{tabular}{|c|c|c|c|c|c|c|c|c|c|c|c|c|c|}
\hline 109 & 102 & 116 & 127 & 127 & 130 & 125 & 119 & 121 & 121 & 118 & 120 & 109 & 119 \\
\hline 311 & 229 & 174 & 150 & 143 & 151 & 144 & 155 & 278 & 278 & 370 & 385 & 347 & 237 \\
\hline 915 & 906 & 879 & 900 & 890 & 867 & 888 & 901 & 929 & 928 & 806 & 979 & 952 & 901 \\
\hline 84 & 73 & 85 & 90 & 83 & 87 & 83 & 83 & 89 & 89 & 90 & 92 & 85 & 85 \\
\hline 1419 & 1309 & 1254 & 1267 & 1243 & 1235 & 1241 & 1258 & 1417 & 1416 & 1383 & 1576 & 1493 & 1341 \\
\hline
\end{tabular}


Oct Nov

Dec

Jan Feb Mar Apr1 Apr16

May Jun Jul Avg

Federal Entities

-NUG: Hydro-

1 Clearwater - State of ID DWR (BPA)

2 Dworshak Small Hydropower (BPA)

3 Elwah Hydro (BPA)

4 Glines Hydro (BPA)

5 Federal -Total NUG: Hydro

\begin{tabular}{|c|c|c|c|c|c|c|c|c|c|c|c|c|c|c|}
\hline 1 & 1 & 1 & 1 & 1 & 1 & 1 & 1 & 1 & 1 & 1 & 1 & 1 & 1 & 1 \\
\hline 2.6 & 2.6 & 2.6 & 2.6 & 2.6 & 2.6 & 2.6 & 2.6 & 2.6 & 2.6 & 2.6 & 2.6 & 2.6 & 2.6 & 2.6 \\
\hline 8.2 & 7.7 & 2.3 & 3.6 & 9.5 & 11 & 12 & 9.1 & 8.8 & 17 & 17 & 9.0 & 8.5 & 5.4 & 8.6 \\
\hline 16 & 15 & 4.1 & 6.7 & 14 & 16 & 15 & 14 & 14 & 25 & 25 & 30 & 16 & 11 & 15 \\
\hline 28 & 26 & 10.0 & 14 & 27 & 31 & 30 & 27 & 26 & 46 & 46 & 42 & 28 & 20 & 27 \\
\hline 0 & 0 & 0 & 0 & 0 & 0 & 0 & 0 & 0 & 0 & 0 & 0 & 0 & 0 & 0 \\
\hline 10 & 10 & 10 & 11 & 13 & 14 & 13 & 12 & 12 & 14 & 14 & 12 & 15 & 11 & 12 \\
\hline 3.3 & 3.3 & 3.8 & 5.6 & 8.3 & 10 & 11 & 9.6 & 7.2 & 5.8 & 5.8 & 4.6 & 4.1 & 3.3 & 6.4 \\
\hline 0.4 & 0.4 & 0.5 & 0.7 & 1 & 1.3 & 1.3 & 1.2 & 0.9 & 0.7 & 0.7 & 0.5 & 0.5 & 0.4 & 0.8 \\
\hline 3.9 & 3.9 & 4.4 & 6.5 & 9.7 & 12 & 12 & 11 & 8.4 & 6.7 & 6.7 & 5.4 & 4.8 & 3.8 & 7.4 \\
\hline 7.9 & 7.9 & 6.2 & 8.1 & 8.8 & 7.8 & 8.3 & 7.8 & 7.8 & 8.6 & 8.6 & 8.8 & 9.1 & 8 & 8.1 \\
\hline 29 & 29 & 23 & 30 & 33 & 29 & 31 & 29 & 29 & 32 & 32 & 33 & 34 & 30 & 30 \\
\hline 55 & 55 & 48 & 62 & 74 & 74 & 76 & 71 & 65 & 68 & 68 & 64 & 67 & 56 & 65 \\
\hline
\end{tabular}

\section{-NUG: Renewables-}

6 Ashland Solar Project (BPA)

7 Condon Wind Project (BPA)

8 Foote Creek 1 (BPA)

9 Foote Creek 2 (BPA)

10 Foote Creek 4 (BPA)

11 Klondike Phase 1 (BPA)

12 Stateline Wind Project (BPA)

\begin{tabular}{|c|c|c|c|c|c|c|c|c|c|c|c|c|c|c|}
\hline 1 & 1 & 1 & 1 & 1 & 1 & 1 & 1 & 1 & 1 & 1 & 1 & 1 & 1 & 1 \\
\hline 2.6 & 2.6 & 2.6 & 2.6 & 2.6 & 2.6 & 2.6 & 2.6 & 2.6 & 2.6 & 2.6 & 2.6 & 2.6 & 2.6 & 2.6 \\
\hline 8.2 & 7.7 & 2.3 & 3.6 & 9.5 & 11 & 12 & 9.1 & 8.8 & 17 & 17 & 9.0 & 8.5 & 5.4 & 8.6 \\
\hline 16 & 15 & 4.1 & 6.7 & 14 & 16 & 15 & 14 & 14 & 25 & 25 & 30 & 16 & 11 & 15 \\
\hline 28 & 26 & 10.0 & 14 & 27 & 31 & 30 & 27 & 26 & 46 & 46 & 42 & 28 & 20 & 27 \\
\hline 0 & 0 & 0 & 0 & 0 & 0 & 0 & 0 & 0 & 0 & 0 & 0 & 0 & 0 & 0 \\
\hline 10 & 10 & 10 & 11 & 13 & 14 & 13 & 12 & 12 & 14 & 14 & 12 & 15 & 11 & 12 \\
\hline 3.3 & 3.3 & 3.8 & 5.6 & 8.3 & 10 & 11 & 9.6 & 7.2 & 5.8 & 5.8 & 4.6 & 4.1 & 3.3 & 6.4 \\
\hline 0.4 & 0.4 & 0.5 & 0.7 & 1 & 1.3 & 1.3 & 1.2 & 0.9 & 0.7 & 0.7 & 0.5 & 0.5 & 0.4 & 0.8 \\
\hline 3.9 & 3.9 & 4.4 & 6.5 & 9.7 & 12 & 12 & 11 & 8.4 & 6.7 & 6.7 & 5.4 & 4.8 & 3.8 & 7.4 \\
\hline 7.9 & 7.9 & 6.2 & 8.1 & 8.8 & 7.8 & 8.3 & 7.8 & 7.8 & 8.6 & 8.6 & 8.8 & 9.1 & 8 & 8.1 \\
\hline 29 & 29 & 23 & 30 & 33 & 29 & 31 & 29 & 29 & 32 & 32 & 33 & 34 & 30 & 30 \\
\hline 55 & 55 & 48 & 62 & 74 & 74 & 76 & 71 & 65 & 68 & 68 & 64 & 67 & 56 & 65 \\
\hline
\end{tabular}

Generating Public Entities

-NUG: Hydro-

14 Don Steffen (SHPD)

15 Eltopia Br Canal 4.6 (SCL)

16 Eltopia Br Canal 4.6 (TPU)

17 John Llewellyn (SHPD)

18 Kevin Duncan (SHPD)

19 Lucky Peak (SCL)

20 Main Canal Headworks (SCL)

21 Main Canal Headworks (TPU)

22 PEC Headworks (GCPD)

23 Pickering Micro Hydro (CKPD)

24 Potholes E Canal 66.0 (SCL)

25 Potholes E Canal 66.0 (TPU)

26 Quincy Chute (GCPD)

27 Russel D. Smith (SCL)

28 Russel D. Smith (TPU)

29 Smith Creek (EWEB)

30 Summer Falls (SCL)

31 Summer Falls (TPU)

32 Woods Creek (SHPD)

33 GPU - Total NUG: Hydro

-NUG: Small Thermal \& Misc.-

34 Grays Harbor Paper (GHPD)

35 Weyerhaeuser Pulp Mill (GHPD)

36 GPU - Total NUG: Small Thermal \& Misc.

$\begin{array}{rrrr}0 & 0 & 0 & \\ 0.9 & 0.9 & 0.6 & 0.3 \\ 0.9 & 0.9 & 0.6 & 0.3 \\ 0 & 0 & 0 & \\ 0 & 0 & 0 & \\ 66 & 66 & 41 & 11 \\ 10 & 10 & 6.1 & 2 . \\ 10 & 10 & 6.1 & 2 . \\ 4.5 & 4.5 & 3.1 & 2.2 \\ 0 & 0 & 0 & \\ 0.9 & 0.9 & 0.9 & 0.8 \\ 0.9 & 0.9 & 0.9 & 0.8 \\ 7.7 & 7.7 & 4 & 2.3 \\ 2 & 2 & 1.3 & 0.7 \\ 2 & 2 & 1.3 & 0.7 \\ 0 & 0 & 0 & \\ 39 & 39 & 24 & 11 \\ 39 & 39 & 24 & 11 \\ 0.1 & 0.1 & 0.1 & 0.1 \\ 185 & 185 & 115 & \mathbf{5}\end{array}$

$\begin{array}{rr}0 & \\ 0.3 & \\ 0.3 & \\ 0 & \\ 0 & \\ 11 & 2.2 \\ 2.6 & \\ 2.6 & \\ 2.2 & \\ 0 & \\ 0.8 & \\ 0.8 & \\ 2.3 & \\ 0.7 & \\ 0.7 & \\ 5 & \\ 11 & \\ 11 & \\ 0.1 & 0 \\ \mathbf{5 1} & \\ & \end{array}$

$\begin{array}{rrr}0 & 0 & 0 \\ 0 & 0 & 0 \\ 0 & 0 & 0 \\ 0 & 0 & 0 \\ 0 & 0 & 0 \\ 2.2 & 3 & 7.9 \\ 0 & 0 & 0 \\ 0 & 0 & 0 \\ 0 & 0 & 0 \\ 0 & 0 & 0 \\ 0 & 0 & 0 \\ 0 & 0 & 0 \\ 0 & 0 & 0 \\ 0 & 0 & 0 \\ 0 & 0 & 0 \\ 5.4 & 3.9 & 0 \\ 0 & 0 & 0 \\ 0 & 0 & 0 \\ 0.1 & 0.1 & 0.1 \\ 7.7 & 7 & 8\end{array}$

$\begin{array}{rrr}0 & 0 & \\ 0 & 0 & \\ 0 & 0 & \\ 0 & 0 & \\ 0 & 0 & \\ 7.9 & 10 & 2 \\ 0 & 0 & 1.4 \\ 0 & 0 & 1.4 \\ 0 & 0 & 0.4 \\ 0 & 0 & \\ 0 & 0 & 0.3 \\ 0 & 0 & 0.3 \\ 0 & 0 & 0.8 \\ 0 & 0 & 0.1 \\ 0 & 0 & 0.1 \\ 0 & 0.8 & 1.5 \\ 0 & 0 & 2.4 \\ 0 & 0 & 2.4 \\ 0.1 & 0.1 & 0.1 \\ 8 & 11 & 32\end{array}$

\begin{tabular}{rr}
0 & \\
0 & 0.7 \\
0 & 0.7 \\
0 & \\
0 & \\
21 & 0 \\
1.4 & 6.8 \\
1.4 & 6.8 \\
0.4 & 5.1 \\
0 & \\
0.3 & 0.9 \\
0.3 & 0 \\
0.8 & 5 \\
0.1 & 1.2 \\
0.1 & 1.2 \\
1.5 & 9.2 \\
2.4 & 25 \\
2.4 & 25 \\
0.1 & 0.1 \\
32 & 141 \\
\hline 3
\end{tabular}

$\begin{array}{rr}0 & 0 \\ 0.7 & 0.7 \\ 0.7 & 0.7 \\ 0 & 0 \\ 0 & 0 \\ 52 & 52 \\ 6.8 & 6.8 \\ 6.8 & 6.8 \\ 5.1 & 5.1 \\ 0 & 0 \\ 0.9 & 0.9 \\ 0.9 & 0.9 \\ 5.5 & 5.5 \\ 1.2 & 1.2 \\ 1.2 & 1.2 \\ 9.2 & 9.2 \\ 25 & 25 \\ 25 & 25 \\ 0.1 & 0.1 \\ 141 & 141\end{array}$

\begin{tabular}{rrrrr}
0 & 0 & 0 & 0 & 0 \\
0.7 & 1 & 1 & 1.1 & 0.5 \\
0.7 & 1 & 1 & 1.1 & 0.5 \\
0 & 0 & 0 & 0 & 0 \\
0 & 0 & 0 & 0 & 0 \\
52 & 84 & 85 & 77 & 38 \\
6.8 & 9.1 & 11 & 12 & 4.9 \\
6.8 & 9.1 & 11 & 12 & 4.9 \\
5.1 & 6.9 & 6.2 & 5.8 & 2.9 \\
0 & 0 & 0 & 0 & 0 \\
0.9 & 0.9 & 0.9 & 0.9 & 0.5 \\
0.9 & 0.9 & 0.9 & 0.9 & 0.5 \\
5.5 & 7.1 & 8.4 & 8.5 & 3.7 \\
1.2 & 1.9 & 2.1 & 1.7 & 0.9 \\
1.2 & 1.9 & 2.1 & 1.7 & 0.9 \\
9.2 & 37 & 37 & 11 & 9.2 \\
25 & 36 & 40 & 44 & 18 \\
25 & 36 & 40 & 44 & 18 \\
0.1 & 0.1 & 0.1 & 0.1 & 0.1 \\
\hline 141 & $\mathbf{2 3 2}$ & $\mathbf{2 4 7}$ & $\mathbf{2 2 1}$ & 105
\end{tabular}

-NUG: Co-Generation-

37 Metro Westpoint (SCL)

38 Scott Paper (SHPD)

39 GPU - Total NUG: Co-Generation

\begin{tabular}{|c|c|c|c|c|c|c|c|c|c|c|c|c|c|c|}
\hline 3.8 & 3.8 & 3.8 & 3.8 & 3.8 & 3.8 & 3.8 & 3.8 & 3.8 & 3.8 & 3.8 & 3.8 & 3.8 & 3.8 & 3.8 \\
\hline 11 & 11 & 11 & 11 & 11 & 11 & 11 & 11 & 11 & 11 & 11 & 11 & 11 & 11 & 11 \\
\hline 15 & 15 & 15 & 15 & 15 & 15 & 15 & 15 & 15 & 15 & 15 & 15 & 15 & 15 & 15 \\
\hline
\end{tabular}

-NUG: Renewables-

40 Foote Creek 1 (EWEB)

41 Nine Canyon Wind Project (CHPD)

42 Nine Canyon Wind Project (COPD)

43 Nine Canyon Wind Project (DOPD)

44 Nine Canyon Wind Project (GCPD)

45 Nine Canyon Wind Project (GHPD)

46 Nine Canyon Wind Project (OKPD)

47 Stateline Wind Project (EWEB)

48 Stateline Wind Project (SCL)

49 GPU - Total NUG: Renewables

\begin{tabular}{|c|c|c|c|c|c|c|c|c|c|c|c|c|c|}
\hline 1.2 & 1.2 & 1.2 & 1.2 & 1.2 & 1.2 & 1.2 & 1.2 & 1.2 & 1.2 & 1.2 & 1.2 & 1.2 & 1.2 \\
\hline 38 & 38 & 27 & 27 & 27 & 27 & 27 & 27 & 38 & 38 & 38 & 38 & 38 & 33 \\
\hline 39 & 39 & 28 & 28 & 28 & 28 & 28 & 28 & 39 & 39 & 39 & 39 & 39 & 34 \\
\hline
\end{tabular}

Non-Generating Public Entities

-NUG: Hydro-

50 Buffalo Hydro (FREC)

51 Burr \& Bouchard (TNEC)

52 City of Cove (OTEC)

53 Falls Creek (CCPD)

54 Lilliwaup Falls Generating Co. (MCPD1)

55 Thomas Burnside (TNEC)

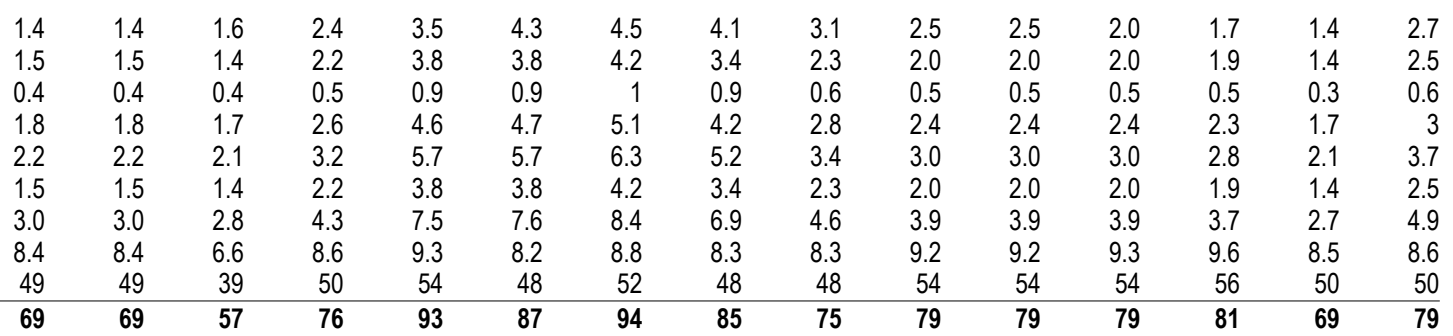

-NUG: Small Thermal \& Misc.-

57 Methane Energy Agricultural D. (TKPD)

$\begin{array}{rrrrr}0.2 & 0.2 & 0.2 & 0.2 & 0.2 \\ 0 & 0 & 0 & 0 & 0 \\ 0.3 & 0.3 & 0.3 & 0.3 & 0.3 \\ 0 & 0 & 0 & 0 & 0 \\ 0.6 & 0.6 & 0.6 & 0.6 & 0.6 \\ 0 & 0 & 0 & 0 & 0 \\ 1.2 & 1.2 & 1.2 & 1.2 & 1.2\end{array}$

$\begin{array}{rr}0.2 & 0.2 \\ 0 & 0 \\ 0.3 & 0.3 \\ 0 & 0 \\ 0.6 & 0.6 \\ 0 & 0 \\ 1.2 & 1.2\end{array}$

$\begin{array}{rr}0.2 & 0.2 \\ 0 & 0 \\ 0.3 & 0.3 \\ 0 & 0 \\ 0.6 & 0.6 \\ 0 & \\ 1.2 & 1.2\end{array}$

\begin{tabular}{rr}
0.2 & 0.2 \\
0 & 0 \\
0.3 & 0.3 \\
0 & 0 \\
0 & 0.6 \\
0 & 0 \\
\hline .2 & 1.2
\end{tabular}

$\begin{array}{rrrrr}0.2 & 0.2 & 0.2 & 0.2 & 0.2 \\ 0 & 0 & 0 & 0 & 0 \\ 0.3 & 0.3 & 0.3 & 0.3 & 0.3 \\ 0 & 0 & 0 & 0 & 0 \\ 0.6 & 0.6 & 0.6 & 0.6 & 0.6 \\ 0 & 0 & 0 & 0 & 0 \\ 1.2 & 1.2 & 1.2 & 1.2 & 1.2\end{array}$




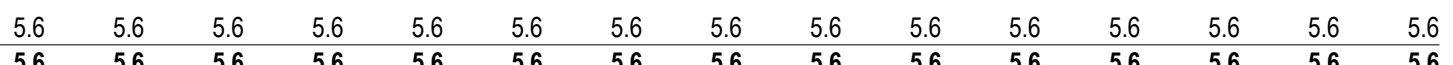

-NUG: Renewables-

61 Ashland Solar Project (ASHL) 62 Nine Canyon Wind Project (BCPD)

63 Nine Canyon Wind Project (ENW)

64 Nine Canyon Wind Project (LCPD)

65 Nine Canyon Wind Project (MCPD3)

66 White Bluffs Solar (ENW)

67 NGP - Total NUG: Renewables

$\begin{array}{rrrrrrrrrrrrrrr}0 & 0 & 0 & 0 & 0 & 0 & 0 & 0 & 0 & 0 & 0 & 0 & 0 & 0 & 0 \\ 0.6 & 0.6 & 0.5 & 0.8 & 1.4 & 1.4 & 1.6 & 1.3 & 0.9 & 0.7 & 0.7 & 0.7 & 0.7 & 0.5 & 0.9 \\ 0.4 & 0.4 & 0.4 & 0.5 & 0.9 & 1.0 & 1.1 & 0.9 & 0.6 & 0.5 & 0.5 & 0.5 & 0.5 & 0.3 & 0.6 \\ 0.2 & 0.2 & 0.2 & 0.3 & 0.5 & 0.5 & 0.5 & 0.4 & 0.3 & 0.2 & 0.2 & 0.2 & 0.2 & 0.2 & 0.3 \\ 0.4 & 0.4 & 0.4 & 0.5 & 0.9 & 1.0 & 1 & 0.9 & 0.6 & 0.5 & 0.5 & 0.5 & 0.5 & 0.3 & 0.6 \\ 0 & 0 & 0 & 0 & 0 & 0 & 0 & 0 & 0 & 0 & 0 & 0 & 0 & 0 & 0 \\ 1.5 & 1.5 & 1.4 & \mathbf{2 . 2} & \mathbf{3 . 8} & \mathbf{3 . 8} & \mathbf{4 . 2} & \mathbf{3 . 5} & \mathbf{2 . 3} & \mathbf{2 . 0} & \mathbf{2 . 0} & \mathbf{2 . 0} & \mathbf{2 . 0}\end{array}$

Investor-Owned Entities

-NUG: Hydro-

68 Barber Dam (IPC)

69 Barney Creek (NWE)

70 Beaverton (PGE)

71 Bell Mountain (UPL)

72 Bingham Engineering (NWE)

73 Birch Creek (IPC)

74 Birch Creek (UPL)

75 Black Canyon \#3 (IPC)

76 Black Creek (AVWP)

77 Blind Canyon Hydro (IPC)

78 Box Canyon (IPC)

79 Boyd James (PPL)

80 Briggs Creek (IPC)

81 Broadwater Dam (NWE)

82 Bypass (IPC)

83 Canyon Springs (IPC)

84 Carlton (PGE)

85 Cascade Creek (NWE)

86 CDM Hydro - Bonneville Pacific (PPL)

87 Cedar Draw (IPC)

88 Central OR Irrigation District (PPL)

89 Clear Springs Trout (IPC)

90 Corbett (PGE)

91 Cornelius (PGE)

92 Crystal Springs (IPC)

93 Curry Cattle Co. (IPC)

94 Dairy Creek (PGE)

95 Deep Creek (AVWP)

96 Derr Creek (AVWP)

97 Dietrich Drop (IPC)

98 Donald Jenni (NWE)

99 Eagle Creek (PGE)

100 Eagle Point Irrigation Dist. (PPL)

101 Elk Creek (IPC)

102 Estacada (PGE)

103 Falls Creek (PPL)

104 Falls River (IPC)

105 Farmers Irrig. (PPL)

106 Faulkner Ranch (IPC)

107 Fisheries Development Co (IPC)

108 Galesville Dam (PPL)

109 Geobon 2 (IPC)

110 Georgetown Power (UPL)

111 Hailey Cspp (IPC)

112 Hazelton A (IPC)

113 Hazelton B (IPC)

114 Horseshoe Bend (IPC)

115 Hutchinson Creek (PSE)

116 Ingram Warm Springs (UPL)

$117 \mathrm{Jim}$ Ford Creek (AVWP)

118 Jim Knight (IPC)

119 John Day Creek (AVWP)

120 Joseph Hydro (PPL)

121 Kasel \& Witherspoon (IPC)

122 Koma Kulshan (PSE)

123 Koyle (IPC)

124 L. Fery (PPL)

125 Lacomb Irrig. (PPL)

126 Lake Oswego (PGE)

2.4
0.1
0.1
0
0.1
0.7
0.4
0.6
1.1

2.
0.1
0.1
1.3
0.1
2.8
0.7
0.4
0.6
1.1
8.3
0.1

$\begin{array}{rrrr}0 & 0 & 0 \\ 0.1 & 0.1 & 0.1\end{array}$

$\begin{array}{rrrr}0.1 & 0.1 & 0.1 & 0.1 \\ 0 & 0 & 0.1 & 0.1\end{array}$

$\begin{array}{llll}1.3 & 1.3 & 1.1 & 1.0 \\ 0.1 & 0.1 & 0.1 & 0.1\end{array}$

$\begin{array}{llll}2.8 & 1.9 & 2.8 & 2.8\end{array}$

$\begin{array}{llll}0.7 & 0.7 & 1.1 & 0.9\end{array}$

$\begin{array}{llll}0.7 & 1.1 & 0.9 & 0.2 \\ 0.4 & 0.4 & 0.4\end{array}$

0

$\begin{array}{llll}0.6 & 0.6 & 0.6 & 0.6\end{array}$

$\begin{array}{llll}1.1 & 1.1 & 1.5 & 1.8\end{array}$

$\begin{array}{llll}8.3 & 8.3 & 6.5 & 2.8\end{array}$

0.1
0

2.92 .9

0.3

3.1

0.4
0

1.3

0.2

0

0.1

4.7

0.1

0

1.2

0.1

6.5

0.6

0.8

0.2

0.6
1.0

1.0

0.1

7

6.6

8.8

0.7

0.2
0

0.4

0.2

1.5

$1.2 \quad 1.2$

4.6

1.1

0.4
0.1

0.1

2.8

$2.9 \quad 3.1 \quad 2.8$

3.1
0.4
0

1.3

0.2

0
0.1

4.7

0.1

0
12

0.1

6.5

0.6

0.8

0.6

0.6
1.0
0

0.1

6.6

8.8
0.7

0.2

$$
3.7
$$$$
0.4
$$

0
0

0
1.6

0.2

0

$3.6 \quad 3$

0.1

0.1

$\begin{array}{rr}0.1 & 0.1 \\ 0 & 0\end{array}$

0.7

0.1
0

3.1

5.0

3.2

0.3

0.3

0.3

0.2

0.3

0.1

0

$\begin{array}{ll}3.5 & 3.9\end{array}$

0.7

0.5

0.5

0.2

0.1

0.3

$6.1 \quad 3.9$

0
1.0
0.4

0.4

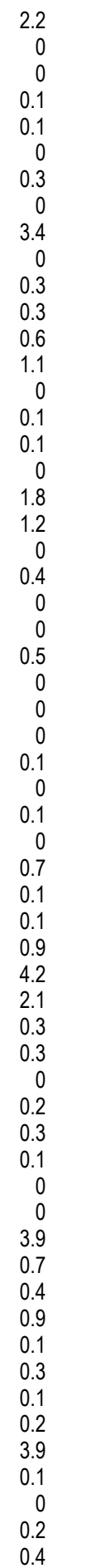

$\begin{array}{llllll}3.2 & 3.2 & 3.1 & 3.2 & 3.3 & 2.3\end{array}$

$\begin{array}{rrrrrrrr}2.6 & 3.3 & 3.2 & 3.2 & 3.1 & 3.2 & 3.3 & 2.3 \\ 0 & 0 & 0 & 0 & 0 & 0 & 0 & 0 \\ 0 & 0 & 0 & 0 & 0 & 0 & 0 & 0\end{array}$

$0.1 \quad 0.1$

0.1
0

$\begin{array}{rr}1.2 & 1.7 \\ 0 & 0.1\end{array}$

3.4
0

$0.3 \quad 0.3$

$0.7 \quad 0.6$

$\begin{array}{rr}1.2 & 1 \\ 0 & 0\end{array}$

$0.1 \quad 0.1$

$\begin{array}{ll}0 & \\ 2 & 2\end{array}$

$1.3 \quad 0.4$

$\begin{array}{ll}0.4 & 0.9 \\ 0.5 & 0.4\end{array}$

0.6

0.6

$\begin{array}{ll}0 & 0 \\ 0 & \\ 0 & \end{array}$

0.1

0.1

0
0.9

0.9
0.1
0.1

0.1

2.7

3.8
4.6

0.3

0.3

0.2

0.3

0.3
0.1

0
4.6

0.7

0.4

0.9

$0.1 \quad 0.2$

0.2

0.2

3.8

0.1
0
0.9

0.9
0.4

0
0.1
0

0
1.6
0.1

0.1
3.1

0.6

0.3
0.8

0.8
0.6

1.8

2.9
0.1

$\begin{array}{ll}0.1 & 0.1 \\ 0.1 & 0.1\end{array}$

0
2.7

2.7
0.4

$\begin{array}{ll}0.8 & 0.4 \\ 0.3 & 0.3\end{array}$

0.3

0

0.9

0.1

0.1

0.2

3.1

0

0.7

0.1

2.7

3.8
3.3

0.5

0.3

0.3
0.6

0.3
0.1

0.1
0.1

5.9

0.7

0.4
1.1

0.2
0.2

0.4
4.4

0.3
0
0.9

0.4

0.4

1
0.4

0.1
0
0
.6
0.1
3.7
0.6
0.3
0.8
0.6
1.8
2.9
0.1
0.1
0
2.7
0.4
0.8
0.3
0

0.1
0.1

0

0.2

$0.1 \quad 0.1$

$\begin{array}{rrr}0.1 & 0.1 & 0.1 \\ 0 & 0 & 0\end{array}$

$\begin{array}{llll}1.6 & 1.3 & 1.2 & 1.2\end{array}$

$\begin{array}{llll}0.1 & 0.1 & 0.1 & 0.1\end{array}$

$\begin{array}{llll}3.4 & 3.1 & 3.1 & 2.9\end{array}$

$\begin{array}{llll}1.6 & 1.0 & 0.7 & 0.6\end{array}$

$\begin{array}{llll}0.3 & 0.4 & 0.4 & 0.4\end{array}$

$\begin{array}{rrrr}0.5 & 0 & 0 & 0.3\end{array}$

$\begin{array}{llll}0.6 & 0.6 & 0.6 & 0.6\end{array}$

$\begin{array}{llll}2.6 & 2.6 & 1.9 & 1.6\end{array}$

$\begin{array}{rrrr}7 & 8.2 & 8.7 & 3.8 \\ 0.1 & 0.1 & 0.1 & 0.1\end{array}$

$\begin{array}{rrrr}0 & 0 & 0 & 0 \\ 0 & 0 & 0 & 0\end{array}$ 
Aug1 Aug16 Sep

127 Lateral \#10 (IPC)

128 Lee Tavenner (NWE)

129 Lemoyne (IPC)

130 Little Wood River Res. (IPC)

131 Littlewood/Arkoosh (IPC)

132 Lowline \#2 (IPC)

133 Lowline Canal (IPC)

134 Magic Reservoir (IPC)

135 Malad River (IPC)

136 Marcos Ranches (IPC)

137 Marsh Valley (UPL)

138 Meyers Falls (AVWP)

139 Middlefork Irrig. (PPL)

140 Mile 28 (IPC)

141 Minikahda (PGE)

142 Mink Creek (UPL)

143 Mitchell Butte (IPC)

144 Mountain Energy (PPL)

$145 \mathrm{Mt}$. Tabor (PGE)

146 Mud Creek/S\&S (IPC)

147 Mud Creek/White (IPC)

148 Nichols Gap (PPL)

149 Nicholson Sunnybar (UPL)

150 North Fork Sprague (PPL)

151 O.J. Power (UPL)

152 Odell Creek (PPL)

153 Opal Springs (PPL)

154 Owyhee Dam (IPC)

155 Pancheri (UPL)

156 Pelton Rereg. Dam (PPL)

157 Phillips Ranch (AVWP)

158 Phillipsburg (NWE)

159 Pigeon Cove (IPC)

160 Pine Creek (NWE)

161 Port Townsend Paper Hydro (PSE)

162 Portland (PGE)

163 Portland Hydro Project (PGE)

164 Preston City (UPL)

165 Pristine Springs (IPC)

166 Pristine Springs \#3 (IPC)

167 Reynolds Irrigation (IPC)

168 Rim View (IPC)

169 Rock Creek \#1 (IPC)

170 Rock Creek \#2 (IPC)

171 Sagebrush (IPC)

172 Salem (PGE)

173 Schaffner (IPC)

174 Sheep Creek (AVWP)

175 Shingle Creek (IPC)

176 Shoshone (IPC)

177 Shoshone II (IPC)

178 Snake River Pottery (IPC)

179 Snedigar Ranch (IPC)

180 Solar Research (PPL)

181 South Dry Creek (NWE)

182 Spokane Upriver (AVWP)

183 Stauffer Dry Creek (UPL)

184 Strawberry Creek (NWE)

185 Sunshine Power \#2 (IPC)

186 Sygitowicz Creek Small Hydro (PSE)

187 TGS/Briggs (UPL)

188 Trout Co. (IPC)

189 Tunnel \#1 (IPC)

190 Twin Falls (PSE)

191 Walla Walla (PPL)

192 Water Street / Santiam (PPL)

193 Weeks Falls (PSE)

194 White James (PPL)

195 White Water Ranch (IPC)

196 Wilson Lake Hydro (IPC)

197 Wisconsin Creek (NWE)

198 Yakima-Tieton (PPL)

199 IOU - Total NUG: Hydro

$1.5 \quad 1.5$

2.7

6.8

6.8

1.

$0.4 \quad 0.4$

1.3

1.2

1
0

0.1

0.1

0
0.2
0

0.2
0

0

2.6

2.8

7

2.0
0.1

0.3
0

2.3

0.3

0.1

0.2

0.2

$\begin{array}{ll}0.2 & 0.2 \\ 1.1 & 1.1\end{array}$

$1.8 \quad 1.8$

$\begin{array}{rr}0.4 & 0.4 \\ 0 & \end{array}$

$0.4 \quad 0.4$

0.4

0.1

0.4
0.6

0

0.2

0.1

0.5

3.4

0.9

0.1

0

$0.2 \quad 0.2$

$\begin{array}{ll}0.1 & 0.1 \\ 4.3 & 4.3\end{array}$

1.5

0
0.8

0.8
0.1

$\begin{array}{llll}0.1 & 0.1 & 0.1 & 0.1 \\ 0.1 & 0.1 & 0.1 & 0.1\end{array}$

$\begin{array}{llll}0.1 & 0.1 & 0.1 & 0.1 \\ 0.1 & 0.1 & 0.1 & 0.1\end{array}$

$\begin{array}{lll}7.8 & 7.5 & 7.3\end{array}$

$\begin{array}{lll}6.8 & 4.4 & 0.5\end{array}$

$\begin{array}{lll}0.5 & 0.6 & 0.5\end{array}$

$\begin{array}{lll}1.1 & 1.2 & 1.1 \\ 0.4 & 0.4 & 0.4\end{array}$

$\begin{array}{lll}1.8 & 0.9 & 0.6 \\ 1.3 & 0.8 & 1.2\end{array}$

$\begin{array}{rrr}1.3 & 1.2 & 0.7 \\ 0 & 0 & 0\end{array}$

$\begin{array}{lll}1.2 & 0.8 & 0.6\end{array}$

$\begin{array}{rrr}0 & 0 \\ 0.1 & 0.1 & 0.1\end{array}$

$\begin{array}{lll}0.4 & 0.5 & 0.5\end{array}$

0
0.2
0

$\begin{array}{rrr}0 & 0.2 & 0.2 \\ 0 & 0.1 & 0 \\ 0 & 0 & 0 \\ 2.6 & 3.0 & 3.6\end{array}$

$\begin{array}{rrr}2.8 & 2.0 & 0.6\end{array}$

7

$\begin{array}{rr}0 & \\ 6.3 & 8.3 \\ 0 & \end{array}$

$\begin{array}{rrr}0 & 0 & 0 \\ 2.0 & 2.1 & 1.3\end{array}$

$\begin{array}{lll}0.1 & 0.1 & 0.1 \\ 0.3 & 0.3 & 0.3\end{array}$

$\begin{array}{rrr}2.3 & 0 & 0 \\ 0.3 & 2.9 & 6.7\end{array}$

$\begin{array}{ll}0.3 & 0.4\end{array}$

$\begin{array}{lll}0.1 & 0.2 & 0.2\end{array}$

$\begin{array}{lll}0.2 & 0.2 & 0.3 \\ 0.2 & 0.2 & 0.2\end{array}$

$\begin{array}{lll}0.2 & 0.2 & 0.3\end{array}$

$\begin{array}{lll}1.6 & 1.7\end{array}$

$0.3 \quad 0.3$

$\begin{array}{rr}0 & 0 \\ 0.3 & 0.2\end{array}$

$\begin{array}{lll}0.4 & 0.3 & 0.3\end{array}$$$
\begin{array}{lll}
0.4 & 0.3 & 0.3 \\
0.4 & 0.2 & 0.2 \\
0.6 & 0.6 & 0.4
\end{array}
$$

$0.6 \quad 0.6$

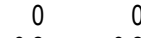

$\begin{array}{lll}0.2 & 0.2 & 0.2 \\ 0.1 & 0.1 & 0\end{array}$

$0.1 \quad 0.1 \quad 0$

$\begin{array}{lll}0.5 & 0.5 & 0.5 \\ 3.4 & 3.4 & 4.7\end{array}$

$\begin{array}{rrr}0.9 & 1 & 0.8 \\ 0.1 & 0.1 & 0.1\end{array}$

$\begin{array}{lll}0.1 & 0.1 & 0.1\end{array}$

$\begin{array}{lll}0.1 & 0.1 & 0.1 \\ 0 & 0 & 0.1\end{array}$

0
1
0

Oct Nov Dec

$\operatorname{Jan}$

Fe

Feb Mar Apr1 Apr16 May Jun Jul Avg

$\begin{array}{llllllllllllll}1.5 & 1.6 & 2 & 2.1 & 1.5 & 1.5 & 1.7 & 1.8 & 1.9 & 1.9 & 1.8 & 1.6 & 1.5 & 1.7\end{array}$

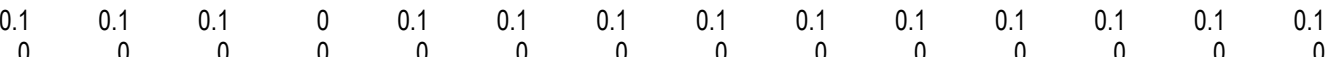

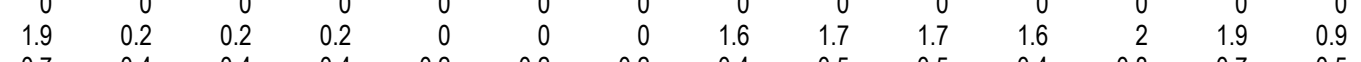

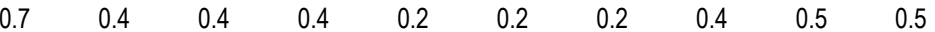

$\begin{array}{llll}2.7 & 2.5 & 1.8 & 1.4\end{array}$

7.5
0.2

0.2
0
0.6

0.5
1.2

0.1

0.1

$\begin{array}{ll}0.9 & 0.9 \\ 1.3 & 1.7\end{array}$

1.3
0.2
0
0.6

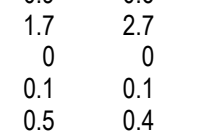

$\begin{array}{rr}0 & 0 \\ 0.1 & 0.1\end{array}$

$\begin{array}{rr}0 & 0 \\ 0 & 0 \\ 0.1 & 0.1\end{array}$

$0.5 \quad 0.5$

$\begin{array}{ll}0.2 & 0.1 \\ 0.4 & 0.6\end{array}$

0.4
0.1

0.1
0.2

$\begin{array}{rrr}0.6 & 0.2 & 0.5 \\ 0 & 0.1 & 0.2 \\ 0 & 0.1 & 0.3\end{array}$

$\begin{array}{rr}0.1 & 0.1 \\ 0 & 0\end{array}$

$\begin{array}{rr}2.9 & 2.9 \\ 0 & 0.2\end{array}$

$\begin{array}{rr}3.2 & 2.9 \\ 0 & 0 \\ 0 & 0\end{array}$

8.7

0
8.0
0

0
7.9
0

$\begin{array}{llll}0.7 & 1.9 & 2.5 & 2.5 \\ 0.7 & 5.3 & 5.5 & 5.5\end{array}$

$$
\begin{aligned}
& 0.4 \\
& 2.7
\end{aligned}
$$

0.8

2.7

8.1
9.1

0.7

2.7
7.8

$\begin{array}{ll}7.8 & 5.3 \\ 9.0 & 3.5\end{array}$

$\begin{array}{ll}7.0 & 3.5\end{array}$

0

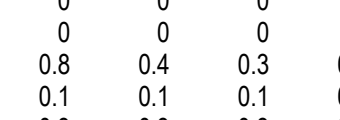

$\begin{array}{lll}0.3 & 0.1 & 0.1 \\ 0 & 0.3 & 0.3\end{array}$

0.3
0
16

$\begin{array}{ll}0.4 & 0.4\end{array}$

$\begin{array}{llll}0.1 & 0.1 & 0.1 & 0.1 \\ 0.2 & 0.2 & 0.2\end{array}$

$\begin{array}{ll}0.2 & 0.2 \\ 0.2 & 0.2\end{array}$

$\begin{array}{ll}0.2 & 0.2 \\ 0.2 & 0.2\end{array}$

$0.2 \quad 0.2$

$\begin{array}{ll}1.2 & 1.3 \\ 1.9 & 1.9\end{array}$

$0.3 \quad 0.1$

$\begin{array}{rr}0 & 0 \\ 0.3 & 0.1\end{array}$

$\begin{array}{ll}0.3 & 0.1 \\ 0.1 & 0.5\end{array}$

0.1

$0.1 \quad 0.1$

$\begin{array}{lll}0.1 & 0.1 \\ 0 & 0\end{array}$

$\begin{array}{rrr}0 & 0 & \\ 0.1 & 0.1 & 0.1\end{array}$

$\begin{array}{rrrr}0 & 0.1 & 0.1 & 0.1 \\ 0.2 & 0.2 & 0.2 & 0.2 \\ 0 & 0 & 0.1 & 0.1\end{array}$

$\begin{array}{llll}0 & 0.2 & 0.4 & 0.4 \\ 0 & 1.1 & 1.2 & 1.2\end{array}$

8.3
0.6

$0.6 \quad 0.4$

0.5

$\begin{array}{ll}0.5 & 0.4 \\ 1.1 & 0.9\end{array}$

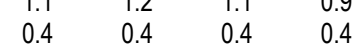

$\begin{array}{llll}0.8 & 1.1 & 0.4 & 0.4 \\ 3.1 & 3.1 & 2.9 & 1.1\end{array}$

$\begin{array}{ll}0.8 & 1.1 \\ 3.1 & 3.1\end{array}$

$\begin{array}{rrrr}0 & 0 & 0.6 & 0.6 \\ 0.1 & 0 & 0.1 & 0.1\end{array}$

1
2.8

2.8
1.2

$\begin{array}{lll}0.2 & 1.1 & 0.9\end{array}$

$\begin{array}{lll}1.2 & 1.1 & 0.9 \\ 2.4 & 2.0 & 2.2\end{array}$

$\begin{array}{lll}1.3 & 1.4 & 0.7\end{array}$

$\begin{array}{rrrrrrrr}0.4 & 0.3 & 0.4 & 0.4 & 1.6 & 2.7 & 1.6 & 0 \\ 0 & 0.3 & 0.8 & 0.8 & 1.2 & 1.3 & 1.1 & 0.6\end{array}$

$\begin{array}{rrrrrrrr}0.4 & 0.3 & 0.4 & 0.4 & 1.6 & 2.7 & 1.6 & 0.9 \\ 0 & 0.3 & 0.8 & 0.8 & 1.2 & 1.3 & 1.1 & 0.6\end{array}$

$\begin{array}{rrrrrrrr}0 & 0 & 0 & 0 & 0 & 0 & 0 & 0 \\ 0.1 & 0.1 & 0.1 & 0.1 & 0.1 & 0.1 & 0.1 & 0.1\end{array}$

$\begin{array}{llllllll}0.4 & 0.3 & 0.3 & 0.3 & 0.3 & 0.3 & 0.4 & 0.4\end{array}$

$\begin{array}{llllllll}0.1 & 0.2 & 0.2 & 0.2 & 0.2 & 0.1 & 0.1 & 0.1 \\ 0.5 & 0.6 & 0.4 & 0.4 & 0 & 0 & 0 & 0.2\end{array}$

$\begin{array}{llllllll}0.5 & 0.6 & 0.4 & 0.4 & 0 & 0 & 0 & 0.2\end{array}$

$\begin{array}{llllllll}0.2 & 0.2 & 0.2 & 0.2 & 0.2 & 0.2 & 0.2 & 0.2 \\ 0.3 & 0.2 & 0.3 & 0.3 & 0.9 & 0.4 & 0.1 & 0.2\end{array}$

$\begin{array}{llllllll}0.3 & 0.2 & 0.3 & 0.3 & 0.9 & 0.4 & 0.1 & 0.2\end{array}$

$\begin{array}{rrrrrrrr}0.1 & 0.1 & 0.1 & 0.1 & 0.1 & 0.1 & 0.1 & 0.1 \\ 0 & 0 & 0 & 0 & 0 & 0 & 0 & 0\end{array}$

$\begin{array}{llllllll}3.5 & 2.6 & 2.7 & 2.7 & 4.5 & 1.6 & 2.2 & 2.9 \\ 0.6 & 1.8 & 3.3 & 3.3 & 3.9 & 3.3 & 3.2 & 1.8\end{array}$

$\begin{array}{rrrrrrrr}0 & 1.8 & 3.3 & 3.3 & 3.9 & 3.3 & 3.2 & 1.8\end{array}$

$\begin{array}{rrrr}10 & 7.7 & 6.8 & 6.8 \\ 0 & 0 & 0 & 0\end{array}$

0

$\begin{array}{ll}0.4 & 0.6 \\ 0.1 & 0.1\end{array}$

$0.3 \quad 0.3$

$\begin{array}{rr}0 & 0 \\ 17 & 16\end{array}$

$\begin{array}{ll}0.4 & 0.3 \\ 0.1 & 0.1\end{array}$

$\begin{array}{ll}0.2 & 0.2 \\ 0.2 & 0.3 \\ 0.2 & 0.2\end{array}$

$\begin{array}{ll}0.2 & 0.2 \\ 0.3 & 0.2 \\ 0.2 & 0.2\end{array}$

$\begin{array}{ll}0.2 & 1.0 \\ 0.6 & 0.4\end{array}$

$\begin{array}{ll}0.2 & 0.2 \\ 1.0 & 1.1 \\ 0.4 & 1.6\end{array}$

-NUG: Small Thermal \& Misc.-

200 Marion Solid Waste (PGE)

\begin{tabular}{|c|c|c|c|c|c|c|c|c|c|c|c|c|c|}
\hline 7.1 & 7.1 & 5.3 & 2.0 & 0.2 & 0 & 0 & 0 & 0 & 2.3 & 2.3 & 6.1 & 6.8 & 7.6 \\
\hline 0 & 0 & 0 & 0.1 & 0 & 0 & 0.1 & 0 & 0 & 0 & 0 & 0 & 0 & 0 \\
\hline 3.0 & 3.0 & 2.5 & 0.4 & 0 & 0 & 0 & 0 & 0 & 0 & 0 & 1.2 & 1.7 & 1.7 \\
\hline 157 & 156 & 133 & 111 & 119 & 110 & 101 & 109 & 130 & 173 & 174 & 212 & 222 & 196 \\
\hline
\end{tabular}

$\begin{array}{rrr}0 & 0 & 0 \\ 6.7 & 13 & 14 \\ 0.4 & 0 & 0.3\end{array}$

$\begin{array}{ll}0.4 & 1.6 \\ 0.1 & 0.3\end{array}$

$\begin{array}{rr}0.1 & 0.3 \\ 0 & 0 \\ 0.2 & 0.3\end{array}$

$\begin{array}{ll}0.5 & 0.9 \\ 0.1 & 0.1 \\ 0 & 0.1\end{array}$

$\begin{array}{rr}0 & 0.1 \\ 0 & 0\end{array}$

$\begin{array}{ll}0.2 & 0.1 \\ 0.1 & 0.1\end{array}$

0.4
0

$\begin{array}{rr}0 & 0 \\ 0.1 & 0 \\ 0.2 & 0.3\end{array}$

$\begin{array}{rrr}0.2 & 0.3 & 0 \\ 0.2 & 0.3 & 0.1\end{array}$

$\begin{array}{rr}0.1 & 0.1 \\ 0 & 0.3 \\ 13 & 14 \\ 0.3 & 0.4\end{array}$

$\begin{array}{rr}13 & 14 \\ 0.3 & 0.4 \\ 0 & 0\end{array}$

\begin{tabular}{|c|c|c|c|c|}
\hline 0.1 & $\begin{array}{l}0.1 \\
0.1\end{array}$ & 01 & 0 & 0 \\
\hline 0 & 0.1 & 0.2 & 0.3 & 0.3 \\
\hline 0.1 & 0.2 & 0.2 & 0.2 & 0.1 \\
\hline 0.1 & 0.1 & 0.1 & 0.1 & 0.1 \\
\hline
\end{tabular}

$\begin{array}{rr}0 & 0.1 \\ 0.4 & 0.3 \\ 0.2 & 0.2\end{array}$

$\begin{array}{ll}0.2 & 0.2 \\ 0.1 & 0.1 \\ 0 & 2.4\end{array}$

$\begin{array}{rrrrrrrrrrr}0 & 0 & 0 & 0 & 2.4 & 5.1 & 5.1 & 5.8 & 5.8 & 5.9 & 2.7 \\ 9.6 & 7.9 & 7.4 & 4.8 & 7.8 & 15 & 15 & 13 & 24 & 19 & 9.6 \\ 1.2 & 1.3 & 1.2 & 1.5 & 1.8 & 1.8 & 1.8 & 1.5 & 1.5 & 1.8 & 1.5 \\ 0.2 & 0.2 & 0.1 & 0.1 & 0.1 & 0.2 & 0.2 & 0.1 & 0.1 & 0.1 & 0.1 \\ 1.7 & 1.8 & 1.2 & 0.6 & 1.2 & 2.6 & 2.6 & 2.4 & 4.4 & 3.5 & 1.7 \\ 0.1 & 0.1 & 0.1 & 0.2 & 0.2 & 0.2 & 0.2 & 0.2 & 0.3 & 0.2 & 0.2\end{array}$

$\begin{array}{rrrrrrrrrrr}0.2 & 0.2 & 0.1 & 0.1 & 0.1 & 0.2 & 0.2 & 0.1 & 0.1 & 0.1 & 0.1 \\ 1.7 & 1.8 & 1.2 & 0.6 & 1.2 & 2.6 & 2.6 & 2.4 & 4.4 & 3.5 & 1.7 \\ 0.1 & 0.1 & 0.1 & 0.2 & 0.2 & 0.2 & 0.2 & 0.2 & 0.3 & 0.2 & 0.2 \\ 0.1 & 0.1 & 0.1 & 0.1 & 0.1 & 0.1 & 0.1 & 0.1 & 0.1 & 0.1 & 0.1 \\ 0.2 & 0 & 0 & 0 & 0 & 2.3 & 2.3 & 6.1 & 6.8 & 7.6 & 3.1\end{array}$

$157 \quad 156$

$\begin{array}{rrrrrr}133 & 111 & 119 & 110 & 101 & 109\end{array}$ 
Table A-24: Regional Non Utility Generating Resources By Project

PNW Loads and Resources Study

2004 - 2005 Operating Year 2003 White Book

Average Energy in Megawatts

Aug1 Aug16 Sep

201 Minnesota Methane (AVWP)

202 Montana One (NWE)

203 Pocatello Waste (IPC)

204 Spokane MSW (PSE)

205 West Boise Waste (IPC)

206 IOU - Total NUG: Small Thermal \& Misc.

-NUG: Combustion Turbines -

207 March Point \#1 Cogeneration (PSE)

208 March Point \#2 Cogeneration (PSE)

209 Rathdrum- Boekel Rd. \#1 (AVWP)

210 Rathdrum -Boekel Rd. \#2 (AVWP)

211 Tenaska Gas Cogen (PSE)

212 IOU - Total NUG: Combustion Turbines

-NUG: Co-Generation-

213 Billings Generation (NWE)

214 Biomass One (PPL)

215 Boise Cascade Medford (PPL)

216 Champion (PPL)

217 DR Johnson - Co-Gen II (PPL)

218 Magic Valley (IPC)

219 Magic West (IPC)

220 PERC Pierce, Wa (PSE)

221 Simplot Pocatello (IPC)

222 Sumas Energy (PSE)

223 Tamarack (IPC)

224 TASCO Nampa ID (IPC)

225 TASCO Twin Falls ID (IPC)

226 Vaagen Bros. (IPC)

227 Warm Springs (PPL)

228 IOU - Total NUG: Co-Generation

\begin{tabular}{rrrrrrrrrrrrrrr}
0.8 & 0.8 & 0.8 & 0.8 & 0.8 & 0.8 & 0.8 & 0.8 & 0.8 & 0.8 & 0.8 & 0.8 & 0.8 & 0.8 & 0.8 \\
9.1 & 9.3 & 8.6 & 8.7 & 6.6 & 7.3 & 7.6 & 7.2 & 6.3 & 6.1 & 6.0 & 8.5 & 8.5 & 8.3 & 7.7 \\
0.1 & 0.1 & 0.2 & 0.1 & 0.2 & 0.1 & 0.1 & 0.2 & 0.1 & 0.2 & 0.2 & 0.1 & 0.2 & 0.1 & 0.1 \\
15 & 15 & 15 & 15 & 15 & 15 & 15 & 15 & 15 & 15 & 15 & 15 & 15 & 15 & 15 \\
0.1 & 0.1 & 0.1 & 0.1 & 0.1 & 0.1 & 0.1 & 0.1 & 0.1 & 0.1 & 0.1 & 0.1 & 0.1 & 0.1 & 0.1 \\
\hline $\mathbf{3 5}$ & $\mathbf{3 5}$ & $\mathbf{3 4}$ & $\mathbf{3 4}$ & $\mathbf{3 2}$ & $\mathbf{3 3}$ & $\mathbf{3 3}$ & $\mathbf{3 3}$ & $\mathbf{3 2}$ & $\mathbf{3 2}$ & $\mathbf{3 2}$ & $\mathbf{3 4}$ & $\mathbf{3 4}$ & $\mathbf{3 4}$ & $\mathbf{3 3}$
\end{tabular}

-NUG: Renewables-

229 Foote Creek 1 (PPL)

230 Healow \#2 (NWE)

231 Josef Staufer (NWE)

232 Lewandowski Farms (IPC)

233 Livingston (NWE)

234 Puyallup Energy Recovery (PSE)

235 Stateline Wind Project (AVWP)

236 Windmill (PGE)

237 IOU - Total NUG: Renewables

\begin{tabular}{|c|c|c|c|c|c|c|c|c|c|c|c|c|c|}
\hline 79 & 79 & 79 & 79 & 79 & 79 & 79 & 79 & 79 & 79 & 55 & 79 & 79 & 77 \\
\hline 59 & 59 & 59 & 59 & 59 & 59 & 59 & 59 & 59 & 59 & 45 & 59 & 59 & 58 \\
\hline 65 & 65 & 65 & 65 & 65 & 65 & 65 & 65 & 65 & 65 & 32 & 65 & 65 & 62 \\
\hline 65 & 65 & 65 & 65 & 65 & 65 & 65 & 65 & 65 & 65 & 32 & 65 & 65 & 62 \\
\hline 503 & 503 & 503 & 503 & 503 & 503 & 503 & 503 & 503 & 503 & 164 & 503 & 503 & 475 \\
\hline
\end{tabular}

Other Entities

-NUG: Co-Generation-

238 SP Newsprint cogen (SPN)

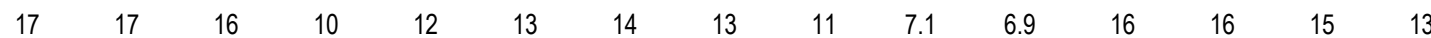

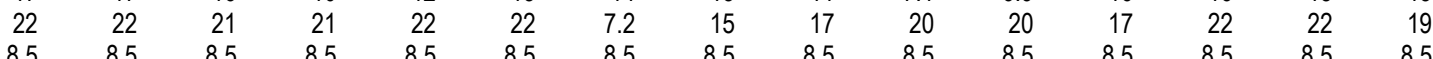

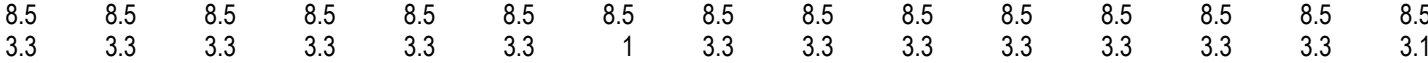

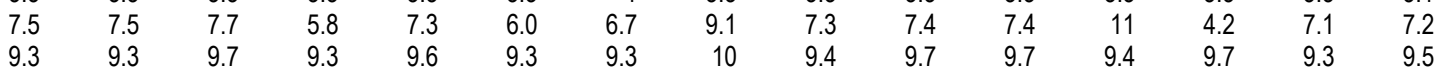

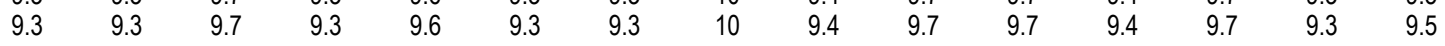

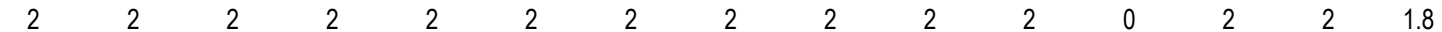

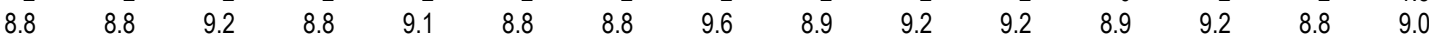

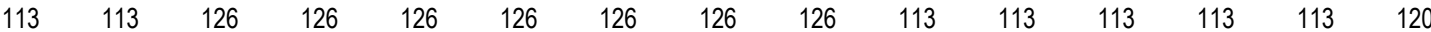

$\begin{array}{lllllllllllllll}3.8 & 3.8 & 4.6 & 4.7 & 4.2 & 3.2 & 3.6 & 3.8 & 4.2 & 4.4 & 4.4 & 3.4 & 4.6 & 4.8 & 4.1\end{array}$

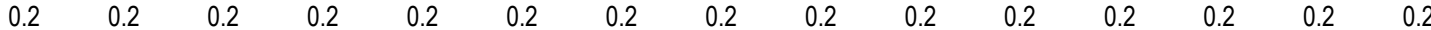

239 Other - Total NUG: Co-Generation

$\begin{array}{rrrrrrrrrrrrrrr}3 & 3 & 3.7 & 3.8 & 3.3 & 2.6 & 29 & 3.1 & 3.4 & 3.6 & 3.6 & 27 & 3.7 & 3.8 & 3.3\end{array}$

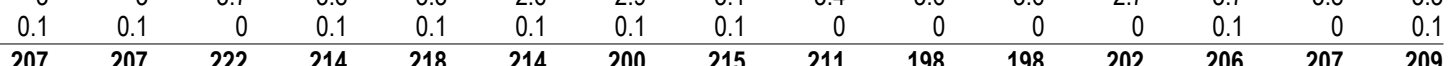

-NUG: Renewables-

240 Stateline Wind Project (PPME)

241 Vansycle Wind (FPLEV)

242 Other - Total NUG: Renewables

$\begin{array}{rrrrrrrrrrrrrrr}4.2 & 4.2 & 4.9 & 7.2 & 11 & 13 & 14 & 12 & 9.2 & 7.4 & 7.4 & 5.9 & 5.2 & 4.2 & 8.1 \\ 0 & 0 & 0 & 0 & 0 & 0 & 0 & 0 & 0 & 0 & 0 & 0 & 0 & 0 & 0 \\ 0 & 0 & 0 & 0 & 0 & 0 & 0 & 0 & 0 & 0 & 0 & 0 & 0 & 0 & 0 \\ 0 & 0 & 0 & 0 & 0 & 0 & 0 & 0 & 0 & 0 & 0 & 0 & 0 & 0 & 0 \\ 0 & 0 & 0 & 0 & 0 & 0 & 0 & 0 & 0 & 0 & 0 & 0 & 0 & 0 & 0 \\ 2 & 2 & 2 & 2 & 2 & 2 & 2 & 2 & 2 & 2 & 2 & 2 & 2 & 2 & 2 \\ 0.3 & 0.3 & 0.2 & 0.3 & 0.3 & 0.3 & 0.3 & 0.3 & 0.3 & 0.3 & 0.3 & 0.3 & 0.3 & 0.3 & 0.3 \\ 0 & 0 & 0 & 0 & 0 & 0 & 0 & 0 & 0 & 0 & 0 & 0 & 0 & 0 & 0 \\ \mathbf{6 . 5} & \mathbf{6 . 5} & \mathbf{7 . 1} & \mathbf{9 . 5} & \mathbf{1 3} & \mathbf{1 5} & \mathbf{1 6} & \mathbf{1 5} & \mathbf{1 2} & \mathbf{9 . 8} & \mathbf{9 . 8} & \mathbf{8 . 2} & \mathbf{7 . 6} & \mathbf{6 . 5} & \mathbf{1 0}\end{array}$

-Total Non-Utility Generating Resources-

243 Federal Entities

244 Generating Public Entities

245 Non Generating Public Entities

246 Investor-Owned Entities

$\begin{array}{llllllllllllll}25 & 25 & 25 & 25 & 25 & 25 & 25 & 25 & 25 & 25 & 25 & 25 & 25 & 25 \\ 25 & \mathbf{2 5} & \mathbf{2 5} & \mathbf{2 5} & \mathbf{2 5} & \mathbf{2 5} & \mathbf{2 5} & \mathbf{2 5} & \mathbf{2 5} & \mathbf{2 5} & \mathbf{2 5} & \mathbf{2 5} & \mathbf{2 5} & \mathbf{2 5}\end{array}$

247 Other Entities

248 Total Non-Utility Generation

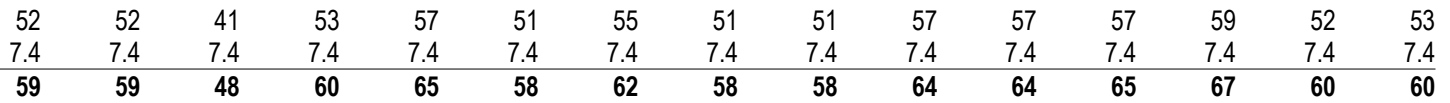


Oct Nov

Dec

Jan Feb Mar Apr1 Apr16

May Jun Jul Avg

Federal Entities

-NUG: Hydro-

1 Clearwater - State of ID DWR (BPA)

2 Dworshak Small Hydropower (BPA)

3 Elwah Hydro (BPA)

4 Glines Hydro (BPA)

5 Federal -Total NUG: Hydro

\begin{tabular}{|c|c|c|c|c|c|c|c|c|c|c|c|c|c|c|}
\hline 1 & 1 & 1 & 1 & 1 & 1 & 1 & 1 & 1 & 1 & 1 & 1 & 1 & 1 & 1 \\
\hline 2.6 & 2.6 & 2.6 & 2.6 & 2.6 & 2.6 & 2.6 & 2.6 & 2.6 & 2.6 & 2.6 & 2.6 & 2.6 & 2.6 & 2.6 \\
\hline 8.2 & 7.7 & 2.3 & 3.6 & 9.5 & 11 & 12 & 9.1 & 8.8 & 17 & 17 & 9.0 & 8.5 & 5.4 & 8.6 \\
\hline 16 & 15 & 4.1 & 6.7 & 14 & 16 & 15 & 14 & 14 & 25 & 25 & 30 & 16 & 11 & 15 \\
\hline 28 & 26 & 10.0 & 14 & 27 & 31 & 30 & 27 & 26 & 46 & 46 & 42 & 28 & 20 & 27 \\
\hline 0 & 0 & 0 & 0 & 0 & 0 & 0 & 0 & 0 & 0 & 0 & 0 & 0 & 0 & 0 \\
\hline 10 & 10 & 10 & 11 & 13 & 14 & 13 & 12 & 12 & 14 & 14 & 12 & 15 & 11 & 12 \\
\hline 3.3 & 3.3 & 3.8 & 5.6 & 8.3 & 10 & 11 & 9.6 & 7.2 & 5.8 & 5.8 & 4.6 & 4.1 & 3.3 & 6.4 \\
\hline 0.4 & 0.4 & 0.5 & 0.7 & 1 & 1.3 & 1.3 & 1.2 & 0.9 & 0.7 & 0.7 & 0.5 & 0.5 & 0.4 & 0.8 \\
\hline 3.9 & 3.9 & 4.4 & 6.5 & 9.7 & 12 & 12 & 11 & 8.4 & 6.7 & 6.7 & 5.4 & 4.8 & 3.8 & 7.4 \\
\hline 7.9 & 7.9 & 6.2 & 8.1 & 8.8 & 7.8 & 8.3 & 7.8 & 7.8 & 8.6 & 8.6 & 8.8 & 9.1 & 8 & 8.1 \\
\hline 29 & 29 & 23 & 30 & 33 & 29 & 31 & 29 & 29 & 32 & 32 & 33 & 34 & 30 & 30 \\
\hline 55 & 55 & 48 & 62 & 74 & 74 & 76 & 71 & 65 & 68 & 68 & 64 & 67 & 56 & 65 \\
\hline
\end{tabular}

-NUG: Renewables-

6 Ashland Solar Project (BPA)

7 Condon Wind Project (BPA)

8 Foote Creek 1 (BPA)

9 Foote Creek 2 (BPA)

10 Foote Creek 4 (BPA)

11 Klondike Phase 1 (BPA)

12 Stateline Wind Project (BPA)

\begin{tabular}{|c|c|c|c|c|c|c|c|c|c|c|c|c|c|c|}
\hline 1 & 1 & 1 & 1 & 1 & 1 & 1 & 1 & 1 & 1 & 1 & 1 & 1 & 1 & 1 \\
\hline 2.6 & 2.6 & 2.6 & 2.6 & 2.6 & 2.6 & 2.6 & 2.6 & 2.6 & 2.6 & 2.6 & 2.6 & 2.6 & 2.6 & 2.6 \\
\hline 8.2 & 7.7 & 2.3 & 3.6 & 9.5 & 11 & 12 & 9.1 & 8.8 & 17 & 17 & 9.0 & 8.5 & 5.4 & 8.6 \\
\hline 16 & 15 & 4.1 & 6.7 & 14 & 16 & 15 & 14 & 14 & 25 & 25 & 30 & 16 & 11 & 15 \\
\hline 28 & 26 & 10.0 & 14 & 27 & 31 & 30 & 27 & 26 & 46 & 46 & 42 & 28 & 20 & 27 \\
\hline 0 & 0 & 0 & 0 & 0 & 0 & 0 & 0 & 0 & 0 & 0 & 0 & 0 & 0 & 0 \\
\hline 10 & 10 & 10 & 11 & 13 & 14 & 13 & 12 & 12 & 14 & 14 & 12 & 15 & 11 & 12 \\
\hline 3.3 & 3.3 & 3.8 & 5.6 & 8.3 & 10 & 11 & 9.6 & 7.2 & 5.8 & 5.8 & 4.6 & 4.1 & 3.3 & 6.4 \\
\hline 0.4 & 0.4 & 0.5 & 0.7 & 1 & 1.3 & 1.3 & 1.2 & 0.9 & 0.7 & 0.7 & 0.5 & 0.5 & 0.4 & 0.8 \\
\hline 3.9 & 3.9 & 4.4 & 6.5 & 9.7 & 12 & 12 & 11 & 8.4 & 6.7 & 6.7 & 5.4 & 4.8 & 3.8 & 7.4 \\
\hline 7.9 & 7.9 & 6.2 & 8.1 & 8.8 & 7.8 & 8.3 & 7.8 & 7.8 & 8.6 & 8.6 & 8.8 & 9.1 & 8 & 8.1 \\
\hline 29 & 29 & 23 & 30 & 33 & 29 & 31 & 29 & 29 & 32 & 32 & 33 & 34 & 30 & 30 \\
\hline 55 & 55 & 48 & 62 & 74 & 74 & 76 & 71 & 65 & 68 & 68 & 64 & 67 & 56 & 65 \\
\hline
\end{tabular}

Generating Public Entities

-NUG: Hydro-

14 Don Steffen (SHPD)

15 Eltopia Br Canal 4.6 (SCL)

16 Eltopia Br Canal 4.6 (TPU)

17 John Llewellyn (SHPD)

18 Kevin Duncan (SHPD)

19 Lucky Peak (SCL)

20 Main Canal Headworks (SCL)

21 Main Canal Headworks (TPU)

22 PEC Headworks (GCPD)

23 Pickering Micro Hydro (CKPD)

24 Potholes E Canal 66.0 (SCL)

25 Potholes E Canal 66.0 (TPU)

26 Quincy Chute (GCPD)

27 Russel D. Smith (SCL)

28 Russel D. Smith (TPU)

29 Smith Creek (EWEB)

30 Summer Falls (SCL)

31 Summer Falls (TPU)

32 Woods Creek (SHPD)

33 GPU - Total NUG: Hydro

-NUG: Small Thermal \& Misc.-

34 Grays Harbor Paper (GHPD)

35 Weyerhaeuser Pulp Mill (GHPD)

36 GPU - Total NUG: Small Thermal \& Misc.

$\begin{array}{rrrr}0 & 0 & 0 & \\ 0.9 & 0.9 & 0.6 & 0.3 \\ 0.9 & 0.9 & 0.6 & 0.3 \\ 0 & 0 & 0 & \\ 0 & 0 & 0 & \\ 66 & 66 & 41 & 11 \\ 10 & 10 & 6.1 & 2 . \\ 10 & 10 & 6.1 & 2 . \\ 4.5 & 4.5 & 3.1 & 2.2 \\ 0 & 0 & 0 & \\ 0.9 & 0.9 & 0.9 & 0.8 \\ 0.9 & 0.9 & 0.9 & 0.8 \\ 7.7 & 7.7 & 4 & 2.3 \\ 2 & 2 & 1.3 & 0.7 \\ 2 & 2 & 1.3 & 0.7 \\ 0 & 0 & 0 & \\ 39 & 39 & 24 & 11 \\ 39 & 39 & 24 & 11 \\ 0.1 & 0.1 & 0.1 & 0.1 \\ 185 & 185 & 115 & \mathbf{5}\end{array}$

$\begin{array}{rr}0 & \\ 0.3 & \\ 0.3 & \\ 0 & \\ 0 & \\ 11 & \\ 2.6 & \\ 2.6 & \\ 2.2 & \\ 0 & \\ 0.8 & \\ 0.8 & \\ 2.3 & \\ 0.7 & \\ 0.7 & \\ 5 & \\ 11 & \\ 11 & \\ 0.1 & 0 \\ \mathbf{5 1} & \\ & \end{array}$

$\begin{array}{rrr}0 & 0 & 0 \\ 0 & 0 & 0 \\ 0 & 0 & 0 \\ 0 & 0 & 0 \\ 0 & 0 & 0 \\ 2.2 & 3 & 7.9 \\ 0 & 0 & 0 \\ 0 & 0 & 0 \\ 0 & 0 & 0 \\ 0 & 0 & 0 \\ 0 & 0 & 0 \\ 0 & 0 & 0 \\ 0 & 0 & 0 \\ 0 & 0 & 0 \\ 0 & 0 & 0 \\ 5.4 & 3.9 & 0 \\ 0 & 0 & 0 \\ 0 & 0 & 0 \\ 0.1 & 0.1 & 0.1 \\ 7.7 & 7 & 8\end{array}$

$\begin{array}{rrr}0 & 0 & \\ 0 & 0 & \\ 0 & 0 & \\ 0 & 0 & \\ 0 & 0 & 0 \\ 7.9 & 10 & 2 \\ 0 & 0 & 1.4 \\ 0 & 0 & 1.4 \\ 0 & 0 & 0.4 \\ 0 & 0 & 0 \\ 0 & 0 & 0.3 \\ 0 & 0 & 0.3 \\ 0 & 0 & 0.8 \\ 0 & 0 & 0.1 \\ 0 & 0 & 0.1 \\ 0 & 0.8 & 1.5 \\ 0 & 0 & 2.4 \\ 0 & 0 & 2.4 \\ 0.1 & 0.1 & 0.1 \\ 8 & 11 & 32\end{array}$

\begin{tabular}{rr}
0 & \\
0 & 0.7 \\
0 & 0.7 \\
0 & \\
0 & \\
21 & 0 \\
1.4 & 6.8 \\
1.4 & 6.8 \\
0.4 & 5.1 \\
0 & \\
0.3 & 0.9 \\
0.3 & 0.9 \\
0.8 & 5.5 \\
0.1 & 1.2 \\
0.1 & 1.2 \\
1.5 & 9.2 \\
2.4 & 25 \\
2.4 & 25 \\
0.1 & 0.1 \\
\hline 32 & 141 \\
\hline
\end{tabular}

$\begin{array}{rr}0 & 0 \\ 0.7 & 0.7 \\ 0.7 & 0.7 \\ 0 & 0 \\ 0 & 0 \\ 52 & 52 \\ 6.8 & 6.8 \\ 6.8 & 6.8 \\ 5.1 & 5.1 \\ 0 & \\ 0.9 & 0.9 \\ 0.9 & 0.9 \\ 5.5 & 5.5 \\ 1.2 & 1.2 \\ 1.2 & 1.2 \\ 9.2 & 9.2 \\ 25 & 25 \\ 25 & 25 \\ 0.1 & 0.1 \\ 141 & 14\end{array}$

$\begin{array}{rrrrr}0 & 0 & 0 & 0 & 0 \\ 0.7 & 1 & 1 & 1.1 & 0.5 \\ 0.7 & 1 & 1 & 1.1 & 0.5 \\ 0 & 0 & 0 & 0 & 0 \\ 0 & 0 & 0 & 0 & 0 \\ 52 & 84 & 85 & 77 & 38 \\ 6.8 & 9.1 & 11 & 12 & 4.9 \\ 6.8 & 9.1 & 11 & 12 & 4.9 \\ 5.1 & 6.9 & 6.2 & 5.8 & 2.9 \\ 0 & 0 & 0 & 0 & 0 \\ 0.9 & 0.9 & 0.9 & 0.9 & 0.5 \\ 0.9 & 0.9 & 0.9 & 0.9 & 0.5 \\ 5.5 & 7.1 & 8.4 & 8.5 & 3.7 \\ 1.2 & 1.9 & 2.1 & 1.7 & 0.9 \\ 1.2 & 1.9 & 2.1 & 1.7 & 0.9 \\ 9.2 & 37 & 37 & 11 & 9.2 \\ 25 & 36 & 40 & 44 & 18 \\ 25 & 36 & 40 & 44 & 18 \\ 0.1 & 0.1 & 0.1 & 0.1 & 0.1 \\ 141 & \mathbf{2 3 2} & \mathbf{2 4 7} & \mathbf{2 2 1} & 105\end{array}$

-NUG: Co-Generation-

37 Metro Westpoint (SCL)

38 Scott Paper (SHPD)

39 GPU - Total NUG: Co-Generation

\begin{tabular}{|c|c|c|c|c|c|c|c|c|c|c|c|c|c|c|}
\hline 3.8 & 3.8 & 3.8 & 3.8 & 3.8 & 3.8 & 3.8 & 3.8 & 3.8 & 3.8 & 3.8 & 3.8 & 3.8 & 3.8 & 3.8 \\
\hline 11 & 11 & 11 & 11 & 11 & 11 & 11 & 11 & 11 & 11 & 11 & 11 & 11 & 11 & 11 \\
\hline 15 & 15 & 15 & 15 & 15 & 15 & 15 & 15 & 15 & 15 & 15 & 15 & 15 & 15 & 15 \\
\hline
\end{tabular}

-NUG: Renewables-

40 Foote Creek 1 (EWEB)

41 Nine Canyon Wind Project (CHPD)

42 Nine Canyon Wind Project (COPD)

43 Nine Canyon Wind Project (DOPD)

44 Nine Canyon Wind Project (GCPD)

45 Nine Canyon Wind Project (GHPD)

46 Nine Canyon Wind Project (OKPD)

47 Stateline Wind Project (EWEB)

48 Stateline Wind Project (SCL)

49 GPU - Total NUG: Renewables

\begin{tabular}{|c|c|c|c|c|c|c|c|c|c|c|c|c|c|}
\hline 1.2 & 1.2 & 1.2 & 1.2 & 1.2 & 1.2 & 1.2 & 1.2 & 1.2 & 1.2 & 1.2 & 1.2 & 1.2 & 1.2 \\
\hline 38 & 38 & 27 & 27 & 27 & 27 & 27 & 27 & 38 & 38 & 38 & 38 & 38 & 33 \\
\hline 39 & 39 & 28 & 28 & 28 & 28 & 28 & 28 & 39 & 39 & 39 & 39 & 39 & 34 \\
\hline
\end{tabular}

Non-Generating Public Entities

-NUG: Hydro-

50 Buffalo Hydro (FREC)

51 Burr \& Bouchard (TNEC)

52 City of Cove (OTEC)

53 Falls Creek (CCPD)

54 Lilliwaup Falls Generating Co. (MCPD1)

55 Thomas Burnside (TNEC)

$\begin{array}{rrrrrrrrrrrrrrr}1.4 & 1.4 & 1.6 & 2.4 & 3.5 & 4.3 & 4.5 & 4.1 & 3.1 & 2.5 & 2.5 & 2.0 & 1.7 & 1.4 & 2.7 \\ 1.5 & 1.5 & 1.4 & 2.2 & 3.8 & 3.8 & 4.2 & 3.4 & 2.3 & 2.0 & 2.0 & 2.0 & 1.9 & 1.4 & 2.5 \\ 0.4 & 0.4 & 0.4 & 0.5 & 0.9 & 0.9 & 1 & 0.9 & 0.6 & 0.5 & 0.5 & 0.5 & 0.5 & 0.3 & 0.6 \\ 1.8 & 1.8 & 1.7 & 2.6 & 4.6 & 4.7 & 5.1 & 4.2 & 2.8 & 2.4 & 2.4 & 2.4 & 2.3 & 1.7 & 3 \\ 2.2 & 2.2 & 2.1 & 3.2 & 5.7 & 5.7 & 6.3 & 5.2 & 3.4 & 3.0 & 3.0 & 3.0 & 2.8 & 2.1 & 3.7 \\ 1.5 & 1.5 & 1.4 & 2.2 & 3.8 & 3.8 & 4.2 & 3.4 & 2.3 & 2.0 & 2.0 & 2.0 & 1.9 & 1.4 & 2.5 \\ 3.0 & 3.0 & 2.8 & 4.3 & 7.5 & 7.6 & 8.4 & 6.9 & 4.6 & 3.9 & 3.9 & 3.9 & 3.7 & 2.7 & 4.9 \\ 8.4 & 8.4 & 6.6 & 8.6 & 9.3 & 8.2 & 8.8 & 8.3 & 8.3 & 9.2 & 9.2 & 9.3 & 9.6 & 8.5 & 8.6 \\ 49 & 49 & 39 & 50 & 54 & 48 & 52 & 48 & 48 & 54 & 54 & 54 & 56 & 50 & 50 \\ \mathbf{6 9} & \mathbf{6 9} & \mathbf{5 7} & \mathbf{7 6} & \mathbf{9 3} & \mathbf{8 7} & \mathbf{9 4} & \mathbf{8 5} & \mathbf{7 5} & \mathbf{7 9} & \mathbf{7 9} & \mathbf{7 9} & \mathbf{8 1} & \mathbf{6 9} & \mathbf{7 9}\end{array}$

-NUG: Small Thermal \& Misc.-

57 Methane Energy Agricultural D. (TKPD)

\begin{tabular}{|c|c|c|c|c|c|c|c|c|c|c|c|c|c|c|}
\hline 0.2 & 0.2 & 0.2 & 0.2 & 0.2 & 0.2 & 0.2 & 0.2 & 0.2 & 0.2 & 0.2 & 0.2 & 0.2 & 0.2 & 0.2 \\
\hline 0 & 0 & 0 & 0 & 0 & 0 & 0 & 0 & 0 & 0 & 0 & 0 & 0 & 0 & 0 \\
\hline 0.3 & 0.3 & 0.3 & 0.3 & 0.3 & 0.3 & 0.3 & 0.3 & 0.3 & 0.3 & 0.3 & 0.3 & 0.3 & 0.3 & 0.3 \\
\hline 0 & 0 & 0 & 0 & 0 & 0 & 0 & 0 & 0 & 0 & 0 & 0 & 0 & 0 & 0 \\
\hline 0.6 & 0.6 & 0.6 & 0.6 & 0.6 & 0.6 & 0.6 & 0.6 & 0.6 & 0.6 & 0.6 & 0.6 & 0.6 & 0.6 & 0.6 \\
\hline 0 & 0 & 0 & 0 & 0 & 0 & 0 & 0 & 0 & 0 & 0 & 0 & 0 & 0 & 0 \\
\hline 1.2 & 1.2 & 1.2 & 1.2 & 1.2 & 1.2 & 1.2 & 1.2 & 1.2 & 1.2 & 1.2 & 1.2 & 1.2 & 1.2 & 1.2 \\
\hline
\end{tabular}




\begin{tabular}{|c|c|c|c|c|c|c|c|c|c|c|c|c|c|c|}
\hline 5.6 & 5.6 & 5.6 & 5.6 & 5.6 & 5.6 & 0 & 0 & 0 & 0 & 0 & 0 & 0 & 0 & 2.3 \\
\hline 5.6 & 5.6 & 5.6 & 5.6 & 5.6 & 5.6 & 0 & 0 & 0 & 0 & 0 & 0 & 0 & 0 & 2.3 \\
\hline
\end{tabular}

-NUG: Renewables-

61 Ashland Solar Project (ASHL) 62 Nine Canyon Wind Project (BCPD)

63 Nine Canyon Wind Project (ENW)

64 Nine Canyon Wind Project (LCPD)

65 Nine Canyon Wind Project (MCPD3)

66 White Bluffs Solar (ENW)

67 NGP - Total NUG: Renewables

\begin{tabular}{rrrrrrrrrrrrrrr}
0 & 0 & 0 & 0 & 0 & 0 & 0 & 0 & 0 & 0 & 0 & 0 & 0 & 0 & 0 \\
0.6 & 0.6 & 0.5 & 0.8 & 1.4 & 1.4 & 1.6 & 1.3 & 0.9 & 0.7 & 0.7 & 0.7 & 0.7 & 0.5 & 0.9 \\
0.4 & 0.4 & 0.4 & 0.5 & 0.9 & 1.0 & 1.1 & 0.9 & 0.6 & 0.5 & 0.5 & 0.5 & 0.5 & 0.3 & 0.6 \\
0.2 & 0.2 & 0.2 & 0.3 & 0.5 & 0.5 & 0.5 & 0.4 & 0.3 & 0.2 & 0.2 & 0.2 & 0.2 & 0.2 & 0.3 \\
0.4 & 0.4 & 0.4 & 0.5 & 0.9 & 1.0 & 1 & 0.9 & 0.6 & 0.5 & 0.5 & 0.5 & 0.5 & 0.3 & 0.6 \\
0 & 0 & 0 & 0 & 0 & 0 & 0 & 0 & 0 & 0 & 0 & 0 & 0 & 0 & 0 \\
\hline $\mathbf{1 . 5}$ & $\mathbf{1 . 5}$ & $\mathbf{1 . 4}$ & $\mathbf{2 . 2}$ & $\mathbf{3 . 8}$ & $\mathbf{3 . 8}$ & $\mathbf{4 . 2}$ & $\mathbf{3 . 5}$ & $\mathbf{2 . 3}$ & $\mathbf{2 . 0}$ & $\mathbf{2 . 0}$ & $\mathbf{2 . 0}$ & $\mathbf{2 . 0}$
\end{tabular}

Investor-Owned Entities

-NUG: Hydro-

68 Barber Dam (IPC)

69 Barney Creek (NWE)

70 Beaverton (PGE)

71 Bell Mountain (UPL)

72 Bingham Engineering (NWE)

73 Birch Creek (IPC)

74 Birch Creek (UPL)

75 Black Canyon \#3 (IPC)

76 Black Creek (AVWP)

77 Blind Canyon Hydro (IPC)

78 Box Canyon (IPC)

79 Boyd James (PPL)

80 Briggs Creek (IPC)

81 Broadwater Dam (NWE)

82 Bypass (IPC)

83 Canyon Springs (IPC)

84 Carlton (PGE)

85 Cascade Creek (NWE)

86 CDM Hydro - Bonneville Pacific (PPL)

87 Cedar Draw (IPC)

88 Central OR Irrigation District (PPL)

89 Clear Springs Trout (IPC)

90 Corbett (PGE)

91 Cornelius (PGE)

92 Crystal Springs (IPC)

93 Curry Cattle Co. (IPC)

94 Dairy Creek (PGE)

95 Deep Creek (AVWP)

96 Derr Creek (AVWP)

97 Dietrich Drop (IPC)

98 Donald Jenni (NWE)

99 Eagle Creek (PGE)

100 Eagle Point Irrigation Dist. (PPL)

101 Elk Creek (IPC)

102 Estacada (PGE)

103 Falls Creek (PPL)

104 Falls River (IPC)

105 Farmers Irrig. (PPL)

106 Faulkner Ranch (IPC)

107 Fisheries Development Co (IPC)

108 Galesville Dam (PPL)

109 Geobon 2 (IPC)

110 Georgetown Power (UPL)

111 Hailey Cspp (IPC)

112 Hazelton A (IPC)

113 Hazelton B (IPC)

114 Horseshoe Bend (IPC)

115 Hutchinson Creek (PSE)

116 Ingram Warm Springs (UPL)

$117 \mathrm{Jim}$ Ford Creek (AVWP)

118 Jim Knight (IPC)

119 John Day Creek (AVWP)

120 Joseph Hydro (PPL)

121 Kasel \& Witherspoon (IPC)

122 Koma Kulshan (PSE)

123 Koyle (IPC)

124 L. Fery (PPL)

125 Lacomb Irrig. (PPL)

126 Lake Oswego (PGE)

2.4
0.1
0.1
0.1
0.7
0.7
0.6
1.1

2.4
0.1
0.1
1.3
0.1
2.8
0.7
0.4
0.6
1.1
8.3
0.1

$\begin{array}{rrrr}0 & 0 & 0 \\ 0.1 & 0.1 & 0.1\end{array}$

$\begin{array}{rrrr}0.1 & 0.1 & 0.1 & 0.1 \\ 0 & 0 & 0.1 & 0.1\end{array}$

$\begin{array}{llll}1.3 & 1.3 & 1.1 & 1.0 \\ 0.1 & 0.1 & 0.1 & 0.1\end{array}$

$\begin{array}{llll}2.8 & 3.1 & 3.1 & 2.8\end{array}$

$\begin{array}{llll}0.7 & 0.7 & 1.1 & 0.9\end{array}$

0

$\begin{array}{llll}0.6 & 0.6 & 0.6\end{array}$

$\begin{array}{llll}1.1 & 1.1 & 1.5 & 1.8\end{array}$

$\begin{array}{lll}8.3 & 8.3 & 6.5\end{array}$

0
0

$2.9 \quad 2.9$

0.3

0.4
0

0
1.3
0.2

0.2

0

0.1

4.7

0.1

0

1.2

0.1

6.5

0.6

0.8

0.2

0.6
1.0

1.0

0.1

7

6.6

8.8

0.7

0.2
0

0.4

0.2

1.5

$1.2 \quad 1.2$

1.1

0
0.1
0
0

$2.9 \quad 3.1 \quad 2.8$

$\begin{array}{ll}0.3 & 0.9\end{array}$

3.1
0.4
0

0

1.3
0.2

0

0.1

0.1

0

1.2

0

6.5

0.8

0.2

0.6
1.0

0

0.1
7

6.6

8.8
0.7

0.2

0

$\begin{array}{ll}0.3 & 0.2\end{array}$

$\begin{array}{lll}0.2 & 0.2 & 0.2\end{array}$

$1.5 \quad 0.9$

$\begin{array}{ll}1.2 & 1.2 \\ 4.6 & 4.3\end{array}$

0
0
0.4

0.4

0
0.4 $\begin{array}{lllll}0.8 & 0.3 & 1.0 & 2.2 & 2.6\end{array}$

0
0.1
0.1
0
0.1
1.1
0.1
1.9
0.2
0.4
0
0.6
1.4

$1.0-0$

0.1

0.1

0
2.4

1.2

0.7

0.6

0
0
0.5

0.5
0

0
0
0

0.1

0.1

0.7

0.1
0

3.1

5.0

3.2

0.3

0.3

0.3

0.2

0.3

0.1

0

3.5

0.7

0.5

0.5

0.2

0.1

0.3

$6.1 \quad 3.9$

0
1.0
0.4

0.4

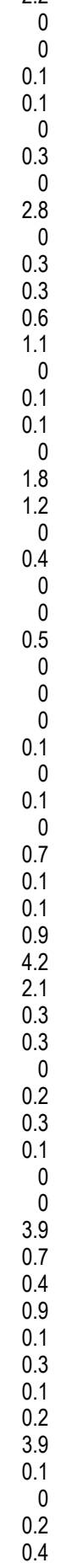

$\begin{array}{ll}0.4 & 0.4\end{array}$ $\begin{array}{rl}0 & \\ 0 & \\ 0.1 & 0.1 \\ 0 & \end{array}$

3

3.2

0
0
0

0
0.1

3.23.

$\begin{array}{rr}1.2 & 1.7 \\ 0 & 0 \\ 1.9 & 2.1\end{array}$

1.9
0
0.3

$0.6 \quad 0.7$

$\begin{array}{ll}0.7 & 0.6 \\ 1.2 & 1.2\end{array}$

$\begin{array}{rr}0 & 0.1 \\ 0.1 & 0.1\end{array}$

$\begin{array}{rr}0.1 & 0.1 \\ 0 & \end{array}$

22

$\begin{array}{ll}1.3 & 0.4 \\ 0.4 & 0.9\end{array}$

$0.5 \quad 0.4$

0
0
0.6

0.6
0
0

0

0.1

0.1

0.9

0.1

0.1

2.7
3.8

4.6

0.3

0.3

0.2

0.3

0.3
0.1

0
4.6

4.6
0.7

0.4

0.9

0.1

0.2

0.2

3.8

$0.1 \quad 0.3$

0.9

0.4

1.7
0.1
2.5
0.3
0.7
0.
1.2
0.1
0.1
0.1
2.7
0.4
0.9
0.4
0.8
0.1
0

0.1

0
0
0

0.6

0.1
27

3.8

3.3

0.5

0.3
0.3

0.6

0.3

0.1
0.1

5.9

0.7

0.4
1.1

0.2

0.2

0.4

4.4

0.3
0
0.9

0.4

0.1
0.1
0.1
0.3
0.1
0.1
0
0.1
0.9
0.1
0
0
0
0

0
0
1.6

0.1

$\begin{array}{ll}3.4 & 3.4 \\ 0.6 & 0.6\end{array}$

$0.8 \quad 0.8$

$0.6 \quad 0.6$

$\begin{array}{ll}1.8 & 1.8 \\ 2.9 & 2.9\end{array}$

0.1

0.1

0.1
0

$2.7 \quad 2.7$

$\begin{array}{ll}0.4 & 0.4 \\ 0.8 & 0.8\end{array}$

$\begin{array}{ll}0.8 & 0.3 \\ 0 & 0\end{array}$

0
0
0.9

0.1

0.9
0.1

0.1
0
0 
Aug1 Aug16 Sep

127 Lateral \#10 (IPC)

128 Lee Tavenner (NWE)

129 Lemoyne (IPC)

130 Little Wood River Res. (IPC)

131 Littlewood/Arkoosh (IPC)

132 Lowline \#2 (IPC)

133 Lowline Canal (IPC)

134 Magic Reservoir (IPC)

135 Malad River (IPC)

136 Marcos Ranches (IPC)

137 Marsh Valley (UPL)

138 Meyers Falls (AVWP)

139 Middlefork Irrig. (PPL)

140 Mile 28 (IPC)

141 Minikahda (PGE)

142 Mink Creek (UPL)

143 Mitchell Butte (IPC)

144 Mountain Energy (PPL)

$145 \mathrm{Mt}$. Tabor (PGE)

146 Mud Creek/S\&S (IPC)

147 Mud Creek/White (IPC)

148 Nichols Gap (PPL)

149 Nicholson Sunnybar (UPL)

150 North Fork Sprague (PPL)

151 O.J. Power (UPL)

152 Odell Creek (PPL)

153 Opal Springs (PPL)

154 Owyhee Dam (IPC)

155 Pancheri (UPL)

156 Pelton Rereg. Dam (PPL)

157 Phillips Ranch (AVWP)

158 Phillipsburg (NWE)

159 Pigeon Cove (IPC)

160 Pine Creek (NWE)

161 Port Townsend Paper Hydro (PSE)

162 Portland (PGE)

163 Portland Hydro Project (PGE)

164 Preston City (UPL)

165 Pristine Springs (IPC)

166 Pristine Springs \#3 (IPC)

167 Reynolds Irrigation (IPC)

168 Rim View (IPC)

169 Rock Creek \#1 (IPC)

170 Rock Creek \#2 (IPC)

171 Sagebrush (IPC)

172 Salem (PGE)

173 Schaffner (IPC)

174 Sheep Creek (AVWP)

175 Shingle Creek (IPC)

176 Shoshone (IPC)

177 Shoshone II (IPC)

178 Snake River Pottery (IPC)

179 Snedigar Ranch (IPC)

180 Solar Research (PPL)

181 South Dry Creek (NWE)

182 Spokane Upriver (AVWP

183 Stauffer Dry Creek (UPL)

184 Strawberry Creek (NWE)

185 Sunshine Power \#2 (IPC)

186 Sygitowicz Creek Small Hydro (PSE)

187 TGS/Briggs (UPL)

188 Trout Co. (IPC)

189 Tunnel \#1 (IPC)

190 Twin Falls (PSE)

191 Walla Walla (PPL)

192 Water Street / Santiam (PPL)

193 Weeks Falls (PSE)

194 White James (PPL)

195 White Water Ranch (IPC)

196 Wilson Lake Hydro (IPC)

197 Wisconsin Creek (NWE)

198 Yakima-Tieton (PPL)

199 IOU - Total NUG: Hydro

$1.5 \quad 1.5$

2.7

6.8

6.8

1.

$0.4 \quad 0.4$

1.3

1.2

1
0

0.1

0.1

0
0.2
0

0.2
0

0

2.6

2.8

7

2.0
0.1

0.3
0

2.3

0.3

0.1

0.2

0.2

0.4

0.4

0.4

0.1

0.4

0.6

0.2

0.1

0.5

3.4

0.9

0.1

0

$0.2 \quad 0.2$

$\begin{array}{ll}0.1 & 0.1 \\ 4.3 & 4.3\end{array}$

1.5

0
0.8

$0.8 \quad 0.8$

$\begin{array}{llll}0.1 & 0.1 & 0.1 & 0.1\end{array}$

$\begin{array}{llll}0.1 & 0.1 & 0.1 & 0.1\end{array}$

$\begin{array}{lll}7.8 & 7.5 & 7.3\end{array}$

$\begin{array}{lll}6.8 & 4.4 & 0.5\end{array}$

$0.5 \quad 0.6 \quad 0.5$

$\begin{array}{lll}1.1 & 1.2 & 1.1 \\ 0.4 & 0.4 & 0.4\end{array}$

$\begin{array}{rrr}1 & 0.9 & 0.6 \\ 1.8 & 0.8 & 1.2\end{array}$

$\begin{array}{rrr}1.3 & 1.2 & 0.7 \\ 0 & 0 & 0\end{array}$

$\begin{array}{rrr}0.2 & 0.2 & 0.2 \\ 0 & 0.1 & 0 \\ 0 & 0.1 & 0\end{array}$

$\begin{array}{rrr}2.6 & 3.0 & 3.6 \\ 2.8 & 2.0 & 0.6\end{array}$

$$
\begin{array}{ll}
0 & \\
7 & 6
\end{array}
$$$$
\begin{array}{rr}
0 & \\
6.3 & 8.3 \\
0 &
\end{array}
$$$$
\begin{array}{rrr}
2.0 & 2.1 & 1.3
\end{array}
$$$$
\begin{array}{lll}
0.1 & 0.1 & 0.1 \\
0.3 & 0.3 & 0.3
\end{array}
$$$$
\begin{array}{rrr}
2.3 & 0 & \\
0.3 & 0.9 & 6.7
\end{array}
$$

$\begin{array}{lll}0.3 & 0.4 & 0.4\end{array}$

$0.1 \quad 0.2$

$\begin{array}{ll}0.2 & 0.2 \\ 0.2 & 0.2\end{array}$

$0.2-0.2$

$\begin{array}{lll}1.1 & 1.6 & 1.7\end{array}$

$\begin{array}{lll}1.8 & 1.8 & 1.9\end{array}$

0.4

$0.3 \quad 0.3$

$\begin{array}{rrr}0.4 & 0.3 & 0.2\end{array}$

$\begin{array}{lll}0.4 & 0.3 & 0.3\end{array}$

$\begin{array}{lll}0.1 & 0.2 & 0.2 \\ 0.4 & 0.5 & 0.4\end{array}$

$\begin{array}{rrr}0.6 & 0.6 & \\ 0 & 0 & 0 \\ 0.2 & 0.2 & 0.2\end{array}$

$\begin{array}{lll}0.5 & 0.5 & 0.5\end{array}$

$3.4-3.4 \quad 4.7$

$\begin{array}{rrr}0.9 & 1 & 0.8 \\ 0.1 & 0.1 & 0.1\end{array}$

$\begin{array}{lll}0.1 & 0.1 & 0.1 \\ 0.1 & 0.1 & 0.1\end{array}$

Oct Nov

Dec

\begin{abstract}
Jan
\end{abstract}

\begin{abstract}
Feb
\end{abstract}
Apr1

Apr16

\begin{abstract}
May
\end{abstract}

Jun

Jul Avg

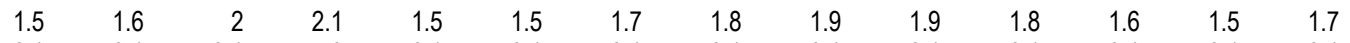

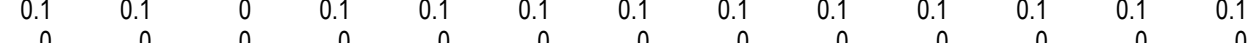

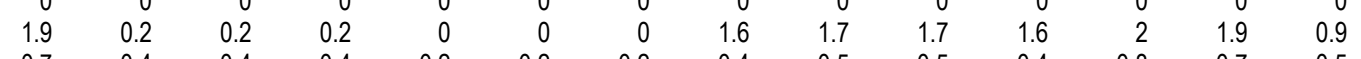

$\begin{array}{llllllllll}0.7 & 0.4 & 0.4 & 0.4 & 0.2 & 0.2 & 0.2 & 0.4 & 0.5 & 0.5\end{array}$

$\begin{array}{llll}2.7 & 2.5 & 1.8 & 1.4\end{array}$

$\begin{array}{rrrr}1.2 & 0.8 & 0 & 0 \\ 1 & 1.1 & 0.6 & 0.6\end{array}$

$\begin{array}{rrr}0 & 0 & 0.7 \\ 0.6 & 0.6 & 0.7\end{array}$

$\begin{array}{llll}7.5 & 0.6 & 0.6 & 0.7 \\ 0.2 & 0.1 & 0.3 & 0.1 \\ 0.5 & 0.1 & 0.1\end{array}$

0.710 .9

$\begin{array}{rr}0 & 0 \\ 0.4 & 0.4 \\ 0.9 & 0.6\end{array}$

$\begin{array}{rrr}1.7 & 2.7 & 3.1 \\ 0 & 0 & 0 \\ 0.1 & 0.1 & 0.1 \\ 0.5 & 0.4 & 0.4\end{array}$

$\begin{array}{rr}0.1 & 0.9 \\ 0 & 0.2\end{array}$

$\begin{array}{ll}0 & 0.2 \\ 0 & 1.1\end{array}$

$\begin{array}{llll}0.4 & 0.4 & 0.4 & 0.4 \\ 0.8 & 1.1 & 1.1 & 1.1\end{array}$

$\begin{array}{ll}0.8 & 1.1 \\ 3.1 & 3.1\end{array}$

$\begin{array}{rrr}0.5 & 0.4 \\ 0 & 0 \\ 0 & 0\end{array}$

$\begin{array}{rr}0 & \\ 0.1 & \\ 0.4 & 0\end{array}$

$\begin{array}{rr}0.4 & 0.3 \\ 0 & 0.3 \\ 0 & 0\end{array}$

$\begin{array}{llllll}0.1 & 0.1 & 0.1 & 0.1 & 0.1 & 0.1 \\ 0.4 & 0.5 & 0.5 & 0.5 & 0.5 & 0.4 \\ 0.1 & 0.2 & 0.2 & 0.2 & 0.1 & 0.1\end{array}$

$0.4 \quad 0.6$

0.1
0.2

$0.4 \quad 0.1$

$\begin{array}{rl}0.2 & 0.1 \\ 0 & 0.1\end{array}$

$0.5 \quad 0.6$

0.1

$\begin{array}{rr}0 & 0 \\ 2.9 & 2.9\end{array}$

$\begin{array}{llll}0.2 & 0.2 & 0.2 & 0.2 \\ 0.3 & 0.2 & 0.3 & 0.3\end{array}$

$\begin{array}{rr}0.1 & 0.1 \\ 0 & 0\end{array}$

$\begin{array}{rr}0 & 0 \\ 3.5 & 2.6 \\ 0.6 & 1.8\end{array}$

$\begin{array}{rrr}0 & 0.2 & 0.6 \\ 0 & 0 & 0 \\ 8.0 & 7.9 \\ 0 & 0\end{array}$

$\begin{array}{rr}0.6 & 1.8 \\ 0 & 0 \\ 10 & 7.7\end{array}$

8.7

$\begin{array}{rr}10 & 7.7 \\ 0 & \\ 0 & 0\end{array}$

$\begin{array}{lllll}0.8 & 0.4 & 0.3 & 0.4 & 0.6 \\ 0.1 & 0.1 & 0.1 & 0.1 & 0.1 \\ 0.3 & 0.3 & 0.3 & 0.3 & 0.3\end{array}$

$0.3 \quad 0.3$

$0.3-0.3$

$\begin{array}{rrrr}0.3 & 0.3 & 0.3 & 0.3 \\ 0 & 0 & 0 & \\ 20 & 18 & 17 & 16 \\ 0.4 & 0.4 & 0.4 & 0.3\end{array}$

$\begin{array}{llll}0.4 & 0.4 & 0.4 & 0.3 \\ 0.1 & 0.1 & 0.1 & 0.1 \\ 0.2 & 0.2 & 0.2 & 0.2\end{array}$

$\begin{array}{lll}0.1 & 0.1 & 0.1 \\ 0.2 & 0.2 & 0.2\end{array}$

$\begin{array}{lll}0.2 & 0.2 & 0.3 \\ 0.2 & 0.2\end{array}$

0.2
0.3
0.2

1.3

$\begin{array}{ll}1.3 & 1.1 \\ 1.9 & 0.6 \\ 0.1 & 0.1\end{array}$

$\begin{array}{rr}0.3 & 0.1 \\ 0 & 0\end{array}$

$\begin{array}{rrr}0.3 & 0.1 & 0 \\ 0.4 & 0.1 & 0.2\end{array}$

$\begin{array}{llll}0.4 & 0.5 & 0.5 & 0.5 \\ 0.1 & 0.1 & 0.1 & 0.1\end{array}$

$\begin{array}{rrrrr}0.4 & 0.1 & 0.1 & 0.1 & 0.1\end{array}$

$\begin{array}{rrrr}0 & 0 & 0 & \\ 0 & 0.1 & 0.1 & 0.1 \\ 0.2 & 0.2 & 0.2 & 0.2 \\ 0 & 0 & 0.1 & 0.1\end{array}$

$\begin{array}{ll}1.0 & 1.1\end{array}$

$\begin{array}{ll}0.4 & 1.6 \\ 0.1 & 0.3\end{array}$

$\begin{array}{rr}0 & 0.3 \\ 0.2 & 0.3\end{array}$

$\begin{array}{rr}0 & 0.1 \\ 0 & \\ 0.1 & 0\end{array}$

$\begin{array}{ll}0.2 & 0.1 \\ 0.1 & 0.1 \\ 0 & 0.3\end{array}$

0
6.7

$\begin{array}{rr}0 & 0 \\ 13 & 14 \\ 0 & 0.3\end{array}$

$\begin{array}{rr}0 & 0.3 \\ 13 & 14 \\ 0.3 & 0.4\end{array}$

$\begin{array}{rr}0.3 & 0.4 \\ 0 & 0\end{array}$

-NUG: Small Thermal \& Misc.-

200 Marion Solid Waste (PGE)

$\begin{array}{rrrr}7.1 & 7.1 & 5.3 & 2.0 \\ 0 & 0 & 0 & 0.1\end{array}$

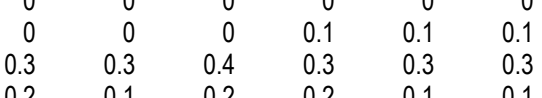

$\begin{array}{rrrrrr}0 & 0 & 0 & 0.1 & 0.1 & 0.1 \\ 0.3 & 0.3 & 0.4 & 0.3 & 0.3 & 0.3 \\ 0.2 & 0.1 & 0.2 & 0.2 & 0.1 & 0.1\end{array}$

$\begin{array}{llll}0.1 & 0.1 & 0.1 & 0.1 \\ 0 & 0 & 0 & 2.4\end{array}$

$\begin{array}{rrrr}0.4 & 0.3 & 0.3 & 0.3 \\ 0.1 & 0.1 & 0.1 & 0.1 \\ 0 & 2.4 & 5.1 & 0.1\end{array}$

$\begin{array}{rrrr}0 & 0 & 0 & 2.4 \\ 7.9 & 7.4 & 4.8 & 7.8\end{array}$

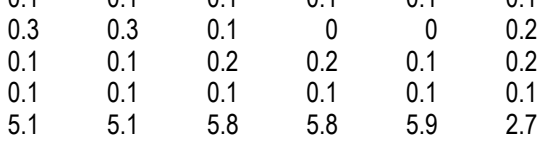

$\begin{array}{rrrrrrrr}4.8 & 7.8 & 15 & 15 & 13 & 24 & 19 & 9.6 \\ 1.5 & 1.8 & 1.8 & 1.8 & 1.5 & 1.5 & 1.8 & 1.5\end{array}$

$\begin{array}{llllllll}1.5 & 1.8 & 1.8 & 1.8 & 1.5 & 1.5 & 1.8 & 1.5 \\ 0.1 & 0.1 & 0.2 & 0.2 & 0.1 & 0.1 & 0.1 & 0.1\end{array}$

$\begin{array}{llllllll}0.1 & 0.1 & 0.2 & 0.2 & 0.1 & 0.1 & 0.1 & 0.1 \\ 0.6 & 1.2 & 2.6 & 2.6 & 2.4 & 4.4 & 3.5 & 1.7\end{array}$

$\begin{array}{llllllll}0.6 & 1.2 & 2.6 & 2.6 & 2.4 & 4.4 & 3.5 & 1.7 \\ 0.2 & 0.2 & 0.2 & 0.2 & 0.2 & 0.3 & 0.2 & 0.2\end{array}$

$\begin{array}{llllllll}0.1 & 0.1 & 0.1 & 0.2 & 0.2 & 0.3 & 0.2 & 0.2 \\ 0 & 0 & 2.3 & 2.3 & 0.1 & 0.1 & 0.1 & 0.1\end{array}$

\begin{tabular}{|c|c|c|c|c|c|c|c|c|c|c|c|c|c|c|}
\hline 7.1 & 7.1 & 5.3 & 2.0 & 0.2 & 0 & 0 & 0 & 0 & 2.3 & 2.3 & 6.1 & 6.8 & 7.6 & 3.1 \\
\hline 0 & 0 & 0 & 0.1 & 0 & 0 & 0.1 & 0 & 0 & 0 & 0 & 0 & 0 & 0 & S \\
\hline 3.0 & 3.0 & 2.5 & 0.4 & 0 & 0 & 0 & 0 & 0 & 0 & 0 & 1.2 & 1.7 & 1.7 & 0.9 \\
\hline
\end{tabular}

$157 \quad 157$

$\begin{array}{rrrrrrrr}3.0 & 2.5 & 0.4 & 0 & 0 & 0 & 0 & 0 \\ 157 & 133 & 111 & 119 & 111 & 101 & 108 & 129\end{array}$

$\begin{array}{rrrrrrrr}3.0 & 2.5 & 0.4 & 0 & 0 & 0 & 0 & 0 \\ 157 & 133 & 111 & 119 & 111 & 101 & 108 & 129\end{array}$

$129 \quad 173$

9.3

$9.3 \quad 9.3$

9.3

9.3

9.3

9.3

9.3

9.3

$\begin{array}{llll}9.3 & 9.3 & 9.3 & 9.3\end{array}$

$9.3 \quad 9.3$ 
Table A-24: Regional Non Utility Generating Resources By Project

PNW Loads and Resources Study

2005 - 2006 Operating Year 2003 White Book

Average Energy in Megawatts

Aug1 Aug16 Sep

201 Minnesota Methane (AVWP)

202 Montana One (NWE)

203 Pocatello Waste (IPC)

204 Spokane MSW (PSE)

205 West Boise Waste (IPC)

206 IOU - Total NUG: Small Thermal \& Misc.

-NUG: Combustion Turbines -

207 March Point \#1 Cogeneration (PSE)

208 March Point \#2 Cogeneration (PSE)

209 Rathdrum- Boekel Rd. \#1 (AVWP)

210 Rathdrum -Boekel Rd. \#2 (AVWP)

211 Tenaska Gas Cogen (PSE)

212 IOU - Total NUG: Combustion Turbines

-NUG: Co-Generation-

213 Billings Generation (NWE)

214 Biomass One (PPL)

215 Boise Cascade Medford (PPL)

216 Champion (PPL)

217 DR Johnson - Co-Gen II (PPL)

218 Magic Valley (IPC)

219 Magic West (IPC)

220 PERC Pierce, Wa (PSE)

221 Simplot Pocatello (IPC)

222 Sumas Energy (PSE)

223 Tamarack (IPC)

224 TASCO Nampa ID (IPC)

225 TASCO Twin Falls ID (IPC)

226 Vaagen Bros. (IPC)

227 Warm Springs (PPL)

228 IOU - Total NUG: Co-Generation

$\begin{array}{rrrrrrrrrrrrrrr}0.8 & 0.8 & 0.8 & 0.8 & 0.8 & 0.8 & 0.8 & 0.8 & 0.8 & 0.8 & 0.8 & 0.8 & 0.8 & 0.8 & 0.8 \\ 9.2 & 9.4 & 8.7 & 8.8 & 6.6 & 7.4 & 7.5 & 7.2 & 6.2 & 6 & 5.9 & 8.5 & 8.5 & 8.2 & 7.7 \\ 0.1 & 0.1 & 0.2 & 0.1 & 0.2 & 0.1 & 0.1 & 0.2 & 0.1 & 0.2 & 0.2 & 0.1 & 0.2 & 0.1 & 0.1 \\ 15 & 15 & 15 & 15 & 15 & 15 & 15 & 15 & 15 & 15 & 15 & 15 & 15 & 15 & 15 \\ 0.1 & 0.1 & 0.1 & 0.1 & 0.1 & 0.1 & 0.1 & 0.1 & 0.1 & 0.1 & 0.1 & 0.1 & 0.1 & 0.1 & 0.1 \\ \mathbf{3 5} & \mathbf{3 5} & \mathbf{3 4} & \mathbf{3 4} & \mathbf{3 2} & \mathbf{3 3} & \mathbf{3 3} & \mathbf{3 3} & \mathbf{3 2} & \mathbf{3 2} & \mathbf{3 2} & \mathbf{3 4} & \mathbf{3 4} & \mathbf{3 4} & \mathbf{3 3}\end{array}$

-NUG: Renewables-

229 Foote Creek 1 (PPL)

230 Healow \#2 (NWE)

231 Josef Staufer (NWE)

232 Lewandowski Farms (IPC)

233 Livingston (NWE)

234 Puyallup Energy Recovery (PSE)

235 Stateline Wind Project (AVWP)

236 Windmill (PGE)

237 IOU - Total NUG: Renewables

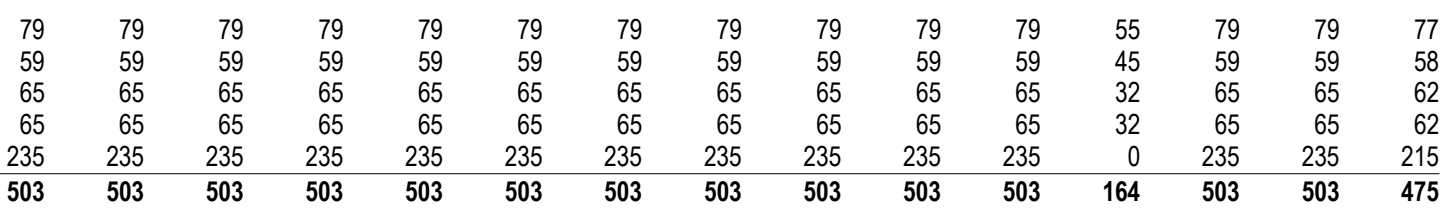

Other Entities

-NUG: Co-Generation-

238 SP Newsprint cogen (SPN)

\begin{tabular}{|c|c|c|c|c|c|c|c|c|c|c|c|c|c|c|}
\hline 17 & 17 & 16 & 10 & 12 & 14 & 14 & 13 & 11 & 7 & 6.9 & 16 & 16 & 15 & 13 \\
\hline 22 & 22 & 21 & 21 & 22 & 22 & 7.2 & 15 & 17 & 20 & 20 & 17 & 22 & 22 & 19 \\
\hline 8.5 & 8.5 & 8.5 & 8.5 & 8.5 & 8.5 & 8.5 & 8.5 & 8.5 & 8.5 & 8.5 & 8.5 & 8.5 & 8.5 & 8.5 \\
\hline 3.3 & 3.3 & 3.3 & 3.3 & 3.3 & 3.3 & 1 & 3.3 & 3.3 & 3.3 & 3.3 & 3.3 & 3.3 & 3.3 & 3.1 \\
\hline 9.3 & 9.3 & 9.7 & 9.3 & 9.6 & 9.3 & 9.3 & 10 & 9.4 & 9.7 & 9.7 & 9.4 & 9.7 & 9.3 & 9.5 \\
\hline 9.3 & 9.3 & 9.7 & 9.3 & 9.6 & 9.3 & 9.3 & 10 & 9.4 & 9.7 & 9.7 & 9.4 & 9.7 & 9.3 & 9.5 \\
\hline 113 & 113 & 126 & 126 & 126 & 126 & 126 & 126 & 126 & 113 & 113 & 113 & 113 & 113 & 120 \\
\hline 3.8 & 3.8 & 4.6 & 4.7 & 4.2 & 3.2 & 3.6 & 3.8 & 4.2 & 4.4 & 4.4 & 3.4 & 4.6 & 4.8 & 4.1 \\
\hline 0.2 & 0.2 & 0.2 & 0.2 & 0.2 & 0.2 & 0.2 & 0.2 & 0.2 & 0.2 & 0.2 & 0.2 & 0.2 & 0.2 & 0.2 \\
\hline 0 & 0 & 0 & 0 & 0 & 0 & 0 & 0 & 0 & 0 & 0 & 0 & 0 & 0 & 0 \\
\hline 3 & 3 & 3.7 & 3.8 & 3.3 & 2.6 & 2.9 & 3.1 & 3.4 & 3.6 & 3.6 & 2.7 & 3.7 & 3.8 & 3.3 \\
\hline 0.1 & 0.1 & 0 & 0.1 & 0.1 & 0.1 & 0.1 & 0.1 & 0 & 0 & 0 & 0 & 0.1 & 0 & 0.1 \\
\hline
\end{tabular}

239 Other - Total NUG: Co-Generation

207207

-NUG: Renewables-

240 Stateline Wind Project (PPME)

241 Vansycle Wind (FPLEV)

242 Other - Total NUG: Renewables

\begin{tabular}{rrrrrrrrrrrrrrr}
4.2 & 4.2 & 4.9 & 7.2 & 11 & 13 & 14 & 12 & 9.2 & 7.4 & 7.4 & 5.9 & 5.2 & 4.2 & 8.1 \\
0 & 0 & 0 & 0 & 0 & 0 & 0 & 0 & 0 & 0 & 0 & 0 & 0 & 0 & 0 \\
0 & 0 & 0 & 0 & 0 & 0 & 0 & 0 & 0 & 0 & 0 & 0 & 0 & 0 & 0 \\
0 & 0 & 0 & 0 & 0 & 0 & 0 & 0 & 0 & 0 & 0 & 0 & 0 & 0 & 0 \\
0 & 0 & 0 & 0 & 0 & 0 & 0 & 0 & 0 & 0 & 0 & 0 & 0 & 0 & 0 \\
2 & 2 & 2 & 2 & 2 & 2 & 2 & 2 & 2 & 2 & 2 & 2 & 2 & 2 & 2 \\
0.3 & 0.3 & 0.2 & 0.3 & 0.3 & 0.3 & 0.3 & 0.3 & 0.3 & 0.3 & 0.3 & 0.3 & 0.3 & 0.3 & 0.3 \\
0 & 0 & 0 & 0 & 0 & 0 & 0 & 0 & 0 & 0 & 0 & 0 & 0 & 0 & 0 \\
\hline 6.5 & $\mathbf{6 . 5}$ & $\mathbf{7 . 1}$ & $\mathbf{9 . 5}$ & $\mathbf{1 3}$ & $\mathbf{1 5}$ & $\mathbf{1 6}$ & $\mathbf{1 5}$ & $\mathbf{1 2}$ & $\mathbf{9 . 8}$ & $\mathbf{9 . 8}$ & $\mathbf{8 . 2}$ & $\mathbf{7 . 6}$ & $\mathbf{6 . 5}$ & $\mathbf{1 0}$
\end{tabular}

-Total Non-Utility Generating Resources-

243 Federal Entities

244 Generating Public Entities

245 Non Generating Public Entities

246 Investor-Owned Entities

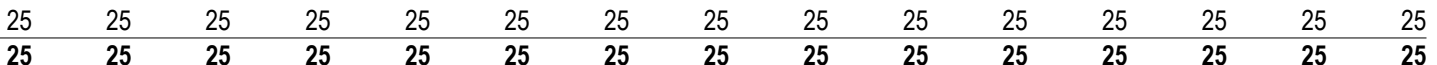

247 Other Entities

248 Total Non-Utility Generation

$\begin{array}{rrrrrrrrrrrrrrr}52 & 52 & 41 & 53 & 57 & 51 & 55 & 51 & 51 & 57 & 57 & 57 & 59 & 52 & 53 \\ 7.4 & 7.4 & 7.4 & 7.4 & 7.4 & 7.4 & 7.4 & 7.4 & 7.4 & 7.4 & 7.4 & 7.4 & 7.4 & 7.4 & 7.4 \\ \mathbf{5 9} & \mathbf{5 9} & \mathbf{4 8} & \mathbf{6 0} & \mathbf{6 5} & \mathbf{5 8} & \mathbf{6 2} & \mathbf{5 8} & \mathbf{5 8} & \mathbf{6 4} & \mathbf{6 4} & \mathbf{6 5} & \mathbf{6 7} & \mathbf{6 0} & \mathbf{6 0}\end{array}$


Oct Nov

Dec

\begin{abstract}
Jan Feb
\end{abstract}

\begin{abstract}
Mar Apr1 Apr16
\end{abstract}

\begin{abstract}
May
\end{abstract}
Jun

Jul Avg

\title{
Federal Entities
}

-NUG: Hydro-

1 Clearwater - State of ID DWR (BPA)

2 Dworshak Small Hydropower (BPA)

3 Federal -Total NUG: Hydro

\begin{tabular}{|c|c|c|c|c|c|c|c|c|c|c|c|c|c|c|}
\hline 1 & 1 & 1 & 1 & 1 & 1 & 1 & 1 & 1 & 1 & 1 & 1 & 1 & 1 & 1 \\
\hline 2.6 & 2.6 & 2.6 & 2.6 & 2.6 & 2.6 & 2.6 & 2.6 & 2.6 & 2.6 & 2.6 & 2.6 & 2.6 & 2.6 & 2.6 \\
\hline 3.6 & 3.6 & 3.6 & 3.6 & 3.6 & 3.6 & 3.6 & 3.6 & 3.6 & 3.6 & 3.6 & 3.6 & 3.6 & 3.6 & 3.6 \\
\hline
\end{tabular}

-NUG: Renewables-

4 Ashland Solar Project (BPA)

5 Condon Wind Project (BPA)

6 Foote Creek 1 (BPA)

7 Foote Creek 2 (BPA)

8 Foote Creek 4 (BPA)

9 Fourmile Hill Geothermal (BPA)

10 Klondike Phase 1 (BPA)

11 Stateline Wind Project (BPA)

12 Federal -Total NUG: Renewables

\begin{tabular}{|c|c|c|c|c|c|c|c|c|c|c|c|c|c|}
\hline 0 & 0 & 0 & 0 & 0 & 0 & 0 & 0 & 0 & 0 & 0 & 0 & 0 & 0 \\
\hline 10 & 10 & 11 & 13 & 14 & 13 & 12 & 12 & 14 & 14 & 12 & 15 & 11 & 12 \\
\hline 3.3 & 3.8 & 5.6 & 8.3 & 10 & 11 & 9.6 & 7.2 & 5.8 & 5.8 & 4.6 & 4.1 & 3.3 & 6.4 \\
\hline 0.4 & 0.5 & 0.7 & 1 & 1.3 & 1.3 & 1.2 & 0.9 & 0.7 & 0.7 & 0.5 & 0.5 & 0.4 & 0.8 \\
\hline 3.9 & 4.4 & 6.5 & 9.7 & 12 & 12 & 11 & 8.4 & 6.7 & 6.7 & 5.4 & 4.8 & 3.8 & 7.4 \\
\hline 0 & 0 & 50 & 50 & 50 & 50 & 50 & 50 & 50 & 50 & 50 & 50 & 50 & 42 \\
\hline 7.9 & 6.2 & 8.1 & 8.8 & 7.8 & 8.3 & 7.8 & 7.8 & 8.6 & 8.6 & 8.8 & 9.1 & 8 & 8.1 \\
\hline 29 & 23 & 30 & 33 & 29 & 31 & 29 & 29 & 32 & 32 & 33 & 34 & 30 & 30 \\
\hline 55 & 48 & 112 & 124 & 123 & 126 & 121 & 115 & 118 & 118 & 114 & 117 & 106 & 107 \\
\hline
\end{tabular}

Generating Public Entities

-NUG: Hydro-

13 Don Steffen (SHPD)

14 Eltopia Br Canal 4.6 (SCL)

15 Eltopia Br Canal 4.6 (TPU)

16 John Llewellyn (SHPD)

17 Kevin Duncan (SHPD)

18 Lucky Peak (SCL)

19 Main Canal Headworks (SCL)

20 Main Canal Headworks (TPU)

21 PEC Headworks (GCPD)

22 Pickering Micro Hydro (CKPD)

23 Potholes E Canal 66.0 (SCL)

24 Potholes E Canal 66.0 (TPU)

25 Quincy Chute (GCPD)

26 Russel D. Smith (SCL)

27 Russel D. Smith (TPU)

28 Smith Creek (EWEB)

29 Summer Falls (SCL)

30 Summer Falls (TPU)

31 Woods Creek (SHPD)

32 GPU - Total NUG: Hydro

$\begin{array}{rr}0 & 0 \\ 0.9 & 0.9 \\ 0.9 & 0.9 \\ 0 & 0 \\ 0 & 0 \\ 66 & 66 \\ 10 & 10 \\ 10 & 10 \\ 4.5 & 4.5 \\ 0 & 0 \\ 0.9 & 0.9 \\ 0.9 & 0.9 \\ 7.7 & 7.7 \\ 2 & 2 \\ 2 & 2 \\ 0 & 0 \\ 39 & 39 \\ 39 & 39 \\ 0.1 & 0.1 \\ 185 & 185\end{array}$

$\begin{array}{rr}0 & \\ 0.6 & 0.3 \\ 0.6 & 0.3 \\ 0 & \\ 0 & \\ 41 & 11 \\ 6.1 & 2 . \\ 6.1 & 2 . \\ 3.1 & 2.2 \\ 0 & \\ 0.9 & 0.8 \\ 0.9 & 0.8 \\ 4 & 2.3 \\ 1.3 & 0.7 \\ 1.3 & 0.7 \\ 0 & \\ 24 & 11 \\ 24 & 11 \\ 0.1 & 0.1 \\ 115 & \mathbf{5}\end{array}$

\begin{tabular}{rrr}
0 & \\
0.3 & \\
0.3 & \\
0 & \\
0 & \\
11 & \\
2.6 & \\
2.6 & \\
2.2 & \\
0 & \\
0.8 & \\
0.8 & \\
2.3 & \\
0.7 & \\
0.7 & \\
5 & \\
11 & \\
11 & \\
0.1 & \\
\hline 51 & & \\
& & \\
& & \\
&
\end{tabular}

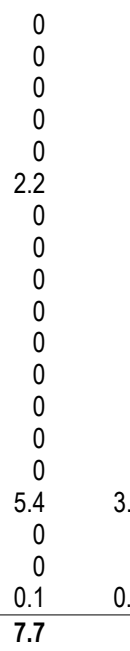

$\begin{array}{rrr}0 & 0 \\ 0 & 0 \\ 0 & 0 \\ 0 & 0 \\ 0 & 0 \\ 3 & 7.9 & \\ 0 & 0 \\ 0 & 0 \\ 0 & 0 \\ 0 & 0 \\ 0 & 0 & \\ 0 & 0 & \\ 0 & 0 & \\ 0 & 0 & \\ 0 & 0 & \\ 3.9 & 0 & 0.8 \\ 0 & 0 & \\ 0 & 0 & 0 \\ 0.1 & 0.1 & 0.1 \\ 7 & 8 & 11\end{array}$

$\begin{array}{rr}0 & \\ 0 & \\ 0 & \\ 0 & \\ 0 & 0 \\ 10 & 21 \\ 0 & 1.4 \\ 0 & 1.4 \\ 0 & 0.4 \\ 0 & \\ 0 & 0.3 \\ 0 & 0.3 \\ 0 & 0.8 \\ 0 & 0.1 \\ 0 & 0.1 \\ 0.8 & 1.5 \\ 0 & 2.4 \\ 0 & 2.4 \\ 0.1 & 0.1 \\ 11 & 32\end{array}$

$\begin{array}{rrrrrrr}0 & 0 & 0 & 0 & 0 & 0 & 0 \\ 0 & 0.7 & 0.7 & 1 & 1 & 1.1 & 0.5 \\ 0 & 0.7 & 0.7 & 1 & 1 & 1.1 & 0.5 \\ 0 & 0 & 0 & 0 & 0 & 0 & 0 \\ 0 & 0 & 0 & 0 & 0 & 0 & 0 \\ 21 & 52 & 52 & 84 & 85 & 77 & 38 \\ 1.4 & 6.8 & 6.8 & 9.1 & 11 & 12 & 4.9 \\ 1.4 & 6.8 & 6.8 & 9.1 & 11 & 12 & 4.9 \\ 0.4 & 5.1 & 5.1 & 6.9 & 6.2 & 5.8 & 2.9 \\ 0 & 0 & 0 & 0 & 0 & 0 & 0 \\ 0.3 & 0.9 & 0.9 & 0.9 & 0.9 & 0.9 & 0.5 \\ 0.3 & 0.9 & 0.9 & 0.9 & 0.9 & 0.9 & 0.5 \\ 0.8 & 5.5 & 5.5 & 7.1 & 8.4 & 8.5 & 3.7 \\ 0.1 & 1.2 & 1.2 & 1.9 & 2.1 & 1.7 & 0.9 \\ 0.1 & 1.2 & 1.2 & 1.9 & 2.1 & 1.7 & 0.9 \\ 1.5 & 9.2 & 9.2 & 37 & 37 & 11 & 9.2 \\ 2.4 & 25 & 25 & 36 & 40 & 44 & 18 \\ 2.4 & 25 & 25 & 36 & 40 & 44 & 18 \\ 0.1 & 0.1 & 0.1 & 0.1 & 0.1 & 0.1 & 0.1 \\ \mathbf{3 2} & \mathbf{1 4 1} & \mathbf{1 4 1} & \mathbf{2 3 2} & \mathbf{2 4 7} & \mathbf{2 2 1} & \mathbf{1 0 5}\end{array}$

-NUG: Small Thermal \& Misc.-

33 Grays Harbor Paper (GHPD)

34 Weyerhaeuser Pulp Mill (GHPD)

35 GPU - Total NUG: Small Thermal \& Misc.

\begin{tabular}{|c|c|c|c|c|c|c|c|c|c|c|c|c|c|}
\hline 3.8 & 3.8 & 3.8 & 3.8 & 3.8 & 3.8 & 3.8 & 3.8 & 3.8 & 3.8 & 3.8 & 3.8 & 3.8 & 3.8 \\
\hline 11 & 11 & 11 & 11 & 11 & 11 & 11 & 11 & 11 & 11 & 11 & 11 & 11 & 11 \\
\hline 15 & 15 & 15 & 15 & 15 & 15 & 15 & 15 & 15 & 15 & 15 & 15 & 15 & 15 \\
\hline
\end{tabular}

-NUG: Co-Generation-

36 Metro Westpoint (SCL)

37 Scott Paper (SHPD)

38 GPU - Total NUG: Co-Generation

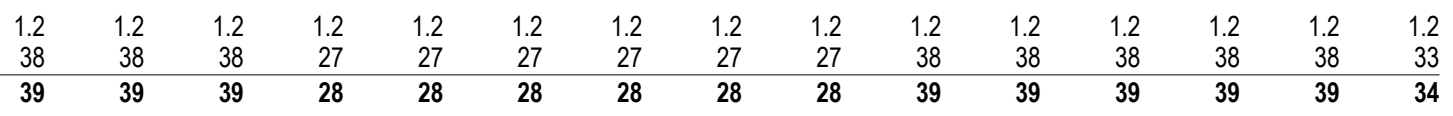

-NUG: Renewables-

39 Foote Creek 1 (EWEB)

40 Nine Canyon Wind Project (CHPD)

41 Nine Canyon Wind Project (COPD)

42 Nine Canyon Wind Project (DOPD)

43 Nine Canyon Wind Project (GCPD)

44 Nine Canyon Wind Project (GHPD)

45 Nine Canyon Wind Project (OKPD)

46 Stateline Wind Project (EWEB)

47 Stateline Wind Project (SCL)

48 GPU - Total NUG: Renewables

Non-Generating Public Entities

-NUG: Hydro-

49 Buffalo Hydro (FREC)

50 Burr \& Bouchard (TNEC)

51 City of Cove (OTEC)

52 Falls Creek (CCPD)

53 Lilliwaup Falls Generating Co. (MCPD1)

54 Thomas Burnside (TNEC)

55 NGP - Total NUG: Hydro

\begin{tabular}{|c|c|c|c|c|c|c|c|c|c|c|c|c|c|c|}
\hline 1.4 & 1.4 & 1.6 & 2.4 & 3.5 & 4.3 & 4.5 & 4.1 & 3.1 & 2.5 & 2.5 & 2.0 & 1.7 & 1.4 & 2.7 \\
\hline 1.5 & 1.5 & 1.4 & 2.2 & 3.8 & 3.8 & 4.2 & 3.4 & 2.3 & 2.0 & 2.0 & 2.0 & 1.9 & 1.4 & 2.5 \\
\hline 0.4 & 0.4 & 0.4 & 0.5 & 0.9 & 0.9 & 1 & 0.9 & 0.6 & 0.5 & 0.5 & 0.5 & 0.5 & 0.3 & 0.6 \\
\hline 1.8 & 1.8 & 1.7 & 2.6 & 4.6 & 4.7 & 5.1 & 4.2 & 2.8 & 2.4 & 2.4 & 2.4 & 2.3 & 1.7 & 3 \\
\hline 2.2 & 2.2 & 2.1 & 3.2 & 5.7 & 5.7 & 6.3 & 5.2 & 3.4 & 3.0 & 3.0 & 3.0 & 2.8 & 2.1 & 3.7 \\
\hline 1.5 & 1.5 & 1.4 & 2.2 & 3.8 & 3.8 & 4.2 & 3.4 & 2.3 & 2.0 & 2.0 & 2.0 & 1.9 & 1.4 & 2.5 \\
\hline 3.0 & 3.0 & 2.8 & 4.3 & 7.5 & 7.6 & 8.4 & 6.9 & 4.6 & 3.9 & 3.9 & 3.9 & 3.7 & 2.7 & 4.9 \\
\hline 3.4 & 8.4 & 6.6 & 8.6 & 9.3 & 8.2 & 8.8 & 8.3 & 8.3 & 9.2 & 9.2 & 9.3 & 9.6 & 8.5 & 8.6 \\
\hline 49 & 49 & 39 & 50 & 54 & 48 & 52 & 48 & 48 & 54 & 54 & 54 & 56 & 50 & 50 \\
\hline 69 & 69 & 57 & 76 & 93 & 87 & 94 & 85 & 75 & 79 & 79 & 79 & 81 & 69 & 79 \\
\hline
\end{tabular}

-NUG: Small Thermal \& Misc.-

56 Methane Energy Agricultural D. (TKPD)

\begin{tabular}{|c|c|c|c|c|c|c|c|c|c|c|c|c|c|}
\hline 0.2 & 0.2 & 0.2 & 0.2 & 0.2 & 0.2 & 0.2 & 0.2 & 0.2 & 0.2 & 0.2 & 0.2 & 0.2 & 0.2 \\
\hline 0 & 0 & 0 & 0 & 0 & 0 & 0 & 0 & 0 & 0 & 0 & 0 & 0 & 0 \\
\hline 0.3 & 0.3 & 0.3 & 0.3 & 0.3 & 0.3 & 0.3 & 0.3 & 0.3 & 0.3 & 0.3 & 0.3 & 0.3 & 0.3 \\
\hline 0 & 0 & 0 & 0 & 0 & 0 & 0 & 0 & 0 & 0 & 0 & 0 & 0 & 0 \\
\hline 0.6 & 0.6 & 0.6 & 0.6 & 0.6 & 0.6 & 0.6 & 0.6 & 0.6 & 0.6 & 0.6 & 0.6 & 0.6 & 0.6 \\
\hline 0 & 0 & 0 & 0 & 0 & 0 & 0 & 0 & 0 & 0 & 0 & 0 & 0 & 0 \\
\hline 1.2 & 1.2 & 1.2 & 1.2 & 1.2 & 1.2 & 1.2 & 1.2 & 1.2 & 1.2 & 1.2 & 1.2 & 1.2 & 1.2 \\
\hline
\end{tabular}

57 NGP - Total NUG: Small Thermal \& Misc.

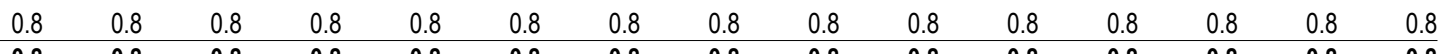


-NUG: Renewables.

58 Ashland Solar Project (ASHL)

59 Nine Canyon Wind Project (BCPD)

60 Nine Canyon Wind Project (ENW)

61 Nine Canyon Wind Project (LCPD)

62 Nine Canyon Wind Project (MCPD3)

\begin{tabular}{|c|c|c|c|c|c|c|c|c|c|c|c|c|c|c|}
\hline 0 & 0 & 0 & 0 & 0 & 0 & 0 & 0 & 0 & 0 & 0 & 0 & 0 & 0 & 0 \\
\hline 0.6 & 0.6 & 0.5 & 0.8 & 1.4 & 1.4 & 1.6 & 1.3 & 0.9 & 0.7 & 0.7 & 0.7 & 0.7 & 0.5 & 0.9 \\
\hline 0.4 & 0.4 & 0.4 & 0.5 & 0.9 & 1.0 & 1.1 & 0.9 & 0.6 & 0.5 & 0.5 & 0.5 & 0.5 & 0.3 & 0.6 \\
\hline 0.2 & 0.2 & 0.2 & 0.3 & 0.5 & 0.5 & 0.5 & 0.4 & 0.3 & 0.2 & 0.2 & 0.2 & 0.2 & 0.2 & 0.3 \\
\hline 0.4 & 0.4 & 0.4 & 0.5 & 0.9 & 1.0 & 1 & 0.9 & 0.6 & 0.5 & 0.5 & 0.5 & 0.5 & 0.3 & 0.6 \\
\hline 0 & 0 & 0 & 0 & 0 & 0 & 0 & 0 & 0 & 0 & 0 & 0 & 0 & 0 & 0 \\
\hline 1.5 & 1.5 & 1.4 & 2.2 & 3.8 & 3.8 & 4.2 & 3.5 & 2.3 & 2.0 & 2.0 & 2.0 & 1.9 & 1.4 & 2.5 \\
\hline
\end{tabular}

64 NGP - Total NUG: Renewables

Investor-Owned Entities

-NUG: Hydro-

65 Barber Dam (IPC)

66 Barney Creek (NWE)

67 Beaverton (PGE)

68 Bell Mountain (UPL)

69 Bingham Engineering (NWE)

70 Birch Creek (IPC)

71 Birch Creek (UPL)

72 Black Canyon \#3 (IPC)

73 Black Creek (AVWP)

74 Blind Canyon Hydro (IPC)

75 Box Canyon (IPC)

76 Boyd James (PPL)

77 Briggs Creek (IPC)

78 Broadwater Dam (NWE)

79 Bypass (IPC)

80 Canyon Springs (IPC)

81 Carlton (PGE)

82 Cascade Creek (NWE)

83 CDM Hydro - Bonneville Pacific (PPL)

84 Cedar Draw (IPC)

85 Central OR Irrigation District (PPL)

86 Clear Springs Trout (IPC)

87 Corbett (PGE)

88 Cornelius (PGE)

89 Crystal Springs (IPC)

90 Curry Cattle Co. (IPC)

91 Dairy Creek (PGE)

92 Deep Creek (AVWP)

93 Derr Creek (AVWP)

94 Dietrich Drop (IPC)

95 Donald Jenni (NWE)

96 Eagle Creek (PGE)

97 Eagle Point Irrigation Dist. (PPL)

98 Elk Creek (IPC)

99 Estacada (PGE)

100 Falls Creek (PPL)

101 Falls River (IPC)

102 Farmers Irrig. (PPL)

103 Faulkner Ranch (IPC)

104 Fisheries Development Co (IPC)

105 Galesville Dam (PPL)

106 Geobon 2 (IPC)

107 Georgetown Power (UPL)

108 Hailey Cspp (IPC)

109 Hazelton A (IPC)

110 Hazelton B (IPC)

111 Horseshoe Bend (IPC)

112 Hutchinson Creek (PSE)

113 Ingram Warm Springs (UPL)

$114 \mathrm{Jim}$ Ford Creek (AVWP)

115 Jim Knight (IPC)

116 John Day Creek (AVWP)

117 Joseph Hydro (PPL)

118 Kasel \& Witherspoon (IPC)

119 Koma Kulshan (PSE)

120 Koyle (IPC)

$121 \mathrm{~L}$. Fery (PPL)

122 Lacomb Irrig. (PPL)

123 Lake Oswego (PGE)

124 Lateral \#10 (IPC)

125 Lee Tavenner (NWE)

126 Lemoyne (IPC)

127 Little Wood River Res. (IPC)

128 Littlewood/Arkoosh (IPC)

129 Lowline \#2 (IPC)
2.4

$\begin{array}{rrr}0 & 0 & 0 \\ 0.1 & 0.1 & 0.1\end{array}$

$\begin{array}{rrrrrrr}0.1 & 0.1 & 0.1 & 0.1 & 0.1 & 0.1 & 0.1\end{array}$

$\begin{array}{llllll}0 & 0 & 0.1 & 0.1 & 0.1 & 0.1\end{array}$

$\begin{array}{lll}0.1 & 0.1 & 0.1 \\ 3.4 & 3.1 & 3.1\end{array}$

$\begin{array}{lll}0.4 & 3.1 & 31\end{array}$

$\begin{array}{llll}0.7 & 0.7 & 1.1 & 0.9\end{array}$

$\begin{array}{rrrr}0.4 & 0.4 & 0.4 & 0.4 \\ 0 & 0 & 0 & 0\end{array}$

$\begin{array}{llll}0.6 & 0.6 & 0.6 & 0.6 \\ 1.1 & 1.1 & 1.5 & 1.8\end{array}$

$\begin{array}{llll}8.3 & 8.3 & 6.5 & 2.8\end{array}$

0.1

0
2.9

0.3

3.1

0.4

0.4
0
0

1.3

0.2

0

0.1

4.7

0.1

0

1.2
0.1

0.1
0

6.5

0.6

0.8
0.2
0.6

0.6

1.0

0
0.1

7

6.6

0.7

0.2
0

0.4

0.2

1.5

1.2

4.6
1.1
0

1.1
0

0

$0.4 \quad 0.4$

$1.5 \quad 1.5$

0.1

1.9

0.7

2.7

$\begin{array}{rrr}0.8 & 0.3 & \\ 0 & 0 & \\ 0 & 0.1 & \\ 0.2 & 0.1 & 0.1 \\ 0.1 & 0 & 0.1 \\ 0.1 & 0.1 & 0.1 \\ 1.0 & 1.1 & \\ 0.1 & 0.1 & \\ 2.8 & 3.1 & 3.1 \\ 0.9 & 0.2 & \\ 0.4 & 0.4 & 0.3 \\ 0 & 0 & 0.4 \\ 0.6 & 0.6 & 0.6 \\ 1.8 & 1.4 & 12 \\ 2.8 & 1.0 & \\ 0.1 & 0.1 & 0.1 \\ 0 & 0 & \\ 0 & 0 & 0\end{array}$

$1.0 \quad 2.2$

$2.6 \quad 3.3$

0.1

0

$3.1 \quad 2.8$

0.3
0.4

$0.6 \quad 0.3$

1.2

0.1

0
0
2.4

0.1
0.1

0.1
1.8

$2.9 \quad 3.1 \quad 2.8$

$\begin{array}{ll}0.3 & 0.9\end{array}$

3.1

3.7

2.8
0.9

2.9
0.9

1.2

0.7
0.6

1.2
0

0.4

$\begin{array}{rr}0 & 0 \\ 0 & 0 \\ 0.5 & 0.5 \\ 0 & 0\end{array}$

$1.6 \quad 1.5$

$\begin{array}{ll}1.6 & 0.5\end{array}$

0
0
0

$0.1 \quad 0.1$

$0.1 \quad 0.1$

0

0.7
0.1

0.7
0.1

0.1

3.1

5.0

3.2

0.3

0.3

0.3

0.2

0.3

0.1

0

3.5
0.7

0.7
0.5

0.5

0.1

0.2

$0.1-0.1$

$\begin{array}{ll}0.3 & 0.2 \\ 6.1 & 3.9\end{array}$

4.2
2.1

0.3

0.3

0.2

0.3

0.1
0
0.2

0.2
0.4

1.5

0.1

0.1
0

$\begin{array}{ll}1.9 & 0.2\end{array}$

$0.7 \quad 0.4$

2.7

0
0.9

0.4

0.1
0

$0.2 \quad 0.2$

0.7 $\begin{array}{llllll}3.2 & 3.2 & 3.1 & 3.2 & 3.3 & 2.3\end{array}$

$\begin{array}{rrrrrr}3.2 & 3.2 & 3.1 & 3.2 & 3.3 & 2.3 \\ 0 & 0 & 0 & 0 & 0 & 0 \\ 0 & 0 & 0 & 0 & 0 & 0 \\ 0.1 & 0.1 & 0.1 & 0.2 & 0.1 & 0.1 \\ 0 & 0 & 0.1 & 0.1 & 0.1 & 0.1\end{array}$

0

$\begin{array}{ll}1.6 & 1.6 \\ 0.1 & 0.1\end{array}$

$0.1 \quad 0.1$

1.9
0.6

0.3
0.8

0.8
0.6

0.6
1.8

2.9

0.1

0.1
0

$2.7 \quad 2.7$

$\begin{array}{ll}0.4 & 0.4 \\ 0.8 & 0.8\end{array}$

$\begin{array}{ll}0.3 & 0.3\end{array}$

0.3
0
0

0.9
0.1

0.1
0
0

0.2

3.1

0
0

0.7

0.1

4.4
6.2

6.2
3.5
0.6

0.6

0.3

0.4
0.6

0.3
0.1

2.6

2.2

8.8

0.7

0.4
1.1

0.3

0.2

0.1

0.5

6.9

$\begin{array}{llll}0.1 & 0.3 & 0.8 & 0.8\end{array}$

0.9

0.4

$1.8-1.9$

0.1
0

$\begin{array}{rrrr}1.6 & 1.7 & 1.7 & 1.6\end{array}$

$\begin{array}{lll}0.4 & 0.5 & 0.5\end{array}$

2.5

2.5

2.7

2
0.8

2.7

1.9

2.7 

2003 White Book

Aug1 Aug16 Sep Oct Nov Dec

Jan Feb Mar Apr1 Apr16

\begin{abstract}
May
\end{abstract}

$$
\text { Jun }
$$
Jul Avg

130 Lowline Canal (IPC)

131 Magic Reservoir (IPC)

132 Malad River (IPC)

133 Marcos Ranches (IPC)

134 Marsh Valley (UPL)

135 Meyers Falls (AVWP)

136 Middlefork Irrig. (PPL)

137 Mile 28 (IPC)

138 Minikahda (PGE)

139 Mink Creek (UPL)

140 Mitchell Butte (IPC)

141 Mountain Energy (PPL)

142 Mt. Tabor (PGE)

143 Mud Creek/S\&S (IPC)

144 Mud Creek/White (IPC)

145 Nichols Gap (PPL)

146 Nicholson Sunnybar (UPL)

147 North Fork Sprague (PPL)

148 O.J. Power (UPL)

149 Odell Creek (PPL)

150 Opal Springs (PPL)

151 Owyhee Dam (IPC)

152 Pancheri (UPL)

153 Pelton Rereg. Dam (PPL)

154 Phillips Ranch (AVWP)

155 Phillipsburg (NWE)

156 Pigeon Cove (IPC)

157 Pine Creek (NWE)

158 Port Townsend Paper Hydro (PSE)

159 Portland (PGE)

160 Portland Hydro Project (PGE)

161 Preston City (UPL)

162 Pristine Springs (IPC)

163 Pristine Springs \#3 (IPC)

164 Reynolds Irrigation (IPC)

165 Rim View (IPC)

166 Rock Creek \#1 (IPC)

167 Rock Creek \#2 (IPC)

168 Sagebrush (IPC)

169 Salem (PGE)

170 Schaffner (IPC)

171 Sheep Creek (AVWP)

172 Shingle Creek (IPC)

173 Shoshone (IPC)

174 Shoshone II (IPC)

175 Snake River Pottery (IPC)

176 Snedigar Ranch (IPC)

177 Solar Research (PPL)

178 South Dry Creek (NWE)

179 Spokane Upriver (AVWP)

180 Stauffer Dry Creek (UPL)

181 Strawberry Creek (NWE)

182 Sunshine Power \#2 (IPC)

183 Sygitowicz Creek Small Hydro (PSE)

184 TGS/Briggs (UPL)

185 Trout Co. (IPC)

186 Tunnel \#1 (IPC)

187 Twin Falls (PSE)

188 Walla Walla (PPL)

189 Water Street / Santiam (PPL)

190 Weeks Falls (PSE)

191 White James (PPL

192 White Water Ranch (IPC)

193 Wilson Lake Hydro (IPC)

194 Wisconsin Creek (NWE)

195 Yakima-Tieton (PPL)

196 IOU - Total NUG: Hydro

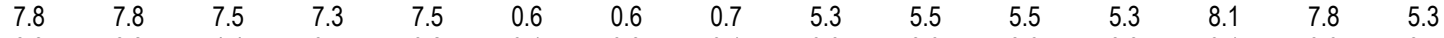

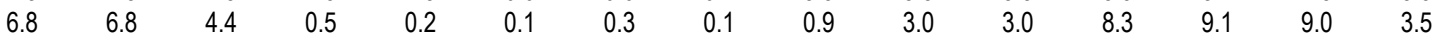

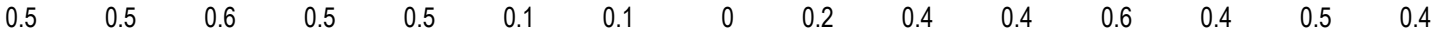

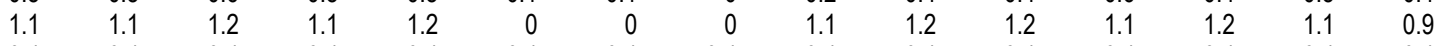

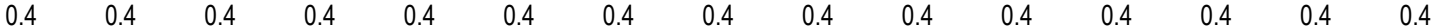

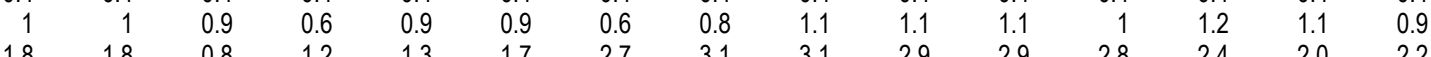

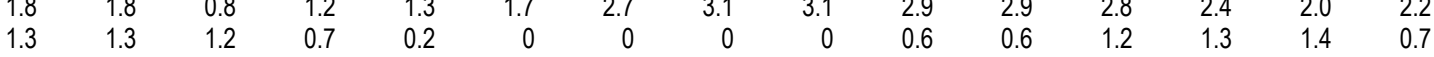

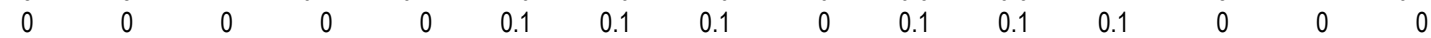

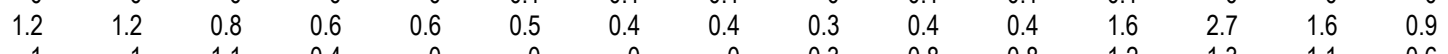

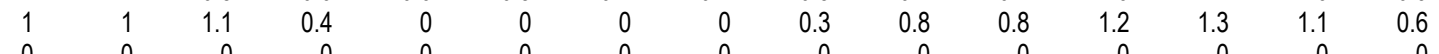

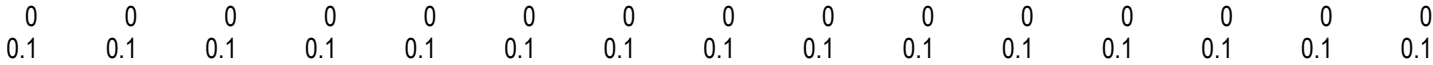

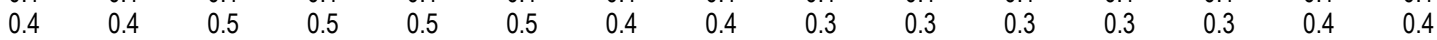

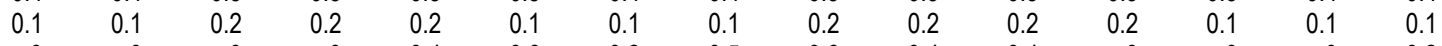

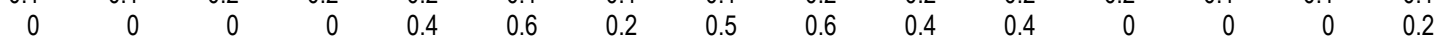

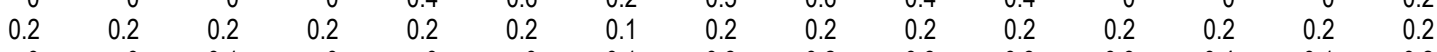

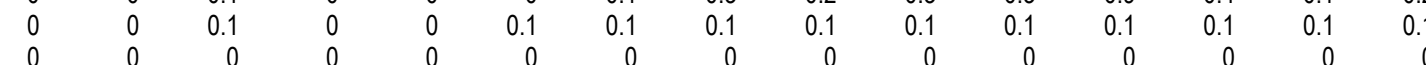

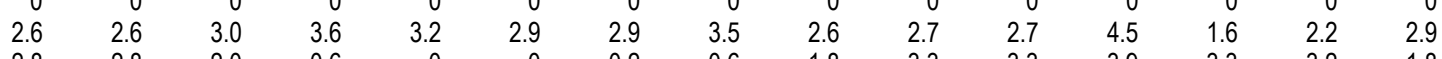

$\begin{array}{rrrrrrr}0 & 0 & 0 & 0 & 0 & 0 & 0 \\ 2.0 & 2.0 & 2.1 & 1.3 & 0.8 & 0.4 & 0.3 \\ 0.1 & 0.1 & 0.1 & 0.1 & 0.1 & 0.1 & 0.1 \\ 0.3 & 0.3 & 0.3 & 0.3 & 0.3 & 0.3 & 0.3\end{array}$

$\begin{array}{rrr}0 & 0 & 0 \\ 3 & 2.3 & 2.9\end{array}$

$\begin{array}{llll}0.3 & 0.3 & 0.4\end{array}$

$\begin{array}{lllllll}0.1 & 0.1 & 0.2 & 0.2 & 0.4 & 0.4 & 0.4 \\ 0.2 & 0.2 & 0.2 & 0.3 & 0.2 & 0.1 & 0.1\end{array}$

$\begin{array}{lllllll}0.2 & 0.2 & 0.2 & 0.3 & 0.2 & 0.2 & 0.2 \\ 0.2 & 0.2 & 0.2 & 0.2 & 0.2 & 0.2 & 0.2\end{array}$

$\begin{array}{lllllll}0.2 & 0.2 & 0.2 & 0.2 & 0.2 & 0.2 & 0.2 \\ 1.1 & 0.2 & 0.2 & 0.3 & 0.2 & 0.2 & 0.2\end{array}$

$\begin{array}{lllllll}1.1 & 1.1 & 1.6 & 1.7 & 1.2 & 1.3 & 1.1 \\ 1.8 & 1.8 & 1.8 & 1.9 & 1.9 & 1.9 & 0.6\end{array}$

$\begin{array}{rrrrrrr}0.4 & 0.4 & 0.3 & 0.3 & 0.3 & 0.1 & 0.1 \\ 0 & 0 & 0 & 0 & 0 & 0 & 0\end{array}$

$\begin{array}{rrrrrrr}0 & 0 & 0 & 0 & 0 & 0 & 0 \\ 0.4 & 0.4 & 0.3 & 0.2 & 0.3 & 0.1 & 0.1 \\ 0.4 & 0.4 & 0.3 & 0.3 & 0.4 & 0.5 & 0.5 \\ 0.1 & 0.1 & 0.2 & 0.2 & 0.1 & 0.1 & 0.1\end{array}$

$\begin{array}{rrrrrrr}0.4 & 0.4 & 0.5 & 0.4 & 0.4 & 0.1 & 0.1 \\ 0.6 & 0.6 & 0.6 & 0 & 0 & 0 & 0\end{array}$

$\begin{array}{rrrrrrr}0.6 & 0.6 & 0.6 & 0 & 0 & 0 & 0 \\ 0 & 0 & 0 & 0 & 0 & 0.1 & 0.1 \\ 0.2 & 0.2 & 0.2 & 0.2 & 0.2 & 0.2 & 0.2 \\ 0.1 & 0.1 & 0.1 & 0 & 0 & 0 & 0.1\end{array}$

$\begin{array}{llll}0.5 & 0.5 & 0.5 & 0.5\end{array}$

$\begin{array}{lllllll}3.4 & 3.4 & 3.4 & 4.7 & 6.7 & 13 & 14\end{array}$

$\begin{array}{rrrrrrr}0.9 & 0.9 & 1 & 0.8 & 0.4 & 0 & 0.3\end{array}$

$\begin{array}{rrrrrrr}0.1 & 0.1 & 0.1 & 0.1 & 0 & 0 & 0.1 \\ 0.1 & 0.1 & 0.1 & 0.1 & 0.1 & 0 & 0 \\ 0 & 0 & 0 & 0.1 & 0.2 & 0.3 & 0.3\end{array}$

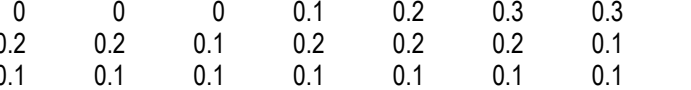

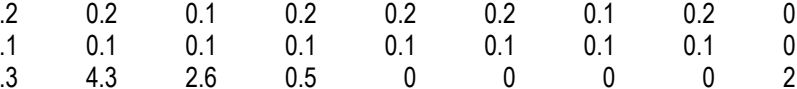

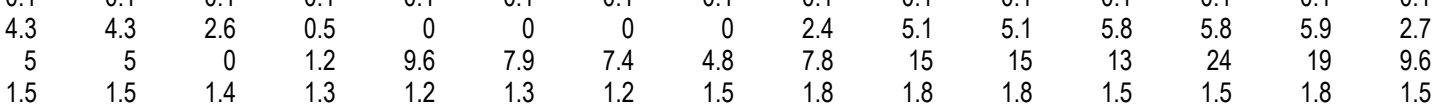

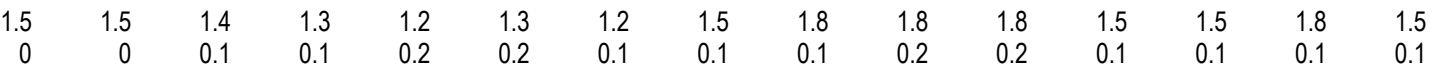

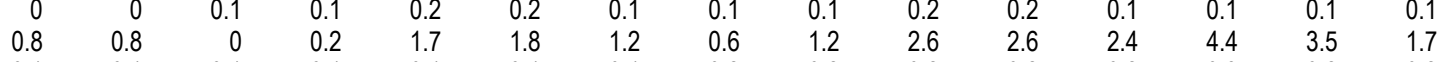

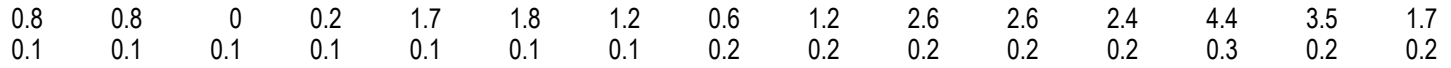

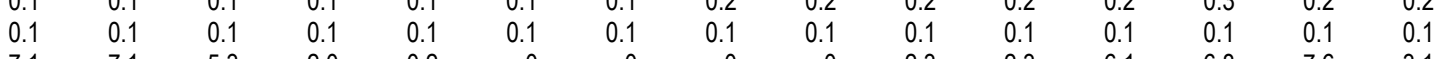

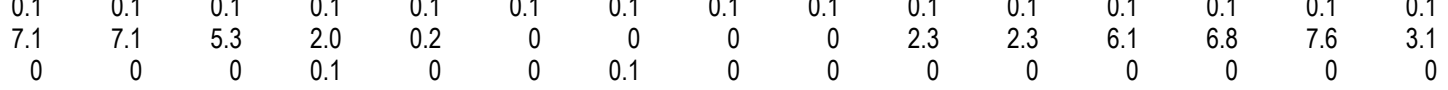

\begin{tabular}{|c|c|c|c|c|c|c|c|c|c|c|c|c|c|c|}
\hline 3.0 & $\begin{array}{r}3.0 \\
157\end{array}$ & 2.5 & 0.4 & 0 & 0 & 0 & 0 & 0 & 0 & 0 & 1.2 & 1.7 & 1.7 & $\frac{0.9}{10}$ \\
\hline & 157 & 133 & 111 & 120 & 111 & 101 & 108 & 129 & 173 & 172 & 212 & 223 & 196 & 148 \\
\hline
\end{tabular}

-NUG: Small Thermal \& Misc.-

197 Marion Solid Waste (PGE)

198 Minnesota Methane (AVWP)

199 Montana One (NWE)

200 Pocatello Waste (IPC)

201 Spokane MSW (PSE)

202 West Boise Waste (IPC)

\begin{tabular}{rrrrrrrrrrrrrrr}
9.3 & 9.3 & 9.3 & 9.3 & 9.3 & 9.3 & 9.3 & 9.3 & 9.3 & 9.3 & 9.3 & 9.3 & 9.3 & 9.3 & 9.3 \\
0.8 & 0.8 & 0.8 & 0.8 & 0.8 & 0.8 & 0.8 & 0.8 & 0.8 & 0.8 & 0.8 & 0.8 & 0.8 & 0.8 & 0.8 \\
9.1 & 9.3 & 8.6 & 8.7 & 6.5 & 7.3 & 7.6 & 7.3 & 6.3 & 6.1 & 6 & 10 & 11 & 8.3 & 8.1 \\
0.1 & 0.1 & 0.2 & 0.1 & 0.2 & 0.1 & 0.1 & 0.2 & 0.1 & 0.2 & 0.2 & 0.1 & 0.2 & 0.1 & 0.1 \\
15 & 15 & 15 & 15 & 15 & 15 & 15 & 15 & 15 & 15 & 15 & 15 & 15 & 15 & 15 \\
0.1 & 0.1 & 0.1 & 0.1 & 0.1 & 0.1 & 0.1 & 0.1 & 0.1 & 0.1 & 0.1 & 0.1 & 0.1 & 0.1 & 0.1 \\
\hline $\mathbf{3 5}$ & $\mathbf{3 5}$ & $\mathbf{3 4}$ & $\mathbf{3 4}$ & $\mathbf{3 2}$ & $\mathbf{3 3}$ & $\mathbf{3 3}$ & $\mathbf{3 3}$ & $\mathbf{3 2}$ & $\mathbf{3 2}$ & $\mathbf{3 2}$ & $\mathbf{3 6}$ & $\mathbf{3 7}$ & $\mathbf{3 4}$ & $\mathbf{3 4}$
\end{tabular}


Table A-24: Regional Non Utility Generating Resources By Project

PNW Loads and Resources Study

2006 - 2007 Operating Year 2003 White Book

Aug1 Aug16 Sep

Oct Nov Dec

Jan Feb Mar Apr1 Apr16

May Jun Jul Avg

-NUG: Combustion Turbines -

204 March Point \#1 Cogeneration (PSE)

205 March Point \#2 Cogeneration (PSE)

206 Rathdrum- Boekel Rd. \#1 (AVWP)

207 Rathdrum -Boekel Rd. \#2 (AVWP)

208 Tenaska Gas Cogen (PSE)

209 IOU - Total NUG: Combustion Turbines

\begin{tabular}{|c|c|c|c|c|c|c|c|c|c|c|c|c|c|c|}
\hline 79 & 79 & 79 & 79 & 79 & 79 & 79 & 79 & 79 & 79 & 79 & 55 & 79 & 79 & 77 \\
\hline 59 & 59 & 59 & 59 & 59 & 59 & 59 & 59 & 59 & 59 & 59 & 45 & 59 & 59 & 58 \\
\hline 65 & 65 & 65 & 65 & 65 & 65 & 65 & 65 & 65 & 65 & 65 & 32 & 65 & 65 & 62 \\
\hline 65 & 65 & 65 & 65 & 65 & 65 & 65 & 65 & 65 & 65 & 65 & 32 & 65 & 65 & 62 \\
\hline 240 & 240 & 240 & 240 & 240 & 240 & 240 & 240 & 240 & 240 & 240 & 180 & 240 & 240 & 235 \\
\hline 508 & 508 & 508 & 508 & 508 & 508 & 508 & 508 & 508 & 508 & 508 & 344 & 508 & 508 & 494 \\
\hline
\end{tabular}

-NUG: Co-Generation-

210 Billings Generation (NWE)

211 Biomass One (PPL)

212 Boise Cascade Medford (PPL)

213 Champion (PPL)

214 DR Johnson - Co-Gen II (PPL)

215 Magic Valley (IPC)

216 Magic West (IPC)

217 PERC Pierce, Wa (PSE)

218 Simplot Pocatello (IPC)

219 Sumas Energy (PSE)

220 Tamarack (IPC)

221 TASCO Nampa ID (IPC)

222 TASCO Twin Falls ID (IPC)

223 Vaagen Bros. (IPC)

224 Warm Springs (PPL)

225 IOU - Total NUG: Co-Generation

\begin{tabular}{|c|c|c|c|c|c|c|c|c|c|c|c|c|c|c|}
\hline 17 & 17 & 16 & 10 & 12 & 13 & 14 & 13 & 12 & 7.1 & 7.0 & 19 & 20 & 15 & 14 \\
\hline 22 & 22 & 21 & 21 & 22 & 22 & 7.2 & 15 & 17 & 20 & 20 & 17 & 22 & 22 & 19 \\
\hline 8.5 & 8.5 & 8.5 & 8.5 & 8.5 & 8.5 & 8.5 & 8.5 & 8.5 & 8.5 & 8.5 & 8.5 & 8.5 & 8.5 & 8.5 \\
\hline 3.3 & 3.3 & 3.3 & 3.3 & 3.3 & 3.3 & 1 & 3.3 & 3.3 & 3.3 & 3.3 & 3.3 & 3.3 & 3.3 & 3.1 \\
\hline 7.5 & 7.5 & 7.7 & 5.8 & 7.3 & 6.0 & 6.7 & 9.1 & 7.3 & 7.4 & 7.4 & 11 & 4.2 & 7.1 & 7.2 \\
\hline 9.3 & 9.3 & 9.7 & 9.3 & 9.6 & 9.3 & 9.3 & 10 & 9.4 & 9.7 & 9.7 & 9.4 & 9.7 & 9.3 & 9.5 \\
\hline 9.3 & 9.3 & 9.7 & 9.3 & 9.6 & 9.3 & 9.3 & 10 & 9.4 & 9.7 & 9.7 & 9.4 & 9.7 & 9.3 & 9.5 \\
\hline 2 & 2 & 2 & 2 & 2 & 2 & 2 & 2 & 2 & 2 & 2 & 0 & 2 & 2 & 1.8 \\
\hline 8.8 & 8.8 & 9.2 & 8.8 & 9.1 & 8.8 & 8.8 & 9.6 & 8.9 & 9.2 & 9.2 & 8.9 & 9.2 & 8.8 & 9.0 \\
\hline 113 & 113 & 126 & 126 & 126 & 126 & 126 & 126 & 126 & 113 & 113 & 113 & 113 & 113 & 120 \\
\hline 3.8 & 3.8 & 4.6 & 4.7 & 4.2 & 3.2 & 3.6 & 3.8 & 4.2 & 4.4 & 4.4 & 3.4 & 4.6 & 4.8 & 4.1 \\
\hline 0.2 & 0.2 & 0.2 & 0.2 & 0.2 & 0.2 & 0.2 & 0.2 & 0.2 & 0.2 & 0.2 & 0.2 & 0.2 & 0.2 & 0.2 \\
\hline 0 & 0 & 0 & 0 & 0 & 0 & 0 & 0 & 0 & 0 & 0 & 0 & 0 & 0 & 0 \\
\hline 3 & 3 & 3.7 & 3.8 & 3.3 & 2.6 & 2.9 & 3.1 & 3.4 & 3.6 & 3.6 & 2.7 & 3.7 & 3.8 & 3.3 \\
\hline 0.1 & 0.1 & 0 & 0.1 & 0.1 & 0.1 & 0.1 & 0.1 & 0 & 0 & 0 & 0 & 0.1 & 0 & 0.1 \\
\hline 207 & 207 & 222 & 214 & 218 & 214 & 200 & 215 & 211 & 198 & 198 & 205 & 210 & 207 & 210 \\
\hline
\end{tabular}

-NUG: Renewables-

226 Foote Creek 1 (PPL)

227 Healow \#2 (NWE)

228 Josef Staufer (NWE)

229 Lewandowski Farms (IPC)

230 Livingston (NWE)

231 Puyallup Energy Recovery (PSE)

232 Stateline Wind Project (AVWP)

233 Windmill (PGE)

234 IOU - Total NUG: Renewables

Other Entities

-NUG: Co-Generation-

\begin{tabular}{|c|c|c|c|c|c|c|c|c|c|c|c|c|c|c|}
\hline 4.2 & 4.2 & 4.9 & 7.2 & 11 & 13 & 14 & 12 & 9.2 & 7.4 & 7.4 & 5.9 & 5.2 & 4.2 & 8.1 \\
\hline 0 & 0 & 0 & 0 & 0 & 0 & 0 & 0 & 0 & 0 & 0 & 0 & 0 & 0 & 0 \\
\hline 0 & 0 & 0 & 0 & 0 & 0 & 0 & 0 & 0 & 0 & 0 & 0 & 0 & 0 & 0 \\
\hline 0 & 0 & 0 & 0 & 0 & 0 & 0 & 0 & 0 & 0 & 0 & 0 & 0 & 0 & 0 \\
\hline 0 & 0 & 0 & 0 & 0 & 0 & 0 & 0 & 0 & 0 & 0 & 0 & 0 & 0 & 0 \\
\hline 2 & 2 & 2 & 2 & 2 & 2 & 2 & 2 & 2 & 2 & 2 & 2 & 2 & 2 & 2 \\
\hline 0.3 & 0.3 & 0.2 & 0.3 & 0.3 & 0.3 & 0.3 & 0.3 & 0.3 & 0.3 & 0.3 & 0.3 & 0.3 & 0.3 & 0.3 \\
\hline 0 & 0 & 0 & 0 & 0 & 0 & 0 & 0 & 0 & 0 & 0 & 0 & 0 & 0 & 0 \\
\hline 6.5 & 6.5 & 7.1 & 9.5 & 13 & 15 & 16 & 15 & 12 & 9.8 & 9.8 & 8.2 & 7.6 & 6.5 & 10 \\
\hline
\end{tabular}

235 SP Newsprint cogen (SPN)

236 Other - Total NUG: Co-Generation

-NUG: Renewables-

237 Stateline Wind Project (PPME)

238 Vansycle Wind (FPLEV)

239 Other - Total NUG: Renewables

$\begin{array}{llll}25 & 25 & 25 & 25 \\ 25 & 25 & 25\end{array}$

$\begin{array}{lll}25 & 25 & 25 \\ 25 & 25\end{array}$
$25 \quad 25$

$25 \quad 25 \quad 25$

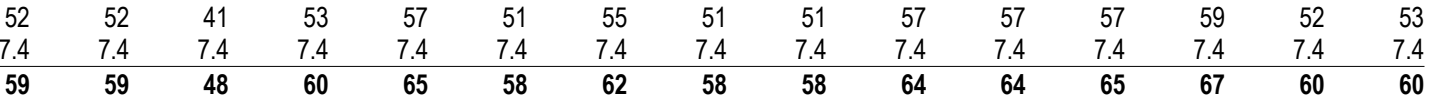

-Total Non-Utility Generating Resources-

240 Federal Entities

241 Generating Public Entities

242 Non Generating Public Entities

243 Investor-Owned Entities

244 Other Entities

245 Total Non-Utility Generation

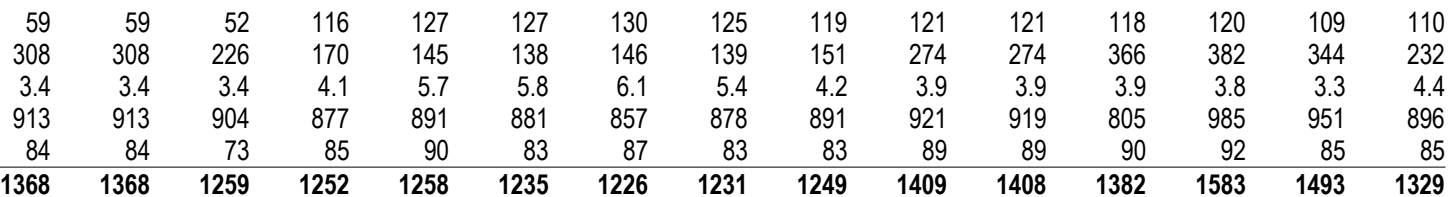


Oct Nov

Dec

\begin{abstract}
Jan Feb
\end{abstract}

\begin{abstract}
Mar Apr1 Apr16
\end{abstract}

\begin{abstract}
May
\end{abstract}
Jun

Jul Avg

\title{
Federal Entities
}

-NUG: Hydro-

1 Clearwater - State of ID DWR (BPA)

2 Dworshak Small Hydropower (BPA)

3 Federal -Total NUG: Hydro

\begin{tabular}{|c|c|c|c|c|c|c|c|c|c|c|c|c|c|c|}
\hline 1 & 1 & 1 & 1 & 1 & 1 & 1 & 1 & 1 & 1 & 1 & 1 & 1 & 1 & 1 \\
\hline 2.6 & 2.6 & 2.6 & 2.6 & 2.6 & 2.6 & 2.6 & 2.6 & 2.6 & 2.6 & 2.6 & 2.6 & 2.6 & 2.6 & 2.6 \\
\hline 3.6 & 3.6 & 3.6 & 3.6 & 3.6 & 3.6 & 3.6 & 3.6 & 3.6 & 3.6 & 3.6 & 3.6 & 3.6 & 3.6 & 3.6 \\
\hline
\end{tabular}

-NUG: Renewables-

4 Ashland Solar Project (BPA)

5 Condon Wind Project (BPA)

6 Foote Creek 1 (BPA)

7 Foote Creek 2 (BPA)

8 Foote Creek 4 (BPA)

9 Fourmile Hill Geothermal (BPA)

10 Klondike Phase 1 (BPA)

11 Stateline Wind Project (BPA)

12 Federal -Total NUG: Renewables

\begin{tabular}{|c|c|c|c|c|c|c|c|c|c|c|c|c|c|}
\hline 0 & 0 & 0 & 0 & 0 & 0 & 0 & 0 & 0 & 0 & 0 & 0 & 0 & 0 \\
\hline 10 & 10 & 11 & 13 & 14 & 13 & 12 & 12 & 14 & 14 & 12 & 15 & 11 & 12 \\
\hline 3.3 & 3.8 & 5.6 & 8.3 & 10 & 11 & 9.6 & 7.2 & 5.8 & 5.8 & 4.6 & 4.1 & 3.3 & 6.4 \\
\hline 0.4 & 0.5 & 0.7 & 1 & 1.3 & 1.3 & 1.2 & 0.9 & 0.7 & 0.7 & 0.5 & 0.5 & 0.4 & 0.8 \\
\hline 3.9 & 4.4 & 6.5 & 9.7 & 12 & 12 & 11 & 8.4 & 6.7 & 6.7 & 5.4 & 4.8 & 3.8 & 7.4 \\
\hline 50 & 50 & 50 & 50 & 50 & 50 & 50 & 50 & 50 & 50 & 50 & 50 & 50 & 50 \\
\hline 7.9 & 6.2 & 8.1 & 8.8 & 7.8 & 8.3 & 7.8 & 7.8 & 8.6 & 8.6 & 8.8 & 9.1 & 8 & 8.1 \\
\hline 29 & 23 & 30 & 33 & 29 & 31 & 29 & 29 & 32 & 32 & 33 & 34 & 30 & 30 \\
\hline 105 & 98 & 112 & 124 & 123 & 126 & 121 & 115 & 118 & 118 & 114 & 117 & 106 & 115 \\
\hline
\end{tabular}

Generating Public Entities

-NUG: Hydro-

13 Don Steffen (SHPD)

14 Eltopia Br Canal 4.6 (SCL)

15 Eltopia Br Canal 4.6 (TPU)

16 John Llewellyn (SHPD)

17 Kevin Duncan (SHPD)

18 Lucky Peak (SCL)

19 Main Canal Headworks (SCL)

20 Main Canal Headworks (TPU)

21 PEC Headworks (GCPD)

22 Pickering Micro Hydro (CKPD)

23 Potholes E Canal 66.0 (SCL)

24 Potholes E Canal 66.0 (TPU)

25 Quincy Chute (GCPD)

26 Russel D. Smith (SCL)

27 Russel D. Smith (TPU)

28 Smith Creek (EWEB)

29 Summer Falls (SCL)

30 Summer Falls (TPU)

31 Woods Creek (SHPD)

32 GPU - Total NUG: Hydro

\begin{tabular}{rrrrrrrrrrrrrrr}
0 & 0 & 0 & 0 & 0 & 0 & 0 & 0 & 0 & 0 & 0 & 0 & 0 & 0 & 0 \\
0.9 & 0.9 & 0.6 & 0.3 & 0 & 0 & 0 & 0 & 0 & 0.7 & 0.7 & 1 & 1 & 1.1 & 0.5 \\
0.9 & 0.9 & 0.6 & 0.3 & 0 & 0 & 0 & 0 & 0 & 0.7 & 0.7 & 1 & 1 & 1.1 & 0.5 \\
0 & 0 & 0 & 0 & 0 & 0 & 0 & 0 & 0 & 0 & 0 & 0 & 0 & 0 & 0 \\
0 & 0 & 0 & 0 & 0 & 0 & 0 & 0 & 0 & 0 & 0 & 0 & 0 & 0 & 0 \\
66 & 66 & 41 & 11 & 2.2 & 3 & 7.9 & 10 & 21 & 52 & 52 & 84 & 85 & 77 & 38 \\
10 & 10 & 6.1 & 2.6 & 0 & 0 & 0 & 0 & 1.4 & 6.8 & 6.8 & 9.1 & 11 & 12 & 4.9 \\
10 & 10 & 6.1 & 2.6 & 0 & 0 & 0 & 0 & 1.4 & 6.8 & 6.8 & 9.1 & 11 & 12 & 4.9 \\
4.5 & 4.5 & 3.1 & 2.2 & 0 & 0 & 0 & 0 & 0.4 & 5.1 & 5.1 & 6.9 & 6.2 & 5.8 & 2.9 \\
0 & 0 & 0 & 0 & 0 & 0 & 0 & 0 & 0 & 0 & 0 & 0 & 0 & 0 & 0 \\
0.9 & 0.9 & 0.9 & 0.8 & 0 & 0 & 0 & 0 & 0.3 & 0.9 & 0.9 & 0.9 & 0.9 & 0.9 & 0.5 \\
0.9 & 0.9 & 0.9 & 0.8 & 0 & 0 & 0 & 0 & 0.3 & 0.9 & 0.9 & 0.9 & 0.9 & 0.9 & 0.5 \\
7.7 & 7.7 & 4 & 2.3 & 0 & 0 & 0 & 0 & 0.8 & 5.5 & 5.5 & 7.1 & 8.4 & 8.5 & 3.7 \\
2 & 2 & 1.3 & 0.7 & 0 & 0 & 0 & 0 & 0.1 & 1.2 & 1.2 & 1.9 & 2.1 & 1.7 & 0.9 \\
2 & 2 & 1.3 & 0.7 & 0 & 0 & 0 & 0 & 0.1 & 1.2 & 1.2 & 1.9 & 2.1 & 1.7 & 0.9 \\
0 & 0 & 0 & 5 & 5.4 & 3.9 & 0 & 0.8 & 1.5 & 9.2 & 9.2 & 37 & 37 & 11 & 9.2 \\
39 & 39 & 24 & 11 & 0 & 0 & 0 & 0 & 2.4 & 25 & 25 & 36 & 40 & 44 & 18 \\
39 & 39 & 24 & 11 & 0 & 0 & 0 & 0 & 2.4 & 25 & 25 & 36 & 40 & 44 & 18 \\
0.1 & 0.1 & 0.1 & 0.1 & 0.1 & 0.1 & 0.1 & 0.1 & 0.1 & 0.1 & 0.1 & 0.1 & 0.1 & 0.1 & 0.1 \\
\hline 185 & 185 & 115 & $\mathbf{5 1}$ & $\mathbf{7 . 7}$ & $\mathbf{7}$ & $\mathbf{8}$ & $\mathbf{1 1}$ & $\mathbf{3 2}$ & $\mathbf{1 4 1}$ & $\mathbf{1 4 1}$ & $\mathbf{2 3 2}$ & $\mathbf{2 4 7}$ & $\mathbf{2 2 1}$ & $\mathbf{1 0 5}$
\end{tabular}

-NUG: Small Thermal \& Misc.-

33 Grays Harbor Paper (GHPD)

34 Weyerhaeuser Pulp Mill (GHPD)

35 GPU - Total NUG: Small Thermal \& Misc.

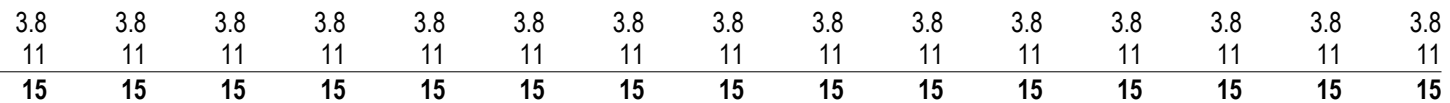

-NUG: Co-Generation-

36 Metro Westpoint (SCL)

37 Scott Paper (SHPD)

38 GPU - Total NUG: Co-Generation

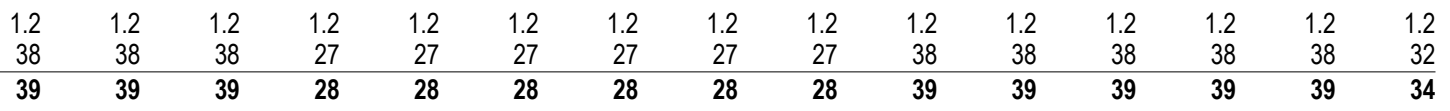

-NUG: Renewables-

39 Foote Creek 1 (EWEB)

40 Nine Canyon Wind Project (CHPD)

41 Nine Canyon Wind Project (COPD)

42 Nine Canyon Wind Project (DOPD)

43 Nine Canyon Wind Project (GCPD)

44 Nine Canyon Wind Project (GHPD)

45 Nine Canyon Wind Project (OKPD)

46 Stateline Wind Project (EWEB)

47 Stateline Wind Project (SCL)

48 GPU - Total NUG: Renewables

Non-Generating Public Entities

-NUG: Hydro-

49 Buffalo Hydro (FREC)

50 Burr \& Bouchard (TNEC)

51 City of Cove (OTEC)

52 Falls Creek (CCPD)

53 Lilliwaup Falls Generating Co. (MCPD1)

54 Thomas Burnside (TNEC)

$\begin{array}{rrrrrrrrrrrrrrr}1.4 & 1.4 & 1.6 & 2.4 & 3.5 & 4.3 & 4.5 & 4.1 & 3.1 & 2.5 & 2.5 & 2.0 & 1.7 & 1.4 & 2.7 \\ 1.5 & 1.5 & 1.4 & 2.2 & 3.8 & 3.8 & 4.2 & 3.4 & 2.3 & 2.0 & 2.0 & 2.0 & 1.9 & 1.4 & 2.5 \\ 0.4 & 0.4 & 0.4 & 0.5 & 0.9 & 0.9 & 1 & 0.9 & 0.6 & 0.5 & 0.5 & 0.5 & 0.5 & 0.3 & 0.6 \\ 1.8 & 1.8 & 1.7 & 2.6 & 4.6 & 4.7 & 5.1 & 4.2 & 2.8 & 2.4 & 2.4 & 2.4 & 2.3 & 1.7 & 3 \\ 2.2 & 2.2 & 2.1 & 3.2 & 5.7 & 5.7 & 6.3 & 5.2 & 3.4 & 3.0 & 3.0 & 3.0 & 2.8 & 2.1 & 3.7 \\ 1.5 & 1.5 & 1.4 & 2.2 & 3.8 & 3.8 & 4.2 & 3.4 & 2.3 & 2.0 & 2.0 & 2.0 & 1.9 & 1.4 & 2.5 \\ 3.0 & 3.0 & 2.8 & 4.3 & 7.5 & 7.6 & 8.4 & 6.9 & 4.6 & 3.9 & 3.9 & 3.9 & 3.7 & 2.7 & 4.9 \\ 8.4 & 8.4 & 6.6 & 8.6 & 9.3 & 8.2 & 8.8 & 8.3 & 8.3 & 9.2 & 9.2 & 9.3 & 9.6 & 8.5 & 8.6 \\ 49 & 49 & 39 & 50 & 54 & 48 & 52 & 48 & 48 & 54 & 54 & 54 & 56 & 50 & 50 \\ \mathbf{6 9} & \mathbf{6 9} & \mathbf{5 7} & \mathbf{7 6} & \mathbf{9 3} & \mathbf{8 7} & \mathbf{9 4} & \mathbf{8 5} & \mathbf{7 5} & \mathbf{7 9} & \mathbf{7 9} & \mathbf{7 9} & \mathbf{8 1} & \mathbf{6 9} & \mathbf{7 9}\end{array}$

-NUG: Small Thermal \& Misc.-

56 Methane Energy Agricultural D. (TKPD)

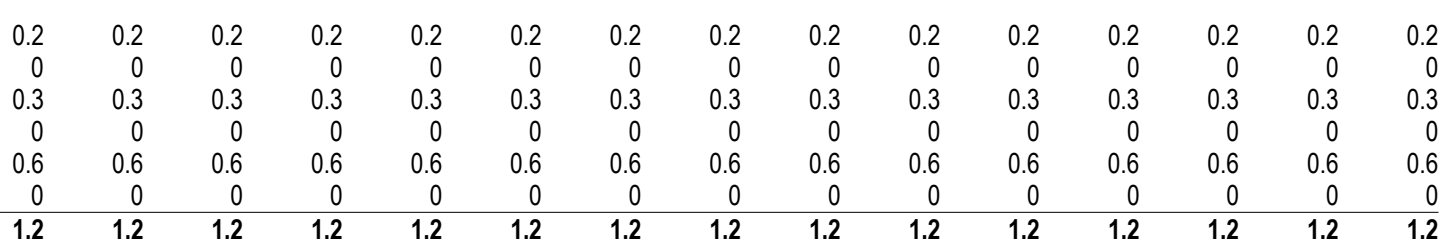

57 NGP - Total NUG: Small Thermal \& Misc.

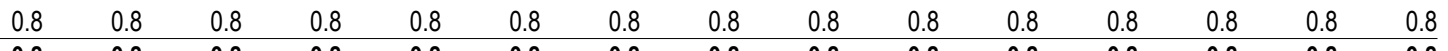


-NUG: Renewables.

58 Ashland Solar Project (ASHL)

59 Nine Canyon Wind Project (BCPD)

60 Nine Canyon Wind Project (ENW)

61 Nine Canyon Wind Project (LCPD)

62 Nine Canyon Wind Project (MCPD3)

64 NGP - Total NUG: Renewables

Investor-Owned Entities

-NUG: Hydro-

65 Barber Dam (IPC)

66 Barney Creek (NWE)

67 Beaverton (PGE)

68 Bell Mountain (UPL)

69 Bingham Engineering (NWE)

70 Birch Creek (IPC)

71 Birch Creek (UPL)

72 Black Canyon \#3 (IPC)

73 Black Creek (AVWP)

74 Blind Canyon Hydro (IPC)

75 Box Canyon (IPC)

76 Boyd James (PPL)

77 Briggs Creek (IPC)

78 Broadwater Dam (NWE)

79 Bypass (IPC)

80 Canyon Springs (IPC)

81 Carlton (PGE)

82 Cascade Creek (NWE)

83 CDM Hydro - Bonneville Pacific (PPL)

84 Cedar Draw (IPC)

85 Central OR Irrigation District (PPL)

86 Clear Springs Trout (IPC)

87 Corbett (PGE)

88 Cornelius (PGE)

89 Crystal Springs (IPC)

90 Curry Cattle Co. (IPC)

91 Dairy Creek (PGE)

92 Deep Creek (AVWP)

93 Derr Creek (AVWP)

94 Dietrich Drop (IPC)

95 Donald Jenni (NWE)

96 Eagle Creek (PGE)

97 Eagle Point Irrigation Dist. (PPL)

98 Elk Creek (IPC)

99 Estacada (PGE)

100 Falls Creek (PPL)

101 Falls River (IPC)

102 Farmers Irrig. (PPL)

103 Faulkner Ranch (IPC)

104 Fisheries Development Co (IPC)

105 Galesville Dam (PPL)

106 Geobon 2 (IPC)

107 Georgetown Power (UPL)

108 Hailey Cspp (IPC)

109 Hazelton A (IPC)

110 Hazelton B (IPC)

111 Horseshoe Bend (IPC)

112 Hutchinson Creek (PSE)

113 Ingram Warm Springs (UPL)

$114 \mathrm{Jim}$ Ford Creek (AVWP)

115 Jim Knight (IPC)

116 John Day Creek (AVWP)

117 Joseph Hydro (PPL)

118 Kasel \& Witherspoon (IPC)

119 Koma Kulshan (PSE)

120 Koyle (IPC)

$121 \mathrm{~L}$. Fery (PPL)

122 Lacomb Irrig. (PPL)

123 Lake Oswego (PGE)

124 Lateral \#10 (IPC)

125 Lee Tavenner (NWE)

126 Lemoyne (IPC)

127 Little Wood River Res. (IPC)

128 Littlewood/Arkoosh (IPC)

129 Lowline \#2 (IPC)

\begin{tabular}{|c|c|c|c|c|c|c|c|c|c|c|c|c|c|c|}
\hline 0 & 0 & 0 & 0 & 0 & 0 & 0 & 0 & 0 & 0 & 0 & 0 & 0 & 0 & 0 \\
\hline 0.6 & 0.6 & 0.5 & 0.8 & 1.4 & 1.4 & 1.6 & 1.3 & 0.9 & 0.7 & 0.7 & 0.7 & 0.7 & 0.5 & 0.9 \\
\hline 0.4 & 0.4 & 0.4 & 0.5 & 0.9 & 1.0 & 1.1 & 0.9 & 0.6 & 0.5 & 0.5 & 0.5 & 0.5 & 0.3 & 0.6 \\
\hline 0.2 & 0.2 & 0.2 & 0.3 & 0.5 & 0.5 & 0.5 & 0.4 & 0.3 & 0.2 & 0.2 & 0.2 & 0.2 & 0.2 & 0.3 \\
\hline 0.4 & 0.4 & 0.4 & 0.5 & 0.9 & 1.0 & 1 & 0.9 & 0.6 & 0.5 & 0.5 & 0.5 & 0.5 & 0.3 & 0.6 \\
\hline 0 & 0 & 0 & 0 & 0 & 0 & 0 & 0 & 0 & 0 & 0 & 0 & 0 & 0 & 0 \\
\hline 1.5 & 1.5 & 1.4 & 2.2 & 3.8 & 3.8 & 4.2 & 3.5 & 2.3 & 2.0 & 2.0 & 2.0 & 1.9 & 1.4 & 2.5 \\
\hline
\end{tabular}

2.4

$\begin{array}{rrr}0 & 0 & 0 \\ 0.1 & 0.1 & 0.1\end{array}$

$\begin{array}{rrrrrrr}0.1 & 0.1 & 0.1 & 0.1 & 0.1 & 0.1 & 0.1\end{array}$

$\begin{array}{rrrrrr}0 & 0 & 0.1 & 0.1 & 0.1 & 0.1\end{array}$

$\begin{array}{llll}0.1 & 0.1 & 0.1 & 0.1 \\ 3.4 & 3.1 & 3.7\end{array}$

$\begin{array}{lll}0.1 & 0.1 & 0.1 \\ 3.4 & 3.1 & 3.7\end{array}$

$\begin{array}{llll}0.7 & 0.7 & 1.1 & 0.9\end{array}$

$\begin{array}{rrr}0 & 0 & 0 \\ 0.6 & 0.6 & 0.6\end{array}$

$\begin{array}{llll}1.1 & 1.1 & 1.5 & 1.8\end{array}$

$\begin{array}{llll}8.3 & 8.3 & 6.5 & 2.8\end{array}$

0
0

$2.9 \quad 2.9$

0.3

3.1
0.4

0.4
0

0
1.3

1.3
0.2

0.2

$\begin{array}{lll}0 & 0 & 0\end{array}$

0.1

4.7

0.1

0
12

1.2
0.1

0.1
0

6.5
0.6

0.6

0.8

0.2
0.6

0.6
1.0

$\begin{array}{rr}1.0 & 1.0 \\ 0 & 0\end{array}$

0.1

6.6

8.8

0.7

0.2

0
0.4

0.2

1.5

1.2

4.6
1.1
0

1.1
0

0

$0.4 \quad 0.4$

$1.5 \quad 1.5$

0.10 .1

1.9

0.7

2.7

$\begin{array}{rr}1.0 & 2.2 \\ 0 & 0 \\ 0 & 0 \\ 0.1 & 0.1 \\ 0.1 & 0.1 \\ 0.1 & 0 \\ 1.1 & 0.3 \\ 0 & 0 \\ 3.1 & 2.8 \\ 0 & 0 \\ 0.3 & 0.3 \\ 0.4 & 0.3 \\ 0.6 & 0.6 \\ 1.2 & 1.1 \\ 0 & 0 \\ 0.1 & 0.1 \\ 0 & 0.1\end{array}$

2.4
1.2

0.7

0.6

$\begin{array}{lll}0.4 & 3.7 & 3.5 \\ 0.4 & 0.5\end{array}$

0
1.3
0.2

$0.1 \quad 0.1 \quad 0.1$

$\begin{array}{lll}0.7 & 3.6 & 3.1\end{array}$

$\begin{array}{rrr}0.1 & 0.1 & 0.1 \\ 0 & 0 & \end{array}$

1.2

0.1
0

6.5
0.6

0.8
0.2

0.2
0.6

0.1

0
0.1

0
6.3

0.7

1.2

$\begin{array}{ll}0.3 & 0.2 \\ 0.4 & 0.2\end{array}$

$\begin{array}{ll}0.4 & 0.2 \\ 0.3 & 0.3 \\ 0.2 & 0.3\end{array}$

0.1
7

$0.1 \quad 0.1$

$\begin{array}{lll}8.8 & 5.2 & 2.4 \\ 0.7 & 0.7 & 3.9\end{array}$

0.7

$\begin{array}{lll}8.8 & 7.5 & 3.9 \\ 0.7 & 0.7 & 0.7 \\ 0.2 & 0.3 & 0.3\end{array}$

0.3

$\begin{array}{ll}0.3 & 0.2\end{array}$

0.1

0.9

0.7

$2.8 \quad 3.5$

$\begin{array}{ll}0.7 & 0.7\end{array}$

$0.5 \quad 0.5$

$\begin{array}{lll}0.4 & 0.3 & 0.2\end{array}$

$\begin{array}{ll}0.3 & 0.5 \\ 0.3 & 0.1\end{array}$

$\begin{array}{ll}0.2 & 0.2\end{array}$

0.2

$\begin{array}{ll}0.2 & 0.2\end{array}$

0.9

$4.6 \quad 4.3$

0.4

$0.1-0.1$

0.2

6.4
1.0

0

$\begin{array}{rrrrr}0 & 0 & 0.2 & 1.0 & 0.2\end{array}$

0.1

$\begin{array}{ll}1.5 & 1.5\end{array}$

0.1

0.1
0

$\begin{array}{lll}1.9 & 0.2 & 0.2\end{array}$

0.2

2.7

2.5

0
0.2
0

$\begin{array}{rr}0.8 & 0.3 \\ 0 & 0 \\ 0 & 0.1 \\ 0.2 & 0.1 \\ 0.1 & 0 \\ 0.1 & 0.1 \\ 1.0 & 1.1 \\ 0.1 & 0.1 \\ 3.4 & 3.1 \\ 0.9 & 0.2 \\ 0.4 & 0.4 \\ 0 & 0 \\ 0.6 & 0.6 \\ 1.8 & 1.5 \\ 2.8 & 1.0 \\ 0.1 & 0.1 \\ 0 & 0 \\ 0 & 0\end{array}$

$\begin{array}{rr}2.6 & 3.3 \\ 0 & \\ 0 & \\ 0.1 & 0.1 \\ 0.1 & \\ 0 & \\ 1.2 & 1.7 \\ 0 & 0.1 \\ 3.1 & 3.1 \\ 0 & \\ 0.3 & 0.3 \\ 0.6 & 0.7 \\ 0.7 & 0.6 \\ 1.2 & 1.2 \\ 0 & 0.1 \\ 0.1 & 0.1 \\ 0.1 & 0.1\end{array}$

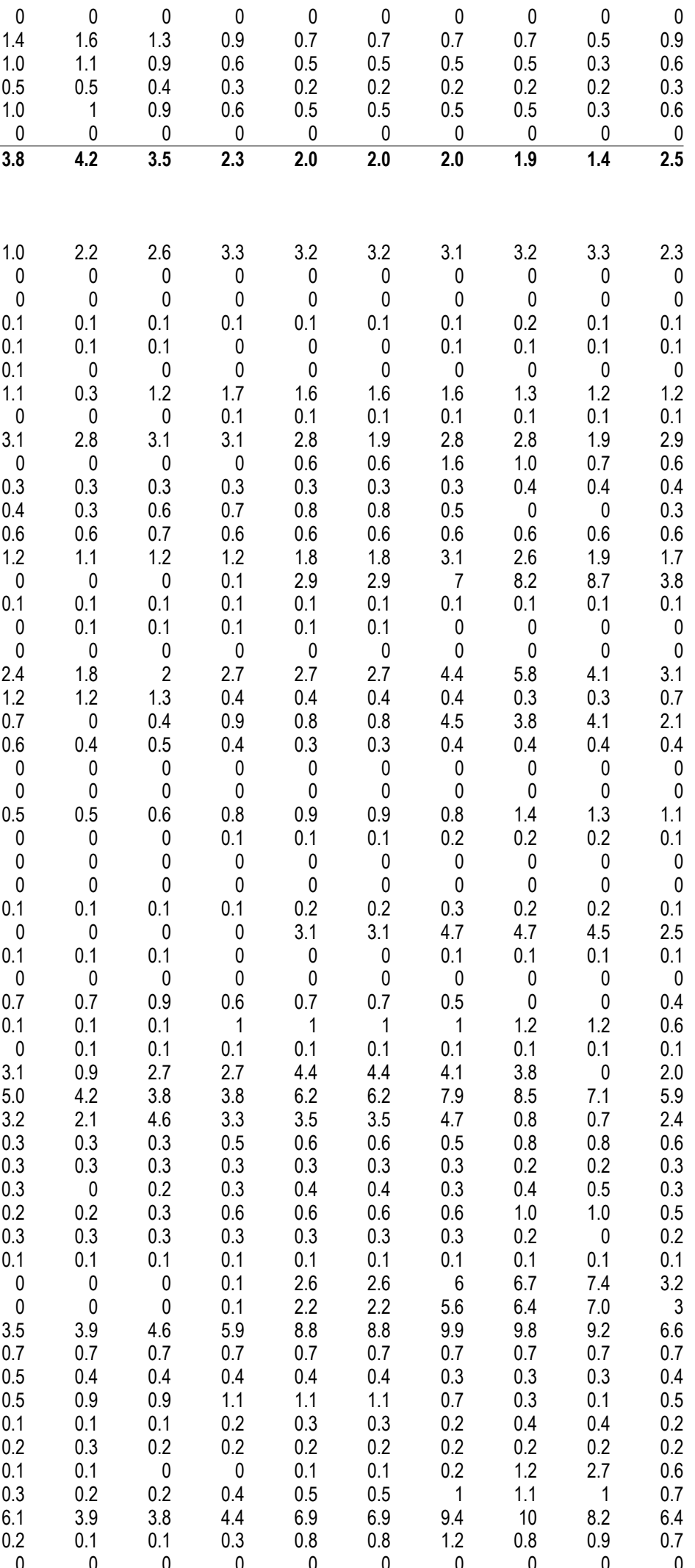

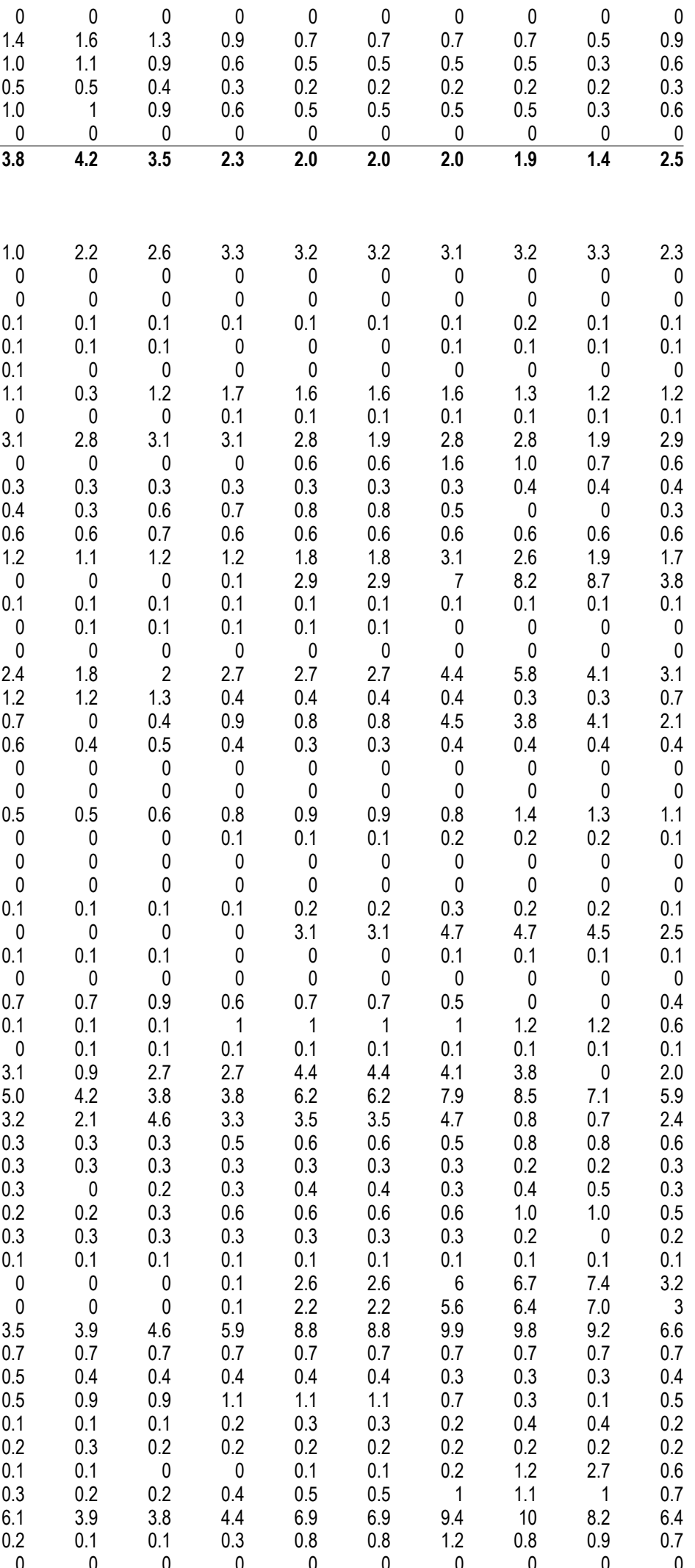

$\begin{array}{ll}0 & \\ 2 & 2\end{array}$

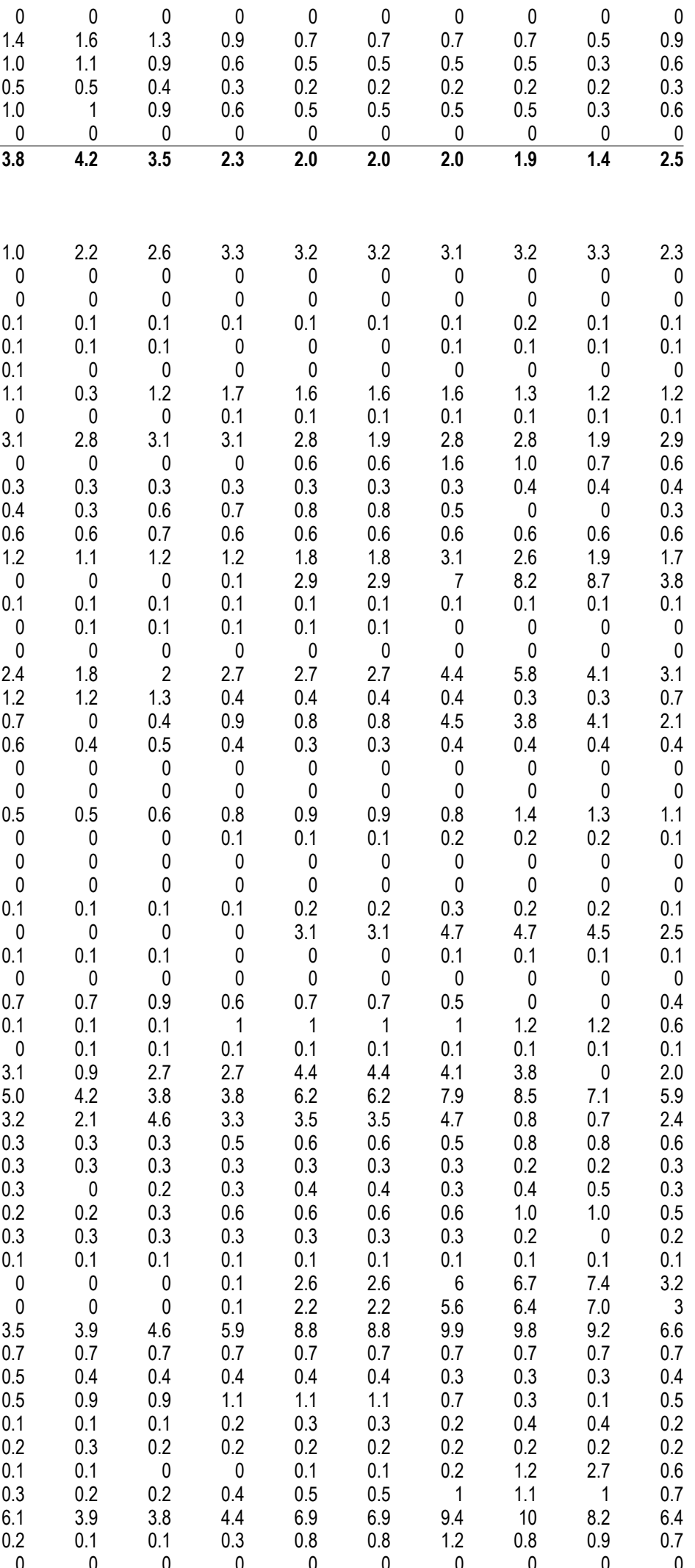

$0.4 \quad 0.9$

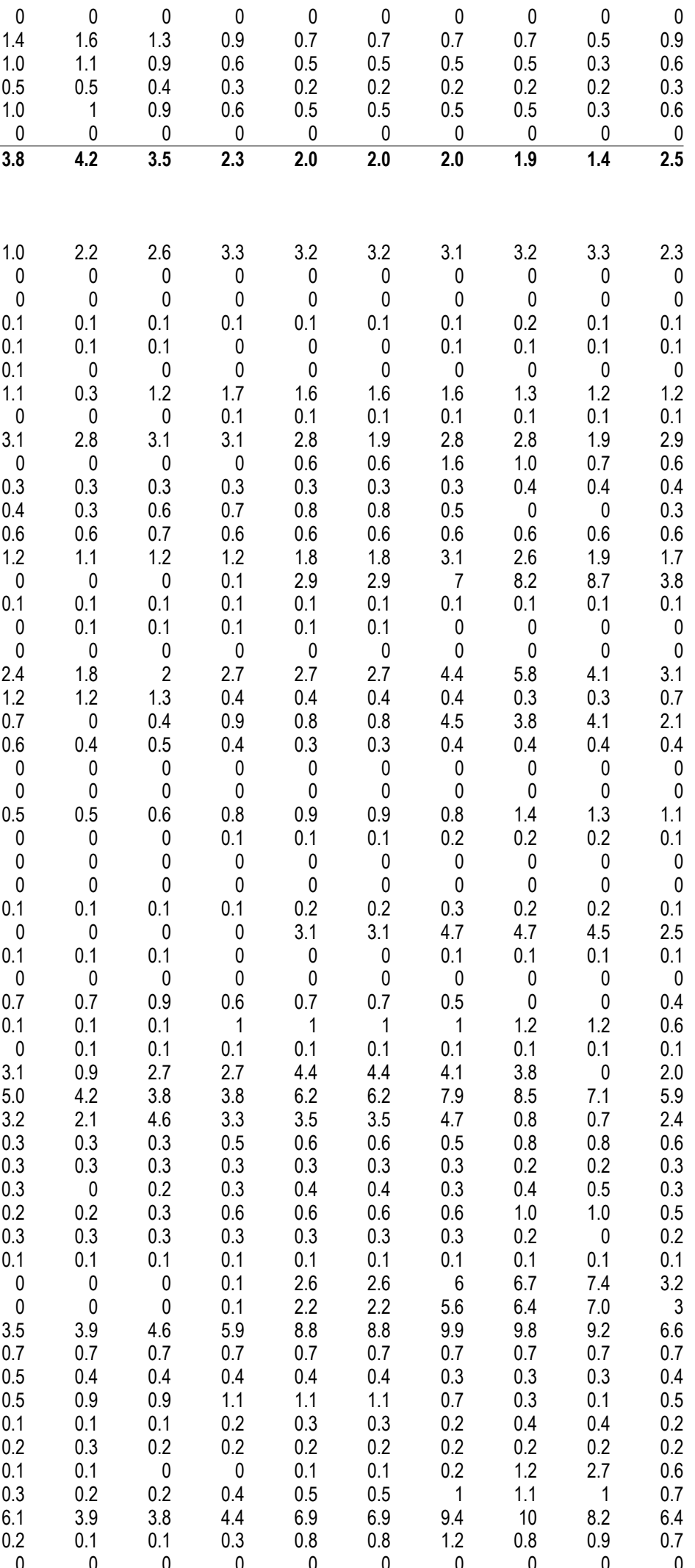

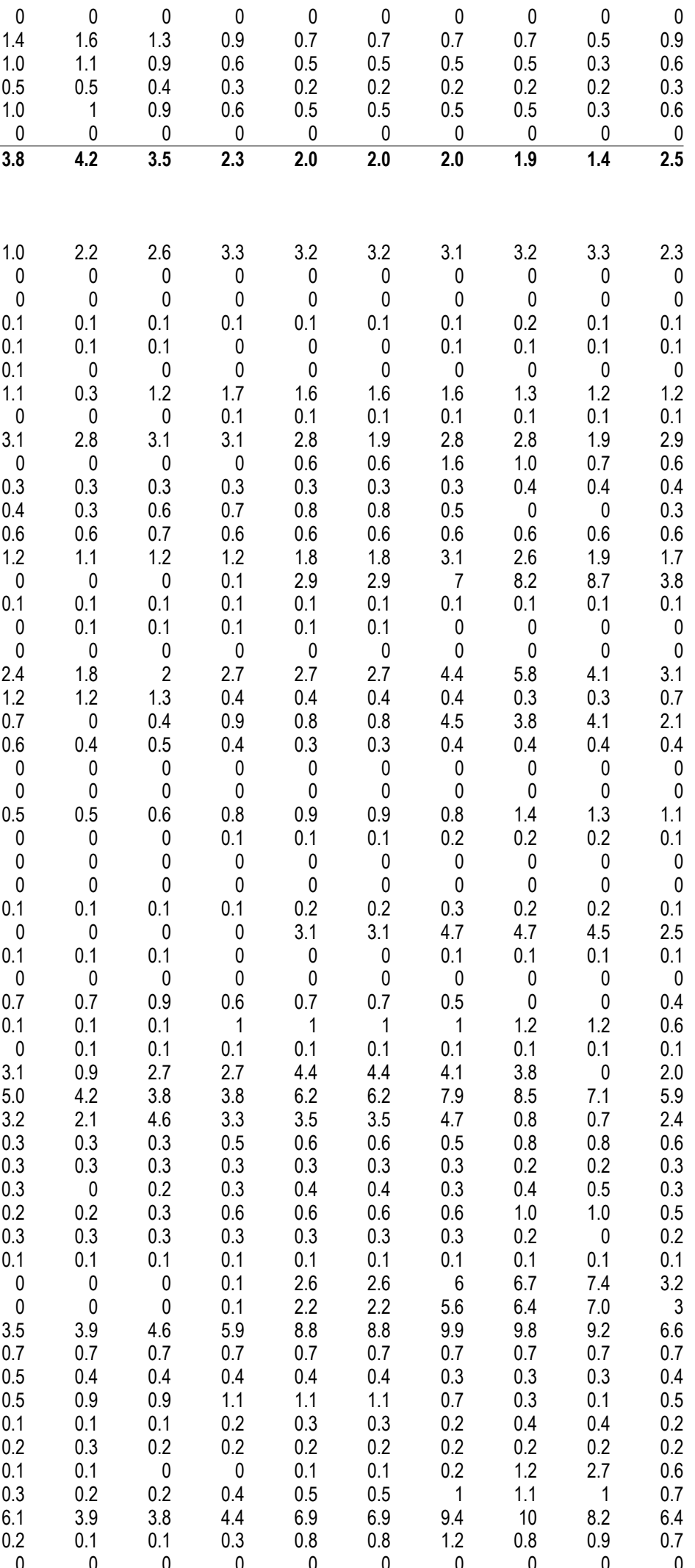

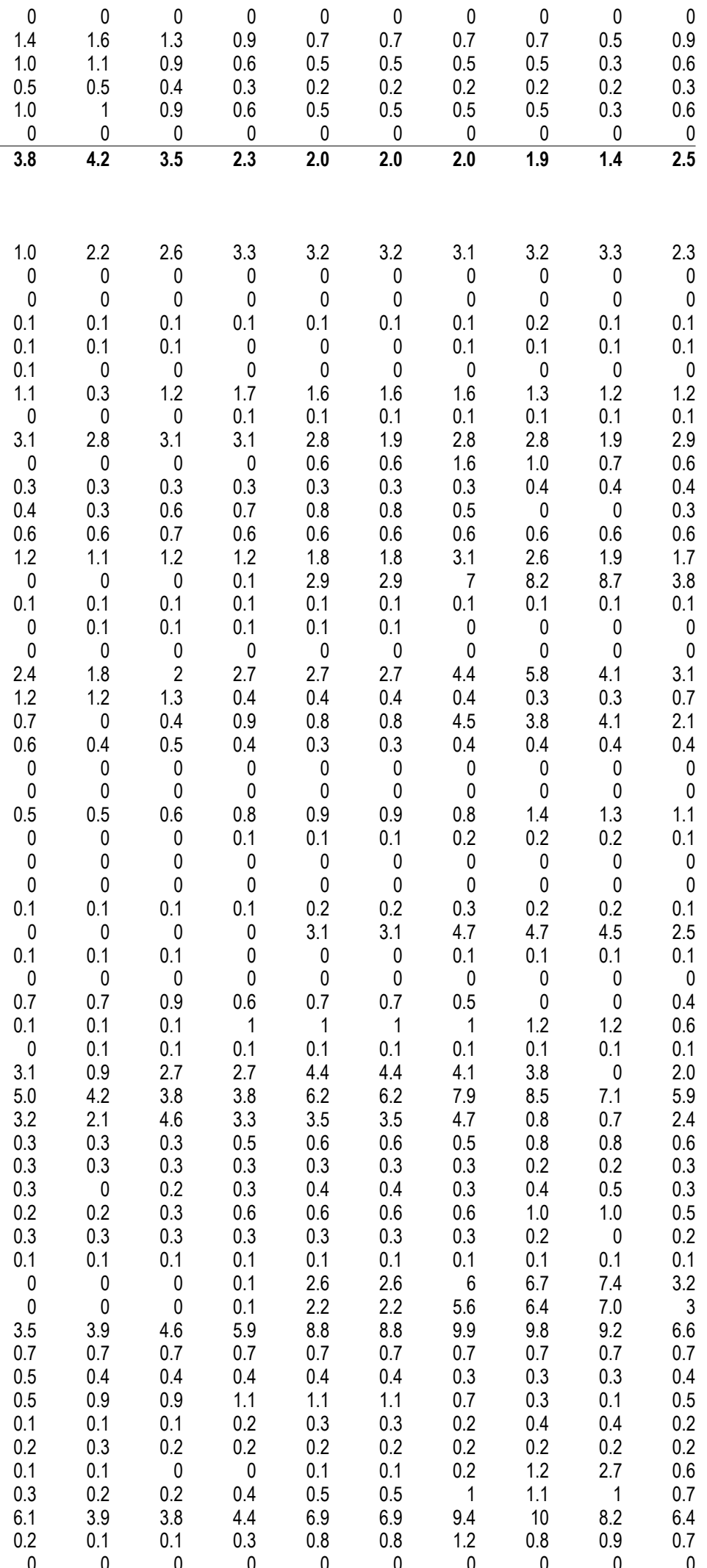

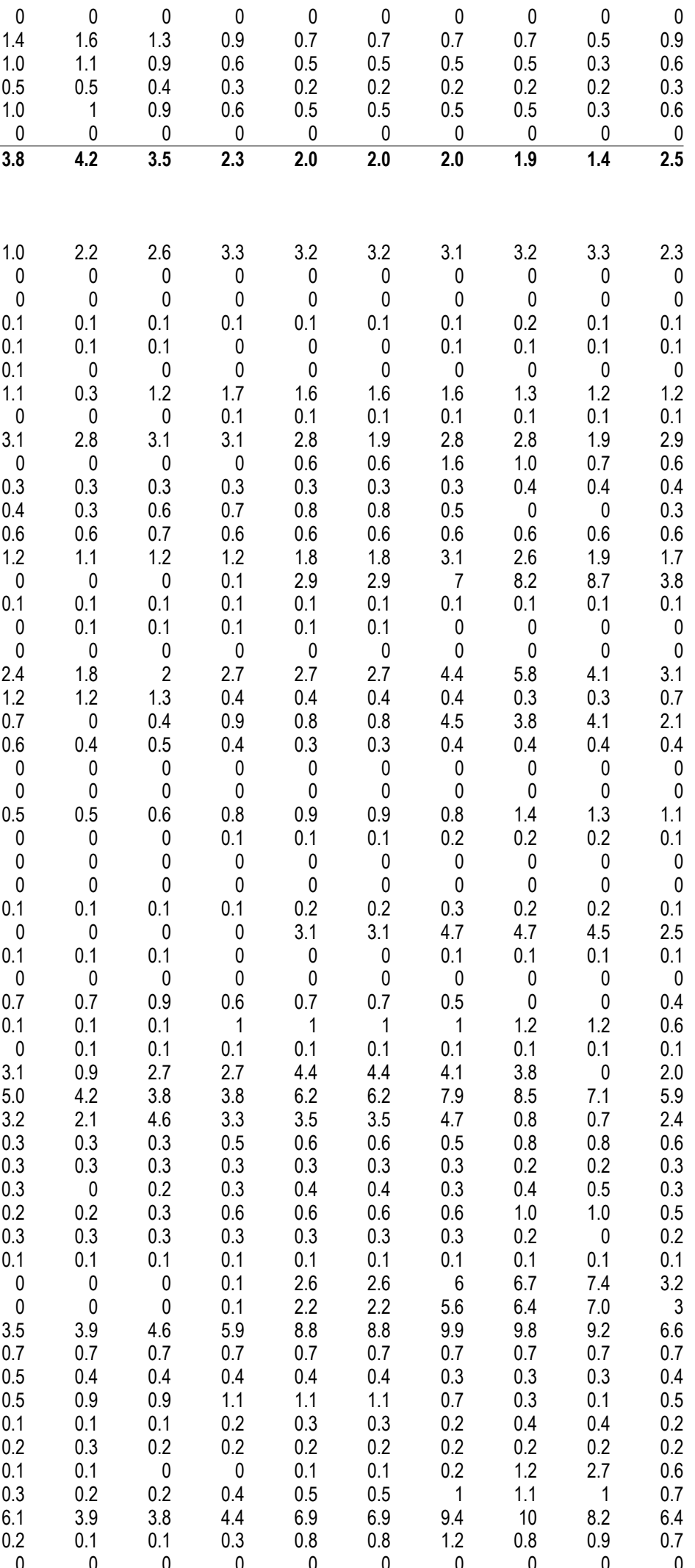

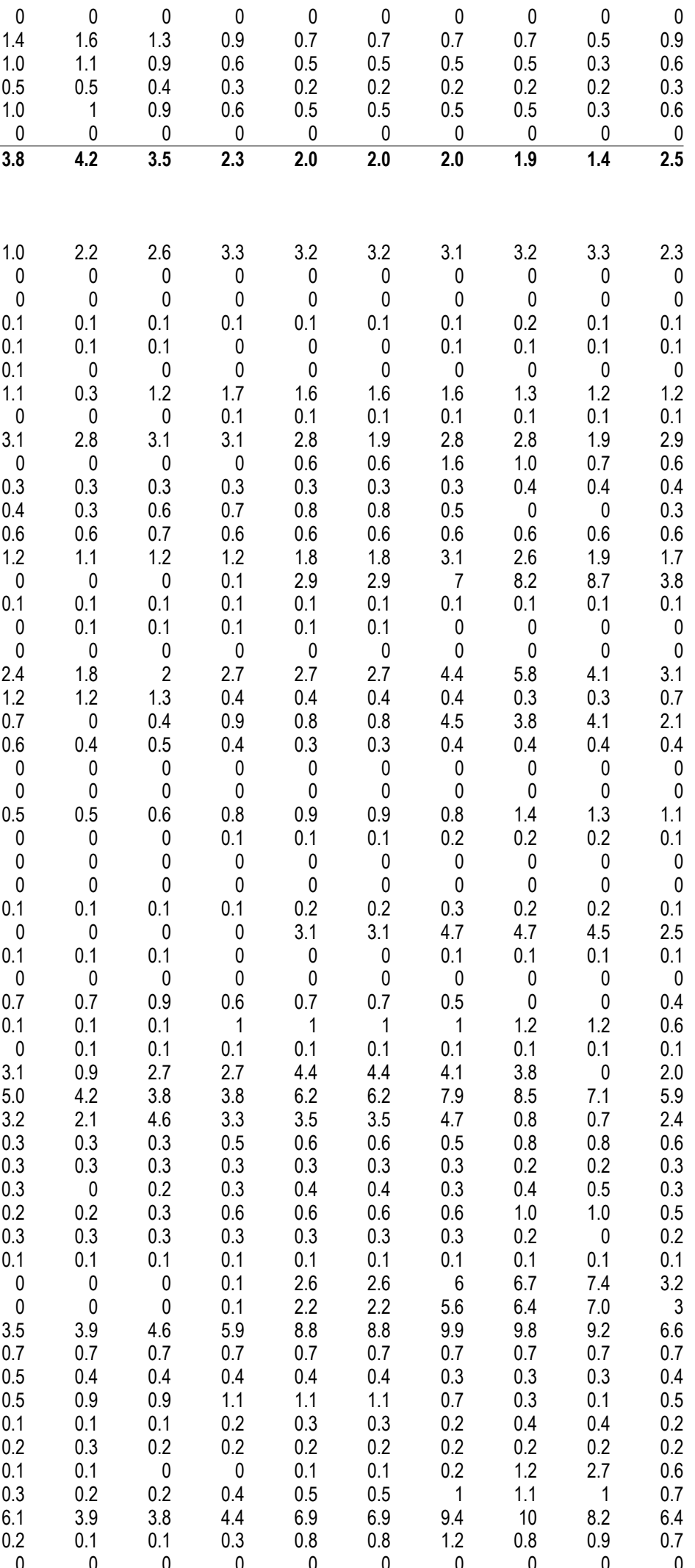

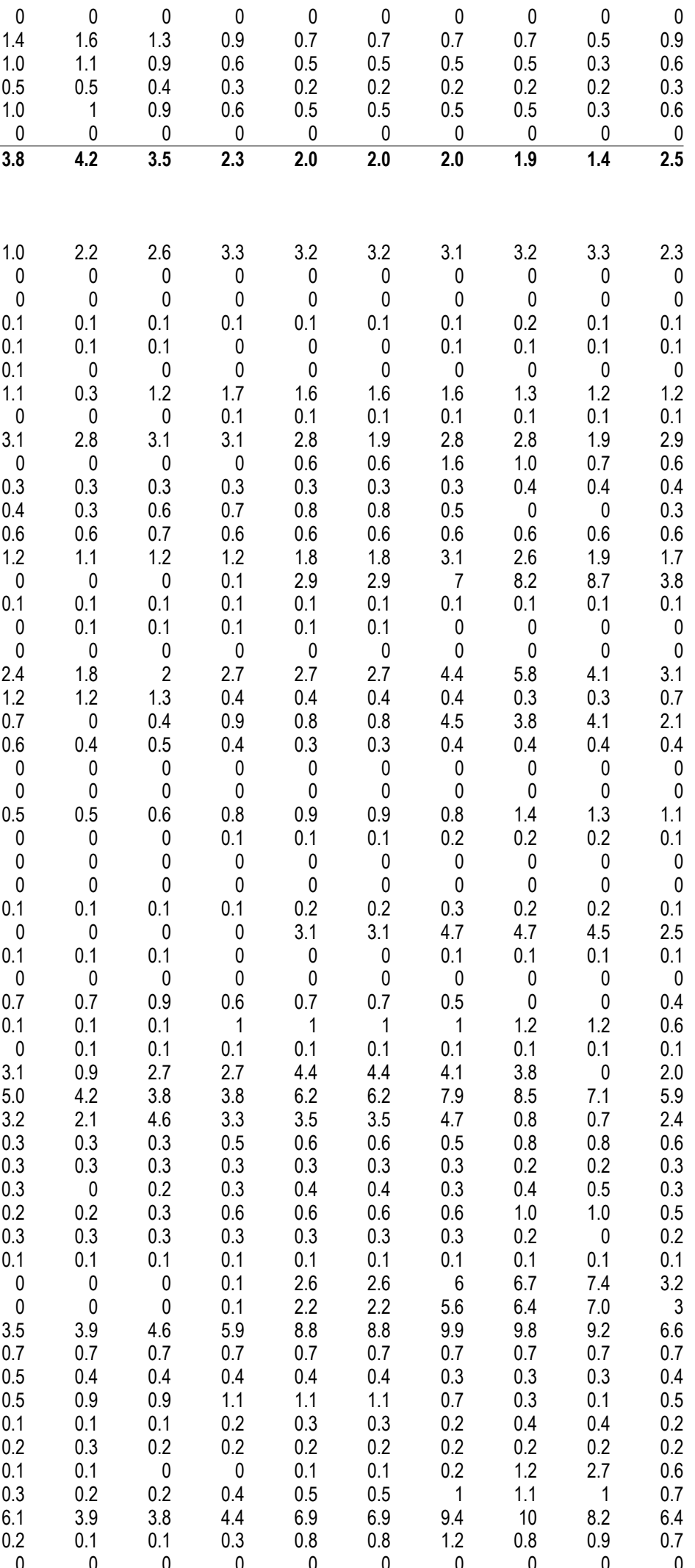

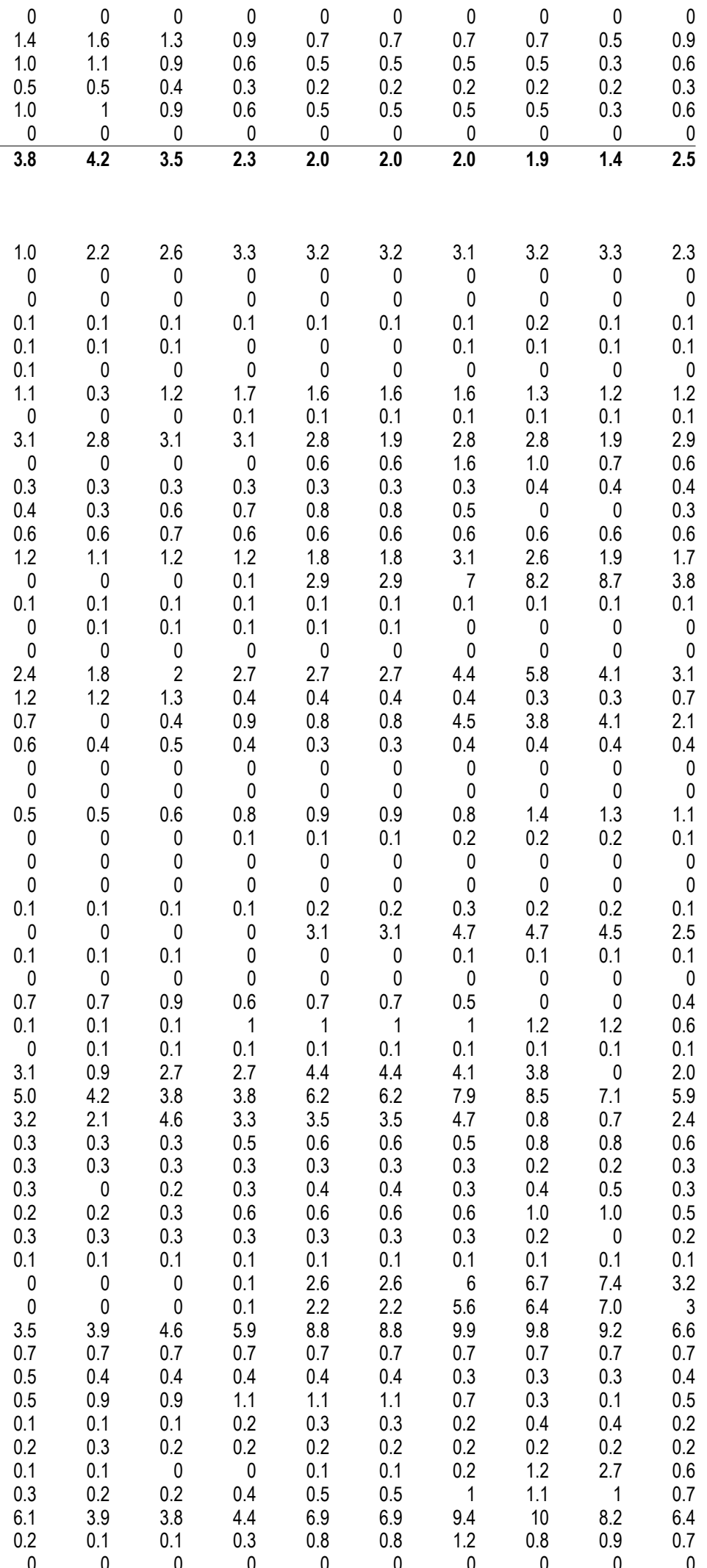

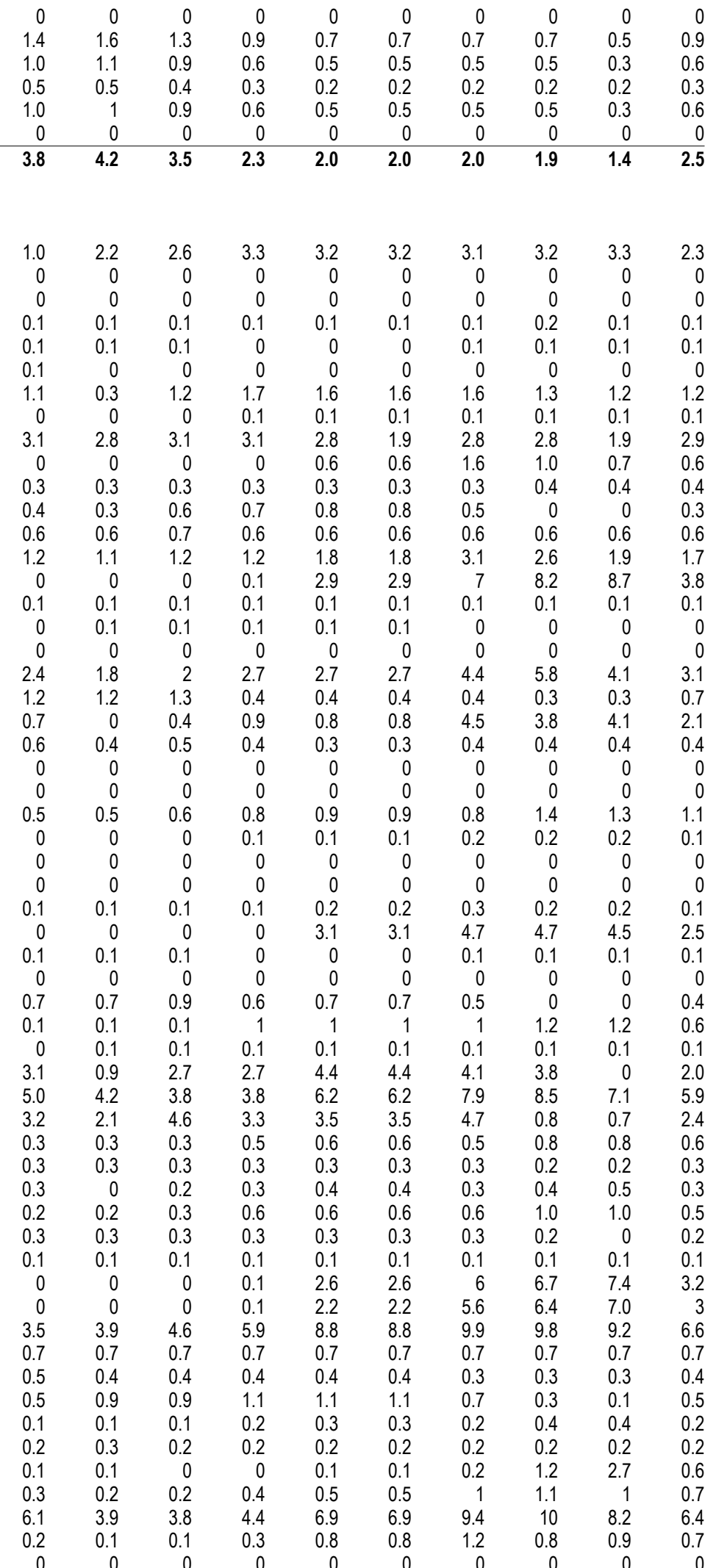

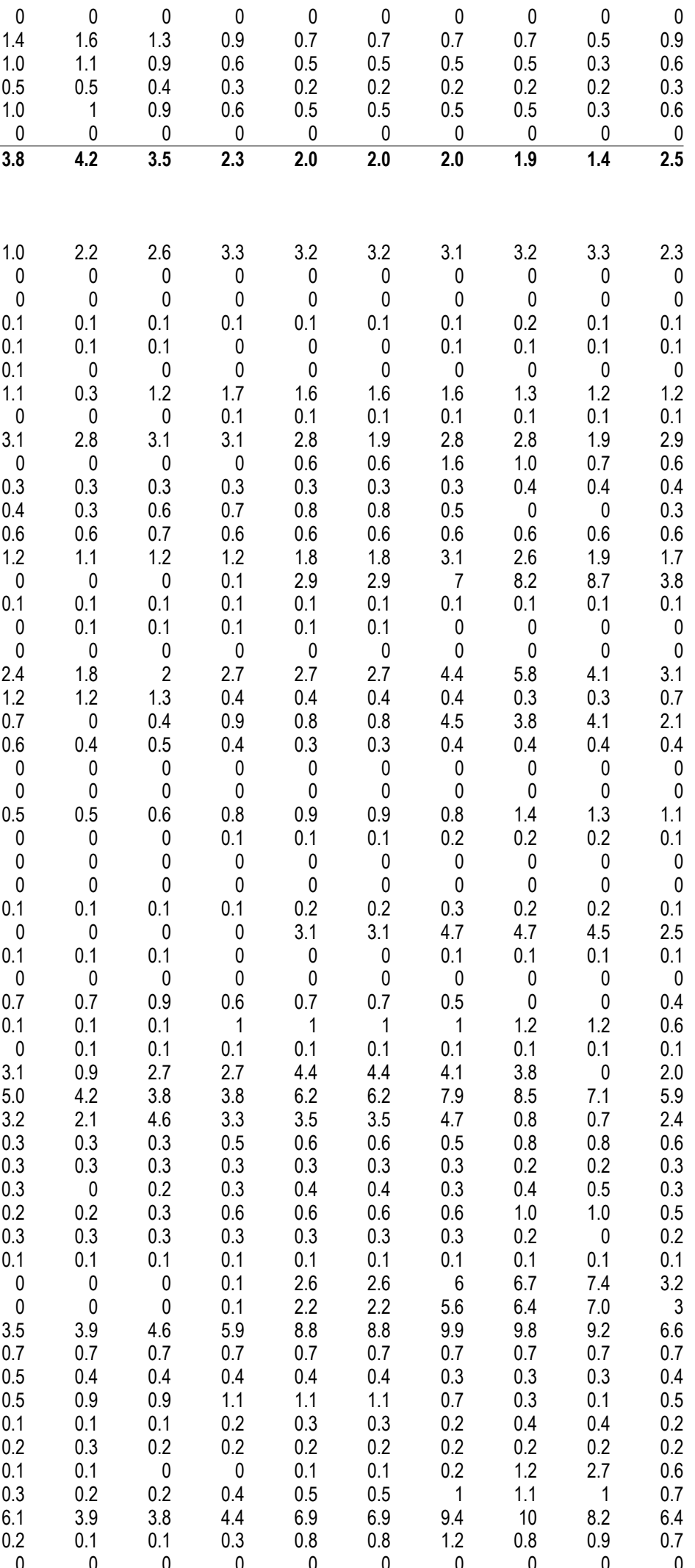

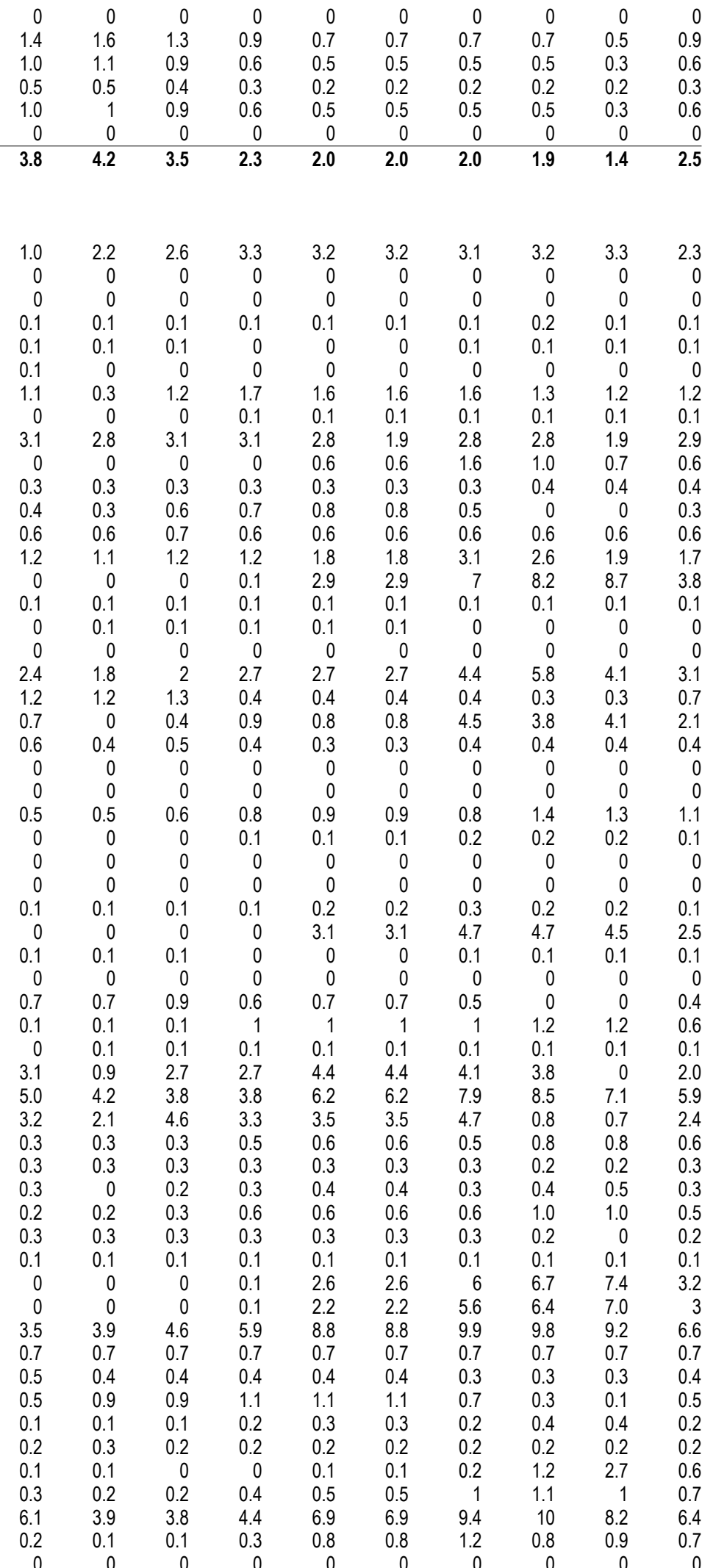

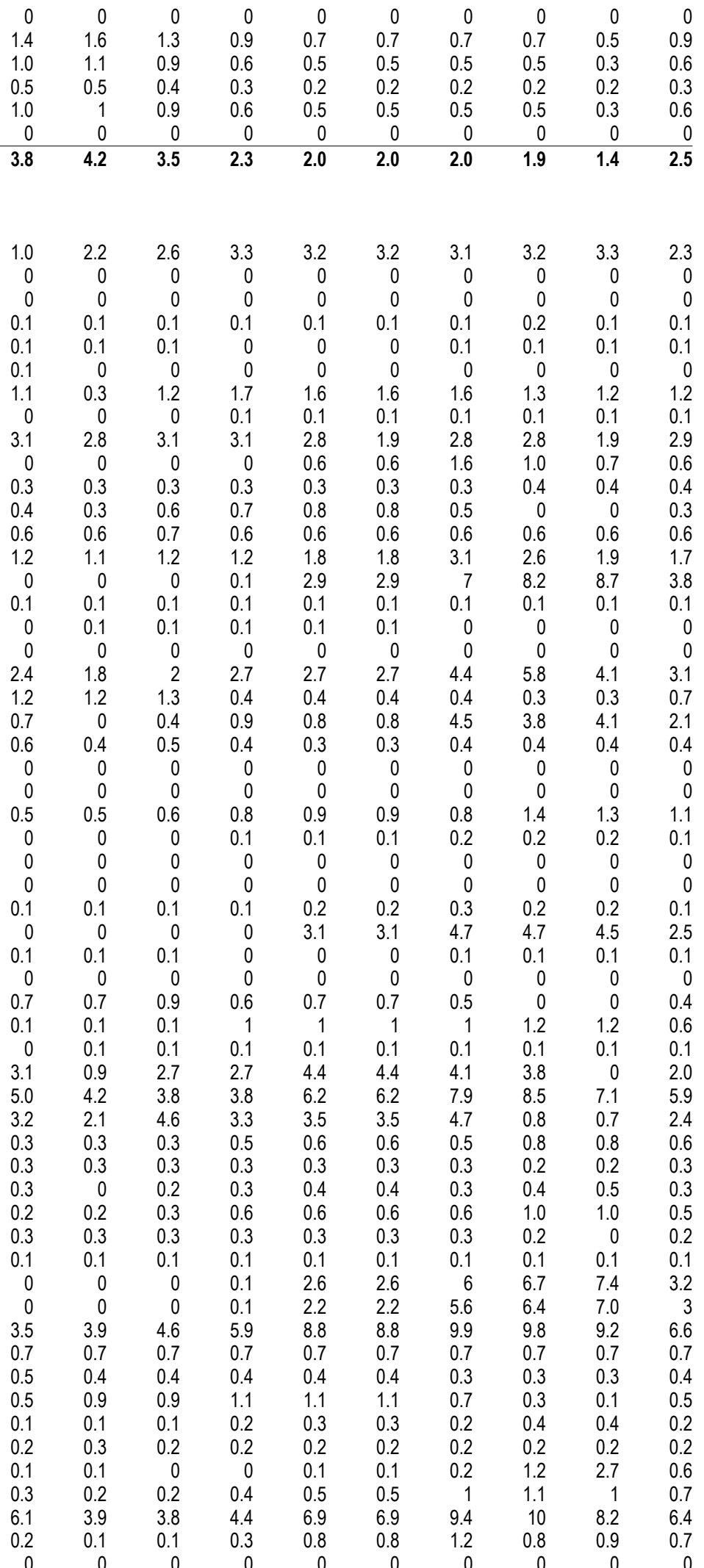

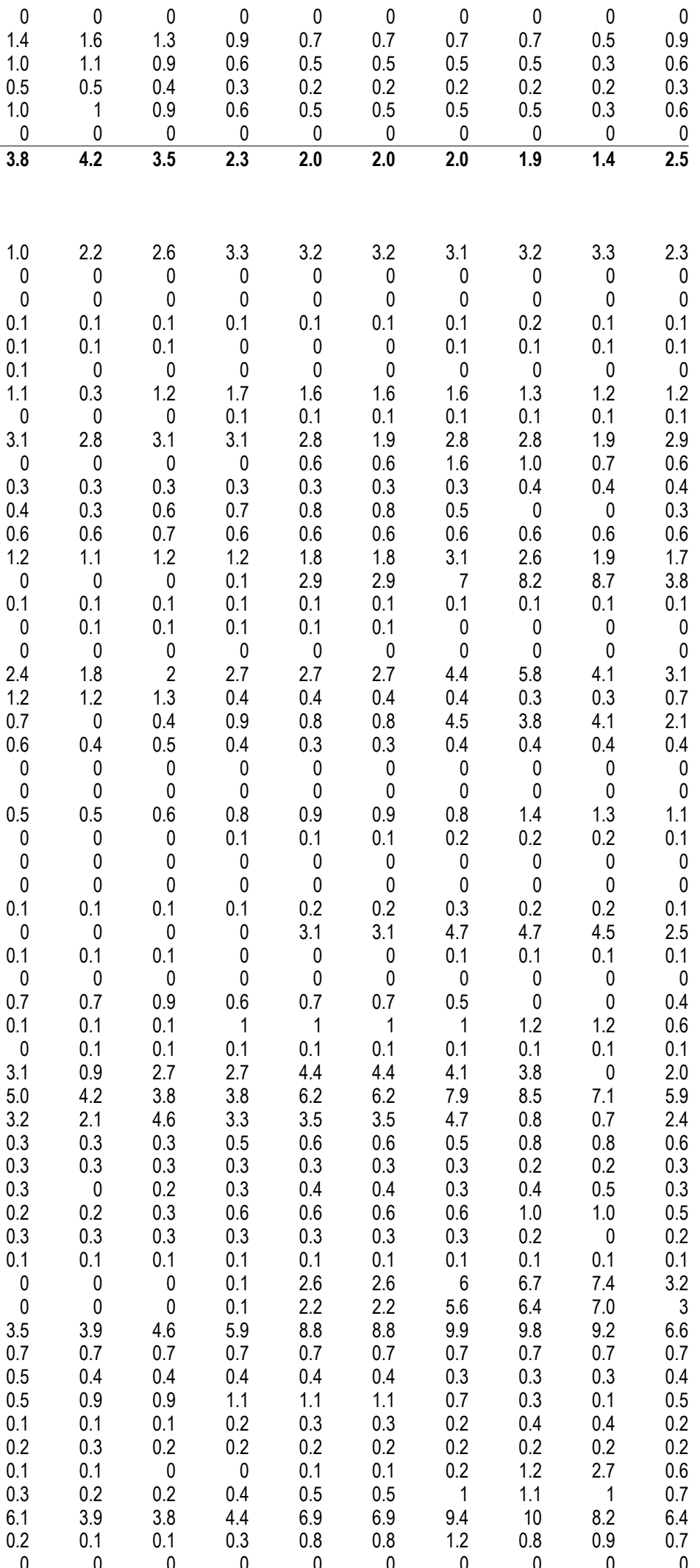

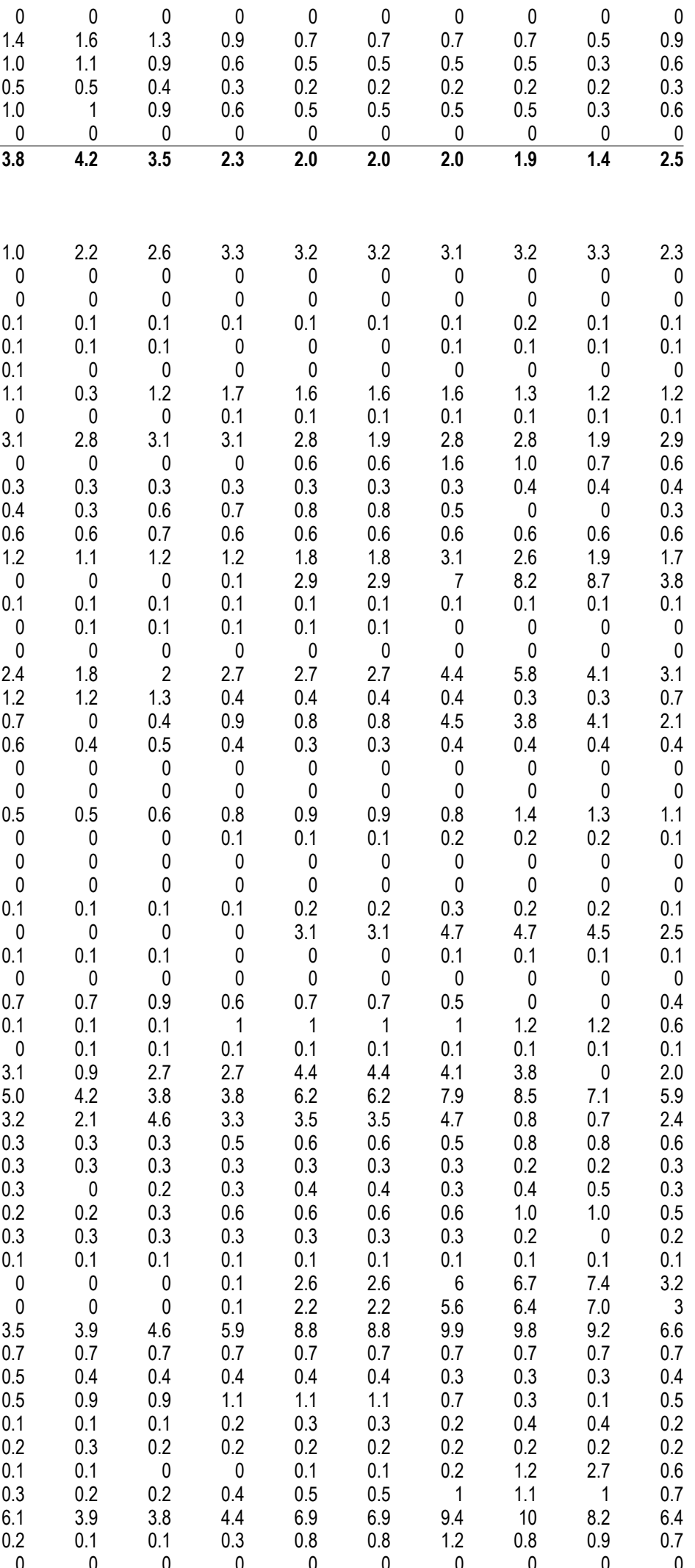

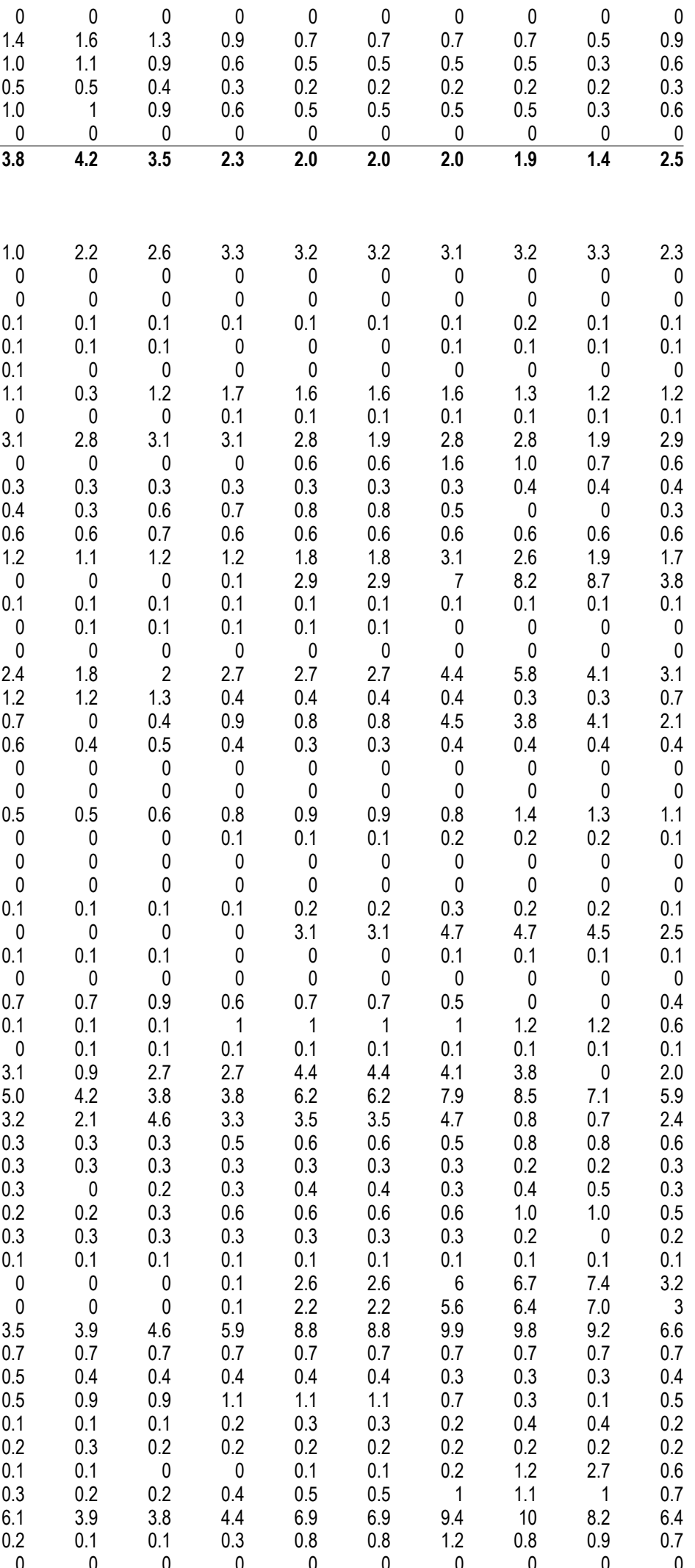

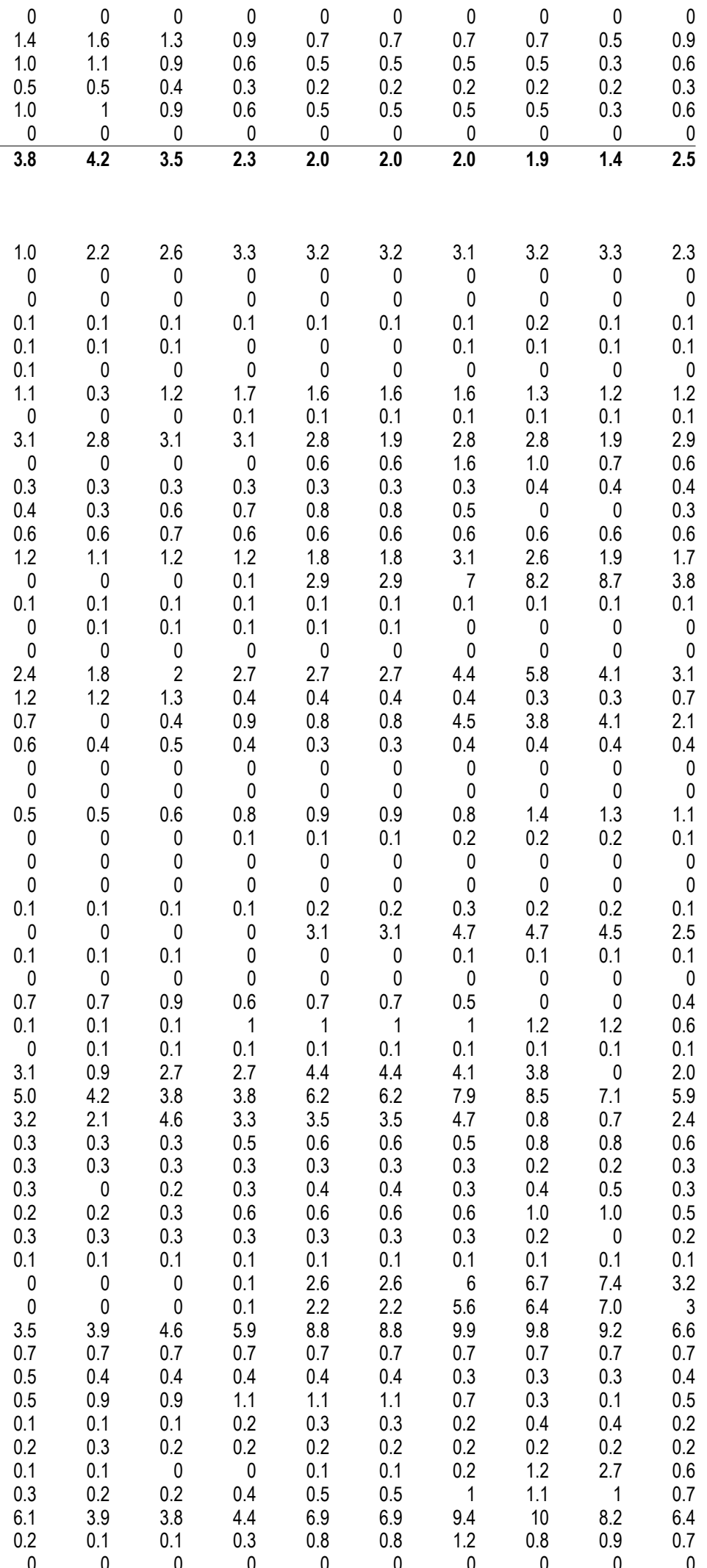

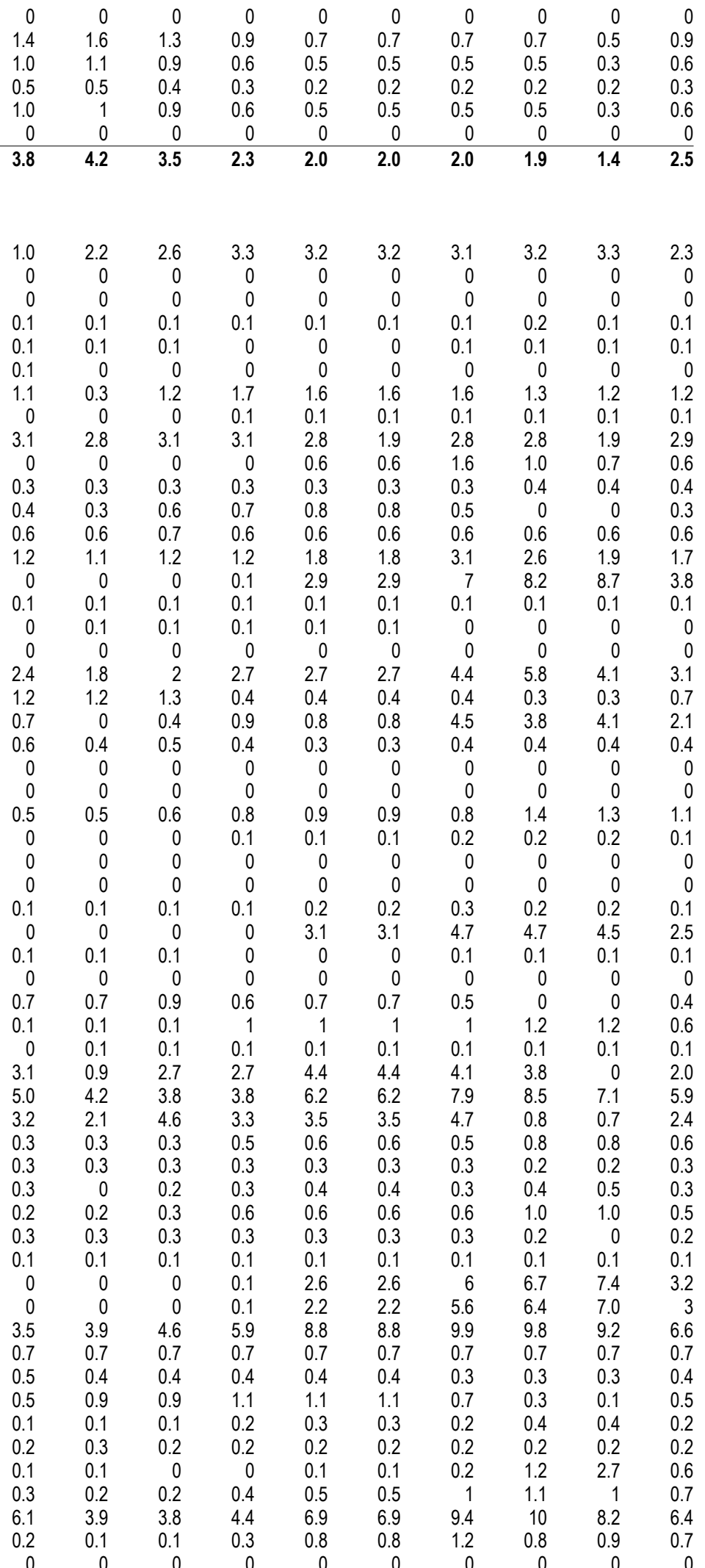

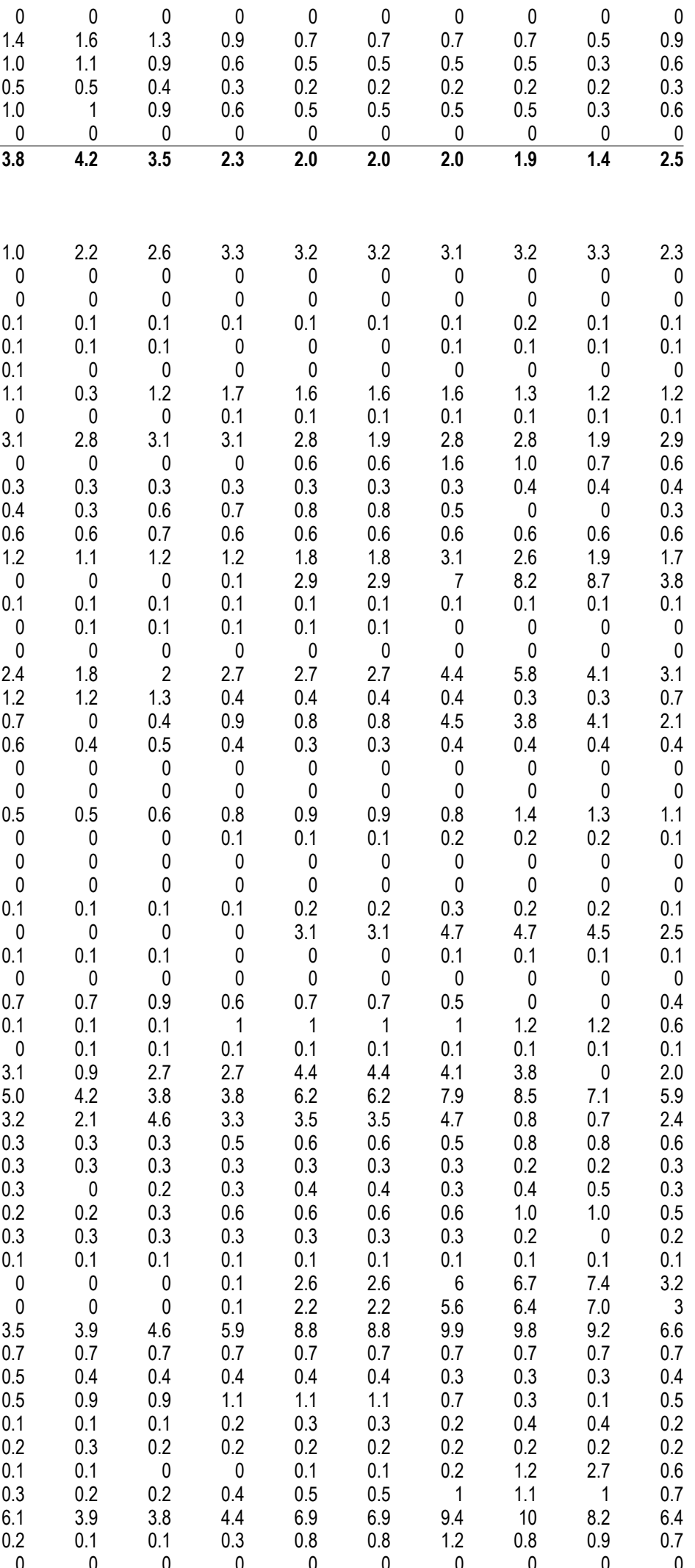

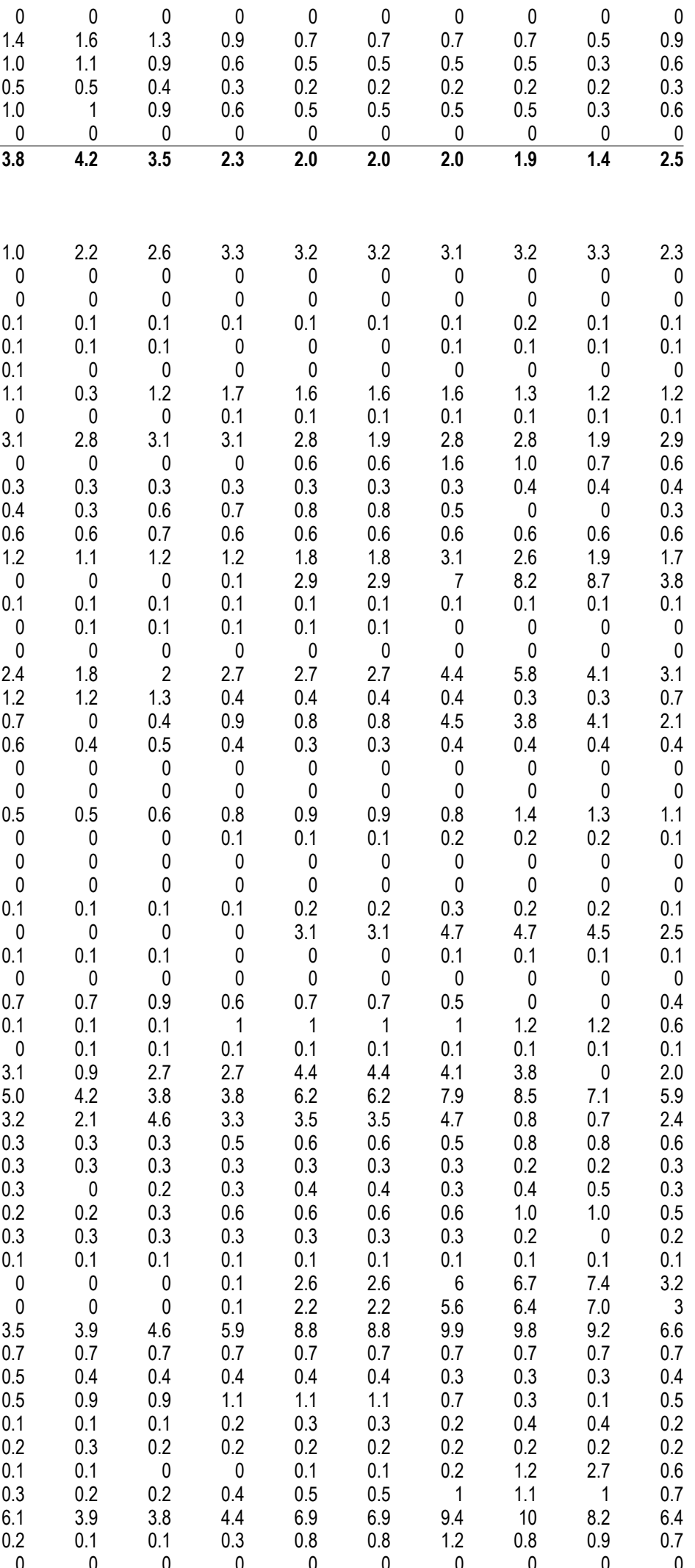

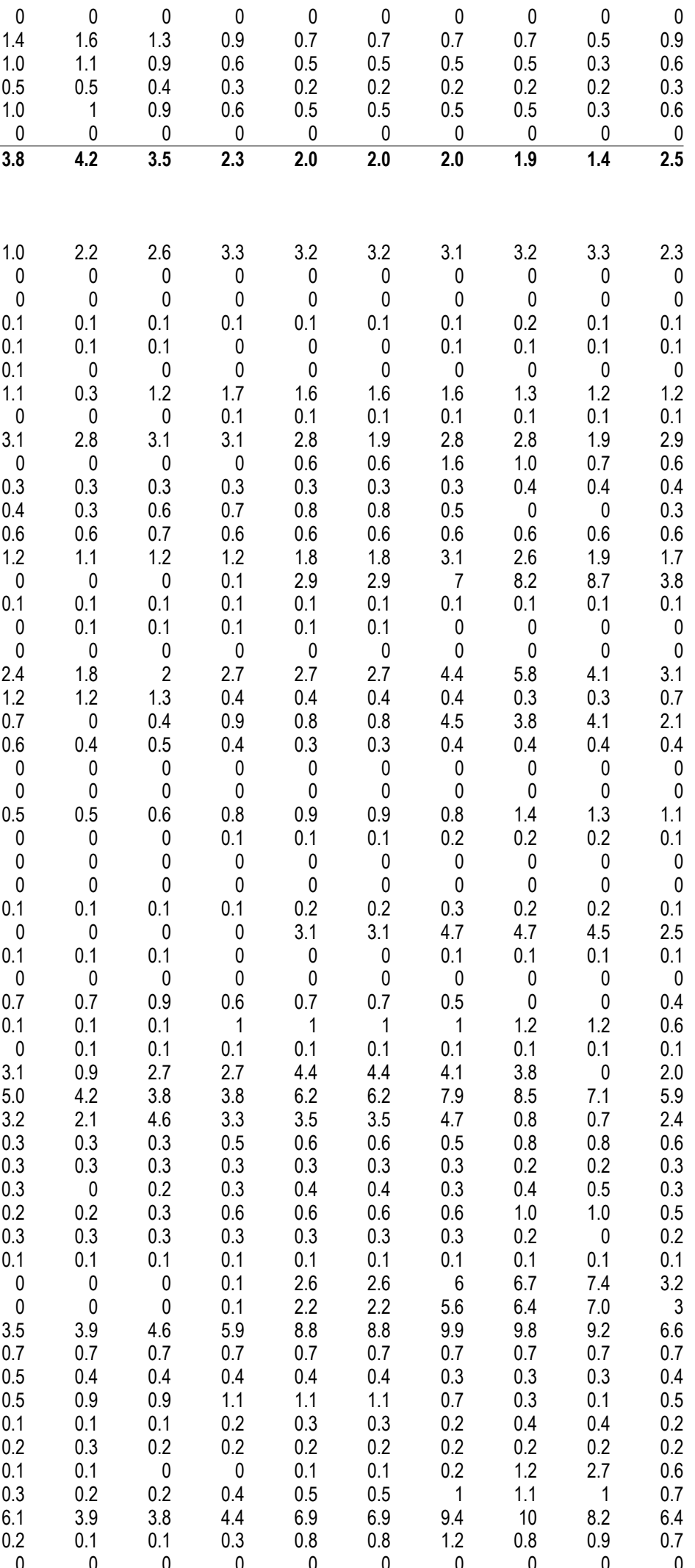

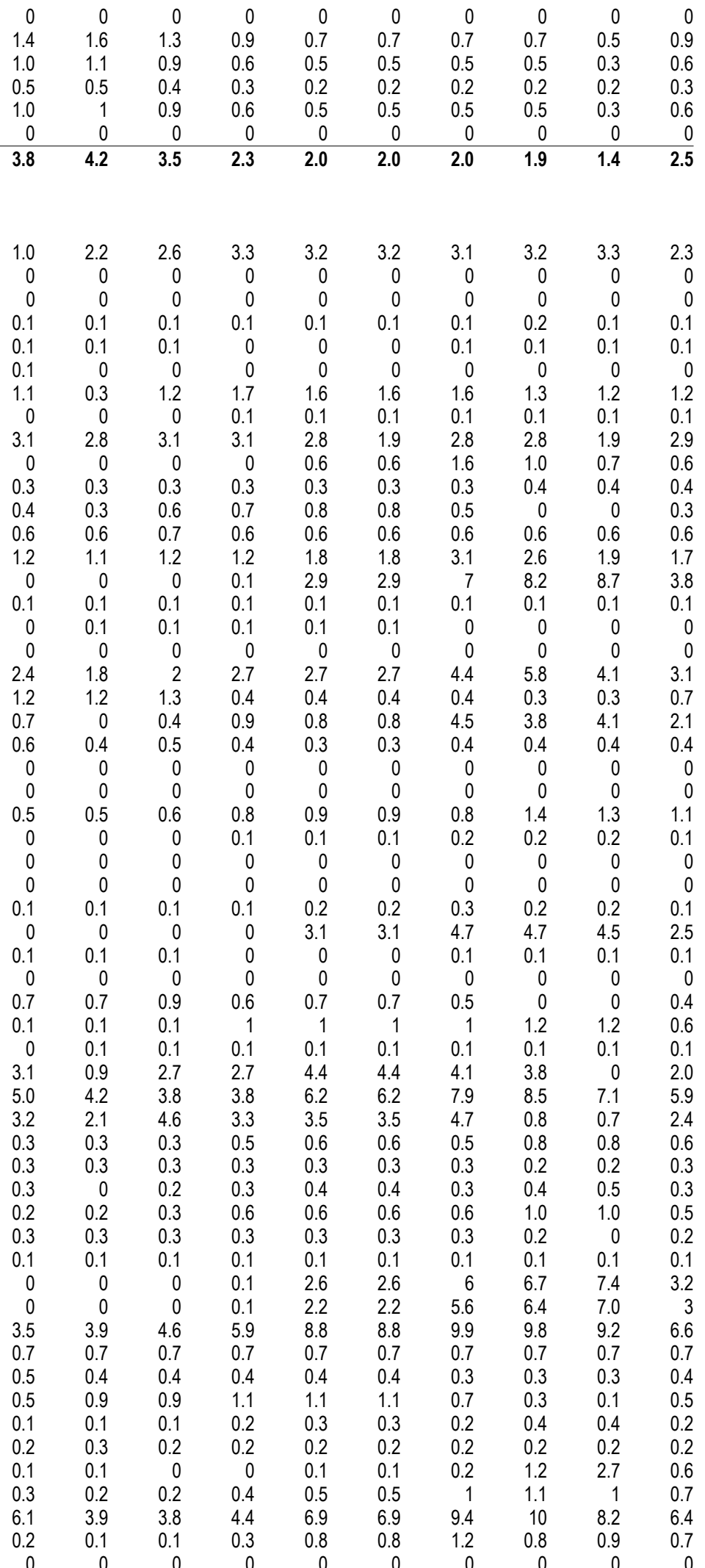

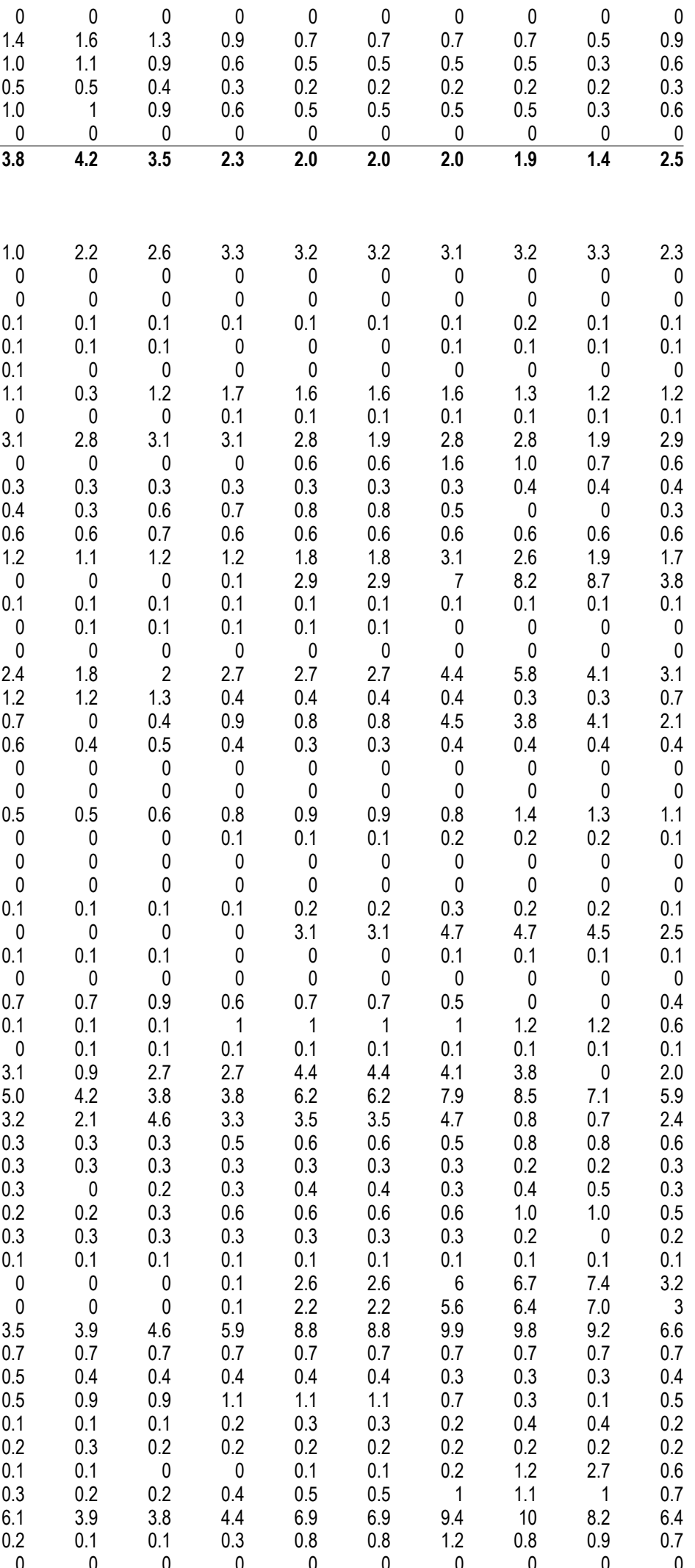

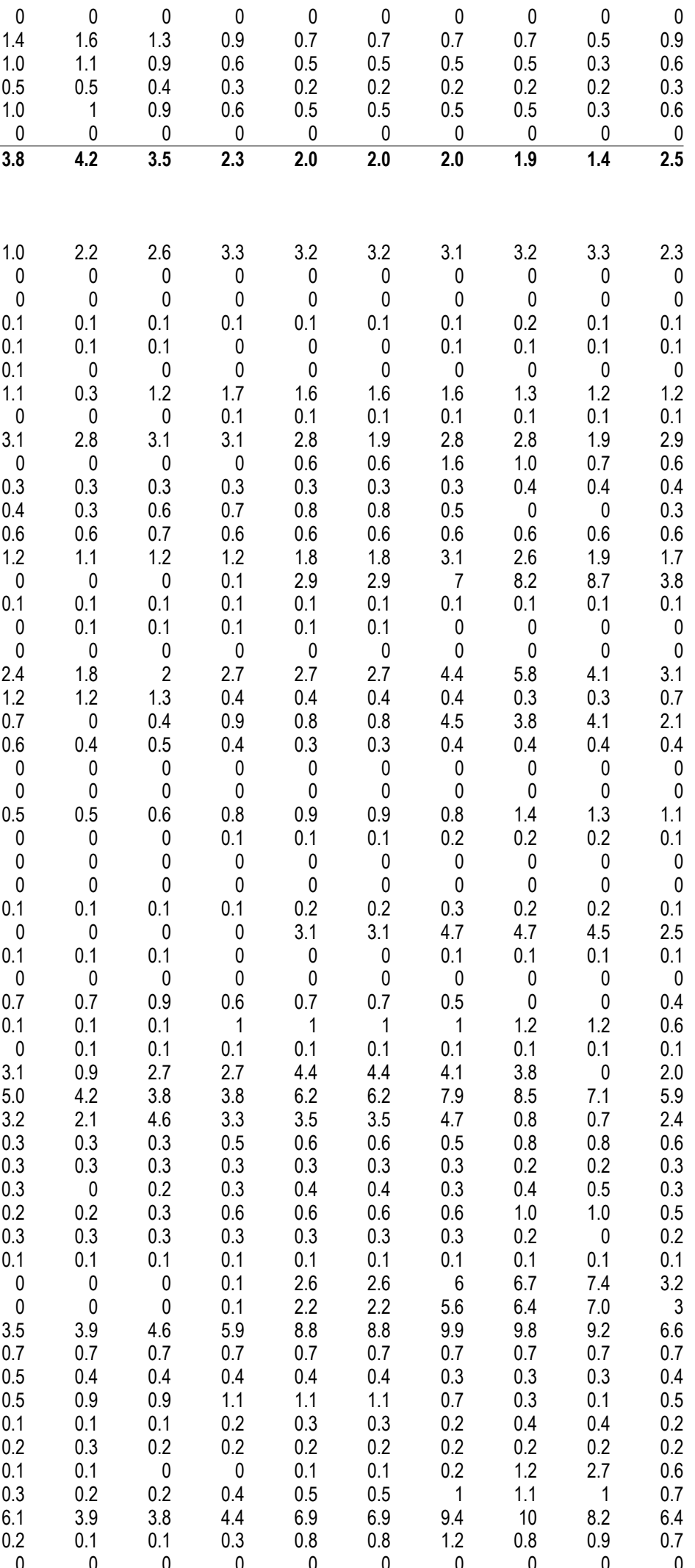

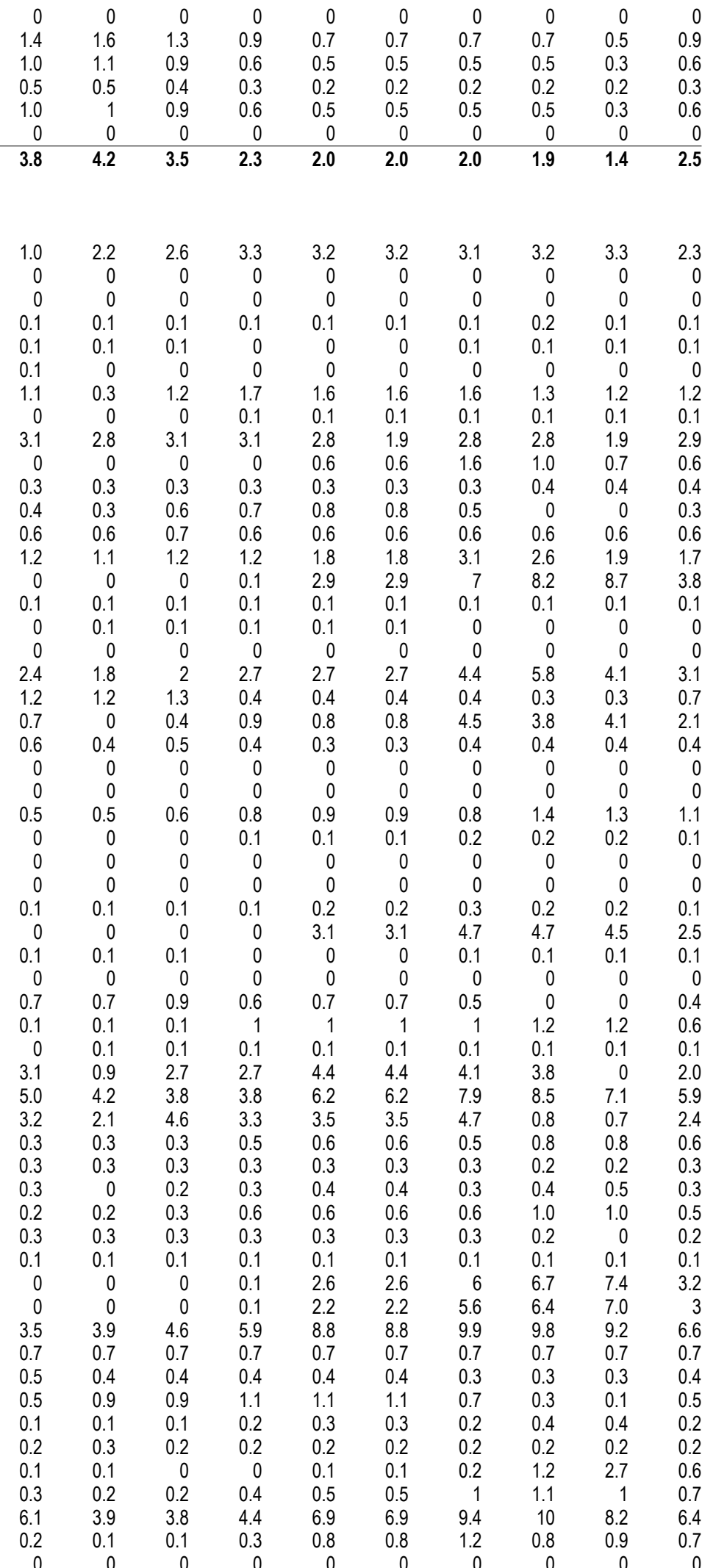

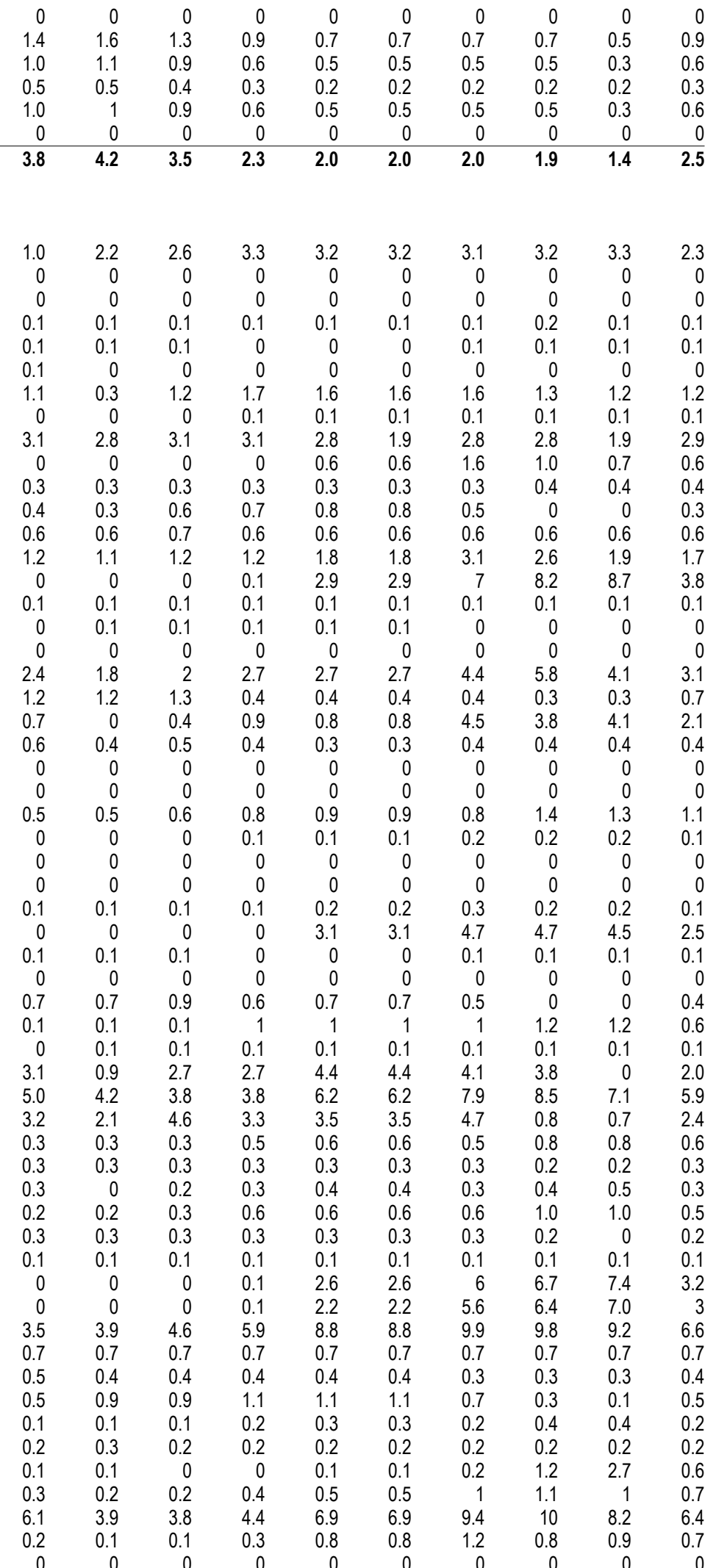

0
0.9

0.9

1.7

0.1

0
0.2
0

0
0.2

0.9
1.8
0.1
1.6

1
0.4

1

0.9

0.9

0.4

1.6

0.1

0.1
0

$1.9 \quad 1.8$

0.1

0

1.7

1.6
0.4

2

2.7

2.7 
Aug1 Aug16 Sep

Oct Nov

130 Lowline Canal (IPC)

131 Magic Reservoir (IPC)

132 Malad River (IPC)

133 Marcos Ranches (IPC)

134 Marsh Valley (UPL)

135 Meyers Falls (AVWP)

136 Middlefork Irrig. (PPL)

137 Mile 28 (IPC)

138 Minikahda (PGE)

139 Mink Creek (UPL)

140 Mitchell Butte (IPC)

141 Mountain Energy (PPL)

$142 \mathrm{Mt}$. Tabor (PGE)

143 Mud Creek/S\&S (IPC)

144 Mud Creek/White (IPC)

145 Nichols Gap (PPL)

146 Nicholson Sunnybar (UPL)

147 North Fork Sprague (PPL)

148 O.J. Power (UPL)

149 Odell Creek (PPL)

150 Opal Springs (PPL)

151 Owyhee Dam (IPC)

152 Pancheri (UPL)

153 Pelton Rereg. Dam (PPL)

154 Phillips Ranch (AVWP)

155 Phillipsburg (NWE)

156 Pigeon Cove (IPC)

157 Pine Creek (NWE)

158 Port Townsend Paper Hydro (PSE)

159 Portland (PGE)

160 Portland Hydro Project (PGE)

161 Preston City (UPL)

162 Pristine Springs (IPC)

163 Pristine Springs \#3 (IPC)

164 Reynolds Irrigation (IPC)

165 Rim View (IPC)

166 Rock Creek \#1 (IPC)

167 Rock Creek \#2 (IPC)

168 Sagebrush (IPC)

169 Salem (PGE)

170 Schaffner (IPC)

171 Sheep Creek (AVWP)

172 Shingle Creek (IPC)

173 Shoshone (IPC)

174 Shoshone II (IPC)

175 Snake River Pottery (IPC)

176 Snedigar Ranch (IPC)

177 Solar Research (PPL)

178 South Dry Creek (NWE)

179 Spokane Upriver (AVWP)

180 Stauffer Dry Creek (UPL)

181 Strawberry Creek (NWE)

182 Sunshine Power \#2 (IPC)

183 Sygitowicz Creek Small Hydro (PSE)

184 TGS/Briggs (UPL)

185 Trout Co. (IPC)

186 Tunnel \#1 (IPC)

187 Twin Falls (PSE)

188 Walla Walla (PPL)

189 Water Street / Santiam (PPL)

190 Weeks Falls (PSE)

191 White James (PPL

192 White Water Ranch (IPC)

193 Wilson Lake Hydro (IPC)

194 Wisconsin Creek (NWE)

195 Yakima-Tieton (PPL)

196 IOU - Total NUG: Hydro

7.8

1.1

0.4

1.3

1.2

1
0

$0.1 \quad 0.1$

$\begin{array}{llll}0.4 & 0.4 & 0.1 & 0.1 \\ 0.1 & 0.1 & 0.5 & 0.5\end{array}$

$\begin{array}{rrrr}0.1 & 0.1 & 0.2 & 0.2\end{array}$

0.2

0

0

2.8

0.3

0
2.3

2.3
0.3

0.1

0.2

0.2

0.2

1.1

1.8

0.4
0

0.4

0.4

0.1

0.4

0.6

0

$0.2 \quad 0.2$

0.10 .1

$\begin{array}{ll}0.5 & 0.5 \\ 3.4 & 3.4\end{array}$

$\begin{array}{ll}3.4 & 3.4 \\ 0.9 & 0.9\end{array}$

0.1

0.1

0

$0.2 \quad 0.2$

$0.1 \quad 0.1$

$4.3 \quad 4.3$

$\begin{array}{rr}5 & \\ 1.5 & 1.5\end{array}$

$\begin{array}{lllllll}0 & 0 & 1.4 & 1.3 & 1.2 & 1.3 & 1.2\end{array}$

$\begin{array}{llll}0.8 & 0.8 & 0 & 0.2\end{array}$

$\begin{array}{rrrr}0.8 & 0.8 & 0 & 0.2 \\ 0.1 & 0.1 & 0.1 & 0.1\end{array}$

$\begin{array}{llll}0.1 & 0.1 & 0.1 & 0.1 \\ 7.1 & 7.1 & 5.3 & 2.0\end{array}$

$\begin{array}{lll}0.2 & 1 \\ 0.1 & 0.1 \\ 0.1 & 0.1 & 0.2 \\ 2.0 & 0.2\end{array}$

-NUG: Small Thermal \& Misc.

197 Marion Solid Waste (PGE)

198 Minnesota Methane (AVWP)

199 Montana One (NWE)

200 Pocatello Waste (IPC)

201 Spokane MSW (PSE)

202 West Boise Waste (IPC)

0.1
0.4
112

$\begin{array}{r}0.2 \\ 0 \\ 0 \\ \hline\end{array}$

$\begin{array}{rr}0 & 0 \\ 0 & 0.1 \\ 0 & 0\end{array}$

0

$\begin{array}{ll}0.1 & 0.1 \\ 2.3 & 2.3\end{array}$

2.3
0
0
173

\begin{tabular}{|c|c|c|c|c|c|c|c|c|c|c|c|c|c|c|c|}
\hline & 9.3 & 9.3 & 9.3 & 9.3 & 9.3 & 9.3 & 9.3 & 9.3 & 9.3 & 9.3 & 9.3 & 9.3 & 9.3 & 9.3 & 9.3 \\
\hline D) & 0.8 & 0.8 & 0.8 & 0.8 & 0.8 & 0.8 & 0.8 & 0.8 & 0.8 & 0.8 & 0.8 & 0.8 & 0.8 & 0.8 & 0.8 \\
\hline & 9.2 & 9.4 & 8.7 & 8.8 & 6.6 & 7.4 & 7.7 & 7.3 & 6.3 & 6.2 & 6.1 & 10 & 8.6 & 8.4 & 8.0 \\
\hline & 0.1 & 0.1 & 0.2 & 0.1 & 0.2 & 0.1 & 0.1 & 0.2 & 0.1 & 0.2 & 0.2 & 0.1 & 0.2 & 0.1 & 0.1 \\
\hline & 15 & 15 & 15 & 15 & 15 & 15 & 15 & 15 & 15 & 15 & 15 & 15 & 15 & 15 & 15 \\
\hline & 0.1 & 0.1 & 0.1 & 0.1 & 0.1 & 0.1 & 0.1 & 0.1 & 0.1 & 0.1 & 0.1 & 0.1 & 0.1 & 0.1 & 0.1 \\
\hline nal \& Misc & 35 & 35 & 34 & 34 & 32 & 33 & 33 & 33 & 32 & 32 & 32 & 36 & 34 & 34 & 34 \\
\hline
\end{tabular}


Table A-24: Regional Non Utility Generating Resources By Project

PNW Loads and Resources Study

2007 - 2008 Operating Year 2003 White Book

Aug1 Aug16 Sep

Oct Nov Dec

Jan Feb Mar Apr1 Apr16

May Jun Jul Avg

-NUG: Combustion Turbines -

204 March Point \#1 Cogeneration (PSE)

205 March Point \#2 Cogeneration (PSE)

206 Rathdrum- Boekel Rd. \#1 (AVWP)

207 Rathdrum -Boekel Rd. \#2 (AVWP)

208 Tenaska Gas Cogen (PSE)

209 IOU - Total NUG: Combustion Turbines

\begin{tabular}{|c|c|c|c|c|c|c|c|c|c|c|c|c|c|c|}
\hline 79 & 79 & 79 & 79 & 79 & 79 & 79 & 79 & 79 & 79 & 79 & 55 & 79 & 79 & 77 \\
\hline 59 & 59 & 59 & 59 & 59 & 59 & 59 & 59 & 59 & 59 & 59 & 45 & 59 & 59 & 58 \\
\hline 65 & 65 & 65 & 65 & 65 & 65 & 65 & 65 & 65 & 65 & 65 & 32 & 65 & 65 & 62 \\
\hline 65 & 65 & 65 & 65 & 65 & 65 & 65 & 65 & 65 & 65 & 65 & 32 & 65 & 65 & 62 \\
\hline 240 & 240 & 240 & 240 & 240 & 240 & 240 & 240 & 240 & 240 & 240 & 180 & 240 & 240 & 235 \\
\hline 508 & 508 & 508 & 508 & 508 & 508 & 508 & 508 & 508 & 508 & 508 & 344 & 508 & 508 & 494 \\
\hline
\end{tabular}

-NUG: Co-Generation-

210 Billings Generation (NWE)

211 Biomass One (PPL)

212 Boise Cascade Medford (PPL)

213 Champion (PPL)

214 DR Johnson - Co-Gen II (PPL)

215 Magic Valley (IPC)

216 Magic West (IPC)

217 PERC Pierce, Wa (PSE)

218 Simplot Pocatello (IPC)

219 Sumas Energy (PSE)

220 Tamarack (IPC)

221 TASCO Nampa ID (IPC)

222 TASCO Twin Falls ID (IPC)

223 Vaagen Bros. (IPC)

224 Warm Springs (PPL)

225 IOU - Total NUG: Co-Generation

-NUG: Renewables-

226 Foote Creek 1 (PPL)

227 Healow \#2 (NWE)

228 Josef Staufer (NWE)

229 Lewandowski Farms (IPC)

230 Livingston (NWE)

231 Puyallup Energy Recovery (PSE)

232 Stateline Wind Project (AVWP)

233 Windmill (PGE)

234 IOU - Total NUG: Renewables

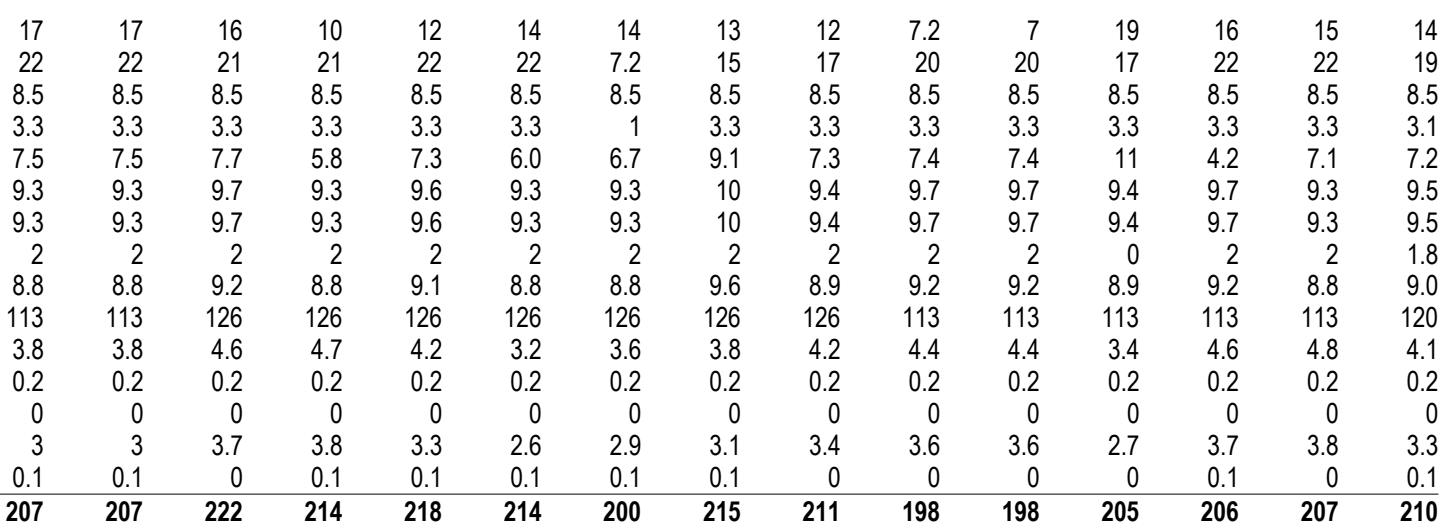

Other Entities

-NUG: Co-Generation-

235 SP Newsprint cogen (SPN)

236 Other - Total NUG: Co-Generation

$\begin{array}{lllllllllllllll}25 & 25 & 25 & 25 & 25 & 25 & 25 & 25 & 25 & 25 & 25 & 25 & 25 & 25 & 25 \\ \mathbf{2 5} & \mathbf{2 5} & \mathbf{2 5} & \mathbf{2 5} & \mathbf{2 5} & \mathbf{2 5} & \mathbf{2 5} & \mathbf{2 5} & \mathbf{2 5} & \mathbf{2 5} & \mathbf{2 5} & \mathbf{2 5} & \mathbf{2 5} & \mathbf{2 5} & \mathbf{2 5}\end{array}$

-NUG: Renewables-

237 Stateline Wind Project (PPME)

238 Vansycle Wind (FPLEV)

239 Other - Total NUG: Renewables

\begin{tabular}{|c|c|c|c|c|c|c|c|c|c|c|c|c|c|c|}
\hline 4.2 & 4.2 & 4.9 & 7.2 & 11 & 13 & 14 & 12 & 9.2 & 7.4 & 7.4 & 5.9 & 5.2 & 4.2 & 8.1 \\
\hline 0 & 0 & 0 & 0 & 0 & 0 & 0 & 0 & 0 & 0 & 0 & 0 & 0 & 0 & 0 \\
\hline 0 & 0 & 0 & 0 & 0 & 0 & 0 & 0 & 0 & 0 & 0 & 0 & 0 & 0 & 0 \\
\hline 0 & 0 & 0 & 0 & 0 & 0 & 0 & 0 & 0 & 0 & 0 & 0 & 0 & 0 & 0 \\
\hline 0 & 0 & 0 & 0 & 0 & 0 & 0 & 0 & 0 & 0 & 0 & 0 & 0 & 0 & 0 \\
\hline 2 & 2 & 2 & 2 & 2 & 2 & 2 & 2 & 2 & 2 & 2 & 2 & 2 & 2 & 2 \\
\hline 0.3 & 0.3 & 0.2 & 0.3 & 0.3 & 0.3 & 0.3 & 0.3 & 0.3 & 0.3 & 0.3 & 0.3 & 0.3 & 0.3 & 0.3 \\
\hline 0 & 0 & 0 & 0 & 0 & 0 & 0 & 0 & 0 & 0 & 0 & 0 & 0 & 0 & 0 \\
\hline 6.5 & 6.5 & 7.1 & 9.5 & 13 & 15 & 16 & 15 & 12 & 9.8 & 9.8 & 8.2 & 7.6 & 6.5 & 10 \\
\hline
\end{tabular}

-Total Non-Utility Generating Resources-

240 Federal Entities

241 Generating Public Entities

242 Non Generating Public Entities

243 Investor-Owned Entities

244 Other Entities

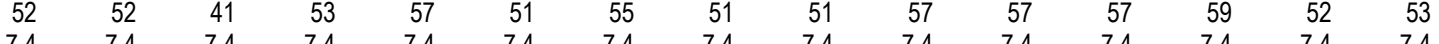

245 Total Non-Utility Generation

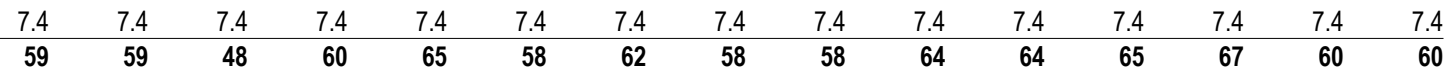


Oct Nov

Dec

\begin{abstract}
Jan Feb
\end{abstract}

\begin{abstract}
Mar Apr1 Apr16
\end{abstract}

\begin{abstract}
May
\end{abstract}
Jun

Jul Avg

\title{
Federal Entities
}

-NUG: Hydro-

1 Clearwater - State of ID DWR (BPA)

2 Dworshak Small Hydropower (BPA)

3 Federal -Total NUG: Hydro

\begin{tabular}{|c|c|c|c|c|c|c|c|c|c|c|c|c|c|c|}
\hline 1 & 1 & 1 & 1 & 1 & 1 & 1 & 1 & 1 & 1 & 1 & 1 & 1 & 1 & 1 \\
\hline 2.6 & 2.6 & 2.6 & 2.6 & 2.6 & 2.6 & 2.6 & 2.6 & 2.6 & 2.6 & 2.6 & 2.6 & 2.6 & 2.6 & 2.6 \\
\hline 3.6 & 3.6 & 3.6 & 3.6 & 3.6 & 3.6 & 3.6 & 3.6 & 3.6 & 3.6 & 3.6 & 3.6 & 3.6 & 3.6 & 3.6 \\
\hline
\end{tabular}

-NUG: Renewables-

4 Ashland Solar Project (BPA)

5 Condon Wind Project (BPA)

6 Foote Creek 1 (BPA)

7 Foote Creek 2 (BPA)

8 Foote Creek 4 (BPA)

9 Fourmile Hill Geothermal (BPA)

10 Klondike Phase 1 (BPA)

11 Stateline Wind Project (BPA)

12 Federal -Total NUG: Renewables

\begin{tabular}{|c|c|c|c|c|c|c|c|c|c|c|c|c|c|}
\hline 0 & 0 & 0 & 0 & 0 & 0 & 0 & 0 & 0 & 0 & 0 & 0 & 0 & 0 \\
\hline 10 & 10 & 11 & 13 & 14 & 13 & 12 & 12 & 14 & 14 & 12 & 15 & 11 & 12 \\
\hline 3.3 & 3.8 & 5.6 & 8.3 & 10 & 11 & 9.6 & 7.2 & 5.8 & 5.8 & 4.6 & 4.1 & 3.3 & 6.4 \\
\hline 0.4 & 0.5 & 0.7 & 1 & 1.3 & 1.3 & 1.2 & 0.9 & 0.7 & 0.7 & 0.5 & 0.5 & 0.4 & 0.8 \\
\hline 3.9 & 4.4 & 6.5 & 9.7 & 12 & 12 & 11 & 8.4 & 6.7 & 6.7 & 5.4 & 4.8 & 3.8 & 7.4 \\
\hline 50 & 50 & 50 & 50 & 50 & 50 & 50 & 50 & 50 & 50 & 50 & 50 & 50 & 50 \\
\hline 7.9 & 6.2 & 8.1 & 8.8 & 7.8 & 8.3 & 7.8 & 7.8 & 8.6 & 8.6 & 8.8 & 9.1 & 8 & 8.1 \\
\hline 29 & 23 & 30 & 33 & 29 & 31 & 29 & 29 & 32 & 32 & 33 & 34 & 30 & 30 \\
\hline 105 & 98 & 112 & 124 & 123 & 126 & 121 & 115 & 118 & 118 & 114 & 117 & 106 & 115 \\
\hline
\end{tabular}

Generating Public Entities

-NUG: Hydro-

13 Don Steffen (SHPD)

14 Eltopia Br Canal 4.6 (SCL)

15 Eltopia Br Canal 4.6 (TPU)

16 John Llewellyn (SHPD)

17 Kevin Duncan (SHPD)

18 Lucky Peak (SCL)

19 Main Canal Headworks (SCL)

20 Main Canal Headworks (TPU)

21 PEC Headworks (GCPD)

22 Pickering Micro Hydro (CKPD)

23 Potholes E Canal 66.0 (SCL)

24 Potholes E Canal 66.0 (TPU)

25 Quincy Chute (GCPD)

26 Russel D. Smith (SCL)

27 Russel D. Smith (TPU)

28 Smith Creek (EWEB)

29 Summer Falls (SCL)

30 Summer Falls (TPU)

31 Woods Creek (SHPD)

32 GPU - Total NUG: Hydro

\begin{tabular}{rrrrrrrrrrrrrrr}
0 & 0 & 0 & 0 & 0 & 0 & 0 & 0 & 0 & 0 & 0 & 0 & 0 & 0 & 0 \\
0.9 & 0.9 & 0.6 & 0.3 & 0 & 0 & 0 & 0 & 0 & 0.7 & 0.7 & 1 & 1 & 1.1 & 0.5 \\
0.9 & 0.9 & 0.6 & 0.3 & 0 & 0 & 0 & 0 & 0 & 0.7 & 0.7 & 1 & 1 & 1.1 & 0.5 \\
0 & 0 & 0 & 0 & 0 & 0 & 0 & 0 & 0 & 0 & 0 & 0 & 0 & 0 & 0 \\
0 & 0 & 0 & 0 & 0 & 0 & 0 & 0 & 0 & 0 & 0 & 0 & 0 & 0 & 0 \\
66 & 66 & 41 & 11 & 2.2 & 3 & 7.9 & 10 & 21 & 52 & 52 & 84 & 85 & 77 & 38 \\
10 & 10 & 6.1 & 2.6 & 0 & 0 & 0 & 0 & 1.4 & 6.8 & 6.8 & 9.1 & 11 & 12 & 4.9 \\
10 & 10 & 6.1 & 2.6 & 0 & 0 & 0 & 0 & 1.4 & 6.8 & 6.8 & 9.1 & 11 & 12 & 4.9 \\
4.5 & 4.5 & 3.1 & 2.2 & 0 & 0 & 0 & 0 & 0.4 & 5.1 & 5.1 & 6.9 & 6.2 & 5.8 & 2.9 \\
0 & 0 & 0 & 0 & 0 & 0 & 0 & 0 & 0 & 0 & 0 & 0 & 0 & 0 & 0 \\
0.9 & 0.9 & 0.9 & 0.8 & 0 & 0 & 0 & 0 & 0.3 & 0.9 & 0.9 & 0.9 & 0.9 & 0.9 & 0.5 \\
0.9 & 0.9 & 0.9 & 0.8 & 0 & 0 & 0 & 0 & 0.3 & 0.9 & 0.9 & 0.9 & 0.9 & 0.9 & 0.5 \\
7.7 & 7.7 & 4 & 2.3 & 0 & 0 & 0 & 0 & 0.8 & 5.5 & 5.5 & 7.1 & 8.4 & 8.5 & 3.7 \\
2 & 2 & 1.3 & 0.7 & 0 & 0 & 0 & 0 & 0.1 & 1.2 & 1.2 & 1.9 & 2.1 & 1.7 & 0.9 \\
2 & 2 & 1.3 & 0.7 & 0 & 0 & 0 & 0 & 0.1 & 1.2 & 1.2 & 1.9 & 2.1 & 1.7 & 0.9 \\
0 & 0 & 0 & 5 & 5.4 & 3.9 & 0 & 0.8 & 1.5 & 9.2 & 9.2 & 37 & 37 & 11 & 9.2 \\
39 & 39 & 24 & 11 & 0 & 0 & 0 & 0 & 2.4 & 25 & 25 & 36 & 40 & 44 & 18 \\
39 & 39 & 24 & 11 & 0 & 0 & 0 & 0 & 2.4 & 25 & 25 & 36 & 40 & 44 & 18 \\
0.1 & 0.1 & 0.1 & 0.1 & 0.1 & 0.1 & 0.1 & 0.1 & 0.1 & 0.1 & 0.1 & 0.1 & 0.1 & 0.1 & 0.1 \\
\hline 185 & 185 & 115 & $\mathbf{5 1}$ & $\mathbf{7 . 7}$ & $\mathbf{7}$ & $\mathbf{8}$ & $\mathbf{1 1}$ & $\mathbf{3 2}$ & $\mathbf{1 4 1}$ & $\mathbf{1 4 1}$ & $\mathbf{2 3 2}$ & $\mathbf{2 4 7}$ & $\mathbf{2 2 1}$ & $\mathbf{1 0 5}$
\end{tabular}

-NUG: Small Thermal \& Misc.-

33 Grays Harbor Paper (GHPD)

34 Weyerhaeuser Pulp Mill (GHPD)

35 GPU - Total NUG: Small Thermal \& Misc.

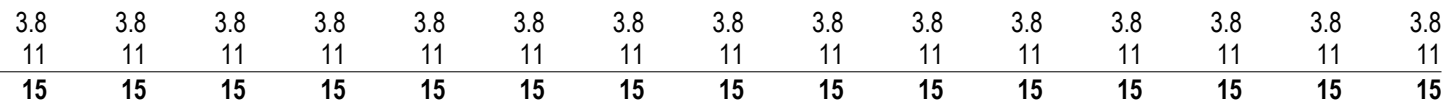

-NUG: Co-Generation-

36 Metro Westpoint (SCL)

37 Scott Paper (SHPD)

38 GPU - Total NUG: Co-Generation

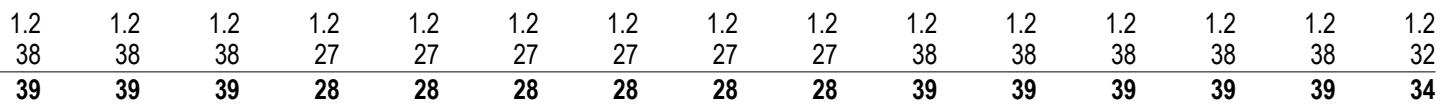

-NUG: Renewables-

39 Foote Creek 1 (EWEB)

40 Nine Canyon Wind Project (CHPD)

41 Nine Canyon Wind Project (COPD)

42 Nine Canyon Wind Project (DOPD)

43 Nine Canyon Wind Project (GCPD)

44 Nine Canyon Wind Project (GHPD)

45 Nine Canyon Wind Project (OKPD)

46 Stateline Wind Project (EWEB)

47 Stateline Wind Project (SCL)

48 GPU - Total NUG: Renewables

Non-Generating Public Entities

-NUG: Hydro-

49 Buffalo Hydro (FREC)

50 Burr \& Bouchard (TNEC)

51 City of Cove (OTEC)

52 Falls Creek (CCPD)

53 Lilliwaup Falls Generating Co. (MCPD1)

54 Thomas Burnside (TNEC)

$\begin{array}{rrrrrrrrrrrrrrr}1.4 & 1.4 & 1.6 & 2.4 & 3.5 & 4.3 & 4.5 & 4.1 & 3.1 & 2.5 & 2.5 & 2.0 & 1.7 & 1.4 & 2.7 \\ 1.5 & 1.5 & 1.4 & 2.2 & 3.8 & 3.8 & 4.2 & 3.4 & 2.3 & 2.0 & 2.0 & 2.0 & 1.9 & 1.4 & 2.5 \\ 0.4 & 0.4 & 0.4 & 0.5 & 0.9 & 0.9 & 1 & 0.9 & 0.6 & 0.5 & 0.5 & 0.5 & 0.5 & 0.3 & 0.6 \\ 1.8 & 1.8 & 1.7 & 2.6 & 4.6 & 4.7 & 5.1 & 4.2 & 2.8 & 2.4 & 2.4 & 2.4 & 2.3 & 1.7 & 3 \\ 2.2 & 2.2 & 2.1 & 3.2 & 5.7 & 5.7 & 6.3 & 5.2 & 3.4 & 3.0 & 3.0 & 3.0 & 2.8 & 2.1 & 3.7 \\ 1.5 & 1.5 & 1.4 & 2.2 & 3.8 & 3.8 & 4.2 & 3.4 & 2.3 & 2.0 & 2.0 & 2.0 & 1.9 & 1.4 & 2.5 \\ 3.0 & 3.0 & 2.8 & 4.3 & 7.5 & 7.6 & 8.4 & 6.9 & 4.6 & 3.9 & 3.9 & 3.9 & 3.7 & 2.7 & 4.9 \\ 8.4 & 8.4 & 6.6 & 8.6 & 9.3 & 8.2 & 8.8 & 8.3 & 8.3 & 9.2 & 9.2 & 9.3 & 9.6 & 8.5 & 8.6 \\ 49 & 49 & 39 & 50 & 54 & 48 & 52 & 48 & 48 & 54 & 54 & 54 & 56 & 50 & 50 \\ \mathbf{6 9} & \mathbf{6 9} & \mathbf{5 7} & \mathbf{7 6} & \mathbf{9 3} & \mathbf{8 7} & \mathbf{9 4} & \mathbf{8 5} & \mathbf{7 5} & \mathbf{7 9} & \mathbf{7 9} & \mathbf{7 9} & \mathbf{8 1} & \mathbf{6 9} & \mathbf{7 9}\end{array}$

-NUG: Small Thermal \& Misc.-

56 Methane Energy Agricultural D. (TKPD)

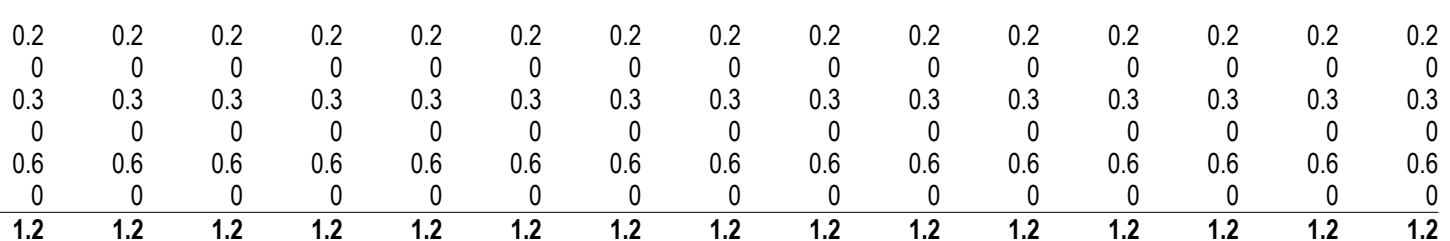

57 NGP - Total NUG: Small Thermal \& Misc.

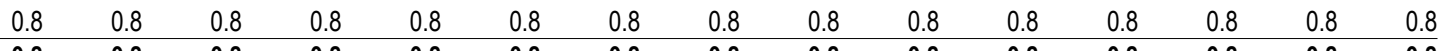


-NUG: Renewables-

58 Ashland Solar Project (ASHL)

59 Nine Canyon Wind Project (BCPD)

60 Nine Canyon Wind Project (ENW)

61 Nine Canyon Wind Project (LCPD)

62 Nine Canyon Wind Project (MCPD3)

\begin{tabular}{|c|c|c|c|c|c|c|c|c|c|c|c|c|c|c|}
\hline 0 & 0 & 0 & 0 & 0 & 0 & 0 & 0 & 0 & 0 & 0 & 0 & 0 & 0 & 0 \\
\hline 0.6 & 0.6 & 0.5 & 0.8 & 1.4 & 1.4 & 1.6 & 1.3 & 0.9 & 0.7 & 0.7 & 0.7 & 0.7 & 0.5 & 0.9 \\
\hline 0.4 & 0.4 & 0.4 & 0.5 & 0.9 & 1.0 & 1.1 & 0.9 & 0.6 & 0.5 & 0.5 & 0.5 & 0.5 & 0.3 & 0.6 \\
\hline 0.2 & 0.2 & 0.2 & 0.3 & 0.5 & 0.5 & 0.5 & 0.4 & 0.3 & 0.2 & 0.2 & 0.2 & 0.2 & 0.2 & 0.3 \\
\hline 0.4 & 0.4 & 0.4 & 0.5 & 0.9 & 1.0 & 1 & 0.9 & 0.6 & 0.5 & 0.5 & 0.5 & 0.5 & 0.3 & 0.6 \\
\hline 0 & 0 & 0 & 0 & 0 & 0 & 0 & 0 & 0 & 0 & 0 & 0 & 0 & 0 & 0 \\
\hline 1.5 & 1.5 & 1.4 & 2.2 & 3.8 & 3.8 & 4.2 & 3.5 & 2.3 & 2.0 & 2.0 & 2.0 & 1.9 & 1.4 & 2.5 \\
\hline
\end{tabular}

64 NGP - Total NUG: Renewables

Investor-Owned Entities

-NUG: Hydro-

65 Barber Dam (IPC)

66 Barney Creek (NWE)

67 Beaverton (PGE)

68 Bell Mountain (UPL)

69 Bingham Engineering (NWE)

70 Birch Creek (IPC)

71 Birch Creek (UPL)

72 Black Canyon \#3 (IPC)

73 Black Creek (AVWP)

74 Blind Canyon Hydro (IPC)

75 Box Canyon (IPC)

76 Boyd James (PPL)

77 Briggs Creek (IPC)

78 Broadwater Dam (NWE)

79 Bypass (IPC)

80 Canyon Springs (IPC)

81 Carlton (PGE)

82 Cascade Creek (NWE)

83 CDM Hydro - Bonneville Pacific (PPL)

84 Cedar Draw (IPC)

85 Central OR Irrigation District (PPL)

86 Clear Springs Trout (IPC)

87 Corbett (PGE)

88 Cornelius (PGE)

89 Crystal Springs (IPC)

90 Curry Cattle Co. (IPC)

91 Dairy Creek (PGE)

92 Deep Creek (AVWP)

93 Derr Creek (AVWP)

94 Dietrich Drop (IPC)

95 Donald Jenni (NWE)

96 Eagle Creek (PGE)

97 Eagle Point Irrigation Dist. (PPL)

98 Elk Creek (IPC)

99 Estacada (PGE)

100 Falls Creek (PPL)

101 Falls River (IPC)

102 Farmers Irrig. (PPL)

103 Faulkner Ranch (IPC)

104 Fisheries Development Co (IPC)

105 Galesville Dam (PPL)

106 Geobon 2 (IPC)

107 Georgetown Power (UPL)

108 Hailey Cspp (IPC)

109 Hazelton A (IPC)

110 Hazelton B (IPC)

111 Horseshoe Bend (IPC)

112 Hutchinson Creek (PSE)

113 Ingram Warm Springs (UPL)

$114 \mathrm{Jim}$ Ford Creek (AVWP)

115 Jim Knight (IPC)

116 John Day Creek (AVWP)

117 Joseph Hydro (PPL)

118 Kasel \& Witherspoon (IPC)

119 Koma Kulshan (PSE)

120 Koyle (IPC)

$121 \mathrm{~L}$. Fery (PPL)

122 Lacomb Irrig. (PPL)

123 Lake Oswego (PGE)

124 Lateral \#10 (IPC)

125 Lee Tavenner (NWE)

126 Lemoyne (IPC)

127 Little Wood River Res. (IPC)

128 Littlewood/Arkoosh (IPC)

129 Lowline \#2 (IPC)
2.4
0.1
0.1
1.3
0.1
2.
0.7
0.4
0.
1.1
8.3

$\begin{array}{rrr}0 & 0 & 0 \\ 0.1 & 0.1 & 0.1\end{array}$

$\begin{array}{rrrrrrr}0.1 & 0.1 & 0.1 & 0.1 & 0.1 & 0.1 & 0.1\end{array}$

$\begin{array}{llllll}0 & 0 & 0.1 & 0.1 & 0.1 & 0.1\end{array}$

$\begin{array}{llll}0.1 & 0.1 & 0.1 & 0.1\end{array}$

$\begin{array}{llll}0.1 & 0.1 & 0.1 & 0.1 \\ 2.5 & 3.4 & 3.4 & 3.4\end{array}$

$\begin{array}{llll}0.7 & 0.7 & 1.1 & 0.9\end{array}$

0.6

$\begin{array}{llll}1.1 & 1.1 & 1.5 & 1.9\end{array}$

$\begin{array}{llll}8.3 & 8.3 & 6.5 & 2.8 \\ 0.1 & 0.1 & 0.1 & 0.1\end{array}$

0.1
0

0
2.9

0.3

3.1
0.4

0.4
0

0
1.3

0.2

0
0

0.1

4.7

0.1

0

1.2
0.1

0.1
0

6.5

0.6

0.8
0.2
0.6

0.6

1.0

0.1

7

6.6

0.7

0.2
0

0.4

0.2

1.5

1.2

4.6
1.1
0

1.1
0

0

$0.4 \quad 0.4$

$1.5 \quad 1.5$

0.1

1.9

0.7

2.7

$\begin{array}{rrr}0 & 0 \\ 29 & 31 & 2.8\end{array}$

$\begin{array}{lll}0.3 & 0.9 & 0.9 \\ 3.1 & 3.7 & 3.5\end{array}$

$\begin{array}{lll}0.4 & 0.4 & 0.5\end{array}$

$\begin{array}{rrr}0 & 0 & \\ 1.3 & 1.6 & 1.5\end{array}$

0.2

$\begin{array}{lll}0 & 0 \\ 0 & 0 & 0\end{array}$

$\begin{array}{lll}0.1 & 0.1 & 0.1 \\ 4.7 & 3.6 & 3.1\end{array}$

$\begin{array}{lll}4.7 & 3.6 & 3.1 \\ 0.1 & 0.1 & 0.1\end{array}$

$\begin{array}{rrrr}0 & 0 & 0 & 0.4 \\ 1.2 & 0 & 0 & 0 \\ 0.1 & 0.1 & 0.1 & 0.1\end{array}$

$\begin{array}{rrrrr}0 & 0 & 0 & 1.9 & 3.1\end{array}$

0.2

$0.6-0.4$

1.0

0.2

0.1

$\begin{array}{lll}6.6 & 5.2 & 2.4 \\ 8.8 & 7.5 & 3.9\end{array}$

$\begin{array}{lll}0.7 & 0.7 & 0.7\end{array}$

0.2

$0.3 \quad 0.3$

$2.9 \quad 2.4$

0
1.8

$0.1-0.1$

2.1

0.3

0.3

0.2

0.3

0.1

0.4

$\begin{array}{lll}0.4 & 0.3 & 0.2\end{array}$

$\begin{array}{lll}0.2 & 0.2 & 0.2 \\ 1.5 & 0.9 & 0.4\end{array}$

1.5

$1.2 \quad 1.2$

$\begin{array}{ll}4.6 & 4.3\end{array}$

0.4

0.8
6.4

1.0

0.4
1.5
0.1

0

$\begin{array}{lllll}0 & 0 & 0.2 & 1.0 & 0.2\end{array}$

$\begin{array}{lllll}0.4 & 0.4 & 0.4 & 0.4 & 0.4\end{array}$

0.1

0.1

$\begin{array}{rrrrr}0.1 & 0.1 & 0 & 0.1 & 0.1\end{array}$

$\begin{array}{llll}1.9 & 0.2 & 0.2 & 0.2\end{array}$

0.7

2.7

0.4
2.5

0.4

0.4

0.2
0

$\begin{array}{rrr}0.8 & 0.3 & \\ 0 & 0 & \\ 0 & 0.1 & \\ 0.2 & 0.1 & 0.1 \\ 0.1 & 0 & 0.1 \\ 0.1 & 0.1 & 0.1 \\ 1.0 & 1.1 & \\ 0.1 & 0.1 & \\ 3.4 & 3.1 & 3.1 \\ 0.9 & 0.2 & \\ 0.4 & 0.4 & 0.3 \\ 0 & 0 & 0.4 \\ 0.6 & 0.6 & 0.6 \\ 1.9 & 1.5 & 12 \\ 2.8 & 1.0 & \\ 0.1 & 0.1 & 0.1 \\ 0 & 0 & \\ 0 & 0 & 0\end{array}$

$\begin{array}{rr}1.0 & 2.2 \\ 0 & 0 \\ 0 & 0 \\ 0.1 & 0.1 \\ 0.1 & 0.1 \\ 0.1 & 0 \\ 1.1 & 0.3 \\ 0 & 0 \\ 3.7 & 3.4 \\ 0 & 0 \\ 0.3 & 0.3 \\ 0.4 & 0.3 \\ 0.6 & 0.6 \\ 1.2 & 1.2 \\ 0 & 0 \\ 0.1 & 0.1 \\ 0 & 0.1\end{array}$

$\begin{array}{rr}2.6 & 3.3 \\ 0 & \\ 0 & \\ 0.1 & 0.1 \\ 0.1 & \\ 0 & \\ 1.2 & 1.7 \\ 0 & 0.1 \\ 3.1 & 3.1 \\ 0 & \\ 0.3 & 0.3 \\ 0.6 & 0.7 \\ 0.7 & 0.6 \\ 1.2 & 1.2 \\ 0 & 0.1 \\ 0.1 & 0.1 \\ 0.1 & 0.1\end{array}$

$\begin{array}{lll}3.3 & 3.2 & 3.2 \\ 0 & 0 & 0\end{array}$

3.2
0
0
0.1
0

$3.2 \quad 3$.

0
0
0.1
0

0.1

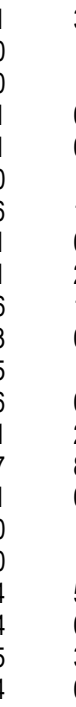

$\begin{array}{lrr}3.2 & 3.3 & 2.3 \\ 0 & 0 & 0\end{array}$

$\begin{array}{rrr}0 & 0 & 0 \\ 0 & 0 & 0 \\ 0.2 & 0.1 & 0.1\end{array}$

$\begin{array}{rrr}0.1 & 0.1 & 0.1 \\ 0 & 0 & 0\end{array}$

$\begin{array}{lllllll}1.7 & 1.6 & 1.6 & 1.6 & 1.3 & 1.2 & 1.2 \\ 0.1 & 0.1 & 0.1 & 0.1 & 0.1 & 0.1 & 0.1\end{array}$

$\begin{array}{lllllll}3.1 & 2.8 & 3.1 & 3.1 & 2.8 & 1.9 & 3.1\end{array}$

0.6

$\begin{array}{ll}0.7 & 0.8\end{array}$

$\begin{array}{lll}0.6 & 0.6 & 0.6\end{array}$

$\begin{array}{lll}1.2 & 1.9 & 1.8\end{array}$

$\begin{array}{lll}1.2 & 1.9 & 1.8 \\ 0.1 & 29 & 2.9\end{array}$

$0.8-0.7$

0.4

$\begin{array}{rr}0 & \\ 2 & 2 . \\ 1.3 & 0.4 \\ 0.4 & 0.9 \\ 0.5 & 0.4\end{array}$

0.1

0.1
0.1

$\begin{array}{rrr}0 & 0 & 0 \\ 0 & 2.7 & 2.7\end{array}$

$\begin{array}{ll}2.7 & 2.7 \\ 0.4 & 0.4\end{array}$

$\begin{array}{lll}0.4 & 0.4 & 0.4 \\ 0.9 & 0.8 & 0.8\end{array}$

0.4
0

$0.3 \quad 0.3$

0
0.6

0.8

0
0.9
0.1

0.9
0.1
0
0

$0.1 \quad 0.1$

0.1

0.2
3.1

0.1

0.9

$0.1 \quad 0.1$

$\begin{array}{ll}0.1 & 0.1 \\ 0.9 & 2.7\end{array}$

$\begin{array}{ll}0.2 & 3.8\end{array}$

3.8
4.6
0.3

0.3

0.3
0.2

0.3

0.3
0.1

0

0
4.6

$\begin{array}{ll}3.9 & 4.6 \\ 0.7 & 0.7\end{array}$

0.7
0.4

0.9

0.1
0.2

0

0.2

$\begin{array}{ll}3.8 & 4.4\end{array}$

$\begin{array}{lll}0.3 & 0.8 & 0.8\end{array}$

0.9

0.4

0.9

0.4

0.1

0.1 

2003 White Book

Aug1 Aug16 Sep Oct Nov Dec

Jan Feb Mar Apr1 Apr16

\begin{abstract}
May
\end{abstract}

$$
\text { Jun }
$$
Jul Avg

130 Lowline Canal (IPC)

131 Magic Reservoir (IPC)

132 Malad River (IPC)

133 Marcos Ranches (IPC)

134 Marsh Valley (UPL)

135 Meyers Falls (AVWP)

136 Middlefork Irrig. (PPL)

137 Mile 28 (IPC)

138 Minikahda (PGE)

139 Mink Creek (UPL)

140 Mitchell Butte (IPC)

141 Mountain Energy (PPL)

142 Mt. Tabor (PGE)

143 Mud Creek/S\&S (IPC)

144 Mud Creek/White (IPC)

145 Nichols Gap (PPL)

146 Nicholson Sunnybar (UPL)

147 North Fork Sprague (PPL)

148 O.J. Power (UPL)

149 Odell Creek (PPL)

150 Opal Springs (PPL)

151 Owyhee Dam (IPC)

152 Pancheri (UPL)

153 Pelton Rereg. Dam (PPL)

154 Phillips Ranch (AVWP)

155 Phillipsburg (NWE)

156 Pigeon Cove (IPC)

157 Pine Creek (NWE)

158 Port Townsend Paper Hydro (PSE)

159 Portland (PGE)

160 Portland Hydro Project (PGE)

161 Preston City (UPL)

162 Pristine Springs (IPC)

163 Pristine Springs \#3 (IPC)

164 Reynolds Irrigation (IPC)

165 Rim View (IPC)

166 Rock Creek \#1 (IPC)

167 Rock Creek \#2 (IPC)

168 Sagebrush (IPC)

169 Salem (PGE)

170 Schaffner (IPC)

171 Sheep Creek (AVWP)

172 Shingle Creek (IPC)

173 Shoshone (IPC)

174 Shoshone II (IPC)

175 Snake River Pottery (IPC)

176 Snedigar Ranch (IPC)

177 Solar Research (PPL)

178 South Dry Creek (NWE)

179 Spokane Upriver (AVWP)

180 Stauffer Dry Creek (UPL)

181 Strawberry Creek (NWE)

182 Sunshine Power \#2 (IPC)

183 Sygitowicz Creek Small Hydro (PSE)

184 TGS/Briggs (UPL)

185 Trout Co. (IPC)

186 Tunnel \#1 (IPC)

187 Twin Falls (PSE)

188 Walla Walla (PPL)

189 Water Street / Santiam (PPL)

190 Weeks Falls (PSE)

191 White James (PPL

192 White Water Ranch (IPC)

193 Wilson Lake Hydro (IPC)

194 Wisconsin Creek (NWE)

195 Yakima-Tieton (PPL)

196 IOU - Total NUG: Hydro

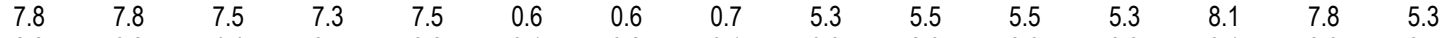

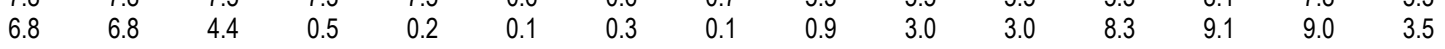

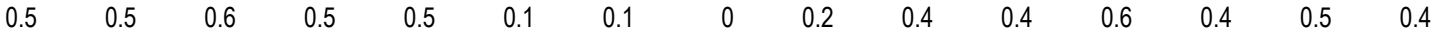

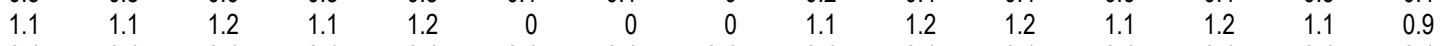

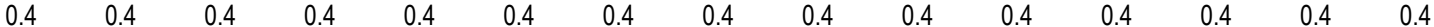

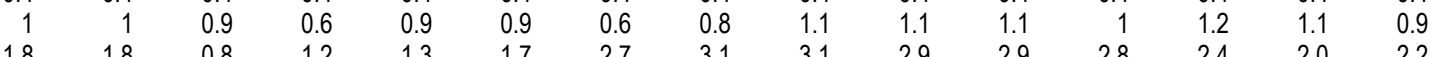

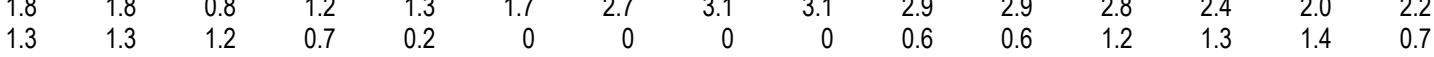

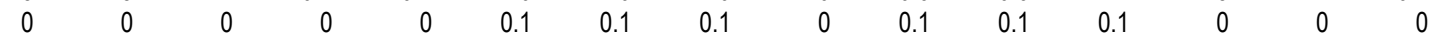

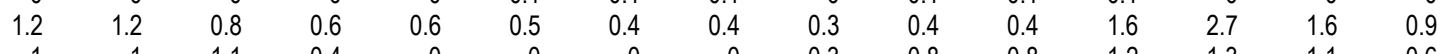

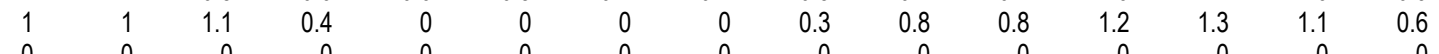

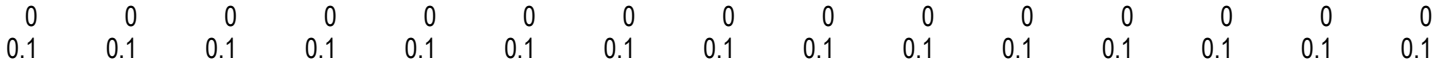

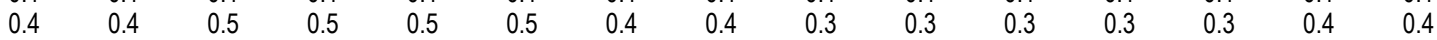

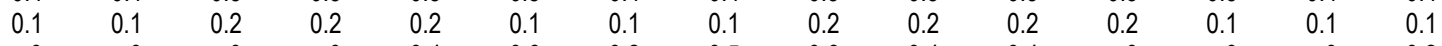

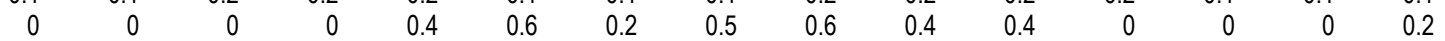

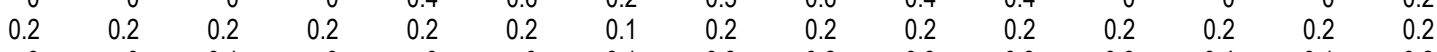

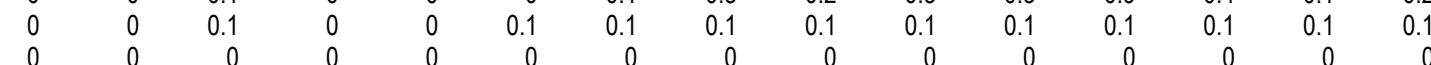

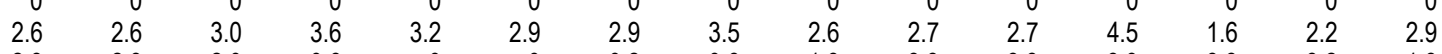

$\begin{array}{lllllll}2.0 & 2.0 & 2.1 & 1.3 & 0.8 & 0.4 & 0.3 \\ 0.1 & 0.1 & 0.1 & 0.1 & 0.1 & 0.1 & 0.1 \\ 0.3 & 0.3 & 0.3 & 0.3 & 0.3 & 0.3 & 0.3\end{array}$

$\begin{array}{rrr}0 & 0 & 0 \\ 2.3 & 2.3 & 2.9\end{array}$

$\begin{array}{llll}0.3 & 0.3 & 0.4 & 0.4\end{array}$

$\begin{array}{lllllll}0.1 & 0.1 & 0.2 & 0.2 & 0.4 & 0.4 & 0.4 \\ 0.2 & 0.2 & 0.2 & 0.3 & 0.2 & 0.1 & 0.1\end{array}$

$\begin{array}{lllllll}0.2 & 0.2 & 0.2 & 0.3 & 0.2 & 0.2 & 0.2 \\ 0.2 & 0.2 & 0.2 & 0.2 & 0.2 & 0.2 & 0.2\end{array}$

$\begin{array}{lllllll}0.2 & 0.2 & 0.2 & 0.2 & 0.2 & 0.2 & 0.2 \\ 1.1 & 0.2 & 0.2 & 0.3 & 0.2 & 0.2 & 0.2\end{array}$

$\begin{array}{lllllll}1.1 & 1.1 & 1.6 & 1.7 & 1.2 & 1.3 & 1.1 \\ 1.8 & 1.8 & 1.8 & 1.9 & 1.9 & 1.9 & 0.6\end{array}$

$\begin{array}{rrrrrrr}0.4 & 0.4 & 0.3 & 0.3 & 0.3 & 0.1 & 0.1 \\ 0 & 0 & 0 & 0 & 0 & 0 & 0\end{array}$

\begin{tabular}{|c|c|c|c|c|c|}
\hline 0 & 0 & 0 & 0 & 0 & 0 \\
\hline 0.4 & 0.3 & 0.2 & 0.3 & 0.1 & 0.1 \\
\hline 0.4 & 0.3 & 0.3 & 0.4 & 0.5 & 0.5 \\
\hline
\end{tabular}

$\begin{array}{lllllll}0.1 & 0.1 & 0.2 & 0.3 & 0.4 & 0.5 & 0.5 \\ 0.4 & 0.4 & 0.5 & 0.4 & 0.1 & 0.1 & 0.1\end{array}$

$\begin{array}{llll}0.4 & 0.4 & 0.5 & 0.4 \\ 0.6 & 0.6 & 0.6 & 0\end{array}$

\begin{tabular}{|c|c|c|c|c|c|}
\hline 0.6 & 0.6 & 0 & 0 & 0 & 0 \\
\hline 0 & 0 & 0 & 0 & 0.1 & 0.1 \\
\hline 0.2 & 0.2 & 0.2 & 0.2 & 0.2 & 0.2 \\
\hline 0.1 & 0.1 & 0 & 0 & 0 & 0.1 \\
\hline
\end{tabular}

$\begin{array}{llll}0.5 & 0.5 & 0.5 & 0.5\end{array}$

$\begin{array}{lllllll}3.4 & 3.4 & 3.4 & 4.7 & 6.7 & 13 & 14\end{array}$

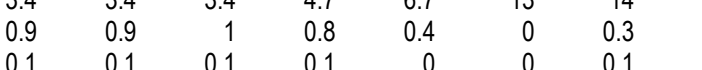

$\begin{array}{rrrrrrr}0.1 & 0.1 & 0.1 & 0.1 & 0.1 & 0 & 0 \\ 0 & 0 & 0 & 0.1 & 0.2 & 0.3 & 0.3\end{array}$

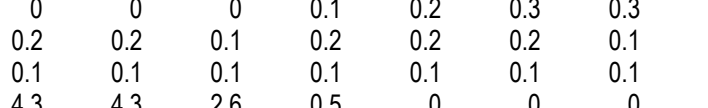

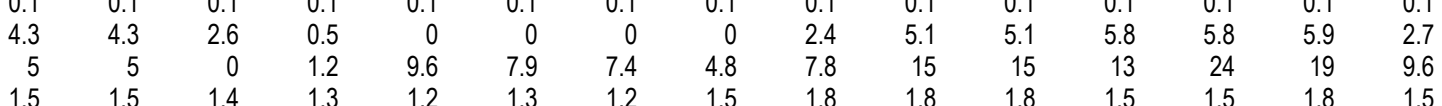

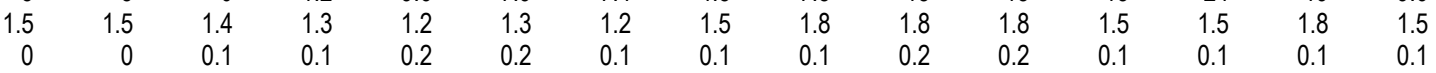

$\begin{array}{rrrllllllllllll}0 & 0 & 0.1 & 0.1 & 0.2 & 0.2 & 0.1 & 0.1 & 0.1 & 0.2 & 0.2 & 0.1 & 0.1 & 0.1 & 0.1 \\ 0.8 & 0.8 & 0 & 0.2 & 1.7 & 1.8 & 1.2 & 0.6 & 1.2 & 2.6 & 2.6 & 2.4 & 4.4 & 3.5 & 1.7 \\ 0.1 & 0.1 & 0.1 & 0.1 & 0.1 & 0.1 & 0.1 & 0.2 & 0.2 & 0.2 & 0.2 & 0.2 & 0.3 & 0.2 & 0.2\end{array}$

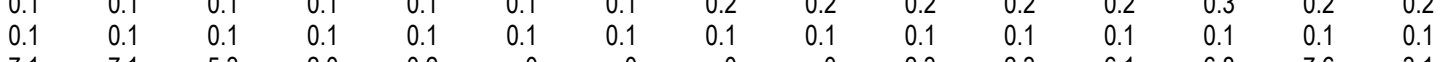

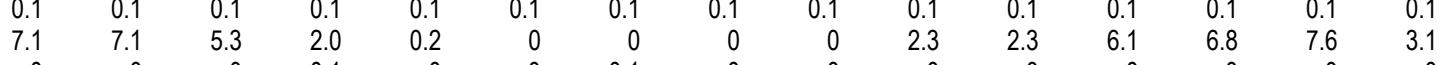

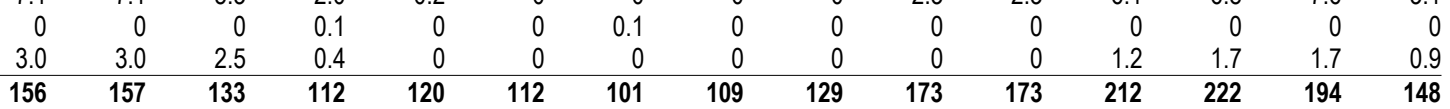

-NUG: Small Thermal \& Misc.-

197 Marion Solid Waste (PGE)

198 Minnesota Methane (AVWP)

199 Montana One (NWE)

200 Pocatello Waste (IPC)

201 Spokane MSW (PSE)

202 West Boise Waste (IPC)

$\begin{array}{rrrrrrrrrrrrrrr}9.3 & 9.3 & 9.3 & 9.3 & 9.3 & 9.3 & 9.3 & 9.3 & 9.3 & 9.3 & 9.3 & 9.3 & 9.3 & 9.3 & 9.3 \\ 0.8 & 0.8 & 0.8 & 0.8 & 0.8 & 0.8 & 0.8 & 0.8 & 0.8 & 0.8 & 0.8 & 0.8 & 0.8 & 0.8 & 0.8 \\ 9.3 & 9.5 & 8.7 & 8.9 & 6.7 & 7.5 & 7.7 & 7.4 & 6.4 & 6.2 & 6.1 & 10 & 8.7 & 8.4 & 8 \\ 0.1 & 0.1 & 0.2 & 0.1 & 0.2 & 0.1 & 0.1 & 0.2 & 0.1 & 0.2 & 0.2 & 0.1 & 0.2 & 0.1 & 0.1 \\ 15 & 15 & 15 & 15 & 15 & 15 & 15 & 15 & 15 & 15 & 15 & 15 & 15 & 15 & 15 \\ 0.1 & 0.1 & 0.1 & 0.1 & 0.1 & 0.1 & 0.1 & 0.1 & 0.1 & 0.1 & 0.1 & 0.1 & 0.1 & 0.1 & 0.1 \\ \mathbf{3 5} & \mathbf{3 5} & \mathbf{3 4} & \mathbf{3 5} & \mathbf{3 2} & \mathbf{3 3} & \mathbf{3 3} & \mathbf{3 3} & \mathbf{3 2} & \mathbf{3 2} & \mathbf{3 2} & \mathbf{3 6} & \mathbf{3 4} & \mathbf{3 4} & \mathbf{3 4}\end{array}$


Table A-24: Regional Non Utility Generating Resources By Project

PNW Loads and Resources Study

2008 - 2009 Operating Year 2003 White Book

Aug1 Aug16 Sep

Oct Nov

Dec Jan Feb Mar Apr1 Apr16

May Jun Jul Avg

-NUG: Combustion Turbines -

204 March Point \#1 Cogeneration (PSE)

205 March Point \#2 Cogeneration (PSE)

206 Rathdrum- Boekel Rd. \#1 (AVWP)

207 Rathdrum -Boekel Rd. \#2 (AVWP)

208 Tenaska Gas Cogen (PSE)

209 IOU - Total NUG: Combustion Turbines

\begin{tabular}{|c|c|c|c|c|c|c|c|c|c|c|c|c|c|c|}
\hline 79 & 79 & 79 & 79 & 79 & 79 & 79 & 79 & 79 & 79 & 79 & 55 & 79 & 79 & 77 \\
\hline 59 & 59 & 59 & 59 & 59 & 59 & 59 & 59 & 59 & 59 & 59 & 45 & 59 & 59 & 58 \\
\hline 65 & 65 & 65 & 65 & 65 & 65 & 65 & 65 & 65 & 65 & 65 & 32 & 65 & 65 & 62 \\
\hline 65 & 65 & 65 & 65 & 65 & 65 & 65 & 65 & 65 & 65 & 65 & 32 & 65 & 65 & 62 \\
\hline 240 & 240 & 240 & 240 & 240 & 240 & 240 & 240 & 240 & 240 & 240 & 180 & 240 & 240 & 235 \\
\hline 508 & 508 & 508 & 508 & 508 & 508 & 508 & 508 & 508 & 508 & 508 & 344 & 508 & 508 & 494 \\
\hline
\end{tabular}

-NUG: Co-Generation-

210 Billings Generation (NWE)

211 Biomass One (PPL)

212 Boise Cascade Medford (PPL)

213 Champion (PPL)

214 DR Johnson - Co-Gen II (PPL)

215 Magic Valley (IPC)

216 Magic West (IPC)

217 PERC Pierce, Wa (PSE)

218 Simplot Pocatello (IPC)

219 Sumas Energy (PSE)

220 Tamarack (IPC)

221 TASCO Nampa ID (IPC)

222 TASCO Twin Falls ID (IPC)

223 Vaagen Bros. (IPC)

224 Warm Springs (PPL)

225 IOU - Total NUG: Co-Generation

\begin{tabular}{|c|c|c|c|c|c|c|c|c|c|c|c|c|c|c|}
\hline 17 & 17 & 16 & 10 & 12 & 14 & 14 & 14 & 12 & 7.2 & 7.1 & 19 & 16 & 15 & 14 \\
\hline 22 & 22 & 21 & 21 & 22 & 22 & 7.2 & 15 & 17 & 20 & 20 & 17 & 22 & 22 & 19 \\
\hline 8.5 & 8.5 & 8.5 & 8.5 & 8.5 & 8.5 & 8.5 & 8.5 & 8.5 & 8.5 & 8.5 & 8.5 & 8.5 & 8.5 & 8.5 \\
\hline 3.3 & 3.3 & 3.3 & 3.3 & 3.3 & 3.3 & 1 & 3.3 & 3.3 & 3.3 & 3.3 & 3.3 & 3.3 & 3.3 & 3.1 \\
\hline 7.5 & 7.5 & 7.7 & 5.8 & 7.3 & 6.0 & 6.7 & 9.1 & 7.3 & 7.4 & 7.4 & 11 & 4.2 & 7.1 & 7.2 \\
\hline 9.3 & 9.3 & 9.7 & 9.3 & 9.6 & 9.3 & 9.3 & 10 & 9.4 & 9.7 & 9.7 & 9.4 & 9.7 & 9.3 & 9.5 \\
\hline 9.3 & 9.3 & 9.7 & 9.3 & 9.6 & 9.3 & 9.3 & 10 & 9.4 & 9.7 & 9.7 & 9.4 & 9.7 & 9.3 & 9.5 \\
\hline 2 & 2 & 2 & 2 & 2 & 2 & 2 & 2 & 2 & 2 & 2 & 0 & 2 & 2 & 1.8 \\
\hline 8.8 & 8.8 & 9.2 & 8.8 & 9.1 & 8.8 & 8.8 & 9.6 & 8.9 & 9.2 & 9.2 & 8.9 & 9.2 & 8.8 & 9.0 \\
\hline 113 & 113 & 126 & 126 & 126 & 126 & 126 & 126 & 126 & 113 & 113 & 113 & 113 & 113 & 120 \\
\hline 3.8 & 3.8 & 4.6 & 4.7 & 4.2 & 3.2 & 3.6 & 3.8 & 4.2 & 4.4 & 4.4 & 3.4 & 4.6 & 4.8 & 4.1 \\
\hline 0.2 & 0.2 & 0.2 & 0.2 & 0.2 & 0.2 & 0.2 & 0.2 & 0.2 & 0.2 & 0.2 & 0.2 & 0.2 & 0.2 & 0.2 \\
\hline 0 & 0 & 0 & 0 & 0 & 0 & 0 & 0 & 0 & 0 & 0 & 0 & 0 & 0 & 0 \\
\hline 3 & 3 & 3.7 & 3.8 & 3.3 & 2.6 & 2.9 & 3.1 & 3.4 & 3.6 & 3.6 & 2.7 & 3.7 & 3.8 & 3.3 \\
\hline 0.1 & 0.1 & 0 & 0.1 & 0.1 & 0.1 & 0.1 & 0.1 & 0 & 0 & 0 & 0 & 0.1 & 0 & 0.1 \\
\hline 207 & 207 & 222 & 214 & 218 & 214 & 200 & 215 & 211 & 198 & 198 & 205 & 206 & 207 & 210 \\
\hline
\end{tabular}

-NUG: Renewables-

226 Foote Creek 1 (PPL)

227 Healow \#2 (NWE)

228 Josef Staufer (NWE)

229 Lewandowski Farms (IPC)

230 Livingston (NWE)

231 Puyallup Energy Recovery (PSE)

232 Stateline Wind Project (AVWP)

233 Windmill (PGE)

234 IOU - Total NUG: Renewables

Other Entities

-NUG: Co-Generation-

235 SP Newsprint cogen (SPN)

236 Other - Total NUG: Co-Generation

\begin{tabular}{|c|c|c|c|c|c|c|c|c|c|c|c|c|c|c|}
\hline 4.2 & 4.2 & 4.9 & 7.2 & 11 & 13 & 14 & 12 & 9.2 & 7.4 & 7.4 & 5.9 & 5.2 & 4.2 & 8.1 \\
\hline 0 & 0 & 0 & 0 & 0 & 0 & 0 & 0 & 0 & 0 & 0 & 0 & 0 & 0 & 0 \\
\hline 0 & 0 & 0 & 0 & 0 & 0 & 0 & 0 & 0 & 0 & 0 & 0 & 0 & 0 & 0 \\
\hline 0 & 0 & 0 & 0 & 0 & 0 & 0 & 0 & 0 & 0 & 0 & 0 & 0 & 0 & 0 \\
\hline 0 & 0 & 0 & 0 & 0 & 0 & 0 & 0 & 0 & 0 & 0 & 0 & 0 & 0 & 0 \\
\hline 2 & 2 & 2 & 2 & 2 & 2 & 2 & 2 & 2 & 2 & 2 & 2 & 2 & 2 & 2 \\
\hline 0.3 & 0.3 & 0.2 & 0.3 & 0.3 & 0.3 & 0.3 & 0.3 & 0.3 & 0.3 & 0.3 & 0.3 & 0.3 & 0.3 & 0.3 \\
\hline 0 & 0 & 0 & 0 & 0 & 0 & 0 & 0 & 0 & 0 & 0 & 0 & 0 & 0 & 0 \\
\hline 6.5 & 6.5 & 7.1 & 9.5 & 13 & 15 & 16 & 15 & 12 & 9.8 & 9.8 & 8.2 & 7.6 & 6.5 & 10 \\
\hline
\end{tabular}

-NUG: Renewables-

237 Stateline Wind Project (PPME)

238 Vansycle Wind (FPLEV)

239 Other - Total NUG: Renewables

$\begin{array}{lllllllllllllll}25 & 25 & 25 & 25 & 25 & 25 & 25 & 25 & 25 & 25 & 25 & 25 & 25 & 25 & 25 \\ \mathbf{2 5} & \mathbf{2 5} & \mathbf{2 5} & \mathbf{2 5} & \mathbf{2 5} & \mathbf{2 5} & \mathbf{2 5} & \mathbf{2 5} & \mathbf{2 5} & \mathbf{2 5} & \mathbf{2 5} & \mathbf{2 5} & \mathbf{2 5} & \mathbf{2 5} & \mathbf{2 5}\end{array}$

-Total Non-Utility Generating Resources-

240 Federal Entities

241 Generating Public Entities

242 Non Generating Public Entities

243 Investor-Owned Entities

244 Other Entities

$\begin{array}{rrrrrrrrrrrrrrr}52 & 52 & 41 & 53 & 57 & 51 & 55 & 51 & 51 & 57 & 57 & 57 & 59 & 52 & 53 \\ 7.4 & 7.4 & 7.4 & 7.4 & 7.4 & 7.4 & 7.4 & 7.4 & 7.4 & 7.4 & 7.4 & 7.4 & 7.4 & 7.4 & 7.4 \\ \mathbf{5 9} & \mathbf{5 9} & \mathbf{4 8} & \mathbf{6 0} & \mathbf{6 5} & \mathbf{5 8} & \mathbf{6 2} & \mathbf{5 8} & \mathbf{5 8} & \mathbf{6 4} & \mathbf{6 4} & \mathbf{6 5} & \mathbf{6 7} & \mathbf{6 0} & \mathbf{6 0}\end{array}$

245 Total Non-Utility Generation

$\begin{array}{rrrrrrrrrrrrrrr}109 & 109 & 102 & 116 & 127 & 127 & 130 & 125 & 119 & 121 & 121 & 118 & 120 & 109 & 119 \\ 308 & 308 & 226 & 170 & 145 & 138 & 146 & 139 & 151 & 274 & 274 & 366 & 382 & 344 & 232 \\ 3.4 & 3.4 & 3.4 & 4.1 & 5.7 & 5.8 & 6.1 & 5.4 & 4.2 & 3.9 & 3.9 & 3.9 & 3.8 & 3.3 & 4.4 \\ 913 & 914 & 905 & 878 & 892 & 882 & 858 & 880 & 892 & 921 & 921 & 806 & 978 & 950 & 896 \\ 84 & 84 & 73 & 85 & 90 & 83 & 87 & 83 & 83 & 89 & 89 & 90 & 92 & 85 & 85 \\ 1417 & \mathbf{1 4 1 9} & \mathbf{1 3 0 9} & \mathbf{1 2 5 3} & \mathbf{1 2 5 9} & \mathbf{1 2 3 6} & \mathbf{1 2 2 7} & \mathbf{1 2 3 2} & \mathbf{1 2 5 0} & \mathbf{1 4 0 9} & \mathbf{1 4 0 9} & \mathbf{1 3 8 3} & \mathbf{1 5 7 5} & \mathbf{1 4 9 2} & \mathbf{1 3 3 7}\end{array}$


Oct Nov

Dec

\begin{abstract}
Jan Feb
\end{abstract}

\begin{abstract}
Mar Apr1 Apr16
\end{abstract}

\begin{abstract}
May
\end{abstract}
Jun

Jul Avg

\title{
Federal Entities
}

-NUG: Hydro-

1 Clearwater - State of ID DWR (BPA)

2 Dworshak Small Hydropower (BPA)

3 Federal -Total NUG: Hydro

\begin{tabular}{|c|c|c|c|c|c|c|c|c|c|c|c|c|c|c|}
\hline 1 & 1 & 1 & 1 & 1 & 1 & 1 & 1 & 1 & 1 & 1 & 1 & 1 & 1 & 1 \\
\hline 2.6 & 2.6 & 2.6 & 2.6 & 2.6 & 2.6 & 2.6 & 2.6 & 2.6 & 2.6 & 2.6 & 2.6 & 2.6 & 2.6 & 2.6 \\
\hline 3.6 & 3.6 & 3.6 & 3.6 & 3.6 & 3.6 & 3.6 & 3.6 & 3.6 & 3.6 & 3.6 & 3.6 & 3.6 & 3.6 & 3.6 \\
\hline
\end{tabular}

-NUG: Renewables-

4 Ashland Solar Project (BPA)

5 Condon Wind Project (BPA)

6 Foote Creek 1 (BPA)

7 Foote Creek 2 (BPA)

8 Foote Creek 4 (BPA)

9 Fourmile Hill Geothermal (BPA)

10 Klondike Phase 1 (BPA)

11 Stateline Wind Project (BPA)

12 Federal -Total NUG: Renewables

\begin{tabular}{|c|c|c|c|c|c|c|c|c|c|c|c|c|c|}
\hline 0 & 0 & 0 & 0 & 0 & 0 & 0 & 0 & 0 & 0 & 0 & 0 & 0 & 0 \\
\hline 10 & 10 & 11 & 13 & 14 & 13 & 12 & 12 & 14 & 14 & 12 & 15 & 11 & 12 \\
\hline 3.3 & 3.8 & 5.6 & 8.3 & 10 & 11 & 9.6 & 7.2 & 5.8 & 5.8 & 4.6 & 4.1 & 3.3 & 6.4 \\
\hline 0.4 & 0.5 & 0.7 & 1 & 1.3 & 1.3 & 1.2 & 0.9 & 0.7 & 0.7 & 0.5 & 0.5 & 0.4 & 0.8 \\
\hline 3.9 & 4.4 & 6.5 & 9.7 & 12 & 12 & 11 & 8.4 & 6.7 & 6.7 & 5.4 & 4.8 & 3.8 & 7.4 \\
\hline 50 & 50 & 50 & 50 & 50 & 50 & 50 & 50 & 50 & 50 & 50 & 50 & 50 & 50 \\
\hline 7.9 & 6.2 & 8.1 & 8.8 & 7.8 & 8.3 & 7.8 & 7.8 & 8.6 & 8.6 & 8.8 & 9.1 & 8 & 8.1 \\
\hline 29 & 23 & 30 & 33 & 29 & 31 & 29 & 29 & 32 & 32 & 33 & 34 & 30 & 30 \\
\hline 105 & 98 & 112 & 124 & 123 & 126 & 121 & 115 & 118 & 118 & 114 & 117 & 106 & 115 \\
\hline
\end{tabular}

Generating Public Entities

-NUG: Hydro-

13 Don Steffen (SHPD)

14 Eltopia Br Canal 4.6 (SCL)

15 Eltopia Br Canal 4.6 (TPU)

16 John Llewellyn (SHPD)

17 Kevin Duncan (SHPD)

18 Lucky Peak (SCL)

19 Main Canal Headworks (SCL)

20 Main Canal Headworks (TPU)

21 PEC Headworks (GCPD)

22 Pickering Micro Hydro (CKPD)

23 Potholes E Canal 66.0 (SCL)

24 Potholes E Canal 66.0 (TPU)

25 Quincy Chute (GCPD)

26 Russel D. Smith (SCL)

27 Russel D. Smith (TPU)

28 Smith Creek (EWEB)

29 Summer Falls (SCL)

30 Summer Falls (TPU)

31 Woods Creek (SHPD)

32 GPU - Total NUG: Hydro

\begin{tabular}{rrrrrrrrrrrrrrr}
0 & 0 & 0 & 0 & 0 & 0 & 0 & 0 & 0 & 0 & 0 & 0 & 0 & 0 & 0 \\
0.9 & 0.9 & 0.6 & 0.3 & 0 & 0 & 0 & 0 & 0 & 0.7 & 0.7 & 1 & 1 & 1.1 & 0.5 \\
0.9 & 0.9 & 0.6 & 0.3 & 0 & 0 & 0 & 0 & 0 & 0.7 & 0.7 & 1 & 1 & 1.1 & 0.5 \\
0 & 0 & 0 & 0 & 0 & 0 & 0 & 0 & 0 & 0 & 0 & 0 & 0 & 0 & 0 \\
0 & 0 & 0 & 0 & 0 & 0 & 0 & 0 & 0 & 0 & 0 & 0 & 0 & 0 & 0 \\
66 & 66 & 41 & 11 & 2.2 & 3 & 7.9 & 10 & 21 & 52 & 52 & 84 & 85 & 77 & 38 \\
10 & 10 & 6.1 & 2.6 & 0 & 0 & 0 & 0 & 1.4 & 6.8 & 6.8 & 9.1 & 11 & 12 & 4.9 \\
10 & 10 & 6.1 & 2.6 & 0 & 0 & 0 & 0 & 1.4 & 6.8 & 6.8 & 9.1 & 11 & 12 & 4.9 \\
4.5 & 4.5 & 3.1 & 2.2 & 0 & 0 & 0 & 0 & 0.4 & 5.1 & 5.1 & 6.9 & 6.2 & 5.8 & 2.9 \\
0 & 0 & 0 & 0 & 0 & 0 & 0 & 0 & 0 & 0 & 0 & 0 & 0 & 0 & 0 \\
0.9 & 0.9 & 0.9 & 0.8 & 0 & 0 & 0 & 0 & 0.3 & 0.9 & 0.9 & 0.9 & 0.9 & 0.9 & 0.5 \\
0.9 & 0.9 & 0.9 & 0.8 & 0 & 0 & 0 & 0 & 0.3 & 0.9 & 0.9 & 0.9 & 0.9 & 0.9 & 0.5 \\
7.7 & 7.7 & 4 & 2.3 & 0 & 0 & 0 & 0 & 0.8 & 5.5 & 5.5 & 7.1 & 8.4 & 8.5 & 3.7 \\
2 & 2 & 1.3 & 0.7 & 0 & 0 & 0 & 0 & 0.1 & 1.2 & 1.2 & 1.9 & 2.1 & 1.7 & 0.9 \\
2 & 2 & 1.3 & 0.7 & 0 & 0 & 0 & 0 & 0.1 & 1.2 & 1.2 & 1.9 & 2.1 & 1.7 & 0.9 \\
0 & 0 & 0 & 5 & 5.4 & 3.9 & 0 & 0.8 & 1.5 & 9.2 & 9.2 & 37 & 37 & 11 & 9.2 \\
39 & 39 & 24 & 11 & 0 & 0 & 0 & 0 & 2.4 & 25 & 25 & 36 & 40 & 44 & 18 \\
39 & 39 & 24 & 11 & 0 & 0 & 0 & 0 & 2.4 & 25 & 25 & 36 & 40 & 44 & 18 \\
0.1 & 0.1 & 0.1 & 0.1 & 0.1 & 0.1 & 0.1 & 0.1 & 0.1 & 0.1 & 0.1 & 0.1 & 0.1 & 0.1 & 0.1 \\
\hline 185 & 185 & 115 & $\mathbf{5 1}$ & $\mathbf{7 . 7}$ & $\mathbf{7}$ & $\mathbf{8}$ & $\mathbf{1 1}$ & $\mathbf{3 2}$ & $\mathbf{1 4 1}$ & $\mathbf{1 4 1}$ & $\mathbf{2 3 2}$ & $\mathbf{2 4 7}$ & $\mathbf{2 2 1}$ & $\mathbf{1 0 5}$
\end{tabular}

-NUG: Small Thermal \& Misc.-

33 Grays Harbor Paper (GHPD)

34 Weyerhaeuser Pulp Mill (GHPD)

35 GPU - Total NUG: Small Thermal \& Misc.

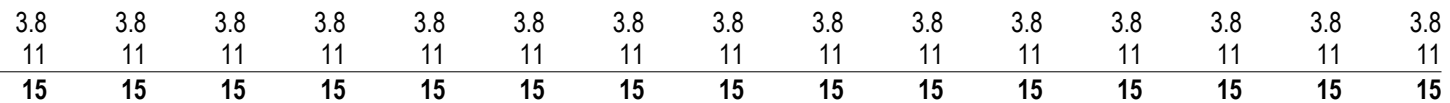

-NUG: Co-Generation-

36 Metro Westpoint (SCL)

37 Scott Paper (SHPD)

38 GPU - Total NUG: Co-Generation

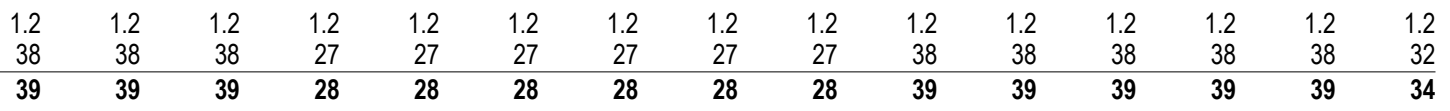

-NUG: Renewables-

39 Foote Creek 1 (EWEB)

40 Nine Canyon Wind Project (CHPD)

41 Nine Canyon Wind Project (COPD)

42 Nine Canyon Wind Project (DOPD)

43 Nine Canyon Wind Project (GCPD)

44 Nine Canyon Wind Project (GHPD)

45 Nine Canyon Wind Project (OKPD)

46 Stateline Wind Project (EWEB)

47 Stateline Wind Project (SCL)

48 GPU - Total NUG: Renewables

Non-Generating Public Entities

-NUG: Hydro-

49 Buffalo Hydro (FREC)

50 Burr \& Bouchard (TNEC)

51 City of Cove (OTEC)

52 Falls Creek (CCPD)

53 Lilliwaup Falls Generating Co. (MCPD1)

54 Thomas Burnside (TNEC)

55 NGP - Total NUG: Hydro

\begin{tabular}{|c|c|c|c|c|c|c|c|c|c|c|c|c|c|c|}
\hline 1.4 & 1.4 & 1.6 & 2.4 & 3.5 & 4.3 & 4.5 & 4.1 & 3.1 & 2.5 & 2.5 & 2.0 & 1.7 & 1.4 & 2.7 \\
\hline 1.5 & 1.5 & 1.4 & 2.2 & 3.8 & 3.8 & 4.2 & 3.4 & 2.3 & 2.0 & 2.0 & 2.0 & 1.9 & 1.4 & 2.5 \\
\hline 0.4 & 0.4 & 0.4 & 0.5 & 0.9 & 0.9 & 1 & 0.9 & 0.6 & 0.5 & 0.5 & 0.5 & 0.5 & 0.3 & 0.6 \\
\hline 1.8 & 1.8 & 1.7 & 2.6 & 4.6 & 4.7 & 5.1 & 4.2 & 2.8 & 2.4 & 2.4 & 2.4 & 2.3 & 1.7 & 3 \\
\hline 2.2 & 2.2 & 2.1 & 3.2 & 5.7 & 5.7 & 6.3 & 5.2 & 3.4 & 3.0 & 3.0 & 3.0 & 2.8 & 2.1 & 3.7 \\
\hline 1.5 & 1.5 & 1.4 & 2.2 & 3.8 & 3.8 & 4.2 & 3.4 & 2.3 & 2.0 & 2.0 & 2.0 & 1.9 & 1.4 & 2.5 \\
\hline 3.0 & 3.0 & 2.8 & 4.3 & 7.5 & 7.6 & 8.4 & 6.9 & 4.6 & 3.9 & 3.9 & 3.9 & 3.7 & 2.7 & 4.9 \\
\hline 3.4 & 8.4 & 6.6 & 8.6 & 9.3 & 8.2 & 8.8 & 8.3 & 8.3 & 9.2 & 9.2 & 9.3 & 9.6 & 8.5 & 8.6 \\
\hline 49 & 49 & 39 & 50 & 54 & 48 & 52 & 48 & 48 & 54 & 54 & 54 & 56 & 50 & 50 \\
\hline 69 & 69 & 57 & 76 & 93 & 87 & 94 & 85 & 75 & 79 & 79 & 79 & 81 & 69 & 79 \\
\hline
\end{tabular}

-NUG: Small Thermal \& Misc.-

56 Methane Energy Agricultural D. (TKPD)

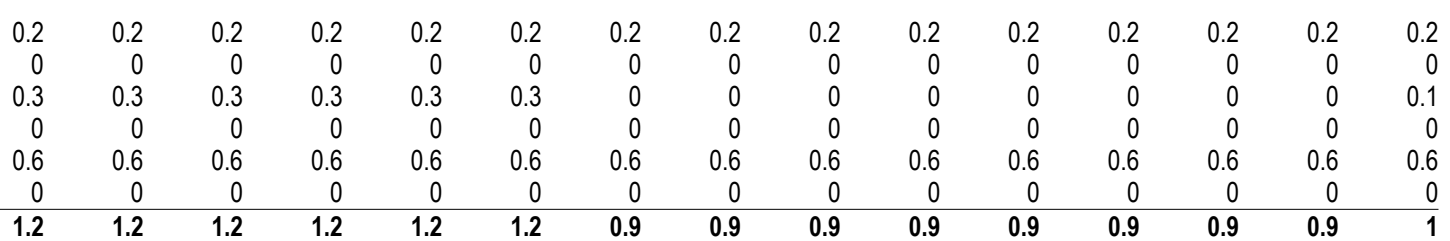

57 NGP - Total NUG: Small Thermal \& Misc.

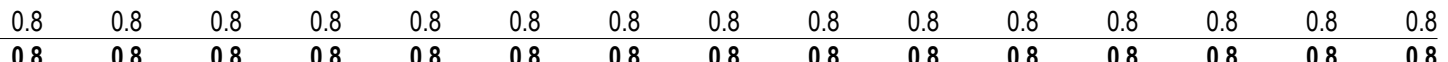


-NUG: Renewables-

58 Ashland Solar Project (ASHL)

59 Nine Canyon Wind Project (BCPD)

60 Nine Canyon Wind Project (ENW)

61 Nine Canyon Wind Project (LCPD)

62 Nine Canyon Wind Project (MCPD3)

\begin{tabular}{|c|c|c|c|c|c|c|c|c|c|c|c|c|c|c|}
\hline 0 & 0 & 0 & 0 & 0 & 0 & 0 & 0 & 0 & 0 & 0 & 0 & 0 & 0 & 0 \\
\hline 0.6 & 0.6 & 0.5 & 0.8 & 1.4 & 1.4 & 1.6 & 1.3 & 0.9 & 0.7 & 0.7 & 0.7 & 0.7 & 0.5 & 0.9 \\
\hline 0.4 & 0.4 & 0.4 & 0.5 & 0.9 & 1.0 & 1.1 & 0.9 & 0.6 & 0.5 & 0.5 & 0.5 & 0.5 & 0.3 & 0.6 \\
\hline 0.2 & 0.2 & 0.2 & 0.3 & 0.5 & 0.5 & 0.5 & 0.4 & 0.3 & 0.2 & 0.2 & 0.2 & 0.2 & 0.2 & 0.3 \\
\hline 0.4 & 0.4 & 0.4 & 0.5 & 0.9 & 1.0 & 1 & 0.9 & 0.6 & 0.5 & 0.5 & 0.5 & 0.5 & 0.3 & 0.6 \\
\hline 0 & 0 & 0 & 0 & 0 & 0 & 0 & 0 & 0 & 0 & 0 & 0 & 0 & 0 & 0 \\
\hline 1.5 & 1.5 & 1.4 & 2.2 & 3.8 & 3.8 & 4.2 & 3.5 & 2.3 & 2.0 & 2.0 & 2.0 & 1.9 & 1.4 & 2.5 \\
\hline
\end{tabular}

64 NGP - Total NUG: Renewables

Investor-Owned Entities

-NUG: Hydro-

65 Barber Dam (IPC)

66 Barney Creek (NWE)

67 Beaverton (PGE)

68 Bell Mountain (UPL)

69 Bingham Engineering (NWE)

70 Birch Creek (IPC)

71 Birch Creek (UPL)

72 Black Canyon \#3 (IPC)

73 Black Creek (AVWP)

74 Blind Canyon Hydro (IPC)

75 Box Canyon (IPC)

76 Boyd James (PPL)

77 Briggs Creek (IPC)

78 Broadwater Dam (NWE)

79 Bypass (IPC)

80 Canyon Springs (IPC)

81 Carlton (PGE)

82 Cascade Creek (NWE)

83 CDM Hydro - Bonneville Pacific (PPL)

84 Cedar Draw (IPC)

85 Central OR Irrigation District (PPL)

86 Clear Springs Trout (IPC)

87 Corbett (PGE)

88 Cornelius (PGE)

89 Crystal Springs (IPC)

90 Curry Cattle Co. (IPC)

91 Dairy Creek (PGE)

92 Deep Creek (AVWP)

93 Derr Creek (AVWP)

94 Dietrich Drop (IPC)

95 Donald Jenni (NWE)

96 Eagle Creek (PGE)

97 Eagle Point Irrigation Dist. (PPL)

98 Elk Creek (IPC)

99 Estacada (PGE)

100 Falls Creek (PPL)

101 Falls River (IPC)

102 Farmers Irrig. (PPL)

103 Faulkner Ranch (IPC)

104 Fisheries Development Co (IPC)

105 Galesville Dam (PPL)

106 Geobon 2 (IPC)

107 Georgetown Power (UPL)

108 Hailey Cspp (IPC)

109 Hazelton A (IPC)

110 Hazelton B (IPC)

111 Horseshoe Bend (IPC)

112 Hutchinson Creek (PSE)

113 Ingram Warm Springs (UPL)

$114 \mathrm{Jim}$ Ford Creek (AVWP)

115 Jim Knight (IPC)

116 John Day Creek (AVWP)

117 Joseph Hydro (PPL)

118 Kasel \& Witherspoon (IPC)

119 Koma Kulshan (PSE)

120 Koyle (IPC)

$121 \mathrm{~L}$. Fery (PPL)

122 Lacomb Irrig. (PPL)

123 Lake Oswego (PGE)

124 Lateral \#10 (IPC)

125 Lee Tavenner (NWE)

126 Lemoyne (IPC)

127 Little Wood River Res. (IPC)

128 Littlewood/Arkoosh (IPC)

129 Lowline \#2 (IPC)
2.4

$\begin{array}{rrr}0 & 0 & 0 \\ 0.1 & 0.1 & 0.1\end{array}$

$\begin{array}{lllllll}0.1 & 0.1 & 0.1 & 0.1 & 0.1 & 0.1 & 0.1\end{array}$

$\begin{array}{llllll}0 & 0 & 0.1 & 0.1 & 0.1 & 0.1\end{array}$

$0.1 \quad 0.1$

$2.8 \quad 2.8$

$0.7 \quad 0.7$

0.4
0

0.6
1.1

8.3

0.1
0

0
2.9

0.3

3.1
0.4

0.4
0

1.3

0.2

0

0.1

4.7

0.1

0

1.2
0.1

0.1
0

6.5

0.6

0.8
0.2
0.6

0.6

1.0

0.1

7

6.6

0.7

0.2
0

0.4

0.2
1.5

1.2
4.6

1.1

0
0

0.4

$1.5 \quad 1.5$

0.1

0
1.9

0.7

2.7

$\begin{array}{rrr}0.8 & 0.3 \\ 0 & 0 \\ 0 & 0.1 & \\ 0.2 & 0.1 & 0.1 \\ 0.1 & 0.1 & 0.1 \\ 0.1 & 0.1 & 0.1 \\ 1.0 & 1.1 & 1 \\ 0.1 & 0.1 & \\ 2.5 & 3.4 & 3.4 \\ 0.9 & 0.2 & \\ 0.4 & 0.4 & 0.3 \\ 0 & 0 & 0.4 \\ 0.6 & 0.6 & 0.6 \\ 1.9 & 1.5 & 1 \\ 2.8 & 1.0 & \\ 0.1 & 0.1 & 0.1 \\ 0 & 0 & \\ 0 & 0\end{array}$

$\begin{array}{rr}1.0 & 2.2 \\ 0 & 0 \\ 0 & 0 \\ 0.1 & 0.1 \\ 0.1 & 0.1 \\ 0.1 & 0 \\ 1.1 & 0.3 \\ 0 & 0 \\ 3.4 & 3.4 \\ 0 & 0 \\ 0.3 & 0.3 \\ 0.4 & 0.3 \\ 0.6 & 0.6 \\ 1.2 & 1.2 \\ 0 & 0 \\ 0.1 & 0.1 \\ 0 & 0.1\end{array}$

$\begin{array}{ll}1.1 & 1.5 \\ 8.3 & 6.5\end{array}$

$\begin{array}{rrr}0.1 & 0.1 & 0.1 \\ 0 & 0 & \end{array}$

$\begin{array}{rrr}0 & 0 & \\ 2.9 & 3.1 & 2.8 \\ 0.3 & 0.9 & 0.9\end{array}$

$\begin{array}{lll}3.1 & 3.7 & 3.5\end{array}$

$\begin{array}{lll}0.4 & 0.4 & 0.5\end{array}$

$\begin{array}{rrr}0 & 0 & \\ 0 & 0 & \\ 1.3 & 1.6 & 1.5\end{array}$

0.2

$\begin{array}{llll}2.8 & 2.9 & 2.4 & 1.8\end{array}$

$\begin{array}{rr}0.9 & 1.2 \\ 0 & 0.7\end{array}$

0.6

0.6

1.2
0

$\begin{array}{rrr}0 & 0 & 0 \\ 1.6 & 0.5 & 0.5\end{array}$

$0 \begin{array}{lll}0 & 0 \\ 0.1 & 0.1 & 0.1\end{array}$

$\begin{array}{lll}0.1 & 0.1 & 0.1 \\ 4.7 & 3.6 & 3.1\end{array}$

$\begin{array}{lll}0.1 & 0.1 & 0.1\end{array}$

$0.1 \quad 0.1$

$0.1 \quad 0.1$

$\begin{array}{ll}0.7 & 0.7 \\ 0.1 & 0.1\end{array}$

0.1

0.1

0
6.3

0.6

$\begin{array}{lll}0.8 & -0.7\end{array}$

0.2

$0.6-0.4$

0.6
1.0
0

0.1
7

0.3

0.2

$\begin{array}{ll}0.1 & 0.1\end{array}$

$\begin{array}{lll}7 & 5.4 & 2.5\end{array}$

$\begin{array}{lll}7.6 & 5.2 & 2.4 \\ 8.8 & 7.5 & 3.9 \\ 0.7 & 0.7 & 0.7\end{array}$

0.7

0.2

$\begin{array}{ll}0.7 & 0.7\end{array}$

$\begin{array}{ll}0.7 & 0.7 \\ 0.3 & 0.3\end{array}$

0
3.1

3.1
5.0

0.9

2.1

0.3

0.3

0.2

0.3

0.1

$\begin{array}{lll}0.4 & 0.3 & 0.2\end{array}$

0.2
1.5

0.9

0.2
0.4

0.1
0

$\begin{array}{lll}1.9 & 0.2 & 0.2\end{array}$

0.7
2.7

0.4

0.4

0.2

$\begin{array}{rr}2.6 & 3.3 \\ 0 & \\ 0 & \\ 0.1 & 0 . \\ 0.1 & \\ 0 & \end{array}$

$\begin{array}{rr}0 \\ 1.2 & 1.7\end{array}$

$\begin{array}{rr}0 & 0.1 \\ 3.1 & 3.7\end{array}$

$\begin{array}{rr}0 & \\ 0.3 & 0.3\end{array}$

$\begin{array}{ll}0.6 & 0.7\end{array}$

$\begin{array}{ll}0.7 & 0.6 \\ 1.3 & 1.3\end{array}$

$\begin{array}{rr}1.3 & 1.3 \\ 0 & 0.1 \\ 0.1 & 0.1 \\ 0.1 & 0.1\end{array}$

$\begin{array}{ll}0.1 & 0.1 \\ 0.1 & 0.1\end{array}$

$3.2 \quad 3.2$

3.23 .1

3.1

$\begin{array}{lll}3.2 & 3.3 & 2.3\end{array}$

$\begin{array}{rr}0 & \\ 0 & \\ 0.1 & 0.1 \\ 0 & \end{array}$

0
0.1
0

0
0.1
0.1

0
0
0.2
0.1

0
0.1
0.1

2.3

0
0.1

0.1
0

$\begin{array}{rrrrrr}1.6 & 1.6 & 1.6 & 1.3 & 1.2 & 1.2\end{array}$

$\begin{array}{llllll}0.1 & 0.1 & 0.1 & 0.1 & 0.1 & 0.1\end{array}$

$\begin{array}{llllll}3.4 & 3.1 & 3.1 & 2.8 & 3.1 & 3\end{array}$

$\begin{array}{llllll}0.6 & 0.6 & 1.6 & 1.0 & 0.7 & 0.6\end{array}$

$\begin{array}{ll}0 & \\ 2 & 2.7\end{array}$

1.3
0.4

0.4

0.5
0

0.6

0.1

0.1

0.9

0.1
0.1

2.7

3.8

4.6
0.3
0.3

0.2

0.3

0.3
0.1

0.1
0
0

2.7

0.4
0.9

0.4
0

0
0.8
0.1

0.1
0

1.9

2.9
0.1

0.1
0

$\begin{array}{ll}2.7 & 2.7\end{array}$

$\begin{array}{ll}0.4 & 0.4 \\ 0.8 & 0.8\end{array}$

$\begin{array}{ll}0.3 & 0.3\end{array}$

0
0

0.9

0.1
0

0.1

0.2

0.1

3.1

$$
0.6
$$

0.1

3.8

3.3

0.5

0.3

0.3
0.6

0.3

0.1

0.1

$$
5.9
$$

0.7

0.7

0.4

0.4
1.1

$0.1 \quad 0.2$

0.2

0.2

0.2

3.8

0.1
0

$\begin{array}{lll}0.4 & 6.9 & 6.9\end{array}$

$\begin{array}{lll}0.3 & 0.8 & 0.8\end{array}$

0.9

0.9

0.4

0.4

0.1

0.

1.6
0.4

0.1
0 

2003 White Book

Aug1 Aug16 Sep Oct Nov Dec

Jan Feb Mar Apr1 Apr16

\begin{abstract}
May
\end{abstract}

$$
\text { Jun }
$$
Jul Avg

130 Lowline Canal (IPC)

131 Magic Reservoir (IPC)

132 Malad River (IPC)

133 Marcos Ranches (IPC)

134 Marsh Valley (UPL)

135 Meyers Falls (AVWP)

136 Middlefork Irrig. (PPL)

137 Mile 28 (IPC)

138 Minikahda (PGE)

139 Mink Creek (UPL)

140 Mitchell Butte (IPC)

141 Mountain Energy (PPL)

142 Mt. Tabor (PGE)

143 Mud Creek/S\&S (IPC)

144 Mud Creek/White (IPC)

145 Nichols Gap (PPL)

146 Nicholson Sunnybar (UPL)

147 North Fork Sprague (PPL)

148 O.J. Power (UPL)

149 Odell Creek (PPL)

150 Opal Springs (PPL)

151 Owyhee Dam (IPC)

152 Pancheri (UPL)

153 Pelton Rereg. Dam (PPL)

154 Phillips Ranch (AVWP)

155 Phillipsburg (NWE)

156 Pigeon Cove (IPC)

157 Pine Creek (NWE)

158 Port Townsend Paper Hydro (PSE)

159 Portland (PGE)

160 Portland Hydro Project (PGE)

161 Preston City (UPL)

162 Pristine Springs (IPC)

163 Pristine Springs \#3 (IPC)

164 Reynolds Irrigation (IPC)

165 Rim View (IPC)

166 Rock Creek \#1 (IPC)

167 Rock Creek \#2 (IPC)

168 Sagebrush (IPC)

169 Salem (PGE)

170 Schaffner (IPC)

171 Sheep Creek (AVWP)

172 Shingle Creek (IPC)

173 Shoshone (IPC)

174 Shoshone II (IPC)

175 Snake River Pottery (IPC)

176 Snedigar Ranch (IPC)

177 Solar Research (PPL)

178 South Dry Creek (NWE)

179 Spokane Upriver (AVWP)

180 Stauffer Dry Creek (UPL)

181 Strawberry Creek (NWE)

182 Sunshine Power \#2 (IPC)

183 Sygitowicz Creek Small Hydro (PSE)

184 TGS/Briggs (UPL)

185 Trout Co. (IPC)

186 Tunnel \#1 (IPC)

187 Twin Falls (PSE)

188 Walla Walla (PPL)

189 Water Street / Santiam (PPL)

190 Weeks Falls (PSE)

191 White James (PPL

192 White Water Ranch (IPC)

193 Wilson Lake Hydro (IPC)

194 Wisconsin Creek (NWE)

195 Yakima-Tieton (PPL)

196 IOU - Total NUG: Hydro

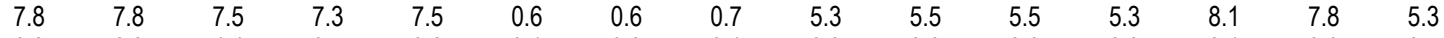

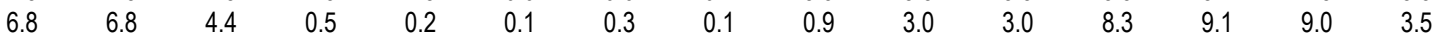

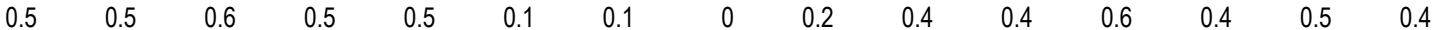

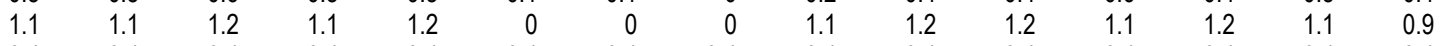

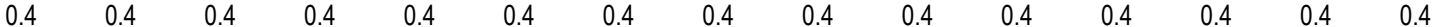

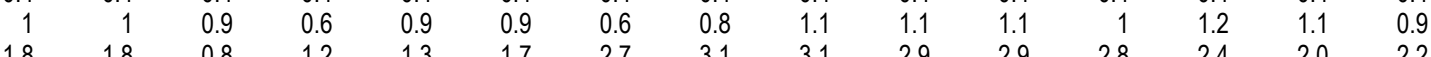

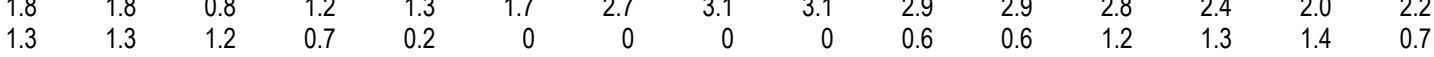

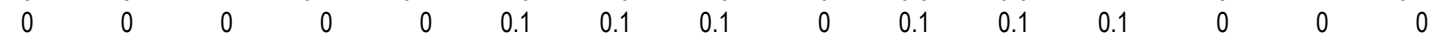

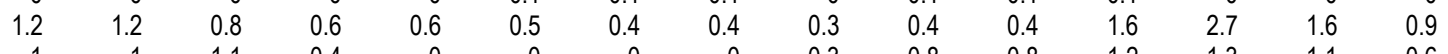

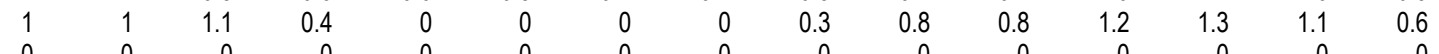

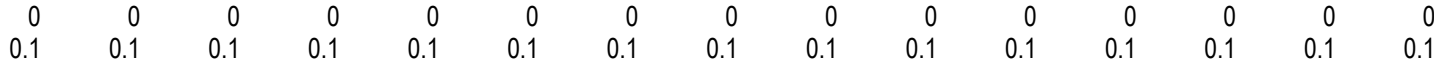

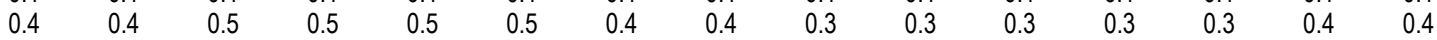

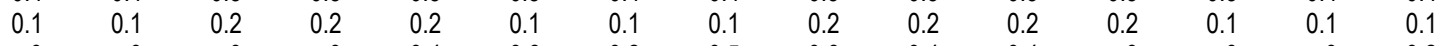

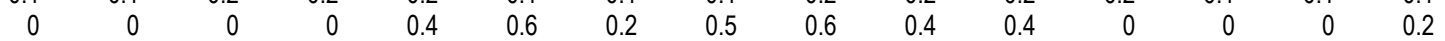

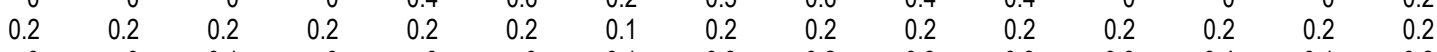

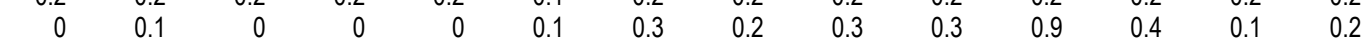

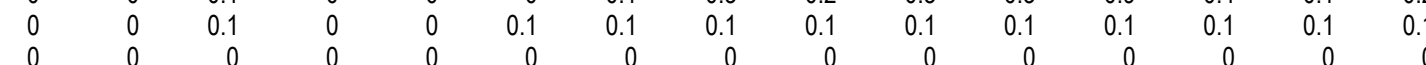

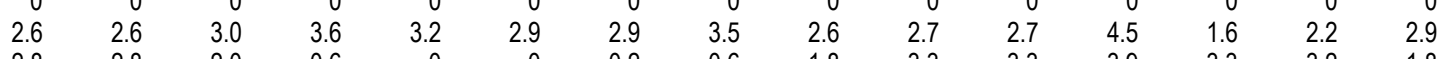

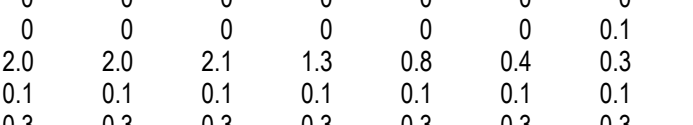

$\begin{array}{rrrrrrr}0.3 & 0.3 & 0.3 & 0.3 & 0.3 & 0.3 & 0.3 \\ 0 & 0 & 0 & 0 & 0 & 0 & 0\end{array}$

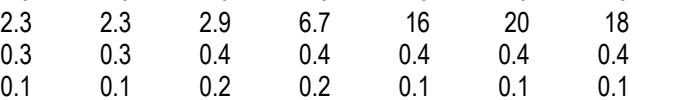

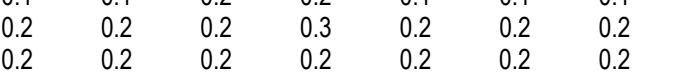

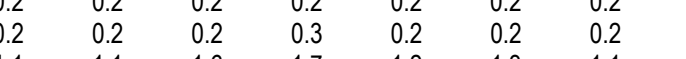

$\begin{array}{llllllll}1.1 & 1.1 & 1.6 & 1.7 & 1.2 & 1.3 & 1.1 & 1.0\end{array}$

$\begin{array}{llllllll}1.8 & 1.8 & 1.8 & 1.9 & 1.9 & 1.9 & 0.6 & 0.4 \\ 0.4 & 0.4 & 0.3 & 0.3 & 0.3 & 0.1 & 0.1 & 0.1\end{array}$

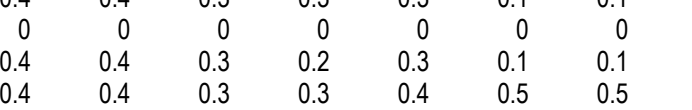

$\begin{array}{llllllll}0.1 & 0.1 & 0.2 & 0.2 & 0.1 & 0.1 & 0.1\end{array}$

$\begin{array}{rrrrrrrrr}0.4 & 0.4 & 0.5 & 0.4 & 0.4 & 0.1 & 0.1 & 0 & 0.1\end{array}$

$\begin{array}{rrr}0.6 & 0.6 & 0.6 \\ 0 & 0 & 0\end{array}$

$\begin{array}{llll}0.2 & 0.2 & 0.2 & 0.2\end{array}$

$\begin{array}{llll}0.1 & 0.1 & 0.1 & 0 \\ 0.5 & 0.5 & 0.5 & 0.5\end{array}$

$\begin{array}{rrrrrrr}0.4 & 3.4 & 0.5 & 0.5 & 0 & 0 & 0 \\ 0.9 & 0.9 & 1.7 & 0.8 & 0.7 & 13 & 14\end{array}$

$\begin{array}{rrrrrrr}0.9 & 0.9 & 1 & 0.8 & 0.4 & 0 & 0.3\end{array}$

$\begin{array}{rrrrrrrrr}0.1 & 0.1 & 0.1 & 0.1 & 0 & 0 & 0.1 & 0 & 0.3\end{array}$

$\begin{array}{rrrrrrrrr}0.1 & 0.1 & 0.1 & 0.1 & 0.1 & 0 & 0 & 0 & 0.1 \\ 0 & 0 & 0 & 0.1 & 0.2 & 0.3 & 0.3 & 0.4 & 0.3\end{array}$

$\begin{array}{rrrrrrrr}0 & 0 & 0 & 0.1 & 0.2 & 0.3 & 0.3 & 0.4 \\ 0.2 & 0.2 & 0.1 & 0.2 & 0.2 & 0.2 & 0.1 & 0.2\end{array}$

\begin{tabular}{|c|c|c|}
\hline 0.2 & 0.1 & 0.2 \\
\hline
\end{tabular}

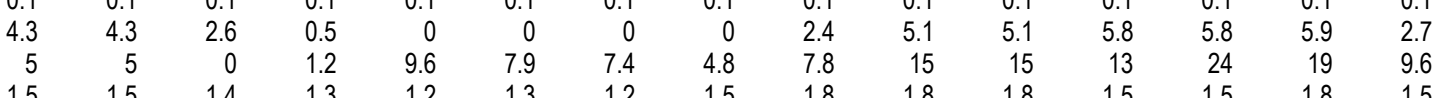

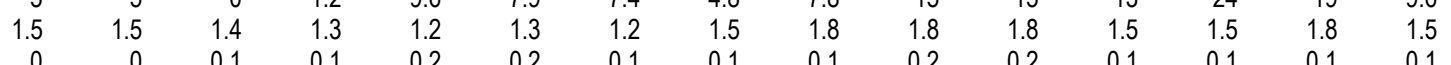

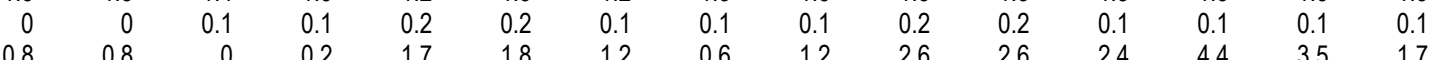

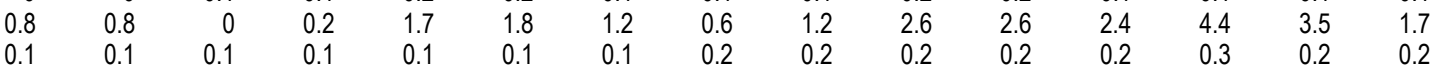

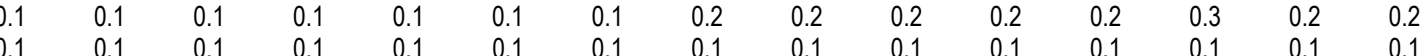

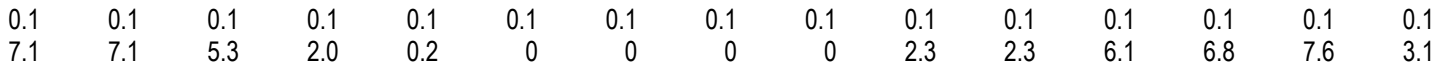

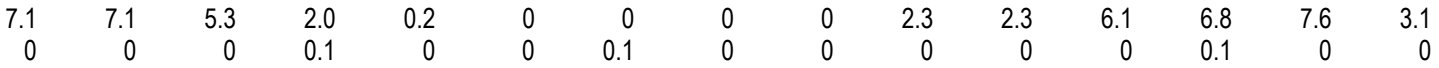

\begin{tabular}{|c|c|c|c|c|c|c|c|c|c|c|c|c|c|}
\hline 157 & 132 & 111 & 121 & 111 & 101 & 109 & 130 & 174 & 173 & $\begin{array}{r}1.2 \\
212\end{array}$ & $\begin{array}{r}1.1 \\
223\end{array}$ & $\begin{array}{r}1.7 \\
196\end{array}$ & 0.9 \\
\hline
\end{tabular}

-NUG: Small Thermal \& Misc.-

197 Marion Solid Waste (PGE)

198 Minnesota Methane (AVWP)

199 Montana One (NWE)

200 Pocatello Waste (IPC)

201 Spokane MSW (PSE)

202 West Boise Waste (IPC)

$\begin{array}{rrrrrrrrrrrrrrr}9.3 & 9.3 & 9.3 & 9.3 & 9.3 & 9.3 & 9.3 & 9.3 & 9.3 & 9.3 & 9.3 & 9.3 & 9.3 & 9.3 & 9.3 \\ 0.8 & 0.8 & 0.8 & 0.8 & 0.8 & 0.8 & 0.8 & 0.8 & 0.8 & 0.8 & 0.8 & 0.8 & 0.8 & 0.8 & 0.8 \\ 9.3 & 9.5 & 8.8 & 8.9 & 6.7 & 7.5 & 7.8 & 7.4 & 6.4 & 6.3 & 6.2 & 10 & 11 & 8.5 & 8.3 \\ 0.1 & 0.1 & 0.2 & 0.1 & 0.2 & 0.1 & 0.1 & 0.2 & 0.1 & 0.2 & 0.2 & 0.1 & 0.2 & 0.1 & 0.1 \\ 15 & 15 & 15 & 15 & 15 & 15 & 15 & 15 & 15 & 15 & 15 & 15 & 15 & 15 & 15 \\ 0.1 & 0.1 & 0.1 & 0.1 & 0.1 & 0.1 & 0.1 & 0.1 & 0.1 & 0.1 & 0.1 & 0.1 & 0.1 & 0.1 & 0.1 \\ \mathbf{3 5} & \mathbf{3 5} & \mathbf{3 4} & \mathbf{3 5} & \mathbf{3 2} & \mathbf{3 3} & \mathbf{3 3} & \mathbf{3 3} & \mathbf{3 2} & \mathbf{3 2} & \mathbf{3 2} & \mathbf{3 6} & \mathbf{3 7} & \mathbf{3 4} & \mathbf{3 4}\end{array}$


Table A-24: Regional Non Utility Generating Resources By Project

PNW Loads and Resources Study

2009 - 2010 Operating Year 2003 White Book

Aug1 Aug16 Sep

Oct Nov Dec

Jan Feb Mar Apr1 Apr16

May Jun Jul Avg

-NUG: Combustion Turbines -

204 March Point \#1 Cogeneration (PSE)

205 March Point \#2 Cogeneration (PSE)

206 Rathdrum- Boekel Rd. \#1 (AVWP)

207 Rathdrum -Boekel Rd. \#2 (AVWP)

208 Tenaska Gas Cogen (PSE)

209 IOU - Total NUG: Combustion Turbines

\begin{tabular}{|c|c|c|c|c|c|c|c|c|c|c|c|c|c|c|}
\hline 79 & 79 & 79 & 79 & 79 & 79 & 79 & 79 & 79 & 79 & 79 & 55 & 79 & 79 & 77 \\
\hline 59 & 59 & 59 & 59 & 59 & 59 & 59 & 59 & 59 & 59 & 59 & 45 & 59 & 59 & 58 \\
\hline 65 & 65 & 65 & 65 & 65 & 65 & 65 & 65 & 65 & 65 & 65 & 32 & 65 & 65 & 62 \\
\hline 65 & 65 & 65 & 65 & 65 & 65 & 65 & 65 & 65 & 65 & 65 & 32 & 65 & 65 & 62 \\
\hline 240 & 240 & 240 & 240 & 240 & 240 & 240 & 240 & 240 & 240 & 240 & 180 & 240 & 240 & 235 \\
\hline 508 & 508 & 508 & 508 & 508 & 508 & 508 & 508 & 508 & 508 & 508 & 344 & 508 & 508 & 494 \\
\hline
\end{tabular}

-NUG: Co-Generation-

210 Billings Generation (NWE)

211 Biomass One (PPL)

212 Boise Cascade Medford (PPL)

213 Champion (PPL)

214 DR Johnson - Co-Gen II (PPL)

215 Magic Valley (IPC)

216 Magic West (IPC)

217 PERC Pierce, Wa (PSE)

218 Simplot Pocatello (IPC)

219 Sumas Energy (PSE)

220 Tamarack (IPC)

221 TASCO Nampa ID (IPC)

222 TASCO Twin Falls ID (IPC)

223 Vaagen Bros. (IPC)

224 Warm Springs (PPL)

225 IOU - Total NUG: Co-Generation

-NUG: Renewables-

226 Foote Creek 1 (PPL)

227 Healow \#2 (NWE)

228 Josef Staufer (NWE)

229 Lewandowski Farms (IPC)

230 Livingston (NWE)

231 Puyallup Energy Recovery (PSE)

232 Stateline Wind Project (AVWP)

233 Windmill (PGE)

234 IOU - Total NUG: Renewables

\begin{tabular}{|c|c|c|c|c|c|c|c|c|c|c|c|c|c|c|}
\hline 17 & 17 & 16 & 11 & 12 & 14 & 14 & 14 & 12 & 7.3 & 7.2 & 19 & 20 & 16 & 14 \\
\hline 22 & 22 & 21 & 21 & 22 & 22 & 7.2 & 15 & 17 & 20 & 20 & 17 & 22 & 22 & 19 \\
\hline 8.5 & 8.5 & 8.5 & 8.5 & 8.5 & 8.5 & 8.5 & 8.5 & 8.5 & 8.5 & 8.5 & 8.5 & 8.5 & 8.5 & 8.5 \\
\hline 3.3 & 3.3 & 3.3 & 3.3 & 3.3 & 3.3 & 1 & 3.3 & 3.3 & 3.3 & 3.3 & 3.3 & 3.3 & 3.3 & 3.1 \\
\hline 7.5 & 7.5 & 7.7 & 5.8 & 7.3 & 6.0 & 6.7 & 9.1 & 7.3 & 7.4 & 7.4 & 11 & 4.2 & 7.1 & 7.2 \\
\hline 9.3 & 9.3 & 9.7 & 9.3 & 9.6 & 9.3 & 9.3 & 10 & 9.4 & 9.7 & 9.7 & 9.4 & 9.7 & 9.3 & 9.5 \\
\hline 9.3 & 9.3 & 9.7 & 9.3 & 9.6 & 9.3 & 9.3 & 10 & 9.4 & 9.7 & 9.7 & 9.4 & 9.7 & 9.3 & 9.5 \\
\hline 2 & 2 & 2 & 2 & 2 & 2 & 2 & 2 & 2 & 2 & 2 & 0 & 2 & 2 & 1.8 \\
\hline 8.8 & 8.8 & 9.2 & 8.8 & 9.1 & 8.8 & 8.8 & 9.6 & 8.9 & 9.2 & 9.2 & 8.9 & 9.2 & 8.8 & 9.0 \\
\hline 113 & 113 & 126 & 126 & 126 & 126 & 126 & 126 & 126 & 113 & 113 & 113 & 113 & 113 & 120 \\
\hline 3.8 & 3.8 & 4.6 & 4.7 & 4.2 & 3.2 & 3.6 & 3.8 & 4.2 & 4.4 & 4.4 & 3.4 & 4.6 & 4.8 & 4.1 \\
\hline 0.2 & 0.2 & 0.2 & 0.2 & 0.2 & 0.2 & 0.2 & 0.2 & 0.2 & 0.2 & 0.2 & 0.2 & 0.2 & 0.2 & 0.2 \\
\hline 0 & 0 & 0 & 0 & 0 & 0 & 0 & 0 & 0 & 0 & 0 & 0 & 0 & 0 & 0 \\
\hline 3 & 3 & 3.7 & 3.8 & 3.3 & 2.6 & 2.9 & 3.1 & 3.4 & 3.6 & 3.6 & 2.7 & 3.7 & 3.8 & 3.3 \\
\hline 0.1 & 0.1 & 0 & 0.1 & 0.1 & 0.1 & 0.1 & 0.1 & 0 & 0 & 0 & 0 & 0.1 & 0 & 0.1 \\
\hline 207 & 207 & 222 & 214 & 218 & 215 & 200 & 215 & 211 & 198 & 198 & 205 & 210 & 207 & 210 \\
\hline
\end{tabular}

Other Entities

-NUG: Co-Generation-

235 SP Newsprint cogen (SPN)

236 Other - Total NUG: Co-Generation

$\begin{array}{lllllllllllllll}25 & 25 & 25 & 25 & 25 & 25 & 25 & 25 & 25 & 25 & 25 & 25 & 25 & 25 & 25 \\ \mathbf{2 5} & \mathbf{2 5} & \mathbf{2 5} & \mathbf{2 5} & \mathbf{2 5} & \mathbf{2 5} & \mathbf{2 5} & \mathbf{2 5} & \mathbf{2 5} & \mathbf{2 5} & \mathbf{2 5} & \mathbf{2 5} & \mathbf{2 5} & \mathbf{2 5} & \mathbf{2 5}\end{array}$

-NUG: Renewables-

237 Stateline Wind Project (PPME)

238 Vansycle Wind (FPLEV)

239 Other - Total NUG: Renewables

\begin{tabular}{|c|c|c|c|c|c|c|c|c|c|c|c|c|c|c|}
\hline 4.2 & 4.2 & 4.9 & 7.2 & 11 & 13 & 14 & 12 & 9.2 & 7.4 & 7.4 & 5.9 & 5.2 & 4.2 & 8.1 \\
\hline 0 & 0 & 0 & 0 & 0 & 0 & 0 & 0 & 0 & 0 & 0 & 0 & 0 & 0 & 0 \\
\hline 0 & 0 & 0 & 0 & 0 & 0 & 0 & 0 & 0 & 0 & 0 & 0 & 0 & 0 & 0 \\
\hline 0 & 0 & 0 & 0 & 0 & 0 & 0 & 0 & 0 & 0 & 0 & 0 & 0 & 0 & 0 \\
\hline 0 & 0 & 0 & 0 & 0 & 0 & 0 & 0 & 0 & 0 & 0 & 0 & 0 & 0 & 0 \\
\hline 2 & 2 & 2 & 2 & 2 & 2 & 2 & 2 & 2 & 2 & 2 & 2 & 2 & 2 & 2 \\
\hline 0.3 & 0.3 & 0.2 & 0.3 & 0.3 & 0.3 & 0.3 & 0.3 & 0.3 & 0.3 & 0.3 & 0.3 & 0.3 & 0.3 & 0.3 \\
\hline 0 & 0 & 0 & 0 & 0 & 0 & 0 & 0 & 0 & 0 & 0 & 0 & 0 & 0 & 0 \\
\hline 6.5 & 6.5 & 7.1 & 9.5 & 13 & 15 & 16 & 15 & 12 & 9.8 & 9.8 & 8.2 & 7.6 & 6.5 & 10 \\
\hline
\end{tabular}

-Total Non-Utility Generating Resources-

240 Federal Entities

241 Generating Public Entities

242 Non Generating Public Entities

243 Investor-Owned Entities

244 Other Entities

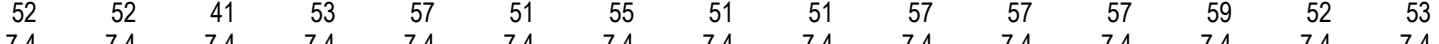

245 Total Non-Utility Generation

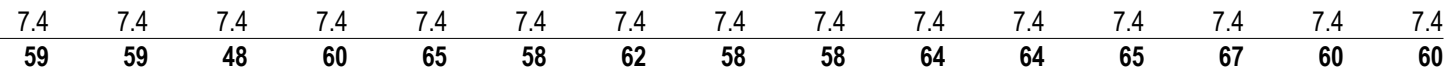


Oct Nov

Dec

\begin{abstract}
Jan Feb
\end{abstract}

\begin{abstract}
Mar Apr1 Apr16
\end{abstract}

\begin{abstract}
May
\end{abstract}
Jun

Jul Avg

\title{
Federal Entities
}

-NUG: Hydro-

1 Clearwater - State of ID DWR (BPA)

2 Dworshak Small Hydropower (BPA)

3 Federal -Total NUG: Hydro

\begin{tabular}{|c|c|c|c|c|c|c|c|c|c|c|c|c|c|c|}
\hline 1 & 1 & 1 & 1 & 1 & 1 & 1 & 1 & 1 & 1 & 1 & 1 & 1 & 1 & 1 \\
\hline 2.6 & 2.6 & 2.6 & 2.6 & 2.6 & 2.6 & 2.6 & 2.6 & 2.6 & 2.6 & 2.6 & 2.6 & 2.6 & 2.6 & 2.6 \\
\hline 3.6 & 3.6 & 3.6 & 3.6 & 3.6 & 3.6 & 3.6 & 3.6 & 3.6 & 3.6 & 3.6 & 3.6 & 3.6 & 3.6 & 3.6 \\
\hline
\end{tabular}

-NUG: Renewables-

4 Ashland Solar Project (BPA)

5 Condon Wind Project (BPA)

6 Foote Creek 1 (BPA)

7 Foote Creek 2 (BPA)

8 Foote Creek 4 (BPA)

9 Fourmile Hill Geothermal (BPA)

10 Klondike Phase 1 (BPA)

11 Stateline Wind Project (BPA)

12 Federal -Total NUG: Renewables

\begin{tabular}{|c|c|c|c|c|c|c|c|c|c|c|c|c|c|}
\hline 0 & 0 & 0 & 0 & 0 & 0 & 0 & 0 & 0 & 0 & 0 & 0 & 0 & 0 \\
\hline 10 & 10 & 11 & 13 & 14 & 13 & 12 & 12 & 14 & 14 & 12 & 15 & 11 & 12 \\
\hline 3.3 & 3.8 & 5.6 & 8.3 & 10 & 11 & 9.6 & 7.2 & 5.8 & 5.8 & 4.6 & 4.1 & 3.3 & 6.4 \\
\hline 0.4 & 0.5 & 0.7 & 1 & 1.3 & 1.3 & 1.2 & 0.9 & 0.7 & 0.7 & 0.5 & 0.5 & 0.4 & 0.8 \\
\hline 3.9 & 4.4 & 6.5 & 9.7 & 12 & 12 & 11 & 8.4 & 6.7 & 6.7 & 5.4 & 4.8 & 3.8 & 7.4 \\
\hline 50 & 50 & 50 & 50 & 50 & 50 & 50 & 50 & 50 & 50 & 50 & 50 & 50 & 50 \\
\hline 7.9 & 6.2 & 8.1 & 8.8 & 7.8 & 8.3 & 7.8 & 7.8 & 8.6 & 8.6 & 8.8 & 9.1 & 8 & 8.1 \\
\hline 29 & 23 & 30 & 33 & 29 & 31 & 29 & 29 & 32 & 32 & 33 & 34 & 30 & 30 \\
\hline 105 & 98 & 112 & 124 & 123 & 126 & 121 & 115 & 118 & 118 & 114 & 117 & 106 & 115 \\
\hline
\end{tabular}

Generating Public Entities

-NUG: Hydro-

13 Don Steffen (SHPD)

14 Eltopia Br Canal 4.6 (SCL)

15 Eltopia Br Canal 4.6 (TPU)

16 John Llewellyn (SHPD)

17 Kevin Duncan (SHPD)

18 Lucky Peak (SCL)

19 Main Canal Headworks (SCL)

20 Main Canal Headworks (TPU)

21 PEC Headworks (GCPD)

22 Pickering Micro Hydro (CKPD)

23 Potholes E Canal 66.0 (SCL)

24 Potholes E Canal 66.0 (TPU)

25 Quincy Chute (GCPD)

26 Russel D. Smith (SCL)

27 Russel D. Smith (TPU)

28 Smith Creek (EWEB)

29 Summer Falls (SCL)

30 Summer Falls (TPU)

31 Woods Creek (SHPD)

32 GPU - Total NUG: Hydro

\begin{tabular}{rrrrrrrrrrrrrrr}
0 & 0 & 0 & 0 & 0 & 0 & 0 & 0 & 0 & 0 & 0 & 0 & 0 & 0 & 0 \\
0.9 & 0.9 & 0.6 & 0.3 & 0 & 0 & 0 & 0 & 0 & 0.7 & 0.7 & 1 & 1 & 1.1 & 0.5 \\
0.9 & 0.9 & 0.6 & 0.3 & 0 & 0 & 0 & 0 & 0 & 0.7 & 0.7 & 1 & 1 & 1.1 & 0.5 \\
0 & 0 & 0 & 0 & 0 & 0 & 0 & 0 & 0 & 0 & 0 & 0 & 0 & 0 & 0 \\
0 & 0 & 0 & 0 & 0 & 0 & 0 & 0 & 0 & 0 & 0 & 0 & 0 & 0 & 0 \\
66 & 66 & 41 & 11 & 2.2 & 3 & 7.9 & 10 & 21 & 52 & 52 & 84 & 85 & 77 & 38 \\
10 & 10 & 6.1 & 2.6 & 0 & 0 & 0 & 0 & 1.4 & 6.8 & 6.8 & 9.1 & 11 & 12 & 4.9 \\
10 & 10 & 6.1 & 2.6 & 0 & 0 & 0 & 0 & 1.4 & 6.8 & 6.8 & 9.1 & 11 & 12 & 4.9 \\
4.5 & 4.5 & 3.1 & 2.2 & 0 & 0 & 0 & 0 & 0.4 & 5.1 & 5.1 & 6.9 & 6.2 & 5.8 & 2.9 \\
0 & 0 & 0 & 0 & 0 & 0 & 0 & 0 & 0 & 0 & 0 & 0 & 0 & 0 & 0 \\
0.9 & 0.9 & 0.9 & 0.8 & 0 & 0 & 0 & 0 & 0.3 & 0.9 & 0.9 & 0.9 & 0.9 & 0.9 & 0.5 \\
0.9 & 0.9 & 0.9 & 0.8 & 0 & 0 & 0 & 0 & 0.3 & 0.9 & 0.9 & 0.9 & 0.9 & 0.9 & 0.5 \\
7.7 & 7.7 & 4 & 2.3 & 0 & 0 & 0 & 0 & 0.8 & 5.5 & 5.5 & 7.1 & 8.4 & 8.5 & 3.7 \\
2 & 2 & 1.3 & 0.7 & 0 & 0 & 0 & 0 & 0.1 & 1.2 & 1.2 & 1.9 & 2.1 & 1.7 & 0.9 \\
2 & 2 & 1.3 & 0.7 & 0 & 0 & 0 & 0 & 0.1 & 1.2 & 1.2 & 1.9 & 2.1 & 1.7 & 0.9 \\
0 & 0 & 0 & 5 & 5.4 & 3.9 & 0 & 0.8 & 1.5 & 9.2 & 9.2 & 37 & 37 & 11 & 9.2 \\
39 & 39 & 24 & 11 & 0 & 0 & 0 & 0 & 2.4 & 25 & 25 & 36 & 40 & 44 & 18 \\
39 & 39 & 24 & 11 & 0 & 0 & 0 & 0 & 2.4 & 25 & 25 & 36 & 40 & 44 & 18 \\
0.1 & 0.1 & 0.1 & 0.1 & 0.1 & 0.1 & 0.1 & 0.1 & 0.1 & 0.1 & 0.1 & 0.1 & 0.1 & 0.1 & 0.1 \\
\hline 185 & 185 & 115 & $\mathbf{5 1}$ & $\mathbf{7 . 7}$ & $\mathbf{7}$ & $\mathbf{8}$ & $\mathbf{1 1}$ & $\mathbf{3 2}$ & $\mathbf{1 4 1}$ & $\mathbf{1 4 1}$ & $\mathbf{2 3 2}$ & $\mathbf{2 4 7}$ & $\mathbf{2 2 1}$ & $\mathbf{1 0 5}$
\end{tabular}

-NUG: Small Thermal \& Misc.-

33 Grays Harbor Paper (GHPD)

34 Weyerhaeuser Pulp Mill (GHPD)

35 GPU - Total NUG: Small Thermal \& Misc.

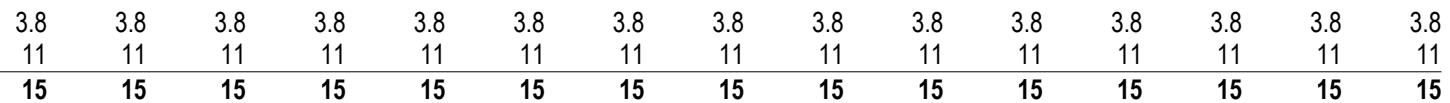

-NUG: Co-Generation-

36 Metro Westpoint (SCL)

37 Scott Paper (SHPD)

38 GPU - Total NUG: Co-Generation

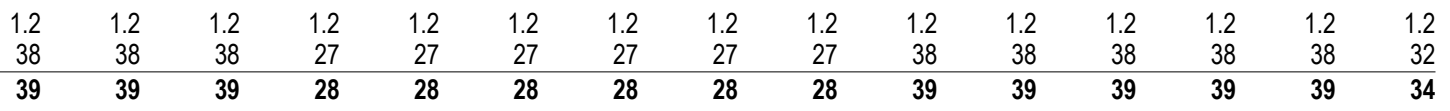

-NUG: Renewables-

39 Foote Creek 1 (EWEB)

40 Nine Canyon Wind Project (CHPD)

41 Nine Canyon Wind Project (COPD)

42 Nine Canyon Wind Project (DOPD)

43 Nine Canyon Wind Project (GCPD)

44 Nine Canyon Wind Project (GHPD)

45 Nine Canyon Wind Project (OKPD)

46 Stateline Wind Project (EWEB)

47 Stateline Wind Project (SCL)

48 GPU - Total NUG: Renewables

Non-Generating Public Entities

-NUG: Hydro-

49 Buffalo Hydro (FREC)

50 Burr \& Bouchard (TNEC)

51 City of Cove (OTEC)

52 Falls Creek (CCPD)

53 Lilliwaup Falls Generating Co. (MCPD1)

54 Thomas Burnside (TNEC)

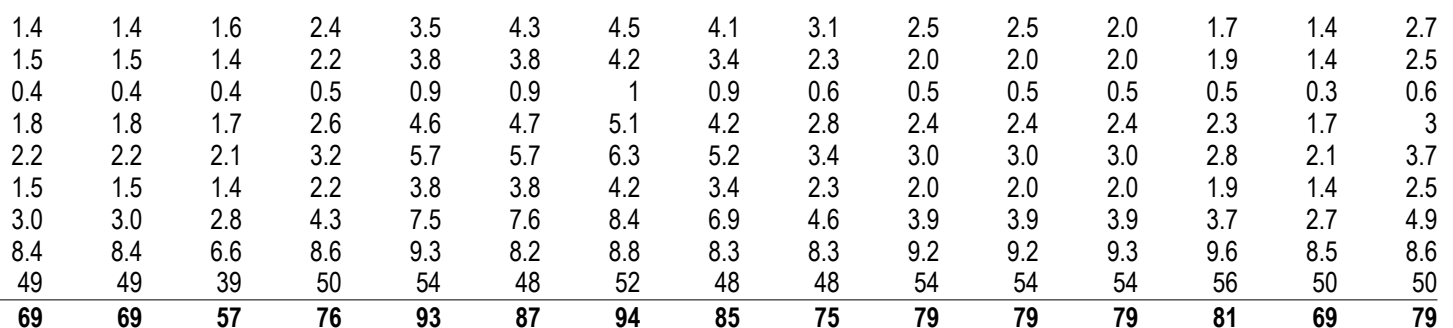

-NUG: Small Thermal \& Misc.-

56 Methane Energy Agricultural D. (TKPD)

$\begin{array}{rrrrrrrrrrrrrrr}0.2 & 0.2 & 0.2 & 0.2 & 0.2 & 0.2 & 0.2 & 0.2 & 0.2 & 0.2 & 0.2 & 0.2 & 0.2 & 0.2 & 0.2 \\ 0 & 0 & 0 & 0 & 0 & 0 & 0 & 0 & 0 & 0 & 0 & 0 & 0 & 0 & 0 \\ 0 & 0 & 0 & 0 & 0 & 0 & 0 & 0 & 0 & 0 & 0 & 0 & 0 & 0 & 0 \\ 0 & 0 & 0 & 0 & 0 & 0 & 0 & 0 & 0 & 0 & 0 & 0 & 0 & 0 & 0 \\ 0.6 & 0.6 & 0.6 & 0.6 & 0.6 & 0.6 & 0.6 & 0.6 & 0.6 & 0.6 & 0.6 & 0.6 & 0.6 & 0.6 & 0.6 \\ 0 & 0 & 0 & 0 & 0 & 0 & 0 & 0 & 0 & 0 & 0 & 0 & 0 & 0 & 0 \\ 0.9 & \mathbf{0 . 9} & \mathbf{0 . 9} & \mathbf{0 . 9} & \mathbf{0 . 9} & \mathbf{0 . 9} & \mathbf{0 . 9} & \mathbf{0 . 9} & \mathbf{0 . 9} & \mathbf{0 . 9} & \mathbf{0 . 9} & \mathbf{0 . 9} & \mathbf{0 . 9} & \mathbf{0 . 9} & \mathbf{0 . 9}\end{array}$

57 NGP - Total NUG: Small Thermal \& Misc.

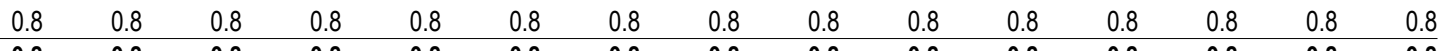



2003 White Book

-NUG: Renewables.

58 Ashland Solar Project (ASHL)

59 Nine Canyon Wind Project (BCPD)

60 Nine Canyon Wind Project (ENW)

61 Nine Canyon Wind Project (LCPD)

62 Nine Canyon Wind Project (MCPD3)

\begin{tabular}{|c|c|c|c|c|c|c|c|c|c|c|c|c|c|c|}
\hline 0 & 0 & 0 & 0 & 0 & 0 & 0 & 0 & 0 & 0 & 0 & 0 & 0 & 0 & 0 \\
\hline 0.6 & 0.6 & 0.5 & 0.8 & 1.4 & 1.4 & 1.6 & 1.3 & 0.9 & 0.7 & 0.7 & 0.7 & 0.7 & 0.5 & 0.9 \\
\hline 0.4 & 0.4 & 0.4 & 0.5 & 0.9 & 1.0 & 1.1 & 0.9 & 0.6 & 0.5 & 0.5 & 0.5 & 0.5 & 0.3 & 0.6 \\
\hline 0.2 & 0.2 & 0.2 & 0.3 & 0.5 & 0.5 & 0.5 & 0.4 & 0.3 & 0.2 & 0.2 & 0.2 & 0.2 & 0.2 & 0.3 \\
\hline 0.4 & 0.4 & 0.4 & 0.5 & 0.9 & 1.0 & 1 & 0.9 & 0.6 & 0.5 & 0.5 & 0.5 & 0.5 & 0.3 & 0.6 \\
\hline 0 & 0 & 0 & 0 & 0 & 0 & 0 & 0 & 0 & 0 & 0 & 0 & 0 & 0 & 0 \\
\hline 1.5 & 1.5 & 1.4 & 2.2 & 3.8 & 3.8 & 4.2 & 3.5 & 2.3 & 2.0 & 2.0 & 2.0 & 1.9 & 1.4 & 2.5 \\
\hline
\end{tabular}

64 NGP - Total NUG: Renewables

Investor-Owned Entities

-NUG: Hydro-

65 Barber Dam (IPC)

66 Barney Creek (NWE)

67 Beaverton (PGE)

68 Bell Mountain (UPL)

69 Bingham Engineering (NWE)

70 Birch Creek (IPC)

71 Birch Creek (UPL)

72 Black Canyon \#3 (IPC)

73 Black Creek (AVWP)

74 Blind Canyon Hydro (IPC)

75 Box Canyon (IPC)

76 Boyd James (PPL)

77 Briggs Creek (IPC)

78 Broadwater Dam (NWE)

79 Bypass (IPC)

80 Canyon Springs (IPC)

81 Carlton (PGE)

82 Cascade Creek (NWE)

83 CDM Hydro - Bonneville Pacific (PPL)

84 Cedar Draw (IPC)

85 Central OR Irrigation District (PPL)

86 Clear Springs Trout (IPC)

87 Corbett (PGE)

88 Cornelius (PGE)

89 Crystal Springs (IPC)

90 Curry Cattle Co. (IPC)

91 Dairy Creek (PGE)

92 Deep Creek (AVWP)

93 Derr Creek (AVWP)

94 Dietrich Drop (IPC)

95 Donald Jenni (NWE)

96 Eagle Creek (PGE)

97 Eagle Point Irrigation Dist. (PPL)

98 Elk Creek (IPC)

99 Estacada (PGE)

100 Falls Creek (PPL)

101 Falls River (IPC)

102 Farmers Irrig. (PPL)

103 Faulkner Ranch (IPC)

104 Fisheries Development Co (IPC)

105 Galesville Dam (PPL)

106 Geobon 2 (IPC)

107 Georgetown Power (UPL)

108 Hailey Cspp (IPC)

109 Hazelton A (IPC)

110 Hazelton B (IPC)

111 Horseshoe Bend (IPC)

112 Hutchinson Creek (PSE)

113 Ingram Warm Springs (UPL)

$114 \mathrm{Jim}$ Ford Creek (AVWP)

115 Jim Knight (IPC)

116 John Day Creek (AVWP)

117 Joseph Hydro (PPL)

118 Kasel \& Witherspoon (IPC)

119 Koma Kulshan (PSE)

120 Koyle (IPC)

$121 \mathrm{~L}$. Fery (PPL)

122 Lacomb Irrig. (PPL)

123 Lake Oswego (PGE)

124 Lateral \#10 (IPC)

125 Lee Tavenner (NWE)

126 Lemoyne (IPC)

127 Little Wood River Res. (IPC)

128 Littlewood/Arkoosh (IPC)

129 Lowline \#2 (IPC)
2.4

$\begin{array}{rrr}0 & 0 & 0 \\ 0.1 & 0.1 & 0.1\end{array}$

$\begin{array}{lllllll}0.1 & 0.1 & 0.1 & 0.1 & 0.1 & 0.1 & 0.1\end{array}$

$\begin{array}{rrrrrr}0 & 0 & 0.1 & 0.1 & 0.1 & 0.1 \\ 3 & 1.3 & 1.1 & 1.0 & 1.1 & 1.1 \\ 0.1 & 0.1 & 0.1 & 0.1 & 0.1\end{array}$

$\begin{array}{llll}0.1 & 0.1 & 0.1 & 0.1\end{array}$

$\begin{array}{llll}0.1 & 28 & 19 & 28 \\ 3.1 & 28 & 19\end{array}$

$\begin{array}{llll}0.7 & 0.7 & 1.1 & 0.9\end{array}$

$\begin{array}{llll}0.7 & 0.4 & 0.9 & 0.2 \\ 0 & 0.4 & 0.4\end{array}$

$\begin{array}{rrrr}0 & 0 & 0 & \\ 0.6 & 0.6 & 0.6 & 0.6\end{array}$

$\begin{array}{llll}1.1 & 1.1 & 1.5 & 1.9\end{array}$

$\begin{array}{llll}8.3 & 8.3 & 6.5 & 2.8 \\ 0.1 & 0.1 & 0.1 & 0.1\end{array}$

0
0

$2.9 \quad 2.9$

0.3

3.1
0.4

$0.4 \quad 0.4$

0

1.3

$0.2 \quad 0.2$

0

0.1

0.1

0

1.2

0.1

6.5

0.6

0.8

0.2

$0.6 \quad 0.6$

1.0

0.1

7
6.6

8.8

0.7

0.2

0.4

0.2

1.5

1.2

4.6
1.1
0

1.1
0

0

$0.4 \quad 0.4$

$1.5 \quad 1.5$

0.10 .1

1.9

0.7

2.7

$\begin{array}{rrr}0.8 & 0.3 & \\ 0 & 0 & \\ 0 & 0.1 & \\ 0.2 & 0.1 & 0.1 \\ 0.1 & 0.1 & 0.1 \\ 0.1 & 0.1 & 0.1 \\ 1.0 & 1.1 & \\ 0.1 & 0.1 & \\ 2.8 & 2.8 & \\ 0.9 & 0.2 & \\ 0.4 & 0.4 & 0.3 \\ 0 & 0 & 0.4 \\ 0.6 & 0.6 & 0.6 \\ 1.9 & 2 & 1.6 \\ 2.8 & 1.0 & \\ 0.1 & 0.1 & 0.1 \\ 0 & 0 & 0 \\ 0 & 0 & 0\end{array}$

$\begin{array}{rr}1.0 & 2.2 \\ 0 & 0 \\ 0 & 0 \\ 0.1 & 0.1 \\ 0.1 & 0.1 \\ 0.1 & 0 \\ 1.1 & 0.3 \\ 0 & 0 \\ 1.9 & 2.5 \\ 0 & 0 \\ 0.3 & 0.3 \\ 0.4 & 0.3 \\ 0.6 & 0.6 \\ 1.6 & 1.5 \\ 0 & 0 \\ 0.1 & 0.1 \\ 0 & 0.1\end{array}$

$\begin{array}{rr}0 & \\ 2.4 & 1.8 \\ 1 & 1\end{array}$

$\begin{array}{lll}3.1 & 3.7 & 3.5\end{array}$

$\begin{array}{rrr}0 & 0.4 & 0.5 \\ 0 & 0 \\ 0 & 0\end{array}$

$\begin{array}{lll}1.3 & 1.6 & 1.5 \\ 0.2 & 0.2 & 0.1\end{array}$

$\begin{array}{ll}0 & 0 \\ 0 & 0\end{array}$

$\begin{array}{lll}0.1 & 0.1 & 0.1 \\ 4.7 & 3.6 & 3.1\end{array}$

$\begin{array}{llll}3.1 & 1.4 & 0 & 0.1 \\ 0.1 & 0.1 & 0.1 & 0.1\end{array}$

0.1

$\begin{array}{rr}0.6 & 0.6 \\ 0 & 0\end{array}$

0.4
0

$\begin{array}{lll}1.6 & 0.5 & 0.5\end{array}$

$\begin{array}{rr}0.1 & 0.1 \\ 0 & 0\end{array}$

0.1

0.1

$\begin{array}{rrr}0 & 0 & 0.1 \\ 0.1 & 0.1 & 0\end{array}$

0
5.7

1.9

3.1
5.0

0.6

0.7

1.2

3.2

3.2

0.3

0.7
0.2

0.3

$0.2 \quad 0.3$

0.2

0.3

$\begin{array}{ll}0.3 & 0.2 \\ 0.3 & 0.3\end{array}$

$\begin{array}{ll}0.2 & 0.3 \\ 0.1 & 0.1\end{array}$

0.1

$\begin{array}{ll}0.3 & 0.2 \\ 0.3 & 0.3\end{array}$

0.1

2.4

$\begin{array}{lll}8.8 & 7.5 & 3.9 \\ 0.7 & 0.7 & 0.7\end{array}$

0.2

$0.3 \quad 0.3$

0.7

$\begin{array}{ll}2.8 & 3.5\end{array}$

$\begin{array}{ll}0.7 & 0.7\end{array}$

$\begin{array}{ll}0.5 & 0.5\end{array}$

$\begin{array}{lll}0.4 & 0.3 & 0.2\end{array}$

$0.3 \quad 0.5$

0.2
1.5

$\begin{array}{ll}0.9 & 0.4\end{array}$

$1.2 \quad 1.2$

$\begin{array}{ll}4.6 & 4.3\end{array}$

0.2
0.4

$0.2 \quad 0.2$

$0.1 \quad 0.1$

$\begin{array}{lll}0.8 & 0.5 & 0.3\end{array}$

6.4

0.4

0
0

$2.6 \quad 3.3$

$\begin{array}{rr}0 & \\ 0 & \\ 0.1 & 0.1\end{array}$

$\begin{array}{rr}0.1 & 0.1 \\ 0 & \end{array}$

$\begin{array}{rr}1.2 & 1.7 \\ 0 & 0.1\end{array}$

$\begin{array}{rr}3.4 & 3.4 \\ 0 & 0\end{array}$

$\begin{array}{ll}0.3 & 0.3 \\ 0.6 & 0.7\end{array}$

$\begin{array}{ll}0.8 & 0.8 \\ 0.6 & 0.6\end{array}$

$\begin{array}{llll}1.7 & 1.7 & 2.7 & 2.6\end{array}$

$\begin{array}{rrrr}0 & 0.1 & 2.9 & 2.9 \\ 0.1 & 0.1 & 0.1 & 0.1\end{array}$

$\begin{array}{llll}0.1 & 0.1 & 0.1 & 0.1\end{array}$

$\begin{array}{ll}0 & \\ 2 & 27\end{array}$

$\begin{array}{rr}1.3 & 0.4 \\ 0.4 & 0.9\end{array}$

$\begin{array}{rr}0 \\ 2.7 & 2.7\end{array}$

$2.7 \quad 2.7$

$\begin{array}{lll}0.9 & 0.8 & 0.8\end{array}$

0.4
0

$0.3 \quad 0.3$

0

0.6

0.6

0.8

$$
\begin{array}{r}
0.1 \\
0 \\
0
\end{array}
$$

0.9

0.1

0.1

0.1

0.1
0

0.9

0.1

\begin{tabular}{l}
0 \\
9 \\
6 \\
3 \\
6 \\
0 \\
\hline 5
\end{tabular}



2003 White Book

Aug1 Aug16 Sep Oct Nov Dec

Jan Feb Mar Apr1 Apr16

\begin{abstract}
May
\end{abstract}

$$
\text { Jun }
$$
Jul Avg

130 Lowline Canal (IPC)

131 Magic Reservoir (IPC)

132 Malad River (IPC)

133 Marcos Ranches (IPC)

134 Marsh Valley (UPL)

135 Meyers Falls (AVWP)

136 Middlefork Irrig. (PPL)

137 Mile 28 (IPC)

138 Minikahda (PGE)

139 Mink Creek (UPL)

140 Mitchell Butte (IPC)

141 Mountain Energy (PPL)

142 Mt. Tabor (PGE)

143 Mud Creek/S\&S (IPC)

144 Mud Creek/White (IPC)

145 Nichols Gap (PPL)

146 Nicholson Sunnybar (UPL)

147 North Fork Sprague (PPL)

148 O.J. Power (UPL)

149 Odell Creek (PPL)

150 Opal Springs (PPL)

151 Owyhee Dam (IPC)

152 Pancheri (UPL)

153 Pelton Rereg. Dam (PPL)

154 Phillips Ranch (AVWP)

155 Phillipsburg (NWE)

156 Pigeon Cove (IPC)

157 Pine Creek (NWE)

158 Port Townsend Paper Hydro (PSE)

159 Portland (PGE)

160 Portland Hydro Project (PGE)

161 Preston City (UPL)

162 Pristine Springs (IPC)

163 Pristine Springs \#3 (IPC)

164 Reynolds Irrigation (IPC)

165 Rim View (IPC)

166 Rock Creek \#1 (IPC)

167 Rock Creek \#2 (IPC)

168 Sagebrush (IPC)

169 Salem (PGE)

170 Schaffner (IPC)

171 Sheep Creek (AVWP)

172 Shingle Creek (IPC)

173 Shoshone (IPC)

174 Shoshone II (IPC)

175 Snake River Pottery (IPC)

176 Snedigar Ranch (IPC)

177 Solar Research (PPL)

178 South Dry Creek (NWE)

179 Spokane Upriver (AVWP)

180 Stauffer Dry Creek (UPL)

181 Strawberry Creek (NWE)

182 Sunshine Power \#2 (IPC)

183 Sygitowicz Creek Small Hydro (PSE)

184 TGS/Briggs (UPL)

185 Trout Co. (IPC)

186 Tunnel \#1 (IPC)

187 Twin Falls (PSE)

188 Walla Walla (PPL)

189 Water Street / Santiam (PPL)

190 Weeks Falls (PSE)

191 White James (PPL

192 White Water Ranch (IPC)

193 Wilson Lake Hydro (IPC)

194 Wisconsin Creek (NWE)

195 Yakima-Tieton (PPL)

196 IOU - Total NUG: Hydrc

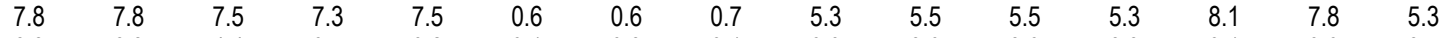

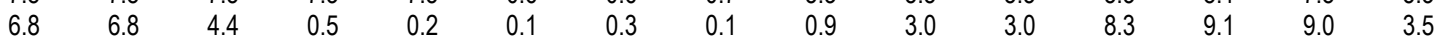

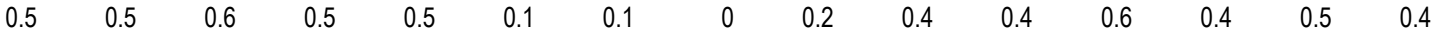

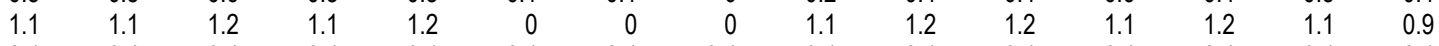

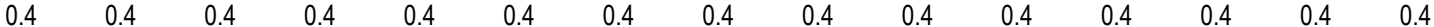

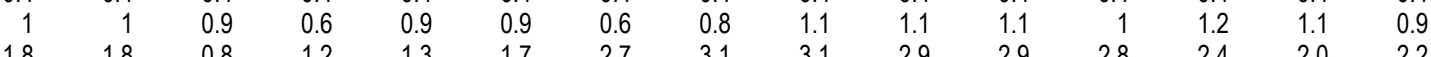

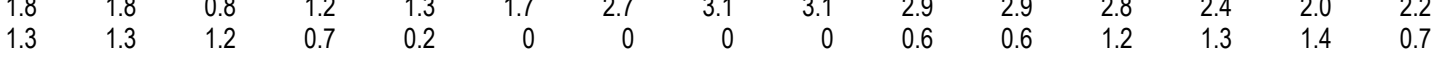

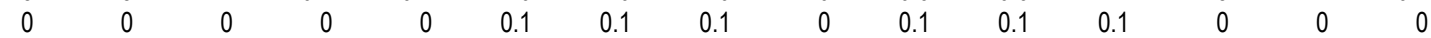

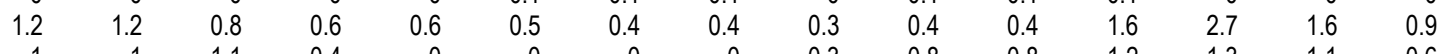

\begin{tabular}{|c|c|}
\hline & 1 \\
\hline
\end{tabular}

\begin{tabular}{|c|c|c|c|c|c|c|c|c|c|}
\hline \multirow[t]{2}{*}{0} & 0 & \multirow[t]{2}{*}{$\begin{array}{r}0 \\
01\end{array}$} & \multirow{2}{*}{$\begin{array}{r}0 \\
0.1\end{array}$} & \multirow[t]{2}{*}{$\begin{array}{r}0 \\
0\end{array}$} & \multirow[t]{2}{*}{$\begin{array}{r}0 \\
01\end{array}$} & \multirow[t]{2}{*}{$\begin{array}{r}0 \\
01\end{array}$} & \multirow[t]{2}{*}{0} & \multirow[t]{2}{*}{0} & \multirow[b]{2}{*}{1} \\
\hline & 0.1 & & & & & & & & \\
\hline
\end{tabular}

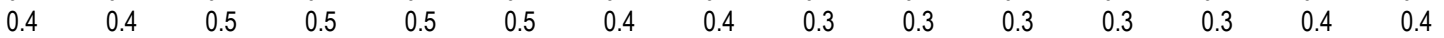

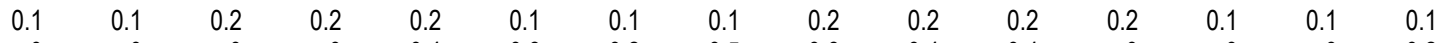

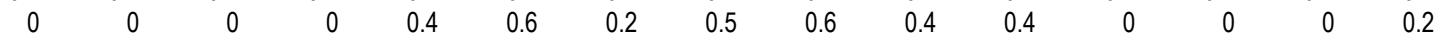

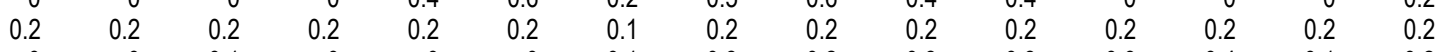

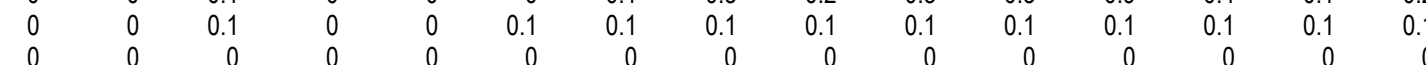

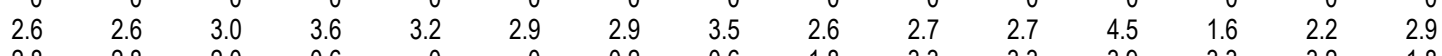

$\begin{array}{rrrrrrrr}0 & 0 & 0 & 0 & 0 & 0.1 & 0.1 & 0.1 \\ 2.0 & 2.0 & 2.1 & 1.3 & 0.8 & 0.4 & 0.3 \\ 0.1 & 0.1 & 0.1 & 0.1 & 0.1 & 0.1 & 0.1 & 0.4 \\ 0.3 & 0.3 & 0.3 & 0.3 & 0.3 & 0.3 & 0.3 & 0.3 \\ 0 & 0 & 0 & 0 & 0 & 0 & 0 & 0\end{array}$

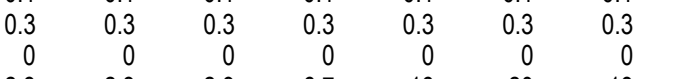

$\begin{array}{lllllllll}2.3 & 2.3 & 2.9 & 6.7 & 16 & 20 & 18 & 17 & 16 \\ 0.3 & 0.3 & 0.4 & 0.4 & 0.4 & 0.4 & 0.4 & 0.4 & 0.3\end{array}$

$\begin{array}{llllllll}0.1 & 0.1 & 0.2 & 0.2 & 0.1 & 0.1 & 0.1 & 0.1\end{array}$

$\begin{array}{lllllll}0.2 & 0.2 & 0.2 & 0.3 & 0.2 & 0.2 & 0.2 \\ 0.2 & 0.2 & 0.2 & 0.2 & 0.2 & 0.2 & 0.2\end{array}$

$\begin{array}{lllllll}0.2 & 0.2 & 0.2 & 0.2 & 0.2 & 0.2 & 0.2 \\ 0.2 & 0.2 & 0.2 & 0.3 & 0.2 & 0.2 & 0.2\end{array}$

$\begin{array}{llllllll}0.2 & 0.2 & 0.2 & 0.3 & 0.2 & 0.2 & 0.2 \\ 1.1 & 1.1 & 1.6 & 1.7 & 1.2 & 1.3 & 1.1 \\ 1.8 & 1.8 & 1.8 & 1.9 & 1.9 & 1.9 & 0.6\end{array}$

$\begin{array}{rrrrrrr}0.4 & 0.4 & 0.3 & 0.3 & 0.3 & 0.1 & 0.1 \\ 0 & 0 & 0 & 0 & 0 & 0 & 0\end{array}$

$\begin{array}{rrrrrrr}0 & 0 & 0 & 0 & 0 & 0 & 0 \\ 0.4 & 0.4 & 0.3 & 0.2 & 0.3 & 0.1 & 0.1 \\ 0.4 & 0.4 & 0.3 & 0.3 & 0.4 & 0.5 & 0.5\end{array}$

$\begin{array}{llllllll}0.1 & 0.1 & 0.2 & 0.2 & 0.1 & 0.1 & 0.1 & 0.1\end{array}$

$\begin{array}{llll}0.4 & 0.4 & 0.5 & 0.4 \\ 0.6 & 0.6 & 0.6 & 0\end{array}$

$\begin{array}{rrrrrrr}0 & 0 & 0.6 & 0 & 0 & 0 & 0 \\ 0.2 & 0.2 & 0 & 0 & 0 & 0.1 & 0.1 \\ 0.1 & 0.1 & 0.1 & 0.2 & 0.2 & 0.2 & 0.2 \\ 0.5 & 0.5 & 0.5 & 0.5 & 0 & 0 & 0.1\end{array}$

$0.3-0.2$

$0.2 \quad 0.2$

$\begin{array}{ll}1.0 & 1.1 \\ 0.4 & 1.6\end{array}$

0.10 .3

0.20 .3

$0.5 \quad 0.9$

$\begin{array}{rr}0.1 & 0.1 \\ 0 & 0.1\end{array}$

$\begin{array}{rrr}0 & 0.1 \\ 0 & 0 & -10 \\ 0.1 & 0\end{array}$

$\begin{array}{rrrrrrr}0.5 & 0.5 & 0.5 & 0.5 & 0 & 0 & 0 \\ 3.4 & 3.4 & 3.4 & 4.7 & 6.7 & 13 & 14\end{array}$

$\begin{array}{llllllll}0.2 & 0.1 & 0.1 & 0.1 & 0.1 & 0.2 & 0.2 & 0.2\end{array}$

$\begin{array}{rrrrrrrr}0.1 & 0.1 & 0.1 & 0.1 & 0.1 & 0 & 0.1 & 0\end{array}$

$\begin{array}{rrrr}0.9 & 0.9 & 1 & 0.8\end{array}$

$\begin{array}{rrrrrrrr}0.1 & 0.1 & 0.1 & 0.1 & 0.1 & 0.1 & 0.1 & 0.1 \\ 0.1 & 0.1 & 0.1 & 0.1 & 0.1 & 0 & 0 & \\ 0 & 0 & 0 & 0.1 & 0.2 & 0.3 & 0.3 & 0.4 \\ 0.2 & 0.2 & 0.1 & 0.2 & 0.2 & 0.2 & 0.1 & 0.2 \\ 0.1 & 0.1 & 0.1 & 0.1 & 0.1 & 0.1 & 0.1 & 0.1 \\ 4.3 & 4.3 & 2.6 & 0.5 & 0 & 0 & 0 & 0\end{array}$

$\begin{array}{rrrrrrrr}0.1 & 0.1 & 0.1 & 0.1 & 0.1 & 0.1 & 0.1 & 0.1 \\ 0.1 & 0.1 & 0.1 & 0.1 & 0.1 & 0 & 0 & \\ 0 & 0 & 0 & 0.1 & 0.2 & 0.3 & 0.3 & 0.4 \\ 0.2 & 0.2 & 0.1 & 0.2 & 0.2 & 0.2 & 0.1 & 0.2 \\ 0.1 & 0.1 & 0.1 & 0.1 & 0.1 & 0.1 & 0.1 & 0.1 \\ 4.3 & 4.3 & 2.6 & 0.5 & 0 & 0 & 0 & 0\end{array}$

\begin{tabular}{|c|c|c|c|c|c|c|c|c|c|c|c|c|}
\hline 0.2 & 0.1 & 0.2 & 0.2 & 0.2 & 0.1 & 0.2 & 0.2 & 0.1 & 0.1 & 0.2 & 0.2 & 0.1 \\
\hline 0.1 & 0.1 & 0.1 & 0.1 & 0.1 & 0.1 & 0.1 & 0.1 & 0.1 & 0.1 & 0.1 & 0.1 & 0.1 \\
\hline 4.3 & 2.6 & 0.5 & 0 & 0 & 0 & 0 & 2.4 & 5.1 & 5.1 & 5.8 & 5.8 & 5.9 \\
\hline 5 & 0 & 1.2 & 9.6 & 7.9 & 7.4 & 4.8 & 7.8 & 15 & 15 & 13 & 24 & 19 \\
\hline 1.5 & 1.4 & 1.3 & 1.2 & 1.3 & 1.2 & 1.5 & 1.8 & 1.8 & 1.8 & 1.5 & 1.5 & 1.8 \\
\hline 0 & 0.1 & 0.1 & 0.2 & 0.2 & 0.1 & 0.1 & 0.1 & 0.2 & 0.2 & 0.1 & 0.1 & 0.1 \\
\hline 0.8 & 0 & 0.2 & 1.7 & 1.8 & 1.2 & 0.6 & 1.2 & 2.6 & 2.6 & 2.4 & 4.4 & 3.5 \\
\hline 0.1 & 0.1 & 0.1 & 0.1 & 0.1 & 0.1 & 0.2 & 0.2 & 0.2 & 0.2 & 0.2 & 0.3 & 0.2 \\
\hline 0.1 & 0.1 & 0.1 & 0.1 & 0.1 & 0.1 & 0.1 & 0.1 & 0.1 & 0.1 & 0.1 & 0.1 & 0.1 \\
\hline 7.1 & 5.3 & 2.0 & 0.2 & 0 & 0 & 0 & 0 & 2.3 & 2.3 & 6.1 & 6.8 & 7.6 \\
\hline 0 & 0 & 0.1 & 0 & 0.1 & 0.1 & 0.1 & 0 & 0.1 & 0.1 & 0 & 0 & 0 \\
\hline 3.0 & 2.5 & 0.4 & 0 & 0 & 0 & 0 & 0 & 0 & 0 & 1.2 & 1.7 & 1.7 \\
\hline 157 & 132 & 111 & 121 & 110 & 101 & 110 & 130 & 175 & 174 & 213 & 222 & 196 \\
\hline
\end{tabular}

-NUG: Small Thermal \& Misc.-

197 Marion Solid Waste (PGE)

198 Minnesota Methane (AVWP)

199 Montana One (NWE)

200 Pocatello Waste (IPC)

201 Spokane MSW (PSE)

202 West Boise Waste (IPC)

$\begin{array}{rrrrrrrrrrrrrrr}9.3 & 9.3 & 9.3 & 9.3 & 9.3 & 9.3 & 9.3 & 9.3 & 9.3 & 9.3 & 9.3 & 9.3 & 9.3 & 9.3 & 9.3 \\ 0.8 & 0.8 & 0.8 & 0.8 & 0.8 & 0.8 & 0.8 & 0.8 & 0.8 & 0.8 & 0.8 & 0.8 & 0.8 & 0.8 & 0.8 \\ 9.4 & 9.6 & 8.9 & 9.0 & 9.2 & 10.0 & 10 & 10.0 & 8.9 & 8.9 & 8.7 & 10 & 8.7 & 8.5 & 9.3 \\ 0.1 & 0.1 & 0.2 & 0.1 & 0.2 & 0.1 & 0.1 & 0.2 & 0.1 & 0.2 & 0.2 & 0.1 & 0.2 & 0.1 & 0.1 \\ 15 & 15 & 15 & 15 & 15 & 15 & 15 & 15 & 15 & 15 & 15 & 15 & 15 & 15 & 15 \\ 0.1 & 0.1 & 0.1 & 0.1 & 0.1 & 0.1 & 0.1 & 0.1 & 0.1 & 0.1 & 0.1 & 0.1 & 0.1 & 0.1 & 0.1 \\ \mathbf{3 5} & \mathbf{3 5} & \mathbf{3 5} & \mathbf{3 5} & \mathbf{3 5} & \mathbf{3 6} & \mathbf{3 6} & \mathbf{3 6} & \mathbf{3 5} & \mathbf{3 5} & \mathbf{3 4} & \mathbf{3 6} & \mathbf{3 4} & \mathbf{3 4} & \mathbf{3 5}\end{array}$


Table A-24: Regional Non Utility Generating Resources By Project

PNW Loads and Resources Study

2010 - 2011 Operating Year 2003 White Book

Aug1 Aug16 Sep

Oct Nov Dec

Jan Feb Mar Apr1 Apr16

May Jun Jul Avg

-NUG: Combustion Turbines -

204 March Point \#1 Cogeneration (PSE)

205 March Point \#2 Cogeneration (PSE)

206 Rathdrum- Boekel Rd. \#1 (AVWP)

207 Rathdrum -Boekel Rd. \#2 (AVWP)

208 Tenaska Gas Cogen (PSE)

209 IOU - Total NUG: Combustion Turbines

\begin{tabular}{rrrrrrrrrrrrrrr}
79 & 79 & 79 & 79 & 79 & 79 & 79 & 79 & 79 & 79 & 79 & 55 & 79 & 79 & 77 \\
$\mathbf{5 9}$ & 59 & 59 & 59 & 59 & 59 & 59 & 59 & 59 & 59 & 59 & 45 & 59 & 59 & 58 \\
65 & 65 & 65 & 65 & 65 & 65 & 65 & 65 & 65 & 65 & 65 & 32 & 65 & 65 & 62 \\
65 & 65 & 65 & 65 & 65 & 65 & 65 & 65 & 65 & 65 & 65 & 32 & 65 & 65 & 62 \\
240 & 240 & 240 & 240 & 240 & 240 & 240 & 240 & 240 & 240 & 240 & 180 & 240 & 240 & 235 \\
\hline $\mathbf{5 0 8}$ & $\mathbf{5 0 8}$ & $\mathbf{5 0 8}$ & $\mathbf{5 0 8}$ & $\mathbf{5 0 8}$ & $\mathbf{5 0 8}$ & $\mathbf{5 0 8}$ & $\mathbf{5 0 8}$ & $\mathbf{5 0 8}$ & $\mathbf{5 0 8}$ & $\mathbf{5 0 8}$ & $\mathbf{3 4 4}$ & $\mathbf{5 0 8}$ & $\mathbf{5 0 8}$ & $\mathbf{4 9 4}$
\end{tabular}

-NUG: Co-Generation-

210 Billings Generation (NWE)

211 Biomass One (PPL)

212 Boise Cascade Medford (PPL)

213 Champion (PPL)

214 DR Johnson - Co-Gen II (PPL)

215 Magic Valley (IPC)

216 Magic West (IPC)

217 PERC Pierce, Wa (PSE)

218 Simplot Pocatello (IPC)

219 Sumas Energy (PSE)

220 Tamarack (IPC)

221 TASCO Nampa ID (IPC)

222 TASCO Twin Falls ID (IPC)

223 Vaagen Bros. (IPC)

224 Warm Springs (PPL)

225 IOU - Total NUG: Co-Generation

\begin{tabular}{|c|c|c|c|c|c|c|c|c|c|c|c|c|c|c|}
\hline 17 & 18 & 16 & 11 & 17 & 18 & 19 & 18 & 16 & 10 & 10 & 19 & 16 & 16 & 16 \\
\hline 22 & 22 & 21 & 21 & 22 & 22 & 7.2 & 15 & 17 & 20 & 20 & 17 & 22 & 22 & 19 \\
\hline 8.5 & 8.5 & 8.5 & 8.5 & 8.5 & 8.5 & 8.5 & 8.5 & 8.5 & 8.5 & 8.5 & 8.5 & 8.5 & 8.5 & 8.5 \\
\hline 3.3 & 3.3 & 3.3 & 3.3 & 3.3 & 3.3 & 1 & 3.3 & 3.3 & 3.3 & 3.3 & 3.3 & 3.3 & 3.3 & 3.1 \\
\hline 7.5 & 7.5 & 7.7 & 5.8 & 7.3 & 6.0 & 6.7 & 9.1 & 7.3 & 7.4 & 7.4 & 11 & 4.2 & 7.1 & 7.2 \\
\hline 9.3 & 9.3 & 9.7 & 9.3 & 9.6 & 9.3 & 9.3 & 10 & 9.4 & 9.7 & 9.7 & 9.4 & 9.7 & 9.3 & 9.5 \\
\hline 9.3 & 9.3 & 9.7 & 9.3 & 9.6 & 9.3 & 9.3 & 10 & 9.4 & 9.7 & 9.7 & 9.4 & 9.7 & 9.3 & 9.5 \\
\hline 2 & 2 & 2 & 2 & 2 & 2 & 2 & 2 & 2 & 2 & 2 & 0 & 2 & 2 & 1.8 \\
\hline 8.8 & 8.8 & 9.2 & 8.8 & 9.1 & 8.8 & 8.8 & 9.6 & 8.9 & 9.2 & 9.2 & 8.9 & 9.2 & 8.8 & 9.0 \\
\hline 113 & 113 & 126 & 126 & 126 & 126 & 126 & 126 & 126 & 113 & 113 & 113 & 113 & 113 & 120 \\
\hline 3.8 & 3.8 & 4.6 & 4.7 & 4.2 & 3.2 & 3.6 & 3.8 & 4.2 & 4.4 & 4.4 & 3.4 & 4.6 & 4.8 & 4.1 \\
\hline 0.2 & 0.2 & 0.2 & 0.2 & 0.2 & 0.2 & 0.2 & 0.2 & 0.2 & 0.2 & 0.2 & 0.2 & 0.2 & 0.2 & 0.2 \\
\hline 0 & 0 & 0 & 0 & 0 & 0 & 0 & 0 & 0 & 0 & 0 & 0 & 0 & 0 & 0 \\
\hline 3 & 3 & 3.7 & 3.8 & 3.3 & 2.6 & 2.9 & 3.1 & 3.4 & 3.6 & 3.6 & 2.7 & 3.7 & 3.8 & 3.3 \\
\hline 0.1 & 0.1 & 0 & 0.1 & 0.1 & 0.1 & 0.1 & 0.1 & 0 & 0 & 0 & 0 & 0.1 & 0 & 0.1 \\
\hline 207 & 207 & 222 & 214 & 222 & 219 & 204 & 220 & 216 & 201 & 201 & 205 & 206 & 207 & 212 \\
\hline
\end{tabular}

-NUG: Renewables-

226 Foote Creek 1 (PPL)

227 Healow \#2 (NWE)

228 Josef Staufer (NWE)

229 Lewandowski Farms (IPC)

230 Livingston (NWE)

231 Puyallup Energy Recovery (PSE)

232 Stateline Wind Project (AVWP)

233 Windmill (PGE)

234 IOU - Total NUG: Renewables

Other Entities

-NUG: Co-Generation-

235 SP Newsprint cogen (SPN)

236 Other - Total NUG: Co-Generation

\begin{tabular}{|c|c|c|c|c|c|c|c|c|c|c|c|c|c|c|}
\hline 4.2 & 4.2 & 4.9 & 7.2 & 11 & 13 & 14 & 12 & 9.2 & 7.4 & 7.4 & 5.9 & 5.2 & 4.2 & 8.1 \\
\hline 0 & 0 & 0 & 0 & 0 & 0 & 0 & 0 & 0 & 0 & 0 & 0 & 0 & 0 & 0 \\
\hline 0 & 0 & 0 & 0 & 0 & 0 & 0 & 0 & 0 & 0 & 0 & 0 & 0 & 0 & 0 \\
\hline 0 & 0 & 0 & 0 & 0 & 0 & 0 & 0 & 0 & 0 & 0 & 0 & 0 & 0 & 0 \\
\hline 0 & 0 & 0 & 0 & 0 & 0 & 0 & 0 & 0 & 0 & 0 & 0 & 0 & 0 & 0 \\
\hline 2 & 2 & 2 & 2 & 2 & 2 & 2 & 2 & 2 & 2 & 2 & 2 & 2 & 2 & 2 \\
\hline 0.3 & 0.3 & 0.2 & 0.3 & 0.3 & 0.3 & 0.3 & 0.3 & 0.3 & 0.3 & 0.3 & 0.3 & 0.3 & 0.3 & 0.3 \\
\hline 0 & 0 & 0 & 0 & 0 & 0 & 0 & 0 & 0 & 0 & 0 & 0 & 0 & 0 & 0 \\
\hline 6.5 & 6.5 & 7.1 & 9.5 & 13 & 15 & 16 & 15 & 12 & 9.8 & 9.8 & 8.2 & 7.6 & 6.5 & 10 \\
\hline
\end{tabular}

-NUG: Renewables-

237 Stateline Wind Project (PPME)

238 Vansycle Wind (FPLEV)

239 Other - Total NUG: Renewables

$\begin{array}{lllllllllllllll}25 & 25 & 25 & 25 & 25 & 25 & 25 & 25 & 25 & 25 & 25 & 25 & 25 & 25 & 25 \\ \mathbf{2 5} & \mathbf{2 5} & \mathbf{2 5} & \mathbf{2 5} & \mathbf{2 5} & \mathbf{2 5} & \mathbf{2 5} & \mathbf{2 5} & \mathbf{2 5} & \mathbf{2 5} & \mathbf{2 5} & \mathbf{2 5} & \mathbf{2 5} & \mathbf{2 5} & \mathbf{2 5}\end{array}$

-Total Non-Utility Generating Resources-

240 Federal Entities

241 Generating Public Entities

242 Non Generating Public Entities

243 Investor-Owned Entities

244 Other Entities

$\begin{array}{rrrrrrrrrrrrrrr}52 & 52 & 41 & 53 & 57 & 51 & 55 & 51 & 51 & 57 & 57 & 57 & 59 & 52 & 53 \\ 7.4 & 7.4 & 7.4 & 7.4 & 7.4 & 7.4 & 7.4 & 7.4 & 7.4 & 7.4 & 7.4 & 7.4 & 7.4 & 7.4 & 7.4 \\ \mathbf{5 9} & \mathbf{5 9} & \mathbf{4 8} & \mathbf{6 0} & \mathbf{6 5} & \mathbf{5 8} & \mathbf{6 2} & \mathbf{5 8} & \mathbf{5 8} & \mathbf{6 4} & \mathbf{6 4} & \mathbf{6 5} & \mathbf{6 7} & \mathbf{6 0} & \mathbf{6 0}\end{array}$

245 Total Non-Utility Generation

\begin{tabular}{|c|c|c|c|c|c|c|c|c|c|c|c|c|c|}
\hline 109 & 102 & 116 & 127 & 127 & 130 & 125 & 119 & 121 & 121 & 118 & 120 & 109 & 119 \\
\hline 308 & 226 & 170 & 145 & 138 & 146 & 139 & 151 & 274 & 274 & 366 & 382 & 344 & 232 \\
\hline 3.1 & 3.1 & 3.8 & 5.4 & 5.5 & 5.8 & 5.1 & 3.9 & 3.6 & 3.6 & 3.6 & 3.5 & 3 & 4.1 \\
\hline 914 & 904 & 877 & 899 & 888 & 865 & 888 & 900 & 928 & 927 & 807 & 978 & 951 & 900 \\
\hline 84 & 73 & 85 & 90 & 83 & 87 & 83 & 83 & 89 & 89 & 90 & 92 & 85 & 85 \\
\hline 118 & 1308 & 1253 & 1266 & 1241 & 1233 & 1240 & 1258 & 1416 & 1416 & 1384 & 1576 & 1493 & 340 \\
\hline
\end{tabular}


Oct Nov

Dec

Jan Fe

\begin{abstract}
Feb
\end{abstract}
Federal Entities

-NUG: Hydro-

1 Clearwater - State of ID DWR (BPA)

2 Dworshak Small Hydropower (BPA)

3 Federal -Total NUG: Hydro

\begin{tabular}{|c|c|c|c|c|c|c|c|c|c|c|c|c|c|}
\hline 1 & 1 & 1 & 1 & 1 & 1 & 1 & 1 & 1 & 1 & 1 & 1 & 1 & 1 \\
\hline 2.6 & 2.6 & 2.6 & 2.6 & 2.6 & 2.6 & 2.6 & 2.6 & 2.6 & 2.6 & 2.6 & 2.6 & 2.6 & 2.6 \\
\hline 3.6 & 3.6 & 3.6 & 3.6 & 3.6 & 3.6 & 3.6 & 3.6 & 3.6 & 3.6 & 3.6 & 3.6 & 3.6 & 3.6 \\
\hline
\end{tabular}

-NUG: Renewables-

4 Ashland Solar Project (BPA)

5 Condon Wind Project (BPA)

6 Foote Creek 1 (BPA)

7 Foote Creek 2 (BPA)

8 Foote Creek 4 (BPA)

9 Fourmile Hill Geothermal (BPA)

10 Klondike Phase 1 (BPA)

11 Stateline Wind Project (BPA)

12 Federal -Total NUG: Renewables

$\begin{array}{rrrrrrrrrrrrrrr}0 & 0 & 0 & 0 & 0 & 0 & 0 & 0 & 0 & 0 & 0 & 0 & 0 & 0 & 0 \\ 10 & 10 & 10 & 11 & 13 & 14 & 13 & 12 & 12 & 14 & 14 & 12 & 15 & 11 & 12 \\ 3.3 & 3.3 & 3.8 & 5.6 & 8.3 & 10 & 11 & 9.6 & 7.2 & 5.8 & 5.8 & 4.6 & 4.1 & 3.3 & 6.4 \\ 0.4 & 0.4 & 0.5 & 0.7 & 1 & 1.3 & 1.3 & 1.2 & 0.9 & 0.7 & 0.7 & 0.5 & 0.5 & 0.4 & 0.8 \\ 3.9 & 3.9 & 4.4 & 6.5 & 9.7 & 12 & 12 & 11 & 8.4 & 6.7 & 6.7 & 5.4 & 4.8 & 3.8 & 7.4 \\ 50 & 50 & 50 & 50 & 50 & 50 & 50 & 50 & 50 & 50 & 50 & 50 & 50 & 50 & 50 \\ 7.9 & 7.9 & 6.2 & 8.1 & 8.8 & 7.8 & 8.3 & 7.8 & 7.8 & 8.6 & 8.6 & 8.8 & 9.1 & 8 & 8.1 \\ 29 & 29 & 23 & 30 & 33 & 29 & 31 & 29 & 29 & 32 & 32 & 33 & 34 & 30 & 30 \\ 105 & \mathbf{1 0 5} & \mathbf{9 8} & \mathbf{1 1 2} & \mathbf{1 2 4} & \mathbf{1 2 3} & \mathbf{1 2 6} & \mathbf{1 2 1} & \mathbf{1 1 5} & \mathbf{1 1 8} & \mathbf{1 1 8} & \mathbf{1 1 4} & \mathbf{1 1 7} & \mathbf{1 0 6} & \mathbf{1 1 5}\end{array}$

Generating Public Entities

-NUG: Hydro-

13 Don Steffen (SHPD)

14 Eltopia Br Canal 4.6 (SCL)

15 Eltopia Br Canal 4.6 (TPU)

16 John Llewellyn (SHPD)

17 Kevin Duncan (SHPD)

18 Lucky Peak (SCL)

19 Main Canal Headworks (SCL)

20 Main Canal Headworks (TPU)

21 PEC Headworks (GCPD)

22 Pickering Micro Hydro (CKPD)

23 Potholes E Canal 66.0 (SCL)

24 Potholes E Canal 66.0 (TPU)

25 Quincy Chute (GCPD)

26 Russel D. Smith (SCL)

27 Russel D. Smith (TPU)

28 Smith Creek (EWEB)

29 Summer Falls (SCL)

30 Summer Falls (TPU)

31 Woods Creek (SHPD)

32 GPU - Total NUG: Hydro

$\begin{array}{rrrrrrrrrrrrrrr}0 & 0 & 0 & 0 & 0 & 0 & 0 & 0 & 0 & 0 & 0 & 0 & 0 & 0 & 0 \\ 0.9 & 0.9 & 0.6 & 0.3 & 0 & 0 & 0 & 0 & 0 & 0.7 & 0.7 & 1 & 1 & 1.1 & 0.5 \\ 0.9 & 0.9 & 0.6 & 0.3 & 0 & 0 & 0 & 0 & 0 & 0.7 & 0.7 & 1 & 1 & 1.1 & 0.5 \\ 0 & 0 & 0 & 0 & 0 & 0 & 0 & 0 & 0 & 0 & 0 & 0 & 0 & 0 & 0 \\ 0 & 0 & 0 & 0 & 0 & 0 & 0 & 0 & 0 & 0 & 0 & 0 & 0 & 0 & 0 \\ 66 & 66 & 41 & 11 & 2.2 & 3 & 7.9 & 10 & 21 & 52 & 52 & 84 & 85 & 77 & 38 \\ 10 & 10 & 6.1 & 2.6 & 0 & 0 & 0 & 0 & 1.4 & 6.8 & 6.8 & 9.1 & 11 & 12 & 4.9 \\ 10 & 10 & 6.1 & 2.6 & 0 & 0 & 0 & 0 & 1.4 & 6.8 & 6.8 & 9.1 & 11 & 12 & 4.9 \\ 4.5 & 4.5 & 3.1 & 2.2 & 0 & 0 & 0 & 0 & 0.4 & 5.1 & 5.1 & 6.9 & 6.2 & 5.8 & 2.9 \\ 0 & 0 & 0 & 0 & 0 & 0 & 0 & 0 & 0 & 0 & 0 & 0 & 0 & 0 & 0 \\ 0.9 & 0.9 & 0.9 & 0.8 & 0 & 0 & 0 & 0 & 0.3 & 0.9 & 0.9 & 0.9 & 0.9 & 0.9 & 0.5 \\ 0.9 & 0.9 & 0.9 & 0.8 & 0 & 0 & 0 & 0 & 0.3 & 0.9 & 0.9 & 0.9 & 0.9 & 0.9 & 0.5 \\ 7.7 & 7.7 & 4 & 2.3 & 0 & 0 & 0 & 0 & 0.8 & 5.5 & 5.5 & 7.1 & 8.4 & 8.5 & 3.7 \\ 2 & 2 & 1.3 & 0.7 & 0 & 0 & 0 & 0 & 0.1 & 1.2 & 1.2 & 1.9 & 2.1 & 1.7 & 0.9 \\ 2 & 2 & 1.3 & 0.7 & 0 & 0 & 0 & 0 & 0.1 & 1.2 & 1.2 & 1.9 & 2.1 & 1.7 & 0.9 \\ 0 & 0 & 0 & 5 & 5.4 & 3.9 & 0 & 0.8 & 1.5 & 9.2 & 9.2 & 37 & 37 & 11 & 9.2 \\ 39 & 39 & 24 & 11 & 0 & 0 & 0 & 0 & 2.4 & 25 & 25 & 36 & 40 & 44 & 18 \\ 39 & 39 & 24 & 11 & 0 & 0 & 0 & 0 & 2.4 & 25 & 25 & 36 & 40 & 44 & 18 \\ 0.1 & 0.1 & 0.1 & 0.1 & 0.1 & 0.1 & 0.1 & 0.1 & 0.1 & 0.1 & 0.1 & 0.1 & 0.1 & 0.1 & 0.1 \\ 185 & 185 & 115 & \mathbf{5 1} & \mathbf{7 . 7} & \mathbf{7} & \mathbf{8} & \mathbf{1 1} & \mathbf{3 2} & \mathbf{1 4 1} & \mathbf{1 4 1} & \mathbf{2 3 2} & \mathbf{2 4 7} & \mathbf{2 2 1} & 105\end{array}$

-NUG: Small Thermal \& Misc.-

33 Grays Harbor Paper (GHPD)

34 Weyerhaeuser Pulp Mill (GHPD)

35 GPU - Total NUG: Small Thermal \& Misc.

\begin{tabular}{|c|c|c|c|c|c|c|c|c|c|c|c|c|c|c|}
\hline 3.8 & 3.8 & 3.8 & 3.8 & 3.8 & 3.8 & 3.8 & 3.8 & 3.8 & 3.8 & 3.8 & 3.8 & 3.8 & 3.8 & 3.8 \\
\hline 11 & 11 & 11 & 11 & 11 & 11 & 11 & 11 & 11 & 11 & 11 & 11 & 11 & 11 & 11 \\
\hline 15 & 15 & 15 & 15 & 15 & 15 & 15 & 15 & 15 & 15 & 15 & 15 & 15 & 15 & 15 \\
\hline
\end{tabular}

-NUG: Co-Generation-

36 Metro Westpoint (SCL)

37 Scott Paper (SHPD)

38 GPU - Total NUG: Co-Generation

\begin{tabular}{|c|c|c|c|c|c|c|c|c|c|c|c|c|c|}
\hline 1.2 & 1.2 & 1.2 & 1.2 & 1.2 & 1.2 & 1.2 & 1.2 & 1.2 & 1.2 & 1.2 & 1.2 & 1.2 & 1.2 \\
\hline 38 & 38 & 27 & 27 & 27 & 27 & 27 & 27 & 38 & 38 & 38 & 38 & 38 & 32 \\
\hline 39 & 39 & 28 & 28 & 28 & 28 & 28 & 28 & 39 & 39 & 39 & 39 & 39 & 34 \\
\hline
\end{tabular}

-NUG: Renewables-

39 Foote Creek 1 (EWEB)

40 Nine Canyon Wind Project (CHPD)

41 Nine Canyon Wind Project (COPD)

42 Nine Canyon Wind Project (DOPD)

43 Nine Canyon Wind Project (GCPD)

44 Nine Canyon Wind Project (GHPD)

45 Nine Canyon Wind Project (OKPD)

46 Stateline Wind Project (EWEB)

47 Stateline Wind Project (SCL)

48 GPU - Total NUG: Renewables

Non-Generating Public Entities

-NUG: Hydro-

49 Buffalo Hydro (FREC)

50 Burr \& Bouchard (TNEC)

51 City of Cove (OTEC)

52 Falls Creek (CCPD)

53 Lilliwaup Falls Generating Co. (MCPD1)

54 Thomas Burnside (TNEC)

$\begin{array}{rrrrrrrrrrrrrrr}1.4 & 1.4 & 1.6 & 2.4 & 3.5 & 4.3 & 4.5 & 4.1 & 3.1 & 2.5 & 2.5 & 2.0 & 1.7 & 1.4 & 2.7 \\ 1.5 & 1.5 & 1.4 & 2.2 & 3.8 & 3.8 & 4.2 & 3.4 & 2.3 & 2.0 & 2.0 & 2.0 & 1.9 & 1.4 & 2.5 \\ 0.4 & 0.4 & 0.4 & 0.5 & 0.9 & 0.9 & 1 & 0.9 & 0.6 & 0.5 & 0.5 & 0.5 & 0.5 & 0.3 & 0.6 \\ 1.8 & 1.8 & 1.7 & 2.6 & 4.6 & 4.7 & 5.1 & 4.2 & 2.8 & 2.4 & 2.4 & 2.4 & 2.3 & 1.7 & 3 \\ 2.2 & 2.2 & 2.1 & 3.2 & 5.7 & 5.7 & 6.3 & 5.2 & 3.4 & 3.0 & 3.0 & 3.0 & 2.8 & 2.1 & 3.7 \\ 1.5 & 1.5 & 1.4 & 2.2 & 3.8 & 3.8 & 4.2 & 3.4 & 2.3 & 2.0 & 2.0 & 2.0 & 1.9 & 1.4 & 2.5 \\ 3.0 & 3.0 & 2.8 & 4.3 & 7.5 & 7.6 & 8.4 & 6.9 & 4.6 & 3.9 & 3.9 & 3.9 & 3.7 & 2.7 & 4.9 \\ 8.4 & 8.4 & 6.6 & 8.6 & 9.3 & 8.2 & 8.8 & 8.3 & 8.3 & 9.2 & 9.2 & 9.3 & 9.6 & 8.5 & 8.6 \\ 49 & 49 & 39 & 50 & 54 & 48 & 52 & 48 & 48 & 54 & 54 & 54 & 56 & 50 & 50 \\ \mathbf{6 9} & \mathbf{6 9} & \mathbf{5 7} & \mathbf{7 6} & \mathbf{9 3} & \mathbf{8 7} & \mathbf{9 4} & \mathbf{8 5} & \mathbf{7 5} & \mathbf{7 9} & \mathbf{7 9} & \mathbf{7 9} & \mathbf{8 1} & \mathbf{6 9} & \mathbf{7 9}\end{array}$

-NUG: Small Thermal \& Misc.-

56 Methane Energy Agricultural D. (TKPD)

$\begin{array}{rrrrrrrrrrrrrrr}0.2 & 0.2 & 0.2 & 0.2 & 0.2 & 0.2 & 0.2 & 0.2 & 0.2 & 0.2 & 0.2 & 0.2 & 0.2 & 0.2 & 0.2 \\ 0 & 0 & 0 & 0 & 0 & 0 & 0 & 0 & 0 & 0 & 0 & 0 & 0 & 0 & 0 \\ 0 & 0 & 0 & 0 & 0 & 0 & 0 & 0 & 0 & 0 & 0 & 0 & 0 & 0 & 0 \\ 0 & 0 & 0 & 0 & 0 & 0 & 0 & 0 & 0 & 0 & 0 & 0 & 0 & 0 & 0 \\ 0.6 & 0.6 & 0.6 & 0.6 & 0.6 & 0.6 & 0.6 & 0.6 & 0.6 & 0.6 & 0.6 & 0.6 & 0.6 & 0.6 & 0.6 \\ 0 & 0 & 0 & 0 & 0 & 0 & 0 & 0 & 0 & 0 & 0 & 0 & 0 & 0 & 0 \\ 0.9 & \mathbf{0 . 9} & \mathbf{0 . 9} & \mathbf{0 . 9} & \mathbf{0 . 9} & \mathbf{0 . 9} & \mathbf{0 . 9} & \mathbf{0 . 9} & \mathbf{0 . 9} & \mathbf{0 . 9} & \mathbf{0 . 9} & \mathbf{0 . 9} & \mathbf{0 . 9} & \mathbf{0 . 9} & \mathbf{0 . 9}\end{array}$

57 NGP - Total NUG: Small Thermal \& Misc.

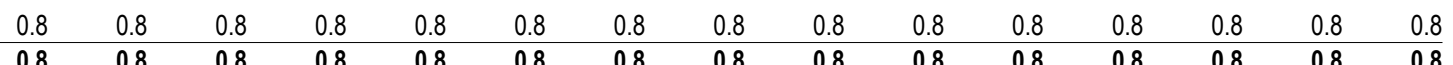


-NUG: Renewables-

58 Ashland Solar Project (ASHL)

59 Nine Canyon Wind Project (BCPD)

60 Nine Canyon Wind Project (ENW)

61 Nine Canyon Wind Project (LCPD)

62 Nine Canyon Wind Project (MCPD3)

\begin{tabular}{|c|c|c|c|c|c|c|c|c|c|c|c|c|c|c|}
\hline 0 & 0 & 0 & 0 & 0 & 0 & 0 & 0 & 0 & 0 & 0 & 0 & 0 & 0 & 0 \\
\hline 0.6 & 0.6 & 0.5 & 0.8 & 1.4 & 1.4 & 1.6 & 1.3 & 0.9 & 0.7 & 0.7 & 0.7 & 0.7 & 0.5 & 0.9 \\
\hline 0.4 & 0.4 & 0.4 & 0.5 & 0.9 & 1.0 & 1.1 & 0.9 & 0.6 & 0.5 & 0.5 & 0.5 & 0.5 & 0.3 & 0.6 \\
\hline 0.2 & 0.2 & 0.2 & 0.3 & 0.5 & 0.5 & 0.5 & 0.4 & 0.3 & 0.2 & 0.2 & 0.2 & 0.2 & 0.2 & 0.3 \\
\hline 0.4 & 0.4 & 0.4 & 0.5 & 0.9 & 1.0 & 1 & 0.9 & 0.6 & 0.5 & 0.5 & 0.5 & 0.5 & 0.3 & 0.6 \\
\hline 0 & 0 & 0 & 0 & 0 & 0 & 0 & 0 & 0 & 0 & 0 & 0 & 0 & 0 & 0 \\
\hline 1.5 & 1.5 & 1.4 & 2.2 & 3.8 & 3.8 & 4.2 & 3.5 & 2.3 & 2.0 & 2.0 & 2.0 & 1.9 & 1.4 & 2.5 \\
\hline
\end{tabular}

64 NGP - Total NUG: Renewables

\subsection{0} -NUG: Hydro-

65 Barber Dam (IPC)

66 Barney Creek (NWE)

67 Beaverton (PGE)

68 Bell Mountain (UPL)

69 Bingham Engineering (NWE)

70 Birch Creek (IPC)

71 Birch Creek (UPL)

72 Black Canyon \#3 (IPC)

73 Black Creek (AVWP)

74 Blind Canyon Hydro (IPC)

75 Box Canyon (IPC)

76 Boyd James (PPL)

77 Briggs Creek (IPC)

78 Broadwater Dam (NWE)

79 Bypass (IPC)

80 Canyon Springs (IPC)

81 Carlton (PGE)

82 Cascade Creek (NWE)

83 CDM Hydro - Bonneville Pacific (PPL)

84 Cedar Draw (IPC)

85 Central OR Irrigation District (PPL)

86 Clear Springs Trout (IPC)

87 Corbett (PGE)

88 Cornelius (PGE)

89 Crystal Springs (IPC)

90 Curry Cattle Co. (IPC)

91 Dairy Creek (PGE)

92 Deep Creek (AVWP)

93 Derr Creek (AVWP)

94 Dietrich Drop (IPC)

95 Donald Jenni (NWE)

96 Eagle Creek (PGE)

97 Eagle Point Irrigation Dist. (PPL)

98 Elk Creek (IPC)

99 Estacada (PGE)

100 Falls Creek (PPL)

101 Falls River (IPC)

102 Farmers Irrig. (PPL)

103 Faulkner Ranch (IPC)

104 Fisheries Development Co (IPC)

105 Galesville Dam (PPL)

106 Geobon 2 (IPC)

107 Georgetown Power (UPL)

108 Hailey Cspp (IPC)

109 Hazelton A (IPC)

110 Hazelton B (IPC)

111 Horseshoe Bend (IPC)

112 Hutchinson Creek (PSE)

113 Ingram Warm Springs (UPL)

$114 \mathrm{Jim}$ Ford Creek (AVWP)

115 Jim Knight (IPC)

116 John Day Creek (AVWP)

117 Joseph Hydro (PPL)

118 Kasel \& Witherspoon (IPC)

119 Koma Kulshan (PSE)

120 Koyle (IPC)

$121 \mathrm{~L}$. Fery (PPL)

122 Lacomb Irrig. (PPL)

123 Lake Oswego (PGE)

124 Lateral \#10 (IPC)

125 Lee Tavenner (NWE)

126 Lemoyne (IPC)

127 Little Wood River Res. (IPC)

128 Littlewood/Arkoosh (IPC)

129 Lowline \#2 (IPC)

\begin{tabular}{|c|c|c|c|c|c|c|}
\hline 2.4 & 2.4 & 1.9 & 0.8 & 0.3 & 1.0 & 2.2 \\
\hline 0 & 0 & 0 & 0 & 0 & 0 & 0 \\
\hline 0 & 0 & 0 & 0 & 0.1 & 0 & 0 \\
\hline 0.1 & 0.1 & 0.1 & 0.2 & 0.1 & 0.1 & 0.1 \\
\hline 0.1 & 0.1 & 0.1 & 0.1 & 0.1 & 0.1 & 0.1 \\
\hline 0 & 0 & 0.1 & 0.1 & 0.1 & 0.1 & 0 \\
\hline 1.3 & 1.3 & 1.1 & 1.0 & 1.1 & 1.1 & 0.3 \\
\hline 0.1 & 0.1 & 0.1 & 0.1 & 0.1 & 0 & 0 \\
\hline 3.1 & 2.8 & 3.1 & 3.1 & 2.8 & 1.9 & 2.8 \\
\hline 0.7 & 0.7 & 1.1 & 0.9 & 0.2 & 0 & 0 \\
\hline 0.4 & 0.4 & 0.4 & 0.4 & 0.4 & 0.3 & 0.3 \\
\hline 0 & 0 & 0 & 0 & 0 & 0.4 & 0.3 \\
\hline 0.6 & 0.6 & 0.6 & 0.6 & 0.6 & 0.6 & 0.6 \\
\hline 1.1 & 1.1 & 1.5 & 1.9 & 2 & 1.6 & 1.5 \\
\hline 8.3 & 8.3 & 6.5 & 2.8 & 1.0 & 0 & 0 \\
\hline 0.1 & 0.1 & 0.1 & 0.1 & 0.1 & 0.1 & 0.1 \\
\hline 0 & 0 & 0 & 0 & 0 & 0 & 0.1 \\
\hline 0 & 0 & 0 & 0 & 0 & 0 & 0 \\
\hline 2.9 & 2.9 & 3.1 & 2.8 & 2.9 & 2.4 & 1.8 \\
\hline 0.3 & 0.3 & 0.9 & 0.9 & 0.9 & 1.2 & 1.2 \\
\hline 3.1 & 3.1 & 3.7 & 3.5 & 0 & 0.7 & 0 \\
\hline 0.4 & 0.4 & 0.4 & 0.5 & 0.6 & 0.6 & 0.4 \\
\hline 0 & 0 & 0 & 0 & 0 & 0 & 0 \\
\hline 0 & 0 & 0 & 0 & 0 & 0 & 0 \\
\hline 1.3 & 1.3 & 1.6 & 1.5 & 1.6 & 0.5 & 0.5 \\
\hline 0.2 & 0.2 & 0.2 & 0.1 & 0.1 & 0 & 0 \\
\hline 0 & 0 & 0 & 0 & 0 & 0 & 0 \\
\hline 0 & 0 & 0 & 0 & 0 & 0 & 0 \\
\hline 0.1 & 0.1 & 0.1 & 0.1 & 0.1 & 0.1 & 0.1 \\
\hline 4.7 & 4.7 & 3.6 & 3.1 & 1.4 & 0 & 0 \\
\hline 0.1 & 0.1 & 0.1 & 0.1 & 0.1 & 0.1 & 0.1 \\
\hline 0 & 0 & 0 & 0 & 0 & 0 & 0 \\
\hline 0 & 0 & 0 & 0 & 0.4 & 0.7 & 0.7 \\
\hline 1.2 & 1.2 & 0 & 0 & 0 & 0.1 & 0.1 \\
\hline 0.1 & 0.1 & 0.1 & 0.1 & 0.1 & 0 & 0.1 \\
\hline 0 & 0 & 0 & 0 & 1.9 & 3.1 & 0.9 \\
\hline 6.5 & 6.5 & 6.3 & 5.7 & 5.6 & 5.0 & 4.2 \\
\hline 0.6 & 0.6 & 0.7 & 1.2 & 3.2 & 3.2 & 2.1 \\
\hline 0.8 & 0.8 & 0.7 & 0.7 & 0.7 & 0.3 & 0.3 \\
\hline 0.2 & 0.2 & 0.3 & 0.2 & 0.3 & 0.3 & 0.3 \\
\hline 0.6 & 0.6 & 0.4 & 0.2 & 0.2 & 0.3 & 0 \\
\hline 1.0 & 1.0 & 0.3 & 0.3 & 0.3 & 0.2 & 0.2 \\
\hline 0 & 0 & 0.2 & 0.3 & 0.3 & 0.3 & 0.3 \\
\hline 0.1 & 0.1 & 0.1 & 0.1 & 0.1 & 0.1 & 0.1 \\
\hline 7 & 7 & 5.4 & 2.5 & 0.9 & 0 & 0 \\
\hline 6.6 & 6.6 & 5.2 & 2.4 & 0.7 & 0 & 0 \\
\hline 8.8 & 8.8 & 7.5 & 3.9 & 2.8 & 3.5 & 3.9 \\
\hline 0.7 & 0.7 & 0.7 & 0.7 & 0.7 & 0.7 & 0.7 \\
\hline 0.2 & 0.2 & 0.3 & 0.3 & 0.5 & 0.5 & 0.4 \\
\hline 0 & 0 & 0 & 0 & 0.3 & 0.5 & 0.9 \\
\hline 0.4 & 0.4 & 0.3 & 0.2 & 0.3 & 0.1 & 0.1 \\
\hline 0.2 & 0.2 & 0.2 & 0.2 & 0.2 & 0.2 & 0.3 \\
\hline 1.5 & 1.5 & 0.9 & 0.4 & 0.1 & 0.1 & 0.1 \\
\hline 1.2 & 1.2 & 1.2 & 0.8 & 0.5 & 0.3 & 0.2 \\
\hline 4.6 & 4.6 & 4.3 & 6.4 & 8 & 6.1 & 3.9 \\
\hline 1.1 & 1.1 & 1.1 & 1.0 & 1 & 0.2 & 0.1 \\
\hline 0 & 0 & 0 & 0 & 0 & 0 & 0 \\
\hline 0 & 0 & 0 & 0 & 0.2 & 1.0 & 0.2 \\
\hline 0.4 & 0.4 & 0.4 & 0.4 & 0.4 & 0.4 & 0.4 \\
\hline 1.5 & 1.5 & 1.6 & 2 & 2.1 & 1.5 & 1.5 \\
\hline 0.1 & 0.1 & 0.1 & 0.1 & 0.1 & 0.1 & 0.1 \\
\hline 0 & 0 & 0 & 0 & 0 & 0 & 0 \\
\hline 1.9 & 1.9 & 0.2 & 0.2 & 0.2 & 0 & 0 \\
\hline 0.7 & 0.7 & 0.4 & 0.4 & 0.4 & 0.2 & 0.2 \\
\hline 2.7 & 2.7 & 2.5 & 1.8 & 1.4 & 0 & 0 \\
\hline
\end{tabular}

$2.6 \quad 3.3$

$\begin{array}{rr}0 \\ 0 \\ 0.1 & 0.1\end{array}$

$\begin{array}{rr}0.1 & 0.1 \\ 0 & \end{array}$

$\begin{array}{rr}1.2 & 1.7 \\ 0 & 0.1\end{array}$

$\begin{array}{rr}2.8 & 1.9 \\ 0 & \end{array}$

$\begin{array}{ll}0.3 & 0.3\end{array}$

$\begin{array}{ll}0.6 & 0.7\end{array}$

$0.7 \quad 0.6$

$\begin{array}{rl}1.7 & 1.7 \\ 0 & 0.1\end{array}$

$\begin{array}{rr}0 & 0.1 \\ 0.1 & 0.1 \\ 0.1 & 0.1\end{array}$

$\begin{array}{ll}0 & \\ 2 & 2\end{array}$

$\begin{array}{ll}1.3 & 0.4 \\ 0.4 & 0.9\end{array}$

$0.5 \quad 0.4$

$\begin{array}{rr}0 & \\ 0.6 & 0.8\end{array}$

$\begin{array}{ll}0 & 0.1 \\ 0 & \\ 0 & \end{array}$

$0.1 \quad 0.1$

$\begin{array}{rr}0.1 & 0.1 \\ 0 & \end{array}$

$\begin{array}{ll}0.9 & 0.6 \\ 0.1 & \end{array}$

$0.1 \quad 0.1$

$\begin{array}{ll}0.7 & 2.7 \\ 3.8 & 3.8\end{array}$

3.8
4.6

0.3

0.3

0.2

0.3

0.3
0.1

0.1
0

0
4.6

4.6
0.7

0.4

0.9

0.1
0.2

0.2
0

0.2

$3.8 \quad 4.4$

$0.1 \quad 0.3$

0.9

0.4

$1.7-1.8$

0.1

0

0.2
0.7

3.8
3.3

0.5
0.3

0.3
0.6

0.3

0.1

0.1
5.9

0.7

0.4
1.1

0.2

0.9

0.1
0

1.9

3.2

$\begin{array}{rr}0 & 0 \\ 0 & 0 \\ 1 & 0.1\end{array}$

$3.2 \quad 3$.

0
0
0.1
0.1

0
1.6

$\begin{array}{ll}0.1 & 0.1\end{array}$

$\begin{array}{ll}2.5 & 3.4 \\ 0.6 & 0.6\end{array}$

$\begin{array}{ll}0.3 & 0.3\end{array}$

$\begin{array}{ll}0.8 & 0.8\end{array}$

$\begin{array}{ll}0.6 & 0.6 \\ 2.7 & 2.6\end{array}$

$\begin{array}{ll}2.9 & 2.9\end{array}$

$\begin{array}{ll}0.1 & 0.1 \\ 0.1 & 0.1\end{array}$

$\begin{array}{rr}0.1 & 0.1 \\ 0 & 0 \\ 27 & 27\end{array}$

$\begin{array}{ll}2.7 & 2.7 \\ 0.4 & 0.4\end{array}$

$0.8 \quad 0.8$

$0.3 \quad 0.3$

0.3
0
0

$\begin{array}{rrr}0 & 0 & 0 \\ 0.9 & 0.9 & 0.8\end{array}$

0.1

0.1
0

0

$\begin{array}{ll}0.2 & 0.2 \\ 3.1 & 3.1\end{array}$

3.1

3.1
0.1

$\begin{array}{lll}0 & 0 & 0\end{array}$

$\begin{array}{ll}0.7 & 0.7 \\ 1 & \end{array}$

$0.1 \quad 0.1$

$4.4 \quad 4.4$

0.122 .6

$\begin{array}{lll}0.2 & 0.2 & 0.2 \\ 0 & 0.1 & 0.1\end{array}$

$\begin{array}{lll}0.4 & 0.5 & 0.5\end{array}$

$\begin{array}{lll}0.3 & 6.9 & 6.9 \\ 0.3 & 0.8 & 0.8\end{array}$

$\begin{array}{ll}4 & 0.4 \\ 1.8 & 1.9\end{array}$

$\begin{array}{lll}0.4 & 0.5 & 0.5\end{array}$

2.5

2.5

2.7

2.7

$\begin{array}{rrr}3.2 & 3.3 & 2.3 \\ 0 & 0 & 0 \\ 0 & 0 & 0 \\ 0.2 & 0.1 & 0.1 \\ 0.1 & 0.1 & 0.1 \\ 0 & 0 & 0 \\ 1.3 & 1.2 & 1.2 \\ 0.1 & 0.1 & 0.1 \\ 3.4 & 3.1 & 2.8 \\ 1.0 & 0.7 & 0.6 \\ 0.4 & 0.4 & 0.4 \\ 0 & 0 & 0.3 \\ 0.6 & 0.6 & 0.6 \\ 2.7 & 2.0 & 2.0 \\ 8.2 & 8.7 & 3.8 \\ 0.1 & 0.1 & 0.1 \\ 0 & 0 & 0 \\ 0 & 0 & 0 \\ 5.8 & 4.1 & 3.1 \\ 0.3 & 0.3 & 0.7 \\ 3.8 & 4.1 & 2.1 \\ 0.4 & 0.4 & 0.4 \\ 0 & 0 & 0 \\ 0 & 0 & 0 \\ 1.4 & 1.3 & 1.1 \\ 0.2 & 0.2 & 0.1 \\ 0 & 0 & 0 \\ 0 & 0 & 0 \\ 0.2 & 0.2 & 0.1 \\ 4.7 & 4.5 & 2.5 \\ 0.1 & 0.1 & 0.1 \\ 0 & 0 & 0 \\ 0 & 0 & 0.4 \\ 1.2 & 1.2 & 0.6 \\ 0.1 & 0.1 & 0.1 \\ 3.8 & 0 & 2.0 \\ 8.5 & 7.1 & 5.9 \\ 0.8 & 0.7 & 2.4 \\ 0.8 & 0.8 & 0.6 \\ 0.2 & 0.2 & 0.3 \\ 0.4 & 0.5 & 0.3 \\ 1.0 & 1.0 & 0.5 \\ 0.2 & 0 & 0.2 \\ 0.1 & 0.1 & 0.1 \\ 6.7 & 7.4 & 3.2 \\ 6.4 & 7.0 & 3 \\ 9.8 & 9.2 & 6.6 \\ 0.7 & 0.7 & 0.7 \\ 0.3 & 0.3 & 0.4 \\ 0.3 & 0.1 & 0.5 \\ 0.4 & 0.4 & 0.2 \\ 0.2 & 0.2 & 0.2 \\ 1.2 & 2.7 & 0.6 \\ 1.1 & 1 & 0.7 \\ 10 & 8.2 & 6.4 \\ 0.8 & 0.9 & 0.7 \\ 0.4 & 0 & 0 \\ 1.6 & 0.4 & 0.5 \\ 0.1 & 0.5 & 0.4 \\ 0 & 0 & 0.1 \\ 2 & 1.9 & 0.9 \\ 0.8 & 0.7 & 0.5 \\ 2.7 & 2.7 & 1.8\end{array}$



2003 White Book

Aug1 Aug16 Sep Oct Nov Dec

Jan Feb Mar Apr1 Apr16

\begin{abstract}
May
\end{abstract}

$$
\text { Jun }
$$
Jul Avg

130 Lowline Canal (IPC)

131 Magic Reservoir (IPC)

132 Malad River (IPC)

133 Marcos Ranches (IPC)

134 Marsh Valley (UPL)

135 Meyers Falls (AVWP)

136 Middlefork Irrig. (PPL)

137 Mile 28 (IPC)

138 Minikahda (PGE)

139 Mink Creek (UPL)

140 Mitchell Butte (IPC)

141 Mountain Energy (PPL)

142 Mt. Tabor (PGE)

143 Mud Creek/S\&S (IPC)

144 Mud Creek/White (IPC)

145 Nichols Gap (PPL)

146 Nicholson Sunnybar (UPL)

147 North Fork Sprague (PPL)

148 O.J. Power (UPL)

149 Odell Creek (PPL)

150 Opal Springs (PPL)

151 Owyhee Dam (IPC)

152 Pancheri (UPL)

153 Pelton Rereg. Dam (PPL)

154 Phillips Ranch (AVWP)

155 Phillipsburg (NWE)

156 Pigeon Cove (IPC)

157 Pine Creek (NWE)

158 Port Townsend Paper Hydro (PSE)

159 Portland (PGE)

160 Portland Hydro Project (PGE)

161 Preston City (UPL)

162 Pristine Springs (IPC)

163 Pristine Springs \#3 (IPC)

164 Reynolds Irrigation (IPC)

165 Rim View (IPC)

166 Rock Creek \#1 (IPC)

167 Rock Creek \#2 (IPC)

168 Sagebrush (IPC)

169 Salem (PGE)

170 Schaffner (IPC)

171 Sheep Creek (AVWP)

172 Shingle Creek (IPC)

173 Shoshone (IPC)

174 Shoshone II (IPC)

175 Snake River Pottery (IPC)

176 Snedigar Ranch (IPC)

177 Solar Research (PPL)

178 South Dry Creek (NWE)

179 Spokane Upriver (AVWP)

180 Stauffer Dry Creek (UPL)

181 Strawberry Creek (NWE)

182 Sunshine Power \#2 (IPC)

183 Sygitowicz Creek Small Hydro (PSE)

184 TGS/Briggs (UPL)

185 Trout Co. (IPC)

186 Tunnel \#1 (IPC)

187 Twin Falls (PSE)

188 Walla Walla (PPL)

189 Water Street / Santiam (PPL)

190 Weeks Falls (PSE)

191 White James (PPL

192 White Water Ranch (IPC)

193 Wilson Lake Hydro (IPC)

194 Wisconsin Creek (NWE)

195 Yakima-Tieton (PPL)

196 IOU - Total NUG: Hydro

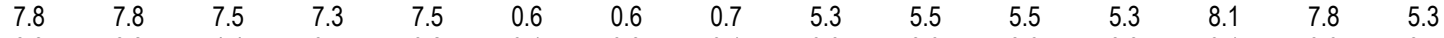

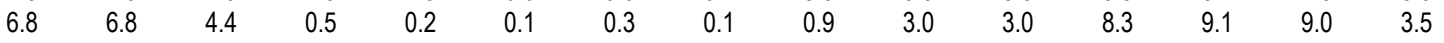

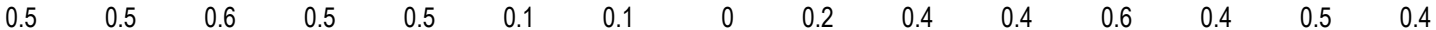

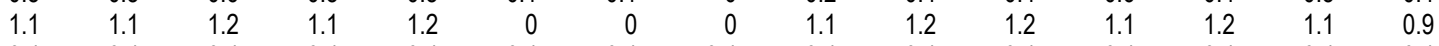

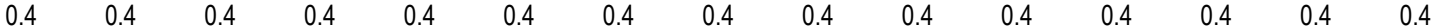

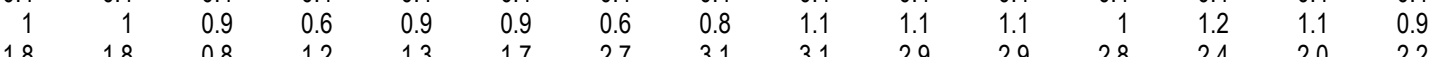

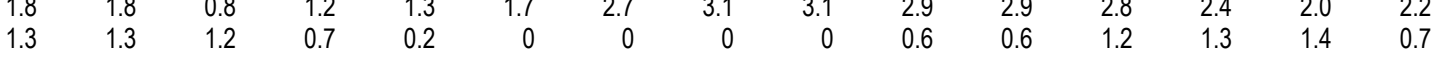

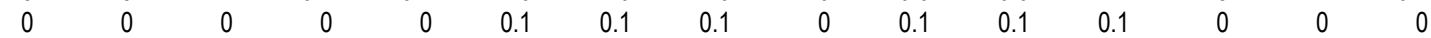

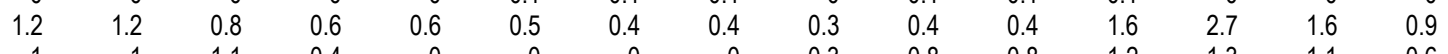

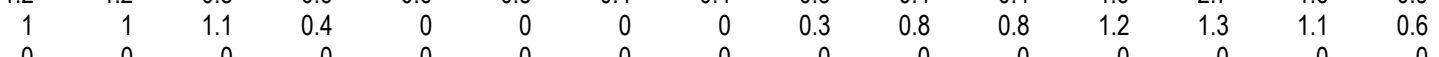

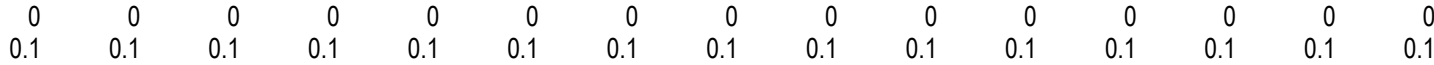

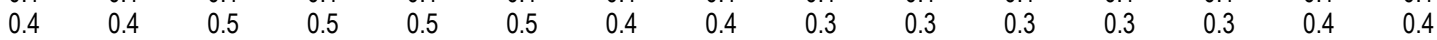

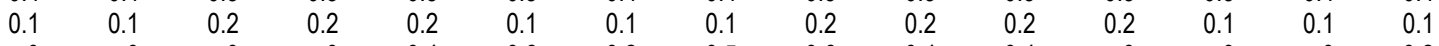

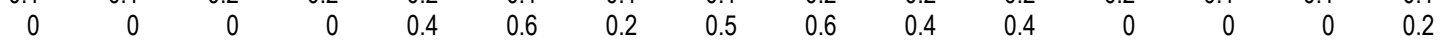

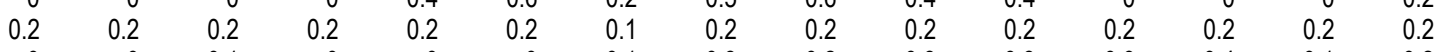

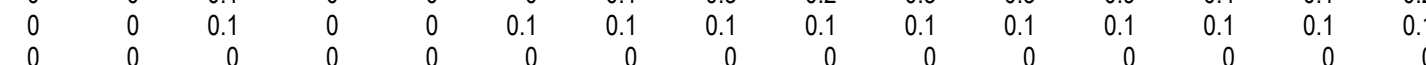

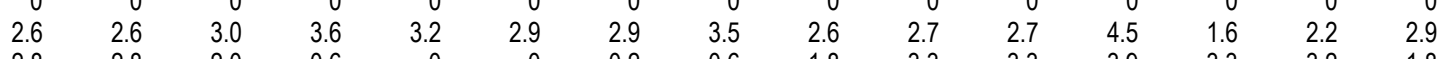

$\begin{array}{rrrrrrrr}0 & 0 & 0 & 0 & 0 & 0.1 & 0.1 & 0.1 \\ 2.0 & 2.0 & 2.1 & 1.3 & 0.8 & 0.4 & 0.3 \\ 0.1 & 0.1 & 0.1 & 0.1 & 0.1 & 0.1 & 0.1 & 0.4 \\ 0.3 & 0.3 & 0.3 & 0.3 & 0.3 & 0.3 & 0.3 & 0.3 \\ 0 & 0 & 0 & 0 & 0 & 0 & 0 & 0\end{array}$

$\begin{array}{rrrrrrrr}0.3 & 0.3 & 0.3 & 0.3 & 0.3 & 0.3 & 0.3 & 0.3 \\ 0 & 0 & 0 & 0 & 0 & 0 & 0\end{array}$

$\begin{array}{rrrrrrrrr}2.3 & 2.3 & 2.9 & 6.7 & 16 & 20 & 18 & 17 & 16 \\ 0.3 & 0.3 & 0.4 & 0.4 & 0.4 & 0.4 & 0.4 & 0.4 & 0.3\end{array}$

$\begin{array}{llllllll}0.1 & 0.1 & 0.2 & 0.2 & 0.1 & 0.1 & 0.1 & 0.1\end{array}$

$\begin{array}{lllllll}0.2 & 0.2 & 0.2 & 0.3 & 0.2 & 0.2 & 0.2\end{array}$

$\begin{array}{lllllll}0.2 & 0.2 & 0.2 & 0.2 & 0.2 & 0.2 & 0.2 \\ 0.2 & 0.2 & 0.2 & 0.3 & 0.2 & 0.2 & 0.2\end{array}$

$\begin{array}{llllllll}1.1 & 1.1 & 1.6 & 1.7 & 1.2 & 0.2 & 0.2 \\ 1.8 & 1.8 & 1.8 & 1.9 & 1.9 & 1.9 & 1.1 \\ 0.4 & 0.4 & 0.3 & 0.3 & 0.3 & 0.1 & 0.1\end{array}$

$\begin{array}{rrrrrrr}0.4 & 0.4 & 0.3 & 0.3 & 0.3 & 0.1 & 0.1 \\ 0 & 0 & 0 & 0 & 0 & 0 & 0\end{array}$

$\begin{array}{rrrrrrr}0 & 0 & 0 & 0 & 0 & 0 & 0 \\ 0.4 & 0.4 & 0.3 & 0.2 & 0.3 & 0.1 & 0.1 \\ 0.4 & 0.4 & 0.3 & 0.3 & 0.4 & 0.5 & 0.5 \\ 0.1 & 0.1 & 0.2 & 0.2 & 0.1 & 0.1 & 0.1\end{array}$

$0.3-0.2$

$0.2 \quad 0.2$

$\begin{array}{ll}1.0 & 1.1 \\ 0.4 & 1.6\end{array}$

$\begin{array}{llll}0.4 & 0.4 & 0.5 & 0.4 \\ 0.6 & 0.6 & 0.6 & 0\end{array}$

$\begin{array}{rrrr}0.6 & 0.6 & 0.6 \\ 0 & 0 & 0\end{array}$

$\begin{array}{llll}0.2 & 0.2 & 0.2 & 0.2\end{array}$

$\begin{array}{llll}0.1 & 0.1 & 0.1 & 0 \\ 0.5 & 0.5 & 0.5 & 0.5\end{array}$

$\begin{array}{rrrrrrr}0.5 & 0.5 & 0.5 & 0.5 & 0 & 0 & 0 \\ 0.4 & 3.4 & 3.4 & 4.7 & 6.7 & 13 & 14\end{array}$

$\begin{array}{rrrrrrr}0.9 & 0.9 & 1 & 0.8 & 0.4 & 0 & 0.3\end{array}$

$\begin{array}{rrrrrrr}0.1 & 0.1 & 0.1 & 0.1 & 0.1 & 0.1 & 0.1 \\ 0.1 & 0.1 & 0.1 & 0.1 & 0.1 & 0 & 0 \\ 0 & 0 & 0 & 0.1 & 0.2 & 0.3 & 0.3\end{array}$

$\begin{array}{rrrrrrr}0.2 & 0.2 & 0.1 & 0.1 & 0.2 & 0.3 & 0.3 \\ 0.1 & 0.1 & 0.1 & 0.1 & 0.2 & 0.2 & 0.1 \\ 4.3 & 4.3 & 2.6 & 0.5 & 0.1 & 0.1 & 0.1\end{array}$

$0.1 \quad 0.3$

$\begin{array}{rr}0 & 0 \\ 0.2 & 0.3 \\ 0.5 & 0.9\end{array}$

$0.1 \quad 0.1$

$\begin{array}{rrrrrrr}4.3 & 4.3 & 2.6 & 0.5 & 0 & 0 & 0 \\ 5 & 5 & 0 & 1.2 & 9.6 & 7.9 & 7.4 \\ 15 & 1.5 & 1.4 & 1.3 & 1.2 & 1.3 & 1.2\end{array}$

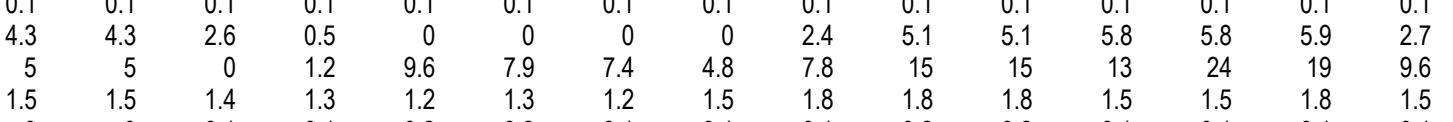

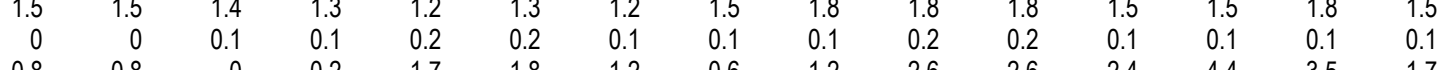

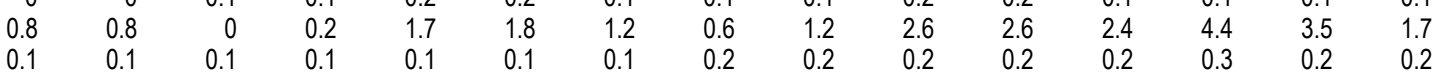

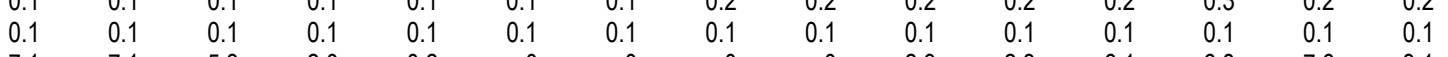

$\begin{array}{rrrrrrrrrrrrrrr}0.1 & 0.1 & 0.1 & 0.1 & 0.1 & 0.1 & 0.1 & 0.1 & 0.1 & 0.1 & 0.1 & 0.1 & 0.1 & 0.1 & 0.1 \\ 7.1 & 7.1 & 5.3 & 2.0 & 0.2 & 0 & 0 & 0 & 0 & 2.3 & 2.3 & 6.1 & 6.8 & 7.6 & 3.1 \\ 0 & 0 & 0 & 0.1 & 0 & 0.1 & 0.1 & 0.1 & 0.1 & 0.1 & 0.1 & 0 & 0 & 0 & 0.1\end{array}$

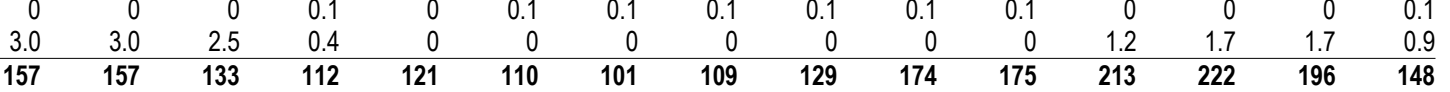

-NUG: Small Thermal \& Misc.-

197 Marion Solid Waste (PGE)

198 Minnesota Methane (AVWP)

199 Montana One (NWE)

200 Pocatello Waste (IPC)

201 Spokane MSW (PSE)

202 West Boise Waste (IPC)

\begin{tabular}{rrrrrrrrrrrrrrr}
9.3 & 9.3 & 9.3 & 9.3 & 9.3 & 9.3 & 9.3 & 9.3 & 9.3 & 9.3 & 9.3 & 9.3 & 9.3 & 9.3 & 9.3 \\
0.8 & 0.8 & 0.8 & 0.8 & 0.8 & 0.8 & 0.8 & 0.8 & 0.8 & 0.8 & 0.8 & 0.8 & 0.8 & 0.8 & 0.8 \\
9.4 & 9.6 & 8.9 & 9.0 & 9.2 & 10.0 & 10 & 10 & 9.0 & 9.0 & 8.8 & 10 & 8.8 & 8.6 & 9.4 \\
0.1 & 0.1 & 0.2 & 0.1 & 0.2 & 0.1 & 0.1 & 0.2 & 0.1 & 0.2 & 0.2 & 0.1 & 0.2 & 0.1 & 0.1 \\
15 & 15 & 15 & 15 & 15 & 15 & 15 & 15 & 15 & 15 & 15 & 15 & 15 & 15 & 15 \\
0.1 & 0.1 & 0.1 & 0.1 & 0.1 & 0.1 & 0.1 & 0.1 & 0.1 & 0.1 & 0.1 & 0.1 & 0.1 & 0.1 & 0.1 \\
\hline $\mathbf{3 5}$ & $\mathbf{3 5}$ & $\mathbf{3 5}$ & $\mathbf{3 5}$ & $\mathbf{3 5}$ & $\mathbf{3 6}$ & $\mathbf{3 6}$ & $\mathbf{3 6}$ & $\mathbf{3 5}$ & $\mathbf{3 5}$ & $\mathbf{3 4}$ & $\mathbf{3 6}$ & $\mathbf{3 4}$ & $\mathbf{3 4}$ & $\mathbf{3 5}$
\end{tabular}


Table A-24: Regional Non Utility Generating Resources By Project

PNW Loads and Resources Study

2011 - 2012 Operating Year 2003 White Book

Aug1 Aug16 Sep

Oct Nov

Dec Jan Feb Mar Apr1 Apr16

May Jun Jul Avg

-NUG: Combustion Turbines -

204 March Point \#1 Cogeneration (PSE)

205 March Point \#2 Cogeneration (PSE)

206 Rathdrum- Boekel Rd. \#1 (AVWP)

207 Rathdrum -Boekel Rd. \#2 (AVWP)

208 Tenaska Gas Cogen (PSE)

209 IOU - Total NUG: Combustion Turbines

\begin{tabular}{|c|c|c|c|c|c|c|c|c|c|c|c|c|c|c|}
\hline 79 & 79 & 79 & 79 & 79 & 79 & 79 & 79 & 79 & 79 & 79 & 55 & 79 & 79 & 77 \\
\hline 59 & 59 & 59 & 59 & 59 & 59 & 59 & 59 & 59 & 59 & 59 & 45 & 59 & 59 & 58 \\
\hline 65 & 65 & 65 & 65 & 65 & 65 & 65 & 65 & 65 & 65 & 65 & 32 & 65 & 65 & 62 \\
\hline 65 & 65 & 65 & 65 & 65 & 65 & 65 & 65 & 65 & 65 & 65 & 32 & 65 & 65 & 62 \\
\hline 240 & 240 & 240 & 240 & 240 & 240 & 240 & 240 & 240 & 240 & 240 & 180 & 240 & 240 & 235 \\
\hline 508 & 508 & 508 & 508 & 508 & 508 & 508 & 508 & 508 & 508 & 508 & 344 & 508 & 508 & 494 \\
\hline
\end{tabular}

-NUG: Co-Generation-

210 Billings Generation (NWE)

211 Biomass One (PPL)

212 Boise Cascade Medford (PPL)

213 Champion (PPL)

214 DR Johnson - Co-Gen II (PPL)

215 Magic Valley (IPC)

216 Magic West (IPC)

217 PERC Pierce, Wa (PSE)

218 Simplot Pocatello (IPC)

219 Sumas Energy (PSE)

220 Tamarack (IPC)

221 TASCO Nampa ID (IPC)

222 TASCO Twin Falls ID (IPC)

223 Vaagen Bros. (IPC)

224 Warm Springs (PPL)

225 IOU - Total NUG: Co-Generation

-NUG: Renewables-

226 Foote Creek 1 (PPL)

227 Healow \#2 (NWE)

228 Josef Staufer (NWE)

229 Lewandowski Farms (IPC)

230 Livingston (NWE)

231 Puyallup Energy Recovery (PSE)

232 Stateline Wind Project (AVWP)

233 Windmill (PGE)

234 IOU - Total NUG: Renewables

\begin{tabular}{|c|c|c|c|c|c|c|c|c|c|c|c|c|c|c|}
\hline 17 & 18 & 16 & 11 & 17 & 18 & 19 & 18 & 16 & 10 & 10 & 19 & 16 & 16 & 16 \\
\hline 22 & 22 & 21 & 21 & 22 & 22 & 7.2 & 15 & 17 & 20 & 20 & 17 & 22 & 22 & 19 \\
\hline 8.5 & 8.5 & 8.5 & 8.5 & 8.5 & 8.5 & 8.5 & 8.5 & 8.5 & 8.5 & 8.5 & 8.5 & 8.5 & 8.5 & 8.5 \\
\hline 3.3 & 3.3 & 3.3 & 3.3 & 3.3 & 3.3 & 1 & 3.3 & 3.3 & 3.3 & 3.3 & 3.3 & 3.3 & 3.3 & 3.1 \\
\hline 7.5 & 7.5 & 7.7 & 5.8 & 7.3 & 6.0 & 6.7 & 9.1 & 7.3 & 7.4 & 7.4 & 11 & 4.2 & 7.1 & 7.2 \\
\hline 9.3 & 9.3 & 9.7 & 9.3 & 9.6 & 9.3 & 9.3 & 10 & 9.4 & 9.7 & 9.7 & 9.4 & 9.7 & 9.3 & 9.5 \\
\hline 9.3 & 9.3 & 9.7 & 9.3 & 9.6 & 9.3 & 9.3 & 10 & 9.4 & 9.7 & 9.7 & 9.4 & 9.7 & 9.3 & 9.5 \\
\hline 2 & 2 & 2 & 2 & 2 & 2 & 2 & 2 & 2 & 2 & 2 & 0 & 2 & 2 & 1.8 \\
\hline 8.8 & 8.8 & 9.2 & 8.8 & 9.1 & 8.8 & 8.8 & 9.6 & 8.9 & 9.2 & 9.2 & 8.9 & 9.2 & 8.8 & 9.0 \\
\hline 113 & 113 & 126 & 126 & 126 & 126 & 126 & 126 & 126 & 113 & 113 & 113 & 113 & 113 & 120 \\
\hline 3.8 & 3.8 & 4.6 & 4.7 & 4.2 & 3.2 & 3.6 & 3.8 & 4.2 & 4.4 & 4.4 & 3.4 & 4.6 & 4.8 & 4.1 \\
\hline 0.2 & 0.2 & 0.2 & 0.2 & 0.2 & 0.2 & 0.2 & 0.2 & 0.2 & 0.2 & 0.2 & 0.2 & 0.2 & 0.2 & 0.2 \\
\hline 0 & 0 & 0 & 0 & 0 & 0 & 0 & 0 & 0 & 0 & 0 & 0 & 0 & 0 & 0 \\
\hline 3 & 3 & 3.7 & 3.8 & 3.3 & 2.6 & 2.9 & 3.1 & 3.4 & 3.6 & 3.6 & 2.7 & 3.7 & 3.8 & 3.3 \\
\hline 0.1 & 0.1 & 0 & 0.1 & 0.1 & 0.1 & 0.1 & 0.1 & 0 & 0 & 0 & 0 & 0.1 & 0 & 0.1 \\
\hline 207 & 208 & 223 & 214 & 222 & 219 & 205 & 220 & 216 & 201 & 201 & 206 & 206 & 207 & 212 \\
\hline
\end{tabular}

Other Entities

-NUG: Co-Generation-

235 SP Newsprint cogen (SPN)

236 Other - Total NUG: Co-Generation

$\begin{array}{lllllllllllllll}25 & 25 & 25 & 25 & 25 & 25 & 25 & 25 & 25 & 25 & 25 & 25 & 25 & 25 & 25 \\ \mathbf{2 5} & \mathbf{2 5} & \mathbf{2 5} & \mathbf{2 5} & \mathbf{2 5} & \mathbf{2 5} & \mathbf{2 5} & \mathbf{2 5} & \mathbf{2 5} & \mathbf{2 5} & \mathbf{2 5} & \mathbf{2 5} & \mathbf{2 5} & \mathbf{2 5} & \mathbf{2 5}\end{array}$

-NUG: Renewables-

237 Stateline Wind Project (PPME)

238 Vansycle Wind (FPLEV)

239 Other - Total NUG: Renewables

\begin{tabular}{|c|c|c|c|c|c|c|c|c|c|c|c|c|c|c|}
\hline 4.2 & 4.2 & 4.9 & 7.2 & 11 & 13 & 14 & 12 & 9.2 & 7.4 & 7.4 & 5.9 & 5.2 & 4.2 & 8.1 \\
\hline 0 & 0 & 0 & 0 & 0 & 0 & 0 & 0 & 0 & 0 & 0 & 0 & 0 & 0 & 0 \\
\hline 0 & 0 & 0 & 0 & 0 & 0 & 0 & 0 & 0 & 0 & 0 & 0 & 0 & 0 & 0 \\
\hline 0 & 0 & 0 & 0 & 0 & 0 & 0 & 0 & 0 & 0 & 0 & 0 & 0 & 0 & 0 \\
\hline 0 & 0 & 0 & 0 & 0 & 0 & 0 & 0 & 0 & 0 & 0 & 0 & 0 & 0 & 0 \\
\hline 2 & 2 & 2 & 2 & 2 & 2 & 2 & 2 & 2 & 2 & 2 & 2 & 2 & 2 & 2 \\
\hline 0.3 & 0.3 & 0.2 & 0.3 & 0.3 & 0.3 & 0.3 & 0.3 & 0.3 & 0.3 & 0.3 & 0.3 & 0.3 & 0.3 & 0.3 \\
\hline 0 & 0 & 0 & 0 & 0 & 0 & 0 & 0 & 0 & 0 & 0 & 0 & 0 & 0 & 0 \\
\hline 6.5 & 6.5 & 7.1 & 9.5 & 13 & 15 & 16 & 15 & 12 & 9.8 & 9.8 & 8.2 & 7.6 & 6.5 & 10 \\
\hline
\end{tabular}

-Total Non-Utility Generating Resources-

240 Federal Entities

241 Generating Public Entities

242 Non Generating Public Entities

243 Investor-Owned Entities

244 Other Entities

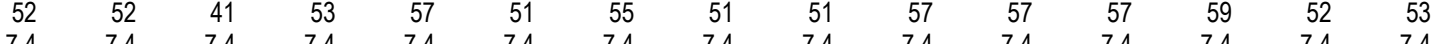

245 Total Non-Utility Generation

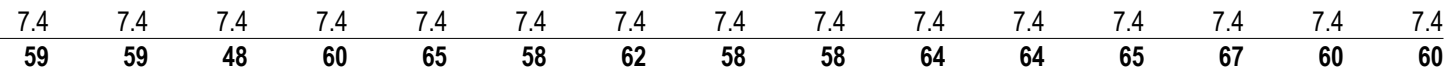

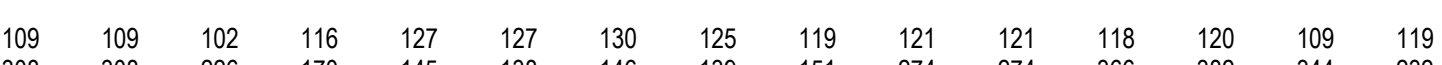

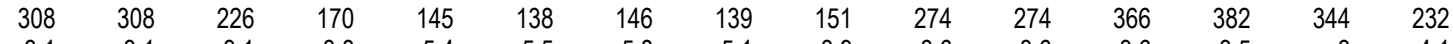

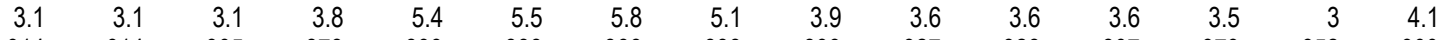

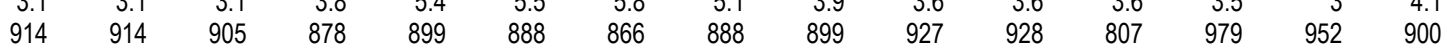

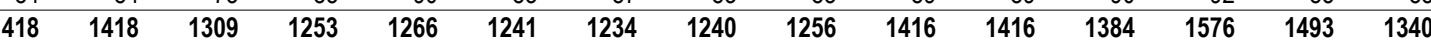


Oct Nov

Dec

Jan Fe

\begin{abstract}
Feb
\end{abstract}
Federal Entities

-NUG: Hydro-

1 Clearwater - State of ID DWR (BPA)

2 Dworshak Small Hydropower (BPA)

3 Federal -Total NUG: Hydro

\begin{tabular}{|c|c|c|c|c|c|c|c|c|c|c|c|c|c|c|}
\hline 1 & 1 & 1 & 1 & 1 & 1 & 1 & 1 & 1 & 1 & 1 & 1 & 1 & 1 & 1 \\
\hline 2.6 & 2.6 & 2.6 & 2.6 & 2.6 & 2.6 & 2.6 & 2.6 & 2.6 & 2.6 & 2.6 & 2.6 & 2.6 & 2.6 & 2.6 \\
\hline 3.6 & 3.6 & 3.6 & 3.6 & 3.6 & 3.6 & 3.6 & 3.6 & 3.6 & 3.6 & 3.6 & 3.6 & 3.6 & 3.6 & 3.6 \\
\hline
\end{tabular}

-NUG: Renewables-

4 Ashland Solar Project (BPA)

5 Condon Wind Project (BPA)

6 Foote Creek 1 (BPA)

7 Foote Creek 2 (BPA)

8 Foote Creek 4 (BPA)

9 Fourmile Hill Geothermal (BPA)

10 Klondike Phase 1 (BPA)

11 Stateline Wind Project (BPA)

12 Federal -Total NUG: Renewables

$\begin{array}{rrrrrrrrrrrrrrr}0 & 0 & 0 & 0 & 0 & 0 & 0 & 0 & 0 & 0 & 0 & 0 & 0 & 0 & 0 \\ 10 & 10 & 10 & 11 & 13 & 14 & 13 & 12 & 12 & 14 & 14 & 12 & 15 & 11 & 12 \\ 3.3 & 3.3 & 3.8 & 5.6 & 8.3 & 10 & 11 & 9.6 & 7.2 & 5.8 & 5.8 & 4.6 & 4.1 & 3.3 & 6.4 \\ 0.4 & 0.4 & 0.5 & 0.7 & 1 & 1.3 & 1.3 & 1.2 & 0.9 & 0.7 & 0.7 & 0.5 & 0.5 & 0.4 & 0.8 \\ 3.9 & 3.9 & 4.4 & 6.5 & 9.7 & 12 & 12 & 11 & 8.4 & 6.7 & 6.7 & 5.4 & 4.8 & 3.8 & 7.4 \\ 50 & 50 & 50 & 50 & 50 & 50 & 50 & 50 & 50 & 50 & 50 & 50 & 50 & 50 & 50 \\ 7.9 & 7.9 & 6.2 & 8.1 & 8.8 & 7.8 & 8.3 & 7.8 & 7.8 & 8.6 & 8.6 & 8.8 & 9.1 & 8 & 8.1 \\ 29 & 29 & 23 & 30 & 33 & 29 & 31 & 29 & 29 & 32 & 32 & 33 & 34 & 30 & 30 \\ 105 & 105 & \mathbf{9 8} & \mathbf{1 1 2} & \mathbf{1 2 4} & \mathbf{1 2 3} & \mathbf{1 2 6} & \mathbf{1 2 1} & \mathbf{1 1 5} & \mathbf{1 1 8} & \mathbf{1 1 8} & \mathbf{1 1 4} & \mathbf{1 1 7} & \mathbf{1 0 6} & \mathbf{1 1 5}\end{array}$

Generating Public Entities

-NUG: Hydro-

13 Don Steffen (SHPD)

14 Eltopia Br Canal 4.6 (SCL)

15 Eltopia Br Canal 4.6 (TPU)

16 John Llewellyn (SHPD)

17 Kevin Duncan (SHPD)

18 Lucky Peak (SCL)

19 Main Canal Headworks (SCL)

20 Main Canal Headworks (TPU)

21 PEC Headworks (GCPD)

22 Pickering Micro Hydro (CKPD)

23 Potholes E Canal 66.0 (SCL)

24 Potholes E Canal 66.0 (TPU)

25 Quincy Chute (GCPD)

26 Russel D. Smith (SCL)

27 Russel D. Smith (TPU)

28 Smith Creek (EWEB)

29 Summer Falls (SCL)

30 Summer Falls (TPU)

31 Woods Creek (SHPD)

32 GPU - Total NUG: Hydro

$\begin{array}{rrrrrrrrrrrrrrr}0 & 0 & 0 & 0 & 0 & 0 & 0 & 0 & 0 & 0 & 0 & 0 & 0 & 0 & 0 \\ 0.9 & 0.9 & 0.6 & 0.3 & 0 & 0 & 0 & 0 & 0 & 0.7 & 0.7 & 1 & 1 & 1.1 & 0.5 \\ 0.9 & 0.9 & 0.6 & 0.3 & 0 & 0 & 0 & 0 & 0 & 0.7 & 0.7 & 1 & 1 & 1.1 & 0.5 \\ 0 & 0 & 0 & 0 & 0 & 0 & 0 & 0 & 0 & 0 & 0 & 0 & 0 & 0 & 0 \\ 0 & 0 & 0 & 0 & 0 & 0 & 0 & 0 & 0 & 0 & 0 & 0 & 0 & 0 & 0 \\ 66 & 66 & 41 & 11 & 2.2 & 3 & 7.9 & 10 & 21 & 52 & 52 & 84 & 85 & 77 & 38 \\ 10 & 10 & 6.1 & 2.6 & 0 & 0 & 0 & 0 & 1.4 & 6.8 & 6.8 & 9.1 & 11 & 12 & 4.9 \\ 10 & 10 & 6.1 & 2.6 & 0 & 0 & 0 & 0 & 1.4 & 6.8 & 6.8 & 9.1 & 11 & 12 & 4.9 \\ 4.5 & 4.5 & 3.1 & 2.2 & 0 & 0 & 0 & 0 & 0.4 & 5.1 & 5.1 & 6.9 & 6.2 & 5.8 & 2.9 \\ 0 & 0 & 0 & 0 & 0 & 0 & 0 & 0 & 0 & 0 & 0 & 0 & 0 & 0 & 0 \\ 0.9 & 0.9 & 0.9 & 0.8 & 0 & 0 & 0 & 0 & 0.3 & 0.9 & 0.9 & 0.9 & 0.9 & 0.9 & 0.5 \\ 0.9 & 0.9 & 0.9 & 0.8 & 0 & 0 & 0 & 0 & 0.3 & 0.9 & 0.9 & 0.9 & 0.9 & 0.9 & 0.5 \\ 7.7 & 7.7 & 4 & 2.3 & 0 & 0 & 0 & 0 & 0.8 & 5.5 & 5.5 & 7.1 & 8.4 & 8.5 & 3.7 \\ 2 & 2 & 1.3 & 0.7 & 0 & 0 & 0 & 0 & 0.1 & 1.2 & 1.2 & 1.9 & 2.1 & 1.7 & 0.9 \\ 2 & 2 & 1.3 & 0.7 & 0 & 0 & 0 & 0 & 0.1 & 1.2 & 1.2 & 1.9 & 2.1 & 1.7 & 0.9 \\ 0 & 0 & 0 & 5 & 5.4 & 3.9 & 0 & 0.8 & 1.5 & 9.2 & 9.2 & 37 & 37 & 11 & 9.2 \\ 39 & 39 & 24 & 11 & 0 & 0 & 0 & 0 & 2.4 & 25 & 25 & 36 & 40 & 44 & 18 \\ 39 & 39 & 24 & 11 & 0 & 0 & 0 & 0 & 2.4 & 25 & 25 & 36 & 40 & 44 & 18 \\ 0.1 & 0.1 & 0.1 & 0.1 & 0.1 & 0.1 & 0.1 & 0.1 & 0.1 & 0.1 & 0.1 & 0.1 & 0.1 & 0.1 & 0.1 \\ 185 & 185 & 115 & \mathbf{5 1} & \mathbf{7 . 7} & \mathbf{7} & \mathbf{8} & \mathbf{1 1} & \mathbf{3 2} & \mathbf{1 4 1} & \mathbf{1 4 1} & \mathbf{2 3 2} & \mathbf{2 4 7} & \mathbf{2 2 1} & 105\end{array}$

-NUG: Small Thermal \& Misc.-

33 Grays Harbor Paper (GHPD)

34 Weyerhaeuser Pulp Mill (GHPD)

35 GPU - Total NUG: Small Thermal \& Misc.

\begin{tabular}{|c|c|c|c|c|c|c|c|c|c|c|c|c|c|c|}
\hline 3.8 & 3.8 & 3.8 & 3.8 & 3.8 & 3.8 & 3.8 & 3.8 & 3.8 & 3.8 & 3.8 & 3.8 & 3.8 & 3.8 & 3.8 \\
\hline 11 & 11 & 11 & 11 & 11 & 11 & 11 & 11 & 11 & 11 & 11 & 11 & 11 & 11 & 11 \\
\hline 15 & 15 & 15 & 15 & 15 & 15 & 15 & 15 & 15 & 15 & 15 & 15 & 15 & 15 & 15 \\
\hline
\end{tabular}

-NUG: Co-Generation-

36 Metro Westpoint (SCL)

37 Scott Paper (SHPD)

38 GPU - Total NUG: Co-Generation

\begin{tabular}{|c|c|c|c|c|c|c|c|c|c|c|c|c|c|}
\hline 1.2 & 1.2 & 1.2 & 1.2 & 1.2 & 1.2 & 1.2 & 1.2 & 1.2 & 1.2 & 1.2 & 1.2 & 1.2 & 1.2 \\
\hline 38 & 38 & 27 & 27 & 27 & 27 & 27 & 27 & 38 & 38 & 38 & 38 & 38 & 32 \\
\hline 39 & 39 & 28 & 28 & 28 & 28 & 28 & 28 & 39 & 39 & 39 & 39 & 39 & 34 \\
\hline
\end{tabular}

-NUG: Renewables-

39 Foote Creek 1 (EWEB)

40 Nine Canyon Wind Project (CHPD)

41 Nine Canyon Wind Project (COPD)

42 Nine Canyon Wind Project (DOPD)

43 Nine Canyon Wind Project (GCPD)

44 Nine Canyon Wind Project (GHPD)

45 Nine Canyon Wind Project (OKPD)

46 Stateline Wind Project (EWEB)

47 Stateline Wind Project (SCL)

48 GPU - Total NUG: Renewables

Non-Generating Public Entities

-NUG: Hydro-

49 Buffalo Hydro (FREC)

50 Burr \& Bouchard (TNEC)

51 City of Cove (OTEC)

52 Falls Creek (CCPD)

53 Lilliwaup Falls Generating Co. (MCPD1)

54 Thomas Burnside (TNEC)

$\begin{array}{rrrrrrrrrrrrrrr}1.4 & 1.4 & 1.6 & 2.4 & 3.5 & 4.3 & 4.5 & 4.1 & 3.1 & 2.5 & 2.5 & 2.0 & 1.7 & 1.4 & 2.7 \\ 1.5 & 1.5 & 1.4 & 2.2 & 3.8 & 3.8 & 4.2 & 3.4 & 2.3 & 2.0 & 2.0 & 2.0 & 1.9 & 1.4 & 2.5 \\ 0.4 & 0.4 & 0.4 & 0.5 & 0.9 & 0.9 & 1 & 0.9 & 0.6 & 0.5 & 0.5 & 0.5 & 0.5 & 0.3 & 0.6 \\ 1.8 & 1.8 & 1.7 & 2.6 & 4.6 & 4.7 & 5.1 & 4.2 & 2.8 & 2.4 & 2.4 & 2.4 & 2.3 & 1.7 & 3 \\ 2.2 & 2.2 & 2.1 & 3.2 & 5.7 & 5.7 & 6.3 & 5.2 & 3.4 & 3.0 & 3.0 & 3.0 & 2.8 & 2.1 & 3.7 \\ 1.5 & 1.5 & 1.4 & 2.2 & 3.8 & 3.8 & 4.2 & 3.4 & 2.3 & 2.0 & 2.0 & 2.0 & 1.9 & 1.4 & 2.5 \\ 3.0 & 3.0 & 2.8 & 4.3 & 7.5 & 7.6 & 8.4 & 6.9 & 4.6 & 3.9 & 3.9 & 3.9 & 3.7 & 2.7 & 4.9 \\ 8.4 & 8.4 & 6.6 & 8.6 & 9.3 & 8.2 & 8.8 & 8.3 & 8.3 & 9.2 & 9.2 & 9.3 & 9.6 & 8.5 & 8.6 \\ 49 & 49 & 39 & 50 & 54 & 48 & 52 & 48 & 48 & 54 & 54 & 54 & 56 & 50 & 50 \\ \mathbf{6 9} & \mathbf{6 9} & \mathbf{5 7} & \mathbf{7 6} & \mathbf{9 3} & \mathbf{8 7} & \mathbf{9 4} & \mathbf{8 5} & \mathbf{7 5} & \mathbf{7 9} & \mathbf{7 9} & \mathbf{7 9} & \mathbf{8 1} & \mathbf{6 9} & \mathbf{7 9}\end{array}$

-NUG: Small Thermal \& Misc.-

56 Methane Energy Agricultural D. (TKPD)

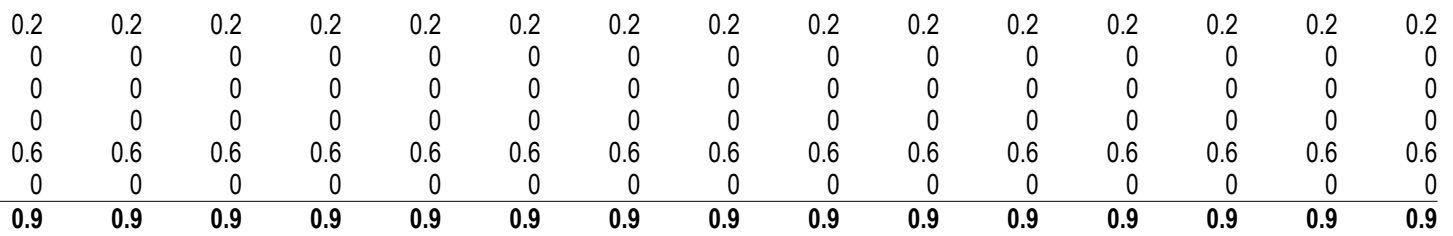

57 NGP - Total NUG: Small Thermal \& Misc.

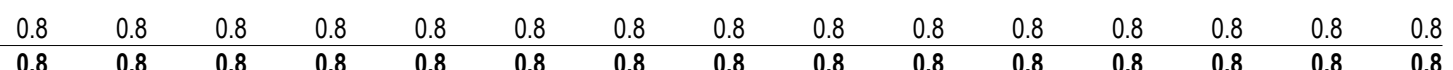


-NUG: Renewables-

58 Ashland Solar Project (ASHL)

59 Nine Canyon Wind Project (BCPD)

60 Nine Canyon Wind Project (ENW)

61 Nine Canyon Wind Project (LCPD)

62 Nine Canyon Wind Project (MCPD3)

\begin{tabular}{|c|c|c|c|c|c|c|c|c|c|c|c|c|c|c|}
\hline 0 & 0 & 0 & 0 & 0 & 0 & 0 & 0 & 0 & 0 & 0 & 0 & 0 & 0 & 0 \\
\hline 0.6 & 0.6 & 0.5 & 0.8 & 1.4 & 1.4 & 1.6 & 1.3 & 0.9 & 0.7 & 0.7 & 0.7 & 0.7 & 0.5 & 0.9 \\
\hline 0.4 & 0.4 & 0.4 & 0.5 & 0.9 & 1.0 & 1.1 & 0.9 & 0.6 & 0.5 & 0.5 & 0.5 & 0.5 & 0.3 & 0.6 \\
\hline 0.2 & 0.2 & 0.2 & 0.3 & 0.5 & 0.5 & 0.5 & 0.4 & 0.3 & 0.2 & 0.2 & 0.2 & 0.2 & 0.2 & 0.3 \\
\hline 0.4 & 0.4 & 0.4 & 0.5 & 0.9 & 1.0 & 1 & 0.9 & 0.6 & 0.5 & 0.5 & 0.5 & 0.5 & 0.3 & 0.6 \\
\hline 0 & 0 & 0 & 0 & 0 & 0 & 0 & 0 & 0 & 0 & 0 & 0 & 0 & 0 & 0 \\
\hline 1.5 & 1.5 & 1.4 & 2.2 & 3.8 & 3.8 & 4.2 & 3.5 & 2.3 & 2.0 & 2.0 & 2.0 & 1.9 & 1.4 & 2.5 \\
\hline
\end{tabular}

64 NGP - Total NUG: Renewables

$1.5-1.4$

-NUG: Hydro-

65 Barber Dam (IPC)

66 Barney Creek (NWE)

67 Beaverton (PGE)

68 Bell Mountain (UPL)

69 Bingham Engineering (NWE)

70 Birch Creek (IPC)

71 Birch Creek (UPL)

72 Black Canyon \#3 (IPC)

73 Black Creek (AVWP)

74 Blind Canyon Hydro (IPC)

75 Box Canyon (IPC)

76 Boyd James (PPL)

77 Briggs Creek (IPC)

78 Broadwater Dam (NWE)

79 Bypass (IPC)

80 Canyon Springs (IPC)

81 Carlton (PGE)

82 Cascade Creek (NWE)

83 CDM Hydro - Bonneville Pacific (PPL)

84 Cedar Draw (IPC)

85 Central OR Irrigation District (PPL)

86 Clear Springs Trout (IPC)

87 Corbett (PGE)

88 Cornelius (PGE)

89 Crystal Springs (IPC)

90 Curry Cattle Co. (IPC)

91 Dairy Creek (PGE)

92 Deep Creek (AVWP)

93 Derr Creek (AVWP)

94 Dietrich Drop (IPC)

95 Donald Jenni (NWE)

96 Eagle Creek (PGE)

97 Eagle Point Irrigation Dist. (PPL)

98 Elk Creek (IPC)

99 Estacada (PGE)

100 Falls Creek (PPL)

101 Falls River (IPC)

102 Farmers Irrig. (PPL)

103 Faulkner Ranch (IPC)

104 Fisheries Development Co (IPC)

105 Galesville Dam (PPL)

106 Geobon 2 (IPC)

107 Georgetown Power (UPL)

108 Hailey Cspp (IPC)

109 Hazelton A (IPC)

110 Hazelton B (IPC)

111 Horseshoe Bend (IPC)

112 Hutchinson Creek (PSE)

113 Ingram Warm Springs (UPL)

$114 \mathrm{Jim}$ Ford Creek (AVWP)

115 Jim Knight (IPC)

116 John Day Creek (AVWP)

117 Joseph Hydro (PPL)

118 Kasel \& Witherspoon (IPC)

119 Koma Kulshan (PSE)

120 Koyle (IPC)

$121 \mathrm{~L}$. Fery (PPL)

122 Lacomb Irrig. (PPL)

123 Lake Oswego (PGE)

124 Lateral \#10 (IPC)

125 Lee Tavenner (NWE)

126 Lemoyne (IPC)

127 Little Wood River Res. (IPC)

128 Littlewood/Arkoosh (IPC)

129 Lowline \#2 (IPC)

\begin{tabular}{|c|c|c|c|c|c|c|}
\hline 2.4 & 2.4 & 1.9 & 0.8 & 0.3 & 1.0 & 2.2 \\
\hline 0 & 0 & 0 & 0 & 0 & 0 & 0 \\
\hline 0 & 0 & 0 & 0 & 0.1 & 0 & 0 \\
\hline 0.1 & 0.1 & 0.1 & 0.2 & 0.1 & 0.1 & 0.1 \\
\hline 0.1 & 0.1 & 0.1 & 0.1 & 0.1 & 0.1 & 0.1 \\
\hline 0 & 0 & 0.1 & 0.1 & 0.1 & 0.1 & 0 \\
\hline 1.3 & 1.3 & 1.1 & 1.0 & 1.1 & 1.1 & 0.3 \\
\hline 0.1 & 0.1 & 0.1 & 0.1 & 0.1 & 0 & 0 \\
\hline 3.7 & 3.4 & 3.1 & 3.1 & 2.8 & 3.1 & 3.1 \\
\hline 0.7 & 0.7 & 1.1 & 0.9 & 0.2 & 0 & 0 \\
\hline 0.4 & 0.4 & 0.4 & 0.4 & 0.4 & 0.3 & 0.3 \\
\hline 0 & 0 & 0 & 0 & 0 & 0.4 & 0.3 \\
\hline 0.6 & 0.6 & 0.6 & 0.6 & 0.6 & 0.6 & 0.6 \\
\hline 1.1 & 1.1 & 1.5 & 1.9 & 2 & 1.6 & 1.6 \\
\hline 8.3 & 8.3 & 6.5 & 2.8 & 1.0 & 0 & 0 \\
\hline 0.1 & 0.1 & 0.1 & 0.1 & 0.1 & 0.1 & 0.1 \\
\hline 0 & 0 & 0 & 0 & 0 & 0 & 0.1 \\
\hline 0 & 0 & 0 & 0 & 0 & 0 & 0 \\
\hline 2.9 & 2.9 & 3.1 & 2.8 & 2.9 & 2.4 & 1.8 \\
\hline 0.3 & 0.3 & 0.9 & 0.9 & 0.9 & 1.2 & 1.2 \\
\hline 3.1 & 3.1 & 3.7 & 3.5 & 0 & 0.7 & 0 \\
\hline 0.4 & 0.4 & 0.4 & 0.5 & 0.6 & 0.6 & 0.4 \\
\hline 0 & 0 & 0 & 0 & 0 & 0 & 0 \\
\hline 0 & 0 & 0 & 0 & 0 & 0 & 0 \\
\hline 1.3 & 1.3 & 1.6 & 1.5 & 1.6 & 0.5 & 0.5 \\
\hline 0.2 & 0.2 & 0.2 & 0.1 & 0.1 & 0 & 0 \\
\hline 0 & 0 & 0 & 0 & 0 & 0 & 0 \\
\hline 0 & 0 & 0 & 0 & 0 & 0 & 0 \\
\hline 0.1 & 0.1 & 0.1 & 0.1 & 0.1 & 0.1 & 0.1 \\
\hline 4.7 & 4.7 & 3.6 & 3.1 & 1.4 & 0 & 0 \\
\hline 0.1 & 0.1 & 0.1 & 0.1 & 0.1 & 0.1 & 0.1 \\
\hline 0 & 0 & 0 & 0 & 0 & 0 & 0 \\
\hline 0 & 0 & 0 & 0 & 0.4 & 0.7 & 0.7 \\
\hline 1.2 & 1.2 & 0 & 0 & 0 & 0.1 & 0.1 \\
\hline 0.1 & 0.1 & 0.1 & 0.1 & 0.1 & 0 & 0.1 \\
\hline 0 & 0 & 0 & 0 & 1.9 & 3.1 & 0.9 \\
\hline 6.5 & 6.5 & 6.3 & 5.7 & 5.6 & 5.0 & 4.2 \\
\hline 0.6 & 0.6 & 0.7 & 1.2 & 3.2 & 3.2 & 2.1 \\
\hline 0.8 & 0.8 & 0.7 & 0.7 & 0.7 & 0.3 & 0.3 \\
\hline 0.2 & 0.2 & 0.3 & 0.2 & 0.3 & 0.3 & 0.3 \\
\hline 0.6 & 0.6 & 0.4 & 0.2 & 0.2 & 0.3 & 0 \\
\hline 1.0 & 1.0 & 0.3 & 0.3 & 0.3 & 0.2 & 0.2 \\
\hline 0 & 0 & 0.2 & 0.3 & 0.3 & 0.3 & 0.3 \\
\hline 0.1 & 0.1 & 0.1 & 0.1 & 0.1 & 0.1 & 0.1 \\
\hline 7 & 7 & 5.4 & 2.5 & 0.9 & 0 & 0 \\
\hline 6.6 & 6.6 & 5.2 & 2.4 & 0.7 & 0 & 0 \\
\hline 8.8 & 8.8 & 7.5 & 3.9 & 2.8 & 3.5 & 3.9 \\
\hline 0.7 & 0.7 & 0.7 & 0.7 & 0.7 & 0.7 & 0.7 \\
\hline 0.2 & 0.2 & 0.3 & 0.3 & 0.5 & 0.5 & 0.4 \\
\hline 0 & 0 & 0 & 0 & 0.3 & 0.5 & 0.9 \\
\hline 0.4 & 0.4 & 0.3 & 0.2 & 0.3 & 0.1 & 0.1 \\
\hline 0.2 & 0.2 & 0.2 & 0.2 & 0.2 & 0.2 & 0.3 \\
\hline 1.5 & 1.5 & 0.9 & 0.4 & 0.1 & 0.1 & 0.1 \\
\hline 1.2 & 1.2 & 1.2 & 0.8 & 0.5 & 0.3 & 0.2 \\
\hline 4.6 & 4.6 & 4.3 & 6.4 & 8 & 6.1 & 3.9 \\
\hline 1.1 & 1.1 & 1.1 & 1.0 & 1 & 0.2 & 0.1 \\
\hline 0 & 0 & 0 & 0 & 0 & 0 & 0 \\
\hline 0 & 0 & 0 & 0 & 0.2 & 1.0 & 0.2 \\
\hline 0.4 & 0.4 & 0.4 & 0.4 & 0.4 & 0.4 & 0.4 \\
\hline 1.5 & 1.5 & 1.6 & 2 & 2.1 & 1.5 & 1.5 \\
\hline 0.1 & 0.1 & 0.1 & 0.1 & 0.1 & 0.1 & 0.1 \\
\hline 0 & 0 & 0 & 0 & 0 & 0 & 0 \\
\hline 1.9 & 1.9 & 0.2 & 0.2 & 0.2 & 0 & 0 \\
\hline 0.7 & 0.7 & 0.4 & 0.4 & 0.4 & 0.2 & 0.2 \\
\hline 2.7 & 2.7 & 2.5 & 1.8 & 1.4 & 0 & 0 \\
\hline
\end{tabular}

$2.6 \quad 3.3$

$\begin{array}{rr}0 \\ 0 \\ 0.1 & 0.1\end{array}$

$\begin{array}{rr}0.1 & 0.1 \\ 0 & \end{array}$

$\begin{array}{rr}1.2 & 1.7 \\ 0 & 0.1\end{array}$

$\begin{array}{rr}2.8 & 1.9 \\ 0 & \end{array}$

$\begin{array}{ll}0.3 & 0.3\end{array}$

$\begin{array}{ll}0.6 & 0.7\end{array}$

$0.7 \quad 0.6$

$\begin{array}{ll}1.7 & 1.8\end{array}$

$\begin{array}{rr}0 & 0.1 \\ 0.1 & 0.1 \\ 0.1 & 0.1\end{array}$

$\begin{array}{ll}0 & \\ 2 & 2\end{array}$

$\begin{array}{ll}1.3 & 0.4 \\ 0.4 & 0.9\end{array}$

$0.5 \quad 0.4$

$\begin{array}{rr}0 & \\ 0.6 & 0.8\end{array}$

$\begin{array}{ll}0 & 0.1 \\ 0 & \\ 0 & \end{array}$

$0.1 \quad 0.1$

$\begin{array}{rr}0.1 & 0.1 \\ 0 & \end{array}$

$\begin{array}{ll}0.9 & 0.6 \\ 0.1 & \end{array}$

$0.1 \quad 0.1$

2.7

3.8
4.6

0.3

0.3

0.2

0.3

0.3
0.1

0.1
0

0
4.6

4.6
0.7

0.4

0.9

0.1
0.2

0.2
0

0.2

3.8

$0.1 \quad 0.3$

0
0.9

0.9
0.4

$0.4 \quad 0.4$

1.8

0.1

0.1
0

1.6
0.4 $\begin{array}{llllll}3.2 & 3.2 & 3.1 & 3.2 & 3.3 & 2.3\end{array}$

$\begin{array}{rrr}3.2 & 3.2 & 3.1 \\ 0 & 0 & 0 \\ 0 & 0 & 0 \\ 0.1 & 0.1 & 0.1 \\ 0.1 & 0.1 & 0.1\end{array}$

0
0
0.2
0.1

$\begin{array}{rrr}0.1 & 0.1 & 0.1 \\ 0 & 0 & 0\end{array}$

$\begin{array}{llllll}1.6 & 1.6 & 1.6 & 1.3 & 1.2 & 1.2\end{array}$

$\begin{array}{llllll}0.1 & 0.1 & 0.1 & 0.1 & 0.1 & 0.1\end{array}$

$\begin{array}{llllll}0.6 & 0.6 & 1.6 & 1.0 & 0.7 & 0.6\end{array}$

$\begin{array}{llllll}0.3 & 0.3 & 0.3 & 0.4 & 0.4 & 0.4\end{array}$

$\begin{array}{rrrrrr}0.8 & 0.8 & 0.5 & 0 & 0 & 0.3\end{array}$

$\begin{array}{rrrrrr}0.6 & 0.6 & 0.6 & 0.6 & 0.6 & 0.6 \\ 2.7 & 2.6 & 3.2 & 3.4 & 2.0 & 2\end{array}$

$\begin{array}{llllll}2.9 & 2.9 & 7 & 8.2 & 8.7 & 3.8 \\ 0.1 & 0.1 & 0.1 & 0.1 & 0.1 & 0.1\end{array}$

$0.1 \quad 0.1$

0.1

0.1

$\begin{array}{ll}8.7 & 3.8 \\ 0.1 & 0.1\end{array}$

$\begin{array}{rrrrrr}0 & 0 & 0 & 0 & 0 & 0 \\ 2.7 & 27 & 4.4 & 5.8 & 4.1 & 3.1\end{array}$

$\begin{array}{llllll}0.4 & 0.4 & 0.4 & 0.3 & 0.3 & 0.7\end{array}$

$\begin{array}{llllll}0.8 & 0.8 & 4.5 & 3.8 & 4.1 & 2.1\end{array}$

$\begin{array}{llllll}0.3 & 0.3 & 0.4 & 0.4 & 0.4 & 0.4 \\ 1 & 0 & 0 & 0\end{array}$

$\begin{array}{rrrrrr}0 & 0 & 0 & 0 & 0 & 0 \\ 0 & 0 & 0 & 0 & 0 & 0 \\ 0.9 & 0.9 & 0.8 & 1.4 & 1.3 & 1.1\end{array}$

$\begin{array}{llllll}0.1 & 0.1 & 0.2 & 0.2 & 0.2 & 0.1\end{array}$

$\begin{array}{llllll}0 & 0 & 0 & 0 & 0 & 0\end{array}$

$\begin{array}{llllll}0.2 & 0.2 & 0.3 & 0.2 & 0.2 & 0.1\end{array}$

$\begin{array}{llllll}3.1 & 3.1 & 4.7 & 4.7 & 4.5 & 2.5\end{array}$

$\begin{array}{llllll}0.1 & 0.1 & 0.1 & 0.1 & 0.1 & 0.1\end{array}$

$\begin{array}{rrrrrr}0.7 & 0.7 & 0.5 & 0 & 0 & 0.4\end{array}$

$\begin{array}{lllllll}0.1 & 0.1 & 0.1 & 0.1 & 0.1 & 0.1 & 0.1\end{array}$

$\begin{array}{lllllll}0.1 & 4.4 & 4.4 & 4.1 & 3.8 & 0 & 2.0\end{array}$

$\begin{array}{lll}3.5 & 3.5 & 4.7\end{array}$

$\begin{array}{lll}0.3 & 0.6 & 0.6 \\ 0.3 & 0.3 & 0.3\end{array}$

$\begin{array}{lll}0.3 & 0.4 & 0.4\end{array}$

$0.3-0.6-0.6$

$\begin{array}{lll}0.1 & 0.1 & 0.1\end{array}$

$\begin{array}{lll}0.1 & 2.6 & 2.6\end{array}$

$\begin{array}{lll}0.1 & 2.2 & 2.2\end{array}$

$\begin{array}{lll}0.9 & 8.8 & 8.8 \\ 0.7 & 0.7 & 0.7\end{array}$

$\begin{array}{lll}0.7 & 0.7 & 0.7\end{array}$

$\begin{array}{lll}0.4 & 0.4 & 0.4 \\ 1.1 & 1.1 & 1.1\end{array}$

$\begin{array}{lll}0.2 & 0.3 & 0.3\end{array}$

$\begin{array}{lll}0.2 & 0.2 & 0.2\end{array}$

$\begin{array}{lll}0.4 & 0.5 & 0.5\end{array}$

$\begin{array}{lll}4.4 & 6.9 & 6.9\end{array}$

$0.8 \quad 0.8$

$0 \quad 0$

$\begin{array}{rrr}1 & 1 & 0.9 \\ 0.4 & 0.4 & 0.4\end{array}$

$\begin{array}{lll}1.9 & 1.9 & 1.8\end{array}$

$\begin{array}{lll}0.1 & 0.1 & 0.1\end{array}$

$\begin{array}{rr}1.7 & 1.7\end{array}$

$0.5 \quad 0.5$

1.6

2.7

2.7

\section{7}

0.8

0.2

0.5

1.0

0
0.1
7.4

7.4

7.0

9.2

0.7

0.3

0.1

0.4

0.2
27

1.2

1.1
10

0.8 

2003 White Book

Aug1 Aug16 Sep Oct Nov Dec

Jan Feb Mar Apr1 Apr16

\begin{abstract}
May
\end{abstract}

$$
\text { Jun }
$$
Jul Avg

130 Lowline Canal (IPC)

131 Magic Reservoir (IPC)

132 Malad River (IPC)

133 Marcos Ranches (IPC)

134 Marsh Valley (UPL)

135 Meyers Falls (AVWP)

136 Middlefork Irrig. (PPL)

137 Mile 28 (IPC)

138 Minikahda (PGE)

139 Mink Creek (UPL)

140 Mitchell Butte (IPC)

141 Mountain Energy (PPL)

$142 \mathrm{Mt}$. Tabor (PGE)

143 Mud Creek/S\&S (IPC)

144 Mud Creek/White (IPC)

145 Nichols Gap (PPL)

146 Nicholson Sunnybar (UPL)

147 North Fork Sprague (PPL)

148 O.J. Power (UPL)

149 Odell Creek (PPL)

150 Opal Springs (PPL)

151 Owyhee Dam (IPC)

152 Pancheri (UPL)

153 Pelton Rereg. Dam (PPL)

154 Phillips Ranch (AVWP)

155 Phillipsburg (NWE)

156 Pigeon Cove (IPC)

157 Pine Creek (NWE)

158 Port Townsend Paper Hydro (PSE)

159 Portland (PGE)

160 Portland Hydro Project (PGE)

161 Preston City (UPL)

162 Pristine Springs (IPC)

163 Pristine Springs \#3 (IPC)

164 Reynolds Irrigation (IPC)

165 Rim View (IPC)

166 Rock Creek \#1 (IPC)

167 Rock Creek \#2 (IPC)

168 Sagebrush (IPC)

169 Salem (PGE)

170 Schaffner (IPC)

171 Sheep Creek (AVWP)

172 Shingle Creek (IPC)

173 Shoshone (IPC)

174 Shoshone II (IPC)

175 Snake River Pottery (IPC)

176 Snedigar Ranch (IPC)

177 Solar Research (PPL)

178 South Dry Creek (NWE)

179 Spokane Upriver (AVWP)

180 Stauffer Dry Creek (UPL)

181 Strawberry Creek (NWE)

182 Sunshine Power \#2 (IPC)

183 Sygitowicz Creek Small Hydro (PSE)

184 TGS/Briggs (UPL)

185 Trout Co. (IPC)

186 Tunnel \#1 (IPC)

187 Twin Falls (PSE)

188 Walla Walla (PPL)

189 Water Street / Santiam (PPL)

190 Weeks Falls (PSE)

191 White James (PPL

192 White Water Ranch (IPC)

193 Wilson Lake Hydro (IPC)

194 Wisconsin Creek (NWE)

195 Yakima-Tieton (PPL)

196 IOU - Total NUG: Hydro

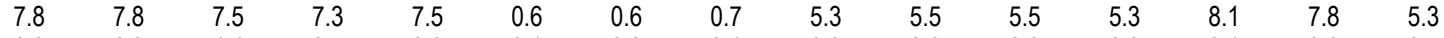

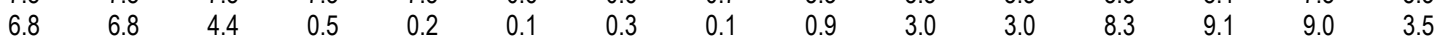

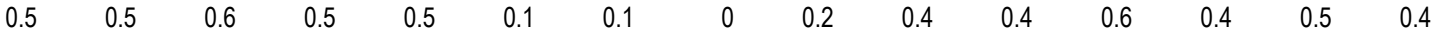

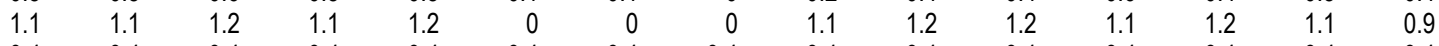

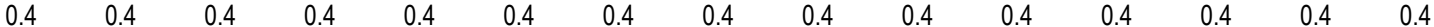

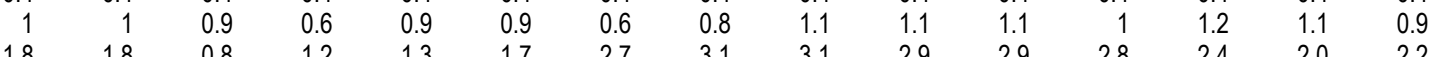

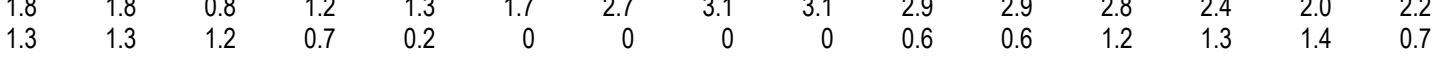

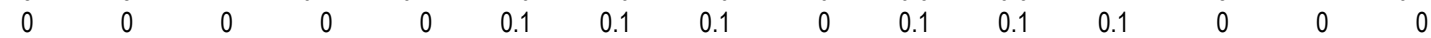

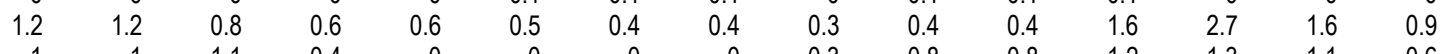

\begin{tabular}{|c|c|}
\hline & 1 \\
\hline
\end{tabular}

\begin{tabular}{|c|c|c|c|c|c|c|c|c|c|}
\hline \multirow[t]{2}{*}{0} & 0 & \multirow[t]{2}{*}{$\begin{array}{r}0 \\
01\end{array}$} & \multirow{2}{*}{$\begin{array}{r}0 \\
0.1\end{array}$} & \multirow[t]{2}{*}{$\begin{array}{r}0 \\
0\end{array}$} & \multirow[t]{2}{*}{$\begin{array}{r}0 \\
01\end{array}$} & \multirow[t]{2}{*}{$\begin{array}{r}0 \\
01\end{array}$} & \multirow[t]{2}{*}{0} & \multirow[t]{2}{*}{0} & \multirow[b]{2}{*}{1} \\
\hline & 0.1 & & & & & & & & \\
\hline
\end{tabular}

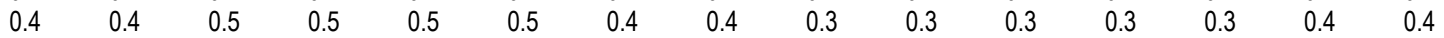

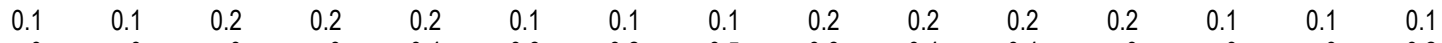

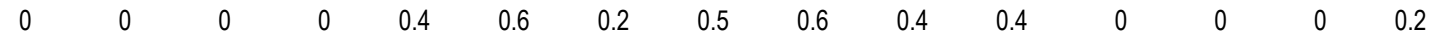

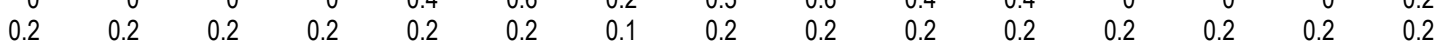

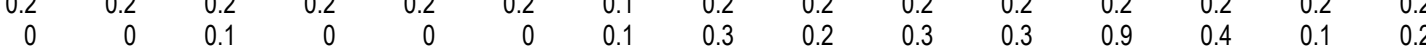

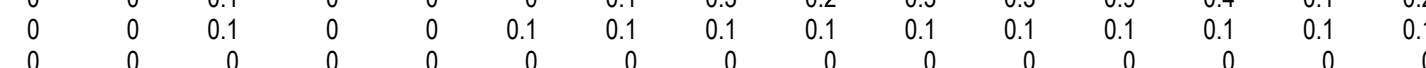

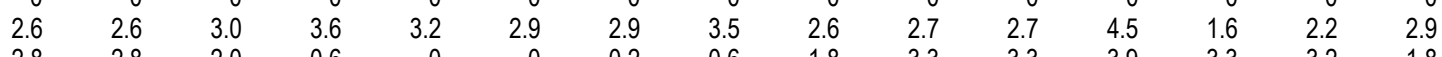

$\begin{array}{rrrrrrrr}0 & 0 & 0 & 0 & 0 & 0.1 & 0.1 & 0.1 \\ 2.0 & 2.0 & 2.1 & 1.3 & 0.8 & 0.4 & 0.3 \\ 0.1 & 0.1 & 0.1 & 0.1 & 0.1 & 0.1 & 0.1 & 0.4 \\ 0.3 & 0.3 & 0.3 & 0.3 & 0.3 & 0.3 & 0.3 & 0.3 \\ 0 & 0 & 0 & 0 & 0 & 0 & 0 & 0\end{array}$

$\begin{array}{rrrrrrr}0.3 & 0.3 & 0.3 & 0.3 & 0.3 & 0.3 & 0.3 \\ 0 & 0 & 0 & 0 & 0 & 0 & 0\end{array}$

$\begin{array}{rrrrrrrrr}2.3 & 2.3 & 2.9 & 6.7 & 16 & 20 & 18 & 17 & 16 \\ 0.3 & 0.3 & 0.4 & 0.4 & 0.4 & 0.4 & 0.4 & 0.4 & 0.3\end{array}$

$\begin{array}{llllllll}0.1 & 0.1 & 0.2 & 0.2 & 0.1 & 0.1 & 0.1 & 0.1\end{array}$

$\begin{array}{llll}0.2 & 0.2 & 0.2 & 0.3\end{array}$

$\begin{array}{llll}0.2 & 0.2 & 0.2 & 0.2\end{array}$

$\begin{array}{lllllll}0.2 & 0.2 & 0.2 & 0.3 & 0.2 & 0.2 & 0.2 \\ 1.1 & 1.1 & 1.6 & 1.7 & 1.2 & 1.3 & 1.1\end{array}$

$\begin{array}{llllllll}1.8 & 1.8 & 1.8 & 1.9 & 1.9 & 1.9 & 0.6 & 0.4 \\ 0.4 & 0.4 & 0.3 & 0.3 & 0.3 & 0.1 & 0.1 & 0.1\end{array}$

$\begin{array}{rrrr}0.4 & 0.4 & 0.3 & 0.3 \\ 0 & 0 & 0 & 0\end{array}$

$\begin{array}{rrrr}0 & 0 & 0 & 0 \\ 0.4 & 0.4 & 0.3 & 0.2\end{array}$

$\begin{array}{llllllll}0.4 & 0.4 & 0.3 & 0.3 & 0.3 & 0.1 & 0.1 & 0.2 \\ 0.1 & 0.1 & 0.2 & 0.2 & 0.4 & 0.5 & 0.5 & 0.5\end{array}$

$\begin{array}{llllllll}0.1 & 0.1 & 0.2 & 0.2 & 0.1 & 0.1 & 0.1 & 0.1\end{array}$

$\begin{array}{llll}0.4 & 0.4 & 0.5 & 0.4 \\ 0.6 & 0.6 & 0.6 & 0\end{array}$

$\begin{array}{rrrrrrr}0 & 0 & 0.6 & 0 & 0 & 0 & 0 \\ 0.2 & 0.2 & 0 & 0 & 0 & 0.1 & 0.1 \\ 0.1 & 0.1 & 0.1 & 0.2 & 0.2 & 0.2 & 0.2 \\ 0.5 & 0.5 & 0.5 & 0.5 & 0 & 0 & 0.1\end{array}$

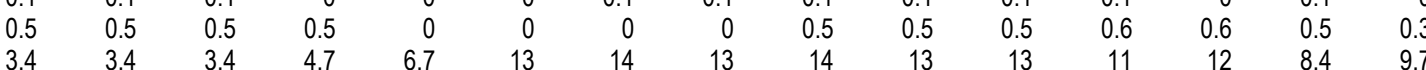

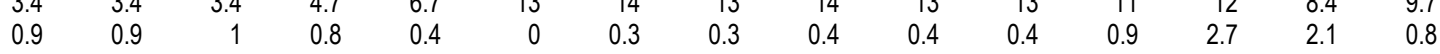

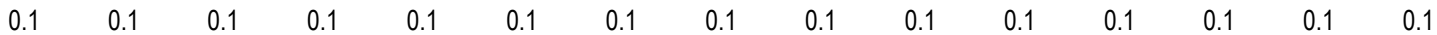

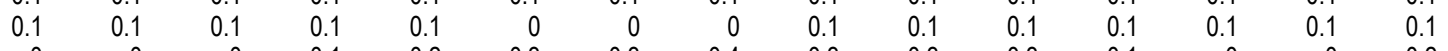

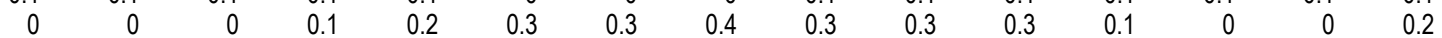

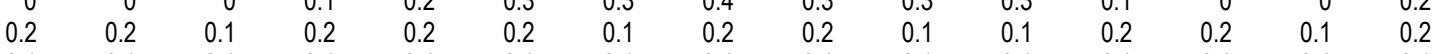

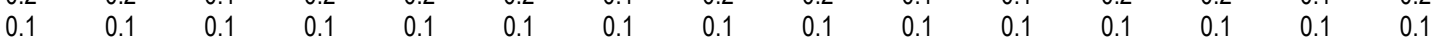

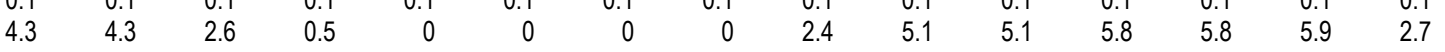

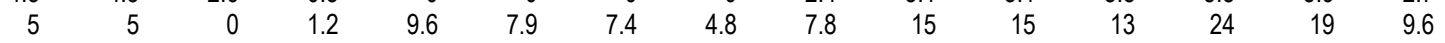

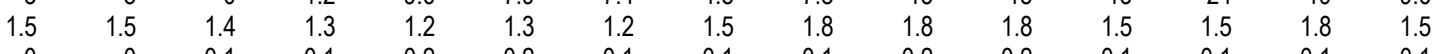

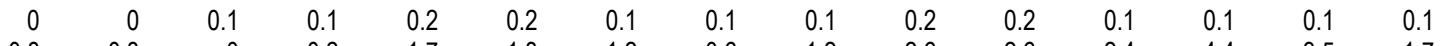

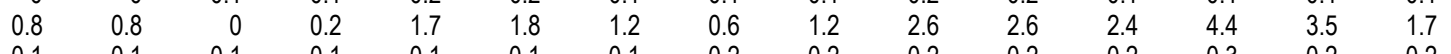

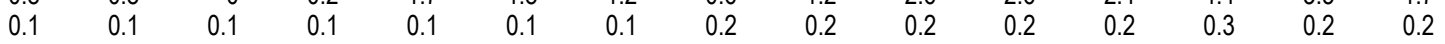

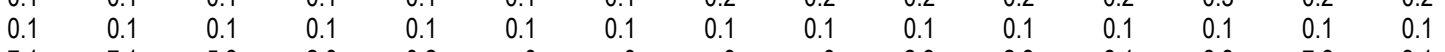

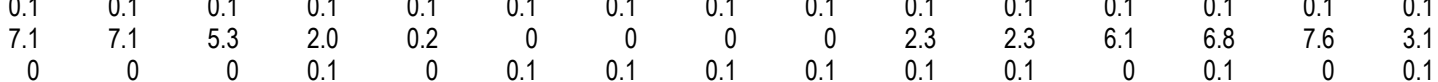

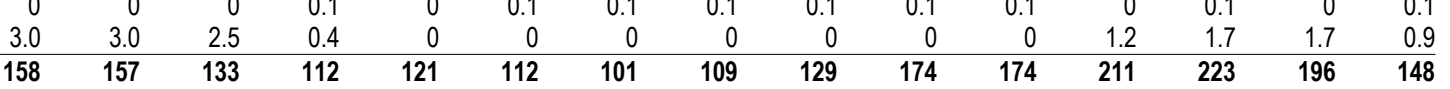

-NUG: Small Thermal \& Misc.-

197 Marion Solid Waste (PGE)

198 Minnesota Methane (AVWP)

199 Montana One (NWE)

200 Pocatello Waste (IPC)

201 Spokane MSW (PSE)

202 West Boise Waste (IPC)

\begin{tabular}{|c|c|c|c|c|c|c|c|c|c|c|c|c|}
\hline 9.3 & 9.3 & 9.3 & 9.3 & 9.3 & 9.3 & 9.3 & 9.3 & 9.3 & 9.3 & 9.3 & 9.3 & 9.3 \\
\hline 0.8 & 0.8 & 0.8 & 0.8 & 0.8 & 0.8 & 0.8 & 0.8 & 0.8 & 0.8 & 0.8 & 0.8 & 0.8 \\
\hline 9.7 & 8.9 & 9.1 & 9.2 & 10 & 10 & 10 & 9.1 & 9.1 & 8.9 & 10 & 11 & 8.6 \\
\hline 0.1 & 0.2 & 0.1 & 0.2 & 0.1 & 0.1 & 0.2 & 0.1 & 0.2 & 0.2 & 0.1 & 0.2 & 0.1 \\
\hline 15 & 15 & 15 & 15 & 15 & 15 & 15 & 15 & 15 & 15 & 15 & 15 & 15 \\
\hline 0.1 & 0.1 & 0.1 & 0.1 & 0.1 & 0.1 & 0.1 & 0.1 & 0.1 & 0.1 & 0.1 & 0.1 & 0.1 \\
\hline 35 & 35 & 35 & 35 & 36 & 36 & 36 & 35 & 35 & 35 & 36 & 37 & 34 \\
\hline
\end{tabular}


Table A-24: Regional Non Utility Generating Resources By Project

PNW Loads and Resources Study

2012 - 2013 Operating Year 2003 White Book

Aug1 Aug16 Sep

Oct Nov

Dec Jan Feb Mar Apr1 Apr16

May Jun Jul Avg

-NUG: Combustion Turbines -

204 March Point \#1 Cogeneration (PSE)

205 March Point \#2 Cogeneration (PSE)

206 Rathdrum- Boekel Rd. \#1 (AVWP)

207 Rathdrum -Boekel Rd. \#2 (AVWP)

208 Tenaska Gas Cogen (PSE)

209 IOU - Total NUG: Combustion Turbines

\begin{tabular}{|c|c|c|c|c|c|c|c|c|c|c|c|c|c|c|}
\hline 79 & 79 & 79 & 79 & 79 & 79 & 79 & 79 & 79 & 79 & 79 & 55 & 79 & 79 & 77 \\
\hline 59 & 59 & 59 & 59 & 59 & 59 & 59 & 59 & 59 & 59 & 59 & 45 & 59 & 59 & 58 \\
\hline 65 & 65 & 65 & 65 & 65 & 65 & 65 & 65 & 65 & 65 & 65 & 32 & 65 & 65 & 62 \\
\hline 65 & 65 & 65 & 65 & 65 & 65 & 65 & 65 & 65 & 65 & 65 & 32 & 65 & 65 & 62 \\
\hline 240 & 240 & 240 & 240 & 240 & 240 & 240 & 240 & 240 & 240 & 240 & 180 & 240 & 240 & 235 \\
\hline 508 & 508 & 508 & 508 & 508 & 508 & 508 & 508 & 508 & 508 & 508 & 344 & 508 & 508 & 494 \\
\hline
\end{tabular}

-NUG: Co-Generation-

210 Billings Generation (NWE)

211 Biomass One (PPL)

212 Boise Cascade Medford (PPL)

213 Champion (PPL)

214 DR Johnson - Co-Gen II (PPL)

215 Magic Valley (IPC)

216 Magic West (IPC)

217 PERC Pierce, Wa (PSE)

218 Simplot Pocatello (IPC)

219 Sumas Energy (PSE)

220 Tamarack (IPC)

221 TASCO Nampa ID (IPC)

222 TASCO Twin Falls ID (IPC)

223 Vaagen Bros. (IPC)

224 Warm Springs (PPL)

225 IOU - Total NUG: Co-Generation

-NUG: Renewables-

226 Foote Creek 1 (PPL)

227 Healow \#2 (NWE)

228 Josef Staufer (NWE)

229 Lewandowski Farms (IPC)

230 Livingston (NWE)

231 Puyallup Energy Recovery (PSE)

232 Stateline Wind Project (AVWP)

233 Windmill (PGE)

234 IOU - Total NUG: Renewables

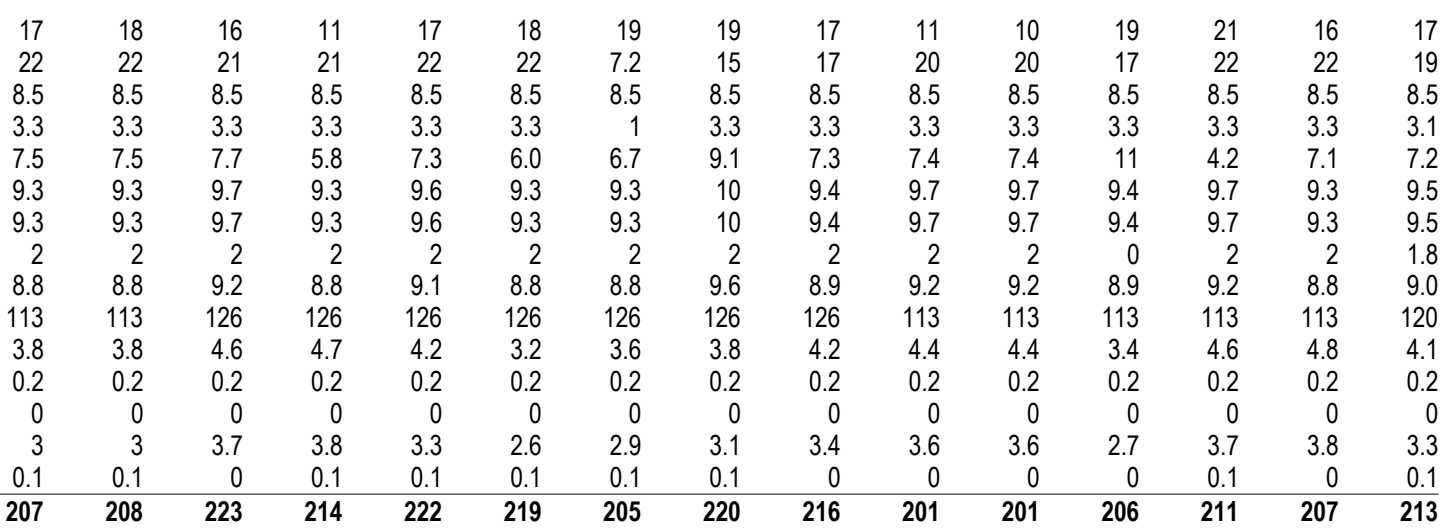

Other Entities

-NUG: Co-Generation-

235 SP Newsprint cogen (SPN)

236 Other - Total NUG: Co-Generation

$\begin{array}{lllllllllllllll}25 & 25 & 25 & 25 & 25 & 25 & 25 & 25 & 25 & 25 & 25 & 25 & 25 & 25 & 25 \\ \mathbf{2 5} & \mathbf{2 5} & \mathbf{2 5} & \mathbf{2 5} & \mathbf{2 5} & \mathbf{2 5} & \mathbf{2 5} & \mathbf{2 5} & \mathbf{2 5} & \mathbf{2 5} & \mathbf{2 5} & \mathbf{2 5} & \mathbf{2 5} & \mathbf{2 5} & \mathbf{2 5}\end{array}$

-NUG: Renewables-

237 Stateline Wind Project (PPME)

238 Vansycle Wind (FPLEV)

239 Other - Total NUG: Renewables

\begin{tabular}{|c|c|c|c|c|c|c|c|c|c|c|c|c|c|c|}
\hline 4.2 & 4.2 & 4.9 & 7.2 & 11 & 13 & 14 & 12 & 9.2 & 7.4 & 7.4 & 5.9 & 5.2 & 4.2 & 8.1 \\
\hline 0 & 0 & 0 & 0 & 0 & 0 & 0 & 0 & 0 & 0 & 0 & 0 & 0 & 0 & 0 \\
\hline 0 & 0 & 0 & 0 & 0 & 0 & 0 & 0 & 0 & 0 & 0 & 0 & 0 & 0 & 0 \\
\hline 0 & 0 & 0 & 0 & 0 & 0 & 0 & 0 & 0 & 0 & 0 & 0 & 0 & 0 & 0 \\
\hline 0 & 0 & 0 & 0 & 0 & 0 & 0 & 0 & 0 & 0 & 0 & 0 & 0 & 0 & 0 \\
\hline 2 & 2 & 2 & 2 & 2 & 2 & 2 & 2 & 2 & 2 & 2 & 2 & 2 & 2 & 2 \\
\hline 0.3 & 0.3 & 0.2 & 0.3 & 0.3 & 0.3 & 0.3 & 0.3 & 0.3 & 0.3 & 0.3 & 0.3 & 0.3 & 0.3 & 0.3 \\
\hline 0 & 0 & 0 & 0 & 0 & 0 & 0 & 0 & 0 & 0 & 0 & 0 & 0 & 0 & 0 \\
\hline 6.5 & 6.5 & 7.1 & 9.5 & 13 & 15 & 16 & 15 & 12 & 9.8 & 9.8 & 8.2 & 7.6 & 6.5 & 10 \\
\hline
\end{tabular}

-Total Non-Utility Generating Resources-

240 Federal Entities

241 Generating Public Entities

242 Non Generating Public Entities

243 Investor-Owned Entities

244 Other Entities

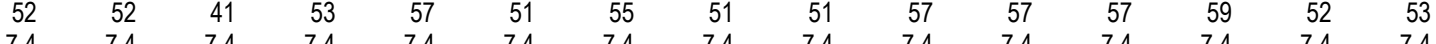

245 Total Non-Utility Generation

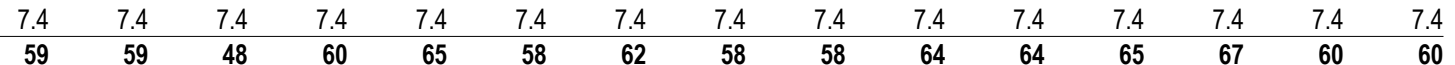

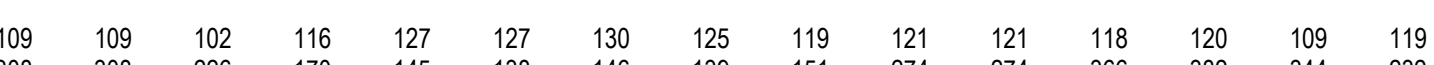

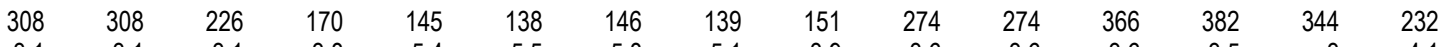

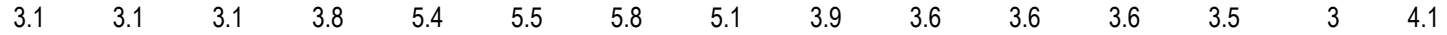

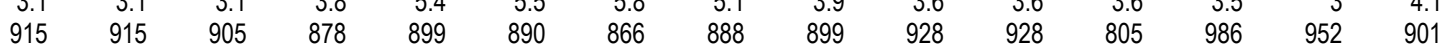

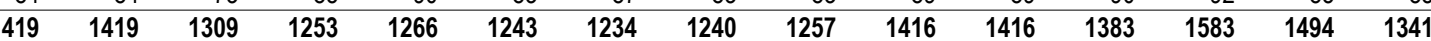


Oct Nov

Dec

\begin{abstract}
Jan Feb
\end{abstract}

\begin{abstract}
Mar Apr1 Apr16
\end{abstract}

\begin{abstract}
May
\end{abstract}
Jun

Jul Avg

\title{
Federal Entities
}

-NUG: Hydro-

1 Clearwater - State of ID DWR (BPA)

2 Dworshak Small Hydropower (BPA)

3 Federal -Total NUG: Hydro

\begin{tabular}{|c|c|c|c|c|c|c|c|c|c|c|c|c|c|c|}
\hline 1 & 1 & 1 & 1 & 1 & 1 & 1 & 1 & 1 & 1 & 1 & 1 & 1 & 1 & 1 \\
\hline 2.6 & 2.6 & 2.6 & 2.6 & 2.6 & 2.6 & 2.6 & 2.6 & 2.6 & 2.6 & 2.6 & 2.6 & 2.6 & 2.6 & 2.6 \\
\hline 3.6 & 3.6 & 3.6 & 3.6 & 3.6 & 3.6 & 3.6 & 3.6 & 3.6 & 3.6 & 3.6 & 3.6 & 3.6 & 3.6 & 3.6 \\
\hline
\end{tabular}

-NUG: Renewables-

4 Ashland Solar Project (BPA)

5 Condon Wind Project (BPA)

6 Foote Creek 1 (BPA)

7 Foote Creek 2 (BPA)

8 Foote Creek 4 (BPA)

9 Fourmile Hill Geothermal (BPA)

10 Klondike Phase 1 (BPA)

11 Stateline Wind Project (BPA)

12 Federal -Total NUG: Renewables

\begin{tabular}{|c|c|c|c|c|c|c|c|c|c|c|c|c|c|}
\hline 0 & 0 & 0 & 0 & 0 & 0 & 0 & 0 & 0 & 0 & 0 & 0 & 0 & 0 \\
\hline 10 & 10 & 11 & 13 & 14 & 13 & 12 & 12 & 14 & 14 & 12 & 15 & 11 & 12 \\
\hline 3.3 & 3.8 & 5.6 & 8.3 & 10 & 11 & 9.6 & 7.2 & 5.8 & 5.8 & 4.6 & 4.1 & 3.3 & 6.4 \\
\hline 0.4 & 0.5 & 0.7 & 1 & 1.3 & 1.3 & 1.2 & 0.9 & 0.7 & 0.7 & 0.5 & 0.5 & 0.4 & 0.8 \\
\hline 3.9 & 4.4 & 6.5 & 9.7 & 12 & 12 & 11 & 8.4 & 6.7 & 6.7 & 5.4 & 4.8 & 3.8 & 7.4 \\
\hline 50 & 50 & 50 & 50 & 50 & 50 & 50 & 50 & 50 & 50 & 50 & 50 & 50 & 50 \\
\hline 7.9 & 6.2 & 8.1 & 8.8 & 7.8 & 8.3 & 7.8 & 7.8 & 8.6 & 8.6 & 8.8 & 9.1 & 8 & 8.1 \\
\hline 29 & 23 & 30 & 33 & 29 & 31 & 29 & 29 & 32 & 32 & 33 & 34 & 30 & 30 \\
\hline 105 & 98 & 112 & 124 & 123 & 126 & 121 & 115 & 118 & 118 & 114 & 117 & 106 & 115 \\
\hline
\end{tabular}

Generating Public Entities

-NUG: Hydro-

13 Don Steffen (SHPD)

14 Eltopia Br Canal 4.6 (SCL)

15 Eltopia Br Canal 4.6 (TPU)

16 John Llewellyn (SHPD)

17 Kevin Duncan (SHPD)

18 Lucky Peak (SCL)

19 Main Canal Headworks (SCL)

20 Main Canal Headworks (TPU)

21 PEC Headworks (GCPD)

22 Pickering Micro Hydro (CKPD)

23 Potholes E Canal 66.0 (SCL)

24 Potholes E Canal 66.0 (TPU)

25 Quincy Chute (GCPD)

26 Russel D. Smith (SCL)

27 Russel D. Smith (TPU)

28 Smith Creek (EWEB)

29 Summer Falls (SCL)

30 Summer Falls (TPU)

31 Woods Creek (SHPD)

32 GPU - Total NUG: Hydro

\begin{tabular}{rrrrrrrrrrrrrrr}
0 & 0 & 0 & 0 & 0 & 0 & 0 & 0 & 0 & 0 & 0 & 0 & 0 & 0 & 0 \\
0.9 & 0.9 & 0.6 & 0.3 & 0 & 0 & 0 & 0 & 0 & 0.7 & 0.7 & 1 & 1 & 1.1 & 0.5 \\
0.9 & 0.9 & 0.6 & 0.3 & 0 & 0 & 0 & 0 & 0 & 0.7 & 0.7 & 1 & 1 & 1.1 & 0.5 \\
0 & 0 & 0 & 0 & 0 & 0 & 0 & 0 & 0 & 0 & 0 & 0 & 0 & 0 & 0 \\
0 & 0 & 0 & 0 & 0 & 0 & 0 & 0 & 0 & 0 & 0 & 0 & 0 & 0 & 0 \\
66 & 66 & 41 & 11 & 2.2 & 3 & 7.9 & 10 & 21 & 52 & 52 & 84 & 85 & 77 & 38 \\
10 & 10 & 6.1 & 2.6 & 0 & 0 & 0 & 0 & 1.4 & 6.8 & 6.8 & 9.1 & 11 & 12 & 4.9 \\
10 & 10 & 6.1 & 2.6 & 0 & 0 & 0 & 0 & 1.4 & 6.8 & 6.8 & 9.1 & 11 & 12 & 4.9 \\
4.5 & 4.5 & 3.1 & 2.2 & 0 & 0 & 0 & 0 & 0.4 & 5.1 & 5.1 & 6.9 & 6.2 & 5.8 & 2.9 \\
0 & 0 & 0 & 0 & 0 & 0 & 0 & 0 & 0 & 0 & 0 & 0 & 0 & 0 & 0 \\
0.9 & 0.9 & 0.9 & 0.8 & 0 & 0 & 0 & 0 & 0.3 & 0.9 & 0.9 & 0.9 & 0.9 & 0.9 & 0.5 \\
0.9 & 0.9 & 0.9 & 0.8 & 0 & 0 & 0 & 0 & 0.3 & 0.9 & 0.9 & 0.9 & 0.9 & 0.9 & 0.5 \\
7.7 & 7.7 & 4 & 2.3 & 0 & 0 & 0 & 0 & 0.8 & 5.5 & 5.5 & 7.1 & 8.4 & 8.5 & 3.7 \\
2 & 2 & 1.3 & 0.7 & 0 & 0 & 0 & 0 & 0.1 & 1.2 & 1.2 & 1.9 & 2.1 & 1.7 & 0.9 \\
2 & 2 & 1.3 & 0.7 & 0 & 0 & 0 & 0 & 0.1 & 1.2 & 1.2 & 1.9 & 2.1 & 1.7 & 0.9 \\
0 & 0 & 0 & 5 & 5.4 & 3.9 & 0 & 0.8 & 1.5 & 9.2 & 9.2 & 37 & 37 & 11 & 9.2 \\
39 & 39 & 24 & 11 & 0 & 0 & 0 & 0 & 2.4 & 25 & 25 & 36 & 40 & 44 & 18 \\
39 & 39 & 24 & 11 & 0 & 0 & 0 & 0 & 2.4 & 25 & 25 & 36 & 40 & 44 & 18 \\
0.1 & 0.1 & 0.1 & 0.1 & 0.1 & 0.1 & 0.1 & 0.1 & 0.1 & 0.1 & 0.1 & 0.1 & 0.1 & 0.1 & 0.1 \\
\hline 185 & 185 & 115 & $\mathbf{5 1}$ & $\mathbf{7 . 7}$ & $\mathbf{7}$ & $\mathbf{8}$ & $\mathbf{1 1}$ & $\mathbf{3 2}$ & $\mathbf{1 4 1}$ & $\mathbf{1 4 1}$ & $\mathbf{2 3 2}$ & $\mathbf{2 4 7}$ & $\mathbf{2 2 1}$ & $\mathbf{1 0 5}$
\end{tabular}

-NUG: Small Thermal \& Misc.-

33 Grays Harbor Paper (GHPD)

34 Weyerhaeuser Pulp Mill (GHPD)

35 GPU - Total NUG: Small Thermal \& Misc.

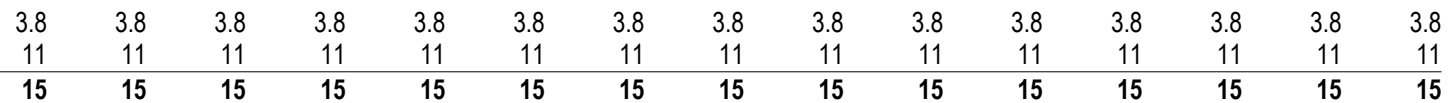

-NUG: Co-Generation-

36 Metro Westpoint (SCL)

37 Scott Paper (SHPD)

38 GPU - Total NUG: Co-Generation

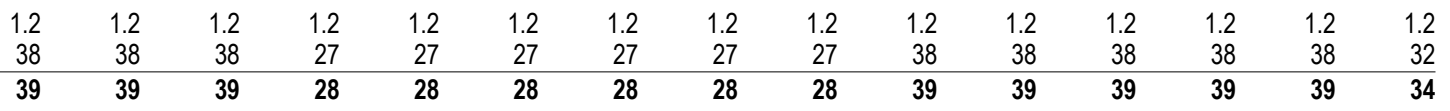

-NUG: Renewables-

39 Foote Creek 1 (EWEB)

40 Nine Canyon Wind Project (CHPD)

41 Nine Canyon Wind Project (COPD)

42 Nine Canyon Wind Project (DOPD)

43 Nine Canyon Wind Project (GCPD)

44 Nine Canyon Wind Project (GHPD)

45 Nine Canyon Wind Project (OKPD)

46 Stateline Wind Project (EWEB)

47 Stateline Wind Project (SCL)

48 GPU - Total NUG: Renewables

Non-Generating Public Entities

-NUG: Hydro-

49 Buffalo Hydro (FREC)

50 Burr \& Bouchard (TNEC)

51 City of Cove (OTEC)

52 Falls Creek (CCPD)

53 Lilliwaup Falls Generating Co. (MCPD1)

54 Thomas Burnside (TNEC)

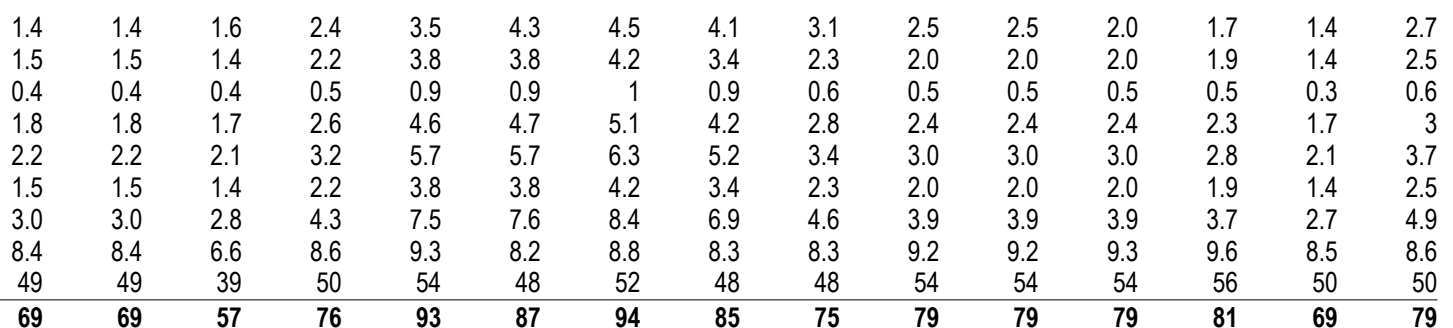

-NUG: Small Thermal \& Misc.-

56 Methane Energy Agricultural D. (TKPD)

$\begin{array}{rrrrrrrrrrrrrrr}0.2 & 0.2 & 0.2 & 0.2 & 0.2 & 0.2 & 0.2 & 0.2 & 0.2 & 0.2 & 0.2 & 0.2 & 0.2 & 0.2 & 0.2 \\ 0 & 0 & 0 & 0 & 0 & 0 & 0 & 0 & 0 & 0 & 0 & 0 & 0 & 0 & 0 \\ 0 & 0 & 0 & 0 & 0 & 0 & 0 & 0 & 0 & 0 & 0 & 0 & 0 & 0 & 0 \\ 0 & 0 & 0 & 0 & 0 & 0 & 0 & 0 & 0 & 0 & 0 & 0 & 0 & 0 & 0 \\ 0.6 & 0.6 & 0.6 & 0.6 & 0.6 & 0.6 & 0.6 & 0.6 & 0.6 & 0.6 & 0.6 & 0.6 & 0.6 & 0.6 & 0.6 \\ 0 & 0 & 0 & 0 & 0 & 0 & 0 & 0 & 0 & 0 & 0 & 0 & 0 & 0 & 0 \\ 0.9 & \mathbf{0 . 9} & \mathbf{0 . 9} & \mathbf{0 . 9} & \mathbf{0 . 9} & \mathbf{0 . 9} & \mathbf{0 . 9} & \mathbf{0 . 9} & \mathbf{0 . 9} & \mathbf{0 . 9} & \mathbf{0 . 9} & \mathbf{0 . 9} & \mathbf{0 . 9} & \mathbf{0 . 9} & \mathbf{0 . 9}\end{array}$

57 NGP - Total NUG: Small Thermal \& Misc.

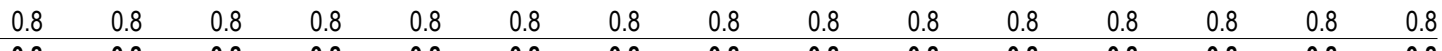


-NUG: Renewables.

58 Ashland Solar Project (ASHL)

59 Nine Canyon Wind Project (BCPD)

60 Nine Canyon Wind Project (ENW)

61 Nine Canyon Wind Project (LCPD)

62 Nine Canyon Wind Project (MCPD3)

\begin{tabular}{|c|c|c|c|c|c|c|c|c|c|c|c|c|c|c|}
\hline 0 & 0 & 0 & 0 & 0 & 0 & 0 & 0 & 0 & 0 & 0 & 0 & 0 & 0 & 0 \\
\hline 0.6 & 0.6 & 0.5 & 0.8 & 1.4 & 1.4 & 1.6 & 1.3 & 0.9 & 0.7 & 0.7 & 0.7 & 0.7 & 0.5 & 0.9 \\
\hline 0.4 & 0.4 & 0.4 & 0.5 & 0.9 & 1.0 & 1.1 & 0.9 & 0.6 & 0.5 & 0.5 & 0.5 & 0.5 & 0.3 & 0.6 \\
\hline 0.2 & 0.2 & 0.2 & 0.3 & 0.5 & 0.5 & 0.5 & 0.4 & 0.3 & 0.2 & 0.2 & 0.2 & 0.2 & 0.2 & 0.3 \\
\hline 0.4 & 0.4 & 0.4 & 0.5 & 0.9 & 1.0 & 1 & 0.9 & 0.6 & 0.5 & 0.5 & 0.5 & 0.5 & 0.3 & 0.6 \\
\hline 0 & 0 & 0 & 0 & 0 & 0 & 0 & 0 & 0 & 0 & 0 & 0 & 0 & 0 & 0 \\
\hline 1.5 & 1.5 & 1.4 & 2.2 & 3.8 & 3.8 & 4.2 & 3.5 & 2.3 & 2.0 & 2.0 & 2.0 & 1.9 & 1.4 & 2.5 \\
\hline
\end{tabular}

64 NGP - Total NUG: Renewables

Investor-Owned Entities

-NUG: Hydro-

65 Barber Dam (IPC)

66 Barney Creek (NWE)

67 Beaverton (PGE)

68 Bell Mountain (UPL)

69 Bingham Engineering (NWE)

70 Birch Creek (IPC)

71 Birch Creek (UPL)

72 Black Canyon \#3 (IPC)

73 Black Creek (AVWP)

74 Blind Canyon Hydro (IPC)

75 Box Canyon (IPC)

76 Boyd James (PPL)

77 Briggs Creek (IPC)

78 Broadwater Dam (NWE)

79 Bypass (IPC)

80 Canyon Springs (IPC)

81 Carlton (PGE)

82 Cascade Creek (NWE)

83 CDM Hydro - Bonneville Pacific (PPL)

84 Cedar Draw (IPC)

85 Central OR Irrigation District (PPL)

86 Clear Springs Trout (IPC)

87 Corbett (PGE)

88 Cornelius (PGE)

89 Crystal Springs (IPC)

90 Curry Cattle Co. (IPC)

91 Dairy Creek (PGE)

92 Deep Creek (AVWP)

93 Derr Creek (AVWP)

94 Dietrich Drop (IPC)

95 Donald Jenni (NWE)

96 Eagle Creek (PGE)

97 Eagle Point Irrigation Dist. (PPL)

98 Elk Creek (IPC)

99 Estacada (PGE)

100 Falls Creek (PPL)

101 Falls River (IPC)

102 Farmers Irrig. (PPL)

103 Faulkner Ranch (IPC)

104 Fisheries Development Co (IPC)

105 Galesville Dam (PPL)

106 Geobon 2 (IPC)

107 Georgetown Power (UPL)

108 Hailey Cspp (IPC)

109 Hazelton A (IPC)

110 Hazelton B (IPC)

111 Horseshoe Bend (IPC)

112 Hutchinson Creek (PSE)

113 Ingram Warm Springs (UPL)

$114 \mathrm{Jim}$ Ford Creek (AVWP)

115 Jim Knight (IPC)

116 John Day Creek (AVWP)

117 Joseph Hydro (PPL)

118 Kasel \& Witherspoon (IPC)

119 Koma Kulshan (PSE)

120 Koyle (IPC)

$121 \mathrm{~L}$. Fery (PPL)

122 Lacomb Irrig. (PPL)

123 Lake Oswego (PGE)

124 Lateral \#10 (IPC)

125 Lee Tavenner (NWE)

126 Lemoyne (IPC)

127 Little Wood River Res. (IPC)

128 Littlewood/Arkoosh (IPC)

129 Lowline \#2 (IPC)
2.

$\begin{array}{rrr}0 & 0 & 0 \\ 0.1 & 0.1 & 0.1\end{array}$

$\begin{array}{lllllll}0.1 & 0.1 & 0.1 & 0.1 & 0.1 & 0.1 & 0.1\end{array}$

$\begin{array}{rrrrrr}0 & 0 & 0.1 & 0.1 & 0.1 & 0.1\end{array}$

$\begin{array}{llll}0.1 & 0.1 & 0.1 & 0.1 \\ 3.4 & 3.4 & 3.1\end{array}$

$\begin{array}{llll}0.4 & 3.4 & 3.1 & 3.7\end{array}$

$\begin{array}{llll}0.7 & 0.7 & 1.1 & 0.9\end{array}$

$\begin{array}{rrr}0 & 0 & 0 \\ 0.6 & 0.6 & 0.6\end{array}$

$\begin{array}{llll}1.1 & 1.1 & 1.6 & 1.9\end{array}$

$\begin{array}{llll}8.3 & 8.3 & 6.5 & 2.8 \\ 0.1 & 0.1 & 0.1 & 0.1\end{array}$

0
0

$2.9 \quad 2.9$

0.3

3.1
0.4

$0.4 \quad 0.4$

0

1.3

$0.2 \quad 0.2$

0

0.1

0.1

0

1.2

0.1

6.5

0.6

0.8

0.2

$0.6 \quad 0.6$

1.0

0.1

7
6.6

8.8

0.7

0.2

0.4

0.2

1.5

1.2

4.6
1.1
0

1.1
0

0

$0.4 \quad 0.4$

$1.5 \quad 1.5$

0.1

1.9

0.7

2.7

$\begin{array}{rrr}0.8 & 0.3 & \\ 0 & 0 & \\ 0 & 0.1 & \\ 0.2 & 0.1 & 0.1 \\ 0.1 & 0.1 & 0.1 \\ 0.1 & 0.1 & 0.1 \\ 1.0 & 1.1 & \\ 0.1 & 0.1 & \\ 3.7 & 3.4 & 3.1 \\ 0.9 & 0.2 & \\ 0.4 & 0.4 & 0.3 \\ 0 & 0 & 0.4 \\ 0.6 & 0.6 & 0.6 \\ 1.9 & 2.1 & 1.6 \\ 2.8 & 1.0 & \\ 0.1 & 0.1 & 0.1 \\ 0 & 0 & \\ 0 & 0 & 0\end{array}$

$2.4 \quad 1.8$

3.12 .8

$\begin{array}{ll}2.9 & 2.4 \\ 0.9 & 1.2\end{array}$

$3.7 \quad 3.5$

$0.4 \quad 0.5$

0.6

0.6

$\begin{array}{ll}0 & 0 \\ 0 & 0\end{array}$

$\begin{array}{ll}0.5 & 0.5\end{array}$

1.5

$\begin{array}{ll}1.6 & 0.5\end{array}$

1.6

$\begin{array}{lll}0 & 0 & 0 \\ 0 & 0 & 0\end{array}$

$\begin{array}{lll}0.1 & 0.1 & 0.1 \\ 4.7 & 3.6 & 3.1 \\ 0.1 & 0.1 & 0.1\end{array}$

$\begin{array}{lll}0.1 & 0.1 & 0.1 \\ 0 & 0\end{array}$

$0.1 \quad 0.1$

$0.1 \quad 0.1$

$\begin{array}{ll}0.7 & 0.7 \\ 0.1 & 0.1\end{array}$

3.1

5.0

3.2

0.3

0.3

0.3

0.2

0.3

0.1

0
0
3.5

$\begin{array}{ll}3.5 & 3.9\end{array}$

0.7

0.5

0.5

$0.1 \quad 0.1$

$\begin{array}{ll}0.2 & 0.3 \\ 0.1 & 0.1\end{array}$

0.3

$\begin{array}{ll}6.1 & 3.9\end{array}$

0.2

0.1
0

0.3

0.2

$\begin{array}{lll}1.9 & 0.2 & 0.2 \\ 0.7 & 0.4 & 0.4\end{array}$

$\begin{array}{lll}0.7 & 0.4 & 0.4\end{array}$

$\begin{array}{llll}2.7 & 2.5 & 1.8 & 1.4\end{array}$

$\begin{array}{rr}1.0 & 2.2 \\ 0 & 0 \\ 0 & 0 \\ 0.1 & 0.1 \\ 0.1 & 0.1 \\ 0.1 & 0 \\ 1.1 & 0.3 \\ 0 & 0 \\ 3.1 & 3.1 \\ 0 & 0 \\ 0.3 & 0.3 \\ 0.4 & 0.3 \\ 0.6 & 0.6 \\ 1.6 & 1.6 \\ 0 & 0 \\ 0.1 & 0.1 \\ 0 & 0.1 \\ 0 & 0\end{array}$

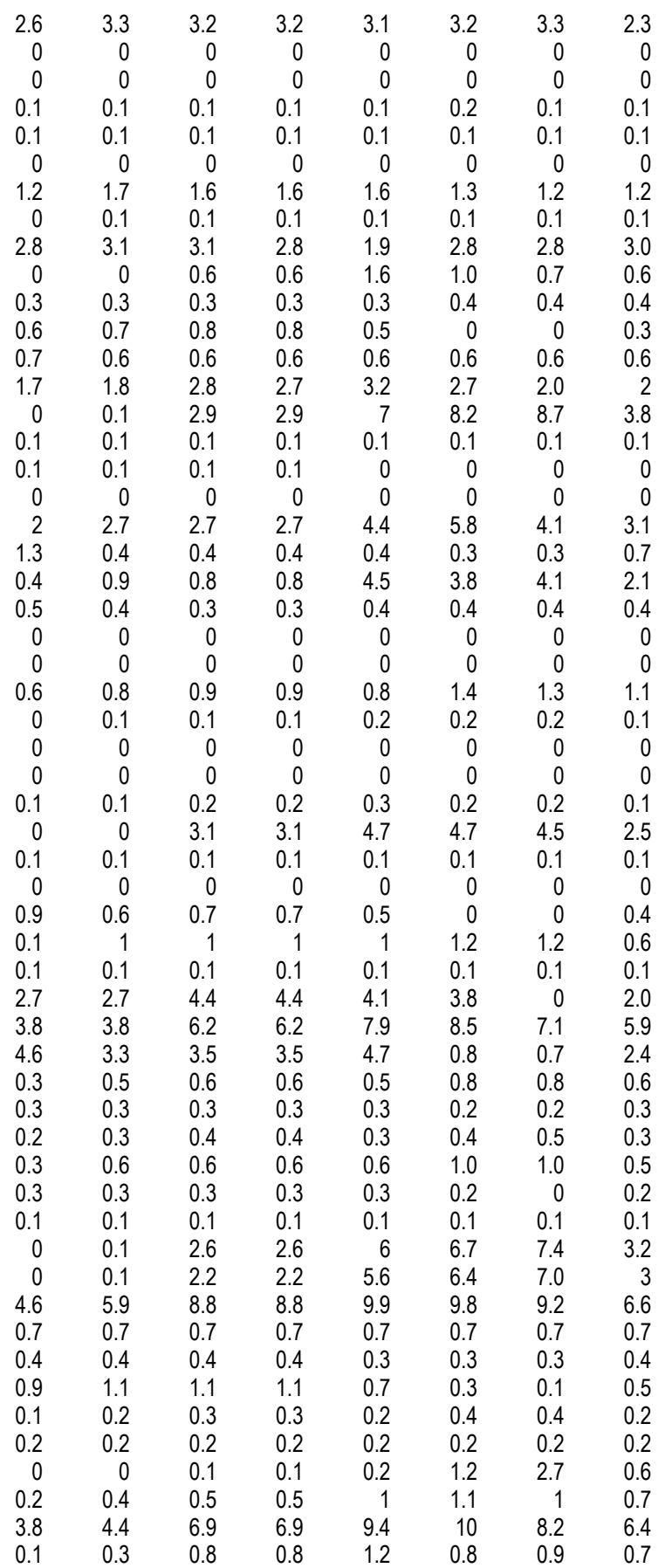

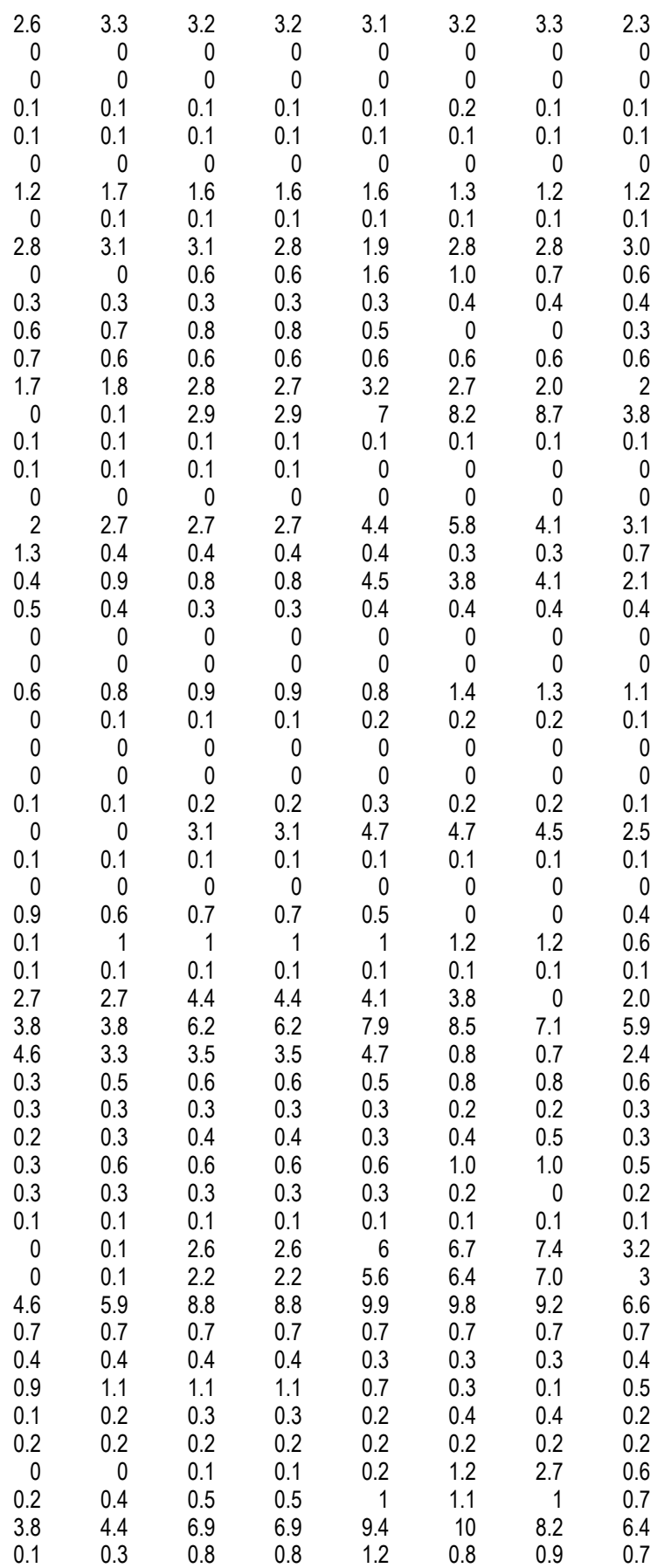

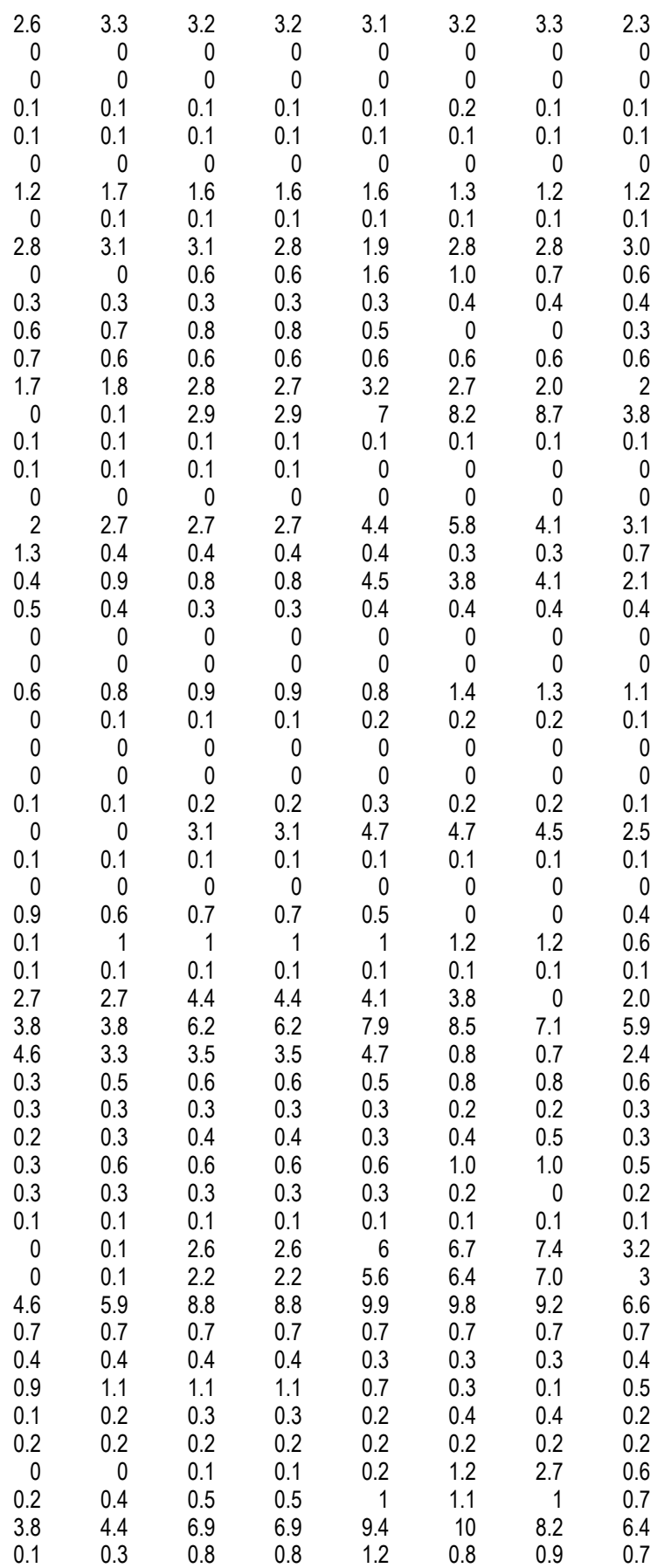

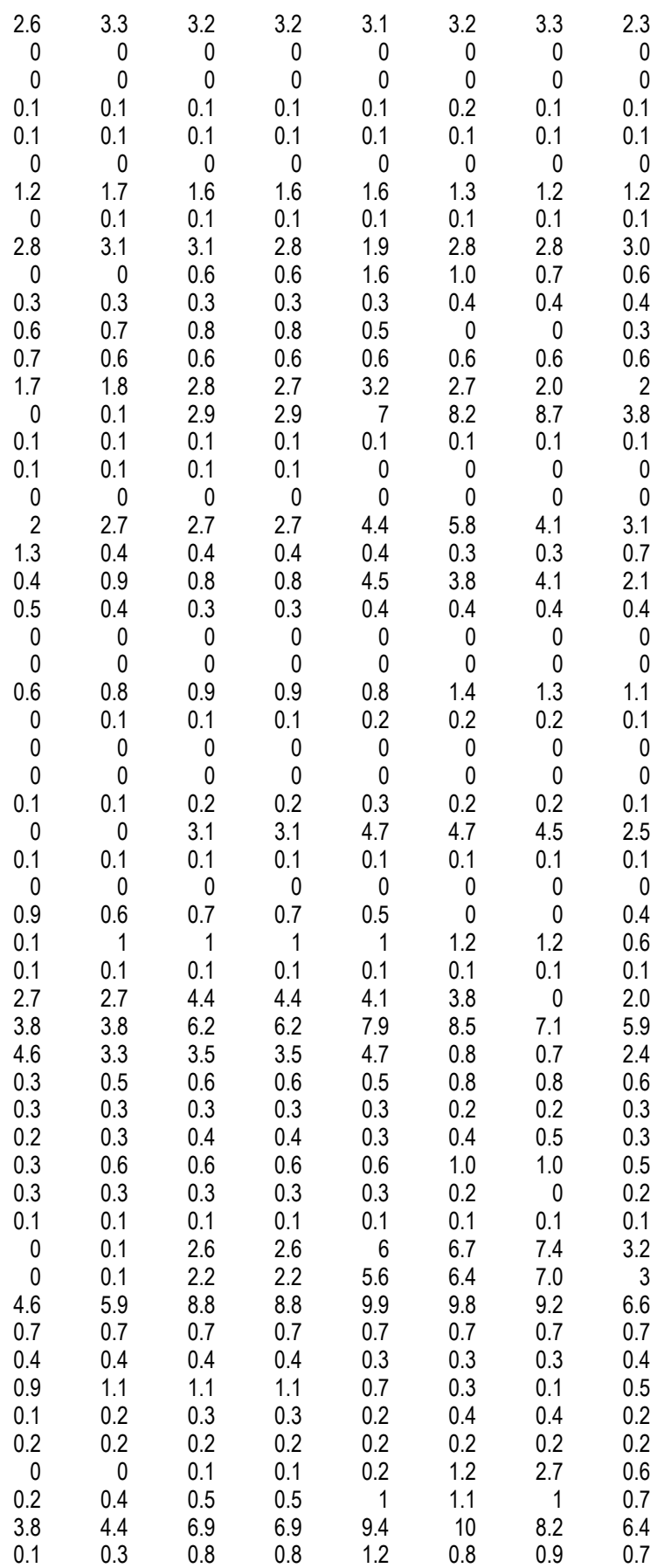

$\begin{array}{rr}2.8 & 3.1 \\ 0 & \end{array}$

$\begin{array}{rrrrrrrr}2.6 & 3.3 & 3.2 & 3.2 & 3.1 & 3.2 & 3.3 & 2.3 \\ 0 & 0 & 0 & 0 & 0 & 0 & 0 & 0 \\ 0 & 0 & 0 & 0 & 0 & 0 & 0 & 0 \\ 0.1 & 0.1 & 0.1 & 0.1 & 0.1 & 0.2 & 0.1 & 0.1 \\ 0.1 & 0.1 & 0.1 & 0.1 & 0.1 & 0.1 & 0.1 & 0.1 \\ 0 & 0 & 0 & 0 & 0 & 0 & 0 & 0 \\ 1.2 & 1.7 & 1.6 & 1.6 & 1.6 & 1.3 & 1.2 & 1.2 \\ 0 & 0.1 & 0.1 & 0.1 & 0.1 & 0.1 & 0.1 & 0.1 \\ 2.8 & 3.1 & 3.1 & 2.8 & 1.9 & 2.8 & 2.8 & 3.0 \\ 0 & 0 & 0.6 & 0.6 & 1.6 & 1.0 & 0.7 & 0.6 \\ 0.3 & 0.3 & 0.3 & 0.3 & 0.3 & 0.4 & 0.4 & 0.4 \\ 0.6 & 0.7 & 0.8 & 0.8 & 0.5 & 0 & 0 & 0.3 \\ 0.7 & 0.6 & 0.6 & 0.6 & 0.6 & 0.6 & 0.6 & 0.6 \\ 1.7 & 1.8 & 2.8 & 2.7 & 3.2 & 2.7 & 2.0 & 2 \\ 0 & 0.1 & 2.9 & 2.9 & 7 & 8.2 & 8.7 & 3.8 \\ 0.1 & 0.1 & 0.1 & 0.1 & 0.1 & 0.1 & 0.1 & 0.1 \\ 0.1 & 0.1 & 0.1 & 0.1 & 0 & 0 & 0 & 0 \\ 0 & 0 & 0 & 0 & 0 & 0 & 0 & 0 \\ 2 & 2.7 & 2.7 & 2.7 & 4.4 & 5.8 & 4.1 & 3.1 \\ 1.3 & 0.4 & 0.4 & 0.4 & 0.4 & 0.3 & 0.3 & 0.7 \\ 0.4 & 0.9 & 0.8 & 0.8 & 4.5 & 3.8 & 4.1 & 2.1 \\ 0.5 & 0.4 & 0.3 & 0.3 & 0.4 & 0.4 & 0.4 & 0.4 \\ 0 & 0 & 0 & 0 & 0 & 0 & 0 & 0 \\ 0 & 0 & 0 & 0 & 0 & 0 & 0 & 0 \\ 0.6 & 0.8 & 0.9 & 0.9 & 0.8 & 1.4 & 1.3 & 1.1 \\ 0 & 0.1 & 0.1 & 0.1 & 0.2 & 0.2 & 0.2 & 0.1 \\ 0 & 0 & 0 & 0 & 0 & 0 & 0 & 0 \\ 0 & 0 & 0 & 0 & 0 & 0 & 0 & 0 \\ 0.1 & 0.1 & 0.2 & 0.2 & 0.3 & 0.2 & 0.2 & 0.1 \\ 0 & 0 & 3.1 & 3.1 & 4.7 & 4.7 & 4.5 & 2.5 \\ 0.1 & 0.1 & 0.1 & 0.1 & 0.1 & 0.1 & 0.1 & 0.1 \\ 0 & 0 & 0 & 0 & 0 & 0 & 0 & 0 \\ 0.9 & 0.6 & 0.7 & 0.7 & 0.5 & 0 & 0 & 0.4 \\ 0.1 & 1 & 1 & 1 & 1 & 1.2 & 1.2 & 0.6 \\ 0.1 & 0.1 & 0.1 & 0.1 & 0.1 & 0.1 & 0.1 & 0.1 \\ 2.7 & 2.7 & 4.4 & 4.4 & 4.1 & 3.8 & 0 & 2.0 \\ 3.8 & 3.8 & 6.2 & 6.2 & 7.9 & 8.5 & 7.1 & 5.9 \\ 4.6 & 3.3 & 3.5 & 3.5 & 4.7 & 0.8 & 0.7 & 2.4 \\ 0.3 & 0.5 & 0.6 & 0.6 & 0.5 & 0.8 & 0.8 & 0.6 \\ 0.3 & 0.3 & 0.3 & 0.3 & 0.3 & 0.2 & 0.2 & 0.3 \\ 0.2 & 0.3 & 0.4 & 0.4 & 0.3 & 0.4 & 0.5 & 0.3 \\ 3.8 & 4.4 & 6.9 & 6.9 & 9.4 & 10 & 8.2 & 6.4 \\ 0.1 & 0.3 & 0.8 & 0.8 & 1.2 & 0.8 & 0.9 & 0.7 \\ 0 & 0 & 0 & 0 & 0 & 0 & 0 & 0 \\ 0.3 & 0.6 & 0.6 & 0.6 & 0.6 & 1.0 & 1.0 & 0.5 \\ 0.3 & 0.3 & 0.3 & 0.3 & 0.3 & 0.2 & 0 & 0.2 \\ 0.1 & 0.1 & 0.1 & 0.1 & 0.1 & 0.1 & 0.1 & 0.1 \\ 0 & 0.1 & 2.6 & 2.6 & 6 & 6.7 & 7.4 & 3.2 \\ 0.6 & 0.1 & 2.2 & 2.2 & 5.6 & 6.4 & 7.0 & 3 \\ 0.7 & 0.7 & 8.8 & 8.8 & 9.9 & 9.8 & 9.2 & 6.6 \\ 0.4 & 0.4 & 0.4 & 0.7 & 0.7 & 0.7 & 0.7 & 0.7 \\ 0.9 & 1.1 & 1.1 & 1.1 & 0.3 & 0.3 & 0.3 & 0.4 \\ 0.1 & 0.2 & 0.3 & 0.3 & 0.2 & 0.4 & 0.1 & 0.5 \\ 0.2 & 0.2 & 0.2 & 0.2 & 0.2 & 0.2 & 0.2 & 0.2 \\ 0.1 & 0.1 & 0.2 & 1.2 & 2.7 & 0.6 \\ 0 & 0.5 & 0.5 & 1 & 1.1 & 1 & 0.7 \\ 0\end{array}$

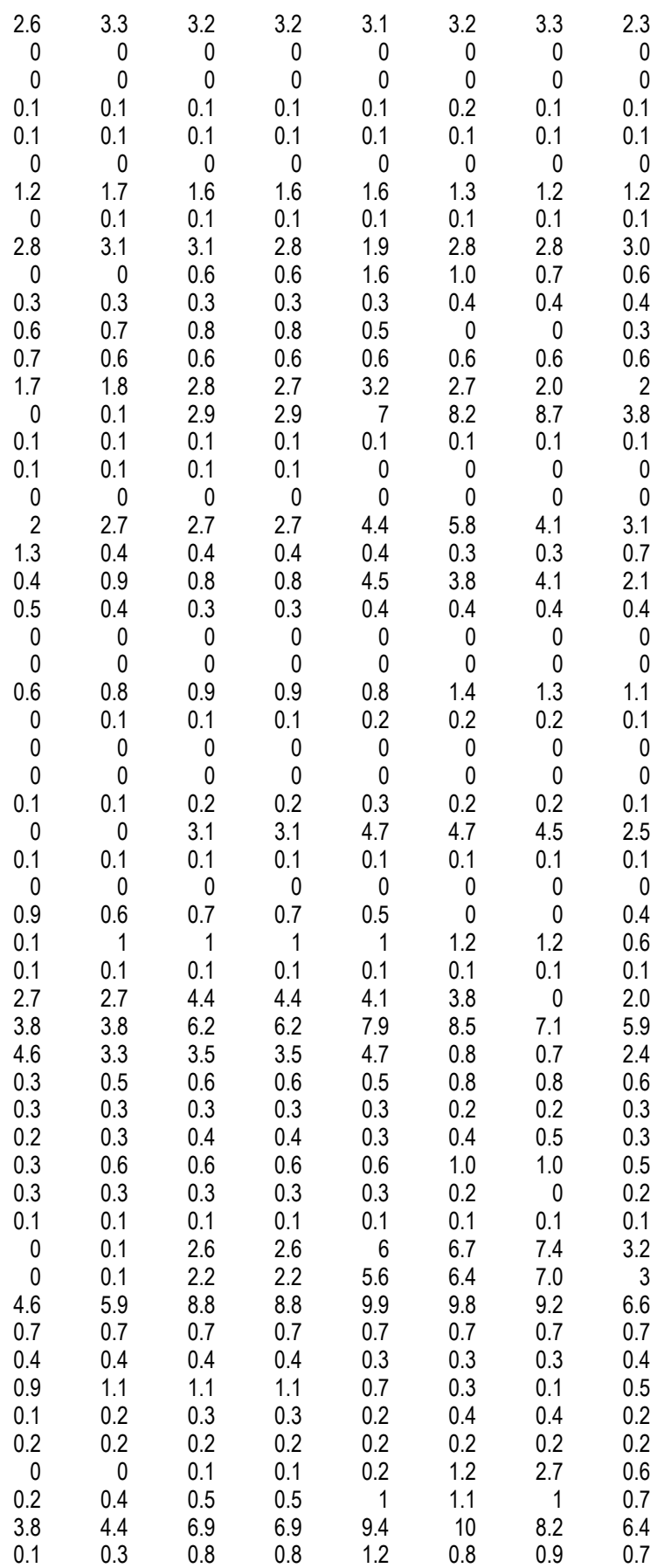

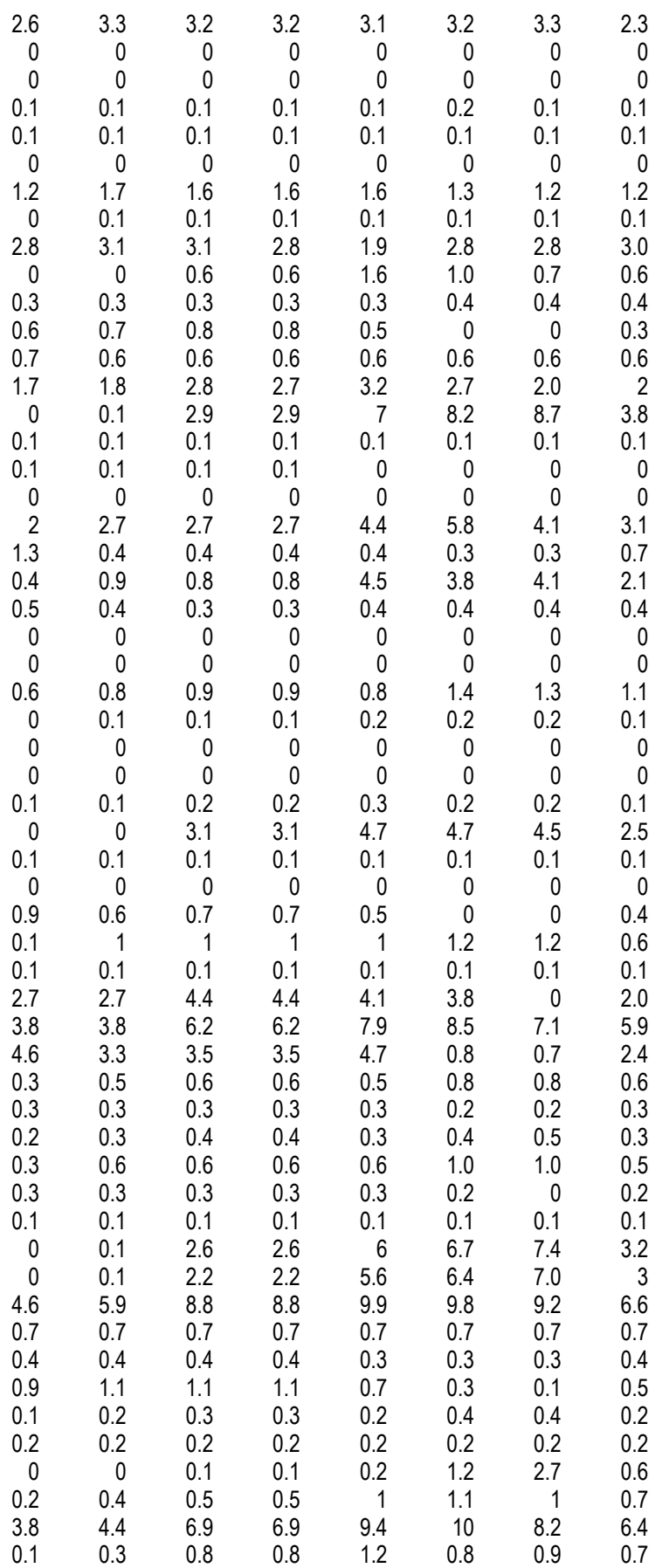

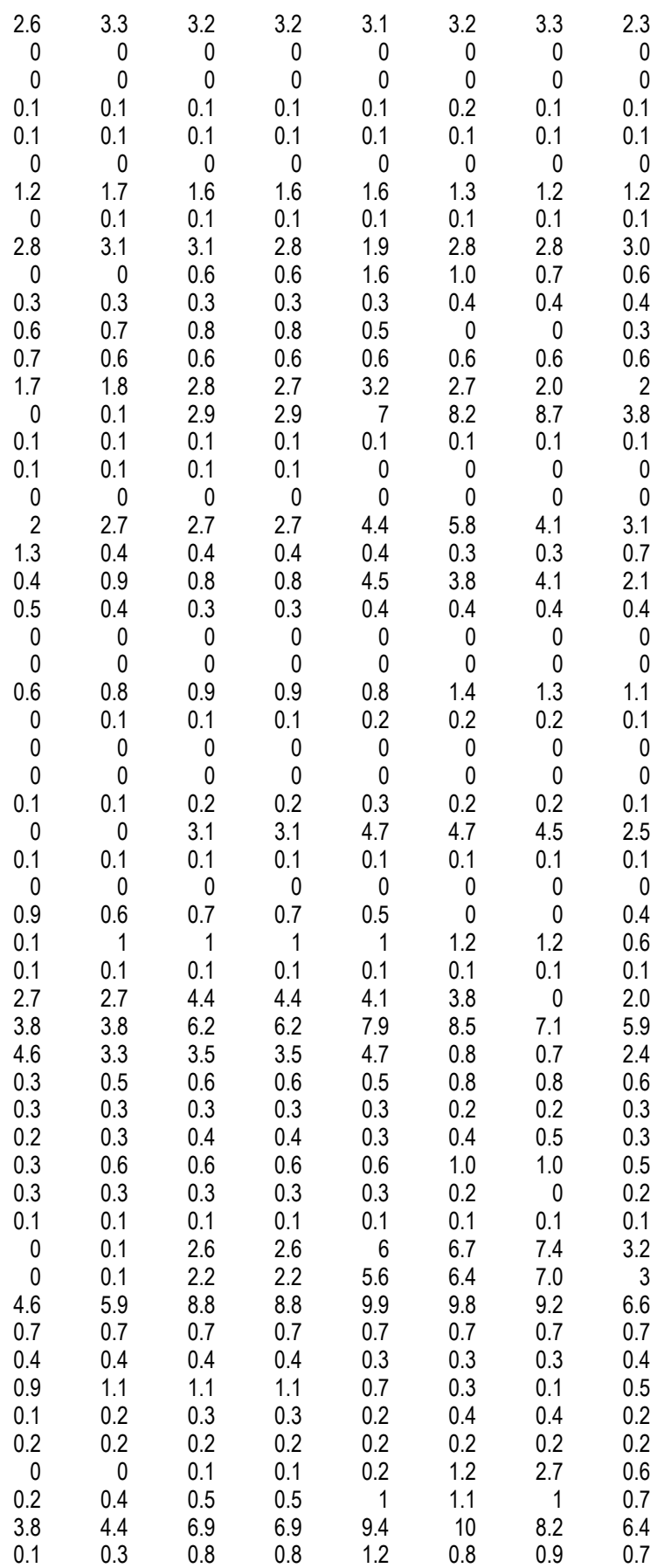

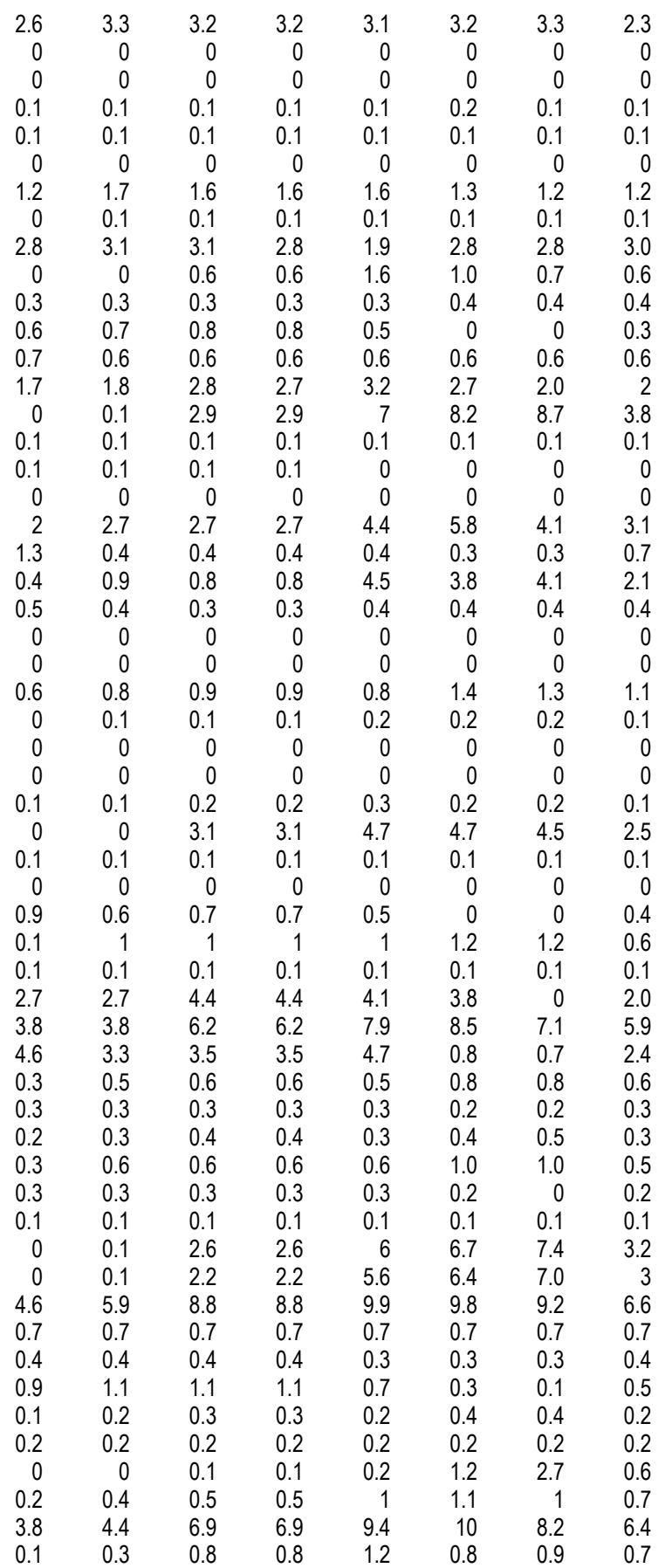

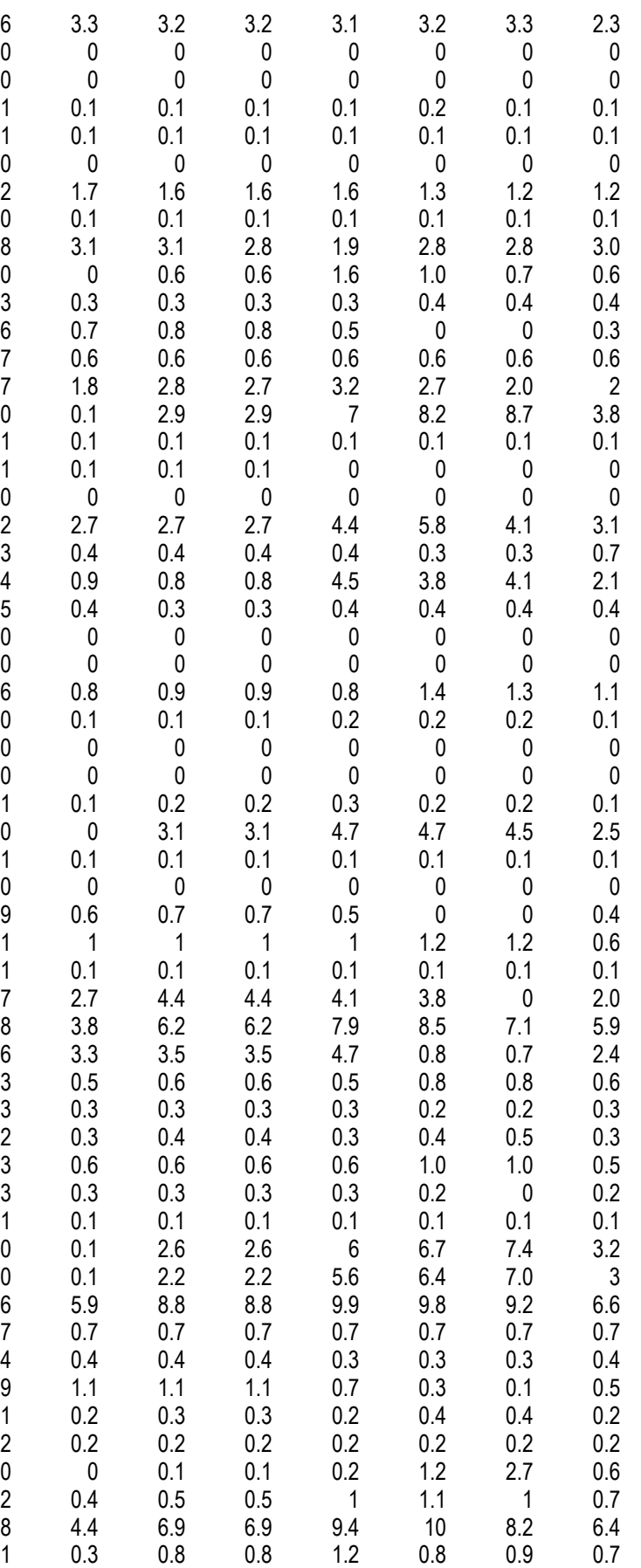

$\begin{array}{rrrrrrrr}6 & 3.3 & 3.2 & 3.2 & 3.1 & 3.2 & 3.3 & 2.3 \\ 0 & 0 & 0 & 0 & 0 & 0 & 0 & 0 \\ 0 & 0 & 0 & 0 & 0 & 0 & 0 & 0 \\ .1 & 0.1 & 0.1 & 0.1 & 0.1 & 0.2 & 0.1 & 0.1 \\ 1 & 0.1 & 0.1 & 0.1 & 0.1 & 0.1 & 0.1 & 0.1 \\ 0 & 0 & 0 & 0 & 0 & 0 & 0 & 0 \\ 2 & 1.7 & 1.6 & 1.6 & 1.6 & 1.3 & 1.2 & 1.2 \\ 0 & 0.1 & 0.1 & 0.1 & 0.1 & 0.1 & 0.1 & 0.1 \\ .8 & 3.1 & 3.1 & 2.8 & 1.9 & 2.8 & 2.8 & 3.0 \\ 0 & 0 & 0.6 & 0.6 & 1.6 & 1.0 & 0.7 & 0.6 \\ 3 & 0.3 & 0.3 & 0.3 & 0.3 & 0.4 & 0.4 & 0.4 \\ .6 & 0.7 & 0.8 & 0.8 & 0.5 & 0 & 0 & 0.3 \\ .7 & 0.6 & 0.6 & 0.6 & 0.6 & 0.6 & 0.6 & 0.6 \\ .7 & 1.8 & 2.8 & 2.7 & 3.2 & 2.7 & 2.0 & 2 \\ 0 & 0.1 & 2.9 & 2.9 & 7 & 8.2 & 8.7 & 3.8 \\ 0 & 0.1 & 0.1 & 0.1 & 0.1 & 0.1 & 0.1 & 0.1 \\ .1 & 0.1 & 0.1 & 0.1 & 0 & 0 & 0 & 0 \\ 0 & 0 & 0 & 0 & 0 & 0 & 0 & 0 \\ 2 & 2.7 & 2.7 & 2.7 & 4.4 & 5.8 & 4.1 & 3.1 \\ 3 & 0.4 & 0.4 & 0.4 & 0.4 & 0.3 & 0.3 & 0.7 \\ 4 & 0.9 & 0.8 & 0.8 & 4.5 & 3.8 & 4.1 & 2.1 \\ .5 & 0.4 & 0.3 & 0.3 & 0.4 & 0.4 & 0.4 & 0.4 \\ 0 & 0 & 0 & 0 & 0 & 0 & 0 & 0 \\ 0 & 0 & 0 & 0 & 0 & 0 & 0 & 0 \\ .6 & 0.8 & 0.9 & 0.9 & 0.8 & 1.4 & 1.3 & 1.1 \\ 0 & 0.1 & 0.1 & 0.1 & 0.2 & 0.2 & 0.2 & 0.1 \\ 0 & 0 & 0 & 0 & 0 & 0 & 0 & 0 \\ 0 & 0 & 0 & 0 & 0 & 0 & 0 & 0 \\ 0 & 0.1 & 0.2 & 0.2 & 0.3 & 0.2 & 0.2 & 0.1 \\ 0 & 0 & 3.1 & 3.1 & 4.7 & 4.7 & 4.5 & 2.5 \\ .1 & 0.4 & 0.1 & 0.1 & 0.2 & 1.2 & 2.7 & 0.6 \\ .1 & 0.1 & 0.1 & 0.1 & 0.1 & 0.1 & 0.1 & 0.1 \\ 0 & 0 & 0 & 0 & 0 & 0 & 0 & 0 \\ .9 & 0.6 & 0.7 & 0.7 & 0.5 & 0 & 0 & 0.4 \\ .1 & 0.4 & 6.9 & 6.9 & 9.4 & 10 & 8.2 & 6.4 \\ 0 & 0.8 & 0.8 & 0.8 & 1.2 & 0.8 & 0.9 & 0.7\end{array}$

$\begin{array}{rrrrr}0.1 & 0.1 & 0.1 & 0.1 & 0 \\ 0 & 0 & 0 & 0 & 0 \\ 2 & 2.7 & 2.7 & 2.7 & 4.4 \\ 1.3 & 0.4 & 0.4 & 0.4 & 0.4 \\ 0.4 & 0.9 & 0.8 & 0.8 & 4.5 \\ 0.5 & 0.4 & 0.3 & 0.3 & 0.4\end{array}$

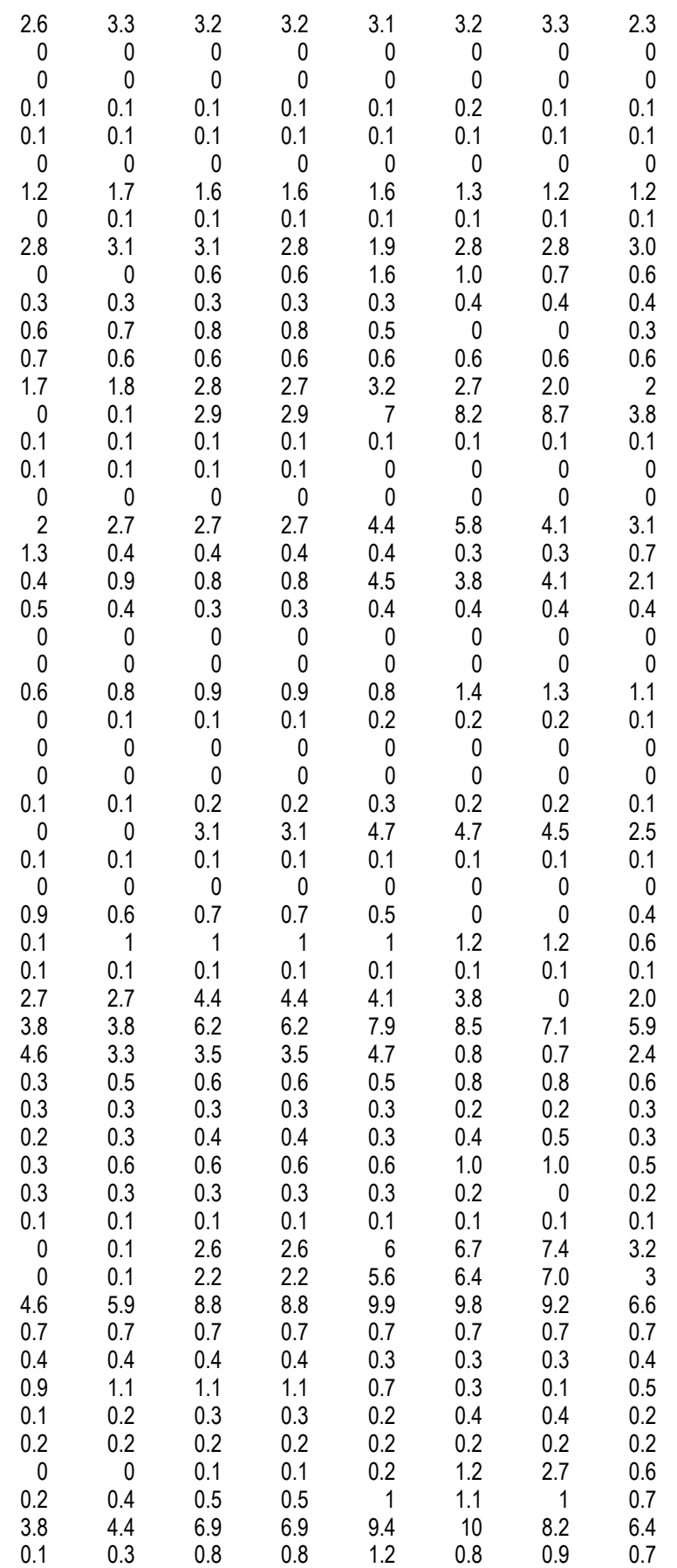

0.6

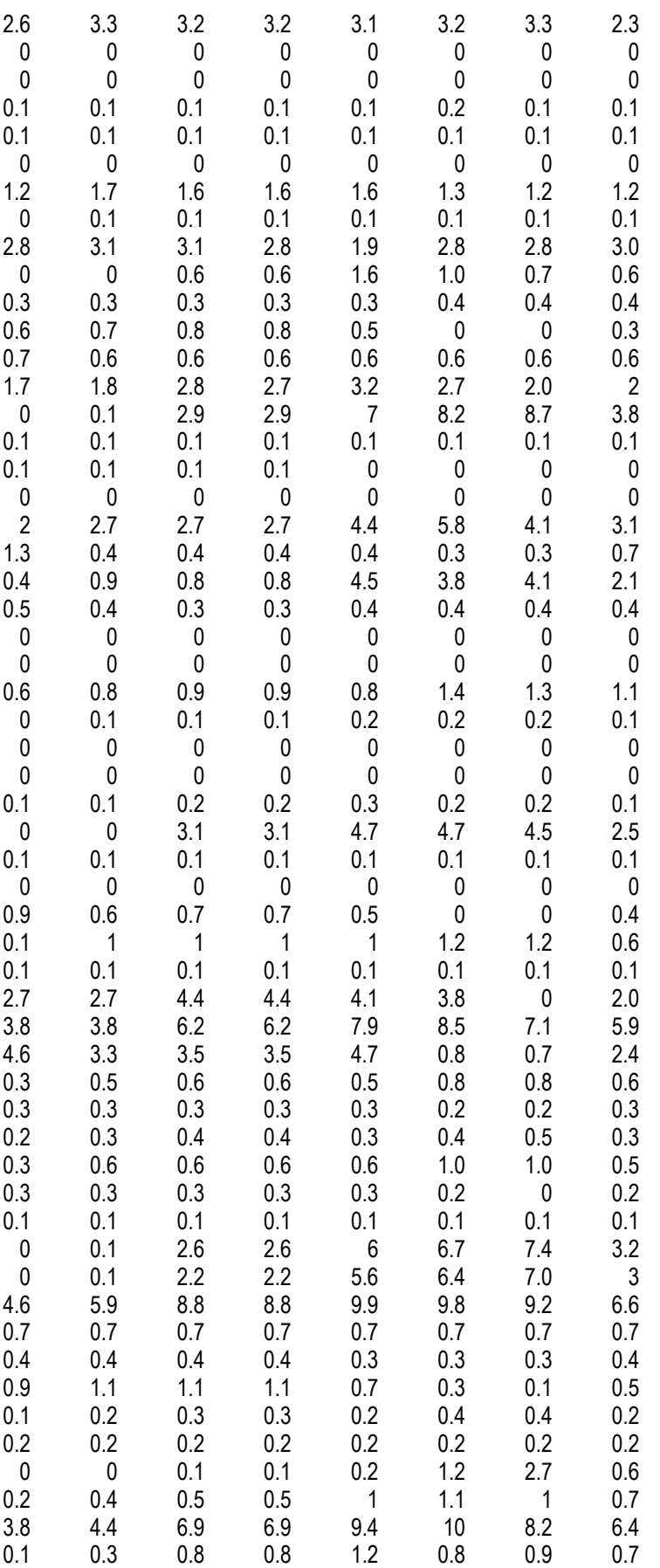

$\begin{array}{rrrrrrrr}2.6 & 3.3 & 3.2 & 3.2 & 3.1 & 3.2 & 3.3 & 2.3 \\ 0 & 0 & 0 & 0 & 0 & 0 & 0 & 0 \\ 0 & 0 & 0 & 0 & 0 & 0 & 0 & 0 \\ 0.1 & 0.1 & 0.1 & 0.1 & 0.1 & 0.2 & 0.1 & 0.1 \\ 0.1 & 0.1 & 0.1 & 0.1 & 0.1 & 0.1 & 0.1 & 0.1 \\ 0 & 0 & 0 & 0 & 0 & 0 & 0 & 0 \\ 1.2 & 1.7 & 1.6 & 1.6 & 1.6 & 1.3 & 1.2 & 1.2 \\ 0 & 0.1 & 0.1 & 0.1 & 0.1 & 0.1 & 0.1 & 0.1 \\ 2.8 & 3.1 & 3.1 & 2.8 & 1.9 & 2.8 & 2.8 & 3.0 \\ 0 & 0 & 0.6 & 0.6 & 1.6 & 1.0 & 0.7 & 0.6 \\ 0.3 & 0.3 & 0.3 & 0.3 & 0.3 & 0.4 & 0.4 & 0.4 \\ 0.6 & 0.7 & 0.8 & 0.8 & 0.5 & 0 & 0 & 0.3 \\ 0.7 & 0.6 & 0.6 & 0.6 & 0.6 & 0.6 & 0.6 & 0.6 \\ 1.7 & 1.8 & 2.8 & 2.7 & 3.2 & 2.7 & 2.0 & 2 \\ 0 & 0.1 & 2.9 & 2.9 & 7 & 8.2 & 8.7 & 3.8 \\ 0.1 & 0.1 & 0.1 & 0.1 & 0.1 & 0.1 & 0.1 & 0.1 \\ 0.1 & 0.1 & 0.1 & 0.1 & 0 & 0 & 0 & 0 \\ 0 & 0 & 0 & 0 & 0 & 0 & 0 & 0 \\ 2 & 2.7 & 2.7 & 2.7 & 4.4 & 5.8 & 4.1 & 3.1 \\ 1.3 & 0.4 & 0.4 & 0.4 & 0.4 & 0.3 & 0.3 & 0.7 \\ 0.4 & 0.9 & 0.8 & 0.8 & 4.5 & 3.8 & 4.1 & 2.1 \\ 0.5 & 0.4 & 0.3 & 0.3 & 0.4 & 0.4 & 0.4 & 0.4 \\ 0 & 0 & 0 & 0 & 0 & 0 & 0 & 0 \\ 0 & 0 & 0 & 0 & 0 & 0 & 0 & 0 \\ 0.6 & 0.8 & 0.9 & 0.9 & 0.8 & 1.4 & 1.3 & 1.1 \\ 0 & 0.1 & 0.1 & 0.1 & 0.2 & 0.2 & 0.2 & 0.1 \\ 0 & 0 & 0 & 0 & 0 & 0 & 0 & 0 \\ 0 & 0 & 0 & 0 & 0 & 0 & 0 & 0 \\ 0.1 & 0.1 & 0.2 & 0.2 & 0.3 & 0.2 & 0.2 & 0.1 \\ 0 & 0 & 3.1 & 3.1 & 4.7 & 4.7 & 4.5 & 2.5 \\ 0.1 & 0.1 & 0.1 & 0.1 & 0.1 & 0.1 & 0.1 & 0.1 \\ 0 & 0 & 0 & 0 & 0 & 0 & 0 & 0 \\ 0.9 & 0.6 & 0.7 & 0.7 & 0.5 & 0 & 0 & 0.4 \\ 0.1 & 1 & 1 & 1 & 1 & 1.2 & 1.2 & 0.6 \\ 0.1 & 0.1 & 0.1 & 0.1 & 0.1 & 0.1 & 0.1 & 0.1 \\ 2.7 & 2.7 & 4.4 & 4.4 & 4.1 & 3.8 & 0 & 2.0 \\ 3.8 & 3.8 & 6.2 & 6.2 & 7.9 & 8.5 & 7.1 & 5.9 \\ 4.6 & 3.3 & 3.5 & 3.5 & 4.7 & 0.8 & 0.7 & 2.4 \\ 0.3 & 0.5 & 0.6 & 0.6 & 0.5 & 0.8 & 0.8 & 0.6 \\ 0.3 & 0.3 & 0.3 & 0.3 & 0.3 & 0.2 & 0.2 & 0.3 \\ 0.2 & 0.3 & 0.4 & 0.4 & 0.3 & 0.4 & 0.5 & 0.3 \\ 0.3 & 0.6 & 0.6 & 0.6 & 0.6 & 1.0 & 1.0 & 0.5 \\ 3.8 & 0.4 & 6.9 & 6.9 & 9.4 & 10 & 8.2 & 6.4 \\ 0.1 & 0.3 & 0.8 & 0.8 & 1.2 & 0.8 & 0.9 & 0.7 \\ 0.3 & 0.3 & 0.3 & 0.3 & 0.3 & 0.2 & 0 & 0.2 \\ 0.1 & 0.1 & 0.1 & 0.1 & 0.1 & 0.1 & 0.1 & 0.1 \\ 0 & 0.1 & 2.6 & 2.6 & 6 & 6.7 & 7.4 & 3.2 \\ 0 & 0.1 & 2.2 & 2.2 & 5.6 & 6.4 & 7.0 & 3 \\ 4.6 & 5.9 & 8.8 & 8.8 & 9.9 & 9.8 & 9.2 & 6.6 \\ 0.7 & 0.7 & 0.7 & 0.7 & 0.7 & 0.7 & 0.7 & 0.7 \\ 0.4 & 0.4 & 0.4 & 0.4 & 0.3 & 0.3 & 0.3 & 0.4 \\ 0.9 & 1.1 & 1.1 & 1.1 & 0.7 & 0.3 & 0.1 & 0.5 \\ 0.1 & 0.2 & 0.3 & 0.3 & 0.2 & 0.4 & 0.4 & 0.2 \\ 0.2 & 0.2 & 0.2 & 0.2 & 0.2 & 0.2 & 0.2 & 0.2 \\ 0 & 0 & 0.1 & 0.1 & 0.2 & 1.2 & 2.7 & 0.6 \\ 0 & 0 & 0 & 0 & 0 & 0 & 0\end{array}$

$\begin{array}{rrrrrrrr}2.6 & 3.3 & 3.2 & 3.2 & 3.1 & 3.2 & 3.3 & 2.3 \\ 0 & 0 & 0 & 0 & 0 & 0 & 0 & 0 \\ 0 & 0 & 0 & 0 & 0 & 0 & 0 & 0 \\ 0.1 & 0.1 & 0.1 & 0.1 & 0.1 & 0.2 & 0.1 & 0.1 \\ 0.1 & 0.1 & 0.1 & 0.1 & 0.1 & 0.1 & 0.1 & 0.1 \\ 0 & 0 & 0 & 0 & 0 & 0 & 0 & 0 \\ 1.2 & 1.7 & 1.6 & 1.6 & 1.6 & 1.3 & 1.2 & 1.2 \\ 0 & 0.1 & 0.1 & 0.1 & 0.1 & 0.1 & 0.1 & 0.1 \\ 2.8 & 3.1 & 3.1 & 2.8 & 1.9 & 2.8 & 2.8 & 3.0 \\ 0 & 0 & 0.6 & 0.6 & 1.6 & 1.0 & 0.7 & 0.6 \\ 0.3 & 0.3 & 0.3 & 0.3 & 0.3 & 0.4 & 0.4 & 0.4 \\ 0.6 & 0.7 & 0.8 & 0.8 & 0.5 & 0 & 0 & 0.3 \\ 0.7 & 0.6 & 0.6 & 0.6 & 0.6 & 0.6 & 0.6 & 0.6 \\ 1.7 & 1.8 & 2.8 & 2.7 & 3.2 & 2.7 & 2.0 & 2 \\ 0 & 0.1 & 2.9 & 2.9 & 7 & 8.2 & 8.7 & 3.8 \\ 0.1 & 0.1 & 0.1 & 0.1 & 0.1 & 0.1 & 0.1 & 0.1 \\ 0.1 & 0.1 & 0.1 & 0.1 & 0 & 0 & 0 & 0 \\ 0 & 0 & 0 & 0 & 0 & 0 & 0 & 0 \\ 2 & 2.7 & 2.7 & 2.7 & 4.4 & 5.8 & 4.1 & 3.1 \\ 1.3 & 0.4 & 0.4 & 0.4 & 0.4 & 0.3 & 0.3 & 0.7 \\ 0.4 & 0.9 & 0.8 & 0.8 & 4.5 & 3.8 & 4.1 & 2.1 \\ 0.5 & 0.4 & 0.3 & 0.3 & 0.4 & 0.4 & 0.4 & 0.4 \\ 0 & 0 & 0 & 0 & 0 & 0 & 0 & 0 \\ 0 & 0 & 0 & 0 & 0 & 0 & 0 & 0 \\ 0.6 & 0.8 & 0.9 & 0.9 & 0.8 & 1.4 & 1.3 & 1.1 \\ 0 & 0.1 & 0.1 & 0.1 & 0.2 & 0.2 & 0.2 & 0.1 \\ 0 & 0 & 0 & 0 & 0 & 0 & 0 & 0 \\ 0 & 0 & 0 & 0 & 0 & 0 & 0 & 0 \\ 0.1 & 0.1 & 0.2 & 0.2 & 0.3 & 0.2 & 0.2 & 0.1 \\ 0 & 0 & 3.1 & 3.1 & 4.7 & 4.7 & 4.5 & 2.5 \\ 0.1 & 0.1 & 0.1 & 0.1 & 0.1 & 0.1 & 0.1 & 0.1 \\ 0 & 0 & 0 & 0 & 0 & 0 & 0 & 0 \\ 0.9 & 0.6 & 0.7 & 0.7 & 0.5 & 0 & 0 & 0.4 \\ 0.1 & 1 & 1 & 1 & 1 & 1.2 & 1.2 & 0.6 \\ 0.1 & 0.1 & 0.1 & 0.1 & 0.1 & 0.1 & 0.1 & 0.1 \\ 2.7 & 2.7 & 4.4 & 4.4 & 4.1 & 3.8 & 0 & 2.0 \\ 3.8 & 3.8 & 6.2 & 6.2 & 7.9 & 8.5 & 7.1 & 5.9 \\ 4.6 & 3.3 & 3.5 & 3.5 & 4.7 & 0.8 & 0.7 & 2.4 \\ 0.3 & 0.5 & 0.6 & 0.6 & 0.5 & 0.8 & 0.8 & 0.6 \\ 0.3 & 0.3 & 0.3 & 0.3 & 0.3 & 0.2 & 0.2 & 0.3 \\ 0.2 & 0.3 & 0.4 & 0.4 & 0.3 & 0.4 & 0.5 & 0.3 \\ 0.3 & 0.6 & 0.6 & 0.6 & 0.6 & 1.0 & 1.0 & 0.5 \\ 3.8 & 0.4 & 6.9 & 6.9 & 9.4 & 10 & 8.2 & 6.4 \\ 0.1 & 0.3 & 0.8 & 0.8 & 1.2 & 0.8 & 0.9 & 0.7 \\ 0.3 & 0.3 & 0.3 & 0.3 & 0.3 & 0.2 & 0 & 0.2 \\ 0.1 & 0.1 & 0.1 & 0.1 & 0.1 & 0.1 & 0.1 & 0.1 \\ 0 & 0.1 & 2.6 & 2.6 & 6 & 6.7 & 7.4 & 3.2 \\ 0 & 0.1 & 2.2 & 2.2 & 5.6 & 6.4 & 7.0 & 3 \\ 4.6 & 5.9 & 8.8 & 8.8 & 9.9 & 9.8 & 9.2 & 6.6 \\ 0.7 & 0.7 & 0.7 & 0.7 & 0.7 & 0.7 & 0.7 & 0.7 \\ 0.4 & 0.4 & 0.4 & 0.4 & 0.3 & 0.3 & 0.3 & 0.4 \\ 0.9 & 1.1 & 1.1 & 1.1 & 0.7 & 0.3 & 0.1 & 0.5 \\ 0.1 & 0.2 & 0.3 & 0.3 & 0.2 & 0.4 & 0.4 & 0.2 \\ 0.2 & 0.2 & 0.2 & 0.2 & 0.2 & 0.2 & 0.2 & 0.2 \\ 0 & 0 & 0.1 & 0.1 & 0.2 & 1.2 & 2.7 & 0.6 \\ 0 & 0 & 0 & 0 & 0 & 0 & 0\end{array}$

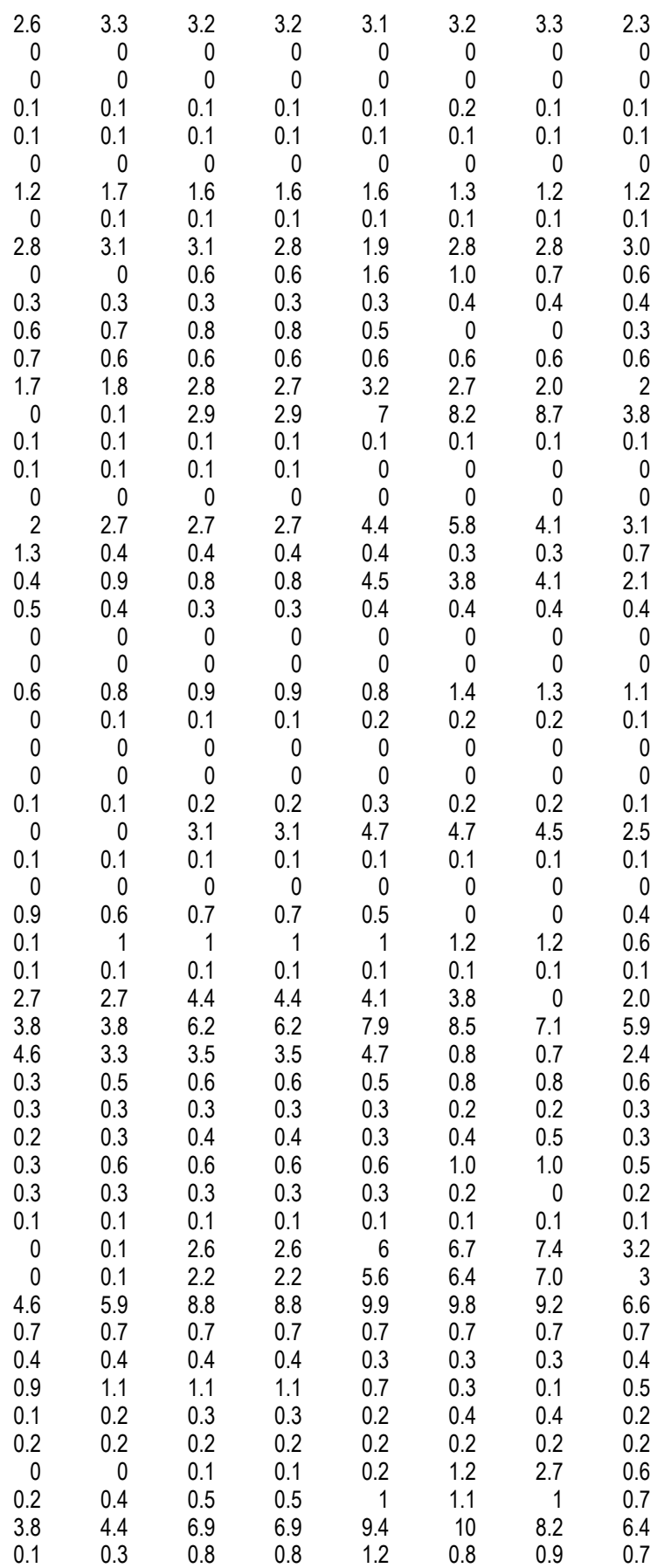

$\begin{array}{ll}0.9 & 0.1 \\ 0.9 & 27\end{array}$

$4.2 \quad 3.8$

$2.1 \quad 4.6$

0.3

0.2

0.3

0.3
0.1

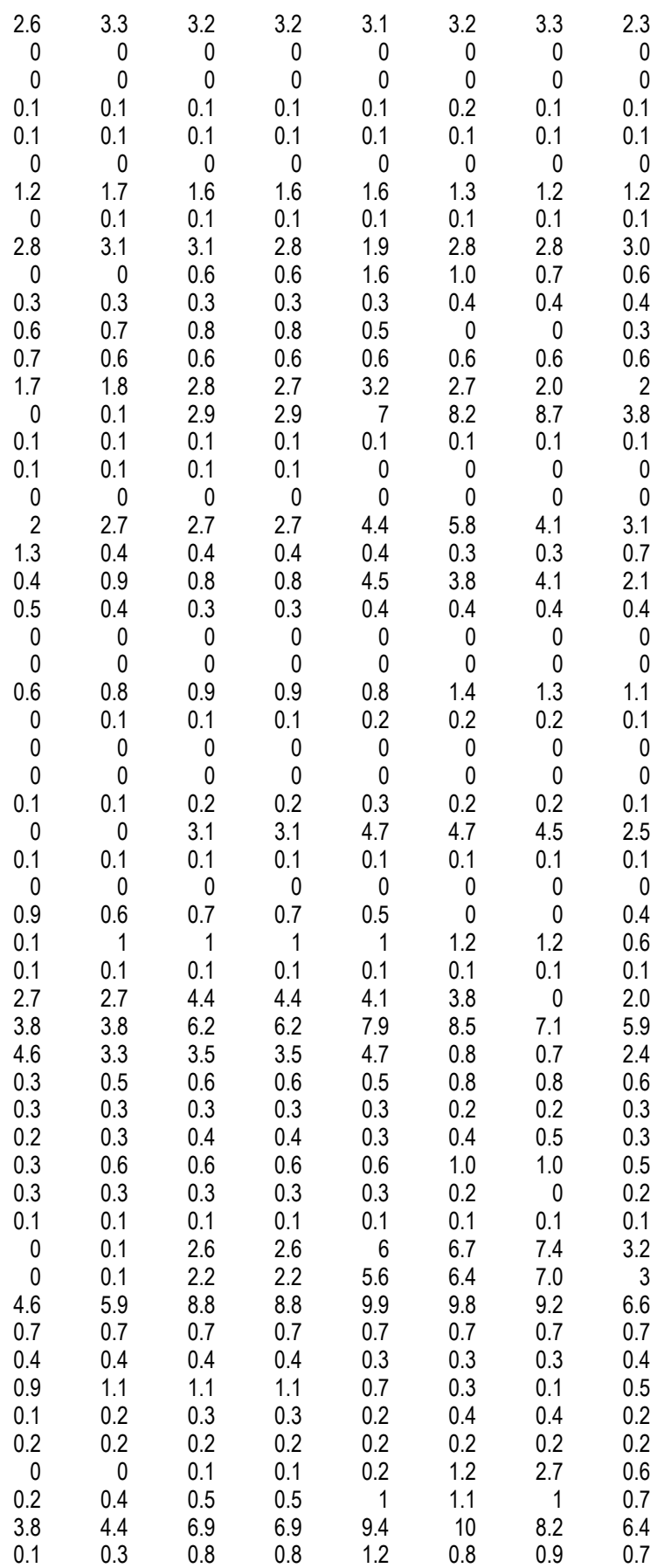

$\begin{array}{rr}0 & 0.1 \\ 4.6 & 5.9\end{array}$

0.7

0.4

0.9

0.9
0.1
0.2

0.2

0.2

$3.8 \quad 4.4$

$\begin{array}{llll}0.1 & 0.3 & 0.8 & 0.8\end{array}$

0.9

0.9
0.4

0.9

0.4

0.1

$\begin{array}{rr}0.1 & 0.1 \\ 0 & \end{array}$

\begin{tabular}{l}
0 \\
9 \\
0 \\
3 \\
0 \\
0 \\
\hline
\end{tabular}



2003 White Book

Aug1 Aug16 Sep Oct Nov Dec

Jan Feb Mar Apr1 Apr16

\begin{abstract}
May
\end{abstract}

$$
\text { Jun }
$$
Jul Avg

130 Lowline Canal (IPC)

131 Magic Reservoir (IPC)

132 Malad River (IPC)

133 Marcos Ranches (IPC)

134 Marsh Valley (UPL)

135 Meyers Falls (AVWP)

136 Middlefork Irrig. (PPL)

137 Mile 28 (IPC)

138 Minikahda (PGE)

139 Mink Creek (UPL)

140 Mitchell Butte (IPC)

141 Mountain Energy (PPL)

142 Mt. Tabor (PGE)

143 Mud Creek/S\&S (IPC)

144 Mud Creek/White (IPC)

145 Nichols Gap (PPL)

146 Nicholson Sunnybar (UPL)

147 North Fork Sprague (PPL)

148 O.J. Power (UPL)

149 Odell Creek (PPL)

150 Opal Springs (PPL)

151 Owyhee Dam (IPC)

152 Pancheri (UPL)

153 Pelton Rereg. Dam (PPL)

154 Phillips Ranch (AVWP)

155 Phillipsburg (NWE)

156 Pigeon Cove (IPC)

157 Pine Creek (NWE)

158 Port Townsend Paper Hydro (PSE)

159 Portland (PGE)

160 Portland Hydro Project (PGE)

161 Preston City (UPL)

162 Pristine Springs (IPC)

163 Pristine Springs \#3 (IPC)

164 Reynolds Irrigation (IPC)

165 Rim View (IPC)

166 Rock Creek \#1 (IPC)

167 Rock Creek \#2 (IPC)

168 Sagebrush (IPC)

169 Salem (PGE)

170 Schaffner (IPC)

171 Sheep Creek (AVWP)

172 Shingle Creek (IPC)

173 Shoshone (IPC)

174 Shoshone II (IPC)

175 Snake River Pottery (IPC)

176 Snedigar Ranch (IPC)

177 Solar Research (PPL)

178 South Dry Creek (NWE)

179 Spokane Upriver (AVWP)

180 Stauffer Dry Creek (UPL)

181 Strawberry Creek (NWE)

182 Sunshine Power \#2 (IPC)

183 Sygitowicz Creek Small Hydro (PSE)

184 TGS/Briggs (UPL)

185 Trout Co. (IPC)

186 Tunnel \#1 (IPC)

187 Twin Falls (PSE)

188 Walla Walla (PPL)

189 Water Street / Santiam (PPL)

190 Weeks Falls (PSE)

191 White James (PPL

192 White Water Ranch (IPC)

193 Wilson Lake Hydro (IPC)

194 Wisconsin Creek (NWE)

195 Yakima-Tieton (PPL)

196 IOU - Total NUG: Hydrc

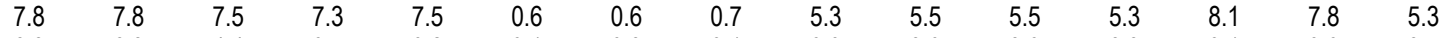

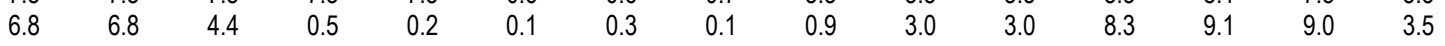

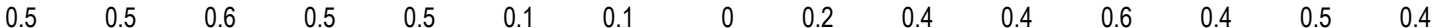

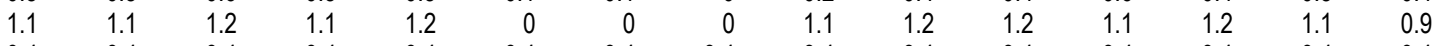

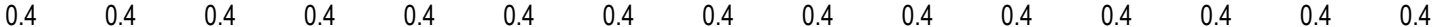

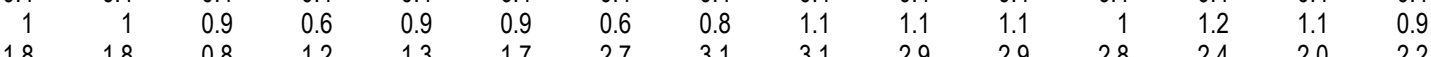

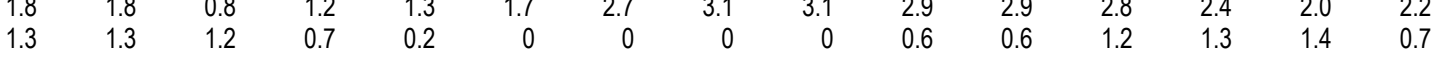

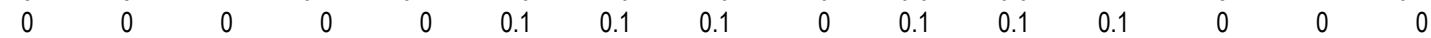

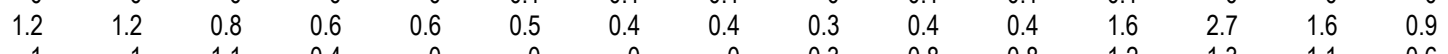

\begin{tabular}{|c|c|}
\hline & 1 \\
\hline
\end{tabular}

\begin{tabular}{|c|c|c|c|c|c|c|c|c|c|}
\hline \multirow[t]{2}{*}{0} & 0 & \multirow[t]{2}{*}{$\begin{array}{r}0 \\
01\end{array}$} & \multirow{2}{*}{$\begin{array}{r}0 \\
0.1\end{array}$} & \multirow[t]{2}{*}{$\begin{array}{r}0 \\
0\end{array}$} & \multirow[t]{2}{*}{$\begin{array}{r}0 \\
01\end{array}$} & \multirow[t]{2}{*}{$\begin{array}{r}0 \\
01\end{array}$} & \multirow[t]{2}{*}{0} & \multirow[t]{2}{*}{0} & \multirow[b]{2}{*}{1} \\
\hline & 0.1 & & & & & & & & \\
\hline
\end{tabular}

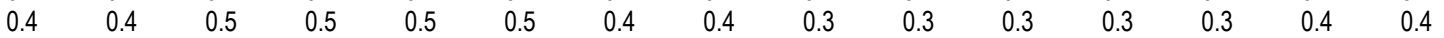

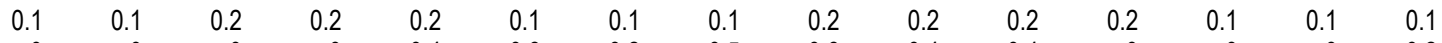

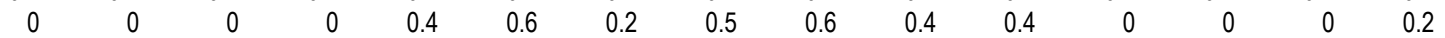

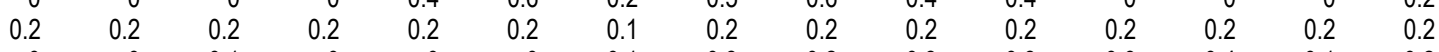

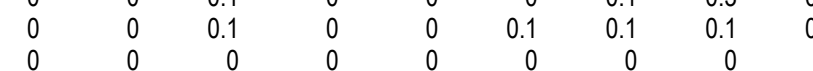

2.8

$\begin{array}{rrrrrrr}0 & 0 & 0 & 0 & 0 & 0.1 & 0.1 \\ 0.1 & 2.0 & 2.1 & 1.3 & 0.8 & 0.4 & 0.3 \\ 0.1 & 0.1 & 0.1 & 0.1 & 0.1 & 0.1\end{array}$

$\begin{array}{rrrrrrr}0.3 & 0.3 & 0.3 & 0.3 & 0.3 & 0.3 & 0.3 \\ 0 & 0 & 0 & 0 & 0 & 0 & 0\end{array}$

$\begin{array}{rrrrrrrrr}2.3 & 2.3 & 2.9 & 6.7 & 16 & 20 & 18 & 17 & 16 \\ 0.3 & 0.3 & 0.4 & 0.4 & 0.4 & 0.4 & 0.4 & 0.4 & 0.3\end{array}$

$\begin{array}{llllllll}0.1 & 0.1 & 0.2 & 0.2 & 0.1 & 0.1 & 0.1 & 0.1\end{array}$

$\begin{array}{lllllll}0.2 & 0.2 & 0.2 & 0.3 & 0.2 & 0.2 & 0.2\end{array}$

$\begin{array}{lllllll}0.2 & 0.2 & 0.2 & 0.2 & 0.2 & 0.2 & 0.2 \\ 0.2 & 0.2 & 0.2 & 0.3 & 0.2 & 0.2 & 0.2\end{array}$

$\begin{array}{lllllll}0.2 & 0.2 & 0.2 & 0.3 & 0.2 & 0.2 & 0.2 \\ 1.1 & 1.1 & 1.6 & 1.7 & 1.2 & 1.3 & 1.1 \\ 1.8 & 1.8 & 1.8 & 1.9 & 1.9 & 1.9 & 0.6\end{array}$

$\begin{array}{rrrrrrr}0.4 & 0.4 & 0.3 & 0.3 & 0.3 & 0.1 & 0.1 \\ 0 & 0 & 0 & 0 & 0 & 0 & 0\end{array}$

$\begin{array}{lllllllll}0.4 & 0.4 & 0.3 & 0.2 & 0.3 & 0.1 & 0.1 & 0.2 & 0.3\end{array}$

$\begin{array}{llllllll}0.4 & 0.4 & 0.3 & 0.3 & 0.4 & 0.5 & 0.5 & 0.5\end{array}$

$\begin{array}{lllllll}0.1 & 0.1 & 0.2 & 0.2 & 0.1 & 0.1 & 0.1 \\ 0.4 & 0.4 & 0.5 & 0.4 & 0.4 & 0.1 & 0.1\end{array}$

$\begin{array}{llll}0.4 & 0.4 & 0.5 & 0.4 \\ 0.6 & 0.6 & 0.6\end{array}$

$\begin{array}{rrrr}0 & 0 & 0 \\ 0 & 0.2 & 0.2\end{array}$

$\begin{array}{llll}0.2 & 0.2 & 0.2 & 0.2 \\ 0.1 & 0.1 & 0.1 \\ 0.5 & 0.5 & 0.5\end{array}$

$\begin{array}{llll}0.5 & 0.5 & 0.5 & 0.5 \\ 0.4 & 3.4 & 3.4 & 4.7\end{array}$

$\begin{array}{rrrrrrr}3.4 & 3.4 & 3.4 & 4.7 & 6.7 & 13 & 14 \\ 0.9 & 0.9 & 1 & 0.8 & 0.4 & 0 & 0.3 \\ 0.1 & 0.1 & 0.1 & 0.1 & 0.1 & 0.1 & 0.1\end{array}$

$\begin{array}{rrrrrrrr}0.1 & 0.1 & 0.1 & 0.1 & 0.1 & 0 & 0 & 0 \\ 0 & 0 & 0 & 0.1 & 0.2 & 0.3 & 0.3 & 0.4\end{array}$

$\begin{array}{llllllll}0.2 & 0.2 & 0.1 & 0.1 & 0.2 & 0.3 & 0.3 & 0.2 \\ 0.1 & 0.1 & 0.1 & 0.1 & 0.1 & 0.1 & 0.1 & 0.1\end{array}$

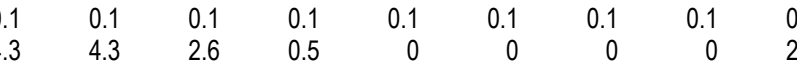

$\begin{array}{rrrrrrrrr}4 & 4.3 & 2.6 & 0.5 & 0 & 0 & 0 & 0 & 2.4 \\ 5 & 5 & 0 & 1.2 & 9.6 & 7.9 & 7.4 & 4.8 & 7.8\end{array}$

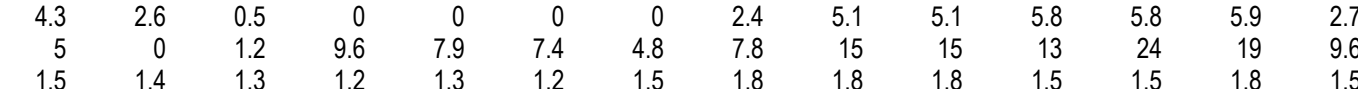

\begin{tabular}{|c|c|c|c|c|c|c|c|c|c|c|c|c|}
\hline 1.5 & 1.4 & 1.3 & 1.2 & 1.3 & 1.2 & 1.5 & 1.8 & 1.8 & 1.8 & 1.5 & 1.5 & 1.8 \\
\hline 0 & 0.1 & 0.1 & 0.2 & 0.2 & 0.1 & 0.1 & 0.1 & 0.2 & 0.2 & 0.1 & 0.1 & 0.1 \\
\hline
\end{tabular}

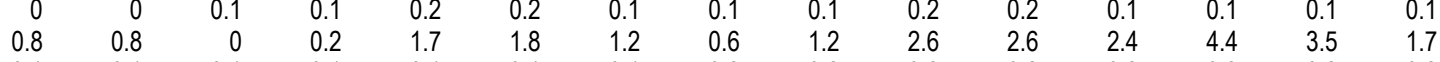

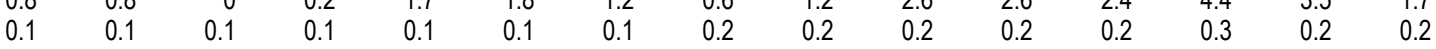

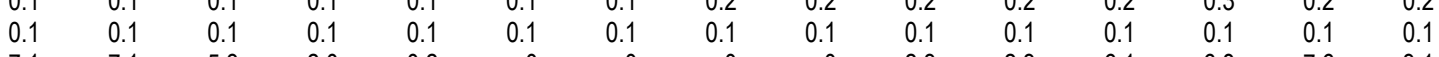

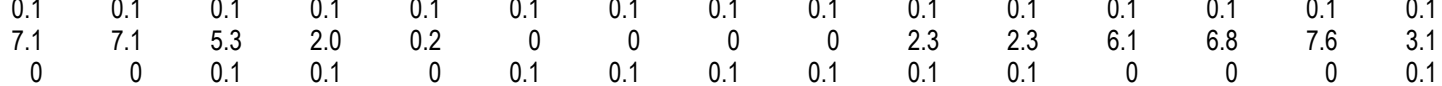

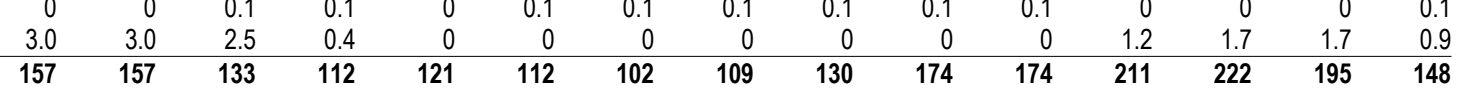

-NUG: Small Thermal \& Misc.-

197 Marion Solid Waste (PGE)

198 Minnesota Methane (AVWP)

199 Montana One (NWE)

200 Pocatello Waste (IPC)

201 Spokane MSW (PSE)

202 West Boise Waste (IPC)

\begin{tabular}{rrrrrrrrrrrrrrr}
9.3 & 9.3 & 9.3 & 9.3 & 9.3 & 9.3 & 9.3 & 9.3 & 9.3 & 9.3 & 9.3 & 9.3 & 9.3 & 9.3 & 9.3 \\
0.8 & 0.8 & 0.8 & 0.8 & 0.8 & 0.8 & 0.8 & 0.8 & 0.8 & 0.8 & 0.8 & 0.8 & 0.8 & 0.8 & 0.8 \\
9.6 & 9.8 & 9 & 9.1 & 9.3 & 10 & 11 & 10 & 9.2 & 9.2 & 9 & 11 & 9.1 & 8.6 & 9.6 \\
0.1 & 0.1 & 0.2 & 0.1 & 0.2 & 0.1 & 0.1 & 0.2 & 0.1 & 0.2 & 0.2 & 0.1 & 0.2 & 0.1 & 0.1 \\
15 & 15 & 15 & 15 & 15 & 15 & 15 & 15 & 15 & 15 & 15 & 15 & 15 & 15 & 15 \\
0.1 & 0.1 & 0.1 & 0.1 & 0.1 & 0.1 & 0.1 & 0.1 & 0.1 & 0.1 & 0.1 & 0.1 & 0.1 & 0.1 & 0.1 \\
\hline $\mathbf{3 5}$ & $\mathbf{3 5}$ & $\mathbf{3 5}$ & $\mathbf{3 5}$ & $\mathbf{3 5}$ & $\mathbf{3 6}$ & $\mathbf{3 6}$ & $\mathbf{3 6}$ & $\mathbf{3 5}$ & $\mathbf{3 5}$ & $\mathbf{3 5}$ & $\mathbf{3 6}$ & $\mathbf{3 5}$ & $\mathbf{3 5}$ & $\mathbf{3 5}$
\end{tabular}


Table A-24: Regional Non Utility Generating Resources By Project

PNW Loads and Resources Study

2013 - 2014 Operating Year 2003 White Book

Aug1 Aug16 Sep

Oct Nov

Dec Jan Feb Mar Apr1 Apr16

May Jun Jul Avg

-NUG: Combustion Turbines -

204 March Point \#1 Cogeneration (PSE)

205 March Point \#2 Cogeneration (PSE)

206 Rathdrum- Boekel Rd. \#1 (AVWP)

207 Rathdrum -Boekel Rd. \#2 (AVWP)

208 Tenaska Gas Cogen (PSE)

209 IOU - Total NUG: Combustion Turbines

\begin{tabular}{|c|c|c|c|c|c|c|c|c|c|c|c|c|c|c|}
\hline 79 & 79 & 79 & 79 & 79 & 79 & 79 & 79 & 79 & 79 & 79 & 55 & 79 & 79 & 77 \\
\hline 59 & 59 & 59 & 59 & 59 & 59 & 59 & 59 & 59 & 59 & 59 & 45 & 59 & 59 & 58 \\
\hline 65 & 65 & 65 & 65 & 65 & 65 & 65 & 65 & 65 & 65 & 65 & 32 & 65 & 65 & 62 \\
\hline 65 & 65 & 65 & 65 & 65 & 65 & 65 & 65 & 65 & 65 & 65 & 32 & 65 & 65 & 62 \\
\hline 240 & 240 & 240 & 240 & 240 & 240 & 240 & 240 & 240 & 240 & 240 & 180 & 240 & 240 & 235 \\
\hline 508 & 508 & 508 & 508 & 508 & 508 & 508 & 508 & 508 & 508 & 508 & 344 & 508 & 508 & 494 \\
\hline
\end{tabular}

-NUG: Co-Generation-

210 Billings Generation (NWE)

211 Biomass One (PPL)

212 Boise Cascade Medford (PPL)

213 Champion (PPL)

214 DR Johnson - Co-Gen II (PPL)

215 Magic Valley (IPC)

216 Magic West (IPC)

217 PERC Pierce, Wa (PSE)

218 Simplot Pocatello (IPC)

219 Sumas Energy (PSE)

220 Tamarack (IPC)

221 TASCO Nampa ID (IPC)

222 TASCO Twin Falls ID (IPC)

223 Vaagen Bros. (IPC)

224 Warm Springs (PPL)

225 IOU - Total NUG: Co-Generation

-NUG: Renewables-

226 Foote Creek 1 (PPL)

227 Healow \#2 (NWE)

228 Josef Staufer (NWE)

229 Lewandowski Farms (IPC)

230 Livingston (NWE)

231 Puyallup Energy Recovery (PSE)

232 Stateline Wind Project (AVWP)

233 Windmill (PGE)

234 IOU - Total NUG: Renewables

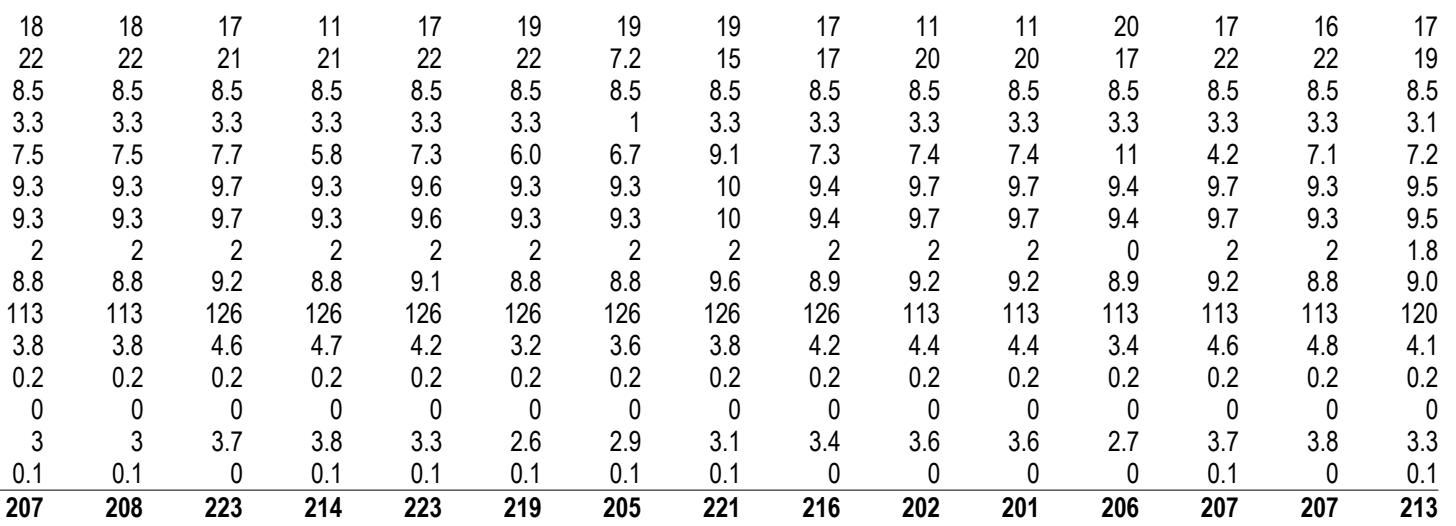

Other Entities

-NUG: Co-Generation-

235 SP Newsprint cogen (SPN)

236 Other - Total NUG: Co-Generation

$\begin{array}{lllllllllllllll}25 & 25 & 25 & 25 & 25 & 25 & 25 & 25 & 25 & 25 & 25 & 25 & 25 & 25 & 25 \\ \mathbf{2 5} & \mathbf{2 5} & \mathbf{2 5} & \mathbf{2 5} & \mathbf{2 5} & \mathbf{2 5} & \mathbf{2 5} & \mathbf{2 5} & \mathbf{2 5} & \mathbf{2 5} & \mathbf{2 5} & \mathbf{2 5} & \mathbf{2 5} & \mathbf{2 5} & \mathbf{2 5}\end{array}$

-NUG: Renewables-

237 Stateline Wind Project (PPME)

238 Vansycle Wind (FPLEV)

239 Other - Total NUG: Renewables

\begin{tabular}{|c|c|c|c|c|c|c|c|c|c|c|c|c|c|c|}
\hline 4.2 & 4.2 & 4.9 & 7.2 & 11 & 13 & 14 & 12 & 9.2 & 7.4 & 7.4 & 5.9 & 5.2 & 4.2 & 8.1 \\
\hline 0 & 0 & 0 & 0 & 0 & 0 & 0 & 0 & 0 & 0 & 0 & 0 & 0 & 0 & 0 \\
\hline 0 & 0 & 0 & 0 & 0 & 0 & 0 & 0 & 0 & 0 & 0 & 0 & 0 & 0 & 0 \\
\hline 0 & 0 & 0 & 0 & 0 & 0 & 0 & 0 & 0 & 0 & 0 & 0 & 0 & 0 & 0 \\
\hline 0 & 0 & 0 & 0 & 0 & 0 & 0 & 0 & 0 & 0 & 0 & 0 & 0 & 0 & 0 \\
\hline 2 & 2 & 2 & 2 & 2 & 2 & 2 & 2 & 2 & 2 & 2 & 2 & 2 & 2 & 2 \\
\hline 0.3 & 0.3 & 0.2 & 0.3 & 0.3 & 0.3 & 0.3 & 0.3 & 0.3 & 0.3 & 0.3 & 0.3 & 0.3 & 0.3 & 0.3 \\
\hline 0 & 0 & 0 & 0 & 0 & 0 & 0 & 0 & 0 & 0 & 0 & 0 & 0 & 0 & 0 \\
\hline 6.5 & 6.5 & 7.1 & 9.5 & 13 & 15 & 16 & 15 & 12 & 9.8 & 9.8 & 8.2 & 7.6 & 6.5 & 10 \\
\hline
\end{tabular}

-Total Non-Utility Generating Resources-

240 Federal Entities

241 Generating Public Entities

242 Non Generating Public Entities

243 Investor-Owned Entities

244 Other Entities

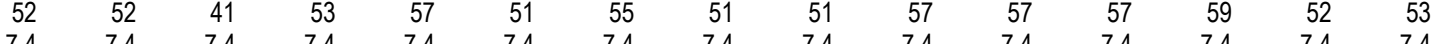

245 Total Non-Utility Generation

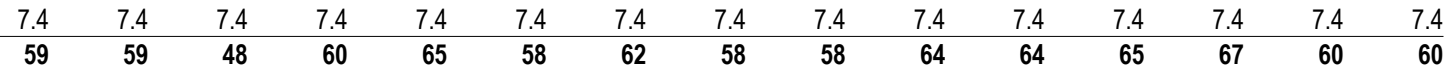

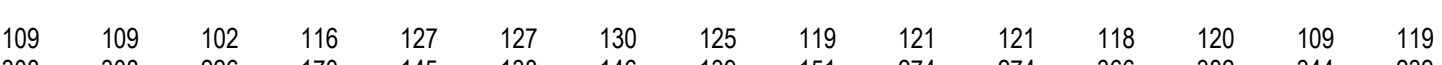

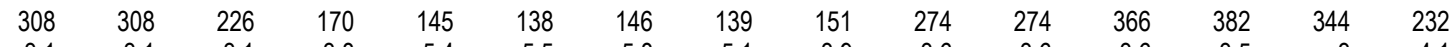

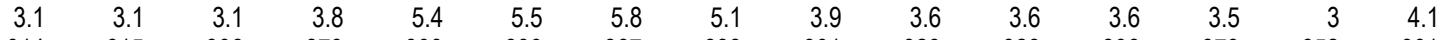

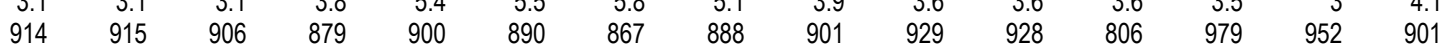

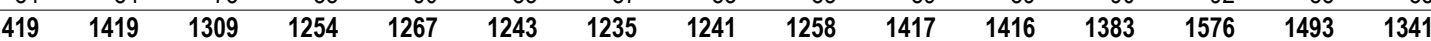


Table A-25: Dedicated Resources

PNW Loads and Resources Study

2004 - 2005 Operating Year

1937 Water Year

Average Energy in Megawatts

-Generating Public Entities-

1 Generating Public Enities

2 Total Generating Public Entities

-Non-Generating Public Entities-

3 Non-Generating Public Enities

4 Total Non-Generating Public Entities

-Investor-Owned Entities-

5 Investor-Owned Enities

6 Total Investor-owned Entities

-Total Dedicated Resources-

7 Generating Public Entities

8 Small \& Non Generating Entities

9 Investor-Owned Entities

10 Regional Total Dedicated Resources
Aug1 Aug16 Sep Oct Nov Dec Jan Feb Mar Apr1 Apr16 May Jun Jul Avg

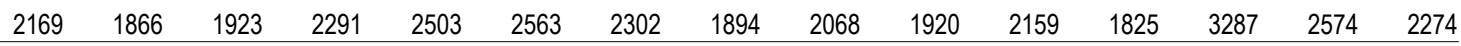

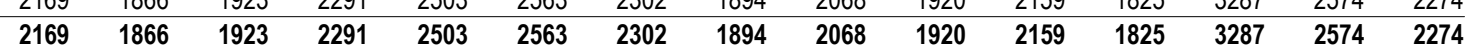

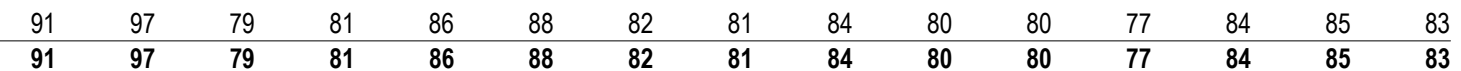

\begin{tabular}{|c|c|c|c|c|c|c|c|c|c|c|c|c|c|c|}
\hline 57 & 681 & 382 & 6653 & 6980 & 7090 & 6840 & 6292 & 6782 & 6791 & 6540 & 6535 & 7431 & 7179 & 6804 \\
\hline 95 & 6681 & 6382 & 6653 & 6980 & 7090 & 6840 & 6292 & 6782 & 6791 & 6540 & 6535 & 7431 & 7179 & \\
\hline
\end{tabular}

$\begin{array}{rrrrrrrrrrrrrrr}2169 & 1866 & 1923 & 2291 & 2503 & 2563 & 2302 & 1894 & 2068 & 1920 & 2159 & 1825 & 3287 & 2574 & 2274 \\ 91 & 97 & 79 & 81 & 86 & 88 & 82 & 81 & 84 & 80 & 80 & 77 & 84 & 85 & 83 \\ 6957 & 6681 & 6382 & 6653 & 6980 & 7090 & 6840 & 6292 & 6782 & 6791 & 6540 & 6535 & 7431 & 7179 & 6804 \\ \mathbf{9 2 1 7} & \mathbf{8 6 4 4} & \mathbf{8 3 8 4} & \mathbf{9 0 2 5} & \mathbf{9 5 6 9} & \mathbf{9 7 4 1} & \mathbf{9 2 2 5} & \mathbf{8 2 6 7} & \mathbf{8 9 3 4} & \mathbf{8 7 9 0} & \mathbf{8 7 7 9} & \mathbf{8 4 3 8} & \mathbf{1 0 8 0 1} & \mathbf{9 8 3 8} & \mathbf{9 1 6 1}\end{array}$


Table A-25: Dedicated Resources

PNW Loads and Resources Study

2005 - 2006 Operating Year

1937 Water Year

Average Energy in Megawatts

2003 White Book

-Generating Public Entities-

1 Generating Public Enities

2 Total Generating Public Entities

Aug1 Aug16 Sep Oct Nov Dec Jan Feb Mar Apr1 Apr16 May Jun Jul Avg

-Non-Generating Public Entities-

3 Non-Generating Public Enities

4 Total Non-Generating Public Entities

$\begin{array}{lllllllllllllll}2088 & 1801 & 1850 & 2197 & 2277 & 2338 & 2104 & 1699 & 1892 & 1908 & 2147 & 1646 & 3018 & 2313 & 2109 \\ \mathbf{2 0 8 8} & \mathbf{1 8 0 1} & \mathbf{1 8 5 0} & \mathbf{2 1 9 7} & \mathbf{2 2 7 7} & \mathbf{2 3 3 8} & \mathbf{2 1 0 4} & \mathbf{1 6 9 9} & \mathbf{1 8 9 2} & \mathbf{1 9 0 8} & \mathbf{2 1 4 7} & \mathbf{1 6 4 6} & \mathbf{3 0 1 8} & \mathbf{2 3 1 3} & \mathbf{2 1 0 9}\end{array}$

-Investor-Owned Entities-

5 Investor-Owned Enities

6 Total Investor-owned Entities

$85 \quad 81 \quad 74$

$74-81$

$\begin{array}{llllll}81 & 91 & 93 & 83 & 81 & 84\end{array}$

\begin{tabular}{lllllll}
84 & 85 & 85 & 84 & 93 & 90 & 85 \\
\hline
\end{tabular}

-Total Dedicated Resources-

7 Generating Public Entities

8 Small \& Non Generating Entities

$85 \quad 81 \quad 74$

7481

\begin{tabular}{|c|c|c|c|c|c|c|c|c|c|c|c|c|c|c|}
\hline 6958 & 6682 & 6388 & 6653 & 6960 & 7090 & 6863 & 6285 & 6849 & 7284 & 6913 & 6475 & 7851 & 7213 & 6879 \\
\hline 6958 & 6682 & 6388 & 6653 & 6960 & 7090 & 6863 & 6285 & 6849 & 7284 & 6913 & 6475 & 7851 & 7213 & 6879 \\
\hline
\end{tabular}

9 Investor-Owned Entities

\begin{tabular}{|c|c|c|c|c|c|c|c|c|c|c|c|c|c|c|}
\hline 2088 & 1801 & 1850 & 2197 & 2277 & 2338 & 2104 & 1699 & 1892 & 1908 & 2147 & 1646 & 3018 & 2313 & 2109 \\
\hline 85 & 81 & 74 & 81 & 91 & 93 & 83 & 81 & 84 & 85 & 85 & 84 & 93 & 90 & 85 \\
\hline 6958 & 6682 & 6388 & 6653 & 6960 & 7090 & 6863 & 6285 & 6849 & 7284 & 6913 & 6475 & 7851 & 7213 & 6879 \\
\hline 9131 & 8564 & 8312 & 8931 & 9328 & 9521 & 9049 & 8064 & 8825 & 9276 & 9145 & 8205 & 10961 & 9616 & 9072 \\
\hline
\end{tabular}


Table A-25: Dedicated Resources

PNW Loads and Resources Study

2006 - 2007 Operating Year

1937 Water Year

Average Energy in Megawatts

2003 White Book

-Generating Public Entities-

1 Generating Public Enities

2 Total Generating Public Entities

Aug1 Aug16 Sep Oct Nov Dec Jan Feb Mar Apr1 Apr16 May Jun Jul Avg

-Non-Generating Public Entities-

3 Non-Generating Public Enities

4 Total Non-Generating Public Entities

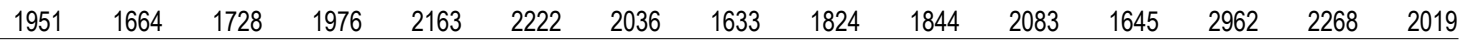

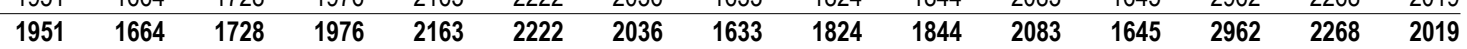

-Investor-Owned Entities-

5 Investor-Owned Enities

6 Total Investor-owned Entities

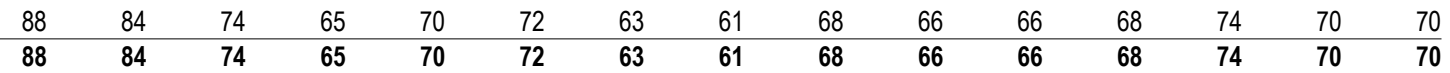

-Total Dedicated Resources-

7 Generating Public Entities

8 Small \& Non Generating Entities

9 Investor-Owned Entities

$\begin{array}{rrrrrrrrrrrrrrr}6941 & 6773 & 6482 & 6685 & 7006 & 7127 & 6853 & 6322 & 6874 & 7277 & 6815 & 6072 & 7015 & 7040 & 6781 \\ \mathbf{6 9 4 1} & \mathbf{6 7 7 3} & \mathbf{6 4 8 2} & \mathbf{6 6 8 5} & \mathbf{7 0 0 6} & \mathbf{7 1 2 7} & \mathbf{6 8 5 3} & \mathbf{6 3 2 2} & \mathbf{6 8 7 4} & \mathbf{7 2 7 7} & \mathbf{6 8 1 5} & \mathbf{6 0 7 2} & \mathbf{7 0 1 5} & \mathbf{7 0 4 0} & \mathbf{6 7 8 1} \\ & & & & & & & & & & & & & & \\ 1951 & 1664 & 1728 & 1976 & 2163 & 2222 & 2036 & 1633 & 1824 & 1844 & 2083 & 1645 & 2962 & 2268 & 2019 \\ 88 & 84 & 74 & 65 & 70 & 72 & 63 & 61 & 68 & 66 & 66 & 68 & 74 & 70 & 70 \\ 6941 & 6773 & 6482 & 6685 & 7006 & 7127 & 6853 & 6322 & 6874 & 7277 & 6815 & 6072 & 7015 & 7040 & 6781 \\ \mathbf{8 9 8 0} & \mathbf{8 5 2 1} & \mathbf{8 2 8 5} & \mathbf{8 7 2 6} & \mathbf{9 2 3 9} & \mathbf{9 4 2 1} & \mathbf{8 9 5 2} & \mathbf{8 0 1 6} & \mathbf{8 7 6 6} & \mathbf{9 1 8 6} & \mathbf{8 9 6 4} & \mathbf{7 7 8 4} & \mathbf{1 0 0 5 1} & \mathbf{9 3 7 7} & \mathbf{8 8 7 0}\end{array}$


Table A-25: Dedicated Resources

PNW Loads and Resources Study

2007 - 2008 Operating Year

1937 Water Year

Average Energy in Megawatts

2003 White Book

-Generating Public Entities-

1 Generating Public Enities

2 Total Generating Public Entities

Aug1 Aug16 Sep Oct Nov Dec Jan Feb

Mar Apr1 Apr16 May Jun Jul Avg

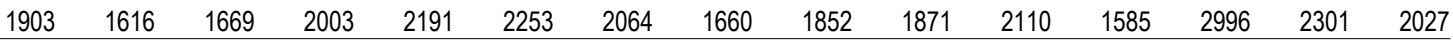

\begin{tabular}{|c|}
\hline 1903 \\
\hline
\end{tabular}

-Non-Generating Public Entities-

3 Non-Generating Public Enities

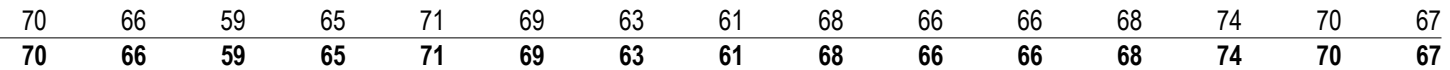

-Investor-Owned Entities-

5 Investor-Owned Enities

6 Total Investor-owned Entities

\begin{tabular}{|c|c|c|c|c|c|c|c|c|c|c|c|c|c|c|}
\hline 0 & 6787 & 6500 & 6697 & 7010 & $\begin{array}{l}7102 \\
710 ?\end{array}$ & 6868 & 6325 & 6887 & $\begin{array}{l}7346 \\
7346\end{array}$ & \begin{tabular}{|l|}
7177 \\
7177
\end{tabular} & $\begin{array}{l}6027 \\
6027\end{array}$ & $\begin{array}{l}7523 \\
7523\end{array}$ & $\begin{array}{l}7314 \\
7314\end{array}$ & \\
\hline 60 & 6787 & 6500 & 6697 & 7010 & 7102 & 6868 & 6325 & 6887 & 7346 & 7177 & 6027 & 7523 & 7314 & \\
\hline
\end{tabular}

-Total Dedicated Resources-

7 Generating Public Entities

8 Small \& Non Generating Entities

$1903 \quad 1616 \quad 1669$

9 Investor-Owned Entities

$\begin{array}{rrr}70 & 66 & \\ 7045 & 6787 & 6500\end{array}$

10 Regional Total Dedicated Resources

9018

$8469 \quad 82$

\begin{tabular}{rrrrrrrrrrrrr}
669 & 2003 & 2191 & 2253 & 2064 & 1660 & 1852 & 1871 & 2110 & 1585 & 2996 & 2301 & 2027 \\
59 & 65 & 71 & 69 & 63 & 61 & 68 & 66 & 66 & 68 & 74 & 70 & 67 \\
500 & 6697 & 7010 & 7102 & 6868 & 6325 & 6887 & 7346 & 7177 & 6027 & 7523 & 7314 & 6869 \\
\hline $\mathbf{8 2 9}$ & $\mathbf{8 7 6 5}$ & $\mathbf{9 2 7 1}$ & $\mathbf{9 4 2 3}$ & $\mathbf{8 9 9 5}$ & $\mathbf{8 0 4 6}$ & $\mathbf{8 8 0 7}$ & $\mathbf{9 2 8 3}$ & $\mathbf{9 3 5 3}$ & $\mathbf{7 6 8 0}$ & $\mathbf{1 0 5 9 3}$ & $\mathbf{9 6 8 5}$ & $\mathbf{8 9 6 3}$
\end{tabular}


Table A-25: Dedicated Resources

PNW Loads and Resources Study

2008 - 2009 Operating Year

1937 Water Year

Average Energy in Megawatts

2003 White Book

-Generating Public Entities-

1 Generating Public Enities

2 Total Generating Public Entities

Aug1 Aug16 Sep Oct Nov Dec Jan Feb Mar Apr1 Apr16 May Jun Jul Avg

-Non-Generating Public Entities-

3 Non-Generating Public Enities

4 Total Non-Generating Public Entities

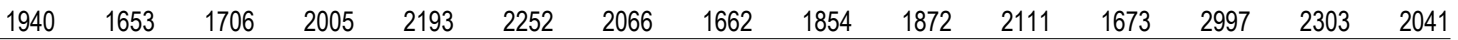

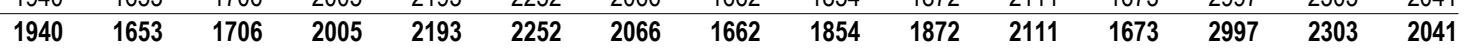

-Investor-Owned Entities-

5 Investor-Owned Enities

6 Total Investor-owned Entities

$\begin{array}{llll}70 & 66 & 59 & 64\end{array}$

$\begin{array}{llllll}64 & 70 & 71 & 62 & 60 & 67\end{array}$

-Total Dedicated Resources-

7 Generating Public Entities

8 Small \& Non Generating Entities

$70 \quad 66$

$\begin{array}{llll}64 & 70 & 71 & 62 \\ 64 & 70 & 71 & 62\end{array}$

$\begin{array}{lllllll}67 & 65 & 65 & 67 & 73 & 69 & 66 \\ 67 & 65 & 65 & 67 & 73 & 69 & 66\end{array}$

9 Investor-Owned Entities

$\begin{array}{lllllllllllllll}7052 & 6828 & 6454 & 6700 & 6984 & 7117 & 6870 & 6337 & 6887 & 7345 & 6890 & 6083 & 7648 & 7313 & 6871\end{array}$

10 Regional Total Dedicated Resources

$\begin{array}{llll}7052 & 6828 & 6454 & 6700\end{array}$

$6984 \quad 7117$

6870

$\begin{array}{rrrrrrrr}1940 & 1653 & 1706 & 2005 & 2193 & 2252 & 2066 & 1662 \\ 70 & 66 & 59 & 64 & 70 & 71 & 62 & 60 \\ 7052 & 6828 & 6454 & 6700 & 6984 & 7117 & 6870 & 6337 \\ \mathbf{9 0 6 2} & \mathbf{8 5 4 7} & \mathbf{8 2 1 9} & \mathbf{8 7 6 9} & \mathbf{9 2 4 6} & \mathbf{9 4 3 9} & \mathbf{8 9 9 7} & \mathbf{8 0 5 9}\end{array}$

1662
60
6337
8059

$\begin{array}{rrr}1854 & 1872 & 2111 \\ 67 & 65 & 65 \\ 6887 & 7345 & 6890 \\ 8807 & 9282 & 9066\end{array}$

\begin{tabular}{rrrr}
1673 & 2997 & 2303 & 2041 \\
67 & 73 & 69 & 66 \\
6083 & 7648 & 7313 & 6871 \\
\hline 7823 & 10718 & 9685 & 8978
\end{tabular}


Table A-25: Dedicated Resources

PNW Loads and Resources Study

2009 - 2010 Operating Year

1937 Water Year

Average Energy in Megawatts

2003 White Book

-Generating Public Entities-

1 Generating Public Enities

2 Total Generating Public Entities

Aug1 Aug16 Sep Oct Nov Dec Jan Feb Mar Apr1 Apr16 May Jun Jul Avg

-Non-Generating Public Entities-

3 Non-Generating Public Enities

4 Total Non-Generating Public Entities

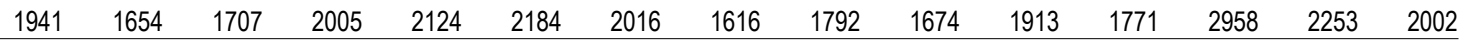

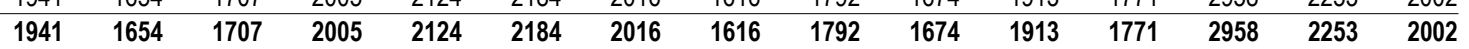

-Investor-Owned Entities-

5 Investor-Owned Enities

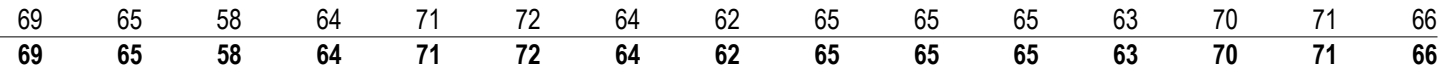

6 Total Investor-owned Entities

\begin{tabular}{|c|c|c|c|c|c|c|c|c|c|c|c|c|c|c|}
\hline 7017 & 6770 & 6456 & 6672 & 6923 & 7043 & 6827 & 6287 & 6795 & 6645 & 6569 & 6648 & 7217 & 7231 & 6800 \\
\hline 7017 & 6770 & 6456 & 6672 & 6923 & 7043 & 6827 & 6287 & 6795 & 6645 & 6569 & 6648 & 7217 & 7231 & 680 \\
\hline
\end{tabular}

-Total Dedicated Resources-

7 Generating Public Entitio

8 Small \& Non Generating Entities

9 Investor-Owned Entities

$\begin{array}{llll}7017 & 6770 \quad 6456 \quad 6672\end{array}$

69237043

\begin{tabular}{|c|c|c|c|c|c|c|c|c|c|c|c|c|c|c|}
\hline 1941 & 1654 & 1707 & 2005 & 2124 & 2184 & 2016 & 1616 & 1792 & 1674 & 1913 & 1771 & 2958 & 2253 & 2002 \\
\hline 69 & 65 & 58 & 64 & 71 & 72 & 64 & 62 & 65 & 65 & 65 & 63 & 70 & 71 & \\
\hline
\end{tabular}

$\begin{array}{llllll}9028 & 8489 & 8221 & 8742 & 9117 & 9299\end{array}$

$8907 \quad 7965$

8653

8384

8547

848210246

$9555 \quad 8868$


Table A-25: Dedicated Resources

PNW Loads and Resources Study

2010 - 2011 Operating Year

1937 Water Year

Average Energy in Megawatts

2003 White Book

-Generating Public Entities-

1 Generating Public Enities

Aug1 Aug16 Sep Oct Nov Dec Jan Feb Mar Apr1 Apr16 May Jun Jul Avg

2 Total Generating Public Entities

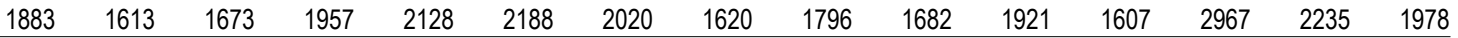

-Non-Generating Public Entities-

3 Non-Generating Public Enities

4 Total Non-Generating Public Entities

$1883 \quad 1613$

$613 \quad 1673$

\begin{tabular}{lllllllllllllll}
72 & 67 & 57 & 63 & 70 & 71 & 63 & 61 & 65 & 65 & 65 & 63 & 70 & 71 & 66 \\
\hline $\mathbf{7 2}$ & $\mathbf{6 7}$ & $\mathbf{5 7}$ & $\mathbf{6 3}$ & $\mathbf{7 0}$ & $\mathbf{7 1}$ & $\mathbf{6 3}$ & $\mathbf{6 1}$ & $\mathbf{6 5}$ & $\mathbf{6 5}$ & $\mathbf{6 5}$ & $\mathbf{6 3}$ & $\mathbf{7 0}$ & $\mathbf{7 1}$ & $\mathbf{6 6}$
\end{tabular}

-Investor-Owned Entities-

5 Investor-Owned Enities

6 Total Investor-owned Entities

\begin{tabular}{lllllllllllllll}
6932 & 6703 & 6381 & 6620 & 6837 & 6967 & 6740 & 6202 & 6720 & 6607 & 6298 & 6334 & 7603 & 7238 & 6743 \\
\hline $\mathbf{6 9 3 2}$ & $\mathbf{6 7 0 3}$ & $\mathbf{6 3 8 1}$ & $\mathbf{6 6 2 0}$ & $\mathbf{6 8 3 7}$ & $\mathbf{6 9 6 7}$ & $\mathbf{6 7 4 0}$ & $\mathbf{6 2 0 2}$ & $\mathbf{6 7 2 0}$ & $\mathbf{6 6 0 7}$ & $\mathbf{6 2 9 8}$ & $\mathbf{6 3 3 4}$ & $\mathbf{7 6 0 3}$ & $\mathbf{7 2 3 8}$ & $\mathbf{6 7 4 3}$
\end{tabular}

-Total Dedicated Resources-

7 Generating Public Entities

8 Small \& Non Generating Entities

9 Investor-Owned Entities

10 Regional Total Dedicated Resources

$\begin{array}{rrrrrrrrrrrrrrr}1883 & 1613 & 1673 & 1957 & 2128 & 2188 & 2020 & 1620 & 1796 & 1682 & 1921 & 1607 & 2967 & 2235 & 1978 \\ 72 & 67 & 57 & 63 & 70 & 71 & 63 & 61 & 65 & 65 & 65 & 63 & 70 & 71 & 66 \\ 6932 & 6703 & 6381 & 6620 & 6837 & 6967 & 6740 & 6202 & 6720 & 6607 & 6298 & 6334 & 7603 & 7238 & 6743 \\ \mathbf{8 8 8 7} & \mathbf{8 3 8 3} & \mathbf{8 1 1 1} & \mathbf{8 6 4 0} & \mathbf{9 0 3 4} & \mathbf{9 2 2 6} & \mathbf{8 8 2 3} & \mathbf{7 8 8 3} & \mathbf{8 5 8 1} & \mathbf{8 3 5 4} & \mathbf{8 2 8 4} & \mathbf{8 0 0 4} & \mathbf{1 0 6 3 9} & \mathbf{9 5 4 5} & \mathbf{8 7 8 7}\end{array}$


Table A-25: Dedicated Resources

PNW Loads and Resources Study

2011 - 2012 Operating Year

1937 Water Year

Average Energy in Megawatts

2003 White Book

-Generating Public Entities-

1 Generating Public Enities

2 Total Generating Public Entities

Aug1 Aug16 Sep Oct Nov Dec Jan Feb Mar Apr1 Apr16 May Jun Jul Avg

-Non-Generating Public Entities-

3 Non-Generating Public Enities

4 Total Non-Generating Public Entities

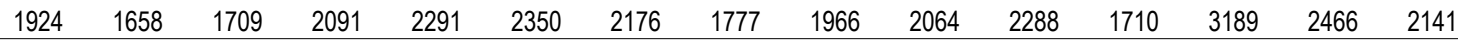

\begin{tabular}{|c|}
\hline 192 \\
\hline
\end{tabular}

-Investor-Owned Entities-

5 Investor-Owned Enities

6 Total Investor-owned Entities

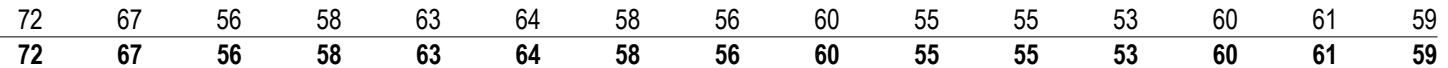

-Total Dedicated Resources-

7 Generating Public Entities

8 Small \& Non Generating Entities

9 Investor-Owned Entities

\begin{tabular}{lllllllllllllll}
6931 & 6674 & 6393 & 6498 & 6721 & 6839 & 6627 & 6088 & 6637 & 7037 & 6871 & 5788 & 7390 & 7062 & 6650 \\
\hline 6931 & $\mathbf{6 6 7 4}$ & $\mathbf{6 3 9 3}$ & $\mathbf{6 4 9 8}$ & $\mathbf{6 7 2 1}$ & $\mathbf{6 8 3 9}$ & $\mathbf{6 6 2 7}$ & $\mathbf{6 0 8 8}$ & $\mathbf{6 6 3 7}$ & $\mathbf{7 0 3 7}$ & $\mathbf{6 8 7 1}$ & $\mathbf{5 7 8 8}$ & $\mathbf{7 3 9 0}$ & $\mathbf{7 0 6 2}$ & $\mathbf{6 6 5 0}$
\end{tabular}

10 Regional Total Dedicated Resources

$\begin{array}{rrr}1924 & 1658 & 17 \\ 72 & 67 & \\ 6931 & 6674 & 6393 \\ \mathbf{8 9 2 7} & \mathbf{8 3 9 9} & \mathbf{8 1}\end{array}$

\begin{tabular}{rrrrrrrrrrrrr}
1709 & 2091 & 2291 & 2350 & 2176 & 1777 & 1966 & 2064 & 2288 & 1710 & 3189 & 2466 & 2141 \\
56 & 58 & 63 & 64 & 58 & 56 & 60 & 55 & 55 & 53 & 60 & 61 & 59 \\
6393 & 6498 & 6721 & 6839 & 6627 & 6088 & 6637 & 7037 & 6871 & 5788 & 7390 & 7062 & 6650 \\
\hline $\mathbf{8 1 5 9}$ & $\mathbf{8 6 4 7}$ & $\mathbf{9 0 7 4}$ & $\mathbf{9 2 5 3}$ & $\mathbf{8 8 6 1}$ & $\mathbf{7 9 2 1}$ & $\mathbf{8 6 6 3}$ & $\mathbf{9 1 5 7}$ & $\mathbf{9 2 1 3}$ & $\mathbf{7 5 5 1}$ & $\mathbf{1 0 6 3 9}$ & $\mathbf{9 5 8 9}$ & $\mathbf{8 8 5 0}$
\end{tabular}


Table A-25: Dedicated Resources

PNW Loads and Resources Study

2012 - 2013 Operating Year

1937 Water Year

Average Energy in Megawatts

-Generating Public Entities-

1 Generating Public Enities

2 Total Generating Public Entities

-Non-Generating Public Entities-

3 Non-Generating Public Enities

4 Total Non-Generating Public Entities

-Investor-Owned Entities-

5 Investor-Owned Enities

6 Total Investor-owned Entities

-Total Dedicated Resources-

7 Generating Public Entities

8 Small \& Non Generating Entities

9 Investor-Owned Entities

10 Regional Total Dedicated Resources
Aug1 Aug16 Sep Oct Nov Dec Jan Feb Mar Apr1 Apr16 May Jun Jul Avg

\begin{tabular}{|c|c|c|c|c|c|c|c|c|c|c|c|c|c|c|}
\hline 2066 & 1801 & 1842 & 2098 & 2297 & 2356 & 2182 & 1783 & 1972 & 2070 & 2293 & 1789 & 3195 & 2472 & 2175 \\
\hline 2066 & 1801 & 1842 & 2098 & 2297 & 2356 & 2182 & 1783 & 1972 & 2070 & 2293 & 1789 & 3195 & 2472 & 2175 \\
\hline
\end{tabular}

$\begin{array}{lllllllllllllll}61 & 57 & 50 & 58 & 62 & 63 & 58 & 56 & 60 & 55 & 55 & 53 & 59 & 61 & 58 \\ \mathbf{6 1} & \mathbf{5 7} & \mathbf{5 0} & \mathbf{5 8} & \mathbf{6 2} & \mathbf{6 3} & \mathbf{5 8} & \mathbf{5 6} & \mathbf{6 0} & \mathbf{5 5} & \mathbf{5 5} & \mathbf{5 3} & \mathbf{5 9} & \mathbf{6 1} & \mathbf{5 8}\end{array}$

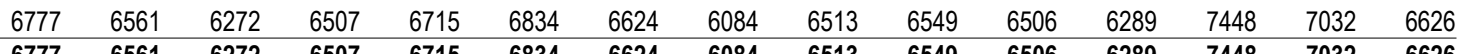

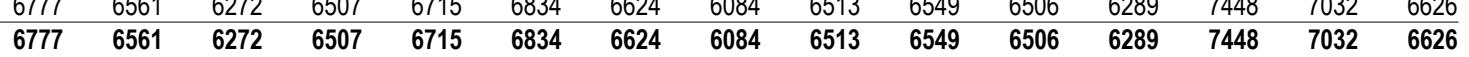

$\begin{array}{rrrrrrrrrrrrrrr}2066 & 1801 & 1842 & 2098 & 2297 & 2356 & 2182 & 1783 & 1972 & 2070 & 2293 & 1789 & 3195 & 2472 & 2175 \\ 61 & 57 & 50 & 58 & 62 & 63 & 58 & 56 & 60 & 55 & 55 & 53 & 59 & 61 & 58 \\ 6777 & 6561 & 6272 & 6507 & 6715 & 6834 & 6624 & 6084 & 6513 & 6549 & 6506 & 6289 & 7448 & 7032 & 6626 \\ \mathbf{8 9 0 4} & \mathbf{8 4 1 8} & \mathbf{8 1 6 4} & \mathbf{8 6 6 2} & \mathbf{9 0 7 4} & \mathbf{9 2 5 4} & \mathbf{8 8 6 4} & \mathbf{7 9 2 3} & \mathbf{8 5 4 5} & \mathbf{8 6 7 3} & \mathbf{8 8 5 4} & \mathbf{8 1 3 1} & \mathbf{1 0 7 0 2} & \mathbf{9 5 6 5} & \mathbf{8 8 5 9}\end{array}$


Table A-25: Dedicated Resources

PNW Loads and Resources Study

2013 - 2014 Operating Year

1937 Water Year

Average Energy in Megawatts

2003 White Book

-Generating Public Entities-

1 Generating Public Enities

Aug1 Aug16 Sep Oct Nov Dec Jan Feb Mar Apr1 Apr16 May Jun Jul Avg

2 Total Generating Public Entities

$\begin{array}{lllllllllllllll}2078 & 1813 & 1854 & 2110 & 2310 & 2369 & 2195 & 1796 & 1985 & 2081 & 2305 & 1790 & 3207 & 2484 & 2186 \\ \mathbf{2 0 7 8} & \mathbf{1 8 1 3} & \mathbf{1 8 5 4} & \mathbf{2 1 1 0} & \mathbf{2 3 1 0} & \mathbf{2 3 6 9} & \mathbf{2 1 9 5} & \mathbf{1 7 9 6} & \mathbf{1 9 8 5} & \mathbf{2 0 8 1} & \mathbf{2 3 0 5} & \mathbf{1 7 9 0} & \mathbf{3 2 0 7} & \mathbf{2 4 8 4} & \mathbf{2 1 8 6}\end{array}$

-Non-Generating Public Entities-

3 Non-Generating Public Enities

4 Total Non-Generating Public Entities

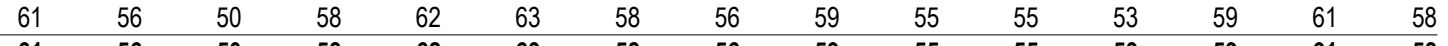

-Investor-Owned Entities-

5 Investor-Owned Enities

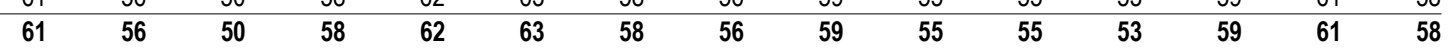

6 Total Investor-owned Entities

\begin{tabular}{|c|c|c|c|c|c|c|c|c|c|c|c|c|c|c|}
\hline 6786 & 6558 & 6280 & 6503 & 6711 & 6831 & 6621 & 6087 & 6638 & 7029 & 6871 & 6200 & 7407 & 7042 & 6662 \\
\hline 6786 & 6558 & 6280 & 6503 & 6711 & 6831 & 6621 & 6087 & 6638 & 7029 & 6871 & 6200 & 7407 & 7042 & \\
\hline
\end{tabular}

-Total Dedicated Resources-

7 Generating Public Entities

8 Small \& Non Generating Entities

9 Investor-Owned Entities

$6780 \quad 6558-6280 \quad 6503$

10 Regional Total Dedicated Resources

\begin{tabular}{rrrrrrrrrrrrrrr}
2078 & 1813 & 1854 & 2110 & 2310 & 2369 & 2195 & 1796 & 1985 & 2081 & 2305 & 1790 & 3207 & 2484 & 2186 \\
61 & 56 & 50 & 58 & 62 & 63 & 58 & 56 & 59 & 55 & 55 & 53 & 59 & 61 & 58 \\
6786 & 6558 & 6280 & 6503 & 6711 & 6831 & 6621 & 6087 & 6638 & 7029 & 6871 & 6200 & 7407 & 7042 & 6662 \\
\hline $\mathbf{8 9 2 6}$ & $\mathbf{8 4 2 7}$ & $\mathbf{8 1 8 4}$ & $\mathbf{8 6 7 0}$ & $\mathbf{9 0 8 2}$ & $\mathbf{9 2 6 3}$ & $\mathbf{8 8 7 3}$ & $\mathbf{7 9 3 8}$ & $\mathbf{8 6 8 2}$ & $\mathbf{9 1 6 5}$ & $\mathbf{9 2 3 1}$ & $\mathbf{8 0 4 3}$ & $\mathbf{1 0 6 7 3}$ & $\mathbf{9 5 8 7}$ & $\mathbf{8 9 0 6}$
\end{tabular}


Table A-26: Other Dedicated Resources

PNW Loads and Resources Study

2005 - 2006 Operating Year

1937 Water Year

Average Energy in Megawatts

2003 White Book

-Generating Public Entities-

1 Generating Public Enities

2 Total Generating Public Entities

Aug1 Aug16 Sep Oct Nov Dec Jan Feb Mar Apr1 Apr16 May Jun Jul Avg

-Non-Generating Public Entities-

3 Small \& Non Generating Public Enities

4 Total Non-Generating Public Entities

$\begin{array}{lllllllllllllll}3613 & 3259 & 3152 & 3431 & 4101 & 4368 & 4168 & 4001 & 3866 & 3394 & 3345 & 3374 & 3382 & 3457 & 3675 \\ \mathbf{3 6 1 3} & \mathbf{3 2 5 9} & \mathbf{3 1 5 2} & \mathbf{3 4 3 1} & \mathbf{4 1 0 1} & \mathbf{4 3 6 8} & \mathbf{4 1 6 8} & \mathbf{4 0 0 1} & \mathbf{3 8 6 6} & \mathbf{3 3 9 4} & \mathbf{3 3 4 5} & \mathbf{3 3 7 4} & \mathbf{3 3 8 2} & \mathbf{3 4 5 7} & \mathbf{3 6 7 5}\end{array}$

-Investor-Owned Entities-

5 Investor-Owned Enities

6 Total Investor-owned Entities

\begin{tabular}{|c|c|c|c|c|c|c|c|c|c|c|c|c|c|c|}
\hline 580 & 3394 & 3030 & 3123 & 3477 & 3892 & 3876 & 3626 & 3396 & 3208 & 3181 & 3213 & 3375 & 3574 & 3439 \\
\hline 568 & 3394 & 3030 & 3123 & 3477 & 3892 & 3876 & 3626 & 3396 & 3208 & 3181 & 3213 & 3375 & 3574 & \\
\hline
\end{tabular}

-Total Dedicated Resources-

7 Generating Public Entitie

8 Non Generating Entities

9 Investor-Owned Entities

\begin{tabular}{lllllllllllllll}
3651 & 3646 & 3581 & 3699 & 3871 & 4026 & 4087 & 3743 & 3507 & 3200 & 3210 & 2060 & 3435 & 3135 & 3500 \\
\hline 3651 & $\mathbf{3 6 4 6}$ & $\mathbf{3 5 8 1}$ & $\mathbf{3 6 9 9}$ & $\mathbf{3 8 7 1}$ & $\mathbf{4 0 2 6}$ & $\mathbf{4 0 8 7}$ & $\mathbf{3 7 4 3}$ & $\mathbf{3 5 0 7}$ & $\mathbf{3 2 0 0}$ & $\mathbf{3 2 1 0}$ & $\mathbf{2 0 6 0}$ & $\mathbf{3 4 3 5}$ & $\mathbf{3 1 3 5}$ & $\mathbf{3 5 0 0}$
\end{tabular}

\begin{tabular}{|c|c|c|c|c|c|c|c|c|c|c|c|c|c|}
\hline 3259 & 3152 & 3431 & 4101 & 4368 & 4168 & 4001 & 3866 & 3394 & 3345 & 3374 & 3382 & 3457 & 3675 \\
\hline 3394 & 3030 & 3123 & 3477 & 3892 & 3876 & 3626 & 3396 & 3208 & 3181 & 3213 & 3375 & 3574 & 3439 \\
\hline 3646 & 3581 & 3699 & 3871 & 4026 & 4087 & 3743 & 3507 & 3200 & 3210 & 2060 & 3435 & 3135 & 3500 \\
\hline 10298 & 9762 & 10253 & 11449 & 12285 & 12131 & 11371 & 10770 & 9802 & 9736 & 8647 & 10193 & 10166 & 10614 \\
\hline
\end{tabular}


Table A-26: Other Dedicated Resources

PNW Loads and Resources Study

2006 - 2007 Operating Year

1937 Water Year

2003 White Book

Average Energy in Megawatts Aug1 Aug16 Sep Oct Nov Dec Jan Feb Mar Apr1 Apr16 May Jun Jul Avg

-Generating Public Entities-

1 Generating Public Enities

2 Total Generating Public Entities

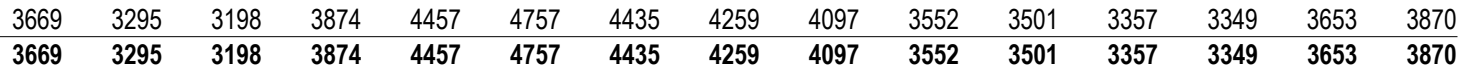

-Non-Generating Public Entities-

3 Small \& Non Generating Public Enities

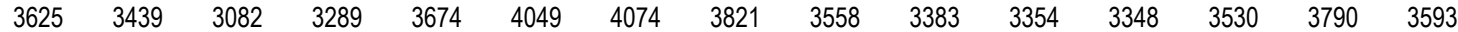

4 Total Non-Generating Public Entities

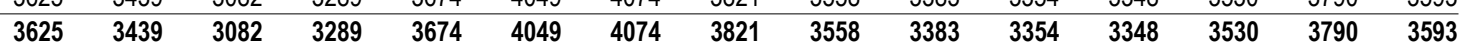

-Investor-Owned Entities-

5 Investor-Owned Enitie

6 Total Investor-owned Entities

$\begin{array}{lll}3263 & 3836 & 37 \\ \mathbf{3 2 6 3} & \mathbf{3 8 3 6} & \mathbf{3 7}\end{array}$

-Total Dedicated Resources-

7 Generating Public Entitio

8 Non Generating Entities

$\begin{array}{lll}3263 & 3836 & 374 \\ 3263 & 3836 & 3742\end{array}$

$\begin{array}{lll}3257 & 347\end{array}$

Investor-Owned Entities

\begin{tabular}{|c|c|c|c|c|c|c|c|c|c|c|c|c|c|c|}
\hline 3669 & 3295 & 3198 & 3874 & 4457 & 4757 & 4435 & 4259 & 4097 & 3552 & 3501 & 3357 & 3349 & 3653 & 3870 \\
\hline 3625 & 3439 & 3082 & 3289 & 3674 & 4049 & 4074 & 3821 & 3558 & 3383 & 3354 & 3348 & 3530 & 3790 & 3593 \\
\hline 3263 & 3836 & 3742 & 3257 & 3474 & 3564 & 3590 & 3408 & 3119 & 2463 & 2391 & 1697 & 3030 & 3299 & 3180 \\
\hline 557 & 10570 & 10022 & 10420 & 11605 & 12371 & 12100 & 11487 & 10773 & 9397 & 9246 & 8402 & 9910 & 10742 & 10643 \\
\hline
\end{tabular}


Table A-26: Other Dedicated Resources

PNW Loads and Resources Study

2007 - 2008 Operating Year

1937 Water Year

2003 White Book

Average Energy in Megawatts Aug1 Aug16 Sep Oct Nov Dec Jan Feb Mar Apr1 Apr16 May Jun Jul Avg

-Generating Public Entities-

1 Generating Public Enities

2 Total Generating Public Entities

\begin{tabular}{|c|c|c|c|c|c|c|c|c|c|c|c|c|c|}
\hline 35 & 3559 & 3427 & 3794 & 4381 & 4682 & 4454 & 4258 & 4114 & 3564 & 3513 & 3467 & 3469 & 3668 \\
\hline & & 3427 & 3794 & 4381 & 4682 & 4454 & 4258 & 4114 & 3564 & 3513 & 3467 & 3469 & 00 \\
\hline
\end{tabular}

-Non-Generating Public Entities-

3 Small \& Non Generating Public Enities

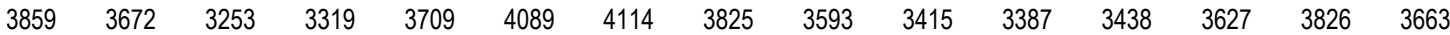

4 Total Non-Generating Public Entities

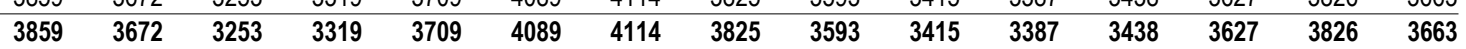

-Investor-Owned Entities-

5 Investor-Owned Enitie

6 Total Investor-owned Entities

$3407 \quad 3404 \quad 3255 \quad 3345$

-Total Dedicated Resources-

7 Generating Public Entitio

8 Non Generating Entities

3407

9 Investor-Owned Entities

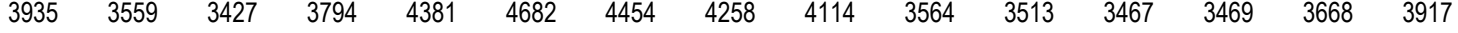

10 Regional Total Dedicated Resources

$\begin{array}{lllll}3407 & 3404 & 3255 & 3345 & 3594\end{array}$

$11201 \quad 10635 \quad 9936$

\begin{tabular}{rrrr}
355 & 3345 & 3594 & 3598 \\
\hline 36 & 10458 & 11684 & 12370
\end{tabular}

$4114 \quad 3825$

$3593 \quad 3415$

3387

$\begin{array}{rrrrrrrrr}3437 & 3291 & 3200 & 2790 & 2687 & 1855 & 2923 & 3446 & 3174\end{array}$

$3438 \quad 3627 \quad 3826 \quad 3663$


Table A-26: Other Dedicated Resources

PNW Loads and Resources Study

2009 - 2010 Operating Year

1937 Water Year

Average Energy in Megawatts

2003 White Book

-Generating Public Entities-

1 Generating Public Enities

2 Total Generating Public Entities

Aug1 Aug16 Sep Oct Nov Dec Jan Feb Mar Apr1 Apr16 May Jun Jul Avg

-Non-Generating Public Entities-

3 Small \& Non Generating Public Enities

4 Total Non-Generating Public Entities

\begin{tabular}{lllllllllllllll} 
& & & & & & & & & & & & & & \\
3961 & 3584 & 3449 & 3820 & 4554 & 4853 & 4588 & 4399 & 4247 & 3680 & 3627 & 3579 & 3626 & 3813 & 4029 \\
\hline 3961 & $\mathbf{3 5 8 4}$ & $\mathbf{3 4 4 9}$ & $\mathbf{3 8 2 0}$ & $\mathbf{4 5 5 4}$ & $\mathbf{4 8 5 3}$ & $\mathbf{4 5 8 8}$ & $\mathbf{4 3 9 9}$ & $\mathbf{4 2 4 7}$ & $\mathbf{3 6 8 0}$ & $\mathbf{3 6 2 7}$ & $\mathbf{3 5 7 9}$ & $\mathbf{3 6 2 6}$ & $\mathbf{3 8 1 3}$ & $\mathbf{4 0 2 9}$
\end{tabular}

-Investor-Owned Entities-

5 Investor-Owned Enities

6 Total Investor-owned Entities

\begin{tabular}{lllllllllllllll}
3925 & 3737 & 3313 & 3379 & 3777 & 4167 & 4201 & 3940 & 3667 & 3486 & 3456 & 3504 & 3694 & 3896 & 3737 \\
\hline 3925 & 3737 & 3313 & 3379 & 3777 & 4167 & $\mathbf{4 2 0 1}$ & $\mathbf{3 9 4 0}$ & $\mathbf{3 6 6 7}$ & $\mathbf{3 4 8 6}$ & $\mathbf{3 4 5 6}$ & $\mathbf{3 5 0 4}$ & $\mathbf{3 6 9 4}$ & $\mathbf{3 8 9 6}$ & $\mathbf{3 7 3 7}$
\end{tabular}

-Total Dedicated Resources-

7 Generating Public Entitie

8 Non Generating Entities

$\begin{array}{llll}3544 & 3577 & 3481 & 3257\end{array}$

3544

$577 \quad 348$

9 Investor-Owned Entities

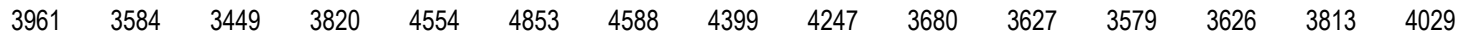

$\begin{array}{lllllllllllllll}3925 & 3737 & 3313 & 3379 & 3777 & 4167 & 4201 & 3940 & 3667 & 3486 & 3456 & 3504 & 3694 & 3896 & 3737\end{array}$

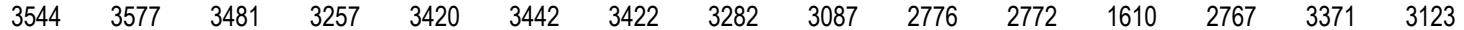

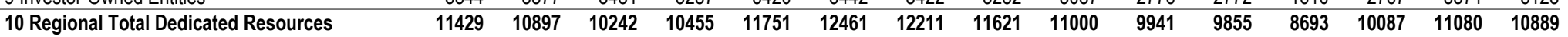


Table A-26: Other Dedicated Resources

PNW Loads and Resources Study

2010 - 2011 Operating Year

1937 Water Year

2003 White Book

Average Energy in Megawatts Aug1 Aug16 Sep Oct Nov Dec Jan Feb Mar Apr1 Apr16 May Jun Jul Avg

-Generating Public Entities-

1 Generating Public Enities

2 Total Generating Public Entities

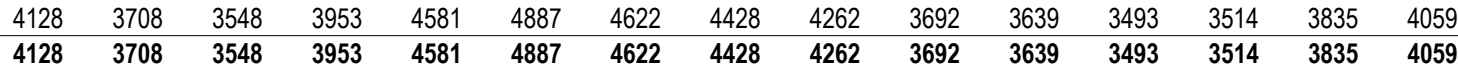

-Non-Generating Public Entities-

3 Small \& Non Generating Public Enities

\begin{tabular}{lllllllllllllll}
3961 & 3773 & 3346 & 3412 & 3814 & 4209 & 4239 & 3978 & 3701 & 3517 & 3487 & 3476 & 3662 & 3929 & 3761 \\
\hline 3961 & 3773 & 3346 & 3412 & $\mathbf{3 8 1 4}$ & $\mathbf{4 2 0 9}$ & $\mathbf{4 2 3 9}$ & $\mathbf{3 9 7 8}$ & $\mathbf{3 7 0 1}$ & $\mathbf{3 5 1 7}$ & $\mathbf{3 4 8 7}$ & $\mathbf{3 4 7 6}$ & $\mathbf{3 6 6 2}$ & $\mathbf{3 9 2 9}$ & $\mathbf{3 7 6 1}$
\end{tabular}

-Investor-Owned Entities-

5 Investor-Owned Enities

6 Total Investor-owned Entities

$3513 \quad 3521 \quad 3324$

-Total Dedicated Resources-

7 Generating Public Entities

3513

35213324

\begin{tabular}{lllllllllllll}
24 & 3291 & 3377 & 3462 & 3331 & 3211 & 3112 & 2570 & 2678 & 1790 & 2656 & 3262 & 3080 \\
\hline
\end{tabular}

9 Investor-Owned Entities

4128

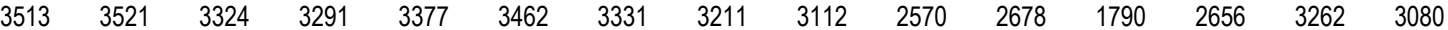

$1160211001 \quad 102$


Table A-26: Other Dedicated Resources

PNW Loads and Resources Study

2012 - 2013 Operating Year

1937 Water Year

Average Energy in Megawatts

2003 White Book

-Generating Public Entities-

1 Generating Public Enities

2 Total Generating Public Entities

Aug1 Aug16 Sep Oct Nov Dec Jan Feb Mar Apr1 Apr16 May Jun Jul Avg

-Non-Generating Public Entities-

3 Small \& Non Generating Public Enities

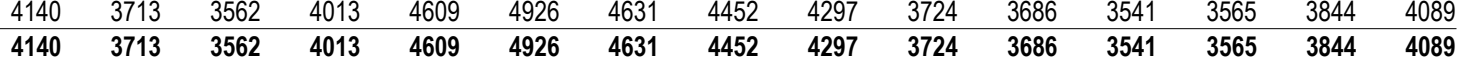

4 Total Non-Generating Public Entities

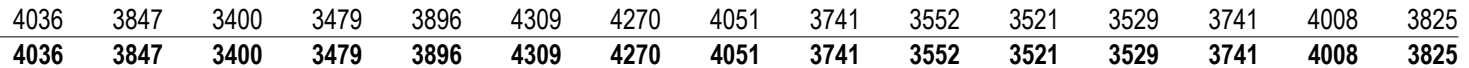

-Investor-Owned Entities-

5 Investor-Owned Enities

6 Total Investor-owned Entities

$\begin{array}{llll}3307 & 3355 & 3263 & 3193\end{array}$

-Total Dedicated Resources-

7 Generating Public Entities

8 Non Generating Entities

3307

$3355 \quad 32$

9 Investor-Owned Entities

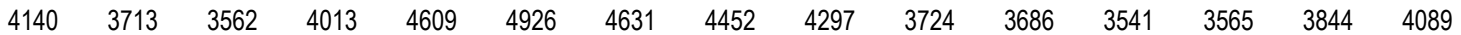

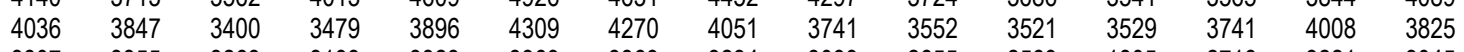

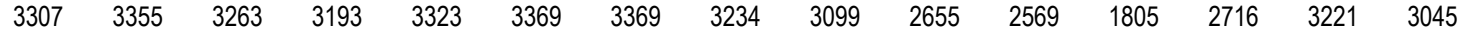

$\begin{array}{llllll}11482 & 10914 & 10226 & 10685 & 11829 & 12604\end{array}$

$\begin{array}{rr}12270 & 11737\end{array}$

1137

99319776

$8875 \quad 10022$

$073 \quad 10959$


Table A-26: Other Dedicated Resources

PNW Loads and Resources Study

2013 - 2014 Operating Year

1937 Water Year

Average Energy in Megawatts

2003 White Book

-Generating Public Entities-

1 Generating Public Enities

Aug1 Aug16 Sep Oct Nov Dec Jan Feb Mar Apr1 Apr16 May Jun Jul Avg

2 Total Generating Public Entities

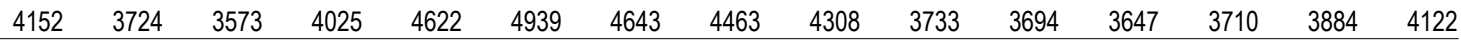

-Non-Generating Public Entities-

3 Small \& Non Generating Public Enities

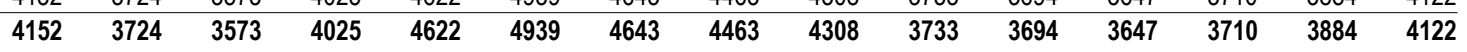

4 Total Non-Generating Public Entities

$\begin{array}{lllllllllllllll}4072 & 3882 & 3434 & 3513 & 3935 & 4349 & 4308 & 4087 & 3775 & 3583 & 3552 & 3617 & 3835 & 4040 & 3870 \\ \mathbf{4 0 7 2} & \mathbf{3 8 8 2} & \mathbf{3 4 3 4} & \mathbf{3 5 1 3} & \mathbf{3 9 3 5} & \mathbf{4 3 4 9} & \mathbf{4 3 0 8} & \mathbf{4 0 8 7} & \mathbf{3 7 7 5} & \mathbf{3 5 8 3} & \mathbf{3 5 5 2} & \mathbf{3 6 1 7} & \mathbf{3 8 3 5} & \mathbf{4 0 4 0} & \mathbf{3 8 7 0}\end{array}$

-Investor-Owned Entities-

5 Investor-Owned Enitio

6 Total Investor-owned Entities

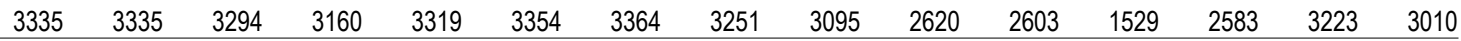

-Total Dedicated Resources-

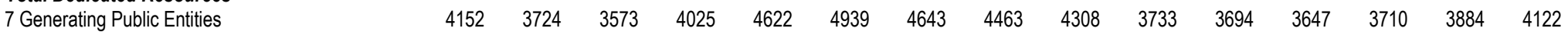

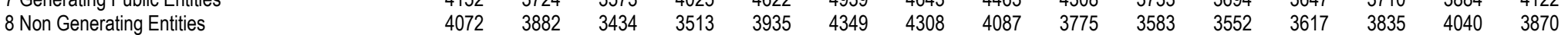

\begin{tabular}{|c|}
\hline 9 Investor-Owned Entities \\
\hline
\end{tabular}

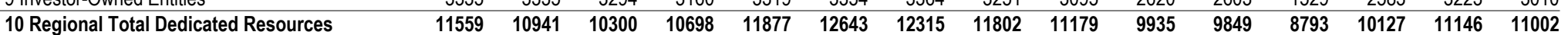


BonnevillePowerAdministration

PO Box 3621 Portland, O regon 97208-3621

DOE/BP-3560 JULY 2004100

(ㄱ) 